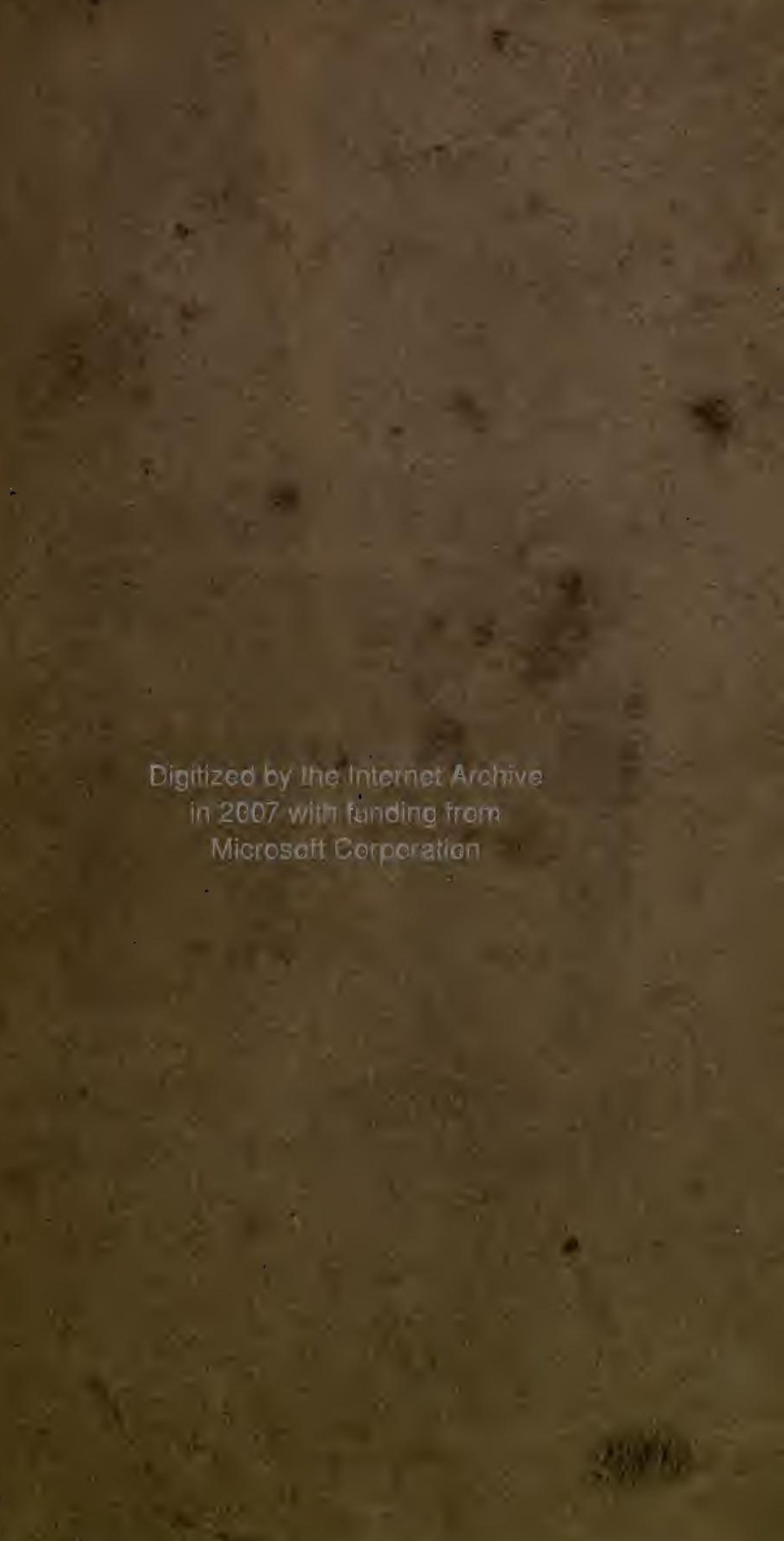

hitto//www archive.oro/details/demonstrationsofocellirich 
The anterior branch $\left({ }^{3}\right)$ passes under the transverse muscle, and accompanies the other to the scrotum. Muscular off'sets are furnished by it to the levator ani and the other superficial muscles.

The superficial perinæal branches communicate with one another, and the posterior is joined by the inferior pudendal nerve. At the scrotum they are distributed by long slender filaments, which reach as far as the under surface of the penis. In the female these nerves supply the labia pudendi.

Other muscular branches of the pudic will be afterwards examined (p. 399).

'The inferior pudendal nerve $\left({ }^{4}\right)$ is a branch of the small sciatic. It pierces the fascia lata about one inch in front of the ischial tuberosity, and enters beneath the superficial fisscia of the perinæum, to end in the outer and fore parts of the scrotum. Communications take place between this nerve, the inferior hamorrhoidal, and the posterior of the two superficial perinæal branches. In the female the inferior pudendal nerve is distributed to the labium.

Dissection. For the display of the muscles, the fatty layer, as well as the vessels and nerves of the left side, must be taken away from the anterior half of the perinæal space. Afterwards a thin subjacent aponeurotic layer is to be removed from the muscles. Along the middle line lies the ejaculator urinæ; and in cleaning it the student is to follow two fasciculi of fibres from it on the same side-one in front, the other behind. On the outer part of the space is the erector penis. And behind, passing obliquely between the other two, is the transverse muscle.

The student should seek, on the right side, the branches of the two superficial perinæal nerves to the underlying muscles; and beneath the transversalis, an offset of the perinaal branch which supplies the deep muscles and the urethra.

Muscles (fig. 130). Superficial to the triangular ligament in the anterior half of the perinæal space, are three muscles, viz., the erector penis, the ejaculator urinæ, and the transversalis perinaci. Other muscles of the urethra are contained between the layers of the triangular ligament, and - will be subsequently seen.

Central point of the perinaum. Between the urethra and the rectum is a white fibrous spot, to which this term has been applied. It occupies the middle line, half an inch in front of the anus. In it the muscles acting on the rectum and the urethra are united; and it serves as a common point of support to the space.

'The ERECTOR PENis (fig. 130, ${ }^{\text {B }}$ ) is the most external of the three muscles, and is narrower at each end than in the middle. It covers the crus penis; and its fibres arise from the ischial tuberosity farther back than the attachment of the penis, and from the bone on each side of the crus. Superiorly the muscle is inserted into the inner and outer surfaces of the crus penis. It rests on the root of the penis and the bone.

Action. The muscle compresses the crus penis against the subjacent bone, and retards the escape of the blood from that organ by the veins : in that way it will contribute to the continuance of distension.

The EJACUlator URIN $x$ muscle (fig. 130, ${ }^{\wedge}$ ) lies on the urethra. The muscles of opposite sides unite by a median tendon along the middle line and in the central point of the perinaum (origin). The fibres are directed outwards, curving around the convexity of the urethra, and give rise to a thin muscle, which has the following insertion:-The most posterior fibres 
are lost on the front of the triangular ligament. The anterior fibres, which are the longest and best marked, are inserted into the penis on its outer aspect before the erector ; and, according to Kobelt, ${ }^{1}$ they send a tendinous expansion over the dorsal vessels of the penis. Whilst the middle or intervening fibres turn round the urethra, surrounding it for two inches, and join its fellow by a tendon.

The ejaculator muscle covers the bulb and the urethra for three inches in front of the triangular ligament. ${ }^{2}$ If the muscle be cut through on the right side, and turned off the urethra, the junction with its fellow above that tube will be apparent.

Action. The two halves, acting as one muscle, can diminish the urethra, and eject forcibly its contents. During the flow of fluid in micturition the fibres are relaxed, but they come into use at the end of the process, when the passage has to be cleared. The action is involuntary in the emission of the semen.

The Transversals PERINex (fig. $130,{ }^{\circ}$ ) is a small thin muscle, which lies across the perinæum opposite the base of the triangular ligament. Arising from the inner surface of the pubic arch near the ischial tuberosity, it is inserted into the central point of the perinæum with the muscle of the opposite side, and with the sphineter ani and the ejaculator urinæ. Behind this muscle the superficial fascia bends down to join the triangular ligament.

Sometimes there is a second small fleshy slip, anterior to the transversalis, which has been named transversalis alter; this throws itself into the ejaculator muscle.

Action. From the direction of the fibres the muscle will draw backwards the central point of the perinæum, and help to fix it, preparatory to the contraction of the ejaculator.

The three muscles above described, when separated from each other by the dissection, limit a triangular space, of which the ejaculator urinæ forms the inner boundary, the erector penis the outer side, and the transversalis perinæi muscle the base. In the area of this interval is the triangular ligament of the urethra, with the superficial perinæal vessels and nerves. Should the knife enter the posterior part of this space during the deeper incisions in the lateral operation of lithotomy, it will divide the transverse muscle and artery, and probably the superficial perinaal vessels and nerves.

Dissection (fig. 131). For the display of the triangular ligament, the muscles and the crus penis, which are superficial to it, are to be detached in the following way:-On the left side the ejaculator urinæ is to be removed completely from the front of the ligament, and the erector muscle from the crus of the penis. Next, the crus penis is to be detached from the bone; but this must be done with care so as not to cut the triangular ligament, nor to injure, near the pubes, the terminal branches of the pudic artery and nerve to the penis.

On the right side the dissector should trace out beneath the transversalis

1 Die Männlichen und Weiblichen Wollust-Organe, von G. L. Kobelt, 1844.

2 Some of the deeper fibres which immediately surround the bulb, have been described as a separate stratum by Kobelt. These are separated from the superficial layer by thin areolar tissue, and join the corresponding part of the other muscle by a small tendon above the urethra. The name compressor hemispherium bulbi has been proposed for it by that anatomist. 

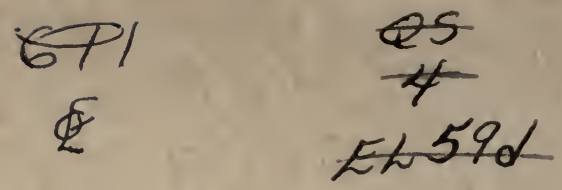

UNIVERSITY OF CALIFORNIA

MEDICAL CENTER LIBRARY SAN FRANCISCO

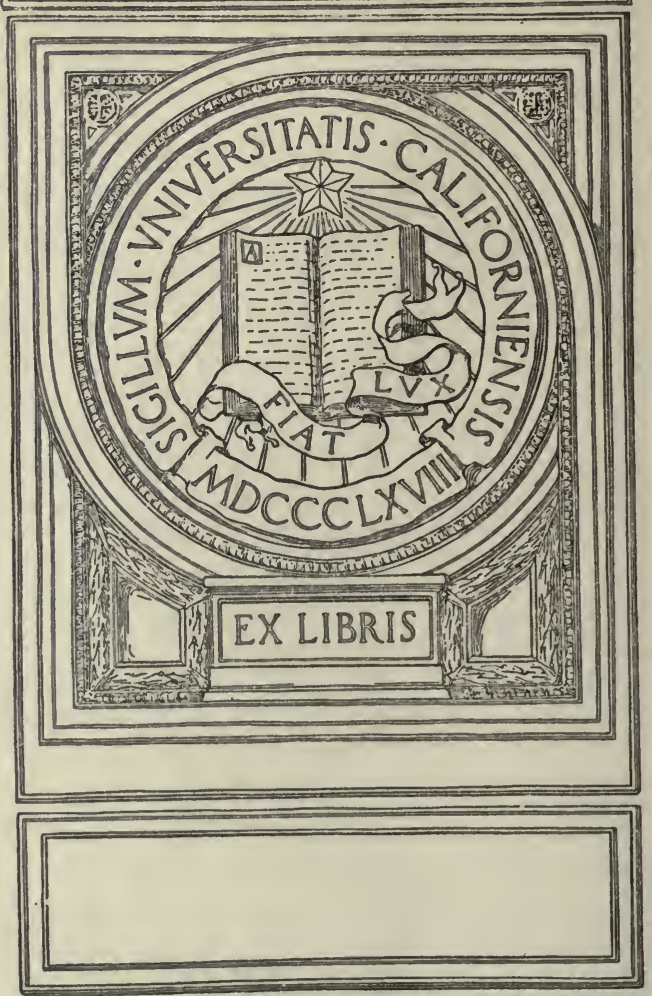


Striasm 



\section{DEIIONSTRATIONS}

\section{or \\ A N A T O M Y;}

BEING A

GUIDE TO THE KNOWLEDGE OF THE HUMAN BODY

BY

\section{DISSECTION.}

BY

GEORGE VINER/ELLIS,

EMERITUS PROFESSOR OF ANATOMY IN UNIVERSITY COLLEGR, LONDON.

FROM THE

EIGHTH AND REVISED ENGLISH EDITION.

ILLUSTRATED BY TWO HUNDRED AND FORTY-NINE ENGRAVINGS ON WOOD.
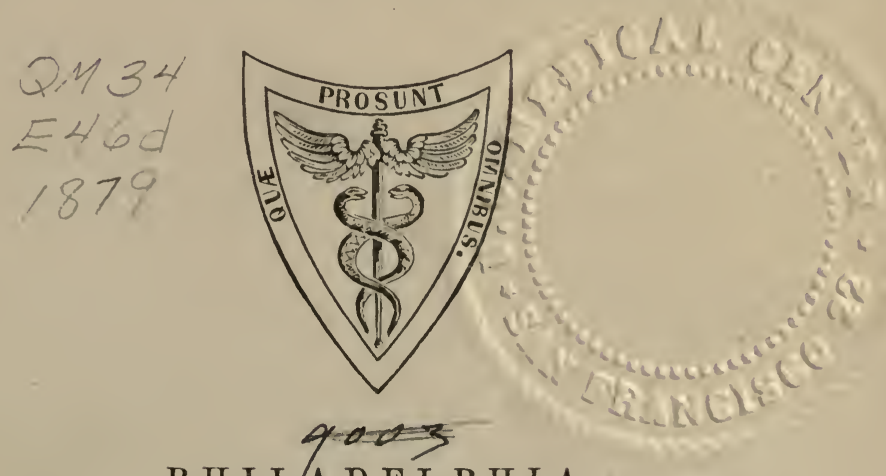

P H I L/A D E L P H I A:

H E N R Y C. L E A. 1879 . 


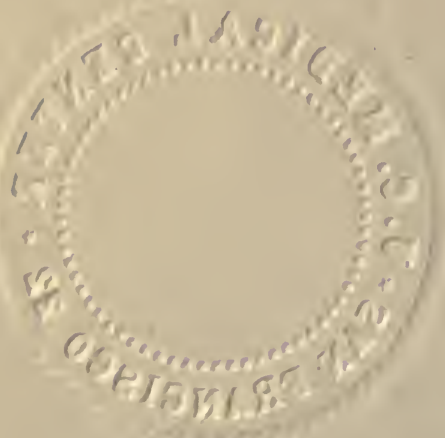

C OLLINS, PRINTER. 


\section{P R E F A C E.}

TIIE plan of this work is designed to teach the Anatomy of the Human Body by dissection in successive stages after the following manner:-

In the dissection of a Part the attention of the Student is directed first to the superficial prominences of bone and muscle, and to the hollows that point out the situation of the subjacent vessels. Next the cutaneous structures, and the different layers of muscles with their appertaining vessels and nerves are examined in succession, so that the several objects between the surface of the Body and the bones may be observed in much the same order as they would be met with in a Surgical operation. And, lastly, the joints and ligaments receive due notice.

In the dissection also of the viscera and the organs of the senses the manner in which the composition of each may be shown, is fully indicated for the guidance of the Student.

The Anatomical description of the Part under examination is arranged in conformity with the dissection in regions, and each muscle, bloodvessel, nerve, or other structure, is described only to such an extent as it may be laid bare.

Since the publication of the last edition great changes have been made in textural Anatomy; and the chief of these, for which I am indebted to Quain's Anatomy, I have introduced into my account of the microscopic structure of the different organs.

In this edition, as in the preceding ones, I have endeavored to make the work more complete by the correction of inaccuracies, and to render it more efficient as a guide to practical work.

G. V. ELLIS. 



\section{CONTENTS.}

\section{CHAPTER I.}

DISSECTION OF THE HEAD AND NECK.

Section 1. External Parts of the Head

2. Internal Parts of the Head

3. The Face

4. The Orbit

5. The Neck, right side

Posterior triangular space

Front of the Neck

Anterior triangular space

6. Pterygo-maxillary Region .

7. Submaxillary Region

8. Superior Maxillary Nerve and Vessels . . . . 103

9. Deep Vessels and Nerves of the Neck . . . . 105

10. Left side of the Neck

11. The Pharynx

12. The Mouth .

13. Cavity of the Nose

14. Spheno-palatine and Otic Ganglia, Facial and Nasal Nerves, and Internal Maxillary Artery $\quad$. $\quad . \quad 0 \quad$ • 138

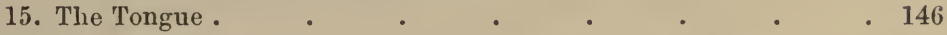

16. The Larynx . $\quad . \quad$. $\quad . \quad$. $\quad$. $\quad . \quad 151$

17. Hyoid Bone, Cartilages and Ligaments of the Larnyx, Structure of the Trachea . . . . . . . . . 158

18. Prevertebral Muscles and the Vertebral Vessels . _ . 163

19. Ligaments of the first two Cervical Vertebræ, and of the Clavicle 166

\section{CHAPTER II.}

DISSECTION OF THE BRAIN.

Section 1. Membranes and Vessels $\quad . \quad \ldots \quad$. $\quad . \quad \therefore \quad$. 172

2. Origin of the Cranial Nerves $\quad$. $\quad$. $\quad$. $\quad$. $\quad 177$

3. Medulla Oblongata and Pons Varolii . . . . 182 
Section 4. The Cerebrum, or Great Brain

The under surface, or the Base

The npper surface and lobes.

Convolutions

The Interior

Ventricles of the Brain

Floor of the lateral Ventricle .

Central Parts of the Cerebrum

Structure of the Cerebrum

5. The Cerebellum, or little Brain

Surfaces and Lobes

190

193

195

199

- 200

- 203

205

- 209

- 211

- 212

Structure of the Mass .

Fourth Ventricle

\section{CHAPTER III.}

\section{DISSECTION OF THE UPPER LIMB.}

Section 1. The Wall of the Thorax

. 224

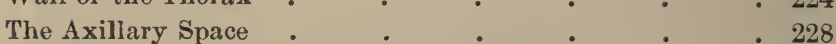

2. Scapular Muscles, Vessels, Nerves, and Ligaments $\quad$ • 240

3. The Front of the Arm . . . . . . . . 248

Back of the Arm . . . . . . . 257

4. The Front of the Forearm . . . . . . 260

5. The Palm of the Hand . . . . . . 272

6. The Back of the Forearm . . . . . . . 282

7. Ligaments of the Shoulder, Elbow, Wrist, and Hand · . 290

\section{CHAPTER IV.}

\section{DISSECTION OF THE THORAX.}

Section 1. Cavity of the Thorax

$\bullet \quad \cdot \quad \cdot \quad \cdot \quad \cdot 305$

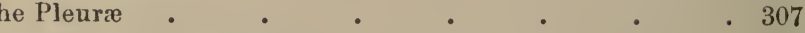

Connections of the Lungs . $\quad$. $\quad$. $\quad 308$

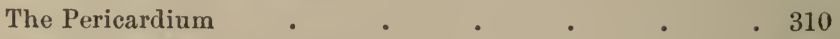

The Heart and its large Vessels o . • . 312

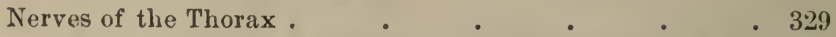

The Trachea and the Characters and Structure of the Lung 333

-Parts in Front of the Spine, and the Cord of the Sympathetic 336

Parieties of the Thorax . . . . . . 342

2. Ligaments of the Trunk . . . . . . 343

Articulation of the Ribs to the Vertebræ . . . 343

Articulation of the Ribs to the Sternum a . . 346

Articulations of the Sternum . $\quad$. $\quad$. $\quad .346$

Articulations of the Vertebra $\quad$. $\quad$. $\quad$. $\quad 346$ 


\section{CHAPTER V.}

DISSECTION OF THE BACK.

First Layer of Muscles .

PAGE

Second Layer of Muscles

Third Layer of Muscles

Fourth Layer of Muscles with Vessels and Nerves

Fifth Muscular Layer, and the Sacral Nerves

\section{CHAPTER VI.}

\section{DISSECTION OF TIIE SPINAL CORD.}

Membranes of the Cord

Roots of the Spinal Nerve

Vessels of the Cord

Form and Divisions of the Cord

Structure of the Cord, and the deep Origin of the Nerves

Intraspinal Vessels

\section{CHAPTER VII.}

\section{DISSECTION OF THE PERINAUM.}

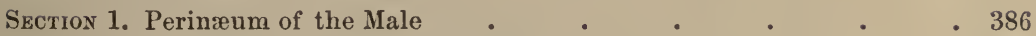

Posterior Half of the Space . $\quad$ - . . $\quad 387$

Anterior Half of the Space $\quad$ - $\quad$. $\quad$ • $\quad$. $\quad 391$

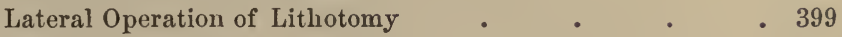

2. Perinæum of the Female . . . . . . 400

\section{CHAPTER VIII.}

DISSECTION OF TIE ABDOMÉN.

Section 1. Wall of the Abdomen

2. Hernia of the Abdomen

3. Cavity of the Abdomen . . . . . . . 430

Connections of the Viscera $\quad$. $\quad$. $\quad . \quad$. $\quad .431$

Peritoneum and its Folds . . . . . 435

Mesenteric Vessels, and Part of the Sympathetic Nerve 439

Comnections of the Aorta and Vena Cava . . . . 444

Connections of the Duodenum and Pancreas . . . 445

Coliac Axis and Vena Portæ . . . . . . 446

Sympathetic and Vagus Nerves . . . . 450

4. Anatomy of the Abdominal Viscera . . . 452

The Stomach . . . . . . . 452

The Small Intestine $\quad . \quad$. $\quad . \quad$. $\quad . \quad .456$

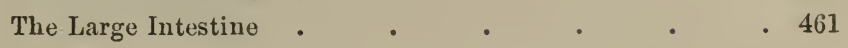


Section 4. Anatomy of the Abdominal Viscera-continued.

PAGE

The Pancreas . • • • • • • •

The Spleen $\quad . \quad$. $\quad . \quad$.

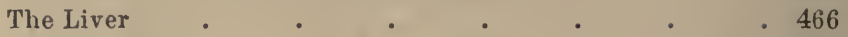

The Gall Bladder . $\quad$. $\quad$. $\quad$. $\quad$. 472

The Kidney and the Ureter . . . . . . . 473

The Suprarenal Body . $\quad$ • $\quad . \quad$. $\quad$. $\quad .478$

The Testis and the Vas Deferens $\quad$. $\quad$ • $\quad$. $\quad .479$

5. Diaphragm with Aorta and Vena Cava . . . . . 484

Deep Muscles of the Abdomen $\quad$ • $\quad$ • $\quad$. 492

6. Lumbar Plexus and the Cord of the Sympathetic Nerve . • 495

\section{DISSECTION OF THE PELVIS.}

Sectiox 1. Fascia of the Cavity and the Muscles of the Pelvic Outlet - 499

2. Connections of the Viscera in the Male . . . 503

3. Connections of the Viscera in the Female . . . . 509

4. Vessels and Nerves of the Pelvis . . . . . 513

5. Anatomy of the Viscera of the Male . . . . $\quad$. 520

The Prostate (*land and the Seminal Vesicles . . 521

The Urinary Bladder . . . . . . 524

The Urethra and the Penis . . . . . 526

The Rectum . . . . . . . $\quad$. 532

6. Anatomy of the Viscera of the Female . . . . 533

Genital Organs . $\quad$. $\quad$. $\quad$. $\quad$. 533

The Vagina . $\quad . \quad$. $\quad . \quad$. 535

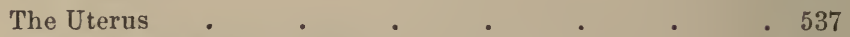

Ovaries and Fallopian Tubes . $\quad . \quad$. $\quad . \quad$. 539

Bladder, Urethra, and Rectum _ . . . . 541

7. Internal Muscles of the Pelvis . . . . . 542

Articulations of the Pelvis . $\quad$. $\quad . \quad$. $\quad .543$

\section{CHAPTER IX.}

\section{DISSECTION OF THE LOWER LIMB.}

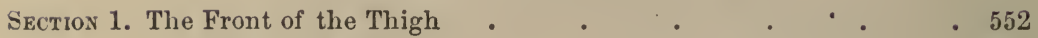

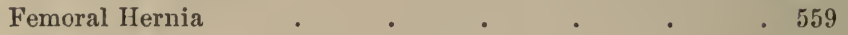

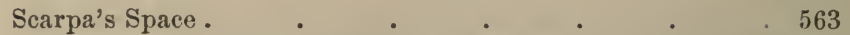

Deep Muscles, Vessels, and Nerves on the Front of the Thigh 565

Deep parts on the inner Side of the Thigh . . . 574

2. The Buttock, or the Gluteal Region _ . _ . 581

3. The Back of the Thigh . . . . . . 592

The Popliteal Space . . . . . . . 593

Hamstring Muscles and Vessels $\quad$. $\quad . \quad$. 597

The Hip-Joint . . . . . . . 600

4. The Back of the Leg $\quad$ - $\quad$. $\quad$. $\quad$. $\quad$. 605

5. The Sole of the Foot . . . . . . . . 614

6. The Front of the Leg and the Dorsum of the Foot a 626

7. Ligaments of the Knee, Ankle, and Foot . • . . 634 


\section{CHAPTER X. \\ DISSECTION OF THE EYE.}

Sclerotic Coat and Cornea

PAGE

Choroid Coat and Ciliary Processes

Ciliary Muscle.

The Iris

Ciliary Vessels and Nerves

Chamber of the Aqueous Humor

The Retina and Jacob's Membrane

- 659

- 660

- 661

- 663

Vitreous Body, and Hyaloid Membrane, with the Suspensory Ligament and the Canal of Petit.

Crystalline Lens and its Capsule

\section{CHAP'TER XI.}

\section{DISSECTION OF TIIE EAR.}

The Auditory Canal

The Tympanum, with its Vessels and Nerves .

Ossicles of the Tympanum and,their Muscles and Ligaments . 674

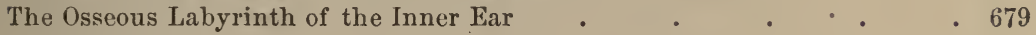

Vestibule .

Semicircular Canals

Cochlea, its Septum and Passages

Organ of Corti

The Membranous Sacs, or Labyrinth.

Utricle

Saccule

Bloodvessels of the Labyrinth .

Nerves of the Cochlea and Membranous Sacs . 



\section{LIST OF ILLUSTRATIONS.}

FIG.

1. Extrinsic muscles of the ear $\quad$ - $\quad$ - $\quad$. $\quad$. 19

2. Cutaneous nerves and arteries of the scalp a $\quad$ - $\quad$ - 22

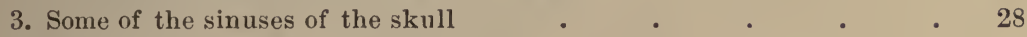

4. Cranial nerves in the base of the skull . $\quad . \quad \ldots \quad . \quad$. 31

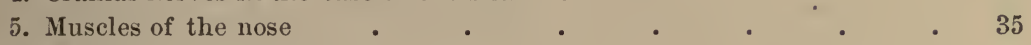

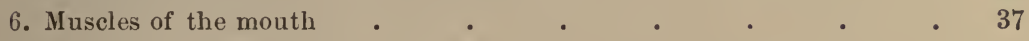

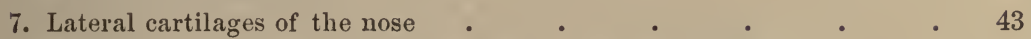

8. Muscles on the outer and inner surface of the ear cartilage . $\quad 46$

9. Cutaneous branches of the fifth nerve in the face . . . 49

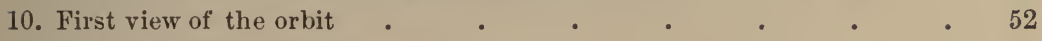

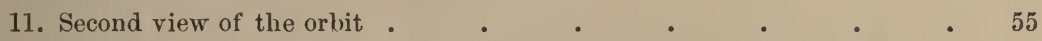

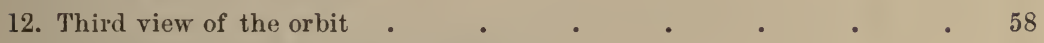

13. The eyelids and lachrymal apparatus $\quad$ • $\quad$ • $\quad$ • 60

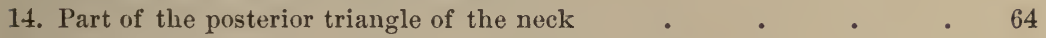

15. View of the anterior triangular space of the neck $\quad . \quad$. $\quad 72$

16. A view of the common carotid and subclavian arteries . . . 74

17. External carotid and its superficial branches . $\quad . \quad$. 84

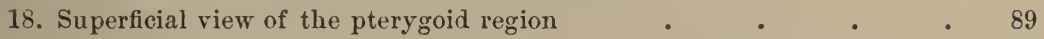

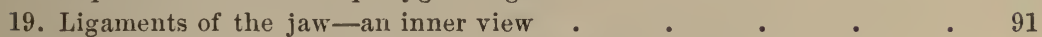

20. A view of the interior of the compound temporo-maxillary joint $\quad$. 91

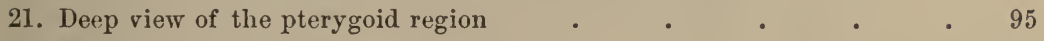

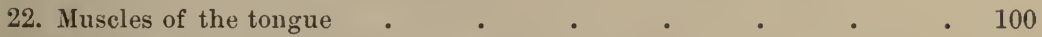

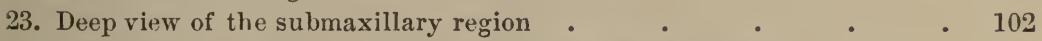

24. Diagram of the upper maxillary nerve and its branches $\quad$ - 105

25. Deep vessels and nerves of the neck . . . . . . 108

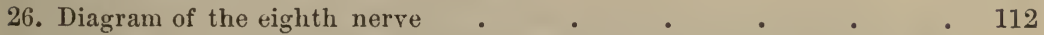

27. Diagram of the ending of the lymph duct and the thoracic duct in the

veins . . . . . . . . . 119

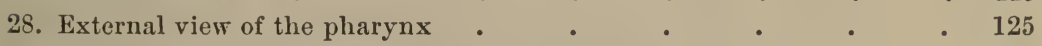

29. Internal view of the pharynx . $\quad . \quad \ldots \quad$. $\quad . \quad 127$

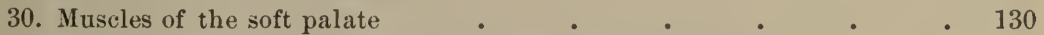

31. Spongy bones and meatuses of the nasal cavity $\quad$. $\quad$ - 135

32. Magnified vertical section of the mucous membrane of the nose - 136

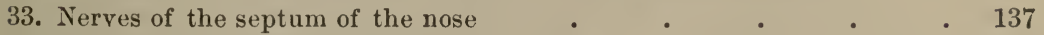

34. Nerves of the nose and palate . . . . . . 140

35. Nerves joining the enlargement of the facial nerve . . . 143

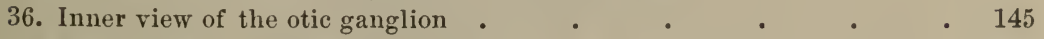

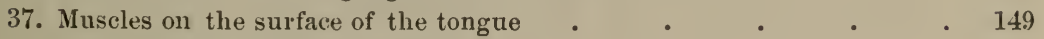

38. Intrinsic muscles of the tongue . $\quad . \quad \ldots \quad$. $\quad . \quad 4 \quad 149$

39. Front view of the larynx . $\quad . \quad$. $\quad . \quad$. $\quad . \quad 152$

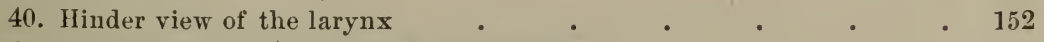

41. View of the internal muscles of the larynx $\quad . \quad \ldots \quad . \quad 154$ 
FIG.

42. Vocal apparatus, on a vertical section of the larynx . . . 156

43. Hyoid bone and the laryngeal cartilages $\quad . \quad$ e $\quad$. $\quad . \quad 160$

44. View of the vocal cords and crico-thyroid ligaments _ _ . 161

45. Deep muscles of the front of the neck and the scaleni muscles . . 164

46. External ligaments in front between the atlas and axis and the occipital bone

47. External ligaments behind between the atlas and axis and the occipital bone

48. Internal ligament between occipital bone and axis

49. Internal ligaments between the occipital bone and the atlas and axis .

50. First vertebra with the odontoid process removed from the socket formed by the bone and the transverse ligament

51. Ligaments of the inner end of the clavicle, and of the cartilage of the second rib

52. Arteries at the base of the brain .

53. Origin of the cranial nerves

54. Anterior view of the medulla oblongata and pons

55. Hinder view of the medulla oblongata .

56. Fibres of the medulla, pons, and crus cerebri .

57. Fibres of the lateral tract and of the olivary body

58. Tranverse section of the medulla oblongata above the middle of the olivary body

59. Under surface of the brain

60. Lobes of the hemisphere, and convolutions and fissures of the outer surface of the brain . $\quad . \quad$. . . 194

61. View of the orbital lobule and the central lobe of the brain . $\quad 196$

62. Convolutions and fissures on the inner face of the hemisphere of the brain

63. View of the lateral rentricles of the brain

64. Second view of the dissection of the brain

65. Connection posteriorly between the cerebrum, the medulla oblongata, and cerebellum .

66. Under part of the cerebellum, seen from behind

67. View from behind of the under surface of the cerebellum _ . 214

68. View of the third and fourth ventricles of the brain . . . 216

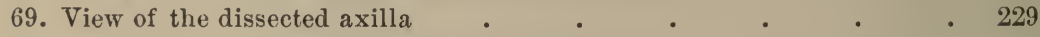

70. Second view of the dissection of the thorax $\quad$. $\quad . \quad$. 233

71. Diagram of the serratus magnus muscle . $\quad$ - $\quad$ • $\quad$. 237

72. View from behind of the attachments of the triangularis sterni muscle 239

73. View of the subscapularis and the surrounding muscles . . 241

74. View of the muscles of the dorsum of the scapula, and of the circumflex vessels and nerve . $\quad . \quad$. . . . . 243

75. Ligaments of the clavicle and scapula, and of the shoulder joint $\quad 246$

76. Cutaneous veins and nerves at the bend of the elbow . . $\quad 250$

77. Axillary and brachial arteries and their branches . . . 253

78. Muscles and deep vessels and nerves of the arm $\quad . \quad$. $\quad 255$

79. Dissection of the dorsal scapular vessels and nerve, and of the triceps muscle of the arm . . . . . . . . .

80. Superficial view of the forearin . . . . . . . 264

81. Dissection of the deep layer of muscles of the forearm, and of the vessels and nerves between the two layers of muscles of the forearm 
FIG.

PAGE

82. The extensor tendon of the finger with its accessory muscles and the sheath of the flexor tendons

83. Dissection of the superficial vessels and nerves of the palm of the hand with some of the superficial muscles.

84. Deep dissection of the palm of the hand

85. Three palmar interosseous muscles . • . . . . 281

86. Four dorsal interosseous museles . . . .

87. Superficial layer of museles on the back of the forearm, with some vessels

88. Dissection of the deep layer of muscles, and the vessels and nerve on the back of the forearm .

89. View of the interior of the shoulder-joint

90. The ligaments of the elbow joint, and the shaft of the radius and ulna

91. View of the orbicular ligament of the radius . . .

92. Front view of the articulations of the wrist joint, and carpal and metacarpal bones. . . . . . . .

93. The wrist joint opened to show the arch formed by the bones of the forearm with the uniting fibro-eartilage

94. Articulations of the carpal bones

95. Posterior ligaments of the wrist, and carpal and metacarpal bones

96. Union of metacarpal bone and first phalanx . . .

97. Diagram to slow the difference in the anterior border of the right and left lung

98. Diagram showing the position of the heart to the ribs and sternum .

99. Back of the heart with the coronary sinus and its veins

100. Diagram of the two cavities of the right side of the heart .

101. Diagram of the two eavities of the left side of the heart . .

102. Muscular fibres of the aurieles . . . . . .

103. A diagram of the arrangement of the fibres in layers in the left ventriele . . . . . . .

104. The formation of the septum ventrieulorum by the fibres of both ventricles

105. Arch of the aorta and its great vessels . $\quad . \quad$. . .

106. View of the thoracic duct, and the intercostal veins . . .

107. Scheme to illustrate the connection between the spinal and sympathetic nerves.

108. Ligaments of the ribs and vertebræ $\quad . \quad \cdots \quad . \quad$.

109. Anterior common ligament of the bodies of the vertebra _ . $\quad 347$

$110 \mathrm{~A}$. View of the posterior eommon ligament of the vertebre of the neck 347

110 в. View of the posterior common ligament of the vertebræ of the loin - 347

111. Intervertebral substance in the lumbar region . . . . . 348

112. Vertieal section of the intervertebral substance . . . . 348

113. A horizontal cut through an intervertebral fibro-cartilage . $\quad 349$

114. An inner view of the neural arehes of the vertebræ, with their inter-

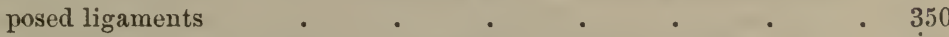

115. Ligaments of the proeesses of the vertebræ, and of the ribs . . . 350

116. Museles of the back . . . . . . . . . 355

117. Part of the third layer of the back-muscles . . . . $\quad$. 360

118. Dissection of the muscles underneath the splenius _ . . . 364

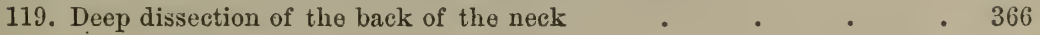


FIG.

120. Dissection of sacral nerves

121. Lower end of the dura mater, with its central and lateral processes

122. View of the membranes of the spinal cord

123. Ronts of the spinal nerves

124. Membranes of the spinal cord laid open

125. A section of the spinal cord, to show its composition, and its divisions

126. The gray substance in the interior of the spinal cord .

127. Intraspinal arteries in the loins .

128. Intraspinal veins in the loins

129. A view of the dissection of the rectal half of the perinrum

130. Superficial dissection of the anterior half of the perinæum

131. Deep dissection of the perinæum

132. The symphysis pubis seen from behind .

133. The female perinæum . . .

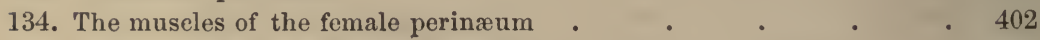

135. Dissection of the first lateral muscle in the wall of the belly . . 409

136. Internal oblique muscle of the abdominal wall

137. The lower part of the internal oblique with the Cremaster muscle and the testicle

138. Dissection of the third lateral muscle in the wall of the belly

139. Rectus muscle of the abdomen .

140. Disseetion for inguinal hernia .

141. View of the parts concerned in femoral hernia . $\quad$ • $\quad$. 428

142. Connections of the liver, stomach, spleen, and large intestine . 432

143. Superior mesenteric artery and its branches . . . . 440

144. The lower mesenteric artery, and the aorta $\quad$ • c c e 442

145. View of the cœliae axis, and of the viscera to which its branches are supplied

146. Vena portæ and the veins joining it . . . . . . 449

147. Diagram representing the arrangement of the muscular fibres of the stomach .

148. Alveolar depressions of the mueous membrane of the stomach

149. Enlarged representation of the tubes of the stomach

150. The duodenum opened showing the valvulæ conniventes, and the opening of the bile duet

151A. Vessels of the villi in the mouse, injected by Gerlach . . 458

151s Lacteals and plexus of vessels in two villi, injected by Teichman . 458

152A. Pateh of Peyer's glands four times enlarged.

152в. Magnified representation of an injection of the vessels surrounding and penetrating the follicles in a patch of Peyer in the rabbit . 459

153A. A piece of mueous membrane enlarged

153в. A piece of mucous membrane enlarged

154. Magnified view of the mucous membrane of the duodenum

155. Interior of the cxeum dried and laid open

$156 \mathrm{~A}$. Enlarged view of "a solitary gland" .

156 в. Enlarged view of "a solitary gland" .

157. A drawing of the trabecular strueture of the spleen of the ox a 466

158. Under surface of the liver $\quad$. $\quad . \quad$. $\quad . \quad 4468$

159. A magnified representation of the hepatic cells . . . . 470

$160 \mathrm{~A}$. Lobules of the liver, magnified 
FIG.

PAGE

$160 \mathrm{~B}$. Lobules of the liver, magnified

161. Vessels in a portal canal, and the lobules of the liver . . . 471

162. Gall-bladder and its duct

163. Section through the kidney

164. Plan of the arrangement of the uriniferal tubes

473

474

165. Plan of contorted urine tubes ending in Malpighian corpuscles

$166 \mathrm{~A}$. Plan of the vessels connected with the urine tubes

166в. Plan of the vessels connected with the urine tubes

167. Vertical section of the suprarenal body .

168. The testis with the tunica vaginalis laid open .

169. Vertical and horizontal sections of the testis

170. Under surface of the diaphragm .

171. Deep view of the muscles, vessels, and nerves of the abdominal cavity

172. Dissection of the lumbar plexus and its branches

173. Side view of the muscles in the outlet of the pelvis . $\quad$. $\quad$ - 501

174. Side view of the dissected male pelvis .

175. Side view of the female pelvis .

176. Dissection of the internal iliac artery .

177. Dissection of the sacral nerves and plexus

476

477

477

479

480

482

485

488

496

501

511

514

178. View of the under part of the bladder with the vesiculæ seminales and vasa deferentia

179. Muscular fibres of the bladder, prostate, and urethra .

180. Section through the bladder, prostate, and urethra . . . 527

181. View of the lower part of the bladder and of the urethra laid open .

182. View of the fibres of the case of the corpus cavernosum .

183. Pectiniform septum of the penis . . . . .

184. Magnified view of the trabecular structure and arteries of the penis .

185. Representation of the clitoris . • • • .

186. Venous plexuses of the genital organs and opening of the vagina .

187. Interior of the uterus, with a posterior view of the broad ligament and the uterine appendages . $\quad . \quad$. $\quad . \quad$. 538

188. Ovary during the child-bearing period laid open _ . $\quad$. 540

189. Irregular piece of cartilage in the sacro-iliac articulation $\quad$. $\quad 545$

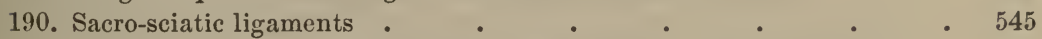

191. Ligaments of the symphysis pubis, thyroid hole, and acetabulum . 546

192. Cutaneous nerves on the front of the thigh _ . $\quad$ • $\quad$ - 556

193. Dissection of the superficial parts of the thigh . $\quad$. 558

194. Dissection of the crural sheath . . . . . . . 561

195. Dissection on Scarpa's triangular space $\quad . \quad$. $\quad . \quad 563$

196. Surface view of the front of the thigh, the teguments and fascia being removed . . . . .

197. Deep part of the femoral artery and its branches, with muscles of the

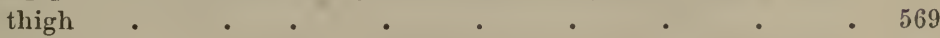

198. Deep dissection of the adductor muscles with their vessels and nerves 575

199. Superficial view of the buttock of the left side . $\quad$ - $\quad 582$

200. Second view of the dissection of the buttock . $\quad$. $\quad$. 585

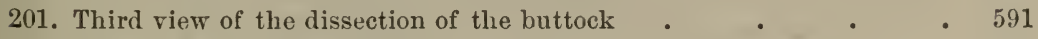

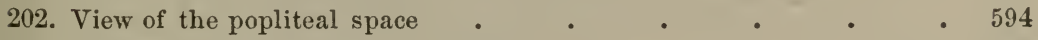

203. Dissection of the back of the thigh . $\quad$. $\quad . \quad$. 598

204. Fore part of the capsule of the hip joint $\quad$ - $\quad$ • $\quad$ - 600 
FIG.

205. Hinder part of the hip-joint capsule

206. Hip joint opened

207. First view of the back of the leg

208. Second view of the back of the leg

209. Deep dissection of the back of the leg .

210. First view of the sole of the foot

211. Second view of the sole of the foot

212. Third view of the sole of the foot

213. Fourth view of the sole of the foot

214. Cutaneous nerves of the front of the leg and foot

215. Anterior tibial vessel and muscles

216. External ligament of the knee-joint

217. Internal ligament of the knee-joint

218. The capsule of the knee-joint cut across, and the patella thrown down to show the named folds of the synovial sac.

219. Interarticular ligaments of the knee-joint

220. View of the head of the tibia with the fibro-cartilages attached

221. Internal lateral ligament of the ankle .

222. External lateral ligament of the ankle .

223. View of the dorsal ligaments of the tarsus

224. Plantar ligaments of the foot

225. View of the inferior ligaments of the tarsal bones

226. Dorsal ligaments uniting the tarsus to the metatarsus, and the metatarsal bones to each other behind

227. Diagram of a horizontal section of the eyeball

228. Vertical section of the cornea

229. Inner view of the front of the choroid coat

230. Pigment cells of the eyeball

231. View of the front of the choroidal coat and iris

232. Distribution of the nerves and vessels of the iris

233. Objects on the inner surface of the retina

234. Magnified vertical section of the retina

235. Enlarged representation of the parts of the eyeball on one side opposite the lens

236. A representation of the laminæ in hardened lens

237. Views of the lens fibres after Henle

242. Plan of the ossicles in position in the tympanum

244. View of the vestibule obtained by cutting away the outer boundary of a fotus

245. Representation of the semicircular canals enlarged 


\title{
DEMONSTRATIONS OF ANATOMY.
}

\author{
CHAPTER I. \\ DISSECTION OF THE HEAD AND NECK.
}

\section{SECTION I.}

\section{EXTERNAL PARTS OF THE HEAD.}

Directions. In the dissection of the head and neck, the student should endeavor to learn the parts described in the first fifty-one pages, before the position of the body is changed; but if want of time necessitates an omission of some part, the examination of the facial nerve (p. 47) can be best deferred till a subsequent stage. The orbit on one side, the posterior triangular space on both sides of the neck, and the exterior and the interior of the head, should be examined whilst the body lies in its first position on the Back.

Position. The student begins with the dissection of the scalp and the muscles of the ear. To obtain the best position, raise the head to a suitable height, and turn the face to the right side. On the left side the muscles are to be seen, and on the opposite half the vessels and nerves are to be displayed.

Extrinsic muscles of the Ear. Three muscles attach the ear to the side of the head. Two are above it, one elevating, the other drawing it forwards; and the third, a retrahent muscle, is behind the ear. There are other special or intrinsic muscles of the cartilage of the ear, which will be afterwards described.

Dissection. When the ear has been drawn down by hooks, the position of the upper muscle will be indicated by a slight prominence between it and the head; and the muscular fibres may be laid bare by means of the two following incisions, made no leeper than the skin : One is to be carried upwards on the side of the head, for about three inches, along the cutaneous ridge before mentioned; and the other, about the same length, is to be directed from before backwards close above the ear, so that the two may join at a right angle. On carefully raising the flaps of skin from below upwards, and removing the subjacent tissue, a thin fan-shaped muscular layer will come into view-the more anterior fibres constituting the attrahens, and the posterior the attollens aurem muscle.

On drawing forwards the ear a ridge marks the situation of the posterior muscle. To remove the integuments, let the scalpel be drawn about an inch behind the ear, from the transverse cut above as far as to a level with 
the lobule of the ear, and then forwards below the lobule. After the piece of skin included by those cuts has been reflected towards the ear, the retrahent muscle must be sought beneath the subcutaneous tissue; it consists of rounded bundles of fibres, and is stronger and deeper than the others.

The attranens Aurem (fig. $1,{ }^{16}$ ) is a small fan-shaped muscle, and, arises from the fore part of the aponeurosis of the occipito-frontalis. Its fibres are directed backwards, and are inserted into a projection on the front of the rim of the ear. Beneath it are the superficial temporal vessels and nerve.

The attollens acrem (fig. $1,{ }^{15}$ ) has the same form as the preceding, thongh its fibres are longer and better marked. Arising also from the tendon of the occipito-frontalis, the fibres converge to their insertion into the inner or cranial surface of the pinna of the ear-into an eminence corresponding with a fossa (that of the anti-helix) on the opposite aspect.

The REtrainens aUrem (musculi retrahentes, Alb., fig. $1,{ }^{17}$ ) consists of two or three roundish but separate bundles of fibres, which are stronger than those of the other muscles. The bundles arise from the root of the mastoid process, and pass almost transversely forwards to be inserted by aponeurotic fibres into the lower part of the ear (concha) at its cranial aspect. The posterior auricular artery and nerve are in contact with this muscle.

Action. The three preceding muscles will move the outer ear slightly in the directions indicated by their names: the anterior drawing it upwards and forwards, the middle one upwards, and the posterior backwards.

'The occipito-Frontalis nuscle (fig. $1,{ }^{1}$ ) covers the arch of the skull, and consists of an anterior and a posterior fleshy part, with an intervening tendon.

Dissection. On the same side of the head (the left) the occipito-frontalis is to be dissected. 'To bring this muscle into view, a cut may be made along the middle line of the skull, from the root of the nose to the occipital protuberance; and it may be connected in front with the transverse incision on the side of the head. The flap of skin, thus marked out, is to be raised with the subjacent fat from before back; whilst doing this the dissector will meet first with the anterior fleshy part of the muscle, next with a white shining thin aponeurosis, and lastly with the posterior fleshy belly towards the lateral aspect of the cranium. The aponeurosis of the muscle is easily taken away with the granular fat superficial to it; and if the under surface of the flap of integuments presents a white instead of a yellow appearance, the student may suspect he is removing that aponeurosis.

The anterior or frontal part $\left({ }^{1}\right)$ is a thin muscular layer over the os frontis, which is said to take its origin below. Along the line of the eyebrow the fibres are blended with the following muscles, orbicularis palpebrarum, corrugator supercilii, and pyramidalis nasi; and they are also fixed to the subjacent bone, viz., to the os nasi internally, and to the outer angular process of the frontal bone externally (Theile). From these attachments the fibres are directed upwards to the aponeurosis, and end in it rather below the level of the coronal suture.

The posterior or occipital part $\left({ }^{4}\right)$ is stronger than the anterior; it arises from the outer half or two-thirds of the upper curver line of the occipital bone, and from the mastoif portion of the temporal bone. 'The fibres are about one inch and a half in length, and ascend to the aponeurosis.

The tendon, or epicranial aponeurosis, extends over the upper part of 
the cranium, and is continuous across the middle line with the same structure of the opposite half of the hearl. On the side it gives origin to the auricular muscles; and a thin membrane is here prolonged from it over the fiscia covering the temporal muscle, to be fixed to the side of the head.

Fig. 1.

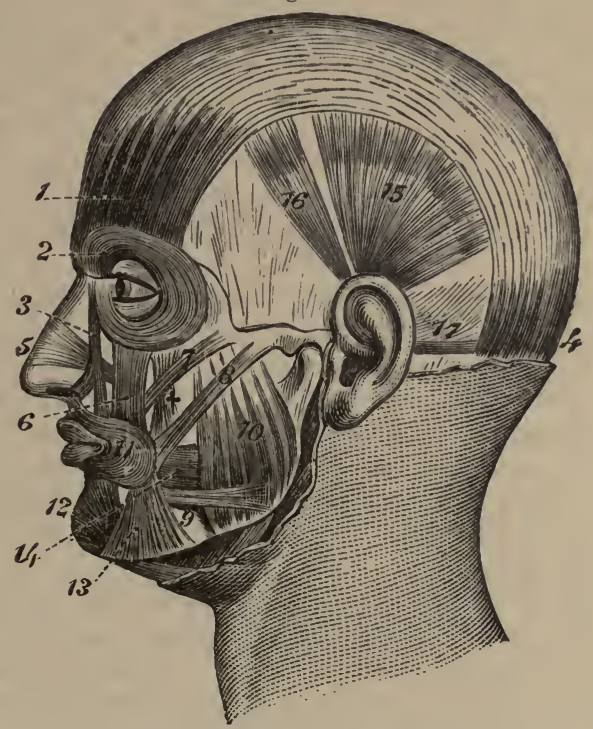

Extrinsic Mescles of the Ear.

15. Attollens aurem.

16. Attrahens aurem.

17. Retrahens aurem, only partly seen.

Posteriorly, the aponeurosis is attached to the superior curved ridge of the ocipital bone between the fleshy parts of the muscles of opposite sides. The aponeurotic expansion is closely united to the skin ; but it is connected to the pericranium only by a loose areolar tissue devoid of fat, so that it moves freely over the skull.

Superficial to the occipito-frontalis are the cutaneous vessels and nerves of the scalp. In front the fleshy fibres of opposite sides are joined above the root of the nose.

Action. When the anterior belly contracts it elevates the eyebrow, making smooth the skin at the root of the nose, and wrinkling transversely that of the forehead; and continuing to contract, it draws forward the scalp. The posterior belly will move back the scalp; and the bellies acting in succession will carry the hairy scalp forwards and backwards.

Dissection. After the removal of the superior auricular muscles and the temporal vessels, together with the epicranial aponeurosis and its lateral prolongation, the attachment of the temporal fascia on the side of the head may be seen.

The temporal fascia is a white, shining membrane, which is stronger than the epicranial aponeurosis, and gives attachment to the subjacent temporal muscle. Superiorly it is inserted into the curved line that limits the temporal fossa on the side of the skull ; and inferiorly, where it is narrower and thicker, it is fixed to the zygomatic arch. By its cutaneous 
surface the faseia is in contact with the muscles already examined, and with the superficial temporal vessels and nerves.

An incision in the fascia, a little above the zygoma, will show it to consist there of two layers, which are fixed to the edges of the upper border of the zygomatic arch. Between the layers is some fatty tissue, with a small branch of the superficial temporal artery, and a slender twig of the orbital branch of the superior maxillary nerve with its artery.

Dissection. The temporal fascia is now to be detached from the skull, and to be thrown down to the zygomatic arch, in order that the origin of the underlying temporal muscle may be examined. A soft areolar tissue which lies beneath it near the zygoma is to be taken away. The difference in thickness of parts of the fascia will be evident.

The TEMPORAL MUSCLE is laid bare only in part. Wide and thin above, it becomes narrower and thicker at the lower end. The muscle arises from the temporal fascia, and from all the surface of the impression on the side of the skull, which is named the temporal fossa. From this origin the fibres descend, converging to a tendon, which is inserted into the under surface and fore part of the coronoid process of the lower jaw.

On the cutaneous surface is the temporal fascia, with the parts superficial to that membrane; and concealed by the muscle are the deep temporal vessels and nerves which ramify in it. The insertion of the muscle underneath the zygomatic arch will be afterwards followed.

The temporal belongs to the group of masticatory muscles; and its action will be referred to with the description of the pterygoid region.

Dissection. For the dissection of the vessels and nerves, let the face be now turned to the left side, and let an incision be carried along the eyebrow and the zygomatic arch to a little behind the ear, so as to allow the skin on the right half of the head to be reflected. The flap of skin is to be raised from before backwards, but the subcutaneous fat slould be left till the nerves are found.

Behind the ear the skin should be raised as on the other side, to uncover the posterior auricular vessels and nerve.

Along the eyebrow seek the branches of vessels and nerves which come from the orbit (fig. 2), viz., the supra-orbital vessels and nerve opposite the middle, and the supra-trochlear nerve and frontal vessels near the inner part of the orbit; they lie at first beneath the occipito-frontalis, and the muscular fibres must be cut through to find them.

On the side of the head, in front of the ear, the superficial temporal vessels and nerve are to be traced to the vertex; and above the zygomatic arch the branches of the facial which join an offset $\left({ }^{10}\right)$ of the superior maxillary nerve, are to be sought.

Behind the ear the posterior auricular vessels and nerve, and below it branches from the great auricular nerve to the tip and back of the ear, are to be found; one or more offsets of the last should be followed to their junction with the posterior auricular nerve.

At the back of the head the ramifications of the occipital vessels, also the large and small occipital nerves, should be denuded; the former nerve lies by the side of the artery, and the latter about midway between this vessel and the ear.

Cutaneous Arteries. The arteries of the sealp (fig. 2), are furnished by the internal and external carotid trunks, and anastomose freely over the side of the head. Only two small branches, the supra-orbital 
and frontal, come from the former; whilst three, viz., the temporal, occipital, and posterior auricular, belong to the latter.

The supra-orbital artery $(c)$ leaves the orbit through the notch in the margin of the orbit, and is distributed on the forehead. Some of its branches are superficial to the occipito-frontalis, and ascend to the top of the head; whilst others lie beneath the muscle, and supply it, the pericranium, and the bone.

The frontal branch $(b)$ is close to the inner angle of the orbit, and is much smaller than the preceding. It ends in branches for the supply of the muscles, integuments, and pericranium.

The superficial temporcal artery $(d)$ is one of the terminal branches of the external carotid. After ascending above the zygomatic arch for about two inches, the vessel divides on the temporal fascia into anterior and posterior :

The anterior branch runs forwards with a serpentine course to the forehead, supplying muscular, cutaneous, and pericranial offsets, and anastomoses with the supra-orbital artery: this is the branch that is opened when blood is taken from the temporal artery.

The posterior branch is larger than the other, and arches backwards above the ear towards the occipital artery, with which it anastomoses. Its offsets to the parts around are similar to those of the anterior, and it communicates with the artery of the opposite side over the top of the head.

Occipital artery $(a)$. The terminal part of this artery, after perforating the trapezius, divides into large and tortuous branches, which spread over the back of the head and the occipito-frontalis muscle. Communications take place with the artery of the opposite side, with the posterior part of the temporal, and with the following artery. Some offsets pass deeply to supply the occipito-frontalis muscle, the pericranium, and the bone.

The posterior auricular artery $(f)$ appears in front of the mastoid process, and divides into two branches. One (mastoid) is directed backwards to supply the occipito-frontalis, and anastomose with the occipital artery. The other (auricular) is furnished to the retrahent muscle and the back of the pinna of the ear; and an offset from this pierces the pinna to be distributed on the opposite surface.

The veins of the exterior of the head are so similar to the arteries, that a full notice of each is not required. All the veins corresponding with branches of the internal carotid artery enter the facial vein, whilst the rest open into the jugular veins. These superficial veins communicate both with the sinuses in the interior of the skull by means of small branches named emissary, and with the veins occupying the spongy substance (diploë) of the cranial bones.

The frontal vein is directed towards the inner angle of the orbit, where it receives the supra-orbital. vein, the two giving rise to the angular vein of the face : near its ending it receives small veins from the eyebrow, and from the upper eyelid and the nose. Both the superficial temporal and posterior auricular veins open into the external jugular; and the occipital joins the internal jugular vein.

Cutaneous Nerves (fig. 2). The nerves of the scalp are furnished from cutaneous offsets of both cranial and spinal nerves. The half of the head anterior to the ear receives branches from three trunks of the fifth cranial nerve, and a few twigs from the facial nerve. All the rest of the 
head is supplied by spinal nerves (anterior and posterior primary branches), except elose behind the ear, where there is an offset of the facial or seventh cranial nerve.

The supra-orbital nerve (fig. $2,{ }^{6}$ ) comes from the first trunk of the fifth nerve, and escapes from the orbit with its companion artery; whilst

Fig. 2.

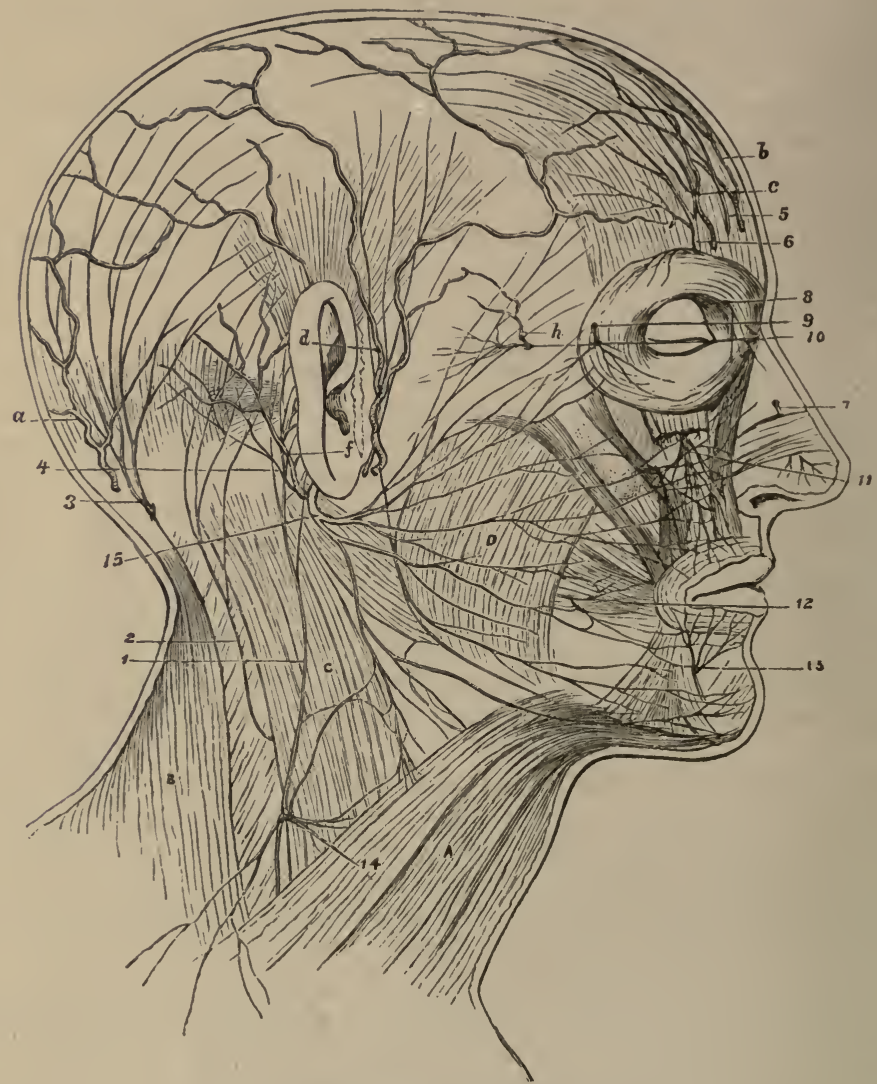

Cutaneous Nerves of the Scatip.

1. Great auricular nerve.

2. small occipital.

3. Great occipital.

4. Posterior auricular of the facial.

A uriculo-temporal (not numbered) in front of the ear, by the side of the temporal artery, $d$.

Cutaneous arteries of the Scalp.

a. Occipital artery.

b. Froutal.

c. Supra-orbital.
5. Supra-trochlear.

6. Supra-orbital.

10. Superficial temporal of the upper maxillary, and crossing it are the superficial temporal branches of the temporal nerve.

beneath the occipito-frontalis muscle, the nerve gives offsets to it and the orbicularis palpebrarum, as well as to the pericranium. In the orbicularis 
a communication is established between this and the facial nerve. Finally the nerve ends in two cutaneous branches, which ramify in the teguments :-

One of these (inner) soon pierces the occipito-frontalis, and reaches upwards as high as the parietal bone. The other branch (outer) is of larger size, and, perforating the muscle higher up, extends over the arch of the head as far as the ear.

As the nerve escapes from the supra-orbital notch it furnishes some palpebral filaments to the upper eyelid.

At the inner angle of the orbit is the small supra-trochlear branch (fig. $2,{ }^{5}$ ) of the same nerve. It ascends to the forehead close to the bone, and piercing the muscular fibres ends in the integument. Branches are given from it to the orbicularis and corrugetor supercilii, and some palpebral twigs enter the eyelid.

The superficial temporal nerves are derived from the second and third trunks of the fifth nerve, and from the facial nerve.

'The temporal branch of the superior maxillary nerve (second trunk of the fifth) is usually a slender twig (fig. $2,{ }^{10}$ ), which perforates the temporal aponeurosis about a finger's breadth above the zygomatic arch. When cutaneous, the nerve is distributed on the temple, and communicates with the facial nerve, also sometimes with the next.

'The auriculo-temporal branch (fig. $2 d$ ) of the inferior maxillary nerve (third trunk of the fifth) lies near the ear, and accompanies the temporal artery to the top of the head. As soon as the nerve emerges firom beneath the parotid gland, it divides into two terminal branches :- The more posterior is the smaller of the two, and supplies the attrahens aurem muscle and the integument above the ear. The other branch ascends vertically in the teguments to the top of the head. The nerve also furnishes an auricular branch (upper) to the anterior part of the ear above the auditory meatus.

The temporal branches of the facial nerve are directed upwards over the zygomatic arch and the temporal aponeurosis to the orbicularis palpebrarum muscle: they will be deseribed with the dissection of the trunk of the facial nerve.

The posterior auricular nerve (fig. 2, ") lies behind the ear with the artery of the same name. It arises from the facial nerve close to the stylo-mastoid foramen, and ascends in front of the mastoid process. Soon after the nerve becomes superficial it communicates with the great auricular nerve, and divides into an occipital and an auricular branch, which are distributed as their names express :-

The occipital branch is long and slender, and ends in the posterior belly of the occipito-frontalis muscle. It lies near the occipital bone, enveloped in dense fibrous structure, and furnishes offisets to the integuments.

'The auricular branch ascends to the back of the ear, supplying the retrahent muscle and the posterior surface of the pinna.

The great auricular nerve of the cervical plexus (fig. $2,{ }^{1}$ ) is seen to some extent at the lower part of the ear, but its anatomy will be afterwards given with the description of the cervical plexus.

The great occipital (fig. $2,{ }^{3}$ ) is the largest cutaneous nerve at the back of the head, and is recognized by its proximity to the occipital artery. Springing from the posterior primary branch of the second cervical nerve, it perforates the muscles of the back of the neck, and divides on the ocei- 
put into numerous large offsets; these spread over the posterior part of the occipito-frontalis muscle, ending mostly in the integument. As soon as the nerve pierces the trapezius, it is joined by an offiset from the third cervical nerve; and on the back of the head it communicates with the small occipital nerve.

The small occipital nerve of the cervical plexus (fig. $2,{ }^{2}$ ) lies midway between the ear and the preceding nerve, and is continued upwards in the integuments higher than the level of the ear. It communicates with the nerve on each side, viz., the posterior auricular and the great occipital. Usually this nerve furnishes an auricular branch to the upper part of the ear at the cranial aspect, which supplies also the attollens aurem muscle.

\section{Section II.}

\section{INTERNAL PARTS OF THE HEAD.}

Dissection. The skull is now to be opened, but before sawing the bone the dissector should detach, on the right side, the temporal muscle nearly down to the zygoma, without separating the fascia of the same name from the fleshy fibres; and all the remaining soft parts are to be divided by an incision carried around the skull, about one inch above the margin of the orbit at the forehead, and as low as the protuberance of the occiput.

The cranium is to be sawn in the same line as the incision through the soft parts, but the saw is to cut only through the outer osseous plate. 'The inner plate is to be broken through with a chisel, in order that the subjacent membrane of the brain (dura mater) may not be injured. 'The skullcap is next to be forcibly detached by inserting the fingers between the cut surfaces in front, and the dura mater will then come into view.

The DURA MATER is the most external of the membranes investing the brain. It is a strong, fibrous structure, which serves as an endosteum to the bones, and supports the cerebral mass. Its outer surface is rough, and presents, now the bone is separated from it, numerous small fibrous and vascular processes; but these are most marked along the line of the sutures, where the attachment of the dura mater to the bone is the most intimate. Ramifying on the upper part of the membrane are branches of the large meningeal vessels.

Small granular bodies, glands of Pacchioni, are also seen along the middle line. The number of these bodies is very variable; they are found but seldom before the third year, but generally after the seventh, and they increase with age. Occasionally the surface of the skull is indented by these so-called glands.

Dissection. For the purpose of seeing the interior of the dura mater, divide this membrane with a scissors close to the margin of the skull, except in the middle line before and behind where the superior longitudinal sinus lies. The cut membrane is then to be raised towards the top of the head; and on the right side the veins connecting it with the brain may be broken through.

The inner surface of the dura mater is smooth and polished; and this appearance is due to an epithelial layer similar to that lining serous membranes. 
This external envelope of the brain consists of white fibrous and elastic tissues so disposed as to give rise to two strata, viz., an external or endosteal, and an internal or supporting. At certain spots those layers are slightly separated, and form thereby the spaces or sinuses for the passage of the venous blood. Moreover, the innermost layer sends processes between different parts of the brain, forming the falx, tentorium, etc.

The falx cerebri is the process of the dura mater, in shape like a sickle, which dips in the middle line between the hemispheres of the large brain. Its form and extent will be evident if the right half of the brain is gently separated from it. Narrow and pointed in front, where it is attached to the erista galli of the ethmoid bone, it widens posteriorly, and joins a horizontal piece of the dura mater named the tentorium cerebelli. The upper border is convex, and is fixed to the middle line of the skull as far backwards as the occipital protuberance; and the lower or free border, concave, is turned towards the central part of the brain (corpus callosum), with which it is in contact posteriorly.

In this fold of the dura mater are contained the following sinuses :- the superior longitudinal along the convex border, the inferior longitudinal in the hinder part of the lower edge, and the straight sinus at the line of junction between it and the tentorium.

The superior longitudinal sinus (fig. $3, b$ ) extends from the ethmoid bone to the occipital protuberance. Its position in the convex border of the falx will be made manifest by the escape of blood through numerous small veins, when pressure is made from before back with the finger along the middle line of the brain.

When the sinus is opened it is seen to be narrow in front, and to widen behind, where it ends in a common point of union of certain sinuses (torcular Iferophili) at the centre of the occipital bone. Its cavity is triangular in form, with the apex of the space turned to the falx; and across it are stretched small tendinous cords-chordæ Willisii-near the openings of some of the cerebral veins. Occasionally small glandulæ Pacehioni are present in the sinus.

The sinus receives small veins from the substance and exterior of the skull, and larger ones from the brain; and the blood flows backwards in it. The cerebral veins open chiefly at the posterior part of the brain, and lie for some distance against the wall of the sinus before they perforate the dura mater; their course is directed from behind forwards, so that the current of the blood in them is evidently opposed to that in the sinus: this disposition of the veins may be seen on the left side of the brain, where the parts are undisturbed.

Directions. Before the rest of the dura mater can be examined, the brain must be taken from the head. To facilitate its removal, let the head incline backwards, whilst the shoulders are raised on a block, so that the brain may be separated somewhat from the base of the skull. For the division of the cranial nerves a sharp sealpel will be necessary; and the nerves are to be cut longer on the one side than on the other.

Removal of the brain. As a first step cut across the anterior part of the falx cerebri, and the different cerebral veins entering the longitudinal sinus; raise and throw backwards the falx, but leave it uncut in the middle line behind. Gently raise with the fingers the frontal lobes and the olfactory bulbs of the large brain. Next eut through the internal carotid artery and the second and third nerves, which then appear; the large second nerve is placed on the inner, and the round third nerve on the 
outer side of the artery. A small branch of artery to the orbit should likewise be divided at this time.

The brain is now to be supported in the left hand, and the pituitary body to be dislodged with the knife from the hollow in the centre of the sphenoid bone. A strong horizontal process of the dura mater (tentorium cerebelli) eomes into view at the back of the eranium. Along its free margin lies the small fourth nerve, which is to be cut at this stage of the proceeding. Make an incision through the tentorium on each side, close to its attachment to the temporal bone, without injuring the parts underneath; the following nerves, which will be then visible, are to be divided in suecession. Near the inner margin of the tentorium is the fifth nerve, consisting of a large and small root; whilst towards the middle line of the skull is the long slender sixth nerve. Below the fifth, and somewhat external to it, is the seventh nerve with its facial and auditory parts, the former being anterior and the smaller of the two. Directly below the seventh are the three trunks of the eighth nerve in one line:- of these, the upper small piece is the glosso-pharyngeal; the flat band next below, the pneumogastric; and the long round nerve aseending from the spinal canal, the spinal accessory. The remaining nerve nearer the middle line is the ninth, which consists of two small pieces.

After dividing the nerves, eut through the vertebral arteries as they wind round the upper part of the spinal cord. Lastly, eut across the spinal cord as low as possible, as well as the roots of the spinal nerves that are attached on each side. Then on placing the first two fingers of the right hand in the spinal canal, the cord may be raised, and the whole brain may be taken readily from the skull in the left hand.

Preservation of the brain. After removing some of the membranes from the upper part, and making a few apertures through them on the under surface, the brain may be immersed in spirit to harden the texture; and methylated spirit may be used on account of its eheapness. Placing the brain upside down on a piece of calico long enough to wrap over it, put it in the spirit.

Examination of the brain. At the end of two or three days the dissector should examine the other membranes, and the vessels. As soon as the vessels have been learnt, the membranes are to be earefully removerl from the surface of the brain, without detaching the different eranial nerves at the under surface. The brain may remain in the spirit till the dissection of the head and neck has been completed, but it should be turned over occasionally to allow the spirit to penetrate its substance.

The description of the brain and its vessels will be found after that of the head and neck.

Directions. After setting aside the brain, the anatomy of the dura mater, and the vessels and nerves in the base of the skull should be proeeeded with. For this purpose raise the head to a convenient height, and fasten the tentorium in its natural position with a few stitches. The dissector should be furnished with the base of a skull while studying the following parts.

Dura mater. At the base of the cranium the dura mater is much more closely united to the bones than it is at the top of the skull. Here it dips into the different inequalities of the osseous surfaces ; and it sends processes through the several foramina, which join for the most part the perieranium, and furnish sheaths to the nerves.

Beginning the examination in front, the membrane will be found to send 
a prolongation into the foramen cæcum, as well as a series of tubes through the apertures in the cribriform plate of the ethmoid bone. Through the sphenoidal fissure it joins the periosteum of the orbit; and through the optic foramen a covering is continued on the optic nerve to the eyeball. Behind the sella 'Turcica, the dura mater adheres closely to the basilar process of the occipital bone; and it may be traced into the spinal canal through the foramen magnum, to the margin of which it is very firmly united.

The tentorium cerebelli is the piece of the dura mater which is interposed in a somewhat horizontal position between the small brain (cerebellum), and the posterior part of the large brain (cerebrum).

Its upper surface is raised along the middle, where it is joined by the falx cerebri, and is hollowed laterally for the reception of the back part of the cerebral hemispheres. Its under surface touches the little brain, and is joined by the falx cerebelli.

The anterior concave margin is free, except at the ends where it is fixed by a narrow slip to each anterior clinoid process. The posterior or convex part is connected to the following bones:-occipital (transverse groove), inferior angle of the parietal, petrous portion of the temporal (upper border), and posterior clinoid process of the sphenoid.

Along the centre of the tentorium is the straight sinus; and in the attached edge are the lateral and the superior petrosal sinus on each side.

Falx cerebri. 'The characters of this fold have been given in page 25.

The Falx cerebelli has the same position below the tentorium as the falx cerebri above that fold. It is much smaller than the falx of the cerebrum, and will appear on detaching the tentorium. Triangular in form, this fold is adherent to the middle ridge of the occipital bone below the protuberance, and projects between the hemispheres of the small brain. Its base is directed to the tentorium; and the apex reaches the foramen magnum, to each side of which it gives a small slip. In it is contained the occipital sinus.

The sinuses are venous spaces between the layers of the dura mater, into which blood is received. All the sinuses open either into a large space named torcular Herophili, opposite the occipital protuberance; or into the two cavernous sinuses on the sides of the body of the sphenoid bone.

A. The torcular Herophili (fig. $3, a$ ) is placed in the tentorium, opposite the centre of the occipital bone. It is of an irregular shape, and numerous sinuses open into it, viz., the superior longitudinal above, and the occipital below; the straight in front, and the lateral sinus on each side.

The superior longitudinal sinus has been already described (see p. 25).

The inferior longitudinal sinus (fig. $3, c$ ) resembles a small vein, and is contained in the lower border of the falx cerebri at the posterior part. This vein receives blood firom the falx and the larger brain, and ends in the straight sinus $(d)$ at the edge of the tentorium.

The straight sinus (fig. 3, d) lies along the middle of the tentorium, and seems to continue the preceding sinus to the common point of union. Its form is triangular, like the superior longitudinal. Joining it are the inferior longitudinal sinus, the veins of Galen from the interior of the large brain, and some small veins from the upper part of the cerebellum.

The occipital sinus (fig. $3, g$ ) is a small space in the falx cerebelli, which reaches to the foramen magnum, and collects the blood from the oceipital fossa. This sinus may be double. 
The lateral sinus (fig. $3, e$ ) is the channel by which most of the blood passes from the skull. There is one on each side, right and left, which extends from the occipital protuberance to the foramen jugulare, where it ends in the internal jugular vein. In this extent the sinus occupies the winding groove in the interior of the skull between the two points of bone before mentioned : and the right is frequently larger than the left.

Fig. 3.

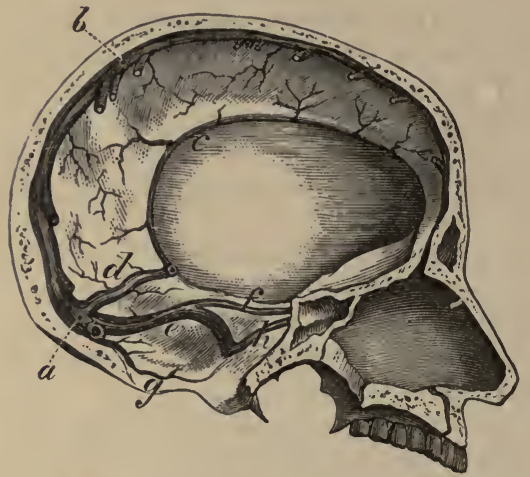

a. Toreular Herophili.

๖. Superior lonyitudinal sinus.

c. Inferior lougitudinal.

d. Straight sinus.

e. Lateral sinus.

g. Occipital sinus.

$f$. Superior, and $h$, inferior petrosal sinus.

Some of the Sinuses of the SKull.

Besides small reins from the brain, it is joined by the superior petrosal sinus $(f)$, opposite the upper edge of the petrous portion of the temporal bone; and by the inferior petrosal $(h)$ at the foramen jugulare. Oftentimes it communicates with the occipital vein through the mastoid foramen, and sometimes with veins of the diploë of the skull.

The foramen jugulare is divided into three compartments by bands of the dura mater. Through the posterior interval the lateral sinus passes; through the anterior the inferior petrosal sinus; and through the central one the pieces of the eighth nerve.

Dissection. To examine the cavernous sinus on the left side, cut through the dura mater by the side of the body of the sphenoid bone from the anterior to the posterior clinoid process, and internal to the position of the third nerve: behind the clinoid process, let the knife be directed inwards for about half the width of the basilar part of the occipital bone. By placing the handle of the scalpel in the opening thus made, the extent of the space will be defined. A probe or a blow-pipe will be required, in order that it may be passed into the different sinuses joining the cavernous centre.

B. The cavernous sinus, which has been so named from the reticulate structure in its interior, is situate on the side of the body of the sphenoid bone. This space, resulting from the separation of the two layers of the dura mater, is of an irregular shape, and extends from the sphenoidal fissure to the tip of the petrous portion of the temporal bone.

The piece of dura mater bounding the sinus externally is of some thickness, and contains in its substance the third and fourth nerves, with the ophthalmic trunk of the fifth nerve; these lie in their numerical order from above down.

The eavity of the sinus is larger behind than before, and in it are shreds of fibrous tissue with small vessels. Through the space winds the 
trunk of the internal carotid artery surrounded by the sympathetic, with the sixth nerve on the outer side of the ressel; but all these are shut out from the blood in the space by a thin lining membrane.

The cavernous sinus receives the ophthalmic vein of the orbit, some inferior cerebral veins, and twigs from the pterygoid veins outside the skull. It communicates with its fellow on the opposite side by the circular and transverse sinuses; and its blood is transmiited to the lateral sinus by the superior and inferior petrowal channels.

The circular sinus lies around the pituitary body, and reaches from the one cavernous sinus to the other across the middle line. Besides serving as the means of communication between those sinuses, it receives small veins from the pituitary body. This sinus is usually destroyed by the removal of the pituitary body.

The transverse or basilar sinus crosses the basilar process of the occipital bone, on a level with the petrous part of the temporal bone, and unites the opposite cavernous sinuses. A second transverse sinus is sometimes found nearer the foramen magnum.

The superior petrosal sinus (fig. $3, f$ ) lies in a groove in the upper edge of the petrous part of the temporal bone, and extends between the eavernous and lateral sinuses. A small vein from the cerebellum, and another from the internal ear, are received into it.

The inferior petrosal sinus (fig. $3, h$ ) extends between the same sinuses as the preceding, and lies in a groove along the line of junction of the petrous part of the temporal with the basilar process of the occipital bone; it is joined by a small vein from the outside of the skull, through the foramen lacerum in the base of the cranium. This sinus passes through the anterior compartment of the jugular foramen, and ends in the internal jugular vein.

Meningeal Arteries. These arteries supplying the cranium and the dura mater come through the base of the skull; they have been named from their situation in the three fossæ, anterior, middle, and posterior meningeal.

The anterior meningeal are very small branches of the ethmoidal arteries (p. 56), which enter the skull by apertures between the frontal and ethmoid bones; they are distributed to the dura mater over and near the ethmoid bone.

The middle meningeal arteries are three in number: two, named large and small, are derived from the internal maxillary trunk ; and the third is an offset of the ascending pharyngeal artery.

a. The large meningeal branch of the internal maxillary artery appears through the foramen spinosum of the sphenoid bone, and ascends towards the anterior inferior angle of the parietal bone. At this spot the vessel enters a deep groove in the cranium, and ends in ramifieations which spread over the side of the head, some of them reaching to the top and the occiput, whilst others perforate the bone, and end on the exterior of the head. Two veins accompany the artery.

Branches. As soon as the artery comes into the cranial cavity, it furnishes branches to the dura mater and osseous structure, and to the ganglion of the fifth nerve. One small offset, petrosal, enters the hiatus Fallopii, and supplies the surrounding bone (Hyrtl). One or two branches pass into the orbit, and anastomose with the ophthalmic artery.

$b$. The small meningeal branch is an offset of the large one outside the 
skull, and is transmitted through the foramen ovale to the membrane lining the middle cranial fossa.

$c$. Another meningeal branch from the ascending pharyngeal artery comes through the foramen lacerum (basis cranii). This is seldom injected, and is not often visible.

The posterior meningeal branches are small, and are furnished by the occipital and vertebral arteries.

That from the occipital, one on each side, enters the skull by the jugular foramen ; and that from the vertebral arises opposite the foramen magnum. Both vessels ramify in the posterior fossa of the skull.

Mexingeal Nerves. Offsets to the dura mater are said to be derived from the fourth, fifth, glosso-pharyngeal, and vagus, cranial nerves, and from the sympathetic nerve. To make these nerves apparent, it would be necessary to steep the dura mater in diluted nitric acid.

Cranial Nerves (fig. 4). The cranial nerves pass from the encephalon through apertures in the base of the skull. As each leaves the cranium it is invested by processes of the membranes of the brain, which are thus disposed:- those of the dura mater and pia mater are lost on the nerve; whilst that of the arachnoid membrane is reflected back, after a short distance, to the interior of the skull. Some of the nerves, those in the middle fossa of the skull for instance, receive sheaths of the dura mater before they approach the foramina of transmission. The nerves will be referred to now as nine pairs, but notice will be subsequently taken of a different mode of enumerating them. Only part of the course of each nerve will be seen at this stage, the rest will be learnt in the dissection of the base of the brain.

The FIRst NERve (fig. 33) ends anteriorly in the enlargement of the olfactory bulb. This swelling lies on the cribriform plate of the ethmoid bone, and supplies about twenty branches to the nose through the small foramina in the subjacent bone. These delicate nerves are surrounded by prolongations of the membranes of the brain, and their arrangement will be noticed in the dissection of the nose.

The sECOND NERVE (fig. $4,{ }^{2}$ ) diverging from its commissure to the eyeball, enters the orbit through the optic foramen; accompanying the nerve is the ophthalmic artery.

Dissection. The third and fourth nerves, and the ophthalmic trunk of the fifth nerve, lie in the outer wall of the cavernous sinus; and to see them, it will be necessary to trace them through the dura mater towards the orbit.

Afterwards the student should follow outwards the roots of the fifth nerve into the middle fossa of the skull, as in fig. 4, taking away the dura mater from them, and from the surface of the large Gasserian ganglion which lies on the point of the petrous portion of the temporal bone. From the front of the ganglion arise other two large trunks besides the oph thalmic, viz., superior and inferior maxillary, and these should also be traced to their apertures of exit from the skull. If the dura mater is removed entirely from the bone near the nerves a better dissection will be obtained.

The THIRD NERve (fig. $4,{ }^{8}$ ) is destined for the muscles of the orbit. It enters the wall of the cavernous sinus near the anterior clinoid process, and is deprived at that spot of its tube of arachnoid membrane. In the wall of the sinus it is placed above the other nerves; but when it is about to enter the orbit through the sphenoidal fissure, it sinks below the fourth and a part of the fifth, and divides into two branches. 
Near the orbit the nerve is joined by one or two delicate filaments of the eavernous plexus (p. 33).

The Fourti Nerve (fig. $4,{ }^{4}$ ) courses forwards, like the preceding, to one muscle in the orbit. It is the smallest of the nerves in the wall of the sinus, and is placed below the third; but as it is about to pass through the sphenoidal fissure it rises higher than all the other nerves.

Fig. 4.

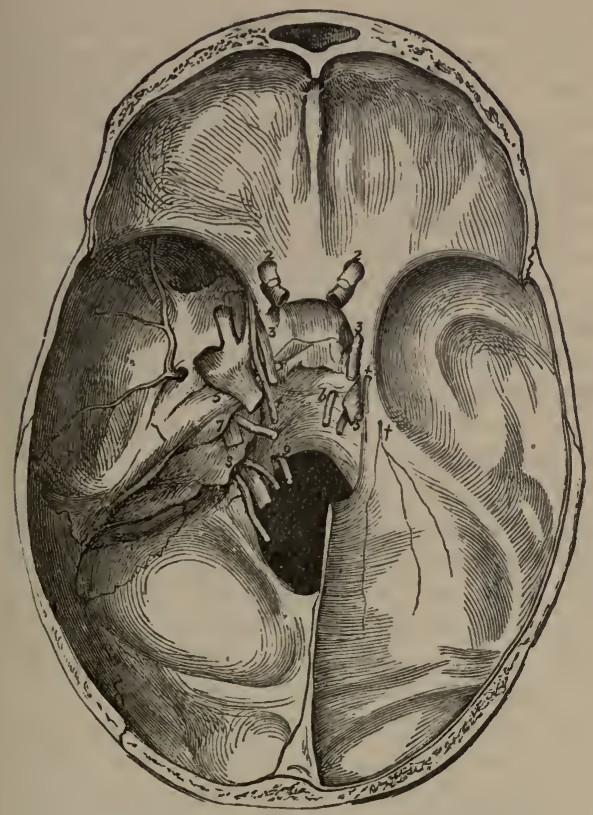

The dura mater has been removed in the middle fossa, on the left side, to show the nerves in the wall of the caveruous sinus, and especially the ganglion, and the three trunks of the fifth nerve. Each nerve, except the first which is absent, is marked by its corresponding numeral. On the right side the dura mater is untouched. $\dagger$ Offsets to the dura mater from the fifth nerve.

Cranial Nerves in the Base of the Skull.

In the wall of the sinus the fourth nerve is joined by twigs of the sympathetic; and it is sometimes united with the ophthalmic trunk of the tifth.

Fiftu Nerve (fig. $4,{ }^{5}$ ). This nerve is distributed to the face and head, and consists of two parts or roots-a large or sensory, and a small or motory.

The large root of the nerve passes through an aperture in the dura mater into the middle fossa of the base of the skull, where it ends immediately in the Gasserian ganglion.

The ganglion of the root of the fifth nerve (Gasserian ganglion), placed in a depression on the point of the petrous part of the temporal bone, is flattened, and is nearly as wide as the thumb-nail. The upper surface of the ganglion is closely united to the dura mater, and presents a semilunar elevation, whose convexity looks forwards. Some filaments from the plexus of the sympathetic on the carotid artery join its inner side.

Branches. From the front of the ganglion proceed the three following trunks:-The ophthalmic nerve, the first and highest, is destined for the orbit and forehead. Next in order is the superior maxillary nerve, which leares the skull by the foramen rotundum, and ends in the face below the 
orbit. And the last, or the inferior maxillary nerve, passes through the foramen ovale to reach the lower jaw, the lower part of the face, and the tongue.

The smaller root, entering the same tube of the dura mater as the large one, passes beneath the ganglion, without communicating with it, and joins only one of the three trunks derived from the ganglion: if the ganglion be raised, this root will be seen to enter the inferior maxillary nerve.

Those branches of the ganglion which are unconnected with the smaller or motor root, viz., the ophthalmic and superior maxillary, are solely nerves of sensibility; but the inferior maxillary, which is compounded of both roots, is a nerve of sensibility and motion. But the whole of the inferior maxillary nerve has not this double function, for the motor root is mixed almost exclusively with the part which supplies the muscles of the lower jaw ; and it is, therefore, chiefly that small piece of the nerve which possesses a twofold action, and resembles a spinal nerve.

The ophthalmic nerve is the only one of the three trunks which needs a more special notice in this stage of the dissection. It is continued through the sphenoidal fissure and the orbit to the forehead. In form it is a flat band, and is contained in the wall of the cavernous sinus below the third and fourth nerves. Near the orbit it divides into three branches (p.51).

In this situation it is joined by filaments of the cavernous plexus of the sympathetic, and gives a small recurrent filament (fig. $4, \dagger$ ) to that part of the dura mater which forms the tentorium cerebelli (Arnold).

The sixtil Nerve (fig. $4,{ }^{6}$ ) enters the orbit through the sphenoidal fissure, and supplies one of the orbital muscles. It pierces the dura mater behind the body of the sphenoid bone, and crosses the space of the cavernous sinus, instead of lying in the outer wall with the other nerves.

In the sinus the nerve is placed close against the outer side of the carotid artery; and it is joined by one or two large branches of the sympathetic nerve surrounding that vessel.

Seventh Nerve according to Willis (fig. $4,{ }^{7}$ ). This cranial nerve consists of two trunks, fascial and auditory, and both enter the meatus auditorius internus. In the bottom of the meatus they separate; the ficial nerve courses through the aqueduct of Fallopius to the face, and the auditory nerve is distributed to the internal ear.

Eighth Nerve (fig. $4,^{8}$ ). Three trunks are combined in the eighth cranial nerve of Willis, viz., glosso.pharyngeal, pneumogastric, and spinal accessory. All three pass through the central compartment of the foramen jugulare, but all are not contained in one tube of the membranes of the brain. The glosso-pharyngeal nerve is external to the other two, being separated from them by the inferior petrosal sinus, and has distinct sheaths of the dura mater and the arachnoid membrane; but the pneumo-gastric and spinal accessory nerves are inclosed in the same tube of the dura mater, only a piece of the arachnoid intervening between them.

The Nistu NERve (fig. $4,{ }^{9}$ ) is the motor nerve of the tongue, and consists of two small pieces, which pierce separately the dura mater opposite the anterior condyloid foramen; these unite after passing through that aperture.

Dissection. The dissector may now return to the examination of the trunk of the carotid artery as it winds through the cavernous sinus.

On the opposite side of the head, viz., that on which the nerves in the wall of the carernous sinus are untouched, an attempt may be made to find two small plexuses of the sympathetic on the carotid artery, though in an injected body this dissection is scarcely possible. 
One of these (eavernous) is near the root of the anterior clinoid process; and to bring it into view it will be necessary to cut off that piece of bone, and to disseet out with eare the third, fourth, fifth, and sixth nerves, looking for filaments between them and the plexus. Another plexus (carotid), joining the fifth and sixth nerves, surrounds the artery as this enters the sinus.

The INTERNAL CAROTID ARTERY appears in the base of the skull at the apex of the petrous part of the temporal bone. In its ascent to the brain the vessel lies in the space of the eavernous sinus, along the side of the body of the sphenoid bone, and makes two remarkable bends, so as to look like the letter $\mathrm{S}$ reelined. At first, the artery ascends to the posterior clinoid process; it is then direeted forwards to the root of the anterior process of the same name; and lastly it turns upwards internal to this last point of bone, perforates the dura mater bounding the sinns, and divides into cerebral arteries at the base of the brain. In this course the artery is enveloped by nerves derived from the sympathetic in the neck.

The branches of the artery here are few. In the sinus there are some small arteries (arterix receptaculi) for the supply of the dura mater and the bone, the nerves, and the pituitary body; and at the anterior clinoid process the ophthalmic branch arises.

The terminal branches of the carotid will be seen in the dissection of the base of the brain.

Srmpatuetic Nerve. Around the carotid artery is a prolongation of the sympathetic nerve of the neck, which forms the following plexuses :-

The carotid plexus is situate on the outer side of the vessel, at its entrance into the eavernous sinus, and communicates with the sixth nerve and the Gasserian ganglion.

The small cavernous plexus is placed below the bend of the artery which is close to the anterior clinoid process, and is connected with that offset of the upper cervieal ganglion which courses along the inner side of the carotid artery. Filaments from the plexus unite with the third, fourth, and ophthalmic nerves. One filament is also furnished to the lenticular ganglion in the orbit, either separately from, or in conjunction with, the nasal nerve.

After forming those plexuses, the nerves surround the trunk of the earotid, and are lost chiefly in the eerebral membrane named pia mater; but some ascend on the cerebral and ophthalmic branches of that vessel, and one offset is said to enter the eyeball with the central artery of the retina.

Petrosal nerves (fig. 34). Beneath the Gasserian ganglion is the large superficial petrosal nerve (fig. $34,{ }^{2}$ ) entering the hiatus Fallopii to join the facial nerve. Externally to this is occasionally seen another" small petrosal nerve (fig. $34,{ }^{4}$ ) (external superficial), which springs from the sympathetie on the middle meningeal artery, and enters the bone to join the facial nerve. A third, the small petrosal nerve (fig. $34,{ }^{3}$ ), is contained in the substance of the temporal bone. The sonree, and the destination of those three small nerves will be afterwards learnt. It will suffice now for the student to note the two first, and to see that they are kept moist and fit for examination at a future time.

Directions. Now the base of the skull has been completed, a preservative fluid or salt should be applied, and the flaps of the teguments should be stitched together over all. 


\section{SECTION III.}

\section{DISSECTION OF THE FACE.}

Directions. The left side of the face may be used for learning the muscles and vessels, and the right side is to be reserved for the nerves.

Position. The previous position of the body for the examination of the base of the skull will require to be changed:- the head is to be lowered, and the side of the face to be dissected is to be placed upwards.

Dissection. As a preparatory step, the muscular fibres of the apertures may be made slightly tense by inserting a small quantity of tow or cottonwool between the eyelids and the eyeball, and between the lips and the teeth.

First lay bare the sphincter muscle of the eyelids by a skin-deep circular incision over the margin of the orbit, and by raising the skin of the lids towards the aperture of the eye. Much care must be taken in detaching the skin from the thin and of tentimes pale fibres of the orbicular muscle in the lids, else they will be cut away in consequence of the little areolar tissue between the two.

Next the integument is to be removed from the side of the face by one incision in front of the ear, from above the zygomatic arch to the angle of the jaw, and then along the base of the jaw to the chin; and by another cut carried backwards horizontally from the corner of the mouth into the first. The flaps of skin are to be raised from behind forwards, and left adherent along the middle line. On the side of the nose the skin is closely united to the subjacent parts, and must be detached with caution. Around the mouth are many fleshy slips extending both upwards and downwards from the orbicular muscle, but they are all marked so distinctly as to escape injury, with the exception of the small risorius muscle which goes from the corner of the mouth towards the ramus of the lower jaw. While removing the fat from the muscles, each fleshy slip may be made tense with hooks.

The facial vessels and their branches will come info view as the muscles are cleaned; but the nerves may be disregarded on this side.

In front of the ear is the parotid gland, whose duct is to be preserved; this is on a level with the meatus auditorius, and pierces the middle of the cheek.

Muscles of the FACE (fig. 6). The superficial muscles of the face are gathered around the apertures of the nose, eye, and mouth. An orbicular or sphincter muscle encircles the apertures of the eye and mouth ; and other muscles are blended with each to enlarge the opening in the centre of the fibres. There are three distinct groups of muscles : one of the eyelids; another of the nostril; and a third of the aperture of the mouth. One of the muscles of mastication, viz., the masseter, is seen between the jaws.

Muscles of tine Nose. These muscles are the following: pyramidalis nasi, compressor naris, levator ala nasi, dilator naris, and depressor ala nasi.

The PYRAMIDALis NASI (fig. $5,{ }^{1}$ ) is a small fleshy slip that covers the nasal bone, and is continuous above with the occipito-frontalis muscle. Over the cartilaginous part of the nose its fibres end in an aponeurosis, 
which joins that of the compressor naris. Along its inner border is the muscle of the opposite side.

Action. This muscle makes tight the skin over the nasal cartilages, but renders lax, and sometimes wrinkles transversely the skin towards the root of the nose.

Compressor Naris. This muscle (fig. $5,{ }^{3}$ ) is not well seen till after the examination of the following one. Triangular in shape, it arises by its apex from the canine fossa of the upper maxillary bone. The fibres are directed inwards, spreading out at the same time, and end in an aponeurosis, which covers the cartilaginous part of the nose, and joins the tendon of the opposite muscle. This muscle is partly concealed by the next-the common elevator of the ala of the nose and the upper lip.

Action. It stretches the skin over the cartilaginous part of the nose.

The Levator LABII SUPERIORIS ALEQUE NASI (fig. $5,{ }^{2}$ ) is placed by the side of the nose, and arises from the top of the nasal process of the upper maxillary bone, internal to the attachment of the orbicularis. As the fibres descend from the inner part of the orbit the most internal are

Fig. 5.

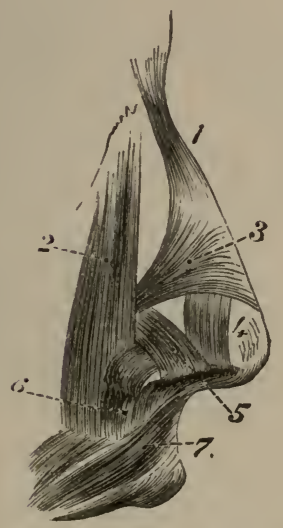

1. Pyramidalis nasi.

2. Common elevator of the nose and lip.

3. Compressor naris.

4 and 5 . The two slips of the dilatator naris.

6. Depressor alæ nasi.

7. Orbicularis oris, attached to the septum nasi.

MUSCles of THE NOSE.

attached by a narrow slip to the wing of the nose, whilst the rest are blended inferiorly with the orbicularis oris. Near its origin the muscle is partly concealed by the orbicularis palpebrarum, but in the rest of its extent it is subcutaneous. Its outer border joins the elevator of the upper lip.

Action. As the name expresses, it can raise the upper lip, and draw outwards the wing of the nose, dilating the aperture; but when the mouth is shut it can enlarge the nostril independently of the lip.

Dilatator Naris. In the dense tissue on the outer side of the nostril are a few muscular fibres, both at the fore and back part of that aperture (fig. $5,{ }^{4}$, and ${ }^{5}$ ), to which the above name has been given by Theile: they are seldom visible without a lens. The anterior slip, ${ }^{4}$, asses from the cartilage of the aperture to the integument of the margin of the nostril ; and the posterior, ${ }^{5}$, arising from the upper jawbone and the small sesamoid cartilages, ends also in the integuments of the nostril. 
Action. The fibres enlarge the nasal opening by raising and everting the outer edge.

The DEPRESSOR ALA NASI (fig. $5,{ }^{6}$ ) will be seen if the upper lip is everted, and the mucous membrane is removed from the side of the frænum of the lip. It arises below the nose from a depression of the upper jawbone above the roots of the second incisor and canine teeth; and asiends to be inserted into the septum nasi and the posterior part of the ala of the nose.

Action. By drawing down and turning in the edge of the dilated nostril, it restores the aperture to its usual size.

Muscles of the Eyelids. The museles of the eyelids and eyebrow are four in number, viz., orbicularis palpebrarum, corrugator supercilii, levator palpebre superioris, and tensor tarsi : ${ }^{1}$ the two latter are dissected in the orbit, and will be described with it.

The orbicularis palpebrarum (fig. $6,{ }^{2}$ ) is the sphineter muscle closing the opening between the eyelids. It is a flat and thin layer, which extends from the margin of the lids beyond the circumference of the orbit. From a difference in the characters of the fibres, a division has been made of them into two parts-outer and inner.

The external fibres (orbital part), the best marked, are fixed only at one point, viz., the inner angle of the orbit. This attachment (origin) is connected with the surface and borders of the small tendo palpebrarum; above that tendon with the nasal process of the upper maxillary, and the internal angular process of the frontal bone; and below the tendon with the superior maxillary bone, and the margin of the orbit. From this origin the fibres are directed outwards, giving rise to ovals, which lie side by side, and increase in size towards the outer edge of the muscle where they project beyond the margin of the orbit.

The internal fibres (palpebral part), paler and finer than the outer, occupy the eyelids, and are fixed at both the outer and inner angles of the orbit. Internally (origin) they are united with the tendo palpebrarum, and externally (insertion) with the external tarsal ligament and the malar bone, and some few may blend with the orbital part. Close to the cilia or eyelashes the fibres form a small pale bundle, which is sometimes called ciliary.

The muscle is subcutaneous; and its circumference is blended above with the occipito-frontalis. Beneath the upper half of the orlicularis, as it lies on the margin of the orbit, is the corrugator supercilii muscle with the supra-orbital vessels and nerve; and beneath the lower half is part of the elevator of the upper lip. The outer fibres are joined occasionally by slips to other contiguous muscles below the orbit.

Action. The inner fibres cause the lids to approach each other, shutting the eye; and in foreed contration the outer commissure is drawn inwards. In closure of the eye the lids move unequally - the upper being much depressed, and the lower slightly elevated and moved borizontally inwards.

When the outer fibres contract, the eyebrow is depressed, and the skin over the edge of the orbit is raised around the eye, so as to protect the ball. Elevation of the upper lip follows contraction of the outer part of the orbicularis, in consequence of fibres being prolonged to the levator labii superioris.

1 The tensor tarsi muscle (p. 59) is sometimes described as a part of the orbicularis. 
The CORRUGATOR SUPERCILII is beneath the orbicularis, near the inner angle of the orbit. Its fibres arise from the inner part of the superciliary ridge of the frontal bone, and are directed outwards to join the orbicular muscle about the middle of the orbital arch. It is a short muscle, and is distinguished by the eloseness of its fibres.

Action. It draws inwards and downwards the mid-part of the eyebrow, wrinkling vertically the skin near the nose, and stretching that outside its point of insertion.

Muscles of Tife Moutir. The muscles of the aperture of the mouth consist of a sphineter; an elevator of the upper lip and angle of the mouth ; an elevator and depressor of the lower lip and angle of the mouth; and retractors of the corner. Lastly, a wide muscle of the cheek closes the space between the jaws.

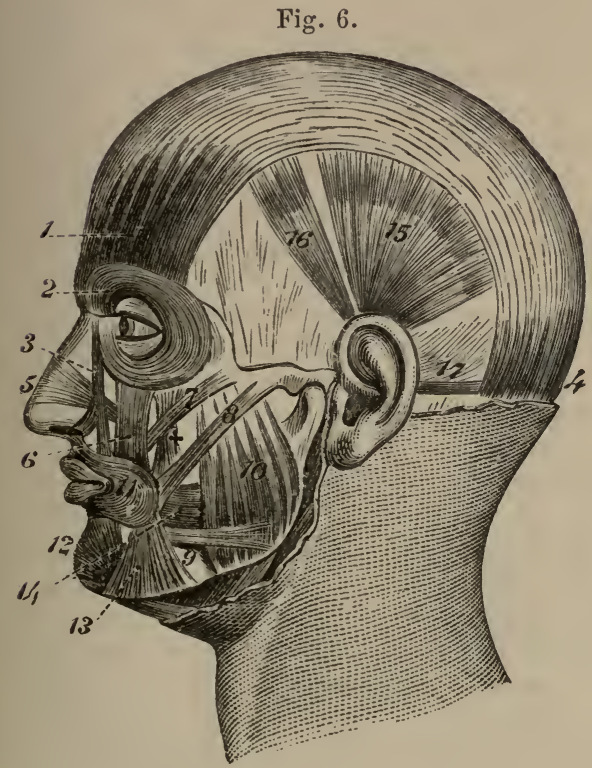

1. Occipito-frontalis, anterior belly. 4. Posterior belly.

2. Orbicularis palpebrarum.

3. Levator labii superioris alæque nasi.

5. Compressor nasi.

6. Levator labii superioris.

7. Zygomaticus minor (too large).

8. Zygomaticus major.

9. Risorius.

10. Masseter.

11. Orbicularis oris.

12. Depressor labii inferioris.

13. Depressor anguli oris.

14. Buccinator.

† Levator anguli oris. See flg. 1 .

The ORBICULARIS ORIS MUSCLE (fig. $6,{ }^{11}$ ) surrounds the opening of the mouth, and is united with the several muscles acting on that aperture. It consists of two parts, inner and outer, which differ in the appearance and arrangement of the fibres, like the sphincter muscle of the eyelids.

The inner part (fig. $5,{ }^{7}$ ), whose fibres are pale in color and fine in texture, forms a rounded thick fasciculus, which corresponds with the red margin of the lip. The fibres of this portion of the muscle, unattached to bone, blend with the buccinator at the corner of the mouth, and some pass from lip to lip.

The outer part is thin, wide, and more irregular. in form, and is connected with the subjacent bone, besides its union with the adjacent muscles. In the upper lip it is attached, on each side of the middle line, by one slip (naso-labial) to the back of the septum of the nose (fig. $5,{ }^{7}$ ); and by a thin stratum to the outer surface of the upper jaw, opposite the canine tooth, and external to the depressor of the wing of the nose. In the 
lower lip it is fixed on each side into the inferior jawbone, opposite the canine tooth, external to the levator labii inferioris muscle. To see these attachments the lip must be everted, and the mucous membrane earefully raised.

The inner margin of the muscle is free, and bounds the aperture of the mouth; whilst the outer edge blends with the different muscles that elevate or clepress the lips and the angle of the mouth. Beneath the orbicularis in each lip is the coronary artery, with the nucous membrane and the labial glands.

Action. Both parts of the muscle contracting, the lips are pressed together and projected forwards, and the aperture of the mouth is diminished transversely by the approximation of the corners towards each other.

The inner fibres acting alone will turn inwards the red part of the lip, and diminish the width of the buccal opening.

The outer fibres press the lips against the dental arehes, the free edges being protruded and somewhat everted. At the same time the centre part of the nose is depressed and the chin raised by means of the fleshy slips conneeted with those parts.

The Levator Labil superioris (fig. $6,{ }^{6}$ ) extends vertically from the lower margin of the orbit to the orbicularis oris. It arises from the upper maxillary and malar bones above the infra-orbital foramen, and blends inferiorly with the orbicularis oris. Near the orbit the muscle is overlapped by the orbicularis palpebrarum, but below that spot it is subcutaneous. By its inner side it joins the common elevator of the ala of the nose and upper lip; and to its outer side lie the zygomatic muscles, the small one joining it. Beneath it are the infra-orbital vessels and nerve.

Action. By the action of this muscle the upper lip is raised, and the skin of the eheek is bulged below the eye.

The DEPRESSOR LABII INFERIORIS (fig. $6,{ }^{12}$ ) is opposite the elevator of the upper lip, and has much yellow fat mixed with its fibres. The muscle has a wide origin from a depression on the front of the lower jaw, reaching backwards from near the symphysis to a little beyond the hole for the labial vessels and nerve; ascending thence it is united with the orbicularis in the lower lip. Its inner border joins the muscle of the opposite side above, and its outer is overlapped below by the depressor anguli oris.

Action. If one musele contracts, the half of the lip of the same side is depressed and everted; but by the use of both muscles, the whole lip is lowered and turned outwards, and rendered tense at the centre.

The LEVATOR LABII INFERIORIS (levator menti) is a sinall muscle on the side of the frenum of the lower lip, which is opposite the depressor of the ala of the nose in the upper lip. When the lip has been everted and the mucous membrane removed, the muscle will be seen to arise from a fossa near the symphysis of the lower jaw, and to descend to its insertion into the integument of the chin. Its position is internal to the depressor of the lip and the attachment of the orbicularis.

Action. It indents the skin of the chin opposite its insertion, and assists in raising the lower lip.

The levator anguli oris (fig. $6, \dagger$ ) has well-marked fibres, and is partly concealed by the levator labii. superioris. Arising from the canine fossa beneath the infra-orbital foramen, its fibres spread out towards the angle of the mouth where they are superficial to the buccinator, and mix 
with the rest of the muscles, but the greater number are continued into the depressor anguli oris and the lower lip.

Action. This muscle elevates the corner of the mouth, and acts as an antagonist to the depressor.

The Depressor ANGULI oris (fig. $6,{ }^{13}$ ) is triangular in shape; it arises from the oblique line on the outer surface of the lower jaw, and ascending to the angle of the mouth, its fibres are prolonged into the elevator of the angle. The muscle conceals the labial branch of the inferior dental vessels and nerve. At its origin the depressor is united with the platysma myoides, and near its insertion with the risorius muscle.

Action. The angle of the mouth is drawn downwards and backwards by it, as is exemplified in a sorrowful countenance.

The ZYGOMATIC MUSCLEs (fig. 6) are directed obliquely from the arch of the same name towards the angle of the mouth and the upper lip. One is longer and larger than the other; they are therefore named major and minor.

The zygomaticus major, ${ }^{8}$, arises from the outer part of the malar bone, and is inserted into the angle of the mouth.

The zygomaticus misor, ${ }^{7}$, is attached to the malar bone anterior to the other, and blends with the fibres of the special elevator of the upper lip.

Action. The large muscle inclines upwards and backwards the corner of the mouth; and the small one assists the levator labii superioris in raising the upper lip.

The risonius MUSCLE (Santorini) (fig. $6,{ }^{9}$ ) is a thin and narrow bundle of fibres, sometimes divided into two or more parts, which arises externally from the fascia over the masseter muscle, and is connected internally with the apex of the depressor anguli oris.

Action. The use of this muscle is indicated by its name, as it retracts the corner of the mouth in laughing.

The Buccinator (fig. $6,{ }^{14}$ ) is the flat and thin muscle of the cheek, and occupies the interral between the jaws. The muscle arises from the outer surface of the alveolar borders of the upper and lower maxilla, as far forwards in each as the first molar tooth; and in the interval between the jaws behind it is attached to a band of fascia-the pterygo-maxillary ligament. From the origin the fibres are directed forwards to the angle of the mouth, where they mix with the other muscles and with both parts of the orbicularis; and as some of the central fibres descend to the lower lip whilst others ascend to the upper lip, a decussation takes place at the corner of the mouth.

On the cutaneous surface of the buccinator are the different muscles converging to the angle of the mouth; and crossing the upper part is the duct of the parotid gland, which perforates the muscle opposite the second upper molar teeth. Internally the muscle is lined by the mucous membrane of the month, and externally it is covered by a fascia (buccopharyngeal) that is continued to the pharynx. By its intermaxillary origin the buccinator corresponds with the attachment of the superior constrictor of the pharynx.

Action. By one muscle the corner of the mouth is retracted, and the cheek wrinkled. By the action of both the aperture of the mouth is widened transversely.

In mastication the cheek is pressed by the muscular contraction against the dental arches, when the corner of the mouth is fixed by the sphincter.

In the expulsion of air from the mouth, as in whistling, the muscle is 
contracted so as to prevent bulging of the eleek; but in the use of a blow-pipe it is distended over the volume of air contained in the mouth, and drives out a continuous stream of air by its contraction.

The vessels of THE FACE (fig. 17) are the facial and transverse facial arteries with their accompanying veins. The arteries are branches of the external carotid; and the facial vein is received into the internal jugular trunk.

The facial artery (fig. $17, f$ ), a branch of the carotid, emerges from the neck, and appears on the lower jaw anterior to the masseter muscle. From this point the artery ascends in a tortuous manner, near the angle of the mouth and the side of the nose, to the inner angle of the orbit, where it anastomoses with the ophthalmic artery. 'The course of the vessel is comparatively superficial in the mass of fat of the inner part of the cheek. At first it is enncealed by the platysma whilst crossing the jaw, but this thin musele does not prevent pulsation being recognized during life; and near the mouth the large zygomatic muscle is superfieial to it. The vessel rests successively on the lower jaw, buecinator muscle, elevator of the angle of the mouth, and elevator of the upper lip. Accompanying the artery is the facial vein, which is nearky a straight tube, and lies to the outer side.

Branches. From the outer side of the vessel unnamed branches are furnished to the muscles and integuments, some of which anastomose with the transverse facial artery. From the inner side are given the following branches:-

The inferior labial branch $(t)$ runs inwards beneath the depressor anguli oris muscle, and is distributed between the lower lip and chin; it communicates with the inferior coronary, and with the labial branch of the inferior dental artery.

Caronary branches ( $r$ and $s$ ). There is one for each lip (superior and inferior), which arise together or separately from the facial, and are directed inwards between the orbicular muscle and the mucous membrane of the lip, till they inosculate with the corresponding branches of the opposite side. From the arterial arches thus formed, offsets are supplied to the lips and labial glands. From the arch in the upper lip a branch is given to each side of the septum of the nose,-_artery of the septum.

The lateral nasal branch $(p)$ arises opposite the ala nasi, and passes beneath the levator labii superioris alæque nasi to the side of the nose, where it anastomoses with the internal nasal branch of the ophthalmic artery.

The angular branch (o) is the terminal twig of the facial artery at the inner angle of the orbit, and joins with a branch (external nasal) of the ophthalmic artery.

The facial vein commences at the root of the nose in a small vein named angular (p. 21). It then crosses over the elevator of the upper lip, and separating from the artery, courses beneath the large zygomatic muscle to the side of the jaw. Afterwards it has a short course in the neck to join the internal jugular vein.

Branches. At the inner side of the orbit it receives veins from the lower eyelid (inferior palpebral), and from the side of the nose. Below the orbit it is joinerl by the infra-orbital vein, also by a large branch, anterior internul maxillary, that comes from the pterygoid region; and thence to its termination by veins corresponding with the branches of the artery in the face and neck. 
The transverse facial artery (fig. 17) is a branch of the temporal, and appears in the face at the anterior border of the parotid gland. It lies by the side of the parotid duct, with branches of the facial nerve, and distributes offsets to the muscles and integuments; some branches anastomose with the facial artery.

Dissection. The parotid gland in front of the ear may be next displayed. To see the gland, raise the skin from the surface towards the ear by means of a cut from the base of the jaw to the anterior border of the sterno-mastoid muscle; this eut may be united with that made for the dissection of the posterior muscle of the ear. A strong fascia covers the gland, and is connected above and behind to the zygomatic arch and the cartilage of the ear, but is continued over the face in front; this is to be removed, so that the gland may be detached slightly from the parts around. The great auricular nerve will be seen ascending to the lobe of the ear; and one or two small glands rest on the surface of the parotid.

The PAROTID (fig. 16, ${ }^{10}$ ) is the largest of the salivary glands. It oceupies the space between the ear and the lower jaw, and is named from its position. Its excretory duct enters the mouth through the middle of the cheek.

The shape of the gland is irregular, and is determined somewliat by the bounding parts. 'Thus inferiorly, where there is not any resisting structure, the parotid projects into the neck, and comes into close proximity with the sub-maxillary gland, though separated from it by a process of the cervical fascia; a line from the angle of the jaw to the sterno-mastoid muscle marks usually the extent of the gland in this direction. Above, the parotid is limited by the zygomatic arch and the temporal bone. Along the posterior part the sterno-mastoid muscle extends; but anteriorly the gland projects somewhat on the face, and in this direction a small accessory part, socia parotidis, is prolonged from it over the masseter.

Connected with the anterior border is the excretory duct-duct of Stenson (ductus Stenonis, fig. 17), which crosses the masseter below the socia parotidis, and perforates the cheek obliquely opposite the second molar tooth of the upper jaw. The duct lies between the transverse facial artery and some branches of the facial nerve, the latter being below it. A line drawn from the meatus auditorius to a little below the nostril would mark the level of the duct in the face; and the central point of the line would be opposite the opening into the mouth. The length of the duct is about two inches and a half; and its eapacity is large enough to allow a small probe to pass, but the opening into the mouth is much less.

The cutaneous surface of the parotid is smooth, and one or two lymphatic glands are seated on it; but from the deep part processes are sent into the inequalities of the space between the jaw and the mastoid process.

Dissection. By removing with caution the parotid gland, the hollows that it fills will come into view : at the same time the dissector will see the vessels and nerves that pass through it. An examination of the processes of the gland, and of the number of important vessels and nerves in relation with it, will demonstrate the dangers attending any operation on it. 'The duct may be opened, and a pin may be passed along it to the mouth, to show the diminished size of the aperture.

Two large processes of the gland extend deeply into the neck. One dips behind the styloid process, and projects beneath the mastoid process and sterno-mastoid musele, whilst it reaches also the deep vessels and nerves of the neck. The other piece is situate in front of the styloid 
process; it passes into the glenoid hollow behind the articulation of the lower jaw ; and sinks beneath the ramus of that bone along the internal maxillary artery.

Passing through the middle of the gland is the external carotid artery, which ascends behind the ramus of the jaw, and furnishes the aurieular, superficial temporal, and internal maxillary branches. Superficial to the artery lies the trunk formed by the junction of the temporal and internal maxillary veins, from which the external jugular vein springs; and this common trunk, receiving some veins from the parotid, is connected with the internal jugular vein by a branch through the gland. ${ }^{1}$ Crossing the gland from behind forwards is the trunk of the facial nerve, which passes over the artery, and distributes its branches through the paroticl. 'The superficial temporal branch of the inferior maxillary nerves lies above the, upper part of the glandular mass; and off'sets of the great auricular nerve pierce the gland at the lower part, and join the facial.

The structure of the parotid resembles that of the other salivary glands. The glandular mass is divided into numerous small lobules by intervening processes of fascia; and each lobule consists of a set of the fine closed saccular extremities of the excretory duct, which are lined by flattened and nucleated epithelium, and surrounded by capillary vessels. These little sacs form by their aggregation the mass of each lobule.

From the lobules issue small ducts, which unite to form larger tubes, and finally all the ducts of the gland are collected into one. The common duct (duct of Stenson) is composed of an external fibrous coat, consisting of white and elastic fibres; and of an internal mucous coat which is clothed with columnar epithelium.

The parotid receives its arteries from the external carotid; and its nerves from the sympathetic, auriculo-temporal of the fifth, facial, and great auricular. Its lymphatics join those of the neck.

Two or three small molar glands lie along the origin of the buccinator, and open into the mouth near the last molar tooth by separate ducts.

Cartilages of the Nose (fig. 7). These close the anterior nasal aperture in the skeleton, and form part of the outer nose and the septum. They are five in number, two on each side-lateral cartilage and cartilage of the aperture; together with a central one, or the cartilage of the septum of the nose. Only the lateral cartilages are seen in this stage of the dissection.

Dissection. The lateral cartilages will be seen when the muscular and fibrous structure of the left side of the nose, and the skin of the lower part of the nostril of the same side, have been taken away. By turning aside the lateral cartilages the septal one will appear in the middle line.

The upper lateral cartilage (fig. $7,{ }^{2}$ ) is Hattened, and is somewhat triangular in form. Posteriorly it is attached to the nasal and upper maxillary bones; and anteriorly it meets the one of the opposite side for a short distance above, but the two are separated below by an interval, in which the cartilage of the septum appears. Inferiorly the lateral cartilage

1 Oftentimes there is a different arrangement of these veins. In such case the external jugular is continued from the occipital (half or all) and posterior auricular veins; whilst the temporal and internal inaxillary veins unite to form a trunk (tempo-maxillary), which receives the facial below the jaw, and opens into the internal jugular vein opposite the upper horder of the thyroid eartilage. When this condition exists, the temporo-maxillary vein accompanies the external carotid artery 
is contiguous to the cartilage of the aperture, and is connected to it by fibrous tissue.

The cartilage of the aperture (fig. 7) forms a ring around the opening of the nose except behind. It has not any attachment direetly to bone; but it is united above to the lateral cartilage by fibrous tissue, and below with the dense teguments forming the margin of the aperture of the nostril.

Fig. 7.

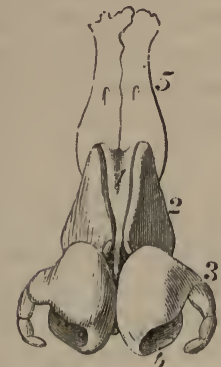

1. Triangular septal cartilage.

2. Upper lateral cartilage.

3. Lower lateral, or the cartilage of the a perture, the outer part.

4. Inner part of the cartilage of the a perture.

5. Nasal bone.

Lateral Cartilages of the Nose.

The part of the cartilage $\left({ }^{3}\right)$ which bounds the opening externally, is narrow and pointed behind, where it ends in two or three small pieces of cartilage-cartilagines minores vel sesamoidea; but swells out in front where it touches its fellow, and forms the apex of the nose.

The inner part $\left({ }^{4}\right)$ projects backwards along the septum of the nose nearly to the superior maxillary bone; it assists in the formation of the partition between the nostrils, and extends below the level of the septum nasi.

The Appendages of the Eye include the eyebrow, the eyelid, and the lachrymal apparatus. Some of these can be examined now on the opposite side of the face. The apparatus for the tears will be dissected after the orbit has been completed.

The eyebrow (supercilium) is a curved eminence just above the eye, which is placed over the orbital arch of the frontal bone. It consists of thickened integuments, and its prominence is in part due to the subjacent oroicularis palpebrarum. It is furnished with long coarse hairs, which are directed outwards, and towards one another.

The eyelids are two movable semilunar parts in front of the eye, which can be approached or separated over the eyeball. The upper lid is the largest and the most movable, and descends below the middle of the eyeball when the two meet; it is also provided with a special muscle to raise it. The interval between the open lids is named fissura palpebrarum. Externally and internally they are united by a commissure or canthus.

The free margin is thicker than the rest of the lid. and is semilunar in form; but towards the inner side, about a quarter of an inch from the commissure, it becomes straighter. At the spot where the two parts join is a small white eminence (fig. $13,{ }^{1}$ ) the papilla lachrymalis: and in this is the punctum lachrymale, or the opening of the canal for the tears.

This margin is providerl anteriorly with the eyelashes, and near the posterior edge with a row of small openings of the Meibomian glands ; but both the cilia and the glands are absent from the part of the lid which is internal to the opening of the punctum lachrymale. The free margin 
of each lid is sharp at the anterior edge where it touches its fellow ; but is sloped at the posterior, so as to leave an interval between it and the eyeball for the passage inwards of fluid.

The eyelashes (cilia) are two or more rows of curved hairs, which are fixed into the anterior edge of the free border of the lid ; they are largest in the upper lid, and diminish in length from the centre towards the sides. The cilia are convex towards one another, and cross when the lids are shut.

The Structure of the Eyelids. Each lid consists fundamentally of a piece of cartilage attached to the bone by ligaments. Superficial to this framework are the integuments with a layer of fibres of the orbicularis palpebrarum, and beneath it the mucous lining of the conjunctiva. The upper lid includes also the tendon of the levator palpebra. Vessels and nerves are contained in the lids.

Dissection. The student may learn the structure of the lids on the left side, on which the muscles are dissected. The bit of tow or wool may remain beneath the lids; and the palpebral part of the orbicularis muscle is to be thrown inwards by an incision around the margin of the orbit. In raising the muscle care must be taken of the thin membranous palpebral ligament beneath, and of the vessels and nerves of the lid.

Orbicularis palpebrarum. The palpebral fibres of this muscle form a pale layer which reaches the free edge of the eyelids (p. 36). A thin stratum of areolar tissue without fat unites the muscle with the skin.

The palpebral ligament is a stratum of fibrous membrane, which is continued from the margin of the orbit to join the lower or free elge of each tarsal cartilage. At the inner part of the orbit the ligament is thin and loose, but at the outer part it is somewhat thicker and stronger.

The tarsal cartilages, one for each eyelid, are elongated transversely, and give strength to the lids. Each is fixed internally by the ligament of the eyelids, and externally by a fibrous band-external tarsal ligament, to the outer part of the orbit. The margin corresponding with the edge of the lid is free, and thicker than the rest of the cartilage. On the inner surface each cartilage is lined by the mucous membrane or conjunctiva.

The cartilages are not alike in the two lids. In the upper eyelid, where the cartilage is largest, it is erescentic in shape, and is about half an inch wide in the centre; and to its fore part the tendon of the levator palpebra is attached. In the lower lid the cartilage is a narrow band, about two lines broad, with borders nearly straight.

Ligament of the eyelids (tendo palpebrarum, internal tarsal ligament) is a small fibrous band at the inner part of the olbit, which serves to fix the lids, and is attached to the anterior margin of the lachrymal groove in the upper jaw. It is about a quarter of an inch long, and divides into two processes, which are united with the tarsal cartilages, one to each. This ligament crosses the lachrymal sae, to which it gives a fibrous expansion ; and the fleshy fibres of the orbicularis palpebrarum arise from it.

The Meibomian glands or follieles are placed in grooves on the ocular surface of the tarsal cartilages. 'They extend, parallel to one another, from the thick towards the opposite margin of the cartilage; and their number is about thirty in the upper, and twenty in the lower lid. The apertures of the glands open in a line on the free border of the lid near the posterior elge.

Each gland is a small yellowish tube, closed at one end, and having 
minute lateral cxcal appendages connected with it. Each contains a sebaceous secretion, and is lined by flattened epithelium.

If the palpebral ligament be cut through in the upper lid, the tendon of the levator palpebra will be seen to be inserted into the fore part of the tarsal cartilage by a wide aponeurotic expansion.

The conjunctiva, or the mucous membrane, lines the interior of the eyelids, and covers the anterior part of the ball of the eye. Inside the lids it is inseparably united to the tarsal cartilages, and has numerous fine papillæ. At the free margin of the lis this membrane joins the common integuments. Through the lachrymal canals and sac it is continuous with the pituitary membrane of the nose.

At the inner commissure of the eyelids the conjunctiva forms a prominent and fleshy-looking body-caruncula lachrymalis (fig. $13,{ }^{4}$ ), which contains a group of mucous follicles, and has a few minute hairs on its surface. External to the caruncle is a small vertical fold of the nucous membrane-plica semilunaris; this extends to the ball of the eye, and represents the membrana nictitans of birds.

Bloodvessels of the eyelids. The arteries of the eyelids are furnished by the oplithalmic artery, and come from the palpebral and lachrymal branches :-

The palpebral arteries, one for each eyelid, run outwards from the inner canthus, lying between the tarsal cartilage and the tendon of the special elevator in the upper lid, and between the cartilage and the palpebral ligament in the lower lid; and they anastomose externally with the lachrymal artery. From the arch that each forms, branches are distributed to the eyelids.

The lachrymal artery furnishes an offset to each lid to form arches with the palpebral arteries, and then perforates the palpebral ligament at the outer part of the orbit to end in the upper lid.

The veins of the lids open into the firontal and angular veins at the root of the nose (pp. 21, 40).

The nerves of the eyelids are supplied from the ophthalmic and facial nerves.

The branches of the ophthalmic nerve (of the fifth) which give offsets to the upper lid, are the following: lachrymal, near the outer part ; supraorbital, abont the middle; and supra-trochlear and infra-trochlear at the inner side (pp. 42,54). In the lower eyelid, about its middle, is a palpebral branch of the superior maxillary trunk of the fifth nerve.

Branches of the facial nerve (p. 48) enter both lids at the outer part, and supply the orbicularis muscle; they communicate with the offsets of the fifth nerve.

External Ear. The outer ear consists of a trumpet-shaped structure, named pinna or auricle, which receives the undulations of the air ; and of a tube-meatus auditorius, which conveys them to the inner ear. The pinna may be examined on the left side of the head: but the anatomy of the meatus will be described with the ear.

The pinna, or auricle, is an uneven piece of yellow fibro-cartilage, which is covered with integument, and is fixed to the margin of the meatus auditorius externus. It is of an oval form, with the margin folded and the larger end placed upwards.

The surface next the head is generally convex ; but the opposite is excavated, and presents the undermentioned elevations and depressions. In the centre is a deep hollow named concha, which is wide above but narrow 
below ; it conducts to the meatus anditorius. In front of the narrowed part of the hollow is a projection of a triangular shape-the tragus, which has some hairs on the under surface; and on the opposite side of the same narrow end, rather below the level of the tragus, is placed another projection-the antitragus.

The round rim-like margin of the ear, which extends into the concha, is ealled the helix; and the depression internal to it is the groove or fossa of the helix. Within the helix, between it and the concha, is the large

Fig. 8.
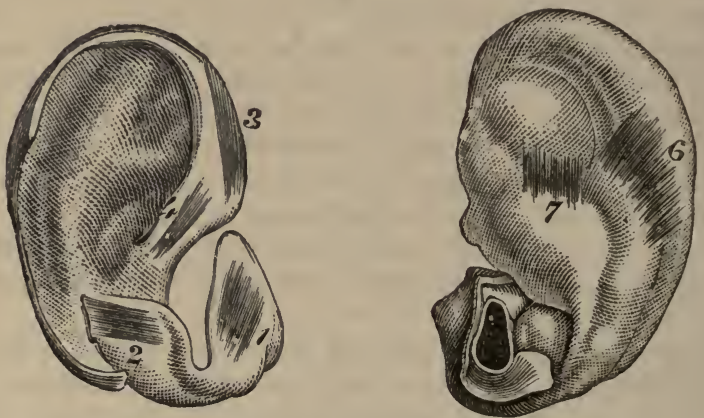

Muscies on the Outer Surface of the Ear Cartilage.

1. Muscles of the tragus.

2. Muscles of the antitragus.
3. Large muscle of the hellx.

4. Small muscle of the helix.

Muscles on the Inver SURface of the Ear Cartilage

6. Transverse inuscle.

7. Oblique muscle (Tod) sometimes seen.

eminence of the antikelix, which presents at its upper part a well-marked depression, the foss a of the antihelix.

Inferiorly the external ear is terminated by a soft pendulous part, the lobule.

The special muscles of the pinna, which extend from one part of the cartilage to another, are very thin and pale. Five small muscles are to be recognized; and these receive their names for the most part from the several eminences of the external ear.

Dissection. In seeking the small auricular muscles, let the integuments be removed only over the spot where each muscle is said to be placed. A sharp knife and a good light are necessary for the display of the muscular fibres. Occasionally the dissector will not find one or more of the number described below.

The muscle of the tragns (fig. $8,{ }^{1}$ ) is always found on the external aspect of the process from which it takes its name. The fibres are short, oblique, or transverse, and extend from the outer to the inner part of the tragus.

The muscle of the antitragus (fig. $8,{ }^{2}$ ) is the best marked of all. It arises from the outer part of the antitragus, and the fibres are directed upwards to be inserted in to the pointerl extremity of the antihelix.

The small muscle of the helix (fig. $8,{ }^{4}$ ) is often indistiuct or absent. It is placed on the part of the rim of the ear that extends into the concha.

The large muscle of the helix (fig. $8,{ }^{3}$ ) arises above the small muscle 
of the same part, and is inserted into the front of the helix, where this is about to eurve backwards. It is usually present.

The transverse muscle of the auricle (fig. $8,{ }^{6}$ ) forms a wide layer, which is situate at the back of the ear in the depression between the helix and the convexity of the concha. It arises from the convexity of the cartilage forming the concha, and is inserted into the back of the helix. The muscle is mixed with much fibrous tissue, but it is well seen when that tissue is removed.

Actions. These muscles are said to alter slightly the condition of the outer ear; the muscles of the helix assisting, and those of the tragus and antitragus retarding the passage of sonorous undulations to the meatus.

Dissection. The pinna may now be detached by cutting it close to the bone. When the integuments are entirely taken off, the eartilage of the pinna will be apparent; but in removing the integuments, the lobule of the ear, which consists only of skin and fat, will disappear as in fig. 8 .

The cartilage of the pinna (fig. 8) resembles much the external ear in form, and presents nearly the same parts. 'The rim of the helix subsides posteriorly in the antihelix about the middle of the pinna; whilst anteriorly a small process projeets from it, and there is a fissure near the projection. The antihelix is divided about two-thirds down into two pieees; one of these is pointed, and is joined by the helix, the other is continued into the antitragus. On the posterior aspect of the concha is a strong vertical process of cartilage.

Inferiorly the eartilage is fixed to the margin of the external auditory aperture in the temporal bone, and forms part of the meatus auditorius ; but it does not give rise to a complete tube, for at the upper and outer part the eanal is elosed by fibrous tissue.

In the piece of eartilage forming the under part of the meatus are two fissures (Santorini), one is at the base of the tragus, the other passes from before backwards.

Some ligaments connect the pinna with the head, but others pass from one point of the eartilage to another.

The external ligaments are condensed bands of fibrous tissue, and are two in number, anterior and posterior. The anterior fixes the fore part of the helix to the root of the zygoma. 'The posterior passes from the back of the eoncha to the mastoid process. The chief special ligament crosses the interval between the tragus and the beginning of the helix, and completes the tube of the meatus.

The FACIAL Nerve (portio dura, fig. 9), or the seventh cranial nerve, confers contraetility on the museles of the face. Numerous communications take place between it and the fifth nerve; the chief of these are found above and below the orbit, and over the body of the lower jaw.

Dissection. The facial nerve is to be displayed on the right side of the face if there is time suffieient before the body is turned, otherwise it is to be omitted for the present (see p. 17). Some of the nerve is concealed by the parotid gland, but the greater part is anterior to the glandular mass.

To expose the ramification of the nerve beyond the parotid gland, let the skin be raised firom the face in the same manner as on the left side. The different branches are then to be sought as they escape from beneath the anterior border of the gland, and are to be followed forwards to their termination.

The highest branches to the temple have been already partly dissected above the zygomatic arch; and their junctions with the temporal branch 
of the superior maxillary and with the supra-orbital nerve have been seen. Other still smaller branches are to be traced to the outer part of the orbit, where they enter the eyelids and communicate with the other nerves in the lids; as these cross the malar bone, a junction is to be found with the subcutaneous malar nerve of the fifth.

With the duct of the parotid are two or more large branches, which are to be followed below the orbit to their junction with the infra-orbital, nasal, and intra-trochlear nerves.

The remaining branches to the lower part of the face are smaller in size. One joins with the buccal nerve at the lower part of the buccinator muscle; and one or two others are to be traced forwards to the lower lip, and to the labial branch of the inferior dental nerve.

To follow backwards the trunk of the nerve through the gland, the integuments should be taken from the surface of the parotid as on the other side, and the gland should be removed piece by piece. In this proceeding the small branches of communieation of the great auricular nerve with offsets of the facial, and the deep branches from the facial to the auriculotemporal nerve, are to be sought.

Lastly, the first small branches of the facial to the ear and the digastric and stylo-hyoid muscles, are to be looked for close to the base of the skull before the nerve enters the parotid.

The Nerve outside the Skull (fig. $9,{ }^{15}$ ). The nerve issues from the stylo-mastoid foramen, after traversing the aqueduct of Fallopius, and furnishes immediately the three following small branches :-

The posterior auricular branch (fig. $9,{ }^{*}$ ) turns upwards in front of the mastoid process, where it communicates with an offset of the great auricular, and is said to be joined by a branch to the ear from the pneumogastric (cranial) nerve; it ends in auricular and mastoid offsets (p. 23).

The branch to the digastric muscle generally arises in common with the next. It is distributed by many offsets to the posterior belly of the muscle near the skull.

The branch to the stylo-hyoideus is a long slender nerve, which is directed inwards and enters its muscle about the middle. This branch communicates with the sympathetic nerve on the external carotid artery.

As soon as the facial nerve has given off those branches, it is directed forwards through the gland, and divides near the ramus of the jaw into two large trunks-temporo-facial and cervico-facial.

The TEMPORO-FACIAL TRUNK furnishes off'sets to the side of the head and face, whose ramifications extend as low as the meatus auditorius. As this trunk crosses over the external carotid artery, it sends downwards branches to join the auriculo-temporal portion of the inferior maxillary nerve; and in front of the ear it gives some filaments to the tragus of the pinna. Three sets of terminal branches, temporal, malar, and infra-orbital, are derived from the temporo-facial part.

The temporal branches ascend obliquely over the zygomatic arch to enter the orbicular muscle, the corrugator supercilii, and the anterior belly of the occipito-frontalis; they are united with offsets of the supra-orbital nerve $\left({ }^{6}\right)$. The attrahens aurem muscle receives a branch from this set; and a junction takes place above the zygoma with the temporal branch of the superior maxillary nerve $\left({ }^{10}\right)$.

The malar branches are directed to the outer angle of the orbit, and are distributed to the orbicularis muscle. In the eyelids communications take place with the palpebral filaments of the fifth nerve; and near the 
outer part of the orbit, with the small subcutaneous malar branch of the superior maxillary nerve $\left({ }^{9}\right)$.

The infra-orbital branches are longer than the rest, and are furnished to the muscles and the integument between the eye and moutl. Close to the orbit, and beneath the elevator of the upper lip, a remarkable communication-infra-orbital plexus, is formed between these nerves and the

Fig. 9.

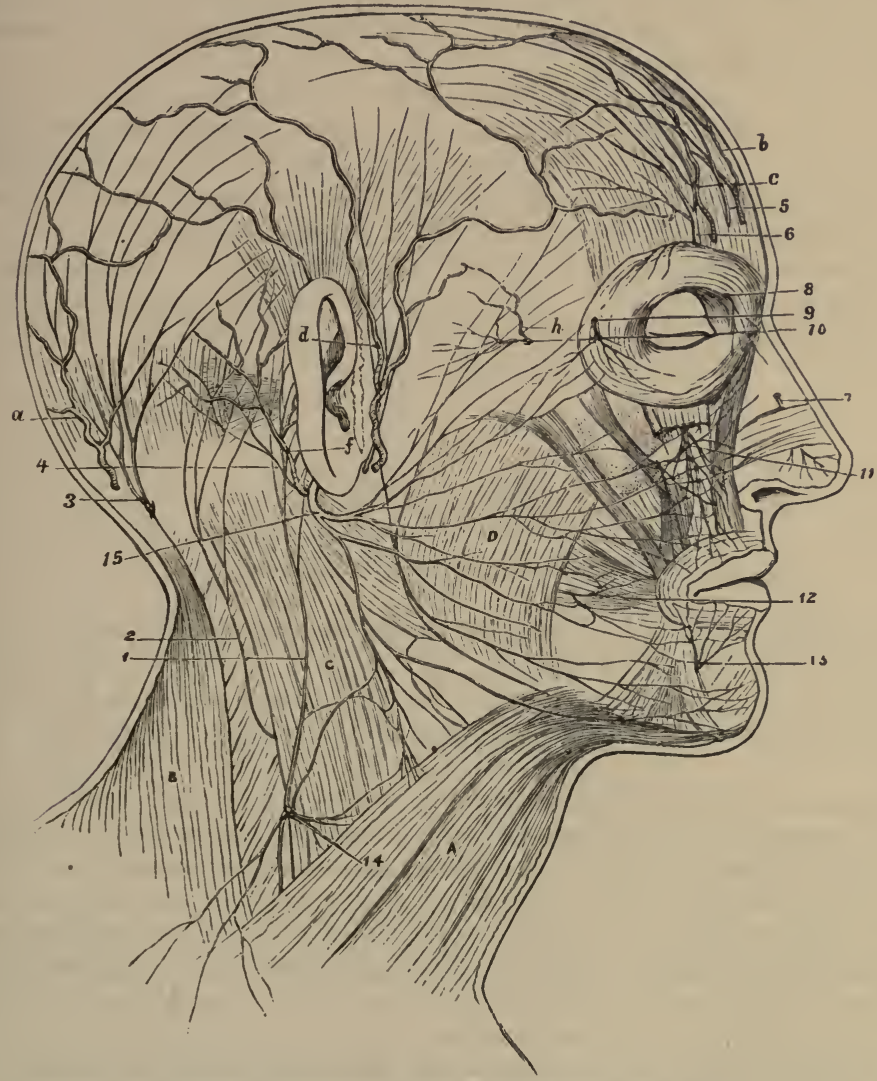

Cutaxeous Braxches of the Fifth Nerte is the Face.

7. Nasal nerve.

8. Infra-trochlear.

9. Subcutaneous malar.

11. Infra-orbital.

12. Buccal.
13. Labial of inferior dental.

15. Facial or seventh cranial, sending back the posterior auricular branch, 4 , and forwards its numerous off'sets to join the branches of the fifth nerve above enumerated.

infra-orbital branches of the superior maxillary $\left({ }^{11}\right)$. After crossing the branches of the fitth nerve, some small offsets of the facial nerve pass inwards to the side of the nose, and others upwards to the inner angle of the orbit, to supply the muscles, and to join the nasal $\left(^{7}\right)$ and infra-trochlear $\left({ }^{8}\right)$ branches of the ophthalmic nerve. 
The CERVICO-FACIAL is smaller than the other trunk, and distributes nerves to the lower part of the face and the upper part of the neck. Its highest branches join the lowest offsets of the temporo-facial nerve, and thus complete the network on the face. This trunk, whilst in the parotid, gives twigs to the gland, and is united with the great auricular nerve. The terminal branches distributed from it are, buecal, supra-maxillary, and infra-maxillary.

The buccal branches pass forwards towards the angle of the mouth, giving offsets to the bucciuator musele, and terminate in the orbicularis oris. On the buccinator they join the branch $\left({ }^{12}\right)$ of the inferior maxillary nerve to that muscle.

The supra-maxillary branches course inwards above the base of the lower jaw to the middle line of the chin, and supply the muscles and the integument between the ehin and mouth. Beneath the depressor anguli oris the branches of the facial join offsets of the labial branch of the inferior dental nerve $\left({ }^{13}\right)$ in their course to the middle line.

The infra-maxillary branches lie below the jaw, and are distributed to the upper part of the neck. The anatomy of these nerves will be given with the dissection of the anterior triangle of the neck.

\section{SECTION IV. \\ DISSECTION OF THE ORBIT.}

Dirertions. The orbit should be learnt on that side on which the muscles of the face have been seen.

Position. In the examination of the cavity the head is to be placed in the same position as for the dissection of the sinuses of the base of the skull.

Dissection. For the display of the contents of the orbit, it will be necessary to take away the cotton wool from beneath the eyelids. To remove the bones forming the roof of the space, two cuts may be made with a saw through the margin of the orbit, one being placed at the outer, the other near the inner angle of the cavity; and these should be continued backward with a chisel, along the roof of the orbit, so as to meet near the optic foramen. The piece of bone included in the incisions is now to be tilted forwards, but is not to be taken away.

Afterwards the rest of the roof of the orbit, which is formed by the small wing of the sphenoid bone, is to be cut away with the bone forceps, except a narrow ring around the optic foramen; and any overhanging bone on the outer side, which may interfere with the dissection, may be likewise removed. During the examination of the eavity the eye is to be pulled gently forwards.

'The periosteum of the orbit, which has been detached from the bone in the dissection, surrounds the eontents of the orbital cavity, and joins the dura mater of the brain through the sphenoidal fissure. It encases the contents of the orbit like a sac, and adheres but loosely to the bones. Apertures exist posteriorly in the membrane for the entrance of the different nerves and vessels; and laterally prolongations of the periosteum accompany the vessels and nerves leaving the cavity. 
Dissection. The periosteum is next to be divided along the middle of the orbit, and to be taken away. After the removal of a little fat, the following nerves, vessels, and muscles come into view ; but it is not needful to remove much of the fat in this stage of the dissection.

The frontal nerve and the supra-orbital vessels lie in the centre; the lachrymal nerve and vessels close to the outer wall; and the small fourth nerve at the back of the orbit: all these nerves enter the cavity above the muscles. The superior oblique muscle is recognized by the fourth nerve entering it: the levator palpebræ and superior rectus lie beneath the frontal nerve; and the external rectus is partly seen below the lachrymal nerve. In the outer part of the orbit, near the front, is the lachrymal gland.

The frontal and lachrymal nerves should be followed forwards to their exit from the orbit, and backwards with the fourth nerve, through the sphenoidal fissure, to the wall of the cavernous sinus. In tracing them back, it will be expedient to remove the projecting clinoid process, should this still remain ; and some care will be required to follow the lachrymal nerve to its commencement.

Contents of the orbit. The eyeball and the lachrymal gland, and a great quantity of granular fat, are lodged in the orbit. Connected with the eye are six muscles-four straight and two oblique; and there is also an elevator of the upper eyelid in the cavity.

The nerves in this small space are numerous, viz., the second, third, fourth, ophthalmic of the fifth, and the sixth nerve, together with the small temporo-malar branch of the superior maxillary nerve, and offsets of the sympathetic; their general distribution is as follows :-The second nerve penetrates the eyeball; the third is furnished to all the muscles of the cavity but two ; the fourth enters the superior oblique (one of the two excepted); and the sixth is spent in the external rectus muscle. The fifth nerve supplies some filaments to the eyeball with the sympathetic, but the greater number of its branches pass through the orbital cavity to the face. The ophthalmic vessels are also contained in the orbit.

The lachrymal gland (fig. 10, F) secretes the tears, and is situate in the hollow on the inner side of the external angular process of the frontal bone. It is of a lengthened form, something like an almond, and lies across the eye. From its anterior part a thin acessory piece projects beneath the upper eyelid. The upper surface is convex, and in contact with the periosteum, to which it is connected by fibrous bands that constitute a ligament for the gland; the lower surface rests on the eyeball and the external rectus muscle.

In structure the lachrymal resembles the salivary glands; and its very fine ducts, from eight to twelve in number, open by as many apertures in a semicircular line on the inner aspect of the upper eyelid towards the outer canthus.

The Fourth Nerve (fig. $10,{ }^{2}$ ) is the most internal of the three nerves entering the orbit above the muscles. After reaching this space, it is directed inwards to the superior oblique muscle, which it pierces at the orbital surface, contrary to the general mode of distribution of the nerves on the ocular surface of the muscles.

The ophthalmic trunk of the fifth nerve (fig. 10, p. 52), as it approaches the sphenoidal fissure, furnishes from its inner side the nasal branch, and then divides into the frontal and lachrymal branches; the 
former passes into the orbit between the heads of the external rectus, but the other two lie, as before said, above the muscles.

The frontal nerve (fig. $10,{ }^{2}$ ) is close to the outer side of the fourth as it enters the orbit, and is much larger than the lachrymal branch. In its course to the forehead the nerve lies along the middle of the orbit, and supplying anteriorly a supra-trochlear branch $\left({ }^{4}\right)$, leaves that eavity by the supra-orbital notch. Taking the name supra-orbital, it ascends on the forehead, and supplies the external part of the head (p.21).

Fig. 10.

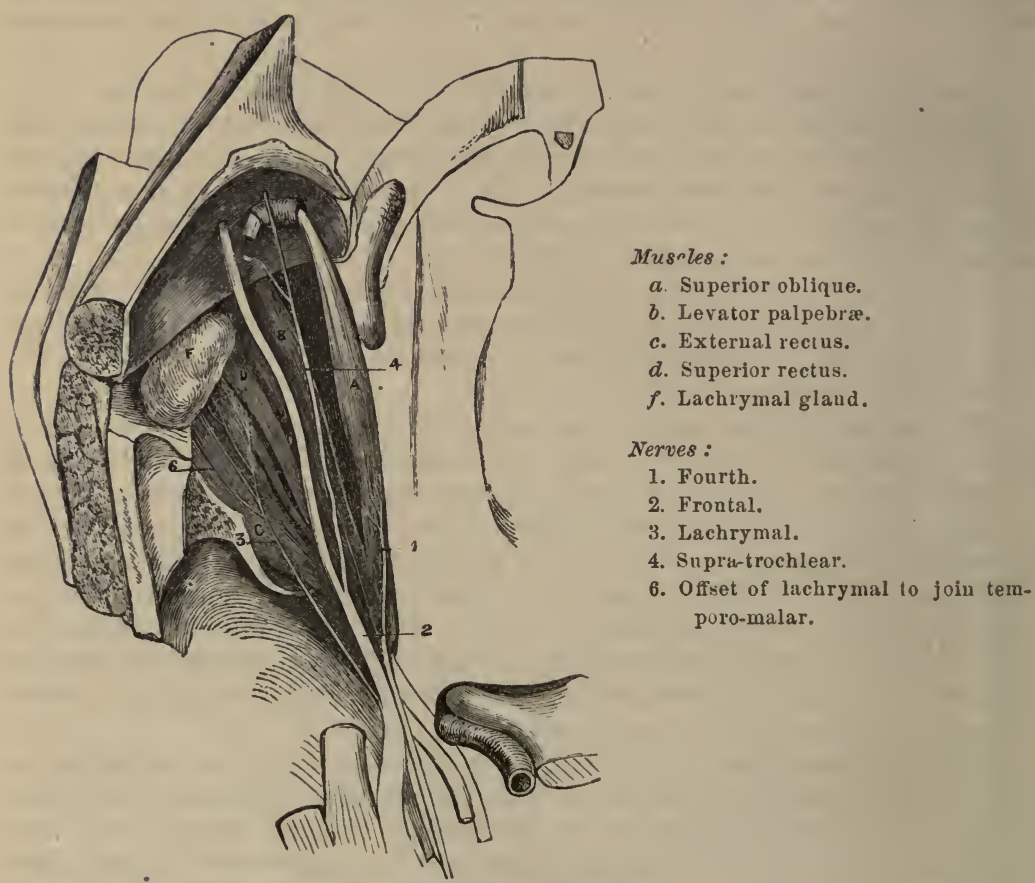

First VIeW OF THE ORBIT (Illustrations of Dissections).

Whilst in the noteh the nerve gives palpebral filaments to the upper lid. The supra-trochlear branch $\left({ }^{4}\right)$ passes inwards above the pulley of the upper oblique muscle, and leaves the orbit to end in the eyelid and forehead (p. 23). Before the nerve turns round the margin of the frontal bone, it sends downwards a branch of communication to the infra-trochlear branch of the nasal nerve. Frequently there are two supra-trochlear branches; in such instances one arises near the back of the orbit.

The lachrymal neve (fig. $10{ }^{3}$ ) after entering the orbit in a separate tube of the dura mater, is direeted forwards in the outer part of the eavity, and beneath the lachrymal gland to the upper eyelicl, where it pierees the palpebral ligament, and is distributed to the structure of the lid.

The nerve furnishes brancles to the lachrymal gland; and near the gland it sends downwards one or two small filaments $\left(^{6}\right)$ to communicate with the temporo-malar or orbital branch of the superior maxillary nerve. 
Occasionally it has a communicating filament behind with the fourth nerve.

The nasal nerve is not visible at this stage of the dissection: it will be noticed afterwards at p. 43.

Dissection. Divide the frontal nerve about its middle, and throw the ends forwards and backwards : by raising the posterior part of the nerve, the separate origin of the nasal branch from the ophthalmic trunk will appear. The lachrymal nerve may remain uncut.

The LEVATOR PALPEBRæ SUPERIORIS (fig. 10, B) is the most superficial muscle, and is attached posteriorly to the roof of the orbit in front of the optic foramen. The muscle widens in front, and bends downwards in the eyelid to be inserted by a wide tendon into the fore part of the tarsal cartilage.

By one surface the muscle is in contact with the frontal nerve and the periosteum; and by the other, with the superior rectus muscle. If it is cut across about the centre a small branch of the third nerve will be seen entering the posterior half at the under surface.

Action. The lid-cartilage is made to glide upwards over the ball by this muscle, so that the upper edge is directed back and the lower forwards, the teguments of the lid being bent inwards at the same time. If the eyeball is directed down, the movement of the lid is less free, because the conjunctiva is put on the streteh.

The ReCTUS SUPERIor (fig. $10,{ }^{D}$ ) is the upper of four muscles that lie arouud the globe of the eye. It arises from the upper part of the optic foramen, and is connected with the other recti muscles around the optic nerve. In front the fleshy fibres end in a tendon, which is inserted, like the other recti, into the sclerotic coat of the eyeball about a quarter of an inch behind the transparent cornea.

The under surface of the muscle is in contact with the globe of the eye, and with some vessels and nerves to be afterwards seen; the other surface is covered by the precerling muscle. The action of the muscle will be given with the other recti (p. 57).

The superior oblique Muscle (fig. $10,{ }^{\wedge}$ ) is thin and narrow, and passes through a fibrous loop at the inner angle of the orbit before reaching the eyeball. The muscle arises behind from the inner part of the optic foramen, and ends anteriorly in a rounded tendon, which, after passing through the loop before referred to (fig. 11) is reflected backwards and outwards between the superior rectus and the globe of the eye, and is inserted into the sclerotic coat behind the middle of the ball.

The fourth nerve is supplied to the orbital surface of the muscle, and the nasal nerve lies below it. The thin insertion of the muscle lies between the superior and the external rectus, and near the tendon of the inferior oblique.

The pulley, or trochlea (fig. 11), is a fibro-cartilaginous ring nearly a quarter of an inch wide, which is attached by fibrous tissue to the depression of the frontal bone at the inner angle of the orbit. A fibrous layer is prolonged from the margins of the pulley on the tendon; and a synovial membrane lines the ring, to facilitate the movement of the tendon through it. To see the synovial membrane and the motion of the tendon, this prolongation must be cut away.

For the use of the muscle, see the description of the inferior oblique, p. 59 . 
Dissection. The superior rectus musele is next to be divided about the middle, and turned backwards (fig. 11), when a branch of the third nerve to its under surface will be found. At the same time the nasal nerve and the ophthalmic artery and vein will come into view as they cross inwards above the optic nerve: these should be traced forwards to the inner angle, and backwards to the posterior part of the orbit.

By taking away the fat between the optic nerve and the external rectus, at the back of the orbit, the student will find easily fine nerve-threads (ciliary) with small arteries lying along the side of the optic nerve; and by tracing the ciliary nerves backwards, they will guide to the small lentieular ganglion (the size of a pin's head) and its branches. 'The dissector should find then two branches from the nasal and third nerves to the ganglion: the nasal branch is slender, and enters the ganglion behind, and that of the third nerve, short and thick, joins the lower part.

Lastly, the student should separate from one another the nasal, third, and sixth nerves, as they pass between the heads of the external rectus muscle into the orbit.

The THIRD NERVE is placed highest in the wall of the cavernous sinus (fig. $4,{ }^{3}$ ); but at the sphenoidal fissure it descends below the fourth, and the two superficial branches (frontal and lachrymal) of the ophthalmic nerve. It eomes into the orbit between the heads of the outer rectus, having previously divided into two parts.

The "pper piece (fig. $11,{ }^{8}$ ), the smallest in size, ends in the under surface of the levator palpebrie and superior rectus museles.

The lower piece supplies some of the other muscles, and will be dissected afterwards (p. 57).

The nasal branch of the ophthalmic nerve (fig. $11,{ }^{1}$ ) enters the orbit between the heads of the rectus, lying between the two parts of the third nerve. In the orbit the nerve is directed obliquely inwards to reach the anterior of the two foramina in the inner wall. Passing through this aperture with the anterior ethmoidal (nasal) artery, the nerve appears in the cranium at the outer margin of the eribriform plate of the ethmoid bone. Finally, it enters the nasal eavity by an aperture at the front of the cribriform plate : and after passing behind the nasal bone, it is directed outwards between that bone and the cartilage, to end on the outer side of the nose.

In the orbit the nasal crosses over the optic nerve, but beneath the superior rectus and levator palpebra muscles, and lies afterwards below the superior oblique; in this part of its course it furnislies the following branches :-

The branch to the lenticular ganglion $\left(^{5}\right)$, is about half an inch long and very slender, and arises as soon as the nerve comes into the orbit: this is the long root of the lenticular ganglion.

Long ciliary branches. As the nasal erosses the optie nerve, it supplies two or more eiliary branches (fig. 11) to the eyeball. These lie on the inner side of the optie, and join the ciliary branches of the lentieular ganglion.

The infra-trochlear branch $\left({ }^{2}\right)$ arises as the nasal nerve is about to leave the cavity, and is direeted forwards below the pulley of the superior oblique muscle, to end in the upper eyelid, the conjunctiva, and the side of the nose. Before this branch leaves the orbit it receives an off'set of communication from the supra-trochlear nerve.

In the nose (fig. 34). Whilst in the nasal cavity the nerve furnishes 
branches to the lining membrane of the septum narium and outer wall; these will be subsequently referred to with the nose.

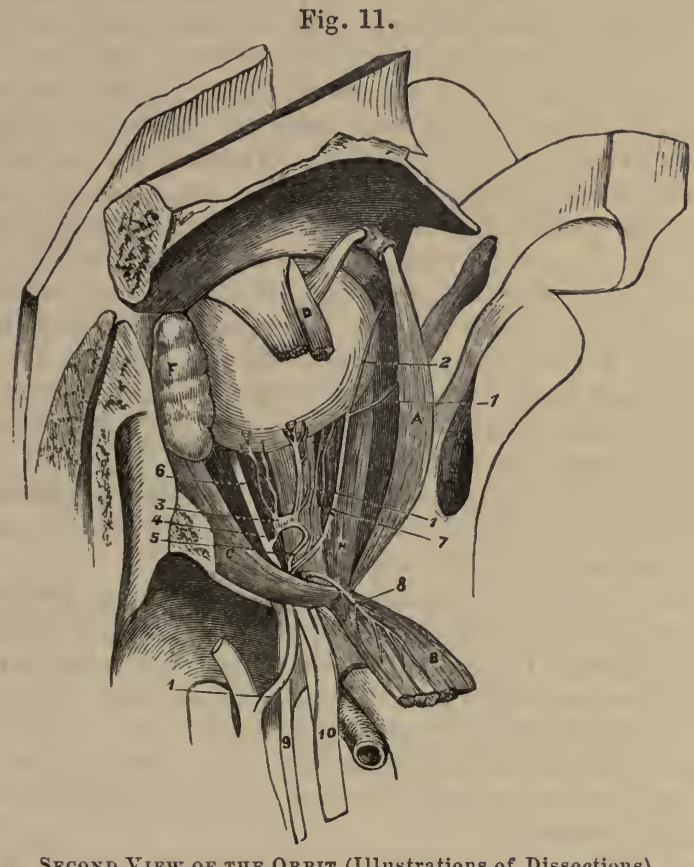

Muscles :

a. Superior oblique.

b. Levator palpebrie and upper rectus thrown back together.

c. External rectus.

d. Fore part of upper rectus.

$f$. Lachrymal gland.

\section{Nerves:}

1. Nasal nerve beginning outside of the orbit.

2. Its infra-trochlear branch.

3. Lenticular ganglion:-

4. Its branch to the third nerve;

8. Its branch to the nasal nerve (too large).

6. Branch of third to inferior oblique muscle.

7. Ciliary branches of the nasal nerve.

8. Upper branch of the third.

9. Sixth nerve.

10. Third nerve, outside the orbit.

Termination of the nasal nerve (fig. 9). After the nerve becomes cutaneous on the side of the nose, as seen in the dissection of the facial nerve (p. 47), it descends beneath the eompressor naris muscle, and ends in the integuments of the wing and tip of the nose.

The OPHTHALMIC or LENTICULAR GANGLION of the sympathetic nerve (fig. 11, ${ }^{3}$ ) is a small round body, of the size of a pin's head, and of a slight red color. It is placed at the back of the orbit between the optic nerve and the external rectus, and eommonly on the outer side of, and close to the ophthalmic artery. By its posterior part the ganglion has branches of communication with other nerves (its roots); and from the anterior part proeed the ciliary nerves to the eyeball. The ganglion rommunieates with sensory, motory, and sympathetic nerves.

The offsets of communication are three in number. One, the long root $\left({ }^{5}\right)$, is the branch of the nasal nerve before noticed, which joins the 
superior angle. A second branch of considerable thickness (short root ${ }^{4}$ ) passes from the inferior angle to join the branch of the third nerve that supplies the inferior oblique muscle. And the third root is derived from the sympathetic (the cavernous plexus), either in union with the long root, or as a distinct branch to the posterior border of the ganglion.

Branches. The short ciliary nerves (fig. 11) are ten or twelve in number, and are collected into two bundles, which leave the upper and lower angles of the front of the ganglion. In the upper bundle are four or five, and in the lower, six or seven nerves. As they extend along the? optic nerve to the eyeball they occupy the outer and under parts, and communicate with the long ciliary branches of the nasal nerve.

The opHTHALMic ARTERY, a branch of the internal carotid, is transmitted into the orbit through the optic formen. At first the ressel is outside the optic nerve, but it then courses inwards, over or under the nerve, to the inner angle of the orbit, where it ends in a nasal branch (external) on the side of the nose (fig. 17, ${ }^{n}$ ), and anastomoses with the angular and nasal branches of the facial.

The branches of the artery are numerous, though inconsiderable in size, and may be arranged in three sets:-one arising outside the optic nerve, another above it, and a third set on the inner side.

'The lachrymal artery accompanies the nerve of the same name to the upper eyelid, where it ends by supplying that part, and anastomosing with the palpebral arches. It supplies branches, like the nerve, to the lachrymal gland and the conjunctiva; and it anastomoses with the middle meningeal by an offset through the sphenoidal fissures.

At the front of the orbit it sends a small branch with each of the terminal pieces of the temporo-malar nerve; and these join the temporal and transverse facial arteries.

The central artery of the retina is a very small branch which pieress the optic nerve, and so reaches its destination in the eyeball.

The supra-orbital branch arises beneath the levator palpebræ and superior rectus muscles; it then takes the course of the nerve of the same name through the notch in the margin of the orbit, and ends in branches on the forehead (p. 21). As it winds round the margin of the orbit it sup)plies the eyelid and the orbicularis muscle.

The ciliary branches are uncertain in their place of origin and enter the eyeball at the front and back :-

The posterior ciliary, about twelve in number, are continued to tlse eyeball around the optic nerve, and perforate the sclerotic coat at the posterior part. 'Two of this set (one on each side of the optic nerve), named long ciliary, pierce the sclerotic farther out than the others, and lie along the middle of the eyeball.

The anterior ciliary arteries arise from muscular branches of the ophthalmic, and perforate the sclerotic coat near the cornea: in the eycball they anastomose with the posterior ciliary. For the ending of these vessels, see the eyeball.

The muscular branches are not fixed in their place of origin ; and those to the lower muscles of ten arise together as one trunk.

The ethmoidal branches are two, anterior and posterior, and are directed through the foramina in the inner wall of the orbit:-

The posterior is the smaller of the two, and furnishing small meningeal offsets (anterior) to the dura mater of the base of the skull, descends into 
the nose cavity through the openings in the cribriform plate of the ethmoid.

The anterior branch (internal nasal) accompanies the nasal nerve to the cavity of the nose (Section XIV.), and gives likewise meningeal offsets to the dura mater, and the fore part of the falx cerebri.

The palpebral branches, one for each eyelid, generally arise together opposite the pulley of the superior oblique muscle, and then separate from one another. The arches they form have been dissected with the eyelids (p. 45).

The frontal branch turns round the margin of the orbit, and is distributed on the forehead (p. 21).

The ophthalmic vein corresponds in its course and most of its branches with the artery of the same name. It begins at the inner angle of the orbit, where it joins the facial vein, and receives tributary branches in its progress to the back of the cavity. Posteriorly it leaves the artery, and escapes from the orbit by the sphenoidal fissure between the heads of the external rectus, to end in the cavernous sinus.

The OPTIC NERVE in the orbit extends from the optic foramen to the back of the eyeball. As the nerve leaves the foramen it is surrounded by the recti muscles; and beyond that spot the ciliary arteries and nerves entwine around it. It terminates in the retinal expansion of the eye.

Dissection (fig. 12). Take away the ophthalmic artery, and divide the optic nerve about its middle, together with the small ciliary vessels and nerves. Turn forwards the eyeball, and fasten it in that position with hooks. On removing some fat the three recti muscles-inner, inferior, and outer, will appear; and lying on the two first, are the offsets of the lower branch of the third nerve.

The lower branch of the third nerve (fig. 12) supplies three muscles in the orbit. Whilst entering this space between the heads of the external rectus, it lies below the nasal, and rather above the sixth nerve. Almost immediately afterwards the nerve divides into three branches. One $\left(^{5}\right)$ enters the internal rectus; another $\left({ }^{4}\right)$ the inferior rectus; and the third $\left({ }^{3}\right)$, the longest and most external, is continued forwards to the inferior oblique muscle which it pierces at the hinder border.

Soon after its origin the last branch communicates with the lenticular ganglion, forming the short root (fig. $11,{ }^{3}$ ) of that body ; and it furnishes two or more filaments to the inferior rectus.

The sixth NERve (fig. $12,{ }^{2}$ ) lies below the other nerves, and above the ophthalmic vein, in the interval between the heads of the external rectus. In the orbit it is distributed to the external rectus muscle.

Recti Muscles. The internal $\mathrm{D}$, inferior $\mathrm{C}$, and external rectus $\mathrm{is}$ (fig. 12) are placed with reference to the eyeball as their names express. They arise posteriorly from the circumference of the optic foramen by a common attachment, which partly surrounds the optic nerve. But the external rectus differs from the others in having two heads:- The upper joins the superior rectus in the common origin. The lower and larger head blends on the one side with the inferior rectus in the common origin, and is attached in addition to a bony point on the lower border of the sphenoidal fissure, near the inner end; whilst some of its muscular fibres are connected with a tendinous band between the heads. All the muscles are directed forwards, but the lower obliquely outwards, and have a tendinous insertion into the ball of the eye about a quarter of an inch from the cornea, and in front of the transverse diameter of the ball. 
Between the heads of origin of the external rectus, the different nerves before mentioned are transmitted into the orbit, viz., the third, the nasal branch of the fifth, and the sixth, together with the ophthalmic vein.

Action. The four recti muscles, attached to the eyeball at opposite sides in front of the transverse diameter, are able to turn the pupil in opposite directions.

The inner and outer recti move the ball horizontally around a vertical axis, the former directing the pupil towards the nose and the latter towards the temple.

\section{Fig. 12.}

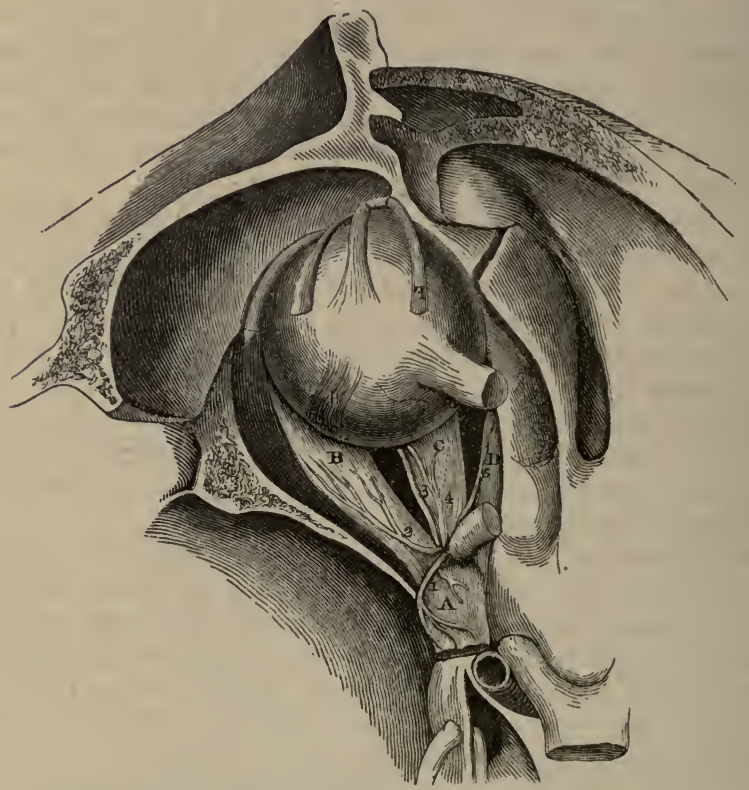

THIRD VIEW OF THE ORBIT (Illustrations of Dissections).

Muscles:

$a$. Upper rectus and levator palpebra thrown back together.

b. External rectus:

c. Inferior rectus.

d. Internal rectus.

$f$. Upper oblique cut, showing the iusertion.

$h$. Insertion of inferior oblique.

Nerves :

1. Upper branch of the hird.

2. Sixth nerve.

3. Inferior oblique branch of the third.

4. Branch of thled to inferior rectus.

5. Branch of third to inteinal rectus.

The upper and lower recti elevate and depress the fore part of the ball around a transverse axis; but as their fibres are directed obliquely outwards, the upper muscle turns the pupil up and in, and the lower musele turns it down and in.

By the simultaneous action of two contiguous recti, the ball will be moved to a point intermediate to that to which it would be directed by either muscle singly.

Dissection. By opening the optic foramen, the attachment of the reeti muscles will be more fully laid bare. 'To dissect out the inferior oblique muscle, let the eyeball be replaced in its natural position ; then by taking 
away the conjunctival lining of the lower eyelid near the inner part of the orbit, and removing some fat, the muscle will appear beneath the eyeball, bending from the inner to the outer side; it may be followed outwards to its insertion into the ball.

The inferior oblique MUSCle (fig. 12, H) is situate near the anterior margin of the orbit, and differs from the other muscles in being directed across, instead of parallel to the axis of the orbit. It arises from the superior maxillary bone betwixt the margin of the orbit and the groove for the lachrymal sac. From this spot the muscle passes outwards between the inferior rectus and the bone, and between the eyeball and the external rectus, to be inserted into the sclerotic coat between the outer and upper recti.

The borders of the muscle look forwards and backwards, and the posterior receives the branch of the third nerve. The tendon of insertion is near that of the superior oblique muscle, but rather closer to the optic nerve.

Action. The oblique muscles rotate the eyeball around an antero-posterior axis, and are supposed to be used in maintaining the parallelism of the axes of the two eyes.

The upper muscle, acting by itself, would rotate the ball in such a way as to cause the inner end of the transverse axis to sink, and the pupil to be directed down and out, as in looking to the shoulder.

The lower muscle would produce by itself rotation of the ball in the opposite direction, viz., the inner end of the transverse axis would be raised, and the pupil would be inclined up and out, as in looking to the outer part of the orbit.

During life the rotary movement is supposed to be chiefly employed in controlling the oblique action of the upper and lower recti. For instance, as the upper rectus alone would turn the pupil upwards and too much inwards, the inner movement will be counteracted by the rotation out of the inferior oblique. And as the lower rectus will incline the pupil down and too much in, the motion inwards will be checked by the rotation out of the superior oblique.

Dissection. To expose the small tensor tarsi muscle, the palpebral ligament uniting the eyelids to the margin of the orbit is to be cut through, where this has not been done; but the lids must be left attached at the inner commissure by means of the tendo palpebrarum. By looking to the posterior aspect of the tendo palpebrarum, after the lids have been placed across the nose, the pale fibres of the tensor tarsi will be recognized.

The TENSOR TARSI MUSCLE arises from the ridge on the os unguis, and slightly from the bone behind the ridge. Its fibres are pale, and form a very small flat band, behind the ligamentum palpebrarum, which divides like that structure into a slip for each eyelid. In the lid the slip lies by the side of the lachrymal canal, and blends with the fibres of the orbicularis allong the free margin of the tarsal cartilage.

Action. By its contraction the muscle draws inwards and backwards the puncta of the lids, so as to favor the reception and passage of the tears.

Dissection. A small nerve, the temporo-malar or orbital branch of the superior maxillary trunk, lies along the outer angle of the floor of the orbit, and may be brought into view after the removal of the eyeball and its muscles. This nerve is very soft and easily broken, and is covered by pale fleshy fibres (orbitalis muscle). Two branches, temporal and malar, 
are to be traced forwards from it : and the junction of a filament of the lachrymal nerve with the former is to be sought in the bone; the outer wall of the orbit may be eut away, bit by bit, to follow the temporal branch to the surface of the head.

The temporo-malar or orbital branch of the superior maxillary nerve (fig. 26) arises in the spheno-maxillary fossa, and divides at the back of the orbit into malar and temporal branches, which ramify in the face and the side of the head with companion vessels.

The malar branch (r. subcutaneus malæ) is directed forwards through a foramen in the malar bone: after emerging from its foramen, this branch supplies the orbicularis, and communicates with the facial nerve (p. 48).

The temporal branch ascends in a groove in the bone on the outer wall of the orbit; and being joined by a filament from the lachrymal nerve, passes into the temporal fossa through a foramen in the malar bone: it is then directed upwards between the temporal muscle and the skull, and perforates the temporal fascia near the orbit (p. 23).

Orbitalis muscle. At the lower and outer angle of the orbit a thin fleshy layer is sometimes well seen. The fibres cross the spheno-maxillary fissure, being attached to the edges, and are pierced by the malar branch of the temporo-malar nerve.

Lachrymal Apparatus (fig. 13). The lachrymal glands, puncta, canals, and sac, constitute the apparatus by which the tears are formed, and conveyed to the nose. The gland has been already described (p. 51).

Dissection. A bristle should be introduced into each lachrymal canal throngh the punctum of the eyelid. The lachrymal sac will appear by removing the tensor tarsi and the areolar tissue from its surface, as it lies: on the os unguis. The prolongation from the ligamentum palpebrarum over the sac should be defined.

The puncta lachrymalia $\left(^{1}\right)$ are two small apertures, one for each lid, by which the lachrymal canals receive the tears. Each is situate in the free margin of the lid, about a quarter of an inch from the inner canthus, and in the elevation of the papilla lachrymalis.

Fig. 13.

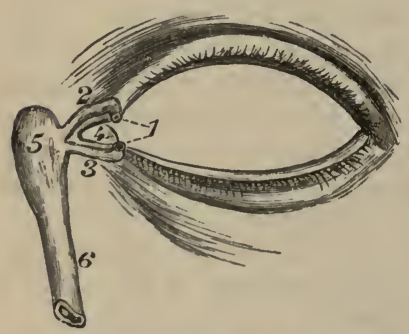

1. Puncta lachrymalia.

3. Upper, and 3, Lower lachrymal canal.

4. Caruncula lachrymalis.

5. Lachrymal sac.

6. Lachrymal duct.

ThE EYRIIDS AND LACHRTMAL APPaRATE.

The lachrymal canals (fig. 13, ${ }^{2}$ and 3 ) lead from the puncta, and convey the tears to the lachrymal sac; their situation is marked by the bristles inserted in them. In their course inwards the canals lie along the ligamentum palpebrarum, one $\left({ }^{2}\right)$ above and the other $\left({ }^{3}\right)$ below it, and they are somewhat arched with the concavity towards each other. Internally they open near together into the lachrymal sac rather above its middle. 
The canal in the upper eyelid is longer and more arched than that in the lower lid.

The lachrymal sac and duct (fig. 13) extend from the inner part of the orbit to the nose, and convey the tears into the latter cavity. 'They form one tube, of which the upper dilated part is the sac, and the lower constricted end the duct.

The $s a c,{ }^{5}$, is situate in the hollow formed by the os unguis and nasal process of the superior maxillary bone. Externally it is crossed by the ligament of the eyelids, and is covered by an expansion derived from that band, which is fixed to the margin of the bony groove. If the aponeurotic eovering be removed, the mucous membrane lining the interior will be seen. Into the outer side of the sac the lachrymal canals open.

The duct, ${ }^{6}$ (ductus ad nasum), is the narrowed part of the tube, and is about half an inch long. It is entirely encased by bone, and corresponds with the passage of the same name in the dried skull. In the nasal eavity it opens into the front of the inferior meatus, where its opening is guarded by a piece of the mucous membrane. A bent probe introduced through the nostril may be passed into the duct from the meatus, but with destruction of the valve.

As the duct is continuous with the mucous membrane of the nose it has the same structure, viz., a fibrous external layer with a mucous lining. The epithelium of the sac and duct is ciliated as in the nose, but in the lachrymal canals it is sealy.

Directions. The examination of the eyeball may be omitted with more advantage to the student till the dissection of the head and neck has been 'ompleted. The description of the eye will be found at the end of the book.

\section{Section $V$.}

\section{DISSECTION OF THE NECK.}

Position. For the dissection of the right side of the neck let the head be supported at a moderate height on a block, and let the face be turned to the left side and fastened in that position with hooks. To obtain a good view of the region, the right arm may be drawn under the body, with the object of depressing the point of the shoulder, and putting the neck parts on the stretch. In some bodies, owing to a difference in the form of the neck, the best position will be obtained by placing the upper linrb over the chest.

Surface-marking. The side of the neck presents a somewhat square outline, and is limited in the following way:-Below is the prominence of the clavicle; and above is the base of the lower jaw with the skull. In front the boundary is a line from the ehin to the sternum; and behind, another line from the oeciput to the acromial end of the clavicle. The part thus included is divided into two triangular spaces (anterior and posterior) by the diagonal line of the projecting sterno-nastoid musele. And in consequence of the position of that muscle the base of the anterior space is at the jaw, and the apex at the sternum; whilst the base of the posterior one is at the claviele, and the apex at the head.

The surface in front of the sterno-mastoid is depressed at the upper part 
of the neck, near the position of the carotid vessels; and behind the muscle, just above the clavicle, is another slight hollow which points to the situation of the subclavian artery.

Along the middle line of the neck the following parts can be recognized through the skin :-About two inclies and a lalf from the base of the jaw is the eminence of the os liyoides, with its cornu extending laterally on each side. Below this may be felt the wide prominence of the thyroirl cartilage, called pomum Adami, which is most marked in man : and between the cartilage and the hyoid bone is a slight interval, corresponding with the thyro-hyoid membrane.

Inferior to the thyroid, is the narrow prominent ring of the cricoid cartilage; and between the two the finger may distinguish another interval, which is opposite the crico-thyroid membrane. In some bodies, especially in women, the swelling of the thyroid gland may be felt by the side of the trachea.

From the cricoid cartilage to the sternum, and between the sterno-mastoid muscles, is a depression, whose depth is much increased in emaciated persons, in which the tube of the trachea can be recognized.

Direction. As the time for turning the body will not allow the examination of the whole side of the neck, the student should lay bare in this stage only the parts behind the sterno-mastoid muscle.

Dissection. To raise the skin from the posterior triangle of the neck, make an incision along the sterno-mastoid muscle from the one end to the other, and afterwards along the clavicle as far as the acromion. The triangular flap of skin is to be reflected from before back towards the trapezius muscle. The superficial fascia which will then be brought into view, contains the platysma; and to see that muscle, it will be necessary to take the subcutaneous fat from the surface of the fibres.

The PLATISMA MYOIDES is a thin subcutaneous muscular layer, which is now seen only in its lower half. The muscle is placed across the side of the neck, and extends from the top of the shoulder to the face. Its fleshy fibres take origin by fibrous bands from the clavicle and the acromion, and below those bones from the superficial fatty layer covering the pectoral and deltoid muscles; ascending through the neck, the fibres are inserted into the jaw.

The lower part of the muscle is more closely united to the skin than the upper, and covers the external jugular vein as well as the lower part of the posterior triangle. At first the fibres of the muscle are thin and scattered, but they increase in strength as they ascend. The oblique direction of the fibres should be noted, because in venesection in the external jugular vein the incision is to be so made as to divide them across.

'The use will be found with the description of the remainder of the muscle.

Dissection. The platysma is now to be cut across near the clavicle, and to be reflected upwards as far as the incision over the sterno-mastoid muscle, but it is to be left attached at that spot. In raising the muscle the student must be careful of the deep fascia of the neck; and he should dissect out the external jugular vein, and the superficial descending branches of the cervical plexus, which are close beneath the platysma.

The external jugular vein (fig. $14,{ }^{8}$ ) commences in the parotid gland (p. 42), and is directed backwards beneath the platysma to the lower part of the neck, where it pierces the deep cervical fascia to open into the subclavian vein. Its course down the neck will be marked by a line from 
the angle of the jaw to the middle of the clavicle. Beyond the sternomastoid muscle the vien is dilated, and the swollen part (sinus) is limited by two pairs of valves_one being situate below at the mouth of the vein, and the other near the muscle. Small superficial branches join the vein, and an offset connects it with the anterior jugular vein. Its size, and the height at which it crosses the sterno-mastoid muscle, are very uncertain.

The deep cervical fascia consists, like the aponeuroses in other regions of the body, of a superficial layer which surrounds the neck continuously, and of processes that are prolonged inwards between the muscles. In some bodies this fascia is thin and indistinct.

In its extent round the neck the membrane incases the sterno-mastoideus, and presents a different disposition before and behind that muscle. As now seen passing backwards from the muscle, the fascia continues over the posterior triangular space, and incloses the trapezius in its progress to the spines of the vertebræ. At the lower part of the neck it is attached to the clavicle and is perforated by the external jugular vein, and the cutaneous nerves.

After the superficial layer has been removed near the clavicle, a deep process may be observed to surround the small omo-hyoid muscle, and to extend under the clavicle, where it is fixed to the back of that bone, and the inner end of the first rib.

POSTERIOR TRIANGULAR SPACE.

This space (fig. 14), having the form and position before noted, is about eight inches in length. It contains the cervical and brachial plexuses, with the portion of the subclavian artery on which a ligature is usually placed, and some offsets of the ressel and the nerves.

Dissection. By the removal of the cervical fascia and the fat between the sterno-mastoid and trapezius muscles, the posterior triangle of the neck will be displayed. In the execution of this task, the student may obtain some assistance by attending to the following remarks :-

Crossing the space obliquely about an inch above the clavicle, and dividing it into two, is the small omo-hyoid muscle (fig. $14,{ }^{3}$ ). Close to or under the upper border of the muscle lie the small nerve and vessels to it: the nerve being traceable to the descendens noni, and the artery to the supra-scapular.

Above the omo-hyoid muscle will be found the ramifications of the branches of the cervical plexus, together with the spinal accessory nerve; the latter will be recognized by its piercing sterno-mastoid muscle. 'The greater number of the branches of the cervical plexus descend in the space to the shoulder; but the small occipital and great auricular nerves ascend to the head, and the superficial cervical branch is directed forwards over the sterno-mastoid muscle.

Below the omo-hyoideus are the subclavian artery and the brachial plexus, which have a deep position. In this part also the following vessels and nerve are to be sought, viz., the supra-scapular vessels behind the clavicle; the transverse cervical vessels which are higher in the neck, but take an outward direction beneath the omo-hyoid muscle; and, lastly, the small braneh of nerve to the subclavius muscle, which lies about the middle of the space between the clavicle and omo-hyoideus.

Underneath the trapezius, where it is attached to the clavicle, the 
serratus magnus muscle appears; and behind the large cervical nerves, towards the lower part of the space, is placed the middle scalenus muscle. 'Through the scalenus issue two muscular nerves; one, formed by two roots, for the serratus magnus; the other smaller, and higher up, for the rhomboidei.

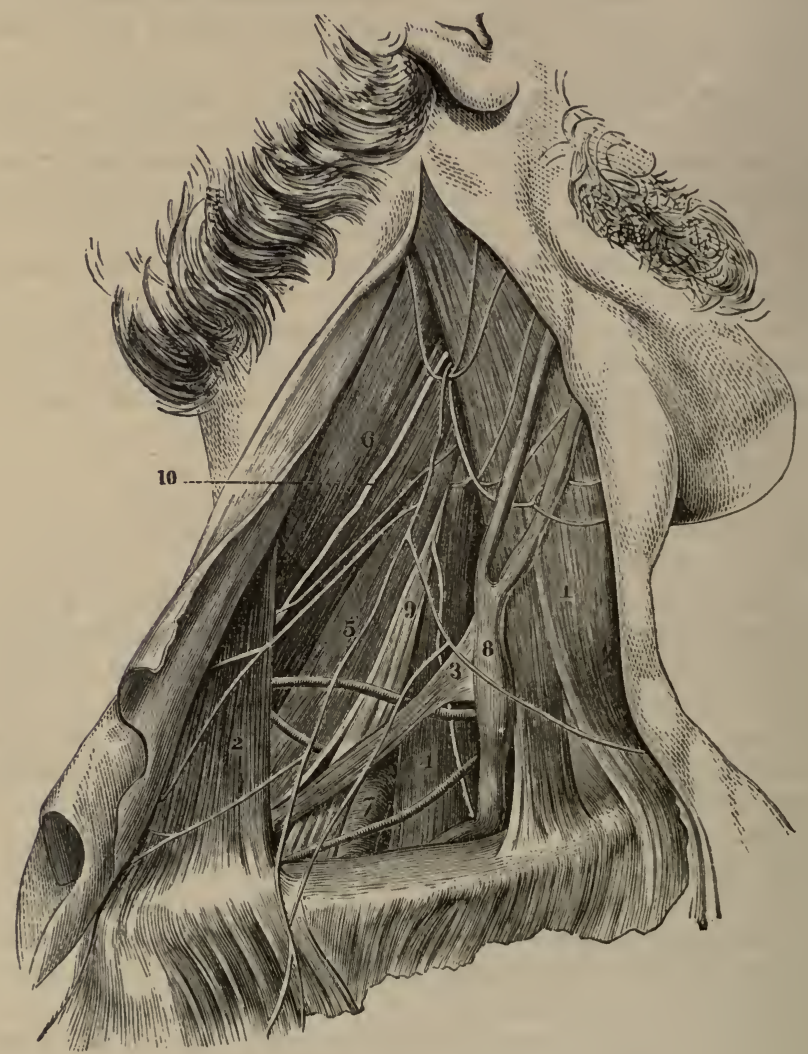

Part of the Posteriok Triangle of the Neck is here displayed, but the student should carry the dissection as higl as the head, so as to lay bare the whole of that space.

1. Steruo-mastoideus.

2 Trapezius.

3. Posterior belly of the omo-hyoid muscle.

4. Anterior scalenus, with the phrenic nerve ou it.

5. Middle scalenus inuscle.
6. Elevator of the angle of the scapula.

7. Third part of the subclaviau artery.

8. External jugular veiu joining the subclil= vian below.

9. Nerves of the brachial plexus.

10. Spinal accessory nerve.

(Blandin's Surgical Anatomy.)

Limits of the space. The space is bounded in the front by the sternomastoid muscle, ${ }^{1}$; and behind by the trapezius, ${ }^{2}$. Its base corresponds with the middle third of the clavicle, and its apex is at the skull. In its 
area are several muscles, which are placed in the following order from above down, viz., splenius capitis, levator anguli scapula ${ }^{6}$; and the middle scalenus ${ }^{s}$; and at the lower and outer angle, somewhat beneath the trapezius lies the upper part of the serratus magnus. Covering the space are the structures already examined, viz., the skin and superficial fascia, the platysna over the lower half or two-thirds, and the deep fascia.

The small omo-hyoid muscle, ${ }^{3}$, crosses the lower part of the space, so as to subdivide it into two-a lower or clavicular, and an upper or oceipital.

The clavicular part is small in size and close to the clavicle, and contains the subelavian artery. It is triangular in form, with its base directed forwards ; and is bounded in front by the sterno-mastoid, ${ }^{1}$; above by the omo-hyoid muscle, ${ }^{8}$; and below by the clavicle. This small space measures commonly about one inch and a half from before backwards, and about half that in front at its base.

Crossing the area of this portion, rather above the level of the clavicle, is the trunk of the subclavian artery, ${ }^{8}$ (its third part), which issues from beneath the anterior scalenus muscle, and is directed over the first rib to the axilla. In the ordinary condition of the vessel the companion subclavian vein is seldom seen, owing to its situation being lower down beneath the claviele. Above the artery are the large cords of the brachial plexus, ${ }^{9}$, which accompany the vessel, and become closely applied to it beneath the clavicle. Behind the artery and the nerves is the middle scalenus muscle, ${ }^{5}$. And below the vessel is the first rib.

Along the lower boundary of the space, and rather beneath the clavicle lie the supra-scapular vessels; and crossing the upper angle, at the meeting of the omo-liyoid and sterno-mastoid muscles, are the transverse cervical vessels. Entering the space from above is the external jugular vein, ${ }^{8}$, which descends over or under the omo-hyoideus near the anterior part, and opens into the subclavian vein; in this spot the rein receives the supra-scapular and transverse cervical branches, and sometimes a small vein, over the clavicle, from the cephalic vein of the arm.

The size of the clavicular part of the space from before back is influenced by the attachment of the trapezius and sterno-mastoid muscles along the clavicle: in some bodies these muscles occupy nearly the whole length of that bone, leaving but a small interval between them; and in others they meet so as to cover the underlying vessels. This space may be further increased or diminished from above down by the position of the omohyoideus in the neck; for this muscle may lie close to the clavicle, being attached thereto, or it may be distant one inch and a half from that bone.

In depth the space varies naturally; and in a short thick neck with a prominent clavicle, the artery is farther from the surface than in the opposite condition of the parts. But the depth may be altered much more by the position of the clavicle, according as the limb may be raised or depressed. And lastly, the artery may be concealed entirely in its usual position by forcing upwards the arm and shoulder, as the collar bone can be raised above the level of the omo-hyoid muscle.

The situation of the trunk of the subclavian artery may vary much, for the vessel may be one inch and a half above the clavicle, or at any point intermediate between this and its usual level just above the prominence of that bone. Further, its position to the anterior scalenus may be changed; and instead of the vessel being beneath, it may be in front of, or even between the fibres of that muscle. 
Commonly there is not any branch connected with the artery in this part of its course; but the posterior scapular branch (fig. 14) may take origin from it at different distances from the scalenus, or there may be more than one branch (Quain).

The subclavian vein rises upwards not unfrequently as high as the artery, or it may even lie with the artery beneath the anterior sealenus in some rare instances. The position of the external jugular vein with regard to the subclavian artery is very uncertain; and the branches connected with the lower end may form a kind of plexus over the arterial trunk.

The occipital part of the posterior triangle is larger than the other. Its boundaries in front and behind are the sterno-mastoid and the trapezius; and it is separated from the elavicular portion by the omo-hyoid muscle.

In it are contained chiefly the ramifications of the cervical plexus; and a chain of lymphatic glands lies along the sterno-mastoid muscle. The spinal accessory nerve, ${ }^{10}$, is directed obliquely across this interval from the sterno-mastoid muscle, which it pierees, to the under surface of the trapezius; and a communication takes place between this cranial and the spinal nerves in the space.

Superficial Branches of the Cervical Plexus. Behind the sterno-mastoid musele appear some of the ramifications of the cervical nerves in the plexus of the same name; and superficial branches are furnished from these both upwards and downwards.

The ASCENDING SET (fig. 14) are three in number, viz., small occipital, great auricular, and superficial cervical.

The small occipital branch (fig. $2,{ }^{2}$ ) comes from the second cervical nerve, and is directed upwards to the head along the posterior border of the sterno-mastoid muscle. At first the nerve is beneath the fascia; but near the occiput it becomes cutaneous, and is distributed between the ear and the great occipital nerve (p. 24). Occasionally there is a second cutaneous nerve to the head.

The great auricular nerve (fig. $2,{ }^{1}$ ) is a branch of the part of the plexus formed by the second and third cervical nerves. Perforating the deep fascia at the posterior border of the sterno-mastoid muscle, the nerve is directed upwards beneath the platysma to the lobule of the ear, where it ends in the following branches:-

The facial branches are sent forwards to the integuments over the parotid, and a few slender filaments pass through the gland to join the facial nerve.

The auricular branches ascend to the external ear, and are chiefly distributed on its cranial aspect; one or more reach the opposite surface by piercing the pinna. On the ear they communicate with branches furnished from the facial and pneumogastric nerves.

The mastoid branch is directed backwards to the integuments between the ear and the mastoid process ; and it joins the posterior auricular branch of the facial nerve (p. 23).

The superficial cervical nerve (fig. $2,{ }^{14}$ ) springs from the same source as the precerling, and turns forwards round the sterno-mastoid muscle about the middle. Afterwards it pierces the fascia and platysma, and ramifies over the anterior triangular space (see p. 68). There may be more than one branch to represent this nerve.

The DESCENDING SET of branches (fig. 2) (supra-clavicular), are derived from the third and fourth nerves of the plexus, and are directed 
towards the clavicle over the lower part of the triangular space. Their number is somewhat uncertain, but usually there are about three on the clavicle.

The most internal branch (sternal) crosses the clavicle near its inner end; the middle branch lies about the middle of that bone; and the posterior (acromial) turns over the attachment of the trapezius to the acromion. All are distributed in the integuments of the chest and shoulder.

Derived from the descending set are two or more posterior cutaneous cervical nerves, which ramify in the integument over the lower two thirds of the fore part of the trapezius.

The lymphatic glands (glandulæe concatenatæ) lie along the sternomastoid muscle, and are continuous at the lower part of the neek with the glands in the cavity of the thorax. 'There is also a superficial chain along the external jugular vein.

Dissection. The dissection of the posterior triangle should be repeated on the left side of the neck, in order that the difference in the vessels may be observed. Afterwards the reflected parts are to be replaced and carefully fastened in their natural position with a few stitehes, preservative fluid or salt having been previously applied.

Directions. It is supposed that the body will now be turned on the fore part for the examination of the Back; and during the time allotted for this position the dissector of the head is to learn the posterior part of the neck. (Dissection of THE BACK.)

After the completion of the Back, the student should take out the spinal cord, and then return to the dissection of the front of the neck, which is described below.

\section{FRONT OF THE NECK.}

Directions. Supposing the thorax and Back finished, the head and neck may be detached from the trunk by dividing the spinal column between the second and third dorsal vertebre, and cutting through the arch of the aorta beyond its large branches (if this is not done), so as to take that piece of the vessel with the head. The dissector continues his work on the remainder of the right side of the neck; but if the facial nerve has been omitted, it should be first learnt (p. 47).

Position. Supposing the facial nerve completed, a small narrow block is to be placed beneath the left side of the neck, and the face is to be turned from the dissector. Further, the neck is to be made tense by means of hooks, the chin being well raised at the same time.

Dissection. An incision along the base of the jaw on the right side (if it has not been made already) will readily allow the piece of integument in front of the sterno-mastoideus to be raised towards the middle line. Beneath the skin is the superficial fascia, containing very fine off'sets of the superficial cervical nerve.

To define the platysma muscle, remove the fat which covers it, carrying the knife down and back in the direction of the fleshy fibres.

Platrsua Mroides. The anterior part of the platysma, viz, from the sterno-mastoid muscle to the lower jaw, covers the greater portion of the anterior triangular space. At the base of the jaw it is inserted between the symphysis and the masseter musele; while other and more posterior fibres are continued over the face, joining the depressor anguli 
oris and risorius, as far as the fascia eovering the parotid gland, or even to the cheek bone.

The fibres have the same appearance in this as in the lower half of the muscle, but they are rather stronger. Below the chin the inner fibres of opposite muscles cross for a distance of about an inch, but those which are superficial do not belong always to the same side.

Action. The ordinary action of this musele is confined to the skin of the neck, which it throws into longitudinal wrinkles; but it can depress the corner of the mouth by the slip prolonged to the face. Through its attachment to the jaw it will assist in opening the mouth.

Dissection. Raise the platysma to the base of the jaw, and dissect out the branches of the superficial cervical nerve, and the cervical branches of the facial nerve that are beneath it. Clean also the deep fascia of the neck, and the anterior jugular vein which is placed near the middle line.

The superficial cervical nerve has been traced from its origin in the cervical plexus to its position on the deep fascia of the neck (p. 66); but the nerve may arise from the plexus by two pieces. Beneath the platysma it divides into an ascending and a descending branch :-

The ascending branch perforates the platysma, supplying it, and ends in the integuments over the anterior triangle, about half way down the neck. Whilst this branch is beneath the platysma it joins the facial nerve.

The descending branch likewise passes through the platysma, and is distributed to the teguments below the preceding, reaching as low as the sternum.

The infra-maxillary branches of the facial or seventh cranial nerve (rami subcutanei colli) (p. 50 ) pierce the deep cervical fascia, and pass forwards beneath the platysma, forming arches across the side of the neck (fig. 9), which reach as low as the hyoid bone. Most of the branches end in the platysma, but a few filaments perforate it, and reach the integuments. Beneath the muscle there is a communication between the branches of the facial and the off'sets of the superficial cervical nerve.

Dissection. Cut across the external jugular vein about the middle, and throw the ends up and down. Afterwards the superficial nerves of the neck may be divided in a line with the angle of the jaw, the anterior ends being removed, and the posterior reflected. The great auricular nerve may be cut through and the ends reflected.

The part of the deep cervical fascia in front of the sterno-mastoideus is stronger than that behind the muscle, and has the following arrangement. Near the sternum the fascia forms a white firm membrane, which is attached to that bone; but higher in the neck it becomes thinner, and is fixed above the lower jaw and the zygoma, covering also the parotid gland. From the ramus of the jaw a piece is prolonged downwards, between the parotid and submaxillary glands, to join the styloid process; this piece is named the stglo-maxillary ligament. And from the angle of the jaw a strong piece is continued to the sterno-mastoideus, which fixes forwards the anterior border of that muscle.

Layers of the membrane are prolonged between the muscles; and that beneath the sterno-mastoid is connected with the sheath of the cervical vessels. One of these beneath the sterno-thyroid musele, descends in front of the great vessels at the root of the neck to the arch of the arta, and the pericardium. 
This space (fig. 15) contains the carotid vessels and their branches, with many nerves; and it corresponds with the hollow on the surface of the neck in front of the sterno-mastoid muscle.

Dissection. To define the anterior triangular space and its contents, take away the deep fascia of the neck, and the fat, but without injuring or displacing the several parts. First clean the surface of the hyoid muscles that appear along the middle line, leaving untouched the anterior jugular vein.

The trunks into which the large carotid artery bifurcates are to be followed upwards, especially the more superficial one (external carotid), whose numerous branches are to be traced as far as they lie in the space. In removing the sheath from the vessels, as these appear from beneath the muscles at the lower part of the neck, the dissector should be careful of the small descending branch of the hypo-glossal nerve in front of it. In the sheath between the vessels (carotid artery and jugular vein) will be found the pneumogastric nerve, and behind the sheath is the sympathetic nerve.

Crossing the space, in the direction of a line from the mastoid process to the hyoid bone, are the digastric and stylo-hyoid muscles, with several nerves directed transversely. Thus lying below them is the hypo-glossal nerve, which gives one branch (descendens noni) in front of the sheath, and another to the thyroid-hyoid muscle. Above the muscles, and taking a similar direction between the two carotid arteries, are the glosso-pharyngeal nerve and the stylo-pharyngeus muscle. Directed downwards and backwards from beneath the same museles to the sterno-mastoideus, is the spinal accessory nerve.

On the inner side of the vessels, between the hyoid bone and the thyroid cartilage, the dissector will find the superior laryngeal nerve; and by the side of the larynx, with the descending part of the superior thyroid artery, the small external laryngeal branch.

Clean then the submaxillary gland close to the base of the jaw ; and on partly displacing it from the surface of the mylo-hyoid muscle, the student will expose the small branch of nerve to that muscle with the submental artery.

The interval between the jaw and the mastoid process is supposed to be already cleaned by the removal of the parotid gland in the dissection of the facial nerve.

Limits of the space. Behind, is the sterno-mastoid muscle, ${ }^{1}$; and in front, a line from the chin to the sternum, along the middle of the neck. Above, at the base of the space, would be the lower jaw, the skull, and the ear; and below, at the apex, is the sternum. Over this space are placed the skin, the superficial fascia with the platysma, the deep fascia, and the ramifications of the facial and superficial cervical nerves.

Muscles in the space. In the area of the triangular interval, as it is above defined, are seen the larynx, and pharynx in part, and many muscles converging towards the hyoid bone as a centre, some being above and some below it. Below are the depressors of that bone, viz., omohyoid, sterno-liyoid, and sterno-thryoid, ${ }^{2}$ to ${ }^{4}$; and above the os hyoides are the elevator muscles, viz., mylo-hyoid, digastric, and stylo-hyoid. Con- 
nected with the back of the hyoid bone and the layrnx are some of the constrictor muscles of the gullet.

Vessels in the triangular space. The carotid bloodvessels, ${ }^{6}$ and 7 , occupy the hinder and deeper part of the space along the side of the sterno-mastoid muscle; and their course would be marked on the surface by a line from the sterno-clavicular articulation to a point midway between the jaw and the mastoid process. As high as the level of the cricoid cartilage they are buried beneath the depressor muscles of the os hyoides; but beyond that spot they are covered by the superficial layers over the space, and by the sterno-mastoid muscle which, before the parts are displaced, conceals the vessels as far as the parotid gland.

For a short distance after its exit from beneath the muscles at the root of the neck, the common carotid artery remains a single trunk, ${ }^{6}$; but opposite the upper border of the thyroid eartilage it divides into two large vessels, external and internal carotid. From the place of division these trunks are continued onwards, beneath the digastric and stylo-hyoid museles, to the interval between the jaw and the mastoid process.

At first the trunks lie side by side, the vessel destined for the internal parts of the head (internal carotid) being the more posterior or external of the two; but above the digastric musele it becomes deeper than the other. The more superficial artery (external carotid) furnishes many branches to the neck and the outer part of the head, viz., some forwards to the larynx, tongue, and face; others backwards to the occiput and the ear; and others upwards to the head.

But the common carotid does not always divide, as here said. For the point of branching of the vessel may be moved from the upper border of the thyroid cartilage, either upwards or downwards, so that the trunk may remain undivided till it is beyond the os hyoides, or end in branches opposite the cricoid cartilage. The division beyond the usual place is more frequent than the branching short of that spot. It may ascend as an undivided trunk (though very rarely), furnishing off'sets to the neck and head.

In close contact with the outer side of both the common and the internal carotid artery, and incased in a sheath of fascia with them, is the large internal jugular vein, which receives branches in the neck corresponding with some of the branches of the superficial artery. In some bodies the vein may cover the artery, and the branches joining it above may form a kind of plexus over the upper part of the common carotid.

Nerves in the space. In connection, more or less intimate, with the large vessels, are the following nerves with a longitudinal direction:-In front of the sheath lies the descending branch of the hypo-glossal nerve; within the sheath, between the carotid artery and jugular vein, is the pneumogastric nerve; and behind the sheath is the sympathetic nerve. Along the outer part of the vessels the spinal accessory nerve extends for a short distanee, till it pierces the sterno-mastoid muscle.

Several nerves are placed across the vessels:-thus, directed transversely over the two earotids, so as to form an arch below the digastric muscle, is the hypoglossal nerve, which gives downwards its branch (descendens noni) most conmonly in front of the sheath. Appearing on the inner side of the earotid arteries, close to the base of the space, is the glosso-pharyngeal nerve, which courses forwards between them. Inside the internal carotid artery, opposite the hyoid bone, the superior laryngeal nerve comes into 
sight; whilst a little lower down, with the descending branches of the thyroid artery, is the external laryngeal branch of that nerve.

Glands in the space. 'Two glandular bodies, the submaxillary, ", and thyroid, ${ }^{12}$, have their seat in this triangular space of the neck. The submaxillary gland is situate altogether in front of the vessels, and is partly concealed by the jaw ; and beneath it on the surface of the mylo-hyoideus is the small nerve to that muscle; with the submental artery. By the side of the thyroid cartilage, between it and the common carotid artery, lies the thyroid body beneath the sterno-thyroid musele: in the female this body is more largely developed than in the male.

At the base of the space, if the parts were not disturbed, would be the parotid gland, which is wedged into the hollow between the jaw and the mastoid process, and projects somewhat below the level of the jaw. Its connections have been noticed at p. 41 .

Directions. The student has to proceed next with the examination of the individual parts that have been referred to with the triangular spaces.

Anterior jugular vein. This small vein occupies the middle line of the neck, and its size is dependent upon the degree of development of the external jugular. Beginning in some small branches below the chin, the vein descends to the sternum, and then bends outwards beneath the sternomastoid muscle, to open into the subclavian vein, or into the external jugular. In the neck the anterior and external jugular veins communicate. 'There are two anterior veins, one for each side, though one is usually larger than the other; and at the bottom of the neck they are joined by a transverse branch.

The sterNo-CLeido-Mastoid muscle (fig. 15, ') forms the superfieial prominence of the side of the neck. It is narrower in the centre than at the ends, and is attached below by two heads of origin, which are separated by an elongated interval. The inner head is fixed by a narrowed tendon to the anterior surface of the first piece of the sternum; and the outer head has a wide fleshy attachment to the sternal third of the clavicle. From this origin the heads are directed upwards, the internal passing backwards, and the external almost vertically, and are blended about the middle of the neck in a roundish belly. Near the skull the musele ends in a tendon, which is inserted into the mastoid process at the outer aspeet from base to tip, and by a thin aponeurosis into a rough surface behind that process, and into the outer part of the upper curved line of the occipital bone.

The muscle divides the lateral surface of the neck into two triangular spaces. On its cutaneous aspect the sterno-mastoid is covered by the eommon integuments, by the platysma and deep fascia, and by the external jugular vein and the superficial branches of the cervical plexus (across the middle). If the muscle be cut through below and raised, it will be seen to lie on the following parts: The clavicular origin is superficial to the anterior scalenus and omo-hyoid muscles; and the sternal head conceals the depressors of the hyoid bone, and the common carotid artery with its vein and nerves. After the union of the heads, the muscle is placed over the cervical plexus, and the middle scalenus and elevator of the angle of the seapula; and near the skull, on the digastric and splenius muscles, the occipital artery, and part of the parotid gland. The spinal accessory perforates the muscular fibres about the upper third.

Action. Both muscles acting bend the head forwards; but one muscle turns the face to the opposite side. In conjunction with the muscles at- 
tached to the mastoid process one sterno-mastoideus will incline the head towards the shoulder of the same side.

Fig. 15.

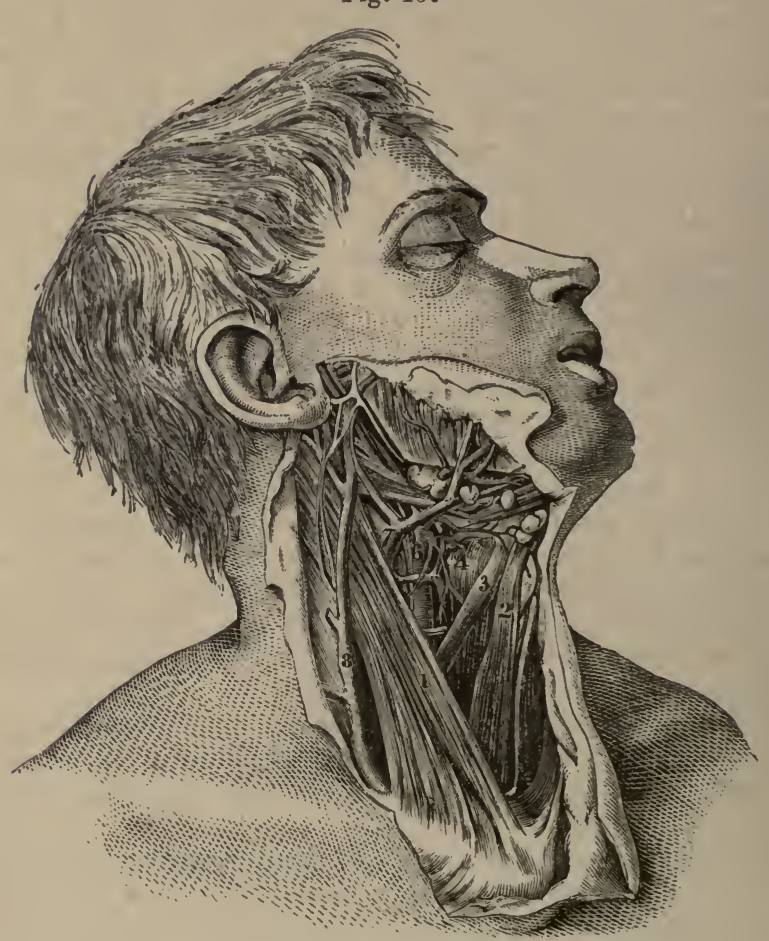

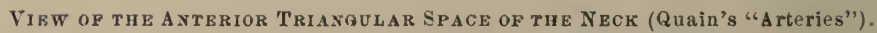

1. Sterno-mastoidens.

2. Sterno-hyoideus.

3. Anterior belly of the omo-hyoideus.

1. Thyro-hyoideus.

6. Common carotid artery dividing.
7 Internal jugular vein.

8. External jugular vein.-In the Drawing from which this cut is copied the sterno-mastoid is partly cut throngh.

In laborious respiration the two muscles will assist in elevating the sternum.

The OMO-HYOID MUSCLE crosses beneath the sterno-mastoidens, and consists of two fleshy bellies united by a small round intermediate tendon (fig. $14,{ }^{3}$ ). The origin of the muscle from the scapula, and the connections of the posterior part, are to be studied in the dissection of the Back. From the intervening tendon the anterior fleshy belly (fig. $15,{ }^{3}$ ) is directed upwards along the outer border of the sterno-lyyoid muscle, and is inserted into the lower part of the body of the hyoid bone, close to the great cormu.

The anterior belly is in contact with the fascia, after escaping from beneath the sterno-mastoid; and rests on the sterno-thyroideus. This part of the muscle crosses the common carotid artery and internal jugular vein on a level with the cricoid cartilage.

Action. The anterior belly depresses the hyoid bone; and the posterior 
is said by Theile to make tense the deep fascia of the neck with which it is connected.

The STERNO-HYOID MUSCLE (fig. $15,{ }^{2}$ ) is a flat thin band nearer the middle line than the preceding. It arises from the posterior surface of the sternum and the cartilage of the first rib. From this spot the fibres ascend, and are inserted into the lower border of the body of the os hyoides, internal to the preceding muscle.

One surface is in contact with the fascia, and is often marked by a tendinous intersection near the clavicle. When the muscle is divided and turned aside, the deep surface will be found to touch the sterno-thyroideus, and the superior thyroid vessels. The museles of opposite sides are separated by an interval which is largest below.

Action. It draws the os hyoides downwards after swallowing; and in laborious respiration it will act as an elevator of the sternum.

The STERNO-THYROID MUSCLE is wider and shorter than the sternohyoid, beneath which it lies. Like the other hyoid muscle, it arises from the posterior surface of the sternum, and the cartilage of the first rib below the former; and it is inserted into the oblique line on the side of the thyroid eartilage, where it is continuous with the thyro-hyoid musele.

The inner border touches its fellow for about an inch, along the middle line of the neck, whilst the outer reaches over the carotid artery. The superficial surface is concealed by the preceding hyoid muscles; and the opposite surface is in contact with the lower part of the common carotid artery, the trachea, and the larynx and thyroid body. A transverse, tendinous line crosses the muscle near the sternum.

Action. Its chief use is to aid the preceding muscle in lowering rapidly the hyoid bone after deglutition; but it can draw down and forwards the thyroid cartilage, and assist in rendering tight the vocal cords.

Like the sterno-hyoid it participates in the movement of the chest in laborious breathing.

The THYRO-HYOIDEus (fig. $15,{ }^{4}$ ) is a continuation in direction of the last muscle. Beginning on the side of the thyroid cartilage, the fibres ascend to the inner half of the great cornu of the os hyoides, and to the outer part of the body of the bone.

On the muscle lies the omo-hyoideus; and beneath it are the superior laryngeal nerve and vessels. It is sometimes considered one of the special muscles of the larynx.

Action. Raising the thyroid cartilage towards the os hyoides, it renders lax the vocal cords, and assists in placing the cartilage under the tongue preparatory to swallowing.

Directions.-The remaining parts included in this SEction are the scaleni muscles and the subclavian bloodvessels, with the cervical nerres and the carotid bloodvessels. The student may examine them in the order here given.

Dissection (fig. 16). Supposing the sterno-mastoid eut, the fat and faseia are to be taken away from the lower part of the neck, so as to prepare the scaleni muscles with the subclavian vessels and their branches. By means of a little dissection the anterior scalenus musele will be seen ascending from the first rib to the neek, having the phrenic nerve and subclavian vein in front of it, the latter erossing it near the rib.

The part of the subelavian artery on the inner side of the scalenus is then to be eleaned, eare being taken not only of its branehes, but of the branches of the sympathetic nerve which course over and along it from the 
neek to the chest. This dissection will be facilitated by the removal of a part or the whole of the clavicle.

All the branches of the artery are in general easily found, except the superior intercostal, which is to be sought in the thorax in front of the neck of the first rib. On the branch (inferior thyroid) ascending to the

Fig. 16.

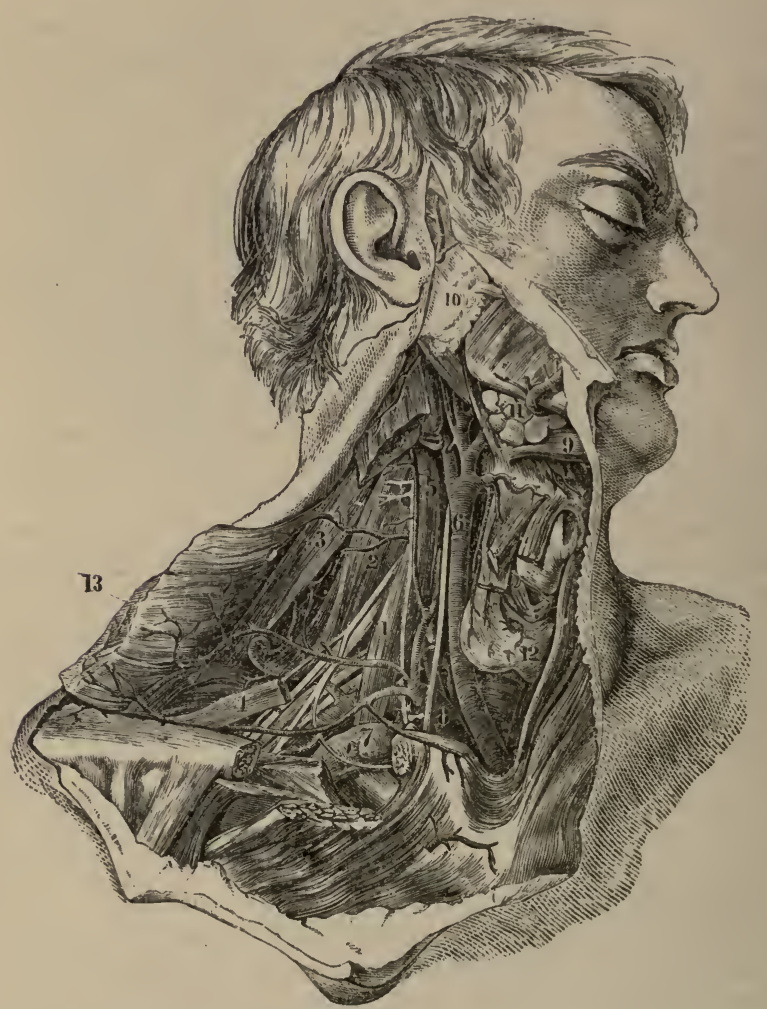

A view of the Common Carotid and Subchavian Arteries (Quain's "Arteries").
1. Anterior scalenus, with the phrenic nerve
7. Subclavian vein. on it.
2. Middle scalenus.
3. Levator anguli scapulæ.
8. Subclavian artery.
4. Onohyoideus.
5. Rectus capitis anticus major.
6. Common carotid artery.
9. Digastric muscle.
10. Parotid gland.
11. Submaxillary gland.
12. Thyroid body.
13. Trapezius muscle, reflected.

thyroid body, or near it, is the middle cervical ganglion of the sympathetic; and the dissector should follow downwards from it a small cardiac nerve to the thorax. Only the origin and first part of the course of the arterial branches can be now seen; their termination is met with in other stages of this dissection, or in the dissection of other parts of the body.

Now the student should seek the small right lymphatic duet that opens into the subclavian vein near its junction with the jugular. A notice of it will be given with the lymphaties of the thorax. 
The outer part of the subclavian artery having been already prepared, let the dissector remove more completely the fibrous tissue from the nerves of the brachial plexus. From the plexus trace the small branch to the subclavius muscle; and the branches to the rhomboid and serratus muscles, which pierce the middle scalenus. If it is thought necessary, the anterior scalenus may be cut through after the artery has been studied.

Clean the cervical plexus, and seek its muscular branches, the small twigs to join the descendens noni, and the roots of the phrenic nerve. Lastly, let the middle sealenus musele be defined, as it lies beneath the eervical nerves.

The SCALENI museles are usually described as three in number, and are named from their relative position, anterior, middle, and posterior; they extend from the first two ribs to the transverse processes of the cervical vertebræ.

The scalenus anticus (fig. 16, ${ }^{1}$ ) extends from the first rib to the lower cervical vertebre, and is somewhat conical in shape. 'It is attached by its apex to the inner border and the upper surface on the first rib, so as to surround the rough surface or projection on this aspect of the bone; and by its base it is inserted into the anterior transverse processes of four of the cervical vertebra, viz., sixth, fifth, fourth, and third (fig. $45,{ }^{\mathrm{c}}$ ).

More deeply seated below than above, the muscle is concealed by the clavicle and the subclavius, and by the elavicular part of the sterno-mastoid : the phrenic nerve lies along its eutaneous surface, and the subclavian vein crosses over it near the rib. Along the inner border is the internal jugular vein. Beneath the scalenus are the pleura, the subclavian artery, and the nerves of the brachial plexus. The insertion into the vertebræ corresponds with the origin of the rectus capitus anticus major musele.

Action. The anterior of these muscles raises strongly the first rib, in consequence of its forward attachment. If the rib.is fixed, it bends forward the lower part of the neck.

The SCALExiUs Medius Muscle (fig. $16,{ }^{2}$ ) is larger than the anterior, and extends farthest of all on the vertebre. Inferiorly it is attached to a groove on the upper surface of the first rib, extending obliquely forwards from the tubercle to the outer border for one inch and a half. The muscle ascends behind the spinal nerves, and is inserted into the tips of the posterior transverse processes of all the cervical vertebræ (fig. $45,{ }^{\mathrm{D}}$ ).

In eontact with the anterior surface are the subelavian artery and the spinal nerves, together with the sterno-mastoid muscle; whilst the posterior surface touches the posterior scalenus, and the deep lateral muscles of the back of the neck. The outer border is perforated by the nerves of the rhomboid and serratus museles.

Action. Usually it elevates the first rib. With the rib fixed, the cervical part of the spine will be inclined laterally by one muscle.

The scalenus posticus (fig. $45,{ }^{\mathrm{F}}$ ) is considerable in size, and appears to be but part of the preceding. It is attached below by a slip, abont half an inch wide, to the second rib, in front of the serratus posticus superior; and it is inserted above with the scalenus medius into two or three of the lower cervieal transverse processes.

Action. It acts as an elevator of the seeond rib; and its fibres having the same direction as those of the medius, it will incline the neck in the same way.

The subclavian artery (fig. 16) is the first part of the large vessel supplying the upper limb with blood, which is thus designated from its 
position beneath the clavicle. This vessel $\left({ }^{8}\right)$ is derived from the branching of the innominate artery behind the sterno-clavicular articulation, and the part of it named subclavian extends as far as the lower border of the first rib. To reach the limb the artery crosses the lower part of the neck, taking an archer course over the bag of the pleura and the first rib, and between the scaleni muscles. For the purpose of describing its numerons connections the vessel may be divided into three parts : the first extending from the sterno-clavicular articulation to the inner border of the interior scalenus; the second, beneath the scalenus; and the third, from the outer border of that muscle to the lower edge of the first rib.

First part. Internal to the anterior scalenus the artery lies deep in the neck, and ascends slightly from its origin. Between the vesscl and the surface will be found the common integumentary coverings with the platysma and the deep fascia, the sterno-mastoid, sterno-hyoid and sternothyroid muscles, and a strong deep process of fascia from the inner border of the scalenus muscle. This part of the subclavian lies over the longus colli muscle, though at some distance from it, and separated from it by fat and nerves. Below the artery is the pleura, which ascends into the arch formed by the vessel.

Veins. The innominate vein, and the ending of the subclavian $\left({ }^{7}\right)$, form an areh below that of the artery. The large internal jugular vein crosses the arterial trunk close to the scalenus; and underneath this vein, with the same direction, lies the vertebral vein. Much more superficial, and separated from the artery by muscles, is the deep part of the anterior jugular vein.

Nerves. In front of the artery lies the pneumogastric nerve, near to the internal jugular vein; and inside this, the lower cardiac branch of the same nerve trunk. Belind the subclavian artery winds the recurrent branch of the pneumogastric ; and still deeper is the cord of the sympathetic nerve with its cardiac branches, one or more of its offsets entwining round the vessel.

Second part. Beneath the scalenus the vessel is less deep than when placed internal to that muscle, and at this spot it rises highest above the clavicle. The second part, like the first, is covered by the integuments, platysma, and deep fascia; then by the clavicular origin of the st.rnomastoideus; and lastly by the anterior scalenus. Behind the vessel is the middle scalenus. Below the artery is the bag of the pleura, which ascends between the scalena.

Veins. Below the level of the artery, and separater from it by the anterior scalenus muscle, lies the arch of the subclavian vein.

Nerves. In front of the scalenus descends the phrenic nerve. Above the vessel, in the interval between the scalena, are placed the large cervical nerves; and the trunk formed by the last cervical and first dorsal is interposed between the artery and the middle sealenus.

Third part. Beyond the sealenus the artery is contained in the clavicular part of the posterior triangular space (p. 64), and is nearer the surface than in the rest of its course: this part of the vessel is incloserl in a tube of the deep cervical fascia, which it receives as it passes from between the sealeni. It is eomparatively superficial whilst in the space before mentioned, for it is covered only by the integuments, the platysma, and deep fascia; but near its termination the vessel gets under cover of the supraseapular artery and vein, and the clavicle and subclavins muscle. In this 
part of its course the artery rests on the surface of the first rib, which is interposed between it and the pleura.

Veins. The arch of the subclavian vein is close to the artery, not being separated by muscle, but lies commonly at a lower level. The external jugular vein crosses it near the scalenus muscle; and the suprascapular and transverse cervical branches, which enter the jugular, form sometimes a plexus over the third part of the artery.

Nerves. The large cords of the brachial plexus are placed above and close to the ressel; and the small nerve of the subclavius crosses it about the middle. Superficial to the cervical fascia lie the descending cutaneous branches of the cervical plexus.

Peculiurities. The artery may spring as a separate trunk from the arch of the aorta; and in such a deviation the vessel takes a deeper place than usual to reach the scaleni muscles.

It has been before said (p. 66) that the subclavian may be in front of the scalenus or in its fibres; and that it may be placed one inch and a half above the level of the clavicle.

Branches of subclavian. Usually there are four chief branches on the subclavian artery. Three branches arise from the first part of the arterial trunk; one (vertebral) ascends to the head; another (internal mammary) descends to the chest; and the remaining one (thyroid axis) is a short thick trunk, which furnishes branches inwards and outwards to the thyroid body and shoulder. These arise commonly near the scalenus muscle, so as to leave an interval at the origin free from offisets. This interval varies in length from half an inch to an inch in the greater number of cases; and its extremes range from somewhat less than half an inch to an inch and three quarters. But in some instances the branches are scattered over the first part of the artery (Quain). ${ }^{1}$

The fourth branch (superior intercostal) arises beneath the anterior scalenus fiom the second part of the artery, and gives off the deep cervical branch; a small spinal artery comes sometimes from this part of the trunk.

If there is a branch present on the third part of the artery, it is commonly the posterior scapular; if more than one, this same branch with the external mammary; and if more than two, an offset belonging to the thyroid axis will be added.

'The vertebral artery is generally the first and largest branch of the subclavian, and arises from the upper and posterior part. Ascending between the contiguous borders of the scalenus and longus colli muscles, this branch enters the aperture in the lateral mass of the sixth cervical vertebra, and is continued upwards to the skull through the foramina in the other cervical vertebræ. Before the artery enters its aperture it is partly concealed by the internal jugular vein, and passes beneath the thyroid artery; it is accompanied by branches of the sympathetic nerve, and supplies small muscular offisets. Its course and distribution will be given afterwards.

The vertebral vein issues with its accompanying artery, to which it is superficial in the neck, and is directed over the subclavian artery to join the subclavian vein; it receives the deep cervical vein, and the branch that accompanies the ascending cervical artery.

The internal mammary branch leaves the lower part of the subclavian

1 The student is referred for fuller information respecting the peculiarities of the vessel, and the practical applications to be deduced from them, to the original and valuable work on the Anatomy of the Arteries of the Human Body, by Richard Quain, F.R.S. 
artery, and coursing downwards beneath the clavicle, subclavius, and the right innominate vein, enters the thorax between the first rib and the bag of the pleura. As the artery disappears in the chest, it is erossed (superficially) by the phrenic nerve. The vessel is distributed to the walls of the chest and abdomen; and its anatoniy will be given with the dissection of those parts.

Thyroid axis. This is a short thick trunk (fig. 16) which arises from the front of the artery near the anterior scalenus muscle, and soon divides into three branches-one to the thyroid body, and two to the scapula.

The suprascapular branch courses outwards across the lower part of the neck, behind the clavicle and subclavins muscle, to the superior costa of the scapula, and entering the supraspinal fossa is distributed on the dorsum of that bone. The connections of this artery are more fully seen in the dissection of the Back.

'The transverse cervical branch, usually larger than the preceding, takes a similar direction, though higher in the neek, and ends beneath the border of the trapezius muscle in the superficial cervical and posterior scipular arteries. ("Dissection OF THE BACK.") In its course outwards through the space containing the third part of the subelavian artery, this branch crosses the anterior scalenus, the phrenic nerve, and the brachial plexus. Some small offsets are supplied by it to the posterior triangular space of the neck.

'Though the transverse cervical artery supplies ordinarily the posterior scapular branch, there are many bodies in which it is too small to give origin to so large an offset. In such instances the diminished artery ends in the trapezius muscle; whilst the posterior scapular branch arises separately from the third, or even the second part of the subclavian artery (fig. 16).

The inferior thyroid branch is the largest offset of the thyroid axis. Directed inwards with a flexuous course to the thyroid body, the branch passes beneath the common carotid artery and the accompanying vein and nerves, and in front of the longus colli muscle and the recurrent nerve. At the lower part of the thyroid body it divides into branches which ramify in the under surface, and communicate with the superior thyroid, and its fellow, forming a very free anastomosis between those vessels.

Near the larynx a laryngeal branch is distributed to that tube, and other offisets are furnished to the trachea.

The ascending cervical branch of the thyroid is directed upwards between the scalenus and rectus capitis anticus major, and ends in branches to those muscles and the posterior triangle of the neck. Some small spinal offsets are conveyed along the spinal nerves to the cord and its membranes.

The veins corresponding with the branches of the thyroid axis have the following destination : those with the suprascapular and transverse cervical arteries open into the external jugular vein. But the inferior thyroid vein begins in the thyroid body, and descends in front of the trachea, beneath the muscles covering this tube, to the innominate vein.

The superior intercostal artery arises from the posterior part of the sub)clavian, and bends downwards over the neck of the first rib : its distribution to the first two intercostal spaces will be seen in the thorax.

Arising in common with this branch is the deep cervical artery (art. profunda eervicis). Analogous to the dorsal branch of an intercostal artery (Quain), it passes backwards between the transverse process of the last cervieal vertebra and the first rib, lying internal to or beneath the two 
hinder scaleni muscles and the fleshy slips continued upwards from the erector spine, to end beneath the complexus muscle at the posterior part of the neck.

A spinal branch (Quain) is frequently given from the second part of the artery; its offsets are continued into the spinal canal through the intervertebral foramina.

The sUbCLAvian vein has not the same limits as the companion artery, reaching only from the lower edge of the first rib to the inner border of the anterior scalenus. It is a continuation of the axillary vein, and ends by joining the internal jugular in the innominate trunk. Its course is arched below the level of the artery, firom which it is separated by the scalenus.

The anterior and external jugular join the subclavian vein outside the sealenus, and the vertebral vein enters it inside that muscle. Into the angle of union of the subclavian and jugular veins the right lymphatic duct opens (fig. $27,{ }^{9}$ ) ; and at the like spot, on the left side, the large lymphatic or thoracie duct ends (fig. $27,{ }^{8}$ ). 'The highest pair of valves in the subclavian trunk is placed outside the opening of the external jugular vein. ${ }^{1}$

It should be borne in mind that not unfiequently the vein is as high in the neek as the third part of its companion artery; and that the vein has been seen twice with the artery beneath the anterior scalenus.

The ANTERIOR PRIMARY BRANCHES OF THE CERVICAL NERVES spring from the common trunks in the intervertebral foramina, and appear on the side of the neck between the intertransverse muscles. 'These nerves are eight in number, and are equally divided between the cervical and the brachial plexus; the highest four being combined in the former, and the remaining nerves in the latter plexus. The nerves receive offsets of communication from the sympathetic at their beginning, and intermix by means of numerous branches near the spine.

To this general statement some addition is needed for the first two nerves; and their peculiarities will be noticed in Section 18.

Brachial Plexus. The four lower cervical nerves and part of the first intercostal are blended in this plexus; and a fasciculus is added to them from the lowest nerve entering the cervical plexus. 'Thus formed, the plexus reaches from the neck to the axilla, where it ends in nerves for the upper limb. Only the part of it above the clavicle can now be seen. In the neck the nerves have but little of a plexiform disposition: they lie at first between the scaleni muscles, opposite the four lower cervical vertebræ, and have the following arrangement:-

The fifth and sixth nerves unite near the vertebræ; the seventh remains distinct as far as the outer border of the middle scalenus; and the last cervical and the piece of the first intercostal are blended in one trunk beneath the anterior scalenus; so that they make at first three cords. Near the attachment of the middle scalenus to the rib, the seventh nerve throws itself into the trunk of the united fifth and sixth, and then there result two cords to the plexus:- the one (upper) formed by the fifth, sixth, and seventh cervical nerves; and the other (lower) by the eightl cervieal and the first intercostal nerve. These two trunks accompany the subclavian artery, lying to its acromial side, and are continued to the axilla where they are more intimately blended.

Branches. The branches of the plexus may be classed into those above

1 See a paper on the Valves in the Veins of the Neck in the Edin. Med. Journal, of Nov., 1856, by Dr. Struthers. 
the clavicle, and those below that bone. The highest set end mostly in muscles of the lower part of the neck and of the scapula; whilst the other set consist of the terminal branches, and are furnished to the upper limb, with which they will be referred to.

Branches above the Cravice. The branch of the subclavins muscle is a slender twig, which arises from the trunk formed by the fifth and sixth nerves, and is directed downwards over the subclavian artery to the under surfice of the muscle; it is often united with the phrenic nerve at the lower part of the neck.

The branch of the rhomboid muscles springs from the fifth nerve in the substance of the middle scalenus, and perforates the fibres of that muscle; it is directed backwards beneath the levator anguli scapulia to its destination. Branches are given usually from this nerve to the levator anguli scapula.

The nerve of the serratus (posterior thoracic nerve) is contained in the scalenus, like the preceding, and arises from the fith and sixth nerves near the intervertrebral foramina. Piercing the fibres of the scalenus lower than the preceding branch, the nerve is continued behind the brachial plexus, and enters the serratus magnus muscle on the axillary surface.

Branches of the scaleni and longus colli muscles. These small twigs are seen when the anterior scalenus is divided; they arise from the beginning of the trunks of the nerves.

The suprascapular nerve is larger than either of the others. It arises near the subclavian branch from the cord of the plexus formed by the fifth and sixth nerves. Its destination is to the muscles on the dorsum of the scapula, and it will be dissected with the arm.

Occasionally an offset from the fifth cervical trunk joins the phrenic nerve on the anterior scalenus muscle.

The CERvical plexus is formed by the first four cervical nerves. Situate at the upper part of the neck, it lies beneath the sterno-mastoid muscle, and on the middle scalenus and the levator anguli scapulæ. It differs much from the brachial plexus, for it resembles a network more than a bundle of large cords. 'The following is the general arrangement of the nerves in the plexus: Each nerve, except the first, divides into an ascending and a descending branch, and these unite with similar parts of the contiguous nerves, so as to give rise to a series of arches. From these loops or arches the different branches arise:-

The branches are superficial and deep. The superficial set has been described with the triangular space of the neck, as consisting of ascending and descending (p. 66). The ascending branches may be now seen to spring from the union of the second and third nerves; and the descending, to take origin from the loop between the third and fourth nerves. The deep set of branches remains to be examined : they are muscular and communicating, and may be arranged into an internal and an external series.

Intersal Series.-The phrenic or muscular nerve of the diaphragm (fig. 16) is derived from the fourth, or third and fourth nerves of the plexus; and it may be joined by a fasciculus from the fifth cervical nerve. Descending obliquely on the surface of the anterior scalenus from the outer to the inner edge, it enters the chest in front of the internal mammary artery, but behind the subclavian vein, and traverses that eavity to reach the diaphragm. At the lower part of the neek the phrenie nerve is joined by a filament of the sympathetic, and sometimes by an offset of the nerve of the subclavius muscle. 
On the left side the nerve crosses over the first part of the subclavian artery.

The branches communicating with the descendens noni are two in number. One arises from the second, and the other from the third cervical nerve; they are directed inwards over the internal jugular vein, and communicate in front of the carotid sheath with the descending muscular branch (descendens noni) of the hypoglassal nerve. Sometimes these nerves pass under the jugular vein.

Muscular branches are furnished to the anterior recti muscles; they arise from the loop between the first two nerves, and from the trunks of the other nerves close to the intervertebral foramina.

Some connecting branches pass from the loop between the first two nerves, and unite with the sympathetic and some cranial nerves near the base of the skull: these will be afterwards described.

External or Posterior Series. Muscular branches are given from the second nerve to the sterno-mastoidens; from the third nerve to the levator anguli scapulæ; and from the third and fourth nerves to the trapezius. Further, some small branches supply the substance of the middle scalenus.

Connecting branches with the spinal accessory nerve exist in three places. First, in the sterno-mastoid muscle; next, in the posterior triangular space; and lastly, beneath the trapezius. The union with the branches distributed to the trapezius has the appearance of a plexus.

The connon CAROTID ARTERY is the leading vessel for the supply of blood to the neck and head (fig. 16, ${ }^{6}$ ). The origin of the vessel differs on opposite sides of the body, beginning at the lower part of the neck on the riglit, and in the thorax on the left side.

The right vessel commences opposite the sterno-elavicular articulation in the bifurcation of the innominate artery, and ends at the upper border of the thyroid cartilage by dividing into the external and internal carotid. The course of the artery is along the side of the trachea and larynx, gradually diverging from the vessel on the opposite side in consequence of the increasing size of the larynx; and its position will be marked by a line from the sterno-clavicular articulation to a point midway between the angle of the jaw and the mastoid process.

Contained in a sheath of cervical fascia with the internal jugular vein and the pneumo-gastric nerve, the carotid artery has the following connections with the surrounding parts:-As high as the cricoid cartilage the vessel is deeply placed, and is concealed by the common coverings of the skin, platysma, and fascix, and by the muscles at the lower part of the neck, viz., sterno-mastoid (sternal origin), sterno-hyoid, omo-hyoid, and sterno-thyroid. But above the circoid cartilage to its termination the artery is less deep, being covered only by the sterno-mastoid with the common investments of the part. The vessel rests mostly on the longus colli muscle, but close to its ending on the rectus capitis anticus major. 'To the inner side of the carotid lie the trachea and larynx, the osopliagus and pharynx, and the thyroid body, the last overhanging the vessel by the side of the larynx. Along the outer side of the carotid sheath is a chain of lymphatic glands.

Veins. The large internal jugular lies on the outer side and elose to the carotid at the upper end, but separated from it below by an interval of about half an inch: on the left side the vein is over the artery below, as will be afterwards seen. One or two upper thyroid veins and their branches 
cross the upper part of the arterial trunk; and opposite the thyroid body another small vein (middle (thyroid) is directed back over the vessel. Near the elavicle the anterior jugular vein passes out under the sternomastoid : it is superficial to the artery, and separated from it by the sternohyoid and thyroid muscles.

Arteries. Offsets of the upper thyroid artery descend over the top of the sheath; and the inferior thyroid crosses under it below the level of the cricoid cartilage.

Nerves. The descendens noni lies in front of the sheath, crossing from the outer to the inner side, and is joined there by the cervical nerves. The pueumogastric lies within the sheath, behind and between the artery and the vein. The sympathetic cord and branches rest on the spine behind the sheath. All the nerves above mentioned have a longitudinal direction; but the inferior laryngeal or recurrent crosses obliquely inwards behind the sheath towards the lower end of the artery.

Branches of carotid. As a rule, the common carotid artery does not furnish any collateral branch, though it is very common for the superior thyroid to spring from its upper end. At the terminal bifurcation into the two earotids the artery is slightly bulged.

The INTERNAL JUGULAR VEIN extends upwards to the base of the skull, but only the part of it that accompanies the common carotid is now seen. Placed behind or external to its artery, the vein ends below by uniting with the subclavian in the innominate vein. Its proximity to the carotid is not equally close throughout, for at the lower part of the neck the vein inclines backwards, leaving a space between it and the artery, in which the vagus nerve is seen about midway between the two. Sometimes the vein is superficial to the earotid, as on the left side.

The lower part of the vein is marked by a dilatation or sinus. Near its ending it becomes contracted, and is provided with a pair of valves (Struthers).

In this part of its course the vein receives the superior and middle thyroid branches.

Peculiarities of the carotid. The origin of the artery on the right side may be above or below the point stated. Mention has been made of the difference in the place of bifurcation, and of the fact that the common carotid may not be divider into two (p. 70). Instead of one, there may be two trunks issuing from beneath the hyoid muscles.

Dissection. The dissector may next trace out completely the trunk of the external carotid (fig. 17), and follow its branches until they disappear beneath different parts. Afterwards he may separate from one another the digastric and stylo-hyoid muscles, which eross the carotid; and may define their origin and insertion.

The DigAstric Muscle (fig. $15^{9}$ ) consists of two fleshy bellies, united by an intervening tendon, whence its name. The posterior, the larger of the two, arises from the groove beneath the mastoid process; whilst the anterior belly is fixed on the side of the symphysis of the lower jaw. From these attachments the fibres are directed to the intervening tendon, which is surrounded by fibres of the stylo-hyoideus, and is united to its fellow, and to the body and part of the great cornu of the os hyoides by an aponeurotic expansion.

The arch formed by the digastric is superficial, except at the outer end, where it is beneath the sterno-mastoid and splenius muscles. The posterior belly covers the carotid vessels and the accompanying veins and 
nerves; and is placed across the anterior triangular space of the neck in the position of a line from the mastoid process to a little above the hyoid bone: along its lower border lie the occipital artery and the hypoglossal nerve; the former passing backwards, the latter forwards. The anterior belly rests on the mylo-hyoid muscle.

The muscle forms the lower boundary of a space between the jaw and the base of the skull, which is subdivided into two by the stylo-maxillary ligament. In the posterior portion are contained the parotid gland $\left({ }^{10}\right)$, and the vessels and nerves in connection with it (p. 41); in the anterior, are the submaxillary gland $\left({ }^{11}\right)$, with the facial and submental vessels, and deeper still, the muscles between the chin and the hyoid bone.

Action. The lower jaw being movable, the muscle depresses that bone and opens the mouth. If the jaw is fixed, the two bellies acting will elevate the hyoid bone.

It is supposed that the posterior belly may assist in moving back the head when the jaw is fixed.

The STYLO-HYOID MUSCLE is thin and slender, and has the same position as the posterior belly of the digastric. It arises from the outer surface of the styloid process, near the base, and is inserted into the body of the os hyoides.

The muscle has the same connections as the posterior belly of the digastric; and its fleshy fibres are usually perforated by the tendon of that muscle. In some bodies the stylo-hyoideus is absent.

Action. This muscle elevates the os hyoides preparatory to swallowing, and checks, with the posterior belly of the digastric, the too forward movement of that bone by the other elevators.

The IYPOGLOSSAL NERVE (ninth cranial) appears in the anterior triangle at the lower edge of the digastric muscle, where it hooks round the occipital artery; it is then directed forwards to the tongue below that muscle, and disappears in front beneath the mylo-hyoideus. In this course the nerve passes over the two carotids; and near the cornu of the os hyoides it crosses also the lingual artery, so as to become higher than the vessel. From this part arise the descendens noni branch, and a small muscular offset to the thryo-hyoideus.

The descending branch (ram. descend. noni) leaves the trunk of the hypoglossal on the outer side of the carotid artery, and descends on the front of (sometimes in) the sheath of the vessel to about the middle of the neck, where it is joined by the communicating branches of the cervical nerves. After the union of the spinal nerves, offsets are supplied to the depressor muscles of the os hyoides, viz., omo-hyoid (both bellies), sternohyoid, and sterno-thyroid: sometimes another offset is continued to the thorax, where it joins the phrenic and cardiac nerves.

The connection between the descendens noni and the spinal nerves is formed by two or more cross filaments, so as to construct an arch with the coneavity upwards; and an interchange of fibrils between the two nerves is supposed to take place.

The extersal Carotid artery (fig. 17, $d$ ) springs from the bifurcation of the common carotid at the upper border of the thyroid cartilage, and furnishes branches to the neck, the face, and the outer parts of the head.

From the place of origin it ascends.in front of the mastoid process, and ends near the condyle of the jaw in the internal maxillary and temporal branches. The artery lies at first to the inner side of the internal carotid, but 
it afterwards becomes superficial to that vessel ; and its direction is somewhat arched forwards, though the position would be marked sufliciently by a line from the front of the meatus of the ear to the cricoid cartilage.

At first the external carotid is overlaid by the sterno-mastoideus, and by the common coverings of the anterior triangular space, viz., the skin, and the superficial and deep fascia with the platysma. But above the

Fig. 17.

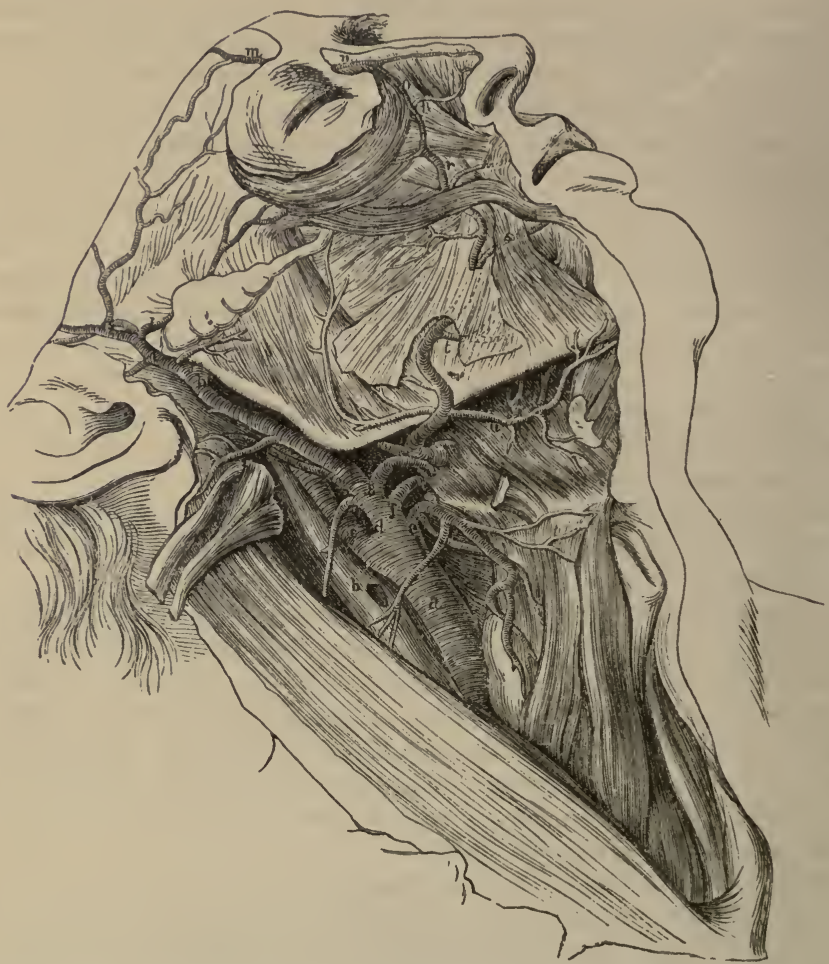

External Carotid and Its SuPerficial Branches ("Anatomy of the Arteries," Quain).

a. Common carotid.

b. Internal jugular vein.

c. Internal carotid.

d. External carotid.

e. Upper thyroid branch.

f. Lingual.

g. Facial.

h. Internal maxillary.

i. Superficial temporal. $m$. Supra-orbital.

$n$. External nasal.

o. Angular branch of facial.

$p$. Lateral nasal.

$r$. Superior coronary.

$s$. Inferior coronary.

$t$. Inferior labial.

$u$. Submental artery.

level of a line from the mastoid process to the hyoid bone, the artery is crossed by the digastric and stylo-hyoid muscles, and still higher the parotid gland conceals it. At its beginning the artery rests against the pharynx; but above the angle of the jaw it is placed over the styloid process and stylo-pharyngeus muscle, which separates it from the internal carotil. To the inner side of the vessel at first is the pharynx; and still higher, are the ramus of the jaw and the stylo-maxillary ligament. 
Veins. There is not any companion vein with the external carotid as with most arteries; but sometimes a vein, formed by the union of the temporal and interual maxillary branches (p. 42), will accompany it. Near the beginning it is crossed by the facial and lingual branches joining the internal jugular vein; and near the ending the external jugular vein lies over it.

Nerves are directed from behind forwards over and under the artery. At the lower border of the digastric muscle the hypoglossal lies over the ressel, and near the ending the ramifications of the facial nerve are superficial to it. Three nerves lie beneath it: beginning below, the small external laryngeal ; a little higher, the superior laryngeal; and near the base of the jaw, the glosso-pharyngeal.

The branches of the external carotid are numerous, and are classed into an anterior, posterior, and ascending set. The anterior set comprise branches to the thyroid body, the tongue, and the face, viz., superior thyroid, lingual, and facial arteries. In the posterior set are the occipital and posterior auricular branches. And the ascending set include the ascending pharyngeal, temporal, and internal maxillary arteries. Besides these, the carotid gives other branches to the sterno-mastoid muscle and the parotid gland.

The origin of the branches of the earotid may be altered by their closer aggregation on the trunk. The usual number may be diminished by two or more uniting into one; or the number may be increased by some of the secondary offsets being transferred to the parent trunk.

Directions. All the branches, except the ascending pharyngeal, lingual, and internal maxillary, may be now examined; but those three will be described afterwards with the regions they occupy.

The superior thyroid artery $(e)$ arises near the cornu of the os hyoides, and passes beneath the omo-hyoid, sterno-hyoid, and sterno-thyroid muscles to the thyroid body, to which it is distributed on the anterior aspect. This artery is superficial in the anterior triangle, and furnishes offsets to the lowest constrictor and the muscle beneath which it lies, in addition to the following named branches :-

a. The hyoid branch is very inconsiderable in size, and runs inwards below the hyoid bone: it supplies the muscles attached to that bone, and anastomoses with the vessel of the opposite side.

b. A branch for the sterno-mastoid muscle lies in front of the sheath of the common carotid artery, and is distributed chiefly to the muscle from which it takes its name.

c. The laryngeal branch pierces the membrane between the hyoid bone and the thyroid cartilage, with the superior laryngeal nerve, and ends in the interior of the larynx.

d. A small crico-thyroid branch is placed on the membrane between the cricoid and the thyroid cartilage, and communicates with the corresponding artery of the opposite side, forming an arch.

The superior thyroid vein commences in the larynx and the thyroid body, and crosses the end of the common carotid artery to open into the internal jugular vein.

The facial artery $(g)$ arises above the lingual, and is directed upwards over the lower jaw to the face. In the neck the artery passes beneath the digastric and stylo-hyoid museles, and is afterwards lodged on the submaxillary gland, on which it makes a sigmoid turn. Its anatomy in the 
face has been examined (p. 40). From the cervical part branches are given to the pharynx, and to the structures below the jaw, viz. :-

a. 'The inferior palatine branch ascends to the pharynx beneath the jaw, passing between the stylo-glossus and stylo-pharyngeus muscles, and is distributed to the soft palate, after furnishing a branch to the tonsil. 'This branch frequently arises fiom the ascending pharyngeal artery.

b. The tonsillar branch is smaller than the preceding, and passes between the internal ptorygoid and stylo-glossus muscles. Opposite the tonsil it perforates the constrictor muscle, and ends in offsets to that borly.

c. Glandular branches are supplied to the submaxillary gland from the part of the artery in contact with it.

d. The submental branch arises near the inferior maxilla, and courses forwards on the mylo-hyoideus to the anterior belly of the digastric muscle, where it ends in offsets: some of these turn over the jaw to the chin and lower lip; and the rest supply the museles between the jaw and the hyoir bone-one or two perforating the mylo-hyoideus and anastomosing with the sublingual artery.

The facial vein (p. 40) joins the internal jugular. In the cervical part of its course it receives branches corresponding with the offisets of the artery. It often throws itself into the temporo-maxillary trunk.

The occipital artery is of considerable size, and is destined for the back of the head. It arises from the carotid opposite the facial branch, near the lower border of the digastric muscle, and ascends to the inner part of the mastoid process of the temporal bone. Next it turns horizontally backwards on the occipital bone, passing above the transverse process of the atlas; and finally becomes cutaneous near the middle line (p. 21). In the neck this artery passes beneath the digastric muscle and a part of the parotid gland; and crosses over the internal carotid artery, the jugular vein, and the spinal accessory and hypoglossal nerves.

The only offiset from the artery in the front of the neck is a small posterior meningeal branch: this ascends along the internal jugular vein, and enters the skull by the foramen jugulare (p. 30). The branches at the back of the neck will be afterwards seen.

The occipital vein begins at the back of the head (p. 21), and has the same course as the artery ; it communicates with the lateral sinus through the mastoid foramen, also with the diploic veins, and coalesces with the internal (sometimes the external) jugular vein.

The posterior auricular artery is smaller than the preceding branch, and takes origin above the digastric muscle. Between the ear and the mastoid process, it divides into two branches for the ear and occiput $(p, 21)$.

A small branch, stylo-mastoid enters the foramen of the same name, and supplies the tympanum of the ear.

The vein with the artery receives a stylo mastoid branch, and terminates in the trunk formed by the temporal and internal maxillary veins. .

The temporal artery $(i)$ is in direction the continuation of the external carotid trunk, and is one of the terminal branches of that artery. Ascending under the parotid gland it divides on the temporal fascia into anterior and posterior branches, about two inches above the zygoma; these are distributed to the front and side of the hear (p.21). 'The trunk of the artery gives offsets to the surromnling parts, viz. :

a. Parotid branches are furnished to the gland of the same name. Articular twigs are supplied to the articulation of the lower jaw; and 
other muscular branches enter the masseter. Some anterior auricular offsets are distributed to the pinna and meatus of the external ear.

$b$. The transverse facial branch quits the temporal artery opposite the condyle of the jaw, and is directed forwards over the masseter muscle (p. 41); on the side of the face it supplies the muscles and integuments, and anastomoses with the facial artery.

c. The middle temporal branch arises just above the zygoma, and pierces the temporal aponeurosis to enter the substance of the temporal muscle: it anastomoses with branches of the internal maxillary artery.

d. A small branch of the temporal artery is likewise found between the layers of the temporal fascia ; this anastomoses with an offset of the lachrymal.

The temporal vein commences on the side of the head ( $p .21)$ and is contiguous to its companion artery. Near the zygoma it is joined by the middle temporal vein; next it receives branches which are companions of the offisets of the artery; and it ends by uniting with the internal maxillary vein.

Directions. The lower part of the neck will not be used again for some days, so that the dissector may stitch together the flaps of skin, when he has applied salt to preserve it.

\section{Section VI.}

\section{PTERYGO-MAXILLARY REGION.}

In this region are included the muscles superficial to and beneath the ramus of the lower jaw, together with the articulation of that bone. In contact with the muscles (pterygoid) beneath the jaw, are the internal maxillary bloodvessels, and the inferior maxillary trunk of the fifth nerve.

Dissection. The masseter muscle, which is superficial to the bones, has been partly laid bare in the dissection of the facial nerve. To see it more fully the branches of the facial nerve, and the transverse facial artery should be cut through, and turned backwards off the face. A little cleaning will suffice to define the origin and insertion of the muscle.

Should there be any tow or cotton wool in the mouth let it be removed.

The MASSETER (fig. $6,{ }^{10}$ ) is partly aponeurotic at the upper attachment. It arises from all the lower border of the zygomatic arch, extending forwards to the upper jaw; and from the inner surface of the arch by fine fleshy fibres. Most of the fibres are inclined down and somewhat back, and are inserted into the outer surface of the coronoid process, ramus, and angle of the lower jaw; but a few are fixed into the contiguous part of the body of the bone as far as the second molar tooth. Some of the hinder and deeper fibres are inclined downwards and forwards across the others:

The lower part of the masseter is subcutaneous, but the upper is partly concealed by the parotid gland (socia parotidis), and is crossed by Stenson's duct, and by the transverse facial vessels and the facial nerve. The anterior border projects over the buecinator muscle, and a quantity of fat resembling that in the orbit is found beneath it. The muscle covers the 
ramus of the jaw, and the masseteric branches of nerve and artery entering it at the under surface.

Action. It raises the lower jaw with the internal pterygoid in the mastication of the food.

Dissection. 'To lay bare the temporal muscle to its insertion, the following dissection may be made: The temporal fascia is to be detached from the upper border of the zygomatic areh, and to be removed from the surface of the muscle. Next, the arch of the zygoma is to be sawn through in front and behind, so as to include all its length; and is to be thrown down (without being cut off) with the masseter muscle still attached to it, by separating the fibres of that muscle from the ramus of the jaw. In detaching the masseter muscle, its nerve and artery, which pass through the sigmoid noteh, will be found.

The surface of the temporal muscle may be then cleaned; and to expose its insertion and deep origin, let the coronoid process be sawn off by a cut passing from the centre of the sigmoid notch nearly to the last molar tooth, so as to include the whole insertion of the muscle. Before sawing the bone let the student find and separate from the muscle the buceal vessels and nerve issuing from beneath it. Lastly, the coronoid process should be raised and the fat removed, in order that the lower fibres of the temporal musele, and their contiguity to the external pterygoid elose below them, may be observed.

The temporal muscle (fig. 18, ${ }^{1}$ ) takes its origin from the whole of the temporal fossa (p.20), reaching up to the semicireular line on the side of the skull, and downwards to the crest on the outer aspect of the great wing of the sphenoid bone. From this extensive attachment, as well as from the fascia over it, the fibres converge to a superficial tendon, which is inserted into the inner surface of the coronoid process, as well as into a groove on the same process which reaches from the apex to near the last molar tooth.

Behind the posterior border of the tendon are the masseteric vessels and nerve, and in front of it the buceal vessels and nerve : the last nerve perforates occasionally some of the fibres of the muscle.

Action. All the fibres contracting the muscle will raise the mandible and press it forcibly against the upper jaw ; but the hinder fibres may retract the lower jaw after it has been moved forwards by the external pterygoid.

Dissection. For the display of the pterygoid muscles (fig. 18), it will be necessary to remove a piece of the ramus of the jaw. But the greater part of the temporal muscle is to be first detached from the subjacent bone with the handle of the sealpel, and the deep temporal vessels and nerves are to be sought in its fibres.

A piece of the ramus is next to be taken away by sawing across the bone close to the condyle, and again close above the dental foramen; and to make the dental vessels and nerve in contact with its inner surface secure from injury, the handle of the sealpel may be inserted between them and the bone, and earried downwards to their entrance into the foramen. The masseteric artery and nerve are liable to be cut in sawing the bone; should these be divider, turn them upwards for the present, and afterwards tie torether the ends.

After the loose piece of bone has been removed, and the subjacent parts freed from inucl fat, the pterygoid muscles will appear, - the external $\left({ }^{2}\right)$ being directed outwards to the condyle of the jaw, and the internal $\left({ }^{3}\right)$, which is parallel in direction to the masseter, being inclined to the angle 
of the jaw. In removing the fatty tissue, the student must be careful not to take away the thin lateral ligament, which lies on the internal pterygoid muscle beneath the ramus.

Position of vessels. Crossing inwards over the external pterygoid muscle, is the internal maxillary artery, which distributes offsets upwards

Fig. 18.'

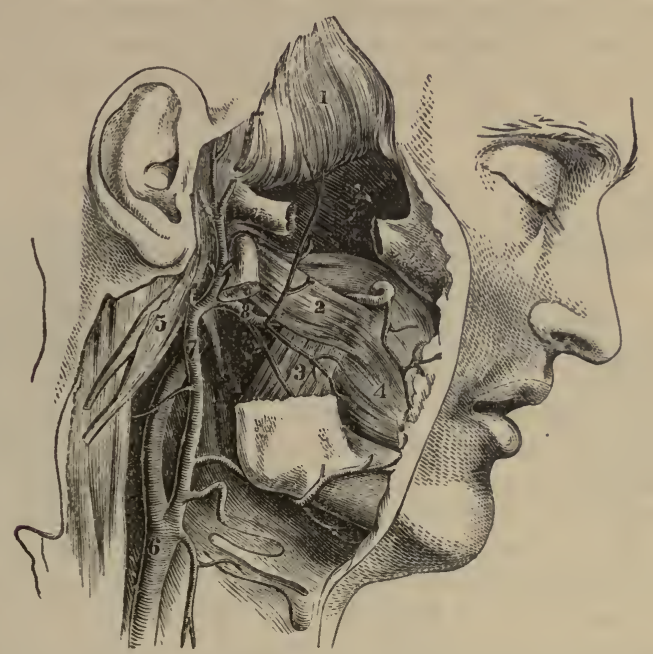

Superfictal View of the Pteryaid Region (Quain's "Arteries").

1. Temporal muscle.

2. External pterygoid.

3. Internal pterygoid.

4. Buccinator.

5. Digastric and stylo-hyoid muscles cut and thrown back.
6. Common carotid dividing into external and interual trunks.

8. Internal maxillary artery (beneath the pterygoid instead of over it) and its brauches.

The nerves are omitted in this woodcut.

and downwards: sometimes the artery will be placed beneath the muscle. The veins with the artery are large and plexiform: and may be taken away.

Position of nerves. Most of the branches of the inferior maxillary nerve appear in this dissection. Thus, issuing from beneath the lower border of the external pterygoid are the large dental and gustatory nerves, the latter being the more internal of the two; and coming out behind the joint of the jaw is the auriculo-temporal nerve. Appearing between the upper border of the muscle and the cranium, are the small masseteric and deep temporal nerves. 'The buccal branch of the nerve perforates the fibres of the same muscle near the inner attachment. Branches of the abovementioned artery accompany the nerves. Coursing along the posterior part of the upper jaw, is the small posterior dental nerve with an artery.

Between the jaws is the whitish narrow band of the pterygo-maxillary ligament, to which the buccinator and superior constrictor muscles are connected.

The exterial pterigoid yuscle (fig. $18,{ }^{2}$ ) extends almost horizontally from the zygomatic fossa to the neck of the lower jaw. Its origin is 
from the outer surface of the great wing of the sphenoid bone below the crest, and from the outer surface of the external pteryoid plate. 'The fibres are directed outwards and somewhat backwards, those attached to the upper margin of the spheno-maxillary fissure forming at first a separate bundle, and are inserted into the hollow in front of the neck of the lower jaw bone, and into the interarticular fibro-cartilage of the joint.

Externally the pterygoid is concealed by the temporal muscle and the lower jaw, and the internal maxillary artery lies on it. By the deep surface it is in contact with the inferior maxillary nerve, with a plexus of veins, and with the internal lateral ligament of the joint of the jaw. The parts in contact with the borders of the muscle have been enumerated before.

Sometimes the slip of the muscle, which is attached to the margin of the spheno-maxillary fissure and the root of the external pterygoid plate, is described as a separate head with an insertion into the interarticular cartilage.

Action. If both muscles act the jaw is moved forwards, so that the lower dental arch is placed in front of the upper, and the grinding teeth are rubbed together in an antero-posterior direction. In order that the lower front teeth may be able to pass the others the jaw is depressed.

One muscle contracting (say the right), the condyle of the same side is drawn inwards and forwards, and the grinding teeth of the lower jaw are moved horizontally to the left across those of the upper. By the alternate action of the two muscles the trituration of the food is effected.

The internal Ptenygoid Muscle (fig. $18,{ }^{3}$ ) is nearly parallel to the ramus of the jaw, and its fibres are longer than those of the preceding muscle. Arising in the pterygoid fossa, and ehiefly from the inner surface of the external pterygoid plate, the muscle is further attached below, outside the fossa, to the outer surface of the tuberosity of the palate bone, and to the tuberosity of the upper jaw bone. The fibres descend to be inserted into the angle, and into the inner surface of the ramus of the jaw as high as the inferior dental toramen.

On the muscle are placed the dental and gustatory nerves, the dental artery, and the internal lateral ligament of the jaw. The deep surface is in relation below with the superior constrictor, and at its origin with the tensor palati muscle.

Action. From the direction and attachment of the fibres the muscle will unite with the masseter in elevating the jaw.

Directions. Before proceeding further in the disseetion, the student may learn the anatomy of the articulation of the lower jaw.

TEMPORO-MAXILLARY ARTICULATION. In this articulation are combined the condyle of the jaw and the anterior part of the glenoid fossa of the temporal bone; but the osseous surfaces are not in contact, for a picee of fibro-cartilage with two synovial sacs is interposed between them. The bones are retained in apposition mostly by the strong museles of the lower jaw ; but the following ligaments serve to unite them.

Capsule. This is a thin fibrous tube inclosing the bones, and is wider above than below. By the upper end it is fixed around the articular surface of the temporal bone in front of the Glaserian fissure; and it is inserted below around the condyle of the lower jaw. The space in the interior is divided into two, upper and lower, by a piece of fibro-cartilage, which is united to the capsule by its eircumference.

'The external lateral is a short ligamentous band, being but a part of the 
capsule, which is attached abore to the tubercle at the root of the zygoma, and below to the outer side of the neck of the inferior maxilla.

The internal lateral ligament (fig. $19,^{1}$ ) is a long, thin, membranous band, which is not in contact with the joint. Superiorly it is connected to a projection inside the glenoid fossa, which consists of the spinous process of the sphenoid and the vaginal process of the temporal bone; and inferiorly it is inserted into the orifice of the dental canal in the lower jaw. The ligament lies between the jaw and the internal pterygoid; and its origin is concealed by the external pterygoid muscle. Between the ligament and the jaw the internal maxillary artery intervenes.

Fig. 19.

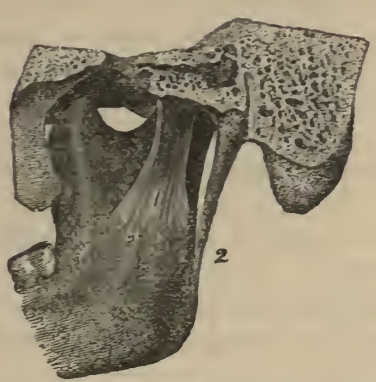

LIGAMEST3 OP THE JAW-AN INIER VIRW (Bourgery and Jacob).

1. Internal lateral ligament.

2 Stylo-maxillary.

3. Stylo-maxillary ligameat.
Fig. 20.

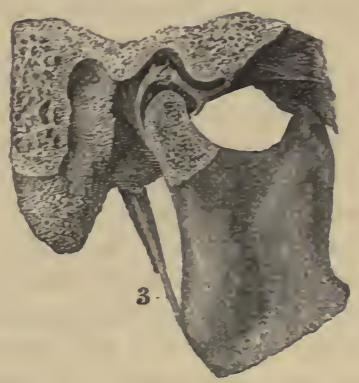

A VIEW OF TBE INTBRIOR OP THE COMPOEND TEMPOBO-MAXILLARY JOIST (Bourgery and Jacob).

4. Interarticular fibro-cartilage-the dark interrals above and below are the hollows containing the synovial membranes.

Dissection. After the external lateral ligament and the capsule of the joint have been examined, an interartieular fibro-cartilage, with a hollow above and below it, will be exposed by taking away the capsule on the outer side (fig. 20).

The interarticular fibro-cartilage (fig. 20,4 ) is adapted to the surfaces of the bones. It is elongated transversely, is thinner in the centre than at the margins, and an aperture is sometimes present in the middle. The upper surface fits into the glenoid fossa, being coneavo-convex from before backwards, and the lower is moulded on the conrexity of the condrle of the jaw. By the cireumference it is connected with the capsule and the external lateral ligament; and in front the external pterygoid muscle is attached to it.

This interarticular pad allows greater freedom of movement in the joint without dislocation; diminishes the injurious effect of pressure; and deadens the sound of the jaw striking the skull.

Two synovial membranes are present in the articulation-one above, and one below the fibro-cartilage. The lower one is the smaller of the two.

Another structure-the stylo-maxillary ligament (fig. $20,{ }^{3}$ ) is described as a uniting band to this articulation. It is a process of the deep cervical fascia, which extends from the styloid process to the hinder part of the ramus of the jaw: it gires attachment to the stylo-glossus muscle, and separates the parotid and submaxillary glands. 
Articular surfaces of the bones. The lower jaw possesses a thin narrow condyle, which is elongated transversely, and directed backwards and in wards.

On the temporal bone is a narrow deep articular hollow (glenoid fossa), which is lengthened from without in, and is placed in front of the Glaserian fissure. In front of this is a prominence of bone (transverse root of the zygomatic process), which is convex from before back and rather hollowed from side to side.

Movements of the joint. This condyloid articulation is provided with an up and down, a to and fro, and a lateral movement.

In depressing the jaw, as in opening the mouth, the articular condyle moves forwards till it is placed under the convexity at the fore part of the articular hollow, but the interposed concave fibro-cartilage gives security to the joint. Even with this provision, a slight degree more of sudden motion throws the condyle off the prominence of the temporal bone into the zygomatic fossa, and gives rise to dislocation.

In this movement the fore and lateral parts of the capsule are made tight; and the fibro-cartilage is drawn forwards with the condyle by the external pterygoid muscle.

When the jaw is elevated and the mouth closed, the condyle and the fibro-cartilage glide back into the glenoid fossa. In this position the jaw is placed in the state of greatest security against dislocation.

The ligaments and the surrounding muscles, which were stretched in the previous movement, are then set at rest.

During the horizontal motion forwards and backwards the condyle is moved successively to the front and back of the temporal articular surface; and the lower jaw is slightly depressed, in order that the fore teeth in the upper dental arch should not imperle those of the lower.

By turns the front and back of the capsule will be stretched; and the fibro-cartilage always follows the condyle of the jaw, even in dislocation.

'Too great motion forwards will be prevented by the coronoid process of the jaw striking against the zygomatic arch; and that backwards, by the meeting of the condyle and the auditory process of the os temporis.

Lateral horizontal movement puts the jaw first to one side and then to the other. When the jaw is forced to the left side, the right condyle sinks into its articular hollow, whilst the left is projected; and the grinding teeth of the lower dental arch are moved to the left across those of the upper. By the alternate aetion to opposite sides the food is triturated.

The inner part of the capsule on the right, and the outer part on the left side, will be put on the stretch when the jaw is carried to the left of the middle line; and the opposite.

With old edentulous jaws the capsule is much enlarged, and permits the condyle to wander backwards behind the Glaserian fissure. Without this provision the altered lower jaw would not meet the upper to crush the food.

Dissection. The condyle of the jaw is next to be disarticulated, the external pterygoid musele being still uneut; and it with the attached muscle is to be drawn forwards so as to allow the fifth nerve to be seen. Whilst eutting through the joint eapsule, the dissector must be eareful of the auriculo-temporal nerve close beneath (fig. 21).

On drawing forwards the pterygoid muscle, and removing some fat, the dissector will find the trunk of the inferior maxillary nerve. All the small muscular branches of the nerve before noted should be traced to the trunk in the foramen ovale of the sphenoid bone. The auriculo-temporal branch 
should be followed backwards with eare behind the articulation, and the dental and gustatory nerves beneath the muscle should be cleaned. The small chorda tympani is then to be found joining the posterior part of the gustatory nerve near the skull.

The middle meningeal artery is to be sought beneath the external pterygoid. Sometimes the trunk of the internal maxillary artery lies beneath that musele, and in such ease, it and its branches are to be traced out.

The INTERNAL MAXILlary ARTERY (fig. $17, l l$ ) is one of the terminal branches of the external carotid, and takes a winding course beneath the lower jaw and the temporal muscle to the spheno-maxillary fossa, where it ends in branches for the face, the interior of the nose, and the palate and pharynx.

At first the artery is directed inwards beneath the jaw, between that bone and the internal lateral ligament of the joint, and crosses the dental nerve. Next, the vessel winds over the external pterygoid muscle, being placed between it and the temporal muscle. And lastly, the artery enters the spheno-maxillary fossa between the proeesses of origin of the external pterygoid. The course of the artery is sometimes beneath, instead of over the external pterygoid: in such a state the artery gains the spheno-maxillary fossa by coming upwards through the origin of the musele, as in the woodeut.

The branches of this artery are very numerous, and are elassed into three sets: thus one set arises beneath the jaw; another between the museles; and another in the spheno-maxillary fossa.

Two branches, viz., the inferior dental and middle meningeal, leave the internal maxillary artery whilst it is in contact with the ramus of the jaw.

The inferior dental branch descends between the internal lateral ligament and the jaw, and enters the foramen on the inner surface of the ramus, along with the dental nerve: it supplies the teeth, and ends in the lower part of the face.

As this artery is about to enter the foramen it furnishes a small twig, mylo-hyoid branch, to the muscle of that name; this is condueted by a groove on the inuer surface of the bone, in company with a branch from the dental nerve, to the superficial surfice of the mylo-hyoid musele, where it anastomoses with the submental artery.

The great meningeal artery is the largest branch, and arises opposite the preceding. It ascends beneath the external pterygoid muscle, and (oftentimes) between the roots of the auriculo-temporal nerve; crossing the internal lateral ligament, it enters the skull through the foramen spinosum of the sphenoid bone. When in the skull the artery ascends to the vertex of the head, and supplies the bone and the dura mater (p. 29). Before the meningeal artery enters the skull, it furnishes the following small branches:

a. The tympanic branch (inferior) passes into the tympanum through the Glaserian fissure, and is distributed to the membrana tympani and that eavity.

b. A deep auricular branch arises with the former or separately enters the meatus through the cartilage, or between this and the bone, and ramifies in the meatus and on the membrana tympani.

$c$. The small meningeal branch begins near the skull, and courses through the foramen ovale with the inferior maxillary nerve; it ramifies in the dura mater in the middle fossa of the skull.

Another small branch springs from the dental artery or the internal 
maxillary trunk, and accompanying the gustatory nerve, ends in the eheek and the mucous membrane of the mouth.

'The branches from the second part of the artery, viz., whilst it is between the temporalis and pterygoideus externus are distributed to the temporal, masseteric, buccal, and pterygoid museles.

The deep temporal arteries are two in number (anterior and posterior); and each occupies the part of the temporal fossa indicated by its name. They ascend beneath the temporal muscle. and anastomose with the superficial temporal artery: the anterior communicates, through the malar bone, with branches of the lachrymal artery.

When the parent trunk has the unusual position beneath the pterygoid, the anterior branch lies under that muscle, instead of over it.

The masseteric artery is directed outwards with the nerve of the same name behind the tendon of the temporal muscle; and passing through the sigmoid notch, enters the under surface of the masseter muscle. Its branches anastamose with the other offsets to the muscle from the external carotid trunk.

The buccal branch quits the artery near the upper jaw, and in the unusual position of the artery it may perforate the fibres of the pterygoid; it descends beneath the coronoid process with its companion nerve, and is distributed to the buccinator muscle, the cheek, and the side of the face, joining the branches of the facial artery.

The pterygoid branches are uncertain in their position; whether derived from the trunk or some of the branches of the internal maxillary, they enter both pterygoid muscles.

Of the branches that arise from the artery when it enters the sphenomaxillary fossa, only one, the superior dental, will be now described. The remainder will be examined with the superior maxillary nerve and Meckel's ganglion; they are infraorbital (p. 105), superior palatine, naso-palatine, vidian, and pterygo-palatine (SECTION 14).

The superior or posterior dental branch takes origin near the top of the upper maxilla, and descends with a tortuous course on the outer surface of that bone, along with a small branch of the superior maxillary nerve. It sends twigs into the foramina in the bone, and supplies the upper molar and bicuspid teeth; but some external offsets are furnished to the gums. A few branches reach the lining membrane of the antrum.

The INTERNAL MAXILLARY VEIN receive the off'sets accompanying the branches of the artery in the first two parts of its course : these veins form a plexus-pterygoid, between the two pterygoid muscles, and in part between the temporal and external pterygoid muscles. This anastomosis communicates with the alveolar plexus; with the facial vein by a large branch (anterior internal maxillary); and with the cavernous sinus in the interior of the skull, by veinules that pass through the base of the cranium.

Escaping from the plexus, the vein accompanies the artery to the parotid gland, and there joins the superficial temporal vein,- - the union of the two giving rise to the external jugular. Sometimes this common vessel enters the internal jugular vein (p. 42).

The inferior maxillary nERve (fig. 21 ) is the largest of the three trunks arising from the Gasserian ganglion (p. 32). It leaves the skull by the foramen ovale in the sphenoid bone, and divides beneath the external pterygoid muscle into two chief pieces, viz., an anterior, small, motosensory part; and a large, posterior, chiefly sensory portion. 
Directions. Should the internal maxillary artery obstruct the view of the nerve, it may be cut through.

The SMALLER PAIT, formed mainly by its contribution from the trunk of the nerve, receives nearly all the fibrils of the motor root, and ends in branches for the muscles of the jaw, viz., temporal, masseter, and one pterygoid ; and for the muscle of the cheek, the buccinator.

Fig. 21.

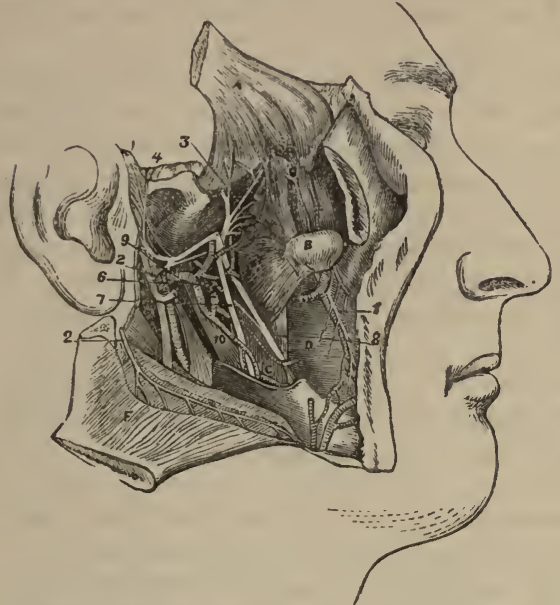

Defp View of the Ptirygoid Region (Illustrations of Dissections).

Muscles :-

a. Temporal reflected.

b. Condyle of the jaw disarticulated with the external pterygoid attached to it.

c. Internal pterygoid.

d. Buccinator.

$f$. Massuter thrown down.

Nerves :-

1. Buccal.

2. Masseteric, cut.

3. Deep temporal.

4. Auriculo-temporal.

6. Chorda tympani.

7. Inferior dental.

8. Gustatory.

10. Internal lateral ligament of the lower jaw. The arteries are not figured, with the exception of the internal maxillary trunk which is marked with 9: the offsets of the artery accompany the nerves, being named like them.

The deep temporal branches $\left({ }^{3}\right)$ are furnished to the under surface of the temporal muscle. Like the arteries, they are two in number, anterior and posterior, and course upwards beneath the external pterygoid muscle.

The posterior branch is the smallest, and is often derived from the masseteric nerve; it is placed near the back of the temporal fossa.

The anterior branch supplies the greater part of the muscle, and communicates sometimes with the buccal nerve.

The masseteric branch $\left({ }^{2}\right)$ takes a backward course above the external pterygoid muscle, and through the sigmoid notch, to the under surface of the masseter muscle: in the masseter the nerve can be followed to near the anterior border. As this branch passes by the articulation of the jaw it gives one or more $t$ wigs to that joint.

The pterygoid branches come from both parts of the inferior maxillary nerve.

The branch or branches to the external pterygoid spring from the small part, or from the buceal nerve, and enter the under surface of its muscle.

The nerve to the internal pterygoid arises from the large part of the maxillary trunk close to the skull, and may be followed beneath the upper border to the deep surface of the muscle; it will be learnt in the dissection of the otic ganglion (Section 14).

The buccal branch $\left({ }^{1}\right)$, longer and larger than the others, perforates the external pterygoid, and is directed inwards, heneath the coronoid process to the surface of the buccinator, where it ends in terminal branches. As it perforates the pterygoid muscle filaments are given to the fleshy sub- 
stance; and after it has passed through the fibres it furnishes a branch to the temporal muscle. 'The nerve is directed towards the angle of the mouth, supplying the integument, the buccinator muscle, and the lining mucous membrane. It is united freely with the facial nerve, the two forming a plexus.

The LARGER PART of the inferior maxillary nerve divides into three trunks-auriculo-temporal, dental, and gustatory. A few of the fibrils of the small (motor) root are applied to it, and are conveyed to certain muscles, viz., tensor tympani, circumflexus palati, pterygoideus, internus, mylohyoideus, and digastricus.

The ACRICULO-TEMPORAL NERVE $\left({ }^{4}\right)$ separates from the others near the base of the skull, and has commonly two roots. Its course to the surface of the head is directed first backwards beneath the external pterygoid muscle, as far as the inner part of the articulation of the jaw; and, then, upwards with the temporal artery in front of the ear. The nerve furnishes branches to the surrounding parts, viz., the joint, the ear, and the parotid gland; and it communicates with the facial nerve. Its ramifications on the head are described at page 23. In the part now dissected its branches are the following:-

a. Branches of the meatus auditorius. Two offsets are given to the meatus from the point of union of the branches of the facial with the auriculo-temporal nerve, and enter that tube between the cartilage and bone.

b. Articular branch. The branch to the joint of the jaw arises near the same spot as the preceding, or from the branches to the meatus.

$c$. The inferior auricular branch supplies the external ear below the meatus auditorius: it sends offsets along the internal maxillary artery, which communicates with the sympathetic nerve.

d. Parotid branches. These small filaments ramify in the gland.

e. Communicating branclies. 'Two or more branches around the external carotid artery communicate with the facial and sympathetic nerves.

The INFERIOR Dental $\left({ }^{7}\right)$ is the largest of the three trunks into which the inferior maxillary nerve divides. In its course to the canal in the lower jaw, the nerve is external to the gustatory, and lies at first beneath the external pterygoid muscle; it is afterwards placed on the internal pterygoid, and on the internal lateral ligament near the dental foramen. After the nerve enters the bone, it is continued forwards beneath the teeth to the foramen in the side of the jaw, and ends at that spot by dividing into an incisor and a labial branch. Only one muscular offset (mylo-hyoid) is supplied by the dental nerve before it enters the bone. Its branches are:-

a. The mylo-hyoid branch arises from the trunk of the nerve near the dental foramen, and is continued along a groove on the inner aspect of the ramus of the jaw to the cutaneous surface of the mylo-hyoideus, and to the anterior belly of the digastric muscle.

$b$. The dental branches arise in the bone, and supply the molar and bicuspid teeth. If the bone is soft, the canal containing the nerve and artery may be laid open so as to expose these branches.

c. The incisor branch continues the trunk of the nerve onwards to the middle line, and furnishes offisets to the canine and incisor teeth, beneath which it lies.

d. The labial branch (mental?) (fig. $9,{ }^{13}$ ) issues on the face beneath 
the depressor of the angle of the mouth. It gives branches to the muscles below the aperture of the mouth, and communicates with the facial nerve; but the greater part of the branch is directed upwards beneath the depressor labii inferioris, and is distributed on the inner and outer surfaces of the lower lip.

The inferior dental artery, after entering the lower jaw, has a similar course and distribution to the nerve. Thus it supplies offsets to the bone, dental branches to the molar and bicuspid teeth, and ends anteriorly in an incisor and a labial branch.

The incisor branch is continued to the symphysis of the jaw, where it ends in the bone: it lies beneath the canine and incisor teeth, to which it furnishes twigs.

The labial branch, issuing by the labial foramen, ramifies in the structures covering the lower jaw, and communicates with the branches of the facial artery.

The GUSTATORY or LINGUAL NERVE $\left({ }^{8}\right)$ is the remaining trunk of the inferior maxillary, and is concealed at first, like the others, by the external pterygoid muscle. It is then inclined inwards with a small artery over the internal pterygoid muscle, and under cover of the side of the jaw to the tongue. The remainder of the nerve will be seen in the dissection of the submaxillary region.

In this course under the jaw the nerve does not distribute any branch to the parts around, but the following communicating branch is received by it.

The chorda tympani is a branch of the facial nerve, and is distributed to the tongue. Escaping from the tympanum by the Glaserian fissure, this small branch $\left({ }^{6}\right)$ is applied to the gustatory nerve at an acute angle. At the point of junction some fibrils communicate with the gustatory, but the greater part of the chorda tympani is conducted along that nerve to the tongue.

The origin of the nerve, and its course across the tympanum to its position beneath the external pterygoid, are described in SECTION 14.

\section{SECTION VII.}

\section{SUBMAXILLARY REGION.}

The submaxillary region is situate between the lower jaw and the hyoid bone. In it are contained the muscles of the os hyoides and tongue, the vessels and nerves of the tongue, and the sublingual and submaxillary glands.

Position. In this dissection the position of the neck is the same as for the examination of the anterior triangle.

Dissection. If any fatty tissue has been left on the submaxillary gland, or on the mylo-hyoid muscle, when the anterior triangular space was dissected, let it be taken away.

The submaxillary gland (fig. $16,{ }^{11}$ ) lies below the jaw in the anterior part of the space limited by that bone and the digastric muscle. Its shape is irregular, and the facial artery winds over the surface. It rests on the mylo-hyoideus, and sends a deep process round the posterior or free border 
of that muscle. In front of it is the anterior belly of the digastric ; and behind is the stylo-maxillary ligament separating it from the parotid. Occupying a position somewhat below the side of the jaw, the gland is very near the surface, being covered only by the integuments and platysma, and the deep fascia.

In structure the submaxillary resembles the parotid gland (p. 4\%); and its duct-duct of Wharton-issuing from the deep process, extends beneath the mylo-hyoid muscle to the mouth.

Dissection. To see the mylo-hyoid muscle, detach the anterior belly of the digastric from the jaw, and dislodge without injury the submaxillary gland from beneath the bone.

The myLO-HYoId MUSCLE (fig. $33,{ }^{3}$ ) is triangular in shape, with the base at the jaw and the apex at the hyoid bone, and unites along the middle line with its fellow of the opposite side. It arises from the mylohyoid ridge on the inner surface of the lower jaw as far back as the last molar tooth; and is inserted into the middle of the body of the os hyoides, as well as into a central tendinous band between that bone and the jaw.

On the cutaneous surface lie the digastric muscle, and the submaxillary gland, the facial artery with the submental offset, and its own branch of nerve and artery. Its fibres are frequently deficient near the jaw, and allow the next muscle to be seen. Only the posterior border is unattached, and round it a piece of the submaxillary gland winds. The parts in contact with the deep surface of the muscle will be perceived after the undermentioned dissection has been made.

Action. The lower jaw being fixed the muscle approaches the os hyoides to the jaw, enlarging the pharynx preparatory to swallowing.

With the hyoid bone immovable, the mylo-hyoideus can help in depressing the jaw, and opening the mouth.

Dissection. 'To bring into view the museles beneath the mylo-hyoid, and to trace the vessels and nerves to the substance of the tongue (as in tigure 23), the student should first divide the facial ressels on the jaw, and remove them with the superficial part of the submaxillary gland; but he should be careful to leave the deep part of the gland which turns beneath the mylo-hyoideus, because the small submaxillary ganglion is in contact with it. Next he should eut through the small branches of vessels and nerve on the surface of the mylo-hyoideus; and detaching that muscle from the jaw and its fellow, should throw it down to the os hyoides, but without injuring the genio-hyoid muscle beneath it.

Afterwards the bone is to be sawn through on the right side of the muscles attached to the symphysis, the soft parts covering the lower jaw having been previously cut. 'The side of the jaw, which will then be loose (tor the ramus of the bone has been sawn before), is to be raised to see the parts beneath, and it may be fastened up out of the way with a stitch; but it should not be detached from the mucous membrane of the mouth.

The apex of the tongue is to be now pulled well out of the mouth over the upper teeth, and fastened with a stitch to the septum of the nose, whilst the left half of the jaw is to be drawn down forcibly with hooks. The scalpel should be then passed from below upwards between the sawn surfaces of the bone, for the purpose of dividing a strong band of the mucous membrane of the mouth; and it should be carried onwards along the middle line of the tongue to the tip.

By means of a stitch the os hyoides may be fastened down, to make 
tight the muscular fibres. All the fat and areolar tissue are to be removed, and in doing this the student is to take care of the Whartonian duct; of the hypoglossal nerve and its branches, which lie on the hyo-glossus muscle, and especially of its small offset aseending to the stylo-glossus muscle; also of the gustatory nerve nearer the jaw. Between the gustatory nerve and the deep part of the submaxillary gland the dissector should seek the small submaxillary ganglion (smaller than a pin's head), with its offsets; and should endeavor to separate from the trunk of the gustatory the small chorda tympani nerve, and to define the offset from it to the submaxillary ganglion.

At the hinder border of the hyo glossus clean the lingual vessels, the stylo-hyoid ligament, and the glosso-pharyngeal nerve, all passing beneath that muscle; and at the anterior border find the issuing ranine vessels which, with the gustatory and hypoglossal nerves, are to be traced on the under surface of the tongue to the tip.

Parts beneath mylo-hyoideus (fig. 23). The relative position of the objects brought into view by the steps of the previous dissection is now apparent:-Extending from the cornu of the hyoid bone to the side of the tongue is the hyo-glossus musele, whose fibres are crossed superiorly by those of the stylo-glossus. On the hyo-glossus are placed, from below upwards, the hypoglossal nerve, the Whartonian duct, and the gustatory nerve, the latter crossing the duct; and near the inner border of the muscle the two nerves are united by branches. Beneath the same muscle lie, from below upwards, the lingual artery with its vein, the stylo-hyoid ligament, and the glosso-pharyngeal nerve. Above the hyo-glossus is the mucous membrane of the mouth, with the sublingual gland attached to it in front, and some fibres of the superior constrictor muscle covering it behind near the jaw.

Between the ehin and the os hyoides, along the middle line, is situate the genio-hyoid muscle; and larger and deeper than it is a fan-shaped muscle, the genio-hyo-glossus. Along the outer side of the last muscle lie the ranine vessels; and a sublingual branch for the gland of the same name springs from the lingual artery at the inner border of the hyo-glossus. On the under surface of the tongue, near the margin, lies the gustatory nerve; and in the fibres of the genio-hyo-glossus runs the liypoglossal nerve.

The rro-gLossus muscle (fig. $22,{ }^{1}$ ) is thin and somewhat square in shape. The muscle arises from the lateral part of the body of the os hyoides (basio-glossus), and from all the great cornu of the same bone (cerato-glossus). The two picces form a thin sheet, and enter the back part and side of the tongue; they will be seen afterwards to mingle with fibres of the palato- and stylo-glossus. ${ }^{1}$

The parts in contact with the surfaces of the hyo-glossus have been already enumerated; and beneath the muscle also are portions of the genio-hyo-glossus and middle constrictor. Along the anterior border is the genio-hyo-glossus musele.

Action. When the tongue is at rest, the muscle can bring that organ to the floor of the mouth, drawing down the sides and giving a rounded

1 A third part (chondro-glossus) is distinct from the others, and is not dissected ; it ends on the upper surface of the tongue near the root. For further details respecting the anatomy of this and the other lingual muscles, reference is to be made to the dissection of the tongue, Sectios 15. 
form to the dorsum; but if the tongue is protruded from the mouth, the fibres will retract it into that cavity.

If the tongue is fixed against the roof of the mouth by other muscles, even though the lower jaw is depressed, this muscle with the geniohyo-glossus will elevate the os hyoides, and allow swallowing to take place.

The STYLO-GLOssus (fig. $22,{ }^{2}$ ) is a slender muscle, whose attachments are expressed by its name. Arising from the styloid process near the apex, and from the stylo-maxillary ligament, the muscle is continued forwards to the side of the tongue. Here it gives filbres to the dorsum, and turning to the under surface, extends to the tip of the tongue. Beneath the jaw this musele is crossed by the gustatory nerve.

Action. Both muscles will raise the back of the tongue against the roof of the mouth, but if the tongue is protruded they will restore it to the cavity.

One muscle can direct the point of the tongue towards its own side of the mouth.

The Genio-Hyoid Muscle (fig. $22,{ }^{4}$ ) arises from the lower of the two

Fig. 22.

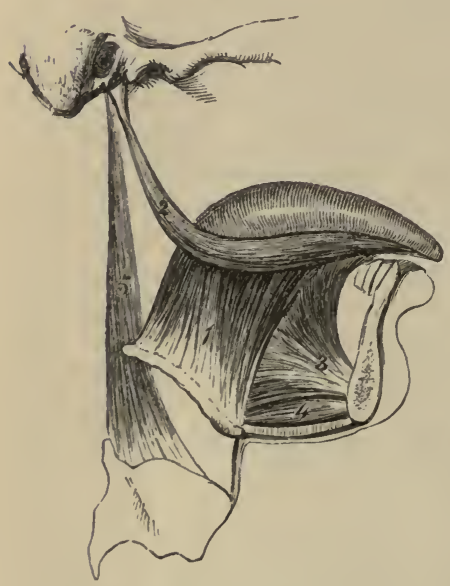

Muscles of the Tonaue.

1. Hyo-glossus.

2. Stylo-glossus.

3. Genio-hyo-glossus.

lateral tubereles on the inner aspect of the symphysis of the jaw, and is inserted into the middle of the hyoid bone.

Covered by the mylo-hyoideus, this muscle rests on the genio-hyo-glossus. The inner border touches the muscle of the opposite side, and the two are often united.

Action. As long as the mouth is shut it raises the hyoid bone; but acting from the os hyoides, and the closers of the mouth being relaxed, it ean depress the jaw and open the moutl.

The Genio-hro-glossus (fig. $22,{ }^{3}$ ) is the largest muscle of this region; it has a triangular form, with the apex at the jaw, and the base at the middle line of the tongue. It takes origin from the upper tuberele behind the symphysis of the jaw. From this spot the filures radiate, the posterior passing downwards to their insertion into the body of the hyoid bone, the anterior forwards to the tip of the tongue, and the intermediate to the tongue from root to point.

Lying along the middle of the tongue, it is in contact with its fellow. The lower border of the muscle corresponds with the genio-liyoideus, and the upper with the frænum linguæ. On its outer side are the ranine vessels, and the hyo-glossus musele; and the hypoglossal nerve perforates the posterior fibres.

Action. By the simultaneous action of all the fibres the tongue is depressed in the floor of the mouth, and hollowed along the middle. But different parts of the muscle are thought to have different uses when they 
act from the jaw :-Thus the fibres attached to the os hyoides advance and fix that bone before swallowing; the hinder tongue fibres raise the root of the tongue and protrude the tip, and the anterior then turn down the tip of the tongue over the teeth.

When the mouth is open swallowing can be performed if the tongue is fixed against the teeth and roof of the mouth, because this muscle and the hyo-glossus can then raise the hyoid bone.

The lingual artery (fig. $17, f$ ), arises from the external earotid between the superior thyroid and fiteial branches. At first it is directed inwards above the os hyoides, and then upwards beneath the hyo-glossus to the under part of the tongue (fig. 23) ; it ends at the anterior border of that musele in the sublingual and ranine branches. Near the hyo-glossus the artery is erossed by the ninth nerve, and by the digastric and stylo-hyoid muscles. Beneath the hyo-glossus, the vessel rests on the middle eonstrictor and genio-hyo-glossus museles, and is below the level of the glossopharyngeal nerve. Its branches are these:-

a. A small hyoid branch is distributed on the upper border of the os hyoides, supplying the muscles; it anastomoses with its fellow of the opposite side, and with the hyoid branch of the superior thyroid artery of the same side.

b. A branch to the dorsum of the tongue arises beneath the hyo-glossus muscle, and aseends to supply the substance of the tongue and the tonsil. The fibres of the hyo-glossus must be divided to see it.

c. The sublingual branch springs from the final division of the artery at the edge of the hyo-glossus, and is directed outwards to the gland of the same name. Some offsets supply the gums and the eontiguous muscles, and one continuès behind the ineisor teeth to join a similar artery from the other side.

$d$. The ranine branch (9) is the terminal part of the lingual artery, and extends forwards along the outer side of the genio-hyo-glossus to the tip of the tongue where it ends. Muscular offsets are furnished to the substanee of the tongue of the same side. This artery lies along the frænum lingux, but is imbedded in the muscular fibres.

The lingual vein commences on both the upper and under surfaces of the tongue. It lies with its eompanion artery, and ends in the internal jugular vein.

The GUSTATORY or Lingual Nerve (fig. $23,{ }^{1}$ ) has been followed in the pterygo-maxillary region to its passage between the ramus of the lower jaw and the internal pterygoid muscle (p. 97). In this dissection the nerve is inclined forwards to the side of the tongue, aeross the mucous membrane of the mouth and the origin of the superior eonstrictor muscle, and above the deep part of the submaxillary gland. Lastly, it is directed across the Whartonian duet, and along the side of the tongue to the apex. Branches are furnished to the surrounding parts, thus :-

Two or more offsets connect it with the submaxillary ganglion, near the gland of that name.

Farther forwards branehes descend on the hyo-glossus to unite in a kind of plexus with twigs of the hypoglossal nerve.

Other filaments are supplied to the mucous membrane of the mouth, the gums, and the sublingual gland.

Lastly, the branches for the tongue aseend through the muscular substance, and are distributed to the eonical and fungiform papilla.

The submaxillary ganglion (fig. $23,{ }^{2}$ ) resembles the other ganglia 
connected with the three trunks of the fifth nerve, and communicates with sensory, motory, and sympathetic nerves. It is smaller in size than the lenticular ganglion, is sometimes rather red, and is placed above the deep process of the submaxillary gland. Offsets proceed upwards to connect it with other nerves; and from the lower part arise the branches to the adjacent structures.

Connection with nerves-roots. Two or three branches, in the form of loops, pass from the ganglion to the gustatory nerve. At the posterior

Fig. 23.

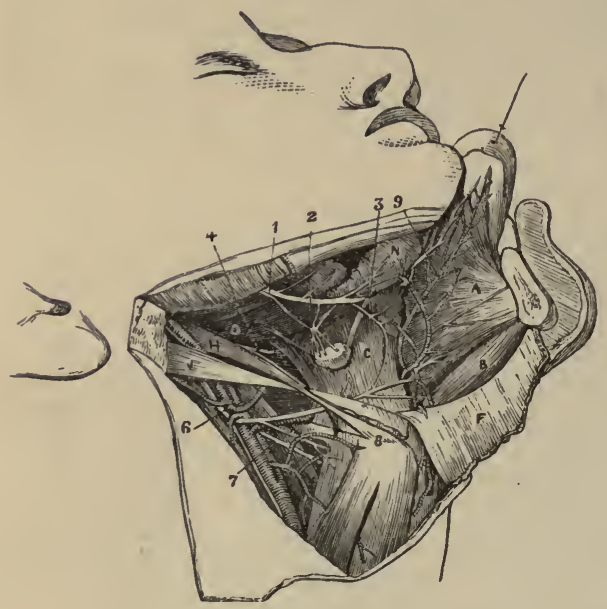

Muscles : A. Genio-hyo-glossus.

B. Genio-hyoideus.

C. Hyo-glossus.

D. Styln-glossus.

E Mylo-hyoideus, reflected.

H. Stylo-hyoideus.

J. Posterior belly of digastricus.

Nerves : 1. Gustatory.

2. Submaxillary ganglion.

3. Wharton's duct.

4. Glosso-pharyugeal nerve.

6. Hy po-glossal.

7. Upper laryngeal. The lingual artery ramifies in this region, lying by the side of the hypoglossal nerve: the ranine offset is marked with 9 .

DeEP VibW of the SUbmaxillaby Region (Illustrations of Dissections).

part the ganglion is further joined by an offset from the chorda tympani. (of the facial nerve) which lies in contact with the gustatory. And its sympathetic branch comes from the nerves around the facial artery.

Branches. From the lower part of the ganglion five or six branches descend to the substance of the submaxillary gland ; and from the anterior part other filaments are furnished to the mucous membrane of the mouth and the Whartonian duct.

Chorda tympani. Joining the gustatory above by fibrils (p. 97), it is applied to the back of that nerve till near the tongue, and can be easily separated from it; but beyond that point it enters amongst the fibres of the gustatory nerve and is conveyed to the tongue. Near the submaxillary gland an offset is sent to the submaxillary ganglion.

The HYPO-GLOSSAL or NINTI NERVE (fig. $23,{ }^{6}$ ) in the submaxillary region lies on the hyo-glossus muscle, being concealed by the mylohyoideus: but at the inner border of the hyo-glossus it enters the fibres of the genio-hyo-glossus, and is continued along the middle line of the tongue to the tip.

Branches. On the hyo-glossus the ninth nerve furnishes branches to the muscles of the submaxillary region, except the mylo-hyoid and the digastric, viz., to the hyo-glossus, stylo-glossus, genio-hyoideus, and geniohyo-glossus. Further, some off'sets communicate with the gustatory nerve on the hyo-glossus.

Along the middle of the tongue the nerve sends upwards long filaments 
which supply the muscular structure, and communicate with the gustatory nerve.

The glosso-pharyngeal cranial nerve (fig. $23,{ }^{4}$ ), issuing between the two carotid arteries, courses over the stylo-pharyngeus and the middle constrictor of the pharynx, and ends under the hyo-glossus in branches for the tongue. See Dissection of the Tongue.

The duct of the submaxillary gland (fig. $23,{ }^{3}$ ), Wharton's duct, issues from the deep part of the glandular mass turning round the border of the mylo-hyoid muscle. It is about two inches in length, and is directed upwards on the hyo-glossus muscle, and beneath the gustatory nerve, to open on the side of the frænum lingux in the centre of an eminence: its opening in the mouth will be seen if a bristle be passed along it. The duct has a thin wall, and consists externally of a fibrous layer with much elastic tissue and a few pale muscular fibres, and internally of a mucous lining with flattened epithelium.

The deep part of the submaxillary gland extends along the side of the duct, reaching in some instances the sublingual gland.

The sublingual gland (fig. $23,{ }^{\mathrm{N}}$ ) is somewhat of the shape of an almond, and the longest measurement, which is about one inch and a half, is directed backwards. It is situate beneath the anterior part of the tongue, in contact with the inner surface of the lower jaw, and close to the symphysis. Separated from the cavity of the mouth by the mucous membrane, the gland is prolonged across the upper border of the genio-hyo-glossus muscle, so as to tonch the one of the opposite side.

The sublingual is an aggregation of small glandular masses, each being provided with a separate duct (Henle). The ducts (ductus Riviniani) are from ten to eighteen in number. Some of them open beneath the tongue along a crescent-shaped fold of the mucous membrane, and others join the Whartonian duct; one or more form a larger tube, which either joins that duct or opens near it.

\section{Section VIII.}

\section{SUPERIOR MAXILLARY NERVE AND VESSELS.}

Directions. The student may examine next the right orbit, and the remaining trunk, superior maxillary of the fifth nerve.

Supposing the right orbit to be untouched, the student may vary his former examination of the left cavity (p. 50) by dissecting it from the outer side.

Dissection. For this purpose divide the margin of the orbit with a saw through the supra-orbital notch, and the roof with a chisel back to the sphenoidal fissure. Cut also with a chisel (from the inside) along the middle fossa of the base of the skull from the sphenoidal fissure in front to the foramen spinosum behind, and outside the line of the foramen rotundum and foramen ovale. The side of the skull is next to be sawn vertically in front of the petrous part of the temporal bone, so that the incision shall end at the posterior extremity of the cut marle in the base. Afterwards the outer wall of the orbit is to be sawn horizontally into the sphenomaxillary fissure. The piece of bone forming part of the cranium and 
orbit is now loose, and is to be removed with the temporal muscle. If the part of the roof of the orbit, which is left, should interfere with the sight of the contents of the cavity, let it be taken away with a bone-forceps.

The description of the orbit (p. 51) will serve in a general way for the right eavity.

The superior maxillary division of the fifth nerve, in its course to the face, occupies successively the skull, the spheno-maxillary fossa, and the infra-orbital canal.

The beginning of the nerve in the cranium has been already demonstrated (p. 31).

Dissection. In the spheno-maxillary fossa the nerve can be partly seen by the dissection already made for the orbit, and its exposure here will be completed by removing the fat, and cutting away some of the wing of the sphenoid bone, so as to leave only an osseous ring round the nerve at its exit from the skull. In the fossa the student seeks the following offsets, the orbital branch entering the cavity of the orbit; branches to Meckel's ganglion which descend in the fossa; and a dental branch along the back of the upper jaw.

To follow onwards the nerve in the floor of the orbit, the contents of the cavity having been taken away, the bony canal in which it lies must be opened to the face. Near the front of the orbit the anterior dental branch is to be traced downwards for some distance in the bone. The infraorbital vessels are prepared with the nerve.

The Superior maxillary nerve (fig. 24) commences in the Gasserian ganglion (p. 31), and leaves the cranium by the foramen rotundum. The course of the nerve is almost straight to the face, across the sphenomaxillary fossa, and along the orbital plate of the superior maxilla and the infraorbital canal. Issuing from the canal by the infraorbital foramen, it is concealed by the elevator of the upper lip, and ends in branches to the eyelid, nose, and upper lip :-

a. The orbital branch $\left({ }^{4}\right)$ arises in the spheno-maxillary fossa, and entering the orbit, divides into malar and temporal branches (p. 60).

b. The spheno-palatine branches $\left({ }^{3}\right)$ descend fiom the nerve in the fossa, and supply the nose and the palate; they are connected with Meckel's ganglion, and will be dissected with it (SEction 14).

c. A posterior dental branch $\left({ }^{5}\right)$ leaves the nerve near the upper jaw. It enters a canal in the maxilla, and supplies branches to the molar teeth and the lining membrane of the antrum; it joins the anterior dental branch near the teeth. Before entering the canal it furnishes one or more off'sets to the gums and the buccinator muscle.

d. 'The anterior dental branch $\left({ }^{6}\right)$ quits the nerve trunk in the floor of the orbit, and descends to the anterior teeth in a special eanal in front of the antrum: it is distributed by two branches. One (inner) gives nerves to the incisor and canine teeth, and furnishes one or two filaments to the lower meatus of the nose; the other (outer) ends by supplying the bicuspid teeth.

$e$. Before the trunk ends in the facial branches, it supplies a small palpebral offset to the lower eyelid; this is directed upwards to the lid in a groove in the margin of the orbit.

f. Infraorbital or facial branches $\left(^{7}\right)$. These are larger than the other offsets of the nerve, and form its terminal ramifications. Some incline inwards to the side of the nose, and the rest descend to the upper lip. Near the orbit they are crossed by branches of the facial nerve (fig. $9,{ }^{11}$ ), 
with which they communicate, the whole forming the infraorbital plexus (p. 49).

g. The branches for the side of the nose supply the muscular and tegumentary structures.

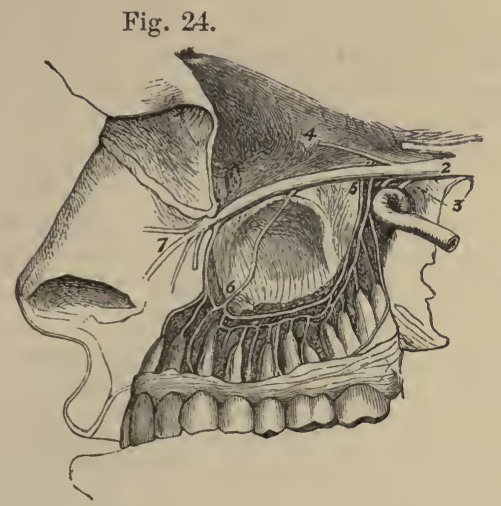

2. Trunk of the nerve leaving the Gasserian ganglion.

3. Spheno-palatine branches.

4. Temporo-malar branch.

5. Posterior dental nerves.

6. Anterior dental.

7. Fucial branches.

Diagram of the UPPER Maxillary Nerve aNd its Branches.

$h$. The branches for the upper lip are three or four in number, which divide as they descend, and are distributed chiefly to the surfaces of the lip, though they supply as well the muscles and the labial glands.

The infraorbital artery is a branch of the internal maxillary (p. 94). Taking the course of the nerve through the infraorbital canal, the vessel appears in the face beneath the elevator muscle of the upper lip; and it ends in branches, which are distributed, like those of the nerve, to the parts between the eye and mouth. In the face its branches anastomose with the facial and buccal arteries. In the canal in the maxilla the artery furnishes branches to the orbit.

Another branch, anterior dental, runs with the nerve of the same name, an supplies the incisor and canine tecth: this gives offsets to the antrum of the maxilla, and near the teeth it anastomoses with the posterior dental artery.

The vein, accompanying the artery, communicates in front with the facial vein; and terminates behind in a plexus of veins (alveolar) corresponding with the offsets of the internal maxilla artery in the sphenomaxillary fossa.

\section{Section IX.}

\section{DEEP VESSELS AND NERVES OF THE NECK.}

Is this Section are included the deepest styloid muscle, the internal carotid and ascending pharyngeal arteries, and some cranial and sympathetic nerves.

Position. The position of the part is to remain as before, viz., the neck is to be fixed over a small block. 
Dissection. To see the stylo-pharyngeus muscle, the posterior belly of the digastric, and the stylo-hyoid muscle, should be detached fiom their origin and thrown down. The trunk of the external carotid artery is to be removed by cutting it through where the hypoglossal nerve crosses it, and by dividing those branches of it that have been already examined; the veins accompanying the arteries are to be taken away. In cleaning the surface of the stylo-pharyngeus muscle, the glosso-pharyngeal nerve and its branches, and the stylo-hyoid ligament may be prepared. The side of the jaw is to be drawn forwards on the face.

The STYLO-PHARYNGEUS MUSCLE (levator pharyngis externus) resembles the other styloid muscles in its elongated form. The fibres arise from the root of the styloid process on the inner surface, and descend between the superior and middle constrictors to be inserted partly into the pharynx, and partly into the upper border (hinder border, Merkel ${ }^{1}$ ) of the thyroid cartilage.

The muscle lies below the stylo-glossus, and between the carotid arteries; and the glosso-pharyngeal nerve turns over the lower part of its fleshy belly.

Action. It elevates and draws outwards the part of the pharynx above the hyoid bone, making the tube ready for the reception of the morsel to be swallowed. From its attachment to the thyroid cartilage it will raise the larynx; and by its position it will control the movement forwards of the air tube.

The stylo-hyoid ligament is a fibrous band, which extends from the tip of the styloid process to the small cornu of the os hyoides. Its position is between the stylo-glossus and stylo-pharyngeus muscles, and over the internal carotid artery; whilst the lower end is placed beneath the hyoglossus muscle. To the posterior border the middle constrictor muscle is attached below. It is frequently cartilaginous or osseous in part of, or in all its extent. Occasionally a slip of fleshy fibres is continued along it.

The INTERNAL CAROTID ARTERY supplies parts within the head, viz., the brain, the eye and orbit, and the nose; and takes a circuitous course through and along the base of the skull before it terminates in the brain.

The arterial trunk in the cranium, and its offset to the orbit, have been already examined; but the portion in the neck and the temporal bone remains to be dissected. The branches of the carotid to the brain are examined with the encephalon.

Dissection. For the display of the cervical part of the artery (fig. 25) there is now but little dissection required. By detaching the styloid process at the root, and throwing it with its muscles to the middle line, the internal carotid artery and the jugular vein may be followed upwards to the skull. Only a dense fascia conceals them, but this is to be taken away carefully, so that the branches of the nerves may not be injured.

In the fascia, and directed inwards over the artery, seek the glossopharyngeal norve and its branches near the skull, and the small pharyngeal branch of the vagus lower down; still lower, the superior laryngeal branch of the vagus, with its external laryngeal off'set crossing beneath the carotid. Between the vein and artery, elose to the skull, will be found the vagus, hypoglossal, and sympathetie nerves; and erossing backwards, over or under the vein, the spinal accessory nerve. External to the ves-

1 Anatomie und Phisiologie des Menschlichen Stimm und Sprach Organs. Leipzig, 1857. Von Dr. Merkel. 
sels a loop of the first and second cervical nerves over the transverse process of the atlas is to be defined ; and from it branches of communication are to be traced to the large ganglion of the sympathetic beneath the artery, and to the vagus and hypoglossal nerves. Ascending to the cranium, on the inner side of the carotid, the ascending pharyngeal artery will be met with.

To open the carotid canal in the temporal bone, and to follow the contained artery into the cranium, make a cut along the side of the skull in the following manner: the saw being placed beneath the mastoid process, cut forwards to the foramen spinosum in the wing of the sphenoid bone (to which spot the side of the skull has been already taken away), and let the instrument be directed through the stylo-mastoid foramen and the root of the styloid process, but rather external to the jugular foramen and the carotid canal. When the piece of bone has been detached, the carotid canal may be opened with the bone forceps.

In cleaning the artery in the canal, large and rather red branches of the superior cervical ganglion of the sympathetic will be found on it ; and in a fresh part two small filaments may be recognized with care-one from Jacobson's nerve, joining the sympathetic at the posterior part of the canal; the other from the vidian nerve, at the front of the canal.

On the piece of bone that has been cut off, the dissector may prepare very readily the tympanum with its membrane and chain of bones, and the chorda tympani nerve.

The internal carotid artery (fig. 25, $d$ ) springs from the bifurcation of the common carotid trunk. It extends from the upper border of the thyroid eartilage to the base of the skull; then through the petrous portion of the temporal bone; and lastly along the base of the skull to the anterior clinoirt process, where it ends in branches for the brain. This winding course of the artery may be divided into three parts : one in the neck, another in the temporal bone, and a third in the cranium.

Cervical part. In the neck the artery ascends almost vertically from its origin to the carotid canal, and is in contact with the pharynx on the inner side. The line of the common carotid artery would mark its position in the neck. Its depth from the surface varies like that of the external carotid; and the digastric muscle may be taken as the index of this difference. 'Thus, below that muscle, the internal carotid is overlapped by the sterno-mastoid and covered by the common teguments, fascia, and the platysma, and is on the same level as the external carotid, though farther back. But, above that muscle, the vessel is placed deeply beneath the external carotid artery and the parotid gland, and is crossed by the styloid process and the stylo-pharyngeus muscle. Whilst in the neck the internal earotid lies on the rectus capitis anticus major muscle, which separates it from the vertebra.

Vein. The internal jugular vein accompanies the artery, being contained in a sheath with it, and placed on the outer side.

Small ressels. Below the digastric muscle the occipital artery is directed back over the carotid ; and the offset from it to the sterno-mastoideus may run down on the earotid trunk. Above the digastric the posterior auricular vessels cross the carotid.

Nerves. The pneumogastric is contained in the sheath between the artery and vein, being parallel to them; and the sympathetic, also running longitudinally, lies behind the sheath of the vessels. Crossing the artery superticially, from below up, is the hypoglossal, which sends the 
descendens noni along it; next the small pharyngeal branch of the vagus; and lastly the glosso-pharyngeal. Directed inwards beneath the carotid is the superior laryngeal nerve, furnishing the external laryngeal branch; together with pharyngeal offsets of the upper ganglion of the sympathetic.

Fig. 25.

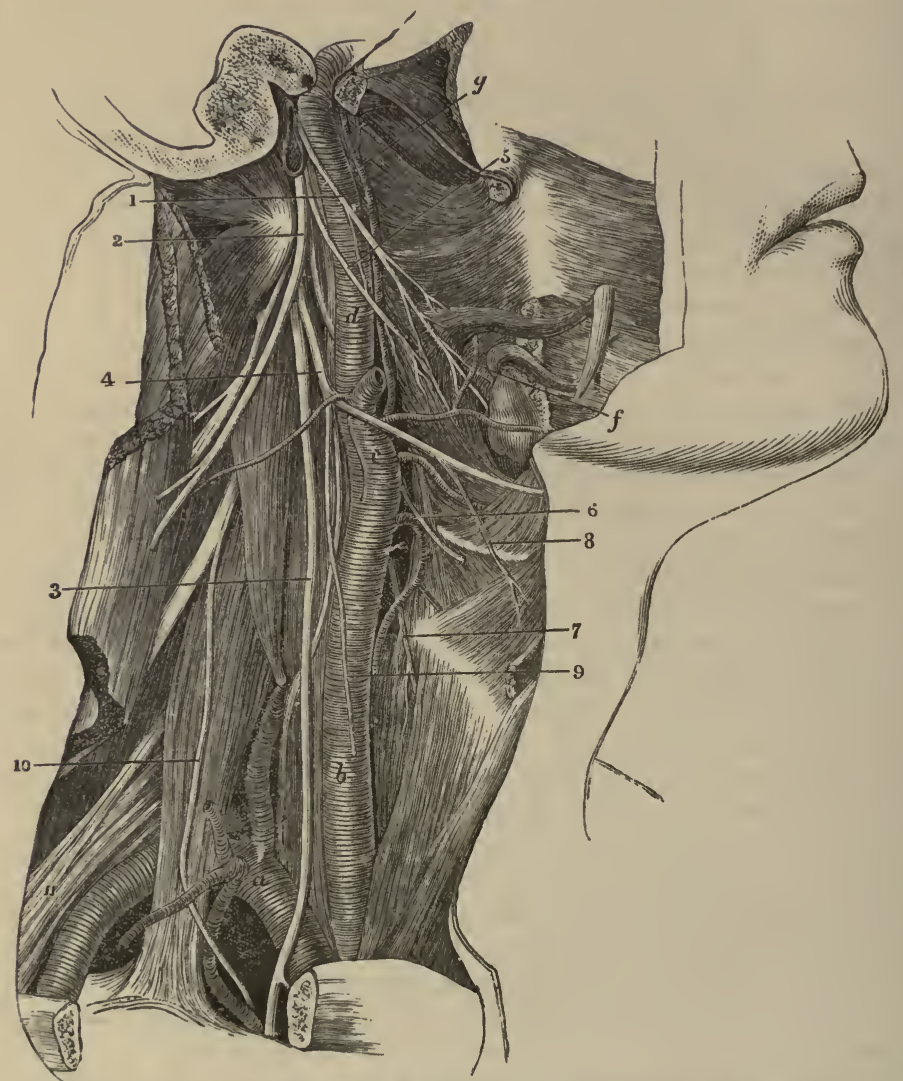

Deep Vesgras and Nerveg of the NeCK (Illustrations of Dissections).

Arteries: $a$. Snbclavian trunk.

b. Common carotid.

c. Exterual carotid, cut.

d. Internal carotid.

$f$. Inferior palatine branch of the facial. g. Ascending pharyngeal.

Nerves: 1. Glosso-pharyngeal.

2. Splnal aсcessory.

3. Pneuino-gastric or vagus.
4. Hy pog loss 11 .

5. Pharyngeal branch of the vagus.

6. Upper laryngeal branch of the vagus.

7. External laryugeal branch of the last.

8. Thyro-lyyold branch of the hypoglossal.

9. Descendens noni of hypoglosial, cut.

10. Phrenic nerve of cervical plexus.

11. Brachial plexus. Recurrent of the vagus, winds rouud the subelavian artery, $a$.

Close to the skull the cranial nerves of the neck are interposed between the artery and the vein. Around the carotid entwine branches of the sympathetic, and offsets of the glosso-pharyngeal nerve.

The cervical part of the artery remains much the same in size to the 
end, though it is sometimes very tortuous; and it usually does not furnish any branch.

Part in the temporal bone. In the carotid canal the winding course of the vessel commences. 'The artery first ascends in front of the inner ear (cochlea and tympanum); next it is directed forwards almost horizontally; and lastly it turns upwards into the cranium opposite the foramen lacerum (basis cranii). Branches of the sympathetic nerve surround the carotid in the bone.

Whilst in the canal the artery supplies a snall branch to the eavity of the typanum.

The cranial part of the artery is described with the base of the skull (p. 33).

The INTERNAL JUGULAR VEIN is continuous with the lateral sinus of the skull, and extends from the foramen jugulare nearly to the first rib. At the lower part of the neck it joins the subclavian to form the innominate vein (p. 79).

As far as the thyroid cartilage the vein accompanies the internal carotid, but below that point it is the companion to the common carotid artery; and it lies on the outer side of each. Its contiguity to the artery is not equally close throughout, for near the skull there is a small interval between them, containing the cranial nerves; and at the lower part of the neck there is a still larger intervening space, in which the pneumogastric nerve with its cardiac branch is found.

The size of the upper part of the vein remains much the same till near the os hyoides, where it is suddenly increased by the addition of those branches of the head and neck, corresponding with branches of the external carotid artery, which do not join the external jugular vein. ${ }^{1}$ Its lower dilatation and its valves have been referred to $(p .82)$.

The following branches open into the internal jugular, viz., the facial, lingual, thyroid (superior), occipital, and pharyngeal; and at the lower part of the neck it receives the middle thyroid vein.

The ascending pharyngeal artery (fig. $25, g$ ) is a long slender branch of the external carotid, which arises near the commencement of that vessel. Directed upwards on the spinal column between the internal carotid and the pharynx, the artery becomes tortuous near the skull, and enters the pharynx above the upper constrictor to end in the soft palate. In the neck the artery gives some small offisets to the surrounding parts, viz., the muscles on the vertebre, the nerves, and the lymphatic glands.

A meningeal branch enters the cranium through the foramen lacerum (basis cranii), and is distributed in the dura mater of the middle fossa of the skull; this is seldom seen in the cranium, because it is but rarely injected.

The palatine branch, which is larger than the preceding, divides in the pharynx into two main pieces, which are directed across the fore part of the palate beneath the mucous membrane, and form arches with like branches of the opposite side; one of these is near the upper, and the other near the lower edge of the soft palate. ${ }^{2}$ The size of the palatine artery depends upon that of the inferior palatine branch of the facial artery.

1 Sometimes the term internal cephalic is applied to the vein between the skull and the hyoid bone; and the name internal jugular, to the part below that bone and the junction of its large branches.

2 The Anatomy of the Arteries. By R. Quain, F.R.S., p. 110. 
Pharyngeal branches. Other small arteries ramify in the upper constrictor, the Eustachian tube, the back of the soft palate, and the tonsil.

'The vein accompanying the pharyngeal artery receives branches from the cranium, the palate, and the pharynx, and ends in the internal jugular vein.

Dissection of the cranial nerves in the neck. By the time this stage of the dissection has been arrived at, the condition of the parts will not permit the tracing of the very minute filaments of the cranial nerves in the foramen jugulare of the skull; and all the paragraphs marked with an asterisk are therefore to be omitted for the present. Afterwards, if a fresh piece of the skull can be obtained, in which the bone has been softened by acid, and the nerves hardened in spirit, the examination of the branches now passed over may be made.

* In the foramen lacerum (fig. 26). Supposing the dissection of the internal carotid to be carried out as is described at page 107 , let the student cut across with care the jugular vein near the skull. Let him then remove bit by bit with the bone forceps, or with a scalpel if the part has been softened, the ring of bone which bounds externally the jugular foramen, proceeding as far forwards as the osseous crest between that foramen and the carotid canal. Between the bone and the coat of the jugular vein, the small auricular branch of the pneumogastric nerve is to be found; it is directed backwards to an aperture near the styloid process.

* Trace, then, the spinal accessory and pneumogastric nerves through the aperture, by opening the fibrous sheath around them. Two parts, large and small, of the spinal accessory nerve should be defined; the latter is to be shown joining a ganglion on the vagus, and applying itself to the trunk of that nerve. A communication between the two parts of the spinal accessory is to be found. On the pneumogastric is a small wellmarked ganglion, from which the auricular branch before referred to takes origin; and from the ganglion filaments are to be songht passing to the smaller portion of the spinal accessory nerve, and to the ascenaing branch of the upper cervical ganglion of the sympathetic.

* Next follow the glosso-pharyngeal nerve through the fore part of the foramen, and take away any bone that overhangs it. This nerve presents two ganglia as it passes from the skull (fig. 26): one (jugular), which is scarcely to be perceived, near the upper part of the tube of membrane containing it; the other, much larger (petrous), is situate at the lower border of the petrous portion of the temporal bone. From the lower one, seek the small nerve of Jacobson, which enters an aperture in the crest of bone between the jugular foramen and the carotid canal; and another filament of communication with the ganglion of the sympathetic. Sometimes the dissector will be able to find a filament firm the lower ganglion to join the auricular branch of the pneumogastric; and another to end in the upper ganglion of the pneumogastric nerve.

Below the foramen of exit from the skull the cranial nerves have been greatly denuded by the dissection of the internal carotid (fig. 25); but the intercommunications of the vagus, hypoglossal, sympathetic, and first two spinal nerves near the skull, are to be traced out more completely.

'The larger part of the spinal accessory has been sufficiently laid bare alrearly; but its small piece is to be traced to the vagus, close to the skull, and onwards by the side of that trunk.

'The chief part of the glosso-pharyngeal has been also dissected; but the offsets on the carotid, and others to join the pharyngeal branch of the vagus and the pharyngeal plexus are to be displayed. 
On the pneumogastric trunk the student should define an enlargement close to the skull (ganglion of the trunk) to which the hypoglossal nerve is intimately united. From the ganglion proceed two branches (pharyngeal and laryngeal), which are to be traced to the parts indicated by their names, especially the first which enters the pharyngeal plexus. The task of disentangling the ramifications of the branch of the vagus, and those of the glosso-pharyngeal and sympathetic in the plexus, is by no means easy, in consequence of the dense tissue in which they are contained. 'Two or more cardiac offsets of the vagus, one at the upper and another at the lower part of the neck, may be recognized readily. Lastly the dissector may prepare more fully the recurrent branch coursing up beneath the lower part of the common carotid : by removing the fat around it, offsets will be seen entering the chest and the windpipe.

Only the first, or the deep part of the hypoglossal nerve remains to be made ready for learning; its communications with the vagus, sympathetic, and the spinal nerves are to be demonstrated.

A dissection for the sympathetic will be given further on (p. 116); but its large ganglion near the skull (upper cervical) should be defined, and the small branches from it to the pharyngeal plexus should be pursued beneath the carotid artery.

The three crunial nerves, glosso-pharyngeal, pneumogastric, and spinal accessory, which constitute the eighth nerve of Willis, leave the cranium by the foramen jugulare (p. 32). Outside the skull the nerves take different directions according to their destination; thus the glosso-pharyngeal is inclined inwards to the tongue and pharynx; the spinal accessory backwards to the sterno-mastoid and trapezius muscles; and the pneumogastric nerve descends to the viscera of the thorax and abdomen.

The GLOSSO-PHARYNGEAL NERVE (fig. $26,{ }^{1}$ ) is the smallest of the three trunks. In the jugular foramen it is placed somewhat in front of the other two, and lies in a groove in the lower border of the petrous part of the temporal bone. In the aperture of exit the nerve is marked by two ganglionic swellings, the upper one being the jugular, and the lower the petrous ganglion.

* Ganglia. The jugular ganglion, ${ }^{4}$, (ganglion superius) is very small, and is situate at the upper part of the osseous groove containing the nerve. It is placed on the outer surface of the glosso-pharyngeal trunk, and includes only some fibrils of the nerve. 'The petrosal ganglion, ${ }^{5}$, (gang. inferius) is much larger than the preceding, and incloses all the fibrils of the nerve. Ovalish in form, it is placed in a hollow in the lower border of the temporal bone; and from it spring the branches that unite the glossopharyngeal with other nerves.

After the nerve has quitted the foramen it comes forwards between the jugular vein and the carotid artery (fig. $25,{ }^{1}$ ), and crossing inwards orer the artery, reaches the lower border of the stylo-pharyngeus muscle. At this spot the nerve becomes almost transverse in direction in its course to the pharynx; it crosses over the stylo-pharyngeus, and forms an arch across the side of the neck above the superior laryngenl nerve. Finally it passes beneath the hyo-glossus muscle, and ends in branches to the pharynx, the tonsil, and the tongue.

The branches of the glosso-pharyngeal may be classed into those connecting it with other nerves at the base of the skull, and those distributed in the neck.

* Connecting branches arise chiefly from the petrosal ganglion; and in this set is the tympanic nerve. 
* A filament ascends from the sympathetic nerve in the neek to join the petrosal ganglion. Sometimes there is an offset from the ganglion to the auricular branch of the vagus, as well as to the upper ganglion of this nerve.

* The tympanic branch (fig. 26, *) (nerve of Jacobson) enters the aperture in the ridge of bone between the jugular and the carotid foramen, and ascends by a special canal to the inner wall of the tympanum, where it ends in branches: its distribution is given with the tympanum of the ear.

a. Banches for Distribution. In the neck the branches are furnished chiefly to the pharynx and the tongue.

b. Carotid branches surround the internal artery of that name, and communicate with the pharyngeal branch of the vagus, and the sympathetic nerve.

c. Some muscular branches enter the stylo-pharyngeus, whilst the nerve is in contact with it.

d. Branches to the pharynx form the pharyngeal plexus by uniting with nerves from the sympathetic and vagus.

$e$. The tonsillitic branches supply the tonsil and the arches of the soft palate. On the former they end in a kind of plexus-circulus tonsillaris.

$f$. Lingual branches. The terminal branches of the nerve supply the root and posterior part of the tongue, as well as the lateral surface. The distribution of these is described with the tongue (SECTION 15).

The pNeumogastric nerve (fig. $26,{ }^{2}$ ) (vagus nerve) is the largest of the cranial nerves in the neck, and escapes through the jugular foramen

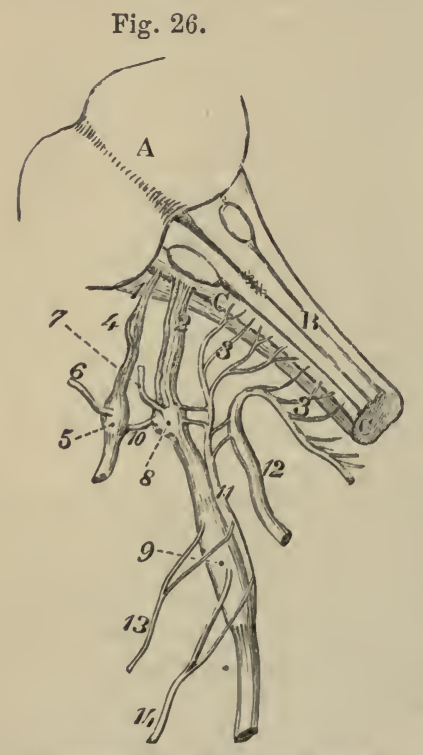

1. Glosso-pharyngeal trunk.

2. Vagus.

3. Spinal accessory.

4. Jugular gangliou.

5. Petrosal ganglion.

6. Jacobson's nerve.

7. Auricular branch.

8. Root ganglion of vagus.

9. Trunk ganglion of vagus.

10. Branch joining the petrosal and upper ganglion of the vagus.

11. Small part of spinal accessory.

12. Chief part of spinal accessory.

13. Pharyngeal branch of vitgus.

14. Superior laryugeal branch of vagus.

DiAgRAM OF THE EIGHTH NERVE.

in the same sheath of dura mater as the spinal accessory. In the aperture of exit it has a distinet ganglion (gang. of the root), to which the smaller part of the spinal accessory nerve is connected. 
When the nerve has left the foramen, it receives the small part of the spinal aceessory, and swells into a ganglion nearly an inch long (gang. of the trunk). Here the nerve lies between the internal carotid artery and jugular vein, and communicates with several nerves. To reach the thorax, the vagus descends almost vertically (fig. $25,{ }^{3}$ ) between the internal jugular vein and the internal and conmon carotid arteries; and enters that cavity, on the right side, by crossing over the subclavian artery, but beneath the innominate vein.

* Ganglia. The ganglion of the root (gang. superius), (fig. 26, ${ }^{8}$ ) is of a grayish color, and in texture is like the ganglion on the large root of the fifth nerve. Small branches in the foramen jugulare come from this ganglion. The ganglion of the trunk (gang. inferius), $\left({ }^{9}\right)$ is cylindrical in form, is reddish in color, and is nearly an inch in length; it communicates with the hypoglossal, spinal, and sympathetic nerves. All the intrinsic fibres of the trunk of the nerve are surrounded by the ganglionic substance, but those derived from the spinal accessory nerve $\left({ }^{11}\right)$ pass over the ganglion without being inclosed in it.

The branches of the pneumogastric nerve may be arranged into those uniting it with other nerves, and those distributed to parts around.

* Connecting branches (fig. 26) arise from the ganglion of the root and the ganglion of the trunk of the vagus.

* From the ganglion of the root. 'The auricular branch $\left({ }^{7}\right)$ is the chief offset, and crosses the jugular fossa to enter an aperture near the root of the styloid process; it traverses the substance of the temporal bone, and reaches the outer ear, on which it is distributed. Its farther course will be described with the anatomy of the ear.

* One or two short filaments unite this ganglion with the spinal accessory nerve; and a branch of the sympathetic nerve in the neck enters it. Occasionally there is an offset $\left({ }^{10}\right)$ to join the lower (petrosal) ganglion of the glosso-pharyngeal nerve.

From the ganglion of the trunk. Communicating filaments connect it with the hypoglossal nerve. Other branches pass between it and the upper ganglion of the sympathetic, and between it and the loop of the first two cervical nerves.

Branches for Distribution (fig. 25). The cervical branches arise from the inner side of the nerve, and are directed inwards, to supply the pharynx, the larynx, and the heart.

a. The pharyngeal branch (fig. $26,{ }^{13}$ ) is an offset from the upper part of the ganglion of the trunk, and terminates in the pharynx. The nerve is directed inwards over the internal carotid artery (fig. $25,{ }^{3}$ ), and joins the branches of the glosso-pharyngeal nerve on that vessel. Finally it courses to the side of the middle constrictor muscle, and communicates with branches of the glosso-pharyngeal, superior laryngeal, and sympathetic nerves, to form the pharyngeal plexus. From the plexus branches are furnished to the constrictors and palato-glossus and pharyngeus, and to the pharyngeal mucous membrane between the tongue and the hyoid bone.

$b$. The superior laryngeal nerve (fig. $26,{ }^{14}$ ) is much larger than the preceding branch, and comes from the middle of the ganglion of the trunk. From this point it inclines obliquely inwards beneath the internal carotid artery (fig. 25, ${ }^{6}$ ), and reaches the larynx opposite the interval between the liyoid bone and the thyroid cartilage. The nerve then perforates the thyro-hyoid membrane, and is distributed to the mucous lining of the 
larynx. (See "LARYxx.") In the neck it furnishes branches to the thyroid body, and the following offset to one laryngeal muscle and the pharyn $x$ :-

The external laryngeal branch (fig. $25,{ }^{7}$ ) arises beneath the internal carotid artery, and runs below the superior laryngeal nerve to the side of the larynx. Here it gives offsets to the pharyngeal plexus, and it is continued beneath the sterno-thyroideus to the crico-thyroid muscle ard the inferior constrictor. Near its origin this branch communicates with the superficial cardiac branch of the sympathetic nerve.

c. Cardiac branches. Some small cardiac nerves spring from the pneumo-gastric at the upper part of the neck, and join cardiac branches of the sympathetic. At the lower part of the neck, on each side, there is a single cardiac nerve : the right one enters the chest and joins the deep nerves to the heart from the sympathetic; and the left nerve terminates in the superficial cardiac plexus of the thorax.

$d$. The inferior laryngeal or recurrent nerve (fig. 25) leaves the pneumogastric trunk on the right side opposite the subclavian artery, and winding round that vessel, takes an upward course in the neck to the larynx, ascending beneath the common carotid and inferior thyroid arteries, and between the trachea and the osophagus. At the larynx it enters beneath the ala of the thyroid cartilage, where it will be afterwards traced. The following branches arise from it :-

Some cardiac branches leave the nerve as it turns round the subclavian artery; these enter the thorax, and join the cardiac nerves of the sympathetic.

Muscular branches spring from it whilst it lies between the trachea and the osophagus, and are distributed to both those tubes. Near the larynx some filaments are furnished to the inferior constrictor muscle.

On the left side the recurrent nerve arises in the thorax, opposite the arch of the aorta, but lies between the trachea and osophagus in the neck, as on the right side.

The SPINAL ACCESSORY NERve (fig. $26,{ }^{12}$ ) courses through the foramen jugulare with the pneumo-gastric, but is not marked by any ganglion. The nerve is constructed of two parts, viz., accessory to the vagus, and spinal, which lave a different origin and distribution. (Origin of the cranial nerves.)

The part accessory to the vagus $\left({ }^{11}\right)$ is the smaller of the two, and finally blends with the pneumo-gastric beyond the skull. In the foramen of exit it lies close to the vagus, and joins the upper ganglion of that nerve by one or two filaments. Below the foramen it is continued over the lower ganglion of the nerve, and blends with the trunk beyond the ganglion. It gives offsets to join the pharyngeal and upper laryngeal branches of the pneumo-gastric, and according to Bendz, to many other branches of that nerve.

The spinal part (fig. 26, ${ }^{12}$ ) is much larger, is round and cord-like, and is connected with the smaller piece whilst it is passing through the foramen jugulare. Beyond the foramen the nerve (fig. $25,{ }^{2}$ ) takes a backward course through the sterno-mastoid muscle, and across the side of the neck to end in the trapezius muscle: at first it is concealed by the jugular vein, but it then passes either over or under that vessel. The connections of the nerve beyond the sterno-mastoideus have been already examined (p. 66). 
The nerve furnishes muscular offsets to the sterno-mastoideus and the trapezius.

The HYPOGLOSSAL NERVE (ninth of Willis), issuing from the cranium by the anterior condyloid foramen, lies deeply between the internal carotid artery and the jugular vein (fig. $25,{ }^{4}$ ). It next comes forwards between the vein and artery, turning round the outer side of the vagus to which it is united. The nerve now descends in the neck, and becomes superficial below the digastric muscle in the anterior triangular space; from this spot it is directed inwards to the tongue and its muscles.

* Connecting branches. Near the skull the hypo-glossal is united by branches with the vagus nerve, the two being almost inseparably joined.

* Rather lower down the nerve is connected by offsets with the sympathetic, and with the loop of the first two spinal nerves.

The branches for distribution have been met with in the foregoing dissections. Thus in the neck it supplies, in union with the spinal nerves, the depressors of the hyoid bone. In the submaxillary region it furnishes branches to one elevator (genio-hyoid) of the os hyoides; to the extrinsic muscles of the tongue except the palato and pharyngeo-glossus; and to all the intrinsic of the tongue.

Dissection. The small rectus capitis lateralis muscle, between the transverse process of the atlas and the base of the skull, is now to be cleaned and learnt. At its inner border the anterior branch of the first cervical nerve, which forms part of a loop on the atlas, is to be found.

The reCtUs CAPITIS LATERALIS (fig. 25 ) is small and thin, and represents an intertransverse muscle. It arises from the anterior transverse process, and the tip of the united transverse processes of the atlas; and is inserted into the jugular eminence of the occipital bone.

On the anterior surface rests the jugular vein; and in contact with the posterior is the vertebral artery. To the inner side lies the anterior primary branch of the first cervical nerve.

Action. It assists the muscles attached to the mastoid process in inclining the head laterally.

Dissection. For the purpose of tracing backwards the anterior branch of the first cervical nerve divide the rectus lateralis muscle, and observe the offset to it ; then cut off the end of the lateral mass of the atlas, and remove the vertebral artery, so as to bring into view the nerve as it lies on the first vertebra.

The anterior primary branch of the first or sub-occipital nerve is slender in size, and arises from the common trunk on the neural arch of the atlas. From that origin it is directed forwards on the arch, beneath the vertebral artery, to the inner side of the rectus lateralis: here it bends down in front of the lateral mass of the bone, and forms a loop by uniting with the second cervical nerve. As the nerve passes forwards it supplies the rectus lateralis muscle, and branches connect the loop with the vagus, hypoglossal, and sympathetic nerves.

Sympatietic Nerve. In the neck the sympathetic nerve consists, on each side, of a gangliated cord, which lies close to the vertebral column, and is continued into the thorax. On this part of the nerve are three ganglia:-One near the skull, another on the neck of the first rib, and a third somewhere between the two; these are named respectively superior, inferior, and middle ganglion. From the ganglia proceed connecting branches with the spinal and most of the cervical cranial nerves; and branches for distribution to viscera and bloodvessels. 
Besides the ganglia above mentioned, there are other ganglia in the head and neck in connection with the three trunks of the fifth nerve.

Dissection. To display the branches of the sympathetic nerve requires greater care than is necessary in tracing the white fibred nerves, for they are softer, more easily torn, and generally of smaller size. In the neck the ganglia and their branches have been partly prepared, and only the following additional dissection will be required to bring them into view :The jugular vein having been cut through, the upper ganglion will be seen by raising the carotid artery, and the trunks of the vagus and hypoglossal nerves, and by cutting through the branches that unite these two to the loop of the first and second spinal nerves. The several branches of the ganglion are to be traced upwards on the carotid artery, inwards to the pharynx, down along the neck, and outwards to other nerves.

The dissector has already seen the middle ganglion on or near the inferior thyroid artery, and its branches to spinal nerves, and along the neck, are now to be traced.

To obtain a view of the inferior ganglion the greater part of the first rib is to be taken away, and the subclavian artery is to be cut through, inside the scalenus, and drawn aside, without however destroying the fine nerves that pass over it. The clavicle is supposed not to be in position. The ganglion is placed on the neck of the first rib; its branches are large, and are easily followed outwards to the vertebral artery and the spinal nerves, and downwards to the thorax.

The superior CERVICAL GANGLion is the largest of the three, and is of a reddish-gray color. Of a fusiform shape, it is as long as the second and third cervical vertebre, and is placed on the rectus capitis anticus major muscle, beneath the internal carotid artery and the contiguous cranial nerves. Branches connect the ganglion with other nerves; and some are distributed to the bloodvessels, the pharynx, and the heart.

* Connecting branches unite the sympathetic with both the spinal and the cranial nerves.

* With the spinal nerves. 'The four highest spinal nerves have branches of communication with the upper ganglion of the sympathetic; but the offset to the fourth nerve may come from the cord connecting the upper to the next ganglion.

* With the cranial nerves. Near the skull the trunks of the vagus (its lower ganglion) and hypoglossal nerves are joined by branches of the sympathetic. In the foramen jugulare also, both the petrosal ganglion of the glosso-pharyngeal and the ganglion of the root of the ragus receive small filaments, one to each, from an ascending offset of the ganglion.

Communications are formed with several other cranial nerres by means of an offset from the ganglion into the carotid canal (p. 33).

Branches for Distribution. This set of branches is more numerous than the preceding, and the nerves are larger in size.

Branches for bloodvessels (nervi molles). These nerves surround the external carotid artery, and ramify on its branches so as to form plexuses on the arteries with the same names as the vessels: some small ganglia are occasionally found on these ramifying nerves. By means of the plexus on the facial artery the submaxillary ganglion communicates with the sympathetic; and through the plexus on the internal maxillary artery the otic ganglion obtains a similar communication.

Another offset from the upper part of the ganglion accompanies the internal carotid artery and its branches. Near the skull it divides into 
two pieces, which enter the canal for the carotid, one on each side of that vessel : and are continued to the eyeball and the pia mater of the brain, forming secondary plexuses on the ophthalmic and cerebral arteries. In the earotid eanal communications are formed with the tympanic nerve ( $p$. 111 ) and with the spheno-palatine ganglion (p. 141); with the former near the lower, and with the latter near the upper opening of the eanal. The communieations and plexuses which these nerves form in their course to the base of the brain are described at p. 33 .

The pharyngeal nerves pass inwards to the side of the pharynx, where they join with other branches of the eranial nerves in the pharyngeal plexus (p. 113).

Curdiac nerves enter the thorax to join in the plexuses of the heart. There are three cardiac nerves on each side, viz., superior, middle, and inferior, each taking its name from the ganglion of which it is an offset.

The superior cardiac nerve (superficial) of the right side courses behind the sheath of the carotid vessels, and enters the thorax beneath the subclavian artery. In the neck the nerve is connected with the eardiae branch of the vagus, with the external laryngeal, and with the recurrent nerve. In some bodies it ends by joining one of the other eardiac nerves.

The MIDDLE CERVICAL GANGLION (gang. thyroideum) is of small size, and is situate opposite the fifth cervieal vertebra, usually on or near the inferior thyroid artery. It has a roundish shape, and lies beneath the great vessels. Its branches are the following:-

* Connecting branches with the spinal nerves sink between the borders of the longus colli and anterior scalenus, to join the fifth and sixth cervical nerves.

Branches for Distribution. These consist of nerves to the thyroid body, together with the middle cardiac nerve.

The thyroid branches ramify around the inferior thyroid artery, and end in the thyroid body; they join the external and recurrent laryngeal nerves.

The middle or great cardiac nerve descends to the thorax across the subclavian artery; its termination in the cardiac plexus will be learnt in the ehest. In the neek it communicates with the upper cardiac and recurrent laryngeal nerves.

The INFERIOR CERVICAL GANGLION is of large size but irregular in shape, and occupies the interval between the first rib and the lateral mass of the last cervical vertebra, its position being internal to the superior intercostal artery. Oftentimes it extends in front of the neck of the rib, and joins the first swelling of the knotted cord in the thorax. Its branches are similar to those of the other two ganglia.

One or two branches surround the trunk of the subclavian artery, and supply filaments to that bloodvessel.

* Connecting branches join the last two cervical nerves. Other nerves aecompany the vertebral artery, forming a plexus-vertebral, around it, and communieate with the spinal nerves as high as the fourth.

Only one branch for distribution, the inferior cardiac nerve, issues from the lower ganglion. It lies beneath the subclavian artery, joining in that position the recurrent laryngeal nerve, and enters the thorax to terminate in the deep cardiae plexus behind the arch of the aorta.

Directions. The student now proceeds to dissect the left side of the neck, but the remains of the right half should be carefully preserved during the time occupied in the examination of the left half. 


\section{Section $\mathbf{X}$. \\ DISSECTION OF THE LEFT SIDE OF THE NECK.}

Directions. In the dissection of the left half of the neck, the differences observable between it and the right side are specially to be studied. When the description of the right side will suffice, reference will be made to it.

After the neck has been made tense over a narrow block, the anterior part of it is to be prepared as on the opposite side. The description of the right side (p. 67 to 73 ) is to be used for the anterior triangular space, the sterno-mastoideus, and the depressor museles of the hyoid bone.

Next the scaleni muscles and the subclavian vessels are to be learnt. The dissection and description of the muscles on the right side (p. 73 to 75 ), will serve for those on the left, except that the student will meet on the left side with the thoracic duct.

The thoracic duct is contiguous to the part of the subclavian artery inside the scalenus muscle. If it is uninjected it looks like a vein, rather smaller than a crow-quill; and it will be found by separating the jugular vein from the carotid artery, about half an inch above the clavicle, coursing from beneath the artery to end in the subclavian vein.

On this side the clavicle may remain articulated, in order that the joint may be learnt.

The LEFT SUBCLAVIAN ARTERY arises from the arch of the aorta, instead of from an innominate trunk, and ascends thence over the first rib in its course to the upper limb. With this difference on the two sides in the origin of the subclavian-the one vessel beginning opposite the sternoclavicular articulation, the other in the thorax-it is evident that the length and connections of the part of the artery on the inner side of the scalenus must vary much on opposite sides.

First part. The part of the artery internal to the anterior scalenus is much longer on the left than the right side, and is almost vertical in direction, instead of being horizontal like its fellow. After leaving the chest it is deeply placed in the neck, near the spine and the cesopliagus, and does not rise usually so high above the first rib as the right subclavian.

Between the artery and the surface are structures like those on the right side, viz., the common teguments with the platysma and deep fascia, and the sterno-mastoid, hyoid, and thyroid muscles. Behind the vessel is the longus colli muscle. To the inner side are the cesophagus and the thoracic duct; and the pleura is in contact with the outer and anterior parts. Its connections lower in the chest are described in the dissection of the thorax.

Veins. 'The internal jugular vein is superficial to the artery and parallel to it.

Ner'es. The pneumo-gastric nerve lies parallel to the vessel instead of across it as on the right side; and the phrenic nerve crosses over it close to the scalenus. Accompanying the artery are the cardiac branches of the sympathetic, which course along its inner side to the chest; and beneath it is the inferior cervical ganglion.

The second and third parts of the artery, viz., beneath and beyond 
the scalenus, are nearly the same as on the right side (p. 76); but the student must note for himself the variations that may exist in the connections.

The branches of this artery resemble so closely those of the right trunk, that one description will serve for both (p. 76 to 78). It may be remarked, that the superior intercostal of the left side is usually internal to, instead of beneath the scalenus as on the right side; in other words, this branch arises sooner.

The thoracic duct (fig. $27,{ }^{8}$ ) conveys the chyle and lymph of the greater part of the body into the venous circulation. Escaping from the thorax on the csophagus, the duct ascends in the neck as high as the seventh or sixth cervical vertebra. At the spot mentioned it issues from beneath the carotid trunk, and arches outwards above or over the subclavian artery, and in front of the scalenus muscle and the phrenic nerve, to open into the subclavian close to the union with the internal jugular vein. Double valves, like those of the veins, are present in the interior of the tube; and a pair guards the opening into the posterior part of the vein, to prevent the passage of the blood into it. Frequently the upper part of the duct is divided; and there may be separate openings into the large vein, corresponding with those divisions.

Fig. 27.

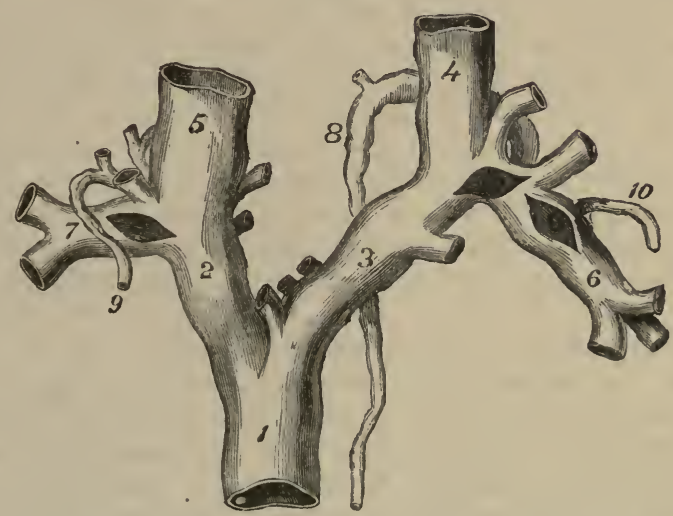

Diagram of the Ending of the Limph Duct and the Thoracic Duct in the Veing.

1. Upper vena cava.

2. Right, und 3 , left innominate vein.

4. Left, and 5, right internal jugular.

6. Left, and 7 , right subclavian vein.
8. Thoracic duct.

9. A lymphatic vessel joining the right lymphatic duct, as thís is about to end in the subclavian vein.

Large lymphatic vessels from the left side of the head and neck, and from the left upper limb, open into the upper part of the duct, and sometimes separately into the vein $\left({ }^{10}\right)$.

Structure. This tube is formed of three eoats like the bloodvessels, viz., inner, middle, and outer. The inner is an elastic layer of longitudinal fibres covered by flattened epithelium; the middle is muscular and elastic with transverse fibres; and the outer is eonstructed ehiefly of fibrous tissues arranged longitudinally and obliquely.

Examine next the brachial and cervical plexuses, using the description of the right side (p. 79 to 81 ). 
Common carotids. On opposite sides these vessels have differences like those between the right and left subclavian arteries; for the left vessel arises from the arch of the aorta, and is therefore deep in the chest, and longer than the right. The description of the artery between its origin and the top of the sternum will be included in the dissection of the thorax.

Beyond the sterno-clavicular articulation the vessels, on both sides, so nearly resemble one another that the same description may serve for the two (p. 81). On the left side, however, the jugular vein and the pneumogastric nerve are much nearer to the carotid than on the right side, and are placed over the artery in the lower third of the neck. ${ }^{1}$

Parts in the upper aperture of the thorax. The relative position of the several parts entering or leaving the thorax by the upper opening may be now observed.

In the middle line lie the remains of the thymus gland, and the trachea and osophagus. In front of the trachea are the lower ends of the sternohyoid and sterno-thyroid muscles with layers of the cervical fascia, and the inferior thyroid veins; and behind the gullet and windpipe is the longus colli muscle. Between the two tubes is the recurrent nerve on the left side.

On each side the bag of the pleura and the apex of the lung project into the neck; and in the interval between the pleura and the trachea and osophagus, are placed the ressels and nerves passing between the thorax and the neck. Most anteriorly on both sides lie the innominate vein, the phrenic nerve, and the internal mammary artery; but the vessels and nerves next met with are different on the two sides. On the right side come the innominate artery, with the vagus, the cardiac nerves, and the right lymphatic duct; but on the left side are the left vagus, the left common carotid artery, the thoracic duct, and the left subclavian artery with the cardiac nerves. Lastly, altogether behind on each side are part of the first intercostal nerve, the cord of the sympathetic, and the superior intercostal artery.

The THYROID BODY is a soft reddish mass, which is situate opposite the upper part of the trachea. It consists of two lobes, one on each side, which are united by a narrow piece across the front of the windpipe. The connecting piece, about half an inch in depth, is named the ist/mus, and is placed opposite the second and third rings of the air tube.

Each lobe is somewhat conical in shape, with the smaller end upwards, and is about two inches in length. It is interposed between the windpipe and the sheath of the common carotid artery, and is covered by the sternothyroid, sterno-hyoid, and omo-hyoid muscles. The extent of the lobe varies; but usually it reaches as high as the middle of the thyroid cartilage, and as low as the sixth ring of the trachea.

From the upper part of the thyroid body, and most commonly from the left lobe, a conical piece-pyramid, ascends towards the hyoid bone, to which it is connected by a fibrous band. Sometimes this part is attached to the os hyoides by a slip of muscle, the levator glandula thyroidea of Sœmmering.

This body is of a brownish red or purple hue, is granular in texture, and weighs from one to two ounces. Its size is larger in the woman than

1 Occasionally these differences will be reversed-the vein and nerve being over the artery on the right side, and away from it on the left. 
in the man. On cutting into the gland a viscid yellowish fluid escapes. It has not any excretory tube or duct.

Structure. The thyroid body is not provided with a distinct capsule; but it is surrounded by arcolar and fine elastic tissues, which project into the substance and divide it into masses.

The substance of the gland consists of spherical or elongated vesicles, which vary in size, some being as large as the head of a small pin, and others only $\frac{1}{8} \frac{1}{5}$ th of an inch. 'These vesicles are simple sacs, distinct from one another, and contain a yellowish fluid with corpuscles. The wall of the vesicles consists of a thin proper membrane with a nucleated epithelial lining. Fine vessels and areolar tissue unite together the vesicles into small irregular masses or lobules of the size of the little finger nail.

The arteries of the thyroid body are two on each side-superior and inferior thyroid. The branches of the external carotids (superior thyroid) ramify chiefly on the anterior aspect; while those from the subclavians (inferior thyroid) pierce the under surface of the body. A very free communication is established between all the vessels; and in the substance of the thyroid body the arteries form a capillary network around the vesicles.

Occasionally there is a third thyroid branch (art. thyroid. ima) which arises from the innominate artery into the thorax, and ascending in front of the trachea assists in supplying the thyroid body.

The veins are large and numerous; they are superior, middle, and inferior thyroid on each side. The first two enter the internal jugular vein (p. 82). The inferior thyroid vein issues from the lower part of the thyroid body, and descends on the trachea - the two forming a plexuse on that tube beneath the sterno-thyroid muscles; it enters finally the innominate vein of its own side.

The tracnea, or air tube, is continued from the larynx to the thorax, and ends by dividing into two tubes (bronchi) one for each lung. It occupies the middle line of the body, and extends commonly from the fifth cervical to the fourth dorsal vertebra, measuring about four inches and a half in length, and nearly one in breadth. 'The front of the trachea is rounded in consequence of the existence of firm cartilaginous bands in the anterior wall, but at the posterior aspect the cartilages are absent, and the tube is flat and muscular.

The cervical part of the trachea is very movable, and has the following relative position to the surrounding parts. Covering it in front are the small muscles reaching from the sternum to the hyoid bone, with the deep cervical fascia: beneath those museles is the inferior thyroid plexus of veins; and near the larynx is the isthmus of the thyroid body. Behind the tube is the ossophagus, with the recurrent nerves. On each side are the common carotid artery and the thyroid body.

The structure of the trachea is described in Section XVII.

The esopitagus, or gullet, reaches from the pharynx to the stomach. It commences, like the trachea, opposite the fifth cervical vertebra, and ends opposite the tenth dorsal vertebra. The tube reaches through part of the neck, and through the whole of the thorax ; and occupies the middle line of the body. In length it measures about nine inches.

In the neck its position is behind the trachea till near the thorax, where it projects to the left side of the air tube, and touches the thyroid body and the thoracic duct. Behind the osophagus is the longus colli muscle. On each side is the common carotid artery, the proximity of the left being greatest because of the projection of the osophagus towards the same side. 
The structure of the oesophagus will be examined in the dissection of the thorax.

Directions. The dissector may learn next the digastric and stylo-hyoid muscles, with the hypoglossal nerve (p. 82). Afterwards he may take the trunk of the external earotid, with the following branches-superior thyroid, facial, occipital, posterior auricular, and superficial temporal ( $p$. 83 to 87 ).

The dissector is not to examine now the pterygo-maxillary or submaxillary regions on the left side, because such a proceeding would interfere with the subsequent dissections. Before learning the pharynx he should lay bare, on this side, the middle and inferior ganglia of the sympathetic with their branches.

Dissection. For the display of the two lower ganglia of the sympathetic and their branches, it will be necessary to take away the great bloodvessels by cutting them across at the lower part of the neck, and near the digastric muscle. In removing the vessels, care must be taken of the sympathetic beneath them.

The middle ganglion must be sought in the fat and areolar tissue near the inferior thyroid artery; and the inferior one will be seen on the neck of the first rib, after the subclavian artery has been divided. The upper cardiac nerve may be found descending beneath the carotid sheath.

The upper end of the sternum with its attached clavicle is to be taken away next, by cutting through the middle of the first rib; and the piece of bone is to be put aside for the subsequent examination of the sternoclavicular articulation.

The middle and inferior cervical ganglia of the sympathetic nerve are so similar to the corresponding ganglia of the right side, that the same description will suffice (p. 117).

The cardiac nerves are three in number on the left as on the right side, viz., superior, middle, and inferior, but they present some differences.

The superior cardiac nerve has a similar course in the neck on both sides; but the left in entering the chest lies between and parallel to the carotid and subclavian arteries.

The middle cardiac nerve unites frequently with the next, and passes beneath the subclavian artery to the deep cardiac plexus.

The inferior cardiac nerve is generally a small braneh, which enters the thorax conjoined with the preceding, to end in the cardiac plexus.

\section{SECTION XI.}

\section{DISSECTION OF THE PHARYNX.}

The pharynx, or the commencement of the alimentary passage, can be examined only when it has been separated from the rest of the head; and it will therefore be necessary to cut through the base of the skull in the manner mentioned below, so as to have the anterior half, with the pharynx connected to it, detached from the posterior half.

Dissection. 'The block being removed from beneath the neck, the head is to be placed downwards, so that it may stand on the cut erge of the skull. Next the trachea and oesophagus, together with the vagus and 
sympathetic nerves, are to be cut near the first rib, and all are to be separated from the spine as high as the basilar process of the occipital bone; and without injuring, on the left side, the vessels and nerves near the skull.

For the division of the skull turn upwards the inner surface of the base, and make the following incisions in the posterior fossa. On the right side a cut, with the chisel, is to be carried along the line of union of the petrous part of the temporal with the occipital bone. On the left side another cut with the chisel is to be made in the same direction, but through the occipital bone internal to the foramen jugulare and the inferior petrosal sinus : this is to begin rather behind that foramen, and to end opposite the one on the other side. The skull is to be sawn through vertically on the left side close behind the mastoid part of the temporal bone, so that the incision shall meet the outer end of the cut made with the chisel.

Finally placing the skull again upside down, let the student chisel through the basilar process of the occipital bone between the attachments of the pharynx and the muscles of the spinal column, the instrument being directed backwards. The base of the skull is now divided into two parts (one having the pharynx attached to it, the other articulating with the spine), which can be readily separated with a scalpel.

The spinal column with the piece of the occipital bone connected with it should be set aside, and kept for after examination.

Dissection of the pharynx (fig. 28). Let the student take the anterior part of the divided skull, and, after moderately filling the pharynx with tow, fasten it with hooks on a block, so that the œesophagus may be pendent and towards him.

On the left side of the pharynx a different view from that of the right side may be obtained of the cranial and sympathetic nerves near the skull (p. 112), when some loose areolar tissue, and the styloid process with its muscles, have been removed: if the lower ends of the nerves are fixed with pieces of thread, a more satisfactory examination can be made of them.

Afterwards the dissector may proceed to remove the fascia from the constrictor muscles of the right side (fig. 28), in the direction of the fibres - these radiating from the side to the middle line. 'The margins of the two lower constrictor muscles (middle and inferior) are to be defined. Beneath the lower one near the larynx, will be found the recurrent nerve with vessels; whilst intervening between the middle and superior, are the stylo-pharyngeus muscle and the glosso-pharyngeal nerve.

To see the attachment of the superior constrictor to the lower jaw and the pterygo-maxillary ligament, it will be necessary to cut through the internal pterygoid muscle of the right side. Above the upper fibres of this constrictor, and near the base of the skull, are two small muscles of the palate $(\mathrm{F}$ and $\mathrm{H})$ entering the pharynx : one-tensor palati, lies between the internal pterygoid plate and muscle; and the other-levator palati, is farther in, and larger.

The PIIARYNx is situated behind the nose, mouth, and larynx. Its extent is from the base of the skull to the ericoid cartilage of the larynx, where it ends in the oesophagus. In form it is somewhat eonical, with the dilated part upwards; and in length it measures from five to six inches.

The tube of the pharynx is incomplete in front, where it communicates with the cavities above mentioned, but is quite closed behind. On each side of it are placed the trunks of the carotid arteries, with the internal 
jugular vein and the accompanying cranial and sympathetic nerves. Behind it is the spinal column, covered by muscles, viz., longus colli and rectus capitis anticus major.

In front the pharynx is united to the larynx, the hyoid bone and the tongue, and to the bony framework of the nasal cavity; but behind it is unattached, and is formed chiefly of thin, fleshy strata. In the posterior wall are contained elevator and constrictor muscles; and at the upper part the bag is further completed by an aponeurotic expansion which fixes it to the base of the skull. The whole is lined by mucous membrane.

The aponeurosis of attachment is seen at the upper part of the pharynx, where the muscular fibres are absent, to connect the tube to the base of the skull, and to complete the posterior boundary. Superiorly it is fixed to the basilar process of the occipital, and the petrous part of the temporal bone, as well as to the cartilage between the two; but inferiorly it becomes thin, and extends between the muscular and mucous strata. On this membrane some of the fibres of the constrictor muscles terminate.

The constrictors are three thin muscles, which are arranged like scales, the lower partly overlaying the middle, and the middle the upper.

The inferior constrictor (fig. $28,{ }^{\wedge}$ ), the most superficial and lowest, arises from the side of the cricoid cartilage; from the oblique line and upper and lower borders of the thyroid eartilage, and from the part of the latter which is behind the oblique line. The origin is small when compared with the insertion, for the fibres are directed backwards, radiating, and are inserted into a raphé along the middle line, where it mects the corresponding muscle of the opposite side.

The outer surface of the muscle is in contact with the sheath of the carotid artery, and with the muscles covering the spinal column. The lower border is straight, and is continuous with the fibres of the cesophagus; whilst the upper border overlaps the fibres of the middle constrictor (в). The recurrent nerve and vessels $\left(^{5}\right)$ enter beneath the lower border.

The middle constrictor (fig. $28^{\mathbf{B}}$ ) has nearly the same shape as the preceding, that is to say, it is narrowed in front and expanded behind. Its fibres arise from the great cornu of the os hyoides, from the small cornu of the same bone, and from the stylo-hyoid ligament. From this origin the fibres radiate, and are blended along the middle line with the other muscles.

The surfaces have connections similar to those of the preceding constrictor. The upper border is separated from the superior constrictor by the stylo-pharyngeus muscle $\mathrm{D}$, and ends on the aponeurosis of the pharynx, about an inch from the base of the skull. The lower border descends beneath the inferior constrictor; and opposite the interval between the two is the upper laryngeal nerve $\left({ }^{3}\right)$.

The superior constrictor (fig. $28^{\mathrm{c}}$ ) is the least strong of the three muscles, and wants the usual conical form. Its origin is extensive, and is connected successively, from above down, with the inner surface of the internal pterygoid plate (the lower third or less), with the pterygo-maxillary ligament, with the posterior part of the mylo-hyoid ridge of the lower jaw, and with the mucous membrane of the mouth and the side of the tongue. 'The fleshy fibres pass backwards, and are inserted on the aponeurosis of the pharyix, as well as into the raple along the middle line.

The parts in contact externally with this muscle are, the deep vessels and nerves of the neck; and internally it is lined by the aponeurosis and the mucous membrane. The upper border consists of arched fibres which 
are directed backwards from the pterygoid plate; and above it the levator palati muscle $\mathrm{F}$ is seen. The lower border is overlaid by the middle constrictor muscle. The attachment to the pterygo-maxillary ligament corresponds with the origin of the buccinator muscle $\mathrm{I}$.

Fig. 28.

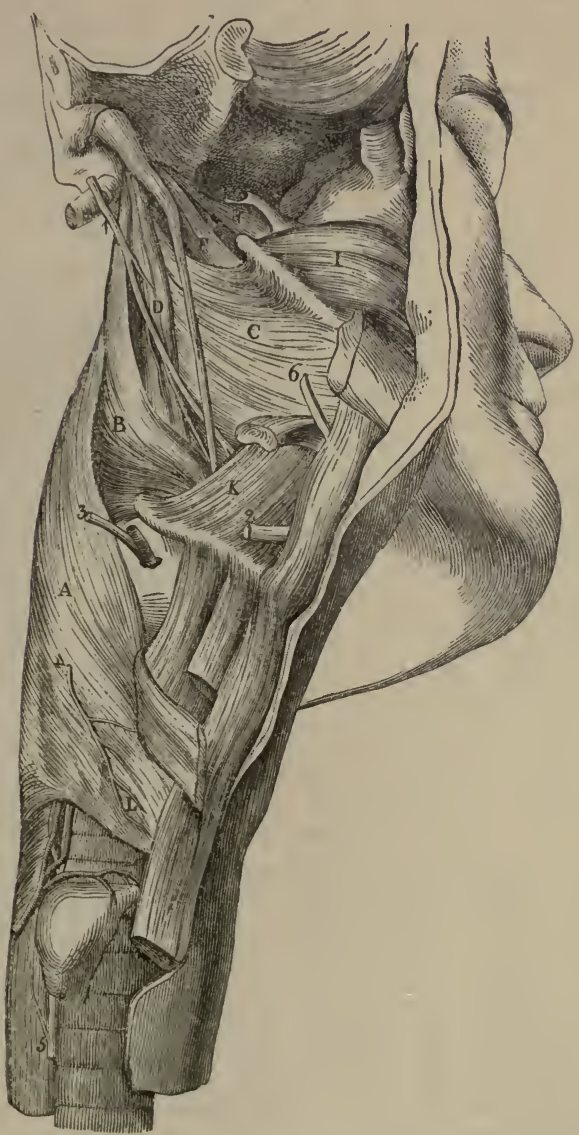

Muscles :

A. Inferior constrictor.

B. Middle constrictor.

C. Upper constrictor.

D. Stylo-pharyngeus.

F. Levator palati.

H. Tensor palati.

I. Buccinator.

K. Hyo-glossus.

Nerves :

1. Glosso-pharyngeal.

2. Hypo-glossal.

3. Superior laryngeal.

4. External laryngeal.

5. Recurrent laryngeal.

6. Gustatory nerve.

Evternal Vigw of the Pharyxx (Illustrations of Dissections).

Action of constrictors. The muscles of both sides contracting will diminish the size of the pharynx; and as the anterior attachments of the lower muscles are nearer together than those of the upper, the tube will be contracted more behind the larynx than near the head.

In swallowing the morsel is seized first by the middle constrictor, and is delivered over to the inferior, by which it is conveyed to the osophagus: both muscles act involuntarily. By the contraction of the upper muscle the space above the mouth will be narrowed, so that the soft palate being raised, the upper portion of the pharyngeal space can be shut off from the lower. 
Dissection (fig. 29). Open the pharynx by an incision along its middle, and, after removing the tow from the interior, keep it open with hooks: a better view of the eavity will be obtained by partly dividing the occipital attachment on each side. The mucous membrane is to be carefully removed below the dilated extremity of the Eustachian tube on the right side, for the purpose of finding some pale muscular fibres, salpingopharyngeus $\mathrm{c}$, which descend from it.

The Elevators of the pharynx are two in number on each side-an external (stylo-pharyngeus), and an internal (salpingo-pharyngeus).

The stylo-pharyngeus (external elevator) may be read again with the pharynx. Its description is given in p. 106.

Salpingo-pharyngeus, c (internal elevator). This little band is fixed by tendon to the lower border of the cartilage of the Eustachian tube near the orifice. Its fleshy fibres end below by joining those of the palatopharyngeus $Q$. If the part is not tolerably fresh the muscle may not be visible.

Action. This thin muscle elevates the upper and lateral part of the pharynx above the spot where the large external elevator enters the wall; but it is probably used chiefly in opening the Eustachian tube in swallowing.

The interior of the pharynx (fig. 29) is wider from side to side than from before back, and its greatest width is opposite the hyoid bone; from that spot it diminishes both upwards and downwards, but much more rapidly in the latter than in the former direction. 'Through the part of the passage above the mouth the air moves in respiration; whilst through that below the mouth both air and food are transmitted-the air passing to the aperture of the windpipe, and the food to the osophagus. In it the following objects are to be noticed:-

At the top are situate the posterior apertures, $G$, of the nasal eavity, which are separated by the septum nasi; and below them hangs the soft palate, partly closing the cavity of the mouth. By the side of each nasal aperture is the trumpet-shaped end of the Eustachian tube, F.

Below the soft palate, the opening into the mouth-isthmus faucium, $\mathrm{H}$, is to be recognized; and on each side of this is the tonsil, $\mathrm{K}$, which is placed in a hollow between two prominences named pillars of the soft palate-the one, $\mathrm{x}$, proceeding from the soft palate to the side of the tongue, and the other, $\mathrm{L}$, from the same part to the side of the pharynx.

Next in order, below the mouth, comes the aperture of the larynx, $\mathrm{s}$; and close in front of it is the epiglottis, or the valve which assists to close that opening during deglutition. Lowest of all is the opening, o, from the pharynx into the oesophagus.

The apertures into the pharynx are seven in number, and have the following position and boundaries:

'The posterior openings of the nasal fossa, $\mathrm{G}$, are oval in form, and measure about an inch from above down, but only half an inch across. Each is constructed in the dried skull by the splienoid and palate bones above, and by the palate below; by the vomer inside, and the internal pterygoid plate outside; and it is lined by mucous membrane.

The Lustactian tube, $\mathrm{F}$, is a canal, partly osseous, partly cartilaginous, by which the tympanic cavity of the ear communicates with the external air.

If the mucous membrane be removed from the tube on the right side, the cartilaginous part appears to be nearly an inch long. It is narrow 
superiorly, where it is fixed to the margins of a groove between the petrons part of the temporal and the sphenoid bone; but it increases in width as it is directed downwards to the pharynx, and ends by a wide aperture inside the internal pterygoid plate, on a level with the inferior meatus. Its opening in the pharynx is oval in form; and the inner side, which is larger than the outer, projects forwards, giving rise to a trumpet-shaped mouth.

Fig. 29.

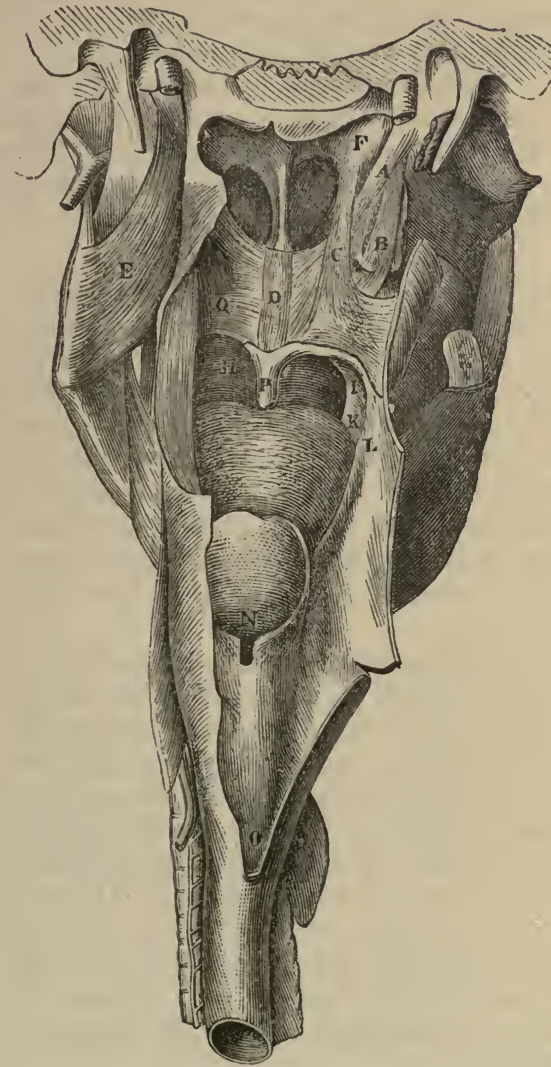

A. Levator palati.

B. Tensor palati.

C. Salpingo-pharyngeus.

D. Azygos uvulæ.

B. Internal pterygoid muscle.

F. End of the Eustachian tube.

f. Posterior uaris.

H. Mouth cavity.

I. Anterior pillar of the fauces.

K. Apertures of the tonsil.

L. Posterior pillar of the fauces.

N. Opening of the larynx.

o. Opening of the oesophagus.

P. Uvula.

Q. Superficial part of Palato-pharyngeus.

Internal View of the Pharix (Illustrations of Dissections). Mesclis of the Palate, and NAMED PARTB.

This part of the tube is constructed by a triangular piece of cartilage, whose margins are bent downwards so as to inclose a narrow space; but at the under aspect the cartilage is deficient, and the wall is formed by fibrous membrane. Closely united to the pterygoid plate, the tube is covered by the mucous membrane; and through it the mucous lining of the cavity of the tympanum is continuous with that of the pharynx.

The space included between the root of the tongue and the arches of the soft palate on opposite sides is called the fauces. It is wider below than above; and on each side lies the tonsil. 
The isthmus faucium, $\mathrm{II}$, is the narrowed aperture of communication between the mouth and the fauces, whose size is altered by the elevated or pendent position of the soft palate. Laterally it is bounded by the anterior arches of the soft palate, which are named pillars of the fauces.

The aperture of the larynx, $\mathrm{N}$, is wide in front, where it is bounded by the epiglottis, and pointed behind between the arytanoid cartilages. The sides are sloped from before back, and are formed by folds of the mucous membrane extending between the arytanoid cartilages and the epiglottis. Posteriorly it is limited by the cornicula laryngis, and by the arytanoid muscle covered by mucous membrane. During respiration this aperture is unobstructed, but in the act of deglutition it is closed by the epiglottis.

The opening into the cesophagus, $o$, is the narrowest part of the pharynx, and is opposite the cricoid cartilage and the fifth cervical vertebra. Internally the mucous membrane in the osophagus is paler than that in the pharynx: and externally the point at which the pharynx ends is marked by a slight contraction, and by a change in the direction of the muscular fibres.

The SOFT PALATE, Q (velum pendulum palati), is a movable structure between the mouth and the pharynx, which can either close the opening of the mouth, or cut off the passage to the nose, according as it is depressed or elevated. In the usual position of the soft palate (the state of relaxation) the anterior surface is somewhat curved, and is continuous with the roof of the mouth, whilst the opposite surface is convex and turned to the pharynx. The upper border is fixed to the posterior margin of the hard palate; and each lateral part joins the pharynx. The lower border is free, and presents in the centre a conical pendulous part-the uvula, $\mathrm{P}$. Along its middle is a slight ridge, indicative of the original separation into two halves.

Springing from the lower part of the soft palate, near the uvula, are two folds on each sides, containing muscular fibres, which are directed downwards on the sides of the fauces. These are named arches or pillars of the palate, and are distinguished from one another by their relative position. The anterior, I, reaches from the fore part to the side of the tongue near the middle; and the posterior, L, longer than the other, is continued from the lower border to the side of the pharynx. As they diverge from their origin to their termination, they limit a triangular space in which the tonsil lies.

The velum consists of an aponeurosis, with muscles, vessels, nerves, and mucous glands; and the whole is enveloped by the mucous membrane.

Dissection.- - Some of the muscles of the palate are readily displayed, but others require care in their dissection.

On the right side the two principal muscles of the soft palate-the elevator and tensor, are very plain. These have been partly dissected on the right side; but to follow them to their termination, let the upper attachment of the pharynx on the same side, and the part of the superior constrictor which arises from the internal pterygoid plate, be cut through. 'The levator will be fully laid bare by the removal of the mucous membrane, and a few muscular fibres covering its lower part. 'The tendon of the tensor palati should be followed round the hamular process of the pterygoid plate; and its situation in the palate beneath the levator should be made evident. The position of the Eustachian tube with respect to those muscles should be ascertained. 
On the left side, the mucous membrane is to be raised with great care from the posterior surface of the palate, to obtain a view of the superficial muscular fibres. Immediately beneath the mucous covering are some fine transverse fibres of the palato-pharyngeus musele; and beneath them, in the middle line, are the longitudinal fibres of the azygos uvulæ. A deeper set of fibres of the palato-pharyngeus is to be followed, on the right side, beneath the levator and azygos muscles.

The student should remove next the mucous membrane from the muscular fibres contained in the arches of the palate, and should follow these upwards and downwards. In order to see them in the anterior fold, it will be necessary to take the membrane from the anterior surface of the palate. If the part is not tolerably fresh, some of the paler fibres may not be visible.

Aponeurosis of the soft palate. Giving strength to the velum is a thin but firm aponeurosis, which is attached to the hard palate. This membrane becomes thinner as it descends in the velum; and it is joined by the tendon of the tensor palati muscle.

The Muscles of the soft palate are four on each side, - an elevator and tensor; with the palato-glossus and palato-pharyngeus, which act as depressors. In addition there is a small median azygos muscle.

The LEvator PALATI (fig. $29,{ }^{\wedge}$ ) is a thick, roundish muscle, which is partly situate outside the pharynx. It arises from the under surface of the apex of the petrous portion of the temporal bone (fig. $30,{ }^{3}$ ), and from the inner and hinder part of the cartilage of the Eustachian tube. The fibres enter the pharynx above the superior constrictor, and then spread out in the soft palate, where they join along the middle line with those of the muscles of the opposite side.

Outside the pharynx this muscle rests against the Eustachian tube. In the palate it forms a stratum that reaches the whole depth of that structure, and is embraced by two planes of fibres of the palato-pharyngeus ( $\left.{ }^{4}\right)$.

Action. It tilts backwards the free edge of the soft palate towards the pharynx so as to enlarge the isthmus faucium, and to shut off with the contracted pharynx the nose openings. In swallowing the palate is raised, and is arched over the bolus passing from the mouth to the pharynx. For its action on the Eustachian tube, see Tensor palati.

The texsor vel circumflexus palati (fig. $29,{ }^{\mathbf{B}}$ ) arises like the preceding outside the pharynx, and is a thin riband-like band, situate between the internal pterygoid plate and muscle. About one inch and a half wide at its origin, it is attached to the slight depression (scaphoid fossa) at the root of the internal pterygoid plate, to the outer and forepart of the Eustachian tube, and still further out to the spinous process of the sphenoid, and the vaginal (tympanic) process of the temporal bone. Inferiorly the fleshy fibres end in a tendon which, entering the pharynx between the attachments of the buccinator muscle, is reflected round the hamular process (fig. $30,{ }^{2}$ ), and is inserted into about one-third of an inch of the posterior border of the palate, viz., from the central spine to a projecting point; and inferiorly into the aponeurosis of the velum.

As the tendon winds round the bone, it is thrown into folds; and between the two is a bursa. In the soft palate it lies beneath the levator muscle. The Eustachian tube is directed inwards between this muscle and the preceding.

Action. Acting from the skull the muscle will fix and make tense the 
lateral part of the soft palate; but its movements will be very limited, seeing that the tendon is inserted partly into the palate bone.

Fig. 30.

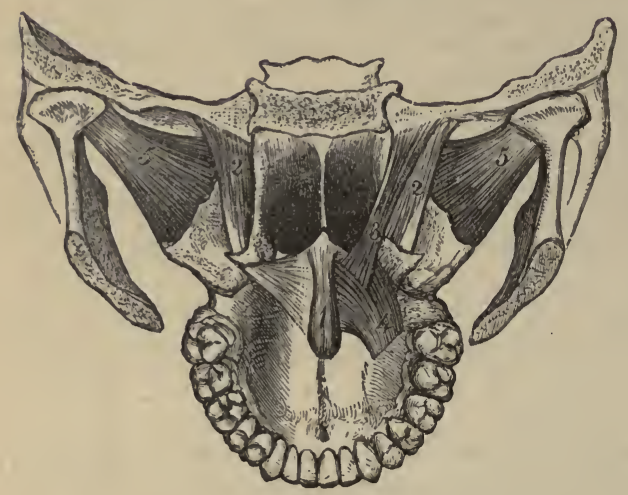

1. Azygos uvulæ.

2. Tensor palati.

3. Levator palati.

4. Palato-pharyngeus-upper end.

5. External pterygoid.

If the soft palate is fixed by the depressor muscles, the levator and tensor, and the salpingo-pharyngeus, taking their fixed points below, open the Eustachian tube in swallowing.

The PALATO-GLOSSUS MUSClE (constrictor isthmi faucium) is a small, pale band of fibres, which is contained in the anterior arch, I, of the soft palate. It is connected inferiorly with the lateral surface and the dorsum of the tongue; from this spot the fibres ascend before the tonsil to the anterior aspect of the soft palate, where they form a thin muscular stratum, and join those of the fellow muscle along the middle line.

At its origin the muscle is blended with the glossal muscles, and at its insertion it is placed before the tensor palati.

Action. The palato-glossus narrows the isthmus of the fauces; the muscles of opposite sides moving inwards towards each other, and separating from the mouth the morsel to be swallowed.

When the tongue is fixed the muscle will render tense and draw down the soft palate.

The PALATO-PHARYNGEUs is much larger in size than the preceding, and gives rise to the eminence of the posterior pillar, $L$, of the sof palate. The muscle is attached below to the posterior border of the thyroid cartilage, some fibres blending with the contiguous portion of the pharynx; and it decussates across the mirldle line with corresponding fibres of the muscle of the opposite side (Merkel). ${ }^{1} \quad$ Ascending thence behind the tonsil, the fibres enter the side of the palate, and separate into two layers (fig. $30,{ }^{4}$ ). The posterior, thin and in contact with the mucous membrane, joins at the middle line a like offset of its fellow. The deeper or anterior stratum, much the strongest, enters the substance of the palate between the levator and tensor, and joins at the middle line the corresponding part of the opposite muscle, whilst some of the upper fibres end on the aponeurosis of the palate.

1 Dr. Merkel, in the work before referred to, states that this muscle has no firm fixed attachment below, and that it ends altogether in the wall of the pharynx, decussating with the muscle of the opposite side. This assertion does not accord with my experience. 
In the palate the muscle incloses the levator palati and azygos uvulæ between its two strati.

Action. Taking its fixed point at the thyroid cartilage the muscle depresses and makes tense the soft palate.

During the act of swallowing both muscles move back the lower edge of the soft palate towards the pharynx; and approaching each other, form an oblique plane for the downward direetion of the food; in that state the uvula lies in the interval between the two.

The Azycos UVlLA (fig. $29,{ }^{D}$ ) is situate along the middle line of the velum near the posterior surface. The muscle consists of two narrow slips of pale fibres, which arise firom the spine at the posterior border of tl.e hard palate, or from the contiguous aponeurosis, and end inferiorly in the tip of the uvula. Belind this muscle, separating it from the mucous membrane, is the thin stratum of the palato-pharyngeus.

Action. Its fibres elevate the urula, shortening the mid part of the soft palate, and direct that process backwards.

The tonsil, $\mathrm{K}$, is a collection of follicular capsules resembling those on the dorsum lingux, which is placed close above the base of the tongue, and between the arches of the soft palate. Each is roundish in shape, but variable in size; and apertures are apparent on its surface. Externally the tonsil is situate opposite the superior constrictor musele and the angle of the lower jaw ; and when enlarged it may press against the internal carotid bloodvessel.

The apertures on the surface lead to rounded terminal recesses or hollows which are lined by mucous membrane. Around each recess is a layer of small elosed capsules, which are seated in the tissue beneath the mucous membrane, and are filled with a grayish substance containing cells and nuclear-looking bodies. No openings from the capsules are to be recognized in the recesses.

Its arteries are numerous and are derived from the facial, lingual, ascending pharyngeal, and internal maxillary branches of the external earotid. Its veins have a plexiform arrangement on the outer side. Nerves are furnished to it from the fifth and glosso-pharyngeal nerves.

The mucons membrane of the pharynx is continuous anteriorly with the lining of the mouth, nose, and larynx. Covering the soft palate and its numerous small glands (palatine), the membrane is continued to the tonsils on each side, and is prolonged by the Eustachian tube to the tympanum. In front of each arytrenoid cartilage it incloses a mass of muciparous glands (arytrenoid). Inferiorly, it is continued by the oesophagus to the stomach.

The mucous membrane is provided with more glands in the upper, than in the lower part of the pharynx; and its character, near the different apertures, resembles that of the membrane lining the cavities communieating with the pharynx. Its epithelium is scaly below the nares (Henle); but is columnar and ciliated above that spot, where only the air is transmitted.

Beginning of the oesophagus. This tube is much smaller than the pharynx, and the walls are flaccid. For the commencement, and its connections in the neck, see p. 121.

The gullet consists of two layers of muscular fibres, with a lining of mucous membrane. The external layer is formed of longitudinal fibres, which begin opposite the cricoid cartilage by three bundles, anterior and two lateral; the former is attached to the ridge at the back of the carti- 
lage, and the others join the inferior constrictor. The internal layer, on the other hand, is formed of circular fibres, which are continuous with those of the inferior constrictor. 'The structure of the osophagus is deseribed more fully in the dissection of the thorax.

\section{SECtion XII.}

\section{CAVITY OF THE MOUTH.}

THE cheeks, the lips, and the teeth are to be examined with the mouth, as all may be considered accessory parts.

The Mouth. 'The cavity of the mouth is situate below that of the nose, and extends from the lips in front to the isthmus of the fauces behind. Its boundaries are partly osseous and partly muscular, and its size depends upon the position of the lower jaw bone. When the lower jaw is moderately removed from the upper, the mouth is an oval cavity with the following boundaries. The roof; concave, is constituted by the hard and soft palate, and is limited anteriorly by the arch of the teeth. In the floor is the tongue, bounded by the arch of the lower teeth; and beneath the tip of that body is the franum lingure, with the sublingual gland on each side. Each lateral boundary consists of the cheek and the ramus of the lower jaw ; and in it, near the second molar tooth in the upper jaw, is the opening of the parotid duct. The anterior opening of the mouth is bounded by the lips; and the posterior corresponds with the anterior pillars of the soft palate.

The mucous membrane is less sensitive on the hard than the soft parts bounding the mouth; it lines the interior of the cavity, and is reflected over the tongue. Anteriorly it is continuous with the tegument, and posteriorly with the lining of the pharynx. The epithelium covering the membrane is of the scaly variety.

Between each lip and the front of the corresponding jaw the membrane forms a small fold-frenulum. On the bony part of the roof it blends with the dense tissue (gums) covering the vessels and nerves. On the soft palate it is smooth and thinner, and along the middle of the palate is a ridge which ends in front in a small papilla. In the floor of the mouth the membrane forms the franum lingua beneath the tip of the tongue, and sends tubes into the openings of the Whartonian and sublingual ducts; whilst on each side of the franum it is raised into a ridge by the subjacent sublingual gland. On the interior of the cheek and lips the mucous lining is smooth, and is separated from the muscles by small buccal and labial glands.

Over the whole cavity, but especially on the lips, are papilla for the purpose of touch.

The снЕкк extends from the commissure of the lips to the ramus of the lower jaw, and is attached above and below to the alveolar process of the jaw on the outer aspect. 'The chief' constituent of the cheek is the fleshy part of the buccinator muscle; on the inner surface of this is the mucous membrane; and on the outer the integuments, with some muscles, vessels, and nerves. The parotid duct perforates the cheek near the second molar tooth of the upper jaw. 
The LIPS surround the opening of the mouth; they consist chiefly of the fleshy part of the orbicularis oris muscle, covered externally by integu-ment, and internally by mucous membrane. The lower lip is the larger and more movable of the two. Between the muscular structure and the mucous covering lie the labial glands; and in the substance of each lip, nearer the inner than the outer surface, and at the line of junction of the two parts of the orbicularis, is placed the arch of the coronary artery.

Tétн. In the adult there are sixteen teeth in each jaw, which are set in the alveolar borders in the form of an arch, and are surrounded by the gums. Each dental arch has its convexity turned forwards; and, commonly, the arch in the maxilla overhangs that in the mandible when the jaws are in contact. The teeth are similar in the half of each jaw, and have received the following names : the most anterior two are incisors, and the one next behind is the canine tooth; two, still farther back, are the bicuspids; and the last three are molar teeth. Moreover, the last molar tooth has been called also "dens sapientix," from the late period of its appearance. The names applied to the teeth indicate very nearly the part they perform in mastication; thus the incisor and eanine teeth act as dividers of the food, whilst the bicuspid and molar teeth serve to grind the aliment.

The several parts of the teeth, viz., the crown, fang, and neck; the general and special characters of those parts in the different groups of teeth; and the structure of the different components of a tooth, must be referred to in some general treatise on anatomy.

\section{Section XIII.}

\section{DISSECTION OF THE NOSE.}

To obtain a view of the interior of the nose, it will be necessary to make a longitudinal section through the base of the skull. Whilst the student is examining the boundaries of the nose he will derive advantage from the use of a vertical section of a dried nasal cavity.

Dissection. Before sawing the bone, the loose part of the lower jaw on the right side should be taken away; further, the tongue, hyoid bone, and larynx, all uniterl, may be detacher from the oppcsite half of the lower jaw, and laid aside till the dissector is ready to use them.

On the right side of the middle line saw through the frontal and nasal bones, the cribriform plate of the ethmoid, and part of the body of the sphenoid bone, without letting the saw descend into the nasal cavity.

Next the roof of the mouth is to be turned upwards, and the soft parts are to be divided on the right of the median line opposite the cut in the roof of the nose. The saw is then to be carried through the floor of the nose and the body of the sphenoid bone in such a direction as to come into the incision above.

The piece of the skull is now separated into two parts, right and left; the right half will serve for the examination of the meatuses, and the left will show the septum nasi, after the mucous membrane has been removed.

The Cavity of THE nOse is placed in the centre of the bones of the face, being situate above the mouth, below the eranium, and between the 
orbits. This space is divided into two parts_nasal fossæ-by a vertical partition.

Each fossa is larger below than above; and is flattened in form, so that the measurement from before back or above down exceeds much that from within out. It communicates with both the face and the pharynx by apertures named nares, and has also apertures of communication with the sinuses in the surrounding bones, viz., frontal, ethmoid, sphenoid, and superior maxillary. The student has to examine in each fossa a roof and floor, an inner and outer wall, and an anterior and posterior opening.

The roof is somewhat arched, and is formed by the cribriform plate of the ethmoid bone in the centre; by the frontal and nasal bones, and the eartilages in front; and by the body of the sphenoid, the sphenoidal spongy bone, and the os palati, at the posterior part. In the dried skull many apertures exist in it; most are in the ethmoid bone for the branches of the olfactory nerve with vessels, and one for the nasal nerve and ressels; in the front of the body of the sphenoid is the opening of its sinus.

The floor is slightly hollowed from side to side, and in it are the palate and superior maxillary bones-their palate processes. Near the front in the dry skull is the incisor foramen leading to the anterior palatine fossa.

The inner boundary (septum nasi) is partly osseous and partly cartilaginous. The osseous part is constructed by the vomer, by the perpendicular plate of the ethmoid bone, and by those parts of the frontal and nasal with which this last bone articulates. 'The irregular space in front in the prepared skull is filled in the recent state by the triangular cartilage of the septum, which forms part of the partition between the nostrils, and supports the cartilages of the anterior aperture. Fixed between the vomer, the ethmoid plate, and the nasal bones, this cartilage rests anteriorly on the median ridge between the superior maxilla, and projects even between the cartilages of each nostril. The septum nasi is commonly bent to one side.

The outer boundary has the greatest extent and the most irregular surface. Six bones enter into its formation, and they come in the following order from before backwards : the nasal and superior maxillary; the small os unguis with the lateral mass of the ethmoid bone; and posteriorly the ascending part of the palate bone, with the internal pterygoid plate of the sphenoid bone: of these, the nasal, ungual, and ethmoidal reach only about half way from the roof to floor, whilst the others extend the whole depth. Altogether in front of the bones, the lateral cartilages may be said to construet part of this boundary.

On this wall are three convoluted osseous pieces, named spongy or turbinate bones, (fig. 31), which project into the cavity:-the two upper $\left({ }^{1}\right)$ and $\left({ }^{2}\right)$, are processes of the ethmoid, but the lower one $\left({ }^{3}\right)$, is a separate bone-the inferior spongy. The spongy bones are confined to a certain portion of the outer wall, and their extent would be limited by a line continued nearly vertically upwards to the roof of the cavity from both the front and back of the liard palate. Between each turbinate bone and the wall of the nose is a longitudinal hollow or meatus; and into these hollows the nasal duct and the sinuses of the surrounding bones open.

The meatuses are the spaces arched over by the spongy bones; and as the bones are limited to a certain part of the outer wall, so are the spaces beneath them.

The upper one (fig. $31,{ }^{6}$ ) is the smallest and straightest of the three 
meatuses, and occupies the posterior half of the space included by the vertical lines before mentioned. Into it the posterior ethmoidal sinuses open at the front; and at its posterior part, in the dried bone, is the sphenopalatine foramen by which the nerves and vessels enter the nose.

The middle meatus (fig. $31,{ }^{7}$ ) is longer than the preceding; it is curved upwards in front, and reaches all across the space referred to on the outer wall. Anteriorly it communicates by a funnel-shaped passage (infundibulum) with the frontal sinus and the anterior ethmoidal cells; and near its middle is a small aperture, which leads into the cavity of the upper jaw.

The inferior meatus (fig. $31,{ }^{8}$ ) is straighter than the middle one, and rather exceeds the width of the included space on the outer wall; and

Fig. 31.

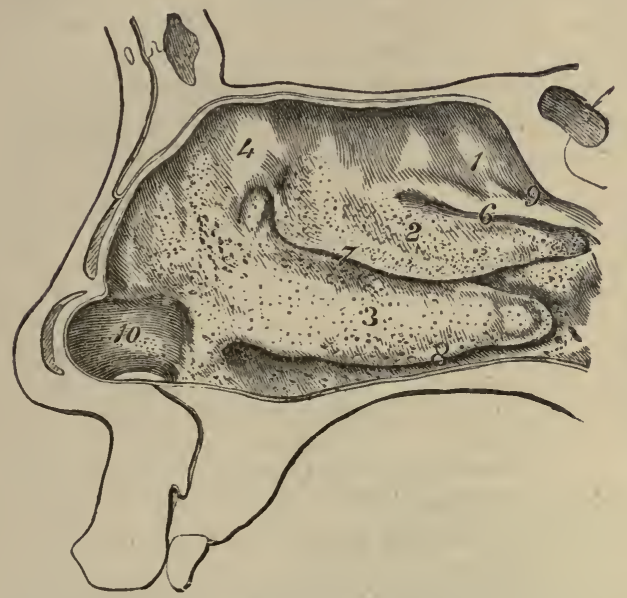

1. Upper spongy bone.

2. Middle spongy bone.

3. Inferior spongy bone.

4. Square part of the ethmoid bone.

6. Upper meatus.

7. Middle meatus.

8. Lower meatus.

9. Rudimentary fourth meatus.

10. Vestibule of the nasal cavity.

The woodcut shows also the apertures of the glands of the nose.

Spongy Bones and Meatuses of the Nasal Cavity.

when the bone is clothed by the mucous membrane it extends still further forwards. In its front is the opening of the ductus ad nasum.

Occasionally there is a small fourth or rudimentary meatus above the rest (fig. $31,{ }^{9}$ ), which communicates with a posterior ethmoidal cell.

The nares. In the recent condition of the nose each fossa has a distinct anterior opening in the face, and another in the pharynx; but in the skeleton there is only one common opening in front for both sides. These apertures, and the parts bounding them, have been before described (pp. 42 and 126$)$.

The mucous membrane lining the nasal fossa is called the pituitary or Schneiderian membrane; and from its blending with the periosteum it acquires much strength. It is continuous with the integument at the nostril, and with the membrane lining the pharynx through the posterior opening: moreover, it is also continuous with the mucous membrane of the eyeball, and with that of the different sinuses, viz., frontal, ethmoidal, sphenoidal, and maxillary.

The foramina in the dry bones, which transmit nerves and ressels, are entirely closed by the membrane, viz., the incisor, spheno-palatine, the holes in the cribriform plate, and the foramen for the nasal nerve and 
vessels; but the apertures that lead to the sinuses and the orbit are only somewhat diminished by the lining they receive. The membrane is stretched over the opening of the ductus ad nasum, forming a flap or valve to close the aperture.

The characters of the membrane in the lower or respiratory part of the nose differ greatly from those of the same layer in the olfactorial region near the roof.

In the lower region of the nose, through which the air passes to the lungs, the membrane is thick, and closely united to the subjacent periosteum and perichondrium; and on the margins of the two inferior spongy bones it is projected somewhat by the large submucous ressels, so as to increase the extent of surface. In the canals and sinuses it is very thin. Near the nostril it is furnished with papilla, and small hairs (vibrissa).

The surface is corered by the apertures of branched mucous glands, which are in greatest abundanee, and of largest size, about the middle and posterior parts of the nasal fossa. In the lower part of the nose, and in the sinuses, the epithelium is of the columnar ciliated kind; but it becomes laminated or scaly in the dilatation or vestibule inside the nastril (fig. $\left.31,{ }^{10}\right)$.

Fig. 32 .
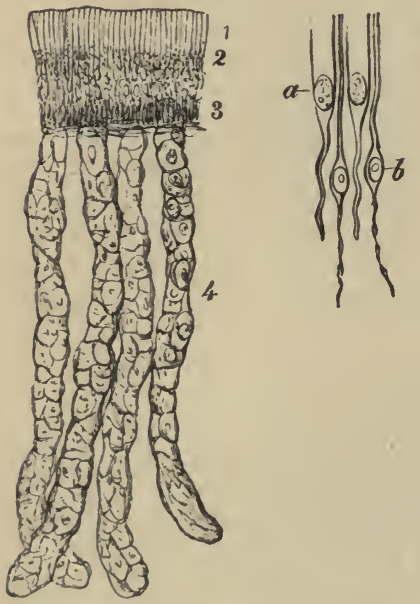

A. 1. Columnar epithelium at the free surface.

2. Granular or middle layer of the same.

3. Deepest layer of elougated cells placed vertically.

4. Secreting tubular glands.

B. a. Pieces of the columnar epithelium greatly enlarged.

b. Olfactorial cells amongst the epithelium particles.

Magnifed Vertical Section of the Mucoú Membrane of the Nosi (altered from Henle).

The olfactory region (fig. 32 ) is situate at the top of the nasal cavity, and is confined to the surface of the roof formed by the cribriform plate of: the ethmoid; to a portion of the outer wall constructed by the lateral mass of the ethmoid bone: and to a corresponding extent of the septum, viz., about one-third.

The mucous membrane in the olfactory region receives the olfactory nerve, and is therefore the seat of the sense of smell. It differs much from that in the lower portion of the nose, for it is less strong and vascular; and is of a yellowish color, which is due to pigment in the epithelium and the glands.

The epithelium (fig. $32 \mathrm{~A}$ ) is thicker but softer here than lower in the cavity, and it is columnar on the surface, but not ciliated. Beneath the 
surface layer are strata of granules and ovalish cells $\left({ }^{2}\right)$, amongst which sink the pointed or attached ends of the pieces of epithelium.

Around the piees of the columnar epithelium stand numerous bodies named olfactorial cells by Schultze (fig. 32 в. $^{8}$ ). They consist of small spindle-shaped nucleated cells, with a rounded filament prolonged from each end towards the attached and free surfaces of the mucous membrane: that to the free surface, the larger, ends on a level with the pieces of the columnar epithelium. But the conneetions of the deeper threads or processes are unknown; they have been supposed to unite with the olfactory nerve.

The glands in the olfactory region are simple lengthened tubes (fig. $32 \mathrm{~A}^{4}$ ) like those in the stomach, but are slightly wavy, and end in the submucous tissue by closed extremities. A flattened epithelium, with colored granular contents, lines the tubes.-(Henle.)

Dissection. At this stage of the dissection, little will be seen of the distribution of the olfactory nerve. If the septum nasi be removed, so as to leave entire the membrane covering it on the opposite side (the left), the filaments of the nerve will appear on the surface, near the cribriform plate. In the membrane, too, near the front of the septum, is an offset of the nasal nerve.

The naso-palatine nerve and artery (fig. $33,{ }^{3}$ ) are to be sought lower down, as they are directed from behind forwards, towards the anterior

Fig. 33.

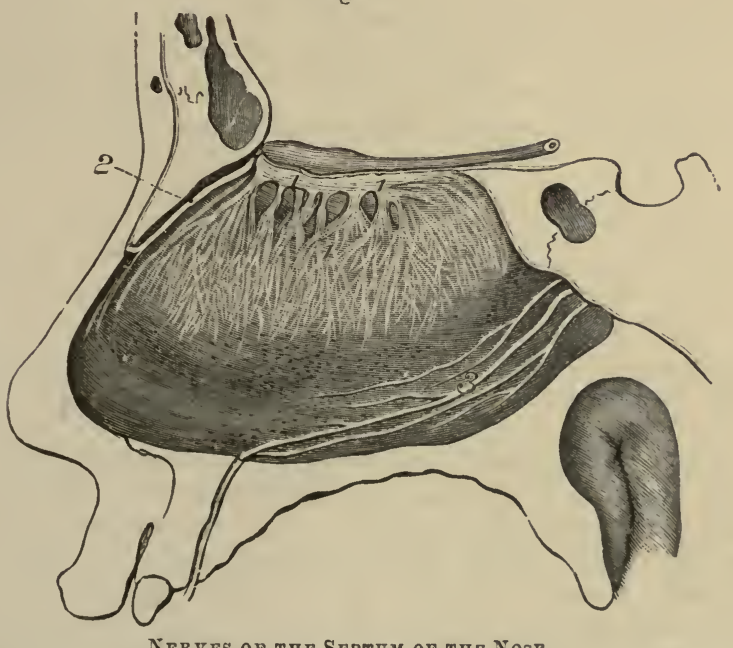

NGRVes of the Septum of the Nose.

1. Olfactory bulb and its ramifications on the septum.

2. Nasal nerve of the ophthalmic trunk.
3. Naso-palatine nerve from Meckel's ganglion (too larke in the cut.)

palatine fossa; the artery is readily seen, especially if it is injected, but the fine nerve, which is about as large as a coarse hair, is imbedded in the membrane and will be found by scraping with the point of the scalpel.

By cutting through the fore and upper part of the membrane that has been detached from the septum nasi, other branches of the olfactory nerve may be traced on the outer wall of the nasal fossa. 
The olfaCtORY NeRve (fig. $33,{ }^{1}$ ) forms a bulb on the cribriform plate of the ethmoid bone, and sends branches to the olfactory region of the nose through the apertures in the roof. 'These branches are about twenty in number, and are divisible into three sets. An inner set, the largest, descend in the grooves on the septum nasi, and branching, extend over the upper third. A middle set is confined to the roof of the nose. And an external set is distributed on the upper spongy bone, on the anterior square surface of the os ethmoides, and on the fore part of the middle spongy bone.

As the branches of the olfactory nerve leave the skull, they receive tubes from the dura mater and pia mater, which are lost in the tissue to which the nerves are distributed. 'The nerves ramify in the pituitary membrane in tufts of filaments which communicate freely with the contiguous twigs, forming a network, but their mode of termination in the tissue is unknown. It has been suggested by Schulze that they join the deep processes or ends of the so-called olfactorial cells; but this union has not been seen.

The olfactory nerve differs in structure from the other cranial nerves; for its branches are deficient in the white substance of Schwann, are not divisible into fibrillæ, and are nucleated and granular in texture. They resemble the ganglionic fibres: and seem to consist of an extension of the nerve substance of the olfactory bulb.

The other nerves in the nose will be described in the following section.

Bloodvessels. For a statement of the different vessels of the nose, see p. 141. The arteries form a network in the pituitary membrane, and a large submucous plexus on the edge of each of the two lower spongy bones, especially on the inferior. The veins have a plexiform disposition like the arteries, and this is largest on the lower spongy bone and the septum nasi.

\section{Section XIV.}

SPHENO-PALATINE AND OTIC GANGLIA, FACLAL AND NASAL NERVES, AND BRANCHES OF THE INTERNAL MAXILLARY ARTERY.

TuE preparation of Meckel's ganglion and its branches (fig. 34), and of the terminal branches of the internal maxillary artery, is a somewhat difficult task in consequence of the nerves and vessels being contained in osseous canals which require to be opened. $A$ s is the case with other dissections, the student seeks first the branches, and traces these to the ganglion and main trunk.

Dissection. The left half of the head is to be used for the display of the ganglion and its branches; but the student may previously acquire some skill by attempting the dissection on the remains of the right side.

To lay bare the branches of the palate, detach the soft parts in the roof of the mouth from the bone, until the nerves and vessels escaping from the posterior palatine foramina are arrived at. Cut off, with a bone forceps, the posterior part of the hard palate to a level with the vessels and nerves; and cleaning these, trace offsets behind into the soft palate, and follow the main pieces forwards to the front of the mouth.

Take away without injury to the naso-palatine nerve and vessels (already 
found), the hinder part of the loose piece of mucous membrane before detached from the septum nasi, and separate the mucous membrane from the outer wall of the nasal fossa, behind the spongy bones, as high as the spheno-palatine foramen. In reflecting forwards the membrane many branches of vessels and nerves will be seen entering it through the foramen; but these may be left for the present, as directions for their dissection will be subsequently given. When the lining membrane of the nose has been removed behind the spongy bones, palatine nerves and ressels will appear through the thin translucent palate bone, and will be readily reached by breaking carefully through it with a chisel. Afterwards the tube of membrane containing the palatine vessels and nerves being opened, these are to be followed down to the soft palate and the roof of the mouth, and upwards to the ganglion which is close to the body of the sphenoid bone.

To bring the ganglion fully into view, it will be necessary to saw through the overhanging part of the sphenoid bone, to eut away pieces of the bones surrounding the hollow in which it lies, and to remove with care the enveloping fat and the periosteum. The ganglion then appears as a flattened reddish-looking body, from which the vidian and pharyngeal nerves pass backwards. Besides the branches referred to, the student should seck two large nerves from the upper part of the ganglion to join the upper maxillary, and smaller offsets to the floor of the orbit.

To trace backwards the vidian branch to the carotid plexus and the facial nerve, the student must lay open the eanal which contains it and its artery in the root of the pterygoid process; and in doing this he must define the small pharyngeal branches of nerve and artery which are superficial to the vidian, and lie in the pterygo-palatine canal. At the back of the pterygoid canal, a small branch from the vidian to the plexus on the internal carotid artery is to be looked for. Lastly, the vidian nerve is to be followed into the skull through the cartilage in the foramen lacerum (basis cranii), after cutting away the point of the petrous portion of the temporal bone, and dividing the internal carotid artery; and it is to be pursued on the surface of the temporal bone, beneath the ganglion of the fifth nerve, to the hiatus Fallopii: its junction with the facial nerve will be seen with the dissection of that nerve.

The branches of the ganglion to the nose will be found entering the outer surface of the detached mucous membrane opposite the spheno-palatine foramen, with corresponding arteries. One of these nerves (nasopalatine), before dissected in the membrane of the septum, is to be isolated, and to be followed forwards to where it enters the floor of the nose. The branches of the internal maxillary artery with the nerves are to be cleaned at the same time.

The spheno-Palatine ganglion (fig. $34,{ }^{7}$ ) (ganglion of Meckel) occupies the spheno-maxillary fossa, close to the spheno-palatine foramen, and is connected with the branches of the superior maxillary nerve to the palate. The ganglionic mass is somewhat triangular in form, and of a reddish-gray color. It is situate for the most part, behind the branches (spheno-palatine) of the superior maxillary nerve to the palate, so as to surround only part of their fibres; and it is prolonged posteriorly into the vidian nerve. Meckel's ganglion resembles the other ganglia in connection with the fifth nerve in having sensory, motor, and sympathetic offiets or roots connected with it.

The Branches of the ganglion are distributed for the most part to the 
nose and palate, but small offsets are given to the pharynx and the orbit. Other offsets connect it with surrounding nerves.

Branches of the nose. The nasal branches, from three to five in number, are for the most part very small and soft, and pass inwards through the spheno-palatine foramen : their distribution is given below :-

The superior nasal branches $(\dagger+)$ are distributed in the mucous membrane on the two upper spongy bones, and a few filaments reach the back part of the septum nasi.

The naso-palatine nerve (nerve of Cotunnius) (fig. $33,{ }^{3}$ ) crosses the roof of the nasal fossa to reach the septum nasi, and descends on that partition to near the front. In the floor of the nose it enters a special canal by the side of the septum, the left being anterior to the other, and is conveyed to the roof of the mouth, where it lies in the centre of the anterior palatine fossa. Finally, the nerves of opposite sides are united in the mouth, and are distributed in the mucous membrane behind the incisor teeth. On the septum nasi filaments are supplied by the naso-palatine nerve to the mucous membrane. To follow the nerve to its termination, the canal in the roof of the mouth must be opened.

Fig. 34 .

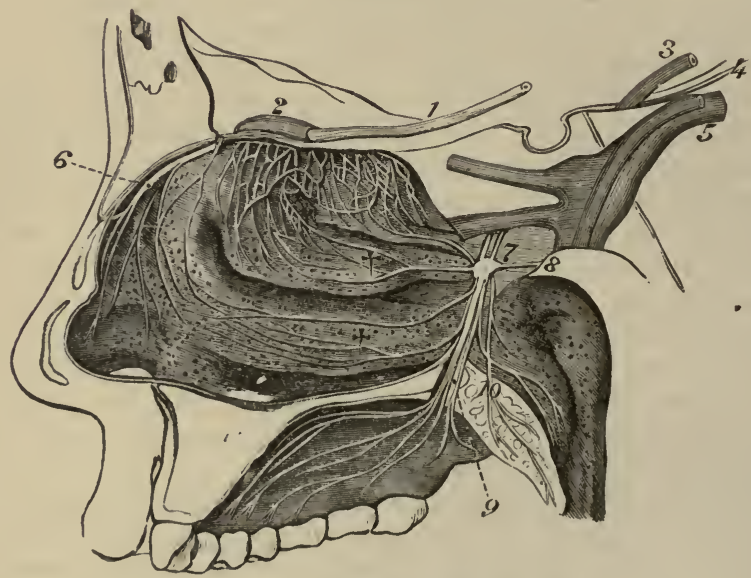

Nerves of the Nose axd Palate.

1. Olfactory nerve.

2. Olfactory bulb giving branches to the nose.

3. Third nerve.

4 Fourth nerve.

5. Fifth nerve.

6. Nasal nerve of the ophthalmic trunk.
7. Meckel's ganglion.

8. Vidian nerve.

9. Larger palatine nerve.

10. Smaller palatine nerve. †† Nasal nerve.

Branches of the palate. The nerves of the palate, though connected in part with the ganglionic mass, are the continuation of the spheno-palatine branches of the superior maxillary nerve (p. 104). Below the ganglion they are divided into three-large, small, and external.

The large palatine nerve (anterior) (fig. $34,{ }^{9}$ ) reaches the roof of the mouth through the largest palatine eanal, and courses forwards nearly to the incisor teeth, where it joins the naso-palatine nerve. Whilst in the eanal, the nerve furmishes two or more filaments (inferior nasal $\dagger$ ) to the 
membrane on the middle and lower spongy bones; in the roof of the mouth it supplies the mucous membrane and glands, and gives an offiset to the soft palate.

The small palatine nerre, 10 (posterior), lies in the smaller canal, and ends inferiorly in the soft palate, and the levator jalati and azygos uvulx muscles; it supplies the uvula and tonsil.

The external palutine nerve is smaller than the other two, and descends in the canal of the same name. Leaving the canal, the nerve is distributed to the velum palati and the tonsil.

The pharyngeal branch is very small, and is directed through the pterygo-palatine canal to the mucous membrane of the pharynx near the Eustachian tube, in which it ends.

Branches to the orbit. Two or three in number, these ascend through the spheno-maxillary fissure, and end in the fleshy layer of the muscuius orbitalis (p. 60). It will be necessary to cut throngh the sphenoid bone to follow these nerves to their termination.

Connerting branches. 'The ganglion is united, as before said, with the spheno-palatine branches of the fifth nerve (fig. $24,{ }^{3}$ ), receiving sensory nerve fibres through them; and through the medium of the vidian, which is described below, it communicates with a motor nerve (facial) and with the sympathetic nerve.

The vidian nerve $\left(^{8}\right)$ passes backwards through the vidian eanal, and sends some small filaments, through the bone, to the membrane of the back of the roof of the nose (upper posterior nasal branches). At its exit from the canal, the nerve receives a soft reddish offset (carotid branch) from the sympathetic on the outer side of the carotid artery. The continuation of the nerve enters the cranium through the cartilaginous substance in the foramen lacerum (basis cranii), and is directed backwards in a groove on the surface of the petrous part of the temporal bone, where it takes the name of large superficial petrosal nerve (fig. $35,{ }^{2}$ ). Lastly it is continued through the hiatus Fallopii, to join the gangliform enlargement on the facial nerve. Whilst in the temporal bone, the vidian receives a twig from the tympanic nerve.

The vidian nerve is supposed to consist of motor and sympathetic fibres in the same sheath, as in the connecting branches between the sympathetic and spinal nerve.

Directions. The student may now give his attention to the remaining nerves in the nasal cavity.

Dissection. The nasal nerve is to be sought in the nose behind the nasal bone (fig. 34), by gently detaching the lining membrane, after having cut off the projecting bone. A branch is given from the nerve to the septum nasi, but probably this, and the trunk of the nerre, will be seen but imperfectly in the present condition of the part.

The terminal branches of the internal maxillary artery in the sphenomaxillary fossa have been laid bare in the dissection of Meckel's ganglion, but they may be now completely traced out.

The nasal nerve (of the ophthalmic) (fig. $34,{ }^{6}$ ) has been already seen in the skull and orbit. Entering the nasal fossa by an aperture at the front of the ethmoid bone, the nerve gives a branch to the membrane of the septum, and is continued in a groove behind the os nasi to the lower margin of this bone where it escapes to the surface of the nose in the face (fig. $9,{ }^{7}$ ). 
Branches. The branch to the septum (fig. 33) divides into filaments that ramify on the anterior part of that partition, and reach nearly to the lower border.

One or two filaments are likewise furnished by the nerve to the mucous membrane on the outer wall of the nasal fossa; these extend as low as the inferior spongy bone.

Terminal braxches of the internal maxillary artery. The branches of the artery in the spheno-maxillary fossa, which have not been examined, are the superior palatine, naso-palatine, pterygo-palatine, and vidian.

The superior or descending palatine is the largest branch, and accompanies the large palatine nerve through the canal, and along the roof of the mouth; it anastomoses behind the incisor teeth with its fellow, and with a branch through the incisor foramen. This artery supplies offsets to the soft palate and tonsil through the other palatine canals, and some twigs are furnished to the lining membrane of the nose. In the roof of the mouth the mucous membrane, glands, and gums, receive their vessels from it.

The nasal or spheno-palatine artery enters the nose through the sphenopalatine foramen, and divides into branches: Some of these are distributed on the spongy bones, and the outer wall of the nasal fossa, and supply offsets to the posterior ethmoidal cells. One long branch, artery of the septum (art. nuso. palatina) runs on the partition between the nasal fossa to the incisor foramen, through which it anastomoses with the superior palatine in the roof of the mouth; this branch accompanies the naso-palatine nerve, and covers the septum with numerous ramifications.

The pterygo-palatine is a very small branch which, passing backwards through the canal of the same name, is distributed to the lining membrane of the pharynx.

The vidian or pterygoid branch is contained in the vidian canal with the nerve of the same name, and ends on the mucous membrane of the Eustachian tube and the upper part of the pharynx.

Some small nasal arteries are furnished to the roof of the nasal fossa by the posterior ethmoidal branch of the ophthalmic (p.56). Also the anterior ethmoidal (internal nasal, p. 57), enters the eavity with the nasal nerve, and ramifies in the lining membrane of the fore part of the nasal chamber as low as the vestibule; a branch passes to the fice between the os nasi and the cartilage, with its nerve. Other offsets from the facial artery supply the part near the nostril.

Veins. The veins accompanying the terminal branches of the internal maxillary artery unite in the spheno-maxillary tossa in the alveolar plexus. Into this plexus off'sets are reeeived from the pterygoid plexus and the infraorbital vein; and from the plexus a large trunk (anterior internal maxillary) is directed forwards below the malar bone to join the facial vein (p. 40). Beneath the mucous membrane of the nose the veins have a plexiform arrangement, as before said.

Facial verve in the temporal bone (fig. 35). This nerve winds through the petrous part of the temporal bone; and it is followed with difficulty in consequence of the extreme density of the bone, and the absenee of marks on the surface to indicate its position. 'To render this dissection easier, the student should be provided with a temporal bone, in which the course of the facial nerve and the eavity of the tympanum are displayed. 
Dissection. The examination of the nerve is to be begun at the stylomastoid foramen, and to be carried forwards from that point. With this view, the side of the skull should be sawn through vertically between the meatus externus and the anterior border of the mastoid process, so as to open the posterior part of the aqueduct of Fallopius. The nerve will be then seen entering deeply into the substance of the temporal bone; and it can be followed by cutting away with the bone forceps all the bone projecting above it. In this last step the eavity of the tympanum will be more or less opened, and the chain of bones in it laid bare.

The nerve is to be traced onwards along the inner side of the tympanum, till it becomes enlarged, and bends suddenly inwards to the meatus auditorious internus. The surrounding bone is to be removed from that enlargement so as to allow of the petrosal nerves being traced to it; and the meatus auditorious is to be laid open, to see the facial and auditory nerves in that hollow.

The course of the chorda tympani nerve (branch of the facial) across the tympanum will be brought into sight by the removal of the central ear bone, the incus. This nerve may be also followed to the facial through the wall of the cavity behind, as well as out of the cavity in front.

The remaining branches of the facial nerve in the bone are very minute, and are not to be seen except on a fresh piece of the skull which has been softened in acid. The student nay therefore omit the paragraphs marked with an asterisk, till he is able to obtain a part on which a careful examination can be made.

The facial nerve (fig. $35,{ }^{1}$ ) is receired into the internal auditory meatus, and entering the aqueduct of Fallopius at the bottom of that hollow, is eonducted through the temporal bone to the stylo-mastoid foramen and the face (p. 48). In its serpentine course through the bone, the nerve is first directed outwards to the inner wall of the tympanum : at that spot it bends backwards, and is marked by a gangliform swelling (intumuscentia gangliformis), to which several small nerves are united. From this swelling the nerve is con-

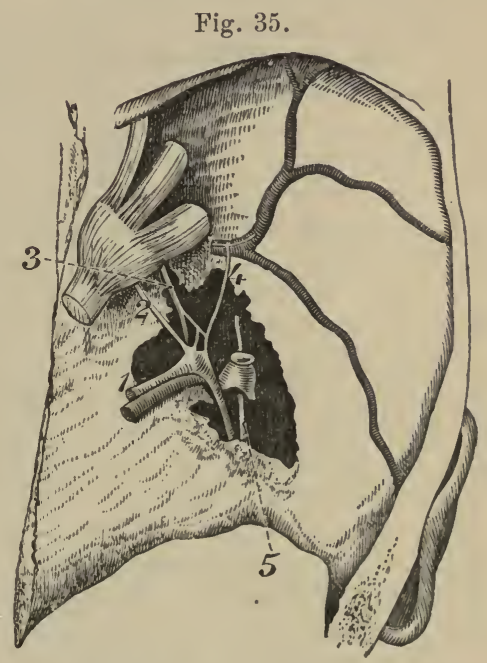

NERTES JOININA THE ENLARGLMENT OF THB FACIAL NERVE.

1. Facial nerve.

2. Large superficial petrosal.

3. Small superflial petrosal from Jacobson's nerve.

4. External superficial petrosal.

5. Chorda tympani of the facial.

tinued through the arched aqueduct, to the aperture of exit from the bone.

The branches of the nerve in the bone serve for the most part to connect it with other nerves; but one supplies the tongue, and another the stapedius muscle.

* Connecting branches communicate with the auditury and glossopharyngeal nerves; and with two trunks (superior and inferior maxillary) of the fifth nerve. 
* Union with the auditory nerve. In the bottom of the meatus the facial and auditory nerves are connected by one or two minute filaments.

* Connecting branches of the gangliform enlargement. The swelling of the facial nerve receives three small twigs. One in front is the large superficial petrosal nerve ${ }^{2}$ (vidian); another is the small superficial petrosal $^{3}$ of the tympanic nerve; and the third is the external superficial petrosal, ${ }^{4}$, which is derived from the sympathetic on the middle meningeal artery.

* The branch of the stapedius muscle arises at the back of the tympanum, and reaches its muscle by a special canal.

Chorda tympani. This long but slender branch of the facial nerve crosses the tympanum, and ends in the tongue. Arising about a quarter of an inch from the stylo-mastoid foramen (fig. $35,{ }^{5}$ ), it enters the tympanum below the pyramid. In the cavity the nerve is directed forwards across the handle of the malleus and the membrana tympani to the Glaserian fissure, or to an aperture on the inner side, through which it leaves the tympanum. As it issues from the cavity it emits a small branch to the laxator tympani muscle (?).

Outside the skull the chorda tympani joins the gustatory nerve, and continues along it to the submaxillary ganglion and the tongue (p. 102).

The AUditory Nenve will be learnt with the ear. Entering the auditory meatus with the facial it divides into two parts, of which one belongs to the cochlea, and the other to the vestibule.

Otic Ganglion (fig. 36). At this stage of the dissection there is little to be seen of the ganglion, but the sturlent should remember that it is one of the things to be examined in a fresh part. Its situation is on the inner aspect of the inferior maxillary nerve, close to the base of the skull, and it must therefore be arrived at from the inner side.

Dissection. Putting the part in the same position as for the examination of Meckel's ganglion, the dissector should define the Eustachian tube and the muscles of the palate, and then take away the levator palati and that tube, using much care in removing the last. When some loose areolar tissue lias been cleared away the internal pterygoid muscle $(b)$ comes into view, with the trunk of the inferior maxillary nerve above it; and a branch (internal pterygoid, ${ }^{5}$ ) descending from that nerve to the muscle. If the nerve to the pterygoid be taken as a guide, it will lead to the ganglion.

To complete the dissection, saw vertically through the petrous part of the temporal bone, near the inner wall of the tympanum, the bone being supported whilst it is divided. 'Taking off some membrane which covers the ganglion, the student may follow backwards a small branch to the tensor tympani muscle; but he must open the small tube that contains the muscle, by entering it below through the carotid canal. Above this small branch there is said to be another minute nerve (small superficial petrosal), which issues from the skull, and joins the back of the ganglion. A small twig is to be sought from the front of the ganglion to the tensor palati muscle; and one, near the same spot, to join the sympathetic nerve on the middle meningeal artery.

The otic Gaxglion (gang. auriculare, Arnold) (fig. 36) is a small reddish body, which is situate on the inner surface of the inferior maxillary nerve close to the skull, and surrounds the origin of the nerve to the internal pterygoid muscle. $13 y$ its inner surface the ganglion is in contact with the Eustachian tube, and at a little distance, behind, lies the midule 
meningeal artery. In this ganglion, as in the others connected with the fifth nerve, filaments from motor, sensory, and sympathetic nerves are blended. Some twigs are furnished by it to muscles.

Connecting branches-roots. The ganglion is joined by a fasciculus from the motor part of the inferior maxillary nerve, and is closely united with

Fig. 36 .

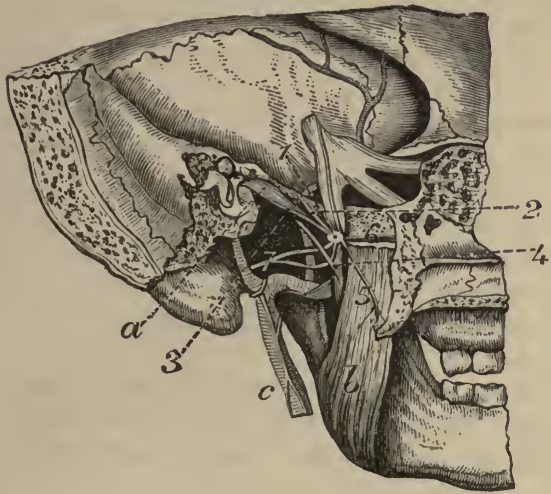

a. Tensor tympani muscle.

b. Internal pterygoid muscle with its nerve entering it.

c. External carotid artery with the sympathetic on it.

1. Otic ganglion.

2. Branch of Jacobson's nerve.

3. Nerve to tensor tympani.

4. Chorda tympani joining gustatury.

5. Nerve to pterygoideus internus.

6. Nerve of tensor palati.

7. Auriculo-temporal nerve.

INNER VIEW OF THE OTIC GANGIION.

the branch of that nerve to the internal pterygoid muscle, thus receiving two of its roots, motor and sensory, from the tifth nerve. Its connection with the sympathetic is established by a twig from the plexus on the middle meningeal artery. ${ }^{1}$

Branches to muscles. 'Two muscles receive their nerves from the otic ganglion, viz., tensor tympani and circumflexus palati. 'The nerve to the tensor tympani, ${ }^{3}$, is directed backwards, and enters the bony canal containing that muscle. The branch for the circumflexus, ${ }^{6}$, arising from the front of the ganglion, may be supposed to be derived from the internal pterygoid nerve.

The nerve of the internal pterygoid muscle, ${ }^{5}$, arises from the inner side of the inferior maxillary nerve near the skull and penetrates the deep surface of the muscle. 'This branch is joined by a fasciculus from the motor root of the fifth nerve.

Directions. The remainder of the pterygo-maxillary region of the left side may be now examined.

1 Further, the ganglion is said to be connected with the tympanic nerre (of the glosso-pharyngeal) by means of the small superficial petrosal nerve, 2 , joining the posterior part. 


\section{Section XV.}

\section{DISSECTION OF THE TONGUE.}

Directions. The tongue and larynx are to remain conneeted with each other whilst the student learns the general form and structure of the tongue.

Dissection. The ends of the extrinsic lingual muscles that have been detached on the right side may be shortened, but enough of each should be left to trace it afterwards into the substance of the tongue.

The TONGUE occupies the floor of the mouth, and is rather flattened, with the larger end turned backwards. It is firee over the greater part of the surface; but at the hinder part, and at the posterior two-thirds of the under surface, it gives attachment to the muscles and the mucous membrane which fix it to the parts around.

The tip of the tongue (apex) touches the incisor teeth; and the base, which looks towards the pharynx, is attacled to the hyoid bone, and is connected likewise with the epiglottis by three folds of nucous membrane -a central and two lateral.

The upper surface of dorsum is somewhat convex, and is received into the hollow of the roof of the mouth; along the anterior two-thirds it is divided into two equal parts by a median groove, which ends belsind in a hollow named foramen cxecum. This surface is covered with papillae over the anterior two-thirds; but is smoother at the posterior third, though even here the surface is irregular in consequence of projecting mucous glands and follieles. The under surface, free only in part, gives attachment to the mucous membrane, and to the different lingual muscles connected with the hyoid bone and the jaw; and in front of those muscles is a fold of the mucous membrane named franum lingux.

The borders of the tongue are thick and round at the base of the organ, where they are marked by vertical ridges and furrows; but gradually become thinner near the tip.

Papilla. On the dorsum of the tongue are the following kinds of papillæ; the conical and filiform, the fungiform, and the circumvallate.

The conical and filiform papillare are the numerous small projections, like the villi on the mucous membrane of the small intestine, which cover the anterior two-thirds of the dorsum of the tongue. Some of the papilla (eonical) are wider at their attached than at their free ends, and these are most developed over the central part of the tongue. Others become longer (filiform), especially towards the sides of the tongue. These papilla are furnished with minuter papilla, and are provided at the tip with hair-like processes of the epithelium. Towards their limit behind, as well as on the side of the tongue, they have a linear arrangement.

The fungiform papilla are less numerous but larger than the precerling set, amongst which they are scattered. They are wider at the free end than at the part fixed to the tongue, and project beyond the other set; they are situate mostly at the tip and sides of the tongue. They are covered witl: small simple papillae.

The circumvallate or caliciform are fewer in number and larger than the others, and are placed at the junction of the two anterior with the 
posterior third of the tongue: their number varies from eight to ten 'These papillæ extend across the tongue in a line resembling the letter $\mathrm{V}$ with the point turned backwards. Each papilla consists of a central truncated part of a conical form, which is surrounded by a fold of the mucous membrane; its wider part or base projects above the surface, whilst the apex is attached to the tongue. Both the papilla and the surrounding fold are furnished with smaller secondary papillæ.

Minute simple papilla exist behind the calciform kind, and on the under surface of the free portion of the tongue; but they are not observed till the epithelium is removed.

Taste buds. Around the circumvallate papilla is a circle of small peculiar bodies, which are covered by the epithelium: they are like a small carafe in shape, the base resting on the corium. They are formed of elongated epithelium-like cells, of which the central, resembling olfactorial cells, are supposed to be connected with the nerve of taste.

A small collection of similar bodies occupies the back of the tongue, on each side, just in fiont of the anterior pillar of the fauces.

Structure of the Papilla. The simple papilla are constructed like those of the skin, viz. of a projecting cone of membrane, which is covered by epithelium, and filled with a loop of capillaries, and a nerve.

The other compound forms of the papillæ may be said to be produced by outgrowths from the simple kind. Thus smaller papillary eminences spring from the common cone of limiting membrane; and each has its separate investment of epithelium, by which the brush-like appearance on the surface is produced. From the plexus of capillary vessels in the interior of the papilla a looped offset is furnished to each smaller papillary projection. The entering nerve sends offsets to the different subdivisions of the papilla, on some of which end-bulbs may be recognized.

Structure. The tongue consists of two symmetrical halves separated by a fibrous layer in the middle line. Each half is made up of muscular fibres with interspersed fat; and entering it are the lingual vessels and nerves. The whole tongue is enveloped by the mucous membrane; and a special fibrous membrane attaches it to the hyoid bone.

Dissection. To define the septum, and the membrane attaching the tongue to the hyoid bone, the tongue is to be placed on its dorsum; and, the remains of the right mylo- and genio-hyoideus having been removed, the genio-hyo-glossi muscles are to be cleaned, and drawn from one another along the middle line. After separating those muscles, except for an inch in front, and cutting across their intercommunicating fibres, the edge of the septum will appear. By tracing the hinder fibres of the genio-hyoglossus muscle towards the os hyoides, the hyo-glossal membrane will be arrived at.

Outside this triangular muscle in the middle line, is the longitudinal bundle of the inferior lingualis, which will be better seen subsequently.

Fibrous tissue. Along the middle line of the tongue is placed a thin lamina of this tissue, forming a septum: its root is attached by another fibrous structure, the hyo-glossal membrane; and covering the greater part of the organ is a submucous layer of the same tissue.

Septum. This structure forms a vertical partition between the two halves of the tongue (fig. $37,{ }^{H}$ ), and extends from the base to the apex. It is thicker posteriorly than anteriorly, and is connected behind with the hyo-glossal membrane. To each side the transverse muscle is connected. Its disposition may be better seen subsequently on a vertical section. In 
some instances a small fibro-cartilage, about a quarter of an inch deep and long, exists in the septum.

The hyo.glossal membrane is a thin but strong fibrous lamina, which attaches the root of the tongue to the upper border of the body of the hyoid hone. On its under or anterior surfice some of the hinder fibres of the genio-hyo-glossi are inserted, as if this was their aponeuroses to attach then to the os hyoides.

The submucous fibrous or aponeurotic stratum of the tongue invests the organ, and is continued into the sheaths of the muscles. Over the posterior third of the dorsum its strength is greater than elsewhere; and in front of the epiglottis it forms bands in the folds of the mucous membrane in that situation. Into it are inserted the muscular fibres which end on the surface of the tongue.

Muscles. Fach half of the tongue is made up of extrinsic and intrinsic muscles. The former or external are distinguished by having only their termination in the tongue; and the latter or internal, by having both origin and insertion within the origin-that is to say, springing from one part and ending in another.

The extrinsic muscles (fig. 37) are the following: palato and styloglossus, hyo and genio-hyo-glossus, and pharyngeo-glossus. Only the lingual endings of these are now to be looked to.

Dissection. After the tongue has been firmly fastened on its left side, the extrinsic muscles may be dissected on the right half. Three of these muscles, viz., palato-, D, stylo-, 13, and hyo-glossus, c, come together to the side of the tongue, at the junction of the middle and posterior third; and, to follow their radiating fibres forwards, it will be necessary to remove from the dorsum, between them and the tip, a thin layer consisting of the mucous membrane and fleshy fibres of the upper lingualis. Beneath the tip a junction between the stylo-glossus muscles of opposite sides is to be traced.

The part of the constrictor muscle, $G$, which is attached to the tongue, and the ending of the genio-hyo-glossus, will come into view on the division of the hyo-glossus.

Only the two parts of the hyo-glossus (basio- and cerato-glossus, p. 99), which arise from the body and great wing of the hyoid bone, are referred to above. To lay bare the third part, or the chondro-glossus, F, which is a small muscular slip attached to the small cornu of the os hyoides, turn upwards the dorsum of the tongue, and feel for the small cornu of the hyoid bone through the mucous membrane. Then remove the mucous membrane in front of the cornu, and the fibres of the muscle radiating forwards will be exposed.

'The palato and stylo-glossus muscles, $\mathrm{D}$ and $\mathrm{B}$, are partly combined at their attachment to the lateral part of the tongue, and form, together with the following muscle, an expansion over the anterior two-thirds of the dorsum beneath the superficial lingualis. In this stratum the fibres radiate from the point of eontact of the muscles with the tongue-some passing almost horizontally inwards to the middle, and others obliquely forwards to the tip of the organ.

$\mathrm{A}^{*}$ great portion of the stylo-glossus is directed along the side of the tongue; and some fibres are inclined to the under surface in front of the hyo-glossus, to join those of the opposite muscle beneath the tip.

Hyo-glossus. The two superficial parts of the muscle (basio and cerato- 
glossus, c, p. 99) enter the under surface of the tongue, between the stylo-glossus and the lingualis. After entering that surface by separate bundles, they are bent round the margin, and form, with the two preceding muscles, a stratum on the dorsum of the tongue.

The third part of the muscle, or the chondroglossus, F, is distinct from the rest. About two or three lines wide at its origin from the root of the small cornu, and from part of the body of the os hyoides, the muscle entering beneath the upper lingualis, passes obliquely inwards over the posterior third of the dorsum, to blend with the hyo glossus.

Cortex of the tongue. 'The muscles above described, together with the superficial lingualis, constitute a cortical layer of oblique and longitudinal fibres, which covers the tongue, except below where some muscles are placed, and resembles "a slipper turned upside down." This stratum is pierced by deeper fibres.

The genio-hyo-glossus (fig. $38,{ }^{\wedge}$ ) enters the tongue vertically on the side of the septum, and perforates the cortical covering to end in the submucous tissue. In the tongue the fibres spread like the rays of a fan from apex to base, and are collected into bundles as they pass through the transversalis. The most posterior fibres end on the hyo-glossal membrane and the hyoid bone; and a slip is prolonged from them, beneath the hyo-glossus, to the up-

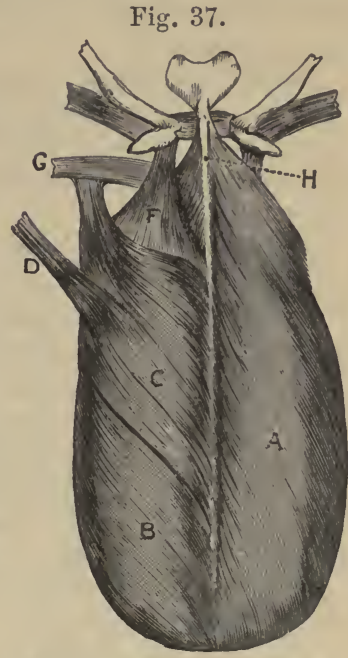

MUSCLES ON THE SURFACB OF THE TONMUE.

A. Superficial lingualis.

B. Stylo-glossus.

C Hyo-glo $\$$ sus.

D. Palato-glossus.

F. Chondro-glossus.

f. Pharyngeo-glossus.

H. Septum lingure (Zaglas).

per constrictor of the pharynx. A vertical section at a future stage will show the radiation of its fibres.

'The pharyngeo-glossus (glosso-pharyngeus), or the part of the upper constrictor attached to the side of the tongue, passes amongst fibres of the hyogrossus, and is continued with the transverse muscle to the septum.

The intrinsic muscles (fig. 38) are three in number in each half of the tongue, viz., transversalis, with a superior and an inferior lingualis.

Dissection. To complete the preparation of the inferior lingualis on the right side, the fibres of the stylo-glossus covering it in fiont, and those of the genio-liyoylossus over it behind, are to be cut through.

The superior lingualis (fig. $37, \stackrel{\wedge}{ }$ ) may be shown, on the left side, by taking the

Fig. 38.

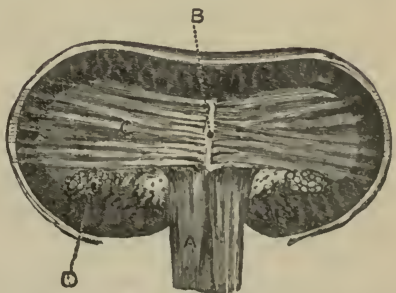

Intrinsic MUacles of the Toxgue.

A. Genio-hyo-glossus.

B. Septum linguæ.

C. Transversalis.

D. Inforior lingualis (Zaglas). thin mucous membrane from the upper surface from tip to base.

The transversalis (fig. $38,{ }^{\mathrm{C}}$ ) may be laid bare on the right side, by cutting away on the upper surface the stratum of the extrinsic muscles 
already seen ; and by removing on the lower surface, the inferior lingualis and the genio-hyo-glossus.

The nerves of the tongue are to be dissected on the left half as well as the part will admit; but a recent specimen would be required to follow them satisfactorily.

The transiersalis muscle (fig. $38,{ }^{\mathrm{C}}$ ) forms a horizontal layer in the substance of the tongue from base to apex. The fibres are attached internally to the side of the septum, and are directed thence outwards, the posterior being somewhat curved, to their insertion into the side of the tongue.

Its fibres are collected into vertical plates, so as to allow the passage between them of the ascending fibres of the genio-hyo-glossus.

Action. By the contraction of the fibres of these muscles the tongue is made narrower and rounder, and is inereased in length.

The superior lingualis (fig. 37, ${ }^{\mathbf{A}}$, noto-glossus of Zaglas) is a very thin layer of oblique and longitudinal fibres close beneath the submucous tissue on the dorsum of the tongue. Its fibres arise from the frænum epiglottidis, and from the fascia along the middle line; from this attachment they are directed obliquely outwards, the anterior becoming longitudinal, to the margin of the tongue, at which they end in the fascia.

Action. Both muscles tend to shorten the tongue; and they will bend the point back and up.

The inferior lingualis (fig. $38,{ }^{D}$ ) is much stronger than the preceding, and is placed under the tongue, between the hyo and genio-hyo-glossus. The muscle arises posteriorly from the fascia at the root of the tongue; and the fibres are collected into a roundish bundle: from its attacherl surface fasciculi are continued vertically through the transverse fibres upwards to the dorsum; and at the anterior third of the tongue, where the muscle is overlaid by the stylo-glossus, some of the fibres are applied to that muscle and distributed with it.

Action. Like the upper lingualis this muscle shortens the tongue, and bends the point down and back.

The mucous membrane is a continuation of that lining the mouth, and is provided with a laminar epithelium. It partly invests the tongue, and is reflected off at different points in the form of folds (p. 1.46). At the epiglottis are three small glosso-epiglottid folds, connecting this body to the root of the tongue; the central one of these is called the fronum of the epiglottis. Like the membrane of the mouth, it is furnished with numerous glands, and some follicles.

The follicles are depressions of the mucous membrane, which are surrounded by closed capsules in the submucous tissue, like the arrangement in the tonsil: they occupy the dorsum of the tongue between the papillx circumvallaix and the epiglottis, where they form a stratum, close beneath the mucous membrane.

The glands (lingual) are racemose or compound in structure, similar to those of the lips and cheek, and are placed beneath the mucous membrane on the dorsum of the tongue behind the papilla vallate. A few are found in front of the circumvallate papilla, where they project into the muscular substance. Some of their ducts open on the surface; others into the hollows around the large papilla, or into the foramen cxcum and the depressions of the follicles.

Opposite the papillae vallatx, at the margin of the tongue, is a small cluster of submucous glands. Under the tip of the tongue, on each side 
of the frenum, is another elongated collection of the same kind of glands imbedded in the muscular fibres, from which several ducts issue.

Nerves. There are three nerves on the under part of each half of the tongue, viz., the gustatory, the hypoglossal, and the glosso-pharyngeal (fig. 23).

The gustatory nerve gives upwards filaments to the muscular substance, and to the two smallest sets of papillx, conical and fungiform; it joins also the hypoglossal nerve.

The hypoglossal nerve is spent in long slender offsets to the muscular substance of the tongue.

The glosso-pharyngeal nerve divides under the hyo-glossus into two branches:-One turns to the dorsum, and ramifies in the mucous membrane behind the foramen cæcum. The other passes beneath the side of the tongue, and ends in branches for the muscular substance; it supplies the papilla circumvallatæ, as well as the mucous membrane covering the lateral part of the tongue.

Vessels. The arteries are derived chiefly from the lingual of each side; these, together with the veins, have been examined (p. 101.)

\section{Section XVI.}

\section{DISSECTION OF THE LARYNX.}

THE LARYNX is the upper dilated part of the air tube, in which the voice is produced. It is constructed of several cartilages united together by ligamentous bands; of muscles for the movement of the cartilages; and of vessels and nerves. The whole is lined by mucous membrane.

Dissection. The tongue may be removed from the larynx by cutting through its root, but this is to be done without injuring the epiglottis.

If the student learns the laryngeal cartilages before he begins the dissection of the larynx, he will obtain more knowledge from the study of this Section.

Occupying the middle line of the neck, the larynx is placed in front of the pharynx, and between the carotid vessels. It is pyramidal in form. The base is turned upwards, and is attached to the hyoid bone; and the apex is continuous with the trachea.

In length it measures abont one inch and a half; in width at the top one inch and a quarter, and at the lower end one inch.

The front is prominent along the middle line of the neck; and the posterior surface is covered by the mucous membrane of the pharynx. The larynx is very movable, and during deglutition is elevated and depressed by the different extrinsic muscles.

Muscles. Commonly five pair and one single muscle are described in the larynx. Three are outside the cartilages, and three are more or less concealed by the thyroid cartilage.

Directions. On one side of the larynx, say the right, the museles may be dissected, and on the opposite side the nerves and vessels; and those superficial muscles are to be first learnt, which do not require the cartilages to be cut.

Dissection. The larynx being extended and fastened with pins, the dissector may clear away from the os hyoides and the thyroid cartilage the 
following extrinsic muscles, viz., constrictor, sterno-hyoid, sterno-thyroid, and thyro-hyoid.

In front, between the thyroid and cricoid cartilages, one of the three small external muscles-crico-thyroid (fig. 39), will be recognized.

The other two external muscles (fig. 40) are situate at the posterior aspect of the larynx: to denude them it will be necessary to turn over the larynx, and to remove the mucous membrane covering it. On the back of the circoid cartilage the dissector will find the crico-arytanoideus posticus muscle; and above it, on the posterior part of the arytænoid cartilages, the arytanoid muscle will appear.

The CRICO-THYRoIdeUs MUsCLE (fig. $39,{ }^{1}$ ) is triangular in form, and is separated by an interval from the one on the opposite side. It arises from the front and the lateral part of the cricoid cartilage; and its fibres

Fig. 39.

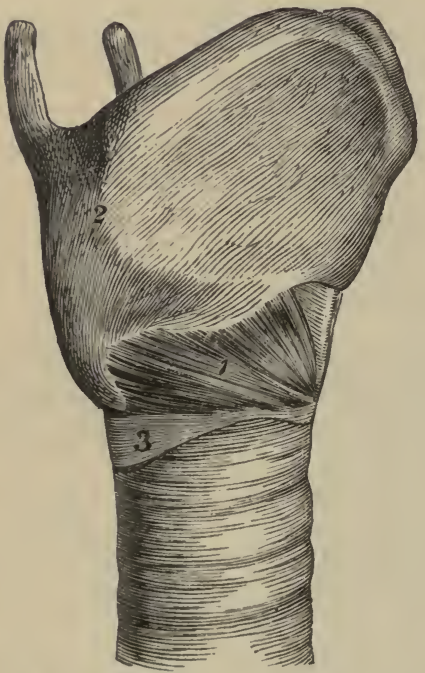

FroNT View OF THE LARYNX.

1. Crico-thyroid muscle

2. Thyroid cartilaye.

3. Cricoid cartilage.
Fig. 40.

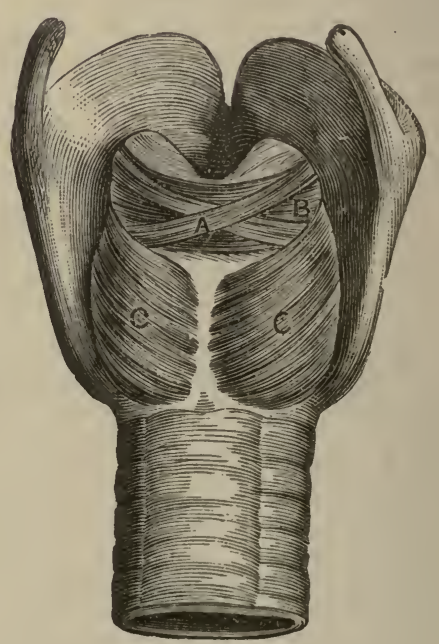

HINDER VIEW OF THE LAKYNX.

A. Superflcial part of the arytænoideus muscle. B. Deep part of the arytanoideus.

C. Crico-arytrenoideus posticus.

ascend to be inserted into the lower cornu, and the lower border of the thyroid cartilage as far forwarls as a quarter of an inch from the middle line; also, for a short distance (a line), into the inuer surface of that cartilage. The muscle rests on the crico-thyroid membrane, and is concealed by the sterno-thyroid muscle.

Action. It approaches the thyroid to the ericoid cartilage, making longer the distance between the thyroid and the arytanoid cartilages, and tightens indirectly the vocal cords.

The crico-arytanoideus posticus muscle (fig. $40,{ }^{\circ}$ ) lies on the posterior part of the cricoid cartilage. Its origin is from the depression on the side of the vertical ridge at the back of that cartilage. From this origin the fibres are directed outwards, and are inserted into a projection at the outer part of the base at the arytanoid cartilage. 
Action. It rotates the arytanoid cartilage, turning out the lateral projection at the base, and enlarges the interval between the cartilages. At the same time the upper orifice of the larynx is widened by the separation from each other of its lateral boundaries.

Musculus kerato-cricoideus (Merkel). This is a small fleshy slip which is occasionally seen below and close to the precedingmuscle; it arises from the cricoid cartilage, and is inserted into the back part of the lower cornu of the thyroid cartilage.

The ARYTanoIdeus is a single muscle in the middle line (fig. $40,{ }^{\mathrm{B}}$ ), and is placed on the posterior surface of the arytænoid cartilages: it possesses two sets of fibres, superficial and deep, with different directions. The deep fibres, $\mathbf{B}$, are transverse, and are inserted into the outer border and the posterior surface of each cartilage; they close the interval between the cartilages. The superficial fibres, $\mathrm{A}$, consist of two oblique fasciculi, which cross like the parts of the letter $\mathbf{X}$, each passing from the base of one cartilage to the apex of the other: a few of these fibres are continued beyond the cartilage to join the thyro-arytanoid muscle, and the depressor of the epiglottis.

Action. The muscle causes the arytanoid cartilages to glide towards one another, and diminishes much, or closes the rima glottidis.

Acting with the depressors of the epiglottis it will assist in closing the upper orifice of the larynx.

Dissection. The remaining muscles and the vocal apparatus would be learnt better on a fresh larynx, if this can be obtained. To bring into view the muscles, which are concealed by the thyroid cartilage (fig. 41) it will be necessary to remove the right half of the cartilage, by cutting through it a quarter of an inch from the middle line, after its lower cornu has been detached from the cricoid. By dividing next the crico-thyroid membrane attached to the lower edge, and the thyro-hyoid ligament connected with the upper margin, the loose piece will come away on separating it from the subjacent areolar tissue.

By the removal of some areolar tissue, the dissector will define inferiorly the crico-arytenoid muscle; above it, the thyro-arytanoideus muscle; and still higher, the thin muscular fibres (depressor of the epiglottis) in the fold of mucous membrane between the epiglottis and the arytrenoid eartilage. On cleaning the fibres of the thyro-arytænoideus near the front of the larynx, the top of the sacculus laryngis with its small glands will appear above the flesliy-fibres.

The CRICO ARYTenordeus lateralis (fig. $41,{ }^{3}$ ) is a small lengthened band, which arises from the upper border of the cricoid cartilage at the lateral part ; its fibres are directed backwards to be inserted into a projection on the outer side of the base of the arytrnoid cartilage, and into the contiguous part of the outer surface.

This muscle is concealed by the erico-thyroideus, and its upper border is contiguous to the succeeding muscle.

Action. Rotating the arytanoid cartilage by moving inwards the projection on the outer part of the base, it replaces the cartilage after this las been everter by the crico-arytanoideus posticus. It may also approach the one vocal cord to the other, and so narrow the glottis.

The THYRO-ARYTexoldeus wuscle (fig. 41) extends from the thyroid to the arytanoid cartilage; it is thick below, but thin and expanded above. The muscle arises from the thyroid cartilage near the middle line, for about the lower half of the depth, and from the erico-thyroid ligament. The fibres are directed backwards with different inclinations:-The external, 4 , 
Fig. 41.

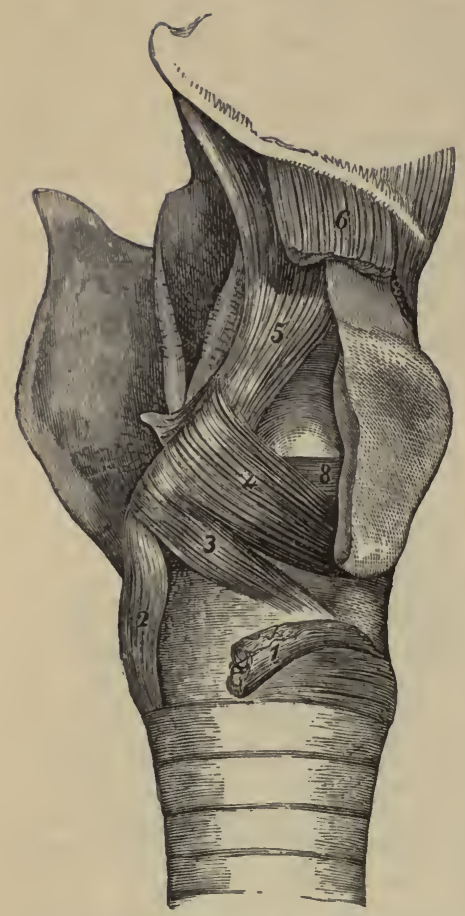

View of The INTERNal MUSCl.eF of THE LARYNX.

1. Crico-thyroidens detached.

2. Crico-arytanoideus p sticus.

3. Crico arytænoideus lateralis.

4. Thyru-arytænoideus, superficial part.

5. Depressor of the epiglottis.

6. Thyro-hyoideus, cut.

8. Deep or transverse part of thyro-arytænoideus.

larynceal pouch, have been described by Mr. Hilton as a separate muscle with the name arytano-epiglottideus inferior.

Action. By the contraction of the fibres, the tip of the arytanoid cartilage will be moved forwards and inwards, and the eppiglottis will be lowered over the orifice of the larynx.

The fibres of the muscle which are spread over the sacculus will compress it, and assist in the expulsion of the contents.

Parts inside the carynx. The parts more immediately concerned in the production of the voice are, the vocal corrls, the glottis, and the ventricle of the larynx and its pouch : these are placed within, and are protected by the laryngeal cartilages.

Dissection. For the purpose of displaying the vocal apparatus, let the tube of the larynx be divided along the posterior part, as in fig. 42 ; and in cutting through the arytenoid muscle, let the incision be rather to the right of the middle line, so as to avoid the nerves entering it.

On looking into the larynx a hollow (ventricle) will appear on each 
side; and bounding the rentricle above and below are the whitish bands of the vocal cords.

If a probe be passed into that hollow, it will enter a small pouch (sacculus laryngis) by an aperture in the anterior and upper part. The dissector should fill the sacculus on the left side by introducing a small piece of cotton wool into it.

The laryngeal space reaches from the epiglottis to the lower border of the cricoid cartilage. It opens above into the pharynx, and below into the trachea; and in the intermediate portion are lodged the parts producing roice.

The upper orifice of the larynx (fig. $29,{ }^{N}$ ) will be evident on placing in contact the cut surfaces. It is triangular in shape, with the base in front and the apex behind, and its sides are sloped obliquely downwards in the antero-posterior direction. Its boundaries are,- the epiglottis in front, the arytanoid muscle and cartilages behind, and the arytieno-epiglottidean fold of mucous membrane on each side. This aperture is closed by the epiglot tis during deglutition.

The lower opening, limited by the inferior edge of the cricoid cartilage, is circular in form, and is of the same size as that cartilage.

The laryngeal cavity is much reduced in size within the thyroid cartilage by the vocal cords, and is dilated above and below them for the purpose of allowing their free vibration. The lower dilatation may be seen to be as large as the ring of the cricoid ; and the upper, much smaller, corresponds with the ventricle of the larynx. Above the upper bulge the wall of the larynx slants up to the epiglottis.

The glottis or rima glottidis, is the interval between the lower vocal cords (fig. 44); it is placed on a level with the base of the arytænoid cartilages, and is the narrowest part of the laryngeal carity.

Its sides are constructed partly of ligament and partly of cartilage :thus, for about the two anterior thirds is the elastic vocal cord (fig. $42,{ }^{\mathrm{B}}$ ), whilst at the posterior third is the smooth inner surface of the arytænoid cartilage, E. Behind it is bounded by the arytenoid muscle; and in front by the thyroid cartilage and the attachments of the vocal cords.

The size of the interval differs in the two sexes. In the male it measures from before back nearly an inch (less a line), and across at the base, when dilated, about a third of the other measurement. In the female the dimensions will be less by two or three lines.

Alterations in the size and form affect the interval where it is bounded by the cartilages, as well as where it is limited by the ligaments. In the former part, the changes are occasioned by the movements of the arytanoid cartilages; but in the latter they are due to the lengthening and shortening of the bands.

In the state of rest it is a narrow fissure which is enlarged a little behind and rounded; but when dilated it is triangular in form, like the upper orifice, though its wider part is turned backwards to the arytænoid muscle. In the living body the fissure is larger in inspiration than in expiration. The muscles too are constantly producing alterations in the fissure, some acting more immediately on the cartilages as dilators and contractors of the base; and others altering the state of the ligaments, by elongating and shortening the sides.

The base is enlarged, and the interval rendered triangular by the posterior crico-arytænoid; and is diminished by the arytænoid, and the lateral crico-arytrenoid. And the ligamentous sides are elongated and made tense by the crico-thyroidei, but are shortened by the thyro-arytanoidei. 
The ventricle of the larynx (fig. $42,{ }^{\wedge}$ ) is best seen on the left side. It is the oval hollow between the vocal cords, whose upper margin is semilunar, and the lower straight. It is lined by the mucous membrane, and on the outer surface are the fibres of the thyro-arytenoid muscle. In the anterior part is the aperture into the laryngeal pouch.

The laryngeal pouch (sacculus laryngis) (fig. $42,{ }^{\mathrm{D}}$ ), has been laid bare in part on the right side by the removal of the half of the thyroid cartilage (p. 153), but it will be seen again in the subsequent dissection for the vocal cords.

It is a small membranous sac, half an inch deep and cylindrical in form, which projects upwards between the upper vocal cord and the thyroid

Fig. 42.

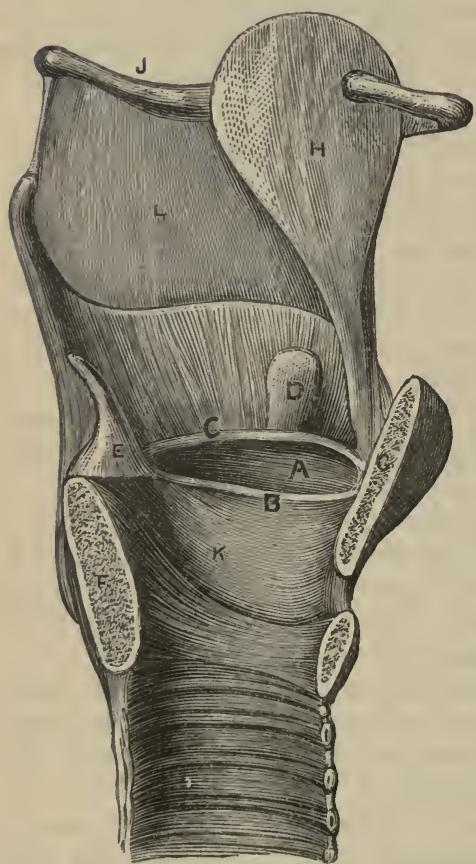

Vocal apparatug, on a Vertical Section OF THE LARYNX.
A. Ventricle of the larynx.
B. True vocal cord.
C. False vocal cord.
D. Sacculus laryngis.
E Arytænoid cartilage.
$\boldsymbol{F}$. Cricoid cartilage.
ब. Thyroid cartilage.
r. Epiglottis.
K. Crico-thyroid ligament.
L. Thyro-hyoid ligameut. slip of the depressor of the epiglottis, and reaches sometimes as high as the upper border of the thyroid cartilage. Its eavity communicates with the front of the ventricle by a somewhat narrow aperture. On the outer surface are numerous small glands, whose ducts are transmitted through the coats of the sac to the inside. Numerous nerves are distributed over the top. Its upper part is corered by the muscular slip before referred to.

Dissection. 'The general shape and position of the vocal cords are evident on the left half of the laryngeal tube, but to show more fully the nature of the lower cord, put the cut surfaces in contact, and detach on the right side the crico-arytanoideus lateralis from its cartilages. Remove in like manner the thyro-arytænoideus, raising it from before back. By the removal of the last muscle, a fibrous membrane, crico-thyroid (fig. $\left.44,{ }^{6}\right)$, comes into view, and its up)per free edge will be perceived to constitute the inferior or true vocal cord. Whilst taking away the thyroarytanoidens, the ventricle and the sacculus laryngis, which are formed chiefly by nucous membrane, will disappear.

The rocal cords or the thyro-arytanoid ligaments (fig. 42), are two bands on each side, which are extended from the angle of the thyroid to the arytanoid cartilage-one forming the upper, the other the lower margin of the ventricle.

The upper ligament (false vocal cord (fig. $42,{ }^{\mathrm{C}}$ ) is semilunar in form, and is much weaker than the other. It is fixed in front to the angle of the thyroid cartilage, near the attachment of the epiglottis; and behind to 
the outer surface of the arytrenoid eartilage. This ligament consists chiefly of white fibrous tissue, which is continuous with that in the arytano-epiglottidean fold of mucous membrane.

The inferior ligament (chorda vocalis, fig. 42, ${ }^{\text {B }}$ ) is attached in front to the angle of the thyroid cartilage, about half-way down below the notch; the ligament is directed backwards, and is inserted into the anterior prominence at the base of the arytanoid cartilage. It is about seven lines long in man, and two less in the woman. Internally this band is covered by thin mucous membrane, and projects towards its fellow into the cavity of the larynx, the interval between it and the opposite one being the glottis. Externally it is connected with the thyro-arytænoid muscle And inferiorly it is continuous with the crico-thyroid ligament, $\mathrm{k}$. 'The edge that bounds the ventricle is straight and well defined, and vibrates to produce sounds. The ligament is composed of fine elastic tissue.

The mucous membrane of the larynx is continued from that investing the pharynx, and is prolonged to the lungs through the trachea. When entering the larynx it is stretched between the epiglottis and the tip of the arytenoid cartilage, forming the arytæno-epiglottid fold on each side of the laryngeal orifice: at this spot it is rery loose, and the submucous tissue abundant. In the larynx the membrane lines closely the cavity, sinks into the ventricle, and is prolonged into the laryngeal pouch. On the thyro-arytenoid ligaments it is very thin and adherent, allowing these to be visible through it.

In the small part of the larynx above the vocal cords, the epithelium is of the laminar kind, and free from cilia. But a columnar ciliated epithelium covers the surface below the level of the superior cords, though it becomes flattened without cilia on the cords : on the epiglottis it is ciliated in the lower half.

Numerous branched glands are connected with the mucous membrane of the larynx, and the orifices will be seen on the surface, especially at the posterior aspect of the epiglottis. In the edge of the arytreno-epiglottidean fold there is a little swelling occasioned by a mass of subjacent glands (arytænoid); and along the upper vocal cord lies another set. None exist over the vocal cords, but close to those bands is the collection of the sacculus laryngis, which lubricates the ventricle and the lower vocal cord.

Dissection of nerres and vessels. The termination of the laryngeal nerves may be dissected on the untouched side of the larynx. For this purpose the other half of the thyroid is to be disarticulated from the cricoid cartilage, care being taken of the recurrent nerve, which lies near the joint between the two. The trachea and larynx should be fastened down next with pins; and after the thyroid has been drawn away from the cricoid cartilage, the inferior laryngeal nerve can be traced over the side of the latter cartilage to the muscles of the larynx, and mucous membrane of the pharynx.

Afterwards the superior laryngeal is to be found as it pierces the thyrohyoid membrane, and branches of it are to be followed to the mucous membrane of the larynx and pharynx. Two communications are to be looked for between the laryngeal nerves; one is beneath the thyroid cartilage, the other in the mucous membrane of the pharynx.

An artery accompanies each nerve, and its offsets are to be dissected at the same time as the nerve. 
Nerves. The nerves of the larynx are the superior and inferior laryngeal branches of the pneumo-gastric (p. 113); the former is distributed to the mucous membrane, and the latter chiefly to the muscles.

The inferior laryngeal nerve (recurrent), when about to enter the larynx, furnishes backwards an offset to the mucous membrane of the pharynx; this joins filaments of the upper laryngeal. The nerve then passes beneath the ala of the thyroid cartilage, and ends in branches for all the special muscles of the larynx except the crico-thyroideus. Its small muscular branches are superficial, but that to the arytrenoid muscle passes beneath the crico-arytrnoideus posticus; Beneath the thyroid cartilage the inferior is joined by a'long offset of the upper laryngeal nerve.

The superior laryngeal nerve pierces the thyro-hyoid ligament, and gives offsets to the mucous membrane of the pharynx; it furnishes also a long branch beneath the ala of the thyroid cartilage to communicate with the recurrent nerve. The trunk then terminates in many branches for the supply of the mucous membrane:- Some of these ascend in the arytanoepiglottid fold to the epiglottis, and the root of the tongue. The others, which are the largest, descend on the inner side of the ventricular pouch, and supply the lining membrane of the larynx as low as the vocal cords. One nerve of this set pierces the arytænoid muscle, and ends in the mucous membrane.

Vessels. The arteries of the larynx are furnished from the superior and in erior thyroid branches (p. 85 and 78 ).

The laryngeal branch of the superior thyroid artery enters the larynx with the superior laryngeal nerve, and divides into ascending and descending branches; some of these enter the muscles, but the rest supply the epiglottis, and the mucous membrane from the root of the tongue to the chorda vocalis. Like the nerves, it unites with the following artery both beneath the ala of the thyroid cartilage, and in the mucous membrane of the pharynx.

The laryngeal branch of the inferior thyroid artery ascends on the back of the cricoid cartilage, and ends in the mucous membrane of the pharynx and the posterior museles of the larynx.

Some other twigs from the superior thyroid artery perforate the cricothyroid membrane, and ramify in the mucous lining of the interior of the larynx at the lower part.

Laryngeal veins. The vein accompanying the branch of the superior thyroid artery, joins the internal jugular or the superior thyroid vein; and the vein with the artery from the inferior thyroid opens into the plexus of the inferior thyroid reins (p. 85 and 78 ).

\section{Section XVII.}

IYYOID BONE, CARTILAGES AND LIGAMENTS OF THE LARYNX, AND STRUCTURE OF THE TRACHEA.

Dissection. All the muscles and the mucous membrane are to be taken away so as to denude the hyoid bone, the cartilages of the larynx, and the epiglottis; but the piece of membrane that joins the hyoid bone 
to the thyroid cartilage, and the ligaments uniting one cartilage to another on the left side, should not be destroyed.

In the arytæno-epiglottidean fold of mucous membrane, a small cartilaginous body (cuneiform) may be recognized; an oblique whitish projection indicates its position.

The hyoid bone (os hyoides) (fig. 43) is situate between the larynx and the root of the tongue. Resembling the letter $\mathrm{U}$, placed horizontally and witl the legs turned backwards, it offers for examination a central part or body, and two lateral pieces or cornua on each side.

The body, c, is thin and flattened, and measures most in the transverse direction. Convex in front, where it is marked by a tubercle, it presents an uneven surface for the attachment of muscles; whilst on the opposite aspect it is concave. To the upper border the fibrous membrane (hyoglossal) fixing the tongue is attached.

The cornua are two in number on each side (large and small). The large cornu, $\mathrm{H}$, continues the bone backwards, and is joined to the body by an intervening piece of cartilage. The surfaces of this cornu look somewhat upwards and downwards; and the size decreases from before backwards. It ends posteriorly in a tubercle. The small cornu, or appendix, $J$, is dissected upwards from the point of union of the great cornu with the body, and is joined by the stylo-hyoid ligament: it is seldom wholly ossified.

Cartilages of the Laryxx (fig. 43). There are four large cartilages in the larynx, which are concerned in the production of the voice, viz., the thyroid, the cricoid, and the two arytænoid. In addition there are some yellow fibro-cartilaginous structures, viz., the epiglottis, a capitulum to each arytænoid cartilage, and a small ovalish piece (cuneiform) in each arytano-epiglottidean fold of mucous membrane.

The thyroid cartilage, $\mathrm{B}$, is the largest of all: it forms the front of the larynx, and protects the vocal apparatus as with a shield. The upper part of the cartilage is considerably wider than the lower, and in consequence of this form the larynx is somewhat funnel-shaped. The anterior surface is prominent in the middle line, forming the subcutaneous swelling named pomum Adami; but the cartilage is concave behind at the same spot, and gives attachment to the epiglottis and the thyro-arytænoid muscles and ligaments. The upper border is notched in the centre.

The cartilage consists of two square halves, which are united in the middle line. Posteriorly each half of the cartilage has a thick border, which terminates upwards and downwards in a rounded process or cornu ( $E$ and $F)$. Both cornua are bent slightly inwards: of the two, the upper, $\mathrm{E}$, is the longest; but the lower one, $\mathrm{F}$, is thicker than the other, and articulates with the cricoid cartilage. The inner surface of each half is smooth ; but the outer is marked by an oblique line for the attachment of muscles, which extends from a tubercle near the root of the upper cornu, almost to the middle of the lower border.

The cricoid cartilage, D, is stronger though smaller than the thyroid, and encircles the cavity of the larynx ; it is partly concealed by the shieldlike cartilage, below which it is placed. It is very unequal in depth before and behind,-the posterior part being three times deeper than the anterior, something like a signet ring. Its hollow is about as large as the fore finger.

The outer surface is rough, and gives attachment to muscles. At the back of the cartilage there is a flat and rather square portion, which is 
marked by a median ridge between two contignous muscular depressions. On each side, immediately in fiont of the square part, is a shallow articular mark, which receives the lower cornu of the thyroid cartilage. The inner surface is smooth, and is covered by mucous membrane.

The lower border is undulating, and is united to the trachea by fibrons membrane. The upper border is nearly straight posteriorly, opposite the

Fig. 43.

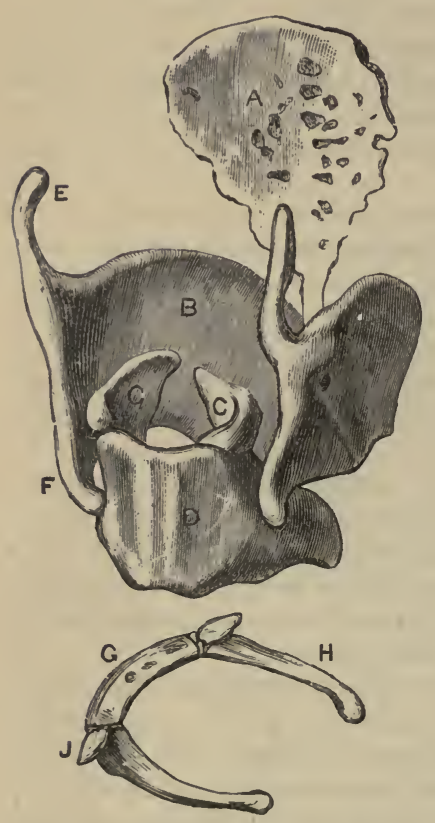

HYOID BONE AND THE LARYNGEAL Cartilages.

G. Body of the hyoid bone.

H. Large cornu.

J. Smail cornu.

A. Epiglottis.

B. Thyroid cartilage.

C. Arytænoid cartilage.

D. Cricoid cartilaye.

E. Upper cornu, and

F. Lower cornu of the thyroid cartilage. deep part of the ring; and this portion is limited on each side by an articular mark for the arytænoid cartilage. In front of that spot the border is sloped obliquely downwards to the middle line. At the middle line behind there is a slight excavation in each border.

The two arytanoid cartilages, $\mathrm{c}$, are placed one on each side at the back of the larynx, on the upper border of the cricoid cartilage. Each is pyramidal in shape, is about half an inch in depth, and offers for examination a base and apex, and three surfaces.

The base has a slightly hollowed surface behind for articulation with the cricoid cartilage, and is elongated in front into a process which gives attachment to the vocal cord. The apex is directed backwards and somewhat inwards, and is surmounted by the cartilage of Santorini.

The inner surface is narrow, especially above, and flat. The outer is wide and irregular, and on it is a small projection at the base, which receives the insertion of some of the muscles. At the posterior aspect the cartilage is concave and smooth.

Cartilages of Santorini. Attached to the apex of each arytanoid cartilage is the small, eonical fibro-cartilage of Santorini (corniculum capitulum), which is bent inwards towards the one of the opposite side. 'The ary tæno-epiglottidean fold is connected with it.

Cuneiform cartilages. Two other small fibro-cartilaginous bodies, one on

each side, which are contained in the arytreno-epiglottid folds, have received this name. Each is somewhat elongated and rounded in form, like a grain of rice; it is situate obliquely in front of the capitulum of the arytanoid cartilage, and its place in the fold of the mucous membrane is marked by a slight whitish projection.

The epiglottis (fig. 43, A) is single, and is the largest of the pieces of yellow fibro-cartilage. In form it is cordate, and it resembles a leaf, with the stalk below and the lamina or expanded part above. Its position is behind the tongue, and in front of the orifice of the larynx. During 
respiration it is placed vertically, but during deglutition it takes a horizontal direction so as to close the opening of the larynx.

The anterior surface is bent forwards to the tongue, to which it is connected by three folds of mucous membrane; and the posterior surface, hollowed laterally, is convex from above down. 'To its sides the arytænoepiglottid folds of mucous membrane are united. After the mucous membrane has been removed from the epiglottis its substance will be seen to be perforated by numerous spaces, which lodge mucous glands.

Between the epiglottis and the hyoid bone is a mass of yellowish fat with some glands; this has been sometimes ealled the epiglottidean gland.

Ligaments of the Larynx. The larynx is connected by extrinsic ligaments with the hyoid bone above and the trachea below. Other ligaments join together the cartilages, forming joints in some cases.

Union of the larynx with the hyoid bone and the trachea. A thin loose elastic membrane (thyro-hyoid) passes from the thyroid cartilage to the hyoid bone; and a second membrane connects the cricoid cartilage with the trachea.

The thyro-hynid ligament (fig. $42, \mathrm{~L}$ ) is attached on the one part to the upper border of the thyroid cartilage; and on the other, to the upper border of the hyoid bone, at the posterior aspect. Of some thickness in the eentre, it gradually becomes thinner towards the sides; and it ends laterally in a rounded elastic cord, which intervenes between the extremity of the hyoid bone and the upper cornu of the thyroid cartilage.

The superior laryngeal nerve and vessels perforate the ligament, and a synovial membrane is placed between it and the posterior surface of the hyoid bone. In the elastic lateral part of the ligament will be found occasionally a small ossific nodule (cartilago triticea).

The membrane joining the lower border of the cricoid cartilage to the first ring of the trachea -crico-tracheal ligament, resembles the band joining the rings of the trachea to each other.

Union of the cricoid and thyroid cartilages. These cartilages are joined in the middle line in front by ligament; and on the side, by a joint with the small cornu of the thyroid cartilage.

The crico-thyroid ligament or membrane (fig. $44,{ }^{6}$ ) closes the space between the thyroid, cricoid, and arytænoid cartilages, and the right half is now visible. It is yellow in color, and is formed mostly of elastic tissue. At the centre it is thick and strong, but is thinner on each side as it is continued backwards. By the lower border it is fixed to the upper edge of the cricoid as Fig. 44.

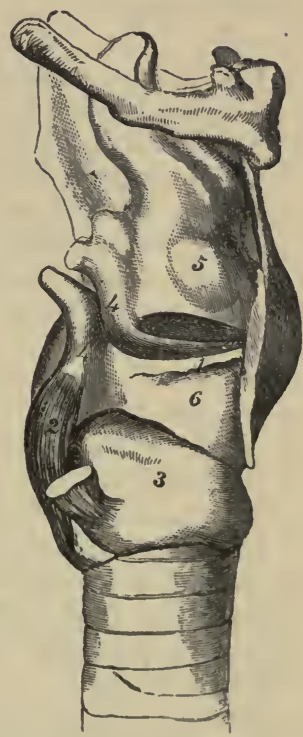

VIEW OF THE Vocal CORDB aND Crico-thromotd LianMENTS.

1. True vocal cord.

2. Post. crico-arytæn muscle.

3. Cricoid cartilage.

4. Arytæuoid cartilage.

5. Sacculus laryngis.

6. Crico-thyroid membrane.

far back on each side as the joint with the arytænoid cartilage. Its upper border, free and rounded, is covered by mucous membrane, and forms the lower vocal cord. In front it is united to the thyroid cartilage; and behind to the base of the arytænoid. 
The ligament is partly concealed by the crico-thyroid muscle, and some small apertures exist in it for the passage of fine arteries into the larynx.

The strong forepart of the ligament serves the purpose of uniting the two large laryngeal cartilages; and the lateral piece, closing the larynx, ends above in the vocal cord.

A capsular ligament surrounds the articular surfaces between the side of the ericoid and the lower cornu of the thyroid cartilage. Its fibres are strongest belind. A synovial membrane lines the capsule.

This joint admits forward and backward movements of the thyroid cartilage, by which the condition of the vocal cords is altered. If that cartilage is moved forwards the cords are stretched, and if backwards the cords are relaxed.

Articulation of the cricoid and arytanoid cartilages. The articular surfaces of the cartilages are retained by a loose capsule, and possess a synovial sac.

The capsular ligament is fixed to each cartilage around its articular surface; and one part-posterior ligament, is strongest on the inner and posterior aspects. A loose synovial membrane is present in the articulation.

The arytanoid cartilage glides freely forwards and backwards, inwards and outwards; but if its horizontal movements are controlled by muscular action, it can be rotated around a vertical axis, the anterior spur being moved inwards and outwards. Obviously the state of the vocal cords will be changed by the movements of the cartilages. When the arytænoids glide in and out the cords will be approximated and separated; when backwards and forwards, the cords will be tightened and relaxed; and in rotation the cords will be moved away from, and bronght towards each other.

A kind of capsule, formed of thin scattered fibres, with a synovial sac, unites the apex of the arytrnoid cartilage with the hollowed base of the capitulum of Santorini. Sometimes these cartilages are blended together.

Fibrous bands (thyro-arytanoid) join the thyroid with the arytanoid cartilages, and have been examined as the vocal cords (p. 156).

Ligaments of the epiglottis. A band, thyro-epiglottidean, connects the lower part of the epiglottis to the thyroid cartilage, close to the excavation in the upper border of the latter (fig. 42). Some fibrous and elastic tissues-hyo-epiglottid ligament, connect likewise the front of the epiglottis to the hyoid bone.

Strccture of tue Trachea. The air tube consists of a series of pieces of cartilage (segments of rings) (fig. 40), which are connected together hy fibrous tissue. The interval between the cartilages at the back of the tube is closed by fibrous membrane, and by muscular fibres and mucous glands. The trachea is lined by mucous membrane with subjacent elastic tissue.

Cartilages. The pieces of eartilage vary in number from sixteen to twenty. Each forms an incomplete ring, which extends about threefourths of a circle; and each is convex forwards, forming the front and sides of the air tube. Both above and below, the cartilaginous pieces are less constant in size and form: for towards the larynx they increase in depth, whilst in the opposite direction they may be slit at their ends or blended together; and the lowest piece of cartilage is shaped like the letter $\mathrm{V}$.

A fibrous tissue is continued from one to another on both aspects, though 
in greatest quantity extermally, so as to incase and unite them ; and it is extended across the posterior part of the air tube.

Dissection. On removing for about two inches the fibrous membrane and the mucous glands from the interval between the cartilages at the back of the trachea, the muscular fibres will appear.

After the muscular fibres have been examined the membranous part of the tube may be slit down, to see the elastic tissue and the mucous membrane.

Muscular fibres. Between the ends of the cartilages is a continuous layer of transverse bundles of unstriated muscle, which is attached to the truncated ends and the inner surface of the cartilages. By the one surface the fleshy fibres are in contact with the membrane and glands, and by the other with the elastic tissue. Some longitudinal fibres are superficial to the transverse; they are arranged in scattered bundles, and are attached to the fibrous tissue.

The elastic tissue forms a complete lining to the tracheal tube beneath the mucous membrane; and at the posterior part, where the cartilages are deficient, it is gathered into strong longitudinal folds. This layer is closely connected with the mucous membrane covering it.

The mucous membrane of the trachea lines the tube, and resembles that of the larynx in being furnished with a columnar ciliated epithelium.

Connected with this membrane are numerous branched mucous glands of variable size. The largest are found at the back of the trachea, in the interval between the cartilages, where some are placed beneath the fibrous membrane with the muscular fibres, and others outside that layer.

Other smaller glands occupy the front and sides of the trachea, being situate on and in the fibrous tissue connecting the cartilaginous rings.

\section{Section XVIII.}

\section{PREVERTEBRAL MUSCLES AND VERTEBRAL VESSELS.}

Directions. On the part of the spinal column that was laid aside after the separation of the pharynx from it, the student is to learn the deep muscles on the front of the vertebra.

Dissection. The prevertebral muscles will be prepared by removing the fascia and areolar tissue. 'The muscles are three in number on each side (fig. 45), and are easily distinguished. Nearest the middle line lies the longus colli-this is the longest; the muscle external to it, which reaches to the heal, is the rectus capitis anticus major; and the small musele close to the skull, which is external to the last and partly concealed by it, is the rectus capitis anticus minor. The smaller rectus muscle is often injured in cutting through the basilar process of the occipital bone.

The LONGUS COLli Muscle (fig. $45, \wedge$ ) is situate on the bodies of the cervical and upper dorsal vertebra, and is pointed above, but larger below. It consists of two parts-internal and external, the former being vertical, and the latter oblique in direction, as on the right side of the figure.

The internal part arises by fleshy and tendinous processes from the bodies of the two upper dorsal and two lower cervical vertebræ; and the external piece takes origin from the anterior transverse processes of four 
cervical vertebra (sixth, fifth, fourth, and third) Both parts of the muscle are blended above, and the whole is inserted by four slips into the bodies of the four upper cervical vertebra.

Some of the lowest fibres of the muscle are attached separately by tendon to the anterior transverse process of one or two of the lower cervical vertebra.

Fig. 45.

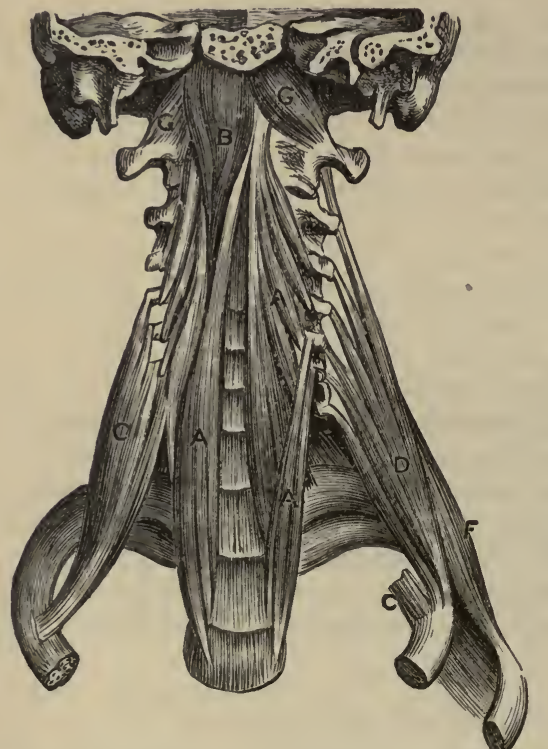

A. Longus medius.

B. Rectus capitis anticus major.

c. Scalenus anticus.

D. Scalenus medius.

F. Scalenus posticus.

G. Rectus capitis anticus minor.

Deep Muscles of the Front of the Neck and the Scaleni Muscles.

In contact with the anterior surface of the muscle is the pharynx. The inner border is at some distance inferiorly from the muscle of the opposite side, but superiorly only the pointed anterior common ligament of the vertebre separates the two. The outer border is contiguous to the scalenus, to the vertebral vessels, and to the rectus capitis anticus major muscle.

Action. Both muscles bend forwards the neck; and the upper oblique fibres of one will turn the neck and head to the same side, by the attachment to the atlas.

The rectus Capitis anticus major (fig. $45,{ }^{\mathrm{B}}$ ) is external to the preceding muscle, and is largest at the upper end. Its origin is by pointed tendinous slips, with the longus colli, from the summits of the anterior transverse processes of four cervical vertehræ (sixth, fifth, fourth, and third); and the fibres ascend to be inserted into the basilar process of the occipital bone, in front of the foramen magnum.

The anterior surface of the muscle is covered by the pharynx, and by the carotid artery and the numerous nerves near the base of the skull. The muscle partly coneeals the following one. At its insertion the rectus is fleshy, and reaches from the middle line to the temporal bone.

Action. Both museles incline forwards the head; and one will bring the face to the same side by rotating the head. 
The RECTUS CApitis ANTICUs minor (fig. $45,{ }^{\mathrm{G}}$ ), is a small flat muscle, which arises from the anterior transverse process and body of the atlas; and is inserted into the basilar process of the occipital bone between the foramen magnum and the rectus major, and half an inch from its fellow.

The anterior primary branch of the suboccipital nerve lies between the borders of this muscle and the rectus capitis lateralis.

Action. Its power is very slight, but it will help in moving forwards the head.

Dissection. The small intertransversales muscles will come into view when the other muscles have been removed from the front and back of the transverse processes. By tracing towards the spine the anterior primary branches of the cervical nerves, the intertransversales will be readily seen on their sides.

After the muscles and nerves have been examined, the tips of the conjoined transverse processes may be cut off to lay bare the vertebral artery.

The INTERTRansverse muscles are slender fleshy slips in the intervals between the transverse processes. In the neck there are seven pairs - the first being between the atlas and axis, and the last between the lowest cervical and the first dorsal vertebra. One set is attached to the anterior, and the other to the posterior tubercles on the tips of the conjoined transverse processes.

Between the muscles, except in the first two spaces, is the anterior primary branch of a cervical nerve; and beneath the posterior muscle is the other primary branch of the same nerve. In the upper space the posterior muscle is often wanting; and in the lowest space, the muscle of the anterior set is smaller than the otliers, or it may be absent.

Action. By approximating the transverse processes these muscles bend the spinal column laterally.

Cervical nerves at their exit from the spinal canal. The trunks of the cervical nerves issue from the spiual canal through the intervertebral foramina, except the first two, and bifurcate into anterior and posterior branches.

The anterior primary branch passes outwards between the intertransverse muscles, and joins in plexuses with its fellows.

The posterior primary branch turns to the Back beneath the posterior intertransverse muscle, and the other muscles attached to the posterior transverse processes; in its course it lies close to the bone between the articular processes of the vertebra.

Peculiarities in the first two. The first two nerves leave the spinal canal above the neural arches of the atlas and axis, and divide at the back of the neck into anterior and posterior branches.

The anterior primary branch of the first or suboceipital nerve has been examined (p. 115). The anterior branch of the second nerve, after perforating the membrane between the neural arches of the first and second vertebra, is directed forwards outside the vertebral artery, and beneath the first pair of intertransverse muscles, to join the cervical plexus.

The posterior primary branches of the first two nerves are described in the dissection of the Back.

The vertebral artery has been seen at its origin in the neck (p. 77); and its termination is described with the vessels of the brain. Entering usually the foramen in the sixth cervical vertebra, the artery ascends ver- 
tically through the corresponding foramina in the other vertebræ. Beyond the atlas, the vessel turns backwards on the neural arch of that bone, and passing beneath the ligament joining the first vertebra with the os occipitis, enters the skull through the foramen magnum. In its course through the foramina the artery lies in front of the anterior trunks of the cervical nerves, except those of the first and second-the former of which crosses on the inner, and the latter on the outer side. The vessel is accompanied by a vein, and by a plexus of nerves of the same name.

In the neck the artery furnishes snall twigs to the surrounding muscles, the spinal canal, and the spinal cord.

The vertebral vein begins by small radicles in the occiput, and in the muscles of the back of the neck, and enters the aperture of the atlas, where it receives sometimes a vein from the skull through the posterior condyloid foramen of the occipital bone. Accompanying the artery, the vein traverses the apertures between the transverse processes, and ends in the subclavian vein.

In its course it is joined by branches from the internal and external spinal veins; its other branches are described at p. 78 .

The vertebral plexus of nerves is derived from the inferior cervical ganglion of the sympathetic (p. 117). It surrounds the artery, and communicates with the spinal nerves as high as the third or fourth.

\section{Section XIX.}

\section{LIGAMENTS OF THE VERTEBR A AND CLAVICLE.}

Directions. On the remaining part of the spine, the ligaments connecting the cervical vertebræ to each other and to the occipital bone are to be learnt.

Dissection. Disarticulate the last cervical from the first dorsal vertebra. Then remove altogether the muscles, vessels, nerves, and areolar tissue and fat from the cervical vertebra. By sawing through the occipital bone, so as to leave only an osseous ring behind the foramen magnum, the ligaments between the atlas and the occipital bone can be more easily cleaned.

The COMmon LIGAMENTS attaching together the cervical vertebre are similar to those uniting the vertebræ in other parts of the spine, viz., an anterior and a posterior common ligament; bands between the lamina and spines; capsular ligaments and synovial membranes for the articulating processes; and an intervertebral ligament between the bodies of the bones.

Directions. 'The common ligaments will be best seen on the dorsal or lumbar portions of the spine where they are more fully developed; their preparation and description will be found at the end of the thorax, with the description of the ligaments of the spine. Should the student examine them in the neek, to see their difference in this region of the spine, he should leave uncut the neural arches of the three highest eervical vertebræ, to which special ligaments are attached.

SPECIAL LigamENTs unite the first two cervical vertebra to eaeh other and to the occipital bone: some of these are external to, and others within the spinal canal. 
The ligaments outside the spinal canal are thin fibrous membranes, which connect the bodies and arches of the first two vertebræ in front and behind; and join the atlas with the occipital bone at the same aspects.

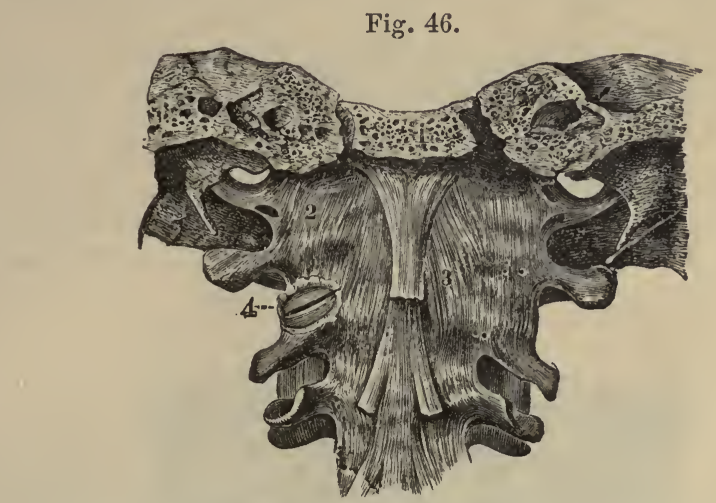

External Ligamente in front betwen the Atras and Axis and the OCCipital BoNe (Bourgery).

1. Sawn basilar process.

2. Anterior occipito-atloid.
3. Anterior atlo-axoid.

4. Articulation of the articular processes cut open.

Capsular ligaments surround the articular surfaces of all the bones; but these will be examined more conveniently after the spinal canal has been opened.

Union of the atlas with the axis. The posterior ligament (atlo-axoid) (fig. $47,{ }^{2}$ ) is a thin loose membrane, which is attached by one margin to the neural arch of the atlas, and by the other to the corresponding arch of the axis. Below the superficial part are some deeper and stronger fibres. The posterior primary branch of the second nerve pierees it.

Fig. 47.

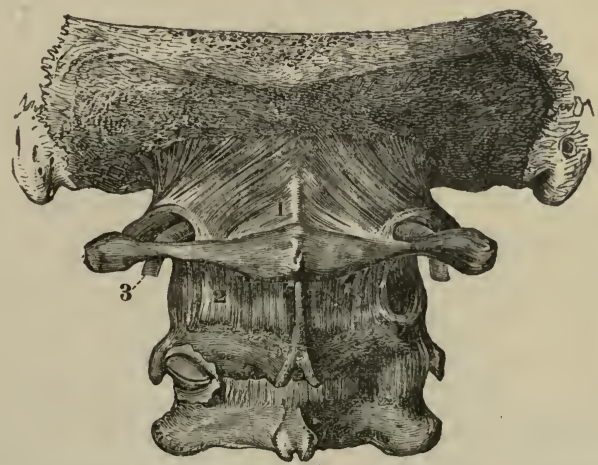

External Ligaments behind between the Atras and Axis and the Occipital Bonis.

1. Posterior occipito-atloid ligament.

2. Posterior atlu-axoid.
3. Vertebral artery entering beneath the occipito-atloid ligament.

The anterior ligament (fig. $46,{ }^{3}$ ) unites the bodies of the first two vertebra in the same manner as the preceding ligament connects their arches. It is thickest in the middle. 
Union of the atlas with the occipital bone. The anterior ligament (occipito-atloid) (fig. $46,{ }^{2}$ ) is thin and wide, and passes from the basilar process of the oceipital bone, in front of the foramen magnum, to the body of the atlas. The middle part of the ligament, which is fixed to the tubercle on the front of the atlas, is much the thickest.

The posterior ligament (fig. $47,{ }^{1}$ ) is fixed to the occipital bone behind the foramen magnum, and to the neural arch of the atlas. It is thin; and at its attachment to the atlas the vertebral artery $\left({ }^{3}\right)$, and the posterior primary branch of the suboccipital nerve, pass beneath it.

The ligaments inside the spinal canal are peculiar in form, and assist in retaining the skull in place during the rotatory and nodding movements of the head. Between the occipital bone and the second vertebra are

\section{Fig. 48.}

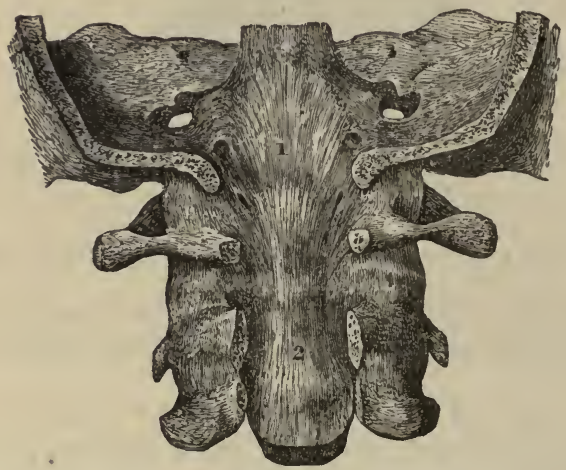

INTERAL LigameNT BetweEN OCcipital Bonf aNd Axis (Bourgery).

1 and 2. Attachments of the occipito-axoidean ligament.

three strong ligaments-a central, and two lateral or check; and the odontoid process of the axis is fixed against the body of the atlas by a strong transverse band.

Dissection. Supposing the neural arches of the cervical vertebre to be removed except in the first three, the arches of these vertebra are to be sawn through close to the articular processes. Nextly the ring of the occipital bone bounding posteriorly the foramen magnum is to be taken away. Lastly, the student should detach the tube of dura mater from the interior of the spinal canal; and, on raising from below the upper part of the posterior common ligament of the bodies of the vertebra, the ligamentous band between the occipital bone and the axis (occipito-ixoid) will come into view.

Union of the occipital bone with the axis. The central ligament (occipito-axoidean) (fig. $48,{ }^{1}$ ) is a strong, thick layer beneath the posterior common ligament of the bodies of the vertebra, and is rather triangular in form with the base uppermost. Above it is attached to the basilar process (on the cranial aspect) near the margin of the foramen magnum, extending as far on each side as the insertion of the check ligaments. From that spot it descends over the odontoid process, and, becoming narrower, is inserted into the body of the axis. Occasionally a bursa is found between it and the transverse ligament of the atlas. 
Dissection (fig. 49). After the removal of the occipito-axoidean ligament, by cutting transversely through it above, and reflecting it, the student should define a strong band, the transverse ligament which crosses

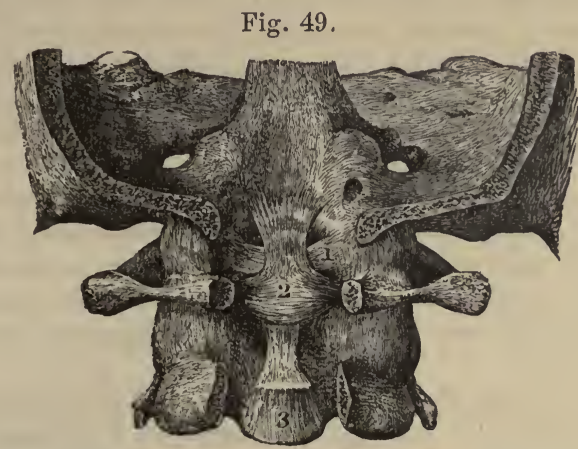

Intronal Lianments between the Occipital Bone and the Atras and Axis (Bourgery).

1. The left cheek ligament.

3. Cut end of the occipito-axoidean ligament.

2. The transverse ligament, sending offsets upwards and downwards.

the root of the odontoid process, and sends upwards and downwards a slip to the occipital bone and the axis. The upper offset from the transverse ligament may be cut through afterwards for the purpose of seeing the check ligaments which diverge, one on each side, from the odontoid process.

The lateral odontoid or check ligaments (fig. $49,{ }^{1}$ ) are two strong bundles of fibres, attached by one end to the side of the head of the odontoid process, and by the other to a depression on the inner surface of the condyle of the occipital bone. These ligaments are covered by the occipitoaxoidean band; their upper fibres are short and almost horizontal, and the lower are longer and oblique.

- Between the lateral bands is a central odontoid ligament, which connects the tip of the odontoid process to the margin of the basilar process of the occipital bone.

Union of the atlas with the axis. (fig. $49,{ }^{2}$ ) is a flat, strong, arched band behind the odontoid process, which is attached on each side to a tubercle below the inner part of the articular process. This ligament is widest in the centre; and at this spot it has a band of longitudinal fibres connected with the upper and lower margins (fig. 49), so as to produce a cruciform appearance: the upper piece is inserted into the basilar process, and the lower into the body of the second vertebra. Its surface towards the cord is concealed by the occipito-axoid ligaThe transverse ligament of the atlas ment.

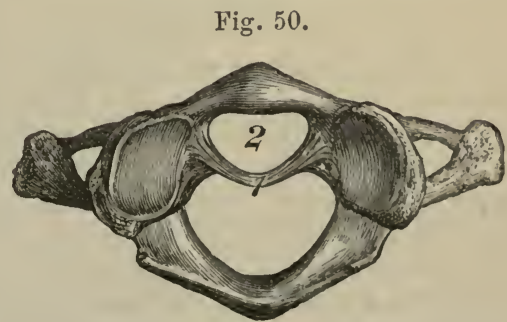

First Vertebra. With The OdONtoid Prockss REMOVED FROM THE SOCKET FORMED BY THE BONE AND THE TRANEVERBE LIAAMENT.

1. Transverse ligament with its offsets cut.

2. Socket for the odontoid process. 
This ligament fixes the odontoid process of the second vertebra against the body of the atlas, confining it in a ring (fig. 50).

When the transverse and check ligaments liave been cut through, the tip of the odontoid process will be seen to have two cartilaginous surfaces; one in front where it touehes the atlas, the other at the opposite aspect, where it is in contact with the transverse ligament. Two synovial mem. branes facilitate the movements of the odontoid process, one serving for the joint between this piece of bone and the atlas; and the other for the joint between it and the transverse ligament.

Union of the articular surfaces. The articular surfaces of the occipital bone and atlas are surrounded by a capsular ligament of scattered fibres, which is strongest externally and in front. When the joint is opened, the condyle of the occipital bone will be seen to look somewhat outwards, and the hollowed surface of the atlas inwards. A synovial membrane is present on each side.

The articular surfaces of the first two vertebræ are inclosed on each side by a capsule (fig. $46,{ }^{4}$ ), which is stronger in front than behind. On opening the joint the surfaces of the bones may be perceived to be almost horizontal. On each side there is a separate loose synovial membrane.

Movements of the head. The head can be bent forwards and backwards; turned from side to side-rotation; and inclined towards the shoulder.

Nodding takes place in the joints between the atlas and the occipital bone, the condyles gliding forwards and backwards. When the head is moved more freely, flexion and extension of the cervical vertebræ come into play.

Rotation is permitted by the several joints between the atlas and axis. In this movement the axis is fixed, and the atlas, bound to it by the transverse ligament, moves to the right and the left, carrying the weight of the head. Too great a movement of the face to the side is checked by the odontoid ligament. Only part of the whole of the rotatory movement to one side is obtained between the atlas and axis, the rest being made up by the neck.

Approximation of the head to the shoulder is effected by the neck movement: perhaps a very slight degree of it may be due to gliding downwards of the occipital condyle of the same side on the articular surface of the atlas.

Sterno-clavicular Articulation (fig. 51). The articular surfaces are somewhat irregular and adapted to each other, with an intermediate fibro-cartilage; and they are retained in contact by a capsular ligament; by a band to the first rib; and by another band between the ends of the clavicles.

Dissection. For the examination of the ligaments of the sterno-clavicular articulation, take the piece of the sternum that was set aside for the purpose (p. 134). If the ligaments have become dry, they may be moistened for a short time. The several ligaments will appear in the situation indicated by their names, after the removal of some fibrous tissue.

Capsular ligament. 'This is a thin membranous expansion (fig. 51, ${ }^{1}$ ), which incases the articular ends of the bones and the fibro-cartilage. It is attached near the articular surface of each bone, and is thinner before than behind. Sometimes the stronger fibres in front and at the back are described as separate ligaments.

The interclavicular ligament (fig. $51,{ }^{3}$ ) extends above the sternum, between the ends of the clavicles. The fibres do not cross in a straight 
line, but dip into the hollow between the collar bones, and are connected with the upper piece of the sternum.

The costo-clavicular ligament (fig. $51,{ }^{2}$ ) is a short strong band of oblique fibres between the first rib and the clavicle. Inferiorly it is fixed to the upper surface of the cartilage of the first rib, and superiorly to a tubercle on the under surface of the clavicle near the sternal end. The subelavius muscle is in front of the ligament.

Sometimes the clavicle touches the rib, and is provided with an articular surface and a synovial membrane.

The interarticular fibro-cartilage (fig. $51,{ }^{7}$ ) will come into view by cutting the ligaments before described, and raising the clavicle. It is ovalish in form and flattened, but is thicker at the circumference than the centre. By its upper margin and surface the cartilage is united to the head of the clavicle which is imbedded in it; and by the opposite surface and margin it is inserted into the cartilage of the first rib. At its circumference it unites with the capsule of the joint. Sometimes there is an aperture in the centre of the fibro-cartilage.

Two synovial sacs are present in the articulation, one on each side of the fibro-cartilage. The sac in contact with the sternum is looser than that touching the clavicle.

Movement. The inner end of the clavicle can be moved up and down, and forwards and backwards, and the direction it takes is the opposite to

Fig. 51.
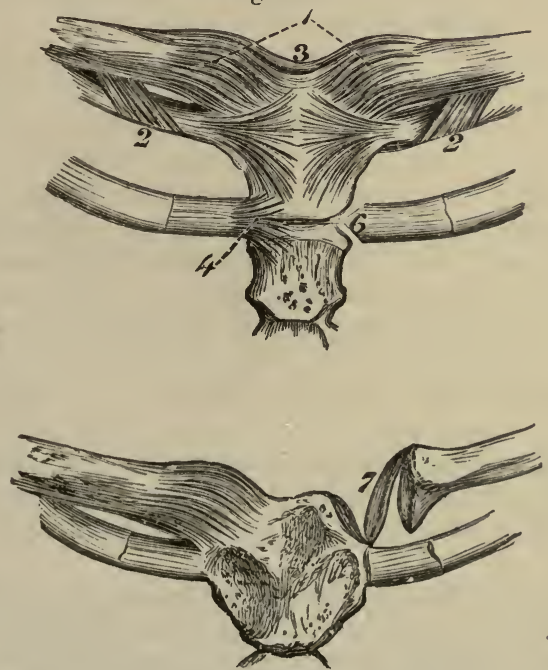

Ligaments of thi InNer ENd of the Claviche, and of the Cartilage of the Second Rib.
1. Capsule.
4. Ligaments of the second rib with the sternum.
2. Costo-cluvicular ligament.
6. Interarticular fibro-cartilage of second rib.
3. Interclavicular ligament.
7. Interarticular fibro-cartilage of the clavicle.

that of the shoulder: thus when the limb is depressed the sternal end of the clavicle is raised; and so on in the other movements. The extent of each movement is but limited, though those forwards and upwards are the freest, and dislocation may ensue in any direction except dowuwards, from force applied to the limb. 


\section{CHAPTER II.}

\section{DISSECTION OF THE BRAIN.}

\section{SECtion I. \\ MEMBRANES AND VESSELS.}

During the examination of the membranes, vessels, and nerves, the brain is to be placed upside down, resting in the coil of a eloth which supports it evenly.

Membranes of tire Brain. The coverings of the brain (meninges) are three in number, viz., dura mater, pia mater, and arachnoid membrane. The dura mater is a firm fibrous investment, which supports part of the brain, and serves as an endosteum to the bones. The pia mater is the most internal layer, and is very vascular. And the arachnoid is a thin serous sac, which is situate between the other two.

Besides enveloping the brain, these membranes are prolonged on the cord into the spinal canal. Only the cranial part of the two last will be now noticed. For the description of the cranial part of the dura mater, see p. 24.

The ARACHNoID is a thin serous membrane, which lines the inner surface of the dura mater, and is reflected over the pia mater and the brain. Around the vessels and nerves that intervene between the skull and the brain, the membrane forms sheaths, which extend a short distance into the several apertures, and then become continuous with the parietal or cranial portion. Like other serous membranes, it forms a sac which eontains a lubricating moisture; and it consists of a parietal and a visceral part.

The parietal part is inseparably united to the inner surface of the dura mater, giving this a smooth and polished surface, and is continued over the pieces of the fibrous membrane projecting between portions of the brain.

The visceral part covers the encephalon loosely, especially at the under surface of the brain, but is united to the underlying pia mater by fibrous processes; beneath it there is a considerable interval (subarachnoid space). On the upper or convex surface of the great brain the membrane passes from one convolution to another, without dipping in to the intervening sulei ; though it lines the great median fissure as low as the extent of the falx. On the lower surface of the cerebrum there is a large space between it and the centre of the brain. Still more posteriorly, between the hemispheres of the little brain, there is an interval, similar to that at the under part of the cerebrum.

The subarachnoid space, or the interval between the arachnoid membrane and the pia mater, is larger in one spot than another; and it con- 
tains more or less fluid, which has been named cerebro-spinal. The space is largest at the under part of the great brain, about its middle, and in the fissure between the hemispheres of it and the cerebellum. Under the arachnoid, in the fissure between the halves of the cerebellum, is the aperture of the fourth ventricle, by which that eavity communicates with the subarachnoid space.

The PIA MATER closely invests the different parts of the brain, and dips into the fissures, as well as into the sulei between the convolutions and laminæ. Besides covering the exterior of the brain, it sends processes into the interior to supply vessels to the walls of the inclosed space: thus, one penetrates into the cerebrum below the corpus callosum, and is named velum interpositum; and two vascular fringes, which project into the fourth ventricle, are known as the choroid plexuses of that cavity.

This membrane is a network of vessels, and is construeted of the minute ramifications of the arteries and veins entering into or issuing from the cerebral substance; whilst the intervals between the vessels are elosed by fine areolar tissue, so as to form a continuous thin layer. From the under surface of the membrane proceed numerous fine vessels for the nutrition of the brain.

Vessels and nerves. The arachnoid membrane has but few vessels, whilst the pia mater is composed almost entirely of vessels. The pia mater is largely supplied by offsets of some cranial nerves, and by branches of the sympathetic which accompany the vessels at the base of the brain (p. 33). Bochdalek has deseribed branches to the arachnoid from some cranial nerves.

Subdivisions of the encephalon. Before the description of the arteries is given, the chief subdivisions of the encephalon will be shortly noticed.

The cranial or encephalie mass of the nervous system consists of cerebrum or great brain, cerebellum or small brain, pons, and medulla oblongata. Each of these parts has the following situation and subdivisions:-

The medulla oblongata, or the upper end of the spinal cord, lies in the groove between the halves of the small brain, and is divided into two symmetrical parts by a median fissure. To it several of the cranial nerves are united.

The pons Varolii is situate in front of the medulla oblongata, and is marked along the middle by a groove, which indicates its separation into halves. Anterior to it are two large processes (erura eerebri) eonnecting it to the great brain; on each side it is united to the small brain by a similar white mass (erus cerebelli); and behind it is the enlarged upper part of the cord.

The cerebellum, or the small brain, is separated into two by a median fissure, and each half will be subsequently seen to consist of lobes.

The cerebrum, or the large brain, is divided into hemispheres by a longitudinal fissure in the middle line; and each half is further subdivided into two by a transverse.sulcus - the fissure of Sylvius. In the centre of the cerebrum, between the hemispheres and in front of the pons, are several small bodies that will be afterwards enumerated.

Dissection. To follow out the arteries, let the brain remain upside down, and let the remains of any arachnoid membrane be removed. Having displayed the trunks of the vertebral arteries on the medulla oblongata, and those of the carotid in front near the median fissure of the large brain, the student should lay bare on one side the branches to the large 
brain. Define first the two arteries lying in the median fissure and joining by a short branch; next, an artery that passes outwards transversely in the fissure of Sylvius, and pursue it to the outer surface of the hemisphere. Look then for a much smaller vessel (choroid), which enters into the brain substance on the outer side of the erus cerebri. By gently raising the cerebellum on the same side, the last artery of the cerebrum may be traced back along the inner aspect of the hemisphere.

Two arteries pass out to the small brain. One on the upper surface may be brought into view on raising the cerebellum; and care is to be taken of the slender fourth nerve which lies by its side. The other artery turns inwards to the median hollow of the cerebellum, and may be easily followed.

Arteries of the Brain (fig. 52). The brain is supplied with blood by the vertebral and internal carotid arteries.

The VERTEBRAL ARTERY, ${ }^{12}$, is a branch of the subclavian trunk (p. 77), and enters the trunk through the foramen magnum ; directed upwards round the medulla oblongata, it blends with its fellow in a common trunk (basilar) at the lower border of the pons. As the vessel winds round the upper part of the cord, it lies between the roots of the suboccipital and hypoglossal nerves; but it is afterwards internal to the last.

Fig. 52.

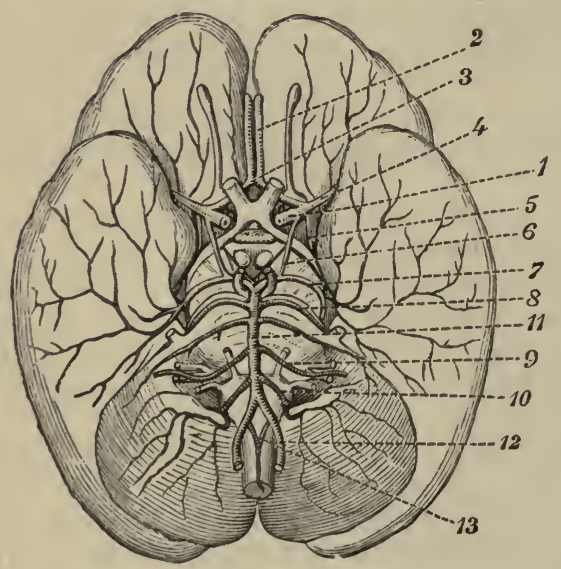

1. Internal carotid trunk.

2. Anterior cerebral branch.

3. Anterior communicating.

4. Middle cerebral branch.

5. Choroidal branch.

6. Posterior communicating.

7. Posterior cerebral branch.

8. Upper cerebellar branch.

9. Internal auditory branch.

10. Inferior cerebellar branch.

11. Basilar artery.

12. Vertebral artery.

13. Anterior spinal branch.

ARTERIEs AT THE BASE OF THB BRAiN.

Branches. Between its entrance into the spinal canal and its termination, each artery furnishes offsets to the spinal cord, the dura mater, and the eerebellum.

a. The posterior spinal branch is of ineonsiderable size, and arises opposite the posterior part of the medulla: it descends along the side of the cord, behind the nerves, and anastomoses with its fellow, and with branches that enter by the intervertebral foramina.

b. The anterior spinal branch $\left({ }^{18}\right)$ is small like the preceding, and springs from the artery opposite the front of the spinal cord. It joins the corresponding twig on the opposite side, and the resulting vessel is continued along the middle of the cord on the anterior aspect.

$c$. The posterior meningeal artery leaves the vertebral trunk opposite 
the foramen magnum, and ramifies in the dura mater lining the fossa of the oceipital bone.

d. The interior cerebellar artery (posterior) $\left({ }^{10}\right)$ is distributed to the under surface of the cerebellum. Taking origin from the end of the vertebral, this branch winds backwards round the medulla between the pneumogastric and spinal accessory nerves, to the median fissure of the cerebellum. Directed onwards along the fissure the artery reaches the upper surface of the small brain, and there anastomoses with the superior cerebellar artery.

An offset of this branch ramifies over the under part of the cerebellum, and ends externally by anastomosing with the artery of the upper surface. As the vessel lies by the side of the aperture of the fourth ventricle it gives a small choroid offset to the plexus of that eavity.

The BASILAR ARTERY $\left({ }^{11}\right)$, formed by the union of the two vertebrals, reaches from the lower to the upper border of the pons, where it ends by dividing into two branches (posterior cerebral) for the cerebrum. The vessel touches the basilar process of the occipital bone, from that circumstance receiving its name, and lies in the median groove of the pons. On each side of, and almost parallel to it, is the sixth nerve.

Branches. Besides the two terminal branches mentioned above, the artery supplies transverse offsets to the pons and the under part of the cerebellum, and a large branch to the upper surface of the cerebellum.

a. The transverse arteries of the pons are four or six small twigs, which are named from their direction, and are distributed to the substance of the pons. One of them $\left({ }^{9}\right)$ gives an offset (auditory) to the internal ear along the auditory nerve.

$b$. Resembling this set of branches is the following artery, the inferior cerebellar (anterior): this arises from the basilar trunk, and extends to the fore part of the under surface of the cerebellum, on which it is distributed.

$c$. The superior cerebellar artery $\left({ }^{8}\right)$ is derived from the basilar so near the termination as to be often described as one of the final branches of that vessel. Its destination is to the upper surface of the small brain, to which it is directed backwards over the third nerve and the crus cerebri, but parallel with the fourth nerve. On the upper surface of the cerebellum the artery spreads its ramifications, which anastomose with the ressel of the opposite side, and with the inferior cerebellar artery.

Some twigs of this vessel enter the piece of the pia mater (velum interpositum), which projects into the posterior part of the cerebrum.

d. The posterior cerebral artery $\left({ }^{7}\right)$ takes on each side a backward course, similar to that of the preceding artery, but separated from it by the third nerve. The vessel is then applied to the inner surface of the cerebrum, and divides into many branches: some of these supply the under part, whilst others turn upwards on both the outer and inner surfaces of the back of the hemisphere, and anastomose with the other cerebral arteries.

Near its origin it is joined by the posterior communicating artery of the carotid; and its branches to the brain are the following:-

Numerous small long branches leave it elose to its origin, and enter the base of the brain between the crura cerebri (posterior perforated spot).

A small choroid artery (posterior) supplies the fold of pia mater that projects into the cerebrum : this small branch is transmitted between the 
crus and hemisphere of the cerebrum to the velum interpositum and the choroid plexus.

From the foregoing examination of the offsets of the vertebral arteries and the basilar trunk, it appears that about half the encephalon-viz., the medulla oblongata, the pons, the cerebellum, and the posterior third of the cerebrum-receives its blood through the branches of the subclavian arteries.

The internal CARotid artery $\left({ }^{1}\right)$ terminates in branches for the remaining part of the cerebrum. The vessel emerges from the cavernous sinus (p. 33) inside the anterior clinoid process, and divides at the inner end of the fissure of Sylvius into cerebral and communicating arteries. At tha base of the brain the carotid lies between the second and third nerves, but nearest the former.

Branches. In the skull the carotid gives off the ophthalmic offset, before it ceases in the following terminal branches to the cerebrum.

a. The anterior cerebral artery $\left({ }^{2}\right)$ supplies the inner part of the cerebral hemisphere. It is directed forwards to the median fissure between the halves of the large brain; and as it is about to enter, it is united to its fellow by a short thick branch-the anterior communicating $\left({ }^{3}\right)$. Then passing into the fissure, it bends round the anterior part of the corpus callosum, so as to be placed on the upper aspect in the natural position of the brain, and is continued backwards distributing offsets nearly to the posterior extremity of the hemisphere.

It gives off numerous branches, and some of them supply the base of the cerebrum, thus :-

Near the commencement it furnishes small branches to the part of the brain (anterior perforated spot) contiguous to the inner end of the fissure of Sylvius: and it distributes some branches to the under part of the frontal lobe.

b. The middle cerebral artery $\left({ }^{4}\right)$ is the largest offset of the carotid, and ramifies over the outer side of the hemisphere. Entering the fissure of Sylvius, the artery divides into many large branches, which issue at the outer end of that groove, and, spreading over the external surface of the hemisphere, inosculate with the other two cerebral arteries at the front, the back, and the upper part of the brain. Only a few fine offsets require special notice :

A set of small branches arise at the inner end of the fissure of Sylvius, and enter the cerebral substance through the substantia perforata antica.

c. The posterior communicating artery $\left({ }^{8}\right)$ is a small twig, which is directed backwards parallel to, and on the inner side of the third nerve, to join the posterior cerebral artery (of the basilar) near the pons.

$d$. The choroid artery (anterior) $\left({ }^{5}\right)$ is small in size, and arises either from the trunk of the carotid, or from the middle cerebral artery : it passes backwards on the other side of the preceding, and finds its way between the hemisphere and the crus cerebri to the choroid plexus of the lateral ventricle.

Circle of Willis. 'The arteries at the under part of the brain are united freely both on their own side and across the middle line in an arterial anastomosis, - the circle of Willis. On each side this circle is formed by the trunk of the internal carotid giving forwards the anterior cerebral, and backwards the posterior communicating artery. In front it is constructed by the converging anterior cerebral, and the anterior communicating 
artery. And behind is the bifurcation of the basilar trunk into the posterior cerebrals. In the area of the circle lie several parts corresponding with the floor of the third ventricle.

The complete inosculation between the cranial vessels in the circle of Willis allows at all times a free circulation of blood through the brain, even though a large vessel on one side of the neck should be obstructed.

The verss of the brain enter the sinuses of the dura mater, instead of uniting trunks as companions to the arteries.

Two sets of veins belong to the cerebrum, viz., superficial or external, and deep or internal.

The external veins of the upper surface are collected into the superior longitudinal sinus (p. 29); and those of the lateral and under parts enter the sinuses in the base of the skull, especially the lateral sinus.

The deep veins of the interior of the cerebrum join the veins of Galen, and reach the straight sinus.

The veins of the cerebellum end differently above and below. On the upper surface they are received by the veins of Galen and the straight sinus; and on the lower surface they terminate in the occipital and lateral sinuses.

Dissection. The pia mater and the vessels are now to be stripped from the brain, and the origin of the cranial nerves is to be defined. Over the greater part of the cerebrum, the pons, and the medulla, the pia mater can be detached with tolerable facility by using two pairs of forceps; but on the cerebellum the membrane adheres so closely as to require some care in removing it without tearing the substance of the brain.

In clearing out the fissure between the laalves of the cerebellum on the under surfice, the membrane bounding the opening of the fourth ventricle will probably be taken away: therefore the position, size, and limits of that opening between the back of the medulla oblongata and the cerebellum should be now noted.

When the surface has been cleaned, the brain is to be replaced in the spirit, but it is to be turned orer occasionally, so that all the parts may be hardened.

\section{SEction II.}

\section{ORIGIN OF THE CRANIAL NERVES.}

TuE cranial nerves take origin from the encephalon, with one exception (spinal accessory), and pass from it through apertures in the skull.

The origin of a nerve is not determined by the place at which it appears on the surfiace of the brain, for fibres or roots may be traced deeply into the brain substance. Each nerve has therefore a superficial or apparent, and a deep or real origin in the encephalon.

With respect to the superficial attachment there cannot be any doubt; but there is much difference of opinion concerning the deep origin, in consequence of the difficulty of tracing the roots. When the roots are followed into the encephalon, they enter masses of gray substance, which are looked upon as ganglia of origin. 
The cranial nerves may be regarded either as nine or twelve pairs, according to the mode of elassifying them. ${ }^{1}$

The several nerves may be designated first, second, third, and so forth : this numerical mode of naming applies to all, and is the one generally used.

But a second name has been derived for some of the nerves from the parts to which they are supplied; as instances of this nomenclature the terms hypoglossal, pneumogastric, may be taken. And a different appellation is given to others, in consequence of the function conferred on the part to which they are distributed, as the terms auditory and olfactory express. In this way two names may be employed in referring to a nerve: one being numerical, the other local or functional, as is exemplified below.

The FIRST OR OLFACTORY NERVE (olfactory process) (fig. $53,{ }^{1}$ ) is soft

Fig. 53.

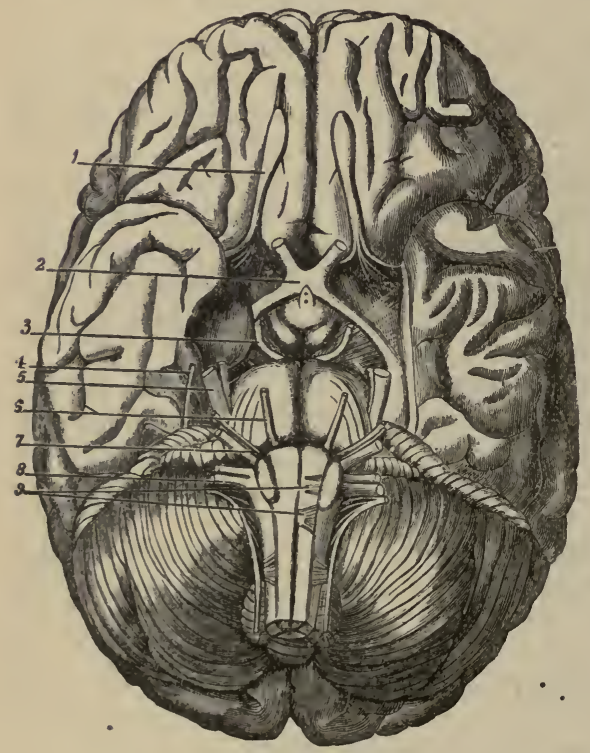

1. Olfactory.

2. uptic.

3. Motor oculi.

4. Trochlear.

5. Trigeminal with small and large root.

6. Abductor oculi.

7. Facial and auditory, the former smaller and internal.

8. Eighth nerve consisting of three trunks iu a line: the upper, glossopharyngeal; the middle, pueumogastric; and the lowest spinal accessory.

9. Roots of attuchment of the hy poglossal nerve.

Orijin of the Crasial Nerves.

and pulpy, being destitute of a neurilemma ; and it may be considered an advanced part of the brain, for it has both gray substance and white fibres in its composition, like the cerebrum.

The olfactory process is a flat-looking band, wider at each end than in the middle, which is lodged in a sulcus on the under aspect of the frontal lobe of the cerebrum, and is kept in position by the reflection of the arachnoid membrane over it. When the so-called nerve is raised from the

1 Those anatomists, who take the smaller number after the example of Willis, include in one nerve all the trunks contained in the same aperture of the skull: as in the case of the eighth nerve, which consists of three trunks in the foramen jugulare. But those who are disposed with Sömmerring to enumerate twelve nerves, consider each of the three trunks of the eiglith nerve before mentioned, to constitute a separate cranial nerve, notwithstanding that it may be combined with otlers in the foramen of exit. 
suleus, it is prismatic in form, the apex of the prism being directed downwards (in this position).

Anteriorly the nervous substance swells into the olfactory bulb,-a pyriform grayish mass, about half an inch in length, which rests on the ethmoid bone, and distributes nerves to the nose.

Posteriorly the olfactory process is connected to the cerebrum by three roots, external, internal, and middle.

The external or long root is a slender white band, which is directed backwards along the outer part of the anterior perforated space, and across the fissure of Sylvius, and sinks into the substance of the cerebrum.

The internal or short root, not always visible, is white and delicate, and comes from the inner part of the cerebrum.

The middle or gray root is connected with the gray matter on the surface of the brain by means of a conical elevation at the back of the sulcus which lodges the nerve.

Deep origin. The external root is said to be traceable to one of the convolutions of the island of Reil. The inner root joins a band of white fibres connected with a convolution (gyrus formicatus) to be afterwards examined. And the middle root, continuous with the gray matter of the convolutions, contains white fibres which enter the corpus striatum.

The SECOND or OPTIC $\left({ }^{2}\right)$ is the largest of the cranial nerves except the fifth, and appears as a flat band on the crus cerebri. Anteriorly the nerves of opposite sides are united in a commissure. The part of the nerve posterior to the commissure is named optic tract; but the part beyond the commissural union, which is round and firm, is called optic nerve. The destination of the nerve is to the eyeball.

The origin of the nerve will be afterwards seen to come from the optic thalamus and the corpora geniculata, and from one of the corpora quadrigemina (nates).

The tract is the flattened part of the optic winding round the peduncle of the cerebrum. In front it ends in the commissure, and behind it splits into two pieces by which it is fixed to the brain. As the tract reaches forwards it crosses the crus cerebri, to which it is attached by its outer or anterior edge; and in front of the crus it is placed between the substantia perforata antica on the outside, and the tuber cinereum on the inside, but whether it receives filaments from the latter is uncertain.

The commissure (chiasma) of the nerves measures half an inch across, and lies on the olivary eminence of the sphenoid bone, within the circle of Willis. It is placed in front of the tuber cinereum; and passing beneath it (in this position of the brain) is the thin lamina cinerea.

In the commissure each tract is resolved into three sets of fibres with the following arrangement :- the outer fibres, few in number, are continued straight to the eyeball of the same side. The middle, the largest, decussate with the corresponding bundle of the other tract,- - those of the right nerve being continued to the left side, to the opposite eye, and vice versâ. And the most internal are continued through the tract of the other side back to the brain without entering the eye. At the front of the commissure are placed some transverse fibres, which are prolonged to the eyeballs through the part of each nerve in front of the commissure, but have not any connection with the tracts and the brain.

The part called nerve extends from the commissure to the eyeball. Leaving the skull by the optic foramen, it receives a tube of dura mater, 
and its course in the orbit is described in p. 56. In the eyeball it ends in the retina.

The THIRD Nerve $\left({ }^{3}\right)$, motor nerve of the eyeball, is round and firm, and is attached by a slanting line of threads to the inner surface of the cerebral peduncle, near the locus perforatus, and close in front of the pons Varolii.

Deep origin. The deep origin is uncertain. According to Stilling, 1 the fibres of the nerve pierce the peduncle, passing through the locus niger, and enter a nucleus of gray substance near the floor of the aqueduct of Sylvius.

The Fovitu or trochlear Nerve $\left({ }^{4}\right)$ cannot be followed backwards at present to its origin. It is the smallest of the cranial nerves, and issues from the valve of Vieussens over the fourth ventricle (fig. $68,{ }^{4}$ ). The nerve appears between the cerebrum and the cerebellum, on the side of the crus cerebri ; and is then directed forwards to enter an aperture in the free edge of the tentorium cerebelli near the posterior clinoid process.

Deep origin. In entering the valve of Vieussens, filaments of the nerves of opposite sides cross and communicate. Each divides into two parts : the anterior enter's a nucleus of gray matter on the side of the aqueduct of Sylvius; the posterior joins a nucleus (upper trigeminal) near the top of the fourth ventricle (Stilling).

The FIFTH or TRIGEMINAL is the largest of all the cranial nerves, and consists of two roots, ganglionic or sensory, and aganglionic or motor, which are partly blended in one trunk beyond the ganglion.

The nerve is attached to the side of the pons Varolii, nearer the upper than the lower border $\left({ }^{5}\right)$.' The small or aganglionic root is highest, and is separated from the large one by two or three of the transverse fibres of the pons. Both roots pass outwards through an aperture in the dura mater, above the petrous part of the temporal bone, and are blended in the peculiar manner stated in page 31 .

Both roots penetrate the fibres of the pons, and are connected with nuclei in the floor of the fourth ventricle.

Deep origin. The large root divides into two parts near the mass of gray matter called locus cæruleus (p. 217). One piece arises from the gray matter near the locus creruleus (upper trigeminal nucleus) ; and the other, from a deeper nucleus, lower trigeminal, opposite the lower border of the pons, within the fasciculus teres (Stilling).

The small root begins with the fourth nerve in the upper trigeminal nucleus near the top of the fourth ventricle (Stilling).

The sixtiI NERve $\left({ }^{6}\right)$, abdueent nerve of the eyeball, springs from the pyramidal body close to the pons, and by a second band from the lower part of the pons.

Deep origin. The fibres of the nerve bend backwards, through the medulla oblongata, to a nucleus in the floor of the fourth ventricle, whose position is on the outer part of the fasciculus teres, and behind the anterior fossa. See Anatony of the Fourth Ventricle (p. 217).

The SEventu Cranial Nerve (Willis) $\left(^{7}\right.$ ) appears at the lower border of the pons near the restiform body. It consists of two distinct trunks, facial and auditory; the former being the motor nerve of the face, and the latter the special nerve of the organ of hearing.

The facial nerve (portio dura, seventh nerve, Sömmerring) is firm and 
round, and smaller than the auditory, internal to which it is placed. It issues from the lateral tract of the medulla at the upper part, and is connected by a slip with the lower border of the pons.

The facial nerve receives a small accessory band of fibres (intermediate portion of Wrisberg) from the same part of the medulla, and enters the internal meatus with the auditory trunk.

The auditory nerve (portio mollis, eighth nerve, Sömmerring) has a surface attachment to the restiform body and the floor of the fourth ventricle. The nerve is very soft, and receives one of its names from that fact.

Deep origin. The facial nerve penetrates to the floor of the fourth ventricle, and arises from the same nucleus as the sixth nerve, which it joins (Clarke ${ }^{1}$ ).

The fasciculus of the auditory nerve which reaches the floor of the fourth ventricle, bends backwards over the restiform body to the auditory nucleus; and some arciform fibres out of the median sulcus are joined with this part of the root. The other fasciculus pierces the restiform body, and takes origin from a network connected with the posterior pyramid (Clarke).

The eiguth CRANial Nerve (Willis) $\left(^{8}\right)$ is placed along the side of the medulla oblongata, and consists of three distinct trunks, glossopharyngeal, pneumogastric, and spinal accessory: the names of the first two indicate their destination; and the last, besides being accessory to the pneumogastric, supplies some muscles.

The glosso-pharyngeal nerve (ninth nerve, Sömmerring) is the smallest of the three, and is situate highest. Its apparent origin is by three or more fibrils, which penetrate the lateral tract of the medulla close to the facial nerve.

The pneumogastric or vagus (tenth nerve, Sömmerring) is connected with the lateral tract of the medulla, below the glosso-pharyngeal nerve, by a series of filaments, which are collected at first into bundles, but are finally gathered into one flat band.

The spinal accessory nerve (eleventh nerve, Sömmerring) consists of two parts-accessory to the vagus, and spinal.

The accessory part is of small size, and arises by fine filaments in a line with the root of the vagus, as low as the first cervical nerve. Finally this fasciculus throws itself into the pneumogastric nerve outside the skull. (See p. 114.)

The spinal part is firm and round, like the third or the sixth nerve, but only a small piece of it can be seen. It arises by a number of fine filaments from the lateral column of the spinal cord near the lateral fissure, as low as the sixth cervical nerve. As the nerve ascends along the side of the cord it lies between the ligamentum denticulatum and the posterior roots of the spinal nerves, with the upper of which it may be connected; and it finally enters the skull by the foramen magnum.

All three nerves converge below the crus cerebelli, and rest on a small lobe of the cerebellum (floceulus). From that spot they are directed outwards to the foramen jugulare (p. 32).

The fibrils of the nerves pierce the medulla; and each nerve, except the spinal part of the last, takes origin from a special deposit of gray matter at the back of the medulla oblongata, and near the lower of the fourth ventricle (p. 217).

I (On the structure of the Medulla Oblongata, by J. Lockhart Clarke, F. R. S., in the Philosophical Transactions for 1858, part i. 
Deep origin. The glosso-pharyngeal penetrates as far as the vagus nucleus, where it ends in fibres which enter that deposit.

The ragus nerve arises in a special nucleus (p. 217); but some fibres pass through the hypoglossal nucleus.

The accessory part of the spinal accessory nerve is transmitted to a special nucleus below that of the vagus; and some fibres decussate across the middle line with their fellows.

The roots of the spinal part of the nerve, piercing the lateral column of the cord, pass through a collection of cells to the anterior cornu of the crescent (Clarke).

The Nintr or HYPogLOSSAL NERVE of Willis (twelfth nerve, Sömmerring) $\left({ }^{9}\right)$ is placed on the front of the medulla oblongata, and arises by a series of filaments from the sulcus between the pyramidal and olivary bodies, in a line with the anterior roots of the spinal nerves.

The filaments of origin unite into two bundles, which pierce separately the dura mater, and do not blend together till they are outside the cranial cavity.

Deep origin. The filaments of the nerve can be traced through the corpus olivare to a nucleus below the level of the fourth ventricle, and in front of the canal of the cord; but some bend inwards to decussate through the raphe with the nerve of the opposite side (Clarke).

\section{Section III. \\ MEDULLA OBLONGATA AND PONS VAROLII.}

THE medulla oblongata and the pons are interposed between the spinal cord and the brain proper.

Directions. On a single brain the student may ascertain nearly all the anatomy of the parts composing the medulla and the pons; but if he can procure one hardened specimen of the medulla and the pons united, and another of a vertical median section through those bodies, he will comprehend much more readily the following description.

Position. The brain is to remain in the same position as for the examination of the nerves and vessels.

The MEDLLLA oblongata is the dilated upper part of the spinal cord which is contained in the cranium (fig. 54). Its limit is the lower border of the pons in one direction, and the upper margin of the atlas in the other. This part of the cord is pyramidal in form, and measures about one inch and a quarter in length; half an inch in breadth below, and about an inch at its widest part.

Its base joins the pons, the transverse fibres of the latter marking its limit; and its apex is blended with the spinal cord. The anterior surface is irregularly convex, and is in contact with the hollowed basilar process of the occipital bone. The opposite surface is somewhat excavated superiorly, where it forms the floor of th' fourth ventricle; and it rests in the fissure between the halves of the cerebellum. On the posterior aspect there are not any cross fibres of the pons, as in front, to mark the extent of the medulla.

The medulla oblongata is divided into halves by a median fissure in front and behind. The fissures are in a line with those along the cord: the anterior ceases at the pons in a dilated part (foramen cacum), but 
the posterior is prolonged, behind the pons, into the floor of the fourth ventricle.

Each half of the medulla is constituted of pieces continuous with those of the spinal cord (fig. 54); and slight grooves indicative of these divisions are to be seen on the surface. Thus at the middle line in front is the anterior pyramid $\left({ }^{1}\right)$ corresponding with the anterior column of the cord; at the middle line behind is the small posterior pyramid $\left(^{4}\right)$ continuous with the posterior median column. On the side is the larger projecting restiform body $\left({ }^{3}\right)$, joining below the posterior column of the corl ; and between this and the anterior pyramid is the lateral tract $\left({ }^{2}\right)$, with an oval projecting body (corpus olivare) $\left(^{5}\right)$ on the exterior, which blends below with the lateral column.

Fig. 54 .

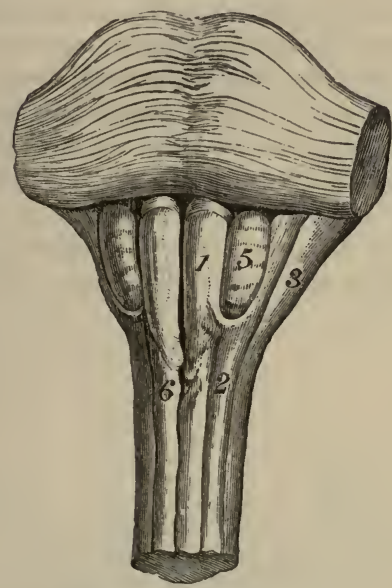

Anterior View of the Medulla Oblongata AND PONS.

1. Anterior pyramid.

2. Lateral tract.

3. Restiform body.

5. Corpus olivare.

6. The decussation.
Fig. 55.

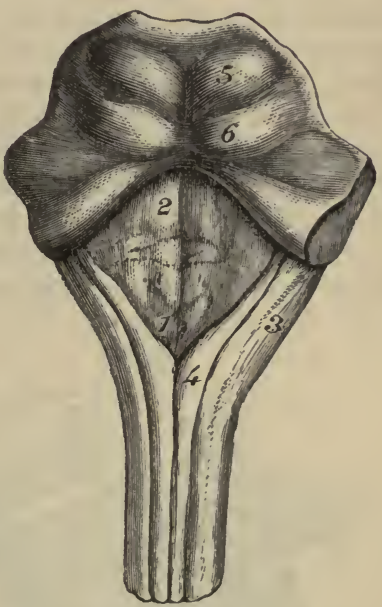

hinder View of the Medella Oblongata.

1. Floor of the fourth ventricle.

2. Eminentia teres.

3. Restiform body.

4. Posterior pyramid.

The anterior pyramid (fig. $54,{ }^{1}$ ) is the most internal eminence and receives its name from its position and form. Situate on the side of the median fissure, it is internal to the olivary body, from which it is separated by a slight groove. Enlarging as it ascends, this body enters the pons, but before disappearing beneath the transverse fibres, it is somewhat constricted and rounded.

Lateral tract and olivary body. The lateral tract (funiculus lateralis) $\left({ }^{2}\right)$ fills the interval between the anterior pyramid and the restiform body. Its surface width is not the same throughout: opposite the lower part of the medulla oblongata it measures as much as the pyramid; but near the pons it is concealed by the olivary body, so that it is seen only in the narrow interval between the outer side of the corpus olivare and the restiform body.

The olivary body (corpus olivare) $\left({ }^{5}\right)$ is an oval projection, about half 
an inch long. A shallow groove separates it from the pyramid, and a deeper and wider one intervenes between it and the restiform body. This eminence is shorter than the pyramid, and does not reach to the pons. Its upper end is most prominent; and arching round the lower end, and over the surface, are some white fibres (fibre arciformes).

Restiform body $\left({ }^{3}\right)$. The restiform body (restis, a rope) forms the largest prominence on the half of the medulla oblongata, and cannot be seen satisfactorily except on a distinct preparation. It is posterior to the lateral tract, and projects on the side, so as to give the width to the upper part of the medulla oblongata. Behind, the restiform bodies diverge above from each other, and between them is the space of the fourth ventricle.

The posterior pyramid (funic. gracilis) (fig. $55,{ }^{4}$ ) lies along the side of the posterior median fissure, and is much narrower than either of the other component pieces. By drawing forwards the medulla it will be seen to be slightly enlarged (clava) at the apex of the fourth ventricle, and then to become gradually indistinct along the inner part of the corpus restiforme.

Structure. From the interposition of the medulla oblongata between the brain and the spinal cord, it is continuous with both. Below, the constituent pieces of the half join directly (as before said) those of the spinal cord; but, above, they are continued chiefly to the cerebrum, only one piece (restiform) entering the cerebellum.

Dissection (fig. 56). The student has now to show the continuity of the constructing parts of one-half of the medulla oblongata with those of the

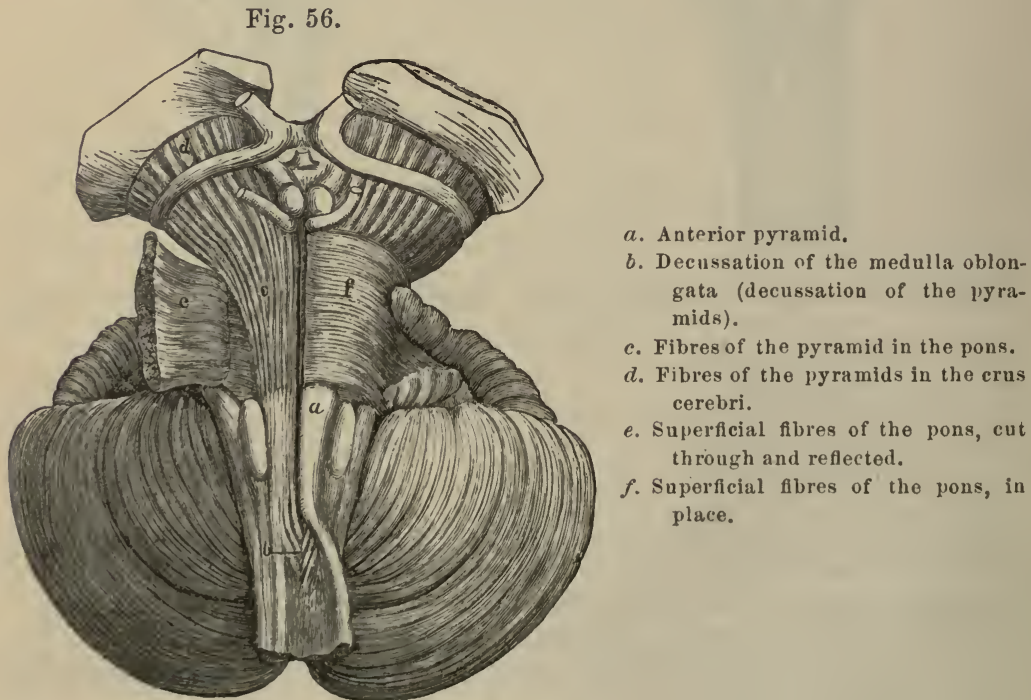

Fibres of the Medulla, Pons and Crug Cerebri.

spinal cord. Let him trace out first the fibres of the pyramid on the right side: in it two sets of fibres have to be shown-one from the same, and one from the opposite side of the cord. The fibres from the opposite half of the cord will appear in the median fissure, when the pyramids are gently drawn from one another, where they are named the decussating fibres; and to lay these bare more completely, the small part of the anterior 
column of the cord on the opposite side, which remains below the cross fibres (for the cord has been eut through near these), may be forcibly turned outwards.

The anterior pyramid (fig. 56,a) receives fibres inferiorly from the anterior column of the cord of its own side, and from the opposite half of the cord. The inner set of fibres, $b$, deep at their origin, become superficial, and are directed upwards, close to the median fissure, joining the fibres continued from the anterior column of the cord; and as the inner fibres of each pyramid are derived from the opposite side, they cross each other in the median line-forming thus the decussation of the medulla oblongata.

The fibres of the pyramid are white and longitudinal, and are collected into a bundle of a prismatic form. Superiorly they enter the pons (fig. 56 ), to reach the cerebrum.

Dissection. For the purpose of seeing the arrangement of the fibres of the lateral tract, the anterior pyramid is to be cut across on the right side, between its decussation and the olivary body (fig. 57), and to be raised towards the pons.

The lateral tract of the medulla is prolonged inferiorly into the portion of the spinal cord between the anterior and posterior roots of the nerves. The continuation of the tract (fig. 57) ascends beneath the olivary body, and leaving the surface of the medulla enters the pons.

The olivary body, and its fillet (fig. 57, c). The olivary mass consists of three parts, viz., a gray incasing layer, a nucleus, and a band prolonged from it-the fillet.

Fig. 57.

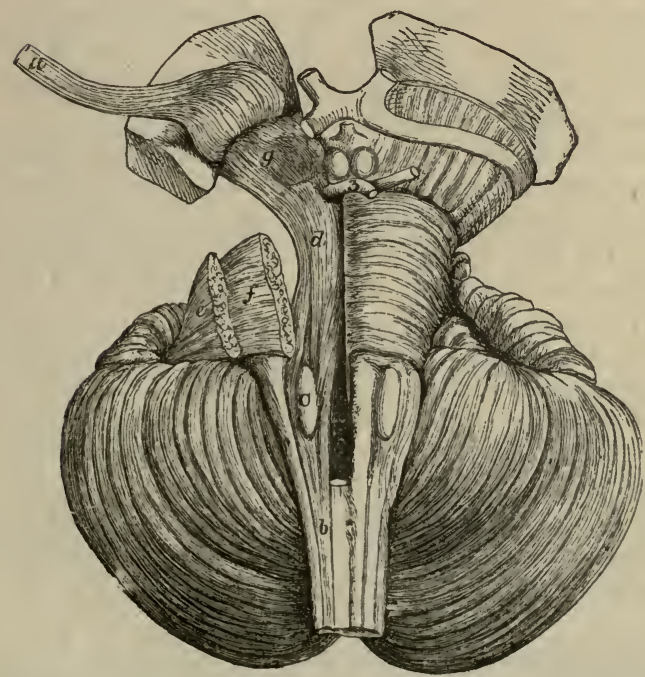

a. Anterior pyramid fibres, cnt through, and raised as far as the optic thalamus.

b. Lateral tract of the medulla oblongata.

c. Olivary body, and fillet of the olivary body issuing at the upper end.

d. Fibres of the lateral tract in the pons and crus cerebri.

e. Superficial, and

$f$. Deep transverse fibres of the pons, cut through, and turned ontwards.

g. Locus niger in the crus cerebri, between the fibres of the lateral traet and pyramid.

Fibres of the Lateral Tract and of the Olivary Body.

When the olivary body has been sliced obliquely, a very thin wavy layer of gray substance surrounding a nucleus of whitish matter will be apparent: this is the corpus dentatum (fig. $58, f^{\prime}$ ). It forms a thin capsule or bag, having a zigzag outline in a section, with the dilated part 
towards the surface, and the narrowed part or neck open and directed backwards near the middle line. It consists of small, nucleated, and ramified nerve cells. ${ }^{1}$

The nucleus is the yellowish white substance filling the eapsule; and from it and the capsule issue transverse fibres, which unite the olivary bodies across the middle line (fig. $58, g$ ) : this uniting band is close below the anterior median fissure.

The fillet is a narrow band of fibres, which ascends from the olivary body to the cerebrum (fig. 57). It is formed in part by longitudinal fibres of the lateral tract which diverge to inclose the corpus olivare, and in part by fibres derived from the sac.

The restiform body (fig. $54,{ }^{3}$ ) is continuous inferiorly with the posterior column of the cord. Superiorly it bends outwards to the cerebellum without entering the pons.

The posterior pyramid runs below into the posterior median column of the cord, and is directed above along the floor of the fourth ventricle, joining the fibres of the lateral tract of the same side.

The decussation of the medulla oblongata (pyramids) (fig. $56 ; b$ ) occupies the anterior groove of the oblong medulla, at the distance of threequarters of an inch from the pons. It is about a quarter of an inch in length, and is constructed by the crossing of three or four bundles of fibres from each side.

In this intercommunication the fibres are derived, according to Mr. Clarke, from all the constituent parts of the half of the spinal cord of the opposite side except from the anterior column; but the fibres from the lateral column of that side, blended with offsets from the contiguous gray substance, form the chief portion of the decussation.

Arched or commissural fibres of the medulla. In each half of the medulla oblongata are fine transverse fibres, both on the exterior and in its substance. At the middle line the fibres of opposite sides unite, and give rise to a commissure between the halves (raphé).

The superficial transverse fibres (fig. 58, s, and fig. 54), more marked in some bodies, issue from the nucleus in the restiform body (Clarke), and advance over the surface of the olivary and pyramidal bodies to the anterior fissure, where they enter the half of the medulla of the same, and of the opposite side. Below the olivary body they form oftentimes a distiact band, the fibre arciformes (fig. 54).

The deep transverse fibres (fig. $58, h$ ) are to be seen with the microscope on thin hardened transverse sections; they begin behind in nuclei of the posterior pyramid and restiform body (Clarke), and penetrate between the longitudinal fibres, and through the corpus olivare, as they reach forwards to enter the raphe in the middle line.

The raphé (fig. 58, $h$ and $g$ ), in which the arched fibres meet, occupies the middle line of the medulla above the decussation of the pyramids, and serves as the commissure between the halves of the medulla and the olivary bodies.

Gray matter of the medulla oblongata. In the medulla oblongata there are the remains of the gray matter of the spinal cord; and some special deposits. Cross sections of the part when hardened would be required to see them.

1 The arrangement of the fibres in the sac is most complicated, and a fuller account may be obtained by consulting Mr. Clarke's Paper in the Philosophical Transactions for 1858 . 
Remains of gray matter of the spinal cord. At the lower part of the medulla the central gray matter resembles that in the spinal cord (see fig. 126), but it undergoes the following changes: ${ }^{1}$ The posterior cornu increases in bulk, and extends towards the side of the cord, where it appears on the surface, in front of the restiform body, as the gray tuberele of Rolando (tuberculo einero) (fig. $58, t$ ). Higher in the medulla it becomes the chief nucleus of the sensory root of the fifth nerve.

The piece of the gray commissure behind the central canal of the cord joins, above, the nueleus of the vagus nerve, and contributes to the auditory nucleus : and the piece in front of the canal is laid bare in the floor

Fig. 58 .

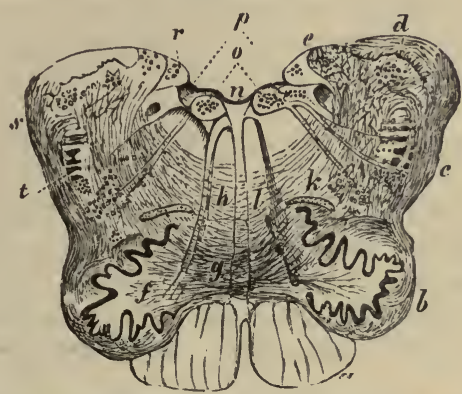

Transperse Section of the Medulta Oblongata above the Middie of thr Olivary Body (Clarke).

a. Anterior pyramid.

$b$ and $c$. Lateral column.

d. Restiform body.

e. Posterior pyramid.

$f$. Corpus olivare with roots of the ninth nerve piercing it.

g. Olivary commissure

$h$. Deep transverse or commissural fibres of the medulla meeting in the raphé (a few more are added in this cut from a second drawing). k. Accessory olivary nucleus.

l. Other gray deposits inside the olivary body.

$n$. Floor of the fourth ventricle covered by epithelium.

o. Nnclei of the ninth nerve.

$p$. Nuclei of the vagus and glosso-pharyngeal nerves.

$r$. Nucleus of the auditory nerve.

8. Superficial transverse fibres.

$t$. Remains of the gelatinous substance (tuberculo cinereo).

of the fourth ventricle by the inclination outwards of the restiform bodies; it disappears above the fasciculi teretes.

Special deposits of gray matter. Other masses of gray substances are deposited in the medulla, both in front and behind: those behind are near the floor of the fourth ventricle, and serve as nuclei of origin for certain nerves; whilst those in front are interspersed amongst the fibres continued from the lateral columns of the cord.

Nuclei at the back of the medulla. A nucleus for the hypoglossal nerve is deposited in firont of the central canal, and extends upwards into the floor of the fourth ventricle close to the median sulcus (fig. 58,o).

'The nurleus of the accessory part of the spinal accessory nerve is placed opposite that of the hypoglossal, behind the central eanal.

The nucleus of the vagus begins on a level with the fourth ventricle

1 The description of the arrangement of the gray matter in the medulla oblongata is a summary of the facts contained in Mr. Clarke's paper in the "Transactions of the Royal Society for 1858." 
(fig. 58, $p$ ), and is continuous below with that of the accessory nerve. On the surface it forms a pyriform swelling along the inner side of the posterior pyramid, and limits laterally the calamus scriptorius.

Above, this sinks under the auditory nucleus, and joins a nucleus for the glosso-pharyngeal nerve in a line with it.

Above the last two nerves is another collection of cells serving as a nucleus for the auditory nerve. This projects on the lateral part of the medulla (fig. $58, r$ ).

At the front of the medulla. Outside the pyramid is the gray layer of the olivary body (fig. 58, $f^{\prime}$ ) already described (p. 185). Behind this is another separate, elongated, and flattened yellowish streak, the accessory olivary nucleus (fig. $43, \mathrm{k}$ ); while at the inner part of the olive, near the raphé, is a second collection (fig. 58, l), which is broken up into pieces. Both of the last deposits Mr. Clarke considers to be but parts of the cut folds of the corpus dentatum.

\section{PONS VAROLII.}

The poxs, or axnular protuberance (pons Varolii, nodus encephali) (fig. 54), is situate above the medulla oblongata, and between the hemispheres of the cerebellum. In its natural position in the skull it lies below the opening in the tentorium cerebelli. It is nearly of a square shape, though it is rather widest from side to side, and measures two inches in the last direction.

The anterior surface is grooved along the middle line, and is reeeived into the basilar hollow in the base of the skull. By the opposite surface the pons forms part of the floor of the fourth ventricle.

The upper border is longest and most eurved, and arches over the cerebral peduncles; and the lower border overlays the medulla oblongata. On each side is the crus cerebelli, whose fibres radiate over the surface.

Structure. In the pons are alternating strata of transverse and longitudinal fibres (fig. 56):-The transverse set are continuous with the fibres of the crus cerebelli, much gray matter being interspersed: and the longitudinal are prolonged from the medulla oblongata.

Dissection. The transverse superficial fibres of the pons being divided along the line of the pyramidal body of the right side (fig. 56), may be turned outwards so as to denude the longitudinal fibres $(c)$ of the pyramid; and this first set of longitudinal fibres, having been eut across alrealy in the medulla oblongata, may be raised as far as the upper border of the pons. Beneath them will appear the second or deep set of transverse fibres of the pons (fig. $57, f$ ).

The deep transverse fibres may be cut through outside the pyramidal (fig. 57); then the deep longitudinal tibres from the lateral column and the posterior pyramid $(d)$ will appear. Amongst this last set of longitudinal fibres is the fillet of the corpus olivare, which the dissector should trace upwards from that bodv.

The superficial fibres of the pons can be seen on the side that is untouched.

The transverse fibres of the annular protuberance (fig. 56), are collecter chiefly into two strata-a superficial and deep, which are united in the middle line: they are commissural fibres of the cerebellum, and are derived from the crus or middle peduncle of that body. There are a few other transverse, which serve also as a commissure. 
The superficial set (fig. $56, f$ ) are mostly horizontal, but some from the upper margin of the pons descend obliquely over the others.

The deep layer (fig. 57, $f$ ) is thickened, and contains much gray matter between its fibres.

The longitudinal fibres consist of two sets, viz., one from the anterior pyramidal body; and another from the lateral tract and the posterior pyramid, to which a slip is added from the corpus olivare. The fibres are not continued simply through the pons, but are increased in number by the addition of others (peduncular) which, beginning in the upper twothirds of the pons, join them on the outer side.

The fibres of the anterior pyramid (fig. 56, c) pass through the pons between the two sets of transverse fibres, but not as one mass, for they are divided into a number of small bundles in their progress. Much increased in number, the fibres enter the crus cerebri at the upper border of the pons, and construct that faseiculated surface of the peduncle $(d)$, which is now uppermost.

The fibres of the lateral column and posterior pyramid are altogether deeper than the transverse fibres of the pons (fig. $\tilde{5} 7, d$ ), and are mixed up with gray matter; they are also more numerous than the preceding set. They project elose to the middle line, in the floor of the fourth ventricle, and form the eminence of the fasciculus teres (fig. $55,{ }^{2}$ ); from that spot they are continued upwards to the crus cerebri, of which they form the deeper or cerebral part (fig. $57, d$ ). In the pons a band from the olivary fasciculus is added to these fibres.

The olivary fasciculus (fillet, fig. 57) divides into two slips the pons. One passes backwards to the upper (in this position deeper) part of the crus cerebri, and ends in and beneath the corpora quadrigemina (p. 208). The other is continued to the crus cerebri with the fibres of the lateral column.

Commissure of the pons. At the back of the pons there is a commissure between the halves, opposite the deep longitudinal fibres. It consists, like that of the medulla oblongata, of fine anterior-posterior and transverse tibres.

\section{SECTION IV.}

\section{DISSECTION OF THE CEREBRUM.}

The cerebrum, or the great brain, is the largest of the subdivisions of the encephalon, and weighs on an average $49 \mathrm{oz}$. in the male, and $44 \mathrm{oz}$. in the female. It fills the upper part of the cavity of the skull; and its under surface would correspond with an oblique line on the head from the eyebrow to the articulation of the jaw ; and from this point to the occipital protuberance.

Taking the general form of the cranium, the cerebrum is convex on the upper aspect, and uneven on the lower. It consists of two hemispheres, which are placed side by side, and are partly separated by a median or longitudinal fissure. Across the middle line the halves are united by certain interior parts (commissures), as well as by several connecting structures at the under surface. Superiorly the surface of the hemisphere is 
without any large cleft; but inferiorly it is divided into two by a transverse fissure-that of Sylvius.

Under Surface or Base of the Cerkbrum (fig. 59). At its under part the cerebrum is very irregular, in consequence of its fitting into inequalities in the base of the skull; and on this aspect the separation into hemispheres is not so complete as on the upper, for the median fissure exists only at the front and back. The following objects are to be recognized at the base of the brain along the middle line.

Fig. 59.

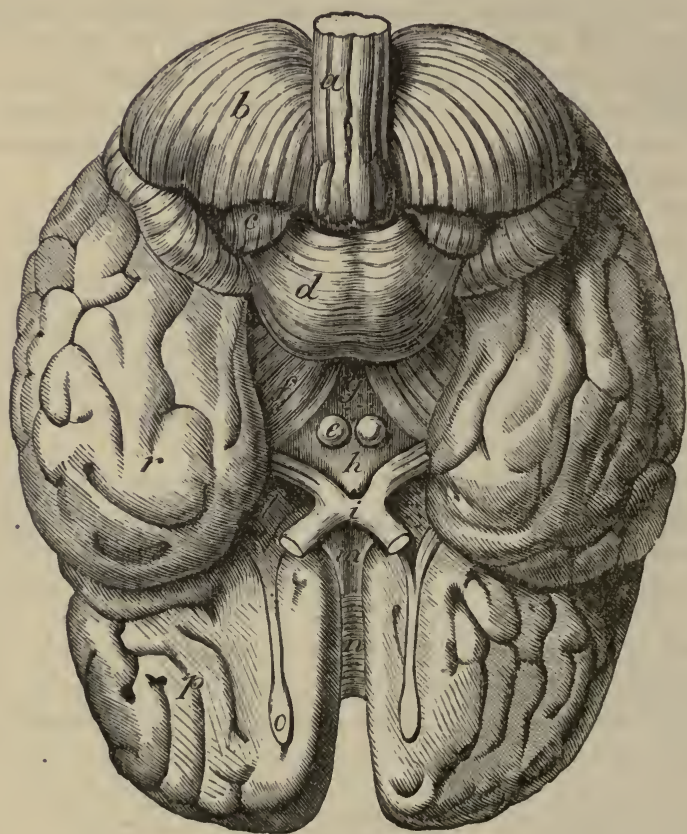

UNDER SURFACE OF THE BRAIN.

b. Cerebollam.

r. Temporo-sphenoldal lobe of the cerebrum.

$p$. Frontal lobe of the cerebrum: the great fissure between the two lobes, is the Sylvian.

\section{a. Medulla oblongata.}

d. Pons Varolii.

f. Crus cerebri.

g. Locus perforatus posticus.

e. Corpus albicans.

h. Tuber cinereum. $i$. Commissure of the optic nerves.

$l$. Locus perforatus anticus.

$m$. Lamina clnerea.

n. Lower end (rostrum) of the corpus callosum in the great median flssure; the point in front where it bends is named genu. On each side of the lamina cinerea a narrow white band is continued from the end of the corpus callosum: this is the flllet of the corpus callosum.

Immediately in front of the pons $(d)$ are two large white masses, the peduncles of the cerebrum (crura cerebri, $f$ ), one belonging to each hemisphere; and between is a space perforated by vessels, which is named locus perforatus posticus $(g)$. Outside the peduncle is the optic tract $(i)$; and between it and the inner part of the hemisphere is a fissure leading into the lateral ventricle.

In front of the peduncles are placed two white bodies like peas, the 
corpora albieantia $(e)$; and anterior to these a grayish mass, called tuber cinereum $(h)$. From the tuber cinereum a conical reddish tube, the infundibulum, descends to the pituitary body in the sella Turcica of the sphenoid bone.

Anterior to the tuber cinereum are the converging optic tracts with their commissure $(i)$. Beneath the commissure lies a thin grayish layer (lamina cinerea, $m$ ); and still further forwards is the great longitudinal fissure between the hemispheres, with the white corpus callosum $(n)$ in the bottom of it.

At the inner end of the transverse fissure (Sylvian) across the hemisphere, is another spot, perforated by vessels, and distinguished by the name substantia perforata, or locus perforatus anticus $(l)$.

Peduncle of the cerebrum (crus cerebri, $f$ ). This is a large, white, stalk-like piece, which reaches from the upper border of the pons to the under part of the cerebral hemisphere of the same side, near the inner margin. In the natural position the two peduncles fill the opening in the tentorium cerehelli. Each is about three-quarters of an inch long, and widens as it approaches the cerebrum. Crossing its outer surface is the optic tract; and between the crura of opposite sides is the interpeduncular space, which contains the locus perforatus, the corpora albicantia, and the tuber cinereum.

Structure. The peduncle consists of longitudinal fibres, continuous with the longitudinal fibres of the pons, which inclose here a mass of graty matter between them.

Dissection. For the purpose of showing the structure of the crus, on the right side (fig. 57), the optic tract should be divided, and the fibres continuous with the anterior pyramid of the medulla oblongata should be raised as far into the crus as the optic thalamus. In this proceeding the mass of gray matter (locus niger) will appear, and beneath it will be seen a second or deeper set of longitudinal fibres.

The superficial fibres, which form the under or free part of the crus, are continued from the anterior pyramidal body (fig. $56, c$ ). They are longitudinal in direction, and coarse in texture, and are directed upwards ralliating to the cerebrum. The surface of the peduncle, which is composed of these fibres, is called the fasciculated portion, or the crust.

The deeper fibres are also prolonged to the cerebrum. They are derived chiefly from the lateral tract and posterior pyramid of the medulla oblongata, with a slip from the olivary fasciculus (fig. $57, d$ ). Others come from the cerebellum, and mix with the former: some of these decussate across the middle line ${ }^{1}$ (p. 215). The fibres obtained from these four sources are situate beneath (as now seen) the gray matter: besides being deeper they are finer than the superficial set, and inclose much gray substance: the part of the crus formed by them is named tegmentum.

The gray matter (locus niger) of the crus (fig. $57, y$ ) forms a thin layer, which reaches nearer the inner than the outer margin of that body ; it is convex towards the free surface, but concave in the opposite direction.

The posterior perforated spot (pons Tarini, $g$ ) is situate between the peduncles of the cerebrum; in the area of the space is a layer of grayish

1 M. Foville describes a median commissure for the medulla oblongata, pons, and cerebral peduncles, which is composed of the interweaving of filles of opposite sides. See the work of M. Foville, entitled: Truite complet del'Anatomie, $\oint^{\circ}$. du Système Nerceux cérébro-spinal, p. 323, 1844. 
matter, and numerous vessels penetrate it. This structure forms the hinder part of the floor of the third ventricle.

The corpora albicantia (corp. mamillaria, $e$ ) are two small, white bodies, about the size of peas, which are constructed in greater part by the crura of the fornix. If one, say the right, is cut across, it will be seen to contain gray matter. In front of them is the gray mass of the tuber cinereum.

The median eminence of the tuber cinereum $(h)$ forms part of the third ventricle, and is continuous with the gray substance in that cavity. In front of it are the optic tracts and commissure, and from its centre projects the following.

The infundibulum (funnel) is a conically-shaped tube which reaches from the tuber cinereum to the upper part of the posterior lobe of the pituitary body. It consists of a layer of gray matter, surrounded by the pia mater; and it is lined by the ependyma of the third ventricle, as far as it is pervious. In the foetus this tube is open between the third ventricle and the pituitary body, but in the adult it is closed inferiorly.

The pituitary body will be very imperfectly seen when it has been dislodged from its resting-place : therefore it should be sometimes examined in the base of the skull by removing the surrounding bone. Its use is unknown.

It is situate in the hollow (sella Turcica) on the sphenoid bone, and consists of two lobes, anterior and posterior. The anterior is the largest, and is hollowed out behind, where it receives the round posterior lobe. In the adult this body is solid, and firm in texture; but in the foctus it is loollow, and opens into the third ventricle through the infundibulum.

Structure. It is firm and reddish externally, but softer and yellowish internally. In it are nucleated cells, mixed with a granular semi-fluid substance; and the whole is contained in roundish spaces, which are constructed by a stroma of areolar tissue with bloodvessels (Sharpey).

Dissection. To see the lamina cinerea and the anterior termination of the corpus callosum, the hemispheres are to be gently separated from each other at the fore part.

The lamina cinerea $(\mathrm{m})$ is a thin concave layer of gray substance, which gradually tapers forwards from the tuber cinereum to the anterior termination of the corpus callosum. This stratum closes the anterior part of the third ventricle, and is continuous laterally with the anterior perforated spot. In consequence of its great thinness, this structure is often broken through in removing the brain.

The corpus callosum $(n)$, bent in front, is continued horizontally backwards in the longitudinal fissure to the lamina cinerea, and ends in two white narrow bands, the fillets or peduncles of the corpus callosum: each band is continued on wards by the side of the lamina cinerea to the anterior perforated spot. To the anterior bend of the corpus callosum the term knee (genu) is applied, and to the prolonged central part the appellation rostrum has been given. Laterally the corpus callosum reaches into the frontal lobe, and forms part of the floor of the lateral ventricle.

Anterior perforated spot (substantia perforata antica, $l$ ) is a space near the inner end of the fissure of Sylvius, which is situate between the frontal and temporo-sphenoidal lobes of the cerebrum, and external to the optic tract. On the inner side it is.continuous with the lamina cinerea; and crossing it, from within outwards, is the fillet of the corpus callosum. 'This space is gray on the surface; it corresponds with the corpus striatum 
in the interior of the brain, and is perforated by numerous vessels for that borly.

Position of the part. Now the base of the cerebrum has been dissected, the brain should be turned over for the examination of the upper part. Something should then be placed beneath the front, in order that it may be raised to the same level as the back; and a rolled-up cloth should loosely encircle the whole, to support the hemispheres.

Upper Surface of the Cerebrum. On the upper surface the cerebrum, taken as a whole, is oval in form, and is convex in its outline, in accurdance with the shape of the skull.

A median longitudinal fissure divides the cerebrum incompletely into two halves. At the front and back the hemispheres are quite separated by it; but at the middle and under parts they are united by connecting pieces, the largest of which is the white corpus callosum. In it the falx cerebri is lodged.

Each hemisphere is larger in front than behind. Its outer surface is convex; but the inner is flat, and in contact in front with the opposite half. On the upper aspect the surface of the hemisphere is divided by fissures into lobes, and on the under aspect it is cleft into two large pieces by the fissure of Sylvius. The superficies of the hemisphere is marked by tortuous eminences, the projections on it being named convolutions or gyri, and the intervening depressions, sulci or anfractuosities. ${ }^{1}$

Fissures of the Hemisphere. The larger fissures separate the lobes, and the smaller sulci mark the extent of particular convolutions. The fissures dividing the hemisphere into lobes are the three following:-

The fissure of Sylvius ( $S$, fig. 60 ) begins below at the anterior perforated spot, and directed out between the frontal and temporo-sphenoidal lobes, divides into two parts, anterior and posterior.

The anterior limb, ' $S$, ascends for a short distance into the frontal lobe.

The posterior, limb, the continuation of the fissure, is directed obliquely upwards and backwards to about the middle of the outer face of the hemisphere. At its extremity it is sometimes divided into smaller sulci.

Fissure of Rolando ( $R$, fig. 60). Beginning above, about half way along the hemisphere, in or near the longitudinal fissure of the cerebrum, it is prolonged outwards between the frontal and parietal lobes nearly to the posterior part of the Sylvian fissure,-about the middle of that limb.

The external parieto-occipital fissure (opposite $P(O$, fig. 60) begins in the median longitudinal fissure half way between the preceding and the hinder end of the hemisphere. It is rery variable in extent, being sometimes an inch long, and at others only a slight indentation ; but it may be always recognized by its continuity with the perpendicular f.siure on the inner face of the hemisphere (fig. 62).

Lobes of the Hemispiere. Each hemisphere is divided into five lobes, which have the following names and limits :-

The frontal lobe ( $\mathrm{Fr}$, fig. $6(1)$ forms the anterior half of the hemisphere. It is limited below by the fissure of Sylvius, $S$, and behind by the fissure

1 In the following description of the surface anatomy of the cerebrum I have followed chiefly the arrangement of Professor Turner in his paper "ON THE Coxvolutions of the Human Cerebrum;" and to him I am indebted for permission to copy the wood-cuts employed in illustration of his publication. 
of Rolando, ${ }^{1} R$. Its under part, which rests on the orbital plate, has been called the orbital lobule.

The parietal lobe (Par, fig. 60) is placed behind the preceding, and reaches down to the Sylvian fissure. It is about half as long as the firontal. In front it is bounded by the fissure of Rolando, $R$, and behind by the parieto-occipital $\left(\begin{array}{ll}P & O\end{array}\right)$. The upper and linder part, elose to the median fissure, is named the parietal lobule $\left(5^{\prime}\right)$.

Fig. 60 .

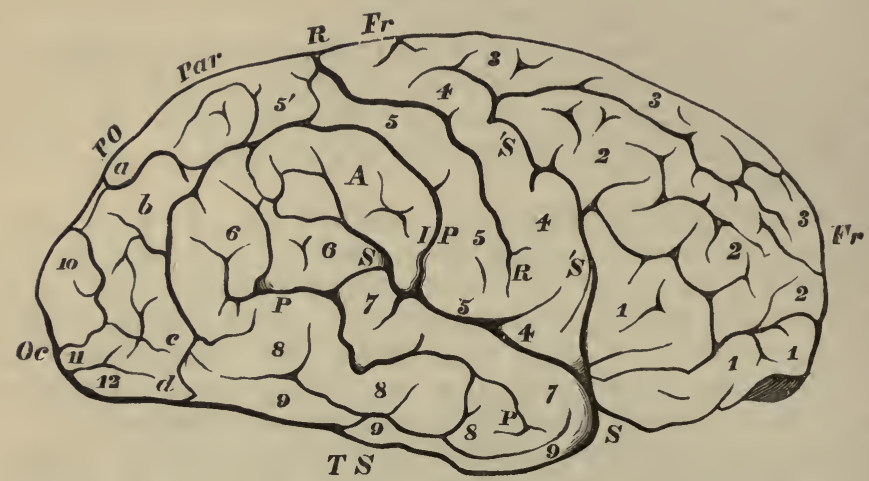

Lobes of thi Hemisphere, Axd Convolctions and Figstres of the OUTER SURFACE.

Fr. Frontal lobe.

Prr. Parietal lobe.

Oc. Occipital lobe.

T S. Teinporo-sphenoidal lobe.

$R$. Fissure of Rolando.

S. Posterior.

'S. Ascending limb of the Sylvian fisstre.

$P$ O. Place of the external parieto-occipital fissure which is not visible in a side view.

$I P$. Intra-parietal sulcus.

P. Parallel sulcus-
1 , Inferior ;
2 , middle; and
3, superior frontal gyrus;

4 , ascending frontal; and

5 , ascending parietal gyrus ;

$5^{\prime}$, parietal lobule;

6 , angular gyrus;

7 , superior ;

8 , middle; and

9 , inferior temporo-sphenoidal gyrus ;

10, superior ;

11 , middle; and

12, inferior occipital couvolution.

A. Supra-marginal convolutiou-

$a$, first ;

$b$, second;

$c$, third; and

$d$, fourth, annectant gyrus.

The occipital lobe (Oc, fig. 60) constitutes the pointed end of the hemisphere, and measures about a fifth of the whole. In front it is separated from the parietal lobe by the parieto-oceipital fissure $(P O)$, but below it blends with the following lobe. It rests on the tentorium. On the inner surface is a triangular piece, the occipital lobule $\left({ }^{25}\right.$, fig. (62).

The temporo-sphenoidal lobe ( $T S$, fig. 60 ) projects into the middle fossa of the base of the skull. It is situate behind the fissure of Sylvius, and below the parietal and oceipital lobes. The outer surface is in contact with the cranium, and the opposite is supported mainly on the tentorium.

The central lobe or the island of Reil ( $C$, fig. 61) lies in the sylvian fissure, and is concealed by the overlapping of the frontal and temporo-sphenoidal lobes. On separating those lobes it will be seen to be bounded in

1 By some the anterior limb of the fissure ' $S$ is made the hinder bound of the lobe; but this is not so good an arrangement as that in the text. 
front and behind by the limbs of the Sylvian fissure, and externally by a deep groove separating it from the frontal and parietal lobes. It is of a triangular form with the apex down.

Convolutions of the Cerebrum. In different brains the convolutions vary slightly in form, and even in the two hemispheres of the same cerebrum they are not exactly alike : but there is always similarity enough for the recognition of the chief eminences. Each lobe possesses convolutions, but these run into each other by means of smaller gyri, either on the surface of the brain or at the bottom of the sulci; and the student may experience some difficulty at first in defining the limits of each. It is in the smaller gyri that the greatest variation will be found.

A. Convolutions of the Outer Surface. About the middle of the hemisphere are two straight vertical convolutions, one on each side of the fissure of Rolando, $R$, which will serve as a starting point. In front of those two the convolutions are longitudinal; and behind they take an oblique direction to the back of the brain.

a. The frontal convolutions (fig. 60) form two sets, one on the outer, the other on the under surface of the frontal lobe: those on the outer aspect are four, viz., one vertical or posterior, and three longitudinal or anterior, as follows :-

The ascending frontal $\left({ }^{4}\right)$ is the vertical, straight convolution, which bounds in front the Rolando fissure. It reaches down from the median to the sylvian fissure (posterior limb). Along the anterior border it is joined by the three frontal convolutions; and below it unites with the most anterior convolution of the parietal lobe round the lower end of the fissure of Rolando, $R$.

The three longitudinal frontal convolutions are much subdivided and blended, and are separated by two intervening sulci. They are named superior $\left({ }^{3}\right)$, middle $\left({ }^{2}\right)$, and inferior $\left({ }^{1}\right)$ : they communicate behind by secondary gyri with the ascending frontal $\left({ }^{4}\right)$, the highest having often two processes; and are directed forwards one outside another to the anterior extremity of the hemisphere.

The under or orbital surface of the frontal lobe, concave, is represented in fig. 61. Near the inner margin is a sulcus, the olfactory, lodging the olfactory nerve ; and internal to it is the lower end of the marginal convolution $\left({ }^{17}\right)$. External to the sulcus lies a convolution, which is pointed behind, but widened and subdivided in front, and incloses smaller gyri and sulci within its coil: this has been subdivided into three parts, an inner $\left({ }^{2}\right)$, a posterior $\left({ }^{3}\right)$, and an external $\left({ }^{6}\right)$.

$b$. The parietal convolutions (fig. 60), like the outer frontal, are four in number; an anterior, or ascending, which is rertical along the fissure of Rolando, and three directed back from it.

The ascending parietal $\left({ }^{5}\right)$, narrow and straight, limits behind the fissure of Rolando, and reaches from the middle line to the hinder limb of the Sylvian fissure, $S$. Above, it runs into the parietal lobule, ${ }^{5 \prime}$; and below, it joins the ascending frontal round the lower end of the fissure of Rolando. Behind it is separated from the other gyri of the parietal lobe by a suclus, I $P .1$

The parietal lobule $\left({ }^{5 \prime}\right)$ appears to be an appendage to the upper end of

1 The intraparietal sulcus ( $I P$, fig. 60, Turner), is placed between the ascending parietal and the supra-marginal convolution, $A$. Above, it is directed back near the upper part of the hemisphere, separating the parietal lobule $\left(5^{\prime}\right)$ and the supra-marginal convolution, $A$. 
the ascending convolution, and is continued back along the upper margin of the hemisphere as far as the parieto-occipital fissure. Subdivided on the surface into two chief parts it is joined behind to the occipital lobe by the small annectant gyrus $(a)$. To its outer side lies the upper part of the intraparietal sulcus ; and here it joins usually the following convolution, $A$.

The supra-marginal convolution $A$, lying outside and below the preceding, is interposed between the intraparietal sulcus, $I P$, and the Sylvian fissure (outer end). Variable in shape it joins, below, the ascending parietal convolution $\left({ }^{5}\right)$; it may communicate above with the parietal lobule, and behind with the following.

The angular convolution $\left({ }^{6}\right)$, rery complicated and not well defined, is placed at the extremity of the hinder limb of the Sylvian fissure, and is

Fig. 61 .

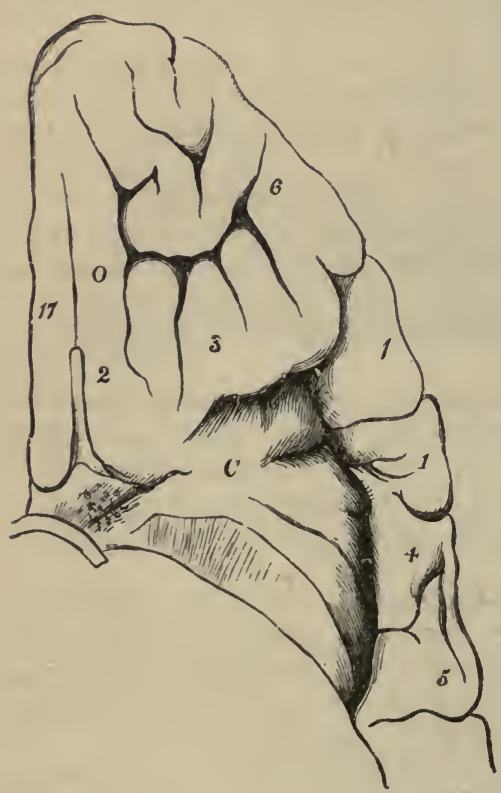

VIEW OF THE ORBITAL LOBELE AND THE Central Lobe.

C. Island of Reil or median loke.

O. Olfactory sulcus.

2. Internal; and

6. External orbital convolution. posterior ;

17. Marginal convolution of the hemisphere. composed of two or three pieces Above it is the parietal lobule; and below, the temporo-sphenoidal lobe which it joins. In front lies the supra-marginal convolution; and behind, the occipital lobe, with which it blends by the small annectant gyrus $(b)$.

$c$. 'The occipital convolutions (fig. $60)$ are small and very much divided, so that their outline is uncertain. They are three in number, lying one above another, and separated by sulei, something like the frontal gyri at the opposite end of the hemispliere.

The upper $\left({ }^{10}\right)$, forming part of the margin of the longitudinal fissure, receives an annectant gyrus from the parietal lobule.

The middle $\left({ }^{11}\right)$, crossing outwards the hemisphere, has two annectant gyri to other convolutions ; one $(b)$ joining it above to the angular convolution, and another (c) passing to the middle temporosphenoidal convolution.

The inferior $\left({ }^{12}\right)$ occupies the tip of the hemisphere between the upper and under surfaces. At the inner end it is continuous with the upper gyrus; and at the outer end with the inferior temporo-sphenoidal convolution $\left({ }^{9}\right)$ by an annectant gyrus $(d)$.

d. The temporo-sphenoidal convolutions (fig. 60 ), three in number, run from above down, and are separated from one another by two sulci.

The superior $\left({ }^{7}\right)$ bounds posteriorly the horizontal limb of the Sylvian fissure. At the upper end it is connected by a gyrus with the angular convolution.

The middle $\left({ }^{8}\right)$ is separated from the first by the parallel sulcus $(P)$. 
Above, it blends commonly with the angular convolution, and is connected to the middle occipital convolution by an annectant gyrus $(c)$.

The inferior $\left({ }^{9}\right)$, less well marked than the other two, forms part also of the inner surface of the temporo-sphenoidal lobe. By the upper end it is united to the third occipital convolution by an annectant gyrus $(d)$.

The parallel sulcus ( $P$, fig. 60 ), named from its position to the Sylvian fissure, extends from the lower end of the temporo-sphenoidal lobe to the angular conrolution.

e. The convolutions of the central lobe ( $C$, fig. 61), about six in number, are straight for the most part, and are separated by shallow sulci : they are directed upwards from apex to base of the lobe. The posterior gyri are the longest and broadest, and the anterior joins the convolution of the under surface of the orbital lobule.

B. The convolutions on the inner surface of the hemisphere (fig. 62) are generally well defined; but some being so long as to reach beyond the extent of a lobe, the arrangement of them in lobes cannot be followed, as on the exterior.

Dissection. Without the use of a separate hardened hemisphere, the parts now to be deseribed will not be seen satisfactorily. If the student possesses only one brain, he may bring into view much of the inner surface by cutting of the left hemisphere as low as the white corpus callosum in the median fissure.

Convolution of the corpus callosum, gyrus fornicatus $\left({ }^{18}\right)$, is long and simple, and arches round the body from which it takes its name. Beginning at the base of the brain in the anterior perforated spot, it bends backwards in contact with the corpus callosum $(\mathrm{Cal})$, and below the back of that body blends by a narrowed part with the uncinate convolution $\left({ }^{19}\right)$ of the temporo-sphenoidal lobe. Anteriorly a sulcus separates it from the following convolution; and smaller gyri often connect the two across that sulcus.

The marginal convolution $\left({ }^{17}\right)$ is named from its position on the edge of the median fissure. Its extent is rather more than half the length of the hemisphere, for it begins in front at the anterior perforated spot, and terminates near the back of the corpus callosum, just behind the fissure of Rolando. It is much subdivided both internally and externally; and on the under part of the frontal lobe (tig. 61) it lies internal to the olfactory sulcus. Between it and the preceding convolution is situate the callosomarginal suleus $(i)$ which marks its hinder limit.

The calloso-marginal sulcus ( $i$, Huxley), designated from its situation, begins in front below the corpus callosum, and ends behind, near the back of the same body, by ascending to the edge of the hemisphere. Smaller gyri uniting the two bounding convolutions, frequently interrupt it, and secondary sulci are prolonged from it into the same convolutions.

The quadrilateral lobule $\left({ }^{18}\right)$ reaches from the marginal convolution in front to the parieto-occipital fissure behind. It is much divided by sulci, and projects above to the edge of the hemisphere; it joins below the gyrus fornicatus.

The occipitat lobule $\left({ }^{25}\right)$ is triangular in shape, with the base upwards, at the margin of the hemisphere. Measuring about an inch and a half in depth, it lies between the internal parieto-occipital fissure, $P O$, and the calcarine sulcus $(l)$. Sulci running from apex to base divide it into four or five narrow convolutions. 
Internal perpendicular or parieto-occipital fissure ( $P O$, fig. 62) separates the two preceding lobules. Continuous with the external fissure of the same name, it opens below into the following.

The calcarine sulcus l, (Huxley) is directed across the back of the hemisphere below the level of the corpus callosum, and ends in front at the gyrus fornicatus $\left({ }^{18}\right)$, whose hinder limit it marks. It receives above

Fig. 62 .

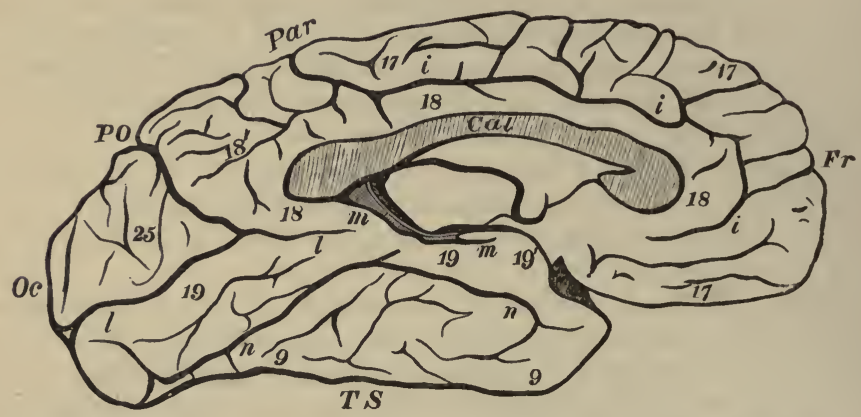

Convolutions and Fissures on the InNer face of the Hemisphere.

$P$. O. Internal parieto-occipital fissure.

Cal. Corpus callosum, cut.

i. Calloso-marginal sulcus.

l. Calcarine sulcus.

$m$. Dentate sulcus.

n. Collateral sulcus.

17. Marginal gyrus.
18. Convolution of corpus callosum.

18'. Quadrilateral lobule.

19. Uncinate gyrus.

19'. Crotchet or hook of the ancinate gyrns.

25. Occipital lobule.

9. Inferior temporo-sphenoidal gyrus partly seen.

the internal perpendicular fissure; and it sinks into the posterior cornu of the lateral ventricle, forming the eminence of the hippocampus minor.

Internal temporo-sphenoidal convolutions (fig. 62) are two in number, viz., the uncinate and dentate, and occupy the tentorial surface of the hemisphere.

The uncinate or hippocampal convolution $\left({ }^{19}\right)$ is prolonged from the posterior end of the hemisphere nearly to the tip of the temporo-sphenoidal lobe. It is somewhat narrowed in the middle, where the gyrus fornicatus blends with it ; and is enlarged at each end, especially at the posterior where it is subdivided by sulci. Below it is a long curved sulcus, the collateral $(n)$; and above it are the calcarine $(l)$, and the dentate sulcus $(m)$. From the anterior extremity a narrow part (19/, uncus) is prolonged back for half an inch on the inner side, like a hook.

Below the uncinate convolution is part of the inferior temporo-sphenoidal convolution $\left({ }^{9}\right)$, before described, which forms the lower edge of the temporo-sphenoidal lobe, appearing more largely on the inner than on the outer face.

The collateral sulcus n, (Huxley) courses along the lower border of the uncinate convolution; it projects into the inferior cornu of the lateral ventricle, and gives rise to the prominence of the eminentia collateralis. secondary sulci emanate from it, and it is often interrupted by cross gyri.

The dentate sulcus $m$, (Huxley) is the deep groove at the upper edge of the uncinate convolution $\left({ }^{19}\right)$, and corresponds with the prominence of the 
hippocampus major in the descending cornu of the lateral ventricle. Upwards it is limited by the corpus callosum ( $\mathrm{Cal}$ ) and downwards it intervenes between the hook and the body of the uncinate convolution.

In the dentate sulcus is the gray substance of the hemisphere, which presents a notehed border at the inner edge of the temporo-sphenoidal lobe; this has been called the dentate convolution, and will be better seen in a subsequent stage of the dissection of the brain.

Structure of the convolutions. Each convolution is continuous with the interior of the brain on the one side (base); and is free on the surface of the brain on the other side, where it presents a summit and lateral parts. On a cross section it will be seen to consist externally of gray cerebral substance as a cortical layer, which is continued from one eminence to another over the surface of the hemisphere; and internally it is composed of white brain substance-the medullary part, which is derived from the fibrous mass in the interior. The cortical layer is composed of two, or in some parts of three strata, which are separated by their intervening paler layers; and an outer white stratum, which covers the surface, is most marked over the internal and lower portions of the uncinate convolution.

Interior of the Cerebrum. The cerebrum consists on each side of a dilated part or hemisphere, and of a stalk or peduncle. In the interior is a large central space, which is subdivided into smaller hollows or ventricles by the before-mentioned connecting pieces. And the whole, except the peduncle, is surrounded by a convoluted erust.

In conducting the dissection of the cerebrum, the student will learn the form and situation of the sereral constituent parts, and the connections between these by means of fibres.

Dissection. Supposing both hemispheres entire, the left is to be cut off to the level of the convolution of the corpus callosum. When this has been done, the surface displays a white central mass of an oval shape (centrum ovale minus), which sends processes into the several convolutions. In a fresh brain this surface would be studded with drops of blood escaping from the divided vessels.

Next, the convolution of the corpus callosum is to be divided about the middle, and the two pieces are to be thrown backwards and forwards. Under it lies a thin narrow band, the covered band of Reil, which bends down before and behind the corpus eallosum.

The same steps of the dissection are to be taken on the opposite side; and the tops of the hemispheres being removed to the level of the corpus callosum, the transverse fibres of that body are to be defined as they radiate to the convolutions.

Now a much larger white surface comes into view (larger ovan centre), which has been named eentrum ovale, Vieussens; and the white mass in each hemisphere is seen to be continuous, across the middle line, through the corpus callosum.

The corpus callosum reaches from the one-half of the cerebrum to the other, and forms the roof of a space (lateral ventricle) in each hemisphere. Between the halves of the brain, where it occupies the longitudinal fissure, it is of small extent, being about four inches in length, and somewhat arched from before backwards. It is narrower in front than behind, and extends nearer to the anterior than the posterior part of the cerebrum.

In front the corpus callosum is bent to the base of the brain (fig. $59, n$ ), as before deseribed (p. 192); and behind it ends in a thick roll, which is connected with the subjacent fornix. 
On the upper surface the fibres are directed from the hemispheres to the middle line-the middle being transverse, but those from the anterior and posterior parts oblique. Along the centre is a ridge or raphé, and close to it are two or more slight longitudinal white lines (nerves of Lancisi). Still further out may be seen other longitudinal lines (covered band), beneath the convolution of the corpus callosum, if all of them have not been taken away in the removal of that convolution. The longitudinal fibres in the middle line are continued downwards in front, and joining the covered band or fillet are continued to the anterior perforated spot.

Dissection. In order to see the thickness of the corpus callosum, and to bring into view the parts in contact with its under surface, a cut is to be made through it on the right side about half an inch from the central ridge : and this is to be extended forwards and backwards, as far as the limits of the underlying ventricle. Whilst cutting through the corpus callosum, the student may observe that a thin membranicorm structure lines its under surface.

The corpus callosum is thicker at each end than at the centre, in consequence of a greater number of fibres being collected from the cerebrum ; and the posterior part is the thickest of all. Connected with its under surface along the middle is the septum lucidum or partition between the ventricles (fig. 63, b), and still posterior to that is the fornix.

This body is the chief commissural part of the halves of the brain, and reaches laterally even to the convolutions, but its fibres are not distinct far in the hemisphere.

Dissection. The left lateral ventricle is to be now opened in the same way as the right; and to prepare for the examination of the cavity on the right side, as much of the corpus eallosum as forms the roof of the space is to be removed. A part of the ventricle extends down in the temporosphenoidal lobe towards the base of the brain ; and to open it, a cut is to be carried outwards and downwards, through the substance of the hemisphere, along the course of the hollow. (See fig. 63.)

VENTRICLES OF THE BRAIN. - The ventricular spaces in the interior of the cerebrum are derived from the subdivision of a large central hollow, and are five in number. One (lateral) is contained in each lemisphere; and these constitute the first and second. The third occupies the middle line of the brain near the under surface; and the small fifth is included in the partition between the lateral ventricles. The fourth is situate between the cerebellum and the posterior suriace of the medulla oblongata and pons.

The lateral ventricles (fig. 63) are two in number, one in each hemispliere; they are separated incompletely in the middle line by a septum, as they communicate by an aperture below that partition. 'The interior is lined by a thin stratum of areolar tissue covered by nucleated epithelium (the ependyma ventriculorum), with cilia at some spots.

Each is a narrow interval, which reaches into the anterior, posterior, and middle regions of the corresponding hemisphere. Its central part (body) is almost straight, but the extremities (cornua) are curved. Thus there are three cornua in each, which have the following disposition:'The anterior is clirected outwards from its fellow in the other liemisphere. 'Tle posterior or the digital eavity is much smaller in size, and is bent inwards in the occipital lobe towards the one on the opposite side. And the inferior cornu, beginning opposite the posterior fold of the corpus cal- 
losum $(a)$, deseends in a curved direction in the temporo-sphenoidal lobe, with the coneavity of the bend turned inwards.

For the purpose of examining its boundaries, the ventricle may be divided into an upper or horizontal, and a lower or descending part.

The upper or horizontal portion reaches from the frontal to the occipital lobe, and is shaped like the italic letter $f$.

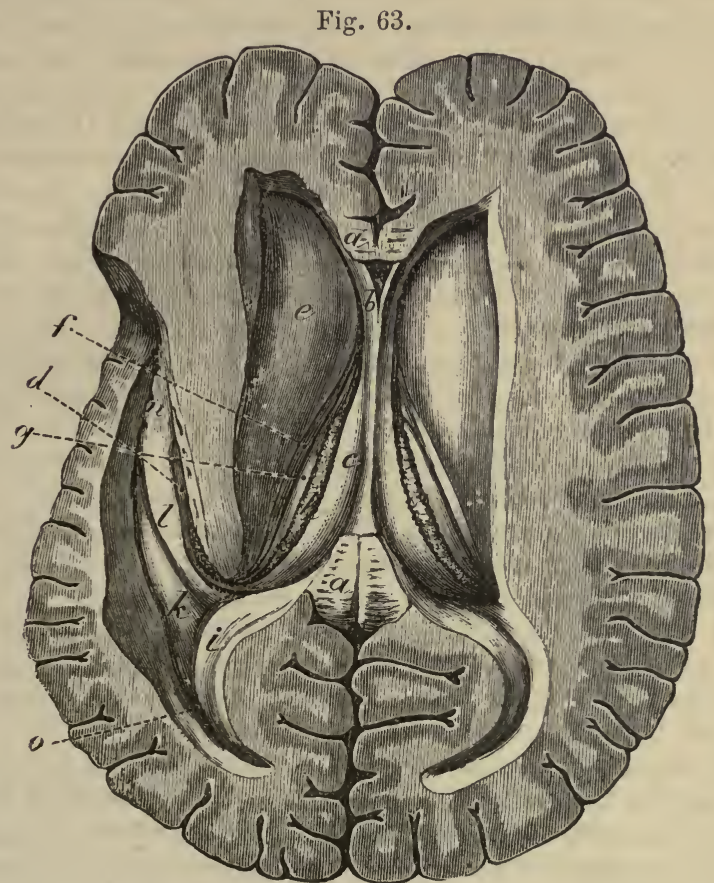

View of the Laterat Ventricles: on the left side the Descending Corye is laid OPEN. (From a cast in a museum of University College, London.)
a. Remains of the corpus callosum.
b. Septum lucidum, inclosing the small space of the fifth ventricle.
c. Fornix.
d. Posterior crus or tænia of the foruix.
e. Corpus striatum.
$f$. Trenia semicircularis.
g. Optic thalamus.
h. Choroid plexus.
$i$ Hippocampus minor.
$k$. Eminentia collateralis.
l. Hippocampus major.
o. Digital fossa.

The roof is formed by the eorpus callosum. The floor is irregular in outline, and presents from before backwards the following objeets:-first, a small piece of the under part of the corpus callosum; next, a large, gray body, the corpus striatum $(e)$; behind this, the large white projection, named optic thalamus $(g)$; and between the two last bodies is a white band $(f)$, trnia semicireularis. On the surface of the optic thalamus is a vascular fold of the pia mater $(h)$ - the plexus choroides, together with the thin white half of the fornix $(c)$. Close behind the thalamus is the beginning of a projection (hippocampus major) (e), in the floor of the deseending part of the lateral ventricle; and in the posterior cornu is an elongated eminence, the hippocampus minor (i). 
The inner boundary (septum ventriculorum) is a thin layer which is sometimes named septum lucidum (fig. $63, b$ ). Its extent corresponds with the central part of the corpus callosum. Below it and the fornix, opposite the front of the optic thalamus, is the aperture of communication (foramen of Monro) between the two lateral ventricles.

The lower or descending part of the ventricle winds beneath the optic thalamus, and forms a curve like the half-bent forefinger. The roof' is formed by the optic thalamus and the contiguous part of the hemisphere. In the floor is a large curved, convex eminence, somewhat indented at the end-the hippocampus major $(l)$; and along its concave margin is a thin white band-trenia $(d)$, which is prolonged from the fornix. External to the projection of the hippocimpus is another white eminence, the eminentia collateralis $(k)$, which tapers from above down. In this part of the ventricle is the vascular fringe of the plexus choroides.

The septum lucidum (fig. $63, b$ ), or the thin structure between the lateral ventricles, is translucent, and hangs vertically in the middle line along the anterior two-thirds of the corpus callosum. It is somewhat triangular in form, with the larger part turned forwards, and the pointed extremity backwards. Its surfaces look to the lateral ventricles. The upper border is attached altogether to the under surface of the corpus callosum; and the lower border is joined in part to the fornix $(c)$, but in front of that body it is inserted into the under or prolonged portion (rostrum) of the corpus callosum. The septum consists of two layers, which inclose a space-the fifth ventricle; and each layer is formed of white substance, with an external coating of gray matter.

Dissection. The space of the fifth ventricle will come into view by cutting through the part of the corpus callosum which remains in the middle line, and by detaching the anterior half from the septum lucidum, and raising it. (See fig. 63.)

The ventricle of the septum, or the fifth ventricle, is a triangular space in the fore part of the ventricular partition, where the depth is greatest. Like the septum containing it, its largest part is in front. Its surface has an epithelial covering like that in the lateral ventricles. In the adult it is closed; but in the foetus it opens inferiorly into the third ventricle between the pillars of the fornix.

Dissection. The fornix is to be next examined. To lay bare this body the posterior part of the corpus callosum should be detached with care from it, and thrown backwards; and the septum lucidum should also be removed from its upper surface.

The fornix, or arch (fig. $63, c$ ), is a thin white horizontal stratum beneath the corpus callosum, which, projecting on each side into the lateral ventricle, forms part of the floor of that cavity. Its central part or body is triangular in shape, with the base turned backwards; and it is continuous with the rest of the brain by processes or erura before or behind.

To the upper surface of the body, along the middle line, the septum lucidum is attached. Each border is free in the corresponding lateral ventricle, where it rests on the optic thalamus; and along it lies the ehoroid plexus. At its posterior part it joins the corpus eallosum in the middle line, whilst on each side it sends off a small riband-like bandtania hippocampi $(d)$, along the concave margin of the hippocampus major. At the anterior part it is arched over the foramen of Monro, opposite the front of the optic thalamus, and ends likewise in two pro- 
cesses or crura, which will be afterwards followed to the corpora albicantia and the optic thalami (p. 208).

If the formix be cut across near its front, the foramen of Monro will be opened, and the descending anterior pillars will be seen (fig. 64). When the posterior part is raised, it will be found to be supported on a process of the pia mater, named velum interpositum. And near its base (on the under aspect) between the two offsets of the tæniæ hippocampi, is a triangular surface, which is marked by transverse lines: the part which is so defined has been ealled the lyra (fig. 64).

The fornix may be described as consisting of two bands, right and left, which are united for a certain distance in the central part or body. According to this view each band, commencing in the optic thalamus, passes over the formen of Monro, and after forming the body of the fornix, is continued as a distinct piece to the uncinate convolution.

The foramen of Monro is the interval beneath the anterior part of the fornix, which opens on each side by a slit between the edge of the fornix and the optic thalamus. In it the plexus choroides lies; and through it the lateral ventricles communicate with one another and with the third ventricle.

Floor of the Lateral Ventricle.-The student may leave untouched, for the present, the membrane on which the fornix rests; and proceed to examine, on the right side, the different bodies which have been enumerated as constituting the floor of the lateral ventricle.

The corpus striatum (superior ganglion of the cerebrum) (fig. 63,e) is the large gray body in the front of the lateral ventricle. It is placed opposite the island of Reil in the fissure of Sylvius; and it has received its name from the striated appearance of a vertical section.

Dissection. To see the composition of the corpus striatum, the student should slice off obliquely the upper and outer part until certain white fibres crossing it obliquely from within outwards are reached. The knife should then be carried through this layer of white fibres until another mass of gray substance, similar to the first, is arrived at.

The striate body is a pyriform mass of gray matter of considerable thickness, which is surrounded by the white substance of the hemisphere, except where it projects into the lateral ventricle. Its position is oblique with respect to the middle line of the brain, for the anterior part is near the septum of the ventricles, whilst the posterior is external to the optic thalamus. By means of the incision in the corpus striatum, white fibres can be seen to be directed through it in such a way as to divide the gray matter into two parts, one being situate in the ventricle (intra-ventricular) above the white fibres, and the other outside the ventricular space (extraventricular), below these fibres.

The intra-ventricular piece (nucleus caudatus) is shaped like a kite, and projects into the floor of the ventricle. The end, directed forwards, is large and rounded; whilst the opposite end is thin and pointed, and is continued backwards, outside the optic thalamus, to the roof of the descending cornu of the lateral ventricle. Numerous veins cover this part of the corpus striatum.

The extra-ventricular part (nuclens lenticularis) will be better seen, afterwards, by sections made from the outer side or from below. It is oval in form, but does not reach so far back as the other, and is bounded inferiorly by a white capsule; through it the anterior commissure of the brain passes very obliquely, as a subsequent dissection will show. 
The tania semicircularis (fig. $63, f$ ) is a thin and narrow white batnd of longitudinal fibres, which lies between the corpus striatum and the optic thalamus. In front this band becomes broader and joins the pillar of the fornix ; and behind it is continued, along with the pointed end of the corpus striatum, into the white substance of the roof of the descending cornu of the lateral ventricle. Superficial to the anterior part of the tania is a yellowish semi-transparent layer (lamina cornea); and beneath this pass some small veins from the corpus striatum, in their course to the veins of Galen.

The optic thalamus is only partly laid bare in this stage of the dissection, and its examination may be omitted till the third ventricle has been learnt.

The hippocumpus minor (calcar avis) resembles a cock's spur (fig. $63, i)$, as it lies in the posterior cornu of the ventricle. It is pointed at its posterior extremity, and is covered on the free surface by a medullary layer continuous with the corpus callosum. When it is cut across a gray stratum will be found beneath the white: and the eminence itself will be seen to be produced by the extension inwards of the calcarine sulens at the inner surface of the hemisphere (p. 198).

The hippocampus major (fig. $63, l$ ) is the eurved projection in the floor of the descending cornu of the lateral ventricle. Convex on the surface that looks to the cavity, this body is curved in the same direction as the cornu, and has its concavity turned inwards. The anterior extremity is the largest, and presents two or three indentations, which give it the appearance of the foot of a feline animal; it is named pes hippocampi.

All along the inner or concave margin is the small band or tania $(d)$ that is prolonged from the fornix; it ends below by joining the small recurved part of the uncinate convolution.

Dissertion. 'To examine more fully the hippocampus, the parts of the corpus callosum and fornix, which remain in the middle line, should be divided longitudinally, and the posterior part of the right hemisphere should be drawn away from the rest of the brain. When the pia mater has been removed from the inner side of the hippocampus, and this projection has been eut across, its structure will be manifest.

The hippocampus is covered on the ventricular surface by a medullary layer, with which the tænia or the band of the fornix blends. On its opposite surface is the hollow of the dentate sulcus on the exterior of the brain, which is filled with gray substance. Along the free margin of the hippocampus the gray matter projects in the form of a notehed ridge, the laminu dentata: this is external to the eavity of the ventricle, beneath the trenia, and has been named the dentate convolution (p. 199).

Transverse fissure of the cerebrum. By drawing the separated right hemisphere away from the crus cerebri and the optic thalamus, and replacing it, the dissector will comprehend the position, and the boundaries of the great cleft at the posterior part of the brain.

This fissure lies beneath the fornix, and opens into the lateral ventricle, on each side, along the edge of the fornix, from the foramen of Monro to the extremity of the descending cornu. The part of the slit entering the lateral ventricle is bounded by the edge of the fornix on the one side, and by the optic thalamus and crus cerebri on the other. A piece of pia mater projects into the transverse fissure, forming a horizontal central piece, velum interpositum, beneath the fornix (fig. $64,{ }^{8}$ ), and thinner lateral 
pieces with terminal fringes in the latter ventricles $(f)$. But the slit through which the membrane enters the rentricle is closed by the lining structure of that cavity being continued on the intruded part.

Fig. 64.

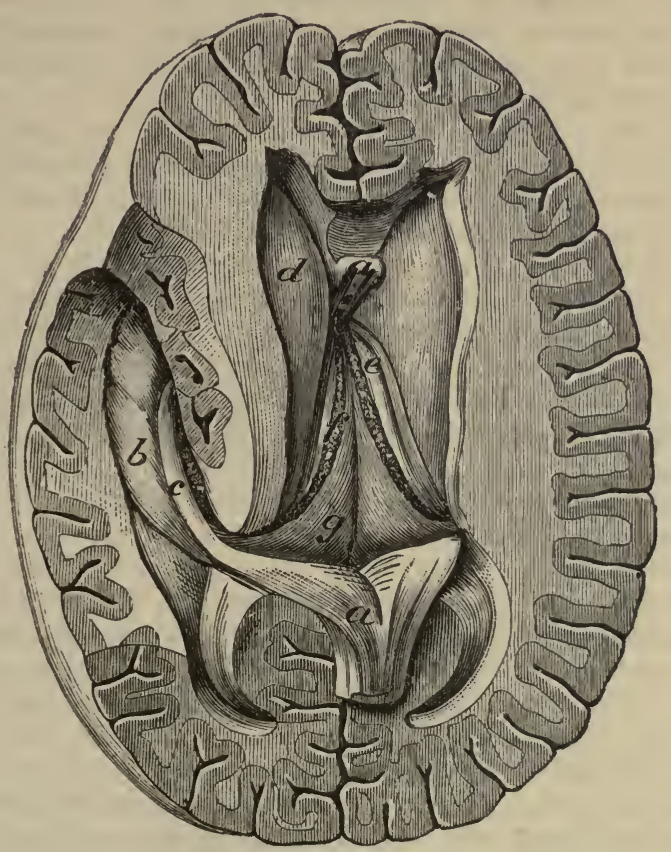

Second View of the Dissection of the Brais, the Fornix bfina Cut thrutgh in Front and RaIsed. (From a cast in the University College, London.)

\section{a. Fornix.}

b. Hippocampus major.

c. Truia hippocampi, or hiıder crus of the fornix.
d. Corpus striatum.
e. Optic thalamus.
$f$. Choroid plexus.
$g$. Velum interpositum.

Parts in the Midde Line of Cerebrum. The student is now to return to the examination of the parts in the centre of the brain, viz., the fold of pia mater and its vessels, with the third ventricle. At the same time the optic thalamus is to be seen.

The velum interpositum (fig. $(j 4, g$ ) is the central part of the fold of pia mater entering the great transverse fissure. 'Triangular in shape, it has the same extent as the body of the fornix, and reaches in front to the foramen of Monro. 'The upper surfice is in contact with the fornix, to which it supplies vessels. And the lower surface, looking to the third ventricle, covers the pineal body, and a part of each optic thalamus: underneath it in the middle line are the two choroid plexuses of the third ventricle. Along each side is another vascular roll of the membrane (choroid plexus).

The choroid plexus of the lateral ventricle (fig. $64, f$ ) is the red, somewhat rounded, and fringed margin of the piece of pia mater in the interior of the laternl ventricle, which extends from the foramen of Monro to 
the extremity of the descending cornu. Its lower end is larger than the upper. On its surface the choroid plexus is villous; and the villi, minutely subdivided, are covered by flattened nucleated epithelium, with the fat granules and pigment in the cells. ${ }^{1}$

Vessels of the velum. Small arteries have been alrearly traced to the velum and the choroid plexus from the cerebral and cerebellar arteries ( $p$. $175)$ : they are three on each side, and supply the surrounding cerebral substance. The veins of the choroid plexus receive branches from the ventricle, and end in the following.

Veins of Galen. Along the centre of the velum are placed two large veins with this name; they begin at the foramen of Monro, by the union of branches from the corpus striatum and the choroid plexus. Lying side by side in the membrane they are usually united into one at the posterior part of the velum; and by this they join the straight sinus.

Dissection. When the velum interpositum has been raised and thrown backwards, the third ventricle will be visible (fig. 68). In reflecting the piece of pia mater the student must be careful behind of the pineal body $(g)$, which would otherwise be detached, as it is surrounded by the membrane. On the under surface of the velum are the choroid plexuses of the third ventricle.

The choroid plexuses of the third ventricle are two short and narrow fringed bodies beneath the relum, which resemble the like parts in the lateral ventricle.

The third ventricle is in the interval between the optic thalami (fig. 68). Its situation is in the middle line of the cerebrum, below the level of the other ventricles with which it communicates; and it reaches to the base of the brain. Its boundaries and communications are the following :-

The roof is formed by the velum interpositum and the fornix. The floor is very oblique from behind forwards, so that the depth of the cavity is about an inch in front and half an inch behind: it corresponds with the parts at the base of the brain, which lie between the crura cerebri and the median fissure (fig. 59), viz., locus perforatus, corpora albicantia, tuber cinereum, commissure of the optic nerves, and lamina cinerea. On the sides of the cavity are situate the optic thalami $(b)$. In front of the space are the descending pillars of the fornix, with the anterior commissure of the cerebrum $(c)$ in the interval between them. Behind are the posterior commissure $(e)$ and the pincal body $(g)$. Crossing the centre of the ventricle, from one optic thalamus to another, is a band of gray matter-the soft commissure $(d)$.

This space communicates with the other ventricles of the brain in the following way:-In front it joins each lateral ventricle through the foramen of IIonro; and in the foetus it opens into the fifth ventricle. Behind is a passage beneath the posterior commissure into the fourth ventricle, which is named aqueduct of Sylvius. At the lower part, in front, there is a depression opposite the infundibulum (iter ad infundibulum).

The lining of the ventricle (ependyma) is continued into the neighboring cavities through the different apertures of communication, and closes the iter ad infundibulum.

Gray matter of the ventricle. of the surface of the ventricle.

A stratum of gray matter covers most At the lower part of each optic thalamus

1 Particles of brain sand, like that in the pineal body, are sometimes present in the choroid plexus. 
it envelops the crus of the fornix, and ascends to the septum lucidum; and in the floor of the cavity it exists in abundance, entering into the corpora albicantia. In the middle of the space it reaches from side to side, and forms the soft commissure (d).

The anterior commissure of the cerebrum $(e)$ is a round bundle of white fibres about as large as a crow-quill, which passes through both corpora striata, and connects the opposite hemispheres. To see it in one-half of its extent, the following dissection should be made:-

Dissection. On the side on which the corpus striatum has been cut into, the commissure is to be followed into the interior of that body, by scraping away the intraventricular gray matter with the handle of the scalpel. 'The commissure may be seer then to perforate below the white fibres of the corpus striatum; and it should be followed through the extraventricular mass of gray matter of the same body.

The anterior commissure is fiee in the middle line for about the eighth of an inch, where it lies before the pillars of the fornix. Laterally it perforates the corpus striatum, passing in succession through the intraventricular gray mass, the white fibres, and the extra gray ventricular gray mass. Lastly, the commissure pierces the white layer bounding externally the corpus striatum, and spreads in the hemisphere over the inferior cornu of the lateral ventricle communicating with the temporo-sphenoidal and central lobes.

The posterior commissure of the cerebrum $(e)$ is smaller than the anterior, and is placed above the passage into the fourth ventricle. Laterally it enters the substance of the optic thalamus; and pierces this body to end in the hemisphere.

The connections of the thalamus opticus (inferior ganglion of the cerebrum) (b) will be best seen on the side on which the inferior cornu of the lateral ventricle has been opened. It has the form of a cube, and bounds the lateral and third ventricles.

The upper surface projects in the floor of the lateral ventricle, and is marked in front by a prominence-anterior tubercle, near the taenia semicircularis. The under surface forms part of the roof of the inferior cornu of the lateral ventricle, and into it the crus cerebri is inserted.

By its inner side it enters into the third ventricle; and along the upper part lies the peduncle of the pineal body. On the outer side are the cor pus striatum, the trenia semicircularis, and the substance of the hemisphere.

The anterior end looks to the foramen of Monro. And the posterior part, which is free in the inferior cornu of the lateral ventricle, presents inferiorly two small roundish tubercles, which are placed one outside and the other inside the bend (genu) of the optic nerve, and are named from their position to it, internal and external geniculate bodies. 211.

'The structure of the optic thalamus will be subsequently referred to, $p$.

The origin of the optic nerve can now be seen. At the back of the crus cerebri the optic tract receives fibres from the thalamus which it touches, and then divides into two terminal bands:-One of these is connected with the gray matter in the external geniculate body, and is continued onwarls to one of the corpora quadrigemina (nates); the other is connected with the internal geniculate body.

Dissection. The origin of the fornix in the optic thalamus may be followed out next. As a preparatory step the anterior commissure, the anterior part of the corpus callosum, and the commissure of the optic 
nerres, should be cut along the middle line, so that the left hemisphere can le separated from the other. On the left hemisphere the crus of the fornix is to be traced downwards through the gray matter of the third ventricle to the corpus albicans, and then upwards into the optic thalamus.

Anterior pillar of the fornix. The fornix begins in the thalamus near the tuberele on the upper surface. From this spot it descends in a curved direction to the corpus albicans, where it makes a turn like half of the figure 8 , and furnishes a white envelope to the gray matter of that body. 'The crus then ascends through the gray substance in front of the optic thalamus, and is applied to the like part of the opposite side to form the borly of the fornix. It is joined by bands of fibres firom the tania semicircular:s and peduncle of the pineal body.

The pineal body and the corpora quadrigemina, which are placed behind the third ventricle, may be next examined.

Dissection. All the pia mater should be earefully removed from the surface of the quadrigeminal bodies, especially on the right side, on which they are to be seen. 'The posterior part of the hemisphere of the same side may be taken away.

The pineal gland (conarium) is a small conical body (fig. $68, g$ ), which is situate above the posterior commissure, and between the anterior pair of the corpora quadrigemina. In shape like the cone of a pine, it is about a quarter of an inch in length, and has the base or wider part turned forwards. It is connected to the optic thalami by two white bands,-peduncles of the pineal body $\left(f^{\prime}\right)$ : these begin at the base, and extending forwards, one on each side along the inner part of the thalamus, end by joining the crura of the fornix. At the base of the gland is a band of transverse white fibres which unites it which the posterior commissure.

'This body is of a red color and vascular, and incloses two or more cells containing a thick fl.:id, with amyloid bodies, and a calcareous material (brain sand) consisting of particles of phosphate and carbonate of lime, and phosplate of magnesia and ammonia. ${ }^{1}$ In its substance are large pale nucleated cells.

The corpora quadrigemina (fig. 68) are four small bodies, which are arranged in pairs, right and left, and are separated by a median groove. Each pair is situate on the upjer aspect of the cerebral peduncle of the same side.

The anterior eminence ( $h$, nates) is somewhat larger than the posterior, from which it is separated by a slight depression; it is oblong from before backwards, and sends forward a white band to join the optic tract and thalamus.

The posterior eminence ( $i$, testis) is rounder in form and whiter in color than the preceding: it has also a lateral white band which is directed beneath the corpus geniculatum internum, and blends with the peduneular fibres in the thalamus opticus.

These bodies are small masses of gray substance enveloped by white, and are placed on the band of the fillet which forms the root of the aqueduct of Sylvius. The processes (brachia) to the optic thalamus are accessory parts to the peduncular fibres of the cérebrum (p. 209).

Fillet of the olivary body. If the upper margin of the cerebellum be

1 These particles are referred to by Kölliker, as pathological products ; and the concentrically arranged masses amongst them are said to be incrustations of fibrin coagula. 
pulled aside, a white band, about a quarter of an inch in width, will be seen to issue from the transverse fibres of the pons, and to bend upwards over the peduncle of the cerebellum to the corpora quadrigemina (fig. $\left.65,{ }^{4}\right)$.

This is the upper or commissural piece of the fillet (p. 128), which passes beneath the corpora quadrigemina, and joins with the similar part of the opposite side over the Sylvan aqueduct.

Structure of the Cerebrum. In each cerebral hemisphere three principal sets of constituent fibres are recognized, viz., diverging, transverse, and longitudinal. The former are in part derived from the spinal cord; while the two latter, joining distant pieces of the cerebrum, are considered to be only connecting or commissural in their office.

Peduncular or diverging fibres (fig. 56). In the crus cerebri two bundles of longitudinal fibres are collected; these are separated, in part, by gray matter, and are derived from the medulla oblongata (p. 191).

Dissection. A complete systematic view of the diverging fibres cannot be now obtained on the imperfect brain. At this stage the chief purpose is to show the passage of the radiating fibres from the crus through the two cerebral ganglia.

To trace the diverging fibres onwards beyonds the crus cerebri, and through the corpus striatum, the nucleus caudatus of this body should be scraped away (fig. 65); and the dissection should be made on the left side on which the striate body and the optic thalamus remain uncut. In this proceeding the pecten of Reil comes into view, viz., gray matter passing between the white fibres in the corpus striatum, and giving the appearance of the teeth of a comb.

On taking away completely the prolonged part of the nucleus caudatus, others of the same set of fibres will be seen issuing from the outer side of the optic thalamus, and radiating to the posterior and inferior lobes.

After tracing those fibres, the upper part of the optic thalamus may be taken away at the posterior end, to denude the accessory bundles to the peduncular fibres, from the corpora quadrigemina and the superior peduncle of the cerebellum (fig. $65,{ }^{1}$ ): the last band lies beneath the corpora quadrigemina.

Their arrangement (fig. $65,{ }^{6}$ ). Some of the diverging fibres radiate from the peduncle of the cerebrum to the surface of the hemisphere, passing in their course through the two cerebral ganglia (optic thalamus $\left(^{6}\right.$ ) and corpus striatum $\left({ }^{9}\right)$ ), and they form a conically-shaped bundle, whose apex is below and base above.

The fibres forming the free or fasciculated part (crust) of the peduncle (fig. 56) pass through the striate body. The fibres on the opposite aspect, which form the tegmentum (fig. 57, d), are transmitted through the under part of the optic thalamus, and through the corpus striatum, reaching as far forwards as, but much farther back than those of the crust.

In the thalanus and the corpus striatum the fibres are greatly increased in number. The upper or sensory set receive also accessory bundles from the superior pedunçle of the cerebellum (fig. $65,{ }^{1}$ ) in the crus cerebri (p. 191); and from the pair of the corpora quadrigemina, and the corpora geniculata of the same side, in the thalamus.

On escaping from the striate body and the thalamus. the fibres decussate with the converging fibres of the corpus callosum, and radiate then into the anterior, middle, and posterior parts of the cerebral hemisphere, forming the corona radiata. In the hemisphere the fibres are continued to 
the convolutions : their expansion in the hemisphere resembles a fan bent down in front and behind, forming thus a layer which is concave on the under side.

Their extent. All the fibres of the peduncle do not reach the surface of the brain, for some end in the corpus striatum and the optic thalamus, especially in the former. And some of the fibres in the convolutions begin in the ganglionic bodies before mentioned, and extend to the surface of the hemisphere. ${ }^{1}$ Thus, in addition to the fibres continued throughout, viz., from the crus to the surface, some unite the peduncle of the cerebrum with the ganglia, and others connect the ganglia with the convolutions on the exterior.

Fig. 65.

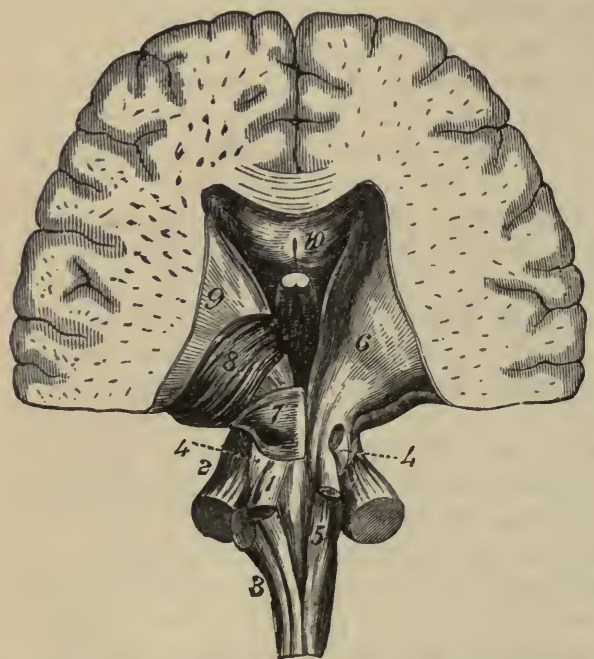

1. Superior peduncle.

2. Middle, and 3 , inferior peduncle of the cerebellum.

4. Process from the fillet of the olivary body to the corpora quadrigemina : on the right side it is cut and reflected.

5 Posterior pyramid.

6. Continuation of the lateral tract into the optic thalainus.

7. Corpora quadrigemiua.

8. Optic thalamus.

9. Corpus striatum.

10. Corpus callosum.

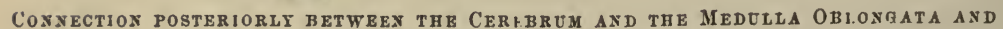
CereBellum.

Their source. The fibres thus entering inferiorly the cerebrum through its peduncle, and continued thence to the periphery of the hemisphere, are derived from the component pieces of the medulla oblongata except the restiform body (fig. 57), viz., from anterior pyramid, lateral column and olivary body, and posterior pyramid (p. 183) : they serve to connect the spinal cord with the cerebrum. The decussation between opposite sides has been before referred to (p. 186).

The transverse or commissural fibres connect the hemispheres of the cerebrum across the middle line. They give rise to the great commissure or the corpus callosum (p. 210): and to the anterior and posterior commissures (p. 207). Those bodies have been already examined.

Longitudinal fibres. Other connecting fibres pass from before backwards, uniting together parts of the same hemispliere. The chief bands of this system are the following, the fornix, the tænia semicircularis, and the peduncles of the pineal body. Other longitudinal fibres may be enu-

1 According to some authors none of the fibres of the peduncle reach farther than the corpus striatum and the optic thalamus. 
merated on the upper and under surfaces of the corpus callosum, along the middle line, together with the band of the convolution of the corpus callosum : these fibres are connected with the anterior perforated spot of the base of the brain.

Structure of the optic thalamus. The thalamus is about an inch deep, and the following is a summary of its structure, as displayed in the previous dissections.

The upper and inner half is formed chiefly of gray matter, with which the undermentioned white bands are connected: Thus through it pass the fornix in front, and the posterior commissure behind; whilst the peduncles of the pineal body lie along the inner side, and the tænia semicircularis along the outer.

The lower and outer part consists mainly of white fibres directed upwards, and these are derived from the peduncle of the cerebrum inferiorly, and fiom the peduncle of the cerebellum and the corpora quadrigemina superiorly : to the hinder part of them two slips of fibres are added from the corpora geniculata.

The corpora geniculata contain gray substance inside. Into these bodies fibres of the optic tract enter; and from each issues a band to join the fibres of the crus cerebri. They seem to serve as accessory ganglia to the peduncular fibres of the cerebrum.

Corpus striatum. By slicing through the corona radiata on the left side, so as to bring into view the extraventricular nucleus of the corpus striatum, the extent and form of that mass, and the situation of the anterior commissure in it, will be apparent.

Crus cerebri. By a vertical section through the left peduncle of the cerebrum, the disposition and the thickness of the two layers of its longitudinal fibres; and the situation and extent of the locus niger between them, may be perceived.

\section{Section V.}

\section{THE CEREBELLUM.}

Dissection. The cerebellum (fig. $59, h$ ) is to be separated from the remains of the cerebrum, by carrying the knife through the optic thalamus so that the small brain, the corpora quadrigemina, the crura cerebri, the pons, and the medulla oblongata, may remain united together.

All the pia mater is to be carefully removed from the median fissure on the under surface; and the different bodies in that fissure are to be separated from one another. Lastly the handle of the scalpel should be passed along a sulcus at the circumference, which is continued from the crus, between the upper and under surfaces.

The cerebellum, little brain (fig. 66), is flattened from above down, so as to be widest from side to side, and measures about foul inches across. This part of the encephalon is situate in the posterior fossæ of the base of the skull, beneath the tentorium cerebelli. Like the cerebrum, it is incompletely divided into two hemispheres; the division being marked by a wide median groove along the under surface, and by a notch at the posterior border which receives the falx cerebelli. 
Upper Surface. On the upper aspect the cerebellum is raised in the centre (fig. 68), but slopes towards the circumference. There is not any median sulcus on this surface; and the halves are united by a central constricted part-the superior vermiform process. Separating the upper from the under surface, at the circumference, is the horizontal fissure, which is wide in front, and extends backwards from the pons Varolii to the middle line of the cerebellum.

The UNDER SURFACE is convex, being received into the fossa of the base of the skull, and is divided into hemispheres (fig. 66) by a median hollow (vallecula).

Fig. 66.

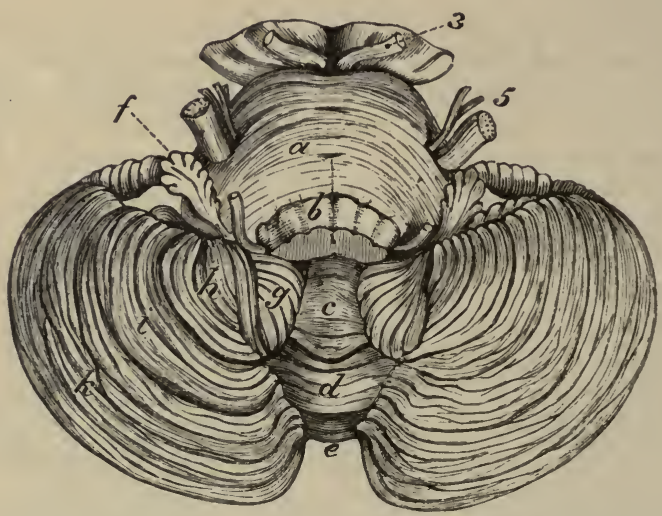

USDBR PART OP THE CRREBELLUM, SEEN FROM BEHIND, THE MEDUlla OblONGATA, $b$, BEING CET AWAY IN GREATER PAKT.

a. Pons Varolii.

b. Medulla oblongata, cut through. $c$ to $e$. Inferior vermiform process, consisting of ;-c. Uvula. d. Pyramid. e. Commissural laminæ.

LOBBS OP EACH HALF OF THE CEREBELLUM ON THE UNDER SURFACE.

\section{f. Subpeduncular.}

g. Amygdaloid.

h. Biventral.

t. Slender.

\author{
k. Posterior. \\ 3 Third nerve attached to the crus cerebri. \\ 5. Two roots of the fifth nerve attached to the \\ side of the pons Varolil.
}

The central fissure, or the vallecula, is wider at the middle tlan at either the anterior or the posterior end, and receives the medulla oblongata. In the bottom of the fissure is a mass named inferior vermiform process (fig. $66, c$ to $e$ ), which corresponds with the central part connecting the halves of the cerebellum on the upper surface. 'The two vermiform processes constitute the general commissure of the halves of the cerehellum.

Constituents of the rermiform process. In the inferior vermiform process are the following eminences, which may be easily separated from one another with the handle of the scalpel :-Mnst anteriorly is a narrow body, the uvula (fig. $66, c$ ), which is named from its resemblance to the same part in the throat; it is longer from before backwards than from side to side, and is divided into laminæ. Its anterior projection into the fourth ventricle is named nodule, or laminated tuberele (fig. 67); and on the side is a band of gray matter with ridges and sulci, the furrowed band (fig. $67, d)$, which unites it with 'the almond-like lobe of the hemisphere. Connected with the nodule is a thin white layer,-the medullary velum 
$(b)$; but this, and the furrowed band will be seen in a subsequent dissection (p. 214). Behind the uvula is a tongue-shaped borly, named pyramid (fig. $66, d$ ), which is elongated from side to side, and is-marked by transverse laminæ. Further back are certain transverse pieces $(e)$, extending between the posterior lobes of the hemispheres, of which they were considered by Reil to be the commissures.

Lamince. The surface of the cerebellum is covered by plates or laminæ (fig. 68), instead of convolutions, which form segments of circles with their convexity directed backwards. On the upper aspect the anterior laminx pass from the one hemisphere to the other, with only a slight bending forwards in the superior vermiform process; but those on the under aspect join the sides of the different commissures in the median fissure.

Sulci. Between the laminæ are sulci, which are lined by the pia mater, and reach to different depths: the shallower separate the laminx; but the deeper limit the lobes, and reach downwards to the white substance of the interior. Here and there the sulci are interrupted by cross laminæ.

Structure of the lamina. On cutting across the lamina of the upper surface on the right side they will be seen to possess a white internal, and a gray external layer (fig. 68). The white part is derived from a central medullary mass; and dividing, like the branching of a tree, it ends in small lateral offsets which enter the subdivisions of the laminæ.

Besides the white stalk of the laminæ, derived from the central mass, there are other white fibres which pass from one lamina to another beneath the sulei.

The stratum of gray matter enveloping the white substance resembles the cortical covering of the convolutions of the cerebrum. It is constructed of two strata, inner and outer, which can be distinguished by a difference in their color. The superficial stratum is elear gray, and about equal to the other in thickness; but the deeper one is of a rust-color, and is generally thickest in the hollows between the laminæ. Between the two a layer of ramified cells (Purkinje) may be recognized with the microscope.

Lobes of the Hemisphere. Each hemisphere is subdivided into lobes on both aspects.

On the upper surface there are two lobes, anterior and posterior, which are separated by a sulcus, but the interval between them is not well marked. The anterior or square lobe extends back to a level with the posterior edge of the vermiform process; and the posterior reaches thence to the great horizontal fissure at the circumference.

On the under surface of the cerebellum (fig. 66), there are five lobes; and three of them are separated by sulci amongst the lamina of the hemisphere, but they are searcely nore distinct than the lobes on the upper surface. Beginning behind, the student will meet first the posterior lobe $(k)$, which joins the commissural laminæ behind the pyramid in the valley. Next in succession is the slender lobe $(i)$, which is connected with the posterior part of the pyramid, as well as with the other transverse lamina behind that body. And lastly, attached to the side of the pyramid, is the biventral lobe $(h)$.

The two other lobes, though smaller are more separate, and appear between the biventral lobe and the medulla oblongata:-One of these is the amygdaloid lobe $(g)$, which projects into the vallecula opposite the uvula, and touches the medulla oblongata. The other is a small pyramidal slip, 
which is directed outwards over (the under surface of the cerebellum being uppermost) the crus cerebelli, and is named flocculus, or subpeduncular lobe $(f)$.

Dissection. To see the flocculus and the posterior medullary velum, the biventral and slender lobes are to be sliced off on the left side, so that the amygdaloid lobe may be everted from the valley (fig. 67 ). 'The floc-

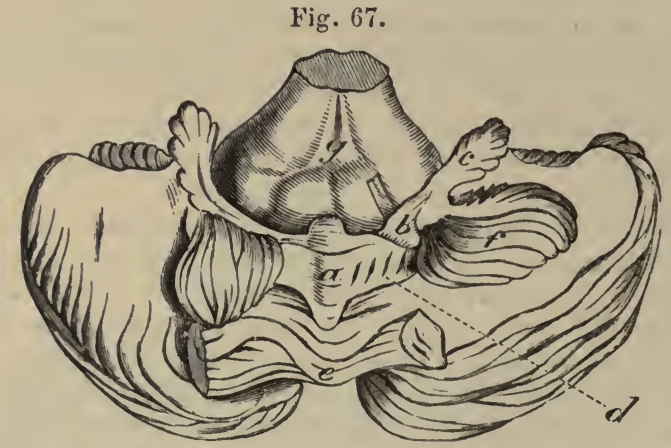

VIEW FROM BEHIND OF THE UNDER SURFACE OF THE CEREBELLUM, WITH SOME OF THE LOBES removed to Show the Posterior MedUllaky Velum.

a. Uvula.

e. Pyramid.

b. Posterior mednllary velum, with a bit of whalebone under it.

c. Subpeduncular lobe or flocculus. d. Furrowed band.

$f$. Amygdaloid lobe, turned aside.

g. Medulla oblongata raised, showing posterior surface.

culus is laid bare by this proceeding, and passing from it to the tip of the uvula is the thin and soft white layer of the posterior velum; beneath the last a bit of paper may be inserted. The furrowed band on the side of the uvula can be fully seen now.

Flocculus and medullary velum. The position of the flocculus to the crus cerebelli has been before mentioned. This body $(c)$ resembles the other lobes in structure, and may be considered a rudimentary lobe; for it is divided on the surface into laminæ, and contains a white medullary centre which furnishes offsets to the lamina.

Passing from the flocculus to the tip of the inferior vermiform process (nodule) is the half of a thin white layer $(b)$, the posterior medullary velum, which serves as a commissure to the floceuli. On each side this band is semilunar in form. Its anterior edge is free; but its posterior border is attached in front of the transverse furrowed band $(d)$. In front of the nodule the pieces of opposite sides are united.

Interior of the Cerebellum. In the cerebellum there is not any cavity or ventricle inclosed as in the cerebrum. In the interior there is a large white centre, corresponding with that of the cerebrum, which furnishes offsets to the laminx, and to other parts of the encephalon.

Dissection. For the purpose of seeing the medullary centre, with its contained corpus dentatum, remove all the lamina from the upper surface on the left side. This dissection may be accomplished by placing the scalpel in the horizontal fissure at the circunference, and carrying it inwards as far as the upper vermiform process, so as to detach the cortical stratum. If the corpus dentatum does not at first appear, thin slices may be made anteriorly till it is reached. 
Medullary Cextre. In the centre of each cerebellar hemisphere is a large white mass, containing in its substance a dentate body. From its surface offsets are furnished to the different laminæ. And from the anterior part proceeds a large stalk-like process, the crus cerebelli, which is subdivided into three pieces or peduncles, an upper for the cerebrum, a middle piece for the pons, and a lower one for the medulla oblongata.

The superior peduncle (processsus ad cerebrum) is directed forwards towards the corpora quadrigemina (fig. $65,{ }^{1}$ ). It is rather flattened in shape, and forms part of the roof of the fourth ventricle: between the processes of opposite sides the valve of Vieussens is situate. Its fibres, continuous behind with the inferior vermiform process, receive an offset from the interior of the corpus dentatum; and passing beneath the band of the fillet and the pair of the corpora quadrigemina of the same side, enter the optic thalamus, and are applied to the fibres of the crus cerebri ( $p$. 209).

Beneath the corpora quadrigemina the internal fibres of the peduncle are directed across the middle line, through the bundle prolonged from the fasciculus teres. ${ }^{1}$ In this way the fibres of each peduncle end partly in the same, and partly in the opposite hemisphere of the cerebrum.

Between the superior peduncles is a thin, translucent, white layer-the valve of Vieussens (vellum medullare anterius), which enters into the roof of the fourth ventricle (fig. $68, k$ ). It is thin and pointed anteriorly, but widens behind, where it is connected with the under part of the vermiform process. Near the corpora quadrigemina the fourth nerve $\left(^{4}\right)$ is attached to the surface of the valve; and close to the cerebellum the surface is marked by some gray transverse ridges.

The middle peduncle (processus ad pontem), commonly named the crus cerebelli (fig. $65,{ }^{2}$ ), is the largest of the three peduncular processes. Its fibres begin in the lateral part of the cerebellum, and are directed forwards to the pons, of which they form the transverse fibres, and unite with the peduncle of the opposite side. This peduncle is supposed to serve as a commissural or connecting band between the halves of the cerebellum.

The inferior peduncle (fig. $65,{ }^{3}$ ) (processus ad medullam) passes downwards to the medulla oblongata, and gives rise to the restiform body. Its fibres begin chiefly in the lamina of the upper surface of the cerebellum. It will be better seen when the fourth ventricle has been opened.

The fibres in the peduncles connect one cerebellar hemisphere with the cerebrum; with its fellow; and with the medulla oblongata of the same side, in the manner mentioned above.

The dentate body (corpus dentatum) is contained in the white mass of the eerebellum, and resembles the like part in the corpus olivare of the medulla oblongata. This body measures nearly an inch from before back, and is situate near the inner part of the white centre. It consists of a small plicated capsule, which, when eut across, appears as a thin, wary, grayish-yellow line; the bag is open at the interior part, and incloses a nucleus of whitish substance. Through its aperture issues a band of fibres from the nucleus to join the superior peduncle.

Dissection. One other section (fig. 68) must be made to show the fourth ventricle, and the structure of the vermiform process. 'The cerebellum still

1 This intercommunication was known to Reil, and was named "ansa" by him, but the decussation has been since noticed by Stilling, Ueber den Bau des Hirnknotens: 1846. 
resting on it under surface, let the knife be carried vertically through the centre of the vermiform processes; and then the structure of the central uniting part, as well as the boundaries of the fourth ventricle, may be observed on separating the halves of the cerebellum.

Fig. 68.

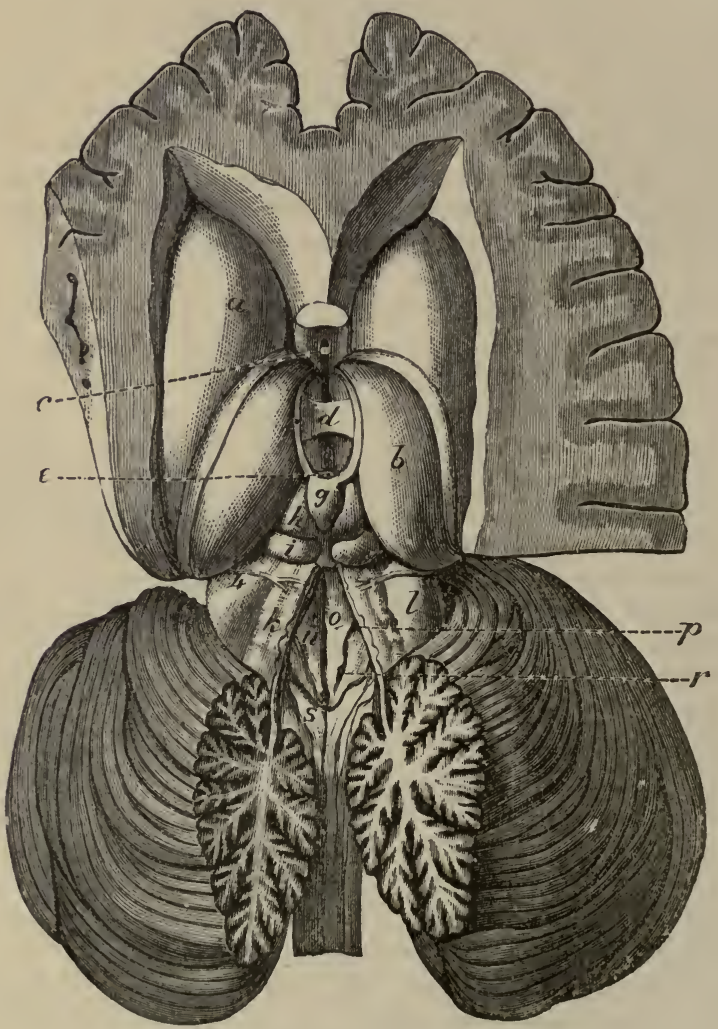

View of the Third and Fourth Ventricles.

The former being obtained by the removal of the velum interpositum; and the latterlyy dividing vertically the vermilorm process of the cerebellnm. (From a cast in the Museum of University College.) The third ventricle is the interval in the middle liue between the optic thulami, $b$.

a. Corpus striatum.

b. Optic thalamus.

c. Anterior commissure.

d. Middle or soft commissure.

e. Posterior commissure.

g. I'ineal body.

$f$. Peduncle of the pineal body.

$h$ and $i$. Left pair of the corporil quadrigemina.

The fourth ventricle, $u$, is at the back of the medulla oblangata. $k$. Valve of Vieussens.

$l$. Upper peduncle of the cerebellum.

o. Eminentia teres.

p. Anterior fossa.

$r$. Posterior fossa.

8. Posterior pyramid.

4. Origin of the fuurth nerve from the valve of Vieussens.

Structure of the vormiform process (fig. 68). The upper and lower vermiform processes of the cerebellum are united in one central part, which connects together the hemispheres. The structure of this connecting 
piece is the same as that of the rest of the cerebellum, viz., a central white portion and investing laminæ. Here the branching appearance of a tree (arbor vitæ) is best seen, in consequence of the laminæ being more divided, and the white central pieces being longer and more ramified.

The Fourth vextricle (fossa rhomboidalis) is a space between the cerebellum and the posterior surface of the medulla oblongata and pons (fig. 68). It has the form of a lozenge, with the points placed upwards and downwards. The upper angle reaches as high as the upper border of the pons; and the lower, to a level with the inferior end of the olivary body. Its greatest breadth is opposite the lower edge of the pons; and a transverse line in this situation would divide the hollow into two triangular portions-upper and lower. The lower half has been named calamus scriptorius from its resemblance to a writing pen.

The lateral boundaries are more marked above than below. For about half way down, the cavity is limited on each side by the superior peduncle of the cerebellum $(l)$, which projecting over it forms part of the roof; and along the lower half lies the eminence of the restiform body (fig. $55,{ }^{3}$ ).

The roof of the space is somewhat arched, and is formed above by the valve of Vieussens $(k)$, and the under part of the vermiform process; and below, by the reflection of the pia mater from that process to the spinal cord.

'The floor of the ventricle is constituted by the posterior surfaces of the medulla oblongata and pons, and is grayish in color. Along its centre is a median fissure, which ends below, near the point of the calamus, in a minute hole - the aperture of the canal of the cord. On each side of the groove is a spindle-shaped elevation, the fasciculus s. eminentia teres $(o)$. This eminenee reaches the whole length of the floor, and is pointerl and little marked inferiorly, where it is covered by gray substance; but it becomes whiter and more prominent superiorly, and its widest point is opposite the lower border of the pons.

The outer border of the eminence is limited externally by a slight groove, which points out the position of two small fossæ (fovea anterior et posterior). The posterior $(r)$ is near the lower end of the groove; and the anterior $(p)$ is opposite the crus cerebelli. Above the anterior fossa is a deposit of very dark gray substance, which has a bluish appearance as it is seen through the thin stratum covering it $;^{1}$ from it a bluish streak is continued upwards, at the outer edge of the eminentia teres, to the opening in the top of the fourth ventricle.

Crossing the floor on each side, opposite the lower border of the pons, are some white lines, which vary much in their arrangement (fig. 55): they issue from the central median fissure, and enter the auditory nerve (p. 181).

Besides the objects above mentioned, there are other eminences in the floor of the ventricle indicating the position of the nuclei of origin of certain nerves.

In the lower half of the space are three slight eminences on each side for the hypo-glossal, vagus, and auditory nerves: that for the hypo-glossal is close to the middle line below, and eorresponds with the lower pointed end of the eminentia teres. The other two, outside that eminence, are placed in a line one above another, but separated by a well-marked groove

1 The term locus ceruleus has been applied to the spot, and the dark vesicular matter in it has beon named substantia ferruginea. 
(fovea posterior); the lower is the nucleus of the vagus and glosso-pharyngeal nerves, and the upper is the nucleus of the auditory nerve. Running into the lower part of the vagus nucleus, is the nucleus of the accessory portion of the spinal accessory nerve. (See p. 189.)

In the upper half of the space some other nerves take origin from nuclei, but there is only one projection. 'This is placed over the common nucleus of the sixth and the facial nerve : it is a rounded elevation on the outer part of the eminentia teres, about a line above the white cross strix on the floor, and close behind the fovea anterior.

The fourth ventricle communicates at the upper part with the third ventricle through the Sylvian aqueduct; and with the subarachnoid space of the cord and brain, through an aperture in the pia mater intervening between the medulla and the cerebellum: laterally, the ventricular space is extended for a short distance between the cerebellum and the side of the medulla oblongata.

The lining of the other ventricles is prolonged into this by the aperture of communication with the third. Covering the floor is a columnar epithelium, which is continuous with that in the upper part of the central canal of the spinal cord (Clarke).

In this ventricle is a vascular fold-choroid plexus, on each side, similar to the body of the same name in the other ventricles. It is attached to the inner surface of the membrane (pia mater) which closes the ventricle between the medulla and the cerebellum, and it extends upwards on the side of the opening into the sub-arachnoid space. Its vessels are supplied by the inferior cerebellar artery.

Gray matter of fourth ventricle. The gray matter forms a surfacecovering for the floor of the fourth ventricle. It is continuous below with the gray commissure of the cord, and extends upwards to the aqueduct of Sylvius (p. 187). The special nuclei have been referred to already (p. 217). 
TABLE OF THE CHIEF ARTERIES OF THE HEAD AND NECK.

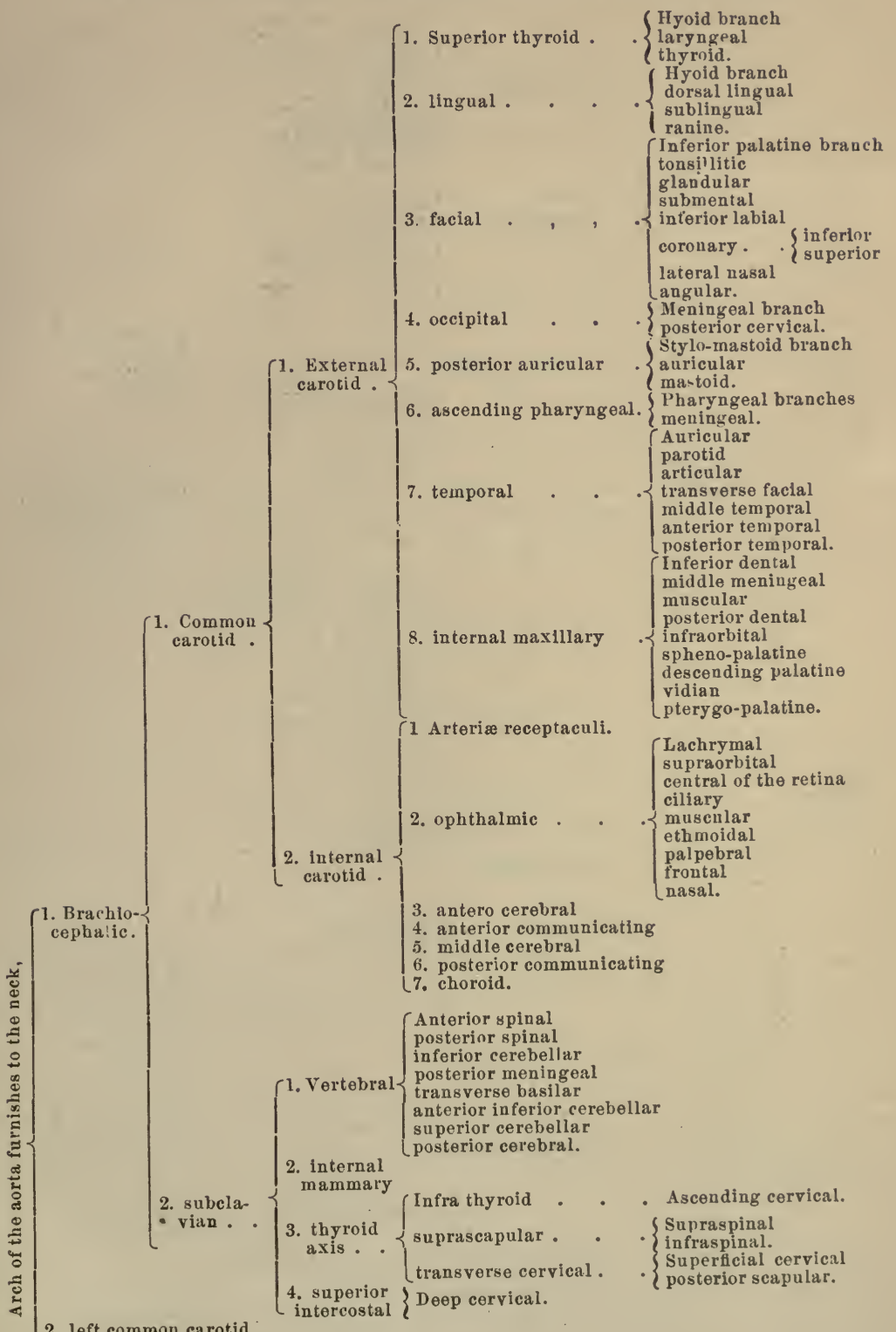

2. left common carotid.

3. left subclavian. 


\section{TABLE OF THE CHIEF VEINS OF THE HEAD AND NECK.}

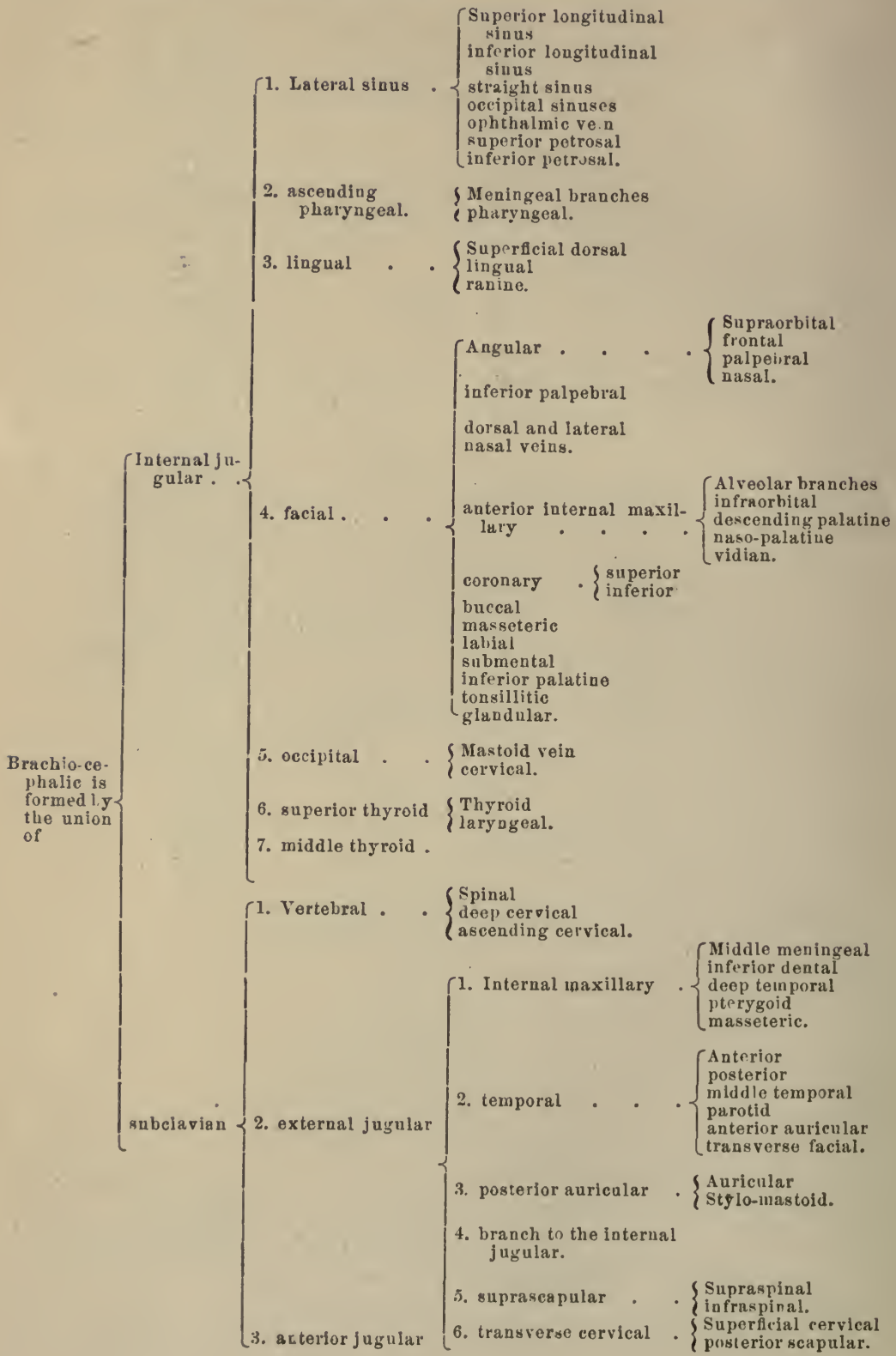


TABLE OF THE CRANIAL NERVES.

1. First nerve . Filaments to the nose.

2. Second nerve . To retina of the eyeball.

3. Third nerve . To muscles of the orbit.

4. Fourth nerve . To superior oblique muscle.

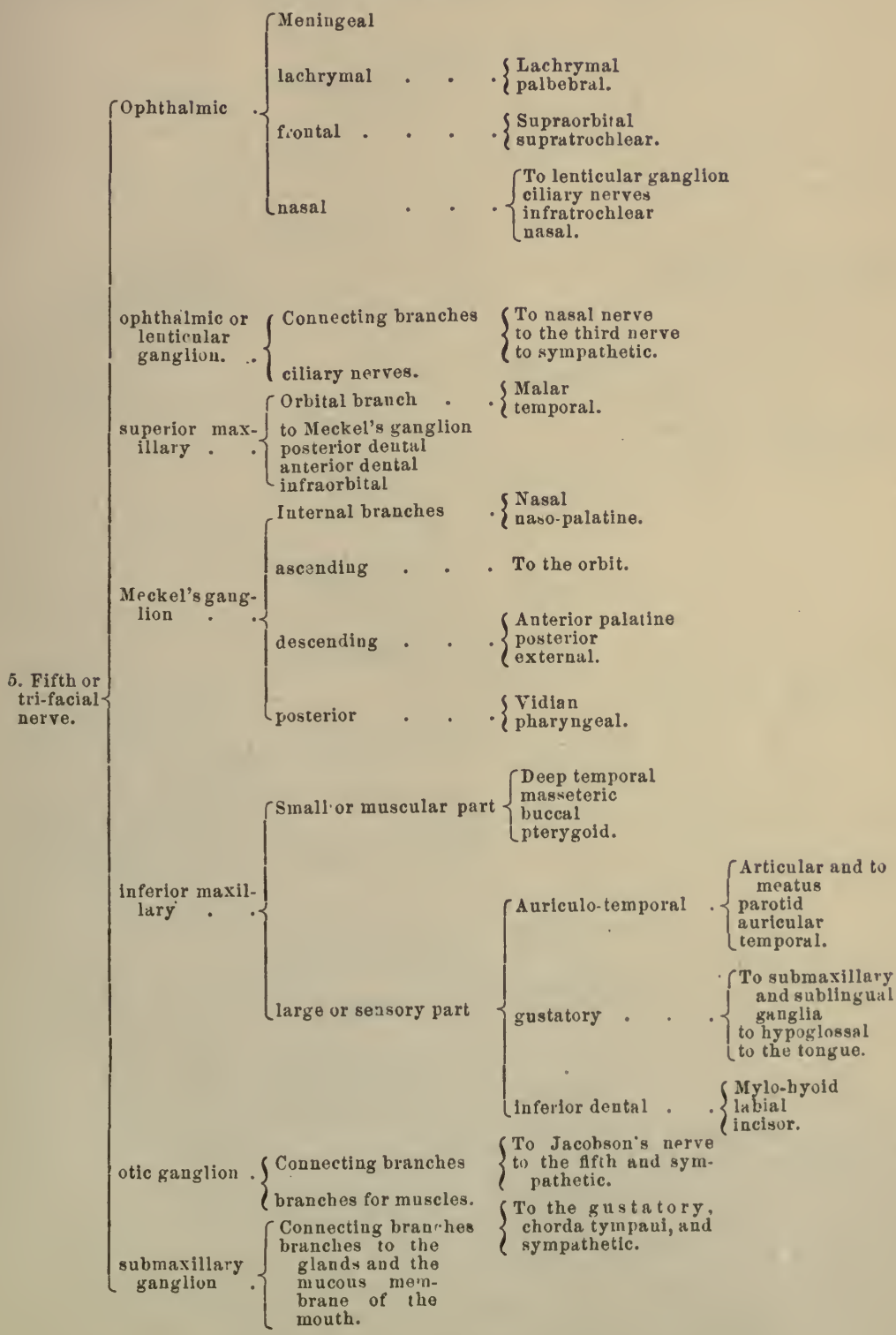




\section{TABLE OF CRANIAL NERVES-Continued.}

6. Sixth nerve . . . To external rectus.

7. Swrenth nerve, or facial

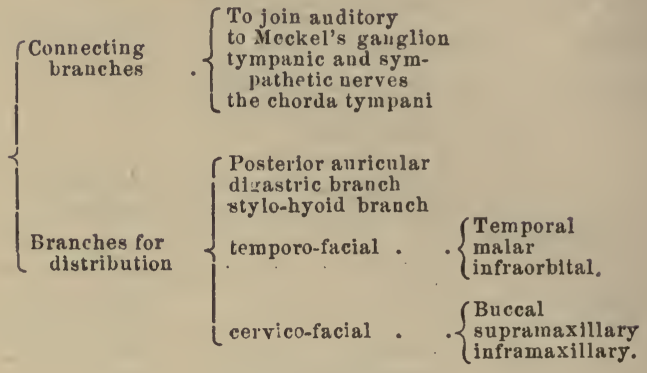

S. Elghth nerve, or auditory $\quad\left\{\begin{array}{l}\text { To the portio dura } \\ \text { nerve to cochlea. }\end{array} \quad \begin{array}{l}\text { To the common sac } \\ \text { to the saccale } \\ \text { to the semicircular } \\ \text { canals. }\end{array}\right.$

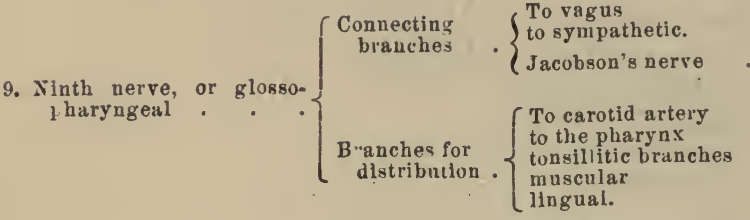

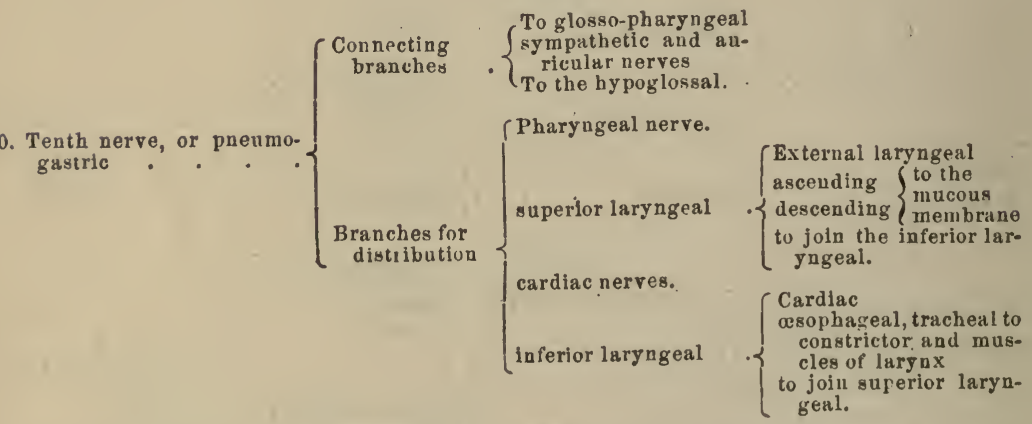

11. Eleventh nerve, or spinal $\left\{\begin{array}{c}\begin{array}{c}\text { Connecting } \\ \text { branches }\end{array} \\ \text { to the cervical plexus. }\end{array}\right.$ accessory $\cdot\left\{\begin{array}{c}\text { Branches for } \\ \text { distribution }\end{array} .\left\{\begin{array}{c}\text { To sterno-mastoidens } \\ \text { and trapezius. }\end{array}\right.\right.$

$\{$ Joins otic ganglion, supplies tympanum.

10. Tenth nerve, or pneumogastric $* .\left\{\begin{array}{l}\text { Branches for } \\ \text { distibution }\end{array}\right.$

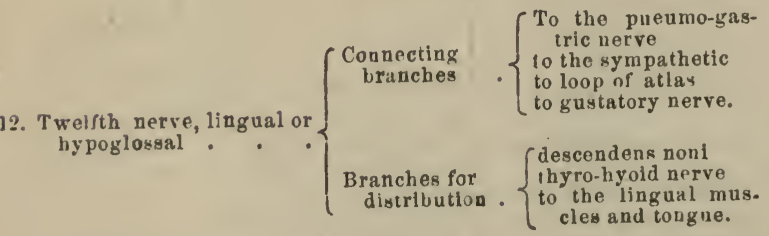


TABLE OF THE SPINAL AND SYMPATHETIC NERVES OF THE HEAD AND NECK.

\section{Spinal Nerves.}

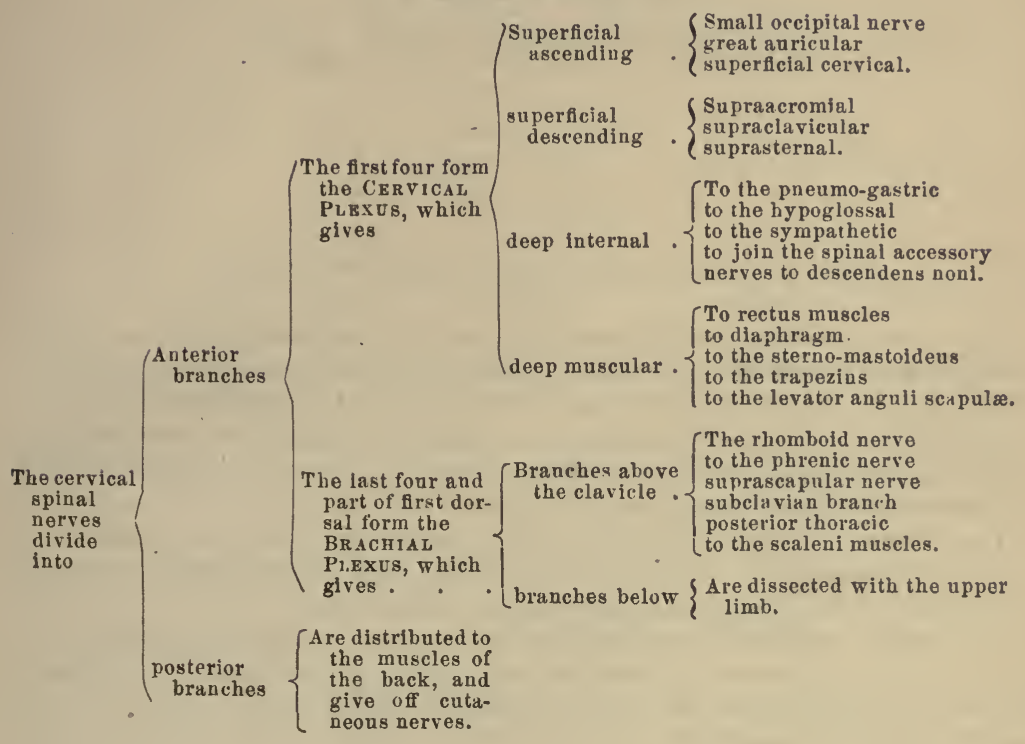

\section{Sympathetic Nerve.}

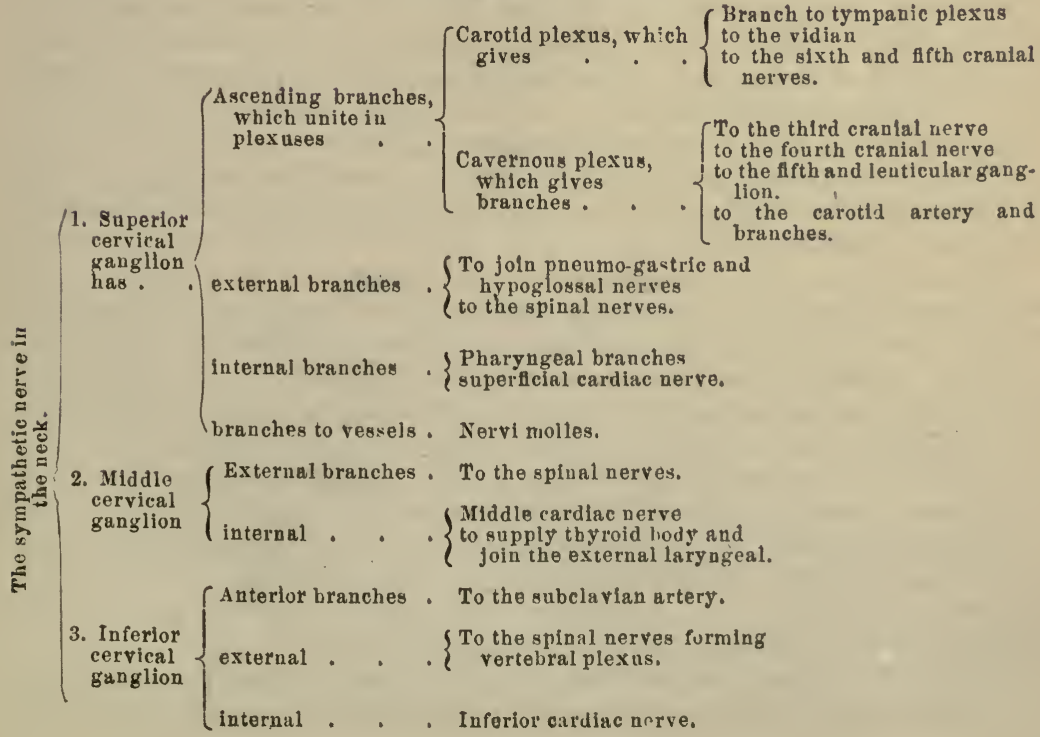




\section{H A P T E R III.}

\section{DISSECTION OF THE UPPER LIMB.}

\section{Section I. \\ THE WALL OF THE THORAX AND THE AXILLA.}

THE parts included in this section, viz., the wall of the chest and the axilla, are to be learnt within a fixed time, in order that the examination of the thorax may be undertaken. Whilst the dissection of the thorax is in progress, the student will have to discontinue his labors on the upper limb ; but, on the completion of that cavity, he must be ready to begin the part of the Back that belongs to him.

Position. Whilst the body lies on the Back, the thorax is to be raised to a convenient height by a block; and the arm, being slightly rotated outwards, is to be placed at a right angle to the trunk.

Directions. Before the dissection is entered on, attention should be given to the depressions on the surface, to the prominences of muscles, and to the projections of the bones; because these serve as guides to the position of parts beneath the skin.

Surface-marking. Batween the arm and the chest is the hollow of the arm-pit, in which the large vesse's and nerves of the limb are lodged. The extent of this hollow may be seen to vary much with the position of the limb to the trunk; for in proportion as the arm is elevated, the fore and hinder boundaries are carried upwards and rendered tense, and the depth of the space is diminished. In this spot the skin is of a dark color, and is furnished with hairs and large sweat glands.

If the arm is forcibly raised and moved in different directions, whilst the fingers of one hand are placed in the arm-pit, the head of the humerus may be recognized.

On the outer side of the limb is the prominence of the shoulder; and immediately above it is an osseous arch, which is formed internally by the clavicle, and externally by the spine and the acromion process of the scapula. Continued downwards from about the middle of the clavicle, is a slight depression between the pectoral and deltoid muscles, in which the coracoid process can be felt near that bone. A second groove, extending outwards from the sternal end of the clavicle, corresponds with the interval between the clavicular and sternal origin of the great pectoral muscle.

Along the front of the arm is the prominence of the biceps muscle; and on each side of that eminence is a groove, which subsides inferiorly in a depression in front of the elbow-joint. The inner of the two grooves, the deepest, indicates the position of the brachial vessels.

If the elbow joint be semiflexed, the prominences of the outer and inner condyles of the humerus will be rendered evident, especially the inner. 
Below the outer condyle, and separated from it by a slight interval, the head of the radius projects; it may be recognized by rotating the bone, the fingers at the same time being placed over it. At the back of the articulation is the prominence of the olecranon.

Dissection. As the first step in the dissection, raise the skin from the side of the chest and the arm-pit, over the great pectoral muscle and the hollow of the axilla, by means of the following incisions:-One is to be made along the middle of the sternum. A second, carried along the elavicle for the inner two-thirds of that bone, is to be continued down the front of the arm rather beyond the anterior fold of the arm-pit, and then to be turnell across the inner surface of the arm as far as the hinder fold of the axilla. From the xiphoid cartilage a third cut is to be directed outwards over the side of the chest, as far back as to a level with the posterior fold of the arm-pit.

The flap of skin now marked out should be reflected outwards beyond the axilla; but it should be left attached to the body, in order that it may be used afterwards for the preservation of the part.

The subcutaneous fatty layer of the thorax resembles the same structure in other parts of the body; but in this region it does not contain much fat.

Beneath the subcutaneous layer is a deeper and stronger special fascia which closely invests the museles, and is continuous with the deep fascia of the arm. It is thin on the side of the chest, but becomes much thicker where it is stretched across the axilla. An incision through it, over the arm-pit, will render evident its increased strength in this situation, and the casing it gives to the muscles bounding the axilla; and if the fore finger be introduced through the opening, some idea will be gained of its eapability of confining an abscess in that hollow.

Dissection. The cutaneous nerves of the side of the chest are to be next sought. At the spots where they are to be found they are placed beneath the fat, so that the student must cut through it ; and those on the clavicle lie also beneath the platysma muscle. Small vessels will indicate the position of the nerves.

Some of them (from the cervical plexus) cross the clavicle at the middle, and the inner part. Others (anterior cutaneous of the thorax) appear at the side of the sternum-one from each intercostal space. And the rest (lateral cutaneous of the thorax) should be looked for along the side of the chest, about one inch below the anterior fold of the axilla, there being one from each intereostal space except the first : as the last-mentioned nerves pierce the wall of the thorax, they divide into an anterior and a posterior piece.

The posterior pieces of the highest two nerves are larger than the rest. They are to be followed across the arm-pit, and a junction is to be found there with a branch (nerve of Wrisberg) of the brachial plexus.

Cutaneous nerves of the cervical plexus. These cross the clavicle and are distributed to the integuments over the pectoral muscle. The most internal branch (sternal) lies near the inner end of the bone, and reaches but a short distance below it. Other branches (clavicular), two or more in number and larger in size, cross the centre of the clavicle and extend to near the lower border of the pectoralis major ; they join one or more of the anterior cutaneous nerves of the thorax.

The cutaneous nerves of the thorax are derived from the trunks of the intercostal nerves between, the ribs (fig. 69). Of these there are two sets: One set, lateral cutaneous nerves of the thorax, arise from the 
trunks of the nerves about midway between the spine and the sternum. The other set, anterior cutaneous nerves of the thorax, are the terminations of the same intercostal trunks at the middle line of the body.

The anterior cutaneous nerves piercing the pectoral muscle, are directed outwards in the integuments as slender filaments. 'The offset of the second nerve joins a cutaneous branch of the cervical plexus; and the others supply the integuments and the mammary gland. Small cutaneous branches of the internal mammary vessels accompany the nerves.

The lateral cutaneous nerves (fig. 69) issue with companion vessels between the digitations of the seratus muscle, and divide into an anterior and a posterior piece. There is not usually any lateral cutaneous nerve to the first intercostal trunk.

The anterior offsets $\left({ }^{9}\right)$ bend over the pectoral muscle, and end in the integuments and the mammary gland: they increase in size downwards, and the lowest give twigs to the digitations of the external oblique muscle. 'The cutaneous nerve of the second intercostal trunk wants commonly the anterior offset.

The posterior offsets $\left({ }^{8}\right)$ end in the integuments over the latissimus dorsi muscle and the back of the scapula, and decrease in size from above down.

Tlie branch of the second intercostal nerve is larger than the rest, and perforates the fascia of the axilla; it supplies the integument of the arm (p. 251), and is named intercosto-humeral. As it crosses the axilla it is divided into two or more pieces, and is connected to the nerve of Wrisberg $\left(^{5}\right)$ by a filament of variable size.

The branch of the third intercostal gives filaments likewise to the armpit and the inner part of the arm.

The mama is the gland for the secretion of the milk, and is situate on the lateral aspect of the fore part of the chest. ${ }^{1}$

Resting on the great pectoral muscle, it is hemispherical in form, but it is rather most prominent at the inner and lower aspects. Its dimensions and weight vary greatly. In a breast not enlarged by lactation, the width is commonly about four inches. Longitudinally it extends from the third to the sixth or seventh rib, and transversely from the side of the sternum to the axilla. Its thickness is about one inch and a half. 'The weight of the mamma ranges firom six to eight ounces.

Nearly in the centre of the gland (rather to the inner side) rises the conical or cylindrical projection of the nipple or mamilla. This prominence is about half an inch or rather more in length, is slightly turned outwards, and presents in the centre a shallow depression, where it is rather redder. Around the nipple is a colored ring,-the areola, about an inch in width, whose tint is influenced by the complexion of the body, and is altered during the times of menstruation, pregnancy, and lactation. The skin of the nipple and areola is provided with numernus papilla and lubricating glands; and on the surface are some small tubercles marking the position of the ducts of the glands.

In the male the mammary gland resembles that of the female in general form, though it is less prominent; and it possesses a small nipple, which is surrounded by an areola provided with hairs. The glandular or secretory structure is imperfect.

1 If the student has a male body, he may disregard the description of the manma; and if the body is a female, he may set aside the breast for a more convenient examination of its structure. 
Structure. In its texture the mamma resembles those compound glands which are formed by the vesicular endings of branched ducts. It consists of small vesicles which are united to form lobules and lobes. Connected with each lobe is an excretory or lactiferous duct.

A layer of areolar tissue, containing fat, surrounds the gland, and penetrates into the interior, subdiving it into lobes; but in the ultimate structure of the gland, and in the nipple and areola, there is not any fatty substance. Some fibrous septa fix the gland to the skin, and support it; these are the ligamenta suspensoria of Sir A. Cooper.

Vesicles. The little vesicles or cells at the ends of the most minute duets are rounded in shape, and when filled with milk or mereury are just visible to the naked eye, being about the size of a small pinhole in paper. (Cooper.) Each is surrounded externally by a close vascular network.

Lobules and lobes. A collection of the vesicles around their ducts form the lobule or glandule, which varies in size from a pin's head to a small tare. By the union of the lobules the lobes are produced, of which there are about twenty altogether, and each is provided with a distinet duct.

The ducts issuing from the several lobes (about twenty) are named from their office galactophorus; they converge to the areola, where they swell into oblong dilations or reservoirs (sacculi) of one-sixth to one-third of an inch in width. Onwards from that spot the ducts become straight ; and, surrounded by areolar tissue and vessels, are continued through the nipple, nearly parallel to one another, and gradually narrowing in size, to open on the summit by apertures varying from the size of a bristle to that of a eommon pin.

Like many other exeretory ducts, the milk tubes consist of an external or fibrous, and of an internal or mucous coat; they and the vesicles are sheathed by a columnar epithelium, which becomes flattened towards the outer openlng.

Beneath the skin of the nipple and areola are branched lubricating glands, which open on the tubercles before mentioned.

Bloodiessels. - The arteries are supplied by the axillary, internal mammary, and intercostal, and enter both surfaces of the gland. The veins end principally in the axillary and internal mammary trunks ; but others enter the intercostal veins.

The nerves are supplied from the anterior and lateral cutaneous branches of the thorax, viz., from the third, fourth, and fifth intercostal nerves.

The lymplatics of the inner side open into the anterior mediastinal glands; but on the outer side they reach the axillary glands.

Dissection (fig. 69). With the limb in the same position to the trunk, the student is first to remove the fascia and the fat from the surface of the great pectoral musele. In cleaning the musele the scalpel should be carried in the direction of the fibres, viz., from the arm to thorax; and the dissection may be begun at the lower border on the right side, and at the upper border on the left side.

The fascia and the fat are to be taken from the axilla, without injury to the numerous vessels, nerves, and glands in the space. The dissection will be best executed by cleaning first the large axillary vessels at the outer part, where these are about to enter the arm: and then following their branches which are direeted to the ehest, viz., the long thoracic under cover of the anterior boundary, and the circumflex and subscapular vessels and nerves along the posterior boundary. Some arterial twigs entering the axillary glands should be traced out.

In taking away the fascia and fat from the muscles in the posterior 
boundary of the space, the small internal cutaneous nerve of the musculospiral should be looked for near the great vessels.

The large nerves of the brachial plexus are then to be defined. The smallest of these, which possibly may be destroyed, is the nerve of Wrisberg: it lies close to the hinder edge of the axillary vein, and joins with the intereosto-humeral nerve.

When cleaning the serratus muscle on the ribs the student is to seek on its surface the posterior thoracic nerve; and to trace the posterior offsets of the intercostal nerves crossing the axilla.

\section{THE AXILLA.}

The axilla is the hollow between the arm and the chest (fig. 69). It is somewhat pyramidal in form, and its apex is directed upwards to the root of the neck. The space is larger near the thorax than at the arm, and its boundaries are as follows :-

Boundaries. In front and behind the space is limited by folds, which are constructed by the muscles passing from the trunk to the upper limb. In the anterior fold are the two pectoral muscles, but these take unequal shares in its construction, in consequence of the difference in their size and shape:thus the pectoralis major a extends over the whole front of the space, reaching from the clavicle to the lower edge of the anterior fold; whilst the pectoralis minor $\mathrm{B}$, which is a narrow muscle, corresponds only with the middle third of the space. In the posterior boundary, from above downwards, lie the subscapularis $\mathrm{F}$, the latissimus dorsi muscle $\mathrm{D}$, and the teres major $\mathrm{E}$ : this boundary reaches further out than the anterior, especially near the humerus; and its lower margin, which is formed by the latissimus dorsi, projects forwards beyond the level of the subseapularis.

On the inner side of the axilla lie the first four ribs, with their intervening intercostal muscles, and the part of the serratus magnus $\mathrm{c}$ taking origin from those bones. On the outer side the space has but small dimensions, and is limited by the humerus and the coraco-brachialis and biceps muscles ( $\mathrm{G}$ and $\mathrm{H}$ ).

The apex of the hollow is situate between the clavicle, the upper margin of the scapula, and the first rib; and the forefinger may be introduced into the space for the purpose of ascertaining the upper boundaries, and the depth. The base or widest part of the pyramid is turned downwards, and is closed by the thick aponeurosis reaching from the anterior to the posterior fold.

Contents of the space. In the axilla are contained the axillary vessels and the brachial plexus, with their branches; some branches of the intercostal nerves; together with lymphatic glands, and a large quantity of loose areolar tissue and fat.

Position of the trunks of vessels and nerves. 'The large axillary artery $(a)$ and vein $(b)$ cross the outer portion of the space in passing from the neck to the upper limb. The part of each vessel now seen lies close to the liumerus, reaching beyond the line of the anterior fold of the arm-pit, and is covered only by the common superficial coverings, viz, the skin, the fatty layer or superficial fascia, and the deep fascia. Behind the vessels are the subscapularis (F) and the tendons of the latissimus and teres muscles ( $D$ and $\mathrm{E}$ ). 'To their outer side is the coraco-brachialis muscle (G). 
artery runs to the side of the chest ; and taking the same direction, thongh nearer the middle of the hollow, are the small external mammary artery and vein.

Extending along the posterior fold, within its lower margin and in contact with the edge of the subscapularis muscle, are the subscapular vessels and nerves $\left(d\right.$ and $\left.{ }^{7}\right)$; and near the humeral end of the sulscapularis the posterior eircumflex vessels and nerve $(e)$ bend backwards beneath the large axillary trunks.

On the inner boundary, at the upper part, are a few snall branches of the superior thoracic artery, which ramify on the serratus muscle; but these are commonly so unimportant, that this part of the axillary space may be considered free from vessels with respect to any surgical operation. Lying on the surface of the serratus magnus, is the nerve to that muscle; and perforating the inner boundary of the space, are the lateral cutaneous nerves of the thorax-two or more offsets of which are directed across the axilla to the arm, and receive the name intercosto-humeral.

The lymphatic glands of the axilla are arranged in two sets: one is placed along the inner side of the bloodvessels; and the other occupies the lower and hinder parts of the space, lying near and along the posterior boundary. Commonly they are ten or twelve in number; but in number and size they vary much. Small vascular twigs from the branches of the axillary vessels are furnished to them.

The glands by the side of the bloodvessels receive the lymphatics of the arm; and those along the hinder boundary are joined by the lymphatics of the fore part of the thorax and posterior surface of the Back, as well as by some from the mamma. Most of the efferent ducts unite to form a trunk, which opens into the lymphatie duct of the neck of the same side; some may enter separately the subclavian vein.

The PECTORALIS MAJOR, A, is triangular in shape, with the base at the thorax and the apex at the arn. It arises internally from the front of the sternum, and the cartilages of the true ribs except the last ; superiorly from the sternal half of the clavicle; and inferiorly from the aponeurosis of the external oblique muscle of the abdomen. From this wide origin the fibres take different directions-those firom the clavicle being inclined obliquely downwards, and those from the lower ribs upwards beneath the former; and all end in a tendon, which is inserted into the outer edge of the bicipital groove of the humerus for about two inches.

This muscle bounds the axilla anteriorly, and is connected sometimes to its fellow by fibres in front of the sternum. Besides the superficial structures and the mamma, the platysma covers the pectoralis major close below the clavicle. A lengthened interval, which corresponds with a depression on the surface, separates the clavicular from the sternal attachment. One border (outer) is in contact with the deltoid muscle, and with the cephalic vein and a small artery; and the lower border forms the margin of the anterior fold of the axilla. 'The parts covered by the muscle will be seen subsequently.

Action. If the humerus is hanging, the muscle will move forwards the limb until the elbow reaches the front of the trunk, and will rotate it in.

When the limb is raised, the pectoralis depresses and adducts it ; and acting with other muscles inserted into the opposite side of the humerus, it may dislocate the head of that bone when the lower end is fixed, as in at fall on the elbow. 
Supposing both limbs fixed as in climbing, the trunk will be raised by both muscles; and the ribs can be elevated in laborious breathing.

Dissection (fig. 70). The great pectoral muscle is to be cut across now in the following manner:-

Only the clavicular part is to be first divided, so that the branches of the nerve and artery to the muscle may be found. Reflect the cut part of muscle, and press the limb against the edge of the table, for the purpose of raising the clavicle and rendering tight the fascia attached to that bone; on carefully removing the fat, and a piece of fascia prolonged from the upper border of the small pectoral muscle, the membranous costocoracoid sheath will be seen close to the clavicle, covering the axillary vessels and nerves.

At this stage the cephalic vein is to be defined as it crosses inwards to the axillary vein. A branch of nerve (anterior thoracic), and the acromial thoracic artery, which perforate the tube of membrane around the vessels, are to be followed to the pectoral muscles.

The remaining part of the pectoralis major may be cut about its centre, and the pieces may be thrown inwards and outwards. Any fat coming into view is to be removed; and the insertion of the tendon of the pectoralis is to be followed to the humerus.

Insertion of the pectoralis. The tendon of the pectoralis consists of two parts, anterior and posterior, at its attachment to the bone; the anterior receives the clavicular and upper sternal fibres, and joins the tendon of the deltoid muscle; and the posterior gives attachment to the lower ascending fibres. The tendon is from two inches to two inches and a half wide, and sends upwards one expansion over the bicipital groove to the capsule of the shoulder-joint, and another to the fascia of the arm.

Parts covered by the pectoralis. The great pectoral muscle covers the pectoralis minor, and forms alone, above and below that muscle, the anterior boundary of the axilla. Between the pectoralis minor and the clavicle it conceals the subclavius muscle, the sheath containing the axillary vessels, and the branches perforating that sheath. Below the pectoralis minor it lies on the side of the chest, on the axillary vessels and nerves, and on the biceps and coraco-brachialis muscles near the humerus.

The PECTORALIS MINOR (fig. $70,{ }^{\mathrm{B}}$ ) resembles the preceding muscle in shape, and is extended like it from the thorax to the arm. Its origin is connected by slips with the third, fourth, and fifth ribs, external to their cartilages; and between the ribs, with the aponeurosis covering the intercostal muscles. The fibres converge to their insertion in the anterior half of the upper surface of the coracoid process of the scapula.

This muscle is placed before the axillary space, and assists the pectoralis major in forming the middle of the anterior boundary: in that position it conceals the axillary vessels and the accompanying nerves. The upper border lies near the clavicle, but between it and that bone is an interval of a somewhat triangular form. The lower border projects beyond the pectoralis major, close to the chest; and along it the long thoracic artery lies. The tendon of insertion is united with the short head of the biceps and the coraco-brachialis.

Action. Acting with the serratus magnus it moves the scapula forwards and somewhat downwards.

In laborious breathing it becomes an inspiratory muscle, as it takes its fixed point at the scapula. 
Dissection. Supposing the clavicle raised by the pressing backwards the arm, as before directed, the tube of fascia around the vessels will be demonstrated by making a transverse cut in the costo-coracoid membrane near the clavicle, so that the handle of the scalpel can be passed beneath it. By raising the lower border of the subclavius this muscle will be seen to be incased by fascia, which is attached to the bone both before and behind it.

The costo-coracoid membrane, or ligament (fig. 70), is a firm membranous band, which receives this name from its attachment on the one side to the rib, and on the other to the coracoid process of the scapula. Between those points it is inserted into the clavicle, inclosing the subclavius muscle ; and is joined by the piece of fascia that incases the small pectoral muscle. From its strength and position it gives protection to the vessels surrounded by their loose sheath.

When traced downwards it is found to descend on the axillary vessels and nerves, joining externally the fascia on the coraco-brachialis muscle, and blending with the sheath of the axillary vessels beneath the small pectoral muscle. Its extent is not so great on the inner as on the outer side, for internally it reaches but a very short distance on the axillary vein.

The sheath of the axillary vessels and nerves, $\mathrm{E}$, is derived from the deep fascia of the neck, being prolonged from that on the scaleni muscles; and resembles, in its form and office, the funnel-shaped tube of membrane surrounding the femoral vessels in the upper part of the thigh. It is strongest near the subclavius muscle, where the costo-coracoid band joins it. The anterior part of the tube is perforated by the cephalic vein $(e)$, the acromial thoracic artery $(a)$, and the anterior thoracic nerve $\left({ }^{1}\right)$.

Dissection. After the costo-coracoid membrane has been examined, the remains of it are to be taken away; and the subclavius muscle, and the axillary vessels and nerves with their branches, are to be carefully cleaned.

The subClavius Muscle (fig. $70,{ }^{D}$ ) is roundish in form, and is placed between the clavicle and the rib. It arises by a tendon from the first rib, at the junction of the osseous and cartilaginous parts, and in front of the costo-clavicular ligament. 'The fibres ascend obliquely, and are inserted into a groove on the under surface of the clavicle, which reaches between the two tubercles (internal and external) for the attachment of the costo and coraco-clavicular ligaments.

The muscle overhangs the large vessels and nerves of the limb, and is inclosed, as before said, in a sheath of fascia.

Action. It depresses the clavicle, and indirectly the scapula; but if the shoulder is fixed it elevates the first rib.

The AXILlary ARTERY (fig. 70) continues the subclavian trunk to the upper limb. The part of the vessel to which this name is applied is contained in the axilla, and extends from the lower border of the first rib to the lower edge of the teres major muscle (II).

In the axillary space its position will be marked by a line from the middle of the clavicle to the inner edge of the coraco-brachialis. Its direction will vary with the position of the limb to the trunk; for when the arm lies by the side of the body the vessel is curved, it convexity being upwards; and in proportion as the limb is removed to a right angle with the chest, the artery becomes straight. In the upper part of the axilla the vessel is deeply placed, but it becomes superficial as it approaches the arm. 
Its connections with surrounding parts are numerous; and the description of these will be methodized by dividing the artery into three partsone above, one beneath, and one below the small pectoral muscle.

Above the small pectoral muscle the artery is contained in the axillary sheath of membrane, $\mathrm{E}$. This part is concealed by the clavicular portion of the great pectoral muscle. Behind it are the intercostal muscles of the first space and the first digitation of the serratus magnus.

Fig. 70 .

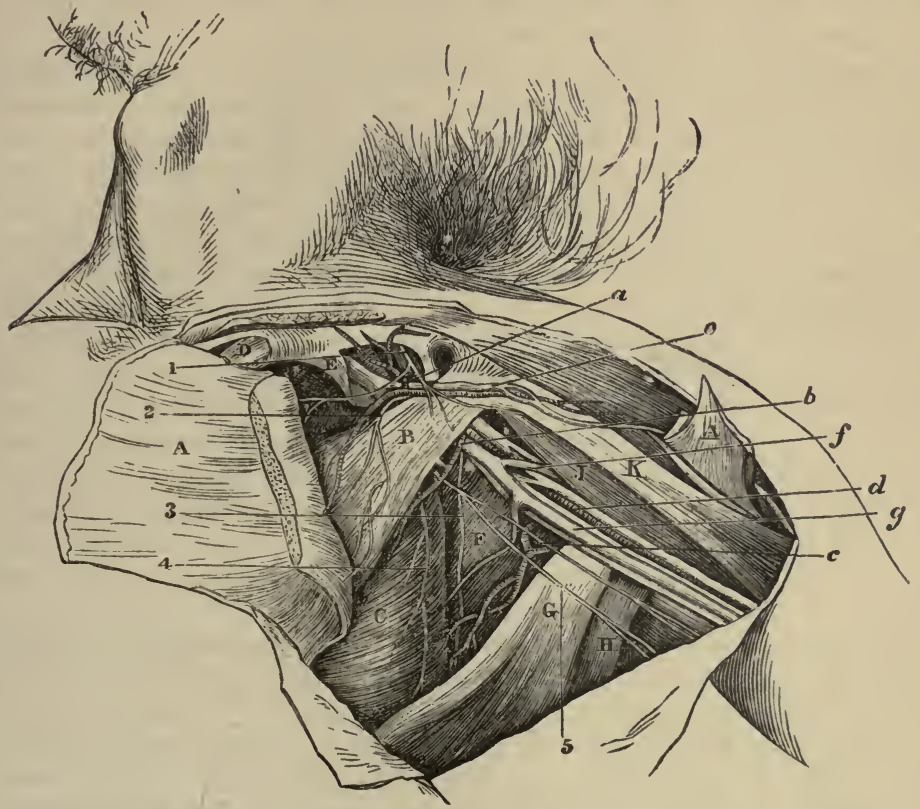

SeCOND VIEW OF THE DISSECTION OF THE THORAX (Illustrations of Dissections).

\section{Muscles :}

A. Pectoralis major, cut.

B. Pectoralis minor.

c. Serratus magnus.

D. Subclavius.

E. Axillary sheath.

F. Subscapularis.

G. Latissimus dorsi.

H. Teres major.

J. Coraco-trachialis.

K. Biceps.
Vessels :

a. Acromial-thoracic branch.

b. Long thoracic branch.

c. Subscapular branch.

d. Axillary artery.

$e$. Cephalic vein.

$f$. Brachial veins joining the axillary veins, $g$.

Nerves:

1 and 2. Anterior thoracic branches.

3. Subscapular branch.

4. Nerve to the serratus.

5. Intercosto-humeral branch.

To the thoracic side is placed the axillary vein $(g)$. The cephalic vein $(e)$, and offsets of the acromial thoracic artery and vein, cross over it.

On the acromial side lie the two cords of the brachial plexus, separated from the vessel by a slight interval. Superficial to it lies an anterior thoracic nerve; and beneath, is the posterior thoracic.

Beneath the pectoralis, the pectoralis minor and major $\mathrm{B}$ and $\mathrm{A}$ are superficial to the axillary vessel. But there is not any muscle immediately 
in contact behind, for the artery is placed across the top of the axilla, particularly when the limb is in the position required by the dissection.

The companion vein $(g)$ lies to the inner side, but separated from the arterial trunk by a bundle of nerves.

In this position the cords of the brachial plexus lie around it, one being outside, a second inside, and a third beneath the artery.

Beyond the pectoralis minor the artery is concealed in part by the lower border of the great pectoral muscle $\mathrm{A}$, but thence to its termination it is covered only by the integuments and the fascia. Beneath it are the subscapularis muscle, F, and the tendons of the latissimus and teres, $\mathrm{G}$ and $\mathrm{II}$. 'To the outer side is the coraco-brachialis muscle, J.

The axillary vein remains as above on the thoracic side of the artery.

Here the artery lies in the midst of the large trunks of nerves into which the brachial plexus has been resolved: On the outer side is the median nerve, with the musculo-cutaneous for a short distance; and on the inner side are the ulnar, and the nerve of Wrisberg. Superficial to the vessel is the internal cutaneous; and behind are the musculo-spiral and circumflex nerves, the latter extending only as far as the border of the subscapular muscle.

The branches of the axillary artery are furnished to the wall of the thorax and the shoulder. The thoracic branches are four in number; two (superior and acromial thoracic) arise from the artery above the pectoralis minor; one (alar thoracic) beneath the muscle; and one (long thoracic) at the lower border. Three branches are supplied to the shoulder, viz., subscapular and two circumflex; the first springs opposite the edge of the muscle of the same name, and the others wind round the neck of the humerus. The last offsets are the external mammary and some muscular twigs.

'The superior thoracic branch is the highest and smallest offset, and arises opposite the first intercostal space; it ramifies on the side of the chest, anastomosing with the intercostal arteries.

The acromial thoracic branch (fig. $70, a$ ) is a short trunk on the front of the artery, which appears at the upper border of the pectoralis minor, and opposite the interval between the large pectoral and deltoid muscles. Its branches are directed inwards, outwards, and upwards:-

a. The inner set supply the thoracic muscles, and give a few offsets to the side of the chest to anastomose with the intercostal and other thoracic arteries.

$b$. The outer or acromial set end mostly in the deltoid; but one small artery accompanies the cephalic vein for a short distance; and another (inferior acromial) perforates the deltoid musele, and anastomoses on the acromion with a branch of the suprascapular artery of the neck.

c. One or two small twigs ascend to the subclavius and deltoid muscles.

'The alar thoracic is very inconstant as a separate branch, and its place is taken by offsets of the subscapular and long thoracic arteries; it is distributed to the glands and fat of the axillary space.

The long thoracic branch is directed along the border of the pectoralis minor (fig. $70, b$ ) to about the sixth intercostal space; it supplies the pectoral and serratus muscles, and anastomoses, like the other branches, with the intercostal and thoracic arteries. In the female it gives branches to the mammary gland.

An external mammary artery is commonly met with, especially in the female; its position is near the middle of the axilla with a companion vein. 
It supplies the glands, and ends in the wall of the thorax below the preceding.

The subscapular branch (fig. $70, c$ ) courses with a nerve of the same name along the subscapularis, as far as the lower angle of the scapula, where it ends in branches for the serratus magnus, and the latissimus dorsi and teres muscles: it gives many offsets to the glands of the space.

Near its origin the artery sends backwards a considerable dorsal branch round the edge of the subscapular muscle: this gives an infrascapular offset to the ventral aspect of the scapula, and then turns to the dorsum of that bone, where it will be afterwards dissected.

The subscapular artery is frequently combined at its origin with other branches of the axillary, or with branches of the brachial artery.

The circumflex branches (anterior and posterior) arise near the border of the subscapular muscle. One turns in front of, and the other behind the humerus. They will be followed in the examination of the arm.

Small muscular offsets enter the coraco-brachialis muscle.

The AXILLARY VEIx $(g)$ continues upwards the basilic vein of the arm and has the same extent and connections as the axillary artery. It lies to the thoracic side of its artery, and receives thoracic and shoulder branches. Opposite the subscapular muscle it is joined externally by a large vein, which is formed by the union of the venæ comites of the brachial artery; and near the clavicle the cephalic vein opens into it.

Dissection. To follow out the branches of the brachial plexus, cut through the pectoralis minor near its insertion into the coracoid process, and turn it towards the chest, but without injuring the thoracic nerves in contact with it. The axillary vessels are next to be cut across below the second rib), ${ }^{1}$ and to be drawn down with hooks; and their thoracic branches may be removed at the same time. A dense fascia is to be cleared away from the large nerves of the plexus.

The BRACHIAL PIEXUs results from the union of the anterior branches of the four lower cervical nerves with the first dorsal (in part); and a slip is added to it above from the lowest nerve in the cervical plexus. It is placed partly in the neck, and partly in the axilla, and is divided opposite the coracoid process into large trunks for the supply of the limb. The part of the plexus above the clavicle is described in the dissection of the head and neck (p. 79). The part below the clavicle has the same connections with the surrounding museles as the axillary artery. The nerve trunks interlace in it generally in the following manner :-

At first the plexus consists of two bundles of nerves, which lie on the outer side of the artery, and are thus constituted;-the one nearest the vessel is formed by the last cervical with the part of the first dorsal nerve; and the other, by the fifth, sixth, and seventh cervical nerves. A little lower down a third or posterior cord is produced by the union of two fitsciculi, one from each of the other bundles; so that, beneath the small pectoral muscle, the plexus consists of three large cords, one being on the outer side, another on the inner side, and the third behind the ressel. Occasionally there may be some deviation from the above mentioned arrangement.

The branches of the plexus below the clavicles arise from the severil cords in the following way :-

1 The student must be careful not to cut the vessels higher than the spot mentioned, otherwise he will injure the dissection of the neck. 
The outer cord gives origin to one anterior thoracic branch, the muscuio cutaneous trunk, and the outer head of the median nerve.

The inner cord produces a second anterior thoracic nerve, the inner head of the median, the internal cutaneous, the nerve of Wrisberg, and the ulnar nerve.

The posterior cord furnishes the subscapular branches, and ends in the circumflex and musculo-spiral trunks.

Only the thoracic and subscapular nerves are dissected to their termina. tion at present; the remaining nerves will be seen in the arm.

The anterior thoracic branches (fig. $70,{ }^{1}$ and ${ }^{2}$ ), two in number, are named outer and inner, like the cords from which they come.

The outer nerve crosses inwards over the axillary artery, to the under surface of the great pectoral muscle in which it ends. On the inner side of the ressel it communicates with the following branch.

The inner thoracic branch turns upwards between the artery and vein, and after receiving the offsets from the other, ends in many branches to the under surface of the pectoralis minor. Some twigs enter the great pectoral muscle, after passing either through the pectoralis minor or above its border.

The subscapular nerves are three in number, and take their names from the muscles supplied:-

The branch of the subscapularis is the highest and smallest, and enters the upper part of that muscle.

The nerve of the teres major gives a small offset to the inferior part of the subscapularis, and ends in its musele.

A long nerve of the latissimus dorsi $\left(^{3}\right)$ takes the course of the subscapular artery along the posterior wall of the axilla, and enters the fleshy fibres near the outer end.

Another small nerve, nerve to the serratus $\left({ }^{4}\right)$ (posterior thoracic), lies on the surface of the serratus muscle. It arises above the clavicle ( $p$. 80 ), from the fifth and sixth cervical nerves; it descends behind the axillary artery, and enters that surface of the serratus magnus which is turned towards the axilla.

The ratissimus DORSi MUSCLE, G, may be examined as far as it enters into the posterior fold of the axilla. Arising from the spinal column and the back of the trunk, and crossing the lower angle of the scapula, the muscle ascends to be inserted into the bottom of the bicipital groove by a tendon, one inch and a half in width, in front of the teres; at the lower border aponeurotic fibres connect the two, but a bursa intervenes between them near the insertion.

Dissection. To lay bare the serratus muscle between the side of the chest and the base of the scapula, the arm is to be drawn from the trunk, so as to separate the scapula firom the thorax. The nerves of the brachial plexus may be cut through opposite the third rib; and the fat and fascia should be cleaned from the muscular fibres.

The serratus magnus muscle (fig. 71, a) extends between the scapula and the thorax. It arises by nine pointed processes from the outer surface of the eight upper ribs, - the second rib having two pieces; and between the ribs it takes origin from the aponeurosis covering the intercostal muscles. The fibres converge towards the base and angles of the scapula, but from a difference in their direction the muscle appears to consist of three parts.

The upper part is attached internally to the first two ribs and an 
aponeurotic areh between them; and externally, to an impression on the ventral surface of the upper angle of the scapula. A middle part, which is very thin, extends from the second, third, and fourth ribs, to the base of the shoulder bone. And a lower part, which is the strongest, is connected on the one side with four ribs (fifth, sixth, seventh, and eighth), where it digitates with like processes of origin of the external oblique muscle; and, on the other side, it is fixed into the special surface on the costal aspect of the lower angle of the scapula.

The serratus is applied against the ribs and the intercostal muscles, and is partly concealed by the pectoral muscles and the axillary vessels and nerves : in the ordinary position of the arm the scapula and subscapularis are in contact with it.

Action. The whole muscle acting, the scapula is carried forwards. But the lower and stronger fibres can move forwards the lower angle, rotating the bone around an axis through the centre, and raise the acromion.

Dissection. The intercostal muscles will be brought into view by detaching the processes of origin of the serratus from the ribs for a couple of inches, and by taking away the loose tissue on the surface. Towards the front of the chest is a thin aponeurosis, which is continued forwards from each external intercostal to the sternum; this is to be retained in the third intercostal space. Some of the lateral cutaneous nerves should be preserved.

The intercostal Muscles are named from their position between the ribs. There are two layers in each space, but neither occupies the whole length of the space. The direction of the fibres differs in each stratum; for, whilst the fibres of the external muscle run very obliquely downwards and forwards, those of the internal have an opposite direction between the osseous parts of the ribs, so that the two sets cross.

The external muscle is fixed to the outer margin of the ribs of each intercostal space, and consists of fleshy and tendinous fibres. Posteriorly the fibres begin at or near the tubercle of the rib; and anteriorly they end short of the middle line, but after a different manner in the upper and lower spaces:-In the intervals between the true ribs, they cease near the costal cartilages, and a thin aponeurosis is continued onwards from the point of ending to the sternum. In the lower spaces they are continued between the cartilages (Theile) reaching the end of the ribs in the last two.

Dissection. The internal interFig. 71.

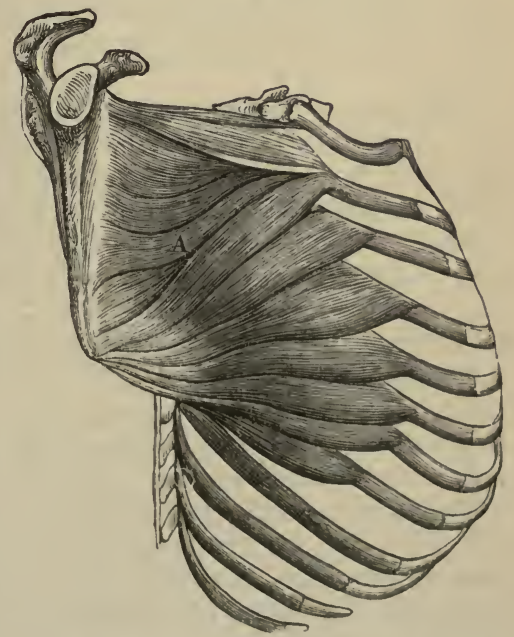

Diagram of the Serratus Magues Muscle. A. Attachments.

costal muscle will be seen by cutting through and removing the external layer and the fascia in one of the widest spaces, say the second; it will be recognized by the difference in the direction of the fibres.

Far back between the two muscles, and close to the rib above, the inter- 
costal nerve and artery will appear. A branch of the nerve to the surface (lateral cutaneous of the thorax) should be followed through the external musele; and the trunk of the nerve is to be traced forwards in one or more spaces to the sternum, and the surface of the thorax.

The linder part of these muscles will be seen in the dissection of the Back and thorax.

'The internal intercostal muscle, attached to the inner border of the ribs bounding the intercostal space, begins in front at the extremity of the ribs, and ceases behind near their angles. Posteriorly they do not end at the same distance from the spine, for the upper and lower approach nearer than the middle; and, anteriorly, in the two lowest spaces, the muscular fibres are continuous with the internal oblique of the abdomen. One surface is covered by the external muscle and in part by the intercostal vessels and nerve; and the opposite surfice is in contact with the pleura.

Action. By the alternate action of the intercostal muscles the ribs are moved in respiration.

The external intercostals elevate the ribs and evert the lower edges, so as to enlarge the thorax in the antero-posterior and transverse directions: they come into play during inspiration.

The internal intercostals act in a diferent way at the side and fore part of the chest.

Between the osseous parts of the ribs they depress and turn in those bones, diminishing the size of the thorax; and they are brought into use in expiration.

Between the rib-cartilages they raise the ribs, and become muscles of inspiration like the outer layer.

If both sets of muscles contract simultaneously, the motion of the ribs will be arrested; or if two or more ribs are broken near the spinal column, the muscles of the space or spaces injured will be unable to move these bones.

Dissection. To bring into view the triangularis sterni muscle and the internal mammary vessels, the cartilages of the true ribs, except the first and seventh, are to be taken away with the intervening muscles on the right side of the body ; but the two ribs mentioned are to be left untouched for the benefit of the dissectors of the abdomen and head and neck. Small arteries to each intercostal space and the surface of the thorax, and the intercostal nerves, are to be preserved. The surface of the triangularis sterni will be apparent when the loose tissue and fat are removed.

The triajgularis sterni (fig. 72, A) is a thin muscle beneath the costal cartilages. It arises internally from the side of the xiphoid cartilage, from the side of the sternum as high as the third costal cartilage, and usually from the inner ends of the lower three true costal cartilages. Its fibres are directed outwards, the upper being most oblique, and are inserted by fleshy fasciculi into the true ribs except the last two and the first, at the junction of the bone and cartilage, and into an aponeurosis in the intercostal spaces.

The muscle is covered by the ribs and the internal intercostals, and by the internal mammary vessels and the intercostal nerves. It lies on the pleura. Its lower fibres touch those of the transversalis abdominis.

I On the left side the vessels and the muscle will have been destroyed by the injection of the body. 
Action. The muscle assists in depressing the anterior ends of the ribs; and by diminishing the size of the thorax, it becomes an expiratory muscle.

The internal mammary artery is a branch of the subclavian (p. 77), and enters the thorax beneath the cartilage of the first rib. It is continued through the thorax, lying beneath the costal cartilages and about half an inch from the sternum, as far as the interval between the sixth and seventh ribs; there it gives externally a large muscular branch (musculo-phrenic), and passing beneath the seventh rib, enters the sheath of the rectus muscle in the wall of the abdomen. In the chest the artery lies on the pleura and the triangularis sterni, and is crossed by the intercostal nerves. It is accompanied by two reins, and by a chain of lymphatic glands. 'The following branches take origin in the thorax:-

a. A small branch (comes nervi phrenici) arises as soon as the artery enters the chest, and descends to the diaphragm along the phrenic nerve.

$b$. A few small mediastinal branches are distributed to the remains of the thymus gland, the pericardium, and the triangularis sterni muscle.

\section{c. Two anterior intercostal}

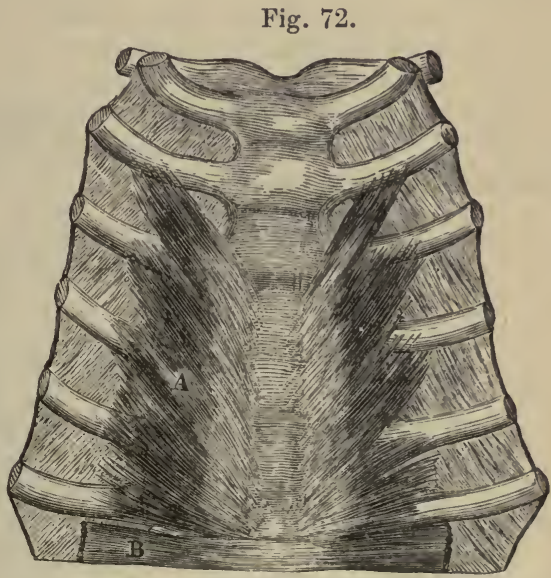

VIEW FROM BEHIND OF THE ATTACHMENTS OF THB Triangularis Sterni Múcle, A.

branches turn outwards in each space, one being placed on the border of each costal cartilage, and terminate by anastomosing with the aortic intercostal arteries. .

d. Perforating branches, one or two opposite each space, pierce the internal intercostal and pectoral muscles, and are distributed on the surface of the thorax with the anterior cutaneous nerves: the lower branches supply the mamma in the female.

e. The musculo-phrenic branch courses outwards beneath the cartilages of the seventh and eighth ribs, and enters the wall of the abdomen by perforating the diaphragm: it supplies anterior branches to the lower intercostal spaces. Its termination will appear in the dissection of the abdomen.

Two veins accompany the artery; these join into one trunk, which opens into the innominate vein.

The intercostal nerves, seen now in the anterior part of their extent, are the anterior primary branches of the dorsal nerves, and supply the wall of the thorax. Placed at first between the layers of the intercostal muscles, each gives off the lateral cutaneous nerve of the thorax, about midway between the spine and the sternum. Diminished in size by the emission of that offset, the trunk is continued onwards, at first in, and afterwards beneath the internal intercostal muscle as far as the side of the sternum, where it ends as the anterior cutaneous nerve of the thorax. Branches supply the intercostal muscles, and the triangularis sterni. 
The aortic intercostal arteries lie with the nerves between the strata of intercostal muscles, and nearer the upper than the lower rib bounding the intercostal space. About the mid point of the space (from before back) the artery bifurcates:-one branch follows the line of the upper rib, and the other descends to the lower rib; both anastomose anteriorly with the intercostal offsets of the internal mammary artery.

A small cutaneous offset is distributed with the lateral cutaneous nerve of the thorax; and other branches are furnished to the thoracic wall.

Directions. The dissector of the upper limb waits now the appointed time for the examination of the thorax. But as soon as the body is turned he is to take his share in the dissection of the Back, and to proceed with the parts marked for him in Chapter V.

After the Back is finished the limb is to be detached from the trunk by sawing the clavicle about the middle, and cutting through the soft parts connected with the scapula.

\section{SECTION II.}

\section{SCAPULAR MUSCLES, VESSELS, NERVES, AND LIGAMENTS.}

Position. After the limb has been separated from the trunk it is to be placed with the subscapularis uppermost.

Dissection. The different muscles that have been traced to the scapula in the dissection of the front of the thorax and the Back, are now to be cleaned, and to be followed to their insertion into the bone. A small part of each, about an inch in length, should be left for the purpose of ascertaining the osseous attachment.

Between the larger rhomboid muscle and the serratus magnus at the base of the scapula, run the posterior scapular artery and vein, whose ramifications are to be traced.

To the borders and the angles of the scapula the following muscles are connected:-

From the upper margin of the scapula arises one muscle, the omo-hyoid (fig. $73, \mathrm{E}$ ). At its origin that musele is about half an inch wide; it is attached to the edge of the bone behind the noteh, and sometimes to the ligament which converts the notch into a foramen.

The lower margin, or costa, gives origin to the long head of the triceps (fig. 79, A), and to some fibres of the teres major; but these attachments will be ascertained in the progress of the dissection.

The base of the bone has many muscles inserted into it (fig. 74). Between the superior angle and the spine is the levator anguli scapulx, $\mathrm{H}$. Opposite the spine the rhomboideys minor, $\mathrm{J}$, is fixed. And between the spine and the inferior angle the rhomboideus major, $\mathrm{k}$, is attached: the upper fibres of this muscle end often in an aponeurotic arch, and are conneeted indirectly to the bone by means of an expansion from it. Interual to those muscles, and inserted into all the base of the scapula, is the serratus magnus muscle (fig. $73, \mathrm{D}$ ).

On the inner surface of the upper and lower angles of the scapula the 
fibres of the serratus magnus are collected. On the outer surface of the inferior angle lies the teres major (fig. $79, G$ ) which will be subsequently seen.

The insertion of the small pectoral muscle into the fore part of the upper surface of the coracoid process may be ascertained at this stage of the dissection (fig. 73, F).

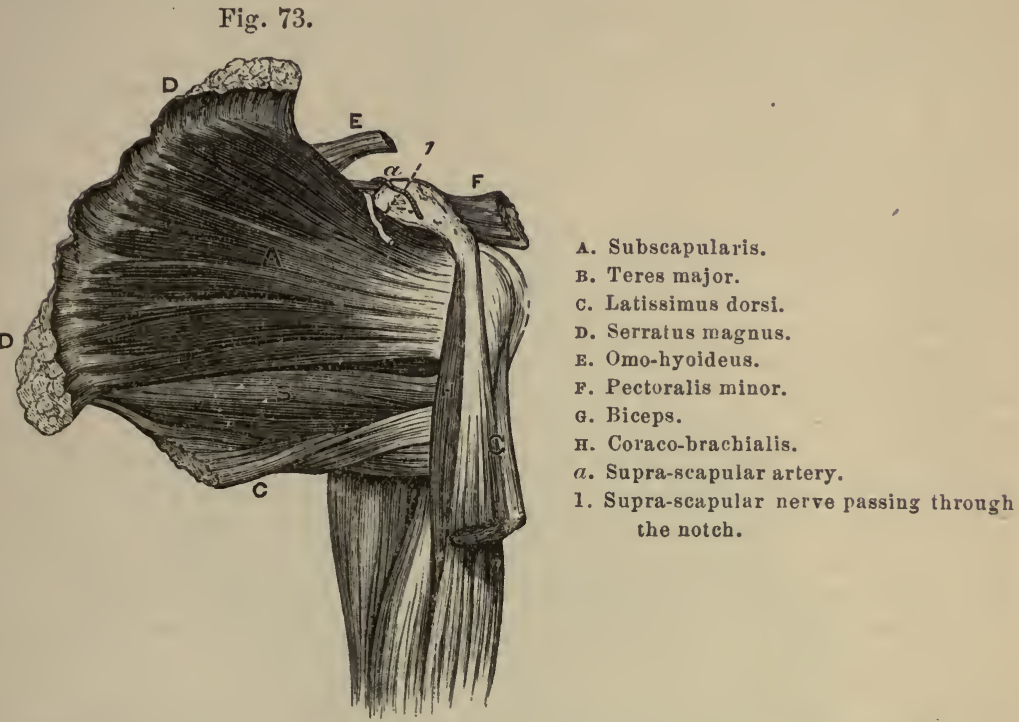

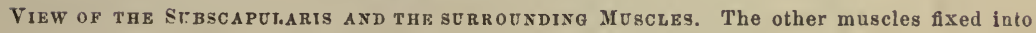
the base of the scapula as shown in fig. 74 .

Dissection. By the separation of the seratus from the subscapularis a thin fascia comes into view, which belongs to the last muscle, and is fixed to the bone around its margins; after it has been observed, it may be removed.

The subscapularis muscle is to be followed forwards to its insertion into the humerus. Next, the axillary vessels and nerves, and the offisets of these to the muscles, should be well cleaned.

The subscapularis MUSClE (fig. 73, a) occupies the under surface of the scapula, and is concealed by that bone when the limb is in its natural position. The muscle arises from the concave surface on the ventral aspect of the scapula, except at the angles, and this attachment reaches forwards nearly to the neck; it is united also to the ridges of the bone by tendinous processes. Externally it is inserted by a tendon into the small tuberosity of the humerus, and by fleshy fibres into the neck for nearly an inch below that process.

By one surface the muscle bounds the axilla, and is in contact with the axillary vessels and nerves and the serratus magnus. By the other, it rests against the scapula and the shoulder joint; and between its tendon and the root of the coracoid process is a bursa, wlich communicates generally with the synovial membrane of the joint. 'The lower border projects much beyond the bone; it is contiguous to the teres major, the latissimus 16 
dorsi, and the long head of the triceps : along this border is the subscapular artery, which gives backwards its dorsal branch.

Action. It rotates in the hanging limb; and when the humerus is raised it depresses the bone.

If the humerus is fixed the subscapularis supports the shoulder joint with the other scapular muscles.

Dissection. The subscapular muscle is to be separated from the scapula, but a thin layer of fibres, in which the vessels lie, is to be left on the bone: as it is raised its tendinous processes of origin, the connection between its tendon and the capsule of the shoulder joint, and the bursa, are to be observed. A small arterial anastomosis on the ventral surface of the bone is to be dissected out of the fleshy fibres.

The infrascapular artery ramifies on the ventral surface of the scapula, and is an offset of the dorsal branch of the scapular ressel (p. 335): entering beneath the subscapularis muscle, it forms an anastomosis with small twigs of the supra and posterior scapular branches.

Position. The examination of the muscles on the opposite surface of the scapula may be next undertaken. For this purpose the scapula is to be turned over; and a block, which is deep enough to make the shoulder prominent, is to be placed between that bone and the arm.

Dissection. The skin is to be removed from the prominence of the shoulder, by beginning at the anterior border of the deltoid muscle. After its removal some small cutaneous nerves are to be found in the fat:- the upper of these extend over the acromion; and another comes to the surlace about half way down the posterior border of the deltoid muscle.

Superficial nerves. Branches of nerves, super-acromial, descend to the surface of the shoulder from the cervical plexus (p. 66). A cutaneous branch of the circumflex nerve (fig. $74,{ }^{2}$ ) turns forwards with a small companion artery from beneath the posterior border of the deltoid, and supplies the integuments covering the lower two-thirds of the muscle.

Dissection. The fat and fascia are now to be taken from the fleshy deltoid, its fibres being made tense at the same time. Beginning at the anterior edge of the muscle, the dissector is to carry the knite upwards and downwards along the fibres, in order that its coarse muscular fasciculi may b s more easily cleaned. As the posterior edge is approached, the cutaneous nerve and artery escaping from beneath it, are to be dissected out.

At the same time the fascia may be remored from the back of the scapula, so as to denude the muscles there.

The DELTOID MUSCLE is triangular in form (fig. $74,{ }^{\mathbf{F}}$ ), with the base at the scapula and clavicle, and the apex at the humerus. It arises from noarly all the lower edge of the spine of the scapula, from the anterior edge of the acromion, and from the outer half or third of the clavicle. Its fibres converge to a tendon, which is inserted into a triangular impression, two or three inches long and about one inch wide at the base, above the middle of the outer surtace of the humerus.

The anterior border is contiguous to the pectoralis major muscle and the cephalic vein; and the posterior rests on the infraspinatus and triceps muscles. The origin of the muscle corresponds with the attachment of the trapezius to the bones of the shoulder; the insertion is united with the tendon of the pectoralis major, and a fasciculus of the brachialis anticus is attached on each side of it.

Action. - The whole muscle raises the humerus, and abducts it from 
the trunk. The limb being raised, the anterior fibres will carry it forwards, and the posterior fibres will move it backwards.

When the humerus is fixed as in climbing, the muscle assists in supporting the weight of the body, and strengthening the shoulder-joint.

Dissection (fig. 74). The deltoid is to be divided near its origin, and is to be thrown down as much as the circumflex vessels and nerve beneath will permit. As the muscle is raised a large thick bursa between it and the head of the humerus comes into sight. The loose tissue and fat are to be taken away from the circumflex vessels and nerve; and the size of the bursa having been looked to, the remains are to be removed. The insertion of the muscle should be examined.

Fig. 74 .

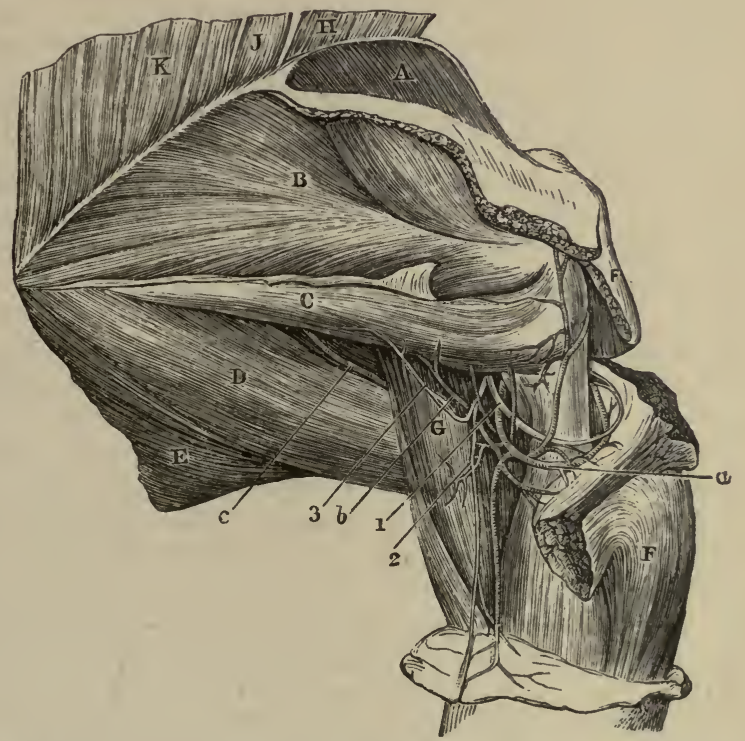

Vifw of the Muscles of the Dorsum of the Scapula, axd of the Circemfibx Vessils A.ND NERVE. (Illustrations of Dissections.)
Muscles :
A. Supra-spinatus.
B. Infra-spinatus.
c. Teres minor.
D. Teres major.
E. Latissimus dorsi.
F. Deltoid.
G. Triceps (long head).
H. Levator anguli scapulæ.
J. Rhomboideus minor.
к. Rhomboideus major.

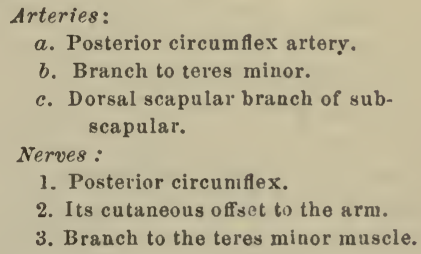

1. Posterior circuniflex.

2. Its cutaneous offset to the arm.

3. Branch to the teres minor muscle.

Parts covered by deltoid. The deltoid conceals the head and upper end of the humerus, and those parts of the dorsal scapular muscles which are fixed to the great tuberosity. A large bursa, sometimes divided into sacs, intervenes between the head of the humerus and the under surface of the deltoid muscle and the acromion process. Below the head of the 
bone are the circumflex vessels and nerve, and the upper part of the biceps muscle. In front of the humerus is the coracoid process with its muscles.

Dissection. By following back the posterior circumflex ressels and nerve through a space between the lumerus and the long head of the triceps, G, their connection with the axillary trunks will be arrived at. In clearing the fat from the space a branch of the nerve to the teres minor muscle is to be sought close to the border of the scapula, where it is surrounded by dense fibrous tissue.

Arching outwards in front of the neck of the humerus, is the small anterior circumflex artery: this is to be cleaned.

The circumflex arteries arise near the termination of the axillary trunk (p. 335); they are two in number, and are named anterior and posterior from their position to the neck of the humerus.

The anterior branch is a small artery, which courses beneath the coraco-brachialis and biceps muscles, and ascends in the bicipital groove to the articulation and the head of the humerus: it anastomoses with small offsets of the posterior circumflex.

The posterior circumflex artery (fig. 74, a), larger in size, winds backwards through a space between the humerus and the long head of the triceps, and is distributed chiefly to the deltoid muscle, in which it anastomoses with the acromial thoracic and upper profunda arteries.

Branches are given from it to the head of the humerus and the shoulder joint, and to anastomose with the anterior circumflex artery. It supplies branches likewise to the teres minor, the long head of the triceps, and the integuments.

The circumflex nerve (fig. $74,{ }^{1}$ ) leaves the arm-pit with the posterior circumflex artery (p. 335), and bends round the neck of the liumerus, beneath the deltoid muscle in which it ends. Many and large branches enter the deltoid, and terminate in it; one or two filaments pierce the fibres and become cutaneous.

Branches. In the axilla it gives an articular filament to the under part of the shoulder-joint. Behind the humerus it furnishes an offset to the teres minor $\left({ }^{3}\right)$, which has a reddish gangliform swelling on it. And at the edge of the deltoid it gives origin to the cutaneous nerve $\left({ }^{2}\right)$ before noticed.

The infraspinatus muscle (fig. $7 t, \mathrm{~B}$ ) occupies the infraspinal part of the scapula, and extends to the head of the humerus. The muscle arises from the infraspinal fossa, except at three spots, viz., the neck, and the lower angle and inferior border where the teres muscles are attached; it arises also from the lower side of the spinous process, and from the special fascia covering the surface. Its fibres converge to a tendon, which is $i n-$ serted into the middle impression on the great tuberosity of the humerus, and joins the tendons of the supraspinatus and teres minor.

A part of the muscle is subcutaneous, and the fibres arising from the spine of the scapula overlay the tendon: the upper portion is concealed by the deltoid; and the lower end, by the latissimus dorsi. The lower border is parallel to the teres minor, with which it is sometimes united. The muscle lies on the scapula and the humero-scapular articulation, but between it and the joint is a small bursa.

Action. With the humerus hanging it acts as a rotator outwards; and when the bone is raised it will move the same backwards in concert with the hinder part of the deltoid. 
The TERES MINOR (fig. 74, c) is a narrow fleshy slip, which is often united inseparably with the preceding muscle, along whose lower border it lies. It arises on the dorsum of the scapula from a special surface along the upper two-thirds of the inferior costa of the bone, and from the investing fascia ; and it is inserted by a tendon into the lowest of the three marks on the great tuberosity of the humerus, as well as by fleshy fibres into the humerus below that spot-about an inch together.

This muscle is partly covered by the deltoid; it rests on the long head of the triceps and the shoulder joint. Underneath it the dorsal branch of the subscapular artery turns.

Action. The arm hanging the muscle rotates it out and moves it back; the arm being raised the teres depresses the humerus.

In climbing it supports the joint like the preceding scapular muscles.

The TERES MAJOR muscle (fig. 74, D) is extended from the inferior angle of the scapula to the humerus. Its origin is from the rough surface on the dorsum of the bone at the inferior angle; from the inferior costa as far forwards as an inch from the long head of the triceps; and from the fascia covering the teres minor. The fibres end in a tendon which is inserted partly into, and partly behind the inner edge of the bicipital groove of the humerus.

This muscle assists in forming the posterior fold of the axilla; and is situate beneath the axillary vessels and nerves near the humerus (fig. 70). At its origin it is covered by the latissimus dorsi. The upper border is contignous to the subscapularis muscle, and the lower is received into a hollow formed by the fibres of the latissimus dorsi At the humerus the tendon of the muscle is one inch and a half to two inches wide, and is placed behind that of the latissimus: the two are separated above by a bursa; but they are united below, and an expansion is sent from them to the fascia of the arm. A second bursa lies between the tendon and the bone.

Action. If the limb hangs it is carried back behind the trunk and is rotated inwards by the muscle. The humerus being raised, the muscle depresses and adducts it.

With the limb fixed by the hand the teres will cause the lower angle of the scapula to move forwards.

Below the scapula (inferior costa), where the teres muscles separate from one another, is a triangular interval, which is bounded in front by the shaft of the humerus, and above and below by the teres muscles. This space is divided into two by the long head of the triceps. Through the anterior part, which is of a quadrilateral shape, the posterior circumflex vessels and the circumflex nerve pass : and opposite the posterior triangular space, the dorsal branch $(c)$ of the subscapular artery bends backwards.

Dissection (fig. 75). In order that the acromion process may be sawn through to expose the supraspinatus muscle, the ligaments of the scapula and clavicle, which would be injured by such a proceeding, should next be dissected.

A ligament (coraco-clavicular) ascends from the coracoid process to the under part of the clavicle: on removing the areolar tissue it will be seen to consist of two parts, anterior and posterior, differing in size, and in the direction of the fibres.

A capsular ligament, connecting the outer end of the clavicle with the acromion, will be recognized by taking away the fibres of the trapezius and deltoid muscles. 
Another strong band (coraco-acromial) passing transversely between the acromion and the coracoid process; and a small fasciculus (posterior proper ligament), placed over the notch in the superior costa, are then to be defined.

Ligaments of the Clavicle and Scapula (fig. 75). The clavicle is connected to the scapula by a distinct joint with the acromion, and by a strong ligament (coraco-clavicular) between it and the coracoid process.

The coraco-clavicular ligament consists of two parts, each having a different direction and designation.

The posterior piece $\left({ }^{1}\right)$, called conoid from its shape, is fixed by its apex to the posterior and inner part of the coracoid process; and by its base to the tubercle and the contiguous part of the under surface of the clavicle, at the junction of the outer with the middle third of the bone.

The anterior part $\left({ }^{2}\right)$, trapezoid ligament, is larger than the conoid piece : it is connected inferiorly to the inner border of the carocoid process along the hinder half; and superiorly to the line on the under suriace of the clavicle which extends outwards from the tubercle before mentioned. The two pieces of the ligament are in apposition posteriorly, but are separated by an interval in firont.

Use. Both pieces of the ligament support the scapula in a state of rest : they serve also to restrain the rotatory movements of that bone; thus

Fig. 75.

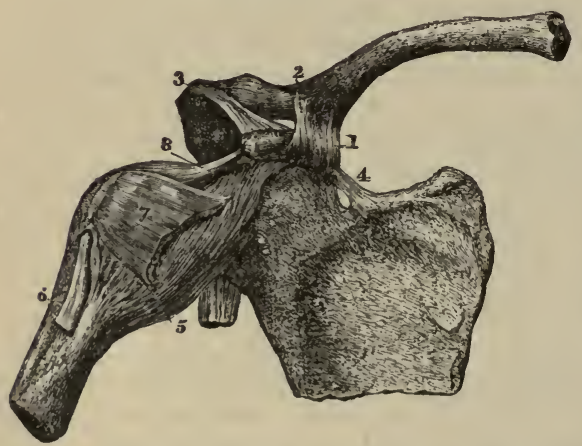

1. Conoid llgament.

2. Trapezoid ligament.

3. Anterior ligament of the scapnla.

4. Posterior scapular ligament.

5. Capsule of the shoulder joint.

6. Tendon of the long head of the biceps entering the joint.

7. Tendon of the subscapular muscle.

8. Coraco humeral ligament.

LiaAMENT OF THE CIAVICIE AND SCAPULA, AND OF THE SHOULDER JOINT

(altered from Bunrgery and Jacob).

when the acromion is rotated down, the motion is checked by the trapezoid band; and when upwards, by the conoid piece.

Acromio-clavicular articulation. The articular surfaces of the clavicle and acromion prozess of the scapula are retained in contact by a capsule formed of strong fibres. Some of the fibres are thicker above and below, and are considered to constitute a superior and an inferior ligament.

An interarticular fibro-cartilage generally exists at the upper part of the joint; but sometimes it forms a complete interarticular septum. If the fibro-cartilage is perfect, there are two synoviol membranes present in the joint; if it is imperfect, there is only one. 'The joint should be opened to see the cartilage and the synovial membrane.

Movements. In this articulation there are limited fore and back and up and down movements of the scapula. 
Besides, there is a gliding movement of the acromion on the clavicle in rotation of the seapula. For instance, when the acromion is depressed, its articular surface moves from above down at the fore part of the joint, and from below up at the back. When the acromion is elevated the surface moves in exactly the opposite way.

Scaptlar Ligaments. The special ligaments of the seapula are two in number, anterior and posterior, and extend from one part of the bone to another.

The posterior ligament $\left({ }^{4}\right)$ is a narrow fasciculus of fibres stretching across the notch in the upper costa of the scapula. By one end it is attached to the base of the coracoid process, and by the other to the costa behind the notch. It converts the notch into a foramen, through which the suprascapular nerve passes.

The anterior or coraco-acromial ligament $\left({ }^{3}\right)$ is triangular in form, and extends transversely between the acromion and the coracoid process. Externally it is inserted by its point or apex into the tip of the acromion; and internally, where it is much wider, it is attached to all the outer border of the coracoid process, reaching backwards to the capsule of the shoulder joint. The ligament consists usually of two thickened bands, anterior and posterior, with a thin intervening part. It forms part of an arch above the shoulder joint, which stops the ascent of the head of the humerus.

Dissection. To lay bare the supraspinatus muscle, the acromion process is to be sawn through, and to be turned aside with the outer end of the clavicle: but in the repetition of the dissection of the upper limb, the bone may be left uncut for the purpose of seeing the use of the coracoacromial arch. A strong fascia covers the surface of the muscle; this is to be taken away after it has been observed.

The supraspinatus nuscle (fig. $74,{ }^{\wedge}$ ) has the same form as the hollow of the bone it fills. It arises from the surfice of the supra-spinal fossa of the scapula, except from the cervical part ; from the upper side of the spine of the bone; and from the fascia covering the surface. Its fibres end in a tendon, which erosses over the shoulder joint, and is inserted into the upper impression of the great tuberosity of the humerus.

The muscle is concealed by the trapezius and the acromion process; and it rests upon the scapula, the shoulder joint, and the suprascapular ressels and nerve. Its tendon joins that of the infraspinatus at the attachment to the humerus.

Action. It comes into use with the deltoid in raising the limb, and supporting the joint.

Dissection (fig. 78). The ressels and nerves on the dorsum of the scapula can be traced by detaching from behind forwards the supra and infraspinatus muscles, so as to leave a thin layer of the fleshy fibres with the ramifying bloodressels on the surface of the bone. In the supraspinal fossa are the suprascapular vessels and nerve, which are to be followed beneath the acromion to the infraspinal fossa; and entering the infraspinal fossa, beneath the teres minor muscle, is the dorsal branch of the subscapular artery. The anastomosis between those vessels should be pursued in the fleshy fibres and cleaned.

The suprascapular artery $(u)$ is derived from the subclavian trunk, and is one of the branches of the thyroid axis (p. 78). After a short course in the neck it passes orer the ligament at the superior costa, and crossing beneath the supraspinatus muscle, ends in the infraspinal tossa, 
where it gives offsets to the infraspinatus muscle and the scapula, and anastomoses with the dorsal branch of the subscapular, and the posterior seapular artery of the subclavian.

Beneath the supraspinatus it furnishes a supraspinal branch for the supply of the muscle, the bone, and the shoulder-joint.

The companion vein of the suprascapular artery joins the external jugular vein.

The suprascapular nerve $\left({ }^{1}\right)$ is a branch of the brachial plexus (p. 80 ). When it reaches the costa of the scapula, it enters the supraspinal fossa beneath the posterior special ligament. In the fossa it supplies two branches to the supraspinatus; and is continued beneath a fibrous band to the infraspinatus muscle, in which it ends.

The nerve gives some articular filaments to the shoulder-joint, and other offsets to the scapula.

The posterior scapular artery runs along the base of the scapula beneath the rhomboid muscles, furnishing offsets to them and the surfaces of the bone. It is more fully noticed with the dissection of the Back.

The dorsal branch of the subscapular artery $(b)$ (p. 335) turns below the inferior costa of the scapula, opposite the posterior of the two spaces between the teres muscles. Entering the infraspinal fossa beneath the teres minor, it supplies that muscle and the infraspinatus, and communicates with the suprascapular artery. This vessel sends a branch along the dorsum of the scapula between the teres muscles, towards the inferior angle of the bone.

\section{Section III.}

\section{THE FRONT OF THE ARM.}

Position. For the dissection of the superficial vessels and nerves on the front of the arm, the limb should lie flat on the table, with the front uppermost.

Dissection. The skin is to be raised from the fore and hinder parts of the arm and elbow-joint. 'To reflect it, make one incision along the centre of the limb as far as two inches below the elbow; and at the termination, a second cut half round the forearm. Strip now the skin from the limb, as low as the transverse incision, so that the fat which contains the cutaneous vessels and nerves may be denuded. Between the skin and the prominence of the olecranon a bursa may be seen.

The cutaneous veins (fig. 76) may be sought first in the fat: they are very numerous below the bend of the elbow, as they issue from beneath the integument. One in the centre of the forearm is the median vein, which bifurcates rather below the elbow. External to this is a small vein (radial); and internal to it are the anterior and posterior ulnar veins, coming from the front and back of the forearm. At the elbow these veins are united into two; one (basilic) is to be followed along the inner side, and the other (eephalic) along the outer side of the arm.

The cutaneous nerves may be next traced out. Where they perforate the deep fascia they lie beneath the fat : and this layer must be scraped through to find them. 
On the outer side of the arm, about its middle, two external cutaneous branches of the musculo-spiral are to be sought. In the outer bicipital groove, in front of the elbow or rather below it, the cutaneous part of the musculo-cutaneous nerve will be recognized.

On the inner part of the limb the nerves to the surface are more numerous. Taking the basilic vein as a guide, the internal cutaneous nerve of the forearm will be found by its side, about the middle of the arm; and rather external to this nerve is a small cutaneous offset from it, which pierces the fascia higher up. Scrape through the fat behind the internal cutaneous, in the lower third of the arm, for the small nerve of Wrisberg; and in the upper third, seek the small nerves which have been already met with in the dissection of the axilla, viz., the intercosto-humeral, and the internal cutaneous of the musculo-spiral.

Superficial fascia. The subcutaneous fatty layer forms a continuous investment for the limb, but it is thicker in front of the elbow than in the other parts of the arm. In that spot it incloses the superficial vessels and the lymphatics.

Cutaneous Verss. The position and the connections of the superficial veins in front of the elbow are to be attentively noted by the dissector, because the operation of venesection is practised in one of them.

The median vein of the forearm (fig. $76,{ }^{2}$ ), divides into two branches, internal and external, rather below the bend of the elbow ; at its point of division it is joined by an offset from a deep vein. The internal branch (median basilic) crosses to the inner border of the biceps, and unites with the ulnar veins $\left({ }^{3}\right)$ to form the basilic vein of the inner side of the arm. The external branch (median cephalic) is usually longer than the other, and by its junction with the radial vein $\left({ }^{7}\right)$ gives rise to the cephalic vein of the arm.

The connections of the two veins into which the median bifurcates, are described below :-

The median cephalic vein (fig. 76) is directed obliquely, and lies over the hollow between the biceps and the outer mass of muscles of the forearm. Beneath it is the trunk of the musculo-cutaneous nerve; and over it some small offsets from the nerve are directed. This vein is altogether removed from the brachial artery, and is generally smaller than the median basilic vein. If opened with a lancet, it does not generally yield much blood, in consequence of its position in a hollow between muscles rendering compression of it very uncertain and difficult.

The median basilic vein (fig. $76,^{1}$ ) is more horizontal in direction than the preceding, and crosses the brachial artery. It is larger than the corresponding vein of the outer side of the arm, and is firmly supported by the underlying fascia - the aponeurosis of the arm, strengthened by fibres from the biceps tendon, intervening between it and the brachial ressels. Branches of the internal cutaneous nerve lie beneath it, and some twigs of the same nerve are placed over it.

The median basilic is the vein on which the operation of blood-letting is commonly performed. It is selected in consequence of its usually larger size, and more superficial position, and of the ease with which it may be compressed; but from its close proximity to the brachial vessels, the spot to be opened should not be immediately over the trunk of the artery.

The basilic vein (fig. $76,{ }^{5}$ ), commencing as before said, ascends near the inner border of the biceps muscle to the middle of the arm, where it 
passes beneath the deep fascia, and is continuous with the axillary vein. In this course it lies to the inner side of the brachial artery.

The cephalic vein (fig. $76,{ }^{2}$ ) is derived chiefly from the external branch of the median, for the radial vein is oftentimes very small : it is continued to the shoulder along the outer side of the biceps, and sinks between the deltoid and pectoral muscles, near the clavicle, to open into the axillary vein.

Fig. 76.

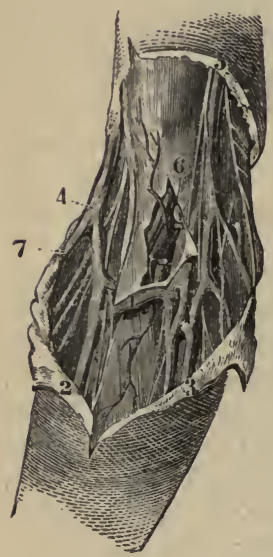

1. Median basilic vein.

2. Median vein of the forearm bifurcating.

3. Anterior ulnar veins.

4. Cophalic vein formed by radial from behind and the median cephalic in front. The musculo-cutaneous nerve is by the side of it.

j. Basilic vein, with large internal cutaneous nerve by its side.

6. Brachial artery, with its companion veins (one cut).

7. Radial vein.

Cetaneots Veins and Nerves. at the Bend of the Elbow. (Quain's "Arteries.")

The superficial lymphatics of the arm lie for the most part along the basilic vein, and enter into the glands of the axilla. A f'ew lymphatics accompany the cephalic vein, and end as the others in the axillary glands.

One or more superficial lymphatic glands are commonly found near the inner condyle of the humerus.

Cutaneous Nerves. The superficial nerves of the arm appear on the inner and outer sides, and spread so as to cover the surface of the limb. With one exeeption (intercosto-humeral) all are derived from the brachial plexus, either as distinet branches, or as offsets of other nerves. On the outer side of the limb are branches of the musculo-spiral and musculocutaneous nerves. On the inner side are two internal cutaneous nerves, large and small (from the plexus), a third internal cutaneous from the musculo-spiral, and the intercosto-humeral nerve.

External cutaneous Nerves. 'The external cutaneons branches of the musculo-spiral nerve are two in number, and appear at the outer side of the limb about the middle. The upper small one turns forwards with the cephalic vein, and reaches the front of the elbow, supplying the anterior part of the arm. The lower and larger pierees the fascia somewhat farther down, and after supplying some cutaneous filaments, is continued to the forearm.

The cutaneous part of the musculo-cutaneous nerve pierces the fascia in front of the elbow; it lies beneath the median cephalie vein, and divides into branches for the forearm.

Internal Cutaxeous Nerves. The larger internal cutaneous nerve perforates the fascia in two parts, or as one trunk that divides almost 
directly into two:-Its external branch passes beneath the median basilic vein to the front of the forearm ; and the internal winds over the inner condyle of the humerus to the back of the forearm.

A cutaneous offset of the nerve pierces the fascia near the axilla, and reaches as far, or nearly as far as the elbow: it supplies the integuments over the inner part of the biceps muscle.

The small ivternal rutaneous nerve (Wrisberg) appears below the preceding, and extends to the interval between the olecranon and the inner condyle of the humerus, where it ends in filaments over the back of the olecranon. The nerve give offsets to the lower third of the arm on the inner and posterior surfaces, and joins above the elbow the inner branch of the larger internal cutaneous nerve.

The internal cutaneous branch of the musculo-spiral nerve becoming subcutaneous in the upper third, winds to the back of the arm, and reaches nearly as far as the olecranon.

The intercosto-humeral branch of the second intercostal nerve (p. 226), perforates the fascia near the axilla, and ramifies in the inner side and posterior surface of the arm in the upper half. But the size and distribution of the nerve will depend upon the derelopment of the small internal cutaneous and the offsets of the musculo-spiral.

The aponeurosis of the arm is a white shining membrane which surrounds the limb, and sends inwards processes between the muscle. Orer the biceps muscle it is thinner than elsewhere. At certain points it receives accessory fibres from the subjacent tendons:-thus in front of the elbow an offset from the tendon of the biceps joins it; and near the axilla the tendons of the pectoralis major, latissimus dorsi, and teres, send prolongations to it.

At the upper part of the limb the fascia is continuous with that of the axilla, and is prolcnged over the deltoid and pectoral muscles to the scapula and the clavicle. Inferiorly it is continued to the forearm, and is connected to the prominences of bone around the elbow joint, especially to the condyloid ridges of the humerus so as to give rise to the intermuscular septa of the arm.

Directions. As the back of the arm will not be dissected now, the skin may be replaced on it until the front has been examined. And to kerep in place the vessels and nerves at the upper part of the limb, these should be tied together with string in their natural position to one another, and fastened to the coracoid process.

Position. The limb is still to lie on the back, but the scapula is to be raised by means of a small block; and the bladebone is to be fixed in such a position as to render tense the muscles. The inner surface of the arm is to be placed towards the dissector.

Dissection. The aponeurosis is to be reflected from the front of the arm by an incision along the centre, like that through the integuments: and it is to be removed on the outer side as far as the onter condyloil ridge of the humerus, but on the inner side rather farther back than the corresponding line, so as to lay bare part of the triceps muscle. In raising the fascia the knife must be carried in the direction of the fibres of the biceps muscle; and to prevent the displacement of the brachial artery and its nerves, fasten them here and there with stitches.

In front of the elbow is a hollow containing the brachial vessels: the artery should be followed into it, to show its ending in the radial and ulnar trunks. 
Muscles on the Front of the Aris. There are only three muscles on the fore part of the arm. The one along the centre of the $\operatorname{limb}$ is the biceps; and that along its inner side, reaching about half way down, is the coraco-brachialis. The brachialis anticus lies beneath the biceps. Some muscles of the forearm are connected to the inner and outer condyles of the humerus, and to the line above the outer condyle.

The BICEPS MUSCLE (fig. $78,{ }^{\mathrm{B}}$ ) forms the prominence observable on the front of the arm. It is wider at the middle than at either end; and the upper part consists of two tendinous pieces of different lengths, which are attached to the scapula. The short head arises from the apex of the coracoid process in common with the coraco-brachialis muscle (fig. 73); and the long head is attached to the upper part of the glenoid cavity of the scapula, within the capsule of the shoulder joint (fig. 89). Muscular fibres spring from each tendinous head, and blend about the middle of the arm in a fleshy belly, which is somewhat flattened from before back. Inferiorly the biceps ends in a tendon, and is inserted into the tubercle of the radius.

The muscle is superficial except at the extremities. At the upper part it is concealed by the pectoralis major and deltoid muscles; and at the lower end the tendon dips into the hollow in front of the elbow, having previously given an offset to the fascia of the arm. Beneath the biceps are the brachialis anticus muscle, the musculo-cutaneous nerve, and the upper part of the humerus. Its inner border is the guide to the brachial artery below the middle of the humerus, but above that spot the coracobrachialis muscle intervenes between them. 'The connection of the long head of the biceps with the shoulder joint, and the insertion of the muscle into the radius, will be afterwards learnt.

Action. It hends the elbow-joint, and acts powerfully in supinating the radius. When the body is hanging by the hands it will apply the scapula firmly to the humerus, and will assist in raising the trunk.

With the arm hanging and the radius fixed, the long head will assist the abductors in removing the limb from the thorax; and after the limb is abducted, the short head will aid in restoring it to the pendent position.

The coraco-Brachial.is (fig. $73,{ }^{\mathrm{H}}$ ) is roundish in form, and is named from its attaehments. Its origin is fleshy from the tip of the coracoid process, and from the tendinous short head of the biceps. Its fibres become tendinous, and are inserted, below the level of the deltoid muscle, into the ridge on the inner side of the humerus: from the insertion an aponeurotic slip is continued upwards to the head of the humerus, and is joined by fleshy fibres.

Part of the muscle is beneath the pectoralis major (fig. 78), and forms a prominence in the axilla; but the rest is superficial, except at the insertion where it is covered by the brachial vessels and the median nerve. The coraco-brachialis conceals the subscapular muscle, the anterior circumflex artery, and the tendons of the latissimus and teres. Along the inner border are the large artery and nerves of the limb. Perforating it is the musculo-cutaneous nerve.

Action. The hanging limb is adducted to the thorax by this muscle; and the action is greater in proportion as the lumerus is removed from the trunk.

The humerus being fixed, the muscle will bring down the scapula, and assist in keeping the articular surfaces of the shoulder joint in apposition. 
The BRACHIAL ARTERY (fig. $77,{ }^{4}$ ) is a continuation of the axillary trunk, and supplies vessels to the upper limb. It begins at the lower border of the teres major muscle, and terminates rather below the bend of the elbow, or "opposite the neck of the radius" (Quain), in two branches - radial and ulnar, for the forearm.

In the upper part of its course, the vessel is internal to the humerus, but afterwards in front of that bone; and its situation is indicated by the surface depression along the inner border of the biceps and coraco-brachialis muscles.

Fig. 77.

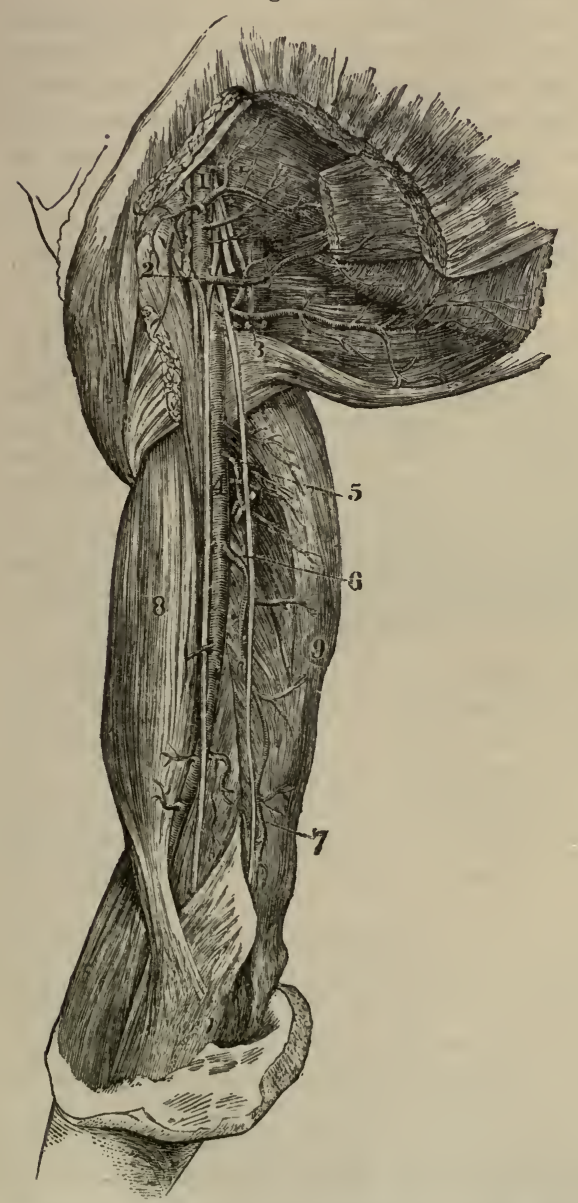

1. Axillary artery and branches : the small branch above the figure is the highest thoracic, and the larger branch close below, the acromial thoracic.

2. Long thoracic branch.

3. Subscapular branch.

4. Brachial artery and branches.

5. Superior profunda branch.

6. Inferior profunda branch.

7. Anastomotic branch.

8. Biceps muscle.

9. Triceps muscle.

The median aud ulnar nerves are shown in the arm; the median is close to the brachial artery.

Aximary axd Brachial Arteribs axd their Braxches. (Quain's "Arteries.")

In all its extent the brachial artery is superficial, being covered by the integuments and the deep fascia; but at the bend of the elbow it becomes deeper, and is crossed by the prolongation from the tendon of the biceps. Posteriorly the artery has the following muscular connections (fig. 78) :- 
Whilst it is inside the humerus it is placed over the long head of the triceps $\mathrm{F}$, for two inches, but separated partly by the musculo-spiral nerve and profunda vessels; and over the inner head, $\mathrm{G}$, of the same muscle for about an inch and a half. But when the vessel turns to the front of the bone, it lies on the insertion of the coraco-brachialis, $\mathrm{c}$, and on the brachialis anticus, Ir. To the outer side are laid the coraco-brachialis and biceps muscles, $\mathrm{c}$, and $\mathrm{B}$, the latter overlapping it.'

Veins. Venæe comites lie on the sides of the artery (fig. $78, d$ ), encircling it with branches, and the median basilic vein crosses over it at the elbow. The basilic vein is near, and inside the artery above, but is superficial to the fascia in the lower half of the arm.

The nerves in relation with the artery are the following:-The internal cutaneous $\left({ }^{2}\right)$ is in contact with the vessel until it perforates the fascia about the middle of the arm. The ulnar nerve: $\left({ }^{4}\right)$ lies to the inner side as far as the insertion of the coraco-brachialis muscle; and the musculospiral (fig. $69,{ }^{4}$ ) is behind for a distance of two inches. The median nerve ( $\left.{ }^{1}\right)$ is close to the vessel throughout, but alters its position in this way:-as low as the insertion of the coraco-brachialis it is placed on the outer side, but it then crosses obliquely either over or under the artery, and becomes internal about two inches above the elbow joint.

Peculiarities in position. The brachial trunk may leave the inner border of the biceps in the lower half of the arm, and course along the internuscular septum, with or without the median nerve, to the inner condyle of the humerus. At this spot the vessel is directed to its ordinary position in front of the elbow, either through or beneath the fibres of the pronator teres, which has then a wide origin. In this unusual course the artery lies behind a projecting bony point of the liumerus.

Muscular covering. In some bodies the humeral artery is covered by an additional slip of origin of the biceps, or of the brachialis anticus muscle. And sometimes a slip of the brachialis may conceal, in cases of high origin of the radial, the remainder of the arterial trunk continuing to the forearm.

Branches spring both externally and internally from the brachial artery (fig. 77). Those on the outer side, muscular, supply the coraco-brachialis, biceps, and brachialis anticus; those on the inner side are named superior and inferior profunda, nutritious, and anastomotic.

The superior profunda branch $\left(^{5}\right)$ is larger than the others, and leaves the artery near the lower border of the teres major; it winds backwards with the musculo-spiral nerve to the triceps muscle, and will be dissected with the back of the arm.

The inferior profunda branch $\left({ }^{6}\right)$ arises opposite the coraco-brachialis muscle, and accompanies the ulnar nerve to the interval between the olecranon and the inner condyle of the humerus. There it anastomoses with the posterior ulnar recurrent and anastomotic branches, and supplies the triceps. It arises often in common with the superior profunda irtery.

A nutritious artery of the bone shaft begins near the preceding branch, and enters the large aperture about the middle of the humerus; it is distributed to the osseous and the medullary substance.

'The anastomotic branch $\left({ }^{7}\right)$ arises one to two inches above the elbow, and courses inwards through the intermuscular septum to the loollow between the olecranon and the inner condyle of the humerus. Here the artery inosculates with the inferior profunda and posterior ulnar recurrent branches, and gives twigs to the triceps muscle: one of the offsets forms 
an arch across the back of the humerus with a branch of the superior profunda.

In front of the elbow joint the anastomotic branch sends an offset to the pronator teres muscle: this joins the anterior ulnar recurrent branch.

Vasa aberrantia. Occasionally long slender vessels connect the brachial or the axillary trunk with the radial artery; the accessory ressel very rarely ends in the ulnar artery.

The BRACHIAL VEINS (fig. $78, d$ ) accompany the artery, one on each side, and have branches of communication across that vessel; they receive contributing veins corresponding with the branches of the arteries. Superiorly they unite into one, which joins the axillary vein near the subscapular muscle.

Nerves of the Aru (fig. 78). The nerres on the front of the arm are derived from the terminal cords of the brachial plexus. Few of them furnish offsets above the elbow, but they are continued, for the most part without branching, to the forearm and the hand. The cutaneous branches of some of them have been referred to (p. 250).

Fig. 78.

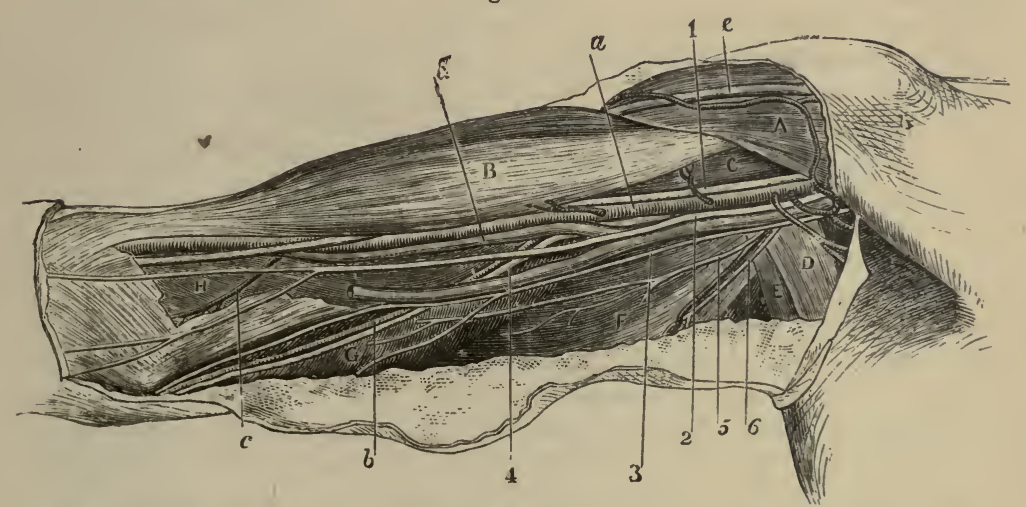

Mugcleg AND DeEp Vesgels and Nervi.s of The ARM. (Illustrations of Dissection*.)

Mescles:

A. Pectoralis major.

B. Biceps.

c. Coraco-brachlalis.

$D$ and $B$. Latissimus and teres.

F. Long head of the triceps.

(t. Inner head of triceps.

H. Brachialis anticus.
Nerves :

1. Median.

2. Internal cutaneous.

3. Nerve of Wrisberg.

4. Uluar.

5. Muscular to the triceps.

6. Internal cutanewns from the musculo-spiral.

The median nerve $\left({ }^{1}\right)$ arises from the brachial plexus by two roots, one from the outer, and the other from the inner cord (p. 236). Its destination is to the palm of the hand; and it accompanies the brachial artery to the forearm. Beginning on the outer side of the artery, the nerve crosses over or under it about the middle of the arm, and is placed on the inner side a little above the elbow. It does not give any branch in the arm; but there may be a fasciculus connecting it with the musculo-cutaneous nerve. Its connections with muscles are the same as those of the artery.

The ulnar nerve ( $\left.{ }^{4}\right)$, derived from the inner cord of the brachial plexus, 
ends at the inner side of the hand. In the arm the nerve lies at first close to the inner side of the axillary, and the brachial artery, as far as the insertion of the coraco-brachialis; then leaving the bloodvessel, it is directed inwards through the inner intermuscular septum to the interval between the olecranon and the inner condyle, being surrounded by the muscular fibres of the triceps. There is not any branch from the nerve till it reaches the elbow-joint.

The internal cutaneous $\left({ }^{2}\right)$ is a tegumentary nerve of the forearm, to which it is prolonged like the others. Arising from the inner cord of the plexus, it is at first superficial to the humeral artery as far as the middle of the arm, where it divides into two branches that perforate the investing fascia and reach the forearm (p. 250). Near the axilla it furnishes a small cutaneous offset to the integuments of the arm.

The small internal cutaneous nerve $\left({ }^{3}\right)$ (nerve of Wrisberg) arises with the preceding. Concealed at first by the axillary vein, it is directed inwards beneath (but sometimes through) that vein, and joins with the intercosto-humeral nerve. Afterwards it lies along the inner part of the arm as far as the middle, where it perforates the fascia to end in the integument (p. 251).

The musculo-cutaneous nerve (nerv. perforans, Casserii), named from supplying muscles and integuments, ends on the surface of the forearm. It leaves the outer cord of the brachial plexus opposite the lower border of the pectoralis minor (fig. 70) and perforates directly the coraco-brachialis: it is then directed obliquely to the outer side of the limb between the biceps and brachialis anticus muscles. Near the elbow it becomes a cutaneous nerve of the forearm.

Branches. The nerve furnishes branches to the muscles in front of the humerus, viz., to the coraco-brachialis as it passes through the fibres, and to the biceps and brachialis anticus where it is placed between them.

Dissection. The brachialis anticus muscle will be brought into view by cutting through the tendon of the biceps near the elbow, and turning upwards this muscle. The fascia and areolar tissue should be taken from the fleshy fibres; and the lateral extent of the muscle should be well defined on each side, so as to show that it reaches the intermuscular septum largely on the inner side, but only for a short distance above on the outer side.

Some care is required in detaching the brachialis externally from the muscles of the forearm, to which it is closely applied. As the muscles are separated the musculo-spiral nerve with a small artery comes into sight.

The brachialis axticus (fig. $78,{ }^{\text {H}}$ ) cover's the elbow-joint, and the lower half of the front of the humerus. It arises from the anterior surface of the humerus below the insertion of the deltoid muscle; and from the intermuscular septa on the sides, viz., from all the inner, but from only the upper part of the outer (about one inch and a half.) The flesliy fibres converge to a tendon, which is inserted into the impression on the front of the coronoid process of the ulna (p. 271).

This muscle is concealed by the biceps. On it lies the brachial artery, with the median, musculo-cutaneous, and musculo-spiral nerves. It covers the humerus and the articulation of the elbow. Its origin embraces by two parts the attachment of the deltoid: and its insertion is placed between two fleshy pieces of the flexor profundus digitorum. 'The inner border reaches the intermuscular septum in all its length; but the outer is separated from the external intermuscular septum below by two muscles of 
the forearm (supinator longus and extensor carpi radialis longior), which extend upwards on the humerus.

Action. The brachialis brings forwards the ulna towards the humerus, and bends the elbow-joint.

If the ulna is fixed, as in climbing with the hands above the head, the muscle bends the elbow-joint by raising the humerus.

\section{BACK OF THE ARM.}

Position. During the examination of the back of the arm, the limb is to be raised in a semiflexed position by means of a block beneath the elbow. The scapula is to be brought nearly in a line with the humerus, so as to tighten the muscular fibres; and it is to be fastened with hooks in that position.

Fig. 79.

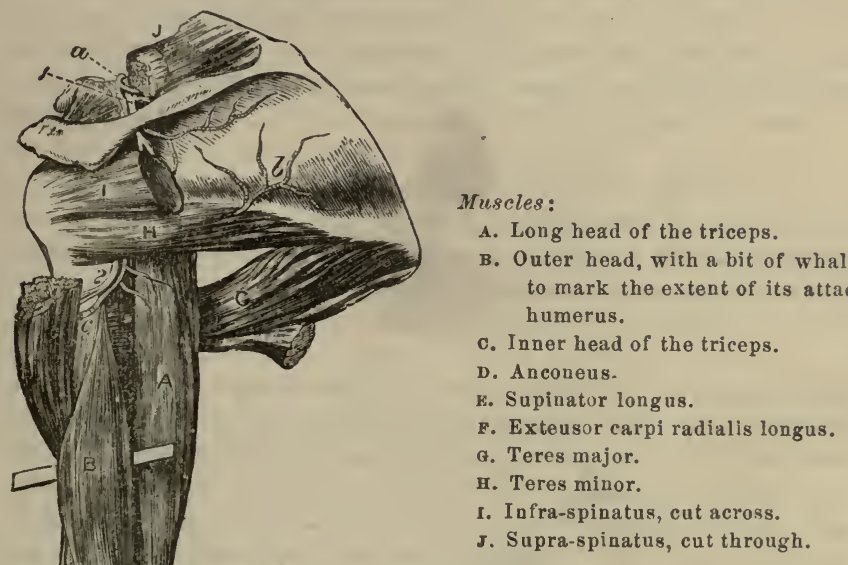

Arteries :

a. Supra-seapular.

b. Dorsal scapular.

c. Posterior circuinflex.

Nerves:

1. Supra-scapular.

2. Posterior circumflex.

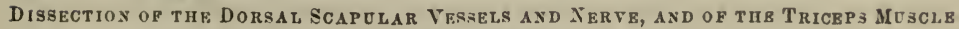
OF THE ARM.

Dissection (fig. 79). On the back of the arm there is one muscle, the triceps, with the musculo-spiral nerve and superior profunda vessels. The skin having been reflected already, the muscle will be laid bare readily, for it is covered only by fascia. To take away the fascia, carry an incision along the middle of the limb to a little below the elbow ; and in reflecting it, the subaponeurotic loose tissue should be removed at the same time. 
Separate the middle from the inner and outer heads of the muscle, and trace the musculo-spiral nerve and vessels beneath it. Define the outer head which reaches down to the spot at which the musculo-spiral nerve appears on the outer side.

'The TRICEPS MUSCLE (fig. 79), is divided superiorly into three parts or heads of origin, inner, outer, and middle: two of these are attached to the humerus, and one to the scapula.

The middle piece, or head, $\mathrm{A}$, is the longest, and has a tendinous origin, about an inch wide, from the inferior costa of the seapula close to the glenoid eavity, where it is united with the capsule of the shoulder joint. The outer head, $\mathrm{B}$, is narrow and arises from the back of the humerus above the spiral groove, extending from the root of the large tuberosity to that groove. The inner head, $\mathrm{c}$, fleshy and wide, arises from the posterior surface of the humerus below the spiral groove, reaching laterally to the intermuscular septa, and gradually tapering upwards as far as the insertion of the teres major (Theile). From the different heads the fibres are directed with varying degrees of inclination to a common tendon at the lower part. Inferiorly the muscle is inserted into the end of the olecranon process of the ulna, and gives an expansion to the ajoneurosis of the forearm. Between the tip of the olecranon and the tendon is a small bursa.

The triceps is superficial, except at the upper part where it is overlapped by the deltoid muscle. It lies on the humerus, and conceals the musculospiral nerve, the superior profunda vessels, and the articulation of the elbow. On the sides the muscle is united to the intermuscular septa ; and the lower fibres are continuous externally with the anconeus-a inuscle of the forearm.

Action. All the parts of the triceps combining in their action will bring the ulna into a line with the humerus, and extend the elbow-joint. As the long head passes the shoulder it can depress the raised humerus, and adduct the bone to the thorax.

The intermuscular septa are fibrous processes continuous with the investing aponeurosis of the arm, which are fixed to the ridges leading to the condyles of the humerus: they intervene between the muscles on the front and back of the limb, and give attachment to fleshy fibres.

The internal is the strongest, and reaches as high as the coraco-brachialis muscle, from which it receives some tendinous fibres. 'The brachialis anticus is attached to it in front, and the triceps behind; and the uhar nerve, and the inferior profunda and anastomotic vessels pierce it.

The external septum is thinner, and ceases at the deltoid muscle. Behind it is the triceps; and in front are the brachialis anticus, and the muscles of the forearm (supinator longus and extensor carpi radialis longus) arising above the condyle of the humerus: it is pierced by the musculo-spiral nerve and the accompanying vessels.

Dissection. To follow the superior profunda ressels and the musculospiral nerve, the middle head of the triceps should be cut across over them, and the fatty tissue should be removed. The trunks of the artery and nerve are to be afterwards followed below the outer head of the triceps to the front of the humerus.

To trace out the branches of the nerve and artery, which descend to the olecranon and the anconeus muscle, the triceps is to be divided along the line of union of the outer with the midtle head.

The superior profunda branch of the brachial artery (p. 254) turns to 
the back of the humerus between the inner and outer heads of the triceps; in this position it supplies branches to the triceps and deltoid muscles, and is continued onwards in the groove in the bone to the outer part of the arm, where it divides into its terminal offsets:-One of these, which is very small, courses on the musculo-spiral nerve to the front of the elbow, anastomosing with the recurrent radial branch: whilst others continue along the intermuscular septum to the elbow, and join the radial and posterior interosseous recurrent branches.

Branches. Most of the muscular offsets of the vessels descend to the olecranon, supplying the triceps, and communicate with the inferior profunda and anastomotic branches of the brachial artery (p. 254); and with the recurrent branches of the arteries of the forearm except the anterior ulnar. One slender offset accompanies a branch of the musculo-spiral nerve, and ends in the anconeus muscle below the outer condyle of the humerus.

Two or more cutaneous offsets arise on the outside of the arm, and accompany the superficial nerves.

The musculo-spiral nerve (fig. 70) is the largest trunk of the posterior cord of the brachial plexus (p. 236) and is continued along the back and outer part of the limb to the hand. In the arm the nerve winds with the profunda artery beneath the triceps muscle. At the outer aspect of the arm it is continued between the brachialis anticus and supinator longus muscles to the external condyle of the humerus, in front of which it divides into the radial and posterior interosseous nerves. The nerve gives muscular branches, and the following cutaneous offsets to the inner and outer parts of the limb.

a. The internal cutaneous branch of the arm (fig. $78,{ }^{6}$ ) is of small size, and arises in the axillary space in common with the branch to the inner head of the triceps; it is directed across the posterior boundary of the axilla to the inner side of the arm, where it becomes cutaneous in the upper third, and is distributed as before said (p. 250).

$b$. The external cutaneous branches springing at the onter side of the limb are two in number: they are distributed in the integuments of the arm and forearm (p. 250).

$c$. 'The muscular branches to the triceps are numerous, and supply all three heads. One slender offset for the inner head, arises in common with the inner cutaneous branch, and lies close to the ulnar nerve till it enters the muscular fibres at the lower third of the arm. Another long and slender branch behind the humerus, appearing as if it ended in the triceps, can be followed downwards to the anconeus muscle.

$d$. On the outer side of the limb the musculo-spiral nerve supplies the brachialis anticus in part, and two muscles of the forearm, viz., supinator longus and extensor carpi radialis longior.

Subanconeus muscle. A thin fleshy stratum of the under part of tlie triceps near the elbow has been so named. It is described as consisting of two fasciculi, inner and outer, which are attached above the fossa for the olecranon, and end in the synovial sae of the joint. A corresponding muscle is placed beneath the extensor of the knee joint.

Action. It is said to raise the synovial membrane in extension of the joint.

Directions. As the dissection of the arm has been completed as far as the elbow, it will be advisable to keep moist the shoulder joint until it is examined with the other ligaments. 


\section{Section IV.}

\section{THE FRONT OF THE FOREARM.}

Position. The limb is to be placed with the palm of the hand uppermost ; and the marking of the surface, and the projections of the bone, are first to be noted.

Surface-marking. On the anterior aspect of the forearm are two lateral depressions, corresponding with the position of the main vessels. The external is placed over the radial artery, and inclines towards the middle of the limb as it approaches the elbow. The internal groove is evident only beyond the middle of the forearm, and points out the place of the ulnar artery.

The bones (radius and ulnar) are sufficiently near the surface to be traced in their whole length: each ends below in a point-the styloid process; and that of the radius is the lowest. A transverse line separates the forearm from the hand, and the articulation of the wrist is about an inch above it.

On each side of the palm of the hand is a lateral projection; the external of these (thenar) is formed by museles of the thumb, and the internal (hypo-thenar) by museles of the little finger. Between the projections is the hollow of the palm, which is pointed towards the wrist. Two transverse lines are seen in the palm, but neither reaches completely across it: the anterior one will direct to the line of the articulations between the metacarpus and the phalanges, but is about a quarter of an inch behind the three inner joints when the fingers are extended.

The superficial palmar arch of arteries reaches forwards a little way into the hollow of the hand, and its position may be marked by a line across the palm from the root of the thumb, when that digit is placed at a right angle to the hand.

Transverse lines are seen on both aspects of the joints of the thumb and fingers. The lines on the palmar surface of the fingers may be used to detect the articulations of the phalanges. Thus the joint between the metacarpal phalanx and the next will be found about a line in front of the chief transverse groove; whilst the articulation between the last two phalanges is situate about a line in front of the single mark.

Dissection. With the limb lying flat on the table, an incision is to be earried through the skin along the middle of the front of the forearm, as far as an inch beyond the wrist ; and at its termination a transverse one is to cross it. The skin is to be reflected earefully from the front and back of the forearm, without injury to the numerous superficial vessels and nerves heneath; and it should be taken also from the back of the hand, by prolonging the ends of the transverse eut along each margin to a little beyond the knuckles. The whole of the forefinger should have the integument removed from it, in order that the nerves may be followed to the end.

The superficial vessels and nerves ean be now traced in the fat; they have the following position, and most of them have been partly dissected: Along the inner side, in front of the forearm with the ulnar veins, is the continuation of the internal cutaneous nerve; and near the wrist there is 
occasionally a small offset from the ulnar nerve. On the outer side with the radial vein is the superficial part of the musculo-cutaneous nerve.

Close to the hand, in the centre of the forearm, and inside the tendon of the flexor carpi radialis which can be rendered prominent by extending the wrist, the small palmar branch of the median nerve should be sought beneath the fat. On the ulnar artery, close inside the pisiform bone, a small palmar branch of the ulnar nerve is to be looked for.

At the back of the forearm the largest external cutaneous branch of the musculo-spiral nerve is to be traced onwards ; and offsets are to be followed to this surface from the nerves in front.

On the posterior part of the hand is an arch of superficial veins. Winding back below the ulna is the dorsal branch of the ulnar nerve; and lying along the outer border of the hand is the radial nerve: these should be traced to the fingers.

Cutaneous Verns. The superficial veins are named median, radial, and ulnar, from their position in the limb.

Superficial arch on the back of the hand. This areh is more or less perfect, and receives the posterior superficial digital veins. At the sides the arch terminates in the radial and ulnar veins.

The radial vein begins in the outer part of the arch above mentioned, and in some small radicles at the back of the thumb. It is continued along the forearm, at first behind and then on the outer border as far as the elbow, where it gives rise to the cephalic vein by its union with the outer branch of the median vein (fig. $76,{ }^{7}$ ).

'The ulnar veins are anterior and posterior, and occupy the front and back of the limb:

The anterior arises near the wrist by the junction of small roots from the hand, and runs on the inner part of the forearm to the elbow; here it unites with the inner branch of the median, and forms the basilic vein (fig. $76,{ }^{3}$ ).

The posterior ulnar vein is situate on the back of the limb. It commences by the union of a branch, "vena salvatella," from the back of the little finger, with an offset of the venous arch; it is continued along the back of the forearm nearly to the elbow, and bends forwards to open into the anterior ulnar vein.

The median vein takes origin near the wrist by small branches which are derived from the palmar surface of the hand; and it is directed along the centre of the forearm nearly to the elbow. Here the vein divides into external and internal branches (median basilic and median cephalic), which unite, as before seen (fig. $76,{ }^{2}$ ), with radial and ulnar veins. At its point of bifureation the median receives a communicating branch from a rein (vena comes) beneath the fascia.

Cutaneous Nerves. Some of the superficial nerves of the forearm are continued from the arm:-those on the inner side from the large internal cutaneous nerve; and those on the outer, from the two external cutaneous nerves of the musculo-spiral, and the musculo-cutaneous. On the forepart of the limb there is occasionally a small offset of the ulnar nerve near the wrist. On the back of the hand is the termination of the radial nerve, together with a branch of the ulnar nerve.

The internal cutaneous nerve (p. 256) is divided into two parts. The anterior branch extends on the front of the forearm as far as the wrist, and supplies the integuments on the inner half of the anterior surface. Near the wrist it communicates sometimes with a cutaneous offset from 
the ulnar nerve. The posterior branch continues along the back of the forearm (ulnar side) to rather below the middle.

The cutaneous part of the musculo-cutaneous nerve (p. 256) is prolonged on the radial border of the limb to the ball of the thumb, over which it terminates in cutaneous offsets. Near the wrist the nerve is placed over the radial artery, and some twigs pierce the fascia to ramify on the vessel and supply the carpus. A little above the middle of the forearm the nerve sends backwards a branch to the posterior aspect, which reaches nearly to the wrist, and communicates with the radial, and the following cutaneous nerve.

The external cutaneous branch of the musculo-spiral nerve (p. 259) after passing the elbow, turns to the hinder part of the forearm, and reaches as far as the wrist. Near its termination it joins the preceding cutaneous nerve.

The radial nerve ramifies in the integument of the back of the hand and some of the digits. It becomes cutaneous at the outer border of the forearm in the lower third, and after giving some filaments to the posterior aspect of the limb, divides into two branches :-

One (external) is joined by the musculo-eutaneous nerve, and is distributed on the radial border and the ball of the thumb.

The other branch (internal) supplies the remaining side of the thumb, both sides of the next two digits, and half the ring finger; so that the radial nerve distributes the same number of digital branches to the dorsum as the median nerve furnishes to the palmar surface. This portion of the radial nerve communicates with the musculo-cutaneous and ulnar nerves; and the offsets to the contiguous sides of the ring and middle fingers is joined by a twig from the dorsal branch of the ulnar nerve.

On the side of the fingers each of these dorsal digital branches is united with an offset from the digital nerve on the palmar surface.

The dorsal branch of the ulnar nerve gives offsets to the rest of the fingers and the back of the hand. Appearing by the styloid process of the ulnar, it joins the radial nerve in an areh across the back of the hand, and is distributed to both sides of the little finger, and to the ulnar side of the ring finger : it communicates with the part of the radial nerve supplying the space between the ring and middle fingers. The ulnar nerve furnishes branches to the same digits on the palmar surface.

The aponeurosis of the forearm is continuous with a similar investment of the arm. It is of a pearly white color, and is formed of fibres which cross obliquely : it furnishes sheaths to the muscles, and is thicker behind than before.

Near the elbow it is stronger than towards the hand; and at that part it receives fibres from the tendons of the biceps and brachialis anticus, and gives origin to the museles attached to the inner condyle of the humerus. On the back of the limb the aponeurosis is connected to the margins of the ulna, so as to leave the upper part of the bone subcutaneous; and it is joined by fibres from the tendon of the triceps.

Horizontal processes are sent downwards from the aponeurosis to separate the superficial and deep layers of muscles, both on the front and back of the forearm; and longitudinal white bands indicate the position of the intermuscular processes which isolate one muscle from another, and give origin to the muscular fibres.

At the wrist the fascia joins the anterior amnular ligament; and near that band the tendon of the palmaris longus pierces it, and reeeives a sheath 
from it. Close to the pisiform bone there is an aperture through which the ulnar vessels and nerve enter the fat of the hand. Behind the wrist it is thickened by transverse fibres, giving rise to the posterior annular ligament; but on the back of the hand and fingers the fascia becomes very thin.

Dissection. The skin is to be replaced on the back of the forearm and hand, in order that the denuded parts may not beeome dry. Beginning the dissection on the anterior surface of the limb, let the student divide the aponeurosis as far as the wrist, and take it away with the cutaneous vessels and nerves, except the small palmar cutaneous offsets of the median and ulnar nerves near the wrist. In cleaning the muscles it will be impossible to remove the aponeurosis from them at the upper part of the forearm without detaching muscular fibres.

In front of the elbow is the hollow, already partly dissected, between the two masses of muscles arising from the inner and outer sides of the humerus. The space should be earefully cleaned, so as to display the brachial and forearm vessels, the median nerve and branches, the musculospiral nerve, and the recurrent radial and ulnar arteries.

In the lower half of the forearm a large artery, radial, is to be laid bare along the radial border; and at the ulnar side, close to the annular ligament, the trunk of the ulnar artery will be recognized, as it becomes superficial. These vessels and their branehes should be carefully cleaned; but the collateral muscles should be fixed with stitches to prevent their displacement.

The anterior'annular ligament of the wrist, which arehes over the tendons passing to the hand, is next to be defined. This strong band is at some depth from the surface; and whilst the student removes the fibrous tissue superficial to it, he must take eare of the small branches of the median and ulnar nerves to the palm of the hand. The ulnar vessels and nerve pass over the ligament, and will serve as a guide to its depth.

Hollow in front of the elbow (fig. 80). This hollow corresponds with the popliteal space at the knee, and is situate between the inner and the outer muscles of the forearm. The interval is somewhat triangular in shape, and the wider part is towards the humerus. It is bounded on the outer side by the supinator longus musele, and on the inner side by the pronator teres. The aponeurosis of the limb is stretched over the space; and the bones, covered by the brachialis anticus and supinator brevis, form the deep boundary.

Contents. In the hollow are lodged the termination of the brachial artery with its veins, and the median nerve; the inuseulo-spiral nerve; the tendon of the biceps muscle; and small recurrent vessels, with much fit and a few glands.

These several parts have the following relative position: The tendon of the biceps is directed towards the outer boundary to reach the radius; on the outer side, concealed by the supinator longus muscle, is the musculospiral nerve. Nearly in the centre of the space are the brachial artery and veins and the median nerve, the nerve being internal; but as the artery is inclined to the outer part of the limb, they soon become distant from one another about half an inch. The brachial artery divides here into two trunks-radial and ulnar; and the recurrent radial and ulnar branches appear in the space, one on the onter and the other on the inner side.

Two or three lymphatie glands lie on the sides of the artery, and one below its point of splitting. 


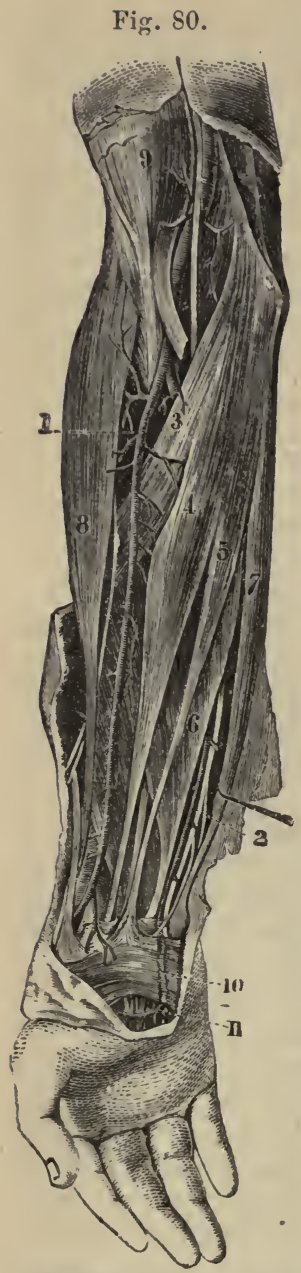

SCPBRPICIAL VIEW OF THE FOREARM (Quain's Arteries).

1. Radial artery with its nerve outside.

2. Ulnar artery and nerve where they are superfleial.

3. Pronator teres.

4. Flexor carpi radialis.

5. Palmaris longus.

6. Flexor sublimis.

7. Flexor carpl ulnaris.

8. Supinator longus.

9. Biceps.
Muscles on the front of the foreari (fig. 80). The muscles on the front of the forearm are divided into a superficial and a deep layer.

In the superficial layer there are five muscles, which are fixed to the inner condyle of the humerus, mostly by a common tendon, and lie in the undermentioned order from the middle to the inner side of the limb: pronator radii teres, flexor carpi radialis, palmaris longus, flexor carpi ulnaris; and deeper and larger than any of these is the flexor sublimis digitorum.

The deep layer will be met with in a subsequent dissection (p. 270).

The PRONATOR RADII TERES (fig. $80^{\circ},{ }^{3}$ ) arises from the inner condyle of the humerus by the common tendon; from the ridge above the condyle by fleshy fibres; from thie inner part of the coronoid process by a second tendinous slip); and from the fascia, and the septum between it and the next muscle. It is inserted by a flat tendon into an impression, an inch in length, on the middle of the outer surface of the radius.

The muscle is superficial except at the insertion, where it is covered by the radial artery, and some of the outer set of muscles, viz., supinator longus, and radial extensors of the wrist. 'The pronator forms the inner boundary of the triangular space in front of the elbow ; and its inner border tonches the flexor carpi radialis. By gently separating the muscle from the rest, it will be found to lie on the brachialis anticus, the flexor sublimis digitorum, and the ulnar artery and the median nerve: the second small head of origin is directed inwards between that artery and nerve.

Action. 'The pronator assists in bringing forwards the radius over the ulna, so as to pronate the hand. When the radius is fixed the muscle raises that bone towards the humerus, bending the elbow-joint.

The FLEXOR CARPI RADIALIS (fig. 80, ${ }^{4}$ ) takes its origin from the common tendon, from the aponeurosis of the limb, and from the intermuscular septum on each side. The tendon of the muscle, becoming free from Heshy fibres about the middle of the forearm, passes through a groove in the os trapezium, outside the anterior annular ligament, to be inserted mainly into the base of the metacarpal bone of the index finger, and by a slip into that of the middle finger.

The muscle rests chiefly on the flexor sublimis digitorum; but near the wrist it lies over the flexor longus pollicis-a muscle of the deep layer. As low as the middle of the forearm the muscle corresponds externally with the pronator teres, and 
below that with the radial artery to which its tendon is taken as the guide. The ulnar border is in contact at first with the palmaris longus muscle, and for about two inches above the wrist, with the median nerve. ${ }^{1}$

Action. The hand being free the muscle flexes first the wrist joint, inclining the hand somewhat to the radial side; and will assist in bringing forwards the lower end of the radius in pronation. Still continuing to contract, it bends the elbow.

The PALMARIS LONGus (fig. $80,{ }^{5}$ ) is often absent: or it may present great irregularity in the proportion between the fleshy and tendinous parts. Its origin is connected, like that of the preceding muscle, to the common tendon, the fascia, and the intermuscular septa. Its long thin tendon is continued along the centre of the forearm ; and piercing the aponeurosis, it passes over the annular ligament to end in the palmar fascia, and to join by a tendinous slip the short museles of the thumb.

The palmaris is situate between the flexor carpi radialis and ulnaris, and rests on the flexor sublimis digitorum.

Action. Rendering tense the palmar fascia, the palmaris will afterwards bend the wrist and elbow, like the other muscles of the superficial layer.

The FLEXOR CARPI ULNARIS (fig $80,{ }^{7}$ ) has an aponeurotic origin from the inner condyle of the humerus; from the inner side of the olecranon; and from the ridge of the ulna between the internal and posterior surfaces for three-fourths of the length. Most of the fibres are continued vertically downwards, but others obliquely forwards to a tendon on the anterior part of the muscle in the lower half, some joining it as low as the wrist; and the tendon is inserted into the pisiform bone, an offset being sent to the muscles of the little finger.

One surface of the muscle is in contact with the fascia; and its tendon, which ean be felt readily through the skin, may be taken as the guide to the ulnar artery. To its radial side are the palmaris and flexor digitorum sublimis muscles. When the attachment to the inner condyle has been divided, the muscle will be seen to conceal the flexor digitorum sublimis and flexor profundus, the ulnar nerve, and the ulnar vessels ; between the attachments to the condyle and the olecranon the ulnar nerve enters the forearm.

Action. The wrist is bent and the hand is drawn inwards by the contraction of the muscle.

The radial artery (fig. $80,{ }^{1}$ ) is one of the vessels derived from the bifurcation of the brachial trunk; and its destination is the palm of the hand. It is placed first along the outer side of the forearm as far as the end of the radius; next it winds backwards below the extremity of that bone: and it enters finally the palm of the hand through the first interosseous space. In consequence of this circuitous course the artery will be found in three different dissections, viz., the front of the forearm, the back of the wrist, and the palm of the hand.

In the front of the forearm. In this part of the limb the position of the artery will be marked, on the surface, by a line from the centre of the hollow of the elbow to the fore part of the styloid process of the radius. At first it lies on the inner side of the radius, but afterwards orer that bone. This vessel is smaller than the ulnar artery, though it appears in direction to be the continuation of the brachial trunk; and it is partly

1 In the body of a woman which was well developed, the muscle was absent on both sides. 
deep and partly superficial, but where it is more superficial in the lower half it can be felt beating as the pulse near the wrist during life.

In its upper half the vessel is placed beneath the supinator longus $\left({ }^{8}\right)$; and rests successively on the following muscles, the fleshy supinator brevis, the pronator teres $\left({ }^{3}\right)$, part of the thin origin of the flexor sublimis $\left({ }^{6}\right)$, and sometimes on the biceps tendon $\left({ }^{9}\right)$.

Veins. Venæ comites lie on the sides, with cross branches over the artery.

Nerve. The radial nerve is parallel to, and outside it, but separated by a sligbt interval.

In its lower half the artery with its venæ comites is stiperficial, being covered only by the teguments and the deep fascia. Here it is placed in a hollow between the tendons of the supinator longus $\left({ }^{8}\right)$ and flexor carpi radicalis $\left({ }^{4}\right)$. It lies from above down on the origin of the flexor sublimis, on two other muscles of the deep layer, viz., flexor pollicis longus and pronator quadratus, and lastly on the end of the radius.

Nerves. The radial nerve is still on the outer side until it passes backwards beneath the tendon of the supinator longus, and becomes eutaneous. Superficial to the lower end are the ramifications of the musculo-cutaneous nerve, some of which reach the vessels (p. 262).

Branches. The radial artery furnishes many unnamed muscular and nutrient offsets to the surrounding parts; and three named branches, viz., recurrent radial, superficial volar, and anterior carpal.

$a$. The radical recurrent is the first branch, and supplies the muscles of the outer side of the limb. Its course is almost transverse to the supinator longus, beneath which it terminates in that muscle and the two radial extensors of the wrist. One offset ascends beneath the supinator, and anastomoses with the superior profunda branch of the brachial artery.

$b$. The superficial volar branch (fig. $83, c$ ) arises usually near the lower end of the radius. It is directed towards the palm of the hand, across or through the mass of muscles in the ball of the thumb, and it either ends in those muscles, or joins the superficial palmar arel.

$c$. The anterior carpal branch is very inconsiderable in size, and will be seen in the examination of the deep layer of museles. Arising rather above the end of the radius, it passes transversely at the lower border of the pronator quadratus, and anastomoses with a similar branch firom the ulnar artery : from the arch thus formed, offsets are given to the corpus.

Peculiarities of the raclial artery. When the radial arises ligh in the arm, its course is close to the brachial artery, along the edge of the biceps muscle; and in passing the bend of the elbow it may be occasionally subcutaneous, viz., above the deep fascia, and be liable to injury in venesection. In the forearm it may be likewise subcutaneous and superficial to the supinator longus muscle.

Dissection. To bring into view the flexor sublimis digitorum, the flexor carpi radialis and palmaris longus must be cut through near the inner condyle of the humerus, and turned to one side. Small branches of the ulnar artery and median nerve may be seen entering the under surfaces of those muscles. For the present, the pronator teres may be left uncut.

The Flexor nigrtorum subimis vel ienforatus (fig. $80,{ }^{6}$ ) is the largest of the muscles of the superficial layer, and is named from its position to another flexor in the deep layer. It arises from the inner condyle of the humerus and the internal lateral ligament, and from the intermuscular septa in common with other muscles; it takes origin in addition fiom 
the bones of the forearm, viz., from the inner part of the coronoid process of the ulna; from the oblique line below the radial tubercle; and from the anterior margin of the radius as far as, or one or two inches below the insertion of the pronator teres. Rather below the middle of the forearm the muscle ends in four tendons, which are continued beneath the annular ligament and across the hand, to be inserted into the middle phalanges of the fingers, after being perforated by the tendons of the deep flexor.

The flexor sublimis is concealed by the other muscles of the superficial layer ; and the radial vessels lie on the attachment to the radius. Along the inner border is the flexor carpi ulnaris, with the ulnar vessels and nerve. The tendons of the muscle are arranged in pairs before they pass beneath the annular ligament of the wrist; - the middle and ring finger tendons being anterior, and those of the index and little finger posterior in position. On dividing the coronoid and condyloid attachments, the muscle will be seen to cover two flexors of the deep layer (flexor digitorum profundus and flexor pollicis), the median nerve, and the upper part of the ulnar artery.

Action. This flexor bends primarily the michlle joint of each finger; and is then able to bring the nearest phalanx towards the palm in consequence of being bound thereto by a sheath. But when the nearest phalanx is fixed by the extensor of the fingers, it remains straight whilst the superficial flexor moves the second phalanx.

After the fingers are bent the muscle will help in flexing the wrist and elbow joints.

The ULAAR ARTERY (fig $81,{ }^{G}$ ) is the larger of the two branches coming from the bifurcation of the brachial trunk; and is directed along the inner side of the limb to the palm of the hand, where it forms the superficial palmar arch, and joins offsets of the radial artery. In the forearm the vessel has an arched direction; and its depth from the surface varies in the first and last parts of its course.

In the upper half the artery is inclined obliquely inwards from the centre of the elbow to the inner part of the limb, midway between the elbow and wrist. It courses between the superficial and deep layers of museles, being covered by the pronator teres, flexor carpi radialis, palmaris longus, and flexor sublimis. Beneath it lie the brachialis anticus, for a short distance, and the flexor profundus, c.

Veins. Two companion veins are situate on the sides of the artery, and join freely over it.

Neries. The median nerve $\left(^{1}\right)$ lies to the inner sirle of the vessel for about an inch, but then crosses orer it to gain the outer side. About the middle of the forearm the ulnar nerve $\left({ }^{4}\right)$ approaches the artery and continues thence on the inner side.

In the lower half it has a straight course to the pisiform bone, and is covered by the teguments and fascia, and the flexor carpi ulnaris as far as the wrist. To the outer side are the tendons of the flexor sublimis. Beneath it is the flexor profundus, c.

Veins. Two companion veins accompany the artery, as in the upper part, and are mited across it at intervals.

Verves. The ulnar nerve $\left({ }^{4}\right)$ lies close to, and on the inner side of the vessels ; and a small branch $\left({ }^{5}\right)$, sending twigs around the artery, courses to the palm of the hand.

On the annular ligament of the wrist the artery has passed through the 
fascia and lies close to the pisiform bone. The ulnar nerve, with its palmar branch, still aceompanies the vessel on the inner side.

Fig. 81.

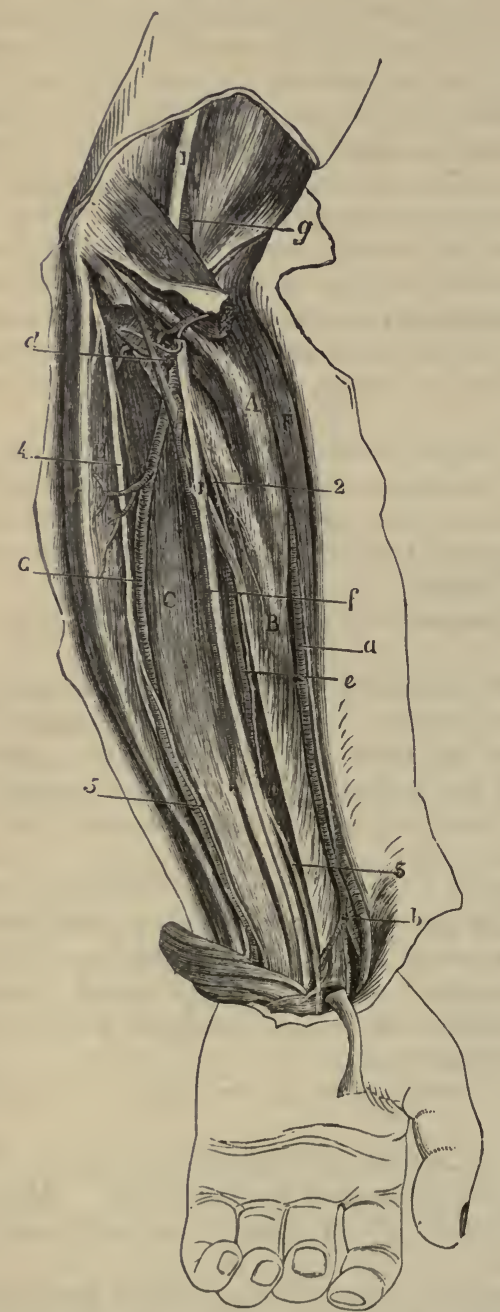

Muscles :

A. Pronator teres.

B. Flexor longus pollicis.

c. Flexor digitorum perforans.

D. Pronator quadratus.

E. Flexor carpi ulnaris.

\section{Arteries :}

a. Radial trunk.

b. Cutaneons branch of it to the palm of the hand.

a. Ulnar trunk.

d. Its recurrent branch.

$f$. Branch with the median nervo.

e. Anterior interosseous.

g. Brachial trunk.

\section{Nerves :}

1. Median.

2. Anterior interosseous.

3. Cutaneous palmar branch.

4. Ulnar trunk.

5. Cutaneous palmar branch of ulnar.

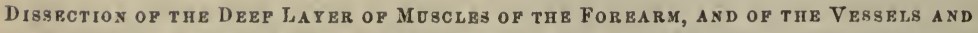
Nerves between the TWo Layeks of MUscles of the Foreara. (Illustrutions of Dissections.)

Branches. The greater number of the collateral offsets of the artery are distributed to the muscles. But the named branches are the following :-

a. The anterior ulnar recurrent branch $(d)$ arises generally in common with the next, and ascending on the brachialis anticus muscle inosculates with the small anastomotic artery beneath the pronator radii teres. It gives offsets to the contiguous muscles. 
b. The posterior ulnar recurrent branch $(d)$, of larger size than the anterior, is inclined beneath the flexor sublimis muscle to the interval between the inner condyle and the olecranon. There it passes with the ulnar nerve between the attachments of the flexor carpi ulnaris, and joins the ramifications of the inferior profunda and anastomotic arteries on the inner side of the elbow joint. Some of its offsets enter the muscles, and others supply the articulation and the ulnar nerve.

c. The interosseous branch is a short thick trunk, about an inch long, which is directed backwards towards the interosseus membrane, and divides into anterior and posterior interosseous : these branches will be afterwards followed.

1. $d$. The metacarpal branch arises from the artery near the lower end of the ulna, and runs along the metacarpal bone of the little finger, of which it is the inner dorsal branch.

e. The carpal branches (anterior and posterior) ramify on the front and back of the carpus, on which they anastomose with corresponding off'sets of the radial artery, and form arches across the wrist.

Peculiarities of the ulnar artery. The origin of the artery may be transferred to any point of the main ressel in the arm or axilla. Once the origin was found between two and three inches below the elbow. (Quain.)

When it begins higher than usual it is generally superficial to the flexor muscles at the bend of the elbow (only one exception, Quain), but beneath the aponeurosis of the forearm; though sometimes it is subcutaneous with the superficial veins.

The UlNar NERVE (fig. $81,{ }^{4}$ ) enters the forearm between the attachments of the flexor carpi ulnaris to the olecranon and inner condyle of the humerus. Under cover of that muscle the nerve reaches the ulnar artery about the middle (in length) of the forearm, and is continued on the inner side of the vessel to the hand. On the annular ligament the nerve is rather deeper than the artery. It furnishes articular, muscular, and cutaneous branches as below :-

a. Articular nerves. In the interval between the olecranon and the inner condyle, slender filaments to the joint arise.

b. Muscular branches. It furnishes offsets near the elbow to two muscles of the forearm; one enters the upper part of the flexor carpi ulnaris, and the other supplies the inner half of the flexor profundus digitorum.

c. Cutaneous nerve of the forearm and hand. About the middle of the forearm arises a small palmar branch $\left({ }^{5}\right)$, which courses on the ulnar artery, sending twigs around that vessel, to the end in the integuments of the palm of the hand: sometimes a cutaneous offset perforates the aponeurosis near the wrist, and joins the internal cutaneous nerve.

$d$. The dorsal cutaneous nerve of the hand arises about two inches above the end of the ulna, and passes obliquely backwards beneath the flexor carpi ulnaris : perforating the aponeurosis it is lost on the back of the hand and fingers (p. 262).

The MEDIAN NERVE (fig. $81,{ }^{1}$ ) leaves the hollow of the elbow between the heads of origin of the pronator teres, and takes the middle line of the limb to the hand. It is placed beneath the flexor sublimis as low as two inches from the annular ligament, where it becomes superficial along the outer border of the tendons of that muscle. Lastly the nerve passes heneath the annular ligament to the palm of the hand. The nerve supplies 
the muscles on the front of the forearm, and furnishes a cutaneous offset to the hand.

a. Muscular offsets leave the trunk of the nerve near the elbow, and are distributed to all the muscles of the superficial layer except the flexor carpi ulnaris; in addition, the nerve supplies the deep layer through the interosseous branch (p. 271), except the inner lalf of the flexor profundus digitorum.

b. 'The cutaneous palmar branch $\left(^{3}\right)$ arises in the lower fourth of the forearm ; it pierees the faseia near the annular ligament, and crosses over that ligament to reach the palm of the hand.

The RADIAL NERVE is the smaller of the two branches into which the musculo-spiral divides at the elbow. This nerve is placed along the outer border of the limb, under cover of the supinator longus (fig. 80), and on the outer side of the radial artery till within three inches of the wrist, where it becomes eutaneous at the posterior border of the supinator tenton. On the surface of the limb it divides into two branches, which are distributed on the dorsum of the hand, and digits $\left(3 \frac{1}{2}\right)$ (p. 262). No muscular offset is furnished by the nerve.

Dissection (fig. 81). To examine the deep layer of muscles it will be necessary to draw well over to the radial side of the forearm the pronator teres; to detach the flexor sublimis from the radius, and to remove its fleshy part. The areolar tissue is to be taken from the deep muscular fibres ; and the anterior interosseous vessels and nerve, which lie on the interosseous membrane, and are concealed by the muscles, are to be traced out.

Above the ends of the forearm-bones the arch of the anterior carpal arteries may be defined.

Deep LAyer of Muscies. Only three deep muscles are present on the front of the forearm. One lies on the ulna, and is the deep flexor of the fingers; a second covers the radius, viz., the long flexor of the thumb; and the third is the pronator quadratus, which lies beneath the other two, near the lower end of the bones.

The Flexor Digitorum PROFundus vel PERForans (fig. $81,{ }^{\circ}$ ) arises from the anterior and inner surfaces of the ulna for three-fourths of the length of the bone; from the inner half of the interosseous ligament for the same distance; and from an aponeurosis common to the muscle and the flexor carpi ulnaris. The muscle has a thick fleshy belly, and ends in tendons which, united together, pass beneath the annular ligament, and are inserted into the last phalanges of the fingers.

'The cutaneous surface of the muscle is in contact with the ulnar nerve and vessels, the superficial flexor of the fingers, and the flexor carpi ulnaris. The deep surtace rests on the ulna and the pronator quadratus musele. The outer border touches the flexor pollicis longus and the anterior interosseous vessels and nerve; and the inner is connected by aponeurosis to the posterior margin of the ulna.

Action. The muscle bends the last joints of the fingers and the wrist; but it does not act on the last phalanx till after the second has been moved by the flexor sublimis.

'The fingers are approximated and the joints bent in the following order:first, the articulation between the metacarpal and the middle phalanx is flexed, secondly, the last phalangeal joint, and thirdly the metacarpo-phalangeal.

The FlEXor LOXges pollicis (fig. $81,{ }^{\mathrm{B}}$ ) arises from the hollowed anterior surface of the radius, as low as the pronator quadratus; from the 
outer part of the interosseous membrane; and by a round distinct slip from the coronoid process of the ulna, internal to the attachment of the brachialis anticus. The fleshy fibres descend to a tendon, which is continued beneath the annular ligament, and is inserted into the last platanx of the thumb.

On the cutaneous sureace of the muscle is the flexor sublimis, with the radial vessels for a short distance inferiorly. The muscle lies on the radius and the pronator quadratus. To the inner side is the flexor profundus digitorum.

Action. It bends both joints of the thumb, but first the distal or ungual. After the phalanges are drawn downwards the muscle flexes the wrist.

The Pronator Quadratus (fig. $81,{ }^{D}$ ) is a flat muscle, and lies on the lower fourth of the bones of the forearm. It arises from the anterior and inner surfaces of the ulna, where it is somewhat widened, and is inserted into the forepart of the radius for about two inches.

'The anterior surface is covered by the tendons of the flexor muscles of the fingers, and by the radial vessels ; and the posterior surface rests on the radius and ulna with the intervening membrane, and on the interosseous vessels and nerve. Along its lower borders is the arch formed by the anterior carpal arteries.

Action. 'The end of the radius is moved forwards over the ulna by this muscle, and the hand is pronated.

The anterior interosseous artery (fig. $81, e$ ) is continued on the front of the interosseous membrane, between the two flexors or in the fibres of the flexor digitorum, till it reaches an aperture beneath the pronator quadratus. At that spot the artery turns from the front to the back of the limb, and descends to the back of the carpus, where it ends by anastomosing with the posterior interosseous and carpal arteries.

Branches. Numerous off'sets are given to the contiguous muscles.

$a$. One long branch $\left(f^{\prime}\right)$, median, accompanies the median nerve, supplying it, and either ends in the flexor sublimis, or extends beneath the annular ligament to the palmar arch.

$b$. About the middle of the forearm two nutrient vessels of the radius and ulna arise from the artery.

c. Where it is about to pass through the interosseous membrane it furnishes twigs to the pronator quadratus; and one branch is continued beneath that muscle to anastomose with the anterior carpal arteries.

The anterior interosseous nerve is derived from the median (fig. $81,{ }^{2}$ ), and accompanies the artery of the same name to the pronator quardratus muscle, the under surface of which it enters. Some lateral branches are distributed by it to the deep flexor muscles.

Dissection. The attachment of the biceps and brachialis anticus to the bones of the forearm may be now cleaned and examined.

The insertion of the brachialis anticus takes place by a broad thick tendon, about an inch in length, which is fixed into the coronoid process of the ulna, except at the inner edge ; and into the contiguous rough part o: the bone.

Insertion of the biceps. The tendon of the biceps is inserted into the inner part of the tubercle of the radius, and slightly into the bone behind it. A bursa intervenes between it and the lorepart of the tubercle. At its attacliment the anterior surface becomes external; and the opposite. The supinator brevis muscle partly surrounds the insertion. 


\section{Section V.}

\section{THE PALM OF THE HAND.}

Dissection (fig. 83). The digits being separated and fixed firmly with tacks, the skin is to be reflected from the palm of the hand by means of two incisions. One is to be earried along the centre from the wrist to the fingers ; and the other is to be made from side to side, at the termination of the first. In raising the inner flap, the small palmaris brevis muscle will be seen at the inner margin of the hand; and its insertion into the skin may be left till the muscle has been learnt. In the fat the ramifications of the small branches (palmar) of the median and ulnar nerves are to be traced.

The student should remove the fat from the palmaris muscle, and from the strong palmar fascia in the centre of the hand; and he should take care not to destroy a transverse fibrous band (transverse ligament) which lies across the roots of the fingers. When cleaning the fat from the palmar fascia he will recognize, near the digits, the digital vessels and nerves; and must be especially careful of two,_-viz., those of the inner side of the little finger and outer side of the index finger, which appear farther back than the rest, and are most likely to be injured. By the side of the vessels and nerves to the fingers four slender lumbricales muscles are to be defined.

Lastly, the skin and the fat may be reflected from the thumb and fingers by an incision along each, in order that the sheaths of the tendons with the collateral vessels and nerves may be laid bare.

Cutaneous palmar nerves. Some unnamed twigs are furnished to the integument from both the median and ulnar nerves in the hand; and two named cutaneous nerves ramify in the palm.

One is an offiset of the median nerve (p. 270), and crosses the annular ligament : it extends to about the middle of the palm, and is united with the palmar branch of the ulnar: a few filaments are furnished to the ball of the thumb.

The other palmar branch is derived from the ulnar nerve (p. 269); it has been traced on the ulnar artery to the hand, and its distribution in the palm may be now observed.

'The PALMARIS BREVIS (fig. $83,{ }^{\text {" }}$ ) is a small subcutaneous muscle, about two inches wide, whose fibres are collected into separate bundles. It is attached on the outer side to the palmar aponeurosis, and its fibres are directed inwards to join the skin at the inner border of the hand.

'This muscle lies orer the ulnar artery and nerve. After it has been examined it may be thrown in wards with the skin.

Action. Drawing inwards the skin of the inner border of the hand towards the centre, it deepens the hollow or cup of the palm.

The palmar fascia or aponeurosis consists of a central and two lateral parts; but the lateral, which cover the muscles of the thumb and little finger, are so thin as not to require separate notice.

'The central part is a strong, white layer, which is pointed at the wrist, but is expanded towards the fingers, where it nearly covers the palm of the hand. Posteriorly the fascia receives the tendon of the palmaris longus, and is connected to the annular ligament; and anteriorly it ends in four 
processes, which are continued forwards, one for each finger, to the sheaths of the tendons. At the point of separation of the processes from one another some transverse fibres are placed, which arch over the lumbricalis muscle, and the digital artery and nerve appearing at this spot. From the pieces of the fascia a few superfieial longitudinal fibres are prolonged to the integuments near the cleft of the fingers.

Dissection. To follow one of the digital processes of the fascia to its termination, the superficial fibres being first removed, it must be divided longitudinally by inserting the knife beneath it opposite the head of the metacarpal bone.

Ending of the processes. Each process of the fascia sends downwards an offset on each side of the tendons, which is fixed to the deep ligament connecting together the ends of the metacarpal bones, and to the edge of the metacarpal bone for a short distance.

The superficial transverse ligament of the fingers is a thin fibrous band, which stretches across the roots of the four fingers, and is contained in the fold of skin forming the rudiment of a web between them. Beneath it the digital nerves and vessels are continued onwards to their terminations.

Sheath of the flexor tendons (fig. 82). Along each finger the flexor tendons are retained in position against the phalanges by a fibrous sheath. Opposite the middle of each of the two nearest phalanges the sheath is formed by a strong fibrous band ( $e$ and $f$ ) (ligamentum vaginale), which is almost tendinous in consistence; but opposite the joints it consists of a thin membrane with scattered and oblique fibres. If the sheath be opened it will be seen to be lined by a synovial membrane, which forms long and slender vascular folds (vincula vasculosa) between the tendons and the bones.

Dissection. The palmar fascia, and the thinner parts of the digital sheaths opposite the joints of the fingers, may be taken away. On the removal of the fascia the palmar arch of the ulnar artery, and the median and ulnar nerves, become apparent.

Palmar Part of tile Ulyar Artery (fig. 83). In the palm of the hand the ulnar artery is directed towards the muscles of the thumb, where it communicates with two offsets of the radial trunk, viz., the superficial volar branch $(c)$, and the branch to the radial side of the forefinger $(f)$.

Fig. 82.

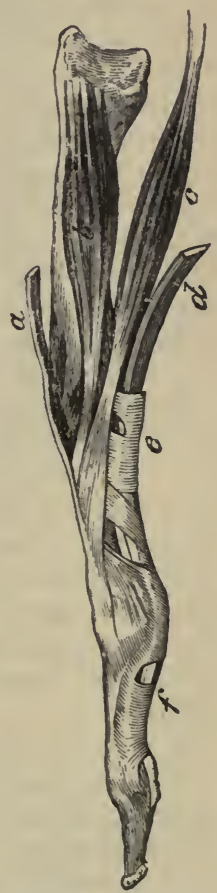

THB Extensor TBNDON OF THB FIXGBR WITH ITS ACCEBSORY MUSCles AND THB SHEATH OF THK FLBXOR TENDONS.

$a$. Extensor tendon, with $b$, interosseous, and $c, 1 u m$ bricales muscles joining it.

d. Flexor tendon; $e$ and $f$, thicker parts of its sheath.

The curved part of the artery, which lies across the hand, is named the superficial palmar arch $(d)$. Its convexity is turned towards the fingers, and its position in the palm would be nearly marked by a line across the hand from the cleft of the thumb.

The arch is comparatively superficial ; it is covered in greater part by 
the integuments and the palmar fascia, but at the inner border of the hand the palmaris brevis muscle (H) lies over it. Beneath it are the flexor tendons and the branches of the ulnar and median nerves. Vena cornites lie on its sides.

Branches. From the convexity of the arch proceed the digital arteries, and from the concavity some small offsets to the palm of the hand. A small branch (profunda) arises as soon as the artery enters the hand.

$a$. The profunda or communicating branch (fig. $84, b$ ), passes downwards with a branch of the ulnar nerve between the abductor and short flexor muscles of the little finger, to inoseulate with the deep palmar arch of the radial artery.

$b$. The digital branches $(g)$ are four in number, and supply both sides of the three inner fingers and one side of the index finger. The branch to the inner side of the hand and little finger is undivided; but the others, corresponding' with the three inner interosseous spaces, bifurcate anteriorly to supply the contiguous sides of the above said digits. In the hand these branches are accompanied by the digital nerves, which they sometimes pierce.

Near the root of the fingers they receive communicating branches from offsets of the deep arch; but the digital artery of the inner side of the little finger has its eommunicating branch about the middle of the hand.

From the point of bifurcation the branches extend along the sides of the fingers; and over the last phalinx the vessels of opposite sides unite in an arch, from whose convexity offsets proceed to supply the ball of the finger. Collateral offsets are furnished to the finger and the sheath of the tendons; and small twigs are supplied to the phalangeal articulations from small arterial arches on the bones-an arch being close behind each joint. On the dorsum of the last phalanx is another arch near the nail, from which the nail-pulp is supplied.

Palmar Part of the Ulnar Nerve (fig. $83,{ }^{1}$ ). The ulnar nerve divides on or near the annular ligament, into a superficial and a deep branch.

The deep branch accompanies the profunda artery to the muscles, and will be subsequently dissected with that vessel (fig. 84).

The superficial branch furnishes an offset to the palmaris brevis muscle, and some filaments to the integument of the inner part of the hand, and ends in two digital nerves, for the supply of both sides of the little finger and half the next.

Digital nerves $\left({ }^{2}\right)$. The more internal nerve is undivided, like the corresponding artery.

The other is directed to the cleft between the ring and little fingers, and bifurcates for the supply of their opposed sides: in the palm of the hand this last branch is connected with an offset $\left({ }^{5}\right)$ of the median nerve.

Along the sides of the fingers the digital branches have the same arrangement as those of the median nerve.

Palyar Part of the Median Nerve (fig. $83,^{3}$ ). As soon as the median nerve issues from beneath the annular ligament it becomes enlarged and somewhat flattened, and divides into two nearly equal parts for the supply of digital nerves to the thumb and the remaining two fingers and a half: the more external of the two portions furnishes a small muscular branch to the ball of the thumb. The trunk of the nerve and its branches are covered by the palmar fascia; and beneath them are the tendons of the flexor muscles. 
$a$. The branch to muscles of the thumb $\left(^{4}\right)$ supplies the outer half of the short flexor, and ends in the abductor, and opponens pollicis muscles.

b. The digital nerves $\left({ }^{5}\right)$ are five in number. Three of them, which are distributed to the sides of the thumb and the radial side of the fore finger, are undivided, and come from the external of the two pieces into

Fig. 83.

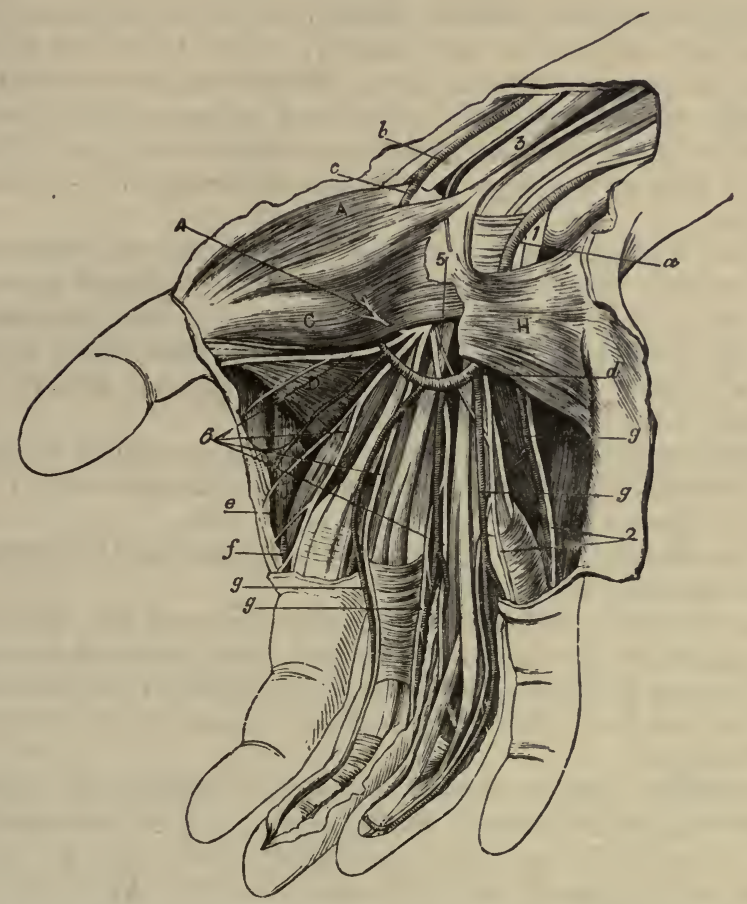

Disgection of the Superficial Vesgels and Nerves of the Patm of the Haxd with some of The Superficial Muscles. (Illustrations of Dissections.)

\section{Muscles :}

A. Abductor policis.

C. Flexor brevis.

D. Adductor policis.

H. Palmaris brevis.

\section{Arteries:}

$a$. Trunk of ulnar, and $b$, of radial.

c. Superficial volar branch.

d. Superficial palmar arch.

$e$. Branch uniting the arch with $f$ the radial digital branch of the foreflnger.

g. Four digital branches of the superflcial arch.

\section{Nerves :}

1. Ulnar, and 2, its two digital branches.

3. Median, and 5 , its digital branches to three flingers and a half.

4. Branch of the median to some muscles of the thumb

5. Communicating branch from the median to the ulnar.

which the trunk of the median splits. The other two spring from the inner piece of the nerve, and are bifurcated to supply the opposed sides of the middle and fore, and the middle and ring fingers.

The first two nerves belong to the thumb, one being on each side, and the most external is said to communicate with branches of the radial nerve. 
The third is directed to the radial side of the index finger, and gives a branch to the most external lumbrical muscle.

The fourth furnishes a nerve to the second lumbrical muscle, and divides to supply the contiguous sides of the fore and middle fingers.

The fifth, like the fourth, is distributed by two branches to the opposed sides of the middle and ring fingers : it communicates with a branch of the ulnar nerve.

On the fingers. On the sides of the fingers the nerves are superficial to the arteries, and reach to the last phalanx, where they end in filaments for the ball and the pulp beneath the nail. In their course forwards the nerves supply chiefly tegumentary branches: one of these is direeted backwards by the side of the metacarpal phalanx, and after uniting with the digital nerve on the back of the finger (p. 262), is continued to the dorsum of the last phalanx.

Dissection. The tendons of the flexor muscles may be followed next to their termination. To expose them the ulnar artery should be cut through below the origin of the profunda branch; and the small superficial volar branch (of the radial) having been divided, the palmar arch is to be thrown towards the fingers. The ulnar and median nerves are also to be cut below the annular ligament, and turned forwards.

A longitudinal incision is to be made through the centre of the annular ligament, without injuring the small muscles that arise from it, and the pieces of the ligament are to be thrown to the sides.

Finally the sheaths of the fingers may be opened for the purpose of observing the insertion of the tendons.

Flexor tendons. Beneath the annular ligament the tendons of the deep and superficial flexors are surrounded by a large and loose synovial membrane, which projects upwards into the forearm and downwards into the hand, and sends an offset into the digital sheath of the thumb and that of the little finger. ${ }^{1}$

Flexor sublimis. The tendons of the flexor sublimis are superficial to those of the deep flexor beneath the ligament; and all four are nearly on the same level, instead of being arranged in pairs, as in the forearm. After crossing the palm of the hand they enter the sheath of the fingers (fig. $82, e$ ), and are inserted each by two processes into the margins of the middle phalanx, about the centre. When first entering the digital sheath, the tendon of the flexor sublimis conceals that of the flexor profundus; but near the front of the first phalanx it is split for the passage of the tendon of the latter muscle.

Dissection. To see the tendons of the deep flexor and the lumbrical muscles, the flexor sublimis must be cut through above the wrist, and thrown towards the fingers. Afterwards the areolar tissue should be taken away.

Flexor profundus. At the lower border of the annular ligament the tendinous mass of the flexor profundus is divided into four pieces, though in the forearm only the tendon of the forefinger is distinct from the rest. From the ligament the four tentons are directed through the hand to the fingers, and give origin to the small lumbricales museles. At the root of the fingers each enters the digital sheath with a tendon of the flexor sublimis, and having passed through that tendon, is inserted into the base of the last phalanx.

1 Theile refers the notice of this fact to M. Maslieurat-Lagémard, in No. 18 of the "Gazette Médicale," for 1839. 
Between both flexor tendons and the bones are thin membranes, one for each. By means of this each tendon is connected with the capsule of the joint, and the fore part of the phalanx immediately behind the bone into which it is inserted.

The lumbricales muscles (fig. 84, I) are four small fleshy slips, which arise from the tendons of the deep flexor near the annular ligament; and are directed to the radial side of each finger, to be inserted into an aponeurotic expansion on the dorsal aspect of the metacarpal phalanx (fig. 82, c).

These muscles are concealed for the most part by the tendons and vessels that have been removed; but they are subcutaneous for a short distance between the processes of the palmar fascia. The outer two arise fiom single tendons, but each of the others is connected with two tendons.

Action. By their insertion into the long extensor tendon they will aid it in straightening the two last phalangeal joints; and when the metacarpophalangeal joints are much bent they may assist in maintaining the flexion of these articulations.

Tendon of the flexor pollicis longus. Beneath the annular ligament this tendon is external to the flexor profundus, and turns outwards between the heads of the flexor brevis pollicis (fig. 84), to be inserted into the last phalanx of the thumb. The common synovial membrane surrounds it beneath the annular ligament, and sends a prolongation, as before said, into its digital sheath.

Dissection (fig. 84). The deep palmar arch of the radial artery, with the deep branch of the ulnar nerve, and the interossei muscles, will come into view if the flexor profundus is cut above the wrist, and thrown with the lumbricales muscles towards the fingers; hut in raising the tendons the student should preserve two fine nerves and vessels entering the two inner lumbrical muscles.

The dissection of the muscles of the ball of the thumb and the little finger is next to be prepared. Some eare is necessary in making a satisfactory separation of the different small thumb muscles; but those of the little finger are more easily defined.

Short Muscles of the Thumb (fig. 84). These are four in number, and are named from their action on the thumb. The most superficial is the abductor pollicis; beneath it is the opponens pollicis, which will be recognized by its attachment to the whole length of the metacarpal bone. To the inner side of the last is the short flexor. And the wide muscle eoming from the third metacarpal bone is the adductor of the thumb.

The ABDuctor poluicis, $A$, is about an inch wide, and is thin, and superficial to the rest. It arises from the upper part of the annular ligament at the radial side, and from the ridge of the os trapezium; and is inserted into the base of the first phalanx of the thumb.

The muscle is subcutaneous, and rests on the opponens pollicis : it is connected oftentimes at its origin with a slip from the tendon of the extensor ossis metacarpi pollieis.

Action. It removes the metacarpal bone of the thumb from the other digits; and when it has so acted it may assist slightly the short flexor in bending the metacarpo-phalangeal joint.

Dissection. The opponens pollicis will be seen on eutting through the abductor. To separate the muscle from the short flexor' on the inner side, the student should begin near the fore part of the metacarpal bone, where there is usually a slight interval. 
The opponexs pollicis, B, arises from the annular ligament beneath the preceding, and from the os trapezium and its ridge; it is inserted into the front and outer border of the metacarpal bone for the whole length.

This muscle is partly concealed by the preceding, though it projects on the outer side. Along its inner border is the flexor brevis pollicis. An insertion into the external sesamoid bone is described by Theile.

Action. From its attachment to the metacarpal bone it is able to draw that bone inwards over the palm of the hand, turning it at the same time, so as to allow the ball of the thumb to be applied to the ball of each of the fingers, as in picking up a pin.

The FLEXOR BREVIS POLLICIS, $\mathrm{c}$, is the largest of the short museles of the thumb : it consists of two pieces (inner and outer) at the insertion, but these are united at the origin. Posteriorly it arises from the os trapezoides and os magnum ; from the bases of the second and third metacarpal bones ; and from the annular ligament, at the lower part. In front it is inserted by two heads into the sides of the base of the first phalanx of the thumb,the inner piece being united with the adductor, and the outer with the abductor pollicis. A sesamoid bone is connected with each head at its insertion.

The tendon of the long flexor lies on this muscle, oceupying the interval between the processes of insertion; and the deep palmar arch of the radial artery issues from beneath the inner head.

Action. The muscle bends the metacarpo-phalangeal joint, and assists the opponens in drawing the thumb forwards and inwards over the palm.

The ADDuctor POLLicis, D, is pointed at the thumb, and wide at the opposite end. Its origin is fixed to the anterior or lower two-thirds of the metacarpal bone of the middle digit, on the palmar aspect; and its insertion is attached, with that of the short flexor, to the inner side of the first phalanx of the thumb.

The cutaneous surface is in contact with the tendons of the flexor profundus and the lumbrical muscles; and the deep surface lies over (in this position) the first dorsal interosseous muscle, $J$, and the second and third metacarpal bones with the intervening muscle.

Actions. By its contraction the thumb is applied to the radial border of the hand, and approximated to the fingers.

Short Muscles of the Little Finger (fig. 84). There are eommonly two muscles in the ball of the little finger,-an abcluetor and an adduetor. Sometimes there is a short flexor between the other two.

The ABDUCtor misimi digiti, $\mathrm{F}$, is the most internal of the short muscles. It arises from the pisiform bone and the tendon of the flexor carpi ulnaris, and is inserted into the ulnar side of the base of the first phalanx of the little finger; an offset from it reaches the extensor tendon on the back of the phalanx. The palmaris brevis partly eonceals the musele.

Action. First it draws the little finger away from the others; but continuing to act it bends the metaearpo-phalangeal joint.

The FLEXOR IBREVIS MINIMI DIGITI, F, appears often to be a part of the abductor. Placed at the radial border of the preeeding muscle, it takes origin from the tip of the process of the unciform bone, and slightly from the annular ligament; it is inserted with the abductor into the first phalanx.

It lies on the adductor; and near its origin it is separated from the abductor muscle by the deep branches of the ulnar artery and nerve. 
Action. The first phalanx is moved towards the palm by this muscle, and the metacarpo-plialangeal joint is bent.

The ADDUCtor vel opponens MINIMI DIGITI, G, resembles the opponens pollicis in being attached to the metacarpal bone. Its origin is from the proeess of the unciform bone, and the lower part of the annular ligament; its insertion is fixed into the ulnar side of the metacarpal bone of the little finger.

Fig. 84 .

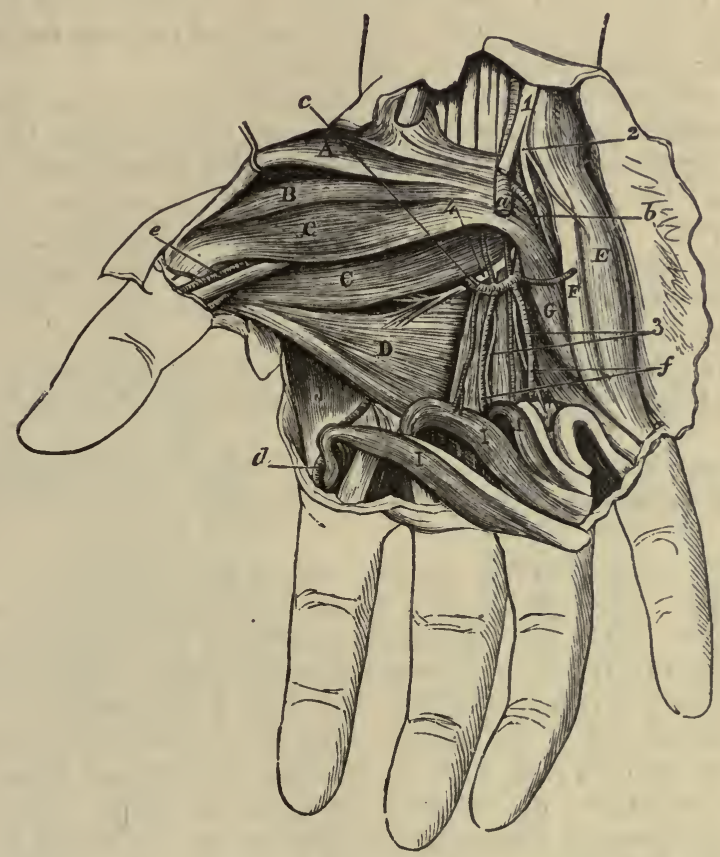

Deep Dissection of the Palm of the Hand. (Illustrations of Dissections.)

\section{Muscles :}
A. Abductor pollicis.
B. Opponens pollicis.
c. Flexor brevis pollicis.
D. Adductor pollicis.
B. Abductor minimi digiti.
P. Flexor brevis minimi digiti.
G. Opponens minimi digiti.
I. Luıbricales.
J. First dorsal interosseous.

Vessels :
a. Ulnar artery, cut.
$b$. Profunda branch.
c. Deep palmar arch.
$d$. Radial digital artery of the index finger.
e. Arteria magna pollicis.
$f$. Interosseous arteries.

\section{Nerves :}

1. UInar nerve, cut.

2. Deep branch of the palm of the hand, and 4, its continuation to end in some of the thumb muscles.

3. Offsets to the inner two lumbricales.

The adductor is partly overlaid by the preceding muscles; and beneath it the deep branches of the ulnar artery and nerve pass.

Action. It raises the inner metacarpal bone, and moves it towards the others, so as to deepen the palm of the hand.

Dissection. The radial artery comes into the hand between the first 
two metacarpal bones; and to lay bare the vessel, it will be requisite to detach the origin of the flexor brevis pollicis. The deep palmar arch, and the branch of the ulnar nerve accompanying it, together with their offsets, are to be dissected out.

A fascia, which covers the interossei muscles, is to be removed, when the dissector has observed its connection with the transverse ligament uniting the heads of the metacarpal bones.

Radial Artery in the Havd (fig. 84). The radial artery enters the palm at the first interosseous space, between the heads of the first dorsal interosseous muscle: and after furnishing one branch to the thumb, and another to the index finger, turns across the hand towards the ulnar side, with its venæ comites, forming the deep arch.

The deep palmar arch $(c)$ extends from the interosseous space to the base of the metacarpal bone of the little finger, where it joins the profunda communicating branch $(b)$. Its convexity, which is but slight, is directed forwards; and its position is nearer the carpal bones than that of the superficial arch. The arch has a deep position in the hand, and lies on the metacarpal bones and the interossei muscles. It is covered by the long flexor tendons, and in part by the flexor brevis pollicis. The branches of the arch are the following:-

a. Recurrent branches pass from the concavity of the arch to the front of the carpus ; these supply the bones, and anastomose with the other carpal arteries.

b. Three perforating arteries pierce the three inner dorsal interossei muscles, and communicate with the interosseous arteries on the back of the hand.

c. Usually there are three palmar interosseous arteries $(f)$, which occupy the three inner metaçarpal spaces, and terminate by joining the digital branches of the superficial palmar arch at the eleft of the fingers. These branches supply the interosseous muscles, and the two or three inner lumbricales; they vary much in their size and distribution.

d. Digital branches of the radial. The large artery of the thumb $(\rho)$ (art. princeps pollicis) runs between the first metacarpal bone and the flexor brevis pollicis, to the interval between the heads of the muscle, where it divides into the two collateral branches of the thumb: these are distributed like the arteries of the superficial arch.

$e$. The digital branch of the index finger (d) (art. radialis indieis) is directed over the first dorsal interosseous muscle, $\mathrm{J}$, and beneath the short flexor and the adductor pollicis, to the radial side of the forefinger. At the free or anterior border of the adductor pollicis, $\mathrm{D}$, this branch is usually connected by an offset with the superficial palmar arch; and at the end of the digit it unites with the branch furnished to the opposite side by the ulnar artery.

The deep branch of the ulnar nerve $\left({ }^{2}\right)$ accompanies the arch of the radial artery as far as the muscles of the thumb, and terminates in offsets to the adduetor pollicis and the inner head of the short flexor.

Branches. Near its origin the nerve furnishes branches to the museles of the little finger. In the palm it gives offsets to all the palmar and dorsal interosseous muscles, and to the inner two lumbrical muscles $\left({ }^{3}\right)$, besides the terminal branches before mentioned.

The transverse metacarpal ligament connects together the heads of the four inner metacarpal bones. Its cutaneous surface is hollowed where the flexor tendons cross it; and beneath the interossei muscles pass to their 
insertion. To the posterior border the fascia covering the interossei muscles is united. The ligament should now be taken away to see the interossei muscles.

The interossei MUSCLEs, so named from their position between the metacarpal bones, are seven in number. Two muscles occupy each space, except in the first where there is only one; they arise from the metaearpal bones, and are inserted into the first phalanx of the fingers. They are divided into palmar and dorsal interossei ; but all the small muscles are evident in the palm of the hand, though some project more than the others.

The palmar muscles (fig. 85), three in number, are "smaller than the dorsal set, and are most prominent in the palm of the hand. They arise from the palmar surface of the metaearpal bones of the fingers on which they act, viz., those of the fore, ring, and little fingers ; and they are inserted into the ulnar side of the fore, and the radial side of the other two digits.

Fig. 85 .

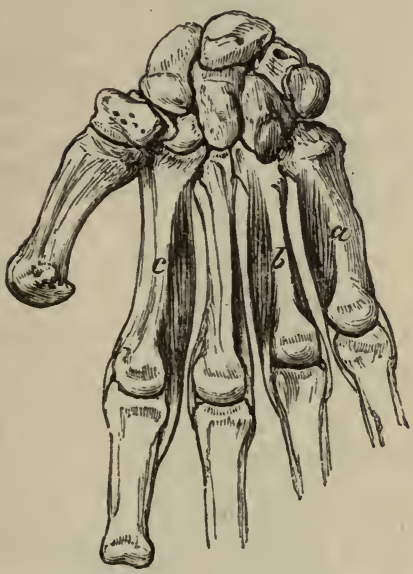

Thre Palmar Interosseous Mugcles.

a. Muscle of the little finger; $b$, of the ring Anger; and $c$, of the forefinger.
Fig. 86.

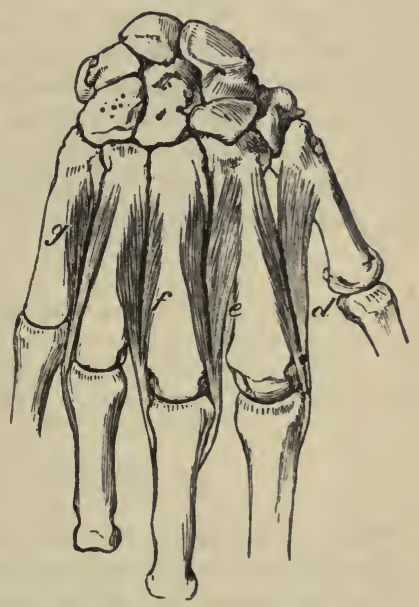

Four Dorsat Interosseots Musclis.

d. Muscle of the forefinger, called sometimes abductor indicis.

$e$ and $f$. Muscles of the middle finger.

g. Muscle of the ring finger.

Both sets of muscles have a similar termination (fig. $82, b$ ):-the fibres end in a tendon, which is inserted into the side of the first or metacarpal phalanx, and sends an expansion to join the extensor tendon on the dorsum of the bone.

The dorsal interossei (fig. 86) extend further back than the palmar set, and arise by two heads from the lateral surfaces of the metacarpal bones between which they lie. The dorsal muscles are thus allotted to the digits :- two belong to the second finger, a third is conneeted with the radial side of the fore, and the fourth with the ulnar side of the ring finger.

Action. They help to bend the metacarpo-phalangeal joints by their 
attachment to the first phalanx : and assist in the extension of the two last phalangeal joints through their union with the extensor tendon.

Further they can separate and approximate the fingers: thus the palmar set adduct to the second finger; and the dorsal abduct from the middle line of the second finger-the two attached to this digit moving it to the right and left of that line.

Dissection. The attachments of the annular ligament to the carpal bones on each side are to be next dissected out by taking away the small muscles of the thumb and little finger. Before reading its description, the ends of the cut ligament rray be placed in apposition.

The anterior annular ligament is a firm ligamentous band, which arches over and binds down the flexor tendons of the fingers. It is attacherl externally to the front of the os scaphoides, and to the fore and inner parts, and ridge of the os trapezium ; and internally to the unciform and pisiform bones. By its upper border it is connected with the aponeurosis of the forearm; and by its anterior surface it joins the palmar fascia. On it lie the palmaris longus and the ulnar artery and nerve.

Dissertion. Next follow the tendon of the flexor carpi radialis through the os trapezium to its insertion into the metacarpal bones.

The tendon of the flexor carpi radialis, in passing from the forearm to the hand, lies in the groove in the os trapezium between the attachments of the annular ligament to the bone, but outside the arch of that ligament; here it is bound down by a fibrous sheath lined by a synovial membrane. The tendon is inserted into the base of the metacarpal bone of the index finger, and sends a slip to that of the middle digit.

\section{SECTION VI.}

\section{THE BACK OF THE FOREARM.}

Position. During the dissection of the back of the forearm the limb lies with the forepart undermost, and a small block is to be placed beneath the wrist for the purpose of stretehing the tendons.

Dissection (fig. 87). The fascia and the cutaneous nerves and vessels are to be reflected from the muscles of the forearm, and from the tendons on the back of the hand; but in removing the fascia in the forearm, the student must be careful not to eut away the posterior interosseous ressels, which are in contact with it on the inner side in the lower third. A thickened band of the fascia opposite the carpus (the posterior annular ligament) is to be left.

Let the integument be removed from the fingers, in order that the tendons may be traced to the end of the digits.

The several muscles should be separated from one another up to their origin, especially the two radial extensors of the wrist.

The posterior annular ligament, $\mathrm{K}$, consists of the special aponeurosis of the limb, thickened by the addition of some transverse fibres, and is situate opposite the lower end of the hones of the forearm. 'This band is connected at the outer part to the radius, and at the inner to the cuneiform and pisiform bones. Processes from it are fixed to the bones beneath, and 
confine the extensor tendons. The ligament will be subsequently examined more in detail.

Superficial layer of muscles (fig. 87). The muscles are arranged in a superficial and a deep layer, as on the anterior part of the forearm.

Fig. 87.

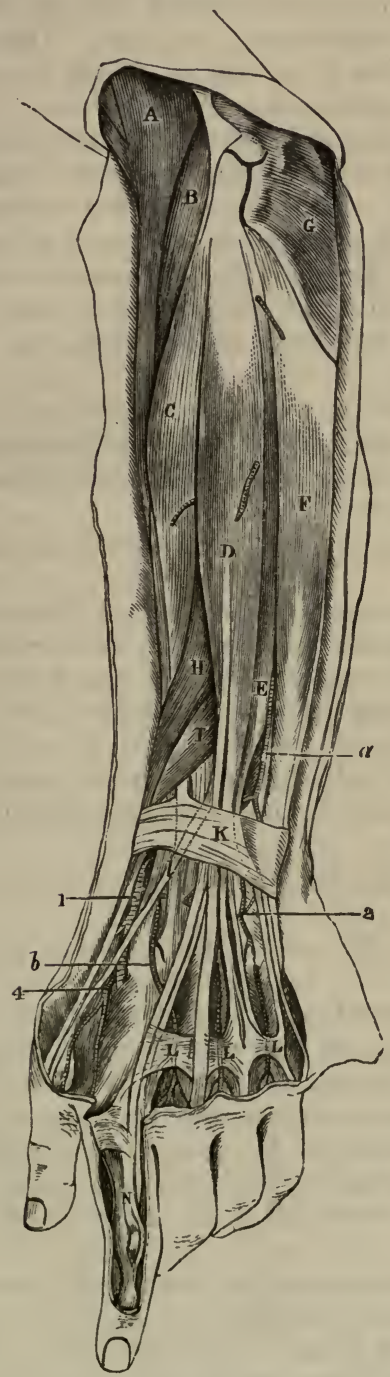

\section{Muscles:}

A. Supinator longus.

B. Extensor curpi radialis longus.

o. Extensor carpi radialis brevis.

D. Extensor communis digitorum.

B. Extensor minimi digiti.

r. Extensor carpi ulnaris.

G. Anconeus.

H. Extensor ossis metacarpi pollicis.

I. Extensor primi internodii.

J. Extensor secundi internodii polticis.

K. Posterior annular ligament.

I. Bands uniting the tendons of the common ex. tensor on the back of the hand.

$\boldsymbol{*}$. Insertion of the common extensor into the last two phalanges.

Arteries :

a. Posterior interosseous.

1. Radial.

2. Posterior carpal arch.

d. Metacarpal branch.

4. Dorsal branches of thumb and forefinger.

Superfictal Layer of MUgCles on the Back of the Foreary, with goue Vegsels.

(Illustrations of Dissections.)

The superficial layer contains seven muscles, which arise mostly by a common tendon from the outer condyle of the humerus, and have the undermentioned position one to another from without inwards; - the long supi- 
nator, A, the two radial extensors of the wrist, B and $\mathrm{c}$ (long and short), the cominon extensor of the fingers, $\mathrm{D}$, the extensor of the little finger, $\mathrm{E}$, and the ulnar extensor of the wrist, $F$. There is one other small muscle near the elbow, the anconeus, G.

The SUPINATOR RADII LONGUS, A, reaches upwards into the arm, and limits on the outer side the hollow in front of the elbow. The muscle arises from the upper two-thirds of the outer condyloid ridge of the humerus, and the front of the external intermuscular septum. The fleshy fibres end about the middle of the forearm in a tendon, which is inserted into the lower end of the radius, close above the styloid process.

In the arm the margins of the supinator are directed towards the surface and the bone, but in the forearm and muscle is flattened over the others, with its edges forwards and backwards. Its anterior border touches the biceps and the pronator teres; and the posterior is in contact with both radial extensors of the wrist. Near its insertion the supinator is eovered by two extensors of the thumb. Beneath the muscle are the brachialis anticus and musculo-spiral nerve, the extensors of the wrist, the radial vessels and nerve, and the radius.

Action. 'The chief use of this supinator is to bend the elbow-joint. But if the radius is either forcibly pronated or supinated the muscle can put the hand into a state intermediate between pronation and supination,- - the thumb being brought above the forefinger.

If the radius is fixed as in climbing, the muscle will bring up the humerus, bending the elbow.

The EXTENSOR CARPI RADIALIS LONGIOR, B, arises from the lower third of the outer condyloid ridge of the humerus, and the front of the contiguous intermuscular septum; and from the septum between it and the next muscle. The muscle lies on the short radial extensor, being partly covered by the supinator longus; and its tendon passes beneath the extensors of the thumb, and the annular ligament, to be inserted into the base of the metacarpal bone of the index finger. Along its outer border lies the radial nerve.

Action. The long extensor straightens first the wrist, and bends next the elbow joint.

If the hand is fixed in climbing, it will act on the humerus like the long supinator.

The extensor CaRPi radialis brevior, c, is attached to the outer condyle of the humerus by a tendon common to it and the three following museles, viz., common extensor of the fingers, extensor of the little finger and ulnar extensor of the wrist: it takes origin also from the capsular ligament of the elbow joint. The tendon of the muscle is closely connected with the preceding, and after passing with it through the same compartment of the annular ligament, is inserted into the base of the metacarpal bone of the middle finger.

Concealed on the outer side by the two preceding muscles this extensor rests on the radius and some of the muscles attached to it, that is to say, on the supinator brevis, and the pronator teres. Along the ulnar side is the common extensor of the fingers; and the extensors of the thumb come between it and the digital extensor. Both radial extensors of the earpus have usually a bursa at the insertion.

Action. 'This muscle resembles its fellow in extending the wrist, but differs from it in extending the elbow. 
Acting with the long extensor of the wrist it will move the lower end of the radius in supination.

The extexsor Communis digitorum, $\mathrm{D}$, is single at its origin, but is divided inferiorly into four tendons. It arises from the common tendon, firom aponeurotic septa between it and the muscles around, and fiom the aponeurosis of the limb. Near the lower part of the forearm the muscle ends in three tendons, which pass through a compartment of the annular ligament with the indicator muscle; below the ligament, the most internal tendon divides into two, and all four are directed along the back of the hand to their insertion into the two last phalanges of the fingers.

On the back of the fingers the tendons have the following arrangement. On the dorsum of the first phalanx each forms an expansion with the tendons of the lumbricales and interossei muscles (fig. 82). At the front of that phalanx it divides into three parts (fig. $87,{ }^{N}$ ): - the central one is fixed into the base of the second phalanx, whilst the lateral pieces unite, and are inserted into the base of the last phalanx. On the fore and little fingers the expansion is joined by the special tendons of those digits; and opposite tlie first two articulations of each finger the tendon sends down lateral bands to join the capsule of the joint.

This muscle is placed between the extensors of the wrist and little finger, and conceals the deep layer. On the back of the hand the tendons are joined by cross pieces, L, which are strongest between the ring finger tendon and its collateral tendons; they prevent the ring finger being raised if the others are closed.

Action. The musele straightens the three phalanges of the fingers from root to tip, and separates the four digits from each other. It can extend the nearest joint of each finger whilst the two farthest are kept bent by the flexors; and it can straighten the last two articulations when the nearest is bent.

The digits being straightened, it will assist the other muscles in extending the wrist and the elbow.

The EXTENSOR MINIMI DIGITI, E, is the most slender muscle on the back of the forearm, and appears to be but a part of the common extensor. Its origin is in common with that of the extensor communis, but it passes through a distinet sheath of the annular ligament. Beyond the ligament the tendon splits into two, and only one unites by a cross piece with the tendon of the common extensor: both finally join the common expansion on the first phalanx of the little finger.

Action. It extends the little finger and moves back the wrist and elbow. As the inner piece of the split tendon is not united with the common extensor it can straighten the digit during flexion of the other fingers.

The EXTENSOR CARPI ULXARIS MUSCLE, F, arises from the common tendon, and the aponeurosis of the forearm; it is also fixed by fascia to the posterior border of the ulna below the anconeus muscle (about the middle third). Its tendon becomes free from fleshy fibres near the annular ligament, and passes through a separate sheath in that structure to be inserted into the base of the metacarpal bone of the little finger.

Beneath this extensor are some of the muscles of the deep layer, with part of the ulna. On the outer side is the extensor of the little finger, with the posterior interosseous ressels.

Action. As the name expresses, the muscle puts back the wrist and inclines the hand towards the ulnar side : it can then extend the elbow joint.

The Axcoxeus, G, is a small triangular muscle near the elbow. It arises 
from the outer condyle of the humerus by a tendon distinct from, and on the ulnar side of the common tendon of origin of the other muscles. From this origin the fibres diverge to their insertion into the outer side of the olecranon, and into the impression on the upper third of the posterior surface of the ulna.

Fig. 88.

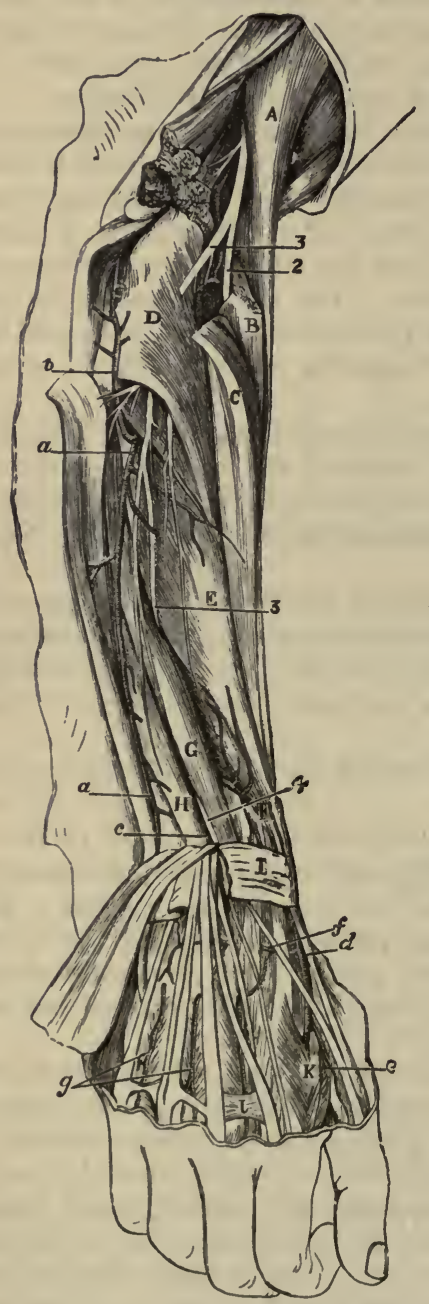

\author{
Muscles : \\ A. Supinator longus. \\ B. and C. Radial extensors of the carpus, cut. \\ D. Supinator brevis. \\ E. Extensor ossis metacarpi pollicis. \\ F. Extensor primi internodii. \\ A. Extensor secundi internodii. \\ н. Extensor indicis. \\ I. Posterior annular ligament.
}

Arteries :

a. Posterior interosseous.

b. Recurrent interosseous.

c. Dorsal part of the anterior interosseous.

d. Dorsal part of the radial.

$e$. Dorsal branches to the thumb and forefinger.

$f$. Dorsal carpal arch.

g. Two posterior interosseous (ulnar) of the hand.

Nerves :

2. Radial.

3. Posterior interosseous at its origin, and 4, near its ending in a swelling on the back of the carpus.

Disgizction of thb Degp Later of Mescles, and the Vegrels and Nervi on the Back of THE FOREARM. (Ill ustrations of Dissections.)

The upper fibres are nearly transverse, and are contiguous to the lowest of the triceps muscle. Beneath the anconeous lie the supinator brevis muscle, and the recurrent interosseous vessels.

Action. Commonly it acts on the ulna, and assists the triceps in extending the elbow. 
Dissertion (fig. 88). For the display of the deep muscles at the back of the forearm, and the posterior interosseous artery and nerve, three of the superficial muscles, viz., extensor communis, extensor minimi digiti, and extensor carpi ulnaris, are to be detached from their origin and turned aside : in this proceeding the small branches of the nerve and artery entering the muscles may be divided.

'The loose tissue and fat are then to be removed from the muscles, and the ramifications of the artery and nerve; and a slender part of the nerve, which sinks beneath the extensor of the second phalanx of the thumb about the middle of the forearm, should be traced beyond the wrist.

The separation of the muscles should be made carefully, because the highest two of the thumb are not always very distinct from each other.

DEEP LAYER OF MUSCLES (fig. 88). In this layer there are five small muscles, viz., one supinator of the forearm, and four special extensor muscles of the thumb and forefinger. The highest muscle, surrounding partly the upper end of the radius, is the supinator brevis, D. Below this are the three muscles of the thumb in the following order:-the extensor of the metacarpal bone, $\mathrm{k}$, the extensor of the first, $\mathrm{F}$, and that of the second phalanx, G. On the ulna the indicator muscle, $\mathrm{H}$, is placed.

The Extersor Ossis METACARPI POLLicis, E, is the largest and highest of the extensor muscles of the thumb, and is sometimes united with the supinator brevis. It arises from the posterior surface of the radius for three inches below the supinator brevis; from the ulna for the same distance by a narrow special impression on the upper and outer part of the posterior surface; and from the intervening interosseous membrane. The tendon is directed outwards over the radial extensors of the wrist, and throngh the annular ligament, to be inserted into the base of the metacarpal bone of the thumb, and by a slip into the os trapezium.

The muscle is concealed at first by the common extensor of the fingers; but it becomes cutaneous between the last muscle and the extensors of the wrist, about two inches above the end of the radius (fig. 87). Opposite the carpus the radial artery winds backwards beneath its tendon. Between the contiguous borders of this muscle and the supinator brevis the posterior interosseous artery $(a)$ appears.

Action. By this muscle the thumb is moved backwards from the palm of the hand, and the wrist is extended on the radial side.

The extexsor primi interNodi politicis, $\mathrm{F}$, is the smallest muscle of the deep layer, and its tendon accompanies that of the preceding extensor. Its origin, about one inch in width, is from the radius and the interosseous membrane, close below the attachment of the preceding muscle. 'The tendon passes through the same space in the annular ligament as the extensor of the metacarpal bone, and is inserted into the metacarpal end of the first phalanx of the thumb. With respect to surrounding parts this muscle has the same connections as the preceding.

Action. It extends first the nearest phalanx, and then the wrist, like its companion.

The ExTENSOR SECUNDi INTERNodi Pollicis, G, arises from the ulna for four inches below the anconeus, along the ulnar side of the extensor of the metacarpal bone; and from the interosseous membrane, below, for one inch. Its tendon passing through a sheath in the annular ligament, distinct from that of the other two extensor muscles, is directed along the dorsum of the thumb to be fixed to the base of the last phalanx.

It is covered by the same muscles as the other extensors of the thumb, 
but it becomes superficial nearer the lower end of the radius. Below the annular ligament its tendon crosses the radial artery, and the extensors of the wrist.

Action. Its use is similar to that of the extensor of the first phalanx. When the phalanges are straight, the two extensors will assist in carrying back the metacarpal bone.

The ExTExSOR INDICIS, I (indicator), arises from the ulna for three or four inches, usually beyond the middle, and internal to the preceding muscles; and from the interosseous ligament below. Near the annular ligament the tendon becomes free from muscular fibres, and passing through that band with the common extensor of the fingers, is applied to, and blends with, the external tendon of that muscle in the expansion on the phalanx of the forefinger.

Until this muscle has passed the ligament it is covered by the superficial layer, but it is afterwards subaponeurotic.

Action. The muscle can point the fore finger even when the three inner fingers are bent, inclining it towards the others at the same time. And it will help the common extensor of the digits in pulling back the hand.

Dissection. To lay bare the supinator brevis, it will be necessary to detach the anconeus from the external condyle of the humerus, and to cut through the supinator longus and the radial extensors of the wrist. After those muscles have been divided, the fleshy fibres of the supinator are to be followed forwards to their insertion into the radius; and that part of the origin of the flexor profundus digitorum, which lies on the outer side of the insertion of the brachialis anticus, is to be removed.

The supinator BREvis, D, surrounds the upper part of the radius, except at the tubercle and along a slip of bone below it. It arises from a depression below the small sigmoid cavity of the ulna, from the external margin of the bone for two inches below that pit, and from the orbicular ligament of the radius and the external lateral ligament of the elbow joint. The fibres pass outwards, and are inserted into the upper third or more of the radius, except at the inner part, reaching downwards to the insertion of the pronator teres, and forwards to the hollowed anterior surface.

The supinator is concealed altogether at the posterior and external aspects of the limb by the muscles of the superficial layer; and anteriorly the radial vessels and nerve lie over it. The lower border is contiguous to the extensor ossis metacarpi pollicis, only the posterior interosseous artery $(a)$ intervening. Through the substance of the muscle the posterior interosseous nerve $\left({ }^{8}\right)$ winds to the back of the limb.

Action. When the radius has been moved over the ulna as in pronation, the short supinator comes into play to bring that bone again to the outer side of the ulna.

The posterior interosseous artery (fig. $88, a$ ) is an offset from the common interosseous trunk (p. 269), and reaches the back of the forearm above the ligament between the bones. Appearing between the contiguous borders of the supinator brevis and extensor ossis metacarpi, the artery descends at first between the superficial and deep layers of muscles; and afterwards, with a superficial position in the lower third of the forearm, along the tendon of the extensor carpi ulnaris as far as the wrist, where it ends by anastomosing with the carpal and anterior interosseous arteries. It furnishes muscular off'sets to the contiguous muscles, except the two or three outer ; and the following recurrent branch:-

The recurrent branch $(b)$ springs from the artery near the commence- 
ment, and ascends on or through the fibres of the supinator, but beneath the anconeus, to supply both those muscles and the elbow joint, and to anastomose with the superior profunda artery in the last-named muscle, as well as with the recurrent radial.

The posterior interosseous nerve $\left({ }^{3}\right)$ takes origin from the musculo-spiral trunk (p. 259), and winds backwards through the fibres of the supinator brevis. Escaped from the supinator, the nerve is placed between the superficial and deep layers of muscles as far as the middle of the forearm. Much reduced in size at that spot, it sinks beneath the extensor of the second phalanx of the thumb, and runs on the interosseous membrane to the back of the carpus. Finally the nerve enlarges beneath the tendons of the extensor comnunis digitorum, and terminates in filaments to the ligaments and the articulations of the carpus.

Branches. It furnishes offsets to all the muscles of the deep layer; and to those of the superficial layer with the exception of the three following, viz., anconeus, supinator longus, and extensor carpi radialis longior.

Radial Artery it the Wrist (fig. 88). The radial artery $(d)$, with its venæ comites, winds below the radius to the back of the carpus, and enters the palm of the hand at the first interosseous space, between the heads of the first dorsal interosseous muscle. At first the vessel lies deeply on the external lateral ligament of the wrist joint, and beneath the tendons of the extensors of the metacarpal bone, $\mathrm{E}$, and first phalanx of the thumb, $\mathrm{F}$; but afterwards it is more superficial, and is crossed by the tendon of the extensor of the second phalanx of the thumb, a.

Offisets of the external cutaneous nerve entwine around the artery, and the radial nerve is superficial to it. Its branches are numerous but inconsiderable in size :-

a. The dorsal carpal branch $(f)$ passes transversely beneath the extensor tendons, and forms an arch with a corresponding offset of the ulnar artery; with this arch the posterior interosseous artery joins.

From the carpal arch branches $(g)$ descend to the third and fourth interosseous spaces, and constitute two of the three dorsal interosseous arteries: at the cleft of the fingers each divides into two, which are continued along the dorsum of the digits. In front they communicate with the digital arteries; and behind they are joined by the perforating branches of the palmar arch.

b. The metacarpal or first dorsal interosseous branch (fig. 87, b), reaches the space between the second and third metacarpal bones, and anastomoses, like the corresponding arteries of the other spaces, with a perforating branch of the deep palmar arch. Finally it is continued to the eleft of the fingers, where it joins the digital artery of the superficial palmar arch, and gives small dorsal branches to the index and second fingers.

c. Two small dorsal arteries of the thumb (c) arise opposite the metacarpal bone, along which they extend, one on each border, to be distributed on its posterior aspect.

$d$. 'The dorsal branch of the index finger is distributed on the radial edge of that digit.

'The different compartments of the annular ligament may be seen more completely by dividing the sheaths of the ligament over the several tendons passing beneath. There are six different spaces, and each is lubricated by a synovial membrane. The most external one lodges the first two extensors of the thumb. The next is a large hollow for the two radial 
extensors of the wrist; and a very small space for the extensor of the second phalanx of the thumb follows on the ulnar side. Farther to the inner side is the common sheath for the extensor of the fingers, and that of the fore finger; and then comes a separate compartment for the extensor of the little finger. Internal to all is the space for the extensor carpi ulnaris. The last muscle grooves the ulna; but the others lie in hollows in the radius in the order mentioned above, with the exception of the extensor minimi digiti, which is situate between the bones.

Dissection. It the supinator brevis be divided by a vertical incision, and reflected from the radius, its attachment to the bone will be better understood.

The posterior interosseous nerve, and the offsets from its gangliform enlargement, may be traced more completely after the tendons of the extensor of the fingers and indicator muscle have been cut at the wrist.

The dorsal surface of the posterior interossei muscles of the hand may be cleaned, so that their double origin, and their insertion into the side, and on the dorsum of the phalanges, may be observed. Between the heads of origin of these muscles the posterior perforating arteries appear.

\section{Section VII.}

LIGAMENTS OF THE SHOULDER, ELBOW, WRIST, AND HAND.

Directions. The ligaments of the remaining articulations of the limb, which are still moist, may be examined at once; but if any of them have become dry, they may be softened by immersion in water, or with a wet cloth, whilst the student learns the others.

Dissection. For the preparation of the external ligaments of the shoulder-joint the tendons of the surrounding museles, viz., subscapularis, supra and infraspinatus, and teres minor, must be detached from the capsule; and as these are united with it, some care will be needed not to open the joint.

Shoulder Jorst. This ball and socket joint (fig. 89) is formed between the head of the humerus and the glenoid fossa of the seapula. Inclosing the articular ends of the bones is a fibrous capsule lined by a synovial membrane. A ligamentous band (glenoid ligament) deepens the shallow scapular cavity for the reception of the large head of the humerus.

The bones are but slightly bound together by ligamentous bands, for, on the removal of the muscles, the head of the humerus may be drawn from the scapula for the distance of an inch.

The capsular ligament (fig. 75, ${ }^{5}$ ) surrounds loosely the articular ends of the bones; it is thickened above and below, and receives fibres from the contiguous tendons.

At the upper edge it is fixed around the articular surface of the scapula, where it is connected with the long head of the triceps. At the lower edge the ligament is fixed (fig. 89) to the neck of the humerus close to the articular surface above, but at a little distance therefrom below; and its continuity is interrupted between the tuberosities $(b)$ by the tendon of the biceps muscle, over which it is continued along the bicipital groove (fig. 75 ). On the inner side there is generally an aperture in the 
capsule, below the coracoid process, through which the synovial membrane of the joint is continuous with the bursa beneath the tendon of the subscapularis.

The following muscles surround the articulation: above and behind are the supraspinatus, infraspinatus, and teres minor; below, the capsule is only partly covered by the subscapularis; but internally it is well supported by the last-named muscle.

On the front of the capsule is a rather thick band of fibres-the coracohumeral or accessory ligament (fig. $75,{ }^{8}$ ), which springs firom the base of the coracoid process of the scapula, and widening over the front of the joint, is attached to the margins of the bicipital groove, and to the tuberosities.

Dissection. To see the interior of the articulation, cut circularly through the capsule near the scapula. When this has been done the attaclıment of the capsule to the bones, the glenoid ligament, and the tendon of the biceps will be manifest.

The tendon of the biceps muscle arches over the head of the humerus, and serves the purpose of a ligament in restraining the upward and outward movements of that bone. It is attached to the upper part of the glenoid fossa of the scapula (fig. 89, $d$ ), and is united on each side with the glenoid ligament. At first flat, it afterwards becomes round, and entering the groove between the tuberosities of the humerus, it is surrounded by the synovial membrane.

Fig. 89 .
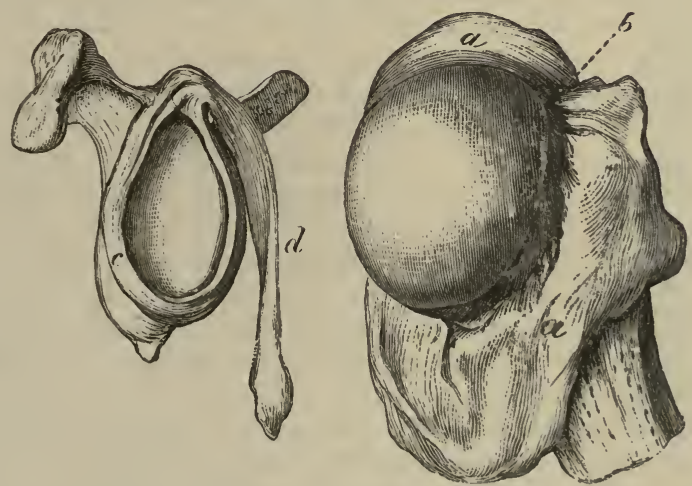

VIEW OF THE INTERIOR OF THE SHOLLDER-JOINT.

a. Attachment of the capsule to the neck of the humerus.

b. Interval of the bicipital groove. $c$. Glenoid ligament around the glenoid fossa. d. Tendon of the biccps fixed to the top of the fossa.

The glenoid ligament (fig. $89, c$ ) is a firm fibro-cartilaginous band, which surrounds the fossa of the same name, deepening it for the reception of the head of the humerus. It is about two lines in depth, and is connected in part with the sides of the tendon of the biceps; but most of its fibres are fixed separately to the margin of the glenoid fossa.

The synovial membrane lines the articular surface of the capsule, and is continued through the aperture on the inner part to join the bursa beneath the subscapular musele. The membrane is reflected around the tendon of the biceps, and lines the bicipital groove of the humerus. 
Articular surfaces (fig. 89). The convex articular head of the humerus is two or three times larger than the hollow in the scapula, and forms rather less than the half of a sphere. The head of the bone is joined to the shaft at an angle as it is in the femur, and a rotatory movement is possessed by the humerus in consequence.

The glenoid surface of the scapula is oval in form with the large end down, and is very shallow; it is not large enough to cover the head of the humerus.

Movements. In this joint there is the common motion in four directions, with the circular or circumductory; and in addition a movement of rotation.

In the swinging or to and fro movement, the carrying forwards of the humerus constitutes flexion, and the moving it backwards, extension. Flexion is freer than extension; and when the joint is most bent. the scapula, rotating on its axis, follows the head of the humerus, so as to keep the centre of the glenoid fossa applied to the middle of the articular surface of the arm-bone. In extension the articular surface of the scapula does not move after the humerus.

During these movements the head of the bone rests in the bottom of the glenoid fossa, turning forwards and backwards around a line representing the axis of the head and neck; and it eannot be dislodged by either the rapidity or the degree of the motion.

The museles have more influence than the loose capsule in controlling the swinging motion.

Abduction and adduction. When the limb is raised, it is abducted, and when depressed, adducted; and in both cases the humerus rolls on the scapula which is fixed.

During abduction the head of the humerus, descends to the lower and larger part of the glenoid fossa, and projects beyond it against the capsule; whilst the great tuberosity rubs against the arch of the acromion. In this condition a little more movement down of the head either by muscles depressing it, or by force elevating the farther end of the bone, will throw it out of place, giving rise to dislocation.

In adduction the head of the bone rises into the socket, the limb meets the trunk, and the tense capsule is set at rest. After the reduction of a dislocation the limb is fixed to the side in this position of security against further displacement.

In circumduction the humerus passes in succession through the four different states before mentioned, and the limb describes a cone, whose apex is at the shoulder and base at the digits.

Rotation. There are two kinds of rotatory movement, viz., in and out; and in each the motion of the head and shaft of the bone has to be considered.

In rotation in, the great tuberosity is turned forwards, and the head rolls from before back across the glenoid fossa so as to project behind. The shaft is moved forwards round a line lying on its inner side, which reaches from the centre of the head to the inner condyle.

In rotation out, the osseous movements are reversed: thus the tuberosity turns back, the head rolls forward so as to project in front, and the shaft is carried back around the line before said.

The upper thickened part of the capsule will be tightened in rotation, but the muscles are the ehief agents in ehecking the movements.

Dissection. 'To make the necessary dissection of the ligaments of the 
elbow, the brachialis anticus must be taken away from the front, and the triceps from the back of the joint. The muscles conneeted with the outer and inner condyles of the humerus, as well as the supinator brevis and the flexor profundus, are to be removed. With a little cleaning the four ligaments-anterior, posterior, and two lateral-will come into view.

The interosseous membrane between the bones of the forearm will be prepared by the removal of the muscles on both surfaces.

Tire Elbow Joint (fig. 90). In this articulation the lower end of the humerus is received into the hollow of the ulna, so as to produce a hingelike arrangement; and the upper end of the radius assists to form part of the joint. Where the bones touch the surfaces are covered with cartilage, and their articular ends are kept in place by the following ligaments :-

The external lateral ligament is a roundish fasciculns, which is attached by one end to the outer condyle of the humerus, and by the other to the orbicular ligament around the head of the radius. A few of the posterior fibres pass backwards to the external margin of the ulna.

The internal lateral ligament is triangular in shape. It is pointed at its upper extremity, and is connected to the inner condyle of the humerus. The fibres diverge, and are inserted in this way:The anterior, which are the strongest, are fixed to the edge of the coronoid process; the posterior are attached to the side of the olecranon; whilst a few middle fibres join a transverse ligamentous band over the notch between the olecranon and the coronoid process. The ulnar nerve is in contact with the ligament; and vessels enter the joint by an aperture beneath the transverse band.

The anterior ligament is thin, and its fibres are separated by intervals in which masses of fat are lodged. By its upper edge the ligament is inserted into the front of the humerus, and by its lower into the front of the coronoid process and the orbicular ligament. The brachialis anticus musele covers it.

The posterior ligament is much thinner and looser than the anterior, and is covered completely by the triceps muscle. Superiorly it is attached to the humerus above the fossa for the olecranon; and inferiorly it is inserted into the olecranon. Some few fibres are transverse between the margins of the fossa before mentioned.

Dissection. Open the joint by an incision across the front near the humerus, and disarticulate the bones, in order that the articular surfaces may be seen.

The synovial membrane of the joint can be traced from one bone to another along the inner surface of the connecting ligaments. It projects between the radius and the orbicular ligament, and serves for the articulation of the head of that bone with the small sigmoid eavity of the ulna. 
Articular surfaces. The humerus presents inferiorly two distinct articular faces for the bones of the forearm. The one for the radius, on the outer side, consists of a rounded eminence (capitellum) on the front of the bone, which is eovered with eartilage only on the interior aspect. The surface in contact with the ulna is limited internally and externally by a prominence, and hollowed out in the centre (trochlea). On the front of the humerus above the articular surface are two depressions which receive the coronoid process and the head of the radius during flexure of the joint; and on the posterior aspect is a large fossa for the reception of the olecranon in extension of the joint.

On the end of the ulna the large sigmoid eavity is narrowed in the centre, but expanded in front and behind (fig. 91). A slightly raised line extends from front to back, and is received into the hollow of the trochlea of the humerus. Across the bottom of the cavity the cartilage is wanting completely, or for a greater or smaller distance.

'The end of the radius presents a circular depression with a raised margin. In the bent state of the joint the hollow of the radius fits on the outer eminence of the humerus, and the bone is supported during rotation of the limb.

Movement. This joint is like a hinge in its movements, and permits only flexion and extension.

In flexion the bones of the forearm move forwards, each on its own articular surface, so as to leave the back of the humerus uncovered. 'The extent is checked by the meeting of the bones of the arm and forearm. Owing to the slanting surface of the humerus the hand falls insicle the limb when the joint is fully bent.

The ligaments are relaxed, with the exception of the following, viz., the posterior, and the hinder part of the internal lateral.

In extension the ulna and radius are carried back over the articular surface of the humerus until they come into a line with the arm-bone. 'This movement is checked by the olecranon touching the humerus.

The anterior ligament, and the fore part of the internal lateral ligament are made tight, but the hinder fibres of the internal lateral are relaxed.

Union of the Radius and Ulna. The radius is connected with the ulna at botlı ends by means of distinet ligaments and synovial membrane; and the shafts of the bones are united by interosseous ligaments.

Upper radio-ulnar articulation. In this joint the head of the radius is received into the small sigmoid cavity of the ulna, and is kept in place by the following ligamentous band:-

The annulur or orbicular ligament (fig. 91, a) is about one-third of an inch wide, and is stronger behind than before; it is placed around the prominence of the head of the radius, and is attached to the anterior and posterior edges of the small sigmoid cavity of the ulna. Its upper border, the widest, is conneeted with the ligaments of the elbow joint; but the lower is free, and is applied around the neck of the radius. In the socket formed by this ligament and the cavity of the ulna, the radius moves freely.

The synovial membrane is a prolongation of that lining the elbow joint; it projects inferiorly between the neek of the radius and the lower margin of the annular ligament.

Ligaments of the shafts of the bones. The aponeurotic stratum conneeting together the bones in nearly their whole length consists of the two following parts:- 
The interosseous membrane (fig. $90,{ }^{3}$ ) is a thin fibrous layer, which is attached to the contiguous margins of the radius and ulna, and forms an incomplete septum between the muscles on the front and back of the forearm. Most of its fibres are directed obliquely inwards towards the ulna, though a few on the posterior surface have an opposite direction. Superiorly the membrane is wanting for a considerable space, and through the interval the posterior interosseous vessels pass backwards. Some small apertures exist in it for the passage of vessels ; and the largest of these $\left({ }^{4}\right)$ is about two inches from the lower end, through which the anterior interosseous artery turns to the back of the wrist. The membrane gives attachment to the deep muscles.

The round ligament (fig. $90,{ }^{2}$ ) is a slender band above the interosseous membrane, whose fibres have a direction opposite to those of the membrane. By one end it is fixed to the front of the coronoid process, and by the other to the radius below the tubercle. The ligament divides the space above the interosseous membrane into two parts. Oftentimes this band is not to be recognized.

Fig. 91.

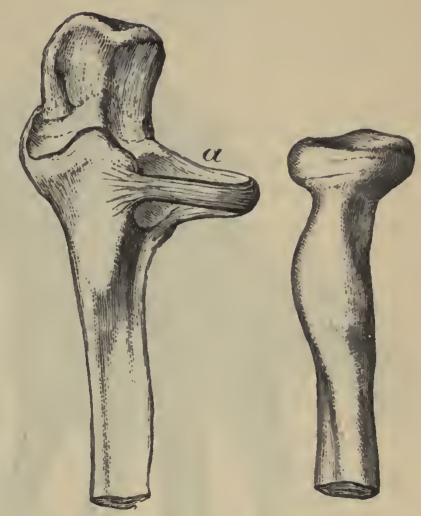

VIEW OF THE ORBICULAR LIGAMENT (a) OF THE RADICs, WHICH RETAINS THE UPPER END OF THE BON B AGAINST THE ULNA.

The lower radio-ulnar articulation cannot be well seen till after the examination of the wrist joint (p. 297).

Movement of the radius. The radius moves forwards and backwards around the ulna. The forward motion, directing the palm of the hand towards the ground, is called pronation; and the backward, by which the palm of the hand is placed upwards, is named supination.

In pronation the upper end of the bone rotates within the hand of the orbicular ligament without shifting its position to the ulna. The lower end, on the contrary, moves over the ulna from the outer to the inner side, describing half a circle; and the shaft crosses obliquely that of the ulna.

In supination the lower end of the radius turns backwards over the ulna; the shafts come to be placed side by side, the radius being external; and the upper end rotates from within out in its circular band.

In these movements the radius revolves round a line, internal to the shaft, which is prolonged upwards through the neck and head of the bone, and downwards through the centre of a circle of which the small sigmoid cavity of the ulna is a segment (Ward).

The upper end of the bone is kept in place by the orbicular ligament ; the lower end by the triangular fibro-rartilage; and the shafts are united by the interosseous ligament, which is tightened in supination, and is relaxed in pronation.

In fracture of either bone the movements cease; in the one case because the radius cannot be moved except it is entire; and in the other, because the ulnar support is wanting for the revolving radius.

Dissection. To see the ligaments of the wrist-joint, the tendons and the annular ligaments must be remored from both the front and baek; and 
the fibrous structures and the small vessels should be taken from the surface of the ligaments.

The WRist Jonnt (fig. 92). The lower end of the radius, and the first row of the carpal bones, except the os pisiforme, enter into the wristjoint. Four ligaments maintain in contact the osseous surfaces, viz., an-

Fig. 92.

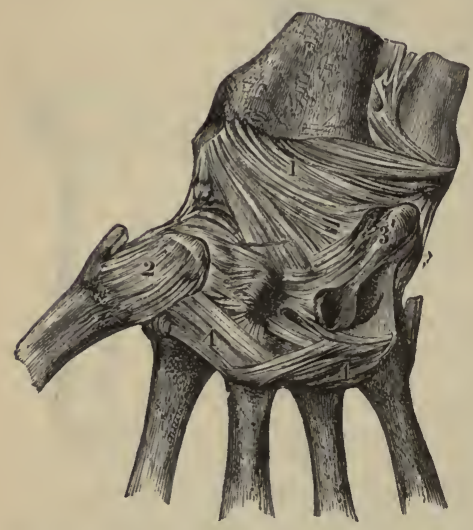

FroNT VinW OF THE ARTICELATIONg OF THE WRIST JoINT, AND CARPAL AND METACARPAL BoNBS (Bourgery and Jacob).

1. Anterior ligament of the wrist joint.

2. Capsule of the joint 0 . the metacarpal bone of the thumb with the os trapezium.

3. Pisiform bone, with its separate joint and ligamentons bands.

4. Transverse bands to the head of the metacarpal bones. terior and posterior, and two lateral. The ulna is shut out from this articulation by means of a piece of fibrocartilage.

The external lateral ligament is a short and strong band, which intervenes between the styloid process of the radius and the outer part of the scaphoid bone.

The internal lateral ligament is smaller than the external, but is longer than it. It is attached by one end to the styloid process of the ulna, and by the other to the rough, upper part of the cuneiform bone. Some of the anterior fibres are continued to the pisiform bone.

The anterior ligament (fig. 92, ${ }^{1}$ ) takes origin from the radius and the fibro-cartilage, and is inserted into the first row of carpal bones, except the pisiform, at the anterior surface.

The posterior ligament (fig. 95, ${ }^{1}$ ) is membranous, like the anterior, and its fibres are directed downwards and inwards. Superiorly it is attached to the radius and the fibro-cartilage; and inferiorly it is fixed, like the anterior,

to the three outer carpal bones of the first row, but on the posterior aspect.

Dissection. To see the form of the articulating surfaces, the joint may be opened by a transverse incision through the posterior liganent, near the bones of the carpus.

Articular surfaces. The end of the radius, and the fibro-cartilage uniting it with the ulna, form an arch for the reception of the carpal bones (fig. 93) ; and the surface of the radius is divided by a prominent line into an external triangular, and an internal square impression. 'The three bones of the first carpal row constitute a convex eminence (fig. 94), which is received into the hollow before mentioned in this way: The scaphoid bone $(a)$ is opposite the external mark of the radius; the semilunar bone $(b)$ touches the square impression, and all or part of the triangular fibro-cartilage; whilst the cuneiform bone $(c)$ is in contact with the capsule (Henle), and sometimes with part of the fibro-cartilage.

The synovial membrane has the arrangement common to simple joints. This joint communicates oceasionally with the lower ralio-ulnar articulation by means of an aperture in the fibro-cartilage separating the two.

Movement. The wrist is a condyloid articulation, and possesses angular motion in four different directions, with circumduction. 
Flexion and extension. In flexion the hand is moved forwards and inwards, whilst the carpus rolls on the radius from before back, and projeets behind, stretehing the posterior ligament. In extension the hand is carried backwards and outwards, and the row of earpal bones moves in the opposite direction, viz., from behind forwards, so as to eause the anterior ligament to be tightened. The hinder movement is freer than the forward.

Abduction and adduction. The row of earpal bones moves transversely inwards in the former, and outwards in the latter state; and the movement is freer towards the ulnar than the radial side.

The latter ligaments are put on the stretch, the inner in abduction and the outer in adduction ; and the motion is limited on the outer side by the meeting of the styloid processes of the radius with the scaphoid bone.

Circumduction. The hand describes a cone in this movement, whose apex is at the wrist and base at the digits; and it moves more freely in extension and adduction than in the opposite directions.

Lower Radio-ulnar Articulation. In this articulation the convexity of the end of the ulna is received into a concavity on the radius; an arrangement just the opposite to that between the upper ends of the bones.

The ehief bond of union between the bones is a strong fibro-cartilage; but a kind of capsule consisting of scattered fibres, surrounds loosely the end of the ulna.

The triangular fibro-cartilage (fig. $93, c$ ) is placed transversely beneath the end of the ulna, and is thiekest at its margins and apex. By its base the cartilage is fixed to the ridge which separates the earpal from the ulnar articulating surface of the radius; and by its apex to the styloid process of the ulna, and the depression at the root of that point of bone. Its margins are united with the contiguous anterior and posterior ligaments of the wrist joint; and its surfaces enter into different joints, viz., the wrist, and the lower

Fig. 93.

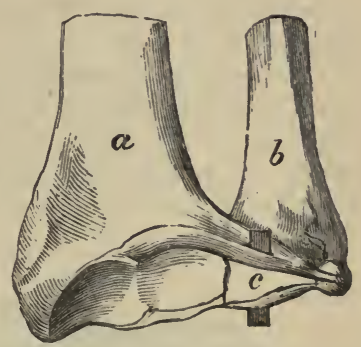

THE WRIST JOINT OPENED TOSHOW THE ARCH FORMEDBY THE BONES OF THE FOREARM WITH THE UNITING FiBRo-CARTILAGE, $c$.

a. Radius.

b. UIna. radio-ulnar. It serves to unite the radius and ulna, and to form part of the socket for the carpal bones. Oecasionally it is perforated by an aperture.

The synovial membrane (membrana saceiformis) is very loose, from which circumstance it has received its name, and ascends between the radius and the ulna: it is separated from that of the wrist-joint by the triangular fibro-cartilage.

The use of this articulation is referred to with the movements of the radius (p. 295).

Union of tire Carpal Bones. The several bones of the earpus are united into two rows by dorsal, palmar, and interosseous bands : and the two rows are connected to each other by separate ligaments.

Dissection. The artieulation of the earpal bones with each other will be prepared by taking away all the tendons from the hand, and cleaning earefully the whole of the connecting ligamentous bands. Two distinet ligaments of the pisiform bone to the unciform and fith metacarpal are to be defined in the palm. 
At the same time the ligamentous bands uniting the metacarpal with the carpal bones and with one another should be dissected.

Bones of the first row (fig. 94). 'The os semilunare is united to the lateral bones, viz., seaphoid and cuneiform by a dorsal (fig. 94, $d$ ), and a palmar transverse band; as well as, it is said, by an interosseous ligament at the upper part of the continuous surfaces. ${ }^{1}$

The pisiform bone is articulated to the front of the euneiform by a distinct capsule and a synovial membrane (fig. $92,{ }^{3}$ ). It has further two special firm ligaments: one of these is attached to the process of the os unciforme, and the other to the base of the fifth metacarpal bone.

The bones of the second row are connected together in the same way as those of the first, viz., by a dorsal and a palmar band of fibres from one bone to another. Between the eontiguous rough surfaces of the several ossicles are interosseous ligaments, one in each interval.

Movement. Only a small degree of gliding motion is permitted between the different carpal bones, in consequence of the flattened articular surfaces, and the interosseous ligaments uniting one to another; and this is less in the second than in the first row.

One row with another (transverse carpal joint, fig. 94). The two rows of carpal bones are connected by an anterior and posterior, and two lateral ligaments.

The anterior ligament $(p)$ consists of strong irregular fibres, and intervenes between the two rows (except the pisiform) on the palmar aspect. The posterior ligament, which is longer and looser, and the greater number of whose fibres are transverse, has a corresponding attachment on the dorsal aspect of the same bones.

Fig. 94.

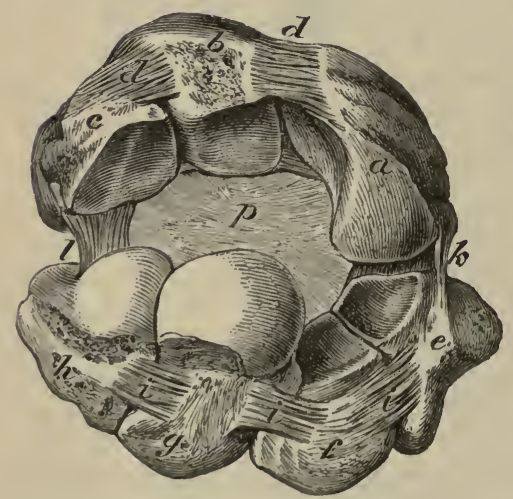

a. Scaphoid bone.

b. Semilunar.

c. Cuneiform.

d. Dorsal trausverse bands between those bones.

e. Trapezium bone.

$f$. Trapezoid.

g. Os magnum.

$h$. Unciform.

i. Dorsal trankverse bands joining the bones.

$k$. External lateral ligament of the intercarpal joint.

l. Internal lateral.

$p$ Anterlor ligaments.

ARticulations of ONE Carpat BoNe With ANOTHFR, AND OF THE TWO ROWS With EACH OTHER. THF JOINT BETWEEN THE TWO ROW IS OPENED BEHIND.

Of the lateral ligaments the external $(k)$ is the best marked, and extends between the os trapezium and the scaphoid bone; the internal ligament (l) reaches between the cuneiform and unciform bones.

Dissection. After the division of the lateral and posterior ligaments,

1 Interosseous ligaments in this row, distinct from the other bands, can scarcely be said to exist. 
the one row of bones may be separated far enough from the other, to allow a sight of the articular surfaces.

Articular surfaces. The first row of carpal bones (except pisiform) forms internally an arch with the scaphoid $(a)$, semilunar $(d)$, and cuneiform bone $(c)$, whose hollow is turned towards the second ; and externally a prominence with the scaphoid $(a)$ which is received into a concavity in the other row. In the second row the os magnum $(g)$ and os uneiforme (h) present a condyloid projection, which is received into the arch before mentioned; but the two outer bones (trapezium and trapezoid, $e$ and $f$ ) are much below the level of the others, and form a slight hollow for the reception of the outer part of the scaphoid bone.

One synovial membrane serves for the articulation of all the carpal bones, except the pisiform with the cuneiform. Lining the joint between the two rows of the carpus, the membrane sends upwards and downwards prolongations between the individual bones. The offsets upwards are two, and they sometimes join the synovial membrane of the wrist joint ; but the offsets in the opposite direction are three, and may be continued to all, or only to some of the articulations between the four inner metacarpal with their carpal bones.

Movements. 'The transverse carpal joint is partly condyloid, but only forward and backward motion is permitted. All lateral and eircumductory movement is arrested, if the rows are closely applied together, by the scaphoid striking against the os magnum on the one side, and the cuneiform against the unciform on the other.

Flexion. As the hand is bent forwards the lower row of earpal bones moves backwards, and renders prominent the posterior ligament. This motion is also brought into play in full bending of the wrist.

Extension. 'The backward movement is freer than flexion. As the lower carpal row moves towards the palm, its progress is checked by the anterior ligament of the joint, and by the strong flexor tendons.

Union of the Metacarpal Boxes. The metacarpal bones of the four fingers are connecter at their bases by the following ligament: A superficial dorsal (fig. 95) and palmar fasciculus of fibres passes transversely from one bone to the next; and the bands in the palm are the strongest (fig. 92). Besides, there is a short interosseous ligament between the contiguous rough surfaces of the bones.

Lateral union. Where the metacarpal bones touch they are covered by cartilage; and the articular sur-

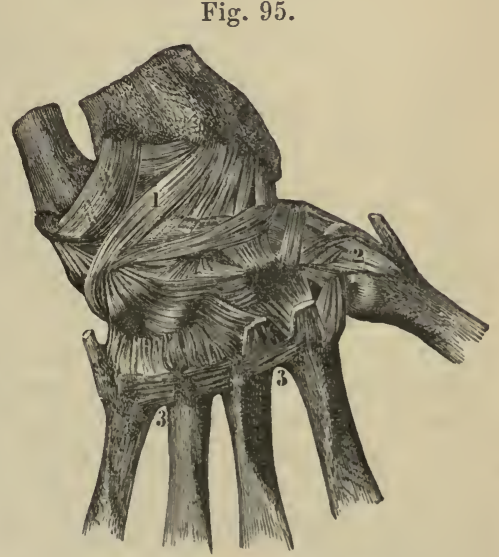

Postekior Lioamexts of THE Wrist, axd Carpal and Metacarpal Bones (Bourgery and Jacob).

1. Posterior radio-carpal.

2. Carpo-metacarpal joint of the thumb.

3,3 . Transverse bands between the bases of the metacarpal bones.

faces are furnished with prolongations of the synovial membrane serving for their articulation with the carpus.

At the anterior extremities the same four metacarpal bones are con- 
nected by the deep transverse ligament which was seen in the dissection of the hand (p. 280)).

Uniox of the Metacarpal and Carpal Bones. The metacarpal bones of the fingers are articulated with the carpal bones after one plan; but the bone of the thumb has a separate joint.

The metorarpal bone of the thumb articulates with the os trapezinm; and the ends of the bonts are incased in a separate capsular ligament (fig. $\left.92,{ }^{2}\right)$. The joint is furnished with a synovial membrane which is simple in its arrangement.

The thumb-joint possesses angular movement in opposite directions, with opposition and circumduction, thus :-

Flexion and extension. When the joint is flexed the metacarpal bone is brought into the palm of the hand, without the ball of the thumb being turned to the tips of the fingers. Extension of the joint is very free, and by it the metacarpal bone is removed from the palm towards the outer border of the forearm.

Abduction and adduction. By these movements the metacarpal bone is placed in contact with, or removed from the fore finger.

Opposition. In this movement the ball of the thumb is turned towards the tip of each finger by a half circumductory motion of the metacarpal bone; and in picking up a pin the joints of the thumb, and the two last joints of the fingers will be bent.

The metacarpal bones of the fingers receive longitudinal bands from the carpal bones on both aspects, thus :-

The dorsal ligaments (fig. 95) are two to each, except to the bone of the little finger. The bands of the metacarpal bone of the fore finger come from the os trapezium and os trapezoides; those of the third metacarpal are attached to the os magnum and os trapezoides; the bone of the ring finger receives its bands from the os magnum and os unciforme; and to the fifth metacarpal bone there is but one ligament from the unciform.

The palmar ligaments (fig. 92) are weaker and less constant than the dorsal. There is one to each metacarpal bone, except that of the little finger. These ligaments may be oblique in direction ; and a band may be divided between two, as in the case of the ligament attached to the os trapezium and the second and third metacarpals. Sometimes one or more may be wanting.

On the ulnar side of the metacarpal bone of the middle digit is a longitudinal lateral band, which is attached above to the os magnum and unciforme, and below to the rough uinar side of the base of the above mentioned bone. Sometimes this band isolates the articulation of the last two metaearpals with the unciform bone from the remaining earpo-metacarpal joint; but more frequently it is divided into two parts, and does not form a complete partition.

This band may be seen by opening behind the articulation between the unciform and the last two metacarpal bones; and by cutting through the transverse ligaments joining the third and fourth metacarpals so as to allow their separation.

Movement. Scarcely any appreciable antero-posterior movement exists in the articulations of the bases of the metacarpal bones of the fore and middle fingers; but in the ring and little fingers the motion is greater, with slight abduction and adduetion.

Dissection. The articulating surfaces of the bones in the carpo-meta- 
carpal articulation may be seen by cutting through the rest of the ligaments on the posterior aspect of the hand.

Articular surfaces. The metacarpal bone of the fore finger presents a hollowed articular surface, which receives the prominence of the os trapezoides, and articulates laterally with the os trapezium and os magnum. The middle finger metacarpal articulates with the os magnum. The metacarpal bone of the ring finger touches the unciform bone and the os magnum. And the little finger bone is opposed to the os unciforme.

Synovial membranes. Usually two synovial membranes are interposed between the carpal and metacarpal bones, viz., a separate one for the bone of the thumb, and offsets of the common carpal synovial sac (p. 299) for the others. Sometimes there is a distinct synovial sac for the articulation of the os unciforme with the two inner metacarpals.

Interosseous ligaments. The interosseous ligaments between the bases of the metacarpal bones may be demonstrated by detaching one bone from another. There are also strong fibrous pieces between all the carpal bones in the second row ; and slight ones are described as present on each side of the os semilunare in the first row.

Dissection. For the examination of the joint between the head of the metacarpal bone and the first phalanx of the finger, it will be requisite to clear away the tendons and the tendinous expansion around it. A lateral ligament on each side, and an anterior thick band are to be defined. One of the joints may be opened to see the articular surfaces.

The same dissection may be made for the articulations between the phalanges of the finger.

Union of Metacarpal Bone and First Phalanx (fig. 96). In this joint the convex head of the metacarpal bone is received into the glenoid fossa of the phalanx, and the two are retained in contact by the extensor and flexor tendons, and by the following ligaments :-

The lateral ligament $(a)$ is the same on both sides of the joint. Each is triangular in form: it is attached by its upper part to the tubercle on the side of the head of the metacarpal bone, and below it is inserted into the side of the phalanx and the anterior ligament.

The anterior ligament $(b)$ is a longitudinal band, which is fixed firmly to the phalanx, but loosely to the metacarpal

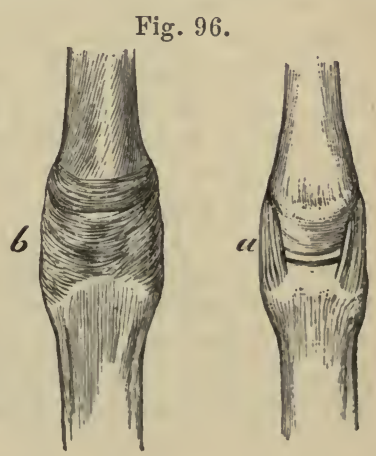
bone. It is fibro-cartilaginous in texture, and is grooved for the flexor tendon: to its sides the lateral ligaments are attached.

Covering the upper part of the joint is the extensor tendon; this takes the place of a dorsal ligament, and sends down an expansion on each side which serves as a capsule to the articulation. The synovial membrane of the joint is a simple sac.

In the articulation of the thumb two sesamoid bones are connected with the anterior ligament, and receive most of the fibres of the lateral ligaments.

Movements. Motion in four opposite directions, and circumduction, exist in these condyloid joints. 
Extension and flexion. The phalanx moves backwards in extension, so as to give an angle with the metacarpal bone. The anterior ligament and the flexor tendons are stretched, and control the movement. In flexion the phalanx glides forwards under the head of the metacarpal bone, and leaves this exposed to form the knuckle when the finger is shut. The lateral ligaments and the extensor tendon are put on the stretch as the joint is bent.

Abduction and adduction are the lateral movements of the finger from or towards the middle line of the hand. 'The lateral ligament of the side of the joint which is convex will be tightened, and the other will be relaxed.

The circumductory motion is less impeded in the thumb, and in the fore and little fingers than in the others; and in the thumb it allows the turning of the last phalanx towards the other digits in the movement of opposition.

Union of the Phalanges. The ligaments of the first joint are similar to those in the metacarpo-phalangeal articulation, viz., two lateral and an anterior.

The lateral ligaments are triangular in form. Each is connected by its apex to the side of the phalanx near the anterior part; and by its base to the contiguous phalanx and the anterior ligament.

The anterior ligament has the same mode of attachment between the extremities of the bones as in the metacarpo-phalangeal joint, but it is not so strong; and the extensor tendon takes the place of a posterior band as in that articulation.

There is a simple synovial membrane present in the joint.

The joint of the second with the last phalanx is like the preceding in the number and disposition of its ligaments; but all the articular bands are much less strongly marked.

Articular Surfaces. The anterior end of each phalanx is marked by a pulley-like surface. The posterior end presents a transversely hollowed fossa, and is provided with a crest which fits into the central depression of the opposed articular surface.

Movements. The two interphalangeal joints can be bent and straightened like a hinge.

Flexion and extension. In flexion, the farther phalan $\mathrm{x}$ moves under the nearer in each joint, and the motion is checked by the lateral ligaments and the extensor tendon: in the joint between the middle and the metacarpal phalanx this movement is most extensive. In extension the farther phalanx comes into a straight line with the nearer one and the motion is stopped by the anterior ligament and the flexor tendons. 
TABLE OF THE CHIEF ARTERIES OF THE UPPER LIMB.

The subclavian is continued in the arm by. .

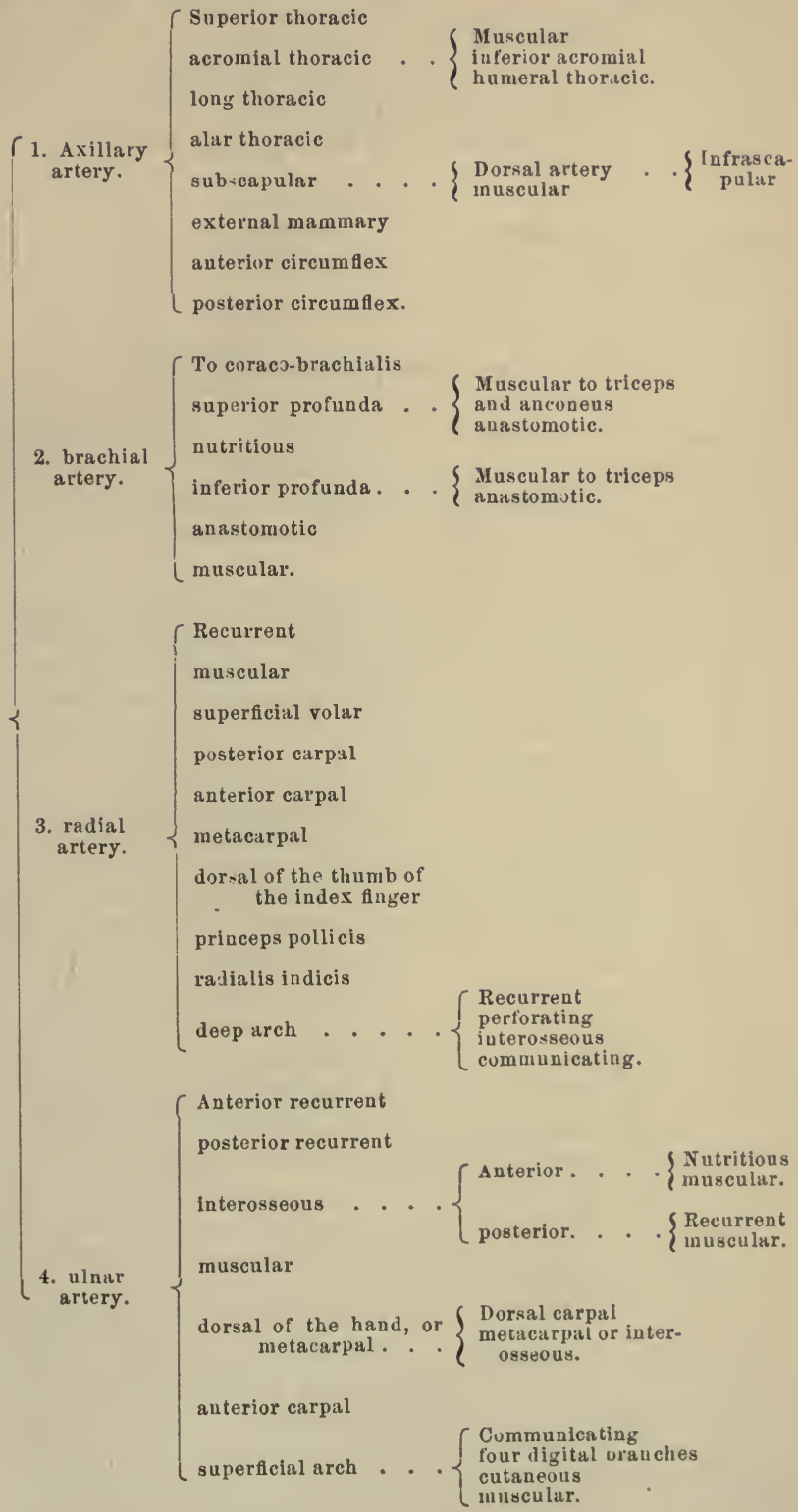


TABLE OF THE SPINAL NERVES OF TIBE UPPER LIMB.

BRACHIAL PLEXUS gives off below the clavicle

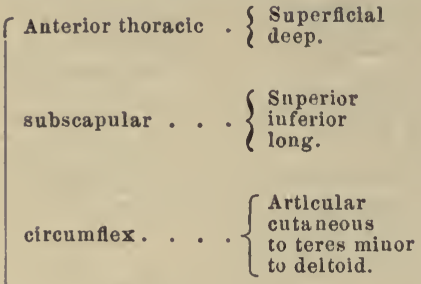




\section{CHAPTER IV.}

\section{DISSECTION OF THE THORAX.}

\section{SECTION I.}

\section{CAVITY OF THE THORAX.}

THE cavity of the thorax is the space included by the spinal column, the sternum and ribs, and by certain muscles in the intervals of the bony framework. In it the organs of respiration, and the heart with its great vessels are lodged : and through it the gullet, and some vessels and nerves are transmitted.

Dissection. When the soft parts covering in front the bony parietes of the thorax have been examined and taken away, the cavity is to be opened by removing a portion of the anterior boundary. To make a sufficient opening in the thorax, the sternum is to be sawn through opposite the interval between the first two ribs, and again between the cartilages of the fifth and sixth ribs. After detaching the lining membrane (pleura) from the inner surface of the chest, the student is to cut through the true ribs, except the first and seventh, ${ }^{1}$ as far back as he can conveniently reach. The loose sternum and the ribs can be removed by dividing the internal mammary vessels, the triangularis sterni, and the intercostal muscles in the first and sixth spaces. The bag of the pleura, and the cavity with its contents will be now ready for examination.

The sternum and the cartilages of the ribs will be required hereafter for the dissection of the ligaments.

Form. The included cavity is irregularly conical, with the apex abore and the base down ; and it appears, from the collapsed state of the lungs, to be only partly filled by the contained viscera, but during life the whole of the now vacant space is occupied by the expanded lungs. On a horizontal section its shape would appear somewhat cordiform ; for the cavity is flattened on the sides, is diminished in the middle line by the prominent spinal column, and is projected backwards on each side of the spine.

Boundaries. On the sides are the ribs with their intercostal muscles; whilst in front is the sternum; and behind is the spine.

The base is constructed at the circumference by the last dorsal vertebra behind, by the end of the sternum before, and by the ribs on the side; whilst the space included by the bones is closed by the diaphragm.

The base is wider transversely than from front to back, and is convex towards the chest; though at certain spots it projects more than at others.

1 The student must be mindful to leave those ribs uncut; the division of them will not be advantageous to him, and will injure the dissection of the neck and abdomen. 
Thus in the centre it is lower than at the sides, and is on a level with the base of the xiphoid cartilage. On the right side it rises to a level with the upper border of the fifth rib near the sternum; and on the left to the corresponding part of the upper border of the sixth rib. ${ }^{1}$ From the lateral projections, the diaphragm slopes suddenly towards its attachment to the ribs, but more behind than before, so as to leave a narrow interval between it and the wall of the chest. The level of this attached part will be marked by an oblique line over the side of the chest from the base of the xiphoid cartilage to the tenth rib; but it differs slightly on the two sides, being rather lower on the left (fig. 97).

The apex of the space is continued higher than the osseous boundary, and reaches into the root of the neck. Its highest point is not in the middle line, for there the windpipe, bloodvessels, \&c., lie; but it is prolonged on each side for an inch or an inch and a half above the first rib, so that the apex may be said to be bifid. Each point projects between the scaleni muscles, and under the subclavian bloodvessels; and in the interval betweeu them lie the several parts passing between the neck and the thorax.

Dimensions. The extent of the thoracic cavity does not correspond with the apparent size externally; for the space included by the ribs below is occupied by the abdominal viscera, and the cavity reaches above into the neck.

In consequence of the arched condition of the diaphragm, the depth of the space varies greatly at different points. At the centre, where the depth is least, it measures about seven inches, but at the back as much again; and the other vertical measurements can be estimated by means of the data given of the level of the base on the wall of the thorax.

Alterations in capacity. The size of the thoracic cavity is constantly varying during life with the condition of the ribs and diaphragm in breathing.

The horizontal measurements are increased in inspiration, when the ribs are raised and separated from one another; and are diminished in expiration as the ribs approach and the sternum sinks.

An alteration in depth is due to the condition of the diaphragm in respiration; for the muscle descends when air is taken into the lungs, increasing thus the cavity; and ascends when the air is expelled from those organs, so as to restore the previous size of the space, or to diminish it in violent efforts. But the movement of the diaphragm is not equal throughout, and some parts of the cavity will be increased more than others. For instance, the central tendinous piece, which is joined to the heart-case, moves but slightly; but the lateral, bulging, fleshy valves descend freely, and add greatly to the size of the lateral part of the chest by their separation from the thoracic parietes.

The thoracic cavity may be diminished by the diaphragm being pushed upwards by enlargement, either temporary or permanent, of the viscera in the upper part of the abdomen; or by the existence of fluid in the latter cavity.

1 This is the height in the dead body. The level to which it may reach in great respiratory efforts during life will be stated with the account of the Diaphragm. 


\section{TIIE PLEURA.}

The pleura are two serous membranes, or closed sacs, which are reflected around the lungs in the cavity of the thorax. One occupies the right, and the other the left half of the cavity; they approach each other along the middle line of the body, forming a thoracic partition or mediastinum.

Each pleura is conical in shape; its apex projects into the neck above the first rib (fig. 97), and its base is in contact with the diaphragm. The outer surface is rough, and is connected to the lung and the wall of the thorax by areolar tissue, but the inner surface is smooth and secerning. Surrounding the lung, and lining the interior of one-half of the chest, the serous membrane consists of a parietal part-pleura costalis, and of a visceral part-pleura pulmonalis.

There are some differences in the shape and extent of the two pleural bags. On the right side the bag is wider and shorter than on the left; and on the latter it is narrowed by the projection of the leart to that side.

The continuity of the bag of the pleura over the lung and the wall may be traced circularly from a given point to the same, in the following manner:-Supposing the membrane to be followed outwards from the sternum, it may be traced on the wall of the chest as far as the spinal column; here it is directed forwards to the root of the lung, and is reflected over the viscus, covering its surface, and connecting together its different lobules. From the front of the root the pleura may be followed over the side of the pericardium to the sternum. Below the root the pleura gives rise to a thin fold, the ligamentum latum pulmonis, which intervenes between the inner surface of the lung and the side of the pericardium.

If the serous sac be traced above the root of the lung, it describes a circle without deflection over a viscus.

The mediastinum. The median thoracic partition, or the mediastinum, is formed by the approximation of the pleural bags along the middle line, and is constructed of two layers - one being derived from each sac. About midway between the sternum and the spine the contiguous strata of the mediastinum are widely separated by the leart; but in front of, and behind the heart they approach near each other. 'To the parts before and behind that viscus the terms "anterior and posterior mediastina" are sometimes applied.

The part in front of the heart (anterior mediastinum) extends from the back of the sternum to the pericardium. Behind the second piece of the sternum the pleural bags touch each other, but above and below that spot they are separated by an interval ; so that the space between them (interpleural) is narrowed at the centre, and is inclined below to the left of the middle line. In the upper part of the space are the remains of the thymus gland, and the origin of some of the hyoid and thyroid muscles; and in the lower part is some areolar tissue, together with the triangularis sterni muscle of the left side.

'The part behind the heart (posterior mediastinum) intervenes between the back of the pericardium with the roots of the lungs, and the spinal column. Its lateral boundaries are the opposite pleural sacs, which are separated here by a larger interpleural interval than in front of the heart. If the pleura be divided behind the lung on the right side, the extent of the space and its contents will appear : in it are contained the different bodies on the front of the spine, viz., the aorta, the vena azygos, the thoracic 
duct, the œsophagus with its nerves, the trachea, the splanchnic nerves at the lower part, and some lymphatic glands.

Dissection. The pleura and the fat are now to be cleaned from the side of the pericardium.

The root of the lung is to be dissected out by taking away the pleura and the areolar tissue from the front and back, without injuring its several component vessels. In this dissection the phrenic artery and nerve will be found in front of the root, together with a few nerves (anterior pulmonary); the last are best seen on the left side. Behind the root of the lung is the vagus nerve, dividing into branches; and arching above it is the large azygos vein.

For the present, the arch of the aorta and the small nerves on it may be left untouched.

The thymus body is a fotal organ, like the thyroid body, whose use is unknown. It occupies chiefly the upper part of the thorax; and it may be best examined in a full-grown foetus.

At birth it is about two inches in length, and is of a grayish color; it possesses two lobes of a conical form, which touch each other. Its upper end is pointed and extends on the trachea as high as the thyroid body; and the lower wider part, reaches as far as the fourth rib. In the thorax it rests on the aortic arch and large vessels, on the left innominate vein, and on the pericardium.

In the adult all that remains of the thymus is a brownish rather firm material in the interpleural space beneath the upper part of the sternum.

In its perfect state it resembles much the thyroid body; and a whitish fluid, containing lymph-like corpuscles, escapes from it when it is cut.

\section{CONNECTIONS OF THE LUNGS.}

The lungs are two in number, and are contained in the cavity of the thorax, one on each side of the spinal column. In these organs the blood is changed in respiration.

The lung is of a somewhat conical form, and takes its shape from the space in which it is lodged. It is unattached, except at the inner part where the vessels enter; and it is covered by the bag of the pleura. Its base and apex, borders and surfaces can be distinguished by differences in form; it is divided also into lobes by fissures; and it has a root formed out of its vessels and nerves.

The lase of the lung is hollow in the centre and thin at the circumference, and fits on the convexity of the diaphragm. Following the shape of that muscle, it is sloped obliquely firom before backwards, and reaches in consequence much lower posteriorly than anteriorly. Its position with respect to the wall of the thorax may be ascertained externally by taking the level of the diaphragm as a guide ( $p .306$ ); and it will be a rib's breadth lower in front on the left, than on the right side (fig. 97). The apex is rounded, and projects an inch to an inch and a half above the first rib, where it lies beneath the clavicle, the anterior scalenus muscle, and the subclavian artery.

The anterior edge or border is thin, and overlays in part the pericardium. On the right side it lies along the middle of the sternum as low as the sixth costal cartilage (fig. 97). On the left side it reaches the midline of the chest as low as the fourth costal cartilage; but below that spot it presents a V-shaped notch (fig. 97), whose apex is opposite the outer 
part of the cartilage of the fifth rib. Two fissures are seen in the border of the right lung, but only one in that of the left. The posterior border is as long again as the anterior, and projects inferiorly between the lower ribs and the diaphragm; it is thick and vertical, and is received into the hollow by the side of the spinal column.

The outer surface of the lung is convex, and is in contact with the wall of the thorax : a large cleft divides it into two pieces (lobes of the lung), and on the right side there is second smaller fissure. 'The inner surface is flat when compared with the outer; altogether in front is the hollow corresponding with the heart and its large vessels, which is greatest on the left lung; and behind this, but nearer the posterior than the anterior border, is a fissure about three inches long, hilum pulmonis, which receives the ressels forming the root of the lung.

Fig. 97.

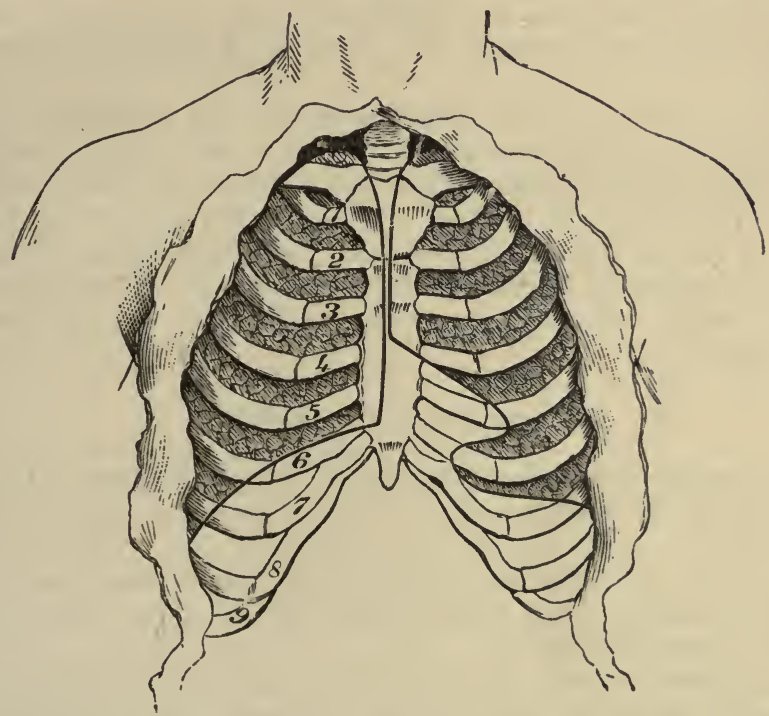

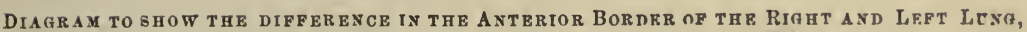
the edge being indicated by the dark line; and to mark the different level of the base on the two sides.

Each lung is divided incompletely into two parts or lobes by an oblique fissure, which hegins near the apex, and ends in the anterior border near the base: from the form of the lung and the direction of the fissure the lower lobe is necessarily the largest. In the right lung a second horizontal fissure is directed forwards from the middle of the oblique one to the anterior border, and cuts off a small triangular piece from the upper lobe : this is the third lobe of the lung. Oceasionally there may be a trace of the third lobe in the left lung.

Besides the difference in the number of the lobes, the right lung is larger and heavier, and is wider and more hollowed out at the base than the left; it is also shorter by an inch. The increased length and the narrowness of the left lung are due to the absence of a large projecting body like the liver under it, and to the direction of the heart to the left side. 
The root of the lung consists of the vessels entering the fissure on the inner surface; and as these are bound together by the pleura and some areolar tissue they form a foot-stalk, which fixes the lung to the heart and the windpipe. The root is situate at the inner surface, about midway between the base and apex, and about a third of the breadth from the posterior border of the lung.

In front of the root on both sides, are the phrenic and the anterior pulmonary nerves, the former being at some little distance from it; and anterior to the right root is the descending eava. Behind on both sides, is the posterior pulmonic plexus; and on the left side there is, in addition, the descending aorta. Above on the right side is the vena azygos; and on the left side, the arch of the aorta. Below each root is the fold of pleura called ligamentum latum pulmonis.

In the root of the lung are collected a branch of the pulmonary artery, two pulmonary veins, and a division of the air tube (bronchus); small nutritive bronchial arteries-and veins, and some nerves and lymphaties. The large vessels and the air tube have the following position to one another:-

On both sides the bronchus is most posterior, the pulmonary veins most anterior, and the pulmonary artery between the other two. In the direction from above down the position on the right side is, bronchus, pulmonary artery, and pulmonary veins; but on the left side the bronchus and artery have changed places, consequently the relative position will there be artery, bronchus, and veins. This difference in the two sides may be accounted for by the left branch of the air tube being at a lower level than the right.

\section{THE PERICARDIUM.}

The bag containing the heart is named the pericardium. It is situate in the middle of the thorax, in the interval between the pleuræ of opposite sides.

Dissection. Supposing the surface of the pericardium to be already cleaned, the student should next dissect out the large vessels connected with the heart, and the nerves.

The large artery curving to the left above the heart is the aorta, which furnishes three trunks to the head and the upper limbs, viz., innominate to the right, next left common carotid, and left subclavian. On its left side lies the pulmonary artery.

Above the arch of the aorta a large venous trunk, left innominate, crosses over the three before said arteries, and ends by uniting on the right side with the right innominate rein in the upper cava. Several small veins, which may be mistaken for nerves, ascend over the aorta, and enter this trunk. Define the branches of this vein, and especially one crossing the aortic arch towards the left side, which is the left superior intercostal vein.

The large vein by the side of the aorta which enters the top of the heart is the upper cava: look for the azygos vein opening into it behind.

Seek the following nerves of the left side which cross the arch of the aorta:- The nerve most to the left, and the largest, is the vagus; the next largest in size on the right of the vagus is the phrenic. Between the preceding nerves, and close to the coats of the artery, are the two following, the left superficial cardial nerve of the sympathetic, and the 
cardiac branch of the left vagus; of the two, the last is the smaller, and on the right of the other.

The cardiac nerves from the left vagus and sympathetic are to be pursued onwards to a small plexus (superficial cardiac) in the concavity of the aorta. An off'set of the plexus is to be traced downwards between the pulmonary artery and the aorta towards the anterior coronary artery of the heart ; and another prolongation is to be found coming forwards from the deep cardiac to the superficial plexus : this dissection is difficult, and will require care.

When the pericardium is afterwards opened the nerves will be followed on the heart. Oftentimes these small nerves are destroyed in injecting the body.

The pericardium is larger than the viscus it contains. Somewhat conical in form, the wider part of the bag is turned towards the diaphragm, and the narrower part upwards to the large vessels of the heart.

Occupying the interpleural space, it is situate behind the sternum, and projects below on each side of that bone, but much more towards the left than the right side. Laterally the pericardium is covered by the pleura, and the phrenic nerve and vessels lie in contact with it. Its anterior and posterior surfaces correspond with the objects in the interpleural space; and on the anterior aspect the bag is partly covered by the margin of the lungs, especially the left.

The heart-case consists of a fibrous structure, which is lined internally by a serous membrane.

The fibrous part surrounds the heart entirely, and is pierced by the different vessels of that organ : it gives prolongations around the vessels, and the strongest of these sheaths is on the aorta. Inferiorly it is united by fibres to the central tendon of the diaphragm.

This membrane is thickest at the upper part, and is formed of fibres crossing in different directions, many being longitudinal. When the pericardium has been cut open, the serous lining will be discernible.

The serous sac lines the interior of the fibrous perieardium, and is reflected over the surface of the heart. Like other serous membranes, the arachnoid for example, it has a parietal and a visceral part. After lining the interior of the fibrons case, to which it gives the shining appearance, the membrane is conducted to the surface of the heart by the different vessels. As it is reflected on the aorta and the pulmonary artery it contains those vessels in one tube, not passing between their contiguous surfaces; and at the posterior part of the pericardium it forms a pouch between the pulmonary veins of opposite sides.

In front of the root of the left lung the serous layer forms a vertical band, the restigial fold of the pericardium (Marshal), which includes the remains of the left innominate vein of the foetus. On separating the pulmonary artery and bronchus, the band will be better seen.

The vessels of the pericardium are derived from the arta, the internal mammary, the bronchial, the oesophageal, and the phrenic arteries.

Nerves. According to Luschka the pericardium receives nerves from the phrenic, sympathetic, and right vagus. 
THE HEART AND ITS LARGE VESSELS.

The heart is a hollow muscular viscus, and is the agent in the propulsion of the blood through the body. Into it, as the centre of the vascular system, veins enter; and from it the arteries issue.

Form. When the heart is distended, its form is conical, but it is rather flattened from before backwards. Its surfaces and borders have the following differences; the anterior surface is slightly convex, whilst the posterior is nearly flat : the left border is thick and round, but the right is thin, sharp, and less firm.

Size. The size varies greatly, and in general the heart of the woman is smaller than that of the man. Its average weight is from ten to twelve ounces in the male, and from eight to ten in the female. The measurements may be said to be about four inches and three-quarters in length, three inches and a half in width, and two inches and a half in thickness.

Position and Direction. The heart lies beneath the lower two-thirds of the sternum, and projects on each side, but more on the left than the right. Its axis is not parallel to, but is inclined obliquely across that of the body; and its position is almost horizontal with the base directed backwards and to the right, and the apex forwards and to the left side. The left margin of the viseus is undermost, whilst the right is foremost.

In consequence of the direction of the heart in the thorax, only some parts can be near, or in contact with the parietes : thus the right lalf and the apex will correspond with the thoracic wall, though mostly with lung intervening, whilst the base is directed away from the sternum and the costal cartilages: and the left half will be undermost and deep in the eavity.

Limits (fig. 98.) The limits of the whole heart are the following: the base is opposite the spinal column, and corresponds with four dorsal vertebra (5th to 8 th). The apex strikes the wall of the thorax during life just below the fifth rib, near the junction with the cartilage.

The upper limit would be shown by a line across the sternum on a level with the upper edge of the third costal cartilage. And the lower limit would be marked by a line over the junction of the sternum with the xiphoid cartilage, drawn from the articulations of the sixth and seventh cartilages of the right side to the spot where the apex touches.

Its lateral limits are the following. On the right it projects from one to one inch and a half beyond the middle line of the sternum, and its increase in this direction is constantly varying with the degree of distension of the right half of the heart. On the left side the apex projects three inches to three inches and a half fiom the centre of the breast bone.

Component parts. The heart is a double organ, and is made up of two similar lialves. In each half are two hollow portions, an auricle and a ventricle; these on the same side communicate, and are provided with vessels for the entrance and exit of the blood. On the surface are grooves indicatory of this composition. Thus, passing circularly round the heart, nearer the base than the apex, is a groove which cuts off the thin auricular from the fleshy ventricular part. A longitudinal sulcus on each surfice marks the situation of a median partition between the ventricles : this sulcus does not occupy the mid space either on the anterior or the posterior aspect, but it is nearer the left border of the heart in front, and the right border behind; so that most of the anterior suriace is formed by the right, and the greater part of the posterior surface by the left ventricle. 
The auricles are two (right and left) and receive their appellation from the resemblance of the appendices to the dog's ears: they are placed so deeply at the base of the heart, that only the tip of the right one comes forwards to the sternum. The auricles are much thinner than the ventricles.

Fig. 98 .

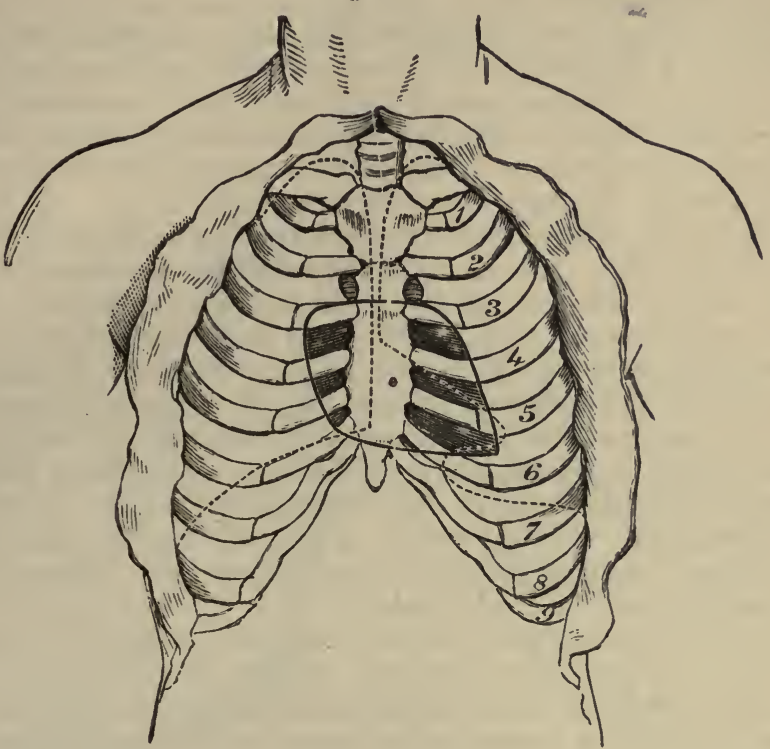

Diagray showixe the Position of the Heart to the Ribs and Sterxem, the soft parts being removed from the exterior of the thorax. The edge of each lung is shown by a dotted liue.

The ventricles reach unequal distances on the two aspects of the heart: thus the right one forms the thin right border and the greater part of the anterior surface; but the left enters alone into the apex, and constructs the left border, and most of the posterior surface of the heart.

Dissection. Before opening the heart the coronary arteries are to be dissected on the surface, with the small nerves and veins that accompany them. The two arteries appear on the sides of the pulmonary artery, and occupy the gronves on the surface of the heart, where they are surrounded by fat : one branches over the right, and the other over the left side. With the anterior artery is a plexus of nerves, which is to be followed upwards to the superficial cardiac plexus; and with the remaining artery another plexus is to be sought.

In the groove at the back of the heart between the auricles and rentricles, the student will find the large coronary vein, and the dilated coronary sinus in which it ends on the right : the last should be defined and followed to its ending in the right auricle.

The coronary arteries are two small vessels which are so named from their course around the heart : they are the first branches of the aorta. One is distributed mostly on the right, and the other on the left side of the heart.

The right coronary branch appears on the right side of the pulmonary 
artery, and is directed onwards in the depression between the right auricle and ventricle to end in the left half of the heart on the posterior aspect. In its course branches are distributed upwards and downwards to the right half of the viscus. Two of these are of larger size than the rest: one runs on the anterior aspect of the right ventricle towards the free margin; the other descends on the back of the heart towards the apex, along the septum between the ventricles.

The left coronary branch is inclined behind the pulmonary artery to the left side of that vessel, and in the groove between the left auricle and ventricle to the back of the same side of the heart. Like the preceding artery, it furnishes offsets to the substance of the auricle and ventricle of its side: the largest of these descends in the anterior sulcus over the septum ventriculorum towards the apex.

The veins of the substance of the heart (cardiac) are not the same in number, nor have they the same arrangement as the arteries. There may be said to be three sets, but for the most part they are collected into one large trunk, the coronary sinus, which opens into the right auricle.

The coronary sinus (fig. $99,{ }^{1}$ ) will be seen on raising the heart to be placed in the sulcus between the left auricle and ventricle. About an inch usually in extent, it is joined at the one end by the great coronary vein $\left({ }^{4}\right)$; and at the other it opens into the right auricle. It is crossed by the muscular fibres of the left auricle. Inferiorly and at its right end it receives some branches from the back of the ventricles $\left({ }^{3}\right.$ and $\left.\dagger\right)$; and nearly at its left extremity another vein $\left({ }^{2}\right)$, - the oblique vein (Marshall), which ascends along the back of the left auricle.

On slitting the sinus with a scissors the openings of its different veins will be seen to be guarded with valves, with the exception of the oblique vein; and at its right end is the large Thebesian valve of the right auricle.

The great cardiac or coronary vein begins in front near the apex of the heart, in the substance of the ventricles. The vessel turns to the posterior surface in the sulcus between the left auricle and ventricle, and opens into the coronary sinus (fig. $99,^{4}$ ). It receives collateral branches in its course, and its ending in the sinus is marked by two valves.

Anterior and posterior cardiac veins. Some small veins on the anterior part of the right ventricle open separately, by one or more trunks, into the lower part of the right auricle. Similar small veins exist over the back of the ventricles; and one, larger than the rest, lies orer the septum ventriculorum : they enter the coronary sinus by separate valved openings.

Smallest cardiac. A third set of veins (venx minima) lie in the substance of the heart, and are noticed in the description of the right auricle.

Cardiac nerves. The nerres for the supply of the heart are derived from a large plexus (cardiac) around the roots of the aorta, and pulmonary artery. Part of this plexus is superficial to the pulmonary artery, and part beneath ; and an offset is sent from each with a coronary artery. Only the superficial part of the plexus can now be seen.

The superficial cardiac plexus is placed below the arch of the aorta, and by the side of the ductus arteriosus. The nerves joining it are the left superficial cardiac of the sympathetic, the lower cardiac of the left vagus (p. 331), and a considerable bundle from the deep cardiac plexus. A small ganglion is sometimes seen in the plexus. Inferiorly it sends off nerves on the right coronary artery to the heart. A few filaments pass on the left division of the pulmonary artery to the left lung. 
The right coronary nerves pass from the plexus to the right coronary artery, and receive near the heart a communicating offset from the deep cardiac plexus.

The left coronary nerves are derived, as will be subsequently seen from the deep cardiac plexus, and accompany the left coronary artery to the heart.

At first the nerves surround the arteries, but they soon leave the vessels, and becoming smaller by subdivision, are lost in the muscular substance of

Fig. 99.

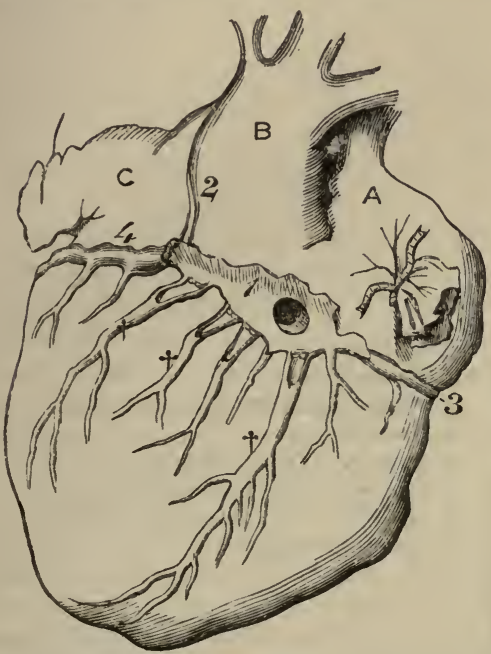

A. Right auricle.

B. Left auricle, with the auricula, c.

1. Coronary sinus.

2. Oblique vein.

3. Vein from the right side of the heart.

4. Left or great cardiac vein.

t† Veins joining the sinus from the back of the ventricles.

Back of the Heart with the Coronary Sinus and its Veins. (Marghall.)

the ventricles. On and in the substance of the heart the nerves are marked by small ganglia.

The Cavities of the heart may be examined in the order in which the current of the blood passes through them, viz., right auricle and ventricle, and left auricle and ventricle.

Dissection. In the examination of its cavities the heart is not to be removed from the body. To open the right auricle, an incision may be made in it near the right or free border, and from the superior cava nearly to the inferior cava; from the centre of that incision the knife is to be earried across the anterior wall to the auricula. By means of those euts an opening will be made of sufficient size; and on removing the coagulated blood, and raising the flaps with hooks or pieces of string, the cavity may be examined.

The CAVity of the RIght AURICLE (fig. 100) is of an irregular form, ${ }^{1}$ though when seen from the right side, with the flaps held up, it has somewhat the appearance of a cone, with the base to the right and the apex below and to the left.

The base or wider part of the cavity is turned towards the right side,

1 The term cavity of the auricle has been sometimes applied to the appendix, and the term sinus venosus to the rest of the space here named auricle. 
and its extremities are the openings of the superior and inferior cavæ. Between those vessels the cavity projects a little, and presents a slight elevation in some bodies (tubercle of Lower). The apex is prolonged downwards towards the junction of the auricle with the ventricle, and in it is the opening into the right ventricular eavity.

The anterior wall is thin and loose. Near its upper part is an opening leading into the pouch of the appendix or auricula (l), which will adnit the tip of the little finger. Around and in the interior of the appendix, are fleshy bands, named musculi pectinati, which run mostly in a transverse direction, and forn a network that contrasts with the general smoothness of the auricle.

Fig. 100.

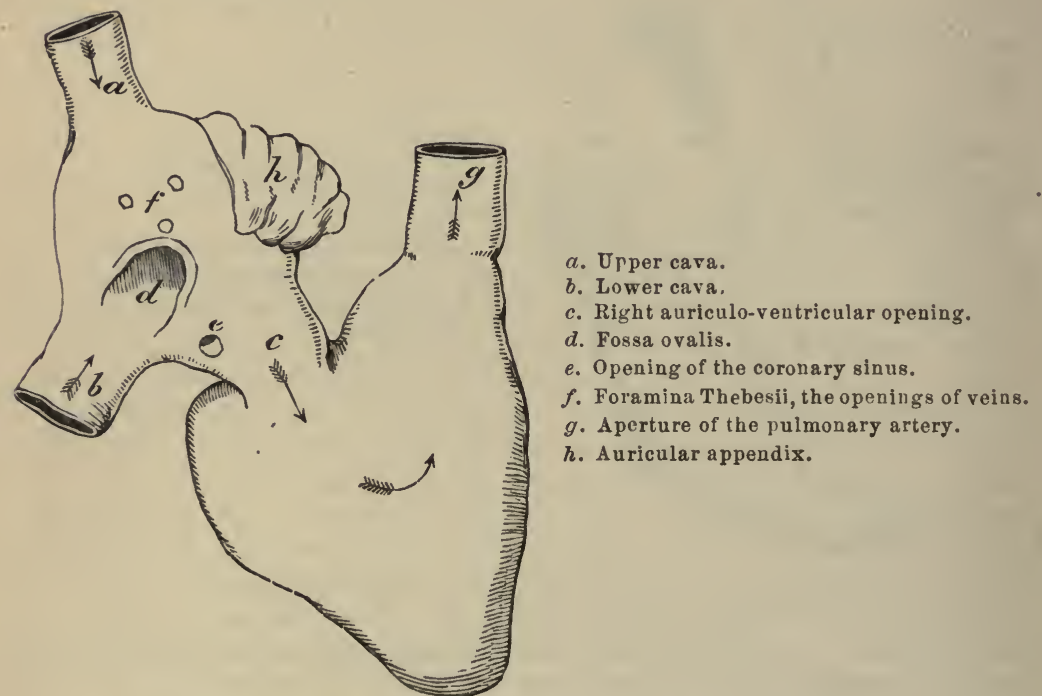

DiAgRAM OF THE TWO CAVITIES OF THE RIGHT SIDE OF THB HEART.

The posterior wall corresponds for the most part with the septum between the auricles, in consequence of the position of the heart. On it, close to the inferior cava, is a large oval depression, the fossa ovalis $(d)$, which is the remains of an opening between the auricles in the fotus. Inferiorly it merges into the lower eava. A thin semi-transparent structure forms the bottom of the fossa; and there is oftentimes a small oblique aperture at its upper part. Around the upper three-fourths of that hollow is an elevated band of muscular fibre, called annulus seu isthmus Vieussenii, which is most prominent above and on the left side, and gradually subsides inferiorly.

Altogether at the lower part of the posterior wall is the aperture of the coronary sinus $(e)$. Other small apertures are scattered over this surface : some lead only into depressions; but others are the mouths of veins of the substance of the heart (venæ cordis minimx), and are named foramina 'Thebesii $(f)$.

The clief apertures in the auricle are those of the two cava, coronary sinus, and ventricle. The opening of the superior eava $(a)$, is in the front 
and top of the auricle, and its direction is forwards. The inferior cava ( $b$ ) enters the back part of the cavity near the septum, and is directed backwards to the fossa ovalis $(d)$. The auriculo-ventricular opening $(e)$ is the largest of all, and is situate at the lowest part of the cavity. Between this and the septum is placed the opening of the coronary sinus $(e)$ which is about as large as a turkev-quill.

All the large vessels, except the superior cava, have some kind of valve. In front of the inferior cava is a thin fold of the lining membrane of the cavity, the Eustachian valve, which is only a remnant of a much larger structure in the foetus. This valve in its perfect state is semilunar in form, with its convex margin attached to the anterior wall of the vein, and the other free in the cavity of the auricle. It is wider than the vein opening, and its surfaces are directed forward and backwards : its free margin is often reticular. The aperture of the coronary sinus is closed by a thin fold of the lining membrane-valve of Thebesius. The auriculo-ventricular opening will be seen, in examining the right ventricle, to be provided with valves, which prevent blood running back into the auricular cavity.

In the adult there is but one current of blood in the right auricle towards the ventricle. But in the fotus there are two streams in the cavity: one of pure, and the other of impure blood, which cross one another in early life, but become more commingled as birth approaches. The placental or pure blood entering by the inferior cava, is directed by the Eustachian valve chiefly into the left auricle, through the foramen ovale in the septum ; whilst the current of systemic or impure blood, coming in by the superior cava, flows downwards in front of the other to the right ventricle.

Dissection. To see the cavity of the right ventricle, the student should pierce it with the scalpel below the opening from the auricle, and cut out inferiorly near the apex of the heart without injuring the septum ventriculorum. A flap is thus formed, like the letter $\mathrm{V}$, of the anterior part of the ventricle. In the examination of the eavity of the right ventricle, both the flap and the apex of the heart should be raised with hooks or string, so that the space may be looked into from below.

The Cavity of the Right ventricle (fig. 100) is triangular in form, and has the base turned upwards to the auricle of the same side. On a cross section the cavity would appear semilunar in shape, with the septum between the ventricles convex towards the cavity.

'The apex of the cavity reaches the right border of the heart at a short distance from the tip. The base of the ventricle is sloped, and is perforated by two apertures; one of these, on the right, leading into the auricle, is the right auriculo-ventricular opening $(c)$; the other on the left, and much higher, is the mouth of the pulmonary artery $(g)$. The part of the cavity communicating with the pulmonary artery is funnel-shaped, and is named infundibulum or conus arteriosus.

The anterior wall, or the loose part of the ventricle, is comparatively thin, and forms most of the anterior surface of the ventricular portion of the heart. The posterior wall corresponds with the septum between the ventricles, and is much thicker.

Over the greater part of the cavity the surface is irregular, and is marked by projecting fleshy bands of muscular fibres, the columna carnere; but near the aperture of the pulmonary artery the wall becomes smooth. The fleshy columns are of various sizes, and of three different kinds. Some form merely a prominence in the ventricle, as on the septum. Others are attached at each end, but free in the middle (trabeculæ carnex). And a 
third set, which are fewer in number and much the largest, project into the cavity, and form rounded bundles, named musculi papillares; these give attachment by their free ends to the little tendinous cords of the valve of the auriculo-ventricular opening.

The auriculo-ventricular orifice $(c)$ is situate in the base of the ventricle, and is opposite the centre of the sternum, between the third costal cartilages. It is slightly larger than the corresponding aperture of the left side of the heart. It is oval from side to side, its shape being maintained by a strong fibrous band around it ; and it measures one inch and a quarter in diameter.

Prolonged from the circumference of the opening is a thin membranons valve, which projects into the cavity of the ventricle. Near its attachment to the heart the valve is undivided, but it presents three chief points at its lower margin, and is named tricuspid; to the lower margin are attached small tendinous cords (chordæ tendinex), which unite it to the muscular bundles of the ventricle. Its three slips or tongues are thus placed:-one touches the front of the rentricle; another is in contact with the posterior wall ; and the remaining slip, the largest and most movable, is interposed between the aperture into the auricle and pulmonary artery.

The tricuspid valve is constructed by the lining membrane of the heart, which incloses fibrous tissue. The central part of each tongue is strong, whilst the edges are thin and notched; and between the prinary pieces there are sometimes secondary points (Kürschner).

The chorda tendina which keep the valve in position ascend from the musculi papillares into the intervals between the pieces of the valve, and are connected with both. ${ }^{1}$ They end on the surface of the valve turned away from the opening; - some reaching the attached upper margin; others entering the central thickened part, and the thin edge and point of the tongue.

As the blood enters the cavity, the valve is raised so as to close the opening into the auricle; and its protrusion into the other cavity during the contraction of the ventricle is arrested by the small tendinous cords. The valve participates in the production of the first sound of the lieart.

The mouth of the pulmonary artery $(g)$ will be seen when the incision in the anterior wall of the ventricle is prolonged into it. Round in shape, it is situate on the left of the opening into the auricle, and is opposite the inner end of the second intereostal space of the left side. Its diameter is rather less than an inch. Into it the funnel-shaped part of the right ventricle is prolonged, and in its interior are three semilunar or sigmoid valves.

Semilunar valves. Each valve is attached to the side of the vessel by its convex border; and is free by the opposite edge, in which there is a slightly thickened nodule-the corpus Arantii. In the wall of the artery opposite each valve is a slight hollow, the sinus of Valsalva, which is better marked in the aorta.

The valves resemble the tricuspid in structure, for they are formed of fibrous tissue with a covering of the lining membrane. In each valve the fibres have this arrangement: there is one band along the attached margin; a second along the free edge, which is connected with the projecting nodule; and a third set of fibres is directed from the nodule across the

1 The papillary muscles are collected into two principal groups, whose tendons enter the interval on each side of the anterior tongue of the valve. In the interval between the left and posterior segments of the valve the tendinous cords are very small, and are connected with the septum ventriculorum. 
valve, so as to leave a semilunar interval on each side near the free edge, which has been named lunula.

The use of the valves is obvious, viz., to give free passage to fluid in one direetion, and to prevent its return. Whilst the blood is entering the artery the valves are placed against the wall; but when the elasticity of the vessel acts on the contained blood, the valves are thrown towards the centre of the vessel, and arrest the flow of the fluid into the ventricle. They are concerned also in giving rise to the second sound of the heart.

Dissection. To open the cavity of the left auricle the apex of the heart is to be raised, and a cut is to be made across the p sterior surface of the auriele from the right to the left pulmonary veins. Another short incision should be made downwards at right angles to the first. The apex of the heart must necessarily be raised during the examination of the cavity.

The Cavity OF THE LEFT AURICle (fig. $101)$ is smaller than that of the right side. Irregularly conical in shape, the wider part is turned towards the spinal column, and receives the pulmonary reins; and the narrowed part $(l)$ opens inferiorly into the left ventricle.

On the left side towards the upper part, is the aperture of the pouch of the auricula $(\mathrm{m})$, which is narrower than that in the right auricle. In the interior of the pouch, as well as around the entrance, are fleshy fibres (musculi peetinati) which resemble those before seen in the other auricle.

On the part of the wall corresponding with the septum auricularum, is a superficial fossa (fig. 101,o), the remains of the oval aperture through that partition; this is bounded below by a projecting ridge, concave upwards, which is the edge of the structure or valve that closed the opening in the foetus. This impression in the left auricle is above the fossa ovalis in the right cavity, because the aperture between the two in the foctus is an oblique canal through the septum.

The apertures in this auricle are those of the four pulmonary veins, two on each side,

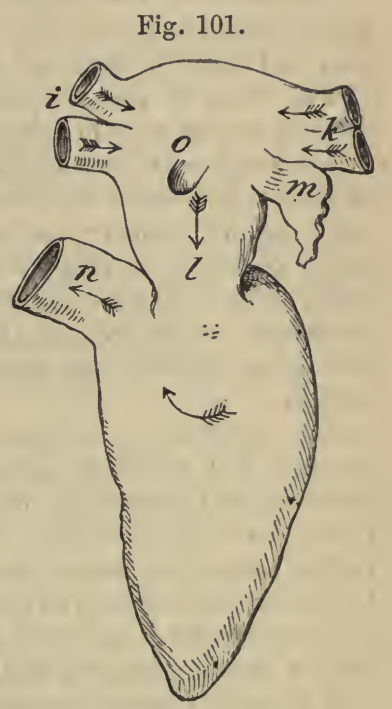

DIAfRAM OF THB TWO CATITIBS OF THE LBFT SIDE OF THB HEART.

$k$. Left pulmonary veins.

i. Right pulmonary veins.

$o$. Remains of the foramen ovale.

l. Left auriculo-ventricular opening.

m. Auricular appendix.

$n$. Aperture of the aorta.

together with the opening of communication with the left ventricle. The months of each pair of pulmonary veins are close to one another; those from the right lung $(i)$ open into the extreme right of the auricle near the septum, and those from the left lung $(h)$ enter the opposite part of the cavity, near the auricula.

The pulmonary veins are not provided with valves. The aperture into the ventricle $(l)$ will be subsequently seen to have a large and complicated valve to guard $i t$, as on the right side.

In the adult the blood enter's this cavity from the lungs by the pulmonary veins, and passes to the left ventricle by the large inferior opening 
between the two. In the foetus the lungs are impervious to the air and the mass of the circulating fluid; and the left auricle receives its pure blood at once from the right auricle through the aperture in the septum (foramen ovale).

Dissection. The left ventricle may be opened by an incision along both the anterior and the posterior surface, near the septum; these are to be joined to the apex, but are not to be extended upwards so as to reach the auricle. On raising the triangular flap the interior of the cavity will be visible.

The cavity of the Left ventricle (fig. 101) is longer, and more conical in shape than that of the opposite ventricle; and is over or almost circular, on a transverse section.

The apex of the cavity reaches the apex of the heart. The base is turned towards the auricle, and is not sloped like that of the right ventricle; in it are the openings into the aorta $(n)$ and the left auricle $(l)$.

'The walls of this ventricle are thickest, and the anterior boundary is formed by the septum ventriculorum.

Its surface is irregular, like that of the right ventriele, in consequence of the projections of the fleshy columns, or earnex columnæ; but near the great artery (aorta) the surface is smoother. There are three sets of fleshy columns ir this as in the right ventricle. But the set (musculi papillares), which project into the eavity, and receive the small tendinous threads of the valve, are the most marked: these are arranged chiefly in two large bundles, and spring from the anterior and posterior walls of the cavity.

The aperture into the left auricle ( $l$ ) (auriculo-ventricular) is placed beneath the orifice of the aorta, but elose to it, only a thin fibrous band intervening between the two. It is rather smaller than the corresponding aperture of the right side, being somewhat more than an inch in dianeter, and is longest in the transverse direction. Placed, as before said, beneath the aortic aperture, it extends also to the right, so as to lie beneath the left extremity of the right auriculo-ventricular opening. It is furnished with a membranous valve (mitral) which projeets into the ventricle.

The mitral valve is stronger and of greater length than the trieuspid, and has also firmer and more tendinous cords: it takes its name from a fancied resemblance to a mitre. Attached to a fibrous ring around the aperture, as well as to the aortic fibrous ring, it is divided below by at notch on each side into two pieces. Its segments lie one before another, with their edges directed to the sides, and their surfaces towards the front and back of the cavity. The anterior tongue of the valve intervenes between the auricular and aortic openings, and is larger and looser than the posterior segment.

The mitral resembles the trieuspid valve in its structure and office. Its segments consist of thicker and thinner parts; and in the notches at the sides there are also secondary pieces between the two primary segments. The tendinous cords ascend to be attached to the valve in the notches between the tongues; and they end on the segments in the same way as in the tricuspid valve. Each of the large papillary muscles acts on both portions of the valve.

When the blood enters the carity, the pieces of the ralve are raised as on the right side, and meet to close the passage into the left auricle. In eombination with the tricuspid it assists in producing the first sound of the heart. 
The opening of the aorta, anterior to that of the auricle, is next the septum of the ventricles. Its aperture is round, and rather smaller than that of the pulmonary artery, and measures about three-quarters of an inch in diameter. It is situate opposite the inner end of the third left intercostal space.

In its interior are three semilunar or sigmoid valves, which are larger and stronger than the corresponding parts in the pulmonary artery, but have a like structure and attachment. The projection in the centre of each valve, viz., the corpus Arantii, is better marked. Opposite each valve the coat of the aorta is bulged as in the pulmonary artery, though in a greater degree, and presents a little hollow on the inner side, named sinus of Valsalva. The apertures of the coronary arteries are placed behind two of the valves.

Like the valves in the pulmonary artery these meet in the middle line to stop the blood passing back into the ventricle, and combine with them in causing the second sound of the heart.

Position of the ventricular apertures. Two openings hare been seen in each ventricle-one of the auricle of its own side of the heart, and one of an artery.

The apertures of the arteries (aorta and pulmonary) are nearest the septum; and as the two vessels were originally formed from one tube, they are close together, but the pulmonary artery is the more anterior of the two. The aperture of communication with each auricle is nearer the circumference of the heart, and is posterior to the artary issuing from the fore part of the ventricle.

The position of the openings to one another from before backwards has been before referred to, viz., the right is partly before the left auriculoventricular; and the opening of the pulmonary artery is anterior to that of the aorta, and rather higher than it.

Structure. The heart is composed of strata of muscular fibres, and of certain fibrous rings with a fibro-cartilage.

The structure may be studied in the heart of the sheep or ox, in which the fibres have been hardened and the connectire tissue destroyed by boiling, so as to allow of the fibres being separated. 'The description of the structure of the heart may be omitted by the student till a suitable preparation of the fibres can be made.

'The fibrous structure forms rings around the auriculo-ventricular and arterial orifices, and is prolonged into the valves connected with those openings.

The auriculo-ventricular rings give attachment to the framework of fibrous tissue in the tricuspid and mitral valves. They are distinct from the bands encircling the mouths of the arteries, except at the front of the left auriculo-ventricular opening, wherethe anricular and arterial circles blend.

An arterial ring surrounds each large artery (aort \& and pulmonary), fixing those vessels, and giving attachment to some muscular fibres. It is a circular band, with three notches in the margin towards the artery; these are filled by corresponding projections of the artery, and give attachment internally to the sigmoid valves along their semilunar edges.

The artery is connected with the band of fibrous tissue in the following manner:-Its middle coat presents three projections, which are received into the notches of the fibrous ring, being joined thereto by fibrous tissue; and the junction between the two is strengthened externally by the outer arterial coat and the pericardium, and internally by the endocardium. 
Behind the aortic aperture, between it and the auriculo-ventricular orifices, is a piece of fibro-cartilage, with which the fibrous rings are united.

The muscular fibres belong to the involuntary class, though marked with transverse strix, and form concentric layers, which inclose the cardiac cavities. In the auricles the fibres are separate from those in the ventricles.

In the wall of the auricles the fibres are mostly transverse (fig. 102,a), and are best marked at the lower part, though they form there but a thin layer; and some of the fibres dip into the septum between the auricular cavities. Besides this set there are annular fibres around the appendages of the auricles, and the endings of the different veins. Lastly a few oblique fibres ( $c$ and $d$ ) pass upwards over the auricles both in front and behind.

Dissection. The auricles having been learnt, separate them from the ventricles by dividing the fibrous auriculo-ventricular rings. Next clean

Fig. 102.

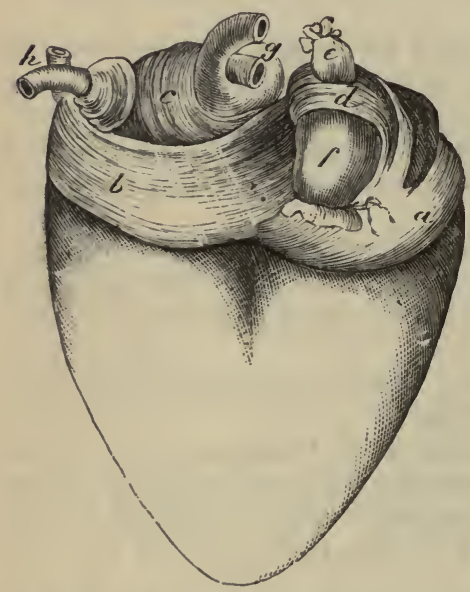

MUSCUI.AR FIBRES OF THE AURICLES.

$f$ Inferior cava, and $e$, superior cava of the right auricle.

$g$. Right, and $h$, left pulmonary veins of the left auricle.

a. Transverse flbres of the right, and $b$, transverse fibres of the left anricle, entering the septum auricularnm.

c. Oblique fibres of the left, and $d$, of the right auricle. Annular fibres surround the auricnlæ and the veins. the fleshy fibres of the ventricles by removing all the fat from the base of the heart around the two arteries (aorta and pulmonary), and from the anterior and posterior surfaces.

Before cutting into the heart let the student note that the anterior surface is to be recognized by the fibres turning in at the septum, with the exception of a small band above about half an inch wide; and that at the posterior aspect the fibres are continued from the left to the right ventricle aross the septum. Separate partly the ventricles in front along the septum by dividing the band near the base, and sinking the knife for about an inch into the groove between them. Disjoin then the aorta and pulmonary artery along the middle line, so as to leave one attached to each ventricle as in fig. 103 .

To show the laminar composition of the left ventricle divide its fibres in front longitudinally near the septum, and transversely about half an inch below the left auriculo-ventricular opening; but the cut is to be very shallow, because seven layers, each about as thick as the thin end of the scalpel, are to be demonstrated. From the lines of incision reflect the different layers downwards to the apex, upwards to the auriculo-ventricular aperture, and backwards into the septum. As the layers are raised the fleshy fibres will be seen to change their direction; and the outer three to be thinner than the three internal.

The LEFT VExTRICLE is a hollow cone, and its wall is formed of layers of fibres, as if a flat muscle liar been rolled up into a conical figure (fig. 103). Seven layers enter into the construction of the wall; and they are 
arranged into three external $(1,2,3)$, three internal $(7,6,5)$, and a central one (4). All are not prolonged equal distances on the rentricle, for the outermost and the innermost reach farthest towards apex and base; and the second external and its corresponding inner layer (sixth) extend farther than the third and the fifth. The fourth is the shortest of all. Consequently the wall is thickest about the middle third where all the layers are present, and gradually becomes thinner upwards and downwards, until there is only the outmost layer at the apex, and the most external and internal (1st and 7 th) at the base. (Dr. Pettigrew, Phil. Trans. 1864.)

Direction of the fibres. Each stratum is formed of fleshy fibres with the undermentioned direction, supposing the ventricle standing on the apex, and the anterior surface towards the dissector.

In the three external strata $(1,2$, $3)$ the fibres are inclined downwards from the base and septum to the apex of the ventricle, and become less vertical in each.

The fourth or mid layer (4) possesses transverse fibres; and it is nearer the outer than the inner surface of the wall.

In the three inner strata the fibres change their direction, as is shown by 5 , and are directed upwards from the apex and septum to the base of the ventricle; so that they cross the fibres of the outer layers like the legs of the letter $\mathbf{X}$, and, becoming more oblique in each layer, are almost vertical in the internal.

Continuity of the fibres. With a piece of muscle rolled into a cone, as before said, the fibres of the different layers would be necessarily continuous at the apex; but in the heart they are united at apex and base. Thus the outermost layer is continuous at the apex and base with the innermost, the fibres being curved in at the tip and out at the base. In like manner the fibres of the second layer are unitel with those of the sixth, and the third stratum with the fifth. From the three outer layers, fibres are continued to the right ventricle at the back of the heart, forming the "common fibres."

Each of the three outer layers consists of two sets of fibres, which occupy the firont and back of the ventricle. By the turning inwards of the two bundles on opposite sides of the apex, the wall is prevented from having a slanting side, like a piece of paper rolled into a cone. And by the turning outwards of two sets of fibres (anterior and posterior) at the base of the ventricle from each inner layer, the sides of the auriculo-ventrieular opening are made level like those of the apex. Many of the fibres of the outer layers are attached to the fibrous ring around the aorta. 
The fibres of the fourth layer are continued into the septum ventriculorum.

Dissection. To display the layers and fibres of the right ventricle, great care will le needed because of the thinness of the wall; but the same number of layers exists in this, as on the other side of the heart.

Make a vertical cut along the anterior aspect from the root of the puimonary artery to the al ex of the ventricle; and reflect the several layers forwards and backwards from that incision. As the three outer are raised let them be traced on the one hand into the part of the septum detached from the left ventricle; and on the other into the left ventricle through the continuity of the common fibres behind.

The Right vextricle possesses seven layers in its wall, like the left, though they are much thinner. They are arranged as in the other ventricle into three external, three internal, and a fourth or intermediate. In like manner the wall decreases from the centre towards the base and apex, but at the tip it is thicker than the apex of the left half of the lieart (Pettigrew).

Directions of the fibres. In this as in the other ventricle, the fibres of the three outer layer's run down from the base to the fore part of the septum and the apex of the heart: the outer being most vertical. In the fourth stratum the fibres have a transverse direction, as in the corresponding layer of the left ventricle. And in the three inner layers they are directed upwards from the apex to the base of the ventricle across the fibres of the three outer strata, the deepest being the most vertical.

Continuity of the fibres. The fibres are not distinct from those of the left ventricle, but are derived in the three outer layers from the "common fibres" and the septum, and from the auriculo-ventricular opening. They are then continued forwards to the front of the septum ventriculorum, where they leave the surface, and bending back construct the right part of the septum : at the back of that partition they blend with the "common fibres" of the left ventricle. 'The fourth layer fibres are continuous for the most part with the "common fibres" crossing the posterior groove. At the apex the three cuter layers do not enter in a whorl as in the left ventricle; but at the base they are continuous with the three inner as on the other side of the heart.

Many of the fibres of the external layer are attached to the ring of the pulmonary artery; and the narrow slip from the right to the left ventricle, near the base in front, receives its fibres from the two outer strata.

In this ventricle the fibres are arranged as if there had been originally one common cavity in the heart-the left ventricle, from which the right bad been detached during the growth by a pushing inwards of a partition from the fore part.

Septum ventriculorum. This partition between the two cavities has been divided anteriorly into right and left parts by the previous dissection; and the layers of the ventricles may be traced into them.

It is rather thicker than the wall of the left ventricle, and is formed by the fibres of both ventricles. About one-third belongs to the right, and two-thirds to the left ventricle. Where the two portions touch the fibres mingle, and altogether behind lie the "common fibres" of the two rentricles.

Endocardium. Lining the interior of the cavities of the heart is a thin membrane, which is named endocardium. It is continuous on the one hand with the lining of the veins, and on the otker with that of the arteries. 
Where the $m$ smbrane passes from an auricle to a ventricle, or from a ventricle to an artery, it forms duplicatures or valves, in which fibrous tissue is inclosed; and in the ventricles it covers the tendinous cords of the valves, and the projecting muscular bundles.

The thickness of the membrane is greater in the auricles than in the ventricles, and in the left than in the right half of the heart. In its structure it resembles a serous membrane.

Great Vessels of the Heart. The arteries which take origin from the heart are the aorta and the pulmonary. The large veins entering the heart, besides the coronary, are the superior anl inferior cava, and the pulmonary.

The Pulmovary ARTERY is a short thick trunk, which conveys the dark or impure blood from the right side of the heart to the lungs. From its commencement in the right ventricle the vessel is directed upwards on the left of the aorta; and at a distance of an inch and a half or two inches, it divides into two branches of nearly equal size for the lungs. The trunk of the pulmonary artery is contained in the pericardium; and beneath it is the beginning of the aorta. On each side are the coronary artery and the auricular appendix.

Fig. 104.

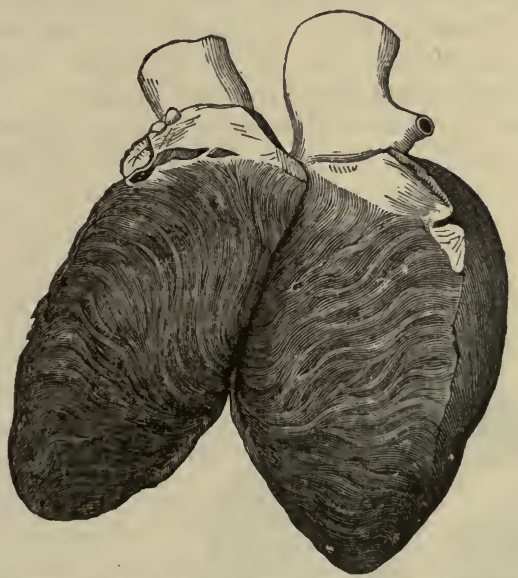

The Formation of the Septum Vextricclorem by the Fibres of both Ventricheg IS REPRESENTED IN THIS CUT.

Near the bifurcation of the artery is a ligamentous cord about as large as a crow-quill, the remnant of the arterial duct, which passes from the left branch of the vessel to the arch of the aorta, and is named ligamentum ductûs arteriosi.

The right branch is longer than the left. In its course to the lung it lies beneath the aorta and the vena cava superior, and rests on the bronchus or piece of the air tube: and as it passes outwards it lies above the level of the right auricle of the heart. At the lung the artery divides into three primary branches, one for each lobe.

The left branch is rather smaller than the right; it is directed in front of the descending aorta and the left bronchus to the fissure of the root of the lung, where it ends in two branches for the two pulmonic lobes. 
As the right and left branches of the pulmonary artery pass outwards, they cross the air-tubes resulting from the division of the trachea, and inclose with them a lozenge-shaped space which contains some bronchial glands.

Ductus arteriosus. The ligamentous structure was the continuation in the foetus of the trunk of the pulmonary artery, and was larger than either branch to the lung. At that period the vessel receives the name arterial eanal or duct (ductus arteriosus, Botalli), and opens into the arta rather beyond the origin of the last great vessel of the head and neck from the arch.

As the lungs do not give passage to the circulating fluid before birth, the impure blood in the pulmonary artery passes throngh the arterial duct into the aorta below the attachment of the vessels of the head and neck, in order that it may be transmitted to the placenta to be purified. But after birth, when the function of the lungs is established, the current of blood is directed along the branches of the pulmonary artery insteal of through the arterial duct; and this tube becoming gradually smaller, is obliterated before the eighth or tenth day, and forms finally the ligament of the arterial duct.

The AORTA is the great systemic vessel which conveys the blood from the heart to the different parts of the body. It arches backwards at first to reach the spinal column, and is continued on the spine through the chest and abdomen. In the thorax the vessel is divided into two parts-arch of the aorta, and the descending or thoracic aorta.

Arch of the aorta (fig. 105). The aorta has its origin in the left ventricle, and curving backwards over the windpipe and the gullet, forms an

Fig. 105.

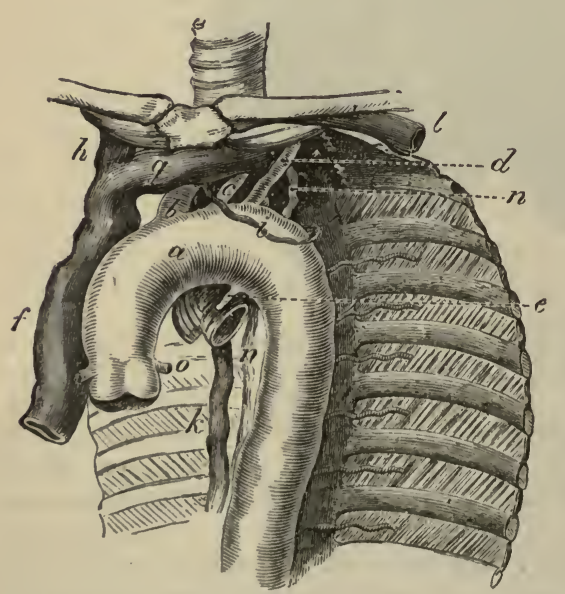

a. Aortic arch.

b. Innominate artery.

c. Left common carotid.

d. Left subclavian.

e. Ligamentum ductus arteriosi.

$f$. Vena cava superior.

g. Left innominate vein.

$h$. Right innominate voin.

i. Left upper intercostal vein.

$k$. Vena azygos major.

l. Left subclavian vein.

$n$. Thoracic duct.

o. Coronary artery.

ARCH OF THE AORTA AND ITS GREAT Vesgels.

arch which ceases on the left side of the spinal column, at the lower border of the fifth dorsal vertebra. The arch has its convexity upwards and to the right, and its concavity to the root of the left lung; and from it the large vessels for the supply of the upper part of the body take their origin. For the purpose of reducing to order the numerous connections of this 
portion of the aorta, it is divided into three parts-ascending, transverse, and descending.

The first or ascending part is about two inches in length, or slightly more, and is directed upwards behind, and close to the sternum : it reaches as high as the upper border of the cartilage of the second rib on the right side, and is contained nearly altogether in the pericardium. At first the pulmonary artery is superficial to it; but, as the vessels take different directions, the aorta is soon uncovered, and remains so to its termination. Behind it are the right branches of the pulmonary vessels. On the right side is the descending cava; and on the left, the pulmonary artery. Near the heart the ressel bulges opposite the semilunar valves (fig. 105). There is sometimes another dilatation along the right side, which is named the great sinus of the aorta.

The second or transverse portion recedes from the sternum and reaches from the second right costal cartilage to the left side of the borly of the fourth dorsal vertebra (the lower border). It rests upon the trachea above the bifurcation, and is placed over the oesophagus and the thoracic duct. Lying in front of this part of the artery are the ragus, phrenic, and superficial cardiac nerves of the left side- the first nerve sending backwards its recurrrent branch beneath the vessel. Along the upper border is the left innominate vein $(g)$, to which the left upper intercostal vein $(i)$ is directed over the left part of the arch ; and to the lower border near the termination, the remnant of the arterial duct $(e)$ is attached. From this part arise the three great vessels of the head and upper limbs.

The third or descending part of the arch is very short, extending from the lower edge of the fourth to that of the fifth dorsal vertebra.' It lies against the fifth vertebra, and the fibro-cartilage between this and the fourth, and is covered by the pleura of the left side of the chest.

In the concavity of the arch of the aorta are contained the root of the left lung, the branching of the pulmonary artery with its arterial duct, and the left recurrent nerve. Deeper than those parts, the oesophagus and the thoracic duct, with some lymphatic glands, may be recognized.

The branches of the arch of the aurta are five in number; two come from the ascending, and three from the transverse part. The first two are the coronary arteries of the heart $(o)$, which have been already noticed (p. 313). The other three are much larger in size, and supply the neck, the head, and the upper limbs. First on the right is the large trunk of the innominate artery $(b)$; close to it is the left carotid $(c)$; and last of all comes the left subelavian $(d)$.

The invominate ARTERY (b) (brachio-cephalic), the first and largest of the three branches, measures from one inch and a half to two inches in length. Ascending to the right beneath the sternum, it divides opposite the sterno-clavicular articulation into the right common carotid and the subclavian artery.

The artery is crossed by the left innominate vein $(g)$, and lies behind the upper piece of the sternum, and the origin of the hyoid and thyroid muscles. At first it rests on the trachea, but as it ascends it is placed on

1 It is usually said that the second part of the aortic arch ends opposite the left side of the body of the second dorsal vertebra, and the third part, opposite the lower edge of the third vertebra. After examination, I have adopted the statement of Mr. Wood respecting the position of the aortic arch to the dorsal vertebræ. (Journal of Anatomy and Physiology for 1868.) 
the right side of the air tube. On its right is the innominate vein of the same side $(h)$. Usually no lateral branch arises from the artery.

Left Common Carotid Artery $(c)$. The common carotid artery of the left side of the neck is longer than the right by the distance between the arch and the top of the sternum.

In the thorax the artery ascends obliquely to the left sterno-clavicular articulation, but not close to the first piece of the sternum and the origin of the depressor muscles of the hyoid bone and larynx. In this course it passes beneath the left innominate vein $(g)$, and the remains of the thymus gland. At first it lies on the trachea, but it erosses afterwards to the left of that tube, so as to be placed over the osophagus and the thoracic duct. To its outer side is the left vagus, with one or more cardiac branches of the sympathetic nerve.

In the neck the connections of the vessels of opposite sides are not the same (p. 120).

The Left SUBCLAvian ARTERY $(d)$ ascends to the neck through the upper aperture of the thorax. Beyond the first rib the vessels of opposite sides are alike (p. 118).

The trunk is directed almost vertically from the arch of the aorta to the inner margin of the first rib. In the thorax the vessel lies deeply, resting at first on the osophagus, and afterwards on the vertebral column and the longus colli muscle. It is invested by the left pleural bag in all its extent. On its inner side is the trachea, and near the upper opening of the thorax the osophagus with the thoracic duct is inside it. Somewhat anterior to the level of the artery, though running in the same direction, are some of the cardiac nerves.

Verns of the Heart (fig. 105). In addition to the cardiac veins (p. $314)$ there are the superior and inferior cava, and the pulmonary veins : the former are the great systemic vessels which return impure blood to the right auricle; and the latter convey pure blood from the lungs into the left auricle.

The superion or Descendixg cava $\left(f^{*}\right)$ results from the union of the right and left innominate veins, and brings to the heart the blood of the head and neck, upper limbs, and thorax.

Its origin is placed on the right side of the sternum, opposite the interval between the cartilages of the first two ribs. From that spot the large vein descends to the pericardium, perforates the fibrous layer of that bag about one inch and a half above the heart, and ends in the right auricle. On its outer surface the vein is covered by the pleura, and the phrenic nerve is in contact with it. To the inner side is the ascending part of the arch of the aorta. Behind the vein is the root of the right lung.

When the cava is about to perforate the pericardium it is joined by the large azygos vein of the thorax $(k)$; and higher up it receives small veins from the perieardium, and the parts in the mediastinal space.

The innominate veins are united inferiorly in the trunk of the descenting cava. They are two in number, right and left; and each is formed near the inner end of the clavicle, by the union of the subclavian and internal jugular veins. The trunks differ in length and direction, and in their connections with the surrounding parts.

The right vein $(h)$ is ahout one inch and a half long, and descends vertically, on the right side of the innominate artery, to its junction with the vein of the opposite side. On the outer surface the pleura covers it, and along it the phrenic nerve is placed. 
The left vein $(g)$ is twice as long as the right, and is direeted obliquely downwards above the level of the arch of the arta. It crosses behind the sternum, and the remains of the thymus gland; and it lies on the three large branches of the aortic arch, as well as on the several nerves de:cending over the arch.

'The branches of the veins are nearly alike on the two sides. Each receives the internal mammary, the inferior thyroid, and the superior intercostal of its own side; and the left vein is joined in addition by some small thymic and pericardiac veins.

Sometimes the innominate veins are not united in the vena cara, but descend. separately to the heart, where each has a distinct opening in the right auricle. When such a condition exists, the right vein takes the course of the upper cava in front of the root of the right lung; but the left vein descends in front of the root of the left lung, and turning to the back of the heart, receives the cardiac veins, before it opens into the right auricle. A cross branch connects the two above the heart. ${ }^{1}$

This occasional condition in the adult is a regular one in a very early period of the growth of the fœtus; and two vessels are also persistent in some mammalia.

Change of the two veins into one. The changes taking place in the veins during the growth of the foetus, to produce the arrangement common in the adult, concern the trunk on the left side. The following is an outline of them. First the cross branch between the two trunks enlarges, and forms the future left innominate vein. Next the left trunk below the cross branch disappears at its middle, and undergoes transformations at each end:-At the upper end it becomes converted into the superior intercostal vein. At the lower part it remains pervious for a short distance as the coronary sinus; and even the small oblique vein opening into the end of that sinus in the adult ( $p .314)$, is a remnant of the trunk of the vein that lay beneath the heart.

In the adult there is a vestige of the occluded vessel in the form of a fold of the serous membrane of the pericardium in front of the root of the left lung; this Mr. Marshall names the vestigial fold of the pericardium (p. 311).2

The INFERIOR or ASCENDING CAVA enters the right auricle as soon as it has pierced the diaphragm. No branches join the vein in the thorax. 'The anatomy of this vein will be given with the vessels of the abdomen.

The pulmonary verss are two on each side. They issue from the fissure of the root of the lung, and end in the left auricle: their position to the other vessels of the root has been noticed at p. 310 .

The right veins are longer than the left, and lie beneath the aorta and the right auricle of the heart. The superior receives its roots from the upper and middle pulmonic lobes, and the inferior vein is formed by branches of the lower lobe.

The left veins cross in front of the descending aorta ; and one springs from each lobe of the lung.

\section{NERVES OF THE THORAX.}

The pneumogastric and the sympathetic nerves supply the viseern of the thorax. Through the cavity courses the phrenic nerve to the diaphragm.

' An example of two large vessels, "double vena cava," opening into the right auricle in the adult, is contained in the Museum of University College.

2 See a paper by Mr. Marshall on the development of the veins of the neck (Philosoph. Transac., 1850). 
Dissection. The phrenic nerve is sufficiently denuded for its examination; but the student should trace the vagus nerves through the thorax.

The vagus is to be followed, on both sides, behind the root of the lung, and its large plexus in that position is to be dissected out : a few filaments of the gangliated cord of the sympathetic coming forwards over the spinal column to the plexus, nust be looked for. In front of the root on the left side, the nerve supplies a few pulmonary filaments. Beyond the root the vagus is to be pursued along the osophagus by raising the lung and removing the pleura.

The PHRENIC NeRve is a branch of the cervical plexus (p. 80). In its course through the thorax it lies along the side of the pericardium, and at a little distance in front of the root of the lung, with a small companion artery. When near the diaphragm it is divided into branches; these perforate the muscle, and are distributed on the under surface. The nerves of opposite sides differ in length, and in their connections above the root of the lung.

The right nerve is deeper at first, and is also shorter and straighter than the left. In entering the chest it crosses behind the subclavian vein, but in front of the internal mammary artery ; and it lies afterwards along the right side of the innominate vein and superior cava till it reaches the root of the lung.

The left nerve crosses the subclavian artery, but has the same position as the right to the mammary vessels when entering the cavity. In the thorax it is directed in front of the arch of the aorta to the root of the lung, and makes a curve lower down around the projecting heart. Before reaching the aorta the nerve is placed external to the left common carotid artery; and crosses the left vagus from without inwards, so as to be internal to that nerve on the arch.

Branches. Some small filaments are said to be furnished from the nerve to the pleura and pericardium.

Internal mammary artery. A small part of this artery, which lies beneath the first rib, and winds round the phrenic nerve and the innominate vein to reach the side of the sternum, is now to be learnt. It gives the following offset :-

The superior plerenic branch (comes nervi phrenici) is a very slender artery, which acompanies the phrenic nerve to the diaphragm, and is distributed to that muscle, anastomosing therein with other branches of the aorta, and with the musculo-phrenic branch of the internal mammary (p. 239).

The PXEUMOgastric Nerve (p. 181) passes through the thorax to the abdomen. In the lower part of the thorax the nerves of opposite sides have a similar position, for they pass behind the root of the lung, each on its own side, and along the nesophagus to the stomach. But above the root of the lung, the right and left nerves differ mueh. Each supplies branches to the viscera, viz., to the heart, the windpipe and lungs, and the gullet.

The right vagus is posterior to the left in position. It enters the thorax between the subelavian artery and the innominate vein, and is directed obliquely backwards, by the side of the trachea, and between this tube and the cesophagus, to the posterior aspect of the root of the lung, where it gives rise to the posterior pulmonary plexus. From the plexus two large offsets are continued to the back of the gullet, and unite below into one trunk, which reaches the posterior surface of the stomach.

The left nerve appears in the thorax on the outer side of the left com- 
mon carotid artery, and courses over the arch of the aorta, and beneath the root of the lung, forming there a larger plexus than on the right side. From the pulmonic plexus one or two branches pass to the front of the wsophagus, and join with offsets of the right nerve in a plexus; but its pieces are collected finally into one trunk, which is continued on the front of the gullet to the anterior part of the stomach.

The branches of the pneumo-gastric nerve in the thorax are the following :-

a. The recurrent or inferior laryngeal nerve, arising on the right side on a level with the subclavian artery, and on the left, at the lower border of the arch of the aorta, bends backwards to the trachea, along which it ascends to the larynx. On each side this branch is freely connected with the cervical cardiac branches of the sympathetic nerve, especially on the left side beneath the arch of the aorta.

b. Cardiac branches (thoracic). Besides the cardiac branches furnished by the vagus in the neck, other offsets pass in front of the trachea to the cardiac plexus. On the right side they come from the trunk of the vagus and the recurrent branch, but they are supplied by the recurrent nerve alone on the left. side.

The termination of the lower cervical cardiac branch of each vagus nerve (p. 114) may be now seen. The branch of the right lies by the side of the innominate artery, and joins a cardiac nerve of the sympathetic of the same side; and the branch of the left vagus crosses over the arch of the aorta, to end in the superficial cardiac plexus.

c. Pulmonary branches. There are two sets of nerves for the lung, one on the anterior and the other on the posterior aspect of the root.

The anterior branches are two or three in number, and small in size, and communicate with filaments of the sympathetic on the pulmonary artery: these nerves are best seen on the left side.

The posterior branches are the largest and much the most numerous. Forming a plexiform arrangement (posterior pulmonary plexus) behind the root of the lung by the flattening and splitting of the trunk of the nerve, they are joined by filaments from the third and fourth ganglia of the knotted cord of the sympathetic, and are conveyed into the lung on the divisions of the air tube.

d. Esophageal branches are furnished to the gullet, but in greatest abundance in the lower half. Below the root of the lung the branches of the pneumo-gastric nerves surround the œesophagus with a network (plexus gula).

Srmpatiletic Nerve. In the thorax the sympathetic nerve consists of a knotted cord along each side of the spinal column, which communicates with the spinal nerves : and of a large prevertebral or cardiac plexus, which distributes branches to the heart and the lungs.

The gangliated cord will be seen in a future stage of the dissection after the heart and the lungs have been removed.

The CARDiac plexus lies at the base of the heart around the great bloodvessels. A part of this network, the superficial cardiac plexus, has been already described (p. 314). The remaining part, or the deep cardiac plexus, is placed beneath the arch of the aorta.

Dissections. The cardiac plexus has been injured by the previous examination of the heart, so that it should be dissected in a body in which the heart and the large vessels are entire.

Dissection. The arch of the aorta is to be cut across near the heart 
and close above the pulmonary artery, and is to be drawn over to the left side: next the upper cava is to be divided above the entrance of the vena azygos, and its lower part is to be thrown down. By the removal of some fibrous and fatty tissues and lymphatic glands, the right part of the plexus, in which the nerves of the right sirle are united, will be seen in front of the trachea, above the right branch of the pulmonary artery. The offsets to the heart should be followed downwards on the trunk of the pulmonary artery; and those to the lung should be traced along the right branch of that vessel.

To lay bare the part of the plexus into which the nerves of the left side of the body enter, the arch is to be cut through a second time, to the right of and close to the junction of the ligamentum arteriosum with it; and the transverse part of the arch is to be turned upwards with the great vessels attached. The lymphatic glands and the areolar and fatty tissue being cleared away from the plexus as on the opposite side, the offsets to the posterior coronary plexus of the heart are to be cleaned.

Deep cardiac plexus. This large centre is situate between the trachea and the arch of the aorta, above the bianches of the pulmonary artery. In it are united the cardiac nerves of the sympathetic of both sides of the neck, except the highest nerve of the left side : and the cardiac branches of the vagus in the neck and chest, with the exception of the lowest cervical branch of the left side. From it nerves are furnished to the heart and lungs.

The several nerves entering the plexus are not intermingled in a plexiform mass in front of the trachea; but those of the right side unite together on the right of the air tube, and those of the left have a like junction on their side.

The right part of the plexus is placed above the right branch of the pulmonary artery, and receives the nerves of the right side, viz., the cardiac nerves of the sympathetic in the neck; the cardiac branches of the trunk of the vagus, in both the neck and chest; and the cardiac offsets of the recurrent branch.

The branches of this half of the plexus are distributed mostly to the right side of the heart, and pass downwards before and behind the right branch of the pulmonary artery: those in front running on the trunk of the pulmonary artery to end in the anterior coronary plexus ( 1.315$)$; and the nerves behind supply the right auricle of the heart. Offsets are sent laterally on the branch of the artery to the root of the lung.

The left half of the plexus lies close to the ligamentum arteriosum, and rather on the left of the trachea. In it are collected the cardiac nerves of the sympathetic ganglia of the left side of the neck, except the highest; and numerous and large branches of the left recurrent nerve of the varus.

Nerves descend from it to the heart around the left branch and trunk of the pulmonary artery, and after supplying branches to the left auricle, terminate in the posterior coronary plexus (p. 315). A considerable offiset is directed forwards by the side of the ligamentum ductus arteriosi to the superficial eardiac plexus; and some nerves reach the lelt anterior pulmonary plexus by passing along the branch of the pulmonary artery.

'Termination of the cardiac branches of the sympathetic nerve of the neck (p. 117).

On the right side there are usually only two cardiac nerves entering the thorax. for the highest nerve is blended commonly with one of the others. The middle and inferior nerves pass beneath the subclavian artery to the 
riglit half of the deep plexus : they communicate with the branches of the recurrent laryngeal nerve of the vagus.

On the left side the highest cardiac nerve lies over the arch of the aorta, and ends in the superficial cardiac plexus; it may give a.branch beneath the arch to the deep plexus. Only one other nerve, the lower cardiac, may be seen entering the left side of the deep plexus, as the middle one throws itself oftentimes into it.

\section{THE TRACHEA AND THE IUNG.}

Dissection. To see the pieces of the air tube in the root of the lung, it will be necessary to remove the pu'monary artery with its branches, and the pulmonary veins. And when the transverse part of the arch of the aorta, which has been already cut through, is turned to one side, the dissector will be able to clear away the bronchial glands, the nerves, and the fibrous tissue from the part of the trachea in the thorax, and from the branches into which it bifureates.

The TRACHEA, or the air tube, reaches from the larynx to the lungs, and lies on the front of the spinal column. The tube begins opposite the fifth cervical vertebra; and it ends commonly at the fourth dorsal vertebra by dividing into two pieces (bronchi), one for each lung. Its loint of splitting may be a vertebra lower.

Its connections in the neck are described in p. 121, and its structure in p. 162. The part in the thorax remains to be studied.

In the thorax the trachea is situate with tlie great vessels in the space between the pleural bags. Here it is covered by the arch of the aorta, by the innominate and left carotid arteries, and by the cardiac plexus of nerves. Behind the air tube is the osophagus, which is slightly inclined to the left near the arch of the aorta. On the right side are the vagus, and the innominate artery, for a short distance, alter this has passed over the trachea; and on the left side lie tlee left subclavian artery, and the vagus with its recurrent branch.

The bronchi, or the branches of the air tube, are contained in the roots of the lungs, and are surrounded by vessels, glands, and nerves. Near the lung each is divided into as many primary pieces as there are lobes. In their structure and form the bronchi resemble the windpipe, for they are round and cartilaginous in front, but flat and muscular and membranous behind. Their position behind the other pulmonary vessels has been described at p. 310 ; but the characters of each are now to be noticed.

The right branch is about an inch in length, and is larger than the left ; it rasses outwards, on a level with the fourth dorsal vertebra, beneath the arch of the aorta and the upper cava, and above the right pulmonary artery: the vena azygos arches above it.

The left branch, about two inches long, is directed obliquely downwards through the arch of the aorta, and joins the root of the left lung a vertebra lower than on the opposite side. The tube lies on the osophagus and thoracic duct, and on the aorta; it is at first below the level of the corresponding pulmonary artery.

Dissection. The lungs are to be removed now from the body by cutting through the vessels of the root.

The remains of the heart and pericardium are to be taken away afterwards. For their removal the inferior cara is to be divided, and the pericardium to be detached from the surface of the diaphragm; in removing 
the pericardium, the dissector should be careful not to injure the parts contained in the interpleural space in front of the spine.

Phisical Ciraracters of the Lung. The surface of the lung is smooth and shining, and is invested by the pleura. 'Through the serous covering the mass of the lung may be seen to be divided by septa, into small irregularly-sized pieces or lobules. On looking closely at it, especially at a thin margin, minute cells will be perceived in it.

The tint of the lung varies with age. In infancy the color is a pale red; but in the adult the texture becomes grayish, and presents here and there dark gray spots or lines of pigment, whose shade deepens with increasing age, and becomes even black in old people. After death, the color of the posterior border may be bluish-black from the accumulation of blood.

To the touch the lung is soft and yielding, and on a section the pulmonary substance appears porous and spongy ; but the lung which is deprived of air by pressure has a tough leathery feel. Slight pressure with the thumb and finger drives the air from the containing cells through the pulmonary structure, and produces the noise known as crepitation. If the lung contains serum, a frothy red fluid will run out when it is cut.

The texture of the lung is very elastic; this elasticity causing the organ to diminish greatly when the thorax is opened, and to expel air that may be blown into it.

The specifie gravity of the lung varies with the conditions of dilatation and collapse, or of infiltration with fluid. When the pulmonary substance is free from fluid, and filled with air, it floats in water; but when it is quite deprived of air it is slightly heavier than water, and sinks in that fluid. 'The weight of the lung is influenced greatly by the quantity of other material contained in its texture; ordinarily it ranges from eighteen to twenty-one ounces, and the right lung is about two ounces heavier than the left. In the male the lungs are larger, and slightly heavier than in the female.

Dissection. By tracing the large branches of the bronchi, and the bloodvessels and nerves into the lung, the mode of branching of the air tubes will be apparent; and by inflating a part of the lung, the cellular structure may be seen. But the arrangement of the small air cells about their tube, and the disposition of the different vessels, camnot be ascertained without fine injections and a microscope.

Structure of the Lung. The spongy pulmonary tissue consists of minute recesses or cells, in which the smallest branches of the air tube terminate; and the mass of the lung is formed by the collection of those cells into small groups or lobules, and by the aggregation of the lobules into larger masses or lobes. Each lobule is distinet from its fellows, and is furnished with its air tube and nerves, and with its set of vessels concerned in the function and nutrition.

The parts of the lung are united by a serous covering, prolonged continuously over the surface; and by a subserous layer of areolar tissue which penetrates into the anterior, subdividing it into pieces. These several parts are examined more in detail below.

Serous and subserous coverings. 'The casing derived from the pleura is thin and transparent, and forms an entire capsule for the lung, except at the root where the vessels enter. The subserous areolar layer contains fibres of elastic tissue, and not only covers the surface, but extends in- 
wards, establishing the division of the mass into lobules: where it separates the lobules it is named interlobular tissue, and is fiee from fat.

Bronchial branches in the lung. When a bronchus is followed into the pulmonary structure it is found to divide generally in a binary order, and to diminish in size at each subdivision, until one terminal offset appertains to a lobule. In the lobule the tube has a diameter of ${ }_{6}^{1} \delta$ to $_{3}^{\frac{1}{0}}$ of an inch. When this last degree of diminution is reached, the tube gives origin to the air cells.

The larger bronchial branches have the same composition as the trachea, but they are round in the lung, instead of being semi-elliptical as in the trachea. The smallest branches want some of the elements found in the larger bronchi; and those from which the cells spring are irregular in shape, appearing to be spaces amongst the cells rather than tubes with continuous walls.

Changes in the bronchi. 'The modifications of the component parts of the bronchi are the following:-The pieces of cartilage are broken up in the smaller bronchial tubes, and are scattered over the wall as irregular fragments. Becoming thinner and smaller as the subdivision of the air tube proceeds, they at last disappear, and are absent from the terminal branches. 'The fibrous and elastic tissues of the bronchial tubes are continued to the air cells, but in the small cell-bearing branches, the bundles of elastic tissue are diffused, and, much diminished in strength, blend with the fibrous or areolar tissue to form the wall. The muscular fibres are diffused over the inner surface of the smaller bronchi, where they have an annular arrangement; they extend beyond the limit of the pieces of cartilage, but they cease where the cells begin to be formed. The mucous membrane becomes thinner as it extends onwards in the bronchial pieces, and is finally continued to the cells, where it is transparent. Its epithelium is columnar and ciliated in the bronchial tubes, but is changed to squamous or laminar in the air cells.

Lobules and lobes. A lobule is a cluster of air cells around a terminal branch of the air tube. Varying in size and shape, each lobule is invested by areolar tissue, and possesses its own offset of the air tube, as well as distinct branches of ressels and nerves. The lobes are produced by the aggregation of the lobules.

The air cells are the little recesses or dilatations connected with the smallest branches of the air tube. They are polyliedral in form, except on the surface of the lung, and are distinct one from another, save through the channel of the air passage. The cells are clustered in groups around the terminal branches of the air tube, with which they communicate by large orifices. These small spaces are about $\frac{1}{\mathrm{~T} v 0}$ of an inch across, but they are larger on the surface and at the edges than in the deeper parts of the lung. 'The cell wall is formed by areolar and elastic tissue, and is lined by a transparent mucous membrane possessing laminar epithelium. Beneath the mucous lining is a network of capillaries of the pulmonary vessels.

Vessels of the Luxg. Two sets of vessels are furnished to the lung, one being concerned in its function, the other in the nutrition. 'The vessels conveying blood to the lung to be aërated, and carrying that fluid away after it has been subjected to the respiratory process, are named pulmonary. The vessels connected with the nutrition of the texture are called bronchial.

'The pulmonary artery divides like the bronchus, which it accompanies 
to the lobule. At the lobule the arterial branch is minutely subdivided, and its ramifications enter the interlobular fissure to end in the cell wall in the following way:-Over the bottom of the cell they form a very fine capillary network, but at the circumference they give rise to a circular vessel; and the circles of several cells communicate with each other.

The pulmonary veins begin in the vascular network before mentioned. The twigs issuing from the several lobules are destitute of valves, and are united in larger tubes which course to the root of the lung. Althongh the small lobular branches of the arteries remain separate from one another, the corresponding veins anastomose together.

The bronchial arteries enter the lung on the air tube, and supply deep branches to it and the contiguous glands, to the large bloodvessels, and to the interlobular areolar tissue of the lung. On the smallest air tubes minute branches anastomose with offsets of the pulmonary arteries.

Superficial tortuous offsets of the artery ramify beneath the pleura, forming a capillary network.

The bronchial vein begins by twigs corresponding with the superficial and deep branches of the artery. Leaving the lung at the root, the vein ends differently on opposite sides of the body (p. 337).

Nerves and lymphatics. The lung receives nerves from the vagus and the sympathetic; and the offsets follow the branches of the air tube, but their ending is uncertain. Remak describes small ganglia on the sympathetic filaments. The lymphatics of the lung are both superficial and deep, and enter the bronchial glands at the root of the lung.

\section{PARTS ON THE SPINE AND THE SYMPATHETIC CORD.}

In front of the spinal column are the several parts lying in the interpleural space of the posterior half of the mediastinum, viz., the aorta, azygos veins, thoracie duct, œsophagus, and splanchnic nerves.

Dissection. The thoracic duct should be found first near the diaphragm by removing the pleural; there it is about as large as a crow quill, and rests against the right side of the aorta : this slender vessel may be injected with tallow.

The areolar tissue and the pleura are to be cleared away from the different parts before mentioned ; and the azygos or intercostal veins, one on the right and two on the left aorta, should be dissected. Next follow the thoracic duct upwards beneath the arch of the aorta, and along the oesophagus beneath the pleura, till it leaves the upper aperture of the thorax.

After raising the pleura also from the inner surface of the chest, the gangliated cord of the sympathetic nerve will be seen lying over the heads of the ribs. Branches are to be followed outwards from the ganglia to the intercostal nerves; and others inwards over the hodies of the vertebre,the lowest and largest of these forming the three trunks of the splanchnic nerves.

The DESCENDING THORACIC AORTA is the part of the great systemic vessel above the diaphragm. Its extent is from the lower border of the tifth dorsal vertebra (the left side), where the arch ceases, to the front of the last dorsal vertebra.

Contained in the interpleural space in front of the spine, the ressel is rather curved, lying at its upper part on the left, but below on the front of the spinal column. Beneath it are the vertebræ and the smaller azygos veins. In front of the vessel is the root of the left lung with the pericar- 
dium. On its left side it is covered throughout by the pleura ; and on its right side are the oesophagus and the thoracic duct, though near the diaphragm the gullet is placed over the aorta.

The branches of the vessel are distributed to the surrounding parts, and are named from their destination bronchial, pericardial, œsophageal, mediastinal, and intercostal.

a. The bronchial arteries supply the structure of the lungs, and adhere to the posterior part of the bronchial tubes, on which they ramify (p. 336); they give some twigs to the bronchial glands and the oesophagus.

For the left lung there are two arteries (superior and inferior), which arise from the front of the aorta at a distance from each other.

The artery of the right lung arises in common with one of the left bronchial arteries (superior), or from the first intercostal artery of the right side.

Bronchial veins. A vein issues from the root of each lung, and ends in the following manner: the right joins the larger azygos vein; and the left ends in the superior intercostal vein of its own side.

$b$. The pericardial branches are some irregular twigs, which are furnished to the posterior part of the cardiac bag.

c. Esophageal branches arise at different points of the aorta, and are four or five in number. Ramifying in the gullet, the vessels anastomose with one another' above, they communicate with branches of the inferior thyroid artery near the pharynx; and below, with twigs of the coronary artery of the stomach.

d. Small mediastinal branches (posterior) supply the areolar tissue and the glands in the interpleural space.

$e$. The intercostal arteries are ten on each side; nine are furnished to the same number of lower intercostal spaces, whilst the last lies below the twelfth rib : to the upper two spaces branches are supplied from the intercostal artery of the subclavian trunk.

These small vessels arise from the posterior part of the aorta, and run outwards on the bodies of the vertebra, beneath the cord of the sympathetic nerve, to the intercostal spaces, where each divides into an anterior and a posterior branch. In this course the upper arteries have a somewhat oblique direstion; and as the aorta lies on the left of the spine the right vessels are the longest: the right also pass beneath the osophagus, the thoracic duct, and the azygos vein. Many twigs are supplied to the bodies of the vertebra.

In the spaces bounded by the true ribs, the anterior branch, the larger of the two, continues onwards between the muscular strata nearly to the anterior third of the intercostal space, where it ends in two pieces, which anastomose with the intercostal arteries of the internal mammary ( $p .239)$. At first the artery lies in the middle of the intercostal space, beneath the pleura and a fascia from the internalintercostal muscle, and resting on the external intercostal layer; but near the angle of the rib it ascends to the upper boundary. Accompanying the artery are the intercostal vein and nerve, the vein being commonly above, and the nerve below it; but in the upper spaces the nerve is, at first, higher than the artery.

Below the true ribs the vessels are contained partly in the thoracic and partly in the abdominal wall. Behind they have the same course and connections as the higher intercostals; but in the wall of the abdomen they lie between the two deep muscles; they will be noticed hereafter.

Branches are furnished to the intercostal and abdominal muscles, and 
to the ribs. About the centre (from front to back) of the intercostal space a superficial twig arises, which accompanies the cutaneous nerve.

The highest artery of the aortic set of intercostals anastomoses with the superior intereostal branch of the subclavian artery; and the lowest (below the true ribs) enters the abdominal wall, and anastomoses with the arteries of that part.

The posterior branch turns backwards between a vertebra and an ascending costo-transverse ligament, and is distributed in the Back. As it passes by the intervertebral foramen it furnishes a small spinal branch to the vertebræ and the spinal cord. (See Vessels of the Spinal Cord.)

The intercostal vein resembles closely the artery in its course and branching. Near the head of the rib it receives a contributing dorsal branch, and then joins an azygos vein.

The superior intercostal artery of the subclavian trunk (p. 78) descends over the neck of the first rib, external to the ganglion of the sympathetic, and supplies a branch to the first intercostal space: continuing to the second space, which it supplies in like manner, it anastomoses with the upper aortic branch.

Its intercostal offsets divide into an anterior and a posterior branch, like the arteries from the aorta.

The vein accompanying the artery opens into the innominate vein of the same side. The left superior intercostal vein (fig. 105, $i$ ), formed by branches from the two or three highest spaces, is joined by the left bronchial vein, and ends in the left innominate vein, after crossing the arch of the arta.

The intercostal or AZYGos veins are two in number on the left, and one on the right side, and receive branches corresponding with the offsets furnished by the thoracic aorta.

The right or larger azygos (fig. $106,{ }^{3}$ ) begins in the lumbar veins on the right side of the spine, and its origin is discribed with the vessels of the abdomen. It enters the thorax through the aortic opening of the diaphragm, and ascends on the right side of the thoracic duct, over the intercostal arteries and the bodies of the vertebrx. Opposite the fourth intercostal space the vein arches forwards above the root of the right lung, and enters the superior cava as this vessel pierces the pericardium. Its valves are very incomplete, so that blood may flow either way; and the intraspinal and intercostal veins may be injected through it.

Branches. In this vein are collected the intercostals of the right side below the upper two spaces; some of the intercostals of the left side of the thorax, through the left azygos veins ; and some small œsophageal, mediastinal, and vertebral veins, with the right bronchial vein.

By means of the right vein the inferior communicates with the superior eava, so that the blood may reach the heart from the lower part of the body, or the opposite, if one of the cava should be obstructed.

The left lower azygos vein (fig. 106, ${ }^{4}$ ) begins in the abdomen in the lumbar veins of the left side of the vertebral column. Entering the thorax along with the aorta, or throngh the crus of the diaphragm, the vein ascends on the left of the aorta as high as the seventh or eighth dorsal vertebra, where it crosses beneath that vessel and the thoracic duct to end in the right azygos. It receives the four or five lower intercostal veins of the left side, and some osophageal and mediastinal branches.

The left upper azygos vein (fig. 106, ${ }^{5}$ ) is formed by offsets from the space between the superior intercostal and the preceding. Receiving three 
or four branches, the trunk either joins the lower azygos of its own side, or crosses the spine to open into the right rein.

'The cesopingus or gullet is a hollow muscular tube, which extends from the pharynx to the stomach: the cervical portion has been described at p. 121, and the thoracic part is now to be examined.

Appearing in the thorax rather to the left of the middle line, the gullet is directed beneath the arch of the aorta, and reaches the middle of the spine about the fifth dorsal vertebra. From that spot it is continued through the interpleural space on the right of the aorta, till near the diaphragm, where it takes a position over the aorta, to gain the œsophageal opening of that muscle.

As far as the aortic arch the œsophagus lies beneath the trachea, though it is inclined somewhat to the left of the air tube; beyond the arch it is crossed by the left bronchus, and is concealed by the pericardium as far as the diaphragm. At the upper part of the thorax the gullet rests on the longus colli muscle and the vertebræ; but below the arch of the aorta it is separated from the spine by the intercostal vessels, and the aorta. Laterally it touches the left pleura above the arch, and both pleuræ below, but more of the right than the left. Below the bronchus the pneumogastric nerves surround the œsophagus with their branches; and above the same spot the thoracic duct is in contact with it on the left.

Structure. If a piece of the gullet be removed and distended with tow, it will be easy to show a muscular, fibrous, and mucous coat, one within another.

The muscular coat is thick and strong, and possesses two layers of fibres, of which the external is longitudinal, and the internal circular in direction, like the muscular tunic of the other parts of the alimentary tube. In the upper third of the oesophagus the fibres are red, but below that spot the color becomes paler.

The external layer is formed of parallel longitudinal fibres, which form an entire covering, and end below on the stomach. The fibres begin opposite the cricoid cartilage (p. 131); and at intervals varying from half an inch to an inch and a half, they are connected with tendinous points ( $\frac{1}{20}$ to $y^{\frac{1}{0}}$ of an inch long) like the fibres of the rectus abdominis muscle.

'The internal layer of circular fibres is continuous above with the fibres of the pharynx; they are more oblique at the middle than at either end of the œesophagus.

'The fibrous layer is situate between the muscular and mucous coats, and attaches the one to the other loosely.

The mucous coat will be seen on cutting open the tube: it is reddish in color above but pale below, and is very loosely connected with the muscular coat, so that it is thrown into longitudinal folds when the osophagus is contracted. Lining the interior is a thick layer of scaly epithelium; and the surface is studded with minute papillæ.

Some compound glands (œsophageal) are scattered along the tube; at the lower part of the gullet they are most numerous, and form a ring (cardiac) close to the stomach.

Lymphatics of tue Thorax. In the thorax are lymphatic ressels of the wall and the viscera, which enter collections of glands, and end in one or other of the two lymphatic ducts. Besides these, the large thoracic duct traverses the thorax in its course from the abdomen to the neck.

Lymphatic glands. Along the course of the internal mammary artery lies a chain of sternal or mediastinal glands, which receive lymphatics 
from the front of the chest, the thymus body, the pericardium, and the upper surfaces of the diaphragm and liver.

On each side of the spine, near the heads of the ribs, as well as between the intercostal muscles, is placed a group of intercostal glands for the re-

Fig. 106.

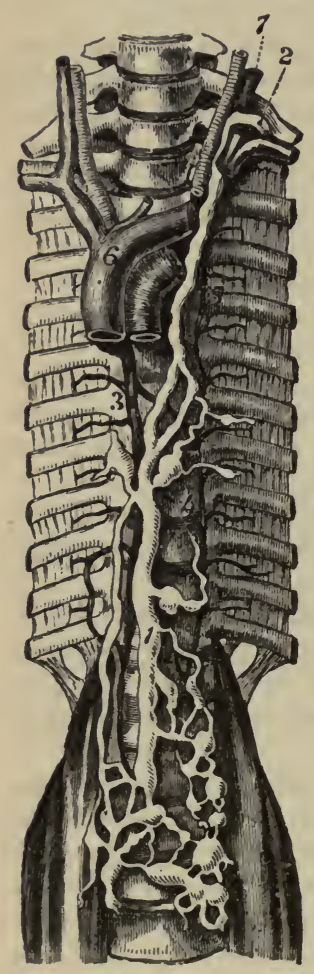

VIEW OF THE THORACIC DUCT, AND THE INTERCOSTAL VEINs.

1. Thoracic duct.

2. Ending of the duct in the left subciavian veln.

3. Large or right azygos vein.

4. Left lower azygos or intercostal vein.

5. Left upper azygos or intercostal.

6. Vena cava superior.

7. Left internal jugular vein, cut through. ception of the lymphaties of the posterior wall of the thorax.

Numerous bronchial glands are situate at the division and along the side of the trachea, through which the lymphatics of the lung pass; and beneath the areh of the aorta are a few cardiac glands, to which the lymphatics of the heart are directed.

Along the side of the aorta and œsophagus is a chain of asophageal glands, which are joined by the lymphatics of the osophagus, and communicate with those of the lungs.

The thoracic duct (fig. 106, ${ }^{1}$ ) is the main channel by which the lymphatic and lacteal fluid of the lower half of the body, and of the left side of the upper half of the body, is conveyed into the blood. The duct begins in the abdomen in an enlargement (chyli receptaculum), and ends in the left subclavian vein of the neck. It is about eighteen inches in length, and is contained in the thorax, except at its origin and termination. It has the undermentioned connections:-

Entering the cavity on the right of the aorta and through the same opening, the duct ascends on the right side of that vessel, as high as the arch. Opposite the fifth dorsal vertebra it passes beneath the aortic arch, and is applied to the left side of the osophagus, on which it is conducted to the neck under the left subclavian artery. At the lower part of the neck the duct arches outwards, as before described (p.119), to open into the left subclavian vein.

In this course the tube is oftentimes divided in two, which unite again; or its divisions may even form a plexus. Near its termination it is frequently branched. It is provided with ralves at intervals, like a vein; and these are in greatest number at the upper part.

Branches. In the thorax the duct receives the lymphaties of the left half of the cavity, viz., from the sternal and intercostal glands; also the lymphatics of the left lung, the left side of the heart, and the trachea and osophagus.

The right lymphatic duct (fig. 27) receives large branches from the viscera of the thor:x. It is a short trunk, about half an inch in length, and opens into the angle of union of the subclavian and jugular veins of the same side (p.79): its opening is guarded by valves.

Branches. Into this trunk the lymphatics of the right upper limb, and 
right side of the head and neck pour their contents. In addition, the lymphatics of the right side of the chest, right lung and right half of the heart, and some from the right lobe of the liver, after passing through their respective glands, unite into a few large trunks, which ascend beneath the innominate vein to reach the duct in the neck.

Structure of the ducts. The wall of the tube resembles that of the bloodvessels in strueture (p. 119).

Cord of the Sympathetic Nerve. The thoracic part of the gangliated cord of the sympathetic nerve is covered by the pleura, and is placed over the heads of the ribs, and the intercostal vessels. The ganglia on it are usually twelve, one being opposite each dorsal nerve, but this number varies much. The upper ganglion is the largest; and the last two are rather anterior to the line of the others, being situate on the side of the bodies of the corresponding vertebræ.

Each ganglion furnishes external branches to communicate with the spinal $\mathrm{n}$ rves, and internal for the supply of the viscera.

External or connecting branches (fig. 107). Two offsets pass outwards from each ganglion to join a spinal nerve (intercostal). In the branches

Fig. 107.

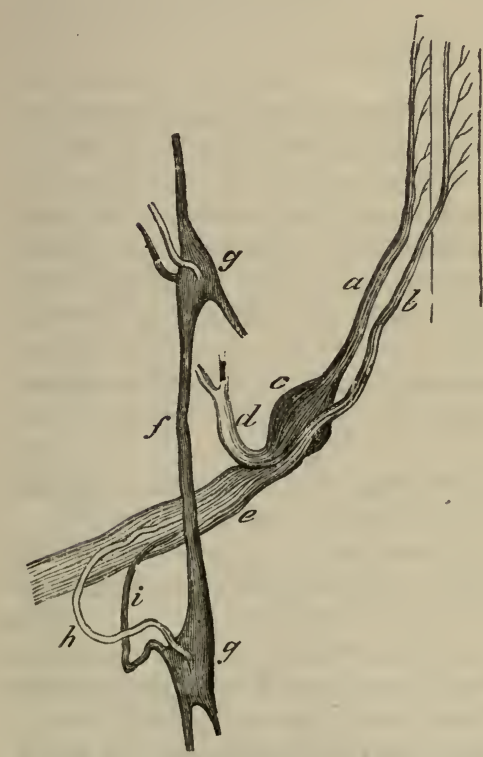

a. Posterior root of a spinal nerve, with a ganglion, $c$.

b. Anterior root.

d. Posterior primary branch.

e. Anterior primary branch of the spinal nerve.

$f$. Knotted cord of the sympathetic.

$g$. Ganglia on the cord.

$h$. White offset from the spinal to the sympathetic nerve.

i. Gray offset from the sympa hetic to the spinal nerve.

Scheme to Illustrate the connection Between the Spinal and Stmpathitic NrRvez. (Todd and Bowman.)

of communication both spinal and sympathetic nerve fibrils are combined: but one $(h)$ consists almost entirely of spinal, and the other (i) nearly altogether of sympathetic nerve fibres.

The internal or visceral branches differ in size and distribution, according as they are derived from the upper or lower six ganglia.

The offisets of the upper six are very small, and are distributed to the aorta, and the vertebre with the ligaments. Mr. Swan describes a plexus in front of the spine, from the union of the branches of opposite sides. 
From the third and fourth ganglia offsets are sent to the posterior pulmonary plexus.

The branches of the lower six are larger and much whiter than the others, and are united to form visceral or splanchnic nerves of the abdomen : these are three in number (large, small, and smallest), and pierce the diaphragm to end in the solar and renal plexuses.

The great splanchnic nerve is a large white cord, which receives roots apparently from only four or five ganglia (sixth to the tenth), but its fibres may be traced upwards on the knotted cord as high as the third ganglion. Descending on the bodies of the vertebræ, it pierces the fibres of the crus of the diaphragm, and ends in the semilunar ganglion of the abdomen. At the lower part of the thorax the nerve may present a ganglion.

The small splanchnic nerve begins in the tenth and eleverith ganglia, or in the intervening cord. It is transmitted inferiorly through the crus of the diaphragm, and enters the part of the solar plexus by the side of the coliac artery.

The smallest splanchnic nerve springs from the last ganglion, and accompanies the other nerves through the diaphragm: in the abdomen it ends in the renal plexus. This nerve may be absent, and its place may be taken by an offset of the preceding.

\section{PARIETES OF TIE THORAX.}

Between the ribs are lodged the two layers of intercostal muscles, with the intervening nerves and arteries; and inside them lies a thin fleshy layer behind,-the infracostals. At the base of the thorax is the diaphragm.

The INFracostal MUSCLES are small slips of fleshy fibres, which are situate on the inner surface of the ribs, where the internal intercostals cease. Apparently part of the inner intercostals, they arise from the inner surface of one rib, and are attached to the like surface of the rib next succeeding.

They are uncertain in number, but there may be ten : they are smaller above than below, and the upper and lower may pass over more than one space.

Action. These thin muscles approach the ribs to one another, diminishing the size of the thoracic cavity, and act thus as expiratory muscles.

Intercostal Muscles. The anterior part of the muscles has been described (p. 237); and the posterior part may be now examined from the inner side.

The inner muscle begins at the sternum, and reaches backwards to the angle of the ribs in the middle spaces, but, higher and lower, the museular fibres approach nearer the spine. Where the fibres cease, a thin fascia is continued backwards over the outer muscle. The inner surface is lined by the pleura, and the opposite surface is in contact with the intercostal nerve and vessels.

External muscle. When the fascia and the infracostal muscles have been removed, the external intercostal will be seen between the posterior border of the internal muscle and the spine. Its fibres cross those of the inner intercostal layer. Whilst this muscle extends backwards to the tubercle of the rib, it does not reach further forwards than the rib cartilages, as before said.

Action. The use of the intercostal muscles in respiration is given in p. 238. 
Dissection. In a few spaces the internal intercostal muscle may be cut through, and the intercostal nerve and artery traced outwards.

The INTERCOSTAL NERVES are the anterior primary branches of the dorsal nerves. Twelve in number, they occupy the intercostal spaces, without communicating in a plexus; and the last is placed below the twelfth rib. The upper six lie between the ribs, and are confined to the wall of the thorax; whilst the lower six are prolonged into the abdominal wall, where the ribs cease in front.

Upper six. At first the nerves lie between the pleura and subjacent fascia and the external intercostal muscle, with an artery and vein ; but they enter soon between the intercostals and extend forwards to the middle line of the body. Near the head of the rib each is joined by filaments from the sympathetic. In its course each supplies branches to the intercostal muscles and the ribs, and cutaneous offsets to the surface; these are described in the dissections of the upper limb and wall of the abdomen.

There are some deviations in the first and second nerves from the arrangement above specified.

The first nerve ascends in front of the neck of the highest rib, and ends in the brachial plexus. Before it leares the chest it supplies to the first intercostal space a branch, which furnishes muscular offsets, and becomes cutaneous by the side of the sternum. There is not any lateral cutaneous offset from this branch, except in those cases in which the second nerve is not as large as usual.

The second nerve may extend a considerable way on the wall of the chest before entering between the intercostal muscles, and may ascend even to the first space. It is remarkable in having a very large lateral cutaneous branch (p. 225). In front it ends like the others.

Upper surface of the diaphragm. The centre of the muscle is tendinous, and the sides are fleshy. In contact with the upper surface are the lungs laterally, and the pericardium in the middle: the phrenic vessels and nerves pierce this surface, external to the pericardium. In the diaphragm are the following apertures: one for the ocsophagus and the pneumogastric nerves, a second for the vena cava, a third for the aorta with the thoracic duct and the vena azygos, and a fourth on each side for the splanchnic nerves. Beneath it the sympathetic passes into the abdomen.

The arch of the diaphragm towards the thorax has been before referred to (p. 306).

Directions. The dissector of the thorax now waits while the examination of the Back is made. Afterwards he is to learn the ligaments of the ribs and spine: a notice of these will be found in the following Section.

\section{SEction II.}

\section{LIGAMENTS OF THE TRUNK.}

Tre ligaments of the vertebræ, ribs, and sternum, are included in this Section.

Articulation of tile Ribs. The osseous part of each rib is united to the spinal column on the one side, and the rib cartilage on the other, by three sets of ligaments, viz., one between the head of the bone and the 
bodies of the vertebra; a second from the neck and tubercle to the transverse processes of the vertebra ; and a third between it and the cartilage.

Dissection. For the purpose of examining the ligaments between the rib and the vertebre, take a piece of the spinal column with three or four ribs attached. After removing the intercostal and other muscles, and the loose tissue from the surface of the bones, the student will be able to define, as below, the ligaments passing from the head and neck of the rib to the bodies and transverse processes of the vertebra.

The ligaments attaching the costal cartilage to the rib and sternum are to be dissected on the part of the thorax which was removed in opening the cavity.

Ligaments of the Head of the Rib. Where the head of the rib is

Fig. 108.

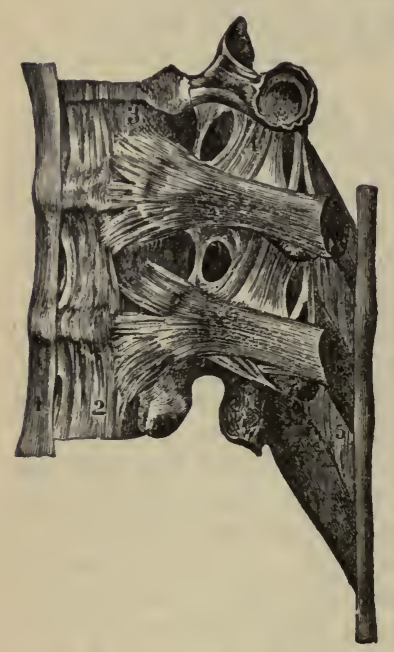

LIGAMBNTS OF THB RIBS AND TERTEBRAB (Bourgery).

1. Anterior ligament of the bodies of the vertebræ.

2. Lateral part of the anterior ligament of the spine.

3. Ligament (stellate) between the vertebræ and head of the rib.

4. Ascending costo-transverse ligament.

5. Interspinous ligament. received into a hollow in the bodies of two continuous vertebre, the articulation is provided with two retaining ligaments-costovertebral and interarticular, with two synovial sacs.

The costo-vertebral ligament (fig. 108, ${ }^{3}$ ), named stellate from its form, is composed of radiating fibres, and is placed in front of the joint. Attached by one end to the rib, it is livided at the other into three sets of fibres: The upper, the largest, ascends to the body of the vertebra above the rib head; the lower descends to the vertebra below the head; and the central part is united with the fibro-cartilage between the vertebra.

Where the rib is in contact with only one vertebra, as in the first, eleventh, and twelfth, the ligament has but two sets of fibres. The chief fibres in those three joints are fixed into the body of that vertebra which is touched by the costal head; whilst the ascending band is attached to the body of the vertebra immediately above. In the first rib-jo $n$ t the ascending band is continued to the last cervical vertebra.

The interarticular ligament will be seen when the stellate is divided. It is a short thin band of fibro-cartilage, which is attached on one side to the ridge separating the articular surfaces on the head of the rib, and on the other to the fibro-cartilage between the vertebra. In the joints of the first, eleventh, and twelfth ribs, where the head is not in contact with the intervertebral substance, the ligament is absent.

Synovial sacs. There are two sacs in the articulation, one on each side of the interarticular ligament. In the three joints before inentioned (1st, 11 th, and 12 th) there is but one sac.

Movements. In the costo-vertebral articulation there is a gliding of the rib up and down, and forwards and backwards. 'The movement of the head is more limited in the first seven ribs which are fixed to the sternum than in the others; and is greatest in the last two, in consequence of the 
ribs being free anteriorly, and not fixed behind by a joint to the transverse process.

During the increase and decrease of the chest the body of the rib is rotated out and in, around a line subtending the arc of the circle of which its fore part is a segment. The degree of motion is greatest in the longest ribs.

Ligaments of the Neck and Tubercle. Three ligaments (costotransverse) pass from the neck and tubercle of the rib to two transverse processes. And there is a synovial sac between the rib and its transverse process.

'The anterior costo transverse ligament (fig. $108,{ }^{4}$ ) is larger and longer than the others. It ascends from the upper edge of the neck to the transverse process of the upper of the two vertebra with which the head articulates: it is wanting to the first rib. Between this ligament and the vertebra the posterior branches of the intercostal vessels and dorsal nerve pass; and externally it is joined by the fibrous membrane between the strata of the intercostal muscles.

The posterior costo-transverse (fig. $115,{ }^{3}$ ) is placed at the posterior aspect of the rib. It is a short band of fibres between the rough part of the tubercle and the tip of the transverse process of the lower of the two vertebræ touched by the rib head. This band is longest in the lowest two articulations.

The middle or interosseous costo-transverse is placed horizontally between the neck of the rib and the transverse process with which the tubercle articulates. It will be best seen by sawing horizontally through the rib and the transverse process. Its fibres are mixed with reddish areolar tissue.

In the lowest two ribs the interosseous costo-transverse blends in one band with the posterior costo-transverse ligament.

Synovial sac. If the posterior ligament is divided, a synovial membrane will be exposed in the joint between the tubercle of the rib and the transverse process.

In the lowest two ribs, which do not touch transverse processes, the synovial sac is absent.

Movement. In the motion of the rib, the bone glides upwards and downwards on the articular facet of the transverse process; and the degree will be limited by the surrounding costo-transverse ligaments.

Ligaments of the Sternal Cartilages. The cartilages of the true ribs except the first are united to the sternum by a fibrous capsule, which covers a synovial membrane; and externally they are joined to the osseous part of the rib. The first is generally ossified to the sternum; and some of the lower cartilages touch each other, and are connected together by fibrous bands with synovial membranes.

In the chondro-sternal articulation (fig. $51,{ }^{4}$ ), the cartilages are received into the depressions on the side of the sternum, and are fixed in their position by a capsule of surrounding fibres. In front and behind the capsule are thickened bands, which have been described as anterior and posterior ligaments.

Between the cartilage and the bone is a synovial membrane.

In the joint of the second cartilage there is an interarticular ligament (fig. $51,{ }^{6}$ ), as in the head of the rib, which joins the cartilage between the pieces of the sternum. A synovial sac exists on each side of the interarticular ligament. 
A separate band of fibres joins the eartilage of the seventh rib to the xiphoid cartilage, and is named costo-xiphoid ligament.

Costal cartilage with the rib. 'The bony part of the rib is hollowed to receive the costal cartilage, and the posterium of the rib is the investing membrane between the two.

Costal Cartilages to one Axotiner. The contiguous surfaces of the costal cartilages from the sixth to the ninth are flattened, and are connected by ligamentous fibres; each articulation is provided with a synovial membrane.

Movement. There is only a limited gliding motion in the chondrosternal and the interchondral articulations. The second rib-cartilage joint is the most movable.

Asternal Cartilages. The cartilages of the three first false ribs are united only by end-bands of fibrous tissue, and are freer to move than those which are attached to the sternum. 'The lowest two, which are separate from one another, are the least fixed of all.

Articulation of the Sternum (fig. 51). As the two pieces of the bone are united by cartilage without any synovial membrane, the kind of articulation is sometimes named synchondrosis. The articulation is strengthened by anterior and posterior longitudinal fibres.

Movement. In articulations throngh the medium of cartilage, as here and in the pelvis, there is very little motion to be perceived, even when the osseous pieces are forcibly pulled by the hands.

Articulation of the Vertebre. The vertebre of the spinal column are united together by two sets of ligaments-one for the bodies, and the other for the arch and processes.

Along the spinal column the ligaments have a general resemblance, and one description will suffice except for those between the first two vertebræ and the head, and in the pelvis.

Dissection. After the articulations of the ribs have been examined, the same piece of the spinal column will serve for the preparation of the ligaments of the bodies of the vertebræ. 'The anterior ligament of the bodies will be defined with very little trouble, by removing the areolar tissue.

It is supposed that the spinal eanal has been opened to examine the spinal cord, and that the posterior ligament of the bodies of the vertebræ is laid bare; but if the canal should not be open; the neural arches of the vertebra are to be removed by sawing through the pedicles.

The remaining ligaments between the neural arches, spines, and articular processes of the bones, may be dissected on the piece taken away in opening the spinal canal.

Ligaments of tire Bonies. The bodies of the vertebræ are united by an anterior and a posterior common ligament, with an intervening piece of fibro-cartilage.

The anterior common ligament (fig. 109, a) reaches from the atlas to the sacrum: it is widest opposite the lumbar vertebræ, and is narrowed upwards. It consists of a central wide and thick part (a), and of a thinner portion $(b)$, on each side of the body of the vertebræ. If the central piece be cut aeross at intervals it will be seen to be thickest opposite the hollow of the bodies.

The fibres of the middle part are longitudinal in direction. By detaching parts of the ligament, the superficial fibres will be seen to reach three or more vertebra, whilst the deep extend from bone to bone. A greater 
number of the fibres are attached to the intervertebral fibro-cartilages than to the bones; and more are fixed to the margins than the centre of the body.

The posterior common ligament (fig. 110), much thinner than the anterior, is contained in the spinal eanal, lying on the posterior surface of the vertebra, and extends from the sacrum to the occipital bone. This ligament is wide above, and diminishes in size downwards, just the opposite of the anterior. In the neck, $\mathbf{A}$, it is loose, and extends across the bodies. In the back and loins it is a narrow band, B, which sends off on each side a pointed process to be attached to the pedicle of the neural arch; and it is wider opposite the intervertebral disks than on the bodies, so that the margins are dentate. One surface of the ligament is in contact with the dura mater; and between the band and the vertebrre are the intraspinal vessels.

Its fibres are superficial and deep as in the anterior ligament, and are more closely united with the intervertebral substance than with the bone.

Dissection. 'To see the intervertebral substance, the anterior and posterior eommon ligaments must be taken away. One vertebra should be detached from the

Fig. 109.

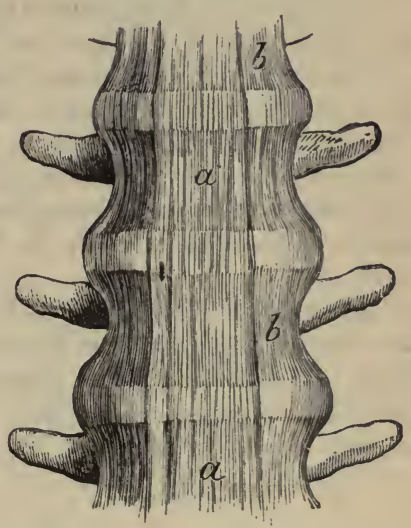

ANTERIOR CoMmon LigameNT OF the BODIES OF THE VeRTBBR.

$a$. Middle strong part, and $b$, lateral thin part of the ligament. fibro-cartilage to obtain a horizontal view of this structure; and two other vertebra should be sawn vertically to see the difference in the consistence and arrangement of the laminæ.

Fig. $110 \mathrm{~A}$.

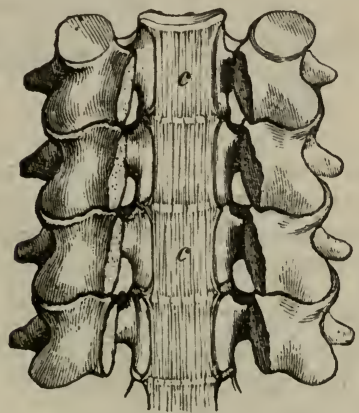

Fig. 110в.

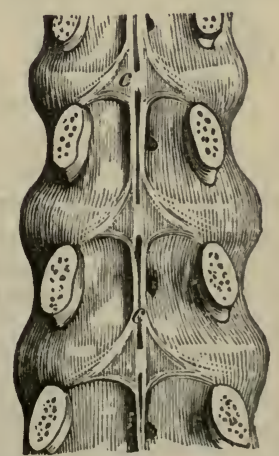

Two Views of the Posterior Common Licament, $c$, of the Bodies of the Vertebri, to SHOW THE DIFFERENCE IN SHAPE.

$\Lambda$, refers to its form in the neck, and $B$, to its shape in the loins.

The intervertebral substance (fig. 111) is placed between the contiguous surfaces of the bodies of the vertebra, from the axis to the sacrum. It 
forms a flattened disk between each pair of the vertebræ, and its width is determined by the size of the bones. It is connected in front and behind with the anterior and posterior common ligaments, and on the sides with the stellate ligament of the head of the rib.

By means of the dissection before made, the intervertebral substance may be observed to consist of two distinet parts ; an external, firm and laminar, and an internal, soft and elastic (fig. 112).

The outer laminar part (fig. 113, a) forms more than half of the disk, and is composed of pieces of fibro-cartilage with layers of fibrous tissue. All the strata are arranged one within another, like the scales of an onion, and are connected by their edges to the bodies of the vertebra; but all have not a vertical direction, for whilst the outer pieces are straight, the inner are bent with the convexity outwards (fig. 112) (Humphry). The laminæ do not form complete rings, but those composed of fibrous tissue reach farther than those of fibro-eartilage: the free ends of both layers overlap.

Each layer is constructed of oblique fibres; and the fibres of one layer are directed across those of another like the parts of the letter $\mathbf{X}$ (fig. 111). This disposition of the fibres will be best seen on the disks between the lumbar vertebræ; and it may be rendered evident by dissecting layer after layer.

Fig. 111.

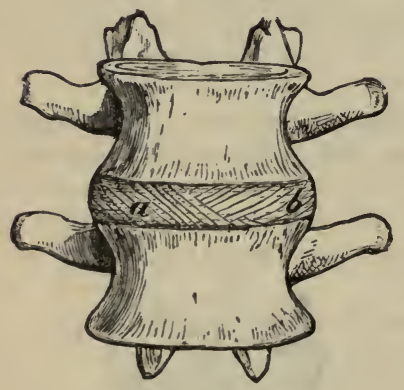

INTERVERTEBRAI, SUBSTANCE IN THK LUMBAR Rrgion, with its lamina displayed.

$a$. Superficial, and $b$, deeper layer, the fibres

in each taking a different direction.
Fig. 112.

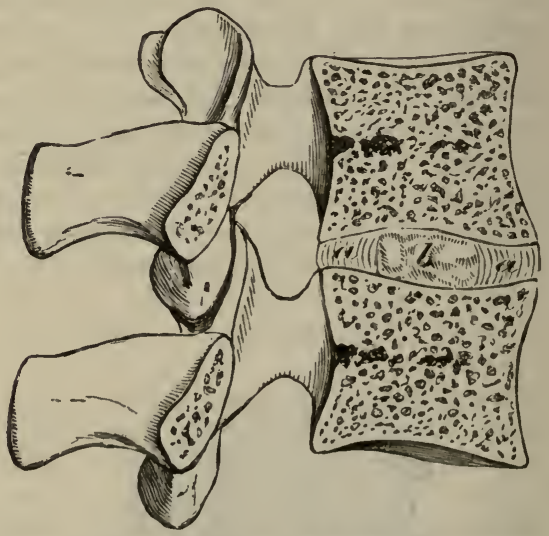

Vertical Section OF THE INTERVERTEBRAL ScBBTANCE, to show the direction of the layers.

a. Circumferential layers bent outwards.

b. Central pulpy part.

The central or pulpy portion of the disk (fig. $113, b$ ) is very soft and elastic, and projects when two vertebra and the interposed mass are sawn through. Placed nearer the back than the front of the disk, it is more marked in the loins and neck than in the dorsal region. It has a yellowish color, and is deficient in the stratiform arrangement so conspicuous at the circumference.

'Towards the confines of the two portions of the intervertebral substance, there is an alternating arrangement of fibrous tissue and fibro-cartilage, though the former is gradually diminishing; but towards the centre a loose fibro-cartilaginous material with large cells preponderates. 
The surfaces of the vertebræ in contact with the disk have a cartilaginous covering; this may be seen by cutting the intervertebral substance from the bone. Over the centre of the osseous surface it forms a continuous layer, but towards the circumference it consists of separate pieces.

The disks are thicker between the lumbar and cervical, than between the upper and middle dorsal vertebre; and where the spinal column is arched forwards, as in the loins and neck, they are deepest at the anterior edge, being wedge-shaped. The thinnest piece is situate between the second and third cervical vertebræ, and the thickest between the fifth lumbar and the sacrum.

$U$ se. The intervertebral substance unites together the vertebra so firmly as to prevent displacement of those bones without rupture of it.

By means of the central elastic part the revolving motion of one bone on another is

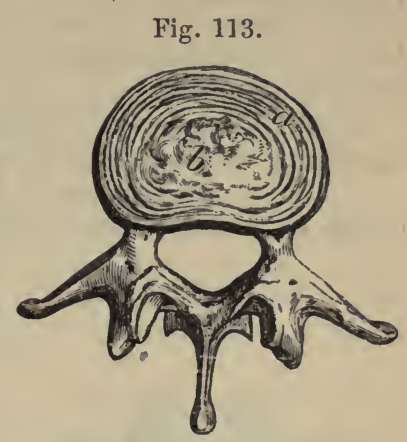

A Horizontal CUt through an INTERVERTEBRAL FIBRO-CARILAGB.

a. Laminar external part.

b. Pulpy central part of the fibro-carti. lage. produced; and the degree of the movement is limited by the circumferential laminar portion.

Through its wedge-shaped form the disk is chiefly instrumental in giving rise to the convexity of the spinal column in the loins and neck; and through its elasticity it moderates the effect of jars or shocks transmitted from bone to bone.

The depth of its several pieces amounts to about a fourth of the length of the movable part of the spinal column ; but as it yields under pressure, the height of the body will be shorter from half an inch to an inch in the course of the day, according to the fatigue undergone.

Liganents of the Neural Arch and Processes. The several processes of the vertebræ have special uniting ligaments: thus the articular processes are joined by a capsule and a synovial membrane; the neural arches are connected by yellow ligaments; the spinous processes have one band along the tip and another between them; and the transverse processes are provided with intervening bands of fibres.

Ligaments of Articulatiny processes. Between the articulating processes there is a movable joint, in which the bones are covered with cartilage, and are surrounded by a loose capsular ligament of scattered fibres, enclosiug a synovial membrane. In the cervical part of the spine the capsular ligaments are looser than in the dorsal or tumbar region.

Movement. With flattened articular surfaces is combined a gliding of one bone upon another. This morement is least limited in the neck, the loins, and the lower dorsal vertebre.

By the difference in the shape of the articulating processes, the kind of motion in the spine is determined; and by their arrangement the degree is limited, and the vertebre are partly maintained in situ. In dislocation of the spinal column they are generally broken before a vertebra cim be dislodged from its imbricated position.

Ligaments of the arches. The ligamenta subflava (fig. $114 .^{2}$ ) so named from their color, are situate between the neural arches of the vertebra, and close the spinal canal behind. Between each pair of aruhes are two 
ligaments, one for each half of the arch, which approach one another along the middle line, and reach on each side from the pedicle of the arch to the ront of the spinous process.

Each consists of elastic yellow tissue. It is attached above to the inner surface of the one, but below to the upper border and outer surfice of the other neural arch.

Between the first two vertebræ and the skull there are special fibrous ligaments in the corresponding situation (see p. 166).

Ligaments of the spines. Along the tips of the spinous processes of the dorsal lumbar vertebræ is a longitudinal band of fibres (fig. $115,{ }^{1}$ ) - the

Fig. 114.

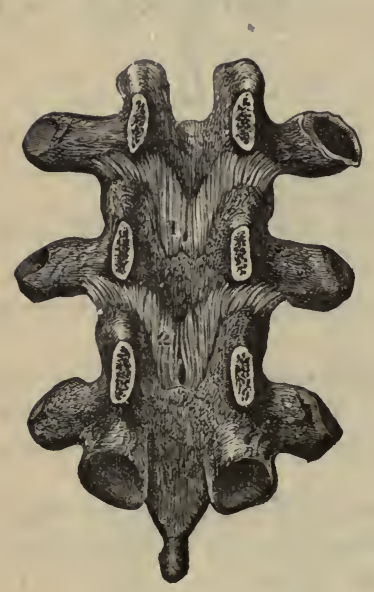

AN INNER VIEW OF THE NECRAI, ARCHES OF THE VERTEBRA, WITH THEIR INTERPOSED LIGAMENTS.

1 and 2. Ligamenta subflava. (Bourgery and Jacob.)
Fig. 115.

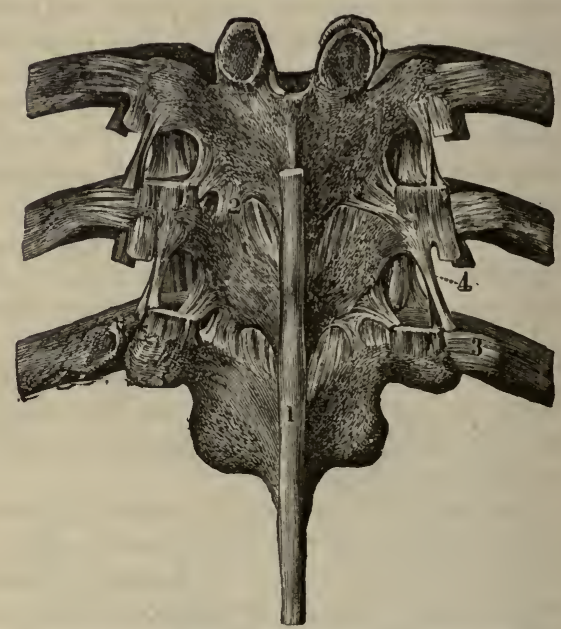

Ligaments OF the Procegges of the Vertebre, AND OF THE RIBS. (Bourgery.)

1. Supraspinous band of ligament.

2. Ligamentum subflarum.

3. Posterior costo-transverse ligament: on the opposite side the band has been removed and the joint opened.

4. Intertransverse ligament.

supraspinous ligament. It is thickest in the lumbar region of the spine, and is formed by the superficial and deep fibres; the former reach over three or more spines, whilst the latter pass from bone to bone. It is closely united with the tendons of the muscles.

The band of the ligamentum nucha, which is composed of fibrous tissue, takes its place in the neck.

Along the spinal column there are also thin membranous interspinous ligaments (fig. $108,{ }^{6}$ ), which reach from the root to the tip of the spinous processes. 'The strength of these bands is dependent upon the interval they fill; they are more marked in the lumbar than the other vertebra, and they are least strong in the cervical region.

Ligaments of the transverse process. In the loins the inter-transverse 
ligaments are thin membranous bands in the intervals between the processes. In the dorsal vertebra they are round bundles of fibres (fig. 115, ${ }^{4}$ ). And in the neck the fibres exist only at the apex of the processes, or they may be wanting here and there.

Ligaments of Special Vertebre. The ligaments of the first two cervical, and the sacral and coccygeal rertebræ, will be given with the dissection of the neck (pp. 166, 167) and of the pelvis (Section vii.).

Movements of Spinal Colums. The spinal column can be bent forwards, backwards, and to each side; and can be rotated.

In flexion the vertebre between the axis and sacrum are bowed forwards. The greatest movement takes place between the last two lumbar vertebre and the sacrum (Humphry); there is an intermediate degree in the neck; and the least is in the upper half of the dorsal region, where the ribs are united to the sternum.

The bodies of the bones are brought nearer together in front, whilst they are separated behind. The inferior pair of articular processes of the second vertebra glide upwards on the upper articular of the third vertebra; the inferior of the third bone move in like manner on the upper processes of the fourth; and so on throughout the movable column.

The ligament in front of the bodies is loose, but the posterior, and those uniting the neural arches and processes, are stretched. The fore part of each intervertebral disk is squeezed and thinner, and the back is elongated.

In extension, the vertebræ are arched backwards, but not to so great a degree as when the spine is bent forwards. The motion is most in the neck; and is least in the dorsal vertebræ, which are fixed by the true ribs and the sternum, and are impeded in their movement by the overlapping spinous processes.

The posterior parts of the vertebre are approximated, whilst the anterior are separated; and the inferior articular processes of each (from the second cervical to the sacrum) glide downwards, on the upper articular processes of the next succeeding bone.

The condition of the ligaments is the opposite to that in flexion. Thus, the intervertebral disks are compressed behind and widened before; the spinous and subflaval are relaxed; the anterior common of the bodies is tightened, and the hinder band is slackened.

Lateral inclination. The spine can be curved to the right or the left side. Like the other movements, this is least in the more fixed upper dorsal vertebræ, and is greatest in the neck.

On the concave side of the curve, say the right, the bodies are brought nearer together; and are carried away from each other on the opposite aspect. The right inferior articular surface glides down, and the left up, in the joints with the vertebra beneath.

On the right side the ligaments will be relaxed and the intervertebral substance compressed; and on the left those structures will be tightened so as to check the movement.

Rotation is the twisting of the bodies of the vertebræ around a line through their centres, the fore part being turned to the right and to the left. Its degree is greatest in the cervical and the upper dorsal vertebrie, but is absent in the lumbar part of the column.

The vertebral bodies are directed horizontally, and the articular processes move differently on the two sides. Supposing the face turned to the right, the processes of the right side glide inwards towards, and those of the left side outwards from the spinal canal. 
The flat ligaments and the intervertebral disks of the bodies, and the ligaments of the neural arches, will be less tight on the side to which the face is directed than on the other.

Dislocation of one vertebra from another without fracture seldom oceurs, in consequence of the way in which the inferior pair of articular processes are placed behind the superior of the next following bone, like scales. In the cervical region, where the articular surfaces are more horizontal, simple displacement may oecasionally take place. 


\section{CHAPTER V.}

\section{DISSECTION OF THE BACK.}

Directions. The dissection of the Back may be undertaken by one student; or conjointly by the dissectors of the head and upper limbs-the former preparing the neck, and the latter making ready the parts in the dorsal and lumbar regions.

If the Back should be allotted to more than one student, the dissector of the upper limb may attend chiefly to the paragraphs marked with an asterisk; and the dissector of the neck may study specially the paragraphs which are not so marked. But as many of the dissections in the earlier stages require the co-operation of the students employed on the same side of the body, a general attention may be given to the whole by each.

The dissector of the abdomen is to examine the arrangement of the fascia lumborum, after the first layer of muscles has been learnt.

Position. The body lies with the face downwards ; and the trunk is to be raised by blocks beneath the ehest and the pelvis, so that the limbs may hang over the end and sides of the dissecting table. To make tense the neck, the head is to be depressed and fastened with hooks.

In this region there are five successive strata of muscles, amongst which vessels and nerves are interspersed.

Dissection. The first step is to raise the skin in two flaps, by means of the following incisions:-One cut is to be made along the middle of the body from the occipital protuberance to the back of the sacrum. Another is to be carried from the last dorsal vertebra to the acromion process of the scapula. The flap of skin above the last cut is to be turned outwards by the dissectors of the head and upper limb.

By another transverse incision opposite the iliac crest, the remaining piece of integument may be detached, and reflected by the dissector of the upper limb in the same direction as the other flap.

Under the upper flap of skin is placed the trapezius, and underneath the lower one the latissimus dorsi muscle.

The cutaneous nerves may now be sought in the superficial fatty layer: they are accompanied by small cutaneous arteries which will guide the student to their position. The nerves vary much in size in the different parts of the Back, and their number is also irregular; as a general rule, there is one opposite each vertebra except in the neck.

To find them in the cervical region look near the middle line, from the $3 \mathrm{~d}$ to the 6th vertebra, and trace an offset from the third nerve upwards to the head: the branch of the second nerve (large occipital) has been laid bare at the back of the head (p. 20).

Opposite the upper part of the thorax, they will be best found near the spines of the vertebre, where they lie at first beneath the fat; but at the lower part, and in the loins, they issue in a line with the angles of the ribs.

The cutaneous branches of the sacral nerves are included in the dissection of the lower limb. 
Cutaneous Nerves. The tegumentary nerves are derived from the posterior primary branches of the spinal nerves, which subdivide amongst the deep muscles into two pieces, inner and outer. Arteries accompanying the greater number of the nerves, bifurcate like them, and furnish cutaneous offsets.

Cervical nerves. In the neck the nerves are derived from the inner of the two pieces into which the posterior trunks bifureate: they perforate the trapezius, and supply the neck and the back of the head. They are four in number, viz., one from each, except the first and the three last.

The branch of the second nerve is named large occipital, and accompanies the occipital artery to the back of the head (p. 23).

The branch of the third cervical nerve supplies a transverse offset to the neck, and then ascends to the lower part of the head, where it is distributed near the middle line, uniting with the great occipital nerve

* Dorsal nerves. These are obtained from both the inner and outer pieces-the upper six from the inner, and the lower six from the outer. On the surface they are directed outwards in the integument over the trapezius and latissimus dorsi muscles.

The upper six perforate the trapezins near the spines of the vertebra; and the branch of the second, which is larger than the rest, reaches as far as the scapula. The lower six pierce the latissimus dorsi mostly in a line with the angles of the ribs; they are oftentimes uncertain in number.

* Lumbar nerves. In the loins the nerves are derived from the outer pieces of the first three; they perforate the latissimus dorsi muscle at the outer border of the erector spina, and crossing the iliac crest of the innominate bone, are distributed in the integuments of the buttock.

* First Layer of Muscles (fig. 116). 'Two muscles, the trapezius and the latissimus dorsi, are included in this layer.

Dissection. The superficial fatty layer is to be taken from the trapezius and latissimus dorsi in the direction of the fibres of each, viz., firom the shoulder to the spinal column; and the upper limb is to be carried backwards or forwards according as it may be necessary to put on the stretch different portions of the museles.

Some of the cutaneous nerves and ressels may be lefi, in order that they may be traced afterwards through the muscles to their origin.

* The Trapezius Muscle (fig. $116,{ }^{\wedge}$ ) is triangular in shape, with the base towards the spine, but the two have a trapezoid form. The muscle has an extensive aponeurotic origin along the middle line from the spines of all the dorsal vertebræ and their supraspinous liganent ; from the spinous process of the seventh cervical vertebra ; from the ligamentum nucha between the last point and the head ; and lastly from the inner third of the superior transverse ridge of the occipital bone. From this origin the fibres are directed outwards, converging to the shoulder, and are inserted into the outer third of the clavicle, at its posterior aspect; into the posterior border of the acromion; and into the upper edge of the spine of the scapula as far as an inch from the root of that process, as well as into a rough impression on the surface of the spine near the hinder part.

The muscle is subcutaneous. At the outer side the lowest fleshy fibres end in a small triangular tendon, which glides over the smooth surface at the root of the spine of the scapula. The anterior border bounds behind the posterior triangular space of the neck. By its insertion the trapezius corresponds with the origin of the deltoid muscle.

Action. If all the fibres of the muscle act, the scapula gliding on the 
ribs is moved upwards and towards the spinal column; but the upper fibres can assist other muscles in elevating, and the lower fibres will help in depressing that bone.

When the scapula is prevented from gliding on the ribs, the trapezius imparts a rotatory movement to it, and raises the acromion.

Fig. 116.

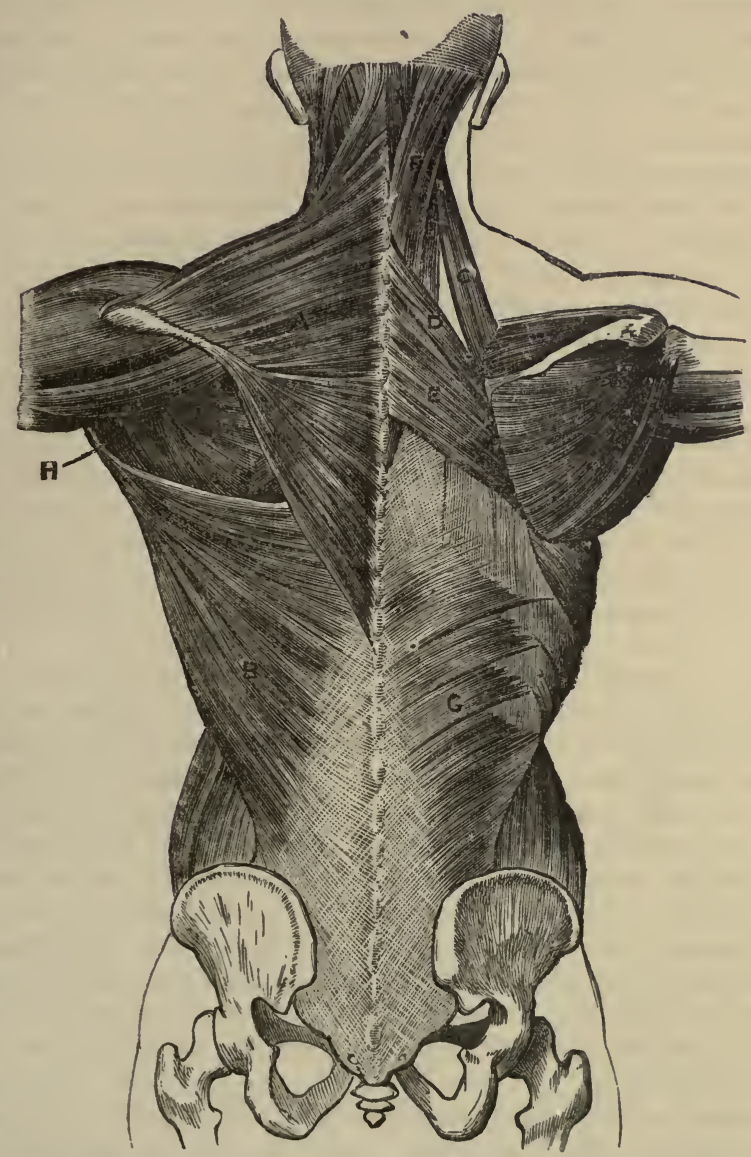

MUSCLES of THE BACK. On the left side the flrst layer is shown, and on the right side the second layer, with part of the third.
A. Trapezius.
B. Latissimus dorsi.
c. Levator anguli scapulx.
D Rhomboideus minor.

E. Rhomboideus major.

F. Splenius.

G. Serratus posticus inferior.

Dissection. The fibres of the trapezius are to be divided near the scapula, over the situation of the spinal accessory nerve, so that the ramifications of that nerve in the musele, and its junction with the branehes of the cervieal plexus may be observed. A small artery to the trapezius (art. superficialis colli) accompanies the nerve. 
The spinal accessory cranial nerve (p. 114), having crossed the posterior triangle of the neck, passes beneath the trapezius, and forms a plexiform union with branclies of the third and fourth nerves of the cervical plexus. The nerve is distributed nearly to the lower border of the musele.

Dissection. To see the parts covered by the trapezius, the pieces of the divided muscle are to be thrown inwards and outwards.

The dissector of the neck should now clean the splenius, and define the parts beneath the clavicle, viz. the posterior belly of the omo-hyoid musele with the suprascapular nerve and vessels; the transverse cervical vessels; and the small branches of nerves to the levator anguli scapula, and rhomboid muscles. If the trapezius be detached along the middle line, the ligamentum nuchæ, from which it takes origin, will be brought into view.

* The dissector of the upper limb should clean the fibres of the rhomboidei and levator anguli scapula muscles, which are fixed to the base of the scapula; and whilst this is being done, the scapula is to be drawn away from the trunk to make tense the fleshy fibres.

* Parts covered by the trapezirs. 'The trapezius conceals in the neck the splenius, a small part of the complexus, and the levator anguli scapulæ; in the dorsal region it covers the following muscles, the rhomboidei, the erector spinæ, and the latissimus dorsi. Near the insertion it lies over the supraspinatus muscle.

The ligamentum nuche is a narrow fibrous band, which extends from the spinous process of the seventh cervical vertebra to the occipital protuberance. From its under part processes are attached to the spines of the six lower cervical vertebra, so that it serves as a partition between the muscles of the opposite sides of the neck. In man it is not formed of elastic tissue.

* 'The LAtissimus Dorsi (fig. $116,{ }^{\mathrm{B}}$ ) is the widest muscle in the back, and is thin and aponeurotic at its inner attachment. It arises along the middle line from the spinous processes of the six lower dorsal, all the lumbar, and the upper two sacral vertebræ, as well as from the supraspinous ligament. On the outer side it arises by an aponeurosis from the outer edge of the posterior half of the iliac crest; and by three or four fleshy processes from as many of the lower ribs, which digitate with pieces of the external oblique muscle of the abdomen. And between the outer and inner attachments it is inseparably blended below with the subjacent tendon of the multifidus spina. All the fibres converge to the inferior angle of the scapula, and after crossing that point of bone, are continued forwards to be inserted by tendon into the bottom of the bicipital groove of the humerus (p. 236).

The muscle is subcutaneous, except a small part of the upper border which is covered by the trapezius. Near the seapula there is a space between the two, in which the rhomboid muscles appear. The lower or anterior overlays the edge of the external oblique muscle of the abdominal wall in the interval between the last rib and the iliac crest, with the exception of a small part below. Frequently the latissimus has a distinet fleshy slip from the inferior angle of the scapula.

Action. If the arm is hanging loose the muscle can move it behind the back, rotating it in at the same time. If the limb is raised, the latissimus, combining with the pectoralis and teres, will depress the humerus. From its attachment to the scapula this bone can be depressed with the arm.

Supposing the arm fixed, the fibres may elevate the ribs as an inspira- 
tory muscle; or they may assist the pectoralis major in drawing the movable trunk towards the humerus, as in the act of climbing.

* Dissection. The latissimus is to be divided about midway between the spines of the vertebræ aud the angle of the scapula, and the pieces are to be reflected inwards and outwards. In raising the inner part of the muscle, care must be taken not to destroy either the thin lower serratus with which it is united, or the aponeurosis continued upwards from the serratus. In the interval between the last rib and the iliac crest the latissimus is adherent to the aponeurosis of the transversalis abdominis muscle, and should not be detached from it.

* Parts covered by the latissimus. The latissimus dorsi lies on the erector spinæ, the serratus posticus inferior, and the lower ribs with their intercostal muscles. As it rests on the angle of the scapula, it conceals the teres major, and part of the rhomboid muscle. Its position to the teres is worthy of note :-at the angle of the scapula it covers the posterior surface of the teres, but nearer the humerus it turns round the lower border, and is inserted in front of that muscle. Between the angle of the scapula and the humerus the latissimus forms part of the posterior boundary of the axilla.

Dissection of fascia lumborum. After the latissimus dorsi has been reflected, the dissector of the abdomen can look to the disposition of the posterior tendon of the transversalis abdominis (fascia lumborum) between the last rib and the innominate bone.

In the spot referred to are portions of the external and internal oblique muscles, left in the dissection of the wall of the abdomen. After the removal of those muscles the aponeurosis of the transversalis musele (fascia lumborum) appears, and perforating it are two nerves-one, the last dorsal with an artery near the last rib: and the other, the ilio-hypogastric with its vessels, close to the iliac erest.

Two offsets are prolonged backwards from this fascia to the transverse processes. To see the more superficial layer which passes beneath the erector spinæ to the apices of the processes, the latissimus dorsi is to be cut through (both its aponeurosis and fleshy part) by a horizontal incision directed outwards from the spinous processes, on a level with the third lumbar vertebra. On raising the outer border of the erector spina muscle which comes into view, the strong process of the fascia will be apparent.

After dividing transversely this first prolongation, another muscle (quadratus lumborum) will be seen; and on raising its outer border the second thin offset of the fascia will be evident on the abdominal aspect of that muscle.

The fascia lumborum (fig. $136,{ }^{\mathrm{C}}$ ) is the posterior aponeurosis or tendon of the transversalis abdominis muscle, and occupies the interval between the last two ribs and the crest of the hip-bone. By its cutaneous surface it gives attachment to the internal oblique muscle, and sometimes to the external oblique. The last dorsal $\left({ }^{1}\right)$ and ilio-hypogastric $\left({ }^{2}\right)$ nerres, accompanied by vessels, pierce it in their course from the abdomen. From the inner part of the aponeurosis two offsets are prolonged to the transverse processes of the lumbar vertebra, and inclose the quadratus lumborum in a sheath.

The more superficial of the two is the strongest; it lies beneath the erector spinx in this position of the body, and is connected to the apices of the transverse processes, but it also fills the intervals between them : at 
the outer border of the erector spinæ it blends with the aponeurosis of the latissimus dorsi and inferior serratus.

The deeper or anterior prolongation passes on the abdominal surface of the quadratus lumborum, and is fixed to the tips and borders of the transverse processes.

In like manner the erector spinæ lies in another sheath, which is formed by the vertebral aponeurosis and the tendons of the latissimus and serratus on the one side, and by the superficial of the two prolongations of the fascia lumborum on the other.

* Second Layer of Muscles (fig. 116). This stratum contains the elevator of the angle of the scapula, and the large and small rhomboid muscles; besides these, the posterior belly of the omo-hyoid muscle, and some ressels and nerves turning backwards towards the scapula are included.

Dissection. By the reflection of the trapezius and latissimus, and by the dissection made subsequently (p. 355), the several parts in this layer will have been sufficiently prepared for learning.

* The levator anguli scapula (fig. $116,{ }^{c}$ ) arises by tendinous slips from the posterior transverse processes of the upper three or four cervical vertebra. The fibres form rather a roundish muscle, and are inserted into the base of the scapula between the spine and the superior angle.

At its origin the muscle lies beneath the sterno-mastoideus, and at its insertion beneath the trapezins, where it touches the serratus magnus muscle; the rest of the muscle appears in the posterior triangular space of the neck. Beneath it are some of the other cervical muscles, viz., splenius colli and cervicalis ascendens.

Action. The muscle raises the angle and hinder part of the scapula, and depresses the acromion; but united with the upper part of the trapezius, which prevents the rotation down of the acromion, it shrugs the shoulder.

When the shoulder is fixed, the neck can be bent laterally to the same side.

* Rhomboider Muscres. The thin muscular layer of the rhomboidei is attached to the base of the scapula, and consists of two pieces, large and small, which are separated by a slight interval.

* The rhomboideus minor (fig. $116,{ }^{D}$ ) is a thin narrow band, which arises from the spines of the seventh cervical and first dorsal vertebra, and the ligamentum nuchæ; it is inserted into the base of the scapula, opposite the smooth surface at the root of the spine.

* 'The rlomboideus major'(fig. 116, ${ }^{\mathrm{E}}$ ) is larger than the preceding by the width of three or more spinous processes. It arises from the spines of four or five dorsal vertebre below the rhomboirleus minor, and from the supraspinous ligament; and its fibres are directed outwards and downwards to be fixed to the base of the seapula between the spine and the lower angle. Sometimes all the fibres do not reach the seapula directly, some ending on a tendinous arch near the bone.

The rhomloidei muscles are covered chiefly by the trapezius and latissimus; but a portion of the larger rhomboid is subcutaneous near the angle of the scapula.

Action. From the direetion of their fibres both rhomboidei will draw the base of the scapula upwards and backwards, so as to depress the acromion. In combination with the trapezius they will carry the scapula 
directly back; for as one tends to raise, and the other to depress the acromion, the bone will be moved in a direction between the two forces. By their united action the muscles help to fix the scapula.

The OMO-HYOID MUSCLE consists of two fleshy bellies, anterior and posterior, which are united by an intervening tendon. Only the posterior half is now seen.

The muscle arises from the upper border of the scapula behind the notch, and from the ligament converting the notch into a foramen. The fibres form a thin, riband-like musele, which is directed forwards across the lower part of the neck, and ends anteriorly in a tendon beneath the sterno-mastoideus (p. 72). The fleshy belly of the muscle is placed partly beneath the trapezius; and is partly superficial in the posterior triangular space of the neck, where it lies abore the clavicle and the subclavian artery. It crosses the suprascapular vessels and nerve, and the brachial plexus near the scapula.

Action. For the supposed use of the posterior belly of the omo-hyoideus, see page 72 .

The suprascapular artery, a branch of the subclavian (p. 78), is directed outwards across the lower part of the neck to the dorsum of the scapula. The vessel courses behind the clavicle with the suprascapular nerve, but beneath the trapezius and omo-hyoid muscles to the supraspinal fossa. Before entering the fossa it furnishes a small branch (supra-acromial) to the upper surface of the acromion.

The suprascapular nerve, an offset of the brachial plexus (p. 79), is inclined backwards to the superior border of the scapula. It passes through the notch in the upper costa of the bone, and terminates beneath the supratspinatus in the muscles of the dorsum of the scapula (p. 248).

The transverse cervical artery, also a branch of the subclavian (p. 78), has the same direction as the suprascapular, towards the upper part of the scapula, but it is higher than the clavicle. Crossing the upper part of the space in which the subclavian artery lies, it passes beneath the trapezius, and divides into the two following branches-superficial cervical and posterior scapular:-

a. The superficial cervical branch is distributed chiefly to the under surface of the trapezius, though it furnishes offsets to the levator anguli scapula and the cervical glands.

$b$. The posterior scapular branch crosses beneath the elevator of the angle of the scapula, and turns along the base of the scapula beneath the rhomboid muscles. If the rhomboid muscles are divided, the artery will be seen to furnish branches to them; and to give small anastomotic twigs to both surfaces of the scapula. 'This branch arises very frequently from the third part of the subclavian trunk.

The supra-scapular and transverse cervical veins have the same course and branches as the arteries above described; they open into the external jugular, near its junction with the subclavian rein.

Nerve to the rhomboid muscles. This slender nerve of the brachial plexus (p. 79) courses beneath the elevator of the angle of the scapula, and is distributed to the rhomboidei on the under surface. Before its termination it supplies one or two twigs to the elevator of the scapula.

* Third Layer (fig. 117). In this stratum are the following muscles :the serratus posticus superior and inferior, with the splenius.

* Dissection. By reflecting the rhomboidei muscles towards the spinous 
processes, and removing loose areolar tissue, the thin upper serratus muscle beneath them will be laid bare.

The splenius and the inferior serratus have been previously exposed by the reflection of the trapezius and

Fig. 117.

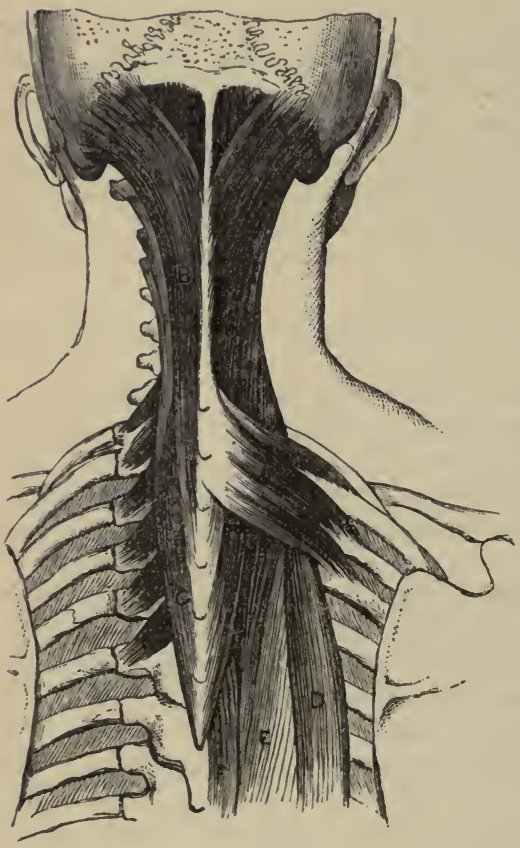

PART OF THB THIRD LAYER OP THE BACKMUSCles.

A. Serratns posticus superior.

B. Splenius capitis.

c. Splenius colli.

D. Ilio-costalis.

B. Longissimus dorsi.

P. Spinalis dorsi.

\section{latissimus.}

* The serrati muscles are very thin, and receive their name from their toothed attachınent to the ribs. They are two in number, superior and inferior, and have aponeurotic origins from the spines of the vertebra.

* The serratus posticus superior (fig. $117,{ }^{A}$ ) arises from the ligamentum nuchæ, and from the spinous processes of the last cervical, and two or three upper dorsal vertebra. The fleshy fibres are inclined down and out, and are inserted by slips into the second, third, and fourth ribs, external to their angles.

The muscle rests on the splenius, and is covered by the rhomboideus major.

* The serratus posticus inferior (fig. $116,{ }^{G}$ ) occupies the lumbar region, and is wider than the preceding muscle. Its aponeurosis of origin is inseparably united with that of the latissimus dorsi, and with the fascia lumborum, and is connected to the spinous processes of the last two dorsal and first three lumbar vertebra. The fleshy fibres ascend to be inserted into the last four ribs in front of their angles, each successive piece extending further forwards than the one below.

This muscle lies on the mass of the erector spinx; and with its tendon the vertebral aponeurosis is united.

Action. The superior serratus raises the upper ribs, and officiates as an inspiratory muscle; and the inferior, depressing the lower ribs, becomes an expiratory muscle. Theile supposes the inferior to act indirectly as a muscle of inspiration, because, by fixing the lower ribs, it enables the diaphragm to contract more effectively.

* The vertebral aponeurosis is a fibrous expansion, which is spread over the fourth layer of museles, and confines the erector spina in the vertebral groove.

Inferiorly it is thickened by the tendons of the latissimus and lower serratus; but it is continued alove beneath the splenius, without joining the upper serratus, and blends with the deep fascia of the neck. 
Internally it is attached to the spinous processes. Externally it is connected to the posterior third of the iliac crest, uniting with the tendon of the latissimus; between the hip-bone and the ribs, to the fascia lumborum; and in the dorsal region to the ribs and a fascia over the intercostal muscles. Below, between the pelvic and vertebral attachments, it blends with the underlying tendon of the erector spinæ and multifidus.

* Dissection. The upper serratus is to be cut through, and the subjacent vertebral aponeurosis to be taken away; and the part of the splenius muscle under the serratus should be cleaned.

The splexius muscle (fig. 117) is thin and elongated; it is undivided internally, but is split externally into two parts. It takes origin along the middle line from the six upper dorsal spines, from the seventh cervical, and from the ligamentum nuche as high as the third cervical vertebra. Some fibres ascend to the head (splenius capitis), and others to the neck (splenius colli).

The splenius colli, c, is inserted by tendinous slips into the posterior transverse processes of the upper three cervical vertebre with, but behind the attachment of the elevator of the angle of the scapula.

The splenius capitis, $\mathrm{B}$, much the largest, is inserted by a thin tendon into the apex and hinder border of the mastoid process, and into the bone behind it for about an inch and a half.

This muscle is situate beneath the trapezius, the rhomboidei, and the serratus superior; and the insertion into the occipital bone is beneath the sterno-mastoidens. The complexus muscle projects above the upper border of the splenius capitis.

Action. The cranial parts of both muscles will carry the head directly back; and one will turn the face to the same side.

The splenius colli of both sides will bend back the upper cervical vertebræ; but one muscle will turn the face to the same side, being able to rotate the head by its attachment to the transverse process of the atlas.

* Fourtir Layer. In this layer are included the spinalis dorsi; the erector spinæ, with its divisions, and accessory muscles to the neck; and the complexus muscle. Most of the vessels and nerves of the Back are to be learnt with this layer of muscles.

Dissection (fig. 118). To lay bare the complexus muscle in the neck, the splenii must be detached firom the spinous processes, and thrown outwards.

And whilst the large erector spine is being displayed in the dorsal and lumbar regions by the dissector of the upper limb, two prolongations from it to the cervical vertebra and the head are to be defined by the dissector of the neck: One, a thin narrow muscle, the cervicalis ascendens, is continued beyond the ribs from the onter piece of the erector (ilio-costalis), and is to be separated from the muscles around. The other is a larger offset of the inner piece of the erector (longissimus dorsi); blended at first with the fibres of the longissimus, it is divided afterwards, like the splenius, into a cranial part (trachelo-mastoid) and a cervical part (transversalis colli).

* The serratus inferior is to be detached with the vertebral aponeurosis from the spines in the dorsal region, and the areolar tissue is to be cleaned from the surfice of the large mass of the erector spina which now comes into view. Opposite the last rib is the beginning of an intermuscular interval, which divides the erector spina into an outer piece (sacro-lumbalis), and an inner (longissimus dorsi). By sinking the knife into this interval 
the sacro-lumbalis may be turned outwards, so as to uncover the fleshy slips of its accessory muscle, which are fixed to the angles of the ribs: a muscular slip (cervicalis ascendens) is prolonged from this to the neck.

* In preparing the sacro-lumbalis muscle, the external pieces of the dorsal nerves with their accompanying arteries will appear.

* Before the longissimus can be displayed, it will be needful to detach, and raise towards the spinous processes the thin muscular fasciculus of the spinalis dorsi, which lies between that muscle and the spines of the vertebræ in the dorsal region. Then the attachments of the longissimus dorsi are to be traced out. Externally it has thin muscular processes of insertion into about the eiglit lower ribs. Internally it is inserted into the transverse processes of the lumbar and dorsal vertebræ by rounded tendons; and for the purpose of seeing these tendons, the longissimus should be drawn away from the spinous processes, and its superficial aponeurosis should be cut through below the ribs, along the line of separation between the muscle and the fleshy multifidus spinæ on the inner side. From this muscle, as from the sacro-lumbalis, a fleshy piece (transversalis colli and trachelo-mastoid) is continued into the neck.

* Between the longissimus and the multifidus spina are the internal pieces of the dorsal and lumbar nerves, and of the intercostal and lumbar arteries and veins.

* The spinalis Dorsi is placed on the side of the spines of the dorsal vertebræ, and is united with the longissimus dorsi. Inferiorly it arises by tendinous processes from the spines of the last two dorsal and first two lumbar vertebre, and by fleshy fibres from the contiguous tendon of the longissimus. From this origin the fibres ascend, forming arches, whose concavity looks inwards, and are connected by tendinous processes to the spines of the dorsal vertebræ as low as the eighth or ninth, or only for half that extent.

Action. Both muscles contracting will extend the dorsal region of the spine. Perhaps the muscle of one side may tend to incline the spine laterally.

* 'The erector SPINe is the muscular mass on the side of the spine in the lumbar region. It is single and pointed below : and its cutaneous surface is covered near the sacrum by a wide and strong tendon, which is common to it and the multifidus spinæ. The muscle arises at the pelvis from the posterior fifth of the crest of the hip-bone at the inner aspect, except opposite the upper spinous process : in the lumbar region it is attached by fleshy and tendinous pieces to the transverse processes, to the tubercle (process. accessorius) at the root, and to the layer of the fascia lumborum external to each. Opposite the last rib it divides into ilio-costalis and longissimus dorsi.

* The ilio-costalis (sacro-lcmibalis) is the smallest of the two pieces resulting from the division of the erector spinæ. Its fibres end in six or seven flat tendons, which are connected together by their margins, and are inserted into the angles of as many of the lower ribs. The muscle is continued onwards to the other ribs and the neck by a fleshy part, which constitutes the two under-mentioned muscles:-

* The musrulus accessorius ad sacro-lumbalum begins by a series of tendinous and fleshy bundles on the angles of the lower six ribs, internal to the insertion of the ilio-costalis ; and it ends in tendons, which are inserted into the remaining ribs (upper six), in a line with the ilio-costalis, and into the posterior transverse process of the seventh cervical vertebra. 
The cervicalis ascendens is a muscular slip prolonging the accessorius into the neck : this muscle is attached to four ribs (third, fourth, fifth, and sixtlı), and is inserted into the posterior transverse processes of three cervical vertebre, viz., sixth, fifth, fourth.

* The Loxgissimus Dorsi gradually decreases in size as it ascends along the thorax. Internally the muscle is inserted into the transverse processes of all the dorsal vertebre by a series of tendinous and fleshy bundles; and externally it is attached to the ribs, except the first two or three, by thin fleshy processes between the tubercle and angle. Its muscular prolongation to the neck is united with the upper fleshy fibres, and splits into the two following pieces:-

The transversalis colli (fig. $118,{ }^{\mathrm{B}}$ ) arises from the transverse processes of the upper six dorsal vertebre, and is inserted into the posterior transverse processes of the certical vertebre, except the first two and the last.

The trachelo-mastoid muscle (fig. $118,{ }^{\mathrm{c}}$ ) (transversalis capitis?) arises in common with the preceding, and is attached, besides, by distinct tendons to the articular processes of the last three or four cervical vertebra. The muscle is thin, and is inserted beneath the splenius into the upper half of the posterior part of the mastoil process : its insertion is about three-quarters of an inch wide. ${ }^{1}$

* Connections of the erector spina. The erector spinæe and its prolongations occupy the lumbar, thoracic, and cervical parts of the Back.

In the loins the muscle is contained in an aponeurotic sheath (p. 358), and has the multifidus spinæ on its inner side: its attachment on the inner surface of the innominate bone corresponds in part with the origin of the gluteus maximus on the outer side. The superfieial tendon, which is common to it and the multifidus, will be described with the last-mentioned muscle (p. 371).

Opposite the ribs the ilio-costalis and longissimus dorsi are concealed by the muscles of the other layers already examined.

In the neck its accessory small muscles lie underneath the splenius and the trapezius:- the cervicalis ascendens is attached in a line with, but below the splenius colli ; and the transversalis colli and trachelo-mastoid are more internal, or between the splenius and cervicalis and the complexus.

Action. Taking their fixed point at the pelvis, both erectors will keep the spine straight in sitting and standing; and, in rising from stooping to the ground, they will bring the trunk into the erect posture. In laborious respiration, the spine being fixed, the muscles are able to depress the ribs, and assist in the expulsion of the air fiom the thorax.

One muscle will incline the spinal column laterally and to its own side.

The cervical prolongations of the erector act on the neck and head, as below :

The cervicalis ascendens. Taking their fixed point below, both muscles will extend the cervical part of the spine: and acting from the transverse processes they will elevate the ribs. One muscle will give a lateral movement to the neek.

1 The anatomy of the prolongation from the longissimus might be simplified by describing it as the transversalis muscle with a double insertion, like the splenius, into the head and neck. In accordance with the nomenclature of the splenius the part to the head might be named transversalis capitis, and the part to the neck transversalis colli, as at present. 
The transversalis colli bends back the neck if the muscles of both sides contract together; or laterally towards its own side, if only one is used.

The trachelo-mastoidens will extend the head in concert with its fellow; or by itself will turn the face to its own side, and then help to approximate the head to the shoulder.

The complexus (fig. 118, D) is internal to the prolongations from the longissimus dorsi, and converges towards its fellow of the opposite side at

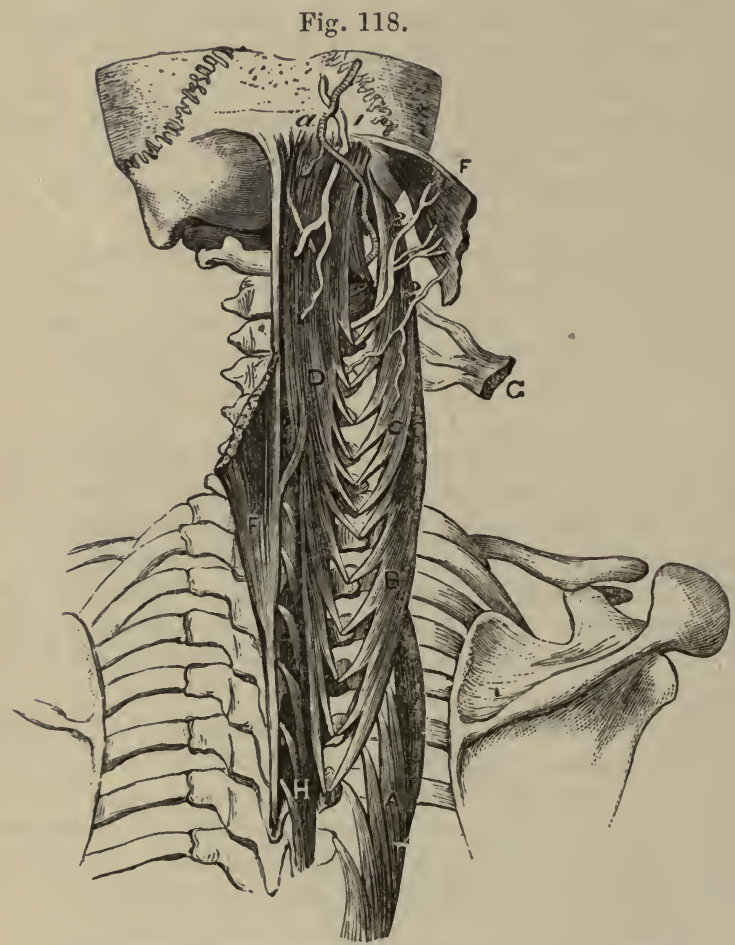

Dissection of the MUSCles Underneatil ThR SPLinidg.
A. Longus colli.
B. Transversalis colli.
C Trachelo-mastoid.
D. Complexus.
P. Splenins capitis, cut.
G. Splenius colli, cut.

H. Semispinalis dorsi.

a. Occipital artery.

1. Great occipital nerve.

2. External piece of the second nerve.

3. Outer piece of the third nerve.

the occipital bone. Narrow at its lower end, the muscle arises by tendinous pieces from the transverse proeesses of the upper six dorsal verte. bra, from the spine of the last cervical, and from the articular processes of the cervical vertebra as high as the third. The fleshy fibres pass upwards to be inserted into an impression between the curved lines of the os occipitis, which reaches outwards nearly two inches from the occipital crest towards the trachelo-mastoideus.

The inner part of the complexus having two fleshy bellies with an intervening tendon, is described often as a separate muscle with the name biventer cervicis. 
The complexus is concealed by the splenis and trapezius : and the cutaneous surface is marked by a tendinous cross intersection towards the upper end. Two or three of the cervical nerves perforate it. Along the inner side is the semispinalis muscle, with the ligamentum nucha. Beneath it are the small recti and obliqui muscles, the semispinalis, and the cervical nerves and vessels.

Action. Both muscles will move the head directly back. One will draw the occiput down and back towards its own side.

Dissection of vessels and nerves (fig. 119). In the neck the nerves and vessels will be brought into view by detaching the complexus from the occipital bone and the spine of the seventh vertebra, and throwing it outwards carefully from the subjacent parts. Beneath the muscle is a dense fascia, in which are contained the ramifications of the cervical nerves, and the deep cervical and other vessels.

Each nerve except the first divides into an inner and an outer piece. Dissect out first the inner pieces of the seven lowest, which lie partly over and partly beneath the fibres of the semispinalis muscle (G). The external pieces $\left({ }^{3}\right)$ are very small; they are given off between the transverse processes close to where the trunks appear; they are to be looked for outside the complexus, and enter the muscles prolonged from the erector spinæ.

The first or suboccipital is the most difficult of the set to find: this little nerve is a short trunk, which is contained in the interval between the small recti and obliqui muscles near the head; it will be best found by looking for the small twigs furnished by it to the muscles around.

The deep cervical artery is met with on the semispinalis muscle; a part of the vertebral artery will be found in contact with the suboccipital nerve; and the occipital artery will be seen crossing the occipital bone.

* Opposite the thorax the dorsal nerves and vessels will be readily displayed on the inner side of the longissimus dorsi muscle, on the removal of a little fatty tissue from between the transverse processes. External and internal pieces are to be traced from each nerve and vessel into the muscles; some of the former have been seen in the interval between the ilio-costalis and the longissimus dorsi.

* The two pieces of the lumbar nerves and ressels will be found in the same line as the dorsal; but the inner set are the most difficult to be discovered.

* The small sacral nerves are placed beneath the multifidus spinæ, and will be dissected after the examination of that muscle (p. 372).

* Posterior Primary Branches of the Spinal Nerves. The spinal nerves, with a few exceptions in the cervical and sacral groups, bifurcate in the intervertebral foramina into anterior and posterior primary branches (p. 165). The posterior supply the integuments and the muscles of the back, and are now to be learnt.

In the neck. The posterior primary branches of the cervical nerves are eight in number, and issue between the transverse processes; but those of the first and second, which begin on the neural arches of the atlas and axis, cross those arches. All, except the first, divide into internal and external pieces.

The external pieces (fig. 118) are very inconsiderable in size, and end in the splenius, and in the muscles prolonged from the erector spinx.

The internal pieces (fig. 119) are larger than the external; they are directed towards the spinous processes, the three lowest nerves passing beneath the semispinalis, and the four next over that muscle. By the side 
of the spine cutaneous branches are furnisher to the neck and the head by the nerves that are superficial to the semispinalis; these cutaneous offsets ascend to the surface through the splenius, the complexus, and trapezius muscles, and are distributed as before seen (p. 354). In their course to

Fig. 119.

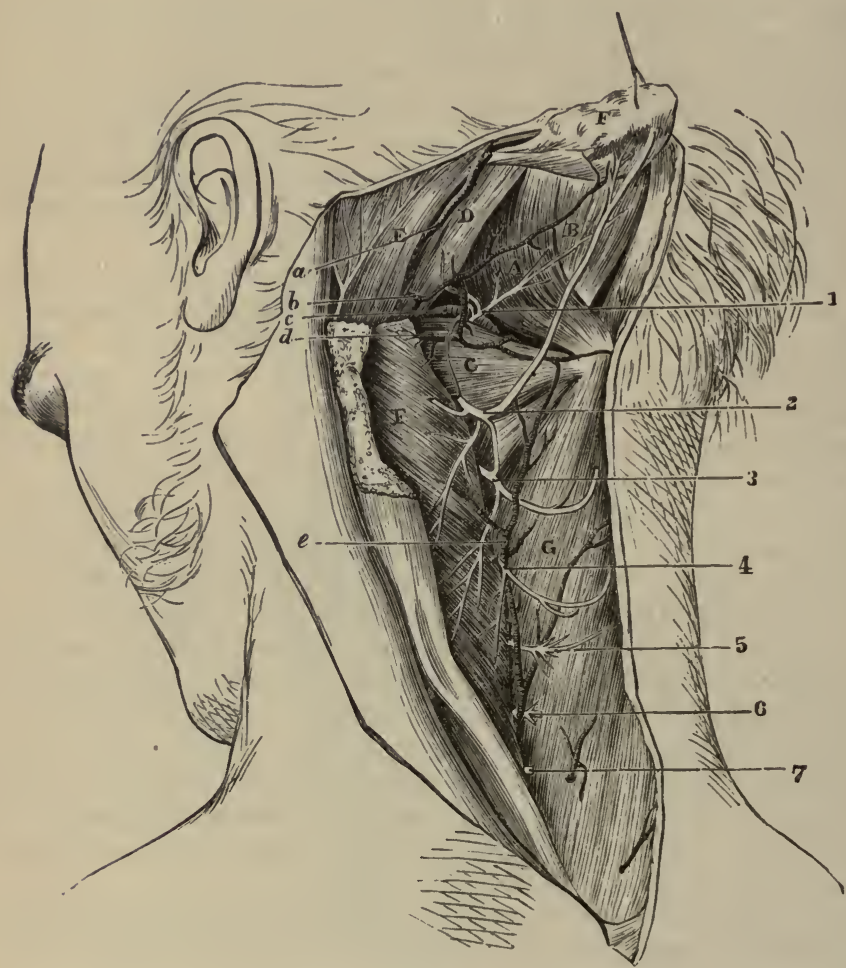

DeEP Dissection of THE BACK OF THE NECK. (Illustrations of Dissections.)

\section{Muscles :}

A. Rectus posticus inajor.

B. Rectus posticus minor.

c. Obliquus inferior.

D. Obliquus superior.

E. Spleuius capltis.

F. Complexus, cut across.

G. Sernispinalis colli.

Arteries :

$a$. Occipital, and $b$, its cervical branch.

c. Vertebral artery, and $d$, its cervical branch.

e. Deep cervical artery of the subclavian.

\section{Nerves :}

1. Suboccipital nerve (posterior branch).

2. Inner piece of the posterior primary branch of the second nerve.

3. Inner piece of the third nerve.

4. Inner piece of the fourth.

And the remaining figures point to the inner pieces of the respective nerves.

the spine the nerves supply the surrounding muscles, viz., complexus, semispinalis, multifidus spina, and interspinales.

The cutaneous branches of the second and third nerves reach the head, and require a separate notice.

That of the second nerve (fig. $119,{ }^{2}$ ), named great occipital, appearing 
beneath the inferior oblique muscle to which it gives offsets, is directed upwards through the complexus and trapezius to end on the occiput (p. 23).

The branch of the third nerve (fig. $119,{ }^{3}$ ) supplies an offset to the integuments of the neck; and ascending to the head through the trapezius, is distributed to the lower part of the occiput, internal to the great occipital nerve. Usually this nerve joins the preceding both beneath, and superficial to the trapezius.

The posterior primary branch of the suboccipital or first spinal nerve (fig. $119,{ }^{1}$ ) is very short, and appears in the interval between the recti and obliqui muscles. In passing from the spinal canal it is placed between the arch of the atlas and the vertebral artery. The following branches radiate from its extremity:-

One enters the under surface of the complexus near the cranial attachment. A slender branch is furnished to each of the small muscles bounding the space in which the nerve is contained, viz., the rectus major and minor, and the superior and inferior oblique : the offset to the last muscle joins the inner branch of the second cervical nerve. Occasionally this nerve gives a cutaneous branch to the occiput.

Posterior cervical plexus. Sometimes there is an intercommunication between the suboccipital nerve and the internal pieces of the next two cervical nerves beneath the complexus; this forms the posterior cervical plexus of M. Cruveilhier.

* In the dorsal region. The posterior primary branches of the dorsal nerves are twelve in number, and appear between the transverse processes. Each divides into an internal and an external piece; and these are distributed after the same plan as in the neck.

* The external pieces increase in size from the first to the last, and are differently distributed above and below. The upper six or eight pass beneath the longissimus and its cervical prolongation, as far as the interval between the longissimus and the ilio-costalis, and end by supplying these muscles and the levatores costarum. The lower six or four have a similar arrangement and distribution with respect to muscles; but, after reaching the interval between the ilio-costalis and the longissimus dorsi, they are continued to the surface through the serratus and latissimus muscles, nearly in a line with the angles of the ribs.

* The internal pieces decrease in size from above downwards, and are directed inwards between the semispinalis dorsi and multifidus spinæ muscles; offsets are supplied to the muscles between which they are placed. The upper six become cutaneous along the sides of the spinous processes by perforating the seratus, rhomboideus, and trapezius. 'The lower six are small in size, and end in the multifidus spinæ muscle.

* In the loins. 'The posterior primary branches of the lumbar nerves, five in number, appear between the erector and multifidus spinae. In their mode of dividing and general arrangement they resemble the dorsal nerves. Cutaneous offsets are furnished by the external set of branches.

* The external pieces enter the erector spinx, and supply it and the small intertransverse muscles. 'The first three pierce the erector spinæ, and become cutaneous after perforating the aponeurosis of the latissimus. The branch of the last nerve is connected with the corresponding part of the first sacral nerve by an offset near the bones.

* The internal pieces are furnished to the multifidus spinæ muscle. Near their origin they are difficult to find, in consequence of being contained in grooves on the articular processes. 
* Vessels in the back. The vessels now dissected are the occipital and the deep cervical; part of the vertebral; and the posterior branches of the intercostal and the lumbar arteries of the aorta. Veins accompany the arteries for the most part.

In the neck. The vessels in the neck are the occipital, the vertebral, and the deep cervical.

The occipital artery (fig. 119, a) courses along the occipital bone. Appearing from beneath the digastric muscle, the vessel is directed backwards beneath the sterno-mastoideus, the splenius, and sometimes the traehelo-mastoideus, but over the obliquus superior and complexus muscles. Near the middle line it perforates the trapezius, and ascends to the occiput, on which it is distributed (p. 21). It supplies the surrounding muscles, and furnishes the following branch to the neck :-

The cervical branch $(b)$ (ram. princeps cerviealis) distributes twigs to the under part of the trapezius, and passing beneath the complexus, anastomoses with the vertebral and deep cervical arteries.

The vertebral artery (fig. 119,c) lies on the neural arch of the first vertebra, behind the articulating process, and appears in the interval between the straight and oblique muscles. Beneath it is the suboccipital nerve. Small branches are given to the surrounding parts, and to anastomose with the contiguous arteries.

The deep cervical artery (fig. $119, e$ ) is a branch of the superior intercostal (p. 78), and resembles the posterior branches of the other intercostal arteries. Passing back between the transverse process of the last cervical vertebra and the neck of the first rib, it ascends between the complexus and semispinalis muscles, as high as the upper border of the latter, and anastomoses with the cervical branch of the occipital artery. The contiguous muscles receive branches from it, and anastomoses are formed between its offsets and those of the vertebral.

* In the dorsal region. The posterior branches of the intercostal vessels (p. 337) pass back between the vertebra and the anterior costo-transverse ligament, and are divided like the nerves into inner and outer pieces.

* The inner branches end in the fleshy mass of the multifidus spinæ and semispinalis, and furnish small cutaneous offsets with the nerves.

* The external branches cross beneath the longissimus dorsi, and supply it and the ilio-costalis. Like the nerves, the lowest branches of this set are the largest and extend to the surface.

As the dorsal branch of the intercostal artery passes by the intervertebral foramen, it furnishes a small intraspinal artery to the spinal canal.

* In the loins. 'The posterior branches of the lumbar' arteries divide, like the intercostal, into internal and external pieces, as soon as they reach the interval between the longissimus dorsi and multifidus spinæ. Each gives also a spinal branch to the spinal canal.

* The internal branches are small, and end in the multifidus spinx muscle.

* The external branches supply the erector spinæ; and offsets are continued onwards to the integuments with the superficial nerves.

Verss. With the deep cervical artery is a large vein, vena profunda cervicis, which communicates with the occipital and other deep veins in this region, forming the posterior plexus of the neck, and passes forwards with its artery, between the transverse processes, to join the vertebral vein. 
The occipital vein lies with its artery, and communicates sometimes with the lateral sinus of the skull through the mastoid foramen.

The dorsal and lumbar veins correspond in their branching and distribution with the arteries they accompany, and end in the intercostal veins and the vena cava.

In contact with the spinous processes and plates of the vertebræ is a deeper set of veins (dorsi spinal), which anastomose freely together, and open into the veins in the interior of the spinal canal.

* Fiftu LaYer. In this layer are the following small muscles: the recti and obliqui, semispinalis, interspinales, multifidus spinæ, and intertransversales.

Dissection. Most of the remaining muscles of the Back are uncovered by the previous dissection. Between the tirst two vertebræ and the occipital bone the small straight and oblique museles extend.

* In the cervical and dorsal regions the semispinalis muscle appears, with the small interspinales internal to it ; and occupying a corresponding position in the loins, is the multifidus spinæ.

* The small intertransverse muscles of the lumbar region will be found by removing the erector spinæ.

The RECTUS CAPITIS POSTICUS MAJOR (fig. 119, A) is the largest of the muscles between the occipital bone and the first two vertebræ, and arises from the side of the spinous process of the axis. It is inserted into the outer part of the inferior curved line of the oceipital bone for about an inch, as well as into the surface below it.

The muscle is directed outwards very obliquely, and forms one side of the triangular space which contains the suboceipital nerve and the vertebral artery. Its upper attachment lies beneath the superiol oblique musele.

Action. By the action of both muscles the head will be put backwards. By one rectus the face will be turned to the same side; and after the head has been so rotated, the muscle will assist in extending the head.

The RECTUS Capitis posticus minor (fig. 119, $\mathrm{B}$ ) is internal to the preceding, and is shorter than it. Arising from the neural arch of the atlas, the muscle is inserted, close to the middle line, into the inferior curved ridge of the occipital bone, and between this and the formen magnum.

This small musele is fan-shaped, and is deeper than the rectus major: it covers the ligament between the atlas and the occipital bone. The two small reeti museles correspond with the inter-spinales between the other vertebræ.

Action. Both muscles, or one, will have the same use, viz., to approach the occiput to the atlas.

The obliques inferion (fig. 119, c) slants between the first two vertebra. It arises from the spinous process of the axis, external to the rectus major muscle, and is inserted into the tip of the transverse processes of the atlas.

Action. One muscle turns the face to the same side by rotating the atlas on the axis. If both muscles act at the same time they will assist in keeping the head straight.

The OBLIQUUS SUPERIOR (fig. 119, D) takes origin from the upper part of the transverse proeess of the atlas, where the preceding muscle terminates; and is directed inwards to be inserted between the eurved lines of the occipital bone, near the mastoid process. 
This muscle is concealed by the complexus and trachelo-mastoideus, and crosses the vertebral artery. Its insertion is beneath the splenius (E), but above the rectus major muscle.

Action. With its fellow the upper oblique will assist in carrying backwards the head. By the action of one muscle the occiput will be inclined backwards to the same side.

The semispinalis (fig. 119, G) occupies the vertebral groove in the dorsal and cervical regions, and extends from the transverse and articular processes to the spines of the vertebre; it is undivided at the outer, but bifurcated at the inner attachment. It arises externally from the transverse processes of the ten upper dorsal vertebra, and from the articular processes of the four lower cervical ; and it is inserted by two pieces, as below :-

The lower piece, semispinalis dorsi, is inserted into the spinous processes of the upper four dorsal and the last two cervical vertebrx.

The upper piece, semispinalis colli, is inserted into the spines of the cervical vertebræ above the attachment of the semispinalis dorsi, the atlas not receiving any slip.

The semispinalis muscle is covered by the complexus, and the deep cervical artery. Some of the cervical nerves are superficial, and others beneath it. To its inner side is the multifidus spinæ muscle.

Action. The muscles of both sides acting together will extend the spine. One muscle will rotate the cervical and dorsal parts of the spine, so as to turn the face to the opposite side.

* The interspinal MUSCLES are placed, as their name expresses : they are arranged in pairs, one on each side of the interspinous ligament; and they are best seen in the neck and loins.

In the cervical region the muscles are absent from the interval between the first two vertebræ. They are small round bundles, and are attached above and below to the bifurcated apices of the spines.

* In the dorsal region the muscles are rudimentary ; they exist between the first two, and tlie lowest two pair of spinous processes, and between the last dorsal and the first lumbar vertebra.

* In the lumbar region they are thin flat muscles, which reach the length of the spines.

Action. By the approximation of the spinous processes these small muscles will help in extending the spine. Necessarily the movement of each pair is very slight, but the aggregate of all would amount to perceptible motion.

* The intertransverse muscles lie between the transverse processes of the vertebræ; but only those in the loins and the back are now dissected.

In the neck they are double, like the interspinal muscles of the same vertebræ (p. 165).

* In the dorsal region they are single rounded bundles, and are found only between the lower processes : their number varies from three to six.

* In the lumbar region the anterior set are four thin and fleshy planes. The posterior set are rounded bundles, which are attached to the accessory points at the roots of the transverse processes: these have been named interaccessorii.

Action. The small intertransversales help to incline laterally the spine by approximating the transverse processes; the motion between a single 
pair of bones would be scarcely appreciable, as in the case of the interspinales.

Dissection. The multifidus spinæ muscle, which fills the hollow by the side of the spinous processes, may be now dissected. The upper part of the muscle is to be prepared and learnt by the dissector of the head and neck. It will be laid bare by cutting through the insertion of the semispinalis, and everting this.

* Over the sacrum the thick aponeurosis covering the multifidus and the erector spina must be turned aside. In the dorsal region the muscle will appear on detaching, and drawing the semispinalis from the spines.

* The MUltifidus sprNe musele extends from the sacrum to the second vertebra, and is much larger towards the pelvis than in the neck.

On the back of the sacrum it takes origin between the central and external row of processes, as low as the fourth aperture; from the inner surface of the iliac spine (posterior superior) of the hip bone; and from the ligaments connecting this bone to the sacrum. It is attached differently along its outer edge (origin) in the several regions of the spine :In the loins it arises by large fasciculi from the accessory and the articular processes. In the dorsal region, from the transverse processes. And in the neck, from the articular processes of the five lower vertebræ. From these attachments the fibres are directed obliquely inwards, some extending more than the length of one vertebra to be inserted into the spines and the neural arches of the vertebræ from the second cervical to the third sacral.

'This muscle fills chiefly the vertebral groove, and is concealed by the erector spinæ and the semispinalis. The internal branches of the vessels and nerves in the back lie along its outer border. The small muscles described below may be said to be parts of the multifidus.

Action. By the use of the muscle of both sides, the spinal column can be extended; and by the contraction of one, the spine will be rotated in the dorsal region and the neck, the face being turned to the opposite side. * Rotatores dorsi ('Theile). These are eleven small muscles beneath the multifidus spinæ in the dorsal region, and are separated from that muscle by fine areolar tissue. Each is attached inferiorly to the tip and upper edge of a transverse process, and superiorly to the lower border of the neural arch of the vertebra next above. The first springs from the transverse process of the second vertebra.

Action. These small rotators will assist the larger muscle (multifidus) in turning the trunk to the opposite side.

* The aponeurosis common to the multifidus and erector spinæ is fixed firmly to the surrounding bones, and furnishes attachment to the muscular fibres. In the middle line it is united with the spines of the lower lumbar vertebræ and sacrum. On the outer side it is attached to the posterior part of the iliac crest, and to the outer row of tubercles on the back of the sacrum, being connected at the last spot with the great sacro-sciatic ligament. Above, it is continued some way on the surface of the erector spina, but furtler on the longissimus dorsi than the ilio-costalis. Below, the latissimus dorsi and the vertebral aponeurosis blent.with its cutaneous surface (p. 360).

* Dissection (fig. 120). To find the branches of the sacral nerves, it will be necessary to remove the part of the multifidus spine which covers the sacrum. The first three are split into two each : their external pieces will be found readily on the great sacro-sciatic ligament, from which they 
may be traced inwards; the inner branches are very slender and difficult to be recognized.

The lowest two nerves are very small, and are to be sought on the back of the sacrum, below the attachment of the multifidus spina. They are not bifurcated like the others, but unite together, and with the coccygeal nerve in loops. The fourth comes through a sacral aperture, the fitth between the sacrum and coccyx; and the coccygeal is still lower.

Sacral Nerves (fig. 120).- The posterior primary branches of the sacral nerves are five in number. Four issue from the spinal canal by the apertures in the back of the sacrum, and the fifth between the sacrum and the coccyx. The first three have the common division into inner and outer pieces, like the other spinal nerves; but the last two are undivided.

* The first three nerves are covered by the multifidus spinæ; and divide regularly.

The inner pieces $\left({ }^{1}\right)$ are distributed to the multifidus; and the last of this set is very fine.

The outer pieces $\left({ }^{2}\right)$ are larger, and have communicating offsets from one to another on the back of the sacrum : further, the branch of the first

Fig. 120.

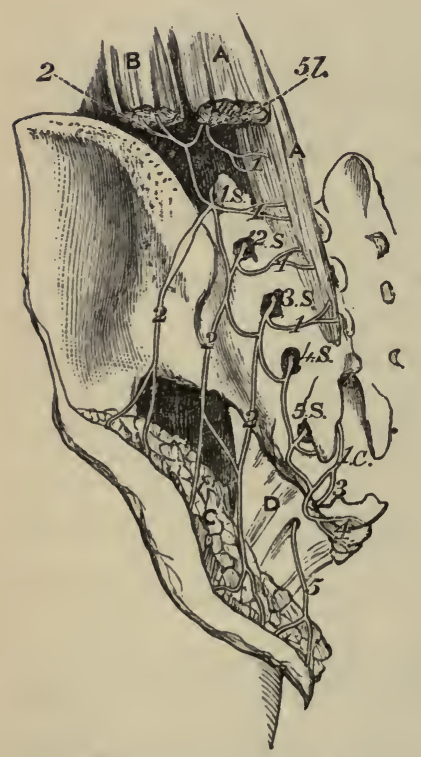

Muscles :

A. Multifidus spinæ, and

B. Erector spinæ: both cut.

c. Gluteus maximus detached from its origin, and thrown down.

D. Great sacro-sciatic ligament.

Nerves :

5 l. Last lumbar.

I $S$ to $5 S$, the five sacral nerves issuing from the sacrum.

$1 c$. The coccygeal nerve, escaping by the opening of the sacral canal.

1. Internal offsets of the last lumbar and first three sacral (these are too large in the cut): and

2. External offsets of the same nerves.

3. Anterior primary branch, and

4. Posterior of the coccygeal nerve.

5. The nerve derived from the anterior primary branches of the lower sacral nerves, which plerces the sacro-sciatic ligament.

Dissection of the Sacral Nerves (Lond. Med. Gazette, 1843).

nerve is connected with the corresponding part of the last lumbar; and the branch of the third joins in a similar manner the sacral nerve next below. After this looping they pass outwards to the surface of the great sacro-sciatic ligament, where they join a second time, and become cutaneous. (Dissection of the Buttock.)

* Last two nerves ( $4 S$ and $5 S$ ). These nerves, which are below the multifidus, are much smaller than the preceding, and want the regular branching of the others : they are connected with each other and the coc- 
cygeal nerve by loops on the back of the sacrum. A few filaments are distributed to the back of the sacrum and the coccyx.

Coccygeal nerve $(1 c)$. Its posterior primary branch issues through the lower aperture of the spinal canal, and appears by the side of the coccyx. It is joined by a loop from the last sacral nerve, and ends on the posterior surface of the coccyx.

* Sacral Arteries. Small branches leave the spinal canal with the sacral nerves; they supply the muscular mass of the erector spinæ, and anastomose with branches on the back of the sacrum from the gluteal and sciatic arteries.

* Dissection. The examination of the posterior part of the wall of the thorax may be made before the body is again turned. By removing, opposite the ribs, the ilio costalis and longissimus dorsi, the small levatores costarum will be uncovered. The hinder part of the external intercostal muscle will be denuded at the same time.

* The Levatores costarum are twelve small fan-shaped muscles, which are connected with the hinder part of the ribs. Each, except the first, arises from the apex and lower border of the transverse process of a dorsal vertebra; and is inserted, the fibres spreading out, into the upper border of the rib beneath, from the tubercle to the angle. The muscles increase in size from above down, and their fibres have the same direction as the external intercostal layer.

The first is fixed above to the transverse process of the last cervical vertebra, and below to the outer border of the first rib. Some of the four lower muscles are continued beyond one rib to that next succeeding: these longer slips have been named levutores longiores costarum.

Action. The muscles raise the hinder part of the ribs, as the name signifies, and the lowermost draw the bones somewhat back.

* The external intercostal muscle is continued backwards along the ribs as far as the tubercle, and is overlaid by the elevator muscle. Beneath the muscle are the intercostal nerve and artery.

Dissection. To trace the anterior and posterior primary branches of the dorsal nerves to their common trunk, the elevator of the rib and the external intercostal muscle are to be cut through in one or more spaces. The intercostal artery with its posterior branch is laid bare by this proceeding.

* The dorsal nerves split in the intervertebral foramina into anterior and posterior primary branches.

* The posterior branches are directed backwards, internal to the anterior costo-transverse ligament, and have been examined (p. 367).

The anterior, named intercostal, is continued between the ribs to the front of the chest: its anatomy is learnt in the dissection of the thorax (p. 343).

* The intercostal artery has an almost exact correspondence with the dorsal nerve in its branching and distribution. 


\section{CHAPTER VI.}

\section{THE SPINAL CORD AND ITS MEMBRANES.}

The spinal cord (medulla spinalis) gives origin to the spinal nerves, and is lodged in the osseous canal formed by the bodies and neural arches of the vertebræ. It is invested by prolongations of the membranes of the brain, which form sheaths around and support it.

Dissection. After all the muscles have been taken from the arches and spines of the vertebrex, the spinal canal is to be opened by sawing through the neural arches, on each side, close to the articular processes; and the cuts of the saw should extend to the lower end of the sacrum, but not higher in the neck than the fourth eervical vertebra. As it is difficult to use the saw in the hollow of the lumbar region, a chisel and a mallet will be found useful to complete the division of the vertebral arches.

The tube of the dura mater is covered by some veins and fat ; and by a loose areolar tissue containing fluid sometimes, especially at the lower part. The fat may be scraped away with the handle of the scalpel; and the lateral prolongations of the membrane through the intervertebral foramina are to be defined.

Membranes of the Cord (fig. 122). Three membranes, like those on the brain, surround the cord, viz., an external tube of dura mater, an internal sheath of pia mater, and an intervening arachnoid or serous covering.

The dura mater (a) forms a strong tube, and is continuous with the membrane lining the interior of the skull. It forms a loose sheath along the spinal canal, as far as the second or third piece of the sacrum (fig. 121); but beyond that point it is continued by a slender impervious cord to the back of the coccyx (fig. 121, b). The capacity of the sheath is greater than is needed for the contents; and its size is larger in the neck and loins than in the back.

On the outer aspect the dura mater is smooth, when a comparison is made between it and the part in the skull, for it does not act as a periosteum to the bones. Between it and the osseous surfaces are some vessels and fat; and it is connected to the posterior common ligament of the vertebræe by a few fibrous bands.

On each side the dura mater sends offsets along the spinal nerves in the intervertebral foramina ; and these several offsets become gradually longer inferiorly (fig. 121), where they form tubes which enclose the sacral nerves, and lie for some distance within the spinal canal. In the midst of the tubes, below, is the slender impervious cord $(b)$, which blends with the periosteum eovering the back of the coceyx.

Dissection. To remove the spinal cord with the sheath of the dura mater from the body, the lateral processes in the intervertebral apertures are to be cut; and one or two of them in the dorsal region should be followed outwards beyond the intervertebral foramen by cutting away the surrounding bone. The eentral prolongation may be now detached from 
the coccyx; and the cord and its membranes are to be divided opposite the lower cervical vertebra, and to be removed by cutting the bands that attach the dura mater to the posterior ligament of the bodies of the vertebrix.

When the cord is taken out, place the anterior surface uppermost with the lateral offsets widely separated. To show the arachnoid eovering, the dura mater is to be slit along the middle as far as the small terminal fibrous cord before referred to; but the membrane is to be raised whilst it is being cut through, so that the loose arachnoid on the cord may not be injured. Lastly, fasten back the dura mater with pins.

Fig. 121.

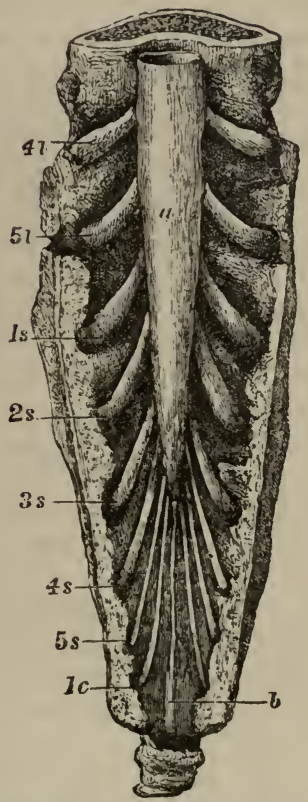

LOWRR END OF THE DURA MATER, WITH ITg Centra l a d Lateral Processes. (Museum University College, London.)

$a$. Large sheath of the dura mater.

b. Central fibrous band fixing it to the coccyx. The lateral offeets encasing the last two lumbar, and the five sacral nerves, with the coccygeal nerve, are also shown. Each nerve is marked by the numeral, and the first letter of its name.
Fig. 122.

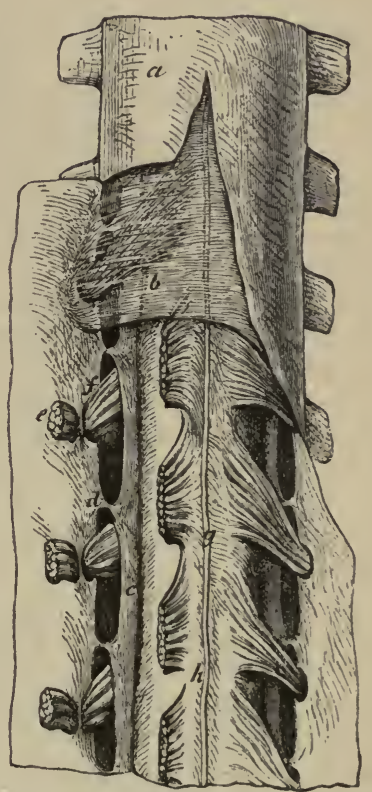

VIEW of the Membranes of the SPINal Cord.

a. Dura mater cut open and reflected.

b. Small part of the translucent arachnoid, left.

$h$. Pla mater closely investing the spinal cord.

c. Ligamentum denticulatum on the side of the cord, shown by cutting through the anterior roots of the nerves.

d. Processes jolning it to the dura mater.

g. Anterior spinal artery and the flbrous band (linea splendens) on the cord.

$e$. Anterior roots of the nerves, cut.

$f$. Posterior, each entering a separate hole in the dura mater.

The arachnoid membrane (fig. $122, b$ ) is the thin serous covering of the cord which is immediately beneath the dura mater. Like the corresponding membrane in the skull, it invests the spinal cord and lines the dura mater, and consists thus of a visceral and a parietal part. 
The outer or parietal part is inseparably joined to the inner surface of the dura mater, and gives to that membrane a shining appearance. ${ }^{1}$

The inner or visceral layer surrounds the cord loosely, so as to leave a considerable interval between the two (sub-arachnoid space). At the lower part of the spinal canal the loose sheath is largest, and envelops the mass of nerves forming the cauda equina. As the different spinal nerves extend to the intervertebral foramina, they receive loose tubes from the arachnoid, but lose the same when they perforate the dura mater.

Dissection. The sub-arachnoid space may be made evident by placing the handle of the scalpel beneath the visceral layer; or by putting a piece of the cord in water, with the posterior aspect uppermost, and blowing air beneath the serous membrane.

The sub-arachnoid space separates the loose or visceral part of the arachnoid membrane, from the spinal cord invested by pia mater. Larger at the lower than the upper part of the spinal canal it contains a special fluid-cerebro-spinal; and it communicates with the cavity in the interior of the brain by an aperture in the fourth ventricle. Crossing the space, at the posterior part of the cord, are bundles of fibrous tissue, which are most marked in the neck. In the space are contained the serrations of the ligamentum denticulatum, and the roots of the spinal nerves, with some vessels.

Dissection. For the purpose of seeing the next covering of the cord with the ligamentum denticulatum, the arachnoid membrane is to be taken away; and two or three of the anterior roots of the upper dorsal nerves may be cut through and reflected, as in the wood-cut 122 .

The pia mater (fig. 122, $h$ ) is much less vascular on the spinal cord than on the brain. Thicker and more fibrous in its nature, the membrane closely surrounds the cord with a sheath, and sends a thin prolongation into the anterior median fissure; it furnishes coverings to the roots of the spinal nerves.

The outer surface of the pia mater is rough. Along the front is a central, anterior fibrous band (linea splendens, Haller); and on each side another fibrous band, the ligamentum denticulatum, is attached to it. Scattered through the membrane are branched pigment cells, which give a dark appearance in the cervical region.

Where the medulla spinalis ceases, viz., about the lower part of the body of the first lumbar vertebra, the tube of the pia mater is suddenly reduced in size, and has the appearance of a round fibrous cord (fig. 124, $b$ ). This cord-like part (filum terminale) is provided with nervous substance above : and blends, below, with the central impervious prolongation of the dura mater. It serves to fix the lower end of the medulla spinalis, and has been named, from that circumstance, the central ligament of the cord. A vein and artery accompany this fibrous piece, and distinguish it from the surrounding nerves.

The ligamentum denticulatum (fig. 122, c) is the white, fibrous toothed band, on each side of the spinal cord. It is named from its serrated appearance; and it has the same structure as the dura mater, except that it wants an epithelial covering.

Situate between the anterior and posterior roots of the nerves, the band reaches upwards to the medulla oblongata, and ends inferiorly on the

1 According to the opinion of Kölliker, the arachnoid membrane is a simple tube corresponding with the visceral layer in the text. 
lower pointed extremity of the cord. Internally it is united to the pia mater. Externally it ends in a series of triangular or tooth-like projections $(d)$, which are fixed at intervals into the dura mater, each being about midway between the apertures of the roots of the spinal nerves. There are twenty or twenty-one denticulations; of which the first is attached to the dura mater opposite the margin of the occipital foramen, and the last, opposite the twelfth dorsal, or the first lumbar vertebra.

This fibrous band supports the spinal cord, and has been called a ligament from that circumstance.

Vessels and nerves of the membranes. The dura mater of the cord has but few vessels in comparison with that in the skull, for its office is different. Nerves are said to be furnished to it from offsets on the vessels supplying the cord.

The arachnoid is sparingly supplied with vessels like serous membranes in general; and proof of its containing nerves, in man, is yet wanting.

The pia mater has a network of vessels in its substance, though this is less marked than in the part on the brain; and from them offsets enter the cord. In the membrane are many nerves, derived from the sympathetic.

Dissection. The arachnoid membrane is to be taken from the fibrils of the roots of the nerves on one side; and the roots are to be traced outwards to their transmission through two apertures in the dura mater for each nerve.

One of the offsets of the dura mater, which has been cut of some length, is to be laid open to show the contained ganglion. The student should define one ganglion, showing its bifid condition at the inner end (fig. $123,{ }^{\mathrm{B}}$ ), and should trace a bundle of threads of the posterior root into each point. 'The anterior root, consisting of two bundles of threads, is to be followed over the ganglion to its union with the posterior beyond the ganglion.

Spinal Nerves. There are thirty-one pairs of spinal nerves; and each nerve is constructed by the blending of two roots (anterior and posterior) in the intervertebral foramen (fig. 123).

They are divided into groups corresponding with the regional subdivisions of the spinal column, viz., cervical, dorsal, lumbar, sacral, and coccygeal. In each group the nerves are the same in number as the vertebra, except in the cervical region of the spine where they are eight, and in the coccygeal region where there is only one. In consequence of the number of nerves in the neck, exceeding that of the vertebre, the last is placed below the seventh vertebra ; and the lowest nerve of each group, except the coccygeal, will be below its corresponding vertebra.

Each nerve divides into two primary branches, anterior and posterior, the former of these is distributed to the front of the body and the limbs, and the latter is confined to the hinder part of the trunk.

Roots of The Nerves (fig. 123). 'Two roots (anterior and posterior) attach the nerve to the spinal cord; and these unite together to form a common trunk in the intervertebral foramen. The posterior root is marked by a ganglion, but the anterior root is aganglionic.

The posterior or ganglionic roots (fig. $123, b$ ) surpass in size the anterior, and are formed by larger and more numerous fibrils. They are attached to the side of the cord between the posterior and lateral columns in a straight line, which they keep even to the last nerve.

In their course to the trunk of the nerve the fibrils converge to an aper- 
ture in the dura mater, opposite the intervertebral foramen; as they approach that aperture they are collected into two bundles (fig. $123 \mathrm{~B}, b$ ) which, lying side by side, receive a sheath from the dura mater, and enter the two points of the intervertebral ganglion.

Fig. 123.

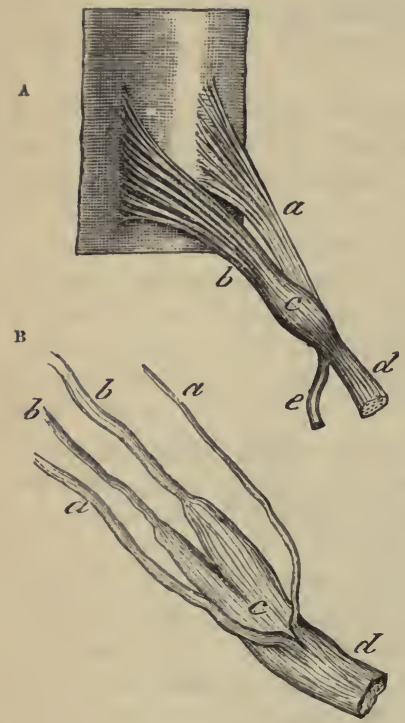

A. Plan of the origin of a spinal nerve from the spinal cord.

a. Anterior root.

b. Posterior root.

c. Ganglion on the posterior root.

d. Anterior primary banch.

e. Posterior primary branch of the nerve trunk.

B. A drawing to show the arrangement of the nerve-roots, and the form of the ganglion in a lumbar nerve.

b. Posterior root gathered into two bundles of threads.

c. The ganglion, bifid at the inner end.

$a$. Threads of the anterior root, also gathered into two bundles.

The intervertebral ganglion (fig. 123). Each posterior root is provided with a ganglion $(c)$. The ganglia are reddish in color, and oval in shape whilst they are surrounded by the dura mater; and their size is proportioned to that of the root. By means of the previous dissection, the ganglion may be seen to be bifid at the inner end (fig. $123 \mathrm{~B}$ ), where it is joined by the bundles of fibrils of the root $(b)$; it might be said to possess two small ganglia, one for each bundle of tibrils, which are blended at their outer ends.

Sometimes the first or suboecipital nerve is without a ganglion.

The anterior or aganglionic roots (fig. $123 \mathrm{~A}$ ) arise from the side of the spinal eord by filaments which are attached irregularly - not in a straight line, and approach near the middle fissure at the lower end of the cord.

Taking the same direction as the posterior root to the intervertebral foramen, the fibrils enter a distinct opening in, and have a separate sheath of the dura mater. In their further course to the trunk of the nerve they are gathered into two bundles (fig. $123, \mathrm{~B}, a$ ), and pass over the ganglion without joining it. Finally the anterior root blends with the posterior beyond the ganglion, to form the trunk.

Characters of the roots. Besides variations in the relative size of the two roots, the following eliaracters are to be noted:-

Union of the fibrils. The fibrils of contiguous anterior roots may be intermingled, and the fibrils of the neighboring posterior roots may be connected in a like manner; but the anterior is never mixed with the posterior root. 
Size of the roots to each other. The posterior root is larger than the anterior, except in the suboccipital nerve; and the number of the fibrils is also greater. Further, the posterior is proportionally larger in the cervical than in any other group; in the dorsal nerves there is but a very slight difference in favor of the hinder root.

Size of both roots along the cord. Both roots are larger where the nerves for the limbs arise, than at any other part of the cord; and they are greater in the nerves to the lower than in those to the upper limbs. They are smallest at the lower extremity of the cord.

Direction and length of the roots. As the apertures of transmission from the spinal canal are not opposite the place of origin of the nerves, the roots must be directed more or less obliquely. This obliquity increases from above down ; for in the upper cervical nerves the roots are horizontal, but in the lumbar and sacral nerves they have a vertical direction around the end of the medulla spinalis. 'The appearance of the long fibrils around the end of the cord (fig. 124) resembles the extremity of a horse's tail, and bears appropriately the term cauda equina.

The length of the roots increases in proportion to their obliquity. Between the origin and the place of exit of the roots of the lower cervical nerve the increase amounts to the depth of one vertebra: in the lower dorsal nerve it equals the depth of two vertebre; and in the lumbar and sacral nerves each succeeding root becomes a vertebra longer, for the cord does not reach beyond the first lumbar vertebra.

Place of union of the roots. Commonly the roots unite as before state in the intervertebral foramina; and the trunk of the nerve bifurcates at the same spot into anterior and posterior primary branches (fig. 123, $d$ and $e$ ). But deviations from this arrangement are found at the upper and lower ends of the spinal column in the following nerves.

The roots of the first two cervical nerves join on the neural arches of the corresponding vertebre; and the anterior and posterior primary branches diverge from the trunks in that situation.

In the sacral nerves the union of the roots takes place within the spinal canal; and the primary branches of the nerves issue by the apertures in the front and back of the sacrum.

The roots of the coccygeal nerve are also united in the spinal canal ; and the anterior and posterior branches of its trunk escape by the lower aperture of that canal.

Situation of the ganglia. The ganglia are placed commonly in the intervertebral foramina, but where the position of those apertures is irregular, as at the upper and lower extremities of the spinal column, they have the following situation: In the first two nerves they lie on the neural arches of the atlas and axis. In the sacral nerves they are contained in the spinal canal. In the coccygeal nerve the ganglion is also within the canal, and about the middle of the long posterior root (Schlemm).

Vessels of the Spinal Cord. 'The arteries on the surface of the cord are anterior and posterior spinal.

The anterior spinal artery occupies the middle line of the cord beneath the fibrous band before alluded to in that position. It begins by the union of two small branches of the vertebral artery (p.174); and it is continued to the lower part of the eord by a series of anastomotic branches, which are derived from the vertebral and ascending cervical arteries in the neck, and from the intercostal arteries in the Back. Inferiorly it supplies the roots of the nerves forming the cauda equina, and ends on the central 
fibrous prolongation of the cord. The branches of this artery ramify in the pia mater, some entering the median fissure.

The posterior spinal arteries, one on each side, are continued from the

Fig. 124.

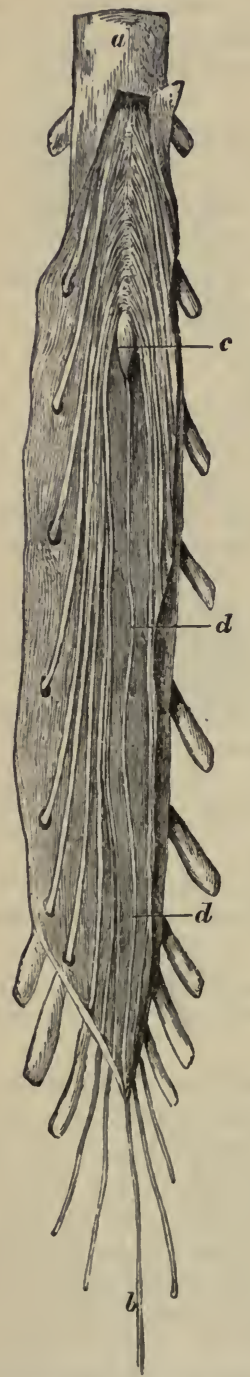

MrMBRANEg OF THR SPINAL CORD I.AID OPBN, to show the lower end of the cord with the flum terminale.

a. Dura mater, and $b$, the fbrous

band fixing it to the coccy $x$.

c. Pointed lower end of the cord.

d. Filum terminale of the cord. upper to the lower part of the eord, behind the roots of the nerves. These vessels are furnished from the same source as the anterior, and their continuity is maintained by a series of anostomotic branches, which enter the canal along the spinal nerves. Dividing into small branches, the vessels of opposite sides form a free anostomosis around the posterior roots, and some offsets enter the posterior fissure of the cord.

The veins of the spinal cord are very tortuous and form a plexus on the surface. At intervals larger trunks arise, which accompany the spinal nerves to the intervertebral foramina, and end in the veins outside the spinal canal. Near the top of the cord the veins are united into two or more small branches; after communicating with the intraspinal veins, these join in the skull the inferior cerebellar veins, or the inferior petrosal sinuses.

The sPINAL CORD (medulla spinalis), is the cylindrical elongated part of the eerebrospinal centre, which is inclosed within the spinal canal. Invested by the membranes before examined, the medulla occupies about two-thirds of the length of the vertebral canal, and is much smaller than the bony case surrounding it.

The extent of the spinal cord is from the upper border of the atlas to the lower border of the first lumbar vertebra, but its termination inferiorly may be a little higher or lower than that spot. In the embryo before the third month the medulla reaches throughout the spinal canal, but it gradually recedes as the surrounding bones enlarge faster than it, until it takes the position it has in the adult. Its length is usually from fifteen to eighteen inches.

Superiorly the cord joins the medulla oblongata; and inferiorly it becomes pointed, being sometimes marked by one or two swellings, and ends in the fibrous prolongation, named the central ligament of the cord or filum terminale (fig. 124, d).

The size of the spinal eord is much increased where the nerves of the limbs are attached. There are therefore two enlargements on it, viz., cervical and dorsal: the one in the neck reaches from the third cer- 
vical to the first dorsal vertebra ; the other in the Back is smaller, and is on a level with the last dorsal vertebra. In the upper enlargement the greatest thickness is from side to side; but in the lower swelling the measurement from before back rather exceeds the other.

Whilst the pia mater remains on the cord, the anterior surface is distinguished from the posterior by a central fibrous band and the anterior spinal artery ; and by the irregular line of the anterior nerve-roots, which approach the middle towards the lower end.

Dissection. For the examination of the structure the student should possess a piece of the medulla which has been hardened in spirit, for the cord which is obtained from the spinal canal at this period, is not fitted for the purpose of dissection. Supposing the pia mater removed from the surface, with the roots of the nerves left on one side, the student will be able to observe the following divisions of the medulla.

Fissures of the Cord (fig. 125). On the anterior and posterior aspects of the cord is a median longitudinal cleft-the anterior and posterior median fissures, which mark its division into halves; and along the line of the posterior roots of the nerves, in each half, is another slit-the lateral fissure.

Fig. 125.

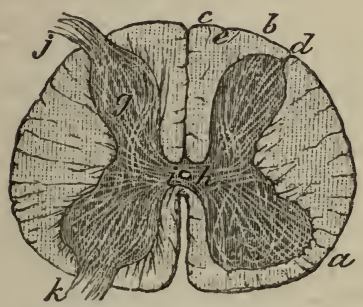

A Shction of the Spinat Cord, TO SHOW ITs CoMposition, AND its Divisions. Fissures IN THE MIDDLE LINE ARE THE ANTERIOR and Posterior Median-thi ANTERIOR BEING THE WIDER. d. The lateral fissure.

$e$. The slight groove between the lateral and the posterior median fissure, which marks the limit of the posterior median column.

\section{Columns :}

a. Antero-lateral.

b. Posterior.

c. Posterior median.

Composition:

g. Gray crescent, surrounded by white fibres.

$h$. Gray transverse comnissure, and $i$, canal of the cord in $1 \mathrm{t}$.

j. Posterior, and $k$, anterior root of a nerve entering the gray crescent.

The anterior median fissure (fig. 125) is wider than the posterior, and penetrates about one-third of the thickness of the medulla: it is lined by a piece of the pia mater, and is deepest towards the lower end of the cord. White medullary substance lines the fissure; and in the bottom of it the white fibres are transverse, and are separated by apertures for bloodvessels.

The posterior median fissure (fig. 125) is not so wide, or so well marked as the anterior; but it is best seen at the upper part of the neck, and in the lower or dorsal enlargement. Vessels of the posterior surface of the cord enter it.

The lateral fissure is situate along the line of attachment of the fibrils of the posterior roots (fig. $125, d$ ).

Between the lateral and posterior median fissures is a slight groove on the surface (fig. 125, e).

Sometimes a lateral fissure is described along the line of origin of the anterior roots $(k)$, but there is not any cleft in that situation. 
Segmexts of the Cord. Each half of the cord between the median fissures is divided into two parts by the lateral sulcus (fig. 125, $d$ ): the piece in front of that slit and the posterior roots of the nerves is called the antero-lateral column $(a)$; and the piece behind, the posterior column $(b)$.

The antero-lateral column (fig. 125, a) includes rather more than twothirds of the half of the cord, extending backwards to the posterior roots of the nerves, and gives attachment to the anterior roots.

The posterior column (fig. 125, b) is situate between the lateral fissure $(d)$, with the posterior roots of the nerves, and the central median fissure. Near the median fissure is a slight groove $(e)$, which marks off a slender piece, the posterior median column $(c)$ : this portion is best seen in the cervical part of the cord.

A central piece, or the commissure of the cord, connects the halves of the medulla, and limits the depth of the median fissures.

Different division of the cord. Each half of the cord is sometimes divided into three columns-anterior, lateral, and posterior, whose limits are the following:-The anterior reaches from the anterior roots of the nerves to the median fissure in front. The lateral column is limited before and behind by the roots of the nerves. The posterior with its small posterior median segment, is placed between the posterior roots and the median fissure behind.

Composition of the Cord (fig. 125). A horizontal section of the medulla shows more distinctly its division into halves, with the commissural or connecting piece between them. The same cut demonstrates the existence of a mass of gray matter in the interior, which is arranged in the form of two crescents (one in each half) united by a cross piece, and surrounded by white substance.

The commissure (fig. $125, h$ ) consists of two parts, viz., a transverse band of gray matter, with a white stratum in front.

The gray transverse band (gray commissure) connects the opposite crescents, and consists of nerve cells, and of transverse nerve fibres derived from the halves of the cord and the roots of the nerves.

In its centre is the shrunken canal of the spinal cord (fig. 125, $g$ ), which is best seen in the fotus. It reaches the whole length of the medulla, and a cross section of the cord shows it as a round spot. Above, it opens on the floor of the fourth ventricle, and below it is continued into the filum terminale. It is lined by a columnar ciliated epithelium, and is obstructed by a granular material near the upper opening (Clarke).

The white piece of the commissure is formed partly by fibres of the anterior column; and partly by fibrils of the anterior roots of the nerves, which here decussate as they cross from the one half to the other. It is best marked opposite the enlargements on the cord, and is least developed in the dorsal region.

The half of the medulla. In the half of the cord as in the commissure, gray and white portions exist; the former is elongated from before back, being crescentic in shape as before said, and is quite surrounded by the latter.

The gray crescent (g) is semilunar in form, with the horns or cornua of the erescent directed towards the roots of the nerves, and the convexity to the middle line (fig. 125). The crescentic masses in the opposite halves of the cord are united by the gray commissure.

The posterior cornu is long and slender (fig. $126,{ }^{1}$ ) and reaches near 
the fissure along the attachment of the posterior nerve-roots. At its extremity, where it is slightly enlarged, it is incased with a rather transparent stratum of small nerve cells, which has been named substantia gelatinosu (fig. $126,{ }^{3}$ ).

The anterior cornu (fig. $126,{ }^{2}$ ) is shorter and thicker than the other, and projects towards the anterior roots without reaching the surface. Its end has an irregular or zigzag outline.

Mr. Clarke describes two special collections of cells, one on the outer and the other on the inner side of the gray crescent, which extend through the cord below the cervical swelling, and with which the roots of the nerves are connected. ${ }^{1}$

The inner group, or the posterior vesicular column (fig. 126, ${ }^{8}$ ) is close behind the transverse commissure $\left(^{5}\right)$; whilst the outer group, or the inter-

Fig. 126.

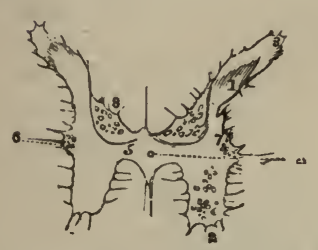

1. Posterior cornu (caput cornu, Clarke) of the crescent.

2. Anterior cornu.

3. Casing of the substantia gelatinosa.

4. Central canal of the cord.

5. Transverse commissure.

6. Tractus intermedio-lateralis.

7. Cervix cornu of Clarke, reaching from the anterior points of the substantia gelatinosa to the level of the canal.

S. Posterior vesicular column.

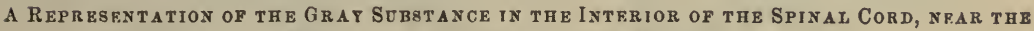
MIDDLE OF THE DORSAL REGION (Clarke).

mediate tract $\left({ }^{6}\right)$, is placed outside the crescent, about midway from front to back. The first is best marked in the dorsal enlargement, and the last in the upper part of the dorsal region. Above the cervical swelling of the cord only rudiments of those tracts remain; and the cells in a line with the intermediate tract are traversed by the roots of the spinal accessory nerie.

The white substance of the cord is composed chiefly of nerve fibres disposed longitudinally in bundles, so as to give passage to intermediate ressels.

A framework of very fine areolar tissue extends through the substance of the spinal cord, supporting the fibres and cells : this is continuous with the pia mater on the surface.

Modifications of the gray and white substance. The white substance exceeds the gray in quantity in the neck and back; but it is less abundant in proportion to the gray matter in the lumbar region.

The cornua of the gray crescents decrease in length from above down, especially the posterior, and towards the end of the cord they blend in one indented or cruciform mass.

Origix of tile Nerves. The deep origin of the spinal nerves is uncertain, like that of the cranial nerves, but the fibrils in each root enter the gray matter of the cord (fig. 125).

The anterior root $(k)$ traverses the longitudinal fibres of the anterolateral column in distinct bundles; and entering the anterior cornu of the

1 Further researches on the gray substance of the spinal cord. By J. Lockhart Clarke, F.R.S., Philosoph. Trans. of the Royal Society for 1859. Part i. 
gray crescent, it is resolved into three sets of fibres, external, internal, and middle.

The outer set penetrate into the antero lateral column.

The inner set pass through the anterior column and across the median fissure to the opposite half of the cord, decussating with like fibres of the opposite side.

The middle set enter the substance of the crescent, and are lost in it and in the cells of the intermediate tract.

The posterior root $(j)$ pierces chiefly the posterior column of the cord, and enters the pcsterior cornu of the crescent : but a few fibres penetrate by the lateral fissure. It terminates in two bundles of fibres.

One bundle courses round the outer side of the vesicular column, some fibres enclosing, and others entering it; and a few fibrils escape into the posterior column of the cord, becoming longitudinal.

The second bundle is prolonged into the crescent and the intermediate tract, as well as into the transverse commissure behind the central canal of the cord.

Intraspinal Vessels (fig. 127). Arteries supply the cord and its

Fig. 127.

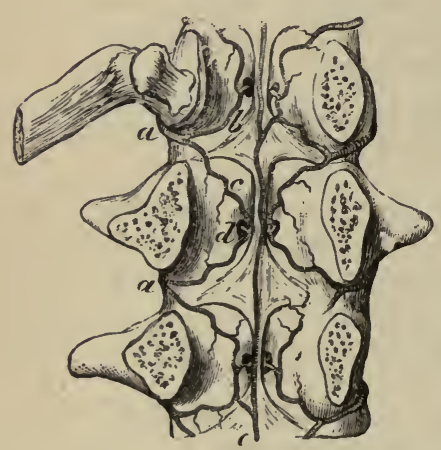

INTRASPINAL ARTRRIES IN THE LoINs (Museum of University College, London).

$a$. Branch of the lumbar artery.

b. Ascending, and $c$, its descending offset.

d. Offisets to the body of the vertebra on each slde.

e. Central artery formed by offsets from the lateral loops.
Fig. 128.

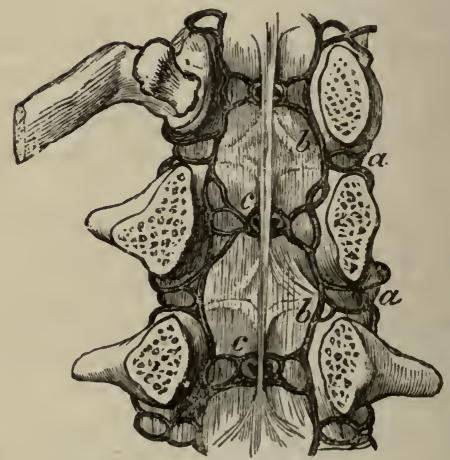

INTRASPINAL VEINS IN THB LOINS.

a. Branch to join a lumbar vein.

b. Anterior longitudinal vein, one on each side.

c. Veins from the bodies of the vertebræ.

membranes, and the bodies of the vertebræ. The veins form a remarkable plexus within the canal, but this will not be seen unless the veins have been specially injected.

The intraspinal arteries $(a)$ are derived from the vessels along the sides and front of the spinal column, viz., from the vertebral and ascending cervical in the neek, from the intercostal in the Back, and from the lumbar and lateral sacral below. They are distributed after the following plan :-

As each artery enters the spinal canal by the intervertebral foramen, it divides into two branches, upper and lower. From the point of division the branches are directed, one $(b)$ upwards and the other $(c)$ downwards, 
behind the bodies of the two contiguous vertebræ, and join in anastomotic loops with an offset of the intraspinal artery above and below. And from the loops offsets $(d)$ are furnished to the periosteum and the bodies of the vertebra. Anastomotic twigs connect the arches across the vertebræ.

The intraspinal vessels produce also a central longitudinal artery $(e)$, like that on the front of the spinal cord, which lies on the bodies' of the vertebre, and is reinforced at intervals by offsets from the loops.

The intraspinal veins (fig. 128) consist of two anterior longitudinal vessels, which extend the whole length of the spinal canal ; of veins inside the bodies of the vertebræ; and of a plexus of veins beneath the neural arches.

The anterior longitudinal $(a)$ are close to the bodies of the vertebræ, one on each side of the posterior common ligament; and they are irregular in outline, owing to certain constrictions near the intervertebral foramina. They receive opposite the body of each vertebra the veins $(c)$ from that bone; and through the intervertebral foramina they have branches of communication $(a)$ with the veins outside the spine in the neck, the dorsal region, the loins, and the pelvis.

Veins of the bodies of the vertebra. Within the canals in the bodies of the vertebræ are large veins, which join on the front of the bone with veins in that situation. Towards the back of the body they are united in an arch, from which two trunks issue by the large apertures on the posterior surface. Escaped from the bone, the trunks diverge to the right and left, and open into the longitudinal veins.

The posterior spinul veins form a plexus between the dura mater and the arches of the vertebræ. A large vein may be said to lie on each side of the middle line, which joins freely with its fellow, and with the anterior longitudinal vein by lateral branches. Offsets from these ressels are directed through the intervertebral foramina, to end in the veins $(a)$ at the roots of the transverse processes. 


\section{CHAPTER VII.}

DISSECTION OF THE PERIN EUM.

\section{Section I.}

\section{PERIN AUM OF THE MALE.}

Directions. The perinæum may be allotted with greatest adrantage to the dissector of the abrlomen ; and its examination should be made first, as the distinctness of many of the parts is destroyed soon after death. Before the body is placed in the position suited for the dissection, the student may practise passing the catheter along the urethra.

Position of the body. Whilst the body lies on the Back it is to be drawn to the end of the dissecting table, till the buttocks project slightly over the edge; and a moderately-sized block is to be placed beneath the pelvis, to raise the perinæum to a convenient height. The legs are to be raised and kept out of the way by the following means: After the knees have been bent, and the thighs bent upon the trunk, the limbs are to be fastened with a cord in their raised position. For this purpose make one or two turns of the cord around one bent knee (say the right); carry the cord beneath the table, and, encircling the opposite limb in the same manner, fasten it finally round the right knee. When the position has been arranged, let the student raise the scrotum, and place a small piece of cotton wool or tow within the anus, but let him avoid distending the rectum.

Superficial limits and marking. The perinæal space in the male is limited, on the surface of the body, by the scrotum in front, and by the thighs and buttocks on the sides and behind.

This region is of a dark color, and is covered with hairs. In the middle line is the aperture of the anus, which is posterior to a line extended from the anterior part of the one ischial tuberosity to the other. In front of the anus the surface is slightly convex over the urethra, and presents a longitudinal prominent line or raphé, which divides this part of the space into two halves. Between the anus and the tuberosity of the hip bone the surface is somewhat depressed over the hollow of the subjacent ischio-rectal fossa, especially in emaciated bodies.

The margin of the anal aperture possesses numerous converging folds, but these are more or less obliterated by the position of the body and the distension of the rectum; and projecting oftentimes through and around the opening are some dilated veins (hamorrhoids).

Deep boundaries. 'The deep boundaries of the perinaal space will be ascertained, in the progress of the dissection, to correspond with the inferior aperture or the outlet of the pelvis. The limits may be seen on a dry 
or prepared pelvis, on which the ligaments remain entire; and the student should trace on the body the individual boundaries with his finger. In front is the arch of the pubes; and at the posterior part is the tip of the coccyx, with the great gluteal muscles. On each side in front is the portion of the innominate bone which forms the pubic arch, viz., from the pubes to the ischial tuberosity; and still further back is the great sacrosciatic ligament extending from the tuber ischii to the tip of the coccyx. 'This region sinks into the outlet of the pelvis as far as the recto-vesical fascia, which forms its floor.

Form and size. The interval included within the boundaries above said has the form of a lozenge, and measures about four inches from before backwards, and three inches between the ischial tuberosities.

Depth. The depth of the perinæum from the surface to the floor may be said to be generally about three inches at the anus, but this measurement varies in different bodies; and it amounts to about an inch near the pubes.

Division. A line from the front of the tuberosity of one side to the corresponding point on the other, will divide the perinaal space into two triangular parts. The anterior half (urethral) contains the penis and the urethra, with their muscles and vessels and nerves. The posterior half (rectal) is occupied by the lower end of the large intestine, with its muscles, etc.

\section{POSTERIOR HALF OF TIIE SPACE.}

This portion of the perinæal space contains the lower end of the rectum, surrounded by its elevator muscle and the museles acting on the anus. The gut does not occupy however the whole of the interval between the pelvic bones; for on each side is a space, the ischio-rectal fossa, in which is contained much loose fat, with the vessels and nerves for the supply of the end of the gut.

Dissection (fig. 129). The skin is to be raised from this part of the perineum by the employment of the following cuts: One is to be made across the perinaum at the firont of the anus, and is to be extended rather beyond the ischial tuberosity on each side. A second is to be carried a little behind the tip of the coccyx in the same direction, and for the same distance. The two transverse cuts are to be connected by carrying the knife along the middle and around the anus. The flaps of skin thus marked out, are to be raised and thrown outwards from the middle line: in detaching the skin from the margin of the anus, the superficial subcutaneous sphineter muscles may be injured without eare, for they are close to the skin, without the intervention of fat. The dissector should trace the sphincter back to the coceyx, and forwards for a short distance beneath the skin; and define a fleshy slip at each side in front and behind to the subcutaneous fatty layer.

The next step is to bring into view the ischio-rectal hollow between the side of the rectum and the tuberosity of the hip bone. On the left side the fat is to be cleaned out of it without reference to the vessels and nerves, but on the opposite side a special dissection is to be made of them. To take out the fat from the left fossa, begin at the outer margin of the sphincter ani, and proceed forwards and backwards. In front the dissection should not extend farther than the anus, whilst behind it should lay bare the margin of the gluteus maximus. On the inner side of the hollow the levator ani (sometimes very pale) is to be dissected. On the outer 
boundary the pudic ressels and nerve should be denuderl: they lie in a canal formed by faseia, and at some distance from the surface.

On the right side it is not necessary to clean the muscular fibres, when following the vessels and nerves. If the student begins at the outer border of the sphincter, he will find the inferior hæmorrhoidal vessels and nerve, which he may trace outwarls to the pudic trunks: some of the branches, which join the superficial perinaal and inferior pudendal nerves, are to be followed forwards. In the posterior angle of the space seek a small offset of the fourth sacral nerve; and external to it, one or more branches of the sciatic nerve and vessels turning round the border of the gluteus. Near the front of the fossa is a superficial perinæal artery and nerve (of the pudic); and the last, after communicating with the hamorrhoidal nerve, leaves the fossa. A second perinæal nerve with a deeper position may be found at the front of the hollow.

The Ischio-RECTAL Fossa (fig. 129) is the space intervening between the rectum and the ischial part of the innominate bone. It is a somewhat

Fig. 129.

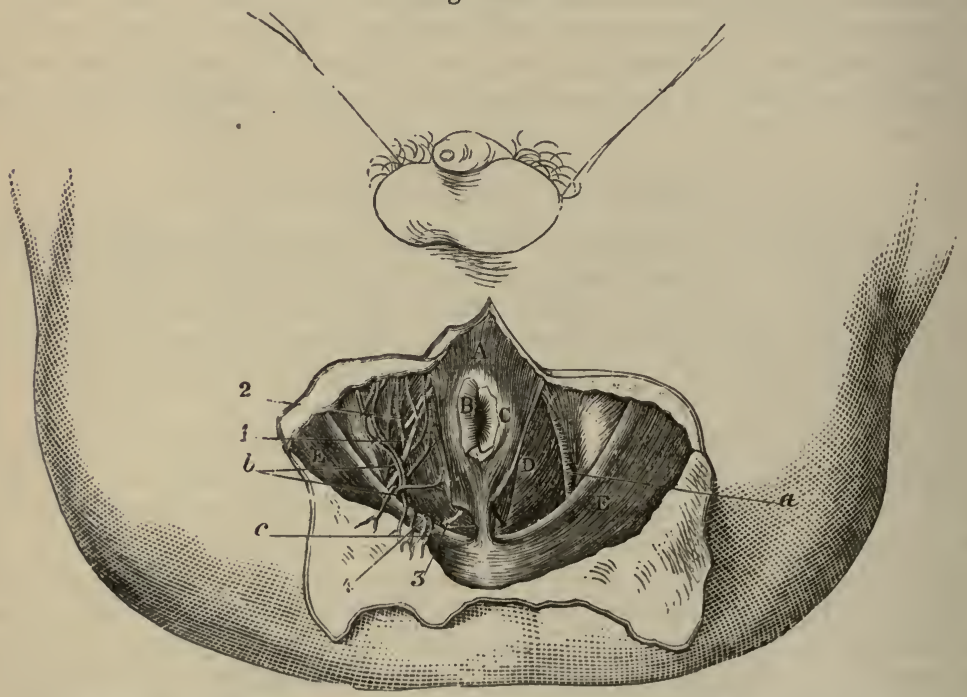

A View of thb Disgection of the Rfctal half of the Peringom. (Illustrationg of Dissections.)

Muscles :

A. External sphincter.

B. Corrugator cutis, only part left.

C. Internal sphincter.

D. Levator ani.

E. Gluteus maximus.
Arteries :

a. Trunk of the pudic artery.

b. Inferior hæmorrhoidal.

c. Branches of the sciatic.

Nerves :

1. Inferior hæmorrhoidal.

2. Superficial perinæal.

3. Peringal branch of the fourth sacral.

4. Small sciatic.

conical interval, uncovered by muscle, which is larger behind than before, and diminishes in width as it sinks into the pelvis. Its width is about one inch at the surface; and its depth about two inches at the outer part. It is filled by a soft granular fat. 
The inner or longest side of the space is very oblique, and is bounded by the levator ani (D), together with the coccygeus muscle posteriorly; but the outer side is vertical, and is formed by the obturator muscle and the fascia covering it. In front it is limited by the triangular ligament (to be afterwards seen); and behind are the great sacro-sciatic ligament, and the largest gluteal muscle. 'Towards the surface it is covered by the teguments, and is overlaid in part by the gluteus $\mathrm{E}$ and the sphineter externus A.

Position of vessels. Along the outer wall lie the pudic vessels and nerve (a), which are contained in a tube of fascia; opposite the ischial tuberosity, they are situate an inch and a half from the surface, but towards the front of the space they approach to about half an inch from the edge of the pubic arch. Crossing the centre of the hollow are the inferior hæmorrhoidal vessels and nerves $(b)$-branches of the pudic. At the anterior part, for a short distance, are two superficial perinæal nerves $\left(^{2}\right)$ (of the pudic); and at the posterior part is a small branch of the fourth sacral nerve $\left({ }^{3}\right)$, with cutaneous offsets of the sciatic vessels $(c)$ and nerve $\left({ }^{4}\right)$ bending round the gluteus.

Into this space the surgeon sinks his knife in the first incisions in the lateral operation of lithotomy ; and as he carries it from before backwards, he will divide the superficial hamorrhoidal vessels and nerve.

Muscles. Connected with the lower end of the rectum are four muscles, viz., a fine cuticular muscle, and two sphincters (external and internal) with the levator ani.

Corrugator cutis ani (fig. 129, ${ }^{\mathrm{B}}$ ). This thin subcutaneous layer of involuntary muscle surrounds the anus with radiating fibres. Externally it blends with the subdermic tissue outside the internal sphincter; and internally it enters the anus and ends in the submucous tissue within the sphincter.

Action. By the contraction of the fibres the skin is raised into ridges radiating from the anus, such as may be seen when that aperture is firmly closed.

The external sphincter (fig. 129, ${ }^{\wedge}$ ) (sphincter ani externus) is a flat, thin, orbicular muscle, which surrounds the lower part of the rectum. The fibres form ellipses around a central aperture, as in other orbicular muscles. It arises posteriorly by a fibrous band from the back of the coccyx near the tip, and by fleshy fibres on each side from the subcutaneous fatty layer. Its fibres pass forwards to the anus, where they separate to encircle that aperture; and in front they are inserted into the central point of the perinæum, and into the superficial fascia by a rather wide fleshy slip on each side.

The sphincter is close beneath the skin, and conceals partly the levator ani. The outer border projects over the ischio-rectal fossa, and the inner is contiguous to the internal sphincter.

Action. The muscle gathers into at roll the skin around the anus, and occludes the anal aperture : by its contraction it makes tense also the central point of the perinæum.

Commonly the fibres are in a state of involuntary slight contraction, but they may be firmly contracted under the influence of the will.

The internal spuncter (fig. 129, ${ }^{\mathrm{C}}$ ) (sphincter ani internus) is situate around the extremity of the intestine, internal to the preceding 
muscle, and its edge will be seen by removing the corrugator and the mucous membrane. The fibres of the muscle are pale, fine in texture, quite separate from the surrounding external sphincter, and encircle the lower part of the rectum in the form of a ring about half an inch in depth. The muscle is a thickened band of the involuntary circular fibres of the large intestines, which is not attached to the bone.

Action. This sphincter assists the external in closing the anus; and its contraction is altogether involuntary.

The Levator ANI muscle (fig. $129,{ }^{D}$ ) can be seen only in part; and the external sphincter may be detached from the coceyx, in order that its insertion may be more apparent. The muscle descends from its origin at the inner aspect of the hip bone, and is inserted along the middle line from the coccyx to the central point of the perinæum :-The most posterior fibres are attached to the side of the coccyx; and between that bone and the rectum the muscles of opposite sides are united in a median tendinous line. The middle fibres are blended with the side of the intestine (rectum). And the anterior are joined with the opposite muscle, in front of the rectum, in the central point of the perinæum.

This muscle bounds the ischio-rectal fossa on the inner side, and unites with its fellow to form a fleshy layer, convex downwards, through which the rectum is transmitted. Deeper than the muscle is the recto-vesical fascia. Along the hinder border is placed the coccygeus.

Action. Its action on the rectum is to elevate and invert the lower end of the gut after this has been protruded and everted in the passage of the feces.

With the description of the muscle in the pelvis its action on the urethra will be referred to.

Arteries (fig. 129). The pudic artery, with its inferior hæmorrhoidal branch, and other small offsets of it and the sciatic, are now visible.

The pudic artery $(a)$ is derived from the internal iliac in the pelvis, and ascending to the genital organs, distributes offsets to the perinæum; one portion will be laid bare in the hinder, and the other in the anterior half of the perinæum.

As now seen, the vessel enters the posterior part of the ischio-rectal fossa, and courses forwards along the outer wall at the depth of one inch and a half behind, but of only half an inch at the fore part. It is contained in an aponeurotic sheath which attaches it to the obturator fascia. The usual companion veins lie by its side; and two nerves accompany it, viz., the trunk of the pudic, and the perinæal branch of the same nerve which is nearer the surface. Its offsets in the posterior half' of its course are the following:-

The inferior homorrhoidal branch (b) arises internal to the ischial tuberosity; it sends offsets inwards across the ischio-rectal fossa to the teguments, and the sphincter and levator ani muscles. On the rectum it anastomoses with the upper hæmorrhoidal branch, and with the artery of the opposite side. In a well injected body cutaneous branches may be seen to run forwards to the anterior part of the perinæum, and to communicate with the superficial perinæal branch.

Small muscular branches cross the front of the ischio-rectal fossin, and supply the anterior part of the levator ani muscle.

The branches of the sciatic artery $(c)$ appear on the inner aspect of the gluteus maximus at the back of the fossa; some end in that muscle, and others are continued round its border to the fat. 
Veins accompany the different arteries, and have a like course and ramification: the pudic end in the internal iliac.

Nerves (fig. 129). The nerves to be learned in this part of the perinæum are, the trunk of the pudic and its inferior hæmorrhoidal and perinæal branches; an offset of the fourth sacral; and some branches of the small sciatic.

The pudic nerve comes from the sacral plexus, and accompanies the artery to the genitals. In the anal half of the perinæum it is placed deeper than the artery, and furnishes the two subjoined branches.

The inferior hamorrhoidal branch (1) crosses the ischio-rectal fossa, and reaches the margin of the anus, where it terminates in offsets to the integument and the sphineter muscle. Other cutaneous offsets of the nerve turn forwards over the fossa, and communicate with one of the superficial perineal nerves, and with the inferior pudendal (of the small sciatic) on the margin of the thigh.

The perinceal branch arises about half way along the fossa, and becomes superficial to the bloodvessels. It is larger in size than the continuation of the nerve to the penis, and divides into cutaneous, muscular, and genital offsets. Its two cutaneous offsets (superficial perinæal) may be seen on the right side, where they lie for a short distance in the ischio-rectal fossa.

'The hamorrhoidal branch of the fourth sacral nerve $\left(^{8}\right)$ reaches the ischio-rectal fossa by piercing the fibres of the levator ani. Appearing in the posterior part of the fossa, near the coceyx, the nerve ends by supplying the external sphincter, and the integuments behind the anus.

One or two cutaneous branches of the small sciatic nerve $\left.{ }^{4}\right)$ turn round the lower border of the gluteus, in their course to the integuments on its surface.

\section{ANTERIOR HALF OF THE PERINAAL SPACE.}

In the anterior part of the perinæal space are lodged the crura of the penis, and the tube of the urethra as it courses from the interior of the pelvis to the surface of the body. Placed about midway between the bones, the urethra is supported by the triangular liganent of the perinæum, and by its union with the penis.

Muscles are collected around the urethra to aid in the expulsion of the contents of that tube: some of these are superficial to, and some within the triangular ligament.

The vessels and nerve lie along the outer side, as in the posterior half, and send inwards offsets.

Dissection (fig. 130). 'To raise the skin from the anterior' part of the perinæum, a transverse cut is to be made at the back of the scrotum, and is to be continued for a short distance (two inches) on each thigh. A second incision along the middle line will allow the Hlaps of skin to be reflected outwards. After the removal of the skin the subcutaneous fat (superficial fascia) which covers the front of the perinæal space, is to be blown up by means of a pipe introduced beneath it posteriorly. Each side is to be inflated to demonstrate a partition along the middle line, and a septum between the perineal space and the thigh which prevents the air passing to the limb.

The student is next to cut through the superficial fascia on the left side from the scrotum to the ischio-rectal fossa; and after reflecting it, and 
removing loose fatty tissue, its line of attachment to the bones externally, and to the triangular ligament posteriorly, will be brought into view. The septum along the middle line should be also defined. To demonstrate more completely the attachment of this subcutaneous layer to the pubic arch between the perinaal space and the thigh, it will be necessary to take away from the left limb the fat on the fascia lata, external to the line of the bone.

In the fat of the thigh on the right side the student should seek the inferior pudendal nerve, which pierces the fascia lata one inch anterior to the tuber ischii, and about the same distance from the margin of the pubic arch; and should trace its junction in the fat with the inferior hamorrhoidal nerve. Afterwards the nerve is to be followed forwards to where it enters beneath the superficial fascia in the middle line.

Fig. 130.

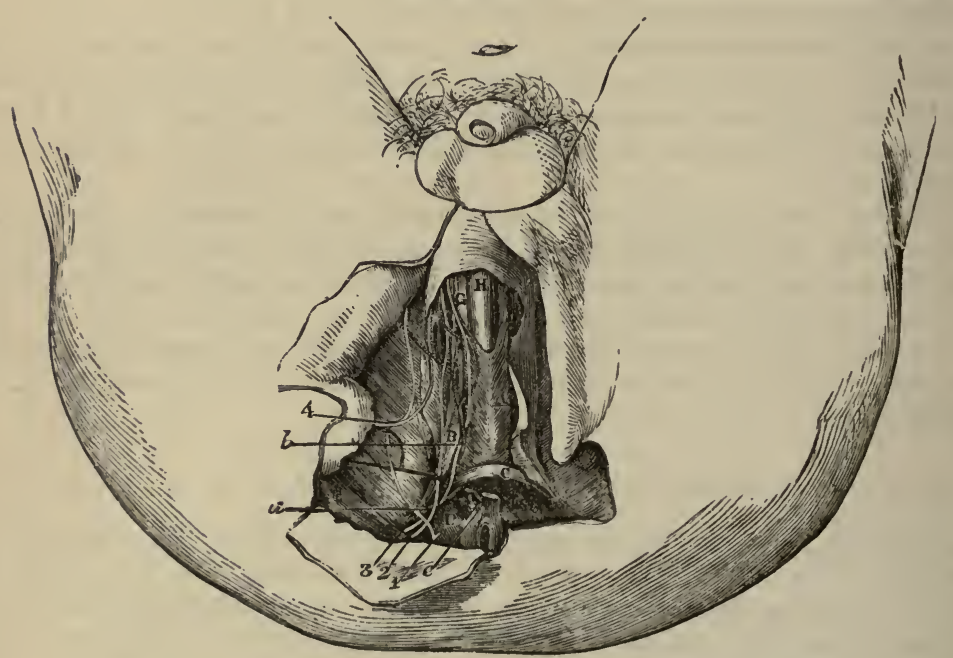

Superficial Digsection of the ANterior Half of the Perin fec.

(Illustrations of Dissections.)

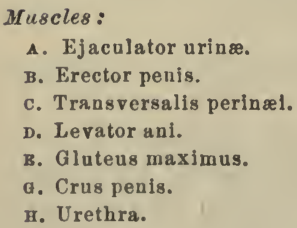

A. Ejaculator urinæ.

B. Erector penis.

c. Transversalis perinæ1.

D. Levator ani.

B. Gluteus maximus.

G. Crus penis.

H. Urethra.
Arteries :
a. Transverse perinæul.
b. Superficial perinæal.
c. Brauch of sciatic. Nerves :
1. Inforior hæmorrhoidal.
2 and 3 . Superficial perinæal.
4. Inferior pudendal.

The subcutaneous fatty layer or the superficial fascia of the anterior half' of the perinæum is continuous with that of the adjoining regions; and its depth, and the quantity of fat in it, will vary with the condition of the borly. It resembles the corresponding stratum of the groin and upper part of the thigh, in consisting of two different portions.

One, a subcutaneous fatty part, continuous with that of the rest of the body, which loses its fat towards the scrotum, and obtains there involuntary muscular tibres. 
The other deeper, but more membranous part, is of limited extent, and is connected with the firm subjacent structures. Externally it is fixed to the pubic arch of the hip-bone, outside the line of the crus penis and its muscle, extending as low as the ischial tuberosity. Posteriorly the stratum bends down to join the triangular ligament of the urethra; but in front it is unattached, and is continued to the scrotum and penis. By means of similar connections of the membrane on both sides, a space is enclosed over the anterior half of the perinæum. From its under surface a septum dips downwards in the middle line, and divides posteriorly the subjacent space into two; but anteriorly this partition is less perfect, or may disappear.

Air blown beneath the fascia passes forwards to the scrotum; and this direction is given to it by the deep connections of that membrane with parts around. Should urine be effused beneath the superficial fascia, the fluid will necessarily be directed forwards, like the air, through the scrotum to the penis and the front of the abdomen.

Dissection. The superficial vessels and nerves are to be dissected on the right side of the perinæum, by cutting through the superficial fascia in the same manner as on the left side. The long slender artery then visible is the superficial perinæal, which gives a transverse branch near its commencement. Two superficial perinæal nerves accompany the artery; and the inferior pudendal nerve is to be traced forwards to the scrotum. Communications are to be sought between these nerves anteriorly, and between one of the perinæal and the inferior hæmorrhoidal posteriorly; and all the nerves are to be followed backwards.

Arteries (fig. 130). The superficial and transverse perinæal arteries beneath the fascia are branches of the pudic, and are two or three in number.

The superficial perincal branch (fig. 130, b), arising at the fore part of the ischio-rectal fossa, runs over or under the transverse muscle, and beneath the superficial fascia to the back of the scrotum, where it ends in flexuous branches. As the ressel lies internal to the pubic arch, it supplies offsets to the muscles beneath; and in front it anastomoses with the external or superficial pudic branches of the femoral artery. Sometimes there is a second perinæal branch.

The transverse artery of the perinæum (fig. 130,a) arises from the preceding, and is directed transversely to the middle of the perinaal space, where it is distributed to the integuments and the muscles between the urethra and the rectum. It anastomoses with the one of the opposite side.

Branches of veins accompany the arteries, and open into the trunk of the pudic vein; those with the superficial perinæal artery are plexiform at the scrotum.

Nerves (fig. 130). There are three cutancous nerres of the scrotam on each side, viz., inferior pudendal of the small sciatic, and two superficial perinæal branches of the pudic nerve.

The superficial perincal nerves, two in number, are named anterior and posterior from their relative position : both arise in the ischio-rectal fossa from the perinaal branch of the pudic nerve (p. 391).

The posterior branch $\left({ }^{2}\right)$ is continued forwards, beneath the superticial fascia, with the artery of the same name to the back of the scrotum. Whilst in the fossa the nerve gives inwards an offset to the integuments in front of the anus ; and this communicates with the inferior hamorrhoidal nerve. 
The anterior branch $\left({ }^{3}\right)$ passes under the transverse muscle, and accompanies the other to the serotum. Muscular otfsets are furnished by it to the levator ani and the other superficial museles.

The superficial perinæal branches communicate with one another, and the posterior is joined by the inferior pudendal nerve. At the scrotum they are distributed by long slender filaments, which reach as far as the under surface of the penis. In the female these nerves supply the labia pudendi.

Other muscular branches of the pudic will be afterwards examined (p. 399).

The inferior pudendal nerve $\left({ }^{4}\right)$ is a branch of the small sciatic. It pierces the faseia lata about one inch in firont of the ischial tuberosity, and enters beneath the superficial fisseia of the perinæum, to end in the outer and fore parts of the scrotum. Communications take place between this nerve, the inferior hæmorrhoidal, and the posterior of the two superficial perinaal branches. In the female the interior pudendal nerve is distributed to the labium.

Dissection. For the display of the muscles, the fatty layer, as well as the vessels and nerves of the left side, must be taken away from the anterior half of the perinæal space. Afterwards a thin subjacent aponeurotic layer is to be removed from the muscles. Along the middle line lies the ejaculator urina; and in cleaning it the student is to follow two fasciculi of fibres from it on the same side-one in front, the other behind. On the outer part of the space is the erector penis. And behind, passing obliquely between the other two, is the transverse muscle.

The student should seek, on the right side, the branches of the two superficial perinæal nerves to the underlying muscles; and beneath the transversalis, an offset of the perinæal branch which supplies the deep muscles and the urethra.

Muscles (fig. 130). Superficial to the triangular ligament in the anterior half of the perinæal space, are three muscles, viz., the erector penis, the ejaculator urinæ, and the transversalis perinæi. Other muscles of the urethra are contained between the layers of the triangular ligament, and - will be subsequently seen.

Central point of the perincum. Between the urethra and the rectum is a white fibrous spot, to which this term has been applied. It oceupies the middle line, half an inch in front of the anus. In it the muscles acting on the rectum and the urethra are united; and it serves as a common point of support to the space.

The ERECTOR PENIS (fig. 130, ${ }^{\mathbf{B}}$ ) is the most external of the three muscles, and is narrower at each end than in the middle. It covers the erus penis; and its fibres arise from the ischial tuberosity farther back than the attachment of the penis, and from the bone on each side of the crus. Superiorly the muscle is inserted into the inner and outer surfaces of the crus penis. It rests on the root of the penis and the bone.

Action. The musele compresses the crus penis against the subjacent bone, and retards the escape of the blood from that organ by the veins : in that way it will contribute to the continuance of distension.

The eJaculator URIN 2 muscle (fig. 130, ${ }^{\text {}}$ ) lies on the urethra. The muscles of opposite sides unite by a median tendon along the middle line and in the central point of the perinæum (origin). 'The fibres are directed outwards, curving around the convexity of the urethra, and give rise to a thin muscle, which has the following insertion:-The most posterior fibres 
are lost on the front of the triangular ligament. The anterior fibres, which are the longest and best marked, are inserted into the penis on its outer aspect before the erector ; and, according to Kobelt, ${ }^{1}$ they send a tendinous expansion over the dorsal vessels of the penis. Whilst the middle or intervening fibres turn round the urethra, surrounding it for two inches, and join its fellow by a tendon.

The ejaculator muscle covers the bulb and the urethra for three inches in front of the triangular ligament. ${ }^{2}$ If the muscle be cut through on the right side, and turned off the urethra, the junction with its fellow above that tube will be apparent.

Action. The two halves, acting as one muscle, can diminish the urethra, and eject forcibly its contents. During the flow of fluid in micturition the fibres are relaxed, but they come into use at the end of the process, when the passage has to be cleared. The action is involuntary in the emission of the semen.

The TRANSVERSAls PERINeI (fig. $130,^{\circ}$ ) is a small thin muscle, which lies across the perinæum opposite the base of the triangular ligament. Arising from the inner surface of the pubic arch near the ischial tuberosity, it is inserted into the central point of the perinæum with the muscle of the opposite side, and with the sphincter ani and the ejaculator urinæ. Behind this muscle the superficial fascia bends down to join the triangular ligament.

Sometimes there is a second small fleshy slip, anterior to the transversalis, which has been named transversalis alter; this throws itself into the ejaculator muscle.

Action. From the direction of the fibres the muscle will draw backwards the central point of the perinæum, and help to fix it, preparatory to the contraction of the ejaculator.

The three muscles above described, when separated from each other by the dissection, limit a triangular space, of which the ejaculator urinæ forms the inner boundary, the erector penis the outer side, and the transversalis perinæi muscle the base. In the area of this interval is the triangular ligament of the urethra, with the superficial perinaal vessels and nerves. Should the knife enter the posterior part of this space during the deeper incisions in the lateral operation of lithotomy, it will divide the transverse muscle and artery, and probably the superficial perinxal vessels and nerves.

Dissection (fig. 131). For the display of the triangular ligament, the muscles and the crus penis, which are superficial to it, are to be detached in the following way:-On the left side the ejaculator urinæ is to be removed completely from the firont of the ligament, and the erector muscle from the crus of the penis. Next, the crus penis is to be detached from the bone; but this must be done with care so as not to cut the triangular ligament, nor to injure, near the pubes, the terminal branches of the pudic artery and nerve to the penis.

On the right side the dissector should trace out beneath the transversalis

1 Die Männlichen und Weiblichen Wollust-Organe, von G. L. Kobelt, 1844.

2 Some of the deeper fibres which immediately surround the bulb, have been described as a separate stratum by Kobelt. These are separated from the superficial layer by thin areolar tissue, and join the corresponding part of the other muscle by a small tendon above the urethra. The name compressor hemisphcorium bulbi has been proposed for it by that anatomist. 
the offsets of the perinaal nerve to the deep muscles and the urethra, with the ressels accompanying them.

The triangular ligament of the urethra (fig. $131{ }^{\circ}$ ) (perinaal aponeurosis) occupies the anterior part of the pubic arch, and supports the urethral canal. The ligament is triangular in form, with its base below; and it is about one inch and a half in depth.

Fig. 131.

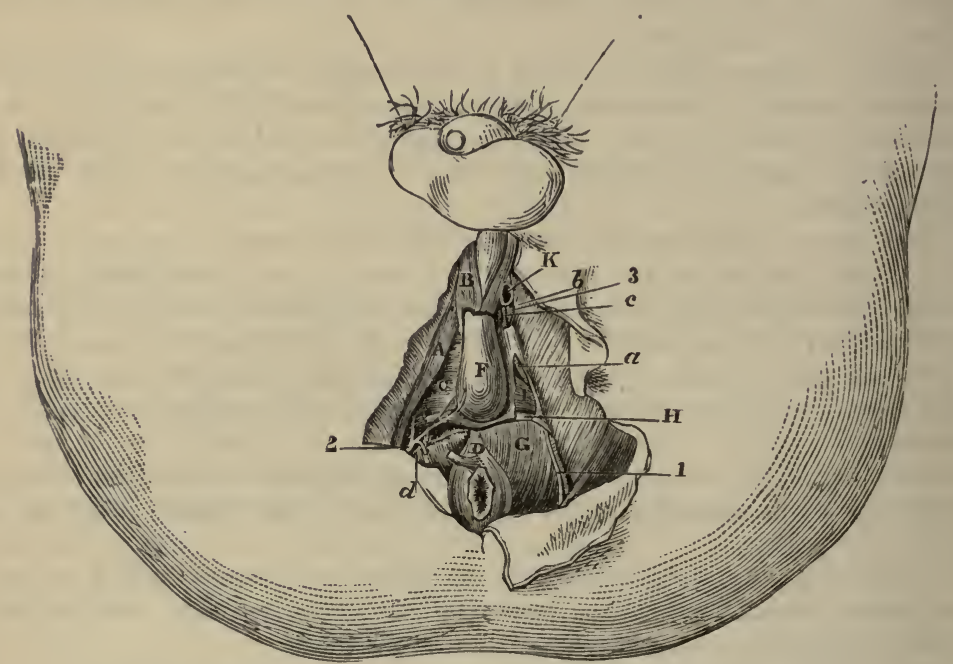

Defe Dissection of the PerixaUM. (Illustrations of Dissections.)

Muscles :

A. Erector penis.

B. Ejaculator urinæ, cut. .

C. Triangular ligament.

D. External sphincter.

F. Bulbous part of the urethra.

G. Levator ani.

H. Deep transverse.

I. Constrictor urethræ.

K. Crns penis, cut.

\section{Arteries :}

a. Pudic, in the triangular ligament.

b. Dorsal of penis.

c. Cavernous.

d. Deep muscular and urethral branch.

\section{Nerves:}

1. Pudic trunk.

2. Deep perinæal or muscular branch.

3. Dorsal of the penis.

On each side it is fixed to the pubie arch beneath the crus penis. Its apex is connected with the symplysis pubis. Its base is turned towards the rectum, and is partly attached and partly free; in the middle line it is connected with the central point of the perinæum, whilst laterally it is s'oped towards the bone, so that it is less deep at the centre than at the sides: connected with the lower border is a thin fascia which covers the surface of the levator ani musele in the ischio-rectal fossa. Superficial to it are the muscles in the anterior half of the perinaal space; and the superficial fascia is united to it near the lower border.

The ligament is composed of two layers of membrane (anterior and posterior) which are united below. The posterior layer is derived from the recto-resical fascia. The anterior is a separate membrane, formed chicfly of transverse fibres, but it is so thin as to allow the vessels and the muscular fibres to be seen through it. 
Perforating the fore part of the ligament, about one inch below the symphysis pubis, is the canal of the urethra, $\mathrm{F}$; but the margin of the opening giving passage to that tube is blended with the tissue of the corpus spongiosum urethræ. About midway between the preceding opening and the symphysis pubis is the aperture for the dorsal vein of the penis; and external to this, near the bone on each side, the terminal parts of the pudic artery and nerve to the penis ( $b$ and 3 ) perforate the ligament by separate apertures.

Between the layers of the ligament are contained the membranous part of the urethra, with its muscles, vessels, and glands; and the bloodvessels and nerves of the penis.

Dissection. The museles between the layers of the ligament will be reached by eutting through with care, on the left side, the superficial stratum near its attachment to the bone, and raising and turning inwards that piece of membrane. By a little cautious dissection, and the removal of some veins, the following objects will come into view with the undermentioned position :-

Parts between the layers of the ligament. Near the base is a narrow transverse muscle, $\mathbf{H}$, which is directed to the bulb of the urethra. Higher up, and erossing inwards from behind the pubic arch, is the fasciculus of fibres of the constrictor urethra muscle, $x$, which surrounds the membranous part of the urethra. And below the urethra are the glands of Cowper. Beneath the bone are the pudic vessels $(a)$ and nerve, the former giving its branch to the bulb, and the latter being deeper in position; and below the pubes is the sub-pubic ligament. Deeper than all, the student will recognize the posterior layer of the ligament, continuous with the recto-vesical fascia, which separates those parts from the eavity of the pelvis.

Muscles. The two muscles conneeted with the membranous part of the urethra are, deep transverse, and constrictor of the urethral passage.

The deep transverse Muscle (fig. 131, H) (elevator urethra Santorini) is a thin flat band on a level with the base of the triangular ligament. It arises externally from the pubic arch of the innominate bone, and is directed below the tip of the bulb and the membranous part of the urethra to the middle line, where it joins the muscle of the opposite side, and is inserted into the central point of the perinæum.

The muscle conceals Cowper's gland, and is frequently placed over the artery of the bulb. The transverse muscle is not always separate from the following.

Action. Like the superficial muscle it will fix the central point of the perinæum.

The constrictor muscle (fig. 131, ${ }^{\mathrm{I}}$ ) (constrictor isthmi urethralis) incloses the membranous part of the urethra, and consists of transverse fibres above and below that tube.

The muscle arises by aponeurotic fibres from the pubic arch above the preceding, and from the posterior layer of the triangular ligament, but this attachment is not evident unless it has been dissected from behind. The fibres pass inwards, and separate near the urethra into two layers (fig. 132), of which one $(c)$ passes over, the other $(d)$ under that canal; in the middle line they unite (sometimes by tendon) with the like parts of the muscle of the opposite side.

It may be considered a single muscle extending across the perinaum 
from one lateral attachment to the other, and inclosing the urethra, like the sphincter ani encircles the rectum.

Action. The muscles of both sides act as a single sphincter in diminishing the membranous part of the urethra, and ejecting the contents of

Fig. 132.

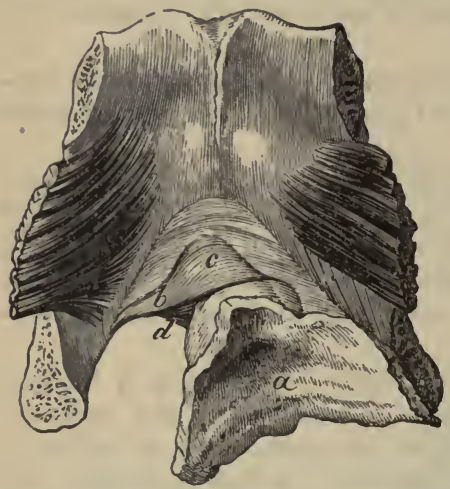

ThE SYMPHYSIS PUBIS gEEN FROM BEHIND WITH PART OF THE URINARY BLADJER OF THE URETHRA SURROUNDED BY THE Constrictor Muscle (Santorini).

a. Bladder cut operi: the swelling of the prostate surrounds it in front.

b. Constrictor urethræ:-

c. Part of the muscle above, and

d. Part underneath the urelhra. ANDThe Prostate, AND With ThK TUBE

the tube. Like the ejaculator, they are relaxed whilst the urine is passing, but the two contract forcibly in expelling the last of that fluid.

Involuntary circular fibres within the constrictor muscle surround the urethra from the bulb to the prostate, and form a layer about $\frac{1}{30}$ th of an inch thick; they are not fixed to bone, and are contintous behind with the circular fibres of the prostate. 'This layer is a portion of the large involuntary muscle, of which the prostate is chief part, surrounding the beginning of the urethra. ${ }^{1}$

Action. This involuntary layer assists in moving forwards the urine and the semen.

The glands of Cowper will be found by cutting through the transverse muscle. They are situate below the membranous part of the urethra, one on each side of the middle line, and close behind the bulb. Each gland is about the size of a pea, and is made up of lobules; and the lobules are composed of small resicles, which are lined by flattened epithelium.

Connected with each is a minute duct, nearly an inch in length, which perforates obliquely the wall of the urethra (corpus spongiosum), and opens into the urethral canal about half an inch in front of the triangular. ligament. Its aperture in the ordinary state does not admit a bristle. In the wall of the duct are unstriated muscular fibres; and the interior is lined by a columnar epithelium. The nature of the secretion of the gland is not known.

These bodies are sometimes so small as to escape detection, and they appear to decrease in size with advancing age.

Dissection. 'The student may complete the examination of the perinæum by tracing out the pudic ressels and nerve, and their remaining branches. From the point of its division beneath the crus into two branches (dorsal of the penis, and eavernous), the artery is to be followed backwards, along the pubic arch of the left side. 'The pudic nerve will be by the side of, but deeper than the artery.

Pudic Artery (a). The posterior half of this artery has been already dissected (p. 390). In the anterior half of the perinaum it ascends between the layers of the triangular ligament, and along the pubic arch nearly to the pubes; there it perforates the superficial part of the liga-

I See a paper on the Arrangement of the Muscular Fibres of the Urethra, in vol. xxxix. 1856, of the Trans. of the Med.-Chir. Society. 
ment, and divides into the arteries of the cavernous structure and dorsum of the penis. In this course it is placed beneath the constrictor urethræ, and is accompanied by venæ comites and the pudic nerve. Its offsets are subjoined :-

a. The artery of the bulb of the urethra is a branch of considerable size, and arises near the base of the triangular ligament. Passing almost transversely inwards between the layers of that ligament, about half an inch from the base, the artery reaches the back of the bulb, and enters the spongy structure. Near the urethra it furnishes a small branch to Cowper's gland.

The distance of this branch from the base of the ligament will be influenced by its origin near the front or back of the perinaal space. If the vessel arises farther behind than usual, it may be altogether below the ligament, and may cross the front of the ischio-rectal fossa, so as to be liable to be cut in the operation of lithotomy.

b. Deep muscular branches $(d)$. As the artery is about to enter between the layer's of the triangular ligament it furnishes one or more branches to the levator ani and sphincter, and fine twigs through the ligament to the constrictor and the urethra.

$c$. The artery of the cavernons structure of the penis (c) (art. corporis cavernosi) is one of the terminal branches of the pudic. At first this small vessel lies between the crus penis and the bone; but it soon enters the crus, and ramifies in the cavernous structure of the penis.

$d$. The dorsal artery of the penis $(h)$ is in direction and size the continuation of the pudic; it runs upwards between the crus and the bone, and reaches the dorsum of the penis by passing through the suspensory ligament. Its distribution with the accompanying nerve is noticed at page 408. It is much smaller in the female than in the male.

Accessory pudic artery. In some cases the pudic artery is not large enough to supply the branches above described to the penis and the urethra. One or more offsets will be then contributed by an accessory ressel, which leaves the pelvis in front by piercing the triangular ligament. The source of this accessory artery is the internal iliac.

'The pudic veins, two in number, have the same connections as the artery; they receive similar branches, except that the dorsal vein of the penis does not join them.

The PUDIC NERve has been examined in the ischio-rectal fossa (p. 391). In the anterior half of the perinæum it is much diminished in size, in consequence of the emission of the large perinæal branch, and courses with the artery between the layers of the triangular ligament; near the pubes it pierces the ligament (fig. $131,{ }^{3}$ ), and is continued to the dorsum of the penis with the dorsal branch of the pudic artery; its termination is described at page 408. The deep muscles and the corpus spongiosum are supplied by the following branch.

Perinaal branch (p. 391). Arising in the ischio-rectal fossa it supplies superficial nerves, and ends near the base of the triangular ligament in deep muscular offsets : some of these (fig. $131,{ }^{2}$ ) pass beneath the transversalis, and piercing the triangular ligament, supply the muscles within it. A long slender branch, nerve of the bulb, is distributed like the artery to the spongy structure investing the urethra: its filaments reach some way on the surface before disappearing in the corpus spongiosum urethræ.

Parts cut in the lateral operation of lithotomy. 'This operation for stone in the bladder may be divided into three stages, viz., cutting down to the 
urethra, opening the canal, and slitting that tube and the neck of the bladder. In the external incisions the knife is entered in the midille line of the perinaum, half an inch in front of the anus, and is drawn backwards on the left side as far as midway between the ischial tuberosity and the anus. The skin and superficial fascia, and the inferior hemorrhoidal vessels and nerve lying across the ischio-rectal fossa, will be eut in this first stage of the operation ; and the transverse perinaal muscle and artery, and, possibly, the superficial perinæal vessels and nerves, may be divided, if the first incision is begun farther forwards.

In the subsequent attempt to reach the staff, when the knife is introduced into the anterior part of the wound, the lower part of the triangular ligament, the deep transverse urethral muscle, and the fore part of the levator ani will be divided; and when the knife is placed within the groove of the staff, the membranous part of the urethra will be cut, with the muscular fibre about it.

Lastly, as the knife is pushed along the staff into the bladder, it incises in its progress the membranous portion of the urethra, part of the prostate with large veins around it, and the neck of the bladder. When the last two parts are being cut, the handle of the knife is to be raised, and the blarle depressed; and the incision is to be made downwards and outwards in the direction of a line from the urethra through the left lateral lobe of the prostate, above the level of the ejaculatory dnet.

Parts to be avoided. In the first incisions in the ischio-rectal fossa, the rectum may be cut if the knife is turned inwards across the intestine, instead of being kept parallel with it; and if the gut is not kept out of the way with the fore finger of the left hand. The pudic vessels on the onter wall of the ischio-rectal fossa can be wounded near the anterior part of the hollow, where they approach the margin of the triangular ligament ; but, posteriorly, they are securely lodged inside the projection of the tuber ischii.

Whilst making the deeper incisions to reach the staff, the artery of the bulb lies immediately in front of the knife, and will be wounded if the incisions are made too far forwards; but the vessel must almost necessarily be cut, when it arises farther back than usual, and crosses the front of the ischio-rectal fossa in its course to the bulb of the urethra.

In the last stage of the operation the neck of the bladder should not be cut to a greater extent than is necessary for the extraction of the stone, lest the recto-vesical fascia separating the perinæum from the pelvis shonld be divided, and the abdominal cavity opened. Too large an incision through the prostate may wound also an unusual accessory pudic artery on the side of that body.

Directions. When the dissection of the perinæum is completed, the flaps of skin are to be fastened together, after salt has been used, and the limbs are to be put down for the examination oi the abdomen.

\section{Section II.}

\section{PERIN EUM OF THE FEMALE.}

TuE perinæum in the female differs from that in the male more in the external form than the internal anatomy. On the surface it has special parts distinguishing it, viz., the aperture of the vagina, and the opening of the vulva with the labia. 
Surface-marking. In the middle line, there are the two apertures of the anus and vulva, which are separated from one another by an interval of about an inch. The anus is situate rather farther back than in the male. And the vulva with the labia majora on the sides is placed in the situation of the scrotum of the other sex.

Within the vulva at the upper part, is the clitoris, and two small membranous folds, labia minora, extend downwards from it. Below the clitoris is the small aperture of the urethra ; and still lower down is the vagina, whose opening is sometimes partly closed by a thin piece of membrane, the hymen.

Deep boundarics. The deep boundaries of the perinæum are alike in both sexes; but in the female the outlet of the pelvis is larger than in the male.

Fig. 133.

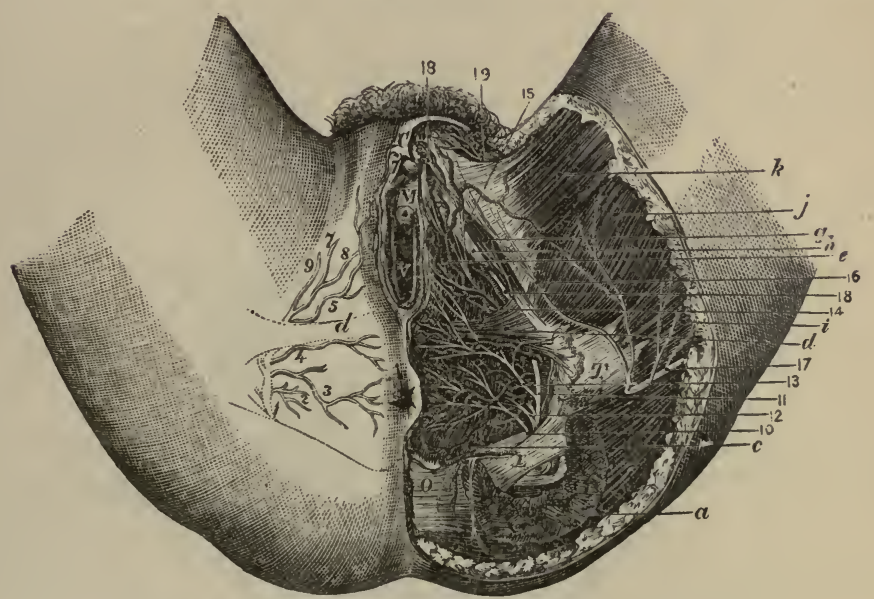

The Female Perin жu (from Dr. Savage's "Illustrations").

1. Pudic artery.

2. Branch to levator ani.

3. Inferior hæinorrhoidal artery.

4 Transverse artery.

5. Great labial (superficial perineal) artery.

7. Dorsal artery of clitoris.

S. Artery of bulb

9. Artery to crus clitoridis.

10. Inferior hæmorrhoidal nerve.

11. Pudic nerve.

12. Muscular branch.

13. Internal superficial perinaul nerve.

14. External superficial perinæal nerve.

15. Its junction with-

16. Inferior pudondal nerve.

17. Small sciatic nerve.

18,15 . Dorsal nerve of clitoris.
19. Ilio-inguinal nerve.

A. Anus.

C. Clitoris.

M. Meatus urinarius.

L. Great sacro-sciatic ligament.

V. Vagina.

o. Coccyx.

T. Tuberosity of i schium.

a. Gluteus miximus

c. Levator ani.

d. Superficial transverse uuscle.

e. Compressor bulbi.

g. Erector clitoridis.

h. Triangular ligament (cut).

$i$. Biceps and semi-tendinosus.

f. Adductor magrus.

k. Gracilis.

Dissection. The steps of the dissection are much the same in both sexes, and the same description will serve, generally, for the male and female perinæum.

First, the dissection of the ischio-rectal fossa is to be made. After26 
wards the muscles, vessels, and nerves of the posterior half of the perinaal space are to be examined. (See description of the male perinæum, p. 386 to 392 .)

Next the skin is to be taken from the interior half of the perinæal space, as in the male; and the transverse incision in front is to be made at the anterior part of the rulva. 'The attachments of the superficial fascia are then to be looked to, and the cutaneous vessels and nerves are to be traced beneath it (p. 392 to 394 ).

Superficial fascia. The description of this fascia in the male will serve for the like part in the female, with these modifications: that in the female it is interrupted in the middle line, and is of less extent, in consequence of the aperture of the vulva; and that it is continued forwards through the labia majora (the representative of the scrotum) to the inguinal region.

Fig. 134.

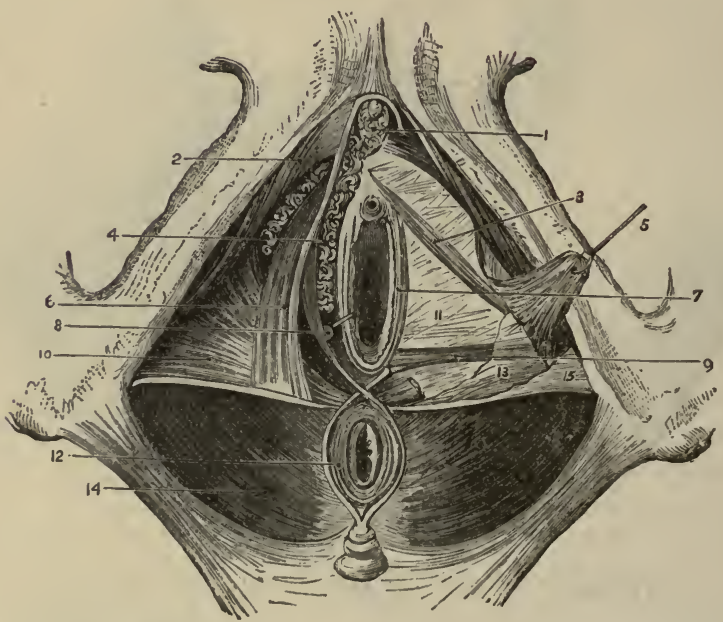

The Mugcles of the Female Perin ad a, Superficial on Right, and Defp on Left Side (from Savage and Luschka).

1. Clitoris.

2. Erector clitoridis.

3. Jarjavay's muscle.

4. Bulb of vagina.

5. Transversus perinæl (reflected).

6. Compressor bulbi (sphincter).

7. Constrictor vaginæ

8. Gland of Bartholin.
9. Deep transversus perinæi.

10. Superficial transversus perinæi.

11. Posterior layer of triangular ligament.

12. Sphincter ani.

13. Triangular ligament.

14. Levator ani.

15. Ischio-perineal ligament.

Dissection. The labia and the superficial faseia are to be removed, to follow the sphincter muscle around the opening of the vagina. Two other museles are exposed at the same time, viz., the erector ciitoridis on the pubic arch, and the transversalis perinæi passing across the perinæum to the central point.

The SPHINCTER VAGINA is an orbicular muscle around the orifice of the vagina, and corresponds with the ejaculator urinæ in the male. Posteriorly it is attached to the central point of the perinxum, where it mixes with the sphineter ani and transversalis museles; and its fibres are directed 
forwards on each side of the vagina, to be inserted into the body of the elitoris, and front of the triangular ligament.

Action. Like the other orbicular muscles the sphincter diminishes that part of the vagina which it encircles; and it assists in fixing the central point of the perinæum.

The ERECTOR CLITORIDIs resembles the erector of the penis in the male, though it is much smaller (p. 394).

The transversalis is similar to the muscle of the same name in the male. The one description will suffice for those muscles in both sexes (p. 395).

Dissection. To see the triangular ligament of the urethra, the erector and the crus clitoridis are to be detached from the bone, and the outer fibres of the sphincter vaginæ are to be removed.

The triangular ligament transmits the urethra, but is not quite so strongly marked as in the male (p. 396); its extent is partly interrupted behind by the large aperture of the vagina.

Dissection. By cutting through the superficial layer of the ligament in the same way as in the male (p. 397), the deep muscles, with the pudic vessels and nerve and their branches, will be arrived at.

The deep transverse muscle (depressor uretlire, Santorini) has the same origin externally as in the male (p. 397); and it meets its fellow at the middle line, like the muscle answering to it in the other sex. Santorini described the muscle as passing over, instead of below the urethra; hence the name given to it by its discoverer.

The CONSTRICTOR MUSCLE of the urethra resembles that of the male in its origin from the pubes, and its disposition around the urethra (p. 397). Within it is a circular layer of involuntary fibres, as in the other sex.

The description of the pudic artery (p. 398) will serve for both sexes, except that the branch in the female, which is the representative of the artery of the bulb in the male, is furnished to the vagina. The terminal branches are much smaller in the female.

The pudic nerve has the same peculiarity as the artery with respect to the branch to the vagina, and the smaller size of the terminal part of the nerve on the clitoris. 


\title{
CHAPTER VIII.
}

\author{
DISSECTION OF THE ABDOMEN.
}

\section{Section I.}

\section{WALL OF THE ABDOMEN.}

THF examination of the abrlomen is to proceed as far as the end of Section III. before the body is turned for the dissection of the Back.

Position. The body will be sufficiently raised by blocks beneath the thorax and head for the dissection of the upper limbs and neck, but the dissector should see that the chest is ligher than the pelvis. If the abdomen is flaccid, let it be inflated by an aperture through the umbilicus, but if it is firm, proceed with the dissection without blowing it up.

Surface-marking. On its anterior aspect the abdomen is for the most part convex, especially in fat bodies; but on the sides, between the ribs and the crista ilii, the surface is somewhat depressed. Along the middle line is a slight groove over the linea alba, which presents about its centre the hollow of the umbilicus. Inferiorly the groove ceases a little above the pelvis in the prominence of the pubes; and superiorly it subsides below the ensiform cartilage in a hollow named the epigastric fossa. On each side of the middle line is the projection of the rectus muscle, and this is intersected in adult well-formed bodies by two or three transverse lines

Underneath the eminence of the pubes the student will be able to recognize with his finger the symphysis pubis, and to trace outwards from it the osseous pubic crest which leads to the pubic spinous process. Rather above and to the outside of the pubes, the opening of the external abdominal ring may be felt; and the prominence of the spermatic cord descending through it to the testicle may be detected. The internal abdominal ring is still to the outer side, though it cannot be recognized on the surface with the finger; but its position may be ascertained by taking a point midway between the symphysis pubis and the crest of the innominate bone, and a little above Poupart's ligament.

If the finger is carried upwards and ontwards between the abdomen and the thigh, it will detect the firm band of Poupart's ligament, and sometimes one or two inguinal glands.

Dissection. 'The requisite incisions for raising the skin from the sides and front of the belly are the following: One cut is to extend outwards over the side of the chest from the ensiform cartilage to about midway between the sternum and the spine. A second incision is to be begun in the middle line midway between the umbilicus and the pubes, and to be carried outwards to the iliac crest, and along the crest till it ends opposite the first cut. Lastly, the hinder extremities of the two incisions are to 
be connected along the side of the chest and the belly. The piece of skin thus marked out is to be raised towards the middle line, but is not to be taken away; and the cutaneous vessels and nerves are to be sought in the fat at the side and middle line of the abdomen.

Along the side of the abdomen look for the lateral cutaneous nerves, five or six in number, which issue in a line with the corresponding nerves of the thorax. At first they lie beneath the fat, and divide into two: one offset is to be traced forwards, and the other backwards, with small cutaneous arteries. On the iliac erest, near the front, is a large branch from the last dorsal nerve; and usually farther back on the crest, and deeper, is a small branch of the iliohypogastric nerve. Near the middle line the small anterior cutaneous nerves will be recognized with small arteries; these are uncertain in number and size, and are to followed outwards in the integuments.

The piece of skin covering the lower part of the abdomen or the groin is next to be thrown downwards, on both sides, by an incision along the middle line to the root of the penis. After its reflection the cutaneous vessels and nerves are to be dissected on the right side, and the superficial fascia on the left.

To make the necessary dissection on the right side, all the fascia superficial to the vessels is to be raised in the same manner as the piece of skin. The vessels which will then appear are the superficial pudic internally, the superficial epigastric in the centre, and an offset of the superficial circumflex iliac artery externally. Some inguinal glands lie along the line of the reflected fascia. 'Two cutaneous nerves are to be sought: one, the ilio-inguinal, comes through the abdominal ring, and descends to the thigh and scrotum; the other, ilio-hypogastric, appears in the superficial fascia above, and rather outside the abdominal ring.

In the examination of the fatty layer on the left side two strata are to be made out, one over and one beneath the vessels. The layer that is superficial to the vessels is to be reflected by means of a transverse cut from the front of the iliac crest, about two inches above Poupart's ligament; and by a vertical one near the middle line to the pubes. The subjacent vessels mark the depth of this layer; and when these are reached, a flap of the fascin, like that of the skin, is to be thrown towards the thigh. To define the thinner under stratum, cut it across in the same manner as the other layer, and detach it with the vessels from the tendon of the external oblique muscle. This stratum, like the preceding, is to be traced around the cord to the scrotum; and as the student follows it downwards, he will find it connected with Poupart's ligament, and blended with the fascia lata close below that structure.

The subcutaneous fat, or the superficial fascia, is a single layer over the greater part of the abdomen; but in the groin it is divided into a subcutaneous and a deeper stratum by the vessels and the glands.

The subcutaneous layer contains fat, and varies therefore in appearance and thickness in different bodies; for it is sometimes divisible into strata, whilst at others it is very thin, and somewhat membranous near the thigh. It is continuous with the fatty covering of the thigh and abdomen; and when traced to the limb, it is separated from Poupart's ligament beneath by the superficial vessels and glands. Internally it is continued to the penis and scrotum, where it ehanges its adipose tissue for involuntary muscular fibre; and after investing the testicle, it is prolongel to the perinæum. 
The deeper layer (aponeurosis of the fascia lata, Scarpa) is thinner and more membranous than the other, and is closely united to the tendon of the external oblique by fibrous bands, especially towards the linea alba. Like the subcutaneous part, this layer is continued upwards on the abdomen, and inwards to the penis and the scrotum : here it becomes very thin, and reaches the perinæum, where it has attachments to the subjacent parts, as before specified (p. 393). Towards the limb, it ends a little below Poupart's ligament in the fascia lata across the front of the thigh ; as it passes over the ligament it is closely joined to that band by fibrous tissue.

Urine effused in the perinæum from rupture of the urethra will be directed through the scrotum and along the spermatic cord to the abdomen (p. 393). From the arrangement of the deeper layer of the fascia across the thigh, it is evident that the fluid cannot pass down the limb, though its progress over the front of the abdomen is uninterrupted.

In the female the fatty layer of the groin is separable into two layers, and the disposition of each is nearly the same as in the male; but the part that is continued to the scrotum in the one sex enters the labium in the other. In the female the round ligament of the uterus is lost in it.

Cutaneous Nerves. The nerves in the teguments are derived from the trunks of the lower intercostal nerves; thus the cutaneous branches, along the side of the belly, are offsets from five or six of those nerves; and the cutaneous branches, along the front, are the terminal parts of the same trunks. 'Two other cutaneous offsets from the lumbar plexus, viz., ilio-hypogastric and ilio-inguinal, appear at the lower part of the abdomen.

The lateral cutaneous nerves of the abdomen emerge between the digitations of the external oblique muscle, in a line with the same set of nerves on the thorax ; and the lowest are the most posterior. As soon as they reach the surface they divide, with the exception of the last, into an anterior and a posterior branch :-

The posterior branches are small in size, and are directed back to the integuments over the latissimus dorsi musele.

The anterior branches are continued nearly to the edge of the rectus muscle, and in increasing in size from above down, supply the integuments on the side of the belly; they furnish offsets to the digitations of the external oblique muscle.

The lateral cutaneous branch of the last dorsal nerve is larger than the rest, and does not divide like the others. After piercing the fibres of the external oblique muscle, it is directed over the iliac crest to the surface of the gluteal region.

The anterior cutaneous nerves of the abdomen pierce the sheath of the rectus : in the integuments they bend outwards towards the lateral cutaneous nerves. The number, and the situation of these small nerves, are very uncertain.

The ilio-hypogastric nerve is distributed by two pieces: one passes over the crista ilii (iliac branch), the other ramifies on the lower part of the abdomen (hypogastric branch):-

The iliac branch lies close to the erest of the hip bone near the last dorsal nerve, and enters the teguments of the gluteal region.

The hypogastric branch pierces the aponeurosis of the external oblique muscle above the abdominal ring, and is distributed, as the name expresses, to the lower part of the abdomen. 
The ilio-inguinal nerve becomes cutaneous through the external abdominal ring, and descends to the teguments of the scrotum, and upper and inner parts of the thigh.

Cutaneous Vessels. Cutaneous vessels run with both sets of nerves on the abdomen : with the lateral cutaneous nerves are branches from the intercostal arteries; and with the anterior cutaneous are offsets from the intercostal, internal mammary, and epigastric vessels. In the groin are three small superficial branches of the femoral artery, viz., pudic, epigastric, and circumflex iliac.

The lateral cutaneous arteries have the same distribution as the nerves they accompany. The anterior or chief offsets are directed towards the front of the abdomen, and end about the outer edge of the rectus muscle.

The anterior cutaneous vessels are irregular in number and in position, like the nerves. After piercing the sheath of the rectus, they run outwards with the nerves towards the other set of branches.

Branches of the femoral artery. Three cutaneous offsets ascend from the thigh between the layers of the subcutaneous fat, and ramify in the integuments of the genital organs and lower part of the abdomen. The greater portion of these vessels appears in the dissection of the thigh.

The external pudic branch (superficial) crosses the spermatic cord, to which it gives offsets, and ends in the integuments of the under part of the penis.

'The superficial epigastric branch ascends over Poupart's ligament, near the centre, and is distributed in the fat nearly as high as the umbilicus.

The circumflex iliac branch lies usually below the level of the iliac crest, and sends only a few offsets upwards to the abdomen.

Small veins accompany the arteries, and join the internal saphenous vein of the thigh.

'The glands of the groin are three or four in number, and lie over the line in Poupart's ligament. They are placed between the strata of the superficial fascia ; and receive lymphatics from the abdominal wall, from the upper and outer portion of the thigh, and from the superficial parts of the genital organs. 'Their efferent ducts pass downwards to the saphenous opening in the thigh to enter the abdomen.

Dissection. After the examination of the superficial fat with its vessels and nerves the student may prepare the cutaneous coverings of the penis and scrotum. The skin may be divided along the dorsum of the penis, and thrown to each side; and the skin of the scrotum is to be reflected on the left side by means of a vertical incision.

Cutaneons coverings of the penis and scrotum. The penis is attached to the front of the pubes by a suspensory ligament, and is provided with a tegumentary covering which is continuous with that of the abdomen, but it loses the fat and acquires special characters.

Around the end of the penis it forms the loose sheath of the prepuce in the following way: When the skin has reached the extremity, it is reflected backwards as far as the base of the glans, constituting thus a sheath with two layers-the prepuce; it is afterwards continued over the glans, and joins the mucous membrane of the urethra at the orifice on the surface. At the under part of the glans, and behind the aperture of the urethra, the integument forms a small triangular fold, franum praprtii.

Where the skin covers the glans, it is inseparably united with that part, is very thin and sensitive, being provided with papilla, and assumes in 
some cases the characters of a mucous membrane. Behind the glans are some sebaceous follicles-glandula odorifera.

In the scrotum the superficial fascia becomes thin, and of a reddish color. The prolongation around the testicle on one side, is separate from that on the other side; and the two pouches coming in contact in the middle line, form the septum scroti.

The subcutaneous layer in the scrotım, penis, and front of the perineum, contains involuntary muscular fibres, to which the corrugation of the skin is owing. This contractile structure is named the dartoid tissue.

Dissection. By removing all the fatty tissue from the root of the penis and the front of the symphysis pubis, the suspensory ligament will be defined. And the dorsal arteries and nerves, with the dorsal vein of the penis, which will be partly laid bare, are to be followed forwards in the teguments.

The suspensory ligament of the penis is a band of fibrous tissue, of a triangular form, which is attached by its apex to the front of the symphysis pubis near the lower part. Widening below, it is fixed to the upper surface of the body of the penis, and is prolonged on it for some distance. In the ligament are contained the dorsal vessels and nerves of the penis.

Dorsal vessels and nerves. The arteries and nerves on the dorsum of the penis are the terminal parts of the purlic trunks of both sides (p. 399). 'The rein accompanying the arteries enters the pelvis through the triangular perinæal ligament.

The dorsal artery, one on each side, pierces the suspensory ligament, and extends forwards to the glans, where it ends in many branches for that structure: in its course the vessel supplies the integuments and the body of the penis. It may be derived from the accessory pudic (p. 399).

The dorsal vein is a single trunk, and commences by numerous branches from the glans penis and the prepuce. It runs backwards by the side of the artery, through the suspensory ligament and the triangular ligament of the urethra, to join the prostatic plexus of veins. The vein receives branches from the erectile structure and the teguments of the penis.

Each dorsal nerve takes the same course as the artery, and ends like it in numerous branches to the glans penis. It furnishes a large branch to the corpus cavernosum penis, and other offsets to the integuments of the dorsum, sides, and prepuce of the penis.

In the female these vessels are much smaller than in the male; they occupy the upper surface of the clitoris-the organ that represents the penis.

Dissection of the muscles. The surface of the external muscle of the abdominal wall (fig. 135) is now to be freed from fascia on both sides of the body.

It is not advisable to begin cleaning this muscle in front, because there it has a thin aponeurosis, which is taken away too readily with the fat. Beginning the dissection at the posterior part, the student is to earry the knite obliquely upwards and downwards in the direction of the fibres. 'The thin aponeurosis before referred to is in front of a line extended upwards from the anterior part of the iliac crest; and as the dissector approaches that spot, he must be careful not to injure it, more particularly at the upper part, where it lies on the margin of the ribs, and is very indistinct.

On the right side the external abdominal ring, c, may be defined, to show the cord passing through it; and on the left side a thin fascia (inter- 
columnar), which is connected with the margin of that opening, is to be preserved. Lastly the free border of the external oblique should be made evident between the last rib and the iliac erest.

Muscles of the abdominal Wall. On the side of the abdomen are three large flat muscles, which are named from their position to one another, and from the direction of their fibres. The most superficial muscle is the external oblique; the underlying one, the internal oblique; and the deepest, the transversalis.

Along the middle line are placed other muscles which have a vertical direction. In front lie the rectus and pyramidalis, and behind is the quadratus lumborum : these are encased by sheaths derived from the aponeuroses of the lateral muscles.

The external oblique mescle (fig. $\left.135,{ }^{\wedge}\right)$ is fleshy on the side, and aponeurotic on the fore part of the abdomen. It arises by fleshy processes from the eight lower ribs; the five highest pieces alternating with similar parts of the serratus magnius, and the lowest three with digitations of the latissimus dorsi muscle. From the attachment to the ribs the fibres are directed over the side of the abdomen in the following manner:- the lower ones descend almost vertically to be inserted into the anterior half or more of the iliac crest, at the outer margin: and the upper and middle fibres are continued forwards obliquely to the aponeurosis on the front of the belly.

The aponeurosis occupies the anterior part of the abdomen, in front of a line from the eighth rib to the fore part of the crista illi; and it is rather narrower about the centre, than either above or below. Along the middle line this expansion ends in the linea alba-the common point of union of the aponeuroses of opposite sides. Ahove, it is thin, and is continued on the thorax to the pectoralis major and the ribs. Below, its fibres are stronger and more separate than above, and are directed obliquely downwards and inwards to the pelvis: some of them are fixed to the front of the pubes; and the rest are collected into a firm band $\mathrm{s}$ (Poupart's ligament) between the pubie spine and the iliac erest.

Connections. The muscle is subcutaneous. Its posterior border is unattached between the last rib and the iliac crest, but is orerlaid commonly by the edge of the latissimus dorsi, except a small part below. Appearing through the aponeurosis, external to the linea alba, is a white line, the linea semilunaris, marking the outer edge of the rectus muscle (fig. 139); and crossing between the two are three or four whitish marks, the linex transversae. Numerous small apertures in the tendon transmit cutaneous vessels and nerves; and near the pubes is the large opening of 
the external abdominal ring, which gives passage to the cord in the male, and the round ligament in the female.

Action. Both muscles taking their fixed point at the pelvis will bend the trunk forwards as in stooping; but supposing the spine fixed they will draw down the ribs. If they act from the thorax they will elevate the pelvis.

Should one muscle contract, it will incline the trunk or the pelvis to the same side, according as the upper or the lower attacliment may be movable : and it will turn the thorax to the opposite side.

Parts of the aponeurosis. Besides the general arrangement of the aponeurosis over the front of the abdomen, the student is to examine more minutely the linea alba in the middle line; the external abdominal ring with the fascia prolonged from its margin; and the rounded border named Poupart's ligament.

Linea alba (fig. 139). This white band on the front of the abdomen marks the place of meeting of the aponeuroses of opposite sides. It extends from the xiphoid cartilage to the pubes, and serves as a ligament between the chest and pelvis. Its breadth is wider above than below; and it is perforated here and there by small apertures, which allow pellets of fat to protrude in some bodies. A little below the centre is the umbilicus, which projects now beyond the surface, though before the skin was removed, a hollow indicated its position.

External abdominal ring (fig. 135, ${ }^{\mathrm{C}}$ ). This opening is situate near the pubes between the diverging fibres of the aponeurosis. It is somewhat triangular in form, with the base of the crest of the pubes, and the apex pointing upwards and outwards. The long measurement of the aperture is about an inch, and the transverse about half an inch.

Its margins are named pillars, and differ in form and strength. The inner one, thin and straight, is attached below to the front of the symphysis pubis, where it crosses the corresponding piece of the opposite sidethat of the right muscle being superficial. The outer margin is the strongest, and is not straight like the inner, but forms a kind of groove for the support of the spermatic cord: this margin is continuous with Poupart's ligament, and is attached below to the pubic spine or tuberosity. A thin membrane (intercolumnar) covers the opening, and is derived from some fibres on the surface of the aponeurosis.

The ring gives passage in the male to the spermatic cord, and in the female to the round ligament; and in each sex the transmitted part lies on the outer pillar as it passes through, and obtains a covering from the intercolumnar fibres. Through this aperture the inguinal hernia protrudes from the wall of the abdumen.

The intercolumnar fibres form a layer orer the aponeurosis, and bind together its parallel fibres, so as to construct a firm membrane. Inferiorly, where they are strongest, a bundle is connected with the outer third of Poupart's ligament, and is continued back to the crista ilii. At the external abdominal ring the fibres stretch from side to side, and becoming stronger and aggregated together, close the upper part of that opening; and as they are prolonged on the cord from the margin of the ring, they give rise to the membrane named intercolumnar fascia. On the left side, where the fascia is entire, this thin covering will be manifest on the surfice of the cord, or on the round ligament in the female.

Dissection. 'To see the attachments and connections of Poupart's ligament, it will be necessary to reflect, on both sides of the body, the lower 
part of the aponeurosis towards the thigh, as in fig. 140. For this purpose an incision is to be carried through the aponeurosis from the front of the iliac crest to about three inches from the linea alba; and the tendon is to be detached from the subjacent parts with the handle of the scalpel. When the aponeurosis cannot be separated farther from the tendons beneath, near the linea alba, it is to be cut in the direction of a vertical line to the symphysis pubis.

After the triangular piece of the aponeurosis has been thrown towards the thigh, the spermatic cord is to be dislodged from the surface of Poupart's ligament, to see the insertion of this band into the pubes, and to lay bare the fibres (triangular ligament) which ascend therefiom to the linea alba.

Poupart's ligament (fig. 136, ${ }^{\mathrm{D}}$ ) is the lower border of the aponeurosis of the external oblique, which intervenes between the front of the crista ilii and the pubes. Externally it is round and cord-like, and is attached to the anterior superior iliac spine. Internally it widens as it approaches the pubes (fig. 135, ${ }^{\mathrm{D}}$ ), and is inserted into the pubic spine and the pectineal line of the hip-bone for about three-quarters of an inch, forming a triangular-looking piece with its base directed outwards, which is named Gimbernat's ligament.

Poupart's ligament is not straight between its outer and inner attachments, but is curved downwards to the thigh; and it retains this position as long as the fascia lata remains uncut. Its outer half is oblique, and is firmly united with the subjacent iliac fascia; along the line of union of the two, the other lateral muscles of the abdominal wall are attached. Its inner half is placed over the vessels passing from the abdomen to the thigh.

Triangular ligament. From the insertion of Gimbernat's ligament into the pectineal line, some fibres are directed upwards and inwards to the linea alba, where they blend with the other tendons. As the fibres ascend, they diverge and form a thin band, to which the above name has been given.

Dissection. The upper part of the external oblique is now to be taken away, on both sides of the body, to see the parts underneath. The muscle may be detached by carrying the scalpel through the digitations on the ribs back to the free border, and then through the insertion into the crista ilii. It may be thrown forwards as far as practicable, after the nerves crossing the iliac crest are dissected out; but in raising it care must be taken not to detach the rectus muscle fiom the ribs above, nor to cut through the tendon of the internal oblique at the upper part. By the removal of the fatty tissue the underlying internal oblique muscle, with some nerves on its surface below, will be prepared.

At the lower border of the internal oblique the cremaster muscle on the cord is to be defined (fig. 137): it is about as wide as the little finger, and consists of fleshy loops which issue through the external abdominal ring. Its inner attachment is tendinous, and is easily taken away.

Parts covered by external oblique (fig. 136). Beneath the external, is the internal oblique muscle, with the ribs and the intercostal muscles. At the lower part of the abdomen the muscle conceals the spermatic cord, and the branches of the lumbar plexus in the abdominal wall.

The intersal oblique muscle (fig. $136, \mathbf{A}$ ) is fleshy below and aponeurotic above, just the reverse of the preceding; and its fibres (except the lowest) ascend across those of the external oblique. The muscle arises 
along the outer half of Poupart's ligament; along the anterior two-thirds of the crest of the hip bone; and from the tendon of the transtersalis muscle, $\mathrm{c}$, (fascia lumborum) in the interval between that bone and the last rib. 'The fibres diverge on the abdomen to their destination: The upper are fleshy and ascend to be inserted into the cartilages of the lower

Fig. 136.

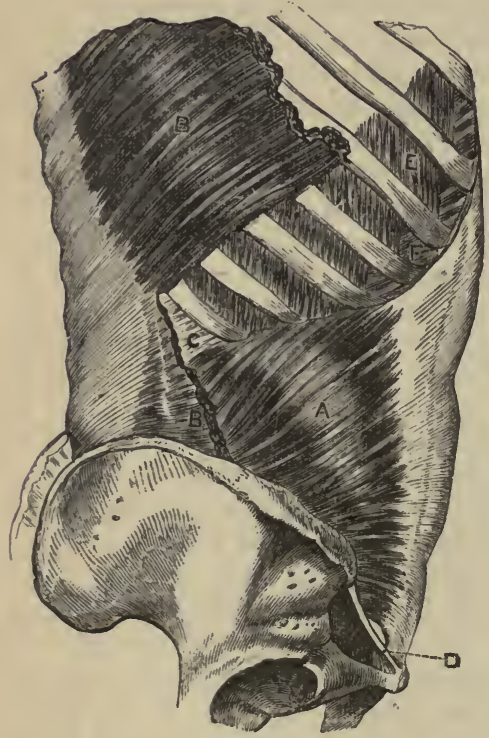

INTERNal OBLIQte MUscre of the ABDomiNA I, WALL.

A. Internal oblique.

B. Latissimus dorsi, cut.

c. Part of the hinder tendon of the transversalis muscle.

D. Poupart's ligament.

B External: F. Internal intercostals. three ribs, where they join the internal intercostal muscles of the lowest two spaces. The remaining fibres pass obliquely to the aponeurosis.

The aponeurosis covers the fore part of the abdomen from the pelvis to the chest, and blends with its fellow along the middle line. For the most part it incases the rectus; but midway between the umbilicus and the pubes it is undivided, and lies in front of that muscle. Superiorly it is attached to the thorax after the following manner: 'The stratum superficial to the rectus is fixed to the ninth rib, and blends with the aponeurosis of the external oblique; and the stratum beneath the muscle joins the cartilages of the eighth and serenth ribs, and the ensiform cartilage. Inferiorly its tibres become more distinct and separate, and are inserted into the front of the pubes, and into the pectineal line for half an inch behind the attachment of Gimbernat's ligament.

Connections. The internal is covered by the external oblique muscle. It is attached on all sides, except between Poupart's ligament and the pubes where it arches over the cord, and has the cremaster muscle contignous to it. The parts covered by the internal oblique cannot be seen till the muscle is reflected.

Action.-Both muscles will depress the ribs; and will assist in forcing back the viscera of the belly, which have been carried down by the descent of the diaphragm.

One muscle may incline the body laterally; and contracting with the external oblique of the other side (the fibres of the two having the same direction) it will rotate the trunk to the same side.

The Cremaster muscle (fig. 137, D) is a fasciculus of fibres, which lies along the lower border of the internal oblique muscle, and is named from suspending the testicle. The muscle has attachments, at the inner and outer sides, similar to those of the internal oblique. Externally it is fleshy, and arises from Poupart's ligament below, and in part beneath the internal oblique, with which some of the fibres are connected. Internally it is small, and is inserted by tendon into the front of the pubes, joining the tendon of the internal oblique. 
Between the two points of attachment the fibres descend on the front and sides of the cord, forming loops, with the convexity downwards, which reach to and over the testis. The bundles of fibres are united by areolar tissue so as to give rise to a covering on the front of the cord, which in hernia is named the fascia cremasterica. Occasionally the fibres may be behind as well as on the sides and front of the cord.

Action. It elevates the testicle towards the abdomen under the influence of the will; but it may be excited to contract involuntarily by cold, fear, etc.

Dissection. On the left side of the body the student is not to make any further dissection of the abdominal wall; and the layers that have been reflected in the groin should be replaced, until the examination of those parts in connection with hernia is resumed.

On the right side the dissection is to be carried deeper. by the removal of the internal oblique and the cremaster. The last muscle may be reflected from the cord by means of a longitudinal incision.

To raise the internal oblique, it will be necessary to cut it through firstly near the ribs; secondly near the crest of the hip bone and Poupart's ligament; and lastly at the hinder part, so as to connect the two first incisions. Its depth will be indicated by a fatty layer between it and the transversalis. In raising the muscle towards the edge of the rectus, let the student separate with great care the lower fibres from those of the transversalis, with which they Fig. 137.

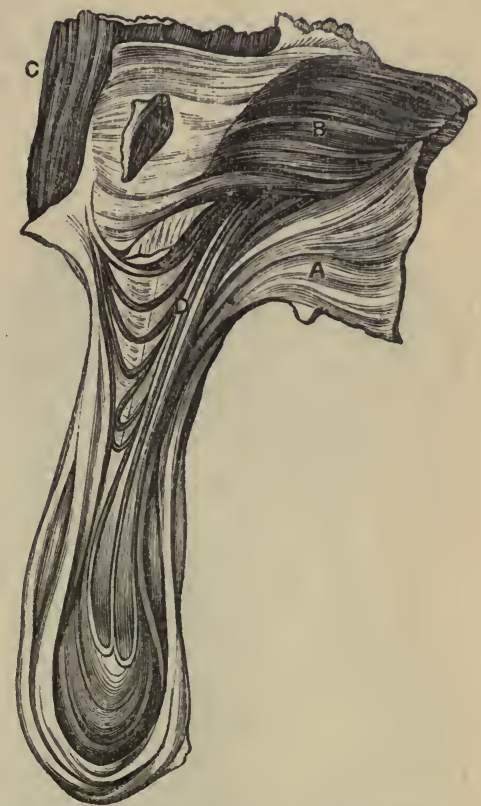

View of the Lower Part of the Internal OBLIQUe With THE Cremaster MÚSCLEAND THE TBSTICLE.
A. External oblique, reflected.
B. Internal oblique.
c. Rectus abdomínis.
D. Cremaster, with its loops over the sperma- tic cord and the testicle.

are often conjoined; and dissect out, between the'two, the intercostal nerves and arteries, and the two branches of the lumbar plexus (ilio-hypogastric and ilio-inguinal) near the front of the crest of the hip bone: the offsets of the intercostals entering the muscle must be cut.

Parts covered by the oblique (fig. 138). The internal oblique conceals the transversalis muscle, and the ressels and nerves between the two. Near Poujart's ligament it lies on the spermatic cord and the fascia transversalis. The rectus muscle is concealed below by the aponeurosis.

The transversalis muscle (fig. $138,{ }^{\mathbf{A}}$ ) forms the third stratum in the wall of the abdomen, and differs from the two oblique in having a posterior as well as an anterior aponeurosis. Like the former muscle it is attached on all sides, except where the spermatic cord lies. At the pelvis it arises along the outer third of Poupart's ligament, and the anterior twothirds of the iliac crest. At the chest it takes origin from the lower six 
ribs, viz., by tendon from the lowest two, and by fleshy processes from the under surface of the cartilages of the four next above. And between the chest and the pelvis it is connected with the lumbar vertebra by means of the posterior aponeurosis or the fascia lumborum. All the fibres are directed to the aponeurosis in front.

Its anterior aponeurosis is widest below, as in the most external muscle. Internally it is continued to the linea alba, passing beneath the rectus as low as midway between the umbilicus and the pubes, but in front of the muscle below that spot. Its attachment below to the pelvis is nearly the same as the internal oblique ; for it is fixed to the front of the pubes, and

Fig. 138.

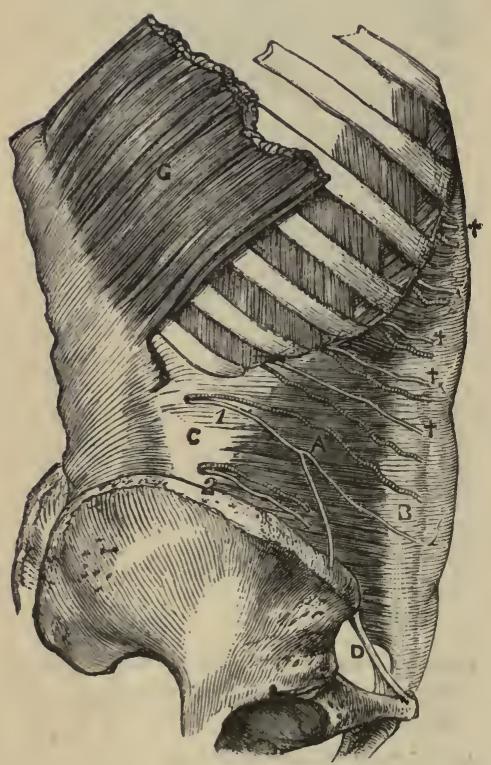

Dissection of the Third Lateral Muscle IN THв WALL OF Tнв BELLY, with the vegsels and nerves on it.

A. Transversalis mnscle, with $B$, its anterior, and $c$, its posterior tendon (fascia lumbornm).

D. Poupart's ligament.

1. Last dorsal nerve with its accompanying artery.

2. Ilio-hypogastric nerve with its artery.

$\dagger \dagger$ Intercostal neves and arteries. to the pectineal line for about an inch, but beneath the oblique muscle: some of the fibres are spent on the transversalis fascia, and are connected with a thickened band of that fascia beneath Poupart's ligament, which is called the deep crural arch.

Action. The chief use of the muscle will be applied to diminishing the size of the abdominal cavity, and compressing the viscera ; but it will assist the internal oblique in replacing the viscera pushed down in inspiration.

Conjoined tendon. The aponeurosis of the internal oblique and transversalis muscles are united more or less near their attachment to the pubes, and give rise to the conjoined tendon. The aponeurosis of the oblique muscle extends about half an inch along the pectineal line; whilst that of the transversalis reaches an inch along the bony ridge, and forms the greater part of the conjoined tendon.

The posterior aponeurosis of the transversalis, or the fascia lumborum, $\mathrm{c}$, is described in the dissection of the Back, p. 357.

Connections. Superficial to the transversalis are the two muscles before examined; and beneath it is the thin fascia transversalis. Its fleshy attachments to the ribs digitate with like processes of the diaphragm. The lower border is fleshy in the outer, but tendinous in the inner half, and is arched above the internal abdominal ring.

Dissection. 'To remove the aponeurotic layer from the rectus muscle of the right side, make a longitudinal incision through the tendinous sheath, and turn it to each side. As the fascia is reflected, its union with three or more tendinous bands across the rectus will have to be cut through ; and near the pubes a small muscle, the pyramidalis, will be ex 
posed. The dissector should leave the nerves entering the outer border of the rectus.

On the left side of the body the rectus should not be laid bare below the umbilicus, for the sake of the hernia to be seen on this side.

The rectus muscle (fig. 139, ${ }^{\mathbf{A}}$ ) extends along the front of the abdomen from the pelvis to the chest. It is narrowest inferiorly, and is attached to the pubes by two tendinous processes;--one, internal and the smaller, arises from the front of the symphysis, where it joins the muscle of the opposite side; and the external process is attached to the pubic crest. Becoming wider towards the thorax, the rectus is inserted by three large fleshy processes into the ensiform cartilage, and the cartilages of the last three true ribs.

The muscle is contained in an aponeurotic sheath, except above and below ; and its fibres are interrupted at intervals by irregular tendinous lines-the inscriptiones tendiner.

Action. It will draw down the thorax and the ribs, or raise the pelvis, according as its fixed point may be above or below. Besides imparting movement to the trunk, it will diminish the eavity of the thorax, and compress the viscera.

Sheath of the rectus, D. This sheath is derived from the splitting of the aponeurosis of the internal oblique at the outer edge of the musele. One piece passes before and the other under the rectus; and the two unite at the inner border so as to inclose it in a sheath. Inseparably blended with the stratum in front of the rectus is the aponeurosis of the external oblique; and joined in a similar manner with that behind, is the aponeurosis of the transversalis.

The sheath is deficient behind, both at the upper, and lower part of the muscle. Above, the muscle rests on the ribs, without the intervention of the sheath which is fixed to the margin of the thorax. Below, midway between the umbilicus and the pubes, the internal oblique ceases to split, and passes

Fig. 139.

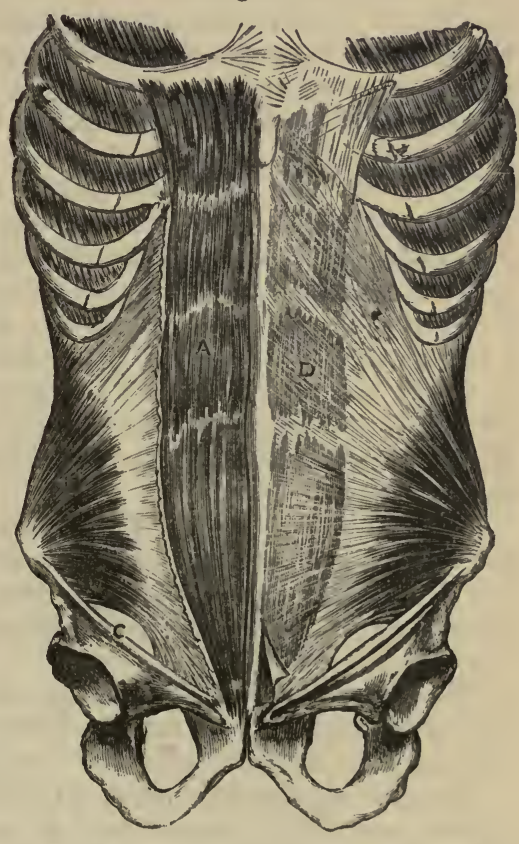

Rectug MUgcle of the AвDomsi, dissected on the right side, and in its sheath on the left. Close to the pelvis is the pyramidalis exposed.
A. Rectus.
B. Interual oblique.
C. Poupart's Hgament.
D. Sheath of the rectus. altogether in front of the rectus, with the other aponeuroses; at the spot where the sheath is wanting inferiorly the muscle is in contact with the fascia transversalis, and a white semilunar line (the fold of Douglas) may be sometimes seen, when the outer edge is raised, marking the cessation.

The linea transversa (fig. 137), on the surface of the abdomen, are caused by tendinous intersections of the rectus. They are usually three 
in number, and have the following position : one is opposite the umbilicus, another at the ensiform cartilage, and the third midway between the two. If there is a fourth, it will be placed below the umbilicus. These markings seldom extend the whole length or breadth of the muscular fibres, more particularly above and below.

Linea semilunaris (fig. 139). This line corresponds with the outer edge of the rectus, and reaches from the eighth rib to the pubic spine of the hip-bone: it marks the line of division of the aponeurosis of the internal oblique muscle.

The PYRaMidalis Muscle (fig. 139) is triangular in form, and is placed in front of the rectus near the pelvis. The muscle arises by its base from the front of the pubes, and is inserted into the linea alba about midway between the umbilicus and the pelvis. This small vessel is often absent.

Action. The muscle renders tight the linea alba; and when large it may assist the rectus slightly in compressing the viscera.

Nerves of the abdominal wall (fig. 138). Between the internal oblique and transversalis muscles are situate the intercostal nerves; and near the pelves are two branches of the lumbar plexus. Some arteries aceompany the nerves, but they will be referred to with the vessels of the abdominal wall.

The lower six intercostal nerves (fig. $138 \dagger \dagger \dagger$ ) enter the wall of the abdomen from the intercostal spaces. Placed between the two deepest lateral muscles, the nerves are directed forwards to the edge of the rectus, and through this muscle to the surface of the abdomen near the middle line. About midway between the spine and the linea alba, the nerves furnish cutaneous branches to the side of the abdomen (lateral cutaneous, p. 406); and whilst between the abdominal muscles they supply muscular branches, and offsets of communication with one another. A greater part of the lower than of the upper nerves is visible, owing to the shortness of the last intercostal spaces.

The last dorsal nerve $\left(^{1}\right)$ is placed below the twelfth rib, and therefore not in an intercostal space, but it has connections and a distribution like the preceding. As it extends forwards to the rectus it communicates sometimes with the ilio-hypogastric nerve. Its lateral cutaneous branch perforates the two oblique muscles (p. 406).

Two branches of the lumbar plexus, viz., ilio-hypogastric and ilio-inguinal, are contained for a certain distance between the muscles of the wall of the abdomen, as they course forwards to the surface of the body.

The ilio-hypogastric nerve $\left({ }^{2}\right)$ perforates the back of the transversalis muscle near the iliac erest, and gives off the iliac branch. The nerve is then directed forwards above the hip bone, and is connected with its companion (ilio-inguinal). Perforating the fleshy part of the internal oblique near the front of the iliac crest, and the aponeurosis of the external oblique near the linea alba, the nerve becomes cutaneous (p. 406).

Its iliac branch pierces both oblique muscles close to the erista ilii, to reach the gluteal region.

The ilio-inguinal nerve perforates the transversalis inuscle near the front of the iliac crest. It pierces afterwards the internal oblique, anil reaches the surface of the thigh through the external abdominal ring ( $p$. 407 ); it furnishes offsets to the internal oblique, the cremaster, and the pyramidalis.

Dissection. For the purpose of seeing the transversalis fascia, it will he necessary to raise, on the right side, the lower part of the transversalis 
muscle by two incisions; one of these is to be carried through the fibres attached to Poupart's ligament; the other, across the muscle from the front of the hip bone to the margin of the rectus. With a little care the muscle may be separated easily from the thin fascia beneath.

The fascia transversalis (fig. $140,{ }^{a}$ ) is a thin fibrous layer between the transversalis muscle and the peritoneum. In the groin or inguinal region, where it is unsupported by muscles, the fascia is considerably stronger than elsewhere, and is joined by fibres of the aponeurosis of the transversalis muscle; but farther from the pelvis it gradually decreases in strength, until at the thorax it becomes very thin.

In the part of the fascia now laid bare, is the internal abdominal ring, which gives passage to the spermatic cord, or the round ligament, according to the sex; this opening resembles the finger of a glove in being visible internally, but indistinguishable externally in consequence of a prolongation fiom the margin. On the inner side of the ring the fascia is thinner than on the outer, and is there fixed into the pubes and the pectineal line of the hip bone, behind the conjoined tendon with which it is united.

At Poupart's ligament the fascia is joined to the posterior margin of that band along the outer half; but along the inner half it is directed down to the thigh, in front of the bloodvessels, to form the anterior part of a loose sheath (crural) around them.

Internal abdominal ring (fig. 140). This opening is situate midway between the symphysis pubis and the anterior superior iliac spine, and half an inch above Poupart's ligament. From its margin a thin tubular prolongation of the fascia is continued around the cord, as before said.

Dissection. The tubiform prolongation on the cord may be traced by cutting the fascia transversalis horizontally above the opening of the ring, and then longitudinally over the cord. With the handle of the scalpel the thin membrane may be reflected to each side, so as to lay bare the subperitoneal fat.

The subperitoneal fat forms a layer between the fascia transversalis and the peritoneum. Its thickness varies much in different bodies, but is greater at the lower part of the abdomen than higher up. This structure will be more specially examined in the dissection of the wall of the abdomen from the inside.

Dissection. After the subperitoneal fat has been seen, let it be reflected to look for the remains of a piece of peritoneum along the cord, in the form of a fibrous thread.

The peritoneum, or the serous sac of the abdominal cavity, projects forwards slightly opposite the abdominal ring. Connected with it at that spot is a fibrous thread (the remains of a prolongation to the testis in the ficetus) which extends a certain distance along the front of the cord. It is generally impervious, and can be followed only a very short way, but it may be sometimes traced as a fine band to the tunica vaginalis of the testis.

In some bodies the process may be partly open, being sacculated at intervals; or it may form occasionally a single large bag in front of the cord. Lastly, as a rare state, the tube of peritoneum accompanying the testis in its passage in the foetus may be unclosed, so that a coil of intestine could descend in it from the abolomen.

In the female the fotal tube of peritoneum remains sometimes pervious for a short distance in front of the round ligament; that unobliterated passage is named the canal of Nuck. 
The spermatic CORD (fig. $140, F_{5}$ ) extends from the internal abdominal ring to the testis, and consists mainly of the ressels and the efferent duct of the gland, united together by coverings from the structures hy or through which they pass.

In the wall of the abdomen the cord lies obliquely, beeause its aperture of entrance amongst, is not opposite its aperture of exit from the muscles; but escaped from the abdomen, it descends almost vertically to its destination. As it lies in this oblique passage named the inguinal canal, it is placed (externally) beneath the internal oblique, and rests against the fascia transversalis; but beyond the lower border of the oblique muscle, it lies on the upper surface of Poupart's ligament, with the aponeurosis of the external oblique between it and the surface of the body, and the conjoined tendon behind it.

Its several coverings are derived from strata in the wall of the abdomen. Thus, from within out come, the subperitoneal fat, the tube of the fascia transversalis, the cremaster muscle continuous with the internal oblique, the intercolumnar fascia from the external oblique muscle, and lastly the superficial faseia and the skin.

The round ligament, or the suspensory cord of the uterus, occupies the inguinal canal in the female, and ends in the integuments of the groin. Its coverings are similar to those of the spermatic cord, except it wants the eremaster.

Dissection. The constituents of the cord will be displayed by cutting through longitudinally, and turning aside the different surrounding layers, and removing the areolar tissue. The dissector should trace branches of the genito-craral nerve and epigastric artery into the cremasteric covering.

Vessels and nerves of the cord. In the cord are collected together the spermatic artery and vein which convey the blood to, and take it away from the testis; the nerves and lymphatics of the testicle; and the vas deferens or the efferent duct.

In the female a branch from the ovarian artery enters the round ligament.

The vas deferens reaches from the testicle to the urethra, and is placed behind the other vessels of the eord; it will be recognized by its resemblance in feel to a piece of whipeord, when it is taken between the finger and the thumb. As it enters the abdomen through the opening in the fascia transversalis (internal ring), it lies on the inner side of the vessels of the testicle; and as it begins its descent to the pelvis, it winds behind the epigastric artery.

Cremasteric artery and nerve. The cremasteric covering of the cord has a separate artery and nerve. The artery is derived from the epigastric, and is distributed to the coverings of the cord. The genital branch of the genito-crural nerve enters the cord by the internal abdominal ring, and ends in the cremaster muscle.

Cutaneous vessels and nerves are supplied to the teguments of the cord from the superficial pudic artery and the ilio-inguinal nerve.

Dissection. By cutting through the spermatic cord near the pubes, and raising it towards the inner abdominal ring, a fibrous band below Poupart's ligament, the deep crural arch, will appear : it passes inwards to the pubes, and is to be defined with some care.

The remaining vessels of the abdominal wall, viz., the epigastric and cireumflex iliac, and the ending of the internal mammary artery are to be next dissected. The epigastric and mammary arteries will be observed on 
raising the outer edge of the rectus, one above and the other below, ramifying in the muscle.

The epigastric, with its earliest branches, may be traced by removing the fascia transversalis from it near Poupart's ligament. The circumflex iliac artery lies behind the outer half of Poupart's ligament, and should be pursued along the iliac crest to its ending.

Deep crural arch. Below the level of Poupart's ligament is a thin band of transverse fibres over the femoral vessels, which has received the name deep crural arch from its position and resemblance to the superficial crural areh (Poupart's ligament). 'This fasciculus of fibres, beginning about the centre of the ligament, is prolonged inwards to the pubes, where it is widened, and is inserted into the pectineal line at the deep aspect of the conjoined tendon of the broad muscles of the abdomen. ${ }^{1}$ It is closely connected with the front of the erural sheath.

Vessels in tife Wall of the Abdomen. On the side of the abdomen are the intercostal and lumbar arteries with the intercostal nerves. In the sheath of the rectus lie the epigastric and internal mammary vessels ; and around the crest of the hip-bone bends the circumflex iliac branch.

The intercostal arteries (fig. 138) issue between the false ribs (p. 337), and enter the abdominal wall between the transversalis and internal oblique muscles: they extend forwards with the nerves, supplying the contiguous muscles, and some end in the teguments. In front they anastomose with the internal mammary and epigastric : behind they communicate with the lumbar arteries.

The lowest artery accompanies the last intercostal nerve below the last rib, and is distributed with the nerve.

The internal mammary artery. The abdominal branch of this vessel (p. 239 ) enters the wall of the abdomen beneath the cartilage of the seventh rib. Descending in the sheath of the rectus, the vessel soon enters the substance of the muscle, and anastomoses in it with the epigastric artery.

The epigastric artery (fig. 140, a) arises from the external iliac about a quarter of an inch above Poupart's ligament; it ascends in the sheath of the rectus and divides above the umbilicus into branches which enter that muscle, and anastomose with the internal mammary.

As the artery courses to the rectus it passes beneath the cord, and on the inner side of the internal abdominal ring; and it is directed obliquely inwards across the lower part of the abdomen, so as to form the outer boundary of a triangular space along the edge of the rectus. It lies at first beneath the fascia transversalis; but soon perforates the finscia, and enters the sheath of the rectus over the semilunar border at the posterior aspect.

'The branches of the artery are numerous, but inconsiderable in size :-

a. 'The pubic branch is a small transverse artery, which runs behind Poupart's ligament to the posterior aspect of the pubes, and anastomoses with a similar branch from the opposite side. Behind the pubes it communicates with a small offset from the obturator artery (fig. 141, $f$ ): the size of this anastomosis varies much, but its situation is internal to the crural ring.

1 Sometimes this structure is a firm distinct band, which is joined by some of the lower fibres of the aponeurosis of the external oblique. At other times, and this is the most common arrangement, it is only a thickening of the fascia transversalis with the fibres added from the tendon of the transversalis muscle. 
b. A cremasteric branch is furnished to the muscular covering of the cord.

c. Muscular branches are given from the outer side of the artery to the abdominal wall, which anastomose with the intercostal arteries (p. 419), and others enter the rectus. Cutaneous offsets pierce the muscle, and ramify in the integuments with the anterior cutaneous nerves.

Two epigastric veins lie with the artery; they join finally into one, which opens into the external iliac vein.

The circumflex iliac artery arises from the outer side of the external iliac, opposite the epigastric, and courses around the iliac crest, as the name expresses. Having perforated the crural sheath, it passes beneath the transversalis muscle to the middle of the crest of the hip-bone. Here it pierces the transversalis, and is continued backwards bctween this and the internal oblique, to anastomose with the ilio-lumbar branch of the internal iliac artery. Its offsets are muscular and anastomotic.

Branches. Near the front of the iliac crest a small branch ascends between the internal oblique and transversalis muscles, supplying them, and anastomoses with the epigastric and intercostal arteries.

As the vessel extends backwards it gives lateral offsets, which supply the neighboring muscles, and communicate on the one side with the iliolumbar, and on the other with the gluteal artery.

The circumflex iliac vein is formed by the junction of two collateral branches, and crosses the external iliac artery nearly an inch above Poupart's ligament, to open into the external iliac vein.

\section{SECTION II.}

\section{HERNIA OF THE ABDOMEN.}

THE lower part of the abdominal wall, which has been reserved on the left side of the body, should now dissected for inguinal hernia.

Dissection. The teguments and the aponeurosis of the external oblique having been thrown down in the previous examination of the wall of the abdomen, the necessary dissection of the inguinal region will be completed by raising the internal oblique muscle, as in fig. 140 .

To raise the oblique muscle, let one incision be made across the fleshy fibres from the iliac crest towards the linea alba; and after the depth of the muscle has bcen ascertained by the layer of arcolar and fatty tissue beneath it, let the lowest fibres be carefully cut through at their attachment to Poupart's ligament. By raising the muscle cautiously, the student will be able to separate it from the subjacent transversalis, so that it may be turned upwards on the abdomen. The separation of the two muscles just mentioned is sometimes difficult, in consequence of their fibres being blended together, but a branch of the circumflex iliac artery will mark their intermuscular interval.

The cremaster muscle is next to be divided along the cord, and to be reflected to the sides. Let the dissector then clean the surface of the transversalis muscle, without displacing its lower arched border; and trace with care the conjoined tendon of it and the internal oblique to show the 
exact extent outwards. The fascia transversalis and the spermatic cord should be likewise nicely cleaned.

Crossing the interval apparent below the border of the transversalis muscle, are the epigastric vessels, which lie close to the inner side of the internal abdominal opening, but beneath the fascia transversalis. A small piece of the fascia may be cut out to show the vessels.

Inguinal Hernia. A protrusion of intestine through the lower part of the abdominal wall near Poupart's ligament (the part answering to the inguinal region), is named an inguinal hernia. The escape of the intestine in this region is predisposed to by the deficiencies in the muscular strata, by the passage of the spermatic cord through the abdominal parietes, and by the existence of fossæ on the inner surface of the wall.

The gut in leaving the abdomen either passes through the internal abdominal ring with the cord, or is projected througl the part of the abdominal wall between the epigastric artery and the edge of the rectus muscle. These two kinds of hernia are distinguished by the names external and internal, from their position to the epigastric artery; or they are called oblique and direct, from the direction they take through the abdominal wall. Thus, the hernia protruding through the internal abdominal ring with the cord is called external from being outside the artery, and oblique from its slanting course; whilst the hernia between the edge of the rectus and the epigastric artery is named internal from being inside the artery, and direct from its straight course.

External or Oblique Inguinal Hernia leaves the cavity of the abdomen with the spermatic cord, and traversing the inguinal canal, makes its exit from that passage by the external abdominal ring.

Anatomy of the external hernia. To acquire a knowledge of the anatomy of this hernia it will be necessary that the space in which it lies (inguinal canal), the apertures by which it enters and leaves the wall of the abdomen (abdominal rings), and the coverings it receives in its progress to the surface of the body, should be studied.

The inguinal canal (fig. 140) is the interval between the flat muscles of the abdominal wall, which contains the spermatic cord in the male, and the round ligament in the female. Its direction is oblique downwards and inwards, being nearly parallel to, but above Poupart's ligament; and its length is about one inch and a half. Superiorly it ceases at the internal abdominal ring; and inferiorly it ends in the external abdominal ring.

Towards the surface of the body the canal is bounded by the teguments, and the two oblique muscles in this way:-The skin with the subjacent fatty layer, and the aponeurosis of the external oblique, A, reach the whole length of the passage; but the internal oblique, B, extends only along its outer third (half an inch).

Towards the cavity of the abdomen the wall of the canal is constructed by the conjoined tendon of the internal oblique and transversalis, and by the deep membranous strata in the wall of the abdomen in this wise:The conjoined tendon, $\mathrm{H}$, placed in front of the other structures, reaches along the inner two-thirds of the space (about an inch); and beneath or behind it come the fascia transversalis, $G$, the subperitoneal fat, and the peritoneum, in the order mentioned, which are continued all along the passage.

Along the lower part, or the floor, the canal is limited by the union of the fascia transversalis with Poupart's liganent, and by the fibres of the 
ligament inserted into the pectineal line; whilst along the upper part its extent is determined only by the apposition of the muscles.

In the female, the canal has the same length and boundaries, though it is usually somewhat smaller. In that sex it lodges the round ligament.

The internal abdominal ring (fig. 140) is an aperture in the fascia transversalis, which is situate midway between the symphysis pubis and the iliac erest, and half an inch above Poupart's ligament. It is oval in form, the extremities of the oval being directed upwards and downwards, and measures about half an inch; the fascia at its outer and lower parts is stronger than at the opposite sides.

Fig. 140.

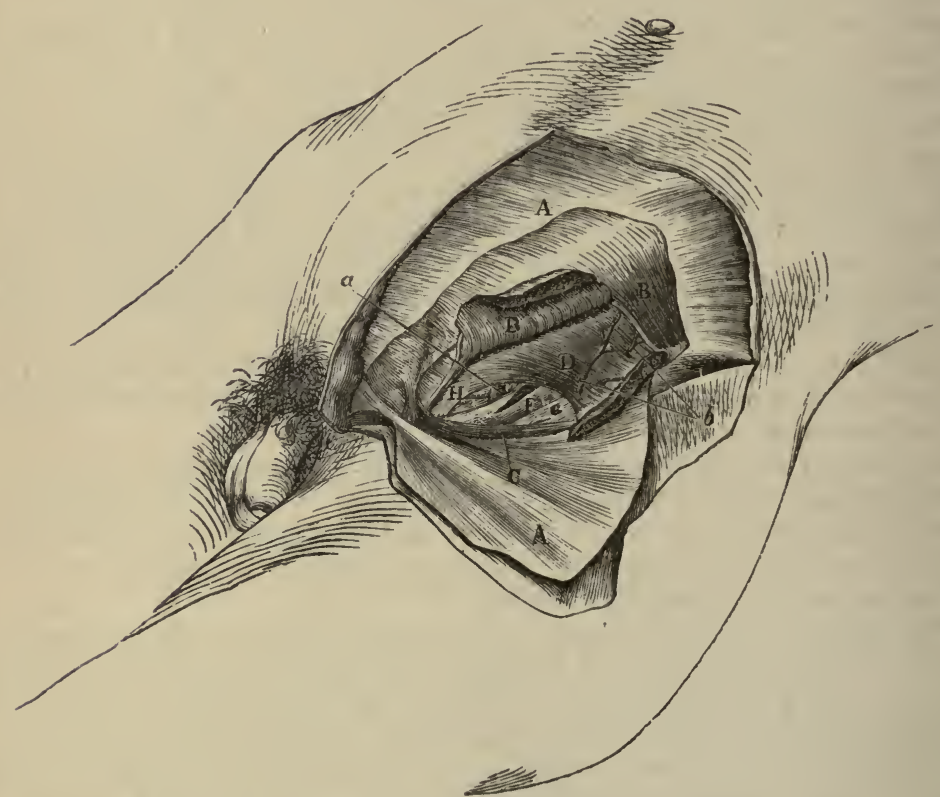

Muscles:

DIssiction for INGUNAI, HeRNia. (Illustrations of Dissections.)
A. External oblique tendon, thrown down.
B. Internal oblique, the lower part raised.
c. Cremaster muscle in its natural position.
D. Transversalis muscle with a free border.
P. Spermatic cord.
G. Fascia transversalis.
H. Conjoined tendon.

Arteries :

a. Epigastric vessels.

Arching above and on the inner side of the aperture, is the lower border of the transversalis muscle, D, which is fleshy in the outer, but tendinous in the inner half. Below, it is bounded by Poupart's ligament. On the inner side lie the epigastric vessels $(a)$.

This opening in the fascia transversalis is the inlet to the inguinal eanal, and through it the cord, or the round ligament, passes into the wall of the abdomen. The external hernia enters the canal the same spot. All the 
protruding parts receive as a covering the prolongation from the margin of the opening.

The external abdominal ring (fig. $135,^{\circ}$ ) is the outlet of the inguinal canal, and through it the spermatic cord reaches the surface of the body. This aperture is placed in the aponeurosis of the external oblique muscle, near the crest of the pubes; and from the margin a prolongation is sent on the parts passing through it (p. 410).

Course and coverings of the hernia. A piece of intestine leaving the abdomen with the cord, and passing through the inguinal canal to the surface of the body, will obtain a covering from every stratum in the lateral part of the wall of the abdomen, except from the transversalis muscle.

It receives its investments in this order: As the intestine is thrust outwards, it carries before it first the peritoneum and the subperitoneal fat, and enters the tube of the fascia transversalis, $\mathrm{F}$ (infundibuliform fascia), around the cord. Still increasing in size it is foreed downwards to the lower border of the internal oblique muscle; where it will have the eremasteric fascia, c, applied to it. The intestine is next directed along the firont of the cord to the external abdominal ring, and in passing through that opening receives the investment of the intercolumnar or spermatic fascia. Lastly, as the hernia descends towards the scrotum, it has the additional coverings of the superficial fascia and the skin.

In a hernia which has passed the external abdominal ring, the coverings from without inwards are the following: the skin and the superficial fascia, the spermatic and cremasteric fascie, the fascia transversalis, the subperitoneal fat, and the peritoneum or hernial sac. Two of the coverings, viz., the peritoneal and subperitoneal, originate as the gut protrudes, but the rest are ready formed around the cord, and the intestine slips inside them. The different layers become much thickened in a hernia that has existed for some time.

Diagnosis. If the hernia is small and is confined to the wall of the belly, it gives rise to an elongated swelling along the inguinal canal. If it has proceeded farther, and entered the scrotum, it forms a flask-shaped tumor with the large end below, and the narrow neck occupying the inguinal passage.

Whilst efforts are being made to force back a piece of protruded intestine during life, the direction of the canal, and the situation of the internal abdominal ring should be borne in mind.

Seat of stricture. The protruded intestine may be constricted at the internal abdominal ring; in the inguinal canal by the fleshy internal oblique muscle ; and at the external abdominal ring.

The stricture is placed usually at the inner abdominal ring, and may be produced in two ways : either by a constricting fibrous band ontside the narrowed neck of the tumor, or, by a thickening and contraction of the peritoneum itself at the inner surface of the neck.

Division of stricture. 'To set free the intestine, an incision is made down to the internal abdominal ring; and, all fibrous bands outside the peritoneum being divided, the intestine is to be returned into the abdomen by gentle pressure.

Supposing the intestine cannot be replaced in the abdomen after the previous steps have been taken, the surgeon proceeds to lay open the peritoneum, and to divide the internal stricture, from within out, on a director 
placed beneath it. With a view of avoiding the surrounding vessels, the cut is directed upwards on the front and mid-part of the hernia.

Other designations. 'This kind of hernia has other names applied to it sometimes by surgeons, according as it has passed certain points in the wall of the abdomen. If the intestine remains in the inguinal canal, the term bubonocele is applied to the swelling; but if it has extended into the scrotum, the appellation scrotal rupture, or oscheocele, is given to the tumor.

Varieties of the external hernia. There are two varieties of the oblique inguinal hernia (congenital and infantile), which are distinguished by the condition of the peritoneal covering.

Congenital hernia. This kind is found for the most part in the infant and the child, though it may occur in the adult male. In it the tube of peritoneum, which accompanies the testicle from the abdomen in the foetus, remaining unclosed, the intestine descends into a sac already formed for its reception.

As it takes the course of the inguinal canal, it will possess the corerings before enumerated for the external hernia; and it passes at the first to the bottom of the scrotum, instead of being arrested at the top of the testis.

With care it may be distinguished, whilst it is of moderate size, by its position in front of the testicle.

For the seat, cause, and division of the stricture, refer to what is before stated for external hernia.

Infantile hernia is much rarer than congenital, and cannot be distinguished from the common external hernia during life. It was first recognized in the young child, and received its name from that circumstance; but, like the congenital, it may be met with in the adult. Its chief differential character is derived from the state of the peritoneum.

The peritoneum has the following condition:- the tube of that membrane passing with the testicle in the foetus, is closed only at the internal abdominal ring, instead of being obliterated from that point down to the testicle, so that a large serous sac will be situate in front of the spermatic cord, and may occupy the inguinal canal. With this state of the peritoneum, should an external hernia with its coverings descend along the cord in the usual way, it will pass behind the unobliterated sac, like a viscus into serous membrane. In this way there will be two sacs; an anterior (the tunica vaginalis), containing serum, and a posterior inclosing the intestine.

The infantile hernia is first recognized during an operation by the knife opening the tunica vaginalis. The operator then proceeds to lay bare the neck of the hinder or hernial sac, and to treat the stricture of it as before described (p. 423).

The Internal or Direct Inguinal. Hervia escapes on the inner side of the epigastric artery, and has a straiglit course through the abdominal parietes. Its situation and coverings, and the seat of stricture, will be better understood after the examination of the part of the abdominal wall through which it passes.

Analomy of the internal hernia. At the lower part of the abdominal wall is a triangular space (fig. 140) which is bounded by the epigastrie artery on one side, the outer edge of the rectns muscle on the other, and the inner half of Poupart's ligament below : it measures about two inches from above down, and one inch and a half across at the base. 
The constituents of the abominal wall in this spot are, the teguments; the strata of the muscles; and the layers lining the interior of the abdomen, viz., fascia transversalis, subperitoneal fat, and peritoneum. The muscles have the undermentioned arrangement:- The aponenrosis of the external oblique is pierced by an aperture (external abdominal ring) towards the lower and inner angle of the space through which the inguinal hernia is transmitted. The internal oblique and transversalis, which come next, are united together in the conjoined tendon; as this descends to its insertion into the pectineal line, it covers the inner two-thirds (about an inch) of the space, and leaves uncovered about half an inch between its outer edge and the epigastric vessels, where the fascia transversalis appears.

Any intestine protruding in this spot must make a new path for itself, and elongate the different structures, because there is not any opening by which it can descend, as in the external hernia. Further, the coverings of the hernia, and its extent and direction in the abdominal wall, must vary according as the gut projects through the portion of the space covered by the conjoined tendon, or through the part external to that tendon.

Course and coverings of the hernia. The common kind of the internal hernia (inferior) passes through the part of the triangular space which is covered by the conjoined tendon.

The intestine in protruding carries before it the peritoneum, the subperitoneal fatty membrane, and the fascia transversalis; next it elongates the conjoined tendon, or as in a sudden rupture, separates the fibres, and escapes between them. Then the intestine advances into the lower part of the inguinal canal, opposite the external abdominal ring; and passes through that opening on the inner side of the cord, receiving at the same time the covering of the fascias permatica. Lastly, it is invested by the superficial faseia and the skin.

In number the coverings of the internal hernia are the same as those of the external; and in kind they are the same, with this exception, that the conjoined tendon is substituted for the cremasteric fascia.

The position of the openings in the abdominal wall should be kept in mind during attempts to reduce this kind of hernia; and the straightness of the course of the internal, in comparision with the external hernia, should be remembered.

Diagnosis. This rupture will be distinguished from external hernia by its straight course through the abdominal wall, and by the neck being placed close to the pubes.

After this hernia has acquired a large size, an examination during life cannot determine whether it began originally in the triangular space, or at the internal abdominal ring; for as an external hernia increases, its weight drags inwards the internal ring into a line with the external, and in this way the swelling acquires the appearance of a direct rupture.

Seat of stricture. The stricture in this form of hernia occurs most frequently external to the neck of the tumor, though it may be inside from thickening of the peritoneum ; and it may occasionally be found at the external abdominal ring.

Division of the stricture. The neek of the tumor is to be laid bare, and all fibrous bands around it are to be divided without injury to the peritoneum ; but if, after this lias been done, the intestine cannot be put backwards into the abdomen, the sac is to be opened, and the internal constricting band is to be divided directly upwards on a direetor.

In the operation on a large rupture appearing to be direct, the operator 
sliould cut on the front and mid-part of the tumor, so as to avoid the epigastric ressels, whose lateral position cannot be known.

Variety of internal hernia. Another kind of internal hernia (superior) occurs through that part of the area of the triangular space which is external to the conjoined tendon. Its existence is determined by the musual position of the obliterated hypogastric artery inside the abdominal wall (p. 427).

The intestine protrudes through the wall of the abdomen close to the epigastric artery, and descends along nearly the whole of the inguinal canal to reach the external abdominal ring; so that the term "direct" would not apply strictly to this form of internal hernia.

Coverings. As the gut traverses nearly the whole of the inguinal canal, it has exactly the same coverings as the external hernia, viz., the skin and the superficial fascia, the spermatic and cremasteric fascix, the fascia transversialis, and the subperitoneal fat and the peritoneum.

Diagnosis. This form of internal hernia would be considered external during life from its course and its form ; and yet it must be remembered that the epigastric vessels are placed on the outer part of its neck, whilst in the hernia which it stimulates, they lie on the inner side. Its nature can be ascertained with certainty only after death.

Seat of stricture. The constriction of the intestine will take place from similar causes, and at the same spots as in the external hernia.

Division of the stricture. From an inability to decide always in the living body whether a small hernia is internal or external, the rule observed in dividing the stricture of the neck of the sac is, to cut down upon the mid-part of the tumor; and if it is necessary to open the peritoneum, to cut directly upwards, as in the other kinds of inguinal hernia.

Unbilical Hernia, or exomphalos, is a protrusion of the intestine through or by the side of the umbilicus. It is very variable in size, and its course is straight through the abdominal wall.

Coverings. The coverings of the intestine are few in number: They are the skin and the superficial fascia; a prolongation from the tendinous margin of the umbilical opening; together with coverings of the fascia transversalis, the subperitoneal fat, and the peritoneum. Over the end of the tumor the superficial fascia blends with the other contiguous structures, and its fat disappears.

If the hernia is suddenly produced, it may want the investment otherwise derived from the edge of the umbilicus.

Seat of stricture. The stricture on the intestine is generally at the margin of the tendinous opening in the abdominal wall; and it may be either outside, or in the neck of the sac, as in the other kinds of hernia. It should be remembered that the narrowed neck is at the upper part and not in the centre of the swelling.

Division of the stricture. 'The constriction may be removed by cutting externally the parts around the neck. Or if the sac is to be opened, the knife may be carried upwards in cutting through the stricture; but there is not any vessel liable to injury in the operation.

Otien Forms of Heinia. At each of the other apertures in the parietes of the abdomen, a piece of intestine may be protruded, so as to form a hernial tumor. For instance there may be femoral hernia below Poupart's ligament, with the femoral vessels; obturator hernia through the thyroid foramen, with the artery of the same name; and ischiatic hernia through the ischiatic notch. 
The femoral hernia, as the most important, will be noticed presently ; but the student must refer to some special treatise for information respecting the other abdominal hernix.

Dissection. The abdomen may be now opened to see the cords and the depressions on the posterior surface of the abdominal wall. A transverse eut may be made through the umbilicus across the front of the abdomen; and on holding up the lower half of the wall, fibrous cords will be seen ascending to the umbilicus from the pelvis.

Cords of the abdominal wall. In the middle line is the prominence of the remains of the urachus, which reaches from the summit of the bladder to the umbilicus. On each side is another cord of the obliterated hypogastric artery; this is directed from the side of the pelvis to the umbilicus, and lies usually behind or close to the epigastric artery, near Poupart's ligament.

Fossa. With this disposition of the cords, two fossæe are seen near Poupart's ligament, one on each side of the obliterated hypogastric artery ; they correspond with the situation of the internal and external abdominal rings, and with the places where the external and internal (common kind) hernix occur.

But occasionally the cord of the obliterated hypogastric is moved inwards from the epigastric artery, to the line of junction of the outer with the inner two-thirds of the triangular space through which the direct hernia comes. In this position of the cord there will be three fossæe on the lower part of the abdominal wall; viz., an inner between it and the urachus, a middle one between it and the epigastric vessels, and an external ontside the epigastric artery. And there may be one, two, or three inguinal hernia, on each side of the body, according to the depth of the fossæ, and the predisposition to protrusion of the intestine.

Femoral Hervia. In this hernia the intestine leaves the abdomen below Poupart's ligament, and descends in a loose membranous sheath around the femoral vessels. Only so much of the structures will be described liere as ean be now seen, the rest are noticed fully in the dissection of the thigh.

Dissection. The dissection for the femoral hernia is to be made on the left side of the body.

The lower portion of the abdominal wall is to be divided from the umbilicus to the pubes. The peritoneum is to be detached from the inner surface of the wall near Poupart's ligament, by means of a transverse cut just above that band; and is to be raised from the iliac fossa. The layer of the subperitoneal fat is to be separated in the same way, but before this ean be done, it will be necessary to cut through the spermatic cord at the abrlominal ring; as this layer is raised, some lymphatic glands will be laid bare by the side of the iliac vessels. Any loose tissue remaining is to be taken away to show the upper opening of the membranous crural sheath containing the femoral vessels, and the interval (crural ring) on their inner side (fig. 141). In this disseetion the genito-crural nerve is seen on the iliac artery.

Afterwards the fascia transversalis and the iliac fascia are to be traced to Poupart's ligament, to see the part that each takes in the production of the crural sheath.

Anatomy of femoral hernia. The membranes concerned in the femoral hernia are, the peritoneum; the subperitoneal fat; the transversalis and 
iliac fascia lining the interior of the abdominal cavity, with the sheath on the femoral vessels to which they give origin at Poupart's ligament.

'The peritoneum lines the inner surface of the abdominal wall, without having any aperture for the escape of the intestine; and its thinness and weakness are apparent now it is detached.

'The subperitoneal fat extends as a continuous layer beneath the peritoneum, but is thickest and most fibrous at the lower part of the abdomen, where the iliac vessels pass under Poupart's ligament. At that spot it extends over the upper opening of the membranous sheaths around the vessels; and internal to the vein, it covers the space of the crural ring, as well as a lymphatic gland which occúpies that space.

Where this layer stretches over the crural ring it is named by M. Cloquet septum crurale; and it is describerl by him as being concave towards the abdomen, and convex towards the thigh. An inguinal gland is generally attached to its under surface.

The fasciu transversalis has been before noticed (p. 417). At Poupart's ligament it joins the iliac fascia, outside the situation of the large iliae artery (fig. 141); but internal to that spot it is continued downwards to the thigh in front of the femoral vessels, and forms the anterior part of the crural sheath.

The iliac fascia covers the iliacus muscle, and lies beneath the iliac vessels. At Poupart's ligament its disposition is similar to that of the transversalis fascia; for, external to the iliac vessels, it joins the faseia transversalis along the line of the ligament; but opposite the vessels it is prolonged into the posterior part of the crural sheath.

Fig. 141.

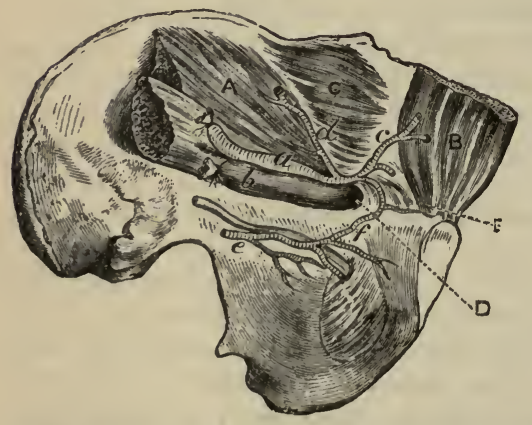

\section{Muscles :}

A. Iliacus covered by the iliac fascia.

B Rectus.

c. Transversalis, covered by the trustersalis fascia.

D. Crural ring.

E. Gimbernat's ligament.

Vessels :

a. Iliac artery.

b. Iliac vein.

c. Epigastric branch.

d. Circumflex lliac.

e. Obturator, with its nerve.

$f$. Small branch joining obturator and epigastric arteries.

Vinw of the Parts concerned in Femoral Hernia (Quain's plates).

The crural sheath is a loose membranous tube, which incloses the femoral vessels as they enter the thigh, and is obtained from the fascia lining the abdomen. Its anterior half is continuous with the fascia transversalis, and its posterior is derived from the fascia iliaca. The sheath is not entirely filled by the ressels, for a space (crural ring) exists on the inner side of the vein, through which the intestine descends in femoral hernia.

The crural ring (fig. 141, D) is referred to also in the dissection of the thigh, but its boundaries are better seen in the abdomen. It is the interval in the sheath, at the inner side of the femoral vein, which is about half an inch wide, and is filled by a lymphatic gland. Bounding it internally, are Gimbernat's ligament, $\mathrm{e}$, and the conjoined tendon; and limit- 
ing it externally is the femoral vein $(b)$ without the intervention of the sheath. In front is Poupart's ligament, with the deep arch ; and behind is the pubes, covered by the pectineus and the fascia lata. Along the front of the space, but at some little distance from it, lies the spermatic cord in the male, and the round ligament in the female.

'Two of the boundaries, anterior and inner, are firm and sharp-edged, though their condition varies with the position of the limb; for if the thigh is raised and approximated to its fellow, those bounding parts will be relaxed.

Position of vessels around the ring (fig. 141). On the outer side is the femoral vein $(b)$; and above this are the epigastric vessels (c). In front is a small branch (pubic) from the epigastric artery to the back of the pubes; and the vessels of the spermatic cord may be said to be placed along the anterior aspect of the ring. The ring is bounded in the male by vessels in front and on the outer sirle.

But in some bodies the obturator artery takes origin from the epigastric, and lies along the ring as it passes to the pelvis. It may have two positions with respect to the crural ring:-either it is placed close to the iliac vein, so as to leave the inner side of that space free from vessels; or it arches over the aperture, descending on the inner side at the base of Gimbernat's ligament; in this last condition the ring will be encircled except at the posterior part.

Course of femoral hernia. The intestine leaves the abdomen by the opening of the crural ring; and it descends internal to the vein in the large crural sheath, as far as the saphenous opening in the thigh, where it projects to the surfice.

Coverings. In its progress the intestine will push before it the peritoneum, and the subperitoneal fat (septum crurale); and it will displace, or cause to be absorbed, the gland which fills the crural ring. Having reached the level of the saphenous opening, the intestine carries before it the inner side of the crural sheath, and a layer called the cribriform fascia ; and, lastly, it is invested by the teguments of the thigh. The dissection of the thigh may be referred to for fuller detail.

Seat of stricture. The stricture of a femoral hernia is placed opposite the base of Gimbernat's ligament, or lower down at the margin of the saphenous opening in the thigh. And the constriction may be caused either by a fibrous band outside the upper narrow end of the tumor, or by the thickening of the peritoneum inside that part, as in inguinal hernia.

Division of the stricture. To free the intestine from the constricting fibrous band arching over it, an incision is to be made down to the neck of the sac at the inner and upper part.

And to relieve the deep stricture within the neck of the sac, the peritoneal bag is to be opened and a director introduced, and the knife is to be carried horizontally inwards, or upwards and inwarrls, through the thickened sac and a few fibres of the edge of Gimbernat's ligament.

Danger to vessels. When the incision is made upwards and inwards to loosen the constricting band in the neck of the tumor, there will not be any vessel injured unless the cut should be made so long as to reach the spermatic cord in the male, or the sinall pubic branch of the epigastric artery.

And when the incision is made directly inwards with the same riew, there is not usually any vessel in the way of the knife. But in some few instances (once in about eighty operations, Lawrence), the obturator artery 
takes its unusual course in front of and on the inner side of the neck of the hernia, and will be before the knife in the division of the stricture. As this condition of the vessel cannot be recognized beforehand, the surgeon will best avoid the danger of wounding the artery by a cautious and sparing use of the knife.

\section{SECTION III.}

\section{CAVITY OF THE ABDOMEN.}

THE abdominal cavity is the space included between the spinal column behind, and the visceral arehes of the vertebræ with their intervening museles in front. It is lined by a serous membrane (peritoneum), and contains the digestive, urinary, and generative organs, with vessels and nerves.

Dissection. To prepare the cavity for examination, the remainder of the abdominal wall above the umbilicus is to be cut, along the left side of the linea alba, as far as the xiphoid cartilage. The resulting flaps may be thrown to the sides.

Size and form. This space is the largest in the body. It is ovoidal in form, with the ends upwards and downwards, so that it measures more in the vertical than the transverse direetion; and it is much wider superiorly than inferiorly.

Boundaries. Above it is limited by the diaphragm; below by the recto-vesical fascia and the levatores ani, and the structures closing the outlet of the pelvis: both these boundaries are concave towards the cavity, and are in part fleshy, so that the space will be diminished by their contraction and flattening.

In front and on the sides the parietes are partly osseous and partly muscular:-thus towards the upper and lower limits is the bony framework of the skeleton, viz., the ribs in one direction and the pelvis in the other; but in the centre are stretched the muscles of the abdominal wall.

Behind is placed the spinal column with the muscles contiguous to it, viz., the psoas and the quadratus lumborum.

Alterations in size. The dimensions of the eavity are influenced by the varying conditions of the boundaries. Its depth is diminished by the contraction and descent of the diaphragm, and the contraction and ascent of the levatores ani; and the eavity is restored to its former dimensions by the relaxation of those muscles.

The width is lessened by the contraction of the abdominal muscles; but it is increased, during their relaxation, by the action of the diaphragm foreing outwards the viscera. 'The greatest diminution of the space is effected by the simultaneous eontraction of all the muscular boundaries, as in the expulsion of the excreta.

Division of the space. A division has been made of the space into the abdomen proper and the eavity of the pelvis.

The abdominal portion reaches from the diaphragm to the brim of the pelvis, and lodges the alimentary tube and its appendages, together with the kidneys. 
The pelvic portion is situate below the brim of the pelvis, and contains chiefly the generative and urinary organs.

The following description concerns the part of the cavity between the diaphragm and the brim of the pelvis. 'Towards the end of the dissection of the abdomen, the pelvic portion will receive a separate notice.

Regions. The upper part of the abdominal cavity is divided into regions by lines extended between certain points of the parietes.

If two circular lines are carried round the body, so that one shall be opposite the cartilage of the ninth rib, and the other on a level with the most prominent point of the crest of the hip-bone, the cavity will be divided into three circles or zones, upper, midule, and lower.

Each of these circles has been further subdivided into three by a line, on each side, from the cartilage of the eighth rib to the centre of Poupart's ligament. The piece marked off, on each side, from the three circles by the vertical line is named respectively, from above downwards, hypochondriac, lumbar, and iliac; whilst the central part of each circle is desig. nated from above down, epigastric, umbilical, and hypogastric.

In addition, the middle and lower part of the hypogastric space is named pubic region, whilst the contiguous portions of the hypogastric and iliac parts constitute the inguinal region.

Contents and their position. The alimentary tube, the liver, pancreas, spleen, and kidney, occupy the abdomen proper.

The alimentary tube presents differences in form, and is divided into stomach, small intestine, and large intestine; and the two last are further subdivided, as it will afterwards appear. 'The several viscera have the following general position: The small intestine is much coiled, and oceupies the greater part of the cavity; whilst the great intestine arches around it. Both are fixed in position by pieces of the serous lining. Above the arch of the great intestine are situate the stomach, the liver, the spleen, and the pancreas; and below it, is the convoluted small gut. Behind the large intestine, on each side, is the kidney with its excretory tube.

Superficial view of the contents. On first opening the abdomen the following viscera appear: On the right side is the liver, which is partly concealed by the ribs. On the left side a piece of the stomach is visible; but this viscus lies for the most part beneath the ribs and the liver. Descending from the stomach is a fold of peritoneum (the large omentum), which reaches to the pelvis, and conceals the small intestine: in some bodies the omentum is raised into the left hypochondriac region, and leaves the intestine uncovered.

If the bladder is distended, a small part of it may come into view just above the pelvis, but commonly it is not seen.

Before the natural position of the viscera is disturbed, their situation in the different regions of the abdomen, and their connections with surrounding parts should be examined.

\section{CONNECTIONS OF THE VISCERA.}

The stomach (fig. 142, a) intervenes between the gullet and the small intestine, and is partly retained in position by pieces of the serous membrane. It is somewhat of a conical form, with the larger end to the left side; and it occupies the left hypochondriac, the epigastric, and part of the right hypochondriac region. 
At the left end it receives the esophagus (b), by which it is firmly fixed to the diaphragm; here it lies beneath the ribs, and is in contact with the spleen $(\mathrm{m})$, to which it is connected by a fold of peritoneum (splenic omentum): when this part of the stomach is distended it pushes

Fig. 142.

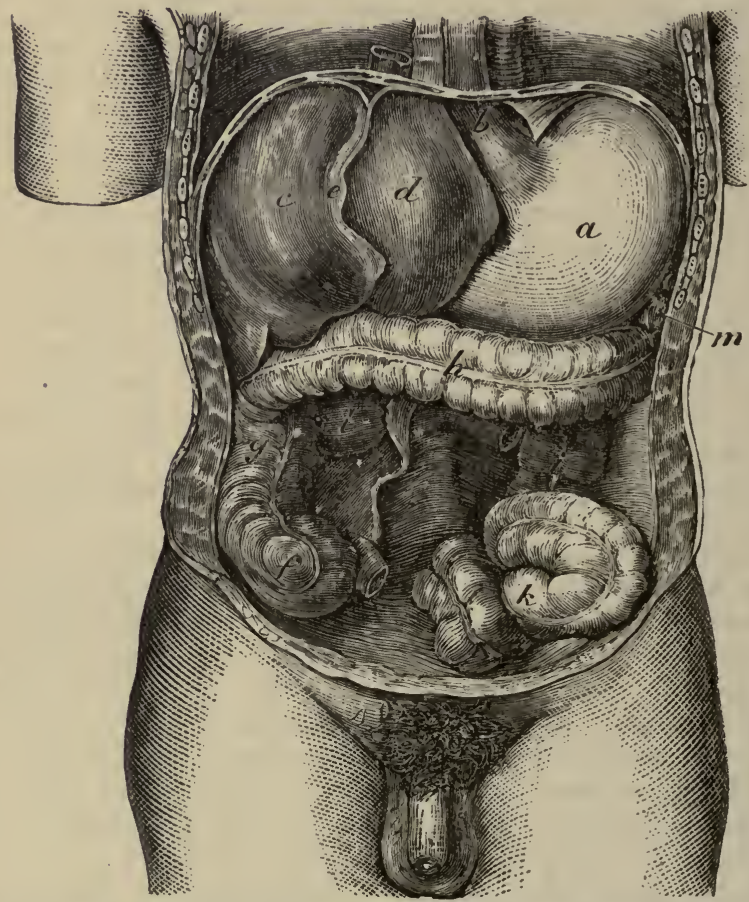

Connections of The Liver, Stomach, Splebs, Ann LARge Intestine, the small intestino having been taken away.

a. Stomach, and $b$, nsophagus.

$c$ and $d$. Right and left lobes of the liver, with

$e$, the suspensory ligament.

f. Cæcum.

g. Ascending colon. h. Transverse.

i. Descending, and $k$, sigmoid flexure of the colon.

l. Duodenum.

$m$. Spleen.

up the diaphragm, and encroaches on the space for the heart and the left lung. The right extremity ends in the small intestine, and reaches towards the gall bladder; it is in contact with the under part of the liver.

The anterior surface touches, from left to right, the diaphragm, the abdominal wall, and the liver; and the posterior surface corresponds with the pancreas, the pillars of the diaphragm, the aorta and vena cava, and the solar plexus.

The upper borler is connected to the liver by a process of peritoneum. the small omentum; and the lower border gives attachment to another peritoneal fo!d, the great om sntum or epiploon, which floats freely over the intestine.

The form, and the connections of the stomach with the surrounding parts will vary with the size. For when the viscus is empty it is flattened, 
its surfaces looking forwards and backwards, and its borders upwards and downwards; but when distended, it becomes somewhat circular, and makes a rotatory movement, so as to bring forwards the border usually lowest, and to turn upwards that surface which is directed forwards at other times.

The position and connections of the stomach may be altered by variations in the size of any of the surrounding organs, or by the accumulation of fluid in the chest, or in the belly. The stomach may be dragged down likewise by the great omentum entering a hernia; or it may be forced down towards the pelvis by the pressure of tight stays. In these different changes in position, the right end moves more than the left, because it is attached mainly by peritoneum to the parts around.

The small intestine (intestinum tenue) reaches from the stomach to the right iliac region, where it ends in the large intestine. It is divided into three parts, duodenum (twelve fingers' length), jejunum, and ileum : of the last two, the former receives its name from its empty condition, and the latter from its numerous coils.

The duodenum (fig. $142, l$ ) cannot be satisfactorily seen at present, and it will be examined afterwards (p. 445).

The jejunum and ileum (fig. 143) begin on the left side of the second lumbar vertebra, without any distinct mark of separation from the duodenum. Two-fifths of the intestine belong to the jejunum, and the remaining three-fifths to the ileum.

This part of the intestinal tube forms many convolutions in the umbilical, hypogastric, lumbar, and iliac regions of the abdomen; and it descends oftentimes, but more frequently in the female, into the cavity of tle pelvis. In front of the convolutions is the great omentum. Beyond the duodenum the intestine is fixed posteriorly to the spine by a process of peritoneum named the mesentery, which contains the vessels and nerves. Surrounding the jejunum and ileum is the large intestine or colon: but on the left side of the body the colon is concealed by the small intestine.

The large intestine, or the colon (fig. 142), is sacculated, and is more fixed than the jejunum and ileum. It begins in the right iliac region in a rounded part or head (caput cæcum coli), and ascends to the liver through the right iliac, lumbar, and hypochondriac regions. Crossing then the abdomen below the stomach, it reaches the left hypochondriac region; and it lies in this transverse part of its course between the epigastric and umbilical regions, or altogether in the latter. Finally, it descends, on the left side, through the regions corresponding with those it occupied on the right, and forms a remarkable bend (sigmoid flexure) in the left iliac fossa ; then becoming straighter it passes through the pelvis to end on the surface of the body.

It is divided into six parts, viz., cxcum, ascending colon, transverse colon, descending colon, sigmoid flexure, and rectum.

The crecum (fig. 142,f) (caput cæcum), or the commencement of the colon, is placed in the right iliac fossa, in which it is fixed by the peritoneum being stretched over it. In front usually are convolutions of the small intestine, but when it is distended it touches the abdominal wall. Behind, it rests on the iliac fascia, only fatty and areolar tissues intervening. On the inner side it is joined by the small intestine; and it presents inferiorly a worm-like piece-the vermiform appendix.

Sometimes the peritoneum surrounds the crecum, and attaches it by a process to the abdominal wall.

The ascending colon (fig. $142, g$ ) reaches from the cxcum to the under 
surface of the liver, on the right of the gall bladder. It lies against the quadratus lumborum inferiorly, but higher up it is placed in front of the kidney. To its inner side are the convolutions of the small intestine. The peritoneum fixes the colon immovably to the wall of the abdomen, and surrounds commonly about two-thirds of the circumference; but it may encircle the tube, and form a fold behind, as in the cxeum.

The transverse colon (fig. 142, $h$ ) passes obliquely upwards and to the left, along the curvature of the stomach, as far as the spleen; in this course it is deeper at each end than in the middle, and forms the arch of the colon by being thus bent.

Above the arch are placed the liver and the gall bladder, the stomach and the spleen : and below, is the small intestine. In front lies the great omentum; and behind is a long process of peritoneum, the transverse meso-colon, which attaches it to the back of the abdomen, and contains the vessels and nerves.

The transverse colon is more movable than any other part of the large intestine,-its peritoneal fold allowing it to be raised on the margin of the ribs. Small pieces of peritoneum, containing fat, the appendices epiploïræe, are attached along it.

The descending colon (fig. 142, i) commences below the spleen, and reaches to the left iliac fossa. At first it is placed deeply in the left hypochondriac region; and its whole course is deeper than that of the right colon. In front of it are the convolutions of the small intestine; and behind it are the diaphragm, the outer part of the kidney, and the quadratus lumborum.

This part of the intestine is smaller than either the right or the transverse portion, and is less surrounded, commonly, by the peritoneum; but its upper end is attached to the diaphragm by a firm process (pleuro-colic) of that membrane.

The sigmoid flexure of the colon (fig, $142, k$ ) is lodged in the left iliac fossa, to which it is attached by a process of the peritoneum, the sigmoid meso-colon, but it olten hangs in the eavity of the pelvis. The intestine makes two turns like the letter $\mathrm{S}$, and has obtained its name from that circumstance. Its extent is from the crest of the hip bone to the sacroiliac articulation, where it ends in the rectum. It is concealed by the small intestine, which is directed more to the left than the right side.

'The rectum, or the termination of the large intestine, which is contained in the pelvis, will be examined in the dissection of that eavity.

The liver (fig. $142, c, d$ ) is situate in the right hypochondriac, and epigastric regions, and reaches slightly into the left hypochondriac. Pieces of peritoneum (ligaments) retain it in place.

The upper surface, convex, is turned to the vault of the diaphragm, and is divided into two parts by the suspensory ligament $(e)$; the right portion, more prominent than the left, reaches to the level of the fifth intercostal space. The under surface is in contact with the stomach and the duodenum, with the ascending colon, and with the right kidney and suprarenal body; attached to this surface is a fold of the peritoneum (small omentum), containing the hepatic vessels.

The anterior border is thin, and lies in the adult male usually within the margin of the ribs, but in women and children it reaches below that line. The gall bladder projects beyond this edge. The posterior border is thick, and is connected to the diaphragm by certain ligaments or pieces 
of the peritoneum; it lies on the large vessels (aorta and cara) and on the pillars of the diaphragm.

The liver changes its situation with the ascent and descent of the diaphragm in respiration ; for in inspiration it descends, and in expiration it regains its former level. In the upright and sitting postures, too, this viscus descends lower than in the lorizontal condition of the body; so that when the trunk is erect, the anterior border may be felt underneath the edge of the ribs, but when the body is reclined it is withdrawn within the margin of the thorax.

The connections of the liver with the surrounding parts may be changed by the growth of tumors, by collections of fluid in the chest or in the abdomen, or by constricting the space for its lodgment, as in tight lacing.

The spleen (fig. 142, $m$ ) lies deeply in the left hypochondrium, between the stomach and the ribs, and is connected by peritoneum to the great end of the stomach on the one side, and to the diaphragm on the other. Its position is almost vertical.

The outer surface is convex, and touches the diaphragm opposite the ninth, tenth, and eleventh ribs. At the inner surface, which is concave, the vessels enter (p. 465), and to it is attached a process of peritoneum, the gastro-splenic omentum: the part in front of the vessels touches the stomach ; and the part behind them is in contact with the tail of the pancreas, the suprarenal capsule, and the left crus of the diaphragm.

Below the spleen is a transverse piece of the peritoneum (pleurocolic fold), the kidney, and the beginning of the descending colon. When the stomach is distended the spleen is somewhat behind it.

The kidney should be examined on the left side of the body, so that the duodenum may not be displaced. In order that it may be seen, the descending colon and the peritoneum must be separated from the abdominal wall, and its casing of fat should be torn through.

This viscus is surrounded with fat, and is situate in the lumbar region opposite the last dorsal, and the upper two or three lumbar vertebre. Its position is somewhat oblique, and the upper end is nearer than the lower to the spinal columu.

In front of the kidney are the peritoneum and the colon; and behind it are the quadratus lumborum and psoas muscles, with the diaphragm and the last rib. Above each kidney and resting on it, is the suprarenal capsule. The inner border looks to the spine and reccives the vessels.

Difference on opposite sides. The right kidney is placed rather lower than the left; it reaches as high as the lower border of the eleventh rib, whilst its fellow is opposite the upper border of the corresponding rib. In front of the right, besides the common connections before specified, is the duodenum : and before the left one is the lower end of the spleen. Above the right is the liver, and above the left the spleen.

The connections of the pancreas may be omitted for the present. This viscus is described at page 446 .

\section{THE PERITONEUM.}

This is the largest serous membrane in the body. Like other membranes of the kind it is a closed sac in the male, but in the female its carity is continuous with the canals of the Fallopian tubes. One part of it lines the wall of the abdomen (parietal layer), and another is reflected over the different viscera (visceral layer), except where the vessels enter. 
The inner surface is smooth; but the outer is rough, when it is detached from the parts with which it is naturally in contact. The membrane forms processes or folds as it passes from viscus to viscus along the vessels; and the folds attaching the viscera to the abdominal wall consist for the most part of two layers, one on each side of the vessels.

'The continuity of the sac may be traced in a horizontal and a vertical direction.

Horizontal circle around the abdomen. The membrane, when followed outwards from the umbilicus, surrounds partly the large intestine on the left side, and fixes it to the abdominal wall. From the colon it may be traced over the kidney as far as the middle line, where it is reflected along the front of the vessels supplying the small intestine, thence around the intestine, and back to the spine along the same vessels. Lastly, it may be pursued outwards to the right kidney, to the colon which it encircles like the left, and along the wall of the abdomen to the umbilicus.

The piece of membrane fixing the colon on each side to the abdominal wall, is named meso-colon, and that attaching the small intestine is the mesentery.

Vertical circle from above downwards. From the under surface of the liver the peritoneum may be followed along the hepatic vessels, one piece before and the other behind them to the upper border of the stomach, the two forming the small omentum. At the stomach the two pieces disunite, one passing before, and the other behind it; but beyond that viscus they are applied to each other to form the great omentum or epiploon. After descending in contact to the lower part of the abdomen they bend backwards, separating to inclose the 'transverse colon like the stomach, and they are then continued to the spine, giving rise to the transverse mesocolon. At the attachment of the transverse meso-colon to the abdominal wall, the two companion pieces part from each other-one passing upwards, the other downwards. ${ }^{1}$

The ascending piece is continued in front of the pancreas and the pillars of the diaphragm, and blends with the peritoneum on the under surface of the liver.

The descending piece or layer may be followed from the transverse meso-colon over the duodenum and the great vessels on the spine (aorta and cava), till it meets with the artery to the small intestine, along which it is continued to form the mesentery, as before explained in tracing the peritoneum in a circular direction.

From the root of the mesenteric artery the peritoneum descends to the pelvis, and covers partly the viscera in that cavity. For instance, surrounding the upper part of the rectum, it attaches this to the abdominal wall by the meso-rectum; next, it is continued forwards between the rectum and the bladder in the male, or between the rectum and the uterus in the fernale, where it forms a pouch. Thence it passes from the pelvis over the back and sides of the bladder.

Lastly, the serous membrane is continued to the inguinal region, where it presents the fossa before alluded to (p. 427); and it can be traced upwards on the wall of the abdomen, and over the diaphragm and upper surface of the liver, to the under surface of this viscus.

1 Sometimes the two pieces ascend over the transverse colon, being slightly attached to it and the transverse meso-colon, as high as the pancreas before they separate. In that case the descending layer would form a distinct mesentery for the transverse colon, like that for the small intestine. 
Folds of the Peritoneum. After tracing the continuity of the serous sac over the viscera, the student is to learn the chief processes or folds of the membrane in connection with the alimentary tube. The pieces of the peritoneum fixing the liver will be examined afterwards; and the processes on the viscera of the pelvis will be seen with the dissection of that eavity.

Folds on the stomach. The processes connected with the stomach are named omenta. They are three in number-one, small omentum, is attached to the upper curve; another, great omentum, to the lower eurve; and the third, splenic omentum, is fixed to the great end of the viscus.

The small or gastro-hepatic omentum is stretched between the under surface of the liver and the upper border of the stomach, and contains the vessels and nerves of the liver. It is formed by two pieces of peritoneum, as before explained, and presents a free border on the right side. Behind it is the space called foramen of Winslow. Its lower edge is fixed to the small curve of the stomach; whilst its upper border is attached to the transverse fissure, as well as to the posterior half of the longitudinal fissure. of the liver, blending behind with the left lateral ligament of that viscus.

The gastro-colic or great omentum is the largest fold of the peritoneum, and consists of two pieces. It is attached above to the spleen and the lower border of the stomach, and descends in front of the large intestine, but lower on the left than the right side of the body. At the lower part of the abdomen the process is bent backwards, and returns to the spine, the pieces of which it is composed separating to inclose the transverse colon. The anterior part of the omental fold is separated from the posterior by a space (cavity of the omentum).

Between its layers are contained some fat, vessels, and nerves; and the power of detaching the one layer from the other diminishes with the increase of the distance from the stomach, until below they are not to be separated, and the membrane they form is thin and net-like.

Cavity of the omentum. When an opening is made through the great omentum near the stomach, and this viscus is raised, a space is seen to extend upwards to the liver, and downwards into the omentum: this is the omental cavity. In front the space is bounded by the small omentum, the stomach, and the anterior part of the great omentum. Behind it, are the posterior part of the great omentum, the transverse colon, and the ascending layer of the transverse meso-colon. This space communicates with the rest of the peritoneal cavity, through a hole (foramen of Winslow), behind the small omentum.

If the sac of the omentum were perfect, it could be inflated through the foramen of Winslow. Supposing it to be detached and removed, there would not be any membrane in the way of the vessels reaching the different viscera; and it may be readily conceived how the peritoneum could be replaced over the viscera, and around the vessels without being perforated by them.

The foramen of Winslow is the space behind the small omentum, through which the omental bag opens into the general cavity of the peritoneum. In front of it is the small omentum, and behind, are the vena cava and the spine. Above it is the liver (lobulus Spigelii), and below is the duodenum.

The splenic omentum reaches from the great end of the stomach to the concave surface of the spleen, and does not consist usually of two strata or 
pieces, like the other omenta. It covers the vessels passing between the two viscera, and is continued inferiorly into the great omentum.

Folds on the large intestine. The large intestine is connected to the wall of the abdomen by processes of the peritoneum (meso-colic), which are formed of two pieces, like the other folds, though they are at some distance from each other. Each part of the colon has a separate mesocolon attaching it, thus there is an ascending, a transverse, a descending, and a sigmoid meso-colon. The cxcum is fixed by a meso-crecum, and the rectum by a meso-rectum.

The meso-cacum attaches the caput cæcum coli to the right iliac fossa. Usually the peritoneum does not surround the gut so as to form a fold behind it, but in some bodies the serous membrane furnishes a suspensory process to this part of the intestine.

By the ascending and descending meso-colon the ascending and the descending part of the colon are kept in place. In these folds, as in that of the cxecum, the peritoneum does not commonly surround the intestine. though it may meet behind the gut and form processes of some length.

The upper end of the left colon has a distinct fold (pleuro-colic), fixing it to the wall of the abdomen. Attached by a wide part to the diaphragm opposite the eleventh or tenth rib, it passes transversely below the spleen, and forms the lower boundary of a hollow in which the spleen rests.

The transverse meso-colon is a more perfect fold than either of the others connected with the large intestine, and serves as a partition between the small intestine and the stomach, liver, and spleen. By one side it is fixed to the colon, and by the other to the abdominal wall below the pancreas. It incloses the vessels of the colon between its layers.

The sigmoid meso-colon is a long process of the serous membrane, and attaches the sigmoid flexure of the colon to the left iliac fossa.

The meso-rectum contains the hemorrhoidal vessels, and connects the rectum to the front of the sacrum.

Small processes of the peritoneum are attached along the tube of the great intestine, chiefly to the transverse colon; they are the appendices epiploìce, and contain fat.

Folds to the small intestine. The small intestine is not enveloped by the peritoneum after the same manner throughout. For whilst the jejunum and ileum are attached to the abdominal wall by one process (mesentery), the duodenum has special connections with the serous membrane.

Serous covering of the duodenum. The first part of the duodenum is surrounded by peritoneum, like the stomach. The second part is covered only in front. And the last part, which crosses the aorta, is but slightly in contact with the serous membrane; for it lies at first between the strata of the transverse meso-colon, and then beneath the superior mesenteric vessels.

Fold of the jejunum and ileum. The mesentery supports the rest of the intestine, and is stronger than any other piece of the serous membrane. Its inner end is narrow, and is attached to the spine from the left side of the second lumbar vertebra to the junction of the right hip bone with the sacrum. 'The other end of the fold is wide, and is connected with the intestine. Between its two layers are the superior mesenteric ressels and nerves, with lymphatic glands and lacteals.

Ligaments of the liver. Along the upper part is a suspensory process of the peritoneum, and there is a wide piece along the posterior border. 
The suspensory or fulciform ligament is placed between the upper convex surface of the liver and the parietes of the abdomen. It is triangular in shape, with its base forwards. The lower border is concave, and is attached to the liver; whilst the upper border is convex, and is connected to the abdominal wall, on the right side of the linea alba, and to the under part of the diaphragm. In its bise or free part is the remains of the umbilical vein, which is named the round ligament.

'This fold allows the umbilical vein to reach the liver without piercing the peritoneum; and with a little care the dissector will be able to detach the serous membrane from the vein, and to trace it on each side into the suspensory ligament.

The coronary ligament is a short but wide process of the peritoneum, which connects the hinder part of the liver to the diaphragm. It reaches all across the liver, but at each side it is enlarged, and forms a triangularly-shaped piece; to these larger portions of it the terms right and left lateral ligaments have been applied.

The left lateral ligament is attached to the liver above the edge of the left lobe, and is formed by two pieces of peritoneum, which are in contact; it lies in front of the osophagean opening in the diaphragm.

The right lateral ligament lies deeply in the hypochondriac region, in front of the vena cava inferior: it consists of two pieces of peritoneum not touching each other.

\section{MESÉNTERIC VESSELS AND SYMPATHETIC NERVE.}

Directions. The vessels and nerves (mesenteric) which are distributed to the greater part of the alimentary tube, may be first dissected. After these have been examined, and the connections of the aorta and vena cava have been learnt, most of the intestine can be taken out to give room for the display of the viscera in the upper part of the abdomen.

Mesenteric Vessels. The superior and inferior mesenteric arteries are two large branches of the aorta, which supply the intestine, except a part of the duodenum and the lower end of the rectum. Each is accomlanied by a vein, and by a plexus of the sympathetic nerve.

Dissection (fig. 143). For the dissection of the superior mesenteric vessels and nerves, the transverse colon and the great omentum are to be placed on the margin of the ribs, and one layer (anterior) of the mesentery is to be removed. Whilst tracing the brarches of the artery to the small intestine, corresponding veins, and offsets of the sympathetic nerve on the arteries, will be met with; these last are removed in cleaning the vessels. Mesenteric glands and a few lacteal vessels will come into view at the same time.

The branches from the right side of the vessel to the large intestine are to be next followed: and after all the branches have been cleaned, the trunk of the artery should be traced back beneath the pancreas. The plexus of surrounding nerves should be also defined.

The superior mesenteric artery (fig. $143, a$ ) supplies branches wholly to the small intestine beyond the duodenum, and to half the large intestine, viz., as far as the end of the transverse colon.

Arising from the aorta near the diaphragm, the vessel is directed downwards between the layers of the mesentery, forming an arch with the convexity to the left side, and terminates in offsets to the ciecum and the end of the small intestine. At first the artery lies beneath the pancreas and 
the splenic vein; and as it descends to the mesentery it is placed in front of the duodenum and the left renal vein. This vessel is surrounded by the mesenteric plexus of nerves, and is accompanied by the vein of the same name.

Branches. Whilst the vessel is covered by the pancreas it gives a small branch to that body and the duodenum. Its other branches are intestinal: those from the left or convex side of the vessel (rami intestinales) are furnished to the jejunum and ileum; and those from the opposite side supply the colon, and are named colic arteries.

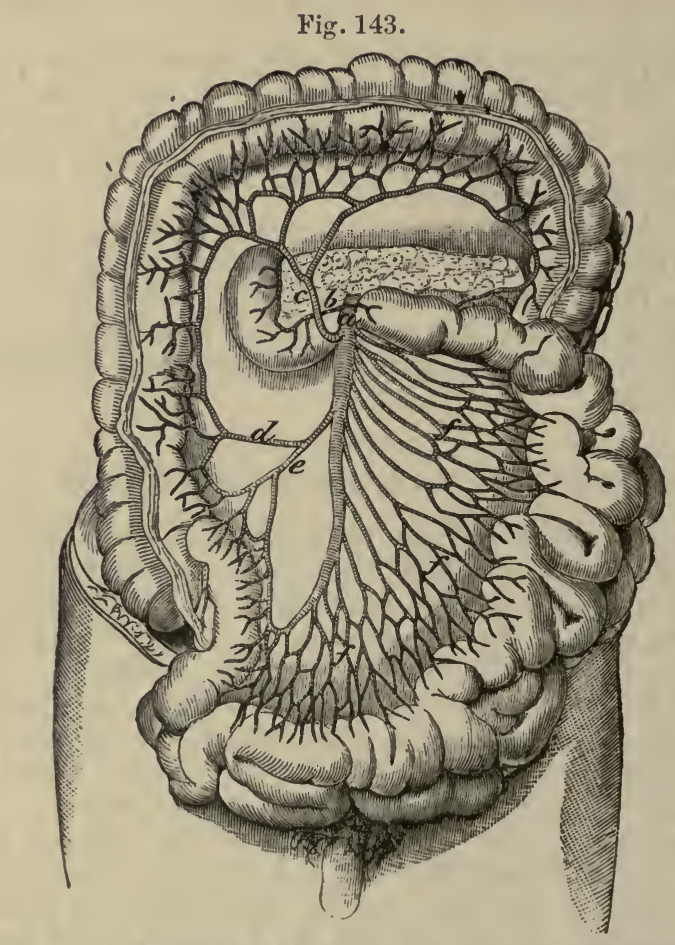

Superior Mesenteric Artery and its Branches (Tiedemuna).

a. Superior mesenteric.

b. Inferior pancreatico-duodenal.

c. Middle colic. d. Right colic.

e. Ileo-colic.

f. Intestinal branches to the jejunum and lleum.

a. The pancreatico-duodenal branch (inferior) is of small size (fig. 143, $b$ ) ; after giving twigs to the pancreas, it runs to the right along the concavity of the duodenum, and anastomoses with the other duodenal branch (p. 448).

$b$. The intestinal branches for the jejunum and i!eum (fig. $143, f$ ) are about twelve in number, and pass from the left side of the artery between the layers of the mesentery. About $t$ wo inches from their origin the branches bifurcate, and the resulting pieces unite with similar offsets from the collateral arteries, so as to form a series of arches. From the convexity of the arches other branches take origin, which divide and unite as before. This process is repeated four or five times between the origin 
and the distribution, but at each branching the size of the vessels diminishes. From the last set of arches twigs are sent to the intestine on both aspects of the tube, and anastomose round it.

The branches of the large intestine are three in number, ileo-colic, right colic, and middle colic arteries.

$c$. The ileo-colic artery $(o)$ arises from the right side of the trunk, and divides at the cxecum into branches which encircle the head of the colon. A descending offset is distributed to the lower part of the ileum, and to the crecum and the vermiform appendix; whilst an ascending offset supplies the beginning of the ascending colon, and anastomoses with the right colic artery.

$d$. The right colic artery $(d)$ is commonly an offset of the preceding, instead of a separate branch from the trunk. Near the ascending colon it divides into ascending and descending pieces, which anastomose with the ileo-colic artery on one side, and with the middle colic on the other.

e. The middle colic branch $(c)$ springs from the upper part of the artery, and entering between the layers of the transverse meso-colon divides into two diverging branches:- the right one anastomoses with the artery to the ascending colon, and the left inosculates on the descending colon with a left colic branch (fig. 144,c) of the inferior mesenteric artery. The intestinal twigs are united in arches before entering the gut, like those to the small intestine.

The superior mesenteric vein (fig. 146, $b$ ) commences in that part of the intestinal tube to which the artery is distributed. Its radicles unite into one trunk, which accompanies the artery beneath the pancreas, and there joins the splenic vein to form the vena portæ.

At the lower border of the pancreas it receives the right gastro-epiploic branch of the stomach, and the pancreatico-duodenal veins (fig. 146, c).

The mesenteric lymphatic gands are numerous between the layers of the me-entery. An upper group lies by the side of the artery, and contains the largest glands; and a lower group, near the intestine, is lodged in the intervascular spaces. The chyliferous vessels of the small intestine, and the absorbents of the part of the large intestine supplied by the superior mesenteric artery, pass through the mesenteric glands in their course to the thoracic duet.

Along the side of the ascending and the transverse colon are a few other small lymphatic glands meso-colic, which receive some absorbents of the large intestine.

Dissection (fig. 144). By drawing the small intestine over to the right side, the dissector will observe the inferior mesenteric artery on the front of the aorta a little above the bifurcation. The peritoneum should be removed from it, and the branches should be traced outwards to the remaining half of the large intestine: a part of the artery enters the pelvis, but this will be dissected afterwards. On the artery and its branches the inferior mesenteric plexus of nerves ramifies.

The mesenteric vein is to be followed upwards, away from the trunk of the artery, to its junction with the splenic, or with the superior mesenteric vein.

On the aorta the dissector will meet with a plexus of nerves, which is to be left uninjured.

The inferior mesenteric artery (fig. $144, b$ ) supplies branches to the part of the large intestine beyond the transverse colon; and communi- 
cating with the superior mesenteric, assists to maintain the chain of anastomosis along the intestinal tube.

This vessel is of smaller size than the superior mesenteric, and arises from the aorta, from one to two inches above the bifureation. At first the vessel descends on the arta, and crosses the left common iliac artery, as it courses to the pelvis to end in branches for the rectum (superior hæmorrhoidal). The following branches are furnished by it to the deseending colon and the sigmoid flexure.

Fig. 144.

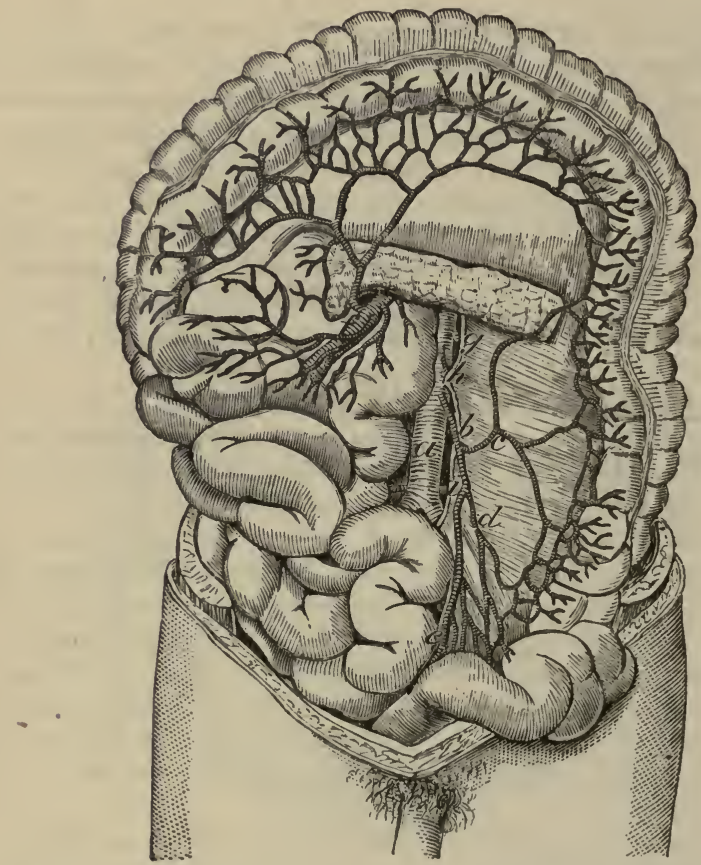

The Lower Mesenteric Artery, aND the Aorta, seen by turning aside the upper mesente: ic artery and the small intestine. (Tiedemann).

a. Aorta.

b. Inferior mesenteric artery.

c. Left colic.

d. Sigmoid, and $e$, Superior hæmorrhoidal branches. f. Upper mesenteric.

g. Renal.

$h$. Spermatic of the left side.

a. The left colic artery $(c)$ ascends in front of the left kidney, and divides into an ascending and a descending branch for the supply of the descending colon: by the ascending offset it anastomoses with the middle colic branch of the superior mesenteric.

$b$. The sigmoid artery $(d)$ is distributed to the sigmoid flexure, and divides into offsets which anastomose above with t'ce preceding colic, and below with the hxmorrhoidal branch. Here, as in the rest of the intestinal tube, arches are formed by the arteries beiore they reach the intestine. 
$c$. The superior hamorrhoidal artery (e) enters between the layers of the meso-rectum, and is distributed to the lower part of the great intestine: it will be described in the dissection of the pelvis.

The inferior mesenteric vein (fig. 146, d) begins in the part of the great intestine to which its companion artery is distributed, and ascends along the psoas muscle to open into the splenic vein beneath the pancreas. Occasionally it joins the superior mesenteric vein.

Both mesenteric veins are without valves, and may be injected from the trunk to the branches, like an artery.

Lymphatic glands are ranged along the descending colon and the sigmoid flexure. The absorbents of the intestine, after passing through those glands, enter the left lumbar lymphatic glands.

Sympatuetic Nerve. The following plexuses of the sympathetic on the vessels, viz., superior mesenteric, aortic, spermatic, and inferior mesenteric, are derived from the solar plexus beneath the stomach. The remaining portion of the sympathetic nerve in the abdomen will be subsequently referred to.

Dissection. On the two mesenteric arteries the dissector will have made out, already, the plexuses of nerves distributed to the intestinal tube beyond the duodenum.

He has now to trace on the aorta the connecting nerves between the mesenteric plexuses, by taking the peritoneum from the aorta between the mesenteric vessels. From the upper part of the aortic plexus an offset is to be followed along the spermatic artery; this may be done, on the left side, where the vessel is partly laid bare.

By removing the peritoneum from the front of the sacrum, and following downwards, over the iliac arteries, the nerves from the aortic plexus and the lumbar ganglia, the dissector will arrive at the hypogastric plexus of the pelvis, opposite the top of the sacrum.

'The superior mesenteric plexus is a large offset, and is distributed to the same extent of the intestinal tube as the mesenteric artery. The nerves surround closely the artery with a sheath, but near the intestine some of them leave the vessels, and divide and communicate before entering the gut. Branches. The secondary plexuses are the same as the offsets of the artery, viz., intestinal nerves to the small intestine; and an ileo-colic, a right colic, and a middle colic plexus to the large intestine.

The aortic plexus is the network of nerves covering the aorta below the superior mesenteric artery; it is stronger on the sides than the front of the aorta, in consequence of its receiving accessory branches from the lumbar ganglia, especially the left. At the upper part the plexus derives an offset, on each side of the aorta, from the solar and renal plexuses. It ends inferiorly, on each side, in branches which cross the common iliac artery, and enter the hypogastric plexus of the pelvis. From it offsets are furnished to the spermatic and inferior mesenteric arteries.

The spermatic plexus, formed by roots from both the aortic and the renal plexus, runs on the spermatic artery to the testicle; in the cord it joins other filaments on the vas deferens.

In the female, the nerves on the ovarian (spermatic) artery are furnished to the ovary and the uterus.

The inferior mesenteric plexus supplies the part of the intestinal tube to which the artery is distributed. This plexus is furnished from the left part of the aortic plexus; and the nerves composing it are whiter and larger than in either of the preceding plexuses of the sympathetic. Near 
the intestine (sigmoid flexure) the branching of the nerves and the union of contiguous twigs are well marked. Branches. Its secondary plexuses are named from the arteries they accompany, viz., left colic, sigmoid, and superior hæmorrhoidal: they ramify on the vessels, and have a like distribution.

The hypogastric plexus, or the large prevertebral centre for the supply of sympathetic nerves to the viscera of the pelvis, is situate in front of the upper part of the sacrum. It is developed more on the sides than in the centre; and the nerves, which are large and flat, have a plexiform arrangement, but without any intermixed ganglionic masses.

By its upper part.it receives the nerves on the aorta, and is joined by some filaments from one or two of the upper saeral ganglia. Inferiorly the plexus ends in two parts, right and left, the last being the largest: each is continued forwards by the side of the internal iliac artery to the pelvic plexus of the same side, and to the viscera.

\section{CONNECTIONS OF AORTA AND VENA CAVA.}

Before the viscera are removed from the body, the connections of the abdominal aorta and vena cava may be learnt.

Dissection. To see the aorta above the origin of the superior mesenteric artery, it will be necessary to detach the great omentum from the stomach, without injuring the gastro-epiploic artery along the great curve ; and after raising the stomach and the spleen, to remove the peritoneum from the surface of the pancreas. A short arterial trunk (coliac axis) above the pancreas is not to be cleaned now, otherwise the nerves about it would be destroyed.

The vena cava on the right side of the aorta may be followed as far as the posterior border of the liver, where it disappears. The connections of its upper part can be better observed after the dissection of the vessels of the liver.

The aorta enters the abdomen between the pillars of the diaphragm, and divides into iliac arteries opposite the left side of the fourth lumbar vertebra. At the beginning the vessel occupies the middle line of the spine, but it gradually inclines to the left as it descends.

In the abdomen the aorta lies behind all the viscera; but it is crossed more immediately by the pancreas and duodenum, which it touches without the intervention of peritoneum. Its connections are the following : at first it is covered by the solar plexus, and by the pancreas and the splenic vein ; still lower (beyond the superior mesenteric artery) by the left renal vein and the duodenum; and thence to its termination by the peritoneum and the aortic plexus. The vessel lies on the lumbar vertebra, with the pillars of the diaphragm embracing it at the beginning. To its right side is the vena cava. Its relation to other deep parts is mentioned in p. 489.

The vena cava inferior commences on the right side of the fifth lumbar vertebra by the union of the common iliac veins, and reaches thence to the heart.

The venous trunk is placed on the right side of the vertebral column. It lies close to the atorta, and is concealed by the same viscera as high as the crus of the diaphragm; but above that spot it is inclined away from the artery, and ascending on the right of the crus of the diaphragm, is imbedded in the posterior part of the liver for an inch or more. Lastly, 
it leaves the abdomen by an aperture in the tendinous centre of the diaphragm, on the right of, and higher than the aortic opening.

Its connections with vessels are not the same as those of the aorta. Beneath it are the right lumbar, renal, capsular, and diaphragmatic arteries; and crossing over it below the kidney is the spermatic. Superficial to it beneath the pancreas is the beginning of the vena portæ. Offsets of the solar plexus of nerves descend on it, as on the aorta.

\section{CONNECTIONS OF THE DUODENUM AND PANCREAS.}

Dissection. To see satisfactorily the duodenum and the pancreas the intestinal tube, beyond the duodenum, is to be removed in the following way :-a double ligature is to be placed on the upper part of the jejunum, another on the lower end of the sigmoid flexure of the colon, and the gut is to be cut through at the points at which it is tied. 'The detached piece of the intestinal tube is to be taken away by cutting through the vessels, and the peritoneum connecting it to the wall of the abdomen. After it has been separated, it is to be set aside for future study whilst the body is turned.

The student should moderately inflate the stomach and duodenum from the cut extremity of the latter, and remove the loose-peritoneum and the fat; whilst cleaning them, he should lay bare the larger vessels and nerves.

On turning upwards the stomach the pancreas may be traced from the spleen on the one hand to the duodenum on the other (fig. 145). By pulling forwards the duodenum, the common bile duct may be found, posteriorly, between the intestine and the head of the pancreas; and some of the pancreas should be removed, to show its duct entering the duodenum.

Duodenux (fig. 145, D). The first part of the small intestine, or the duodenum, begins at the pyloric end of the stomach, and crossing the spinal column, ends on the left side of the second lumbar vertebra. It makes a curve around the head of the pancreas, and occupies the right hypochondriac, right lumbar, and umbilical regions of the abdomen. From its winding course around the pancreas it is divided into three parts - superior transverse, vertical, and inferior transverse.

The superior transverse part is free and movable, like the stomach; it measures about two inches in length, and is directed from the pylorus to the neck of the gall bladder, ascending slightly between one point and the other. In front it is overlapped by the liver, as well as by the gall bladder when this is distended; and behind it are the bile duct and the vena portæ.

The vertical part is fixed almost immovably by the peritoneum and the pancreas. It is nearly three inches in length, and descends from the gall bladder as far as the third lumbar vertebra. Superficial to this part is the right bend of the colon; and beneath it are the kidney and its vessels. On its inner side is the head of the pancreas, with the common bile-duct. The ducts of the liver and pancreas pour their contents into this portion of the duodenum.

The inferior transverse part is the longest of the three, and is continued across the spinal column to end in the jejunal portion of the small intestine. As it crosses the spine, it ascends trom the third to the level of the 
second lumbar vertebra, and lies between the layers of the transverse meso-colon. It has the following connections with the parts around :-

In front of it are the superior mesenteric vessels with their plexus of nerves. Beneath it lie the vena cava and the aorta, with the pillars of the diaphragm; and the left renal vein is sometimes, between it and the aorta. Above it is the pancreas.

Pancreas (fig. 145, ${ }^{\mathrm{F}}$ ). The pancreas is situate behind the stomach, and has numerous and complicated connections. Of an elcngated form, it extends across the spine from the spleen to the duodenum, and oceupies the left liypochondriac, the umbilical, and the right lumbar region of the abdomen.

The gland is covered anteriorly by the ascending layer of the transverse meso-colon. It is in contact posteriorly with the aorta, the vena cava, and the pillars of the diaphragm ; and it conceals likewise the splenic vein and the commencement of the vena portæ.

Projecting above the upper border, near the centre, is the arterial trunk of the cœlic axis: to the left of that vessel, along the same border, is placed the splenic artery; whilst to the right of it lie the hepatic artery and the first part of the duodenum. At the lower border the superior mesenteric ressels emerge opposite the colic axis; to the right of that spot lies the third part of the duodenum, and to the left the inferior mesenteric ascending to join the splenic vein.

'The left end or the tail of the pancreas touches the spleen, and is placed over the left kidney. The right extremity or the head is received into the coneavity of the duodenum, the two being partly separated by the common bile duct and the pancreatico-duodenal arteries. 'This part projects above and below the body of the gland, like the head of a hammer beyond the handle; and the lower projecting piece is directed to the left along the duodenum beneath the superior mesenteric vessels.

\section{CUELIC AXIS AND VENA PORTA.}

A short branch of the aorta-the cœliac axis, furnishes arteries to the stomach and duodenum, the liver, pancreas, and spleen; it subdivides into three chief branches-coronary, hepatic, and splenic.

The veins corresponding with the arteries (except the hepatic) are collected into one trunk-the vena portæ.

Dissection. The vessels have been in part laid bare by the previous dissection, and in tracing them out fully the student should spare the plexuses of nerves around each. Supposing the liver well raised, he may first follow to the left side the small coronary artery, and show its branches to the osophagus and the stomach. Next the hepatic artery, with the vena portæ and the bile duct, may be traced to the liver and the gall bladder; and a considerable branch of it should be pursued beneath the pylorus to the stomach, duodenum, and pancreas. Lastly, the splenic artery, which lies along the upper border of the pancreas, is to be cleaned; and its branches to the pancreas, stomach, and spleen should be defined; this is a difficult task without the aid of some one to hold aside the stomach and spleen.

The veins will have been dissected for the most part with the arteries; but the origin of the vena porte is to be made out beneath the pancreas, and in front of the vena cava.

The C(Eliac AXIS (fig. 145, c) is the first visceral branch of the ab- 
dominal aorta, and arises between the pillars of the diaphragm. It is a short thick trunk, about half an inch long, which projects above the upper border of the pancreas, and is surrounded by the solar plexus of the sympathetic. Its branches-coronary, hepatic, and splenic-radiate from the trunk (whence the name axis) to their distribution to the riscera in the upper part of the abdomen.

$\alpha$. The coronary artery $(d)$ is the smallest of the three, and passes between the layers of the little omentum to the left end of the stomach. At that spot it furnishes some oesophageal branches, and turns from left to right, along the upper border of the stomach, to anastomose with a branch (pyloric) (o) from the hepatic artery. Its off'sets to the ocsophagus and the stomach are thus distributed:-

OEsophageal branches ascend on the gullet through the opening in the diaphragm, supplying that tube; and they anastomose on it with branches of the thoracic aorta.

Gastric branches are given to both sides of the stomach, and those.on the left end communicate with twigs (vasa brevia) of the splenic artery.

b. 'The splenic artery $(e)$ is the largest branch of the coliac axis in the adult. It is a tortuous vessel, and runs almost horizontally to the spleen, along the upper border of the pancreas. Near the spleen it divides into terminal branches, about seven in number (from four to ten), which enter that viscus by the surface towards the stomach. It is accompanied by the splenic vein, which is below it; and it distributes branches to the pancreas and the stomach.

Pancreatic branches. Numerous small branches are supplied to the gland; and one of these, art. pancreatica magna, arises near the left end, and runs to the right in the substance of the viscus with the duct.

Branches for the stomach arise from the divisions of the artery near the spleen. Some of these, vasa brevia, turn upwards to the left end of the stomach, beneath the gastro-splenic omentum, and ramify in the coats of that organ.

Another longer branch, art. gastro-epiploirca sinistra $(f)$ turns to the righ.t between the layers of the great omentum along the great curvature of the stomach, and inosculates with the right gastro-epiploï branch of the hepatic artery. This artery distributes twigs to both surfaces of the stomach, and between the pieces of peritoneum forming the great omentum.

$c$. The hepatic artery $(g)$ is intermediate in size between the other two, and is encircled by the largest plexus of nerves. In its course to the liver the vessel is bent first to the right towards the small end of the stomach, where it supplies its principal branches (superior pyloric and gastro-epiploïc). It ascends then between the layers of the little omentum, on the left side of the bile duct and vena portae, and divides near the transverse fissure of the liver into two-the right and left hepatic. Branches are distributed not only to the liver, but freely to the stomach, the duodenum, and the pancreas, as below:-

The superior pyloric branch $(o)$ descends to the upper border of the stomach, and running from right to left anastomoses with the coronary artery; it distributes small arterial twigs on both surfaces of the stomach.

The right gastro-epipluïc branch $(h)^{1}$ (art. gast. epiploïca dextra) de-

1 This artery is named commonly gastro-duodenal as far as to the spot where it gives off the branch to the duodenum and pancreas. 
scends beneath the duodenum near the pylorus, and turning to the left along the great curvature of the stomach, inosculates with the left gastro-epiploic of the splenic artery. To the surfaces of the stomach some offsets are given; and others descend between the layers of the omentum. It furnishes the following named branches to the stomach, and the pancreas and duodenum:-

Small inferior pyloric branches end in the small extremity of the stomach.

Fig. 145 .

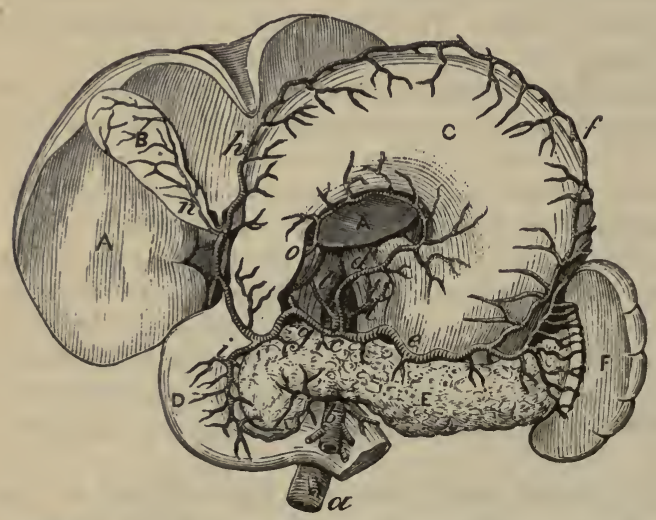

View of the Certiac Axis, AND OF the Viscera to which ITS BRANCHES ARE SUPPLIED (Tídmann).
A. Liver.

B. Gall-bladder.

c. Stomach.

D. Duodenum.

E. Pancreas.

F. Spleen.

Vessels :

a. Aorta.

b. Upper mesenteric.

c. Cœliac axis.

$d$. Coronary.

e. Splenic.

f. Left gastro-epipiolc.

g. Hepatic.

h. Right gastro-epiploĩc.

$i$. Superior, and $k$, inferior pancreatico-duodenal.

l. Diaphragmatic arteries.

$n$. Cystic.

o. Superior pyloric.

The pancreatico-duodenal branch (i) (superior) arises opposite the duodenum, and runs between the intestine and the pancreas; it anastomoses below with the pancreatico-duodendal branch (inferior) of the superior mesenteric (fig. 146, $b$ ). Both the duodenum and the pancreas receive offsets from this vessel. On the posterior aspect of the same viscera is another small offset of the pancreatico-duodenal with a similar position and distribution.

The hepatic branches (right and left) sink into the liver at the transverse fissure, and ramify in its substance :-

The right branch is divided when about to enter the transverse fissure, and supplies the following small artery to the gall bladder. The cystic artery $(n)$ bifurcates on reaching the neck of the gall bladder, and its two twigs ramify on the opposite surfaces.

The left branch is smaller than the other, and enters the liver at the left end of the transverse fissure: a branch to the Spigelian lobe of the liver arises from this piece of the artery.

Portal Veins. The veins of the intestinal tube, and of the spleen and pancreas, pour their blood into the vena portæ. The two mesenteric veins and their branches have been referred to (p. 441); and the two following, with the trunk of the portal vein, remain to be noticed.

The superior coronary vein (fig. $146, f$ ) lies along the upper border of the stomach. It begins in the oesophagus and the left part of the stomach, and joins the vena portæ at the pylorus.

The splenic vein (fig. 146) is large in size, and is formed by the union of branches from the spleen. It takes much the same course as, but below 
the artery, and runs beneath the pancreas to the front of the vena cava, where it joins the superior mesenteric vein $(b)$ to form the vena portæ.

Between its origin and termination it receives branches corresponding with the following arteries:-vasa brevia $(g)$, left gastro-epiploïc $(e)$, and pancreatic. The inferior mesenteric vein $(d)$ opens into it about its middle.

The vena porta (fig. $146, a$ ) is formed by the union of the splenic and superior mesenteric veins. Its origin is placed in front of the vena cava, but beneath the pancreas, and two inches from the riglit end. The vessel is about four inches long, and is directed upwards in the small omentum, behind the bile duct and the hepatic artery, to the transverse fissure of the liver, where it divides into a right and a left branch.

Fig. 146.

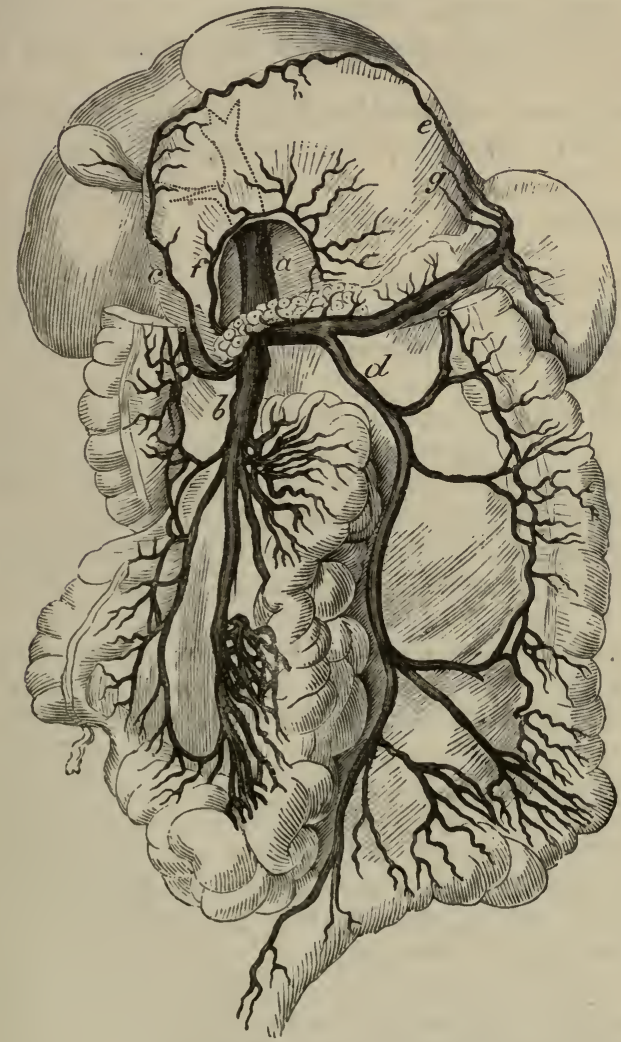

a. Trunk of the vena portæ.

b. Upper mesenteric.

c. Right gastro-epiploīc.

d. Inferior mesenteric.

e. Left gastro-epiploric.

$f$. Corouary of the stomach.

g. Vasa brevia.

Vexa Porta and the Veins Joining it (Henle: Anatomie des Menschen).

In its course it is joined by the coronary vein $(f)$, and by the cystic vein near the liver.

The right branch enters the transverse fissure to ramify in the right lobe of the liver.

The left branch is distributed to the left part of the liver, and gives a small branch to the Spigelian lobe. 
This vein commences by roots in the viscera above mentioned, like any other vein, but it is deficient in valves; and it ramifies through the structure of the liver in the same manner as an artery. Its radicles communicate with the systemic veins on some parts of the intestinal tube, but more particularly on the rectum.

Bile Ducts. Two hepatic ducts issue at the transverse fissure of the liver (fig. 158), one from each lobe, and unite to form the following:-

The common hepatic duct is an inch and a half long, and receives at its termination the duct of the gall bladder, the union of the two giving origin to the common bile duct.

The common bile duct (ductus communis choledochus) is about three inches long. It descends almost vertically beneath the upper transverse portion of the duodenum; then passing between the pancreas and the vertical piece of the duodenum, it opens into this portion of the intestine at the inner side, and above the middle. Whilst in the small onentum the duct lies to the right of the hepatic artery, and somewhat before the portal vein.

Before piercing the coats of the intestine it is joined commonly by the pancreatic duct, but the two may enter the duodenum separately.

\section{SYMPATHETIC AND VAGUS NERVES.}

Sympathetic Nerve. In the abdomen, as in the thorax, the sympathetic nerve consists of a gangliated cord on each side of the vertebral column, and of prevertebral centres or plexuses, which furnish branches to the viscera.

Two prevertebral plexuses exist in the abdomen. One of these, the epigastric, is placed behind the stomach, and supplies nerves to all the viscera above the cavity of the pelvis. The other, the hypogastric plexus, is situate in the pelvis, and distributes nerves to the pelvic viscera.

The knotted or ganoliated cord will be met with in a subsequent stage of the dissection; and only the great solar plexus with its offsets is to be now examined.

Dissection. To denude the epigastric plexus, the following dissection is to be made: After the air lias been let out of the stomach and duodenum, the portal vein, the common bile duct, and the gastro-epiploï vessels are to be cut through near the pylorus; and the stomach, duodenum, and pancreas are to be drawn over to the left side. On raising the liver the vena cava appears: the vein is to be cut across above the junction of the renal vein with it, and the lower end is to be drawn down with hooks.

Beneath the vein the dissector will find the large reddish semi-lunar ganglion; and mixed up with the nerves of the plexus are numerous lymplatic glands, and a dense tissue, which require to be removed with care. From its inner part he can trace the numerous nerves and ganglia around the coeliac and superior mesenteric arteries, and the secondary plexuses on the branches of those arteries. From the outer part of the ganglion offsets are to be followed to the kidney, the suprarenal body, and the diaphragmatic arteries : at its upper part the junction with the large splanchnic nerve may be seen; and deeper than the last, one or two smaller splanchnic nerves may be observed in a fissure of the diaphragm, which throw themselves into the coeliac and renal plexuses.

The student should then trace the ending of the pneumogastric nerves on the stomach. 'The left nerve will be found at the upper border in front 
near the osophagus; and the right nerve will be seen at a corresponding point on the opposite aspect. Branches from the right nerve are to be followed to the plexus of the sympathetic by the side of the coliac axis, and from the left, to the hepatic plexus.

The EPIgAstric or solar plexus is a large network of nerves and ganglia, which lies in front of the aorta and the pillars of the diaphragm: it fills the space between the suprarenal capsules of opposite sides, and extends downwards to the pancreas, surrounding the coeliac axis and the superior mesenteric artery. The plexus is connected on each side with the large and small splanchnic nerves; and it is joined also by an offset of the right pneumo-gastric nerve. Large branches are furnished to the different viscera along the vessels.

The semilunar ganglia, one on each side, are the largest in the body, and each is joined at the upper end by the great splanchnic nerve. Each is situate at the outer part of the plexus, close to the suprarenal body, and on the pillar of the diaphragm : the ganglion on the right side is beneath the vena cava. Irregular in shape, the mass is oval, or divided into smaller ganglia : from its outer side nerves are directed to the kidney and the suprarenal capsule.

Offsets of the plexus. The nerves supplied to the viscera form plexuses around the vessels; thus there are coliac, mesenteric, renal, spermatic, diaphragmatic, \&c.

Diaphragmatic plexus. This plexus comes from the upper part of the semilunar ganglion, but it soon leaves the artery to enter the substance of the diaphragm: a communication takes place between the phrenic nerve of the cerrical plexus (p. 80) and these branches of the sympathetic. On the right side is a small ganglion where the plexus is joined by the spinal nerve; and from it filaments are supplied to the vena cava and the suprarenal body. The ganglion is absent on the left side. (Swan.)

The suprarenal nerves are very large and numerous, in comparison with the size of the part supplied, and are directed outwards to the suprarenal body. One of the splanchnic nerves communicates with this plexus.

The renal plexus is derived from the semilunar ganglion and outer part of the plexus, and is joined by the smallest splanchnic uerve. The nerves surround the renal artery, having small ganglia on them, and enter the kidney with the vessels. An offset is given from the renal to the spermatic plexus (p. 443).

The coliac plexus is a direct continuation of the plexus around its artery: it is joined by the small splanchnic nerve on each side, and by an offset from the right pneumo-gastric nerve. The plexus divides like the artery into three parts-coronary, splenic, and hepatic.

a. The coronary plexus accompanies the vessel of the same name to the upper border of the stomach, where it ends : it communicates with the left vagus nerve.

$b$. The splenic plexus furnishes offsets to the pancreas, and to the stomach along the left gastro-epiploïc artery : and it is joined by an offset from the right pneumo-gastric nerve.

c. The hepatic plexus is continued on the rena porte, the hepatic artery, and the bile duct into the liver, and ramifies on those vessels : in the small omentum, the plexus is joined by offsets from the left vagus. The following secondary plexuses are furnished around the branches of the hepatic artery, and hare the same name and distribution as the vessels :-

A pyloric plexus is distributed along the upper border of the stomach. 
Two other plexuses_gastro-epiploïc (right) and pancreatico-duodenal, correspond in distribution with the branches of each artery.

A cystic plexus ramifies in the coats of the gall bladder with its artery.

The remaining offsets of the plexus, viz., superior and inferior mesenteric, aortic, and spermatic, have been already noticed (p. 443); but the derivation of the superior mesenteric and aortic plexuses from the epigastric centre can be now seen.

Ending of the splanchnic nerves. The large nerve perforates the crus of the diaphragm, and generally ends altogether in the semilunar ganglion.

The small nerve comes through the same opening in the diaphragm as the preceding, and joins the coeliac plexus.

The smallest nerve, which is often absent, throws itself into the renal plexus.

Ending of the vagus nerve. The pneumo-gastric nerves end in the stomach :-

The left nerve divides into branches, which extend along the small curvature, and over the front of the stomach; these send offsets to the hepatic plexus.

The right nerve is distributed to the posterior surface of the stomach near the upper border; it communicates with its fellow, and with the coeliac and splenic plexuses.

Dissection. The viscera are now to be removed from the abdomen, in order that the body may be turned for the dissection of the Back and lower limbs.

The stomach and the spleen, with the duodenum and the pancreas, are to be taken away together by cutting through the ocosphagus near the diaphragm, as well as the vessels and nerves they receive. The liver is to be removed from the abdomen by dividing its ligaments, and incising the vena cava between the posterior border and the diaphragm.

At the same time the left testicle, and the right kidney with the suprarenal body, should be removed for examination whilst the body is turned; the former can be taken out by cutting through the spermatic cord, and the latter by dividing the vessels about the middle.

Directions. Supposing the body to be now turned for the dissection of the Back, and to lie with the face downwards for the usual time, the dissector may look first to the fascia lumborum, which is described in the Dissection of the Back, p. 357 .

The rest of the time should be occupied in learning the viscera included in the following Section.

\section{Section IV. \\ ANATOMY OF THE VISCERA OF THE ABDOMEN.}

TIIE STOMACII.

TIE stomach is the dilated part of the alimentary tube between the asophagus and the small intestine, into which the masticated food is received to be changed into chyme.

-Dissection. To see the form, the stomach must be blown up moder- 
ately, and the surface cleaned; but, previously, let the student detach the spleen, and cut through the duodenum close to the pylorus.

Forms and Divisions. 'The stomach is somewhat conical in form (fig. 147). Its size varies much in different bodies, and is sometimes much diminished by a constriction in the centre; when it is moderately distended, it is about twelve inches long and four wide. There are two ends, two orifices, two surfaces, and two borders or curves to be examined.

Extremities. The left end or tuberosity (fundus ventriculi) is the largest part of the stomach, and projects about three inches to the left of the opening of the esophagus. The right or pyloric end, much smaller than the other, is cylindrical, and forms the apex of the cone to which the stomach is likened.

Openings. 'The left opening (cardiac), which communicates with the œesophagus, is at the highest part of the stomach, and is funnel-shaped towards the cavity of the organ. The right or pyloric orifice opens into the duodenum, and is guarded internally by a muscular band (pylorus): at the same spot the stomach is slightly constricted externally, where a firm circular ring may be felt.

Surfaces. The surfaces (anterior and posterior) are somewhat flattened when the viscus is empty, but rounded when it is distended : the parts in contact with them have been referred to (p. 431).

Borders. The upper border or small curve is concave towards the left opening, but convex at the opposite end; and the lower border or large curve is convex, except near the right end, where it is concave-the concavity of the one border corresponding with the convexity of the other. An arterial arch, and a fold of peritoneum (omentum) are fixed to each border.

Structure. In the wall of the stomach are four coats, viz., serous, muscular, fibrous, and mucous; and belonging to these there are vessels, nerves, and lymphatics.

Serous coat. The peritoneum gives a covering to the stomach, and is adherent to the surface, except at each margin, where an interval exists corresponding with the attachment of the small and large omentum : in those spaces are contained the ressels, nerves, and lymphatics. During distension of the stomach the spaces above mentioned are much diminished.

The muscular coat will be laid bare by the removal of the serous covering. It consists of three sets of fibres-longitudinal, circular, and oblique; these lie from without inwards in the order mentioned, and are unstriated or involuntary.

The longitudinal fibres (fig. 147,a) are derived from the oesophagus; they spread over the surfaces, and are continued to the pylorus and the small intestine. The fibres are most marked along the borders, particularly at the smaller one; and at the pylorus they are stronger than in the centre of the stomach.

The circular fibres (fig. 147, b) form the middle stratum, and will be best seen by removing the longitudinal fibres near the pylorus. They reach from the left to the right end of the stomach; but at the pylorus they are most numerous and strongest, and form a ring or sphincter (c) around the opening.

The oblique fibres (fig. $147, e$ ) are continuous with the circular or deep layer of the osophagus. On the left and right of the cardic orifice they are so arranged as to form a kind of sphincter ( $d$ and $e$ ) (Henle); but 
those on the left (e), the strongest, arch over the great end of the stomach, and spread out on the anterior and posterior surfaces, gradually disappearing on them.

Fibrous coat. By removing the muscular layer over a small space, the fibrous eoat will appear as a white shining stratum of areolar tissue. This coat gives strength to the stomach, and serves as a bed in which the larger vessels and nerves ramify before their distribution to the mucous layer. If a small opening is made in this membrane, the mucous coat will project through the stomach to be distended with air.

Fig. 147.

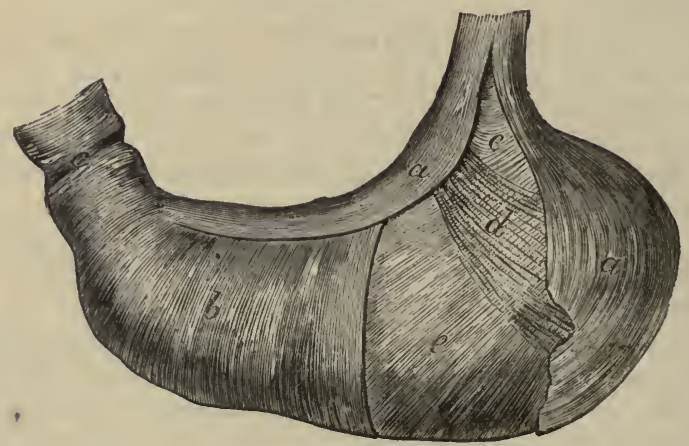

Diagram Repregexting the ARRANGemext of the MUgcular Fibres of the Stomach. Part of each of the two external coats is removed.

a. External or longitudinal fibres.

b. Middle or circular.

c. Sphincter of the pylorus.

d. Oblique fibres on the left of the cardiac opening. $e$. Oblique fibres, more numerous, on the right of the cardiac orifice, and covering the great end of the stomach.

The mucous coat will come into view on cutting open the stomach, but the appearances now described can be recognized only in a recent stomach.

This coat is a thickish layer, of a pale rose color soon after death in the healthy condition. In the empty state of the stomach the membrane is less vascular than during digestion; and in infancy the natural redness is greater than in ehildhood or old age. When the stomach is contracted the membrane is thrown into numerous wavy ridges or ruga, which become longitudinal along the great curve, towards the pylorus.

The thickness of the mucous membrane is greatest near the pylorus; and at that spot it forms a fold, opposite the muscular ring, which assists in elosing the opening. If this membrane and its submucous layer are removed from the pyloric part of the stomach, the ring of muscular fibres (sphincter of the pylorus) will be more perfectly seen.

Microscopic-structure of the mucous membrane. With the aid of a lens, the surface of the mucous membrane, when well washed, may be seen to be covered by shallow depressions or alveoli (fig. 148), which measure from $\frac{1}{2} \frac{1}{0}$ th to $1_{1}^{\frac{1}{0} 0}$ th of an inch across. Generally hexagonal or polygonal in outline, the hollows become larger and more elongated towards the small end of the stomach; and near the pylorus the margins of the alveoli project, and become irregular. In the bottom of each depression are the apertures of minute tubes. 
By means of a thin section under the microscope the membrane may be observed to be composed almost altogether of minute vertical tubes, which lie side by side, and project into the submucous tissue. Measuring from $\frac{1}{60}$ th to $\frac{1}{30}$ th of an inch in length, the tubes are closed at the deep end; but they open on the surface of the stomach both in the alveoli (fig. 148 ) and in the interalveolar spaces. They are formed of a homogeneous membrane, and are for the most part straight, but towards the pylorus they increase in length, and are somewhat sacculated at the deep extremity; some are divided into two or more pieces (fig. 149).

Fig. 148.

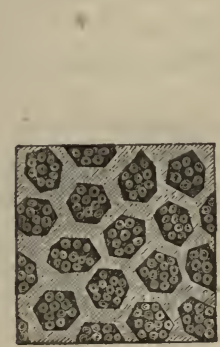

AtVEOLAR DEPRESSIONS OF THE Mccous Mrmbrane of The SToмАCH, magnified 32 diameters, with the minute tubes opening into them (Sprott Boyd.)
Fig. 149.
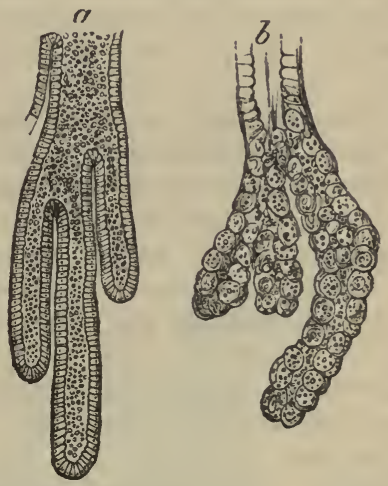

ENLARGED REPRESENTATION OF THE TUBES OF THB STOMACH.

a. Gastric tube from the pyloric end lined, throughout, by columnar epithelium (Kölliker).

b. Gastric tube from the cardiac end, with a columnar epithelium lining near the mouth, and flattened nucleated granular cells beyond (Thomson).

At the cardiac end of the stomach the tubes are lined at the free end by columnar epithelium (fig. 149,b); but in the closed end by flattened and granular nucleated cells, which are named peptic glands, and are supposed to give origin to the gastric fluid. Towards the pylorus the tubes are closed throughout by columnar epithelium (fig. 149,a) and secrete mucus.

A columnar epithelium covers the surface of the mucous membrane, and enters the small tubules.

On the attached surface of the mucous membrane is a thin layer of involuntary muscular fibres, separating it from the fibrous coat : it is said to send offsets amongst the tubules.

Bloodvessels. The arteries of the stomach, after supplying the muscular coat, ramify in the submucous tissue; from this anastomosis fine offsets are continued on the tubes to the inner surface of the mucous membrane, where they form a network. The veins begin in the mucons membrane, receive branches from the muscular coat, and deliver their blood into the portal system.

Lymphatics. 'Two layers of absorbents, superficial and deep, exist in the stomach : the latter begin in a plexus beneath the tubules, and form a 
network in the fibrous layer. Both sets leave the stomach with the bloodvessels.

Nerves. The nerves are derived from the pneumo-gastric and sympathetic (p. 451), and can be followed to the fibrous coat; small ganglia have been observed on them.

\section{SMALL INTESTINE.}

The three parts into which the small intestine is divided, have the following characters:-

The duodenum measures as much as the breadth of twelve fingers, viz., about ten inches, and is more fixed than the rest of the intestinal tube. It is wider than either the jejunum or the ileum, and its muscular coat is also thicker. Into it the common bile and pancreatic ducts pour their contents.

The jejunum and the ileum together measure about twenty feet in length, and are connected with the mesentery. There is not any perceptible difference between the termination of the one and the commencement of the other, but two-fifths of the length are assigned to the jejunum, and three-fifths to the ileum. Between the ends, however, a marked difference may be perceived; for the upper part of the jejunum is thicker and more vascular than the lower part of the ileum, and its width is also greater.

Structure. In the small intestine the wall is formed by the same number of layers as in the stomach, viz., serous, muscular, fibrous, mucous, and submucous.

Dissection. The different layers are to be examined on pieces, about three inches long, taken from the duodenum, the upper part of the jejunum, and the lower end of the ileum. After the pieces have been cut off, they are to be distended with air ; and the serous covering is to be torn off for a short distance, to show the muscular coat, but in doing this, the external longitudinal fibres will be taken away without great care.

The serous coat is closely connected with the subjacent muscular layer. To the jejunum and ileum it furnishes a covering, except at the attached part where the vessels enter : at this spot the peritoneum is reflected off to form the mesentery, and a space exists resembling that at the borders of the stomach. The peritoneum surrounds the duolenum only partly; this peculiarity has been described at p. 438 .

The muscular coat is constructed of two sets of fibres, a superficial or longitudinal, and a deep or circular. The fibres are pale in color, and are not striated.

The longitudinal fibres form a thin covering, which is most marked at the free border of the gut.

The circular fibres are much more distinct than the others, and give the chief strength to the muscular coat : they do not form complete rings around the intestine.

Dissection. On the removal of a part of the muscular stratum from the jejunum or the ileum, the submucous fibrous layer will come into view.

'The fibrous coat has the same position and use as the corresponding layer in the stomach.

Dissection. In the upper part of the duodenum the student is to seek some small compound glands - those of Brunner, which are imbedded in the submueous tissue. They lie beneath the mucous membrane, and will 
be seen shining through the fibrous layer, when the muscular coat has been taken away.

The pieces of intestine may be opened and washed to show the mucous coat, but the gut should be cut along the line of attachment of the mesentery, so as to avoid Peyer's glands on the opposite side.

Mucous coat. The lining membrane is thicker and more rascular at the beginning than at the ending of the small intestine. It is marked by numerous prominent folds (valvula conniventes); and the surface of the membrane is covered with small processes (villi) like the pile of velvet. Occupying the substance of the mucous coat are numerous glands; and covering the whole is a columnar epithelium.

A thin layer of non-striated muscular fibres (muscularis mucosa) covers the outer surface of this coat (fig. 154, $d$ ), and sends inwards prolongations between the tubules into the villi.

The valvula conniventes (fig. 150) (valves of Kerkring) are permanent ridges of the mucous membrane, which are arranged circularly in the intestine, and project into the alimentary mass. Crescentic in form, they extend round the intestine for half or two-thirds of its circle, and

Fig. 150.

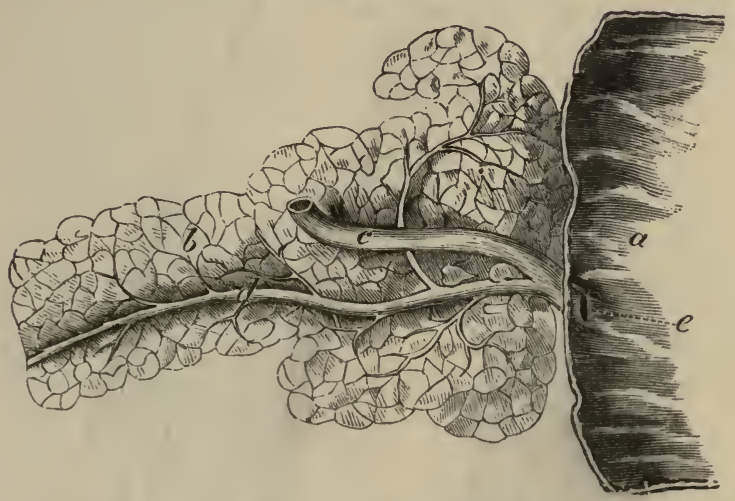

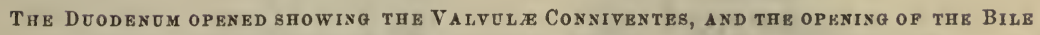
D $\mathbf{C}$ T. The duct of the paucress is also represented in greater part of its course.
a. Duodenum.
d. Pancreatic duct.
b. Pancreas.
c. Common bile duct.
e. Opening of the common duct in the intestine (Henle).

some end in bifurcated extremities. Larger and smaller folds are met with, sometimes alternating; and the larger are about two inches long, with one-third of an inch in depth towards the centre. Each is formed of a doubling of the mucous membrane, which incloses vessels between the layers.

They begin in the duodenum, about one or two inches beyond the pylorus, and are continued in regular succession to the middle of the jejunum; but beyond that point they become smaller and more distant from one another, and finally disappear about the middle of the ileum, having previously become irregular and rudimentary. The folds are largest and most uniform beyond, and not far from the opening of the bile duct.

The aperture of the common bile and pancreatic ducts (fig. 150,e) is a 
narrow orifice, from three to four inches from the pylorus, and is situate in a small prominence of the mucous membrane, at the inner and posterior part of the duodenum (p. 445). A probe passed into the bile duct will show the oblique course (half an inch) under the mucous coat. Sometimes the pancreatic duct opens by a distinct orifice.

Microscopic structure of the mucous membrane. With the use of the microscope, and with pieces of fresh intestine, the student will be able to make out the nature of the villi, the glandular bodies, and the epithelium.

Villi. When a piece of the lower part of the duodenum, from which the mucus is washed away, is examined in water, the mucous membrane will be seen to be studded over thickly with small projections, like those on velvet. These bodies exist along the whole of the small intestine, and are irregular in form (fig. 153, ${ }^{\wedge}$ ) some being triangular, others conical or cylindrieal with a large end. Their length is from $\frac{1}{40}$ th to $\frac{1}{20}$ th of an inch; and they are best marked where the valvula conniventes are largest. In the duodenum their number is estimated at 50 to 90 in a square line, but in the lower end of the ileum at only 40 to 70 on the same surface (Krause).

Fach villus is an extension of the mucous coat, and is covered by columnar epithelium. One or sometimes two arterial twigs form a capillary network beneath the mucous eovering (fig. $144,{ }^{\wedge}$ ), and end generally in a single emerging vein. A single lacteal, or two forming a loop with cross branches (fig. $151,{ }^{\mathbf{B}}$ ), occupies the centre, and communicates with

A

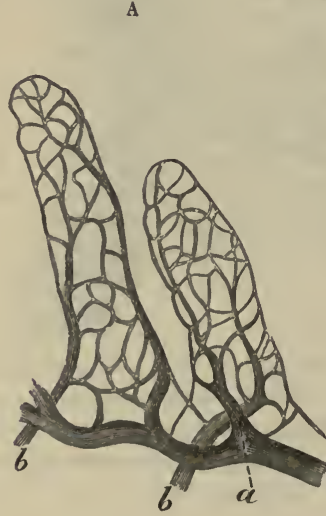

A. Vessels of the Villi in the Mousb, INJECtEd By GrRlach (Köllicker).

$a$. Artery, and $b$, vein.
Fig. 151.

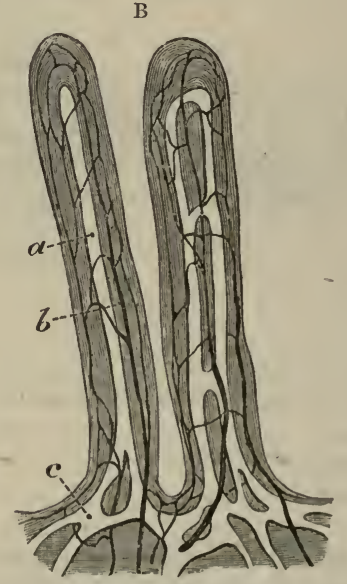

B. Lacteals and Plexug of Vesgels in two VILLI, INJECTED BY TEICHMAN.

a. Lacteal vessel, single in one villus, double in the other.

b. Plexus of vessels.

c. Plexus of lacteals below the villi. (Quain's Anatorny.)

plexus below the villus. Around the lacteals a thin layer of unstriated muscular fibre is arranged longitudinally (Brücke). Nerves lave not been detected in the villus.

Glands. In the glandular apparatus of the small intestine are included 
the crypts of Lieberkühn, solitary glands, and Peyer's and Brunner's glands.

The crypts of Lieberkiihn (fig. 154,a) are minute simple tubes, similar to those in the stomach, though not so closely aggregated, which exist throughout the small intestine. They open on the surface of the mucous membrane by small orifices between the villi, and around the larger glands; but closed at the opposite end, they project into the submueous layer, and are seldom branched. Their length is from $\frac{1}{70}$ th to $\frac{1}{50}$ th of an inch: they are filled with a translucent fluid containing granules, and are lined by a columnar epithelium.

The so-called solitary glands (fig. $153,{ }^{\mathrm{B}}$ ) are roundish white eminences, about the size of mustard seed if distended, which are scattered along the small intestine, but in greatest numbers in the ileum. Placed on all parts of the intestine, and even on or between the valvulæ conniventes; they are covered by the villi of the mucous membrane, and are surrounded at

\section{Fig. 152.}

A

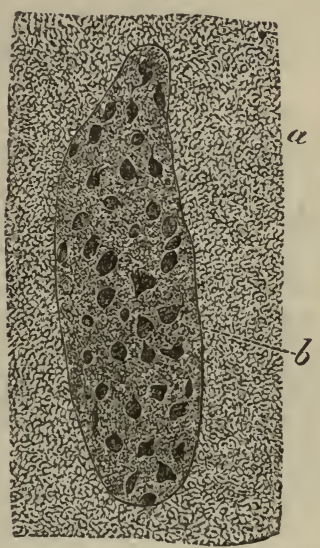

A. Patch of Pexer's Glands four times enLARGED.

a. Surface of the mucous membrane covered with villi.

b. Pits over the follicles where the villi are absent.
B

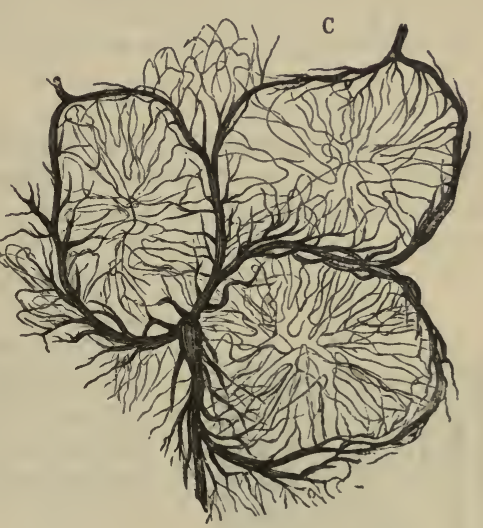

b. Magnified Representation of an InjecTION IN THE RABBit, By Frit, OP THE Vessels Surrounding and Penetrating th f Follicles in a Patch of Peyer (Köllicker).

the: $r$ circumference by apertures of the erypts of Lieberkiihn. They are closed lymph follieles beneath the mucous coat, which project into the gut; and they are formed of a network of reticular connective tissue with lymph-corpuscles between the meshes. Fine capillary vessels permeate the mass; and it is surrounded by a plexus of lymphatic vessels.

The glands of Peyer (fig. 153, ^) (glandula agminata) exist chiefly in the ileum, in the form of oval patehes, which measure from half an inch to two inches or more in length, and about half an inch in width. They are situate on the part of the intestine opposite to the attachment of the mesentery, and their direetion is longitudinal in the gut: usually they are from twenty to thirty in number. In the lower part of the ileum they are largest and most numerous; but they decrease in number and size up- 
wards from that spot, till at the lower part of the jejunum they become irregular in form, and may consist only of small roundish masses.

The mucous membrane over them is hollowed into pits (fig. 152, b), and is generally destitute of villi on the subjacent follicles (fig. 153, $\mathbf{A}$ ). but between the pits it has the same characters as in other parts.

Fig. 153.

A

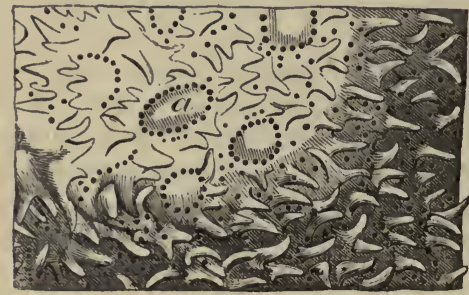

B

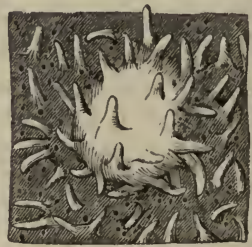

A. A piece of Mucots Membrane eniaraed, with its villi and tubules. Part of a patch of Peyer's glands is also represented with the follicles $(a)$, each having a ring of tubes at the circumference. B. A "golitary gland" of the small intestine, also enlarged, covered by vill (Buebm).

A pateh, when examined by the microseope, appears to be but a collection of lymph follicles like the "solitary glands" (fig. 153), which are round or oval in form, and are covered by the mucous membrane. Around each follicle is a ring of apertures of the crypts before described. The

Fig. 154.

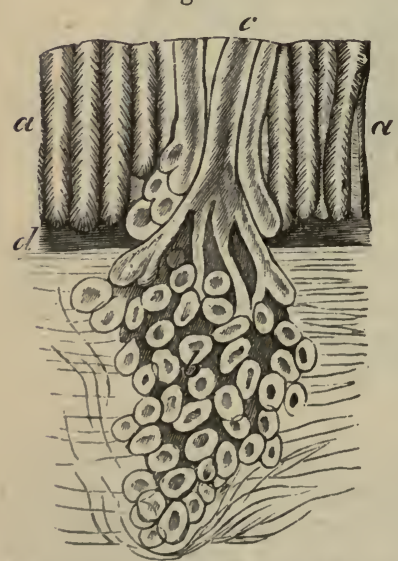

Magnified View op thb Mucoug Membrane of the DÉonexum, with the tubules of Lieberkahn and a gland of Brunner.

a. Tubules.

b. Gland of Brunner.

c. Duct of the gland.

d. Submucous layer of muscular fibres (Kolliker). follicles have the same composition as the scattered "solitary glands."

Fine arterial twigs (fig. 152, ${ }^{\mathrm{B}}$ ) ramify on the follicles, and send inwards aapillary offsets which form a network in the interior, and converge to the centre. Lacteal vessels form plexuses around and beneath the follicle, but do not penetrate the wall.

The Glands of Brunner (fig. 154, b) are small compound bodies, similar to the buccal and labial glands of the mouth, which exist in the duodenum. For a few inches near the pylorus they are most numerous, and there they are visible without a lens, being nearly as large as hemp seed. 'The glands consist of lobules, with appertaining excretory tubes: and each ends on the surface of the mucous membrane by a duct $(c)$, whose aperture is slightly larger than the mouths of the contiguous crypts of Lieberkühn; they secrete mucus.

Epithelium. 'The epithelial lining of the mucous membrane of the small intestine is of the columnar or eylindrical kind. On the villi it forms a distinet covering of elongated pieces. It sinks into the crypts 
of Lieberkühn, and into the ducts of the glands of Brunner, and gives them a lining.

Dissection. To demonstrate the areolar tissue between the coats of the intestine, a piece of the bowel turned inside out is to be inflated forcibly; and to insure the success of the attempt, a few cuts may be previously made through the peritoneal coat. The air enters the wall of the intestine where the peritoneal covering is injured, and spreads through the whole gut; but opposite the solitary glands, and the patches of Peyer, the nucous coat is more closely connected with the contiguous structures, and the subjacent portion will not be distended with the air. The piece of the intestine may be examined when it is dry.

Vessels of the intestine. The branches of arteries ramify in the submucous layer, and end in a network of small twigs in the mucous membrane, which supplies the folds, the villi, and the glands. Opposite Peyer's patches the intestine is most vascular; and the vessels form circles around the follieles, before supplying offsets to them. 'The veins have their usual resemblance to the companion arteries.

The absorbents consist of a superficial set (lymphatics) in the muscular coat; and of a deep plexiform set (lacteals) in both the mucous and submucous layers. The two sets join, and all end in larger trunks in the mesentery.

Nerves of the small intestine come from the upper mesenteric plexus, and entering the coats by the side of the arteries, form plexuses with interspersed ganglia. One such plexus is contained in the muscular coat between the longitudinal and circular fibres (Auerbach); and another is placed in the submucous layer (Meissner): they join freely by branches through the intestinal coats, and reach from the pylorus to the extremity of the alimentary tube.

Structure of the common bile duct. The bile duct consists of an external or strong fibrous layer, and of an internal or mucous coat which is lined by columnar epithelium. On the surface of the inner membrane are the openings of numerous branched mucous glands, which are inbedded in the fibrous coat; some of them are aggregated together, and are visible with a lens.

\section{LARGE INTESTINE.}

The large intestine is the part of the alimentary canal between the termination of the ileum and the anus. Its division and its attachment by peritoneum to the abdominal wall, have been deseribed (p. 433).

In length this part of the alimentary canal measures about five or six feet-one-fifth of the length of the intestinal tube. The diameter of the colon is largest at the commencement in the cæcum, and gradually decreases as far as the rectum, where there is a dilatation near the end.

When compared with the small intestine, the colon is distinguished by the following characters : It is of greater capacity, being in some parts as large again, and is more fixed in its position : it is also free from convolution, except in the left iliac fossa, where it forms the sigmoid flexure. Instead of being a smooth cylindrical tube, the colon is sacculated, and is marked by three longitudinal muscular bands, which alternate with as many rows of dilatations; but at the lower part of the large intestine (rectum), the surface is smooth, and the longitudinal bands have disappeared. Attached to the surface at intervals, especially along the trans- 
verse colon, are processes of peritoneum containing fat-the appendices epiploïcæ.

Dissection. For the purpose of examining the large intestine the student should cut off and blow up the crecum, with part of the ileum

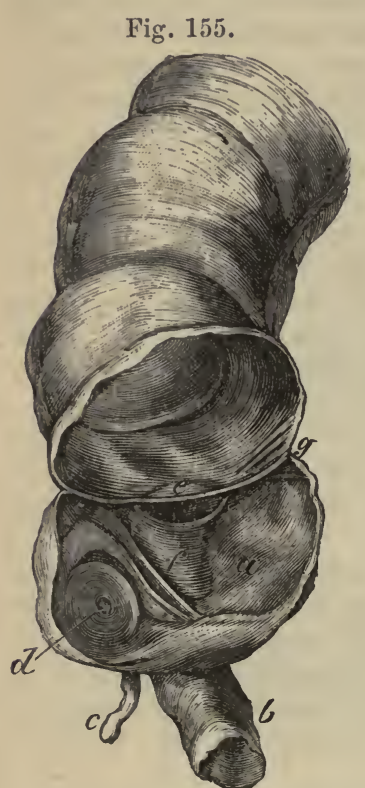

INTERIOR OF A CACUM DRIER AND I.AID OPEN.

и. Cæcum.

b. Small intestine.

c. Vermiform appendix, und $d$, its aperture.

e. Ilio-colic piece of the valve at the junction of the small intestine.

f. Ilio-cæcal piece of the valve.

$g$. Retinaculum of the valve on each side.

side of the crecum, opposite the entrance of the small intestine.

Ileo-cacal valve (fig. 155). 'This valve is situate at the entrance of th:e ileum into the crecum. It is composed of two pieces, each with a different inclination, which project into the interior of the cxcum, and bound a narrow, nearly transverse aperture of communication between the two differently-sized portions of the alimentary canal.

The upper piece of the valve, ileo-colic $(e)$ projects horizontally into the large intestine, opposite the junction of the ileum with the colon. And the lower piece, ileo-cacal $(f)$, which is the larger of the two, has a vertical direction between the ileum and the cxeum. At each extremity of the opening the pieces of the valve are blended together; and the resulting prominence $(g)$ extends transversely on each side of the intestine, forming the frena or retinacula of the valve. 
The size of the opening depends upon the distension of the intestine; for when the retinacula of the valve are stretched the margins of the aperture are approximated, and may be made to touch.

Each piece of the valve is formed by circular muscular fibres of the int'stinal tube, covered by mucous membrane; as if the ileum was thrust obliquely through the wall of the cæcum, after being deprived of its peritoneal coat and layer of longitudinal fibres. This construction is easily seen on a fresh specimen by dividing the peritoneum and the longitudinal fibres, and gently drawing out the ileum from the cæcum.

The opening of the appendix into the cxcum $(d)$ is placed below that of the ileum. A piece of mucous membrane partly closes the aperture, and acts as a valve.

Folds or ridges are directed transversely in the interior of the gut, and correspond with depressions on the outer surface: these folds result from the doubling of the wall of the intestine, and the largest inclose ressels.

Structure of the Colos. The coats of the large are similar to those of the small intestine, viz., serous, muscular, fibrons, and mucous.

Serous coat. The peritoneum does not clothe the large intestine, throughout, in the same degree. It covers the front of the cæcum, and the front and sides of the ascending and descending colon; but in neither does it reach commonly the posterior aspect (p. 438). The transverse colon is incased like the stomach, and has intervals along the borders, where the transverse meso-colon and the great omentum are attached.

The muscular coat is formed by longitudinal and circular fibres, as in the small intestine.

The longitudinal fibres may be traced as a thin layer over the surface, but most are collected into three longitudinal bands, about a quarter of an inch in width. On the vermiform appendix the fibres form a uniform layer; but they are continued thence into the bands on the cæcum and colon: on the rectum they are diffused over the surface. When the bands are divided the intestine elongates-the sacculi, and the ridges in the interior of the gut, disappearing at the same time.

The circular fibres are spread over the whole surface, but are most marked in the folds projecting into the intestine. In the rectum (to be afterwards seen) they form the band of the internal sphincter muscle.

The fibrous coat resembles that of the small intestine. It will be exposed by removing the peritoneal and muscular coverings.

The mucous coat, which may be examined on opening the intestine, is smooth, and of a pale yellow color; and it is not thrown into special folds, except in the rectum. The surface is free from villi ; and by this circumstance the mucous membrane of the large, can be distinguished from that of the small intestine. This difference in the two portions of the alimentary tube is well marked on the ilio-cæcal valve; for the surface looking to the small intestine is studded with villi, whilst the lower surface, covered by the lining membrane of the cæcum, is free from those small eminences.

Microscopic appcarances. In a piece of fresh intestine the microscope will show the mucous membrane to possess small tubes or erypts, and some larger solitary follicles; with an epithelial covering on the free surface, and a thin muscular layer (muscularis mucosa) on the other, whose arrangement is similar to that of the small intestine.

The tubules (fig. 156, ${ }^{\mathrm{B}}$ ) occupy the whole length of the large gut, and resemble those of the small intestine, but are more numerous and closer together. Their orifices on the surface are circular $\left({ }^{1}\right)$, and are more uni- 
A

Fig. 156.
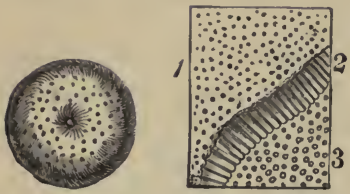

ENLARGBD VIBW OF "A SOLITARY GLAND," and of the tubules of the mucous coat. (Boehm.)

A. Gland of the large intestine.

B. Tubules of the mucous membrane.

1. Surface opening.

2. Side view of the tubes.

3. Pits for the closed ends of the tubes in the submucous tissue. formly diffused than the apertures of the tubules in the small gut. A vertical section of the membrane $\left({ }^{2}\right)$ will show the tubes to extend vertically from the surface into the submucous coat, and to be longer than the crypts of Lieberkühn in the jejunum and ileum ; they measure from $\frac{1}{5}$ th $_{1}$ to $\frac{1}{40}$ th of an inch in length.

The so-called solitury glands (fig. 156, ${ }^{\wedge}$ ) are scattered here and there through the large intestine; but they are in greatest number in the exeum and vermiform appendix. They are whitish rounded bodies from $\frac{1}{20}$ th to $\frac{1}{10}$ th of an inch in diameter, and are situate in the submucous layer amongst the tubules. 'They are lymph follicles with a structure like that in the small intestine.

The epithelium is of the columnar kind, and enters the tubules.

Vessels. The distribution of the vessels in the wall of the large intestine is the same as in the smaller bowel.

Nerves. In the coats of the large intestine the nerves have the plexiform arrangement like that in the small gut.

The absorbent vessels form two sets as in the small intestine; after leaving the gut they join the lymphatic glands along the side of the colon.

\section{THE PANCREAS.}

The pancreas (fig. 145, e) is a narrow flattened gland, from six to eight inches in length, which has some resemblance to a dog's tongue. It is larger at the right than the left end; and it is divided into head, tail, and body.

The head, or the right extremity, occupies the concavity of the duo. denum; and the left extremity, or the tail, is rounded, and touches the spleen.

The body of the gland is narrowest a little to the right of the vertebral column, and is thickest at the upper border; it measures about one inch and $\mathbf{a}$ half in breadth, and from half an inch to an inch in thickness. 'The connections of the pancreas with surrounding parts are described at p. 446 .

Dissection. Let the pancreas be placed on the anterior surface, and let the excretory duct be traced from the head to the tail by cutting away the substance of the gland. 'The small duct will be recognized by its whiteness.

Structure. The pancreas is a gland consisting of separate lobules, and is provided with a special duct. It is destitute of a distinct capsule; but it is surrounded by areolar tissue, which projects into the interior, and connects togrether its smaller pieces. The fluid secreted by it assists in the digestion of the aliment.

The lobules are soft and loose, and of a grayish-white color, and are united into larger masses by areolar tissue, vessels, and ducts. Each consists ultimately, as in the parotid, of the branchings of the excretory duct, which end in closed vesicular extremities, and are surrounded by a plexus of vessels. In the vesicles the epithelium is spheroidal. 
The duct of the pancreas (fig. 150,d) (canal of Wirsung) extends the entire length of the gland, and is somewhat nearer the lower than the upper border. It begins in the tail of the pancreas, where it presents a bifurcated extremity; and as it continues onwards to the head, it receives many branches. It finally ends by opening into the duodenum, either in union with, or separate from the common bile duct (p. 457). Of the tributary branches, the largest is derived from the head of the pancreas.

The duct measures from $\frac{1}{15}$ th to $\frac{1}{10}$ th of an inch in diameter near the duodenum. It is formed of a fibrous and a mucous coat: the latter is lined by a cylindrical epithelium, and is provided with small glands in the duct and its largest branches.

Vessels, lymphatics, and nerves. The arteries and veins have been described (p. 447); and the lymphatics join the lumbar glands. The nerves are furnished by the solar plexus.

\section{THE SPLEEN.}

The spleen is a vascular spongy organ of a bluish or purple color, sometimes approaching to gray. Its texture is friable, and easily broken under pressure. The use of the spleen is unknown.

The viscus is somewhat elliptical in shape, and is placed vertically against the great end of the stomach. Its size varies much. In the adult it measures commonly about five inches in length, three or four inches in breadth, and one inch to one inch and a half in thickness. Its weight lies between four and ten ounces, and is rather less in the female than the male.

At the outer aspect it is convex towards the ribs, the inner surface is marked by a longitudinal ridge, nearer the posterior than the anterior border, into which the vessels plunge to ramify in the interior. Before and behind the ridge, the surface is flattened or somewhat hollowed. The spot where the vessels enter is named the hilum of the spleen.

The anterior border is thinner than the posterior, and is often notched. Of the two extremities, the lower is more pointed than the upper.

Small masses or accessory spleens (splenculi), varying in size from a bean to a moderate-sized plum, are found occasionally, near the fissure of the spleen, in the gastro-splenic omentum, or in the great omentum.

Structure. Enveloping the spleen are two coverings, a serous and a fibrous. It is formed by a network of fibrous or trabecular tissue, which contains in its meshes the splenic pulp, with the Malpighian corpuscles. Throughout the mass the bloodvessels and the nerves ramify. No duct exists in connection with this organ.

The serous or peritoneal coat incases the spleen, and covers the surface except at the hilum and the posterior border. It is closely connected to the subjacent fibrous coat.

The fibrous coat (tunica propria) gives strength to the spleen, and forms a complete case for it. At the fissure on the inner surface this investment passes into the interior with the ressels, to which it furnishes sheaths: and if an attempt is made to detach this coat, numerous fibrous processes will be seen to be connected with its inner surface. Its color is whitish; and its structure is made up of areolar and elastic tissues.

Dissection. The spongy or trabecular structure will best appear, by washing and squeezing a piece of fresh bullock's spleen under water, so as to remove the grumous-looking material. 
The trabecular tissue (fig. 157) forms a network through the whole interior of the spleen, similar to that of a sponge, which is joined to the external easing, and forms sheaths around the vessels. Its processes or threads are white, flattened or cylindrical, and average from ${ }_{Y}^{\frac{1}{0}} 0^{\text {th }}$ to $\frac{1}{30}$ th of an inch : they consist of fibrous and elastic tissues, with a few muscular fibres. The interstices communicate freely together, and contain the proper substance of the spleen, and the vessels.

Microscopic appearances. The characters of the spleen substance cannot be ascertained without the aid of the microscope.

The splenic pulp is a soft red-brown mass, which is lodged in the areola of the trabecular structure. Under the microscope this material is seen

Fig. 157.

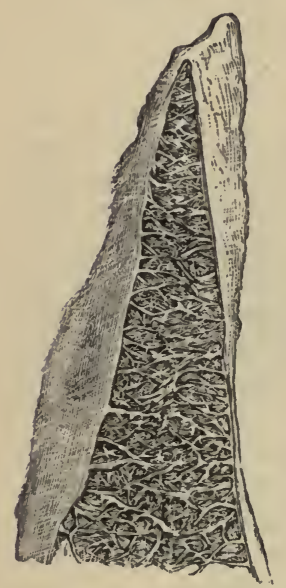

A DRAWING OF THB TRABECILAR STRUCTURB OF THE SPLEEx OP THE OX, at some distance from the hilum.

to be composed of a fine network of ramifying connective tissue corpuscles, with blood-cells in its meshes.

The Malpighian corpuscles are small rounded whitish bodies, about $\frac{1}{6} 0$ th of an inch in diameter, and are connected with the outer coat of the smallest branches of the arteries; they project into the pulp of the spleen, and are surrounded by it. In structure they are like the lymph follicles of the intestine, consisting of reticular tissue, with lymph corpuscles in its meshes, through which blood-capillaries pass.

Bloodvessels. The larger branches of the splenic artery are surrounded by sheaths of fibrous tissue in the trabecula; but the smallest branches leave the sheathing, and break up into tufts of capillaries, which open into the fine meshes of the spleen substance. In the smallest branches, with which the Malpighian corpuscles are united, the outer coat is thickened by lymphoid tissue, and is directly continuous with those bodies in structure.

The splenic vein begins in the meshes of the splenic pulp by open channels. From the union of these radicles arise small branches, which unite into trunks larger than the accompanying arteries, and issue by the fissure of the spleen ; in their course they receive accessory branches, some joining at a right angle.

Nerves and lymphatics. 'The lymphatics are superficial and deep, and enter the glands in the gastro-splenic omentum. In the spleen they begin in the corpuscles of Malpighi, and in the outer coat of the smallest arteries; they are conveyed to the hilum of the spleen on the vessels. The nerves come from the solar plexus, and surround the artery and its branches.

\section{THE LIVER.}

The liver secretes the bile, and is the largest gland in the body. Its duct opens into the duodenum with that of the pancreas.

Dissection (fig. 158). Preparatory to examining the liver, the vessels at the under surface should be dissected out. 'This proceeding will be facilitated by distending the vena cava and vena porta with tow or cotton wool, and the gall-bladder with air through its duct. The several vessels and the ducts are then to be defined, and the gall-bladder to be cleaned. 
On following outwards the left branch of the vena porta to the longitudinal or antero-posterior fissure, it will be found united anteriorly with the round ligament or the remains of the umbilical vein, and posteriorly with the fine fibrous remnant of the ductus venosus.

The liver is of a red-brown color and firm consistence; and weighs commonly in the adult from three to four pounds (fifty to sixty ounces). Transversely the gland measures from ten to twelve inches; from front to back between six and seven inches; and in thickness, at the right end, about three inches; but this last measurement varies with the spot examined.

In shape the liver is somewhat square. It has many named parts, viz., two surfaces, two borders, and two extremities; and the under surface is further marked by lobes and fossa, and by fissures which contain vessels.

The connections and the ligaments of the liver are described at p. 434 and p. 438.

Surfaces. On the upper aspect the liver is convex: extending from front to back in the suspensory ligament, which divides the upper surface into two unequal parts, of which the right is the larger. The under surface is rendered irregular by lobes, fissures, and fosse : in contact with it is the gall-bladder; and a longitudinal sulcus divides it into a right and a left lobe.

Borders. The anterior border is thin, and is marked by two notches: one is opposite the longitudinal sulcus on the under surface before alluded to, and the other is over the large end of the gall-bladder. The posterior border is much thicker at the right than at the left end; and at the thickened part it touches the right kidney and the diaphragm. Opposite the vertebral column is a hollow in this border; and the vena cava is partly imbedded in it on the right of the spine.

Extremities. The right extremity is thick and rounded; and the left is thin and flattened.

Lobes. On the under surface the liver is divided primarily into two lobes, a right and a left, by the antero-posterior or longitudinal fissure; and occupying this surface of the right lobe are three others, viz., the square, the Spigelian, and the caudate lobe:-

The left lobe. $\mathrm{B}$, is smaller and thinner than the right, and there is a slight depression inferiorly where it touches the stomach.

The right lobe, A, forms the greater part of the liver, and is separated from the left by the longitudinal fissure on the one aspect, and by the suspensory ligament on the other. To it the gall-bladder is attached below ; and the following lobes are projections on its under surface :-

The square lobe, $\mathrm{c}$ (lobulus quadratus), is situate between the gallbladder and the longitudinal fissure. It reaches anteriorly to the margin of the liver, and posteriorly to the fissure (transverse) by which the vessels enter the interior of the viscus.

The Spigelian lobe, $\mathrm{D}$, lies behind the transverse fissure, and forms a roundish projection on the surface. On its left side is the longitudinal fissure; and on its right, the vena cava inferior.

The caudate lobe, $\mathrm{E}$, is a slight, elongated eminence, which is directed from the Spigelian lobe behind the transverse fissure, so as to form the posterior boundary of that suleus. Where the fissure terminates this projection subsides in the right lobe.

Fissures. Extending horizontally half across the right part of the liver between the Spigelian and caudate lobes on the one hand, and the square 
lobe on the other, is the transverse or portal fissure. It is situate nearer the posterior than the anterior border, and contains the vessels, nerves, ducts, and lymphaties of the liver. At the left end it is united at a right angle with the longitudinal fissure.

Fig. 158.

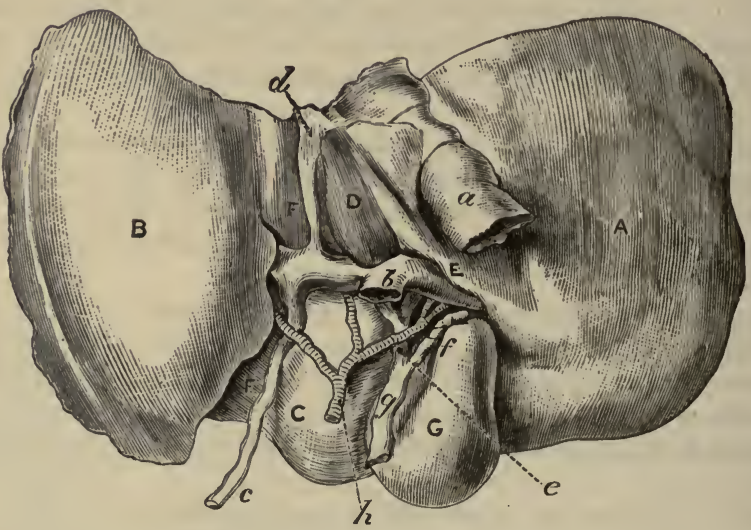

UNDER SURFACE OF THE LIVER.

A. Right, and B, left lobe.

C. Quadrate lobe.

D. Spigelian, and E, caudate lobe.

F. Longitudinal fissure.

G. Gall-bladder.

a. Vena cava.

b. Vena portæ. c. Round ligament.

d. Obliterated ductus venosus.

e. Common hepatic duct.

f. Cystic duct.

g. Common bile duct.

$h$. Hepatic artery.

The longitudinal fissure, F, extends from the front to the back of the liver, between the right and left lobes. In the part anterior to the transverse fissure lies the remnant of the umbilical vein $(c)$, which is called round ligament, and is oftentimes arched over by a piece of the hepatic substance (pons hepatis). In the part behind that fissure is contained a small obliterated cord $(d)$, the remains of the vessel named ductus venosus in the foetus.

The groove for the vena cava is placed on the right side of the Spigelian lobe, and is frequently bridged over by the liver: If the cava be opened, two large and some smaller hepatic veins will be observed entering it.

Fossa. On the under surface of the right lobe are three depressionsone for the gall-bladder to the right of the square lobe; another for the colon, near the anterior edge; and a third for the kidney near the posterior border.

Vessels of the transverse fissure. The vessels in the transverse fissure, viz., vena portæ, hepatic artery and duct, have the following position: the duct is anterior, the portal vein posterior, and the artery between the other two.

The hepatic duct (fig. $158, e$ ) is formed by two branches from the liver, one from each lobe, which soon blend in a common tube. After a distance of one inch and a half it is joined by the duct of the gall-bladder $(f)$; and the union of the two gives rise to the common bile duct $(g)$. 
The hepatic artery $(h)$ is divided into two, one for each lobe, and its branches are surrounded by nerves and lymphatics.

The vena porta $(b)$ branches, like the artery, into two trunks for the right and left lobes, and gives an offset to the Spigelian lobe; its left branch is the longest.

Fotal condition of the umbilical vein. Before birth the previous umbilical vein occupies the longitudinal fissure, and opens posteriorly into the vena cava; the portion of the vessel behind the transverse fissure receives the name ductus venosus. Branches are supplied from it to both lobes of the liver; and a large one, directed to the right lobe, is continuous with the left piece of the vena portæ. Purified or placental blood circulates through the vessel at that period.

Adult state. After birth the part of the umbilical vein in front of the transverse fissure is closed, and becomes eventually the round ligament (fig. 158, $)$. The ductus venosus is also obliterated, only a thin cord $(d)$ remaining in its place. Whilst the lateral branches, which are in the same line as, and continuous with the left branch of the vena portæ, remain open, and subsequently form part of the left division of the vena portæ. Occasionally the ductus venosus is found more or less pervious.

Structure of the Liver. The substance of the liver consists of small bodies called lobules or acini; together with vessels which are concerned both in the production of the secretion, and in the nutrition of the organ. The whole is surrounded by a fibrous and a serous coat.

Serous coat. The peritoneum invests the liver almost completely, and adheres closely to the subjacent coat. At certain spots intervals exist between the two, viz., in the fissures occupied by vessels, along the line of attachment of the ligaments, and at the surface touching the gall-bladder.

The fibrous covering is very thin, but it is rather stronger where the peritoneum is not in contact with it. It invests the liver, and is continuous at the transverse fissure with the fibrous sheath (capsule of Glisson) surrounding the vessels in the interior. When the membrane is torn from the surface, it will be found connected with fine shreds entering into the liver.

Size and form of the lobules. 'The lobules (fig. 161, l) constitute the proper secreting substance, and can be seen either on the exterior of the liver, on a cut surface, or by means of a rent in the mass. As thus observed, these bodies are about the size of a pin's head, and measure from $\frac{1}{2} 0^{t}$ th to $\frac{1}{10}$ th of an inch in diameter. Closely massed together they possess a dark central point; and there are indications of lines of separation between them, though they communicate by vessels. By means of transverse and vertical sections of the lobules, their form will appear flattened on the exterior, but many sided in the interior of the liver. They are clustered around the smallest divisions of the hepatic vein, to which each is connected by a small twig issuing from the centre, something like the union of the stalk with the body of a small fruit.

'To study the minute structure of the lobules, a microscope will be necessary; and the different vessels of the liver should be minutely injected.

Constituents of the lobules. Each lobule is composed of minute hepatic cells, which are arranged web-like amongst the ducts and vessels; and it is provided with a capillary network of vessels, and with a plexus of the bile duct.

Cells of the lobules. The hepatic or biliary cells (fig. 159, a) form the chief part of the lobule; they are irregular in form, being rounded or 
many sided, and possess a bright nucleus, or even more than one. In size they vary from $\frac{1}{100} 0^{\text {th }}$ to $\frac{1}{80} \pi^{\text {th }}$ of an inch. They are of a yellowish color, and inclose granular particles, together with fat and yellow coloring

Fig. 159.

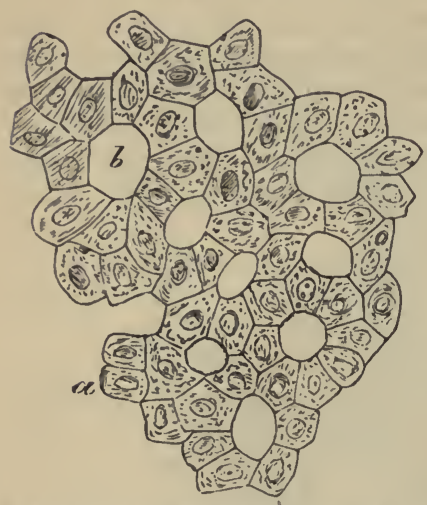

A Magnified RePResentation of the HePATIC Cells, with their arrangement in the lobule (Henle).

a. Cells.

$b$. Intercellular spaces. matfer. These nucleated cells arhere together by their surfaces so as to form rows radiating from the centre, with spaces $(b)$ between them for the bloodvessels and ducts. The cells are concerned in the secretion of the bile.

Vessels of the lobule (fig. 160, в). The smallest branches of the vena porta, after uniting in a circle around the lobule, where they are named interlobular $(a)$, enter its substance, and form therein a network of capillaries $(c)$ near the circumference.

A small branch of the hepatic vein $(d)$ occupies the centre of the lobule; its radicles communicate with the portal network, and it issues from the base of the lobule as the intralobular vein.

The radicles of the bile duct (fig. 160 , A) begin within the lobule in a fine intralobular plexus of ducts $(g)$ between

Fig. 160.

A.

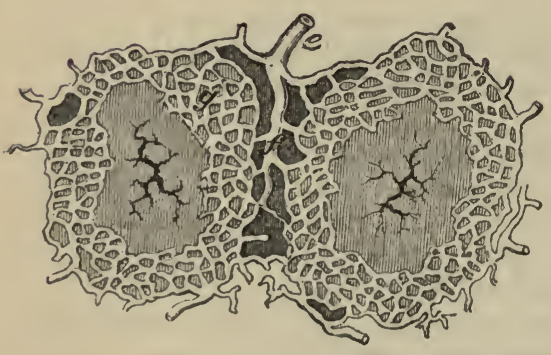

B.

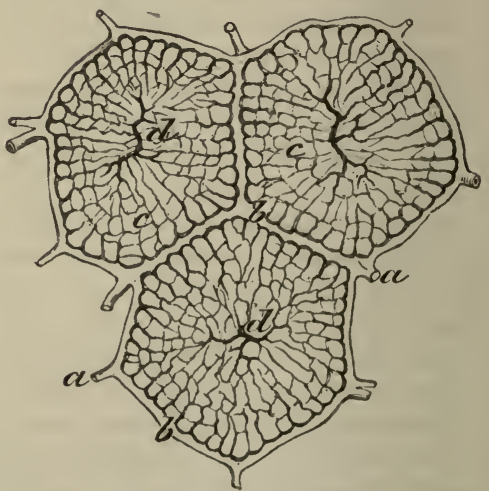

A. Two lobnles of the liver showing the plexus of ducts in the interior, near the circum. ference (Kiernan); recent Inquiries demonstrate the existence of a plexus throughout the lobule.

g. Intralobular plexus.

$f$. Interlobular ducts.

e. Sinall branches of the hepatic ducts.
B. Lobules of an injected liver to show the urrangement of the veins.

a. Smallest branches of the vena portx ending in the interlobular veins, $b$.

c. Plexus of portal veins within the lobule.

d. Intralobular commencement of the hepatic vein joining the plexus of the portal veín.

the hepatic cells (Chrzonszczewsky); they leave the lobule at the circumference, and are joined togrether outside it in the smallest interlobular branches $(f)$. 
From the arrangement of the vessels, it appears that the portal vein conducts the blood from which bile is secreted; that the hepatic vein carries away the superfluous blood; and that the secreted bile is received by the plexus of the biliary duct.

Vessels of the Liver. Two sets of bloodvessels ramify in the liver: One enters the transverse fissure, and is directed transversely in spaces (portal canals) where it is enveloped by areolar tissue. The other set (hepatic veins) run from the anterior to the posterior border of the liver without a like sheath. The ramifications of these different vessels are to be followed in the liver.

The capsule of Glisson is a layer of areolar tissue, which envelops the vessels and the ducts in the transverse fissure. In this sheath the vessels ramify, and in it they are minutely divided before their termination in the lobules. If a transverse section is made of a portal canal, the ressels will retract somewhat into the loose surrounding tissue.

The vena porta ramifies in the liver like an artery; and the blood circulates through it in the same manner, viz., from trunk to branches. After entering the transverse fissure the vein divides into large branches; these lie in the portal canals or spaces, with offsets of the hepatic artery, the hepatic duct, and the nerves and lymphatics (fig. 161, P). The division is repeated again and again until the last branches of the vein (interlobular, fig. $160, b)$ penetrate between the lobules; there they unite, and end in the interior as before explained.

In the portal canals the offsets of the e'ena porta. are joined by small vaginal and surface veins, which convey blood from branches of the hepatic artery.

The hepatic artery (fig. 161, c), whilst surrounded by the capsule, furnishes vaginal branches, which ramify in the sheath, giving it a red appearance in a well-injected liver, and supply twigs to the coats of the vena portæ and biliary ducts, and to the areolar tissue: from the vaginal branches a few offsets (capsular) are given to the coat of the liver. Finally the artery ends in fine interlobular branches, from which offsets enter the lobule, and convey blood into the network between the branches of the vena porte and hepatic vein (Chrzonszczewsky).

Fig. 161.

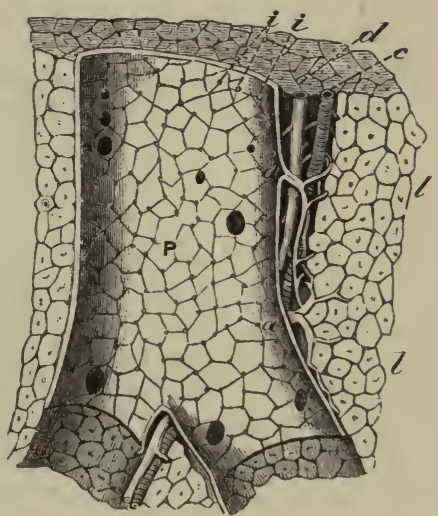

Vesgetis in a PORTal Canat, and ThB LOBULRS OF THE LIVER (Kiernan).

l. Lobules of the liver.

P. Branch of the vena portæ, with, $a, a$, raginal branches which supply interlobular offsets.

c. Hepatic artery.

d. Hepatic duct.

$i, i$. Openings of the interlobular brauches of the portal vein.

The hepatic vein (venæ cavæ hepaticx) begins by a plexus in the interior of each lobule (fig. 160, d), and its smallest radicle issues from the base of the lobule as the intralobular vein; these are received into the sublobular branches, which anastomose together, and unite into larger vessels. Finally, uniting with neighboring branches to produce larger trunks, the hepatic veins are directed from hefore backwards to the vena cava inferior, into which they open by large orifices. 'The venæ cavæ he- 
paticx may be said to be without a sheath, except in the larger trunks; so that when they are cut across the ends remain patent, in consequence of their close connection with the liver structure.

Hepatic duct (fig. 160,e). The duct commences in the biliary plexus within the lobules. On leaving the lobules the radicles communicate by the interlobular branches $\left(f^{*}\right)$; and the smaller ducts soon unite into larger vaginal branches $(d)$, which lie in the portal canals with the other vessels. Lastly, the ducts are collected into a right and a left trunk (fig. 158,e), and leave the liver at the transverse fissure. ${ }^{1}$

Structure. The moderately-sized hepatic ducts consist of a fibrous coat, lined by a mucous layer with eylindrical epithelium; and penetrating the wall is a longitudinal row of openings, on each side, leading into sacs, and into branched tubes which sometimes communicate. In the fine interlobular ducts the coat is formed by a homogeneous structure, with columnar epithelium (Henle).

Lymphatics of the liver are superficial and deep. The superficial of the upper surface join the lymphatics in the thorax by piercing the diaphragm, and end for the most part in the sternal glands; those on the under surface enter chiefly the glands by the side of the abdominal aorta, a few uniting with the deep lymphatics, and the coronary of the stomach.

The deep lymphatics accompany the vessels through the liver, and communicate with one of the large contributing trunks of the thoracic duct.

Nerves come from the sympathetic and the pneumo-gastric, and ramify with the vessels; but their mode of ending is not ascertained.

\section{THE GALL-BLADDER.}

The gall-bladder (fig. 162) is the receptacle of the bile. It is situate in a depression on the under surface of the right lobe of the liver, and to the right of the square lobe. It is pear-shaped, and its larger end (fundus) is directed forwards beyond the margin of the liver; whilst the smaller end (neck) is turned in the opposite direction, and bends downwards to termidate in the cystic duct by a zigzag part.

In length the gall-bladder measures three or four inches, and in breadth rather more than an inch at the widest part. It holds rather more than an ounce.

By one surface it is in contact with the liver, and on the opposite it is covered by peritoneum. The larger end touches the abdominal wall opposite the tip of the cartilage of the tenth rib, where it is contiguous to the transverse colon; and the small end is in contact with the duodenum.

Structure. The gall-bladder possesses a peritoneal, a fibrous and muscular, and a mucous coat.

The serous cout is stretehed over the under or free surface of the gallbladder, and surrounds the large end.

The fibrous coat is strong, and torms the framework of the sac; intermixed with it are some involuntary muscular fibres, the chief being longitudinal, but others circular.

1 Aberrant ducts exist between the pieces of the peritoneum in the left lateral ligament of the liver, and in the pons bridging over the vena porte and vena cava; they anastomose together, and are accompanied by branches of the vessels of the liver, viz., vena portæ, hepatic artery, and hepatic vein. 
The mucous coat is marked internally by numerous ridges and intervening depressions, which give an areolar or honeycomb appearance to the surface. On laying open the gall-bladder this condition will be seen, with the aid of a lens, to be most developed about the centre of the sac, and to diminish towards each extremity. In the bottom of the larger pits are depressions leading to recesses. The surface of the mucous membrane is covered by a columnar epithelium.

Where the gall-bladder ends in the cystic duct (fig. 162 ) its coats project into the interior, and give rise to ridges resembling those in the sacculated large intestine.

The cystic duct (b) joins the hepatic duct at an acute angle, to form the ductus communis choledochus. It is about an inch and a half long, and is distended and somewhat sacculated near the gall-bladder.

Structure. The coats of the duct are formed like those of the sac from which it leads, but the muscular fibres are very few. The mucous lining is provided with glands, as in the hepatic and common bile ducts (p. 472).

On opening the duct the mucous membrane may be observed to form about twelve semilunar projections (fig. 162,c), which are arranged obliquely around the tube, and increase in size towards the gall-bladder. This structure is best seen on a gall-bladder which has been inflated and dried: as in this state the parts of the duct between the ridges are most stretched.

Bloodiessels and nerves. The vessels of the gallbladider are named cystic. The artery is a branch of the hepatic; and the cystic vein opens into the vena portæ near the liver. The nerves are derived from the hepatic plexus, and entwine around the vessels. The lymphatics follow the cystic duct, and join the deep lymphatics on the spinal column.

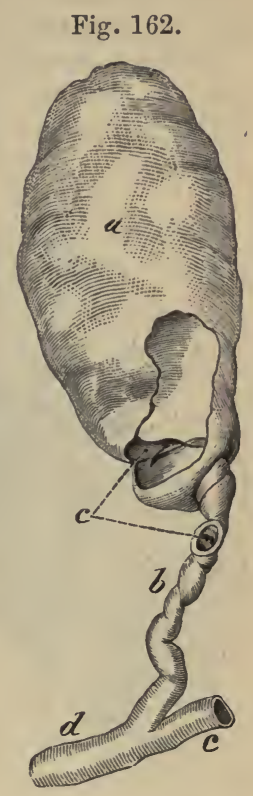

GALL-BLADDER AND IT DUCT

a. Gall-bladder.

b. Bile duct sacculated. c. Ridges in the interior. d. Common bile duct.

e. Common hepatic duct.

\section{THE KIDNEY AND THE URETER.}

The kidney has a characteristic form: flattened on the sides, it is larger at the upper than the lower extremity, and is hollowed out at one part of its circumference. For the purpose of distinguishing between the right and left kidneys, let the excavated margin be turned to the spinal column, with the ureter or the excretory tube behind the other vessels ; and let that end be directed downwards, towards which the ureter is naturally inclined.

With the special form above mentioned, the kidney is of a deep red color, with an even surfice. Its average length is about four inches; its breadth two; and its thickness about one inch; but the left is commonly longer and more slender than the right kidney. Its usual weight is about five ounces and a half in the male, and rather less in the female.

The upper extremity of the kidney is rounded, is thicker than the lower, and is surmounted by the suprarenal body. The lower end is flat, and 
more pointed. The position with respect to the spinal column has been before detailed ( $\mu .435$ ).

On the anterior surface the viscus is rounded, but on the opposite surface it is generally flattened.

The outer border is convex; but the inner is excavated, and is marked by a longitudinal fissure, hilum. In the fissure the vessels are thus placed with respect to one another: The divisions of the renal vein are in front,

Fig. 163.

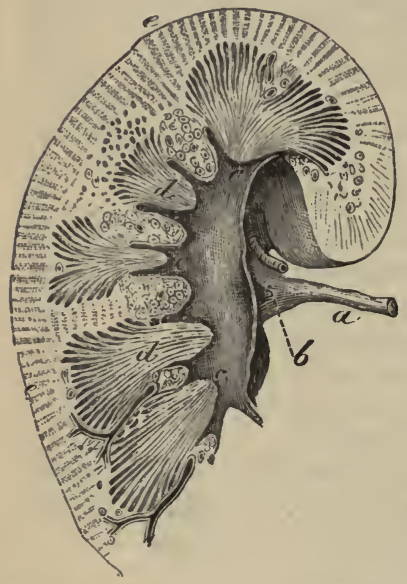

SECton thROLGH THE KIDNEY, SHOWING THB MEDULLART AND COKTICAL Portions, and The Beginning of THi URETER (Henle).

a. Ureter.

b. Pelvis of the nreter.

c. Calyx of the excretory tube.

d. Pyramidal portions.

e. Curtical portion of the kidney. the ureter is behind, and the branches of the artery lie between the two. On the vessels the nerves and lymphatics ramify; and areolar tissue and fat surround the whole. Opposite the fissure is a hollow in the interior of the kidney, named siuus, in which the vessels and the duct are contained before they pierce the renal substance.

Dissection. To see the interior it will be necessary to cut through the kidney from the inner to the outer border; and to remove the loose tissue from the vessels, and from the divisions of the excretory duct. The hollow or sinus containing the bloodvessels now comes completely into view.

The interior of the kidney fig. 163) appears on a section to consist of two different materials, viz., of an external granular or cortical part; and of internal, darker colored, pyramidal masses, which converge towards the centre. But these unlikelooking parts are constructed of urine tubes, though with a different arrangement.

The pyramidal masses $(d)$ (pyramids of Malpighi), are twelve or eighteen in number, and converge to the sinus of the kitlney. The apex of each mass, which is free from cortical covering, is directed to the sinus, and ends in a smooth, rounded part, named mamilla or papilla $(c)$. In it are the openings of the urine tubes, which are about twenty in number, some being situate in a central depression and the others on the surface; and it is surrounded by one of the divisions (calyx) of the excretory tube. Occasionally two of the masses are united in one papillary termination. The base is embedied in the cortical substance, and is resolved into bundles of tubes which are prolonged into the cortical covering.

Each pyramidal mass is constructed of uriniferal tubes (tubes of Bellini) which open below at the apex of the papilla (fig. $164, a$ ) ; and the cut surface lias a grooved appearance indicatory of its construction. If the mass is compressed, urine will exurle from the tubes through the apertures in the apex.

The cortical or investing part (fig. $163, e$ ) forms about three-fourths of the kidney; it covers the pyramidal masses with a layer about two lines in thickness, and sends prolongations between the same nearly to their appices. Its color is of a light red, unless the kidney is blanched; and its consistence is so slight that the mass gives way beneath the finger. In 
the injected kidney red points (Malpighian bodies) are scattered amongst the cortex, giving it a granular appearance.

Structure of tIIE KidNey. The mass of the kidney consists of minute convoluted tubes, intermixed with bloodvessels, lymphatics, nerves, and an intertubular matrix. The whole is incased by a fibrous coat.

The fibrous coat is a white layer, which is connected with the kidney by fine processes and vessels, but is readily detached from it by slight force. At the inner margin of the kidney it sinks into the sinus, and sends processes on the entering vessels and excretory duct.

Stroma or matrix. Between the tubules and the vessels of the kidney is a uniting materal, which surrounds and isolates them, and is most abundant in the cortical substance. It somewhat resembles areolar tissue in its nature, and is fibrous at some spots.

To obtain a knowledge of the anatomy of the secreting tubes, and of the bloodvessels, the dissector will require a microscope, and good fine injections of the kidney.

Secretory tubules. The uriniferous tubes (tubuli uriniferi) occupy successively the cortical substance, and the Malpighian pyramids; but they have a different arrangement in each part as below (fig. 164,a).

In the Malpighian pyramid tubes are straight, and ascend from the apertures in the apex, bifurcating repeatedly, as far as the base, and forming a cone which resembles the stem and branches of a tree. At the wide end of the pyramid they are collected into bundles which reach nearly to the surface of the kidney, and become convoluted as they enter the cortex, but some unite in arches (Henle). Near the apex they measure $\frac{1}{30} \sigma^{\text {th }}$ of an inch across, but the last subdivisions are only half that size, or $\frac{1}{600}$ th of an inch.

Descending between the straight tubes are the small "looped tubes" of Henle (fig. $364, g$ ). These run down from the cortical substance nearly to the apex of the pyramid, where they turn upwards, forming loops with the convexity down, and ascend to open into the straight tubes : their size is about a third of the others.

In the cortical substance the tubes are more numerous and very convoluted (fig. 165); they have an average width of $\frac{1}{60}$ th $^{\text {th }}$ of anch, and are surrounded by a capillary plexus of bloodvessels (fig. 166, A). At the one end (farther) each tube is dilated into the Malpighian corpuscle $(b)$; and at the other it passes into a straight tube or joins an "arch" at the base of the pyramid. The "looped tubes" of Henle (fig. 164) have the same arrangement as the larger tubuli uriniferi in the cortical substance.

The wall of the convoluted tubes consists of a thin basement membrane, and is lined by a thick, nucleated, and granular epithelium.

Malpighian corpuscles (fig. 166, A). These small bodies are connected with the free ends of the convoluted tubes, one to each : and are arranged in double rows in the cortical substance between the pyramids, one row being on each side of an interpyramidal branch of artery, from which they receive trigigs; each measures about $\frac{1}{12} 0^{t h}$ of an inch, and consists of an incasing capsule with an inclosed tuft of bloodvessels (glomerulus).

The capsule $(b)$ is the dilated end of the convoluted tube, and is perforated at the extremity by two small bloodvessels. Its wall consists of a thin basement membrane, and is lined by a transparent laminar epithelium.

The glomerulus (fig. 166, в) is formed by the intercommunications of two vessels piercing the capsule, and is clothed with epithelium. One 
of the two, the afferent vessel $(d)$, is an off'set of the renal artery, and divides into convoluted loops of the fineness of capillaries, which form the exterior of the tuft. The other, the efferent vessel $(f)$, begins in the interior of the tuft by the union of the loops on the outer surface; and

Fig. 164 .

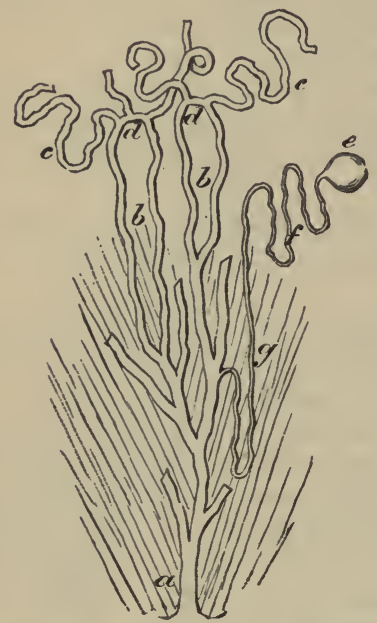

Plan of the Arrangement of the UriniFERAL TUBES.

a. Tube at its ending in the pyraunid.

b. Divisions of the same tube in the pyramid.

d. Arches of Henle.

c. Twisted tubes joining the "arches."

g. Looped tube of Henle, with its convoluted part, $f$, and Malpighian corpuscle, $e$, in the cortical substance.
Fig 165.

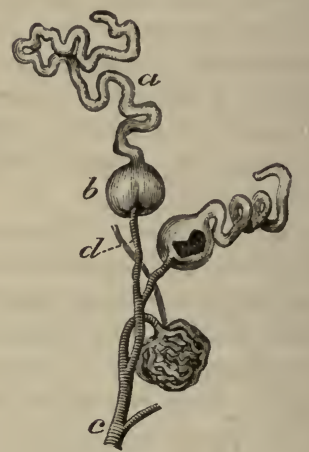

Plan of Contorted Urine Tubes ending in Malpiahian CoRPUACLes, which are connected with the small arteries $(d)$.

a. Urine tube.

b. Malpighian corpuscles.

c. Interpyramidal branch of renal artery (Bowman).

passing out is distributed in a close network of capillaries $(g)$ on the convoluted urine tubes; and in one with elongated meshes on the straighter tubes near the pyramids. The office of the glomerulus is to secrete the watery part of the urine.

Bloonvessecs. The artery and vein distributed to the kidney are very large in proportion to the size of the organ they nourish.

Renal artery. As the artery enters the kidney it divides into four or five branches, which are invested by sheaths of the fibrous capsule, and reach the bases of the pyramids of Malpighi, where they form arches. From those arches arise small interpyramidal branches, which ascend towards the surface, and furnish the afferent twigs to the Malpighian bodies, whose arrangement has been referred to. Other offsets are supplied to the capsule and matrix of the kidney; the former anastomose with the subperitoneal branches of the lumbar arteries.

Straight vessels descend amongst the tubes in the pyramids of Malpighi, and some form loops like the tubes of Henle.

Renal vein. This vein begins in the capillary plexuses on the eonvoluted urine tubes; and its small branches, receiving twigs from the matrix 
and the fibrous coat, unite into larger veins, which anastomose freely around the bases of the pyramids of Malpighi. At this spot they are joined by offsets from capillary plexuses in the pyramids; and the larger trunks then accompany the arteries to the sinus of the kidney. Finally all are united into one trunk, which opens into the vena cava.

Fig. 166.

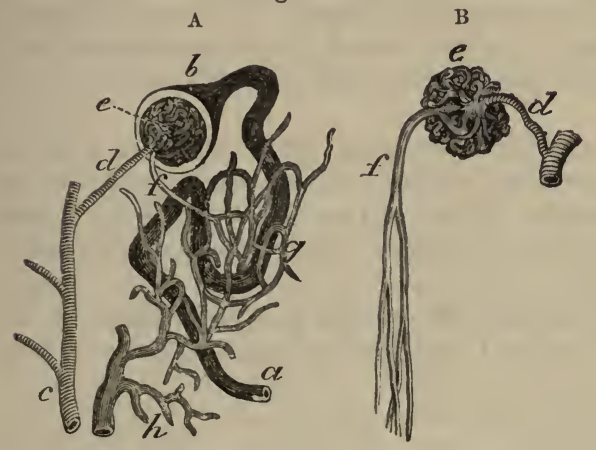

A. a. Urine tube.

b. End-dilatation.

c. Interpyramidal artery.

d. Afferent branch.

e. Glomerulus.

$f$. Efferent vessel.

g. Plexus of capillaries around the urine tube.

$h$. Radicles of the veins.

B. e. Glomerulus.

d. Afferent, and

$f$. Efferent vessel of the glomerulus (Bowman).

Pla of the Vessels connected With the URine Tebes.

Nerves. The ramifications of the sympathetic nerve may be traced to the smaller branches of the artery.

'The absorbents are superficial and deep :-The deep absorbents are supposed to hegin in a plexus between the urine tubes. Both unite at the hilum of the kidney, and join the lumbar glands.

The URETER is the tube by which the fluid secreted in the kidney is conveyed to the bladder. Between its origin and termination the canal neasures from sixteen to eighteen inches in length. Its size corresponds commonly with that of a large quill. Near the kidney it is dilated into a funnel-shaped part, named pelvis; and near the bladder it is again somewhat enlarged, though the lower aperture by which it terminates is the narrowest part of the tube. Its relative anatomy must be studied afterwards, when the body is in a suitable position.

In its course from the one viscus to the other, the ureter is close beneath the peritoneum, and is directed obliquely downwards and inwards along the posterior wall of the abdomen as far as the pelvis; here it changes its direction, and becomes almost horizontal. At first the ureter is placed over the psoas, inclining on the right side towards the inferior vena cava; and about the middle of the muscle it is crossed by the spermatic vessels. Lower down it lies over the commun or the external iliac artery, being beneath the sigmoid flexure on the left side, and the end of the ileum on the right side. Lastly, it lies below the level of the obliterated hypogastric artery.

Sometimes the ureter is divided into two for a certain distance.

Part in the kidney (fig. 163, $b$ ). Near the kidney the ureter is dilated into a pouch called pelvis; and it begins by a set of cup-shaped tubes, named calices or infundibula, which vary in number from seren to thirteen. Each cup-shaped process embraces the rounded end of a pyramidal mass, and receives the urine from the apertures in that projection; sometimes a calyx surrounds two or more masses. The several calices are 
united together to form two or three larger tubes; and these are finally blended in the ureter.

Structure. Besides an external fibrous layer, the ureter possesses a muscular and a mucous coat.

The muscular covering is composed of an external or longitudinal, and of an internal or circular stratum.

The mucous coat is thrown into longitudinal folds during the contracted state of the ureter. Its epithelium consists of layers of cells of different shapes; thus, at the free surface they are squarish, at the attached sur.. face rounded, whilst in the intermediate strata they are cylindrical (Kölliker).

The calices resemble the rest of the duct in having a fibrons, a muscular, and a mucous coat. Around the base of the pyramid the calyx is continuous with the enveloping tunic of the kidney; and at the apex the mucous lining is prolonged into the uriniferal tubes through the small openings.

Vessels. The urteries are numerous but small, and are furnished by the renal, spermatic, internal iliac, and inferior vesical. The veins correspond with the arteries.

The lymphatics are received into those of the kidney.

\section{THE SUPRARENAL BODY.}

This small body, whose use is unknown, has received its name from its position to the kidney. Its vessels and nerves are numerous, but it is not provided with any excretory duct.

One on each side, it is situate on the upper extremity and fore part of the kidney; and without care it may be removed with the surrounding fat, which it resembles. Its color is a brownish-yellow. It is like a cocked hat in form, with the upper part convex, and the base or lower part hollowed, where it touches the kidney.

In the adult it measures about one inch and a half in depth, and rather less in width; and its weight is between one and two drachms, but the left is commonly larger than the right.

Areolar tissue attaches the suprarenal body to the kidney; and large vessels and nerves retain it in place. The connections with the surrounding parts are the same as those of the upper end of the kidney. Thus it rests on the diaphragm on both sides; whilst above the right is the liver, and above the left the pancreas and the spleen. On the inner side of the right capsule is the vena cava, with part of the solar plexus; and internal to the left is the aorta, with the same plexus of nerves.

Structure (fig. 167). A perpendicular section shows the suprarenal body to be formed of a firm or external (cortical) part, and of an internal (medullary) soft and dark material. With the microscope this body may be seen to consist of cells, which are lodged in spaces formed by a stroma of areolar tissue, with vessels and nerves.

The whole is surrounded by a thin fibrous capsule (a), which sends processes into the interior, and along the bloodvessels.

The cortical part, yellowish in color and striated, forms about twothirds of the thickness of the whole body. Its stroma of areolar tissue (a) forms a thin surface layer, and is connected internally with processes or septa, which are so arranged as to build up spaces elongated from without inwards, and arranged vertically around the centre; but near the surface 
there are smaller oval spaces, some of them crossing the deeper and larger. The spaces or loculi $(b)$ are filled by columnar masses of cells, but slight force readily removes these from their containing hollows.

The central or medullary portion $(c)$ is rather red in color, or it may be dark brown or black from the presence of blood. About half as thick as the cortical part, it possesses internally small round or oval spaces, on a section, which are vein trunks cut across. The areolar tissue of its stroma is very fine, and forms a network with small but regular meshes; and the medullary is separated generally from the cortical portion by a layer of areolar tissue. Cells fill the meshes.

Cells. The cells filling the loculi of the stroma in the cortex are nucleated with oil globules and fine granules; and being packed in masses, they take on a polygonal form: they measure about the $\frac{1}{700}^{\text {th }}$ of an inch. In the medullary portion the cells resemble those of the cortex, except that they do not contain oil particles; and they are rather larger and more granular, measuring about $\frac{1}{100} \sigma^{\text {th }}$ of an inch.

Bloodvessels. Numerous arteries are furnished to the suprarenal body from the diaphragmatic and renal vessels, and from the aorta. In the interior the arteries ramify in the cortex along the septa between the cell masses; and frequently anastomosing together, end in a fine capillary network, with elongated meshes, around the loculi. In the medullary part the fine arteries are distributed through the stroma.

The veins originate in capillary plexuses; and the several radicles, uniting in large branches which pass through the centre of the medullary

Fig. 167.

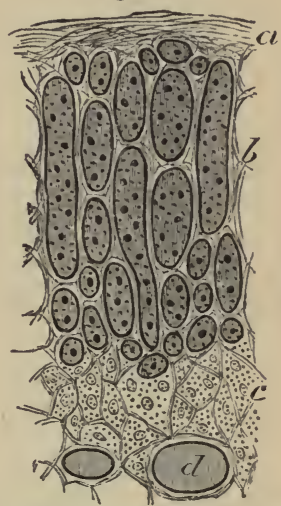

Vertical Section of the SUPRARENAL BODY.

a. Surface layer of stroma.

b. Cells in the cortex.

c. Medullary portion.

$d$. Spaces in the medullary part (Harley). part, are collected finally into one trunk; this opens on the right side into the vena cava, and on the left into the renal vein. Other smaller veins pass out through the cortex to the renal vein and the vena cava.

Nerves. The nerves are very numerous and large, and pass inwards along the septa of the cortical part : branching, they extend between the cortical and medullary parts in the layer of areolar tissue, and in the medullary substance they form a network in the areolar structure, but their ending is unknown.

Lymphatics are superficial and deep, and both join those of the kidney; the arrangement of the deep is undetermined.

\section{THE TESTES.}

The testes are the glandular organs for the secretion of the semen. Each is suspended in the scrotum by the spermatic cord and its coverings (p. 418), but the left is usually lower than the right ; and each is provided with an excretory duct named vas deferens. A serous sac partly surrounds each organ.

Dissection. For the purpose of examining the serous covering of the testicle (tunica vaginalis), make a small aperture into it at the upper part, and inflate it. The sac and the spermatic cord are then to be cleaned; 
and the vessels of the latter are to be followed to their entrance into the testicle.

The tunica vaginalis (fig. $168, d$ ) is a serous bag, which is continuous in the foetus with the peritoneal lining of the abdomen, but becomes subsequently a distinct sac through the obliteration of the part connecting the two.

It invests the testicle after the manner of other serous membranes: for the testicle is placed behind it, so as to be partly enveloped by it. 'The sac,

Fig. 168.

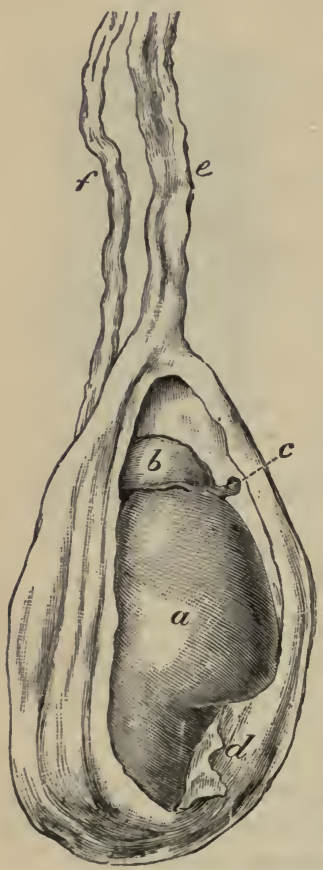

THE TEsTis WITH THE TUNICA VAGINALIS LAID OPEN.

a. Testicle.

b. Head of the epididymis.

c. Corpus Morgagni.

d. Parietal part of the tunica vaginalis.

e. Vessels of the spermatic cord

f. Vas deferens.

Its weight nearly an oune, alde to side rather less than an inch. other.

Structure. The substance of the testicle is composed of minute secerning tubes, around which the bloodvessels are disposed in plexuses. Surrounding and supporting the delicate seminiferal tubes is a dense covering - the tunica albuginea. Its excretory or efferent duct is named. vas deferens,

Dissection. With the view of examining the investing fibrous coat, let the testis be placed on its outer side, viz., that on which the epididymis 
lies, and let it be fixed firmly in that position with pins. The fibrous coat is to be cut through along the anterior part, and thrown backwards as far as the entrance of the bloodvessels. Whilst raising this membrane a number of fine bands will be seen traversing the substance of the testicle, and a short septal piece (mediastinum) may be perceived at the back of the viscus, where the vessels enter ; but it will be expedient to remove part of the mass of tubes in the interior, to bring more fully into view the mediastinum, and to trace back some of the finer septa to it.

'The tunica albuginea, or the fibrous coat of the testicle, is of a bluishwhite color, and resembles in appearance the sclerotic coat of the eyeball. This membrane protects the secreting part of the testicle, and maintains the shape of the organ by its dense and unyielding structure: it also sends inwards processes to support and separate the seminal tubes. These several offsets of the membrane appear in the dissection; and one of them at the back of the testicle, which is larger than the rest, is the mediastinum.

The mediastinum testis (fig. $169, d$ ) (corpus Highmorianum) projects into the gland for a third of an inch with the bloodvessels. It is situate at the back of the testis, extending from the upper nearly to the lower part, and is rather larger and deeper above than below. It is formed of two pieces, which are united anteriorly at an acute angle. To its front and sides the finer septal processes are connected; and in its interior are contained the bloodvessels behind, and a network of seminal ducts in front (rete testis).

Of the finer processes of the tunica albuginea (fig. 169, $b$ ) which enter the testis, there are two kinds. One set round and cord-like, but of different lengths, is attached posteriorly to the mediastinum, and serves to maintain the shape of the testis. The other set form; delicate membranous septa, which divide the mass of seminal tubes into lobes, and join the mediastinum, like the rest.

Within the tunica albuginea is a thin vascular layer, tunica vasculosa (Sir A. Cooper), which lines the fibrous coat, covering the different septa in the interior of the gland. It is formed of the ramifications of the bloodvessels, united by areolar tissue, like the pia mater of the brain ; in it the arteries are subdivided before they are distributed on the secerning tubes, and the small veins are collected into larger trunks.

Form and length of the seminal tubes (tubuli seminiferi). The secerning or seminal tubes are very convoluted, and are but slightly held together by fine areolar tissue and surrounding bloolvessels, so that they may be readily drawn out of the testis for some distance: their length is said by Lauth to be two feet and a quarter.

Ending, size, and structure. Within the lobes of the testis some tube; end in distinct closed extremities; but the rest communicate, forming loops or arches. 'The diameter of the tubules varies from $\frac{1}{20} \frac{1}{0}$ th to $\frac{1}{50}$ th of an inch. The wall of the tubule is formed of a thin translucent membrane, but it las considerable strength. Lining the interior in the child is a nucleated granular epithelium, with polygonal cells; but in the adult the tube is filled by cells in different stages of development of the spermatic corpuscles. On the exterior is a plexus of bloodvessels.

Names from the arrangement of the tubes. To different parts of the seminal tubes, the following names have been applied. Where the tubules are collected into masses, they form the lobes of the testis. As they enter the fibrous mediastinum they become straight, and are named tubuli recti. Communicating in the mediastinum, they produce the rete testis. And, 
lastly, as they leave the upper part of the gland they are convoluted, and are called coni vasculosi, or vasa efferentia.

The lobes of the testis (fig. 169, a) are formed by bundles of the seminiferal tubes, and are situate in the intervals between the processes of the

Fig. 16.7.

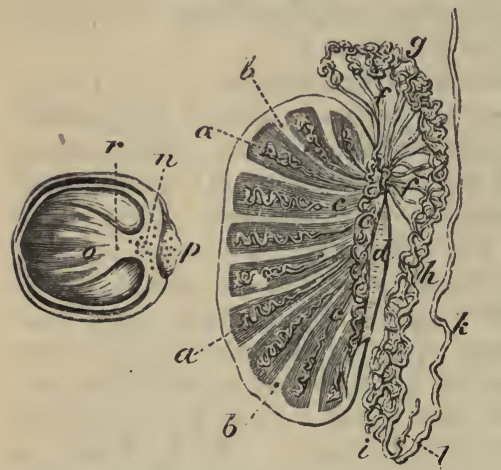

a. Lobes of the testis.

b. Septa between the lobes.

c. Tubuli recti.

d. Mediastinum testis.

e. Rete testis.

$f$. Vasa efferentia.

g. Globus major.

$h$. Body, and $i$, globus minor of the epididymis.

$k$. Vas deferens.

l. Vas aberrans.

$n$. Rete testis, cut.

o. Finer septa.

$p$. Epididymis, cut a ross.

$r$. Mediastinum, cut across.

Verticil and HoRizontal Sections of the Testis to ghow the arRangement of the SBMINAL TUBBS, AND SEPTA.

tunica albuginea: their number is differently stated:-according to one authority (Berres) they are 250; but according to another (Krause) 400 or more. They are conical in form, with the base of each at the circumterence, and the apex at the mediastinum testis; and those in the centre of the testicle are the largest.

Each is made up of one, two, or more tortuous semiral tubules; and the minute tubes in one lobe are united with those in the neighboring lobes. 'Towards the apex of each lobe the tubules become less bent, and are united together; and the tubuli of the several lobes are further joined at the same spot into larger canals-the tubuli recti.

Tubuli recti (fig. $169, c$ ). The seminal tubes unite together, becoming larger ( $\frac{1}{60}$ th of an inch) and straighter in direction, and are named tubuli recti or vasa recta: they are about twenty in number, and piercing the tibrous mediastinum enter into the rete testis.

Rete testis (fig. $169, e$ ). In the mediastinum the seminal tubes have very thin walls, and are situate in the anterior part, in front of the bloodressels; they communicate freely so as to form a network.

Vasa efferentia (fig. 169,f). About twelve or twenty seminal tubes issue from the top of the rete, and leave the upper part of the testicle as the vasa efferentia: these are larger than the tubes with which they are continuous, and end in the common excretory duct. 'Though straight at first they soon become convoluted, and have been named coni vasculosi. In the natural state they are about half an inch in length, but when unravelled they measure six inches; and they join the excretory duct at intervals of about three inches. They have a muscular coat of longitudinal and circular fibres; and the epithelium of the mucous lining is columnar and ciliated.

The EXCRETORY DUCT receives the vasa efferentia from the upper part of the gland, and extends thence to the urethra. Its first part, which is in contact with the testis, is very flexuous, and forms the epididymis: but the remainder is straight, and is named vas deferens. 
The epididymis (fig. $169, h$ ) extends in the form of an arch along the outer side of the testis, from the upper to the lower end, and receives its name from its situation. Opposite the upper part of the testicle it presents an enlarged portion or head, the globus major $(g)$; and at the lower end of that organ it becomes more pointed or tail-like-globus minor (i), before ending in the vas deferens. The intervening narrow part of the epididymis is called the body $(h)$. Its head is attached to the testis by the vasa efferentia; and its lower part is fixed to the tunica albuginea by fibrous tissue, and by the reflection of the tunica vaginalis.

The epididymis is formed of a single tube, bent in a zigzag way, whose coils are united into a solid mass by fibrous tissue. After the removal of the serous membrane and some fibrous tissue this part of the tube may be uncoiled; it will then measure twenty feet in length. The diameter of its canal is about $\frac{1}{70}$ th of an inch, though there is a slight diminution in size towards the globus minor, but it is increased finally in the vas deferens.

The vas deferens (fig. 169, $k$ ) begins opposite the lower end of the testis, at the termination of the globus minor of the epididymis. At first this part of the excretory duct is slightly wavy, but afterwards it becomes for the most part a firm round tube: near its termination it is enlarged again and sacculated, but this condition will be referred to with the viscera of the pelvis.

In its course to the urethra it ascends on the inner side of the testicle, and along the bloodvessels of the spermatic cord with which it enters the internal abdominal ring; it is then directed over the side of the bladder, and through the prostate to open into the urethra. The length of this part of the excretory duct is about two feet, and the width of its canal about $\frac{1}{30}$ th of an inch.

Opening sometimes into the vas deferens, at the angle of union with the epididymis, is at small narrow creal appendage, the vas aberrans of Haller (tig. $169, l$ ). It is convoluted, and projects upwards for one or two inches annongst the ressels of the cord. Like the epididymis, it is longer when it is uncoiled: its capacity is greatest at the free end. Its use is unknown.

Structure. The excretory duct of the testis has a thick muscular coat, which is covered externally by fibrous tissue, and lined internally by mucous membrane. To the feel the duct is firm and wiry, like whip-cord. (On a section its wall is dense and of a rather yellow color, but it is thinnest at the head of the epididymis.

The muscular coat is composed of longitudinal and circular fibres arranged in strata. Both externally and internally is a longitudinal layer, the latter being very thin; and between them is the layer of circular fibres.

The mucous membrane is marked by longitudinal folds in the straight part of the canal, and by irregular ridges in the sacculated portion. A columnar epithelium, though not ciliated, covers the inner surface; but in the epididymis it is ciliated (Becker).

Organ of Giraldès. ${ }^{1}$ In the spermatic cord of the foetus and child, close above the epididymis, is a small whitish granular-looking body ("Corps Innominé," Giraldès), about half an inch long-the remains of the lower part of the Wolffian body of the embryo. With slight magnifying power its component white granules are resolved into small vesicles, and convoluted tubes of varying shape, filled with a clear thick fluid; their wall

1 Sur un Organe placé dans le Cordon Spermatique, et dont l'existence n'a pas été signalée par les Anatomistes. Par F. Giraldès. Proceedings of the Rnyal Society for May, 1858. 
consists of a thin membrane, lined by flattened epithelium, with plexuses of bloodvessels ramifying on the exterior.

Bloodvessels and nerves of the testicle. The branches of the spermatic artery supply offsets to the epididymis, and pierce the back of the testicle to enter the posterior part of the mediastinum. The vessels are finely divided in the vascular structure lining the interior of the tunica albuginea; and offsets are continued on the fine septa to the seminal tubules, on which they are distributed in capillary plexuses.

The spermatic vein begins by radicles in the plexuses around the seminal tubes, and issues from the gland at the posterior part, being there joined by veins from the epididymis. As it ascends along the cord its branches form the spermatic plexus; it joins the vena cava on the right side, and the renal vein on the left (p. 492).

The arrangement of the lymphatics in the testicle is unknown; external to that body they ascend on the bloodvessels, and join the lumbar glands.

The nerves are derived from the sympathetic, and accompany the arteries to the testis : their ending las not been seen.

Vessels of the vas deferens. A special artery is furnished to the excretory duct from the upper or lower vesicle, and reaches as far as the testis, where it anastomoses with the spermatic artery. Veins from the epididymis enter the spermatic vein. The nerves are derived from the hypogastric plexus.

\section{Section V.}

\section{DIAPHRAGM WITH AORTA AND VENA CAVA.}

Directions. After the body is replaced in its former position on the Back, the student should prepare first the diaphragm, next the large vessels and their branches, and then the deep muscles of the abdomen.

Dissection. For the dissection of the diaphragm it will be necessary to remove the peritoneum, defining especially the central tendinous part, and the fleshy processes or pillars which are fixed to the lumbar vertebra. Whilst cleaning the muscle the student should be careful of the vessels and nerves on the surface, and of others in and near the pillars.

On the right side two aponeurotic bands or arches, near the spine, which give attachment to the muscular fibres, should be dissected: one curves over the internal muscle (psoas); the other extends over the external muscle (quadratus), and will be made more evident by separating the faseia covering the quadratus from it.

The Diaphragm (fig. $168,{ }^{\wedge}$ ) forms the vaulted movable partition between the thorax and the abdomen. It is fleshy externally, where it is attached to the surrounding ribs and the spinal columu, and has its tendon in the centre.

'The origin of the muscle is at the circumference, and is similar on each side of the middıe line. Thus, it is connected by fleshy slips with the posterior part of the xiphoid cartilage, and the inner surface of the six lower. ribs; with two aponeurotic arches between the last rib and the spinal columil-one being placed over the quadratus lumborum, and the other over the psoas muscle; and, lastly, it is connected with the lumbar vertebre by a thick muscular part or pillar. From this extensive origin the 
fibres are directed inwards, with different degrees of obliquity and length, to the central tendon, but some have a peculiar disposition in the pillars which will be afterwards noted.

The abdominal surface is coneave, and is covered for the most part by the peritoneum. In contact with it on the right side are the liver and the kilney; and on the opposite side. the stomach, the spleen, and the left kidney : in contact also with the pillars is the pancreas, together with the solar plexus and the semilunar ganglia. The thoracic surface is covered by the pleura of each side and the pericardium, and is convex towards the thorax (p. 343). At the circumference of the midritf the fleshy processes of origin alternate with like parts of the transversalis musele; but a slight interval separates the slips to the xiphoid cartilage and seventh rib, and a second space exists sometimes between the fibres firom the last rib and the arch over the quadratus lumborum muscle. In it are certain aperiures for the transmission of parts from the thorax to the abdomen.

The muscle is convex towards the chest, and concave to the abdomen. Its arch reaches higher on the right than the left side (p. 306), and is con-

Fig. 170.

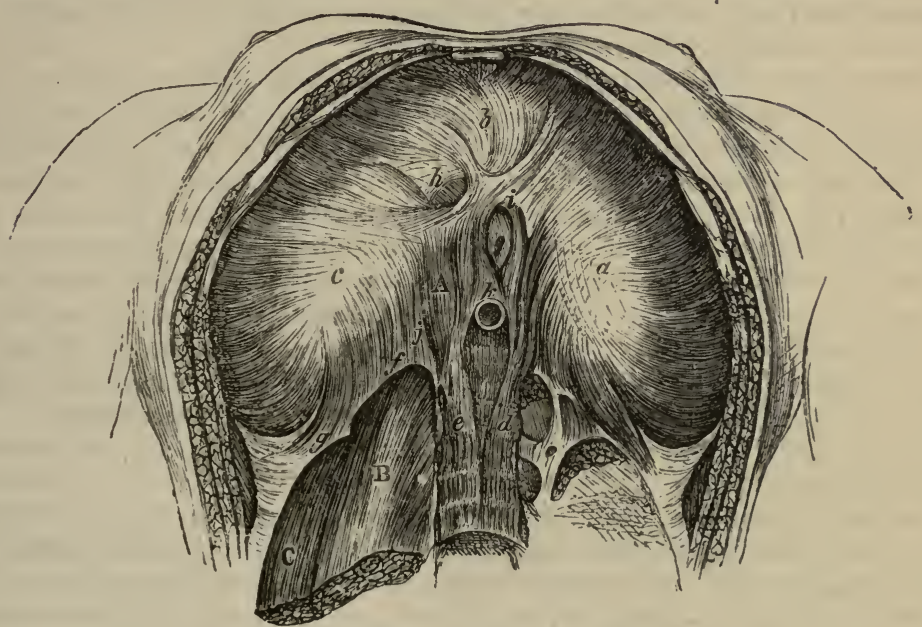

UNDER SLRFACE OF THE DIAPHRATM.

\section{A. Diaphragm.}

B. Psoas maguus.

c. Quadratus lumborum.

a. Left piece of the teudon of the diaphragm; $b$, middle; and $c$, right piece. d. Left, and $e$, right crus.

$f$. Inner, and $g$, outer arched ligament. $h$. Opening for vena cava; $i$, for osophagus, $\boldsymbol{i}$, for aorta, $j$, for sympathetic nerves.

stantly varying during life in respiration. In forced expiration the muscle ascends, and reaches as high as the upper border of the right fourth rib at the sternum, and the upper edge of the fiftl ${ }^{\circ}$ rib on the left side, close to the sternum. In forced inspiration it descends, and its slope would be represented by a line drawn from the middle of the ensiform cartilage to the eleventh lib.

Action. As the muscle moves up and down during respiration, it is depressed by the contraction of the fleshy fibres which are attached to the ribs and spine, and is raised during their relaxation. 
When the diaphragm descends it changes its shape. The central tendon, which moves but slightly, remains the highest part of the arch, whilst the sides which contract freely are sloped from the tendon to the wall of the thorax. During the ascent the midriff retains nearly the same form as in a state of rest, for the tendon is the lowest part of the arch, and the bulges on the sides reach rather higher.

With the movement of the diaphragm the size of the cavities of the abdomen and thorax will be altered. In inspiration the thorax is enlarged, and the abdomen diminished; and the viscera in the upper part of the latter cavity, viz., liver, stomach, and spleen, are partly moved from beneath the ribs. In expiration the cavity of the thorax is lessened, and that of the abdomen is restored to its former size; and the displaced viscera return to their usual place. By the contraction of the fibres the aperture for the oesophagus will be rendered smaller, and that tube may be compressed; but the other openings for the vena cava and aorta do not experience change.

Preparatory to the making of a great muscular effort, the midriff contracts, and descends for the purpose of permitting a full quantity of air to enter the thorax. Till the effort is over the diaphragm remains in a depressed position. Its action is commonly involuntary, but the movement can be controlled by the will at any stage.

Parts of the diaphragm. 'The following named parts, which have been noticed shortly in describing the muscle, are now to be referred to more fully, viz., the central tendon, the pillars, the arches, and the apertures.

The central tendon (cordiform tendon) occupies the middle of the diaphragm (fig. 170), and is surrounded by muscular fibres; the large vena cava pierces it. It is of a pearly white color, and its tendinous fibres cross in different directions. In form it resembles a trefoil leaf : of its three segments the right $(c)$ is the largest, and the left $(a)$ the smallest.

The pillars (crura) are two large muscular and tendinous processes $(d$ and $e$ ), one on each side of the abdominal aorta. They are pointed and tendinous below, where they are attached to the upper lumbar vertebræ, but large and fleshy above; and between them is a tendinous arch over the aorta.

In each pillar the fleshy fibres pass upwards and forwards, diverging from each other: the greater number join the central tendon without intermixing, but the inner fibres of the two erura cross one another in the following manner :- Those of the right $(e)$ ascend by the side of the aorta, and pass to the left of the middle line decussating with the fibres of the opposite crus between that vessel and the opening of the cesophagus. The fibres of the other crus (d) may be traced in the same way, to form the right half of the osophagean opening. In the decussation the fasciculus of fibres from the right crus is generally larger than that from the left, and is commonly anterior to it.

The pillars differ somewhat on opposite sides. The right $(e)$ is the larger of the two, and is fixed by tendinous processes to the bodies of the first three lumbar vertebra, and their intervertebral substance, reaching to the disk between the third and fourth vertebra. The left pillar $(d)$ (sometimes absent) is situated more on the side of the spine, is partly concealed by the aorta, and does not reach so far as the right by the depth of a vertebra, or of an intervertebral substance.

'The arches (ligamenta arcuata) are two tibrous bands on each side over 
the quadratus lumborum and psoas muscles, which give origin to fleshy fibres.

The arch over the psoas (lig. arcuat. internum $f$ ) is the strongest, and is connected by the one end to the tendinous part of the pillar of the diaphragm, and by the other to the transverse process of the first or the second lumbar vertebra.

The arch over the quadratus lumborum (lig. areuat. externum $g$ ) is only a thickened part of the fascia covering that muscle, and extends from the same transverse process (first or second lumbar) to the last rib.

Apertures. There are three large openings for the aorta, the vena cava, and the osophagus; with some smaller fissures for nerves and vessels.

The opening for the arta $(k)$ is rather behind, than in the diaphragm, for it is situate between the pillars of the muscle and the spinal column : it transmits the aorta, the thoracic duct, and the venla azygos.

The opening for the oesophagus and the pneumo-gastric nerves $(i)$ is rather above and to the left of the anrtic aperture; it is placed in the muscular part of the diaphragm, and is bounded by the fibres of the pillars as above explained.

The opening for the vena cava $(h)$ (foramen quadratum) is situate in the right division of the central tendon; and its margins are attached to the vein by tendinous fibres, except at the inner part.

There is a fissure $(j)$ in each pillar for the three splanchnic nerves; and through that in the left crus passes also the small azygos vein.

Dissection. After the diaphragm has been learnt, the ribs that support it on each side may be cut through, and the pieces of the ribs with the fore part of the diaphragm may be taken away, to make easier the dissection of the deeper vessels and muscles. But the posterior part of the diaphragm with its pillars and arches should be left; and the vessels ramifying on it should be followed back to their origin.

The large vessels of the abdomen, viz., the aorta and the vena cava, are to be cleaned by removing the fat, the remains of the sympathetic, and the lymphatic glands; and their branches are to be followed to the diaphragm, to the kidney and suprarenal borly, and to the testicle. In like manner the large iliac branches of the aorta and cava are to be laid bare as far as Poupart's ligament. The ureter and the spermatic vessels are to be cleaned as they cross the iliac artery to the limb; and on the iliac trunk near the thigh, branches of a small nerve (genito-crural) are to be songht.

The muscles are to be laid bare on the right side, but on the left side the fascia covering them is to be shown; and the fat is to be cleared away fiom about the kidney.

The psoas muscle, the most internal of all, lies on the side of the spine, with the small psoas superficial to it oceasionally. On its surface, and in the fat external to it, the following branches of the lumbar plexus will be found: 'The genito-crural nerve lies on the front. Four other nerves issue at the outer border-the ilio-hypogastric and ilio-inguinal near the top, the external cutaneous about the centre, and the large anterior crural at the lower part. Along the inner border of the psoas the gangliated cord of the sympathetic is to be sought, along with a chain of lumbar lymphatic glands; and somewhat below the pelvie part of the muscle the obturator nerve may be recognized. External to the psoas is the quadratus lumborum, and crossing it near the last rib is the last dorsal nerve, with an 
Fig. 171.

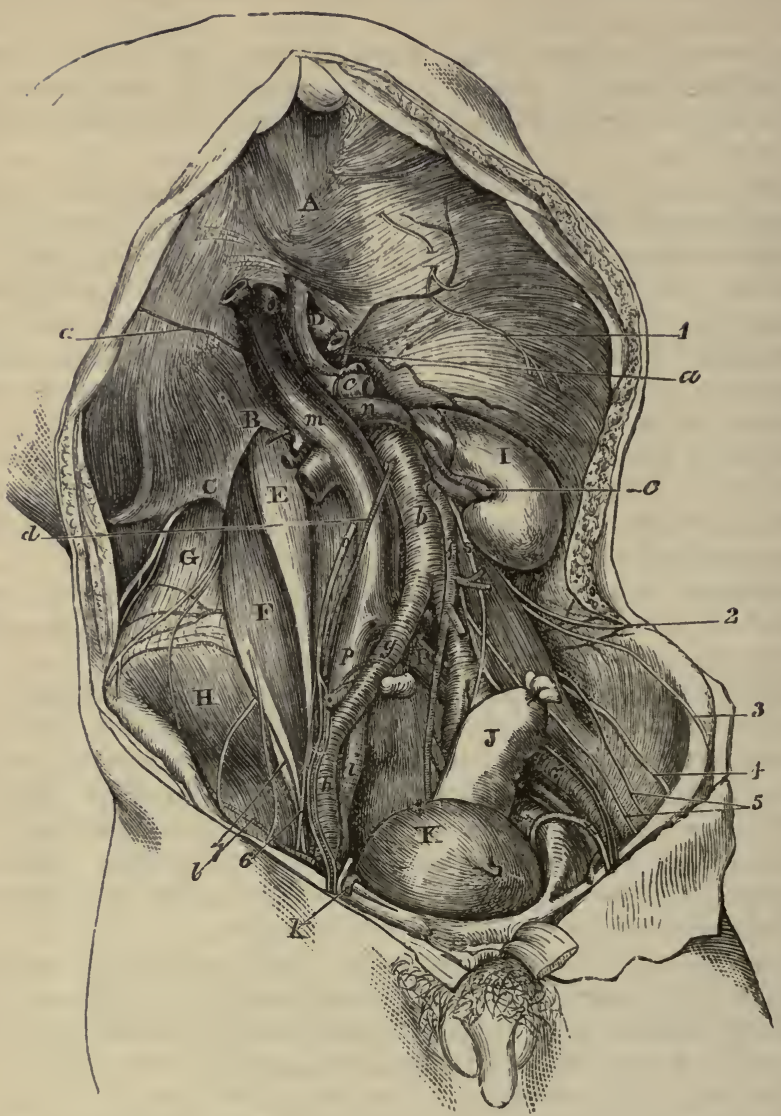

Degp view of the Muscles, Vessels, and Nerves of the A bomisal Cavity.

(Illustrations of Dissections.)

Muscles and viscera:

A. Diaphragm with $B$, internal, and c, external arched ligament.

D. End of the osophagus, cut.

v. Small psoas.

F. Large psous.

f. Quadrutus lumborum.

H. Iliacus.

I. Kldney.

J. Rectum.

K. Bladder.

Vessels :
a. Diaphragmatic artery.
b. Aorta.
c. Renal.
d. Spermatic.
e. Upper mesenteric, cut.
f. Lower mesenteric.

$g$ Common iliac, and $h$, external iliac artery.

k. Epigastric urtery, cut; by its side is the vas deferens, bending in to the pelvis.

l. Circumflex iliac.

in. Vena cava.

n. Renal vein.

o. Right spermatic vein, $p$, common lliac vein, and $r$, external iliac (this letter is put on the left artery lustead of the vein just below it).

8. Ureter.

Nerves :

1. Phrenic.

2. Ilio hypogastric.

3. Illo-inguinal.

4. External cutaneons of the thigh.

5 and 6 . Genito-crural.

7. Anterior crural. 
artery. In the hollow of the hip-bone is the iliacus muscle which unites below with the large psoas.

The a BDominal aORTA (fig. $171, b$ ) extends from the last dorsal vertebra to the left side of the body of the fourth lumbar vertebra, where it divides into the common iliac arteries. Its commencement is between the pillars of the diaphragm, and its termination is placed on the left side of the umbilicus, and nearly on a level with the highest part of the crest of the hip-bone.

The chief eonnections of the vessels with surrounding parts have been before referred to (p. 444), but some deep vessels in relation with it come now into view. As the aorta lies on the spine it rests on the left lumbar veins, which end in the inferior eava. And between it and the right crus of the diaphragm lie the vena azygos major, and the thoracic duct. Along the sides of the vessel are the lumbar lymphatic glands, from which large vessels run beneath it to end in the beginning of the thoracic duct.

The branches of the aorta are nunierous, and arise in the following order : First, are the diaphragmatic arteries, two in number, which leave the sides of the vessel immediately it appears in the abdomen. Close to the tendinous ring of the diaphragm, the single trunk of the coliac axis arises from the front : and about a quarter of an inch lower down, also on the front, the trunk of the superior mesenteric artery begins. IIalf an inch lower the renal arteries, right and left, take origin from the sides of the aorta. On the lateral part of the vessel, close above each renal, is the small capsular branch; and below the renal is the spermatic artery. From the front of the arterial trunk, one to two inches above the bifureation, springs the inferior mesenteric artery; and from the angle of division the small middle sacral artery runs downwards. Four small lumbar branches on each side come from the posterior part of the vessel, opposite the bodies of the lumbar vertebra.

The branches may be classified into two sets-one to the viscera of the abrlomen (visceral), and another to the abclominal wall (parietal).

'The visceral branches are coliac axis, superior and inferior mesenteric, renal, eapsular, and spermatic. This set has been examined, except the renal, capsular, and spermatic.

The renal arteries (fig. $171, c$ ) leave the aorta nearly at a right angle, and are directed outwards, one on each side. Near the kidney each divides into four or five branches, which enter the renal substance between the vein and the ureter. Each artery lies beneath its companion vein, being surrounded by a plexus of nerves, and supplies small twigs to the suprarenal body (inferior eapsular), to the ureter, and to the fatty layer about the kidney.

The arteries of opposite sides have some differences. The left is the shortest, owing to the position of the aorta: the right crosses the spine, and passes beneath the vena cava.

The middle capsular artery is a small branch which runs almost transversely outwards to the suprarenal body : this offset anastomoses with the? other branches of the suprarenal body from the renal and diaphragmatic arteries. It is of large size in the foetus.

The spermatic artery of the testicle (fig. $171, d$ ) is remarkable for its small size in proportion to its length; for leaving the cavity of the abdomen; and for having the part in the abdomen straight, but that in the cord tortuous.

From its origin below the renal, the ressel passes downwards along 
the posterior wall of the abdomen to the internal ahdominal ring, where it enters the spermatic cord (p. 418). In its course beneath the peritoneum the vessel runs along the front of the psoas, crossing over the ureter; and on the right side it passes over the vena cava. It is accompanied by the spermatic vein, and the spermatic plexus of nerves. In the foctus before the testicle leaves the abdomen the spermatic artery is very short, but the vessel elongates as the part supplied is removed from its former site.

In the female the corresponding artery (ovarian) descends into the pelvis to end in the ovary and the uterus.

The parietal branches of the aorta are the diaphragmatic, lumbar, and middle sacral.

The diaphragmatic arteries (inferior plirenic) (fig. 171, a) are directed outwards along the under surface of the diaphragm near the posterior part, the left artery passing behind the ocsophageal opening, and the right behind the vena cava. Each ends in two branches: One (internal) passes onwards towards the fore part of the diaphragm, and anastomoses with its fellow, and with the branch (superior phrenic) to the diaphragm from the internal mammary (p. 239). The other (external) is larger, and is directed outwards to the side of the muscle, where it communicates with the musculo-phrenic and intercostal arteries.

Branches. Small offsets to the suprarenal body from the external division of this artery are named superior capsular. Some twigs are given by the left artery to the œesophagus, and by the right to the vena cava.

On the under surface of the diaphragm are two branches of the internal mammary artery ; one (superior phrenic, p. 239) accompanies the phrenic nerve, and ramifies over the middle of the musele; the other (musculophrenic, p. 239) appears opposite the ninth rib, and passing along the edge of the thorax, gives offsets to the lower intercostal spaces.

The other parietal branches, viz., lumbar and middle sacral, are not learnt in this stage : the former will be seen after the lumbar plexus, and the latter in the pelvis.

The CoMmon ILIAC ARTERY (fig. 171, g) is directed ontwards from the hifurcation of the aorta, and divides into two large trunks opposite the fibro-cartilage between the base of the sacrum and the last lumbar vertebra :- one of these (external iliac) supplies the lower limb, and the other (internal iliac) enters the pelvis. Placed obliquely on the vertebral column, the vessel measures about two inches in length. It is covered by the peritoneum, and is crossed by branches of the sympathetic nerve, and sometimes by the ureter. It is accompanied by a vein of the same name. Usually it does not furnish any named branch, but it may give origin to the ilio-lumbar, or a renal artery. On opposite sides the vessels have some differences.

'The right artery is rather the longest, in consequence of the position of the aorta on the left side of the spine. 'T'o its outer side at first is the vena cava, and near its termination is the psoas muscle. The companion vein $(p)$ is at first beneath, but becomes external to the artery at the upper part ; and beneath the right artery also is the left common iliac vein. The left artery is crossed by the colon and the inferior mesenteric vessels; and its companion vein is situate below it.

'The length of the common iliac ranges from less than half an inch (in one case) to four inches and a half; but in the majority of instances it varies between one inch and a half and three inches (Qaain). 
The external iliac artery (fig. $171, h$ ) is the first part of the vessel leading to the lower limb, and is contained in the cavity of the abdomen. Its extent is from the bifureation of the common iliac to the lower border of Poupart's ligament, where it becomes femoral. And its direction would be indicated, on the surface of the abdomen, by a line from the left of the umbilicus to the middle of the space between the symphysis pubis and the front of the iliac crest.

The vessel lies above the brim of the pelvis in its eourse to Poupart's ligament, and is covered closely throughout by the peritoneum and the subperitoneal fat. To its outer side is the psoas, except at its termination under Poupart's ligament, where the muscle lies beneath it. A chain of lymphatic glands is placed along the front and inner side of the artery.

Near its origin it is crossed sometimes by the ureter ; and near Poupart's ligament the vas deferens bends down along its inner side, whilst the spermatic vessels, and part of the genito-crural nerve lie on it for a short distance.

The position of the external iliac vein $(r)$ is not the same on both sides. The left vein is altogether internal to the artery; whilst the right, though internal in position on the pubes, afterwards lies beneath the arterial trunk. The eircumflex iliac vein crosses it nearly an inch above Poupart's ligament.

Branches. Two branches, epigastric and circumflex iliac, arise about a quarter of an inch from the end of the artery, and are distributed to the wall of the abdomen (p. 419,420).

Some small unnamed twigs are given to the psoas muscle and the lymphatic glands.

Peculiarities in usual branches. The epigastric and circumflex branches may wander over the lower inch and a half or two inches of the artery.

In unusual branches. Though the trunk of the vessel is commonly free from any unusual branch, it may be occupied between the middle and the end by the obturator artery, or by the internal circumflex artery of the thigh.

Iliac Veins and Vexa Cava (fig. 171). The larger veins of the abdomen correspond so closely with the arteries, hoth in number, extent, and connections, as to render unnecessary much detail in their description. As the veins increase in size from the circumference towarls the centre of the body, those most distant from the heart will be first referred to.

The external iliac $(r)$ is a continuation of the femoral vein beneath Poupart's ligament. It has an extent like the artery of the same name, and ends by uniting with the vein from the pelvis (internal iliac), to form the common iliac vein. On the pubes it is inside its companion artery, and lies between the psoas and pectineus muscles; the left vein remains internal to, but the right slips beneath its artery.

The veins opening into it are the epigastric and circumflex iliac (1.420).

The common iliac vein (fig. $171, p$ ) ascends by the side of its accompanying artery, the right almost vertically, and the left obliquely, to the right side of the body of the fifth lumbar vertebra (the upper part), where it blends with its fellow in one trunk - the vena cava.

The right rein is the shortest, and lies at first behind, but afterwards outside the artery of the same name. "The left is altogether below the artery of its own side, and crosses beneath the right common iliac artery.

Each vein receives the ilio-lumbar, and the lateral sacral branch; and the common iliac of the left side is joined by the middle sacral rein. 
The rena cava inferior (fig. $171, \mathrm{~m}$ ) collects and conveys to the heart the blood of the lower half of the body. Taking the origin on the right side of the fifth lumbar vertebra, below the bifurcation of the aorta, this large vein ascends on the right side of the vertebral column, and reaches the heart by perforating the diaphragm. Its connections with the surrounding parts have been already noticed (p. 444), but the description may be referred to, as the position of the branches of the aorta to it can be better seen now.

Branches. The cava receives parietal branches (lumbar and diaphragmatic), from the wall of the abdomen and the diaphragm; and visceral branches from the testicle, the kidney, the suprarenal body, and the liver.

The veins belonging to the digestive apparatus, viz., the intestinal canal, the spleen, and the pancreas, are united to form the vena porta (p. 448); and the blood contained in those veins reaches the cava by the vena cava hepatice, after it has circulated through the liver.

The spermatic vein (fig. $171, o$ ) enters the abdomen by the internal abdominal ring, after forming the spermatic plexus in the cord (p. 484). At first there are two branches in the abdomen, which lie on the sides of the spermatic artery; but these soon join into one trunk. On the left side it opens into the renal vein at right angles, and a small valve exists sometimes over the aperture; on the right side it enters the inferior cava below the renal vein. As the vein ascends to its destination, it receives one or more branches from the wall of the abdomen, and from the fat about the kidney.

In the female this vein (ovarian) has the same ending as in the male, and it forms a plexus in the broad ligament of the uterus. Valves are absent from the vein and its branches, but occasionally there is one at its union with the renal.

The renal or emulgent vein (fig. $171, n$ ) is of large size, and joins the vena cava at a right angle. It commences by many branches in the kiclney; and the trunk resulting from their union is superficial to the renal artery.

The right is the shortest, and joins the cava higher up usually than the other. The left vein crosses the aorta close to the origin of the superior mesenteric artery: it receives separate branches from the left spermatic and suprarenal veins.

The suprarenal vein is of consilerable size when it is comparerl with the body from which it comes. 'The right opens into the cava, and the left into the renal vein.

The hepatic veins enter the vena cava where it is in contact with the liver. These veins are described in the dissection of the liver (p. 468).

The limbar veins correspond in number and course with the arteries of the same name: they will be dissected after.

The diaphragmatic veins (inferior), two with each artery, spring from the under surface of the diaphragm. They join the eava either as one trunk or two.

DEEP MUSCLES OF THE ABDOMEN.

The deep muscles in the interior of the abdomen are the psoas, iliacus, and quadratus lumborum.

The P'SOAS MAGNes (fig. $171,{ }^{\mathrm{r}}$ ) reaches from the lumbar vertebra to the femur, and is situate partly in the abdomen and partly in the thigh. 
The muscle arises from the front of the transverse processes of the lumbar vertebræ; from the bodies and intervertebral disks of the last dorsal and all the lumbar vertebra by five fleshy pieces_each piece being connected with the intervertebral substance and the borders of two contignous vertelire, and with tendinous bands over the bloodvessels opposite the mildle of the vertebrie. 'The fibres give rise to a roundish belly, which gradually diminishes towards Poupart's ligament, and ends inferiorly in a tendon on the outer aspect, which receives fibres of the iliacus, and passes beneath Poupart's ligament to be inserted into the small trochanter of the femur.

The abdominal part of the musele has the following connections:-In front are the internal arch of the diaphragm, the kidney with its vessels and duct, the spermatic vessels, and the genito-crural nerve, and, near Poupart's ligament, the ending of the external iliac artery. Posteriorly the muscle is in contact with the transverse processes, with part of the quadratus lumborum, and with the innominate bone.

The outer border touches the quadratus and iliacus; and branches of the lumbar plexus issue from beneath it. The inner border is partly connected to the vertebra, and is partly free along the margin of the pelvis: -along the attached part of this border lies the sympathetic nerve, with the cava on the right, and the aorta on the left side; along the free or pelvic part are the external iliac artery and vein.

Action. If the femur is free to move it is raised towards the belly; and as the flexion proceeds, the limb is rotated out by the attachment of the muscle to the trochanter minor. The psoas is always combined with the iliacus in flexion of the hip-joint.

When the lower limbs are fixed the two muscles will draw down the lumbar part of the spine, and bend the hip-joints, as in stooping to the ground. One muscle under the same circumstances can incline the spine laterally.

The PSOAS PARVUs (fig. $171,{ }^{\mathrm{E}}$ ) is a small muscle with a long and flat tendon, which is placed on the fiont of the large psoas, but is rarely present. Its tibres urise from the bodies of the last dorsal and first lumbar vertebre, with the intervening fibro-cartilage. Its tendon becomes broader inferiorly, and is inserted into the ilio-pectineal eminence and the brim of the pelvis, joining the fascia over the iliacus muscle.

Action. If the spine is immovable the two muscles will make tense the pelvic fascia. The pelvis being fixed they may assist in bending the lumbar part of the spinal column.

The ILIACUS MUSCLE (fig. $171,{ }^{\mathrm{H}}$ ) occupies the iliac fossa on the inner aspect of the hip-bone, and is blended inferiorly with the psoas. It is triangular in form, and has a fleshy origin from the iliac fossa and the ilio-lumbar ligament, from the base of the sacrum, and from the capsule of the hip-joint in front. The fibres pass inwards to the tendon of the psoas, uniting with it even to its insertion into the femur, but some reach separately the femur near the sinall trochanter.

Above Poupart's ligament the muscle is covered by the iliar fascia; but over the right iliacus is placed the cecum, and over the left, the signoid flexure. Beneath it are the innominate bone and the capsule of the hipjoint; and between it and the grooved anterior margin of the bone, above the joint, is a bursa. The inner margin is in contact with the psoas and the anterior crural nerre. The connections of the united psoas and iliacus below Poupart's ligament are given with the dissection of the thigh. 
Action. The iliacus flexes the hip-joint with the psoas when the femur is movable, and bends forwards the pelvis when the limb is fixed. In consequence of its union with the psoas, the two are described as the flexor of the hip-joint by Theile.

The QUADratus Lumborum (fig. $171^{a}$ ) is a short thick muscle between the crest of the hip-bone and the last rib. About two inches wide inferiorly, it arises from the ilio-vertebral ligament, and from the iliac crest of the hip-bone behind, and an inch outside that band. The fibres ascend to be inserted by distinct fleshy and tendinous slips into the apices of the transverse processes of the four upper, or all the lumbar vertebre; and into the body of the last dorsal vertebra, and the lower border of the last rib for a variable distance.

This muscle is encased in a sheath derived from the fiscia lumborum. Crossing the surface are branches of the lumbar plexus, together with the last dorsal nerve and its vessels. Beneath the quadratus is the mass of the erector spinæ musele.

Action. Both muscles keep straight the spine (one muscle antagonizing the other) ; and by fixing the last rib they aid in the more complete contraction of the diaphragm.

One muscle will incline laterally the lumbar part of the spine to the same side, and depress the last rib.

Fascia of the quadratus. Covering the surface of the quadratus is a thin membrane, which is derived from the hinder aponeurosis of the transversalis abdominis (fascia lumborum, p. 357); it passes in front of the quadratus to be fixed to the tips and borders of the lumbar transverse processes, to the ilio-lumbar ligament below, and to the last rib above. 'This fascia forms the thickened band called ligamentum arcuatum externum, to which the diaphragm is connected.

Fascia of the iliacus and psoas. A fascia covers the two flexor muscles of the hip-joint, and extends in different directions as far as their attachments. Over the iliacus muscles the membrane is thickest; and a strong aceession is received fiom the tendon of the small psoas. Its disposition at Poupart's ligament, and the part that it takes in the formation of the crural sheath, have been before explained (p. 428).

Opposite the pelvis the membrane is inserted into the brim of that cavity for a short distance, and into the hip bone along the edge of the psoas. Opposite the spinal column it becomes thin, and is fixed on the one side to the lumbar vertebræ and the ligamentum arcuatum internum, but is blended on the other with the fascia on the quadratus. The fascia should be divided over the psoas on the left side, and reflected towards the brim of the pelvis.

Dissection. The student is now to clean the lymphatic glands lying along the vertebrx, and to trace upwards some lymphatie vessels to the thoracic duct.

To show the origin of the duct, the diaphragm is to be divided over the. aorta, and its pillars are to be thrown to the sides; a piece may be eut out of the aorta opposite the first lumbar vertebra. The beginning of the duet (chyli receptaculum), and of the vena azygos, may be well seen; and the two may be followed upwards into the thorax.

On the left side the student may trace the splanchnic nerves and the small vena azygos through the pillar of the diaphragm; and may show the trunk of the sympathetic nerve entering the abdomen beneath the arch over the psoas muscle. 
Lymphatic glands. A chain of glands is placed along the side of the external iliac artery, and along the front and sides of the lumbar vertebrx; they are connected by short tubes, which increase in size and diminish in number, until at the upper part of the lumbar vertebræ three or four trunks unite in the thoracic duct. Into the glands the lymphatics of the lower limbs, and those of the viscera and wall of the abdomen are received.

Receptaculum chyli (Pecquet). The thoracic duct begins in the abdomen by the union of three or four large lymphatic vessels. Its commencement is marked by a dilatation, which is placed on the right side of the aorta, about opposite the first lumbar vertebra. The duct enters the thorax by passing through the diaphragm with the aorta.

Beginning of the azygos veins. The right vein (vena azygos major) begins opposite the first or second lumbar vertebra by a small branch, which is united with a lumbar vein. It enters the thorax with the thoracic duct and the aorta, to the right of which it lies.

The left or small azygos vein begins on the left side of the spine, joining one of the lumbar veins, and passes through the pillar of the diaphragm, or through the aortic opening.

The anatomy of these veins is given in the description of the thorax, p. 338.

\section{Section VI. \\ SPINAL AND SYMPATHETIC NERVES.}

THE spinal nerves of the loins are united in a plexus, and supply the limb and the contiguous parts of the trunk.

Dissection. The lumbar nerves and their plexus are to be learnt on the left side, although the woodcut shows them on the right side; and to bring them into view, the dissector should eut through the external iliac vessels, and afterwards scrape away the psoas. For the most part the fleshy fibres may be removed freely; but a small branch (accessory of the obturator) should be first looked for at the inuer border of the muscle. In the substance of the quadratus lumborum a communication may be sometimes found between the last dorsal and the first lumbar nerve.

The cord of the sympathetic nerve lies along the edge of the psoas, and offsets of it join the spinal nerves; these are to be followed back along the lumbar arteries.

On the right side the psoas is to be left untouched, in order that the place of emergence of the different nerve branches from it may be noticed.

Lumbar Spinal Nerves. The anterior primary branches of the lumbar nerves enter the lumbar plexus, with the exception of the last. Five in number, they increase in size from the first to the last, and are joined by filaments of the sympathetic near the intervertebral foramina. Before entering the plexns they supply branches to the psoas and quadratus lumborum muscles.

The fifth nerve (fig. 177) receives a communicating branch from the fourth, and is to be followed into the pelvis to its junction with the sacral plexus. After the two are united, the name lumbo-sacral is applied to 
the common trunk ; and from this, before it enters the sacral plexus, arises the superior gluteal nerve $\left({ }^{1}\right)$.

The Lumbar plexus (tig. 172) is formed by the intercommunication of the first four lumbar nerves. Contained in the substance of the psoas near the posterior surface, it consists of communicating loops between the several nerves, and increases in size from above downwards, like the individual nerves. Superiorly it is sometimes united by a small branch with the last dorsal nerve; and inferiorly it joins the sacral plexus through the large lumbo-sacral cord.

Fig. 172.

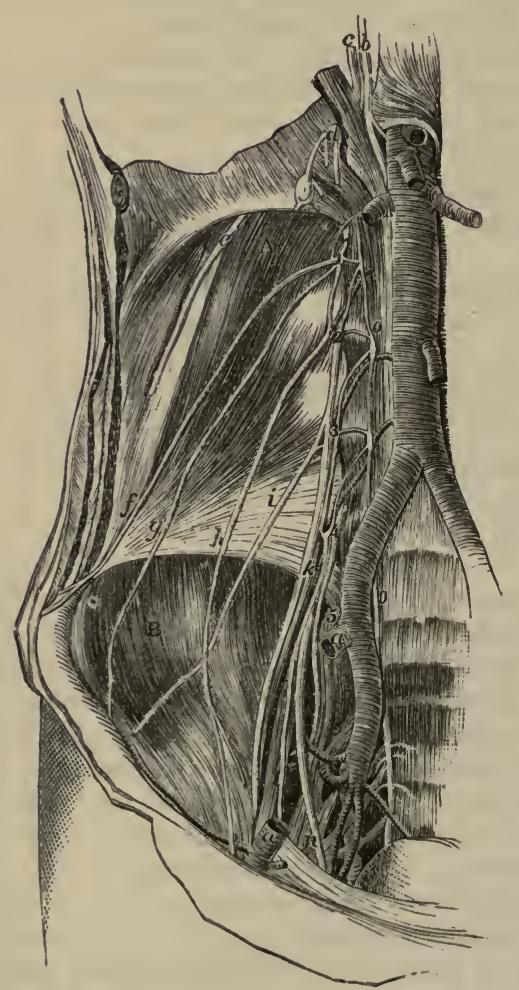

a. External 1liac artery, cut ac:oss.

b. Thoracic duct.

c. Azygos vein.

Nerves: The figures 1 to 5 mark the trunks of the five lumbar nerves.

d. Splanchnic nerves.

e. Last dorsal.

f. Ilio-hypogastric.

g. Ilio-inguinal.

h. Gevito-crural.

i. External cutaneous.

k. Anterior crural.

l. Accessory to obturator.

$n$. Obturator.

o. Gangliated cord of the sympathetic.

Disscction of the LUMBar Ploxes axd its Branches. (Illustrations of Dissectiony.)

The branches of the plexus are six in number, and supply the lower part of the abdominal watl and muscular covering of the spermatic corl, the fore part of the thigh, and the inner side of the leg.

The first two branches (ilio-hypogastric and ilio-inguinal) end as cutaneous nerves of the buttock, lower part of the abdomen, and the serotum.

a. The ilio-hypogastric branch (fig. $172, f$ ) is derived from the first nerve, and appears at the outer border of the psoas muscle, near the upper part. It is directed over the quadratus lumborum to the iliac erest, and enters the wall of the abdomen by penetrating the transversalis abdominis. 
Its termination in the integuments of the buttock and abdomen, by means of an iliac and a hypogastric branch, has been already mentioned (p. 416).

$b$. The ilio-inguinal branch $(g)$ arises with the preceding from the first nerve, and issues from the psoas nearly at the same spot. Of smaller size than the ilio-hypogastric, this branch courses outwards over the quadratus and iliacus muscles towards the front of the crest of the hip bone, where it pierces the transversalis abdominis. 'The farther course of the nerve in the abdominal wall, and its distribution over the scrotum and the groin, are before noticed (p. 416).

$c$. The genito-crural nerve $(h)$ is distributed to the cremaster muscle and the limb. It arises from the second lumbar nerve, and firom the connecting loop between the first two ; it pierces the fibres of the psoas, and descending on the surface of the muscle divides into the two following pieces. Sometimes the nerve is divided in the psoas, and the pieces perforate separately the muscle.

The cremasteric branch descends on the external iliac artery, and furnishes offsets around it: it passes from the abdomen with the spermatic vessels, and is distributed in the cremaster muscle. In the female the nerve is lost in the round ligament.

The crural branch issues beneath Poupart's ligament to supply the integument of the thigh.

$d$. The external cutaneous nerve of the thigh (i) arises from the second nerve of the plexus, or from the loop between it and the third, and appears about the middle of the outer border of the psoas. The nerve then crosses the iliaeus to the interval between the anterior iliac spinous processes, and leaves the abdomen beneath Poupart's ligament, to be distributed on the outer aspect of the limb.

$e$. The anterior crural nerve $(k)$ is the largest offset of the plexus, and supplies branches mainly to the extensor muscles of the knee joint, and to the teguments of the front of the thigh and inner side of the leg. Taking origin from the third and fourth nerves, and receiving a fasciculus also firom the second, this large trunk appears towards the lower part of the psoas, where it lies between that muscle and the iliacus. It passes from the abdomen beneath Poupart's ligament; but before the final branching in the thigh, the nerve furnishes the following twigs :-

Some small branches are furnished to the iliacus from the upper part of the nerve.

A branch to the femoral artery, whose place of origin varies much, is distributed around the upper part of that vessel.

$f$. The obturator nerve $(n)$ appertains to the adductor muscles of the thigh. Derived from the third and fourth nerves in the plexus, it is directed beneath the psoas to the inner or pelvic border; escaped firom beneath the muscle the nerve crosses the pelvic cavity below the external iliac, but above the obturator vessels, and enters the thigh through the aperture in the upper part of the thyroid foramen. Occasionally the obturator gives origin to the following branch:-

'I he accessory obturator nerve $(l)$ arises from the trunk of the obturator, or from the third and fourth nerves of the plexus. Its course is along the inner border of the psoas beneath the investing fascia, and over the hip bone to the thigh, where it ends by joining the obturator nerve, and supplying the hip joint.

Gangliated cord of the sympathetic. The lumbar part of the gangliated cord of the sympathetic in the abdomen is placed on the side 
of the spinal column (fig. 172); it lies along the inner border of the psoas muscle, nearer the front of the vertebra than in the thorax, and is somewhat concealed on the right side by the rena cava. The cord possesses four or five oblong ganglia opposite the bodies of the vertebra, which sup)ply connecting and visceral branches.

Connecting branches. From each ganglion two small branches are directed backwards along the centre of the body of the rertebra, with the lumbar artery; these unite with one or two spinal nerves near the intervertebral foramen. The connecting branches are long in the lumbar region in consequence of the gangliated cord being raised by the psoas muscle to the fore part of the vertebræ.

Branches of distribution. Most of the internal branches throw themselves into the aortic and hypogastric plexuses, and so reach the viscera indirectly. Some filaments enter the vertebræ and their connecting ligaments.

Last dorsal nerve (fig. $172, e$ ). The anterior primary branch of the last dorsal resembles the intereostal nerves in its distribution, but differs from them in not be:ng contained in an intercostal space. Lying below the last rib, the nerve is directed outwards across the upper part of the quadratus lumborum, and beneath the fascia covering that muscle, as far as the aponeurosis of the transversalis abdominis (fascia lumborum); here it enters the wall of the abdomen, and ends in an abdominal and a cutaneous branch (p. 416). The lowest intercostal artery accompanies it.

Near the spine it furnișhes a small branch to the quadratus muscle; and it may communicate by means of this with the first lumbar nerve.

The lumbar arteries of the aorta (p. 489), are furnished to the Back, the spinal canal, and the wall of the abdomen: they resemble the aortic intercostals in their course and distribution. Commonly four in number on each side, they arise opposite the centre of the lumbar vertebræ, and the vessels of opposite sides are sometimes joined in a common trunk; they then pass backwards beneath the pillar of the diaphragm and the psoas, to reach the interval between the transverse processes, where each ends in an abdominal and a dorsal branch. The arteries of the right side lie beneath the vena cava.

The dorsal branch continues to the Back between the transverse processes, and supplies an offset to the spinal canal (pp. 368, 384).

The abdominal branches are directed outwards beneath the quadratus lumborum, and enter the posterior part of the abdominal wall, where they anastomose with the lower intercostal above, and with the circumflex iliac and ilio-lumbar arteries below : these branches supply the psoas and quarlratus muscles; and the last furnishes an off'set to the teguments with the ilio-hypogastric nerve.

The LUMBAR VEINS are the same in number, and have the same course as the arteries. Commencing by the union of a dorsal and an abdominal branch at the root of the transverse process, each trunk is directed forwards to the vena cava. They open into the posterior part of the cava, cither singly, or eonjointly with those of the opposite side. On the left side the veins are longer than on the right, and pass beneath the aorta.

Around the transverse processes, and beneath the psoas muscle, the lumbar veins communicate freely with one another, with the ilio-lumbar, and sometimes with the common iliac, so as to form a plexus of veins. Issuing from the upper part of the plexus is a small branch, the ascending lumbar vein, which joins the azygos vein of the corresponding side of the body. 


\section{CAVITY OF THE PELVIS.}

Tre cavity of the pelvis is a part of the general abdominal space ( $p$. 431 , and is situate below the brim or inlet of the true pelvis.

Boundaries. The space is surrounded by the firm bony ring of the pelvic bones: it is bounded behind by the sacrum and the coceyx, with the pyriformis muscles and the sacro-sciatic ligaments; and laterally and in front, by the innominate bones covered by the obturator muscles.

Inferiorly, or towards the perinæum, the cavity is limited by the fascia reflected from the wall to the viscera, and by the levatores ani and coccygei muscles: only in this last direction, where the bounding structures are movable, can alterations be made in the size of the space.

Contents. In the pelvis are contained the urinary bladder, the lower end of the large intestine or the rectum, and some of the generative organs, according to the sex. All the viscera possess ressels, nerves, and lymphatics; and the serous membrane is reflected over them.

\section{Section I.}

\section{PELVIC FASCIA AND MUSCLES OF THE OUTLET.}

Ox the wall of the pelvis is a thin fascia (pelvic) which extends from the brim to the outlet, and covers the obturator muscle.

Dissection. To bring into view the pelvic fascia, the internal iliac vessels, and the psoas (if this has not been removed in the dissection of the lumbar plexus), are to be taken away on the left side of the body. The obturator vessels and nerve are to be cut through on the same side; and the peritoneum being detached from the wall of the pelvis, the fascia will be seen on scraping away with the handle of the scalpel a large quantity of fat. By this proceeding the membrane is dissected in its upper half, or as low as the situation of a piece of fascia (recto-resical) which is attached to the viscera.

To display the lower half, the student must ra: se the outlet of the pelvis ; and, should the perinæum be undissected, the fat must be taken from the ischio-rectal hollow. The lower part of the fascia will now appear on the outer side of that fossa, as it covers the obturator muscle.

An additional step for showing the arrangement of the fascia may be taken, by removing the external obturator muscle and the obturator membrane, and then scraping away through the thyroid hole the obturator internus muscle, so as to look at the fascia through that aperture. 
The PELVIC FAscia is a thin membrane in close contact with the obturator muscle, and is fixed to the bone around the attachment of the fleshy fibres, so that it might be called the sjecial fascia (obturator) of that muscle.

Superiorly it is fixed into the brim of the pelvis for a short distance at the lateral aspect of the cavity. In front of that spot it does not extend so high as the brim, but is inserted into the bone around the attachment of the muscle, except opposite the hole for the obturator vessels and nerve, where it is united witl the obturator membrane. Inferiorly the fascia is attached to the hip hone along the side of the pubic arch, and to the margin of the great sacrosciatic ligament where the obturator internus issues from the pelvis.

The outer surface of the fascia is in contact with the obturator muscle. The inner surface corresponds above with the cavity of the pelvis, and below, with the ischio-rectal fossa. With this surface the thin membrane (recto-vesical) supporting the viscera of the pelvis is united ; the place of union being indicated, on looking into the pelvis, by a whitish line near to, and on a level with the ischial spine. At the posterior border of the obturator muscle the fascia is joined by a thin membrane (fiascia of the pyriformis) which covers the pyriformis muscle and the sacral plexus, but is beneath the iliac vessels by branches of which it is perforated.

The recto-vesical fascia may be now seen in part; but it will be better displayed after the hip bone has been taken away.

Dissection. To obtain a side view of the pelvis it will be necessary to detach the left innominate bone. The pelvic fascia is first to be separated from the bone and the obturator muscle. The innominate bone is next to be sawn through, in front, rather external to the symphysis, and, behind, at the articulation with the sacrum. After the bone has been pulled somewhat away from the rest of the pelvis, the ischial spine with the rectovesical fascia attached to it may be cut off with a bone-forceps; and the rest of the bone may be then removed by cutting through the pyriformis muscle, the vessels and nerves passing through the sacro-sciatic notch, and any other structure that may retain it.

A block is afterwards to be placed beneath the pelvis. The bladder is to be moderately distended with air through the ureter, and the urethra is to be tied. Some tow is to be introduced into the rectum, also into the vagina if it is a female pelvis; and a small piece is to be placed in the pouch of peritoneum between the bladder and the reetum. After the viscera are thus made prominent, the ischial spine and the recto-vesical fascia should be raised with hooks whilst the levator ani and coceygeus muscles below it are cleaned.

Parts closing the pelvis below. In ardition to the recto-vesical fascia, the following parts close the pelvic cavity on each side, between the sacrum and the pubic symiphysis.

Behind, the student will meet with the pyriformis passing through the great sacro-sciatic notch, with the gluteal vessels and nerve above it. Next comes the coccygeus musele, c, on the small sacro-sciatic ligament, between the ischial spine and the coccyx: one horder of the muscle reaches towards the pyriformis, the other to the levator ani ; and between its hinder border and the pyriformis lie the sacral plexus of nerves $\left({ }^{1}\right)$, and the sciatic and pudic vessels.

The greater part of the rest of the pelvic outlet is closed by the levator ani, D, which extends forwards from the coceygeus to the symphysis pubis. 
It meets its fellow inferiorly, but the muscles of opposite sides are separated in front by the urethra, with the vagina in the female; and the interval between them is closed by the triangular ligament of the perineum, $\mathrm{H}$.

The Coccygeus muscle (fig. $173,{ }^{\circ}$ ) is flat and triangular, and assists to close the outlet of the pelvis. It arises from the upper part of the ischial spine, and some fibres are attached to the small sacro-sciatic ligament. Widening as it passes inwards the muscle is inserted into the side, and the contiguons anterior surface of the coceyx, and into the side of the lower piece of the sacrum.

Fig. 173.

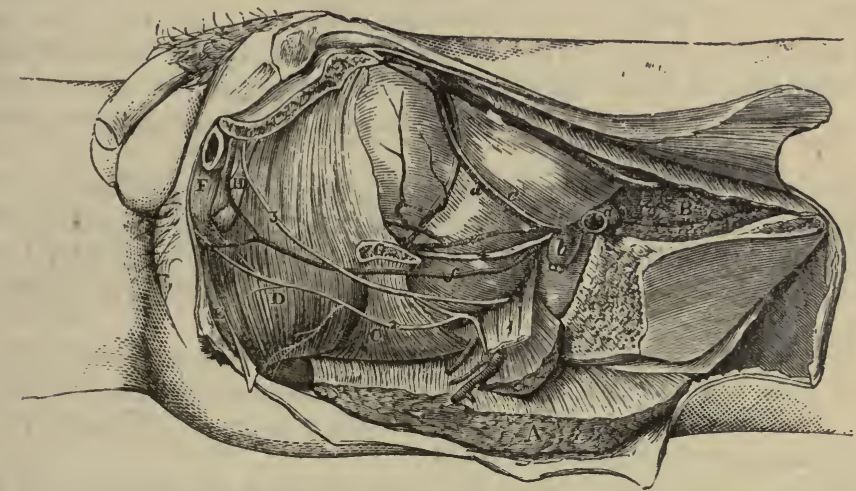

Side View of the MUSCles in the outret of the Pelvis (11lustrations of Dissectious).

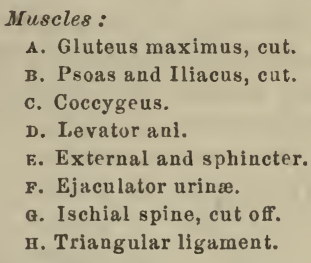

A. Gluteus maximus, cut.

B. Psoas and Iliacus, cut.

c. Coccygeus.

D. Levator ani.

E. External and sphincter.

F. Ejaculator urinæ.

G. Ischial spine, cut off.

H. Triangular ligament.

Arteries :
a. Exterual iliac artery, cut.
$b$. Its accompanying vein.
c. Obliterated hypogastric cord.
d. Upper vesical, and
e. Lower vesical.
$f$. Internal pudic.
1. Sacral plexus.
2. Inferior hemorrhoidal.
3. Pudic.

Nerves:

The inner surface looks to the pelvis, and is in contact with the rectum on the left side; the opposite surface rests on the small sacro-sciatic ligament. The upper or hinder border is contiguous to the pyriformis muscle, vessels and nerves intervening; and the anterior or lower border is parallel with the levator ani muscle.

Action. Uniting in its action with the hinder fibres of the levator ani, the muscle will draw slightly forwards the coccyx.

The Levator ANI (fig. $173,{ }^{D}$ ) is a thin flat muscle, which is attached above to the side of the pelvis, and descends below into the outlet of the eavity, where it joins its fellow and supports the viscera.

It arises anteriorly by fleshy fibres from an oblique line above the obturator internus ; lower down from the fascia on that muscle, and from the back of the triangular ligament. Posteriorly it is fixed to the lower and inner surface of the ischial spine. And between those osseous attac.'1ments the musele takes origin from the under part of the recto-vesical tiascia, but not in a straight line. All the fibres are directed downwards to 
be inserted after the following manner: 'The anterior, the longest, descend by the side of the prostate and join, in front of the rectum, with the muscle of the opposite side in the central point of the perinæum; the middle fibres blend with the side of the rectum; whilst the posterior meet the opposite muscle behind the gut, and are attached in part to the side of the coccyx (p. 390).

The anterior fibres of the levator are in contact with the triangular perineal ligament $;^{1}$ and there is an interval between the two muscles, which allows the urethra, with the ragina in the female, to pass from the pelvis. The posterior border is parallel to the coccygeus muscle. "The upper surface is contiguous to the recto-vesical fascia ; and the under surface looks to the perinaum (ischio-rectal fossa). 'The two muscles, by their union, form a fleshy layer or diaphragm across the outlet of the pelvis, similar to that which separates the abdomen from the chest; this partition is convex below and concave above, and gives passage to the rectum.

Action. By the union of the muscles of opposite sides below the urethra this tube can be raised, and compressed during their contraction. Whilst the urine is flowing the fibres are passive, but towards the end of micturition they contract suddenly, and help the other muscles in clearing the passage.

As the levatores descend by the side of the vesiculæ seminales, and the prostate, they will compress and evacuate the contents of those viscera.

The hindmost fibres, which are fixed to the coccyx, will assist the coccygeus in moving forwards that bone.

Dissection. The recto-vesical fascia will be seen by detaching the fleshy fibres of the levator ani and the coccygeus at their origin, and throwing both downwards. The thin membrane descends on the levator ani to the side of the bladder and the rectum, and sends downwards sheaths around the prostate and the gut. 'To demonstrate those sheaths one incision is to be made along the prostate, and another along the lower end of the rectum, below the fascia; and the tubes are to be isolated from the viscera.

The recto-vesical fascia supports and partly invests the viscera of the pelvis. Covering the pelvic surface of the levator ani it is fixed above, like that muscle, to the wall of the pelvis in front and behind, and between those attachments it joins the pelvie fascia. Below it meets the fascia of the opposite side, in the centre of the pelvis, and forms a partition across the cavity, like that of the levatores ani, which is perforated by the bladder and the rectum. 'The partition is supported anteriorly by being fixed to the pubes, and posteriorly, where it blends with the fascia on the cocygeus, by being inserted into the coccyx : it is concave above and convex below, and divides the eavity of the pelvis from the perineal space. This septal piece is attuched to the viscera which pierce it, forming ligaments for them; and from the under surface tubes are prolonged on the rectum and the prostate.

The sheath on the rectum incloses the lower three inches of the intestine, and gradually becomes very thin towards the anus; between it and the intestine are interposed the branches of the upper hemorrhoidal vessels, with a layer of fat.

1 The anterior part of the muscle which descends by the side of the prostate, and unites with its fellow below the inembranous part of the urethra, thus supporting that canal as in a sling, is named sometimes levator seu compressor prostatee. 
On the prostate the sheath is thinner than on the rectum, and is continued forwards to the apex of that body, where it blends with the triangular ligament of the urethra: it is separated from the eapsule of the prostate by a plexus of veins, and by some small arteries.

In the female the fascia has much the same arrangement as in the male; but the vagina perforates the membrane, and receives a tube from it, like the prostate.

The true ligaments of the bladder are two on each side, anterior and lateral, and are parts of the recto-vesical fascia.

The anterior reaches from the posterior part of the pubes to the upper surface of the prostate, and the neck of the bladder; it is a narrow white band, and incloses some muscular fibres of the bladder. Between the ligaments of opposite sides, the recto-vesical fascia dips down to reach the triangular ligament of the perinæum, and closes the pelvis between the levatores ani.

The lateral ligament is a piece of the same fascia, which is fixed to the upper border of the prostate gland, to the side of the bladder close above the vesicula seminalis, and to the back of the bladder between the vesicula ; from this part of the fascia an offset is continued downwards over the vesicula seminalis, so as to join a like piece from the other side, and form a sheath for those bodies.

There are other ligaments of the bladder (false ligaments), which are derived from the peritoneum investing it, and will be described in the following Section.

Ligament of the rectum. On each side of the rectum is a strong wide piece of the recto-vesical fascia, which is connected externally to the ischial part of the hip bone, and supports that viscus like the bladder.

\section{Section II.}

\section{CONNECTIONS OF THE VISCERA IN THE MALE.}

Directions. If the student dissects a female pelvis, he will find the description of it at page 509.

Contents and position (fig. 174). The viscera in the cavity of the male pelvis are, the lower end of the large intestine (rectum); the bladder with its excretory tube-the urethra; together with some generative organs. These have the following relative situation:-

The rectum $(k)$ is behind all, and takes a curved course, with the convexity backwards, along the front of the sacrum and coecy $x$. The bladder $(a)$ is placed in the concavity of the reetum, its neck being surrounded by the prostate gland $(b)$; and the urethra $(d)$ curves forwards from it above the intestine. Beneath the bladder-between it and the rectumare the little seminal sacs $(g)$ with the vasa deferentia $f$. Some of these organs are partly surrounded by the peritoneum.

Dissection. All the recto-vesical fascia, except the anterior true ligament of the bladder, may be taken from the prostate and rectum. The obliterated (hypogastric) cord from the internal iliac artery should be followed forwards along the bladder from the back of the pelvis; and the branches of the same artery to the bladder should be cleaned. When the 
fat has been cleared from the rectum without injuring its arteries, the pouch of the peritoneum, in which tow has been placed, will be brought into view, with the ureter $(h)$ passing to the bladder across its side.

The part of the lladder below the peritoneum is to be cleaned, and the vas deferens $(f)$, which lies on the lateral aspect of the viscus, is to be followed down to the seminal sac. Take away with care the remains of the sheath of the vesicula seminalis, defining at the same time the ras deferens inside the latter.

The peritoneum does not envelop the viscera in the pelvis so completely as in the upper part of the abdomen. After partly surrounding the upper portion of the rectum, and fixing it by a process-meso-rectum, the membrane elothes the back of the bladder, projecting for some way between this viseus and the rectum, where it forms the recto-vesical ponch : on each side the serous membrane is arresterl by the obliterated hypogastric artery, and gives rise to a fold, the posterior ligament of the bladder. It covers the posterior surface, and each lateral region of the bladder as far forwards as the obliterated hypogastric ; but at that cord it is reflected to the wall of the pelvis and abdomen. All the anterior surface of the bladder is therefore uncovered by peritoneum; and when the bladder is distended it rises above the pubes, so as to allow of its being punctured in front without injury to the serous membrane.

The recto-vesical pouch is wide behind, where it corresponds with the interval between the iliac arteries, and is narrow in front between the rectum and the bladder. Anteriorly it extends slightly into the interval between the vesiculæ seminales, and ends usually about one inch and a half from the tip of the coceyx. The distance of the pouch from the anus is commonly about four inches; but it will vary with the state of the bladder, for if this viscus is distended the peritoneum will be raised, and removed farther from the end of the intestine.

False ligaments of the bladder. Where the peritoneum is reflected from the bladder to the pelvic wall, it gives rise to a wide piece of membrane, which constitutes the false ligaments of that viscus, though without any subdivision of it into pieces. These are said to be five in numbertwo posterior, two lateral, and one superior.

The posterior ligament (one on each side) reaches from the back of the pelvis to the bladder, and contains the obliterated hypogastric artery, the ureter, and smaller vessels, and nerves. Between these is the hollow of the recto-vesical pouch.

The lateral ligament, also one on each side, the widest, is reflected from the side of the bladder to the iliac-fossa and the wall of the pelvis. Along its line of attachment to the bladder is the obliterated hypogastric artery.

The supevior ligament is prolonged from the top of the bladder to the abdominal wall, along the remains of the obliterated hypogastric vessels.

The rECTUm, or the lower part of the great intestine (fig. 174, $k$ ) extends from the articulation between the sacrum and the left hip bone to the anus, and is kept in place by the peritoneum, the recto-resical fascia, and the levatores ani. The intestine is about eight inehes long, and has a winding course, for it follows the curve of the sacrum and coccyx : it is divided into three parts-upper, middle, and lower.

The upper part, longer than the others, extends obliquely from the sacro-iliac articulation to the centre of the thirl piece of the sacrum. Surrounded almost entirely by the peritoneum, it lies against the sacrum, and on the pyriformis muscle and sacral plexus of the left side. In contact 
with it, are the branches of the left internal artary, and the le?t ureter. In some bodies this part of the intestine is much curved to the right side. $^{1}$

The middle piece lies beneath the bladder, and reaches to the tip of the coccyx: it is about three inches in length, and is covered by peritoneum on the front and sides above, but only in front below. Resting on it is the triangular part of the bladder, with the back of the prostate and the vesieula seminales and vasa deferentia; and beneath it are the sacrum and coceyx. On each side is the coccygeus muscle.

Fig. 174.

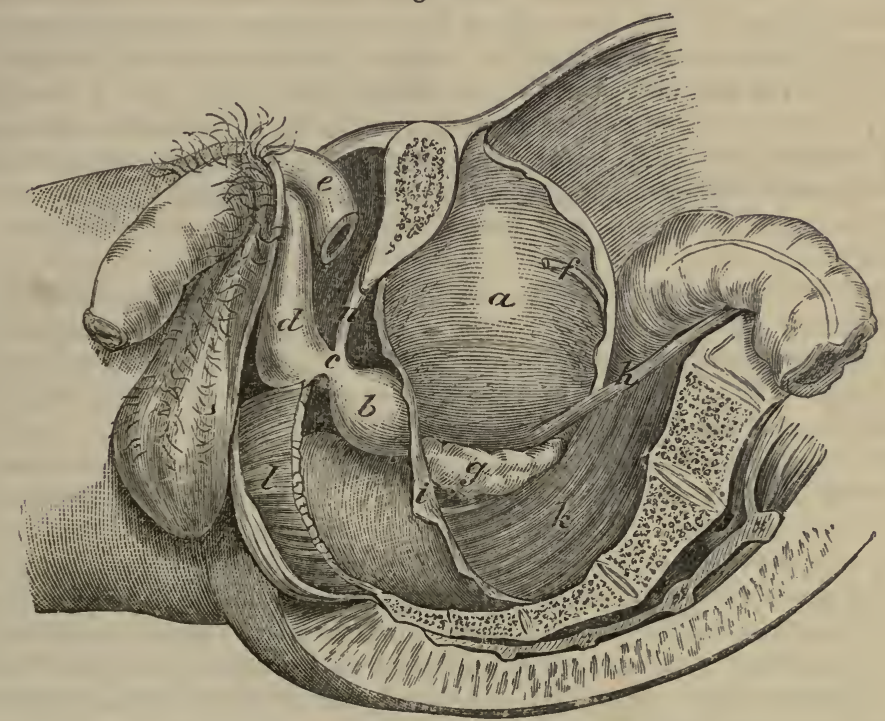

Side view of the Disgected Malb Pelvis.

a. Urinary bladder.

b. Prostate.

c. Membranous part of the urethra.

d. Spougy part of the urethra.

$e$ Crus penis, detached.

$f$. Vas deferens. g. Vesicula seminalis.

h. Ureter.

i. Recto-vesical fascia.

$k$. Rectum.

l. Levator ani, cut.

The lower part is about an inch and a half long, and is curved from the tip of the coceyx to the anus: at first it is dilated, but at the anus it is contracted. This end of the intestine is without peritoneal covering, and is supported by the lower part of the triangular ligament of the urethra, and by the levatores ani muscles.

Above the extremity of the rectum (in this position of the body) are the fore part of the prostate, the membranous part of the urethra, and the

' It is not uncommon to see the rectum on the right side of the sacrum. In the dissecting-room of University College in one winter (1854-1855), I saw three examples of the rectum in that situation. In two bodies the lower end of the left colon crossed the spine at the top of the sacrum, and the rectum descended through the pelvis, on the right of the middle line, to the end of the coccyx. In the third the large intestine crossed the spine twice, once at top of the sacrum, and again about the middle of that bone. 
hulb of the corpus spongiosum urethra; but as the gut recedes from the urethra there is an angular interval left between them. The levatores ani muscles descend on its sides, and unite beneath it, supporting it in a sling; and the sphincter muscles surround the aperture. Sometimes the end of the intestine within the anus is very much enlarged, especially in women and old men; and in that condition in the male it rises up on each side of the prostate.

The URINARY BLADDER (vesica urinaria) is situate in the fore part of the pelvis (fig. 174, a), and is the receptacle for the fluid secreted by the kidneys.

When the bladder is contracted it is flattened, and of a triangular form, and lies against the anterior wall of the pelvis; but when distended it becomes of a conical shape, with the larger part towards the rectum. and the apex to the abdominal wall. In distension during life it is slightly curved over the anterior part of the pelvis, as it projects above the bone; and if a line through its centre were prolonged, it would tonch anteriorly the abdominal wall somewhere (according to the distension) between the umbilicus and the pubes, and posteriorly the end of the sacrum or the coceyx.

The position and form of the bladder are not the same in early life as in the adult. For in the ehild this viscus rises above the brim of the pelvis into the hypogastric region of the abdomen, and the cervix is the lowest part. But in the adult the bladder is contained within the space inclosed by the pelvic bones, and the base projects inferiorly.

The organ is maintained in position by the recto-vesical fascia and the peritoneum, which form its ligaments (pp. 502 and 504). The connections of the bladder moderately full are the following:-

The summit or apex is rounded, and from its anterior part three ligamentous cords are prolonged to the umbilicus; the central one of these is the remains of the urachus; and the two lateral are formed by the obliterated liypogastric arteries. If the bladder is distended the apex is above, but otherwise below the pubes. All the surface behind the obliterated vessels is covered by peritoneum.

The base (fundus) is large, and rests on the middle piece of the rectum: in the state of emptiness of the blalder the base is scarcely prominent, but in distension of the visens, it extends lower, and becomes widened. Connected with the fundus are the vesiculæ seminales and the vasa deferentia; and between these is a triangular space, from which the peritoneum is partly absent.

Surfaces of the body. The front of the body is in contact with the posterior surface of the symphysis pubis, and with the abdominal wall if it is distended, and is altogether free from peritoneum; whilst the posterior surface is entirely covered by the serous membrane, and is toucherl often by the small intestine.

Extending along the upper part of each lateral region is the obliterated hypogastric vessel; and running down behind this is the vas deferens, which passes internal to the ureter. Near the under part the ureter enters the bladder. All the side of the bladder behind the hypogastric vessel is covered by peritoneum, but the rest is uncovered.

The neck (cervix) is the narrow anterior part of the bladder that joins the urethra: in the state of contraction it is the lowest part of the viscus, but in distension it is above the level of the fundus. It is surrounded by the prostate gland. 
The ureter (fig. $174, h$ ) erosses the common or the external iliac artery, and forms an arch below the level of the obliterated hypogastric vessel; it enters the bladder near the lower part, and somewhat on the side, at the distance of one inch and a half or two inches from the prostate gland.

The PRostate GLAND (fig. $17 t, b$ ) surrounds the neck of the bladder. It is placed about an inch below the level of the symphysis pubis, and is supported by the rectum. Its shape is conical with the base turned backwards, and its size equals nearly that of a large chestnut. In the present position of the pelvis, a line from the apex through the middle of the gland would be directed obliquely downwards and backwards towards the end of the sacrum; but in the erect state of the body, upwards and backwards from the triangular ligament.

The upper surface is about an inch below the symphysis pubis, and is connected to it by the anterior true ligaments of the bladder. On this surface are branches of the dorsal vein of the penis.

The under surface has the greatest extent, and is contiguous to the rectum; this is the part that is felt by the finger introduced into the bowel through the anus.

The apex touches the posterior layer of the triangular ligament; and the base surrounds the neck of the bladder, and the vesiculæ seminales with the vasa deferentia.

The prostate is enveloped by a sheath obtained from the recto-vesical fascia (p. 502), and a plexus of veins (prostatic) surrounds it. Through the gland the urethra takes its course to the penis; and the ejaculatory ducts pierce it obliquely to open into the urethra (fig. $180, f$ ). The size of the prostate alters much, and in old men it may acquire a considerable magnitude.

The VESICULA SEMINALEs (fig. $174, g$ ) are two small sacculated bodies, each about two inches long, between the under part of the bladder and the rectum. Each is pyramidal in form, and has the larger end turned backwards towards the ureter, whilst the smaller is surrounded by the prostate. Along the inner side is the vas deferens. At the prostate gland the vesicula approach one another, only the vasa deferentia intervening; but further backwards they diverge, and inclose with the pouch of the peritoneum the triangnelar space at the under aspect of the bladder. The vesiculæ are contained in a membranous sheath, which is derived from the recto-vesical fascia, and is lined by involuntary muscular fibres.

The VAS DEFERENS or the excretory duct of the testis (fig. 174, $f$ ) in its course to the urethra enters the abdomen by the internal abdominal ring; and crossing the obliterated hypogastric artery, is directed inwards along the side and under part of the bladder to the base of the prostate, where it forms the common ejaculatory duct by joining with the duct from the vesicula seminalis. 'The position of this tube to the external iliac artery has been noticed; on the bladder it passes internal to the ureter and the vesicula of the same side. By the side of the vesicula the duct is much enlarged, and is saceulated.

The URETHRA is the excretory passage for the urine and semen (fig. 174), and reaches from the blarder to the end of the penis. In length it measures about eight inches, and it presents one or two curves according to the state of the penis. At first the canal is directed forwards through the triangular ligament of the perinæum to the body of the penis, forming a large curve with the eoncavity to the pubes. Thence to its termination the urethra is applied to the penis ; and whilst this body remains pendent 
the canal forms a second bend with the concavity downwards, but if the penis is raised the tube makes but one curve. The canal is divided into three parts-prostatic, membranous, and spongy.

The prostatic part (b) is contained in the prostate gland, and receives its name from that cireumstance. Its length is about one inch and a quarter, and in the ereet posture it is inclined downwards to the triangular perineal ligament. Its connections are the same as those of the gland (p. 507).

The membranous part (c), about three-quarters of an inch long, intervenes between the apex of the prostate and the front of the perineal triangular ligament. It slants downwards in the erect posture to the fore part of the triangular ligament; and as the bulb of the next portion of the urethral tube is directed backwards below it, the under part measures only half an inch.

This division of the urethra is the weakest: but it is supported by the triangular ligament $(n)$. Surrounding it are the muscular fibres of the constrictor urethræ; and close below it are Cowper's glands with the rectum.

The spongy part $(d)$ is so named from its being surrounded by a cellular and vascular structure. It is applied to and assists to form the body of the penis, and terminates anteriorly in the orifice named meatus urinarius in the end of the glands. It is the longest part of the urethra, and measures about six inches. At its commenerment this division of the excretory eanal is covered for two inches by the ejaculator urinæ muscle (fig. 130).

The curve of the urethra is the fixed bend at the inner extremity, which lies below the pubes. It extends from the bladder to an inch and a half in front of the triangular ligament, and consists of the prostatic and membranous portions, with a fourth of the spongy part. Its covexity, which is turned downwards, is greatest at the fore part of the triangular ligament in the ereet posture: and from this point it curves up and back to the bladder, and up anteriorly to the penis.

It is surrounded by voluntary and involuntary museular fibres: thus, behind the ligament, by the involuntary muscular tissue of the prostate; within the ligament by the voluntary eonstrictor urethra, with a thin involuntary layèr inside that muscle; and before the ligament by the voluntary ejaculator urinæ.

Its size is smallest where the tube pierces the perinxal ligament $(n)$, and lies between the layers; and is largest in the middle of the prostatic part.

Dissection. The tegumentary covering of the penis may be removed, to see the component parts of that body ; and after its removal the spongy part of the urethra will be better seen: the teguments should be replaced after the part has been learnt.

The PENis is attached to the fore part of the pelvis, and hangs in front of the scrotum. It is construeted of two firm fibrons bodies (fig. 174,e) named corpora cavernosa, which are filled with a plexus of vessels, and make up the principal part. Below these is a soft spongy substance (corpus spongiosum) which surrounds the urethra, and forms the heal or the glans penis. The tegumentary investment, which covers the whole, is noticed at p. 407 .

The body of the penis is grooved above and below along the middles line, and is covered anteriorly by the glans penis; along its under surface 
the urethra is conducted. Besides the attachment of the corpora cavernosa to the bone, the borly of the penis is connected with the front of the symphysis pubis by the suspensory ligament.

The corpus spongiosum urethra incloses the urethral canal in front of the triangular ligament, and forms the head of the penis. It is a vascular and erectile texture, like the corpora carernosa, but is much less strong. Commencing posteriorly by a dilated part-the bulb, it extends forwards around the urethra to the extremity of the penis, where it swells into the conical glans penis.

The bulb (fig. 174, d) is directed backwards, slightly, below the membranous part of the urethra, and is fixed by fibrous tissue to the front of the triangular ligament. The ejaculator urinæ muscles cover it. This enlargement presents usually a central depression, with a bulging on each side, and is subdivided into two lobes.

The glans penis (fig. $181, l$ ) is somewhat conical in form, and covers the truncated end of the corpora cavernosa. Its base is directed backwards, and is marked by a slightly prominent border-the corona glandis; it is sloped obliquely along the under aspect, from the apex to the base. In the apex is a vertical slit, in which the urethral canal terminates; and below that aperture is an excavation, which contains the piece of the teguments named franum preputii.

\section{Section III.}

\section{CONNECTIONS OF THE VISCERA IN THE FEMALE.}

In the pelvis of the female are contained the lower end of the intestinal tube, and the bladder and urethra, as in the male; but there are in addition the uterus with its accessories, and the vagina.

Position. The rectum is posterior to the rest as in the male pelvis, and forms a like curve. In the concavity of the bent intestine lie the uterus with its appendages, and the tube of the ragina. And in front of all are the bladder and the urethral canal. Thus there are three tubes connected with the viscera in this sex, viz., the urethra, the vagina, and the rectum; and all are directed forwards, one above another, to the surface.

Directions. The description in Section I. (p. 499) must be used for instructions respecting the removal of the innominate bone, and the distension of the viscera; also for the muscles of the pelvic outlet and the anatomy of the fascia. After the student has learnt the muscles and the fascia, p. 499, which are nearly alike in both seres, he may make the following special dissection of the viscern of the female pelvis.

Dissection. On taking away the recto-vesical fascia and much fat, the viscera will come into view. To maintain the position of the uterus, raise it up with a piece of string passed throngh the upper part. The reflections of the peritoneum on the viscera are to be preserved; and a piece of cotton wool is to be placed between the rectum and the uterus.

The obliterated cord of the internal iliac artery is to be followed on the bladder; and the ureter is to be traced forwards by the side of the uterus to the bladder. Afterwards the urethra, the ragina, and the rectum are 
to be cleaned and partly separated from one another at the anterior part of the pelvis; but the arteries on the rectum are to be preserved.

The peritoneum gives a partial covering to the viscera, as in the male pelvis. Investing the upper part of the rectum, and forming behind it the meso-rectum, the membrane is continued to the posterior part of the vagina, and the back of the uterus. It covers the posterior, and the greater part of the anterior surface of the uterus, and can be tracerl to the bladder without again touching the vagina: on each side of the uterus it forms a wide fold (broad ligament), which attaches that viscus to the wall of the abdomen. As the peritoneum is followed upwards it may be observed to cover the posterior surface of the bladder, and the lateral part behind the position of the obliterated hypogastric artery.

In the pelvis the serous membrane forms the following ligaments for the uterus and bladder.

The broad ligament of the uterus (fig. 175) passes from the side of the uterus to the wall of the abdomen, and supports that organ. By its position across the pelvis, it divides the cavity into an anterior and a posterior portion: in the former are placed the bladder, urethra, and vagina; in the latter the upper part of the rectum, and the small intestine when it reaches the pelvis.

Each ligament shows traces of a subdivision into three pieces, corresponding with the bodies contained between its two layers. 'Thus there is a posterior piece belonging to the ovary and its ligament, $\mathrm{L}$; an anterior, near the upper part, which is appropriated to the round ligament, $\mathbf{x}$; and a middle piece, the highest of all, surrounds the Fallopian tube, M.

Anterior and posterior ligaments of the uterus. As the peritoneum is reflected from the rectum to the uterus, and from the uterus to the bladder, it forms two anterior and to posterior folds or ligaments. The anterior or vesico-uterine pair is smaller than the posterior or recto-uterine.

The recto-uterine pouch corresponds with the recto-vesical in the male. On each side it is bounded by the obliterated hypogastric artery : and below, it reaehes beyond the uterus, so as to touch the back of the ragina.

The false ligaments of the bladder are the same as in the male, and are five in number, viz., two posterior, two lateral, and a superior : they are all blended in one large piece of peritoneum that reaches firom the bladder to the side and front of the pelvis. In the female the posterior ligament, containing the vessels of the bladder, is less marked than in the male.

The REстUм (fig. $175,{ }^{\mathrm{K}}$ ) is not so curved in the female as in the male, and is generally larger. Descending along the middle of the sacrum and coccyx to the anus, the intestine is divided into three parts :-

The first part ends over the third piece of the sacrum, and is enveloped by the peritoneum, except posteriorly: its colinections are similar to those of the rectum in the male, p. 504 .

The middle part reaches to the tip of the eoceyx, and has the vagina above and in contact with it. The peritoneum extends on the front for a short distance.

The lower part curves to the anus away from the vagina so as to leave between the two a space, which corresponds, on the suriace of the body, with the part of the perinxum between the anus and the vulvil. The levatores ani are on the sides, and unite below it, and the splineter muscles surround the extremity.

The uterus (fig. $175,{ }^{\circ}$ ) is somewhat conical in shape, and flattenerl from before backwards. Unless enlarged, it lies below the brim of the 
pelvis, between the bladder and the rectum; and it is retained in place by the ligaments. Its wider end is free and placed upwards, and the lower end communicates with the vagina.

This viscus is directed forwards, so that its position is oblique in the cavity of the pelvis; and a line through its centre would correspond with the axis of the inlet of the pelvic eavity.

Fig. 175.

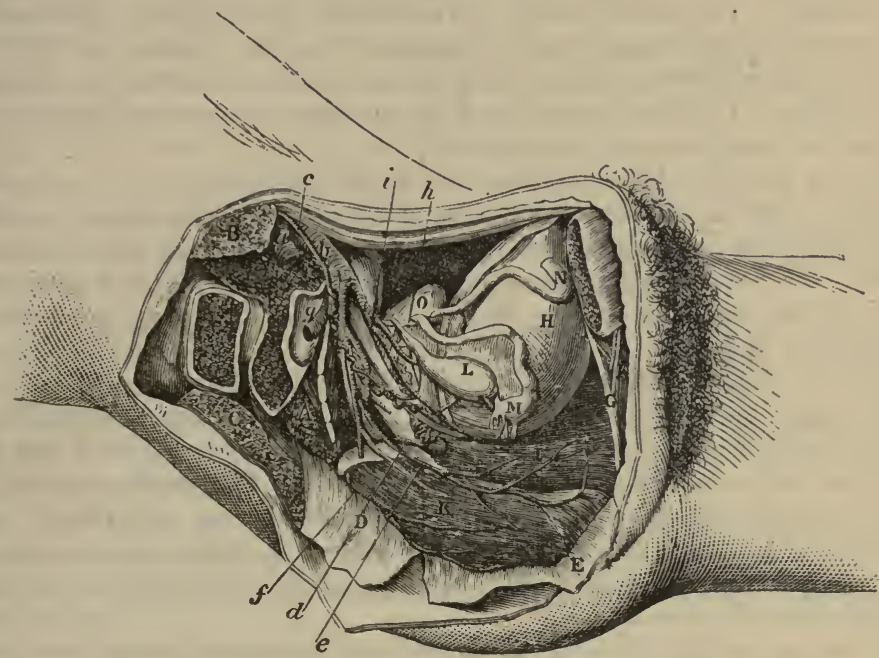

Side View of the Female Pelivis. (Illustrations of Dissections.)

Muscles and Viscera:

A. Pyriformis muscle, cut.

B. Large psoas, cut.

C. Gluteus maximus, cut.

D. Coccygeus, and $\mathrm{E}$, Levator ani, thrown down.

F. Sphincter vaginæ.

G. Urethra.

н. Urinary bladder.

I. Vagina.

K. Rectum.

L. Ovary and its ligament.

M. Fallopian tube.

s. Round ligament.

o. Uterus.

\section{Arteries :}

a. External iliac.

b. Internal iliac.

c. Middle sacral.

d. Uterine.

e. Vaginal.

$f$. Upper hæmorrhoidal.

g. Gluteal, cut.

$h$. Obliterated hypogastric.

i. Inferior vesical.

The anterior flattened surface is covered by peritoneum, except in the lower fourth where it is in contact with the bladder. The posterior surface, rounded, is invested altogether by the serous membrane.

The upper end (fundus) is the largest part of the organ, and is in contact with the small intestine. The lower end, or the neck (cervix) is received into the vagina.

'To each side are attached the broad ligament with the Fallopian tube, the round ligament, and the ovary.

The Fallopian tube, in, four inches long, is contained in the upper or free border of the ligament. One end is connected to the upper angle of 
the uterus, whilst the other is loose in the cavity of the pelvis. At the uterine end the tube is of small size, but at the opposite extremity it is dilated like a trumpet, and fringed, forming the corpus fimbriatum.

The round or suspensory ligament, $\mathbf{N}$, is a fibrous cord about five inches long, which is directed outwards through the internal abdominal ring and the inguinal canal to end in the groin. 'This cord lies over the obliterated hypogastric, and the external iliac artery; and it is surrounded by the peritoneum, which accompanies it a short way into the canal.

The ovary, $\mathrm{L}$; is placed nearly horizontally, and bulges at the posterior aspect of the broad ligament ; it is connected to the uterus at the inner end by a special fibrous band, one inch and a half in length, the ligament of the ovary. Its form is oval, and its margins are turned forwards and hackwards. Its size is very variable.

The VAGINA (fig. $175,{ }^{\mathrm{I}}$ ) is the tube by which the uterus communicates with the exterior of the body. It is somewhat cylindrical in shape, though flattened from above down; and its length is about five inches. As it follows the bend of the rectum it is slightly curved; and its axis corresponds at first with the centre of the outlet, but higher up with that of the cavity of the pelvis.

Above the vagina are the base of the bladder, and the urethra; and beneath or below it is the rectum. To each side is attached the rectovesical fascia, which sends a sheath along the lower half of the tube. The upper end receives the neck of the uterus by an aperture in the anterior or upper wall ; and the lower end, the narrowest part of the canal, is encircled by the sphincter vaginæ muscle. A large plexus of veins surrounds the vagina. In children, and in the virgin, the external aperture is partly closed by the hymen.

The BLADDER (fig. $175,{ }^{\mathrm{B}}$ ) is placed at the anterior part of the pelvis, ahove the vagina and in contact with the back of the pubes. Its position and connections so closely resemble those of the bladder in the male borly, as to render unnecessary any further description of them (p. 506). The chief differences in the bladder of the two sexes are the following.

In the female the bladder is larger than in the male, and its transverse exceeds its vertical measurement. The base is of less extent, and is in contact with the vagina and the lower part of the uterus; and it does not reach below the orifice of the urethra. On the side of the viscus there is not any vas deferens; and the prostate does not project around the neck.

The ureter has a longer course in the female than in the male pelvis before it reaches the bladder. After crossing the internal iliac vessels, it passes by the neck of the uterus ere it arrives at its destination.

The urethra (fig. $175,{ }^{a}$ ) is a small narrow tube about one inch and a half long, which curves slightly below the symphysis pubis, the concavity being upwards. Its situation is above the vagina, and its external opening is placed within the vulva.

In its course to the surface it is embedded in the tissue of the vaginal wall, and perforates the triangular ligament of the perinaum. It is surrounded by the muscular fibres of the constrictor and orbicularis urethre (p. 398); and corresponds with the two hinder parts (prostatic and membranous) of the male urethra. A plexus of veins surrounds the urethra as well as the vagina. 


\section{Section IV.}

\section{VESSELS AND NERVES OF THE PELVIS.}

Is the pelvis are contained the internal iliac vessels, and their branches to the viseera; the sacral plexus and its nerves; and the sympathetic nerve. 'This Section is to be used by the dissectors of both the male and female pelvis.

Directions. The internal iliac vessels are to be dissected on the right side. But the air should be previously let ont of the bladder; and this viseus and the rectum, with the uterus and the vagina in the female, should be drawn aside from their situation in the centre of the pelvis.

Dissection. The loose tissue and fat are to be removed from the trunks of the vessels, as well as from the branches of the artery that leave the pelvis, or supply the viscera ; and the obliterated cord of the hypogastric artery is to be traced on the bladder to the umbilieus.

With the vessels are offisets of the hypogastrie plexus of nerves, but these will probably not be seen; but in dissecting the vessels to the bladder and rectum, branches of the sacral spinal nerves will come into view. The veins may be removed in a general dissection, to make elean the arteries.

When the vessels are prepared the bladder may be again distended, and the viscera replaced.

The internal iLIAC ARtery (fig. $176, g$ ) is one of the trunks resulting from the division of the common iliac artery, and furnishes branches to the viscera and wall of the pelvis, to the generative and genital organs, and to the limb.

In the adult the vessel is a short trunk of large eapacity, which measures about an inch and a lialf in lengtl. Direeted downwards as far as the sacro-sciatic notch, the artery terminates generally in two large pieces, from which the several offsets are furnished. From the extremity a partly obliterated vessel (hypogastric) extends forwards to the bladder.

In entering the pelvis the artery lies in front of the lumbo-sacral nerve. It is accompanied by the internal iliac vein, which is posterior to it, and somewhat to the outer part on the right side.

The branches of the artery are numerous, and arise usually in the following manner: From the posterior piece of the trunk three arise, viz., the ilio-lumbar, lateral sacral, and gluteal. And from the anterior portion come the vesical (upper and lower), hæmorrhoidal, obturator, sciatic, and pudic: in the female there are in addition the uterine and vaginal branches.

Artery in the foetus. In the foetus the hypogastric artery takes the place of the internal iliae, and leaves the abdomen by the umbilicus. At that time it is larger than the external iliac artery; and, entering but slightly into the eavity of the pelvis, it is directed forwards to the back of the bladder, and along the side of that viseus to the apex.

Beyond the bladder the artery ascends along the posterior aspeet of the abdominal wall with the urachus, eonverging to its fellow. Finally at the umbilicus the vessels of opposite sides come in contact with the umbilieal vein, and passing from the abdomen through the aperture at that spot, enter into the placental cord, and receive the name umbilical.

In the fortus, branches similar to those in the adult are furnished by the artery, though their relative size at the two periods is very different. 
Change to adult state. When uterine life has ceased the hypogastric artery diminishes in consequence of the arrest of the current of blood through it, and finally becomes obliterated, more or less completely, as far back as an inch and a half of its commencement. 'The part of the trunk which is unobliterated becomes the internal iliac; and commonly a portion of the vessel remains pervious as far as the upper part of the bladder, and gives origin to the vesical arteries.

Peculiarities. The length of the internal iliac arteries varies from half an inch to three inches, its extreme measurements; but in two-thirds of a certain number of hodies (Quain) it ranged from an inch to an inch and a half.

Size. When the femoral trunk is derived from the internal iliac, and is placed at the back of the thigh, the parent vessel is larger than the external iliac.

Fig. 176.

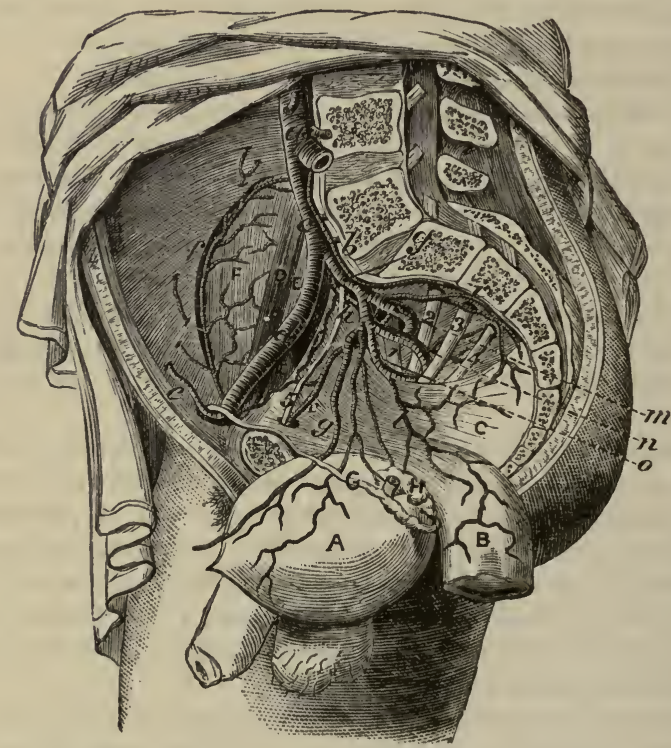

Dissection of the INternal, Iliac Artery (Tiedemana).

A. Bladder.

A. Vas deferens.

H. Vesicula seminalis.

B. Lower end of the rectum.

C. Levator ani.

D. Proas magnus.

E. Psoas parvus.

P. Hliacus.

Arteries :

a. Aorta splitting into the common fliacs.

b. Middle sacral branch.

c. Common iliac. d. External, and $g$, internal iliac trunk continued by an irupervious part along the bladder.

$e$. Epigastric, and $f$, circumflex iliac.

h. Ilio lumbar.

i. Lateral sacral.

l. Middle hæmorrholdal.

k. Obturator.

m. Gluteal.

$n$. Sciatic.

o. Pudic.

Nerves :

1, 2, 3, 4. Four highest sacral nerves. 5. Obturator.

A. The three branches arising from the posterior portion of the internal iliac may be first examined.

The ilio-lumbar branch (fig. $176, h$ ) passes outwards beneath the psoas 
muscle and the obturator nerve, but in front of the lumbo-sacral nerve, and divides into an ascending and a transverse branch in the iliac fossa :-

The ascending or lumbar branch, which is beneath the psoas, supplies that muscle and the quadratus lumborum, and anastomoses with the last lumbar artery: it sends a small spinal branch through the foramen between the sacrum and the last lumbar vertebra.

The transverse or iliac offset divides into branches that ramify in the iliacus muscle, some running over and some beneath it. At the iliac erest these branches anastomose with the lumbar and circumflex iliac arteries; and some deep twigs communicate with the obturator artery, and enter the innominate bone.

The lateral sacral arteries (fig. 176, $i$ ) are two in number, superior and inferior, but the upper is the larger; they correspond in situation with the lumbar arteries, and form a chain of anastomoses by the side of the apertures in the sacrum. These arteries supply the pyriformis and coccygeus muscles, and anastomose with each other, as well as with the middle sacral. A small spinal branch enters each aperture in the sacrum.

The gluteal artery (fig. $176, m$ ) is a short thick trunk, which appears to be the continuation of the posterior division of the internal iliac. Its destination is to the gluteal muscles on the dorsum of the hip bone; and it is transmitted from the pelvis above the pyriformis muscle, with its accompanying vein, and the superior gluteal nerve. In the pelvis the artery gives small branches to the contiguous muscles, viz., iliacus, pyriformis, and obturator, and a nutritious artery to the hip bone.

13. The branches of the anterior portion of the internal iliac artery are the following:-

The vesical arteries, superior and inferior, are distributed to the upper and lower parts of the bladder.

The upper, three or four in number, arise at intervals from the partly obliterated hypogastric trunk; the lowest of these is sometimes called middie vesical branch. Offsets are furnished from those branches to all the body and upper part of the bladder.

The lower artery arises from the internal iliac in common with a branch to the rectum, or with one to the vagina in the female. It is distributed to the base of the bladder, the vesicula seminales, and the prostate. A small offset from this artery or from the upper vesical is furnished to the vas deferens.

'The branch to the rectum (middle hamorrhoidal) is commonly supplied by the inferior vesical. It is spent on the anterior and lower part of the rectum, and on the vagina in the female, and anastomoses with the hæmorrhoidal arteries.

The obturator artery (fig. $176, k$ ). The branch is directed forwards below the brim of the pelvis to the aperture in the upper part of the thyroid foramen; passing through that opening it ends in two branches, which ramify on the membrane closing the thyroid foramen, and lie beneath the muscle in that situation. In the pelvis the artery has its companion nerve above, and vein below it ; and it gives origin to the following small branches.

Iliac branch: this small offset enters the iliac fossa to supply the bone and the iliacus muscle; it anastomoses with the ilio-lumbar artery.

The pubic branch (fig. $141, f$ ) ascends on the posterior aspect of the pubes, and communicates with the corresponding branch of the opposite side, and with an offset from the epigastric artery. 
Sometimes the obturator may take origin from the external iliac artery.

The sciatic artery (fig. $176, n$ ) is the next largest branch to the gluteal, and is continued over the pyriformis muscle and the sacral plexus to the lower part of the sacro-sciatic notch, where it issues between the pyriformis and the coccygeus. External to the pelvis it divides into branches beneath the gluteus maximus, and is distributed to the buttock: in the pelvis it supplies the pyriformis and coccygeus muscles.

The pudic artery (fig. 176,o) supplies the perineum and the genital organs, and has nearly the same connections in the pelvis as the sciatic, from which it often springs. It accompanies the sciatic, though external to it, and leaves the pelvis between the pyriformis and coccygens. At the back of the pelvis it winds over the ischial spine of the hip bone, and enters the perineal space (p. 390). The artery gives some unimportant offsets in the pelvis, and frequently the middle hæmorrhoidal branch arises from it.

Accessory pudic (Quain). 'The pudic artery is sometimes smaller than usual, and fails to supply some of its ordinary perinaal branches, especially the terminal for the penis. In those cases the deficient branches are derived from an accessory artery, which takes origin from the internal iliac (mostly from the trunk of the pudic), and courses forwards on the side of the bladder and the prostate gland, to perforate the triangular perinaal ligament. It furnishes branches to the penis to supply the place of those that are wanting.

c. The branches of the internal iliac artery which are peculiar to the female are two, the uterine and vaginal.

'The uterine artery (fig. $17 j, d$ ) passes inwards between the layers of the broad ligament to the neck of the uterus, where the vessel changes its direction, and ascends to the fundus. Numerous branches enter the substance of the uterus, and, ramifying in it, are remarkable for their tortnons condition.

At the neck of the uterus some small twigs are supplied to the vagina and the bladder; and opposite the ovary a branch bends outwards to anastomose with the ovarian artery (spermatic) of the aorta.

The vaginal artery (fig. $\mathbf{1 7 5}, e$ ) seldom arises separately from the internal iliac. Combined with the preceding, or with the branch to the rectum, this artery extends along the vagina, and ramifies in its wall as low as the outer orifice.

Other arteries in the pelvis. The remaining arteries in the pelvis, which are not derived from the internal iliac, are the ovarian, superior hamorrhoidal, and middle sacral.

The ovarian artery (p. 490), after passing the brim of the pelvis, becomes tortuous, and enters the broad ligament to be distributed to the ovary : it supplies an offset to the Fallopian tube, and another to the round ligament; and a large branch anastomoses internally with the uterine artery.

The superior hamorrhoidal artery, the continuation behind the rectum of the inferior mesenteric (p. 441), divides into two branches near the middle of the sacrum. From the point of division the branches are continued along the rectum, one on each side, and each ends finally in about three branches, which pierce the layer of the gut three inches from the anus; they terminate opposite the inner sphincter in anastomotic loops beneath the mucous membrane, and anastomose with the middle and inferior hamorrhoidal arteries. 
The middle sacral artery of the aorta (p. 489), (fig. 176, b) descends along the middle of the last lumbar vertebra, the saerum, and the coceyx. The artery gives small branches laterally, opposite each piece of the sacrum, to anastomose with the lateral sacral arteries, and to supply the nerves, and the bones with the periosteum. Sometimes a small branch is furnished by it to the lower end of the rectum, to take the place of the middle hæmorrhoidal artery.

The INTERNAL ILIAC VEIN reeeives the blood from the wall of the pelvis, and the pelvic viscera, by branehes corresponding for the most part with those of the arteries. The vein is a short thick trunk, which is situate at first on the inner side of the internal iliac artery; but as it ascends to join the external iliac, it passes behind, and to the outer aspect of its companion artery on the right side

Branches. Most of the branches entering the trunk of the internal iliac vein, have the same anatomy as the arteries; but the following visceral branches-the pudic and dorsal of the penis, the vesical and hrmorrhoidal, the uterine and vaginal, have some peculiarities.

The pudic vein receives roots eorresponding with the branches of the purlic artery in the perinaum, but not those corresponding with the offsets of the artery on the dorsum of the penis.

'The dorsal vein of the penis receives veins from the corpora cavernosa and corpus spongiosum of the penis, and piereing the triangular ligament of the urethra, divides into two, a right and a left branch, which enter a plexus around the prostate.

The superior hamorrhoidal vein eommences in a large plexus (hæmorrhoidal) around the lower end of the rectum beneath the mucous membrane (p. 533).

The vesical veins begin in a plexus about the lower part of the bladder, and anstomose with the prostatic and hrmorrhoidal veins.

The uterine veins are numerous, and form a plexus in and by the side of the uterus: this plexus inoseulates above with the ovarian plexus, and below with one on the vagina.

The vaginal veins surround their tube with a large vaseular plexus.

Parietal veins of the pelvis. 'Three veins of the wall of the pelvis, viz., ilio-lumbar, lateral sacral, and middle sacral, open into the common iliac vein.

Dissection (fig. 177). To dissect the nerves of the pelvis, on the right side, it will be necessary to detach the triangular ligament with the urethra from the areh of the pubes; and to eut through, on the right side, the recto-vesical fascia and the levator ani, together with the visceral arteries, in order that the viscera may be drawn somewhat from the side of the pelvis. If the bladder is distended let the air escape from it.

By means of the foregoing dissection the sacral nerves may be found as they issue from the sacral foramina. The dissector should follow the first four into the sacral plexus, and some branches from the fourth to the viscera. The last sacral and the eoeeygeal nerve are of small size, and will be detected coming through the coceygeus muscle, close to the coccyx; these are to be disseeted with eare; and the student will sueeeed best by traeing the eomnecting filaments which pass from one to another, beginning above with the offset from the fourth nerve.

Opposite the lower part of the rectum, bladder, and vagina is a large plexus of the sympathetic (pelvie plexus), which sends branches to the viscera along the arteries. This plexus is generally destroyed in the pre- 
vious dissections; but if any of it remains the student may trace the offsets distributed from it, and its communicating branches with the spinal nerves.

Sacral Spinal Nerves (fig. 177). The anterior primary branehes of the sacral nerves are five in number, and decrease suddenly in size fiom above downwards. Issuing by the apertures in the front of the sacrum (the fifth nerve excepted) they receive short filaments of communication from the gangliated cord of the sympathetic. The first three nerves and part of the fourth enter the sacral plexus, but the fifth ends on the back of the coccyx.

The coccygeal nerve, and the peculiarities of the fourth and fifth sacral, will be noticed before the plexus is described.

The fourth nerve $(4 S)$ divides into two parts:-one communicates with the sacral plexus; the other joins the fifth nerve and distributes the following branches to the viscera and the surrounding muscles:-

The visceral branches (3) supply the bladder and the vagina, and communicate with the sympathetic nerve to form the pelvic plexus. Sometimes these branches come from the third sacral nerve.

The muscular branches are three in number. One (2) rather long branch enters the levator ani on the visceral aspect; another (4) supplies the coccygeus; and the third (5) reaches the perinaum by piercing the levator ani muscle (p. 391).

The fifth nerve $(5 S)$ comes forwards between the sacrum and coccyx, and receives the communicating branch from the fourth nerve; it is then directed downwards in front of the coccygeus, where it is joined by the coccygeal nerve, and perforates that muscle, the sacro-sciatic ligament, and the gluteus maximus, to end on the posterior surface of the coccyx.

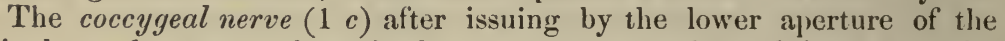
spinal canal, appears through the coccygeus muscle, and joins the fifth sacral nerve as above stated.

Sacral Plexus (fig. 177). This plexus is formed by the lumbo-sacral cord, the first three sacral nerves, and part of the fourth sacral. It is situate on the pyriformis muscle, beneath the sciatic and pudic branches of the internal iliac artery; and the nerves entering it converge towards the large sacro-sciatic notch, to unite in a flat band. From that spot the plexus becomes gradually smaller towards the outer end; and, leaving the pelvis below the pyriformis, terminates in branches for the limb at the lower border of that muscle.

Branches. Most of the branches arise outside the pelvis, and are distributed to the back of the lower limb; for, only two internal pelvie muscles (pyriformis and obturator internus) receive nerves from it.

'The nerve of the obturator internus springs from the part of the plexus formed by the union of the lumbo-sacral with the first sacral nerve; it leaves the pelvis with the pudic artery, and winding over the ischial spine of the hip bone and through the small sacro-sciatic notch, enters the muscle on the perinaal surtace.

The nerves of the pyriformis are commonly two in number, and arise from separate parts of the plexus: they enter the muscle at its visceral aspect.

The pudic nerve, like the artery of the same name, supplies the parts in the perinæum, and the genital organs. The nerve arises in the lower part of the plexus, and courses over the small sacro-sciatic ligament, to accompany its artery through the small sacro-sciatic notch. 
The remaining branches of the plexus, viz., the small and great sciatic nerves, and muscular offsets to the gluteus, gemelli, and quadratus femoris, are described with the lower limb. (Dissection of the Buttock.)

Dissection. The gangliated cord of the sympathetic that lies in front of the sacrum may be examined now : its several ganglia (three or four), and their branches, will come into view on the removal of the areolar tissue.

Fig. 177.

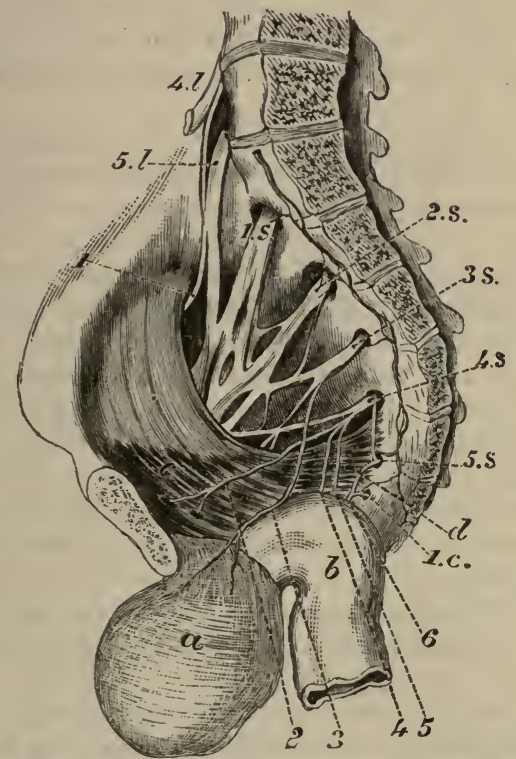

a. Urinary bladder.

b. Rectum.

c. Levator ani.

d. Coccygeus.

Nerves:

$4 l$ and $5 l$, the last two lumbar nerves, which form by their union the lumbo-sacral cord.

$1 S$ to $5 S$, the five sacral nerves, forming the sacral plexus.

1 c. Coccygeal nerve.

1. Upper gluteal nerve.

2. Branch of levator anl.

3. Branch to the bladder.

4. Branch of sphincter ani.

5. Branch of coceygeus.

6. Cominon branch of $4 S, 5 S$, and 1 $c$, for the back of the coccyx.

Disaction of the Sacral Nervas and Plexus (altered from Henle).

Sympathetic Nerve. In the pelvis the sympathetic nerve consists of a gangliated cord, and of a plexus on each side.

The Gaxgliated Cord (fig. 177) lies on the front of the sacrum and internal to the series of apertures in that bone. Inferiorly it converges to its fellow, and is united with it by a loop in front of the coccyx, on which is situate a single median ganglion (gang. impar). Each cord is marked by ganglia at intervals, the number varying from three to five: from them branches of communication pass outwards to the spinal nerves, and some filaments are directed inwards in front of the sacrum.

The connecting branches are two to each ganglion, gray and white, and are very short: the gray cord unites the ganglion with the spinal nerve, but the white one is continued over the ganglion to the visceral plexus (Beck).

The internal branches are small, and communicate around the middle sacral artery with the branches of the opposite side. From the first, or first two ganglia, some filaments are furnished to the hypogastric plexus; and from the terminal connecting branches, and from the ganglion in front of the coccyx, offsets descend over that bone.

The PELViC Plexuses (lateral inferior hypogastrie) are two in number, right and left, and are continuous with the lateral prolongations of the 
hypogastric plexus (p. 444). Each is situate by the side of the bladder and rectum in the male, and by the side of the uterus and vagina in the female, and is united with offsets of the third and fourth sacral nerves. Numerous ganglia are found in the plexus, especially at the points of union of the spinal and sympathetic nerves.

Offsets. From each plexus offsets are furnished along the branches of the internal iliac artery to the viscera of the pelvis, and the genital organs ; these form secondary plexuses, and have the same name as the vessels on which they are placed.

The inferior hamorrhoidal plexus is an offset from the back of the plexus to the rectum, and joins the sympathetic on the superior hamorrhoidal artery.

The vesical plexus contains large offsets, with many white fibred or spinal nerves, and passes forwards to the side and lower part of the bladder. It gives one plexus to the vesicula seminalis, and another to the vas deferens.

The prostatic plexus leaves the lower part of the pelvic plexus, and is distributed to the substance of the prostate. At the front of the prostate an offset (cavernous) is continued onwards to the dorsum of the penis, to supply the cavernous structure. On the penis the cavernous nerves join the pudic nerve.

In the female there are the following additional plexuses for the supply of the viscera peculiar to that sex :-

Ovarian plexus. The chief nerves to the ovary are derived from the renal and aortic plexuses, and accompany the artery of that body; but the uterine nerves supply some filaments to it.

Vaginal plexus. The nerves of the vagina are large, and are not plexiform, but consist in greater part of spinal nerve fibres; they end in the lower part of the tube.

The uterine nerves are furnished to the uterus without direct admixture with the spinal nerves : they ascend along the side of the uterus, and are, for the most part, long slender filaments without ganglia or communications. The Fallopian tube receives its branches from the uterine nerves.

Some few nerves surrounding the arteries of the uterus are plexiform and ganglionic.

The lymphatic glands of the pelvis form one chain in front of the sacrum, and another along the internal iliac artery: their efferent ducts join the lumbar glands. Into these glands the deep lymphatics of the penis, those of the genital organs in the female, and the lymphatics of the viscera and wall of the pelvis are collected.

\section{Section V.}

ANATOMY OF THE VISCERA OF THE MALE PELVIS.

Directions. The bladder and the parts at its base, viz., the vesicula seminales, and the prostate gland, are to be taken first for examination.

Dissection. To study the form and structure of the viscera, it will be necessary to remove them from the pelvis. For this purpose the student should carry the scalpel around the pelvic outlet, close to the osseous 
boundary, so as to detach the crus of the penis from the bone, and the end of the rectum from the parts around. When the viscera are removed, the rectum is to be separated from the other organs; but the bladder, the penis, and the urethra are to remain united.

After the bladder has been distended with air, the areolar tissue is to be removed from the muscular fibres. The prostate gland and the vesiculæ seminales are to be then cleaned; and the duct of the latter, with the vas deferens, is to be followed to the gland.

If any of the integument has been left on the penis and the urethra it is to be taken away.

THE PROSTATE GLAND AND SEMINAL VESICLES.

Prostate gland (fig. 178). This is a firm museular body, with glands in it secreting a special fluid, which surrounds the neck of the bladder and the beginning of the urethra. Its connections with parts around have been enumerated (p. 507 ).

The prostate is conical in form like a chestnut, with the base or larger end directed backwards. Its dimensions in different directions are the following :- Transversely at the base it measures about an inch and a half; from apex to base an inch and a quarter; and in depth about three-quarters of an inch or an inch: so that an incision directed obliquely outwards and backwards laterally, from the apex to the base, will be the longest that can be practised in the half of this body. Its weight is about an ounce, but in this respect it varies greatly.

The upper surface of the prostate is narrow and rounded. The under surface, which is larger and flatter, is marked by a median hollow which indicates the division into lateral lobes.

The posterior part, or the base, is thick, and in its centre is a notch which receives the common seminal ducts. The forepart or apex is pierced by the urethra.

Three lobes are described in the prostate, viz., a middle and two lateral, though there is no fissure in the firm mass. The lateral parts or lobes (fig. $178, b, c$ ) are similar on both sides, and are separated only by the hollow on the under surface; they form the chief part of the prostate, and are prolonged back, on each side, beyond the notch in the base. The middle lobe $(d)$ will be brought into view by detaching the vesicula seminales and the vasa deferentia from the bladder; it is the piece of the gland between the neck of the bladder and the seminal ducts, which extends across between the lateral lobes. Oltentimes the middle lobe is enlarged in old people, and projects upwards into the bladder, so as to interfere with the flow of the urine from that viscus, or the passage of a catheter into it.

The urethra and the two common seminal ducts are contained in the substance of the prostate (fig. 180). The former is transmitted through the gland from base to apex ; and the latter perforate it obliquely to terminate in the urethral canal.

Structure. On a section the gland appears reddish gray in color, is very firm to the feel, and is scarcely lacerable. It is made up of a mass of unstriated muscular and fibrous tissues, with interspersed glandular structure; and the whole is enveloped by a fibrous coat.

Fibrous covering. 'This forms a thin investment for the gland, and sends offsets into the interior. It is quite distinct from the denser sheath 
derived from the pelvic fascia, and is separated from that sheath by a plexus of veins.

Muscular tissue. The firm part of the gland consists of involuntary muscular fibres, intermixed with elastic and fibrous tissues. The muscular fibres are arranged circularly around the tube of the urethra: they are continuous behind with the annular fibres of the bladder, and in front with a thin layer of circular fibres around the membranous part of the urethra.

At the lower and outer parts the texture is looser and more spongy, especially where the glands are situate, and the larger vessels enter (Roy. Med. Chir. Trans., 1856). This arrangement will be better seen when the urethra has been opened.

Glandular structure. Small branched glands project from the tube of the urethra amongst the muscular fibres; they form but a small part of the prostate, and are most numerous in the middle lobe. The final radicles of the ducts are surrounded by small sessile vesicles: on the exterior of the vesicles and ducts the bloodvessels ramify. Lining the interior of the tubes is an epithelium of the columnar kind. The duets of the glands vary in number from twelve to twenty, and open into the prostatic part of the urethra (p. 527).

Bloodvessels. The arteries are rather small, and are furnished by the inferior vesical and middle hrmorrhoidal (p. 515). The veins form a plexus around the gland, which communicates in front with the dorsal vein of the penis, and behind with the venous plexus at the base of the bladder. In old men this vascular intercommunication gives rise to considerable hemorrhage in the operation of lithotomy.

The nerves are supplied by the hypogastric plexus. The lymphatics of this body and of the vesicula seminales are received into the glands placed along the internal iliac artery.

Vesicula Semivales (fig. $178, e$ ). These vesicles are two membranous sacs, which secrete a fluid to mix with the semen. They are placed on the under part of the bladder behind the prostate, and diverge from one another so as to limit laterally a triangular space in that situation: their form and connections have been already described (p. 507). Though saceulated and bulged behind, the vesicula becomes straight and narrowed in front (luct $d$ ); and at the base of the prostate it blends with the vas deferens to form the common seminal or ejaculatory duct $(g)$.

The vesicula seminalis consists, like the epididymis, of a tube bent into a zigzang form, so as to produce lateral sacs or pouches, whose bends are bound together by fibrous tissue; this eell structure will be shown by means of a cut into it. When the bends of the vesicle are undone, its formative tube, which is about the size of a quill, measures from four to six inches, and ends posteriorly in a closed extremity : connected with the tube at intervals, are lateral cacal appendages (fig. 178).

Structure. 'The wall of the seminal vesicle has the same number of layers as the vas deferens (p. 483); but the muscular coat is thinner.

Within the casing of the recto-vesical fascia, the vesicula and vasa deferentia are covered by a layer of transverse and longitudinal plain muscular fibres. The transverse are the more superfieial (the base of the bladder being upwards), are strongest near the prostate, and act most on the vasa deferentia. 'The longitudinal fibres, placed chiefly on the sides of the vesicula, are continued forwards with the common seninal ducts to the urethra. (Med. Chir. 'Trans. 1856.) 
The mucous membrane is thrown into ridges by the bending of the tube, and presents an areolar or honeycomb appearance; it is provided with tubular glands, as in the vas deferens, and is covered by a flattened epithelium.

End of vas deferens (fig. 178). Opposite the vesicula the vas deferens is increased in eapacity, and is rather sacculated like the contiguous vesiele : but before it joins the tube of that body to form the common seminal duct, it diminishes in size, and becomes straight. In the mucous lining are numerous tubular glands like those of the intestine (Henle); and the epithelium is columnar as in the rest of the tube.

Fig. 178.

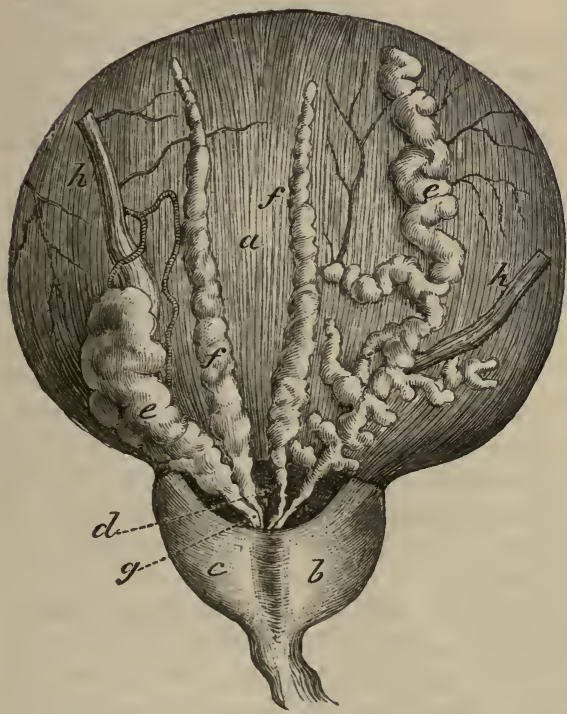

a. Bladder.

$b$ and $c$, right and left lateral lobes of the prostate.

d. Middle lobe.

$e$. Vesicula seminalis, the right one unravelled.

$f$. Vas deferens.

$g$. Common seminal duct, formed by the union of the vas deferens with the duct of the vesicula.

$h$. Ureter.

View of the Under Part of the Bladder with the Vesicula Semisales axd VAsA Deferentia (Slightly altered from Haller).

Common seminal ducts (fig. 180, $f$ ). These tubes (right and left) are formed by the junction of the narrowed part or duct of the vesicula seminalis with the vas deferens of the same side. They begin opposite the base of the prostate, and are directed upwards and forwards through an aperture in the circular prostatic fibres, and along the sides of a hollow (vesicula prostatica), to open into the urethral tube. Their length is rather less than an inch, and their course is convergent to their termination close to each other in the floor of the urethra (p. 527).

Structure 'The wall of the common duct is thinner than that of the vesicula seminalis; but it possesses similar coats. It is surrounded by longitudinal involuntary muscular fibres, which blend in the urethra with the submucous stratum. It possesses the same glands and epithelium as the dilated part of the vas deferens; but at the end of the tube the mucous membrane wants glands and is smooth (Henle). 
THE BLADDER.

After the bladder has been separated from the surrounding parts its form and the extent of its different regions can be more conveniently studied.

Whilst the bladder is in the body, it is conical in shape, and rather

Fig. 179 .

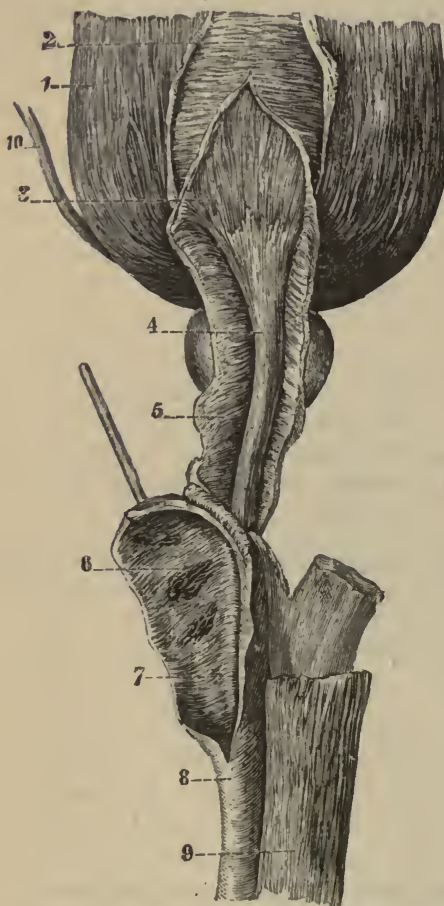

Mtecular fibres of the Bradder, ProsTATE, AND URETHRA.

1. Exterual or longituninal fibres of the bladder.

2. Circular fibres of the middle coat.

3. Submucous layer.

4. Muscular layer around the urethra.

5. Circular fibres of the prostate and urethra continuous with the circular of the bladder.

6, 7. Septum of the corpus spongiosum.

8. Corpus spongiosum.

9. Corpus cavernosum.

10. Ureter. through the anterior true ligament of the blatder. On the front and back of the bladder the muscular layer is stronger, and its fibres more vertical than on the lateral parts. Sometimes this outer layer of fibres is called detrusor urina from its action in the expulsion of the urine.

The circular fibres (fig. $179,{ }^{2}$ ) are thin and scattered on the botly of out of the body it is more circular than when in its natural position, and it loses that arched form by which it adapts itself in distension to the curve of the pelvis.

If this viscus is moderately dilated, it measures about five inches in length, and three inches across (Huschke). Its capacity is greatly influenced by the age and sex, and by the habits of the inclividual. Ordinarily the bladder holds about a pint without inconvenience during life, though it can contain much more when distended. As a general rule it is larger in the female than in the male.

Structure. A muscular and a mucous coat, with an intervening fibrous layer, exist in the wall of the bladder : at certain parts the peritoneum may be also enumerated as a constituent of the wall. The vessels and nerves are large.

The imperfect covering of peritoneum has been described (p. 504).

The muscular coat is formed of thin layers of unstriated muscular fibres, viz., an external or longitudinal, a middle or circular, and an internal or submucous.

The longitudinal fibres (fig. 179, ${ }^{1}$ ) form a continuous covering, with the usual plexiform disposition of the muscular bundles, and extend from apex to base. Above, some are connected with the urachus, and the subperitoncal fibrous tissue. Below, the posterior and lateral fibres enter the prostate; whilst the anterior are attached to the fascia covering the prostate, with the exception of a fasciculus on each side, which is united to the back of the pubes 
the bladder; but around the cervix they are collected into a thick bundle, the sphincter vesica, and are continnous before, with the fibres of the prostate. When the fibres are hypertrophied, they project into the interior of the organ, forming the fasciculated bladder; and in some bodies the mucous coat may be forced outwards here and there between them, in the form of sacs, producing the sacculated bladder.

The submucous stratum (fig. $179,{ }^{8}$ ) forms a continuous layer over the lower half of the bladder, but its fibres are scattered above. In the lower third of the viscus the fibres are longitudinal, and are continued around the urethra, but they become oblique above that point. At the back of the bladder the layer is increased in strength by the longitudinal fibres of the ureters blending with it. The projection of the uvula vesicx is due to this submucous stratum.

The muscular strata communicate freely, so that one cannot be separated from another without division of the connecting bundles of fibres. In both sexes the disposition of the fibres is similar (Med. Chir. Trans., 1856).

Fibrous coat. A fibrous layer is placed between the muscular and mucous strata, and is enumerated as one of the coats of the blarlder; it is composed of areolar and elastic tissues as in other hollow viscera, in which the vessels and nerves ramify.

Dissection. The bladder is now to be opened by an incision down the front; and the cut is to be continued along the upper part of the prostate gland.

The mucous membrane of the bladder is continuous posteriorly with the lining of the ureters, and anteriorly with that of the urethra. It is very slightly united to the muscular layer; and it is thrown into numerous folds in the flaccid state of the viscus, except over a small triangular surface behind the urethral opening.

The membrane is of a pale rose color in the healthy state soon after death. Its surface is studded with mucous follicles and branched glands, particularly towards the neck of the bladder. In the epithelium covering the surface are three kinds of cells : the superficial are roundish and flattened; the middle are pyriform; and the deeper are conical and cylindrical.

Objects inside the bladder. Within the bladder are the following named parts, viz., the orifices of the ureters and urethira, with the triangular surface (fig. 179).

Orifices. At the anterior part of the bladder is the orifice of the urethra, surrounded by the prostate gland. The mucous membrane presents here some longitudinal folds; and the aperture is partly closed by a small prominence below, uvulu vesica, occasioned by a thickening of the submucous muscular and fibrous layer. This eminence is placed in firont of the middle lobe of the prostate; and from its anterior part a slight ridge is continued on the floor of the urethra.

About an inch and a half behind the orifice of the urethra, and rather more than that distance apart, are the two narrow openings of the ureters (fig. 181). These excretory tubes for the urine perforate the wall of the bladder obliquely, lying in it for the distance of one-third of an inch, so that the reflux of fiuid through them towards the kidney is prevented as the bladder is distended. Each terminates by a slit-like opening in a prominence formed by subjacent muscular fibres.

Triangular surface. Inmediately behind the orifice of the urethra is a smooth triangular surface, which is named trigone (trigonum vesica). Its 
apex reaches the prostate, and its base the ureters. Its boundaries may be marked out by a line on each side from the urethra to the ureter, and by a transverse one, behind, between the openings of the ureters. 'This surface corresponds with the triangular space externally at the base of the bladder, between the prostate in front and the vesicula and vasa deferentia on the sides. Over it the mucous coat is more closely united to the fibrous and muscular, so as to prevent the accidental folds occurring in the other parts of the empty bladder.

Dissection. 'The arrangement of the fleshy fibres of the ureters will come into view on the removal of the mucous membrane from the lower third of the bladder.

Ending of the fibres of the ureters. As soon as the ureter pierces the outer and middle coats of the bladder, its longitudinal fibres are thus disposed :- the more internal and strongest are directed transversely, and join the corresponding fibres of the other urine tube; whilst the remainder are continued down over the triangular surface, and blend with the submucous layer.

Bloodvessels and nerves. The source of the vesical arteries, and the termination of the veins have been detailed (p. 515). The vessels are disposed in greatest number about the base and neck of the bladder. Most of the nerves distributed to the bladder, though supplied from the pelvic plexus of the sympathetic ( $p .519)$, are derived directly from the spinal nerves. The lymphatics enter the glands by the side of the internal iliac artery.

\section{THE URETHRA AND PENIS.}

Uretura (fig. 181). The tube of the urethra extends from the neck of the bladder to the end of the penis, and has an average length of about eight inches; but it is shorter by one inch during life ('Thompson). It is supported by the prostate, the triangular ligament, and the spongy structure of the penis. The tube is divided, as before said (p. 508), into a prostatic, a membranous, and a spongy part.

Dissection. To open the urethra, let the incision through the npper part of the prostate be continued onwards to the extremity along the line of union of the urethral canal with the body of the penis.

The prostatic part (fig. 181) is nearer the upper than the lower surface of the muscular mass surrounding it. It is one inch and a quarter in length, and is altogether the widest portion of the urethral canal. Its form is spindle-shaped, for it is larger in the middle than at either end. Its transverse measurement at the neck of the bladder is a quarter of an inch; at its centre a line or two more; and at the front rather less than at the back.

Separating this part of the passage from the bladder is the eminence of the uvula vesica with its slight ridge. In front of this is a central longitudinal eminence of the mucous lining $(d)$, about three-quarters of an inch in length, and larger behind than before, which is prolonged anteriorly towards the membranous part of the canal, and is named crest of the urethra (veru montanum, caput gallinaginis): it is formed like the uvula by a bundle of the submucous muscular and fibrous tissue. In that crest of the mucous membrane, near its posterior extremity, is a slight hollow (e) named vesicula prostatica or sinus pocularis.

The vesicula prostatica or utricle (fig. $180, g$ ) is directed backwards 
and downwards in the prostate, for a quarter of an inch, passing beneath the middle and between the lateral lobes. Its orifice in the urethra is about a line wide, and its closed extremity is about as large again. Along the wall, on each side, is placed the common seminal duct $\left(f^{\prime}\right)$, which terminates on or within the margin of the mouth of the sae; and if bristles are introduced into the common seminal duct behind the prostate, they will render evident the apertures. Small glands open on the surface of the mucons membrane lining the utricle. The vesicula is the remains of the united lower ends of the ducts of Müller in the foctus, and represents the uterus in the female.

Fig. 180.

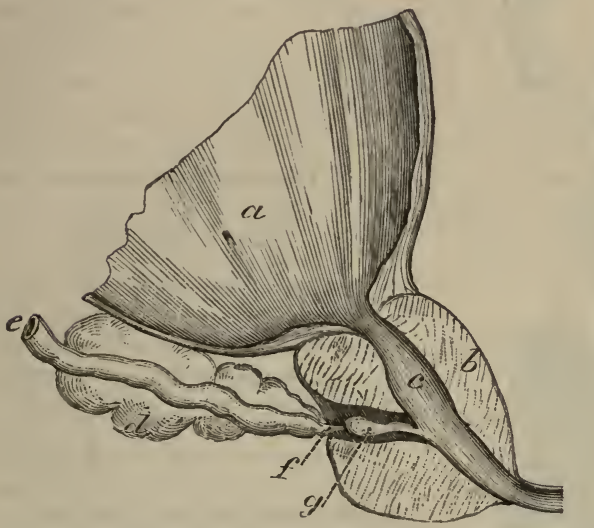

a. Bladder.

b. Prostate, and $c$, prostatic part of the urethra.

d. Vesicula seminalis.

e. Vas deferens.

$f$. Common ejaculatory duct.

g. Vesicula prestatica.

SECTION THROUGH THE BLADDRR, Prostate, AND URETHRA, to show the vesicula prostatica and the common seminal duct.

On each side of the central crest is an exeavation, which is named the prostatic sinus (fig. 181, $f$ ). Into this hollow the greater number of the ducts in the prostate open; but the apertures of some are seen at the posterior part of the central eminence.

The membranous part of the urethra (fig. $181, g$ ) is three-quarters of an inch in length, and intervenes between the apex of the prostate gland and the bulb $(k)$ of the corpus spongiosum urethræ. In its interior are slight longitudinal folds. This is the narrowest portion of the whole tube, with the exception of the outer orifice, and measures rather less than a quarter of an inch across. It is the weakest of the three portions of the canal, and is supported by a thin stratum of erectile tissue, by a thin layer of unstriated circular fibres (p. 397), and outside all by the constrictor urethre muscle.

The spongy part (fig. $181, i$ ) reaches to the end of the penis. It is about six inches in length. And its strength depends upon a surrounding material named corpus spongiosum urethre.

The average size of the cinal is about a quarter of an inch in diameter, though at the vertical slit (meatus urinarius), by which it terminates on the glans penis, the tube is smaller than elsewhere. On a cross section it appears as a transverse slit, but in the glans, as a vertical interval. Two dilatations exist in the floor of the spongy portion: One is close to the triangular ligament, being contained in the bulb or bulbous part of the 
urethra, and is named sinus of the bulb ; the other is an elongated hollow, situate in the glans penis, which has been called fossa navicularis ( $n$ ) from its shape.

Fig. 181.

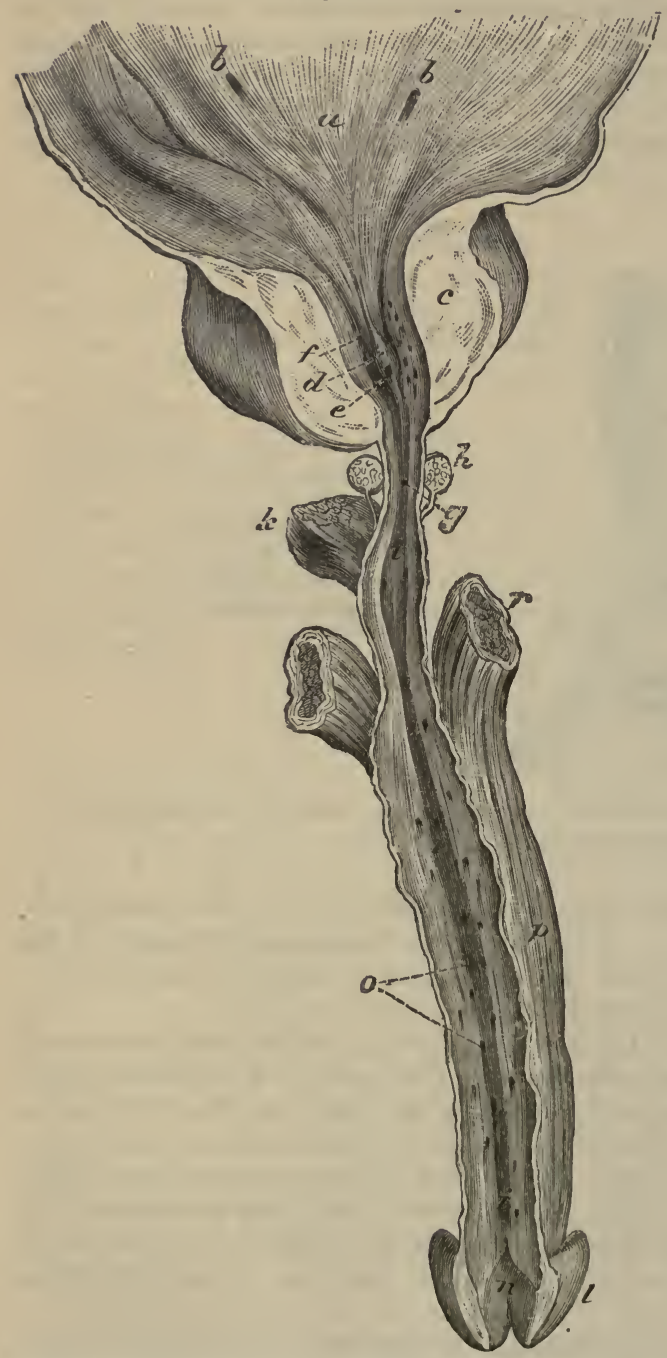

a. Triangular surface of the bladder.

b. Openings of the ureters.

c. Prostate, cut.

d. Caput gallinaginis.

e. Sinus pocularis.

$f$. Prostatic sinus, with openings of the glauds of the prostate.

g. Membranous part of the urethra.

$h$. Cowper's glands, a duct from each opening in to the urethra.

$i$. Spongy part of the urethra.

k. Bulb of the urethra.

l. Glans penis, and $n$, fossa navicularis.

o. Openings of lacunæ and glands.

$r$. Corpus cavernosum of the penis.

VIEW OF THE LOWER PART OF THE BLADDER AND OF THE URETHRA LAID OPEN.

There are many small pouches or lacuna $(o)$ in the eanal, as far back as the membranous part, which have their apertures turned towards the outer orifice of the urethra. One of these, larger than the rest, lacuna magna, is placed, generally, on the upper boundary or roof of the urethra, opposite the fossa navicularis.

The ducts of Cowper's glands (fig. 181, $h$ ) are two in number, and 
terminate, one on each side, on the floor of the urethra near the front of the bulb, but they are generally too small to be recognized.

Mucous lining of the urethra. The mucous membrane of the urethra is continued into the bladder, as well as into the ducts opening into the canal, and joins in front the tegumentary covering of the glans penis. It is of a reddish color in the spongy and membranous portions, but in the prostate it becomes whiter. In the spongy and membranous parts it is thrown into longitudinal folds during the contracted state of the penis.

Its surface is studded throughout with follicles, and with the apertures of branched glands, which are lodged in the submucous tissue, and whose ducts are inclined obliquely forwards; and it is provided with papilla towards the external orifice. Its epithelial covering is of the columnar kind, but near the meatus it becomes laminar.

Submucous tissue. Beneath the mucous lining of the urethra is a stratum of longitudinal unstriated muscular fibres, mixed with elastic and fibrous tissues. It is continuous behind with the sulmmeous fibres of the bladder, and is joined in the prostate by the muscular fibres accompanying the common seminal ducts. The stratum differs along the canal :- It is most developed in the prostate, where it forms the projection of the crest ; in the membranous portion the muscular structure is less abundant; and in the spongy part fibrous tissue forms the greater portion of the submucous layer.

In the prostatic and membranous divisions of the urethra there is, in addition, a thin enveloping layer of vascular or erectile tissue, which is continued backwards from the corpus spongiosum urethre to the neck of the bladder.

Structure of the Pexis. The form and the connections of the penis having been described in lage 508 , the tissues of which it is composed

$a$

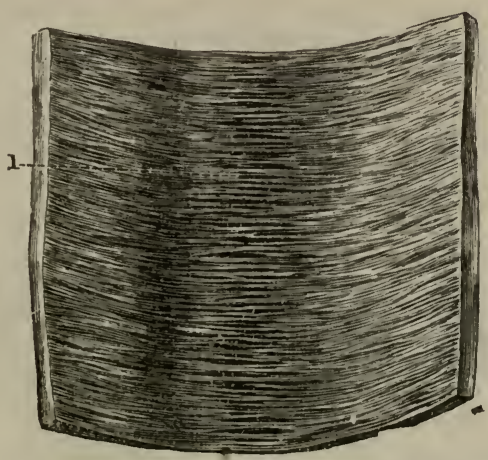

Vifw of the Fibres of thr Case of the Corpes Cavernosum.
Fig. 182.

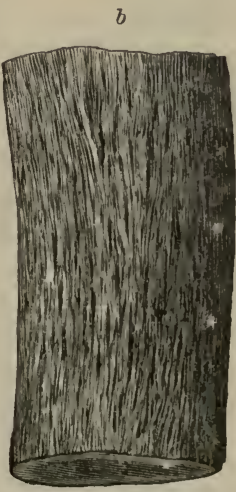

1. The pectiniform septum.

b. The external or longitudinal layer.

a. Inner or circular fibres.

remain to be noticed. If a section is made along one side of the pen's. it will show this body to be composed of two masses of spougy and rascu'ar tissue (corpora cavernosa) incased in a fibrous covering, with an imperfect septum between them. 
Corpora cavernosa (fig. 181, $r$ ). These bodies form the bulk of the penis, and are two dense cylindrical tubes of fibrous tissue, containing erectile structure. Each is fixed behind by a pointed process, crus penis, to the upper part of the pubic arch for about an inch ; and blends with its fellow in the body of the penis, after a distance of an inch and a half. There is a slight swelling on the crus, called the bulb of the corpus cavernosum (Kobelt).

Each corpus cavernosum is composed of a fibrous case with a cavernous or trabecular structure inside, with vessels in the intervals of the spongy mass. An incomplete median septum exists along the body of the penis.

The fibrous case is a white, strong, elastic covering (fig. 182), from half a line to a line in thickness. Along the middle of the penis a septal

Fig. 183.

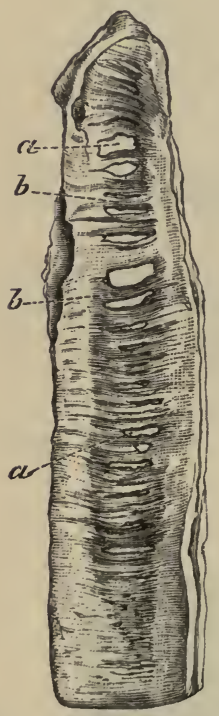

PeCTINIFORM SEPTUM OF THE PENIs.

a. Apertures in the partition.

b. Separate fibrous processes like the teeth of a comb, which are formed by the circular fibres.

process is sent inwards from it; and numerous other finer threads, - the trabeculæ of the spongy structure, are connected with its inner surfice.

It is formed of white shining fibres which are disposed in two layers, outer and inner. The outer stratum (fig. 182,b) is formed of longitudinal fibres with close meshes. The inner stratum (fig. 182, a) consists of circular fibres, with a like plexiform disposition; and the circular fibres of each cavernous body meeting in the middle line give rise to the septum penis. Both strata are inseparably united by communicating bundles.

The septal process (fig. 183) is placed vertically along the borly of the penis, and is thicker and more perfect behind than in front. Near the junction of the crura this partition divides the inclosed cavity into two parts; but as it reaches forwards it becomes less strong, and is pierced by elongated apertures, which give it the appearance of a comb, from which the name septum pectiniforme is derived. Through the intervals in the septum the vessels in the corpora carernosa communicate. It is formed by the circular fibres of the fibrous case.

The cavernous or trabecular structure is a network of fine threads, which fills the interior of the corpora cavernosa. Its processes are thinner towards the centre than at the circumference; and the areolar spaces are larger in the middle and at the fore part of the contained cavity, than at the circumference or in the crura of the penis. In addition to white fibrous tissue, the trabeculæ contain elestic fibres, and unstriated muscular fibres (Müller). The spongy structure may be demonstrated by sections of the penis, after it has been distended with air and dried.

Bloodvessels. The bloodvessels of the penis are large in size, and serve to nourish as well as to minister to the function of the organ. Having entered the cavernous mass, they ramify in the trabecular structure.

'The arteries of the corpora cavernosa are offsets of the pudic: the chief branch (art. corp. eavernosi) enters at the crus, and runs forwards through 
the middle of the cavernous structure, distributing offsets; and the rest pierce the fibrous case along the dorsum of the penis.

In the anterior they divide into branches, which ramify in the trabeculæ (fig. 184, a), becoming finer, until they terminate in very minute branehes, which open into the veins in the intertrabecular spaces without an intervention of capillaries. Some of the finest twigs end in tufts of short, slightly curled and dilated vessels-the helicine arteries of Miuller (fig. $184, e)$, which project into the intertrabecular spaces, and are imberded in the thin coat of the veins $(f)$ : with the extremities of these twisted vascular bodies fine fibrous processes are connected. The helicine arteries exist in greatest number at the posterior part of each corpus cavernosum.

The veins fill the interstices of the areolar structure, and anastomose freely together to form venous plexuses; and into them the terminal branches of the arteries pour their contents. By means of the apertures

Fig. 184.
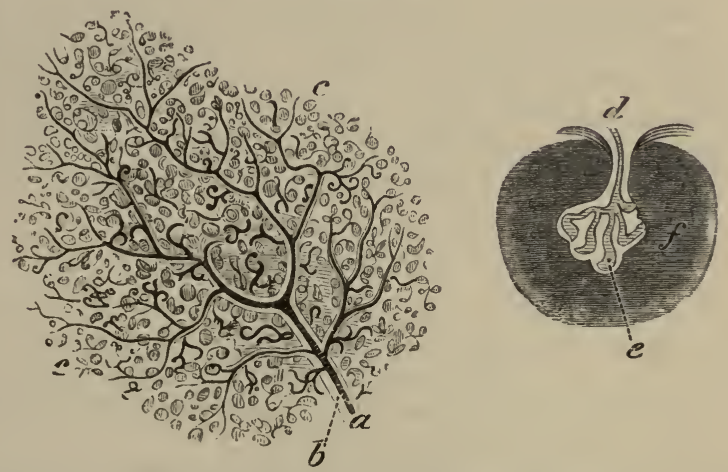

Magnified view of the Trabecular Strccture and Arterieg of the Penis.

a. Branch of an artery and its ramiflcations in the bands of the spougy structure of the penis, $b$. e. A tuft of the twisted or helicine arteries. The dark shadowing, $f$, represents a vein incasing the bundle of vessely (Müller).

$c$. Trabecular structure.

in the septum the veins of opposite sides communicate freely. The erectile condition of the corpus cavernosum is produced by the distension of the plexuses.

'The chief veins of the corpus cavernosum eseape at the crus penis with the artery, and join the pudic vein; but others issue along the upper and under aspects, to end in the dorsal vein and the prostatic plexus.

Curpus spongiosum urethra (fig. 179). 'This constituent part of the penis surrounds the urethra, but not equally on all sides; for at the bulb) only a thin stratum is above the canal, whilst at the glans penis (fig. 181, l) it is placed chiefly above the urethral tube. Posteriorly an offset of the corpus spongiosum is continued beyond the bulb around the urethra

Structure. The tissue of the corpus spongiosum is similar to that of the corpus cavernosum : thus it consists of a fibrous tunic inclosing a trabecu-. lar structure and bloodvessels.

The fibrous covering is less dense and strong than in the corpora carernosa, and consists only of circular fibres. A septal piece (fig. 179, ${ }^{6}$ ) projects inwards from it in the middle line, opposite the tube of the urethrit; 
this is best marked for a short distance in front of the bulb, and assists in dividing that part into two lobes. The trabecular bands are much finer, and more uniform in size than in the corpora cavernosa.

Bloodvessels. The arrangement of the bloodvessels in the erectile structure of the corpus spongiosum is similar in the bulb to that in the corpora cavernosa; but in the rest of the spongy substance the arteries are said to end in capillaries in the usual way. The helicine terminations of the arteries are absent from the glans penis, where the veins form a very close and regular plexus.

The arteries are derived from the pudic on each side :-one, arteries of the bulb, enters the bulb behind; and several in front, offsets of the dorsal artery, penetrate into the glans. Kobelt describes another branch to the bulb at the upper aspect.

Most of the veins, including those of the glans, end in the large dorsal vein of the penis, some communicating with veins of the cavernous body; others issue from the bulb, and terminate in the pudic vein.

Nerves and lymphatics. The nerves of the penis are large and are supplied by both the spinal and sympathetic nerves: on the glans penis they are furnished with Pacinian bodies. The superficial lymphatics of the integuments, and those beneath the mucous membrane of the urethra, join the inguinal glands; the deep accompany the reins beneath the arch of the pubes, to end in the lymphatic glands in the pelvis.

\section{THE RECTUM.}

Dissection. The rectum is to be washed out, and then distended with tow ; and the peritoneum and the loose fat are to be removed from it.

The lower end of the large intestine, which is contained in the pelvis, is not sacculated like the colon, but is smooth on the surface, the longitudinal bands of the eolon being absent from it.

It is about eight inches in length; and its average diameter is that of the sigmoid flexure of the colon. Its size is uniform as far as the lower extremity, where it is dilated, particularly in old people; but at the aperture of termination in the anus the gut is smaller than elsewhere.

Structure. The rectum contains in its wall a peritoneal, a muscular, a mucous, and a submucous stratum; and the muscular and mucous layers have certain characters which distinguish this part of the intestinal tube.

The peritoneum forms but an incomplete covering, and its arrangement is referred to in the description of the connections of the pelvic viscera (p. 505).

The muscular coat consists of two planes of pale or unstriated fibres, viz., a superficial or longitudinal, and a deep or circular. The longitudinal, continuous with those in the bands on the colon, are here diffused to form a stratum around the gut. The circular describe arches around the intestine, and become thicker and stronger towards the anus, where they are collected in the band of the internal sphincter muscle.

The mucous coat is more movable than in the colon, and resembles in this respect the lining of the osophagus; it is also thicker and more vascular than in the rest of the large intestine.

When the bowel is contracted the mucous lining is thrown into numerous accidental folds; but some near the anus are longitudinal, inclosing submucous muscular fibres, and form the columns of Morgagni. There are other three permanent folds (Houston) which are half an inch or less 
in depth, and contain circular fibres of the gut. The most constant of these is about three inches from the anus, on the front of the rectum, opposite the base of the bladder; another is placed on the right side of the intestine, towards the top; and the third is on the left side, midway between the other two. These folds will be seen by laying open the gut along the side, provided it is tolerably fresh.

The mucous membrane has the same general structure as in the colon, but towards the anus the secretory apparatus gradually disappears.

Bloodvessels. The arteries are supplied from three different sources, viz., superior hæmorrhoidal of the inferior mesenteric, middle hæmorrhoidal of the internal iliac, and inferior hæmorrhoidal of the internal pudic. All three sets anastomose on the lower end of the gut, and only the upper hæmorrhoidal, which is the largest, requires further notice. The final branches of this artery (p. 516), about six in number, pierce the muscular layer three inches from the anus, and descend between the mucous and muscular coats as far as the internal sphincter, where they unite in loops just within the anus.

The veins are deficient in valves, and communicate freely, like the arteries, in a plexus between the muscular and mucous coats, around the lower end of the gut. Above, they join the inferior mesenteric vein, and through it reach the vena portæ; and, posteriorly, they pour some blood into the internal iliac vein by the middle hæmorrhoidal branch.

Nerves and lymphatics. The nerves of the intestine are obtained from the sympathetic; and those of the sphincter come from the spinal cord. The lymphatics terminate in the chain of glands on the sacrum.

\section{Section VI.}

\section{ANATOMY OF THE FEMALE VISCERA.}

In the pelvis are contained the viscera, alike in both sexes, viz., the bladder, urethra, and rectum; and those special to the female, or the organs of generation.

Dissection. The contents of the pelvis are to be removed, together with the genital organs. For this purpose the student should keep the scalpel close to the osseous boundary of the pelvic outlet, to avoid injuring the end of the rectum; and he should also detach the crus of the clitoris from the bone.

After the parts are taken from the body, the rectum is to be separated from the uterus and vagina, but the rest of the viscera may remain united until after the genital organs are examined. The bladder and rectum may be moderately distended; and the fat and areolar tissue are to be removed from the viscera.

\section{GENITAL ORGANS.}

The genital organs consist of the following parts: the mons Veneris and external labia, the clitoris and internal labia, and the vestibule with the meatus urinarius. Within the external labia is the aperture of the vagina, with the hymen. Sometimes the term vulva or pudendum is applied to these parts as a whole. 
Mons Veneris and labia pudendi. In front of the pubes the integument is covered with hair, and is raised into a slight eminenee-mons Veneris, by a layer of subjacent fat.

Extending downwards from the prominence are two folds of integument, the labia pudendi (labia majora), which correspond with the scrotum in the male. Above and below, the labia are united, the points of junetion being named commissures; and between them is an interval called rima. The labia decrease in thickness inferiorly; they are covered externally with a few hairs, but are lined internally with mucous membrane. In them is a dartoid tissue resembling that in the male serotum.

Within the lower commissure of the labia is a small thin transverse fold of integuments named fourchette or franulum; and between this fold and the lower commissure is an interval-the fossa navicularis.

Clitoris and nympha. Beneath the upper commissure of the labia majora is the projection of the clitoris, with the nymphæ or smaller labia descending from it.

Dissection. To see the clitoris, the integuments forming the upper commissure must be removed; and, after the body of the organ has been laid bare, the crura, one on each side, are to be followed outwards.

'The clitoris (fig. $185 \mathrm{~b}$ ) is a small erectile body, and is the representative of the penis. It has the same composition as the penis, except that the urethra is not continued below it. Its anterior extremity is terminated by a rounded part or glans $(c)$, and is covered by a fold of the skin corresponding with the prepuce of the male.

Fig. 185.

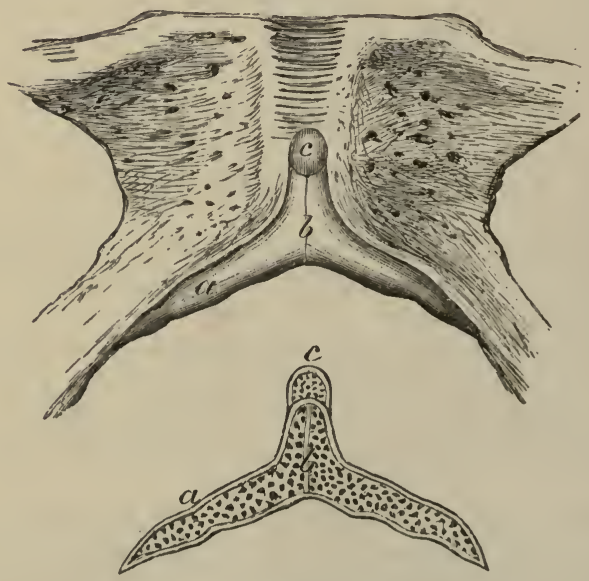

REPRESENTATION OF THE CLITORIS.

a. Cras, and $b$, body of the corpas cavernosum.

c. Glans clitoridis. The lower figureshows the structure on a rertical section; the same letters refer to like parts.

In its structure this organ resembles the penis in the following particulars : it consists of corpora eavernosa, which are attached by crura (one on each side $(a)$ ) to the pubic arch, and are blended in the body. Along the middle is an imperfect pectiniform septum. Further, it possesses it portion of corpus spongiosum, but this structure is limited to the glans clitoridis $(c)$. 
Structure. The outer fibrous casing and the septum are alike in both penis and clitoris; and in the interior of the clitoris is an erectile tissue, like that in the male organ (p. 533).

'The bloodvessels of the corpus cavernosum are like those of the penis; and the glans receives the ending of the dorsal artery (p. 408).

The nympha (fig. 186, ${ }^{\circ}$ ) (labia minora) are two folds of mucous membrane, which descend from the end of the clitoris, one on each side of the orifice of the vagina; they are continuous above with the preputial covering of the glans, and extend down about one inch and a half. The inner surface is continuous with the lining of the vestibular space and vagina, and the outer, with the external labium. Bloodvessels are contained in each fold.

Vestibule and orifice of the urethra. Within the nymphæ, between the clitoris above and the vagina below, is an interval, about one inch and a half deep, which is called the vestibule. In the middle line of the vestibular space is the round orifice of the urethra, which is placed in a median eminence about one inch below the elitoris, and near the aperture of the vagina.

Orifice of the vagina (fig. 186), and the hymen. The aperture of the vagina is close below the meatus urinarius, and varies much in size. In the ebild, and in the virgin, it is partly closed below by a thin semilunar fold of mucous membrane, named the hymen. After the destruction of that membrane small irregularly-shaped projections, caruncula myrtiformes, exist around the opening of the vagina.

Mucous membrane. The mucous covering of the external genitals is furnished throughout with papillx, but these are largest on the labia minora and the clitoris. Its epithelium is laminar.

Sebaceous glands open on the contiguous surfaces of the labia majorat and minora, and beneath the prepuce; and mucous glands and follicles exist on the vestibule and the inner surface of the nymphæ.

\section{GENERATIVE ORGANS.}

The generative organs are the uterus and vagina, and the ovaries with the Fallopian tubes.

Dissection. The viscera are now to be separated, so that the bladder and the urethra may be together, and the vagina and the uterus remain united. The bladder is to be set aside for subsequent use. 'The surface of the vagina and the lower part of the uterus should be cleaned; but the peritoneal investment of the latter is to be left untouched for the present.

\section{TIIE VAGINA.}

The tube of the vagina is connected with the uterus at one end, and with the vulva at the other (fig. $175,{ }^{1}$ ). It has a curved course between the two points mentioned; and the anterior and posterior walls are not equal in length, for the former measures about four inches, and the latter five or six.

In the body the vagina is flattened from above downwards, so that the opposite surfaces may be in contact, but the upper end is rounded where it is joined to the uterus. Its size varies at different spots: thus the external orifice which is surrounded by the constrictor vaginæ muscle is the narrowest part; the middle portion is the largest; and the upper end is intermediate in dimensions between the other two. 
After the vagina has been laid open by an incision along the upper wall, the position of the uterus in that wall, instead of the extremity of the passage, may be remarked; and the tube may be seen to extend higher on the posterior than the anterior aspect of the cervix uteri. On the inner surface, towards the lower part, is a slight longitudinal ridge both in front and behind, named columns of the vagina. Before the tissue of the vagina has been distended, other transverse ridges or ruga pass between the columns. The wall of the vagina is thicker anteriorly around the urethra than at any other part of the canal.

Structure. The vaginal wall is formed by a spongy erectile tissue, covered externally by a layer of unstriated muscle, and lined by mucous membrane. At its lower end the tube is surrounded by a band of the fibres of the sphincter vaginæ muscle (p. 402).

The erectile tissue is more abundant at the ends than the middle of the vagina, and is greatest in quantity below where it gives increased thickress to the wall. Two masses, one on each side of the opening of the vagina, have been described as the semi-bulbs by Taylor (bulbi vestibuli, Kobelt, fig. 186,a). These are elongated bundles of plexiform veins, inclosed in tibrous membrane; they are about an inch in length from above down, and are situate one on each side of the vestibule, where they are covered on the outer side by the constrictor vaginæ (A). At the upper end each is pointed, and communicates with the vessels of the clitoris: and at the lower rounded extremity it joins the venous plexus of the vagina.

These bodies would answer to the divided bulb of the corpus spongiosum urethræ in the male, each lateral half being thrust aside in the female towards the crus clitoridis by the large aperture of the vagina.

Fig. 186.

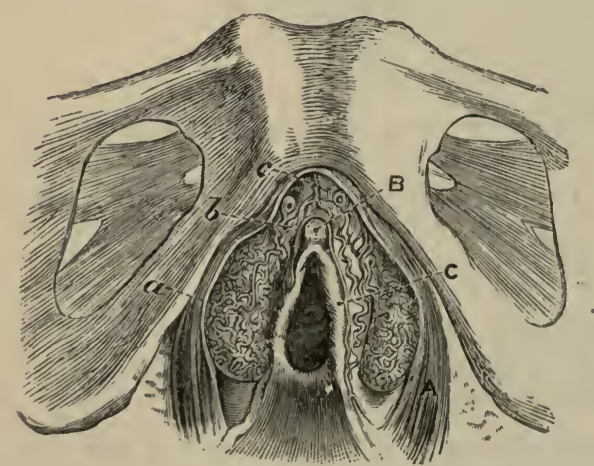

A. Sphincter vaginæ muscle.

B. Clitoris.

c. Nymphæ.

a. Semi-bulbs or bulbi vestibuli.

b. Venous plexus continuous with veins of the clitoris (pars intermedia, Kobelt).

c. Dorsal vein of the clitoris.

Viboug Piexuges of the Ginital Organs and Opening of the Vagina (Kobelt).

The mucous membrane is continued through the lower aperture to join the integument on the labia majora, and through the os uteri, at the opposite end, to the interior of the uterus. Many muciparous glands and follicles open on the surface, but these are in greatest abundance at the upper part. Conical and filiform papilla exist on the membrane; and a laminar epithelium gives a covering to it.

The muscular layer is outsile the erectile structure, and consists of longitudinal fibres. Some reach all along the vagina; others, and these are 
the strongest, only as far upwards as the recto-vesical faseia, to which they are attached on each side. Above they end in the superficial layer of the uterus, and in the subperitoneal fibrous tissue; and below in the subdermic tissue.

Bloodvessels and nerves. The arteries are derived from the vaginal, uterine, and vesical branches of the internal iliac. The veins form a plexus around the vagina, as well as in the genital organs, and open into the internal iliac vein. For a description of the nerves, see page 520 .

The lymphatics accompany the bloodvessels to the glands by the side of the internal iliac artery.

Glands of Bartholin. On the outer part of the vagina, near the lower end, are two small yellowish glandular bodies, one on each side, which represent Cowper's glands in the male. Each is about as large as a small bean ; and its duct is directed forwards to open on the inner aspect of the nympha of the same side. The duet resembles that of Cowper's gland in its structure (p. 398).

\section{THE UTERUS.}

The uterus or womb is formed ehiefly of unstriated muscular fibres. Its office is to receive the ovum, and to retain for a fixed period the developing foetus.

This viseus in the virgin state is somewhat pear shaped, the body being flattened (fig. 187), and possesses inferiorly a rounded narrow part or neek.

Before impregnation the uterus measures about three inches in length, two in breadth at the upper part, and an inch in greatest thickness. Its weight varies from an ounce to an ounce and a half. But after gestation its size and volume exceed always the measurements here given.

The upper end is convex, and is covered by peritoneum: the term fundus is applied to the part of the organ above the attachment of the Fallopian tube $(e)$.

The lower end is small and rounded, and in it is a transverse aperture of communication between the uterus and the vagina, named os uteri (os tinca) : its margins or lips (labia) are smooth, and anterior and posterior in situation, but the hinder one is the longest. Towards the lower part the uterus is constricted, and this diminished portion is called the neck (b) of the uterus (cervix uteri); it is surrounded by the vagina, and is covered by this tube to a greater extent behind than in front. The neck is about half an inch in length, and gradually tapers towards the extremity.

The body $(a)$ of the nterus is more convex posteriorly than anteriorly, and decreases in size down to the neck. It is corered on both aspects by the peritoneum, except at the lower part in front (about half an inch), where it is connected to the bladder. To each side, which is straight, the parts contained in the broad fold of the peritoneum are attached (fig. 175):-viz., the Fallopian tube at the top (x); the round ligament (x), rather below and before it; and the ovary (L), and its ligament below and behind the others.

Dissection. 'To examine the interior of the uterus, a cut is to be made along the anterior wall from the fundus to the os uteri; and then some of the thick wall is to be removed on each side of the middle line to show the contained artery. 
The thickness of the uterine wall is greatest opposite the middle of the body. It is greater at the centre than at the angles of the fundus (fig. 187), the wall becoming thinner towards the attachment of the Fallopian tubes.

Interior of the uterus (fig. 187). Within the uterus is a small space, which is divided artificially into two-that of the body, and that of the neck.

The space occupying the body of the viscus $(c)$ is triangular in form, and is larger than the other. Its base is at the fundus, where it is convex towards the cavity, and the angles are prolonged towards the Fallopian tubes. The apex is directed downwards, and joins the eavity in the cervix by a narrowed circular part, isthmus, which may be narrower than the opening of the uterus into the vagina.

The space within the neck $(d)$ terminates inferiorly at the os uteri, and is continuous above with the space within the body. It is larger at the middle than at either end, being spindle-shaped, and is somewhat flattened

Fig. 187.

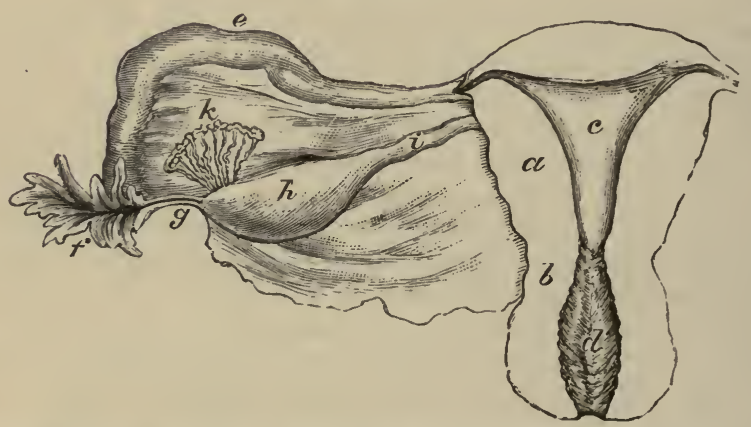

INTERIor of the Uterus, With a Posterior View of the Broad Lieament and the UTERINE APPENDAGES.

a. Body, and $b$, neck of the uterus.

$c$. Cavity of the body, and $d$, of the neck.

e. Fallopian tube, and $f$, its trumpet-shaped end. $g$. The fimbria attached to the ovary.

$h$. Ovary, and $i$, ligament of the ovary.

$k$. Parovarium.

like the cavity of the body. Along both the anterior and the posterior wall is a longitudinal ridge; and the other ridges (ruga) are directed obliquely from these on each side: this appearance has been named arbor vita uterinus. In the intervals between the rugae are mucous follicles, which sometimes become distended with fluid, and give rise to rounded clear sacs.

STRucture. The dense wall of the uterus is composed of layers of unstriated muscular fibre, intermixed with areolar and elastic tissues, and large bloodvessels. On the exterior is the peritoneum; and lining the interior is a thin mucous membrane.

The muscular fibres can be demonstrated at the full period of gestation to form three strata in the wall of the uterus, viz., external, internal, and mirldle :-

The external layer contains fibres which are mostly transverse; but at the fundus and sides they are oblique, and are more marked than along 
the middle of the organ. At the sides the fibres converge towards the broad ligament; some are inserted into the subperitoneal fibrous tissue; and others are continued into the Fallopian tube, the round ligament, and the ligament of the ovary.

The internal fibres describe circles around the openings of the Fallopian tubes, and spread from these apertures till they meet at the middle line. At the neck of the uterus they are arranged in a transverse direction.

The middle or intervening set of fibres are more indistinct than the others, and have a less determinate direction.

The mucous lining of the uterus is continued into the vagina at one end, and into the Fallopian tubes at the other.

In the body of the uterus it is of a reddish-white color, and is thin, smooth, and adherent, but without papillæ. Like the mucous membrane of the intestine, it possesses tubular glands, which may be either straight and simple, or twisted and branched; they are lined by ciliated epithelium, and their minute apertures are scattered over the surface.

In the cervix uteri, between the rugæ, mucous follicles and glands are collected, and near the outer opening are papillæ.

The epithelicil covering of the mucous membrane consists of a single layer of cells, which are columnar and ciliated throughout the cavity of the uterus.

The bloodvessels of the uterus are large and tortuous and occupy canals in the uterine substance, in which they communicate freely together. The arteries are furnished from the uterine and ovarian branches (p. 516).

The veins correspond with the arteries: they are large in size, and form plexuses in the uterus.

The nerves are derived from the sympathetic (p. 519), and are very small in proportion to the size of the uterus : in the cervix they are traced to the papillæ.

Lymphatics. One set accompanies the uterine vessels to the glands on the iliac artery. Another set issues from the fundus, enters the broad ligament, and accompanies the ovarian artery to the glands on the aorta: the last are joined by lymphatics of the ovary and Fallopian tube.

Round ligament of the uterus (fig. 175, $\mathrm{N}$ ). This firm cord supports the uterus, and is contained partly in the broad ligament, and partly in the inguinal canal. It is about five inches in length, and is attached to the upper part of the uterus close below, and anterior to the Fallopian tube. A process of the peritoneum accompanies it in the inguinal canal, and remains pervious sometimes for a short distance.

The ligament is composed of unstriated muscular fibres, derived from the uterus, together with vessels and areolar tissue.

\section{OVARIES AND FALLOPIAN TUBES.}

Ovary (fig. 187). The oraries are two bodies, corresponding with the testes of the male. They are contained in the broad ligaments of the uterus, one in each.

Each ovary is of an elongated form, and somewhat flattened from above down. It is of a whitish color, with either a smooth or a scarred surface. Its volume is variable; but in the virgin state it is about one inch and a half in length, half that size in width, and a third of an inch in thickness. Its weight varies from one to two drachins.

The ovary is placed at the back of the broad ligament, and is connected 
with that membrane by its anterior margin, where the vessels enter the stroma. Its outer end $(g)$ is rounded and is connected with one of the fimbriae at the mouth of the Fallopian tube. The inner extremity is narrowed, and is attached to the side of the uterus by a fibrous cord (i)-the ligament of the ovary, below the level of the Fallopian tube and round ligament.

Structure. The ovary consists of a stroma of areolar tissue containing small sacs named Graafian, and the whole is inclosed within a fibrous tunic. The peritoneum surrounds it except at the attached margin.

The fibrous coat is adherent to the contained stroma. Along the attached margin of the ovary is a slit, by which the vessels and nerves enter. Sometimes a yellow spot (corpus luteum), or some cicatrices, may be seen in this covering.

Stroma (fig. 188). The substance of the ovary is spongy, vaseular, and fibrous. At the centre the fibres radiate from the hilum towards the circumference. But at the exterior is a granular material (cortical layer) which contains very many small cells, about ${ }_{10} \frac{1}{0}$ th of an inch in size-the nascent Graafian vesicles.

The Graafian vesicles or ovisacs (fig. 188) are round and transparent cells, seattered through the stroma of the ovary below the cortical layer. During the child-bearing period some are larger than the rest $(a)$; and of this larger set ten to thirty, or more, may be counted at the same time;

Fig. 188.

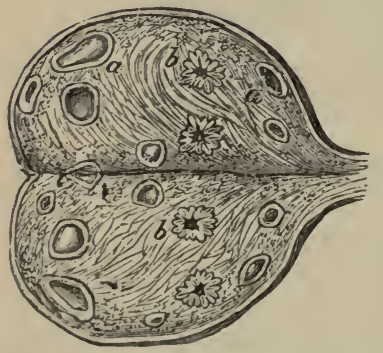

Ofart dURing the Chit, D-Bearing PERIOD LAID OPEN.

$a$. Graaflan vesicles in different stages of growth.

b. Plicated body remaining after the escape of the ovum (Farre). these vary in size from a pin's head to a pea. The largest are situate at the circumference of the organ, and sometimes they may be seen projecting through the fibrous coat.

Each consists of a transparent coat with a fluid inside. The coat of the vesicle named ovi-capsule (tunica fibrosa), is formed of fine areolar tissue, and is united to the stroma of the ovary by bloodvessels, which ramify in the wall. Lining it is a layer of nucleated granular cells - the membrana granulosa, which is thickened at one spot, and surrounds the ovum at the discus proligerus (Von Baer), fixing it to the wall. 'Ths fluid in the interior is transparent and albuminous; it contains the minute ovum, together with molecular granules.

When the Graatian vesicle is matured it bursts on the surface of the ovary, and the contained ovum escapes into the Fallopian tube. After the shedding of the ovum the ruptured vesicle gives origin to a yellow substance, corpus luteum, which finally changes. into a cicatrix $(b)$.

Bloodvessels and nerves. The ovarian artery pierces the ovary at the anterior or attached border, and its branches run in zigzag lines through the stroma, to which and the Graafian vesicles they are distributed. The veins begin in the vesicles and the texture of the ovary, and after escaping from its substance, form a plexus (pam-piniform) within the fold of the broad ligament. The nerves are derived from the sympathetic on the ovarian and uterine vessels.

Appendage to the ovary (fig. 187,k) (Parovarium, Organ of Rosen- 
miiller). On holding up the broad ligament of the uterus to the light, a collection of small tortuous tubules will be seen between the ovary and the Fallopian tube. 'This is the remnant of the upper part of the Wolffian body of the fretus; it is about one inch broad, with its base to the Fallopian tube, and apex to the attached part of the ovary. The small tubes are from twelve to twenty in number; at the wider end they are joined more or less perfectly by a tube crossing the rest, which is prolonged sometimes a short way into the broad ligament. Each tube is a closed fibrous capsule with a clear fluid within, and with a lining of epithelium.

Fallopian Tubes (fig. 187,e). Two in number, one on each side, they convey the ova from the ovaries to the uterus, and correspond in their office with the vasa deferentia in the male.

Each is about four inches in length: cord-like at the inner end, where it is attached to the upper part of the uterus, it increases in size towards the outer end, and terminates in a wide extremity $(f)$, like the mouth of a trumpet. This dilated end is fringed, and the pieces are called fimbria. When the fimbriated end is floated out in water, one of the processes $(g)$ may be seen to be connected with the outer end of the ovary. In the centre of the fimbrix is a groove leading to the orifice of the Fallopian tube.

On opening the tube with care, the size of the contained space, and its small aperture into the uterus can be observed. Its canal varies in size at different spots: the narrowest part is at the orifice into the uterus (ostium uterinum), where it scarcely gives passage to a fine bristle; towards the outer end it increases a little, but it is rather diminished in diameter at the outer aperture (ostium abdominale).

Structure. This excretory tube has the same structure as the uterus with which it is connected, viz., a muscular layer covered externally by peritoneum, and lined by mucous membrane.

The muscular coat is formed of an external or longitudinal, and an internal or circular layer; both these are continuous with similar strata in the wall of the uterus.

The mucous membrane forms some longitudinal folds, particularly at the outer end. At the inner extremity of the canal it is continued into the mucous lining of the uterus, but at the outer end it joins the peritoneum. A columnar and ciliated epithelium covers the surface, as in the uterus, and is said by Henle to be detected on the outer surface of the fimbriae.

The bloodvessels and nerves are furnished from those supplied to the ovary and uterus.

THE BLADDER, URETHRA, AND RECTUM.

BLADDER. The peculiarities in the form and size of the female bladder have been detailed in the description of the connections of the viscera of the female pelvis (p. 513). For a notice of its structure, the anatomy of the male bladder is to be referred to (p. 524).

Dissection. To prepare the bladder, distend it with air, and remove the peritoneal covering and the loose tissue from the muscular fibres.

After the external anatomy of the bladder, and urethra has been learnt, they are to be slit open along the upper part.

Urethra. The length and the connections of the excretory tube are given in page 512 .

The average diameter of the urethra is rather more than a quarter of an inch, and the canal is enlarged and funnel-shaped towards the neck of the 
bladder; near the external aperture is a hollow in the floor. In consequence of not being surrounded by resistant structures, the female urethra is much more dilatable than the corresponding passage in the male.

Structure. Like the urethra of the male, it consists of a mucous coat, which is enveloped by a plexus of bloodvessels, and by muscular fibre.

The muscular layer extends the whole length of the urethra. Its fibres are circular, corresponding with the prostatic enlargement in the other sex, and continuous behind with the middle layer of the bladder. In the perineal ligament this stratum is covered by the constrictor urethre as in the male (p. 403).

The mucous coat is pale except near the outer orifice. It is marked by longitudinal folds; and one of these, in the floor of the canal, resembles the median crest in the male urethra (p. 526). Around the outer orifice are some mucous follicles; and towards the inner end are tubular mucous glands, whose apertures are arranged in lines between the folds of the membrane. A laminar epithelium is spread over the surface, and beneath it are deeper conical cells as in the bladder.

A submucous stratum of longitudinal elastic and muscular tissues lies close beneath the mucous membrane, as in the male.

Dissection. The rectum may be prepared for examination by distending it with tow, and by removing the peritoneal covering and the areolar tissue from its surface.

RECTUM. The structure of the rectum is similar in the two sexes; and the student may use the description in the Section on the viscera of the male pelris (p. 522).

\section{Section VII.}

INTERNAL MUSCLES OF THE PELVIS.

Two muscles, the pyriformis and obturator internus, have their origin within the cavity of the pelvis.

Dissection. Take away any fascia or areolar tissue which may remain on the muscles; and define their exit from the pelvis, the pyriformis passing through the great sacro-sciatic notch, and the obturator through the sniall notch of the same name. On the right side the dissector may look to the attachment of the levator ani muscle to the pubic part of the hipbone.

The PYRIFORMIS MUSCLE is directed outwards through the great sacrosciatic notch to the great trochanter of the femur. The muscle has received its name from its form.

In the pelvis the pyriformis arises by three slips from the second, third, and fourth pieces of the sacrum, between the anterior aperture:, and from the lateral part of the bone external to those holes; as it passes from the pelvis, it takes origin also from the surface of the hip-bone forming the upper part of the large sacro-sciatic notch, and firom the great sacro-sciatic lig.ument. From this origin the fibres converge to the tendon of insertion into the troclianter. (Dissection of the B.ttock.)

The anterior surface is in contact with the rectum on the left side, with the sacral plexus, and with the sciatic and pudic branches of the internal 
iliac vessels. The opposite surface rests on the sacrum, and is covered by the great gluteal muscle outside the pelvis. The upper border is near the hip-bone, the gluteal vessels and the superior gluteal nerve being between : and the lower border is contiguous to the coceygeus muscle, the sacral plexus, and the sciatic and pudic vessels intervening.

Action. The pyriformis belongs to the group of external rotators of the hip-joint; and its use will be given with the description of the rest of the muscle in the dissection of the Buttock.

The obTURATor internus Muscle has its origin in the pelvis, and insertion at the great trochanter of the femur, like the preceding: but the part outside is almost parallel in direction with that inside the pelvis.

The muscle arises by a broad fleshy attachment from the obturator membrane, except a small part behind ; from the pelvic fascia covering its surface; slightly from the bone anterior to the thyroid hole, but from all the smooth inclinerl surface of the pelvis behind and above that aperture, though opposite the small sacro-sciatic foramen a thin layer of fat separates the fleshy fibres from the bone. The fibres are directed backwards and somewhat downwards, and end in three or four tendinous pieces, which turn over the sharp edge of the hip-bone corresponding with the small sacro-sciatic notch. Outside the pelvis the tendons blend into one, which is inserted into the great trochanter.

The muscle is in contact by one surface with the wall of the pelvis and the obturator membrane; by the other surface with the pelvic fasciab, and towards its lower border with the pudic vessels and nerve.

Action. The musele draws the trochanter towards the back of the hipbone over which it bends, and rotates out the hip joint. For further notice of its use, see the dissection of the Buttock.

Coccygeus Muscle. The position and the connections of this muscle may be studied with advantage in the interior of the pelvis. The muscle is described at p. 501 .

\section{LIGAMENTS OF THE PELVIS.}

The following are the articulations between the bones of the pelvis:The several pieces of the sacrum and coccyx are united with one another. The sacrum is joined at its base to the last lumbar vertebra, at its apex to the coccyx, and laterally to the two innominate bones. And the innominate bones are connected together in front, as well as to the sacrum and the spinal column posteriorly.

Union of Pieces of Sacrum and Coccyx. Whilst the pirces of the sacrum and coccyx remain separate they are articulated as in the other vertebræ by an anterior and posterior common ligament, with a disk of intervertebral substance for the bodies; and by other ligaments for the neural areh and processes (p. 346).

After the sacral vertebre have coaleseed, only rudiments of the ligaments of the bodies are to be recognized; but when the pieces of the coccy $x$ unite by bone, those ligaments disappear in the adult male.

Sacro-vertebral Articulation. The base of the sacrum is articulated with the last lumbar vertebra by ligaments similar to those uniting one vertebra to another (p. 346 ); and by one special ligament-the sacrovertebral.

Dissection. For the best manner of bringing these different ligaments 
into view, the dissector may consult the directions already given for the dissection of the ligaments of the vertebræ (p. 346).

The common ligaments for the bodies of the two bones are an anterior and a posterior, with an intervening fibro-cartilaginous substance. Between the neural arches lic the ligamenta subflava; and between the spines the supra and interspinous bands are situate. The articular processes are united by capsular ligaments with synovial membranes.

The sacro-vertebral ligament is a rather strong bundle of fibres, which reaches from the under surface of the tip of the transverse process of the last lumbar vertebra to the lateral part of the base of the sacrum. Widening as it descends, the ligament joins the fibres in front of the articulation between the sacrum and the innominate bone.

Sacro-coccygeal Articulation. The sacrum and coccyx are united at the centre by a fibro-cartilage, and by an anterior and posterior common ligament. And there is a separate articulation for the cornua of the bones.

Dissection. Little dissection is needed for these ligaments. When the areolar tissue has been removed altogether fiom the bones, the ligaments will be apparent.

The anterior ligament (sacro-coccygeal) consists of a few fibres that pass between the bones in front of the fibro-cartilage.

The posterior ligament is wide at its attachment to the last piece of the sacrum, but narrows as it descends to be inserted into the coccyx.

. The fibro-cartilage resembles that between the bodies of the other vertebræ, and is attached to the surfaces of the bones.

Articulation of the cornua. The cornua of the first piece of the coccyx are united with the cornua of the last sacral vertebra by ligamentous bands, and not by joints as in the articular processes of the other vertebræ.

Movement. Whilst the coccyx remains separate from the sacrum, a slight antero-posterior movement will take place between them.

Sacro-iliac Articulation. The irregular surfaces by which the sacrum and the innominate bone articulate, are united by cartilage (synchondrosis), and are maintained in contact by anterior and posterior sacroiliac ligaments. Inferiorly the bones are further connected, without being in contact, by the strong sacro-sciatic ligaments.

Dissection. To see the posterior ligaments, the mass of muscle at the back of the sacrum is to be removed on the side on which the innominate bone remains. The anterior bands will be visible on the removal of some areolar tissue. The small sacro-sciatic ligament will be bronght into view by removing the coccygeus; and the large ligament is dissected with the lower limb.

The anterior sacro-iliac ligament consists of a few thin scattered fibres between the bones, near their articular surfaces.

The posterior ligaments (sacro-iliac) are much stronger than the anterior, and the fibres are collected into bundles: these ligaments pass from the rough inner surface, at the posterior end of the innominate bone, to the first two pieces of the sacrum. One bundle, which is distinct from the others, and more superficial, is named the oblique or long posterior ligament; it is attached to the posterior upper iliac spinous process, and descends almost vertically to the third piece of the sacrum.

Articular cartilage. Between the irregular surfaces of the bones is a thin uneven layer of eartilage (fig. 189, a). It fits into the inequalities of the osseous surfaces, uniting them very firmly together. On separating 
the bones after the other ligaments are examined, the cartilage may be detached with a knife.

Movement. There is scarcely any appreciable motion in the sacro-iliac articulation, even when the hip-bone is seized by the hand, and forcibly pulled in different directions. The articulation seems designed for security and little movement, inasmuch as the surfaces are not in contact, are very irregular, and have a firm and inextensible piece of cartilage interposed between them. In some instances, and especially during pregnancy, there is a greater degree of motion perceptible.

Two sacro-sciatic ligaments pass from the lateral part of the sacrum and coccyx to the hinder border of the os innominatum, across the space between the bones at the back of the pelvis : they are named large and small.

The large ligament (fig. 190,a) reaches from the back of the hip bone, and the side of the sacrum and coccyx to the ischial tuberosity. As this

Fig. 189.

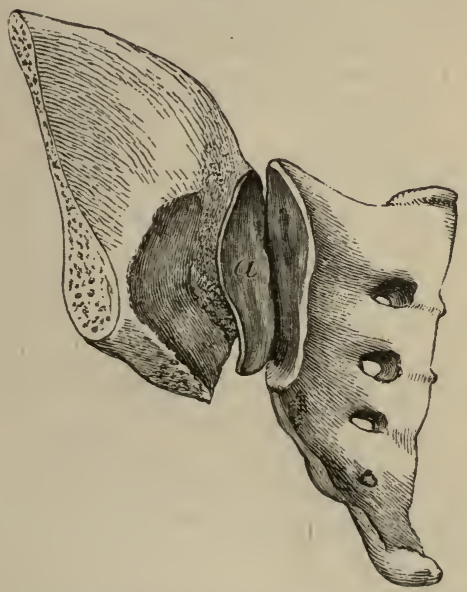

This figure shows the irregular piece of cartilage $(a)$ in the sacro-iliac articulation.
Fig. 190 .

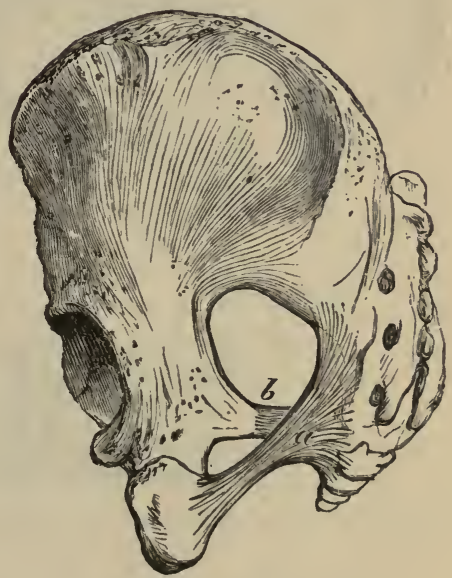

SACR 0-SCIATIC LIGAMRNTS. a. Large or posterior ligament. b. Small or anterior ligament.

may have been cut in the examination of the gluteal region, no further notice is given here; but if it remains entire, see Dissection of the Buttock for its description.

The small ligament (fig. 190, $b$ ) is attached internally by a wide piece to the border of the sacrum and coccyx, where it is united with the origin of the preceding band. The fibres are directed outwards, and are inserted by a narrowed part into the ischial spine of the hip bone. Its pelvic surface is covered by the coccygeus muscle; and by tle opposite surface it is in contact with the great sacro-sciatic ligament. Above it is the large sacro-sciatic foramen; and below it is the small foramen of the same name, which is bounded by the two ligaments.

By their position these ligaments convert into two foramina (sacrosciatic), the large sacro-sciatic excavation in the dried bones: the openings, and the parts they give passage to, are described with the Buttock. 
Ligaments of the Innominate Bones (fig. 191). The innominate bones are united in front, at the pubic symphysis, by an interposed piece of cartilage and special ligaments; and behind, each is connected with the transverse process of the last lumbar vertebra by a separate band (iliolumbar). In the centre of the bone is a membranous structure closing the thyroid aperture.

The ilio-lumbar or ilio-vertebral ligament is triangular in form and is divided into fasciculi. Internally it is attached to the tip of the transverse process of the last lumbar vertebra ; externally the fibres spread out,

Fig. 191.

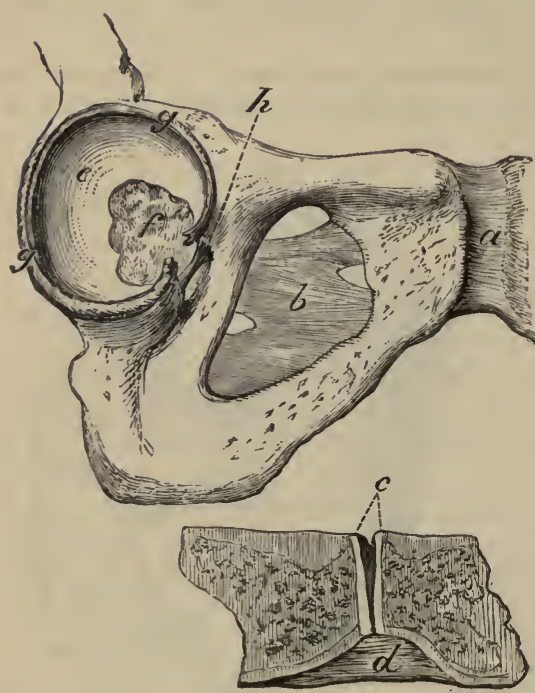

a. Anterior ligament of the symphysis ; $d$, inferior, and $c$, cartilage of the symphysis, with a slit in the middle.

b. Obturator membrane.

$e$. Surface of the acetabulum covered with cartilage.

$f$. Fatty substance in the acetabulum.

g. Cotyloid ligament, which is cut where it forms part of the transverse band over the notch.

$h$. Deep part of the ligament over the cotyloid notch.

Ligaments of the Symphysis Pubis, Thyroid Hole, and Acetabulum.

and are inserted into the iliac-crest for about an inch, opposite the posterior part of the iliac fossa. To the upper border of the ligament the fuscia lumborum is attached; its posterior surface is covered by the quadratus lumborum, and its anterior by the iliacus muscles.

The thin obturator membrane (fig. 191, b) closes almost entirely the thyroid foramen, and is composed of fibres crossing in different directions. It is attached to the bony margin of the foramen, except above where the obturator vessels pass through ; and towards the lower part of the aperture it is connected to the pelvic aspect of the hip-bone. The surfaces of the ligament give attachment to the obturator muscles. Branches of the obturator vessels and nerve perforate it.

Pubic Articulation (fig. 191, a) (symphysis pubis). The oval pubic surfaces of the hip bones are united by cartilage, and by fibres in front of, and above the bones: they are also connected by a strong subpubic ligament.

The anterior pubic ligament (fig. $191, a$ ) is very strong and is formed of different layers of fibres. The superficial are oblique, and cross one another, joining with the aponeurosis of the external oblique muscle of 
the abdomen; but the deeper fibres are transverse between the surfaces of the bones. Some of the deepest fibres contain cartilage cells.

There is not any strong posterior band; but beneath the periosteum are a few scattered fibres in contact with the articular cartilage.

The superior ligamentous fibres fill the interval between the bones above the cartilage.

The subpubic ligament (fig. 191, d) (ligam. arcuatum) is a strong triangularly-shaped band below the symphysis, and occupies the upper part of the pubic areh. The apex of the ligament touches the articular cartilage, and the base, contained within the triangular perinæal ligament, is turned towards the membranous part of the urethra.

Dissection. The cartilage will be best seen by a transverse section of the pubes, which will show the disposition of the anterior ligament of the articulation, and the thickness of the cartilage, with its toothed mode of insertion into the bone; but when opportunity offers, a vertical section may be made.

Cartilage (fig. 191, c). The cartilage is firmly fixed to the ridged bony surfaces of the sympliysis: it is wider above than below, and is generally as thick again before as behind. Variations in its size depend on the shape of the bones.

Towards the posterior part of the cartilage there is a narrow fissure with uneven walls; and a fibrous structure with large interspersed compound cells is to be recognized in the wall. It extends usually the whole depth of the cartilage and through a half or a third of the thickness : it is said to increase in pregnancy. In some bodies it reaches through the cartilage so as to divide this into two collateral pieces.

Movement. As the bones are not in contact in the pubic symphysis, but are united by an intermediate cartilage, the motion is usually very slight. The kind of movement of the hip bone is inward and outward, so as to increase or diminish the pelvic eavity.

When the pubic cartilage is divided into two by a larger central space than usual, greater freedom of motion is present in the symphysis; and in pregnancy the looseness of the innominate bone is sometimes so great as to interfere seriously with locomotion. 
TABLE OF THE ARTERIES OF THE ABDOMEN.

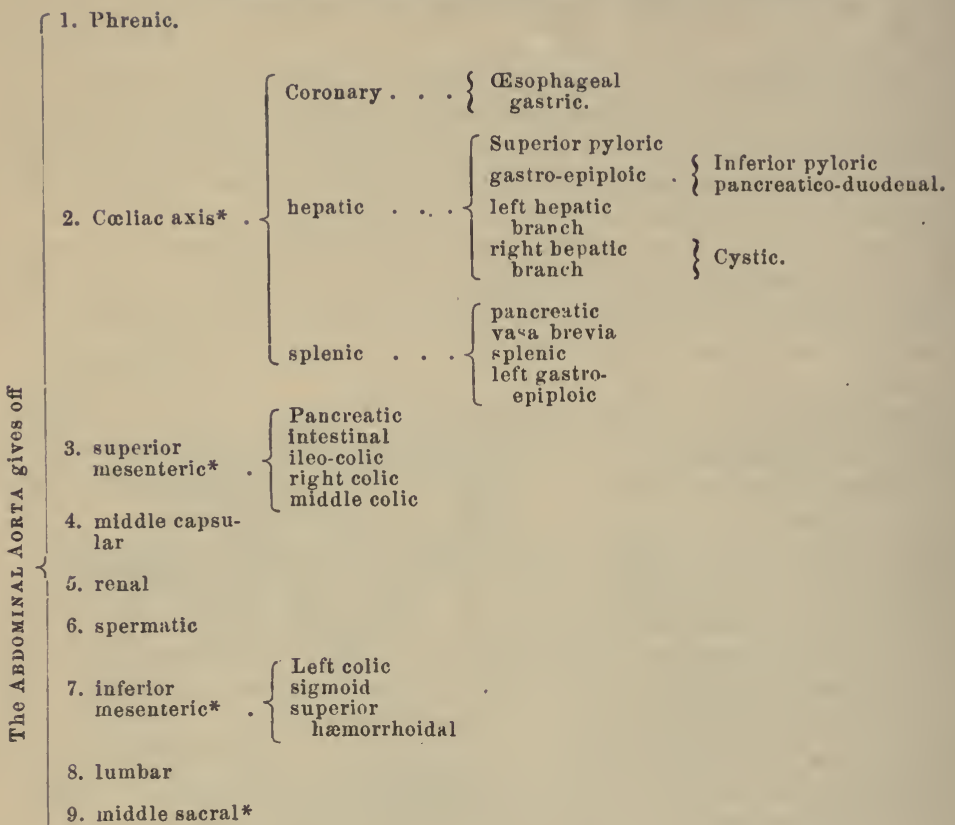

10. common iliac.

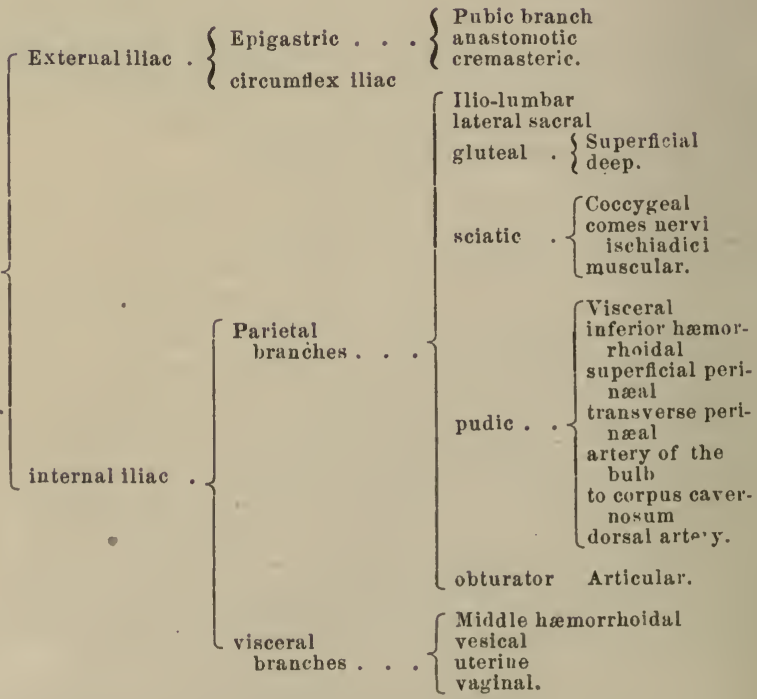

* The branches marked with an asterisk are single. 
TABLE OF THE VEINS OF THE ABDOMEN.

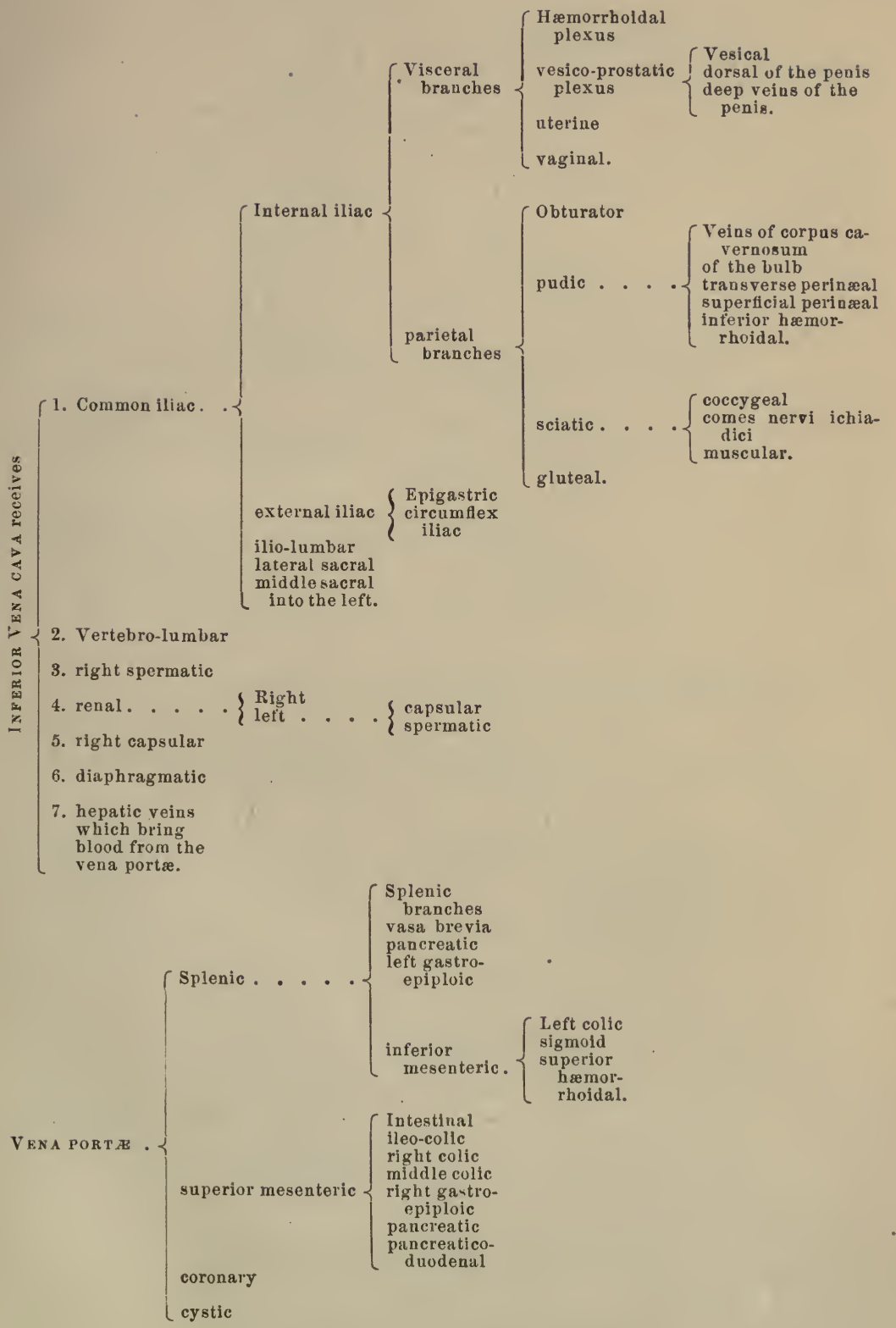


TABLE OF TIIE SPINAL NERVES IN THE ABDOMEN.

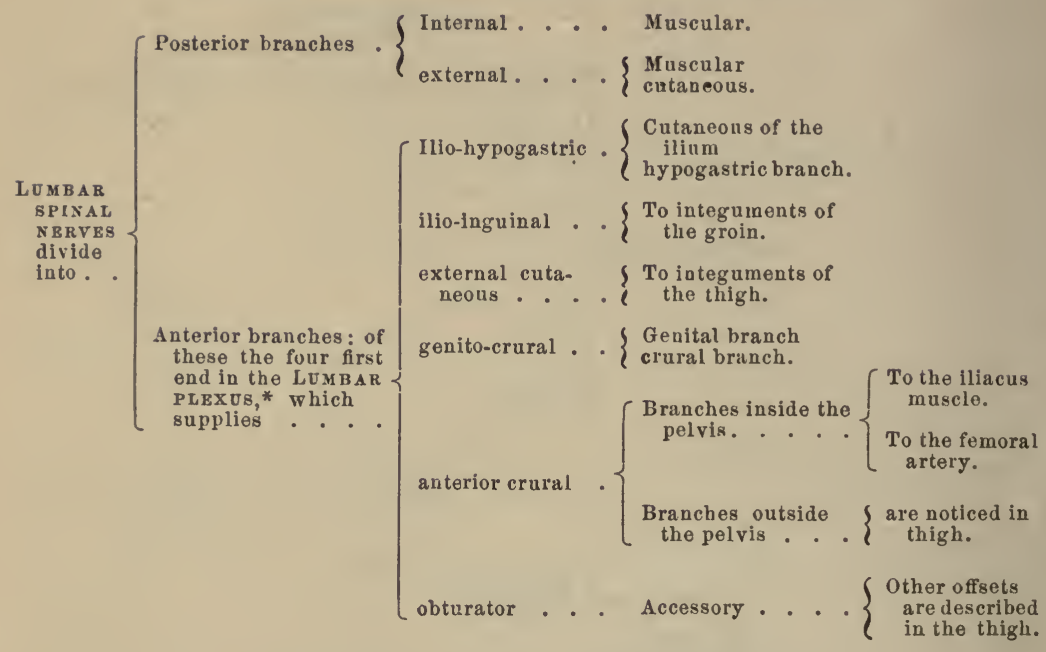

* The lumbo-sacral gives off the superior gluteal nerve.

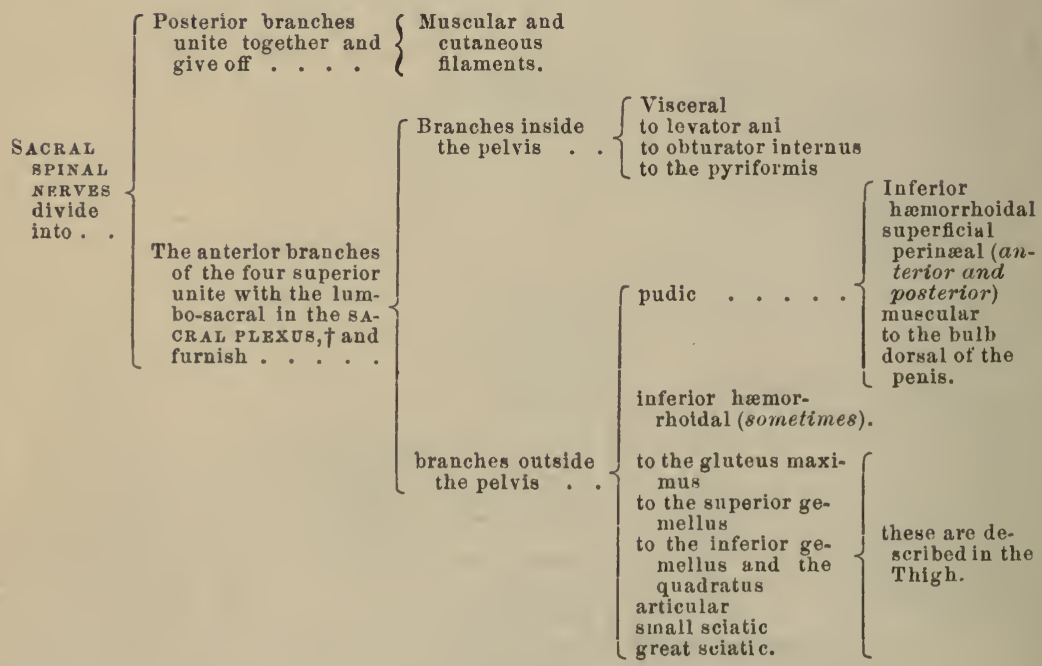

† The other sacral nerves are described at p. 518 . 
TABLE OF SYMPATHETIC NERVE OF THE ABDOMEN.
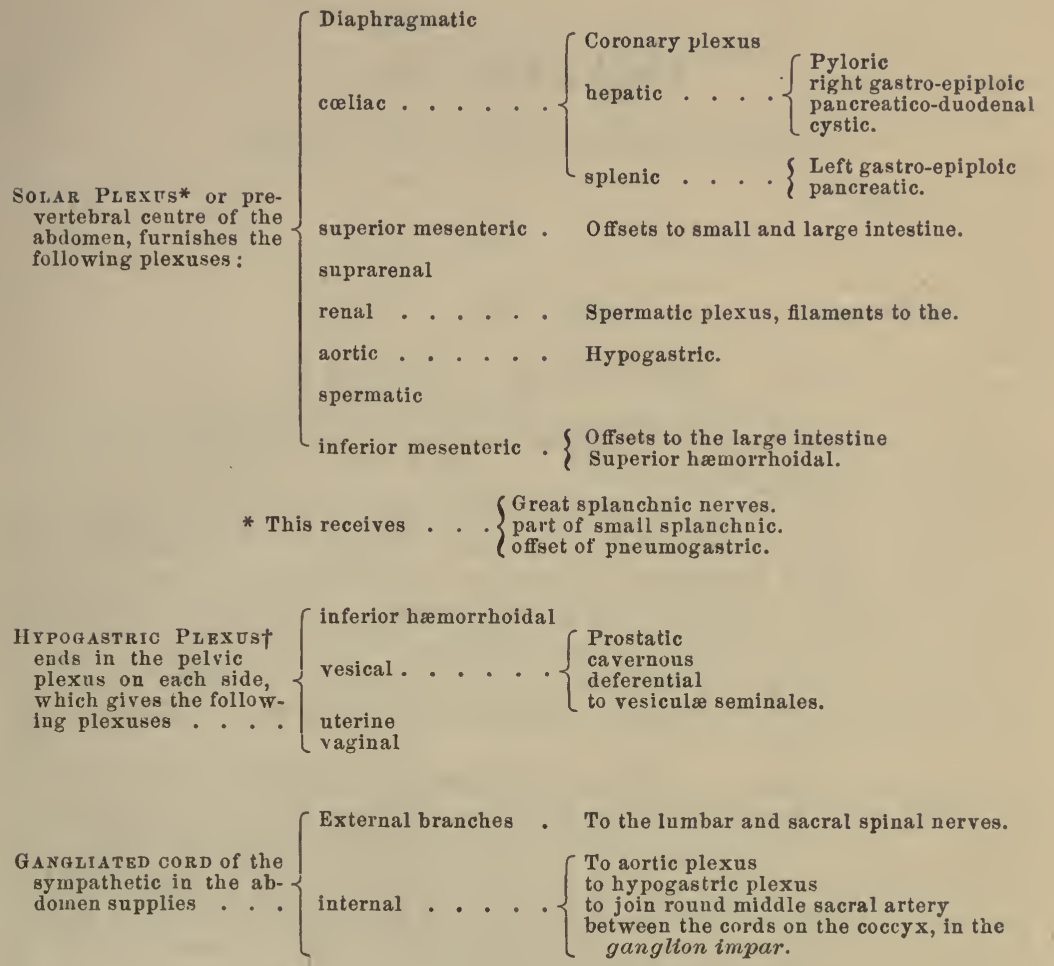

+ This is joined above by .. $\quad \begin{aligned} & \text { The aortic plexus } \\ & \text { flaments from the lumbar ganglia. }\end{aligned}$

\section{PNEUMOGASTRIC NERVE IN THE ABDOMEN.}

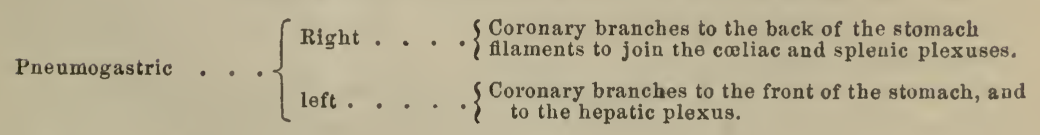




\title{
CHAPTER IX.
}

\author{
DISSECTION OF THE LOWER LIMB.
}

\section{Section I.}

\section{THE FRONT OF THE THIGH.}

AlL the parts described in Section I. are to be examined before the time for turning the body arrives.

Position. During the dissection of the front of the thigh the body lies on the back, with the buttocks resting on the edge of the table, and with a block of suitable size beneath the loins. 'The lower limb should be supported in a half-bent position by a stool beneath the foot, and should be rotated outwards to make evident a hollow at the upper part of the thigh.

Surface marking. Before any of the integument is removed from the limb, the student is to observe the chief eminences and hollows on the surface of the thigh.

The limit between the thigh and abdomen is marked, in front, by the firm band of Poupart's ligament reaching from the erest of the hip bone to the pubes. On the outer side, the separation is indicated by the convexity of the iliac erest of the lip bone, which subsides behind in the sacrum and cocсyx. On the inner side is the projection of the pubes, from which a line of bone (pubic arch) may be traced backwards along the inner and upper parts of the limb to the ischial tuberosity.

On the anterior aspect of the thigh, and close to Poupart's ligament, is a slight hollow, corresponding with the triangular space of Searpa, in which the larger vessels of the limb are contained; and extending thence obliquely towards the inner side of the limb, is a slight depression marking the situation of the femoral artery beneath. The position of the arterial trunk may be ascertained by a line from the centre of the interval between the symphysis pubis and the crest of the hip bone, to the inner condyle of the femur.

At the outer side of the athigh, about four inches below and behind the anterior part of the iliac crest, the well-markerl projection of the great trochanter of the femur will be recognized. In a thin body the hearl of the femur may be felt by rotating the limb inwards and outwards, whilst the thumb of one hand is placed in the hollow below Poupart's ligament, or the fingers behind the great trochanter.

At the knee the outline of the several bones entering into the formation of the joint may be traced with ease. In front of the joint, when it is half-bent, the rounded prominent patella may be perceived; this bone is firmly fixed whilst the limb is kept in the same position, but is moved with great freedom when the joint is extended, so as to relax the muscles 
inserted into it. On each side of the patella is the projection of the condyle of the femur, but that on the inner side is the largest. If the fingers are passed along the sides of the patella whilst the joint is half-bent, they will be conducted to the tuberosities of the head of the tibia, and to a slight hollow between the bones.

Behind the joint is a slight dspression over the situation of the ham or popliteal space; and on its sides are firm boundaries, which are formed by the tendons (hamstrings) of the flexor muscles of the leg.

Dissection. With the position of the limb the same as before directed, the student begins the dissection with the examination of the subcutaneous fatty tissue with its nerves and vessels.

At first the integument is to be reflected only from the hollow on the front of the thigh close below Poupart's ligament. To raise the skin from this part, an incision about four inches in length, and only skin deep, is to be made from the pubes along the inner border of the thigh. At the lower end of the first incision, another cut is to be directed outwards across the front of the limb to the outer aspect; and at the upper end the knife is to be carried along the line of Poupart's ligament as far as the crest of the hip bone. 'The piece of skin included by these incisions is to be raised and turned outwards, without taking with it the subcutaneous fat.

The subcutaneous fatty tissue, or the superficial fascia, forms a general investment for the limb, and is constructed of a network of areolar tissue, with fat or adipose substance amongst the meshes. As a part of the common covering of the body, it is continuous with that of the neighboring regions, consequently it may be followed inwards to the scrotum or labium, and upwards on the abdomen.

Its thickness varies in different bodies, according to the quantity of fat in it; and at the upper part of the thigh it is divisible into two strata (superficial and deep) by some cutaneous vessels and inguinal glands. The superficial of the two layers is apparent after the removal of the skin, but its connections will be made more evident by the following dissection.

Dissection. To reflect the superficial stratum of the fascia, incisions similar to those in the skin are to be employed, though the transverse one is not to reach so low on the thigh by a couple of inches; and the separation from the subjacent structures is to be begun at the lower part, where the large saphenous vein, and a condensed or membranous appearance on the under surface, will mark the depth of the stratum. 'This layer of the fat may be thrown upwards readily, by a few touches of the knife along the middle line of the limb; but where vessels and glands are not found, viz., along the outer and inner borders of the thigh, the separation of the superficial fascia into two layers cannot be easily made.

'The subcutaneous layer of the fat decreases in thickness near Poupart's ligament, becoming more fibrous at the same spot; and at its under aspect is a smooth and membranous surface. It conceals the superficial vessels and the inguinal glands, and is separated by these from Poupart's ligament.

Dissection (fig. 193). The inguinal glands and the superficial vessels are to be next laid bare by the removal of the surrounding fat; but the student is to be careful not to destroy the deeper, very thin layer of the superficial fascia, which is beneath them, and is visible chiefly on the inner side of the centre of the limb. Three sets of ressels are to be dissected out:-One set (artery and vein) is directed inwards to the pubes, 
and is named external pudic ; another, superficial epigastric, ascends over Poupart's ligament; and the third, or the superficial circumflex iliac, appears at the outer border of the limb. The large vein in the middle line of the thigh to which the branches converge, is the internal saphenous.

Some of the small lymphatic vessels may be traced from one inguinal gland to another.

A small nerve, the ilio-inguinal, is to be sought on the inner side of the saphenous vein, close to the pubes; and the branch of the genito-crural nerve, or an offset of it, may be found a little outside the vein.

Superficial Vessels. The small cutaneous arteries at the top of the thigh are the first branches of the femoral trunk; they pierce the deep fascia (fascia lata), and are distributed to the integuments and the glands of the groin.

The external pudic artery (b) (superior) crosses the spermatic cord in its course inwards, and ends in the integuments of the penis and scrotum, where it anastomoses with offsets of the internal pudic artery.

Another external pudic branch (inferior, p. 565) pierces the fascia lata at the inner border of the thigh, and ramifies also in the scrotum. In the female both branches supply the labium pudendi.

The superficial epigastric artery (c) passes over Poupart's ligament to the lower part of the abdomen (p. 407), and communicates with branches of the deep epigastric artery.

The superficial circumflex iliac artery $(d)$ is the smallest of the three branches: appearing as two or more pieces on the outer border of the thigh near the iliac crest, it is distributed in the integuments : it supplies an offset with the external cutaneous nerve.

A vein accompanies each artery, having the same name as its companion vessel ; and ends in the upper part of the saphenous vein, with the exception of that with the inferior pudic artery : but the description of these veins will be given in a subsequent page.

The superficial inguinal glands $(e)$ are arranged in two lines. One set lies across the thigh, near Poupart's ligament; and the other is situate along the side of the saphenous vein. In the lower or femoral group the glands are larger than in the upper, and the lymphatic vessels from the surface of the lower limb enter them. The upper or abdominal group is joined by the lymphatics of the penis, by those of the lower part of the abdomen, and by those of the buttock. The glands vary much in number and size; and not unfrequently the longitudinal set by the side of the vein are blended together.

Dissection. The deeper layer of the superficial fascia is to be detached from the subjacent fascia lata. Internal to the saphenous vein a thin membrane can be raised by transverse cuts above and below, and by a longitudinal one on the inner side of the thigh ; but external to that vessel there exists scarcely a continuous layer. The handle of the scalpel may be employed in the separation; and the dissector is to avoid injuring the nerves and vessels. In reflecting the stratum the margin of an aperture (saphenous) in the fascia lata will become apparent.

The deeper layer of the superficial fascia is a very thin membraniform stratum, which is most evident near Poupart's ligament, and on the inner side of the saphenous vein. About one inch below the ligament it conceals the large saphenous opening in the faseia lata; and as it stretches across the aperture it is attached to the circumference-internally by loose areolar 
tissue, but externally by firm fibrous bands; it is also connected with the loose crural sheath of the subjacent vessels in the aperture.

The part of the stratum over the saphenous opening is perforated by many small apertures for the transmission of the lymphatics; and it has been named cribriform fascia from its sieve-like appearance. In a hernial protrusion through that opening the cribriform portion is projected forwards by the tumor, and forms one of the coverings.

Dissection. Now the student has observed the disposition of the superficial fascia near Poupart's ligament, he may proceed to examine the remainder of the subcutaneous covering of the thigh, together with the vessels and nerves in it.

To raise the skin from the front of the thigh, a cut is to be carried along the centre of the limb, over the knee joint, to rather below the tubercle of the tibia. At the extremity a transverse incision is to be made across the front of the leg, but this is to reach farthest on the inner side. The skin may be reflected in flaps inwards and outwards; and as it is raised from the front of the knee a superficial bursa between it and the patella will be opened.

The saphenous vein is first to be traced out in the fat as far as the skin is reflected, but in removing the tissue from it the student should be careful of branches of the internal cutaneous nerve.

The cutaneous nerves of the front of the thigh (fig. 192) are to be sought in the fat, with small cutaneous arteries, in the following positions: On the outer margin, below the upper third, is placed the external cutaneous nerve. In the middle of the limb, below the upper third, lie the two branches of the middle cutaneous nerve. At the inner margin are the ramifications of the internal cutaneous nerve-one small offset appearing near the upper part of the thigh; one or more about half way down ; and one of the terminal branches (anterior) about the lower third.

On the inner side of the knee three other cutaneous nerves are to be looked for: One, a branch of the great saphenous, is directed outwards over the middle of the patella. Another, the trunk of the great saphenous nerve, lies by the side of the vein of the same name, close to the lower part of the surface now dissected. And the third is a terminal branch (inner) of the internal cutaneous nerve, which is close behind the preceding, and communicates with it.

Vrssels. All the cutaneous veins on the anterior and inner aspects of the thigh are collected into one; and this trunk is named saphenous from its manifest appearance on the surface.

The internal saphenous vein (fig. 193, $a$ ) is the cutaneous vessel of the inner side of the lower limb, and extends from the foot to the upper part of the thigh. In the part of its course now dissected, the vessel lies inferiorly somewhat behind the knee-joint ; but as it ascends to its termination, it is directed along the inner side and the front of the thigh. Near Poupart's ligament it pierces the fascia lata by a special opening named saphenous, and enters the deep vein (femoral) of the limb.

Superficial branches join it both externally and internally; and near Poupart's ligament the three veins corresponding with the arteries in that situation, viz., external pudic $(b)$, superficial epigastric $(c)$, and circumflex iliac $(d)$, terminate in it. Towards the upper part of the limb the veins of the inner side and back of the thigh are most frequently united into one branch, which enters the saphenous trunk near the aperture in the fascia lata; and sometimes those on the outer side of the thigh are collected to- 
gether in a similar way. When this arrangement exists three large veins will be present on the front of the thigh, near the saphenous opening. On the side of the knee the vein receives a deep branch from the joint.

Some unnamed cutaneous arteries are distributed to the integuments along with the nerves; and the superficial branch of the anastomotic artery (p. 568) accompanies the saphenous nerve and its branches near the knee.

Nerves. The cutaneous nerves of the thigh are derived from branches of the lumbar plexus, and are distributed in greater number on the inner than the outer side.

Ilio-inguinal. This nerve (p. 497) is small in size, and reaches the

Fig. 192.

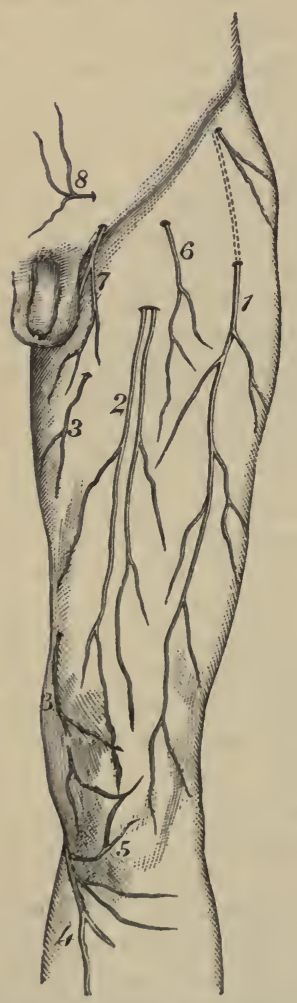

Cutaneots Nerveb ON the Front OF THЕ THIGH.

1 External cutaneons.

2. Middle cutaneous.

3. Internal cutanenus.

4. Internal saphenous.

5. Patellar branch of saphenous.

6. Genito-crural.

7. Ilio-inguinal.

\&. Ilio-hypogastric on the belly. surface by passing through the external abdominal ring (fig. 192, ${ }^{7}$ ); it supplies the scrotum, and ends in the contiguous part of the thigh, internal to the saphenous vein.

The Genito-crural. The crural branch of this nerve (p. 497) pierces the fascia lata near Poupart's ligament (fig. 192, ${ }^{6}$ ), rather external to the line of the femoral artery. After or before the nerve has become superficial it communicates with the middle cutaneous nerve; and it extends on the anterior aspect of the thigh as far as midway between the knee and the pelvis.

Occasionally this branch is of large size, and takes the place of the external cutaneous nerve on the outer side of the limb.

The external cutaneous nerve (p. 497) ramifies on the outer aspect of the limb (fig. $\left.192,{ }^{1}\right)$. At first it is contained in a prominent ridge of the fascia lata on the outer margin of the thigh, where it divides into an anterior and a posterior branch.

The posterior branch subdivides into two or three others, which arch backwards to supply the integuments of the outer part of the thigh as low as the middle.

The anterior branch appears on the surface of the fascia lata about four inches from Poupart's ligament, and is continued to the knee; it distributes branches laterally, but those towards the posterior surface are the most numerous, and the largest in size.

Middle cutaneous (fig. $192,{ }^{2}$ ). The nerve of the centre of the thigh is a cutaneous offset of the anterior crural (p. 497), and divides into two branches. It is transmitted through the fascia lata about three inches from Poupart's ligament, and its branches are continued to the knee. In the fat this nerve is united with the genito-crural and internal cutaneous nerves.

Internal cutaneous. Derived from the anterior crural trunk, this nerve is furnished to all the inner side of the 
thigh. It is divided into two branches (anterior and inner), which perforate the fascia in separate places.

The anterior branch becomes cutaneous in the lower third of the thigh, in the line of the inner intermuscular septum (fig. $192,{ }^{3}$ ), along which it is continued to the knee. This branch is distributed in the lower third of the thigh, as well as over the patella and the inner side of the kneejoint, and is united with the patellar branch of the internal saphenous nerve.

The inner branch (fig. $207,{ }^{8}$ ) perforates the fascia at the inner side of the knee behind the internal saphenous nerve, with which it communicates; it furnishes offsets to the inside of the knee, and to the upper half of the leg on the inner surface.

Other small offsets of the nerve supply the inner side of the thigh, and appear by the side of the saphenous vein. One or two come into view near the upper part of the vein, and reach as far as the middle of the thigh ; and one, larger in size than the rest, becomes cutaneous where the others cease, and extends as far as the knee.

The internal saphenous (fig. 192, ${ }^{4}$ ), a branch of the anterior crural, is continued to the foot, but only a small part of it is now visible. The nerve pierces the fascia on the inner side of the knee; and, after communicating with the inner branch of the internal cutaneous, gives forwards some offsets over the knee-joint. Finally it accompanies the saphenous vein to the leg and foot.

Its patellar branch (fig. $192,{ }^{5}$ ) appears on the inner side of the knee above the preceding, and is soon joined by the internal cutaneous nerve. It ends in many branches over the patella; these communicate with offsets from the middle and external cutaneous nerves, and form an interlacement_plexus patella_-over the joint.

Dissection. Let the fat and the inguinal glands be now removed from the surface of the fascia lata, the cutaneous nerves being thrown aside to be traced afterwards to their trunks.

At the upper part of the thigh the student is to define the saphenous opening in the fascia lata by detaching the superficial fascia. The inner side is easily shown. But the outer border is blended with the superficial fascia and with the subjacent crural sheath; and it is only after the uniting fibrous bands are broken or cut through that its semilunar edge comes into view.

The fascia lata is the deep aponeurosis of the thigh. It surrounds the limb with a firm sheath, and sends inwards septa between the different muscles. This membranous investment is of a bluish-white color, but in fat bodies is sometimes so slight as to be taken away with the subcutaneous fat.

It is much stronger on the outer than the inner aspect of the limb where it receives the insertion of the tensor vaginæ femoris, and the greater part of the gluteus maximus muscle. This thickened part (ilio-tibial band) is attached above to the hip-bone and below to the bones of the leg, and helps the extensor muscle to keep the knee-joint straight in standing.

Numerous apertures exist in the fascia for the transmission of the cutaneous nerves and vessels; and the largest hole is near Poupart's ligament, to permit the passage of the internal saphenous vein.

Processes prolonged from the under surface form septa between, and fibrous sheaths around the several muscles. Two of the processes are larger than the rest, and are named outer and inner intermuscular septa of 
the thigh : they are fixed to the femur, so as to limit on the sides the extensor of the knee. The position of these partitions is marked on the surface by white lines.

At the top of the thigh the fascia is fixed to the prominent borders of the pelvis. Thus it is connected externally with the iliac crest, and internally with the pubes and the pubic arch. In the middle line behind it is joined to the lower end of the sacrum and coccyx ; and in front, to Poupart's ligament between the pubes and the iliac crest. Behind the kneejoint the fascia passes uninterruptedly to the leg; but in front of the articulation it blends with an expansion from the extensor muscle, and is continued over the joint and the patella, though separated from that bone by a bursa, to be inserted into the heads of the tibia and fibula.

On each side of the patella is a band of almost transverse fibres (retinaculum) which is attached to and supports the knee-cap. The outer,

Fig. 193.

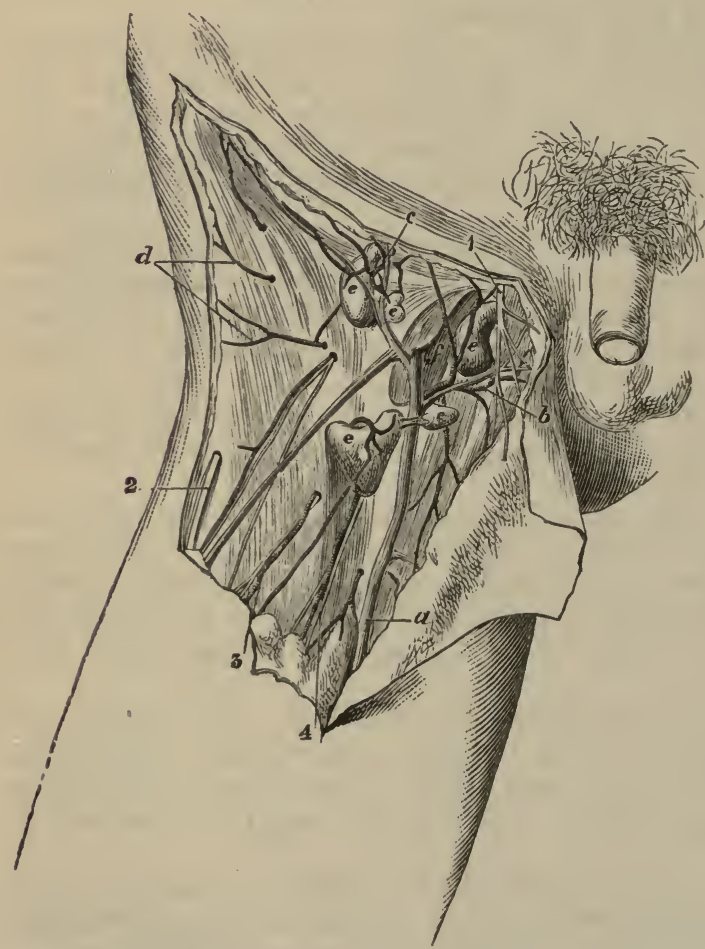

Vessels:

a. Saphenous vein.

b. Superficial pudic.

c Superficial epigastric.

d. Superficial circumflex iliac.

e. Inguinal glands.

$f$. Saphenous opening.

Nerves:

1. Ilio-inguinal.

2. External cutaneous.

3. Genito-crural.

4. Middle cutaneous.

Small unuamed vessels uccompany the different nerves to the teguments.

DIssection OF THE SUPERFICIAI, PARTS OF THE THIGH (Illustrations of Dissections).

thick and strong, is continuous externally with the ilio-tibial band, and joins the insertion of the vastus externus at its attachment to the upper part of the patella: it guides the patella outwards when the joint is bent. The inner band, of slight strength, is fixed to the patella lower than the other, and unites with the insertion of the inner vastus.

Directions. The flaps of skin which were removed from the front of the thigh, to follow the cutaneous vessels and nerves, are to be now 
stitched together to keep moist the subjacent parts; and the saphenous opening is to be learnt.

The saphoneous opening in the fascia lata (fig. 193, $f$ ) is a narrow semilunar slit, which is situate rather to the inner side of the middle line of the thigh. It measures about a third of an inch in width, and one inch and a half in length. Its upper extremity (superior cornu) is at Poupart's ligament; and its lower extremity (inferior cornu) is distant from that structure about one inch and a half, and presents a well-defined margin.

The inner part of the opening is posterior to the level of the femoral vessels, and is flattened over the subjacent muscle (pectineus); but it is marked below by a thin and sharp border.

The outer boundary is much stronger, and has a semilunar border when detached, whose coneavity is turned downwards and inwards. This edge is named from its shape falciform margin of the saphenous opening (falciform process of Burns); it is superficial to the femoral vessels, and is connected by fibrous bands to the crural sheath, and to the deeper layer of the superficial fascia. Traced upwards, the outer edge blends with the base of Gimbernat's ligament (part of Poupart): the upper end of this border, where it is internal to the subjacent femoral vein, has been named the femoral ligament.

The rigidity of the margin of the opening is much influenced by the position of the limb; for with the finger beneath the upper part of the falciform border, whilst the thigh is moved in different directions, this band will be perceived to be most unyielding when the limb is extended and rotated out, and most relaxed when the thigh is bent and turned in the opposite direction.

Through the lower part of the opening the saphenous vein is transmitted: and through the upper part, close to the falciform edge, a femoral hernia projects. Lymphatics and one or two superficial vessels also pass through it.

\section{PARTS CONCERNED IN FEMORAL HERNIA.}

To obtain a knowledge of the hernial protrusion in the thigh, the dissector has to study the undermentioned parts, viz., the crural arch and Gimbernat's ligament, the crural sheath with its crural canal and ring, together with a partition (septum crurale) between the thigh and the abdomen.

Dissection (fig. 194). To examine Poupart's ligament and a loose membranous sheath around the femoral vessels, the piece of the fascia lata outside the saphenous opening is to be reflected inwards by the following incisions: One cut is to be begun near the edge of the falciform border, and to be carried outwards for one inch and a half, parallel and close to Poupart's ligament. Another is to be directed obliquely downwards and inwards from the termination of the first, to a little below the inferior cornu of the opening. When the fascia marked out by those incisions has been raised and turned inwards, and the fat removed, the tube on the vessels (crural sheath) will be brought into view as it descends beneath Poupart's ligament.

With the handle of the scalpel the crural sheath is to be separated carefully from the fascia lata beneath, from Poupart's ligament in front, and from Gimbernat's ligament on the inner side.

Poupart's ligament (fig. 194, ${ }^{\circ}$ ), or the crural arch, is the firm band of 
the aponeurosis of the external oblique muscle of the abdomen, which stretches from the front of the iliac crest to the pubes (p. 411). When viewed on the surface, the arch is curved downwards towards the limb, whilst the fascia lata remains on the thigh. The outer half is oblique. But the inner half is almost horizontal, and widens as it approaches the pubes, where it is inserted into the pubic spine and pectineal line of the hip bone, forming Gimbernat's ligament.

The space between the crural arch and the innominate bone is larger in the female than in the male, and is closed by parts passing from the abdomen to the thigh. 'The outer half of the interval is filled by the fleshy psoas and iliacus muscles, to which the arch is closely bound by fascia ; and the inner half is occupied by the femoral vessels and their sheath.

Gimbernat's ligament, or the part of the tendon of the external oblique muscle which is inserted into the pectineal line, is about three-fourths of an inch in length, and is triangular in shape. Its apex is at the pubic spine; whilst its base is in contact with the crural sheath, and is joined by the fascia lata. By one margin (anterior) it is continuous with the crural areh, and by the opposite it is fixed to the pectineal line. In the erect position of the body the ligament is almost horizontal.

The crural sheath (fig. 194, ${ }^{\mathrm{B}}$ ) is a loose tube of membrane around the femoral vessels. It has the form of a funnel, sloped unequally on the sides. The wide part or base of the tube is upwards; and the narrow part ceases about two inches below Poupart's ligament, by blending with the common areolar sheath of the bloodvessels. Its outer border is nearly straight, and is perforated by the genito-crural nerve $\left(^{1}\right)$. Its inner border is oblique, and is pierced by lymphatics, superficial vessels, and the saphenous vein $(f)$; this part of the sheath appears in the saphenous opening, and is connected to the falciform margin and the superficial fascia. In front of the crural sheath and behind it is the faseia lata of the thigh.

The sheath is continuous with the fascia lining the abdomen in this way; the anterior part is prolonged beneath Poupart's ligament into the fascia transversalis, and the posterior half is continued into the fascia iliaca (p. 428).

Crossing the front of the sheath beneath the arch of Poupart's ligament, is a fibrous band, the deep crural arch. A notice of it is included in the description of the fascia transversalis (p. 419).

Dissection (fig. 194). The student is to open the sheath by an incision across the front, and to raise the loose anterior part with hooks. Inside the tube are contained the femoral vessels, each surrounded by its covering of areolar tissue, together with an inguinal gland; and if a piece of the areolar casing be cut out over both the artery and the vein, there will be an appearance of two thin partitions, the one being situate on the inner side of the vein, separating this vessel from the gland, and the other ( $\left(\begin{array}{l}+ \\ +\end{array}\right)$ between the vein and the artery. A fatty stratum stretches over the upper aperture of the sheath, closing it towards the abdomen.

Interior of the crural sheath (fig. 194). The sheath is said be be divided into three compartments by two partitions; and the position of the socalled septa has been before referred to--one being internal to the femoral vein, and the other between the two large vessels. In the outer compartment is contained the femoral artery $(a)$, lying close to the side of the sheath ; in the middle one is placed the femoral vein $(b)$; and in the inner space (crural canal) only a lymphatic gland $(c)$ is situate.

The crural canal (fig. 192) is the innermost space in the interior of the 
crural sheath : Its length is about a third of an inch, and it reaches from the base of Gimbernat's ligament to the upper part of the sapenous opening. It decreases rapidly in size from above down, and is closed below. 'The aperture by which the space communicates with the cavity of the abdomen is named in the crural ring.

Fig. 194.

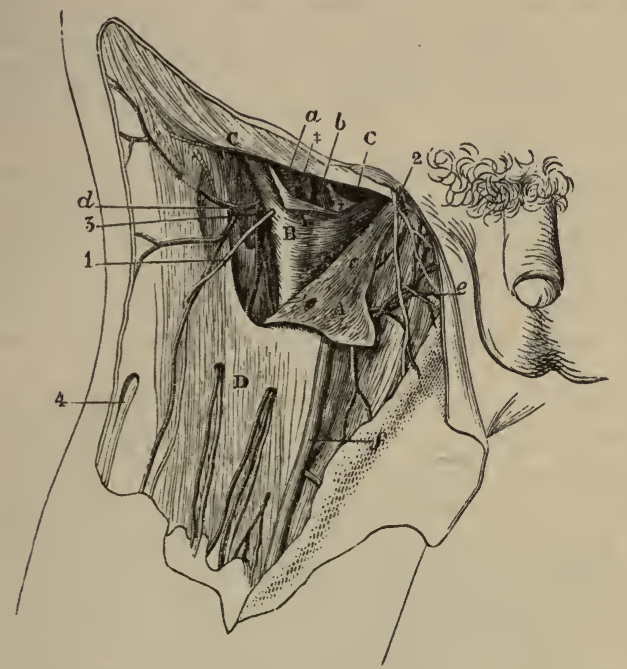

A. Fascia lata reflected.

B. Crural sheath opened.

C. Poupart's ligament.

D. Fascia late of the thigh in place.

$\ddagger$ Two septa dividing the space of the crural sheath into three compartments.

Vessels :

a. Femoral artery.

b. Femoral vein; and

c. A lymphatic gland, all in the crural sheath.

d. Superficial circumflex iliac.

e. Superficial pudic.

$f$. Saphenous vein.

Nerves :

1. Genito-crural.

2. Ilio-inguinal.

4. External cutaneous.

Dissection of thi CruRal Shrath (Illustrations of Dissections).

Anterior to the canal, are Poupart's ligament and the upper end of the falciform margin of the saphenous opening; whilst behind it is the pectineus inuscle, covered by fascia lata. On the outer side of the canal, but in the sheath, is the femoral vein. Through this channel the intestine passes from the abdomen in femoral hernia.

The crural ring ${ }^{1}$ is the upper opening of the crural canal. It is on a level with the base of Gimbernat's ligament (fig. 142, ${ }^{\mathrm{D}}$ ), and is larger in the female than in the male. Oral in shape, its greatest measurement is from side to side, in which direction it equals about half an inch; and it is filled by a lymphatic gland.

The structures around the ring, outside the crural sheath, are similar to those bounding the canal, viz, in front the superficial and the deep crural arch, and behind, the pubes covered by the pectineus muscle and the fascia lata. Internally is Gimbernat's ligament with the conjoined tendon; and externally (but within the sheath) is the femoral vein. 'The position of vessels on the several sides of the ring is stated at page 429 .

Septum crurale. That part of the subperitoneal fatty layer which is placed over the opening of the crural ring, has been named crural septum from its position between the thigh and abdomen (Cloquet). 'The situa-

1 Gimbernat used the name crural ring, and Mr. Lawrence proposes to call it femoral aperture. Might not the nomenclature be made to resemble more that used in describing inguinal hernia, by calling this opening the internal crural aperture, and the saphenous opening the external crural aperture? 
tion of the septum is now visible, but its characters are ascertained in the dissection of the ablomen (p. 428).

Femoral Hernia. In this kind of hernia there is a protrusion of intestine into the thigh beneath Poupart's ligament. And the gut descends in the crural sheath, being placed on the inner side of the vein.

Course. At first the intestine takes a vertical direction in its progress from the abdomen, and passes through the crural ring, and along the crural canal as far as the saphenous opening. At this spot it changes its course, and is directed forwards to the surface of the thigh, where it becomes elongated transversely; and should the gut protrude still farther, the tumor ascends on the abdomen, in consequence of the resistance being less in this direction than on the front of the thigh.

The winding course of the hernia may suggest to the dissector the direction in which attempts should be made to replace the intestine in the abdominal cavity. With the view of making the bowel retrace its course, it will be necessary if the protrusion is small to direct it backwards and upwards; but if the hernia is large it must be pressed down first to the saphenous opening, and afterwards backwards and upwards towards the crural canal and ring.

During the manipulation to return the intestine to its cavity the thigh is to be raised and rotated inwards, in order that the margin of the saphenous opening and the other structures may be relaxed.

Coverings. As the intestine protrudes it is elothed by the following layers, which are elongated and pushed before it from within outwards. First is a covering of the peritoneum lining the abdomen, which forms the hernial sac. Next one from the septum erurale across the crural ring. Afterwards comes a stratum from the crural sheath, unless the hernia bursts through an aperture in the side. Over this is spread a layer of the cribriform fascia. And, lastly, there is an investment of the superficial fat or fascia, together with the skin.

The coverings may vary, or may be conjoined in different degrees according to the condition of the hernia. In some instances the prolongation from the crural sheath is wanting. Further, in an old hernia the covering derived from the septum crurale is united usually with that from the erural sheath, so as to form one layer, the fascia propria (Cooper). In general, in an operation for the relief of the strangulated bowel, the surgeon, after dividing the subcutaneous fat, can recognize but little of the coverings enumerated by anatomists until he meets with that of the subperitoneal fat or septum crurale.

Diagnosis. This hernial tumor is generally smaller than inguinal, and does not extend into the scrotum in the male, or the labium in the female; and if its neck can be traced below Poupart's ligament, it can be distinguished eertainly from an inguinal hernia.

Seat of stricture and division of it. The strangulation of a femoral hernia may be situate either outside or inside the neck of the sac.

The external stricture may be found opposite the margin of the saphenous opening, or deeper in, opposite Poupart's ligament. It may be removed by cutting down on the neck of the tumor at the inner side, and dividing the eonstricting band arching over the neek of the hernia in this situation, without opening the sac.

The stricture inside the neck of the sac is occasioned by the thickening of the peritoneum. For its relief the neck of the sac is to be laid bare, as if there was an external stricture; and if the intestine cannot be passed 
into the abdomen after division of all constricting bands on the exterior of the neek, the sac of the peritoneum is to be opened; and a director having been introduced through the constriction, a cut is made horizontally inwards for the extent of one or two lines. The several vessels that may be wounded in attempting to relieve the deep stricture are enumerated at page 429.

\section{SCARPA'S TRIANGULAR SPACE.}

This hollow is situate at the upper part of the thigh, and lies beneath the depression observable near Poupart's ligament. It corresponds with the axilla in the upper limb.

Dissection (fig. 195). The space will appear on removing the fascia lata near Poupart's ligament. The muscular boundaries on the sides may be first dissected, and the muscle on the outer side (sartorius) should be fixed in place with stitches. Afterwards, the remains of the crural sheakh

Fig. 195.

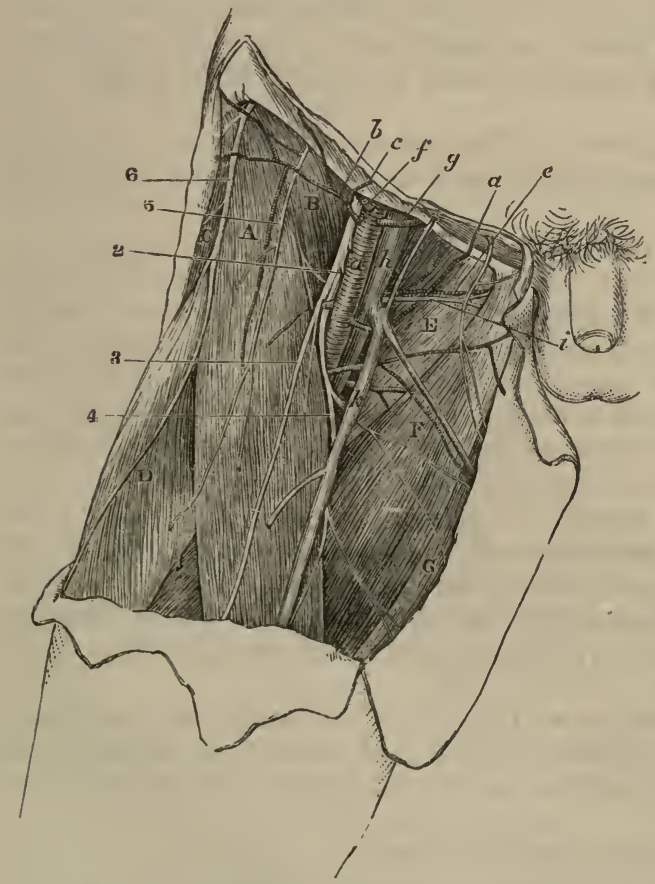

Muscles :

A. Sartorius.

B. Iliacus.

C. Tensor fasciæ latæ.

D. Rectus femoris.

E. Pectineus.

F Adductor longus.

G. Gracilis.

\section{Vessels :}

a. Femoral artery.

b. Superficial circumflex iliac.

c. Superficial epigastric.

e. Superficial pudic (iuferior), and $i$, the accompanying veins.

f. Deep circumflex iliac.

g. Deep epigastric.

h. Femoral vein.

$i$. Inferior external pudic veln.

$k$. Saphenous vein.

\section{Nerves :}

The large anterior crural is close ontside the artery.

2. Offset from the same to the pectineus.

3. Middle cutaneous.

4. Internal cutaneous.

5. Genito-crural.

6. External cutaneous.

Dissection on Scarpa's Triangular SPaCe (Illustrations of Dissections).

are to be taken away; and the femoral vessels are to be followed downwards as far as the sartorius musele. On the outer side of the vesse's clean the divisions of the anterior erural nerve, together with the branches of an artery (profunda) which are buried in the fat. In removing the fat from beneath the femoral artery, the student is to look for one or two small nerves to the pectineus musele.

This intermuscular space (fig. 195) contains the trunks of the bloodvessels of the thigh, and the anterior crural nerve, with lymphatics and 
fat. It measures commonly three inches from above down; but the length varies with the breadth of the sartorius, and the height at which this muscle crosses inwards.

The base of the space is at Poupart's ligament; and the apex is at the meeting of the sartorius with the adductor longus muscle.

Towards the surface it is covered by the fascia lata, and by the teguments with inguinal glands and superficial vessels. The floor slopes towards the middle, where it is deepest ; it is constructed externally by the sartorius, A, and by the conjoined psoas and iliacus, B, for about two inches; and internally by the pectineus and adductor longus muscle, $\mathrm{E}$ and $\mathrm{F}$, and between and beneath these near the large vessels, is a small piece of the adductor brevis.

The femoral artery runs through the centre of the hollow, and supplies small cutaneous offsets, as well as a large deep branch, the profunda: a small offset (external pudic) is directed from it to the scrotum across the inner boundary. On the inner side of the artery and close to it is placed the femoral vein, which is here joined by the saphenous and profunda branches. About a third of an inch external to the vessel is situate the large anterior crural nerve, which lies deeply at first between the iliacus and psoas, but becomes afterwards more superficial and divides into branches.

Deep lymplatics accompany the femoral vessels, and are continued into the iliac glands in the abdomen; they are joined by the superficial lymphatics.

Femoral Artery (fig. 197.) This vessel is a continuation of the external iliac, and reaches from the lower of Poupart's ligament to the margin of the opening in the adductor magnus muscle; at that spot it passes into the ham, and takes the name popliteal. Occupying two-thirds of the thigh, the course of the ressel will be indicated, during rotation outwards of the limb with the knee-joint half bent, by a line drawn from a point midway between the symphysis pubis and the front of the iliac crest, to the inside of the inner condyle of the femur.

In the upper part of its course the artery lies rather internal to the head of the femur, and is comparatively superficial, being uncovered by muscle; but, in the lower part, it is placed along the inner side of the shaft of that bone, and is beneath the sartorius muscle. 'This difference in its connections allows of a division of the arterial trunk into two portions, superficial and deep.

The superficial part of the artery (fig. 195, a), which is now laid bare, is contained in Scarpa's triangular space, and is about three inches long. Its position in that hollow may be ascertained by the line before mentioned.

Incased at first in the crural sheath for about two inches, it is covered by the skin and the superficial fascia, and by the fascia lata and some inguinal glands. At its beginning the artery rests on the psoas muscle; and it is subsequently placed over the pectineus, E, though at some distance from it in this position of the limb, and separated from it by fat, and the profunda and femoral veins.

Its companion vein $(h)$ is on the inner side and close to it at the pubes, but is placed behind the artery at the apex of the space.

The anterior crural nerve lies on the outer side, being distant about a third of an inch near Poupart's ligament; and the internal cutaneous branch of the nerve approaches the artery, or lies on it, near the apex of 
the containing space. Crossing beneath the vessels is the nerve of the pectineus $\left({ }^{2}\right)$.

Unusual position. Four examples of transference of the main artery of the limb from the front to the back of the thigh have been recorded. In these cases the vessel passed from the pelvis through the great sacro-sciatic notch, and accompanied the great sciatic nerve to the popliteal space.

The branches of the first part of the artery are the superficial epigastric and circumflex iliac, two external pudic, and the deep femoral branch. The cutaneous offsets have been seen (p. 554), with the exception of the following, which lies at first beneath the fascia lata.

The inferior external pudic artery (fig. 195,e) arises separately from, or in common with the other pudic branch (superior). It courses inwards over the pectineus muscle to end in the teguments of the scrotum or the labium pudendi, according to the sex, and it perforates the fascia lata at the inner border of the thigh to reach its destination: in the fat it anastomoses with branches of the superficial perineal artery.

The deep femoral branch (fig. 197, ${ }^{2}$ ) or the profunda, is the largest offset of the femoral artery, and arises from the outer part of that trunk one to two inches (Quain) below Poupart's ligament. It is consumed in the muscles of the thigh, and its distribution will be afterwards ascertained. In the present dissection it may be seen to lie over the iliacus muscle, where it gives the external circumflex artery to the outer part of the thigh; and then to turn, with a large vein, beneath the trunks of the femoral vessels to the inner side of the limb. ${ }^{1}$

Variation in origin. The origin of the profunda may approach riearer to Poupart's ligament until it arrives opposite that band; or may even go beyond, and be fixed to the external iliac artery (one example, Quain). And the branch may recede farther and farther from the ligament, till it leaves the parent trunk at the distance of four inches from the commencement; but in this case the circumflex branches usually arise separately from the femoral. In applying a ligature to the femoral artery in the upper part of the thigh, the thread should be placed four inches below Poupart's ligament, in order that the spot chosen may be free from the disturbing influence of so large an offset.

Femoral Vein (fig. 195, $h$ ). The principal vein of the limb, whilst in the triangular space, has almost the same relative anatomy as the artery: its position to that vessel, however, is not the same throughout. Beneath Poupart's ligament it is on the inner side of the arterial trunk, and on the same level, and is supported on the pubes between the psoas and pectineus muscles; but it soon winds beneath the artery, and appears on the outer side opposite the upper border of the adductor longus muscle. Occasionally it is inside the artery throughout. In this space it receives the internal saphenous and deep femoral veins, and a small branch (i) with the inferior external pudic artery.

\section{DEEP PARTS OF THE FRONT OF THE TIIGH.}

The muscles on the front of the thigh are to be learnt next: they are the sartorius, and the extensor of the knee; and at the top of the thigh is the small tensor of the fascia lata. Three muscles are combined in the extensor, viz., rectus, vastus externus, and vastus internus.

1 Sometimes the term common femoral is applied to the part of the trunk above the origin of the profunda, and the names superficial and deep femoral to the nearly equal parts into which it divides. 
The external circumflex branch of the profunda artery lies amongst the muscles and supplies them with branches; and a large nerve, the anterior crural, furnishes offisets to them.

Dissection. To proceed with the deep dissection, the limb is to be retained in the same position as before, and the flaps of skin on the front of the thigh are to be thrown aside. The fascia lata is to be cut along the middle line of the thigh and knee, and to be reflected to each side nearly to the same extent as the skin. Over the knee-joint the student is to note its attachment to the edges of the patella, and its union with a prolongation from the tendon of the extensor muscle to the leg.

In raising the inner piece of the fascia the narrow muscle appearing (sartorius) should be followed to its insertion into the tibia : and to prevent its displacement, it should be fixed with stitches along both edges. Care should be taken of the small nerves in contact with the sartorius;viz., a plexus beneath it at the middle of the thigh from the saphenous, internal cutaneous, and obturator; two branches of the internal cutaneous below its middle,--one crossing the surface, and the other lying along the inner edge of the muscle; and the trunk of the great saphenous escaping from beneath it near the knee, with the patellar branch of the same perforating it rather higher.

Internal to the sartorius some strong muscles (adductors) are inclined downwards from the pelvis to the femur. The student is to lay bare the fore part of those muscles; and beneath the most superficial (adductor longus), near where it touches the sartorius, he is to seek a branch of the obturator nerve to the plexus before mentioned in the middle of the thigh. On the outer side of the sartorius is the large extensor of the knee. For its dissection the knee is to be bent, to make tense the fibres: and an expansion below from the common tendon to the fascia lata and the knee-joint is not to be removed now,-its arrangement will be noticed after.

The little muscle at the upper and outer part of the thigh,-_tensor of the fascia lata, is to be cleaned; and a strip of the fascia, corresponding with its width, should be left along the outer aspect of the thigh. After this slip has been separated, the rest of the fascia on the outer side of the thigh is to be divided by one or two transverse cuts, and is to be followed backwards to its attachment to the femur.

'The sartorius (fig. $196,{ }^{A}$ ) is the longest muscle in the body, and extends from the pelvis to the leg. It arches over the front of the thigh, passing from the outer to the inner side of the limb, and lies in a hollow between the extensor on the one side, and the adductors on the other.

Its origin is tendinous from the upper anterior iliac spinous process of the hip bone, and from about half the interval between this and the inferior process. The fibres constitute a ribbon-like muscle, which ends in a thin tendon below the knee, and is inserted into the inner surface of the tibia-mainly into a slight depression by the side of the tubercle for an inch and a half, but also, by its upper edge, as far back as the internal lateral ligament of the knee-joint.

The musele is superficial throughout, and is perforated by some cutaneous nerves and vessels. Its upper part is oblique, and forms the outer boundary of the triangular space containing the femoral artery: it rests on the following muscles, iliacus, $\mathrm{B}$, rectus, $\mathrm{D}$, and adductor longus, $\mathrm{G}$, as well as on the anterior crural nerve and the femoral vessels. The middle portion is vertical, and lies in a hollow between the vastus internus $\mathrm{E}$, and 
the adductor museles as low as the opening for the femoral artery; but beyond that aperture, where it bounds the popliteal space, it is placed between the vastus with the great adductor in front, and the gracilis, II, with the inner hamstrings behind. The femoral ressels and their accompanying nerves are concealed by this portion of the muscle. The lower or tendinous piece, I, rests on the internal lateral ligament of the knee-joint,

Fig. 196.

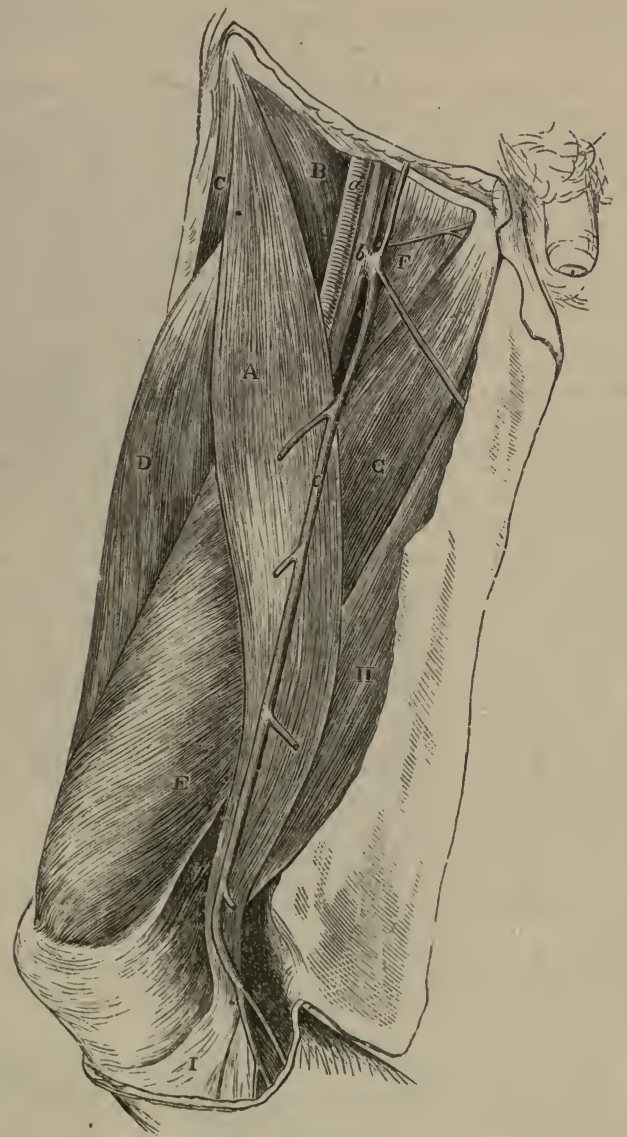

\author{
Muscles: \\ A. Sartorius. \\ B. Hliacus. \\ c. Tensor fasciæ latæ. \\ D. Rectus femoris. \\ E. Vastus internus. \\ F. Pectineus. \\ G. Adductor longus. \\ H. $G$ racilis. \\ I. Tendon of Sartorius.
}

Vessels:

a. Femoral artery.

b. Femoral vein.

c. Saphenous vein.

Strface VibW of the Front of the Thigh, the TegUments and Fascia betNg Removed. (Illustrations of Dissections.)

being superficial to the tendons of the gracilis and semi-tendinosus, and separated from them by a prolongation of their synovial bursa: from its upper border there is an aponeurotic expansion to join that from the extensor over the knee; and from its lower border is given another which blends with the fascia of the leg. Below the tendon the great saphenous nerve appears with vessels; and piercing it is the patellar branch of the same nerve.

Action. The tibia and femur being free to move, the muscle bends the 
knee and hip-joints over which it passes, giving rise to rotation inwards of the tibia; and makes tense finally the fascia of the thigh.

With the limbs fixed, the two muscles will support the pelvis in standing, and will assist in bringing forwards the pelvis in stooping and walking.

When standing on one leg the muscle will help to rotate the body, so as to turn the face to the opposite side.

Dissection (fig. 197). The sartorius is to be turned aside, or cut through if it is necessary, to follow the remaining part of the femoral artery.

Beneath the muscle is an aponeurosis between the adductor and extensor muscles; this is thin above, and when it is divided the internal saphenous nerve will come into view. Parallel to the upper part of the saphenous nerve, but outside it, is the nerve to the vastus internus muscle, which sends an offset on the surface of the vastus to the knee-joint; this may be traced now, lest it should be destroyed afterwards. The plexus of nerves on the inner side of the thigh may be more completely dissected in this stage.

The femoral vessels and their branches are to be nicely cleaned. Where the femoral artery passes to the back of the limb its small anastomotic b:ancl arises: this branch is to be pursued through the fibres of the vastus internus, and in front of the adductor magnus tendon to the knee; an offset of it is to be followed with the saphenous nerve.

The aponeurotic covering over the femoral vessels (fig. 197, ${ }^{7}$ ) exists only where these are covered by the sartorius. It is thin above, but below it is formed of strong fibres, which are directed transversely between the vastus internus and the tendons of the adductor muscles. Inferiorly the membranous structure ceases at the opening in the adductor magnus by a defined border, beneath which the saphenous nerve and its vessels escape.

The deep part of the femoral artery (fig. 197, ${ }^{1}$ ) lies in a hollow between muscles (Hunter's canal) until it reaches the opening in the adductor magnus. Here it is covered by the sartorius muscle and the subjacent aponeurosis, in addition to the integuments and the superficial and deep fascix. Beneath it are the pectineus, the adductor brevis in part, the adductor longus, and a small piece of the adductor magnus. On the outer side is the vastus internus.

External to the artery and close to it is the femoral vein; and in the integuments oftentimes an offset of the saphenous passes across the line of the arterial trunk.

Crossing over the artery from the outer to the inner side is the internal saphenous nerve, which is beneath the aponeurosis before noticed, but is not contained within the areolar sheath of the vessels.

Splitting of the artery. Occasionally the femoral artery is split into two below the origin of the profunda. Four examples of this peculiarity have been met with; but in all, the trunks were blended into one above the opening in the adductor muscle.

Branches. One named branch, anastomotic and muscular offsets, spring from this part of the artery.

The anastomotic branch (fig. 198, $k$ ) (arter. anastomotica magna) arises close to the opening in the adductor muscle, and divides at once into two parts, superficial and deep :-

The superficial offset $(n)$ continues with the saphenous nerve to the 
lower border of the sartorius, and piercing the fascia lata, ramifies in the integuments.

The deep branch $(l)$ is concealed in the fibres of the vastus internus, and descends in front of the tendon of the adductor magnus to the inner side of the knee-joint, where it anastomoses with the articular branches of

Fig. 197.

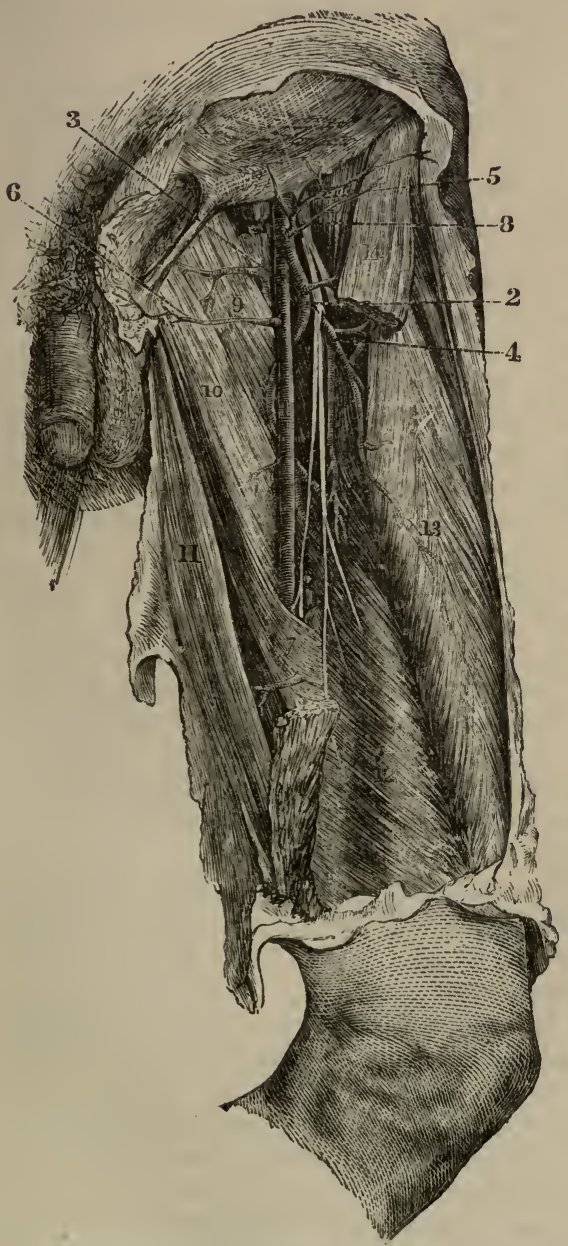

1. Femoral artery.

2. Profunda artery.

3. Internal circumflex.

4. External circumflex.

5. Superficial circumflex iliac and epigastric branches.

6. External pudic artery.

7. A poneurosis over the lower part of the femoral artery.

8. Anterior crural nerve.

9. Pectineus muscle.

10. Adductor longus.

11. Gracilis.

12. Vastus internus.

13." Rectus femoris.

14. Sartorius cut across.

Deep Part of the Femoral Artert and its Branches, With Musches of the Thigh (Quain's Arteries).

the popliteal and anterior tibial arteries. A branch passes outwards from it in the substance of the vastus, and forms an arch in front of the lower end of the femur with an offset of the upper external articular artery; from this loop twigs descend to the joint.

Muscular branches. Branches for the supply of the muscles come 
mostly from the outer side of the femoral artery ; they enter the sartorius, the vastus internus, and the adductor longus.

The FEMORAL VEIN corresponds closely with the femoral artery in its connections with the parts around, and in its branches.

Dissection. The femoral artery and vein are to be cut across below the origin of the profunda, and are to be thrown downwards preparatory to the deeper dissection. Afterwards all the fat, and all the veins, are to be carefully removed from amongst the branches of the profunda artery and anterior crural nerve. Unless this dissection is completed, the upper part of the vastus internus will not be prepared for learning.

The TENSOR VAGINA FEMORIS (fig. 198, L) occupies the upper third of the thigh, and is the smallest and most external of the outer set of muscles. It takes origin from the front of the crest of the hip bone at the outer aspect; from the anterior upper iliac spine, and from part of the notch between this and the inferior spine as far as the attachment of the sartorius. Its fibres form a fleshy belly about two inches wide, and are inserted into the fascia lata about three inches below, and rather in front of the line of the great trochanter of the femur.

At its origin the muscle is situate between the sartorius and the gluteus medius. Beneath it are the ascending offsets of the external circumflex artery; and a branch of the superior gluteal nerve enters its under surface. A strong sheath of fascia surrounds the muscle.

Action. Supposing the limb movable the muscle abducts the thigh, making tense at the same time the fascia lata; and finally it will help in rotating inwards the femur.

When the limb is fixed it will support the pelvis, and assist in balancing the same on the femur in walking.

Dissection. After the tensor has been learnt, the slip of fascia extending from it to the knee may be cut through; and when it is detached from the muscles around, the head of the rectus may be followed upwards to the pelvis.

The TRICEPS Extexson of the knee (fig. 197) consists of three fleshy parts or heads, outer (vastus externus), inner (vastus internus), and niddle (rectus), which are united below in a common tendon.

The RECTUS FEMORIS gives rise to a fleshy prominence on the front of the thigh (fig. 197, ${ }^{13}$ ). At its origin from the pelvis the muscle consists of two tendinous pieces: one arises from the anterior inferior iliac spinous process; the other (to be afterwards seen) is fixed into a depression on the back of the hip bone, close above the acetabulum. The fleshy fibres terminate inferiorly in another tendon, which joins the aponeurotic parts of the other two muscles in the common tendon.

The rectus is larger at the middle than at the ends; and its fibres are directed from the centre to the sides, as in a quill, giving rise to that condition called penniform. It is subcutaneous, except above where it is overlaid by the sartorius. It conceals branches of the external circumflex artery and anterior crural nerve, and rests on the vasti. The upper tendon of the rectus reacliest furthest on the anterior surface where the sartorius touches, whilst the lower tendon is most extensive on the posterior aspect, or towards the subjacent vasti.

Dissection. To see the remaining muscles, cut across the rectus near the lower end, and raise it without injuring the branches of vessels and nerves beneath. The muscular mass on the front of the femur is to be divided into two, above, along the situation of some descending vessels and 
nerves: the part external to the vessels is the vastus externus, and the larger mass, internal to them, is the vastus internus.

To make out the lower separation of the two, look to the outer aspect of the thigh about half way down, where the long and vertical fibres of the vastus externus descending to their tendon, cross over others (deeper), which are continued obliquely inwards, and belong to the inner vastus.

The rastus Exterixus has a very narrow attachment to the femur in comparison with its size. It takes origin along the upper half of the femur, by a piece from half an inch to an inch thick, which is attached to the root of the neck of the femur, and the fore and outer parts of the root of the great trochanter; to the line connecting the trochanter with the linea aspera; and to the upper half of the linea aspera, and the contiguous external intermuscular septum. Inferiorly the fibres of the muscle end in an aponeurosis which blends with the tendons of the rectus and vastus internus in the common tendon, and sends a slip to the outer edge of the patella.

The muscle is pointed at the upper end; but enlarged below where it produces the prominence on the outer side of the thigh. Its cutaneous surface is aponeurotic above, and is covered by the rectus, tensor vaginæ femoris, and gluteus muscles. The deep surface rests on the vastus internus, and receives branches of the external circumflex artery and anterior crural nerve.

The vastus Internus (fig. $196,{ }^{\mathrm{F}}$ ) form the large head of the extensor. ${ }^{1}$ The fleshy mass arises from the anterior and two lateral surfaces of the shaft of the femur, except where the vastus externus is attached, and its limits may be thus indicated: Upwards it reaches as far as the anterior introchanteric liné; downwards, in the middle, to about two inches from the articular end of the femur; and laterally to both intermuscular septa. At the lower end of the muscle the fibres terminate in an aponeu rosis, which blends in the common tendon of insertion, and is attached to the patella lower than the vastus externus.

The upper part of the muscular mass is buried beneath the sartorius and rectus muscles; but the lower part is superficial, and projects more than the vastus externus; some of the lowest fibres are almost transverse, and will be able to draw inwards the patella. The adductor muscles are almost inseparably joined with this vastus along the attachment to the linea aspera.

Dissection. The tendon of the extensor will appear by dividing along the middle line of the patella and knee-joint a thin aponeurotic layer, which is derived from the lower fleshy fibres of the muscle, and covers the joint. On reflecting inwards and outwards that fibrous layer the tendon will be laid bare to its insertion into the tibia.

The tendon of the extensor muscles of the leg is common to the rectus, the vastus externus, and vastus internus. It is placed in front of the knee-joint, to which it serves the office of an anterior ligament. Wide above where the muscular fibres terminate, it narrows as it descends over the joint, and is inserted inferiorly in to the prominence of the tubercle of the tibia, and into the bone below it for an inch; close to its attachment to the tibia a synovial bursa is beneath it. In it the patella is situate,

1 Sometimes the part of the mass, inside a line continued upwards from the inner border of the patella, is named crureus : naturally there is not any separation at that spot. 
some few scattered aponeurotic fibres passing over the cutaneous surface, but none being continued over the articular surface of the bone. (See Ligament of the Patella.)

From the lower part of the musele a superficial aponeurotic expansion is derived: this prolongation, which is strongest on the inner side, is united with the fascia lata and the other tendinous offsets to form a capsule in front of the joint, and is fixed below to the heads of the tibia and fibula.

Subcrureus muscle. Beneath the strong fibres of the vastus, near the knee joint, is a thin layer of pale fibres, which is but a part of the inner vastus, separated from the rest by areolar tissue. Attached to the femur in the lower fourth, and often by an outer and inner slip, it ends in aponeurotic fibres on the synovial sac of the knee joint.

Action. All three heads of the triceps extend the knee joint, when the tibia is movable; and the rectus can flex the hip joint over which it passes. The fleshy bellies are strong enough to break the patella transversely over the end of the femur, or to rupture sometimes the common tendon.

When the tibia is fixed the vasti will bring forwards the femur, and straighten the knee, as in walking or standing; and the rectus will prop the pelvis on the femur, or assist in moving it forwards in stooping.

The subcrureus contracts in extension of the knee, and elevates the synovial membrane above the patella.

Intermuscular septa. The processes of the fascia lata, which limit laterally the extensor muscle of the knee, are thus named, and are fixed to the linea aspera and the lines leading to the condyles of the femur.

The external septum is the strongest, and reaches from the outer condyle of the femur to the insertion of the gluteus maximus. It is situate between the vastus internus and externus on the one side, and the short head of the biceps on the other, to which it gives origin; and it is perforated near the outer condyle by the upper external articular vessels and nerve.

The inner partition is very thin along the side of the vastus internus; and its place is supplied by the strong tendon of the adductor magnus between the inner condyle and the linea aspera: the internal articular vessels are transmitted through it to the front of the knee joint.

The EXTERNAL CIRCUMFLE ARTERY (fig. 197, ${ }^{4}$ ) is the chief vessel for the supply of the muscles of the front of the thigh. It arises from the outer side of the profunda (deep femoral) artery, but often from the femoral trunk. It is directed outwards through the divisions of the anterior crural nerve, and beneath the sartorius and rectus muscles to the outer part of the thigh, where it ends in branches. Offsets are given from it to the rectus and sartorius; and its terminal muscular branches consist of ascending, transverse, and descending :-

The ascending branch is directed beneath the tensor vagina femoris to the back of the hip bone, where it anastomoses with the gluteal artery, and supplies the contiguous muscles.

The transverse, the smallest in size, divides into two which perforate the vastus externus, and anastomose with arteries on the back of the thigh.

The descending branch is the largest, and ends in pieces which are distributed to the vasti muscles. One considerable branch enters the outer part of the vastus internus, and reaching the knee, anastomoses on this joint with the external articular arteries; a small offset courses over the muscle with a nerve to the joint.

The anterior crural nerve (fig. $197,{ }^{8}$ ) of the lumbar plexus (p. 
497 ) supplies the muscles, and most of the teguments of the front of the thigh, and the integuments of the inner side of the leg. Soon after the trunk of the nerve leaves the abdomen it is flattened, and is divided into superficial and deep parts.

A. The superficial part ends in three tegumentary branches: the middle and internal cutaneous of the thigh, and the great saphenous.

The middle cutaneous nerve (fig. $192,{ }^{2}$ ) perforates the fascia lata, sometimes also the sartorius, about three inches below Poupart's ligament, and extends to the knee (p. 556).

The internal cutaneous nerve (fig. $192,{ }^{3}$ ) sends two or more small twigs through the fascia lata to the integument of the upper third of the thigh, and then divides in front of the femoral artery, or on the inner side, into the two following branches, anterior and inner. Sometimes these branches arise from the anterior crural trunk at separate spots :-

The anterior branch $\left({ }^{3}\right)$ is directed to the inner side of the knee. As far as the middle of the thigh it lies over the sartorious, but it then pierces the fascia lata, and ramifies in the integuments (p. 557.)

The inner branch remains beneath the fascia lata as far as the knee ( $p$. 557 ). Whilst underneath the fascia the nerve lies along the inner border of the sartorius, and joins in a plexus, about the middle of the thigh, with offsets of the obturator and, nearer the knee, with a branch of the internal saphenous nerve.

The internal saphenous nerve (fig. 197) is the largest of the three superficial branches. In the thigh the nerve takes the course of the deep bloodvessels, and is continued along their outer side, beneath the aponeurosis covering the same, as far as the opening in the adductor magnus muscle. At that spot the nerve passes from beneath the aponeurosis, and is prolonged under the sartorius muscle to the upper part of the leg, where it becomes cutaneous (fig. 192, ${ }^{4}$ ). It supplies two offsets whilst it is contained in the thigh beneath the fascia :-

A communicating branch arises about the middle of the thigh, and crosses inwards beneath the sartorius to join in the plexus of the internal cutaneous and obturator, or with the internal cutaneous nearer the knee : this branch is often absent.

The patellar branch springs from the nerve near the knee joint, and perforating the sartorius muscle and the fascia lata, ends in the integument over the knee (fig. $192,{ }^{5}$ ).

B. The deep or muscular part of the anterior crural nerve (fig. 197) gives branches to all the muscles of the front of the thigh, except the tensor vagine femoris; and it supplies also an offset to one of the adductor muscles, viz., the pectineus.

A slender nerve (fig. $195,{ }^{2}$ ) crosses beneath the femoral artery, and enters the anterior surface of the pectineus: sometimes there are two.

Branches to the sartorius are furnished by the middle, or by the internal cutaneous nerve, whilst it is in contact with that muscle.

A nerve enters the under surface of the rectus at the upper part, and divides into branches as it is about to penetrate the fibres.

The nerve to the vastus externus separates into two or more branches as it enters the muscle. From one of these an articular filament is continued downwards to the knee joint, which it enters on the anterior aspect.

The nerve to the vastus internus (fig. 197) is nearly as large in size as the internal saphenous, in common with which it often arises. To the 
upper part of the vastus it furnishes one or more branches, and is then continued as far as the middle of the thigh, where it ends in offsets to the muscle and the knee joint.

Its articular branch is prolonged on or in the vastus, and on the tendon of the adductor magnus to the inner side of the knee joint; and it is distributed over the synovial membrane on the front of the articulation. This small nerve accompanies the deep branch of the anastomotic artery.

A branch of nerve to the tensor vagina femoris is derived from the superior gluteal; it enters the under surface of the muscle, and extends nearly to the lower end.

Directions. After the examination of the muscles of the front of the thigh, with their vessels and nerves, the student is to learn the adductor muscles, and the ressels and nerves which belong to them.

\section{PARTS OF THE INNER SIDE OF THE THIGH.}

The muscles in this position are the three adductors,-longus, brevis, and magnus, with the gracilis and pectineus; these have the following position with respect to one another. Internal to all and the longest, is the gracilis. Superficial to the others, are the pectineus and the adductor longus; and beneath the last two are the short adductor and the adductor magnus.

In connection with the muscles, and supplying them are the profunda artery (of the femoral) and its branches, with the accompanying vein.

The obturator nerve lies amongst the adductor muscles, and furnishes branches to them.

Dissection. For the preparation of the muscles, the investing fascia and tissue are to be taken away; and the two superficial adductor's are to separaterl from one another.

Let the student be careful of the branches of the obturator nerve in connection with the muscles, viz., those entering the muscular fibres, and one issuing beneath the adductor longus, to join the plexus at the imner side of the thigh.

Lastly, should any fat and veins be left with the profunda and its branches they must be removed.

The GRACILIS reaches from the pelvis to the tibia (fig. $198,^{\circ}$ ), and is fleshy and ribbon-like above, but tendinous below. The muscle arises by a thin aponeurosis, two or three inches in depth, from the pubic border of the hip bone close to the margin, viz., opposite the lower half of the symphysis, and the upper part of the pubic arch. Inferiorly it is inserrted by a flat tendon, about one-third of an inch wide, into the inner surface of the tibia, beneath and close to the sartorius.

The muscle is superficial throughout. At the upper part of the thigh it is flattened against the adductors brevis and magnus, so as to have its borders directed forwards and backwards; and in the lower third, it intervenes between the sartorius and semi-membranous muscles, and forms part of the inner boundary of the popliteal space. At its insertion the tendon is nearer the knee than that of the semitendinosus, though at the same depth from the surfuce, and both lie over the internal lateral ligament; and from the tendon an expansion is continued to the fascia of the leg, like the sartorius. A bursa separates the tendon from the ligament, and projects above it to the sartorius.

Action. It bends the knee joint if the tibia is not fixed, rotating in 
Fig. 198.

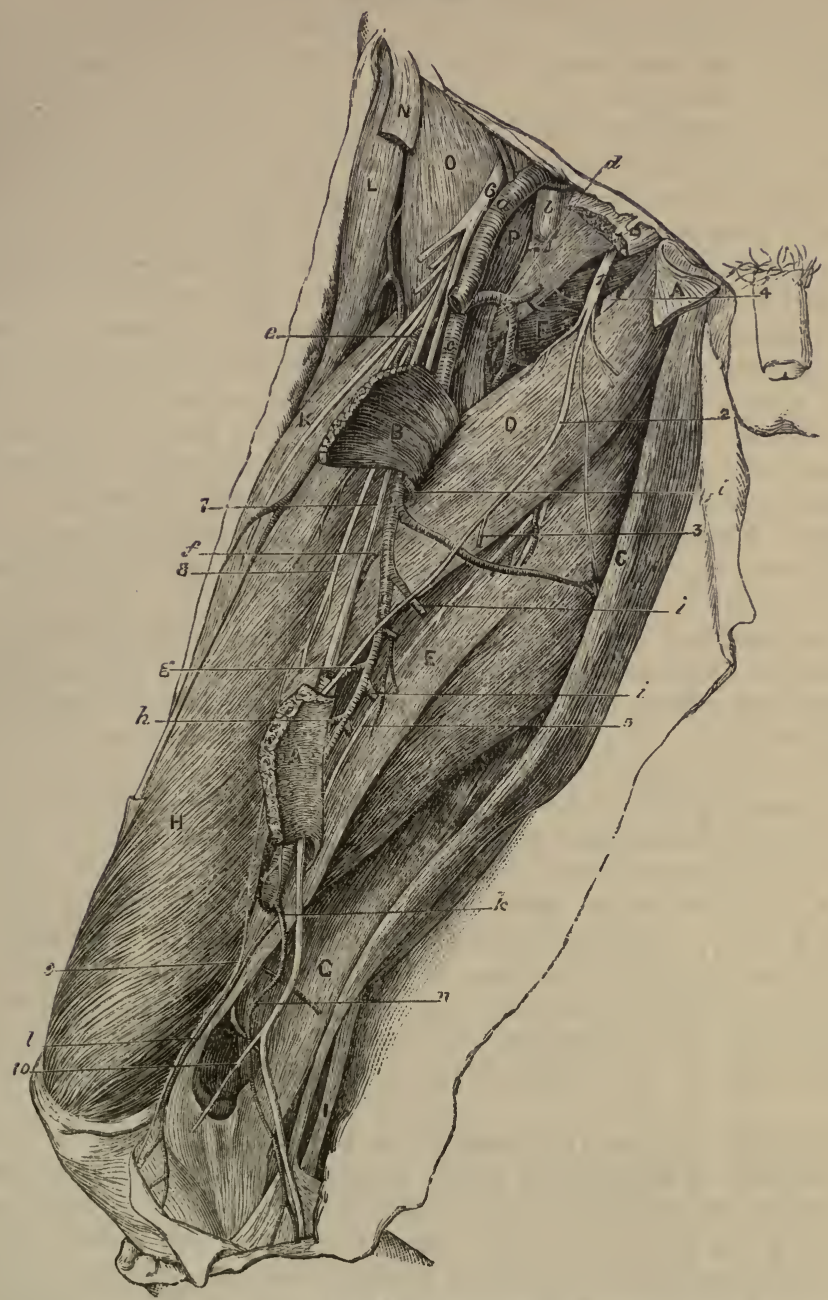

Deep Dissection of the Adductor MUsches With their Vessels avo Nerfes.

(Illustrations of Dissections.)

\section{Muscles :}

A. Adductor longus, cut.

B. Pectinens, cut.

c. Gracilis.

D. Adductor brevis.

B. Adductor magnus.

F. Obturator externus.

$\boldsymbol{~}$. Semimembranosus.

н. Vastus internus.

K. Rectus femoris.

L. Tensor fasciæ latæ.

v. Piece of the sartorius.

o. Iliacus.

P. Psoas.

Vessels :

$a$. Feinoral artery, and $b$, femoral vein. d. Internal, and $e$, external circumflex

$f$. First, $g$, second, and $h$, third perforating.

$i$. Muscular and anastomotic of the profunda.

$k$. Anastomotic of the femoral, with $l$, the articular, and $n$, the cutaneous piece.

Nerves :

1. Obturator, joined by the accessory obturator nerve, with 2 , the superficial, and 4, the deep part.

3. Cutaneous branch of the obturator.

5. Articular branch to the kee from the deep piece.

6. Anterior crural nerve.

7. Internal saphenous, and 10 , its patellar branch.

8. Nerve to the vastus internus, and 9 its 
that bone; and then brings the movable femur towards the middle line with the other adductors.

Supposing the foot resting on the ground the gracilis will aid in propping the pelvis on the limb.

The pectinfus (fig. $196,{ }^{\mathrm{r}}$ ) is the highest of the muscles directed from the pelvis to the inner side of the femur. It has a fleshy origin from the ilio-pectineal line of the hip bone, and from the triangular smooth surface in front of that line; and it is inserted inferiorly by a tendon, about two inches in width, into the femur behind the small trochanter, and into the upper part of the line which extends from that process to the linea aspera.

The muscle is $t w i s t e d$, so that the surfaces which are directed forwards and backwards near the pelvis are turned inwards and outwards at the femur. One surface is in contact with the fascia lata; and the opposite touches the obturator muscle and nerve, and the adductor brevis. The pectineus lies between the psoas and the adductor longus; and the internal circumflex vessels pass between its outer border and the psoas.

Action. It adducts the limb and bends the hip-joint. When the fernur is fixed it can support the pelvis in standing; or it can draw forwards the pelvis in stooping.

The ADDUCTOR LONGus lies helow the pectineus (fig. $196,{ }^{\circ}$ ), and is triangular in form, with the apex at the pelvis and the base at the fermur. It arises by a narrow tendon from the front of the pubes below the angle of union of the crest and the symphysis; and it is inserted into the inner edge of the linea aspera.

This muscle is situate between the gracilis and the pectineus, and forms part of Scarpa's triangular space. Its anterior surface is covered near the femur by the femoral vessels and the sartorius: the posterior rests on the other two adductors, on part of the obturator nerve, and on the deep femoral artery. Aponeurotic bands connect the tendon of insertion with the adductor magnus and vastus internus.

Action. With the femur movable, it will flex the hip-joint, and with the aid of the other adductors will carry inwards the limb, so as to cross the thigh bones. In walking it helps the other adductors to project the limb.

With the femur fixed, the muscle props and tilts forwards the pelvis.

Dissection. The adductor brevis muscle, with the obturator nerve and the profunda vessels, will be arrived at by reflecting the two last muscles. On cutting through the pectineus near the pubes, and throwing it down, the dissector may find occasionally the small accessory nerve of the obturator which turns beneath the outer border; if this is present, its branches to the hip joint and the obturator nerve are to be traced out. The adductor longus is then to be divided near its origin, and raised with care, so as not to destroy the branches of the obturator nerve beneath; its tendon is to be detached from that of the adductor magnus beneath it, to see the branches of the profunda artery.

Now the adductor brevis will be laid bare. A part of the obturator nerve crosses over this musele to the femoral artery, and sends an offset to the plexus at the inner side of the thigh: a deeper part of the same nerve lies beneath this adductor. The muscle should be separated from the subjacent adductor magnus, where the lower branch of the nerve with an artery issues. In this last ste $_{\mathbf{1}}$ of the dissection the student should trace on and in the fibres of the adductor magnus a slender articular branch of the obturator nerve to the knee. 
The accessory obturator nerve (Schmidt) is derived from the trunk of the obturator, near the lumbar plexus (p. 497), and passes from the abdomen over the brim of the pelvis. In the thigh it turns beneath the pectineus, and joins the superficial part of the obturator nerve; it supplies an offset to the hip-joint with the articular artery, and occasionally one to the under surface of the pectineus.

The ADDUCtor BREvis (fig. 198, D) has a thin fleshy and aponeurotic attachment, about two inches in depth, to the front of the hip-bone with the gracilis. The muscle arises from the pubic border of the bone close to and outside the gracilis, reaching upwards as high as the adductor longus, and not quite so low as the gracilis. It is inserted, behind the pectineus, into all the line leading from the linea aspera to the small trochanter.

In firont of the muscle are the pectineus and the adductor longus, with the superficial piece of the obturator nerve, and the profunda artery; but it is gradually uncovered by the adductor longus below, and the contiguous borders of the two are side by side at their insertion into the femur. Behind the muscle is the adductor magnus, with the deep piece of the obturator nerve and a branch of the inner circumflex artery. In contact with the upper border is the obturator externus, $\mathrm{F}$, and the internal circumflex artery passes between the two.

Action. This muscle adducts the limb with slight flexion of the hipjoint, like the pectineus. And if it acts from the femur it will balance and move forwards the pelvis.

The OBTURATOR NERve (fig. $198,{ }^{1}$ ) is a branch of the lumbar plexus (p. 497), and supplies the adductor muscles of the thigh, as well as the hip and knee joints. The nerve issues from the pelvis through the aperture in the upper part of the thyroid foramen; and it divides in that opening into two pieces, which are named superficial and deep from their position with respect to the adductor brevis muscle.

A. The superficial part $\left({ }^{1}\right)$ of the nerve is directed over the adductor brevis, but beneath the pectineus and the adductor longus, to the femoral artery, on which it is distributed ; at the lower border of the last muscle it furnishes an offset or two to join in a plexus with the internal cutaneous and saphenous nerves (p. 573), and supply the teguments. ${ }^{2}$

Near the pelvis or in the aperture of exit, this piece of the nerve sends outwards an articular twig to the hip joint with the joint-artery.

Muscular branches are furnished to the adductor longus, the adductor brevis, and the gracilis.

B. The deep part $\left({ }^{4}\right)$ of the obturator nerve pierces the fibres of the external obturator muscle, and continuing beneath the adduetor brevis is consumed chiefly in the adductor magnus. The following offsets are supplied by it:-

Muscular branches enter the obturator externus as the nerve pierces it; others are furnished to the large, and sometimes to the short adductor.

A slender articular branch (fig. 198, ${ }^{5}$ ) enters the fibres of the adductor magnus, and passes through it near the linea aspera to reach the popliteal

1 This small nerve is often absent; it was found only four or five times in nine or ten bodies which were examined by its discoverer. The name given to it by Schmidt refers to this irregularity, viz., nerv, ad obturatorem accessorius inconstans. Commentarius de Nervis Lumbalibus.

2 In some bodies the superficial part of the nerve is of large size, and has a distribution similar to that of the inner branch of the internal cutaneous nerve, whose place it takes : in such instances it joins freely in the plexus. 
artery, by which it is conducted to the back of the knee-joint : its termination is seen in the dissection of the popliteal space.

Dissection. To prepare the profunda artery and its branches, supposing the veins and the fat removed, it will be requisite to follow backwards the internal circumflex artery above the upper border of the adductor brevis, and to trace the perforating branches to the apertures in the adductors near the femur.

The PROFUNDA (fig. 198, $c$ ) is the chief muscular artery of the thigh, and arises from the femoral about one inch and a half below Poupart's ligament (p. 565). At its origin the ressel is placed on the outer side of the parent trunk; but it is soon directed inwards beneath the femoral vessels to the inner side of the femur, and ends at the lower third of the thigh in a small branch that pierces the adductor magnus.

Where the vessel lies in the triangular space of the thigh it rests on the iliacus muscle. But on the inner side of the femur it is parallel to the femoral artery, though deeper in position; and it is placed first over the pectineus and adductor brevis, and thence to its termination between the adductors longus and magnus.

Its branches are numerous to the surrounding muscles on the front and back of the thigh, and maintain free anastomoses with other vessels of the thigh and leg; through these communications the blood finds its way to the lower part of the limb when the tube of the chief artery is obliterated either above or below the origin of the profunda. The named branches are these :-

The external circumflex artery (fig. 198,e) has been described in the dissection of the muscles of the front of the thigh (p. 572).

The internal circumflex branch (fig. 198, d) arises from the inner and posterior part of the profunda, and turns backwards between the psoas and pectineus, but above the adductor brevis and magnus. Opposite the small trochanter it ends in two branches, which will be seen in the dissection of the buttock (p. 590). It supplies the undermentioned offsets to the inner side of the thigh :-

An articular artery may enter the hip joint through the notch in the acetabulum.

At the border of the adductor brevis two muscular branches arise : one ascends to the obturator and the superficial adductor muscles; the other, which is larger, descends with the deep piece of the obturator nerve beneath the adductor brevis, and ends in this and the largest adductor.

The perforating branches, three in number, pierce the tendons of some of the adductor muscles close to the linea aspera of the femur: they supply muscles on the back of the thigh, and wind round the thigh-bone to end in the rasti.

The first $(f)$ begins opposite the lower border of the pectineus, and perforates the short and large adductors.

The second branch $(g)$ arises below the middle of the adductor brevis, and passes through the same muscles as the preceding: from it a nutritious vessel is supplied to the shaft of the femur.

The third artery $(h)$ springs from the deep femoral trunk below the adductor brevis, and is transmitted through the adductor magnus.

The terminal branch of the profunda (fourth perforating) pierces the adductor magnus near the aperture for the femoral artery.

Muscular or anastomotic branches (i) to the back of the thigh (three or four in number) pass through the adductor magnus at some distance 
from the linea aspera, and end in a chain of anastomoses in the hamstrings.

The Profunda veIn results from the union of the different branches corresponding with the offsets of its companion artery. It accompanies closely the artery of the same name, to which it is superficial, and ends above in the femoral vein.

Dissection. To bring into view the remaining muscles, viz., adductor magnus, obturator externus, and the psoas and iliacus insertion, the adductor brevis is to be cut through near the pelvis, and to be thrown down. Then the investing layer of fascia and areolar tissue is to be removed from each muscle.

After the adductor magnus has been learnt, it will be needful to detach a few of the upper fibres to examine the obturator externus.

The ADDUCTOR MAGNus (fig. $128,{ }^{\mathrm{E}}$ ) is narrow at the pelvis, and wide at the femur. It is triangular in form, with its base directed upwards, one side being attached to the femur, and the other free at the inner part of the thigh.

The muscle arises along the pubic arch of the innominate bone outside the other adductors, reaching from the symphysis to the lower part of the ischial tuberosity. The anterior fibres diverge from their origin, being horizontal above but more oblique below, and are inserted (from above down) into the line from the great trochanter to the linea aspera; into the linea aspera; and into the line leading from that crest of bone to the inner condyle for about an inch. The posterior fibres from the ischial tuberosity are vertical in direction, and end at the lower third of the thigh in a tendon, which is inserted into the inner condyle of the femur, and is connected by a fibrous expansion to the inner condyloid ridge.

'The muscle consists of two parts, which differ in their characters. The anterior one, thin and fleshy, forms a septum between the other adductors and the muscles on the back of the thigh; but the posterior piece, partly fleshy and partly tendinous, constitutes the inner thick margin of the muscle. On the anterior surface are the other two adductors and the pectineus, with the obturator nerve and the profunda artery. The posterior surface touches the ham-string muscles and the great sciatic nerve. In contact with the upper border are the obturator externus and the quadratus femoris, with the internal circumflex vessels; and along the inner border lie the gracilis and the sartorius. At its attachment to the femur the muscle is closely united with the other adductors, particularly the adductor longus, and is there pierced by apertures for the passage of the femoral and perforating arteries.

Action. This musele is used as an adductor, but chiefly as a projector forwards of the femur in walking: in the last office it receives help from the other adductors internally, and from the gluteus medius and minimus externally.

The femur being fixed it will act powerfully in keeping the pelvis erect on the head of the thigh bone.

The opening in the adductor for the transmission of the femoral vessels into the popliteal space is tendinous at the anterior, but fleshy at the posterior aspect. It is situate at the point of junction of the middle with the lower third of the thigh, and is larger than is necessary for the passage of the vessels. On the outside it is bounded by the vastus internus; and on the inside by the tendon of the adductor magnus, with some fibres added from the tendon of the long adduetor. 
The PSOAS and ILIACUS (fig. 198) arise separately in the abdomen (p. 493), but are united in the thigh - the conjoined portion of the muscles coming beneath Poupart's ligament. The psoas, $\mathrm{P}$, is inserted by tendon into the small trochanter of the femur: and the fleshy iliacus, 0 , joins partly the tendon of the psoas, tut the rest of its fibres are fixed into a special triangular surface of bone in front of and below that trochanter.

Beneath the ligament the muscles occupy the interval between the iliopectineal eminence and the anterior superior iliac spinous process-the iliacus resting on a small bursa; and below the pelvis the mass covers the eapsule of the hip joint, and a larger intervening bursa. On the front of the psoas is the femoral artery, and between the two muscles lies the anterior crural nerve. The pectineus and the internal circumflex vessels are contiguous to the inner border; and the sartorius and vastus internus touch the outer edge.

Action. These muscles act as flexors of the hip joint, and their use is given with the description of the part in the abdomen ( $p .493$ ).

The oBTURATOR EXTERNus (fig. $198,{ }^{\mathrm{F}}$ ) is triangular in form, with the base at the pelvis and the apex at the femur. The fibres of the muscle take origin from the outer surface of the obturator membrane for the anterior half; and from the anterior half or more of the bony circumference of the thyroid foramen - the attachment being an inch wide opposite the symphysis pubis. The tibres are directed obliquely backwards to be inserted by a tenrlon into the pit at the root of the great trochanter.

This muscle is concealed by the pectineus, and adductor brevis and magnus. It covers the obturator nembrane and vessels, and is pierced by part of the obturator nerve. As it winds back it is in contact with the inner and lower parts of the hip joint. The insertion of the musele will be seen in the dissection of the Buttock.

Action. The muscle is an external rotator of the thigh : and its action will be given in full with the other muscles of the same group in the Buttock.

Dissection By detaching a small part of the obturator muscle from the pelvis, the branches of the artery and nerve of the same name will be seen amongst its fibres. A better view will he obtained if the dissection of the vessel and nerve is deferred till after the limb is detached.

The obturator artery is a branch of the internal iliac (p. 515), and enters the thigh through the upper part of the thyroid foramen. In the aperture the artery divides into two pieces, which form a circle beneath the muscle around the obturator membrane:-

The upper branch extends along the inner half of the membrane; and the lower, perforating the membrane below the level of the other, turns downwards and forms a circle by uniting with the upper branch. An articular twig to the hip-joint is supplied from the lower branch.

Muscular offsets of the artery are furnished to the obturator museles, and some small twigs reach the upper part of the adductors.

Branches of nerve to the external obturator muscle come from the deep portion of the obturator trunk, and perforate the membrane with the lower branch of the artery. 


\section{SECTION II.}

\section{THE BUTTOCK, OR THE GLUTEAL REGION.}

Directions. Both this Section and the following one are to be completed by the student in the time appointed for the body to lie in the prone position.

Position. During the dissection of the back of the thigh the body is placed with the face down; and the pelvis is to be raised by blocks, until the lower limbs hang almost vertically over the end of the dissecting table. When the body is turned, the points of bone marking posteriorly the limit between the thigh and the abdomen can be better ascertained.

Dissection. The integument is to be raised from the buttock by means of the following incisions: One is to be made along the iliac erest of the hip bone, and is to be continued in the middle line of the sacrum to the tip of the coccyx. Another is to be begun where the first terminates, and is to be carried outwards across the thigh till it is about six inches below the great trochanter. The flap of skin thus marked out is to be thrown down.

Many of the cutaneous nerves of this region will be found in the fat along the line of the iliac crest. Thus in front, but rather below the crest, are branches of the external cutaneous, if these have not been cut in the dissection of the thigh. Crossing the crest towards the fore part is a large offset of the last dorsal nerve; and usually farther back, but close to the bone, a smaller branch from the ilio-hypogastric nerve. In a line with the outer border of the erector spina, are two or three branches of the lumbar nerves.

By the side of the sarcum and coccyx two or three offsets of the sacral nerves are to be looked for beneath the fat.

The remaining cutaneous nerves are derived from the small sciatic, and must be sought beneath the fat along the line of the lower incision, where they come from underneath the gluteus maximus. A few turn upwards over that muscle; the rest are directed down the thigh, and one (inferior pudendal) bends below the ischial tuberosity to reach the perinæal space.

Cutaneous arteries aecompany all the nerves, and will serve as guides to their situation.

Cutaneous Nerves (fig. 199). The nerves distributed in the integuments of the buttock are small but numerous, and are derived from the spinal nerves (posterior primary pieces); from branches of the lumbar and sacral plexuses; and from the last dorsal nerve.

Branches of the lumbar nerves $\left({ }^{6}\right)$. The offsets of the posterior primary pieces of the lumbar nerves (p. 367) are two or three in number, and cross the crest of the hip bone near the anterior edge of the erector spinæ: they ramify in the integuments of the middle of the buttock, and some branches may be traced nearly to the trochanter major.

The branches of the sacral nerves $\left(^{5}\right)$ perforate the gluteus maximus near the sacrum and coccyx, and are then directed outwards for a short distance in the integuments over the muscle. These offsets are usually two in number: the largest is opposite the lower end of the sacrum, and the other by the side of the coccyx.

The last dorsal nerve $\left({ }^{8}\right)$ supplies the buttock by means of its lateral 
cutaneous branch (p. 416). This offset perforates the muscles of the abdomen, and crosses the anterior part of the iliac crest to be distributed over the fore part of the gluteal region, as low as the great trochanter.

Neries of the lumbar plexus. Parts of two nerves of the lumbar plexus (p. 496), viz. ilio-hypogastric and external cutaneous, are spent in the integuments of this region.

The iliac branch of the ilio-hypogastric $\left({ }^{7}\right)$ crosses the iliac crest in front of the branches from the lumbar nerves, lying generally in a groove in the bone, and extends only a short distance below the crest.

Fig. 199.

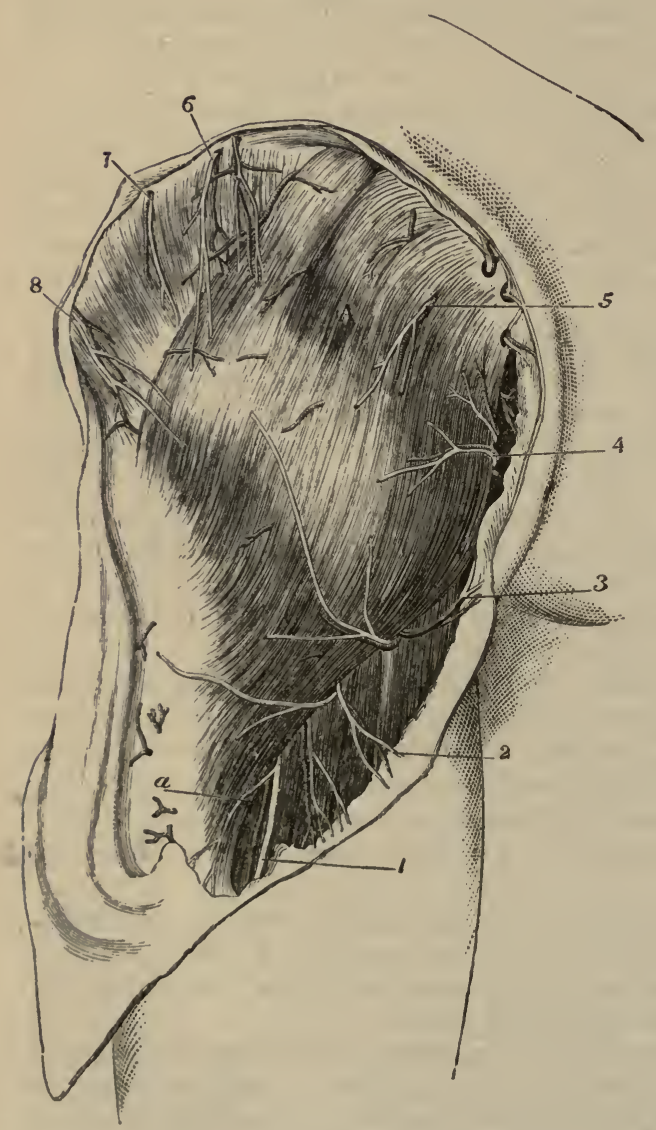

A. Glutens maximus muscle, with the glnteus medius projecting above it.

a. Continuation of small sciatic artery along the back of the thigh. Nerves and vessels, most of them cut from the teguments:-

1. Small sciatic nerve-the trunk.

2. Its cutaneous thigh branches; \&

3. Inferior pudendal.

4. Small sciatic offsets in the perinæum.

5. Cutaneous of the sacral.

6. Offsets of the lumbar nerves.

7. Ilio-hypogastric

8. Branch of the last dorsul.

SUPBR PICIAL View OP THE BUTtock OF THE LEFT Side (Illustrations of Dissections).

Offsets of the external cutaneous nerve of the thigh bend backwards to the integuments above the great trochanter, and cross the ramifications of the last dorsal nerve.

Small sciatic $\left({ }^{1}\right)$. This nerve of the sacral plexus (p. 518) sends superficial branches to the buttock. Its cutaneous offsets appear along the lower border of the gluteus maximus, accompanied by superficial branches 
of the sciatic artery : two or three ascend round the edge of the muscle, and are lost in the integuments of the lower part of the buttock; the remaining branches $\left({ }^{2}\right)$ descend to the thigh, and will be afterwards noticed on it.

Dissection. 'The thin and unimportant deep fascia of this region may be disregarded, in order that the great gluteal muscle, which is the most difficult in the body to clean, may be well displayed. Supposing the student desirons to lay bare the muscle, let him turn aside the cutaneous nerves, and adduct and rotate inwards the limb to make tense the muscular fibres. Having cut through the fat and fascia from the origin to the insertion, let him earry the sealpel along one bundle of fibres at a time in the direction of a line from the sacrum to the femur, until all the coarse fasciculi are cleaned. If the student has a right limb, the dissection may be begun at the upper border; but if a left limb, at the lower margin of the muscle.

The fasia of the buttock is a prolongation of that enveloping the thigh, and is fixed to the crest of the hip bone, and to the sacrum and coceyx. It is much thicker in front of, than on the gluteus maximus, and gives attachment anteriorly to the gluteus medius which it covers. At the edge of the gluteus maximus, the fascia splits to incase the muscle.

The Gluteus maximus (fig. 199, ${ }^{\wedge}$ ) is the most superficial muscle of the buttock, and reaches from the pelvis to the upper part of the femur. Its origin from the pelvis is partly connected with bone and partly with aponeurosis: Thus, the muscle is attached, from above down, to the posterior third of the iliac crest, and to a special impression on the hip bone below it; next, to the aponeurosis covering the multifidus spina muscle; then to the back of the lowest piece of the sacrum, and the back of the coccyx; and lastly to the great sacro-sciatic ligament. From this extensive origin the fibres are directed outwards to their insertion: About twothirds of the upper tibres, and a few of the lowest, end in the fascia lata of the outer part of the thigh: and the remainder are fixed for three inches into the lower part of the line leading from the linea aspera to the great trochanter of the femur.

The gluteus forms the prominence of the buttock, and resembles the deltoid muscle of the arm in the situation, and in the coarseness of its texture. Its eutaneous surface is eovered by the common teguments and investing fascia of the limb, and by the superticial nerves and vessels. The parts in contact with the under surface will be seen when the muscle is cut through. The upper border overlays the gluteus medius. And the lower edge, which is longer and thicker than the upper, forms the fold of the nates, and bounds posteriorly the perinæal space; beneath the lower border the ham-string muscles and the sciatic vessels and nerves issue.

Action. With the femur hanging the muscle extends the hip-joint by putting back that bone, and abducts and rotates out the limb.

When the limb is fixed, and the body is raised from a sitting into a standing posture, the gluteus acts as an extensor of the articulation by moving back the pelvis.

In standing both muscles assist in keeping the pelvis balaneed on its props; and in rising from stooping they are the active agents in bringing upright the pelvis. When the body is supported on one leg the muscle can draw the sacrum towards the femur, so as to turn the face to the opposite side.

Dissection (fig. 200). The gluteus maximus is to be cut across near the pelvis, and without injury to the subjacent sacro-sciatic ligament to 
which the lower fibres are closely joined. The depth of the muscle will be ascertained by the fascia and some vessels beneath it. When this intermuscular layer is arrived at, the outer part of the gluteus is to be thrown towards its insertion, and the sciatic artery and nerves are to be detached from the under surface, though the branches of the gluteal vessels entering the muscle must be cut.

The loose fat is to be taken away from the hollow between the pelvis and the trochanter, without injuring the ressels and nerves; and the several muscles are to be cleaned, the fibres of each being made tense at the time of its dissection by rotating the femur. 'The ressels, nerves, and muscles, which are to be defined, may be ascertained by referring to the enumeration below of the parts beneath the gluteus. In removing the areolar tissue from the ischial tuberosity and the great trochanter, the bursa on each prominence of bone will be observed.

Lastly the origin of the muscle is to be removed; and the sacral nerves are to be dissected out of the gluteus, and to be followed to the surface of the great sacro-sciatic ligament, where they will be afterwards seen.

Parts beneath the gluteus (fig. 200). At its origin the gluteus maximus rests on the pelvis, and conceals part of the hip bone, sacrum, and coceyx, also the ischial tuberosity with the origin of the hamstring muscles, L, and the great sacro-sciatie ligament, $\mathrm{k}$. At its insertion it covers the upper end of the femur, with the great trochanter, and the origin of the vastus externus, I. Between the muscle and each prominenee of bone, viz. the tuberosity and the trochanter, is a large, loose synovial membrane; and between it and the vastus externus is another synovial sac.

In the hollow between the pelvis and the femur the musele conceals, from above downwards, the undermentioned parts :-First, a portion of the gluteus medius, A; and below it the pyriformis, B, with the superficial branch $(a)$ of the gluteal vessels between the two. Coming from beneath the pyriformis are the sciatic vessels $(b)$, and the large and small seiatic nerves $\left({ }^{3}, 6\right)$, which descend to the thigh between the great troehanter and the ischial tuberosity; and internal to the sciatic are the pudic vessels and nerve $\left(d,{ }^{5}\right)$, and the nerve to the obturator internus musele $\left({ }^{4}\right)$ with its vessels, which are directed inwards through the small sacro. sciatic notch. Still lower down is the tendon of the obturator internus muscle, $\mathrm{D}$, with a fleshy fasciculus-the gemellus ( $\mathrm{C}$ and $\mathrm{k}$ )-above and below it. Next comes the thin quadratus femoris muscle, $\mathrm{G}$, with the upper part of the adductor magnus, $\mathrm{I}$ : at the upper border of the quadratus is the tendon of the obturator externus, $\mathrm{F}$; and at the lower borler, between it and the adductor, issues one of the terminal branches of the internal circumflex artery $(c)$ with its veins.

Dissection. Tracing back the offsets of the sacral nerves which perforate the gluteus, and removing a fibrous stratum which covers them, the looped arrangement of the first three nerves on the great sacro-seiatic ligament will appear. Finally the nerves may be followed inwards beneath the multifidus spina to the posterior sacral formmina.

Sacral nerves. The external pieces of the posterior primary branches of the first three sacral nerves, after passing outwarls beneath the multifidus spina (p. 372), are joined by loops on the surface of the great sacrosciatic ligament (fig. 120).

Two or three cutaneous offsets are derived from this intercommunication, and pierce the fibres of the gluteus maximus to be distributed on the surface (p. 581). 
The GLUTEus medrus (fig. 200, ${ }^{\mathrm{A}}$ ) is triangular in form, with its base at the innominate bone, and apex at the femur. It arises from the outer surface of the hip bone between the crest and the superior curved line,

Fig. 200.

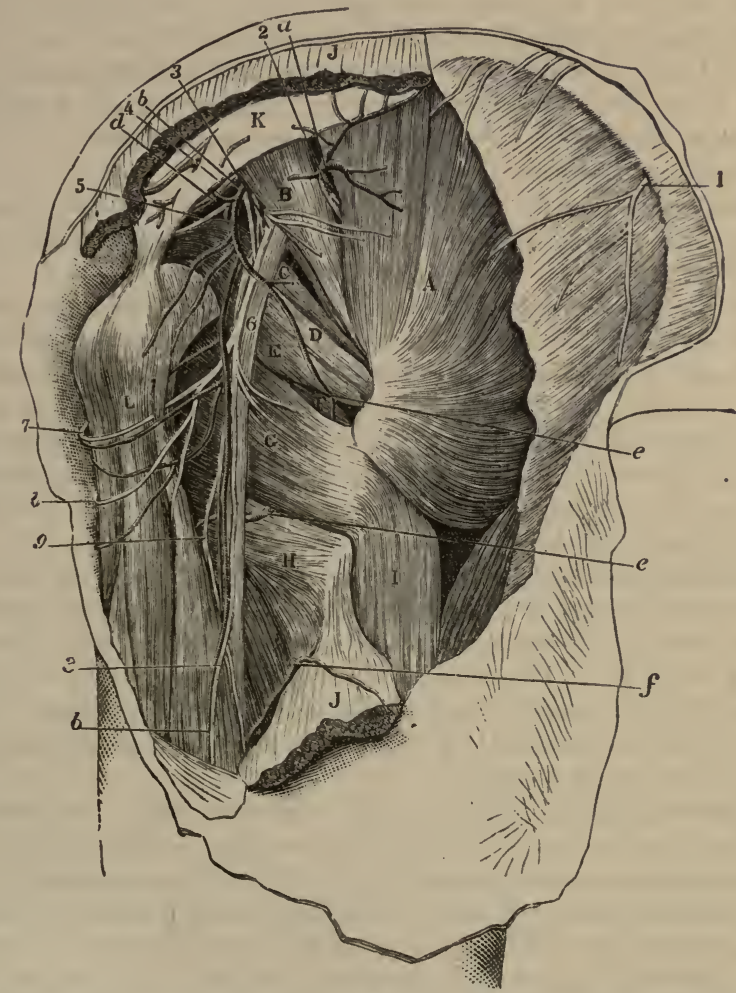

Second View of the Dibsection of thB Butrock (Illustrations of Dissections).

Muscles :

A. Gluteus medius.

B. Pyriformis.

c. Upper gemellus.

D. Obturator internus.

B. Gemellus inferior.

F. Obturator externus.

G. Quadratus femoris.

H. Adductor maguus.

I. Vastus externus.

J. Gluteus maximus, cut.

K. Great sacro sciatic ligament.

I. Hamstring muscles.

Arteries :

a. Gluteal. b. Seiatic.

c. Internal circumflex.

d. Pudic.

e. Anastomotic branch of sciatic.

$f$. First perforating. Nerves :

1. Last dorsal.

2. Upper gluteal.

3. Small sciatic.

4. Nerve to the obturator internus.

5. Pudic.

6. Great sciatic.

7. Inferior pudendal.

8. Cutaneous of the thigh of small sciatic.

9. Muscular branch of great sciatic.

except behind where there is a surface of bone free from muscular fibres ; and many superficial fibres come from the strong fascia covering the anterior part of the muscle. The fibres converge to a tendon, which is inserted 
into an impression across the outer surface of the great trochanter, extending from the tip behind to the root in front.

The superficial surface is eoneealed in part by the gluteus maximus; and the deep is in eontact with the gluteus minimus, and the gluteal vessels and nerve. The anterior border lies over the gluteus minimus, and is in contact with the tensor of the fascia lata. The posterior is contiguous to the pyriformis, only the gluteal vessels intervening. A small bursa is interposed between the tendon of insertion and the trochanter.

Action. The whole muscle abducts the harging femur; and the anterior fibres rotate in the limb. In walking it is combined with the adduetors in moving forwards the femur.

Both limbs resting on the ground the muscles assist in fixing the pelvis. In standing on one leg this gluteus will aid in balancing the pelvis on the top of the femur.

Dissection. When the gluteus medius is detached from the pelvis, and partly separated from the gluteus minimus beneath, the gluteal vessels and nerve will come into view. The two chief branches of the artery-one being near the iliac erest, and the other lower down-are to be traced through the fleshy fibres as the reflection of the gluteus is proceeded with; and the main part of the nerve is to be followed at the same time to the tensor vaginæ femoris muscle. The branches of the artery and nerve to the gluteus medius will be cut in removing that muscle.

The gluteal artery (fig. 200,a) is the largest branch of the internal iliac (p. 513), and issues from the pelvis above the pyriform muscle. On the dorsum of the hip bone it ends in offsets which supply the gluteal muscles and the bone. Its namerl branches are superficial and deep:-

The superficial branch supplies offisets to the integuments, and some deeper twigs over the sacrum; it ends in the gluteus maximus, which it penetrates on the under surface.

The deep branch (fig. $201, a$ ) is the continuation of the artery, and subdivides into two pieees which run between the two smaller glutei. One (b) (superior) courses along the origin of the gluteus minimus (supplying mostly the medius) to the front of the iliac erest, where it anastomoses with the ascending branch of the external circumflex artery. The other portion (c) (inferior) is directed forwards over the middle of the smallest gluteal musele, with the nerve, towards the anterior lower iliac spine where it enters the tensor of the fuscia lata, and communicates with the external circumflex branch (p. 572): many offsets are furnished to the gluteus minimus, and some pierce that muscle to supply the hip joint.

Vein. The companion vein with the artery enters the pelvis, and ends in the internal iliac vein.

The superior gluteal nerve (fig. $201,{ }^{1}$ ) is a branch of the lumbo-sacral cord (p. 495-6). It accompanies the gluteal artery, and divides into two branches for the supply of the two smallest gluteal muscles: its lowest. branch terminates anteriorly in the tensor vaginæ femoris, $\mathbf{B .}$

The GLuteus minmes (fig. $201, \mathrm{c}$ ) is triangular in shape, and arises from the dorsum of the hip bone between the superior and inferior curved lines, extending backwards as far as the middle of the hip joint. Its tendon is inserted into an impression along the fore part of the great trochanter, where it is united inferiorly with the gluteus medius: some fibres are attaclied to the capsule of the hip joint.

One surface is in contact with the gluteus medius, and the gluteal vessels and nerve; the other with the hip bone, the hip joint, and the outer. 
head of the rectus femoris muscle. The anterior border lies by the side of the other gluteus; and the posterior is covered by the pyriformis muscle. A bursa is placed between the tendon and the bone.

Action. It acts as an abductor and rotator out of the femur when this bone is hanging; and in walking, it and the medius will be employed in bringing forwards the limb.

Both legs being fixed, the muscles are used in balancing the pelvis. In standing on one leg the gluteus pitches the pelvis over the supporting limb with the preceding muscle.

Dissection. Cut through the smallest glutens muscle near the innominate bone, and define the tendinous part of the rectus femoris underneath it, close above the hip joint. Whilst detaching the gluteus from the parts underneath, the student cannot fail to notice the connection between its tendon and the eapsule of the joint.

The deep vessels to the articulation may be observed and followed as the muscle is removed.

The outer head of the rectus femoris is a tendon as wide as the little finger, and about two inches long, which is fixed into the groove above the margin of the acetabulum. In front it joins the other tendinous piece of the rectus, which is attached to the anterior inferior iliac spine; and below, it is connected with the capsule of the hip joint.

The pYriformis (fig. $200,{ }^{\text {B }}$ ) arises in the pelvis from the front of the sacrum (p. 542), and leaves that cavity through the great sacro-sciatic notch. Outside the pelvis it ends in a rounded tendon, which is inserted into the upper edge of the great trochanter, between the two smaller glutei.

As the muscle passes through the sacro-sciatic notch it divides that space into two parts-the upper giving passage to the gluteal vessels and nerve, and the lower transmitting the sciatic and pudic vessels and the sacral plexus. Its upper border is contiguous to the gluteus medius, and its lower, to the gemellus superior. Like the other rotator muscles in this situation, it is covered by the gluteus maximus, and by the gluteus medius at the insertion; it rests on the gluteus minimus, which separates it from the hip joint. Its tendon is united by fibrous tissue to that of the obturator and gemelli.

Action. The use of this and the other external rotators is altered by the position of the femur. If that bone hangs the pyriformis rotates it out; but if the hip joint is bent the muscle abducts the limb from its fellow.

Both limbs being fixed the muscles balance the pelvis, and help to make the trunk erect after stooping to the ground. In standing on one leg, besides assisting to support the trunk, the pyriformis turns the face to the opposite side.

Dissection (fig. 201). The pyriformis may be cut across and raised towards the sacrum, to allow the dissector to follow upwards the sciatic and pudic vessels, and to trace the accompanying nerves to their origin in the lower part of the sacral plexus.

Some small nerves to the obturator internus $\left({ }^{5}\right)$, the gemellus superior $\left({ }^{7}\right)$, and the hip joint, are to be sought in the fat at the lower part of the plexus. A branch to the inferior gemellus and the quadratus $\left({ }^{6}\right)$ will be found by raising the trunk of the great sciatic nerve; but it will be followed to its termination after the muscles it supplies have been seen. 
Sciatic and Pudic Vessels. The vessels on the back of the pelvis, below the pyriformis muscle, are branches of the internal iliac (p. 513).

The sciatic artery (fig. 200, $b$ ) supplies the buttock below the gluteal. After escaping from the pelvis helow the pyriformis, it descends with the small sciatic nerve over the gemelli and obturator muscles, as far as the lower border of the gluteus maximus: here the artery gives off many branches with the superficial offsets of its companion nerve; and much reduced in size, it is continued with that nerve along the back of the thigh. In this course it furnishes the following named branches :-

a. The coccygeal branch, arising close to the pelvis, perforates the great sacro-sciatic ligament and the gluteus maximus, and ramifies in this muscle, and on the back of the sacrum and coccyx.

$b$. The branch to the great sciatic nerve (comes nervi ischiadici) is very slender, and entering the nerve near the pelvis, ramifies in it along the thigh.

c. Muscular branches enter the gluteus maximus, the upper gemellus, and obturator internus; and by means of a branch to the quadratus, which passes with the nerve of the same name beneath the gemelli and obturator internus, it gives offsets to the hip joint and the inferior gemellus.

d. Anastomotic branch (fig. 200,e). Varying in size this artery is directed outwards to the root of the great trochanter, where it anastomoses with the gluteal and internal circumflex.

The pudic artery (fig. 200,d) belongs to the perinæum and the genital organs; it is smaller than the sciatic, internal to which it lies. Only the small part of the vessel which winds over the ischial spine is seen on the back of the pelvis, for it enters the perinaal space through the small sacro-sciatic notch, and is there distributed (p. 390).

It supplies a small branch over the back of the sacrum, which anastomoses with the gluteal and sciatic vessels; and a twig from it accompanies the nerve to the obturator internus muscle.

The veins with the sciatic and pudic arteries receive contributing twigs corresponding with the branches of those arteries at the back of the pelvis, and open into the internal iliac vein.

Sciatic and Pudic Nerves. The nerves appearing at the back of the pelvis, below the pyriformis, are branches of the sacral plexus to the lower limb (p. 518); they are furnished mostly to parts beyond the gluteal region, but a few are distributed to the muscles at the back of the pelvis.

The small sciatic (fig. $200,{ }^{3}$ ) is a cutaneous nerve of the back of the thigh, for it supplies only one muscle of the buttock. It springs from the lower part of the sacral plexus, generally by two pieces, and takes the course of the sciatic artery as far as the lower border of the great gluteus, where it gives many cutaneous branches : much diminished in size at that spot, the nerve is continued along the back of the thigh beneath the fascia, and ends below the knee in the integuments of the back of the leg. The branches which are distributed to, or near the buttock, are museular and cutaneous :-

The muscular branches (inferior gluteal) enter the under surface of the gluteus maximus near the lower border.

'The cutaneous branches are directed upwards and downwards at the border of the gluteus :-

The ascending set (fig. 199) are distributed in the fat over the lower third of the muscle. 
The descending set (fig. $199,{ }^{2}$ ) supply the integuments of the upper third of the thigh at the inner and posterior aspects. One of these branches $\left({ }^{8}\right)$, which is larger than the others, is distributed to the genital organs, and is named inferior pudendal (p. 394); as it courses to the perinæum, it turns below the ischial tuberosity, and perforates the fuscia lata at the inner part of the thigh to end in the scrotum.

The great sciatic (fig. $200,{ }^{6}$ ) is the largest nerve in the body. It is the source of all the muscular, aud most of the cutaneous branches distributed to the limb beyond the knee, as well as of the muscular branches at the back of the thigh.

At its origin it appears to be a prolongation of the sacral plexus. It is directed through the buttock to the posterior part of the thigh, and rests on the external rotator muscles below the pyriformis. Commonly it does not supply any branch to the buttock, but it may give origin to one or two filaments to the hip joint. Frequently the nerve is divided into two large trunks at its origin, and one of them pierces the fibres of the pyriformis muscle.

The pudic nerve (fig. $200,{ }^{5}$ ) winds over the small sacro-sciatic ligament by the side of its companion artery, and is distributed with this vessel to the perinaum and the genital organs (p. 391). No branch is supplied to the buttock.

Muscular branches of the sacral plexus are furnished to the gluteus maximus, and to the external rotators except the obturator externus.

Branches of gluteus. One or more branches of the plexus enter the top of the gluteus maximus (fig. 200).

Two branches of the pyriformis enter the under surface, and are learnt with the sacral plexus in the pelvis.

The nerve to the obturator internus (fig. $201,{ }^{5}$ ) arises from the upper part of the plexus, and is directed to its muscle through the small sacrosciatic notch with the pudic nerve: its termination is seen in the dissection of the pelvis.

The nerve to the superior gemellus (fig. $201,{ }^{7}$ ) is a very small twig, and arises separately from the following: it enters the inner end of the muscle on the superficial surface.

The nerve to the inferior gemellus and the quadratus (fig. $201,{ }^{6}$ ) is a slender branch, which passes with a companion artery beneath the gemelli and the obturator internus, to end in the two muscles from which it receives its designation. This nerve will be seen more fully in a subsequent dissection, when articular filaments from it to the hip-joint may be recognized.

Dissection. To see the remaining small rotator muscles, hook aside the great sciatic nerve, and take away the branches of the sciatic artery if it is necessary. In cleaning these muscles the limb should be rotated inwards. The gemelli are to be separated from the tendon of the obturator internus.

The superior GeMellus (fig. $200,{ }^{c}$ ) is the highest of the two muscular slips along the sides of the tendon of the obturator muscle. Internally it is attached to the outer and lower part of the ischial spine, and externally it is inserted with the obturator into the great trochanter. Ottentimes the muscle is absent.

The INFERIOR GeMelues (fig. $200,{ }^{\mathrm{E}}$ ) is larger than its fellow. Its origin is connected with the upper part of the ischial tuberosity, along the lower edge or lip of the hollow for the obturator internus muscle; 
and its insertion is the same as that of the obturator tendon. This musele is placed between the obturator internus and quadratus, but near the femur the tendon of the obturator externus comes into contact with its lower border.

Action. These small fleshy slips seem to be but accessory pieces of origin to the internal obturator, with which they eombine in use.

The obturator internus (fig. 200, ${ }^{D}$ ) alises from the innominate bone inside the pelvis (p. 543), and passes to the exterior through the small sacro-sciatic notch. The tendon of the muscle is directed outwards over the hip-joint, and is inserted with the gemelli into the upper part of the great trochanter, in front of the pyriformis, as well as into the contiguous portion of the neck of the femur.

Outside the pelvis the obturator is mostly tendinous, and is embraced by the gemelli muscles in the following way: near the pelvis the gemelli meet beneath, but near the trochanter they cover the tendon. Beneath the obturator is a synovial sac. Crossing the muscle are the large and small sciatic nerves and the sciatic vessels; and covering the whole is the gluteus maximus. On cutting through the tendon and raising the inner end, it will be found divided into three or four pieces as it turns over the margin of the pelvis (fig. $201,{ }^{\mathrm{H}}$ ); at this spot the pelvis is marked by ridges of fibro-cartilage, which correspond with the intervals between the tendons, and the surfaces are lubricated by a synovial membrane.

Action. Acting from the hinder border of the pelvis round which it turns, it rotates out or abducts the femur according as this bone may be hanging or raised. It will erect the pelvis after stooping, and will balance the same in standing; and it will rotate to the opposite side the trunk supported on one limb.

The QUADRATUS FEMORIS (fig. $200,{ }^{\circ}$ ) has the form expressed by its name, and is situate between the inferior gemellus and the adductor magnus. Internally it arises from the outer border of the tuber ischii for two inches, along the origin of the semi-membranosus and adductor magnus; externally it is inserted into a tubercle in the posterior intertrochanteric ridge, and slightly into the neck of the femur; and into a line on the upper end of the bone for about two inches above the attachment of the great adductor.

By one surface it is in contact with the sciatic vessels and nerves, and the gluteus. By the other it rests on the obturator externus, the internal circumflex vessels, and its small nerve and vessels. Between its lower border and the adductor magnus one of the terminal branches of the internal circumflex artery issues. Between it and the small trochanter is a bursa, which is eommon also to the upper part of the adductor magnus.

Action. Though the muscle has but slight power, it will be associated with the other muscles on the back of the pelvis in rotation out of the pendent femur, and in abduction of the femur when the hip-joint is bent.

And its femoral attachment being fixed, it will help in supporting the pelvis; or it will turn the fice to the opposite side, the body being supported on one limb.

Dissection (fig. 201). The quadratus and the gemelli muscles may be now cut across, in order that their small nerve and artery, the ending of the internal circumflex artery, and the obturator externus may be dissected out.

The internal circumflex branch (fig. 201, $f$ ) of the profunda artery ( $p$. 578 ) divides finally into two parts. One $(g)$ ascends beneath the quad- 
ratus (in this position of the body) to the pit of the trochanter, where it anastomoses with the gluteal and sciatic arteries, and supplies the bone. The other $(h)$ passes between the quadratus and adductor magnus to the hamstring muscles, and communicates with a branch of the profunda artery.

The obturator externus (fig. 201, $\mathrm{L}$ ) has been dissected at its origin in the front of the thigh (p. 580). The part of the muscle now laid bare, winds below the hip-joint, and ascends to be inserted into the pit at the root of the great trochanter.

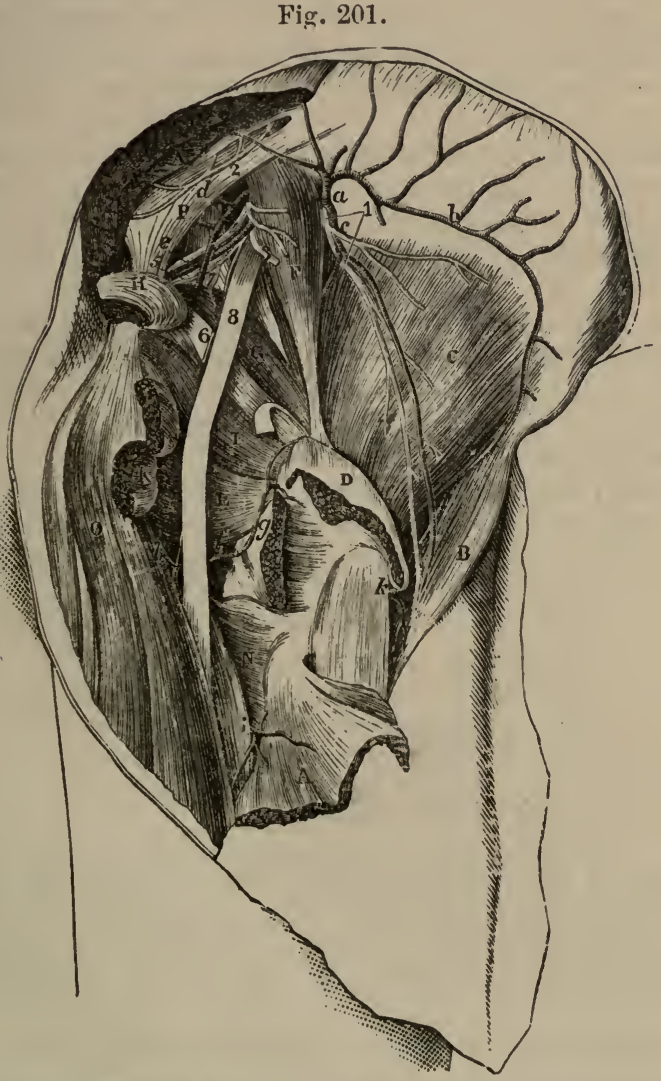

Muscles.

A. G]uteus maximus, cut.

B. Tensor fasciæ latæ.

c. Gluteus minimus.

D. Gluteus medius, cut.

F. Pyiformis.

G. Gemellus superior

H. Obturator internus, cut.

I. Geinellus inferior.

K. Quadratus femoris, cut.

L. Obturator exteruus.

N. Adductor magnus.

o. Hamstrings.

P. Great sacro-sciatic ligament.

Arteries.

a. Gluteal.

b. Its upper and, and $c$, its lower piece.

d. Sciatic.

e. Pudic.

$f$. Internal circumflex; $g$, its ascending, and $h$, its transverse offset.

i. First perforating.

k. External circumflex.

Nerves.

1. Superior gluteal.

2. Sacral.

3. Small sciatic, cut.

4. Pudic.

5. Nerve to obturator internus.

6. Nerve to quadratus and infexior gemellus.

7. Nerve to upper gemellus.

8. Large sciatic nerve.

THIRD View OF THE Dissection OF THE BUTTOCK (Illustrations of Dissections).

On the back of the pelvis the obturator externus is covered by the quadratus, except near the femur where the upper border is in contact with the inferior gemellus. As it turns back to its insertion it supports the hip-joint.

Action. Like the other muscles of the same group it rotates out the hanging limb; but it differs from them in having the same action even when the hip-joint is bent.

With the limb fixed, Theile supposes it to help in bending the hip-joint 
in stooping, instead of extending it and raising the trunk like the other external rotator muscles.

The SACRo-SCIATIC Ligamexts pass from the innominate bone to the sacrum and the coceyx ; they are two in number, and are named large and small.

The large or posterior ligament (fig. $201,{ }^{\mathrm{P}}$ ) is attached internally to the posterior part of the hip bone, and to the side of the sacrum and coccyx ; and externally it is inserted into an impression on the inner and anterior part of the ischial tuberosity, sending upwards a prolongation along the pubic arch. It is wide next the sacrum, but is contracted towards the middle, and is expanded again at the tuberosity. On the cutaneous surface are the branches of the sacral nerves; and the gluteus maximus conceals and takes origin from it. Branches of the gluteal and sciatic arteries perforate it.

The small ligament will be seen on dividing the other near the hip bone. At the sacrum and coccyx it is united with the large band, but at the opposite end it is inserted into the ischial spine. It is less strong than the superficial ligament, by which it is concealed; and it rests on the coccygeus muscle.

By their attachments these ligaments convert the large sacro-sciatic notch of the dried pelvis into two apertures or foramina. Between their insertion into the spine and tuberosity of the innominate bone, is the small sacro-sciatic foramen, which contains the internal obturator muscle with its nerve and vessels, and the pudic vessels and nerve. And above the smaller ligament is the large sacro-sciatic foramen, which gives passage to the pyriformis muscle, with the gluteal vessels and the superior gluteal nerve above it, and the sciatic and pudic vessels and the sacral plexus below it.

\section{Section III.}

\section{THE BACK OF THE THIGH.}

Directions. The ham or the popliteal space may be taken after the buttock, in order that it may be seen in a less disturbed state than if it was dissected after the examination of the muscles at the back of the thigh. When this space has been learnt the student will return to the dissection of the thigh. '

Position. The limb is to remain in the same position as in the dissection of the buttock.

Dissection (fig. 202). To remove the skin from the popliteal region let an incision be made behind the knee for the distance of six inches above, and four inches below the joint. At each extremity of the longitudinal cut make a transverse incision, and raise the skin in two flaps, the one being turned outwards and the other inwards.

In the fat are some small cutaneous nerves and vessels, viz., one or two twigs in the middle line of the limb from the small sciatic nerve and artery beneath the fascia; and some offsets of the internal cutaneous nerve towards the inner part. After the subcutuneous fat is removed, the special fiscia of the limb will be brought into view. 
Fascia lata. Where this fascia covers the popliteal space it is strengthened by transverse fibres, particularly on the outer side ; and it is connected laterally with the tendons bounding that interval. The short saphenous vein perforates it sometimes opposite the knee, but usually at a spot lower down.

Dissection (fig. 202). The fascia over the ham is now to be removed without injuring the small seiatic nerve and artery, and the short saphenous vein, which are close beneath it. A large quantity of fat may be next taken out of the space, but without injury to the several small vessels and nerves in it.

In cleaning the space the student will come upon the large internal popliteal nerve in the middle line; and nearer the outer side, on the external popliteal. Both nerves give branches; and the numerous offsets of the inner will be recognized more certainly by tracing them from above down along the trunk of the nerve, than by proceeding in the opposite direction; in fat bodies the two small nerves from the inner popliteal trunk to the knee joint are difficult to find. Under cover of the outer boundary, and deep in the space, is an articular nerve from the external popliteal, which sometimes arises from the great sciatic.

In the bottom of the space are the popliteal vessels, the vein being more superficial than the artery. The student is to seek an articular branch (superior), on each side, close ahove the condyle of the femur; and to clean numerous other branches of the vessels to the muscles around, especially to those of the leg. On the upper part of the artery, the branch of nerve from the obturator to the knee joint is to be found : and on the sides of the artery are three or four lymphatic glands in the fat.

After the ham has been cleaned, the sartorius and the gracilis are to be replaced in their natural position on the inner side.

The popliteal SPaCe, or the ham (fig. 202), is the hollow behind the knee: it allows of the free flexion of the joint, and contains the large vessels of the limb. When dissected, this interval has the form of a lozenge, and extends upwards along one-third of the femur, and downwards along one-sixth of the tibia; but in the natural condition of the parts the sides are approximated by the fascia of the limb, and the space is limited, apparently, almost to the region of the joint.

This hollow is situate between the muscles on the back of the limb; and the lateral boundaries are therefore formed by the muscles of the thigh (hamstrings), and leg. Thus, on the outer side, is the biceps muscle $\left({ }^{6}\right)$ as far as the joint; and the plantaris and the external head of the gastrocnemius $\left({ }^{8}\right)$ beyond that spot. On the inner side, as low as the articulation, are the semimembranosus $\left({ }^{4}\right)$ and semitendinosus $\left({ }^{5}\right)$ muscles, with the graeilis and sartorius between them and the femur; and beyond the joint is the inner head of the gastrocnemius $\left({ }^{7}\right)$. 'The upper point of the ham is limited by the apposition of the inner and outer hamstrings; and at the lower point the heads of the gastrocnemius tonch each other.

Stretched across the cavity are the fascia lata and teguments. Forming the deep boundary, or the floor, are the following parts-the posterior surface of the femur included between the lines to the condyles, the posterior ligament of the knee-joint, and part of the popliteus muscle with the upper end of the tibia.

The popliteal space is widest opposite the femoral condyles, where the muscles are most drawn to the sides; and is deepest above the articular 
end of the femur. Above and below it communicates, beneath the muscles, with the back of the thigh and leg.

In the hollow are contained the popliteal vessels with their branches,

Fig. 202.

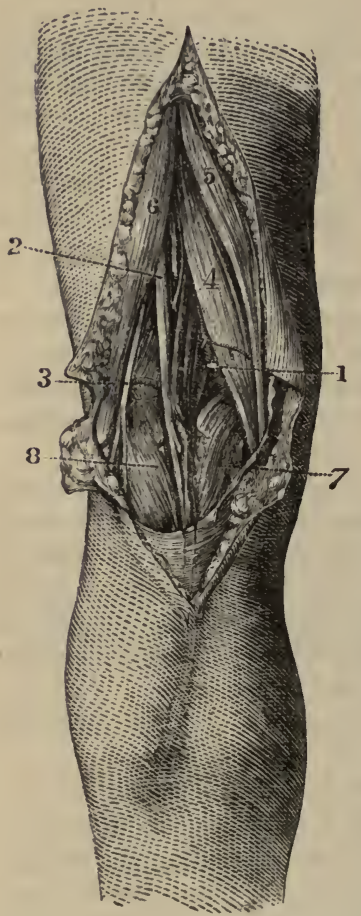

Virw of the Popliteal Space (Quain's Arterles).

1. Popliteal vessels.

2. Internal popliteal nerve.

3. External popliteal nerve.

4. Semimembranosus muscle.

5. Semitendinosus muscle.

6. Biceps muscle.

7, 8. Inner and outer heads of the gastrocnemius muscle. The superficial vein on the gastrocnemius is the short saphenous, which enters the popliteal. and the ending of the external saphenous rein ; the popliteal trunks of the great sciatic nerve, and some of their branches; together with lymphatic glands, and a large quantity of fat. The small sciatic nerve and its ressels are placed superficially in the ham ; and a branch of the obturator nerve lies on the artery in the bottom of the space.

'The POpliteal artery (fig. 202, ${ }^{1}$ ) is the continuation of the femoral, and reaches from the opening in the adductor magnus to the lower border of the popliteus muscle, where it terminates by bifurcating into the anterior and posterior tibial vessels. A portion of the artery lies in the ham, and is uncovered by muscle; but the rest is beneath the gastrocnemius, and beyond the limits of the popliteal space as above defined. The description of the artery may be divided therefore into two parts, corresponding with this difference in the connections.

In the ham the vessel is inclined obliquely from the inner side of the limb to the interval between the condyles of the femur; and is then directed along the middle of the space over the knee-joint. As far as the inner condyle the artery is overlaid by the belly of the semimembranosus muscle; but thence onwards it is situate between the heads of the gastrocnemius, and is covered only by the fascia lata and the integuments. Beneath it is the femur with the posterior ligament of the knee-joint.

In contact with the vessel, and somewhat on the outer side at first, lies the popliteal vein, so that, on looking into the space, the arterial trunk is almost covered; but in the interval between the heads of the gastrocnemius, the vein and its branches conceal altogether the artery. Below the knee the short saphenous vein (fig. 203, $i$ ), and the museular branches of the artery, are laid over the popliteal trunk.

More superficial than the large vessels, and slightly external to them in position, is placed the internal popliteal nerve, which with its branches lies over the artery, like the vein, between the heads of the gastrocnemius. In the bottom of the hollow the small obturator nerve runs on the artery to the joint.

Dissection. To sce the deep part of the artery the inner head of the gastrocnemius should be cut through, and raised firom the subjacent parts. On removing the areolar tissue the vessels and nerves will appear. The lower articular branches of the vessels and nerve are now brought into 
view ; - the inner artery is below the head of the tibia, and the outer higher up between the tibia and fibula, each with a vein, and the first has a companion nerve.

Beyond the ham. Whilst the artery is beneath the gastrocnemius (fig. 208 ) it sinks deeply into the limb; here it is crossed by a small musclethe plantaris $\mathrm{c}$, and the ending is concealed by the soleus $\mathrm{B}$. It rests on the popliteus muscle.

Both the companion vein and the internal popliteal nerve change their position to the artery, and gradually cross over it, so as to lie on its inner side at the lower border of the popliteus.

Sometimes the artery is divided as high as the back of the knee joint; and then the anterior tibial artery may lie beneath the popliteus muscle.

Branches (fig. 203) are furnished by the artery to the surrounding museles, and to the articulation;-those that belong to the joint are five in number, and are called articular, viz., two superior, inner and outer ; two inferior, also inner and outer; and a central or azygos branch.

$a$. The muscular branches are upper and lower. The upper set, three or four in number, arise above the knee, and end in the semi-membranosus and biceps museles, communicating with the perforating and muscular branches of the profunda. The lower set (sural) are furnished to the museles of the calf, viz., gastrocnemius, soleus, and plantaris.

b. A superficial or cutaneous branch arises near the knee joint, and accompanies the external saphenous nerve over the muscles of the leg to end in the teguments (fig. 203).

c. The superior articular arteries arise from the popliteal trunk, one from the inner and one from the outer side, above the condyles of the femur; they are directed almost transversely beneath the hamstring muscles, and turn around the bone to the front of the joint.

The external one $(k)$ perforates the intermuscular septum, and divides in the substance of the vastus internus. Some of the branches end in that muscle, and anastomose with the external circumflex (of the profunda): others descend to the joint; and one offset forms an areh across the fore part of the bone with the anastomotic artery.

The internal artery $(l)$, oftentimes very small, winds beneath the tendon of the adduetor magnus, and terminates in the vastus internus; it supplies this and the knee joint, and communicates with the anastomotic artery.

d. The inferior articular branches (fig. 208) lie beneath the gastroenemius, but are not on the same level on opposite sides of the limb; for the inner one descends below the head of the tibia, whilst the outer one is placed above the fibula. Each lies beneath the lateral ligament of its own side.

The external branch $(c)$ supplies she outer side of the knee joint, anastomosing with the other vessels on the articulation, and with the recurrent branch of the anterior tibial artery: it sends an offset beneath the ligament of the patella to join a twig from the lower internal branch.

The internal artery $(b)$ ascends at the anterior border of the internal lateral ligament, and after taking its share in the free anastomoses orer the joint, ends in offsets for the articulation and the head of the tibia.

$e$. The azygos branch enters the back of the joint through the posterior ligament, and is distributed to the ligamentous structures, the fat, and the synovial membrane of the interior. 
The popliteal vein (fig. 203, $h$ ) originates in the union of the venæe eomites of the anterior and posterior tibial vessels, and has the same extent and connections as the artery it accompanies. At the lower border of the popliteus muscle the vein is internal to the arterial trunk; between the heads of the gastrocnemius it is superficial to that vessel; and thence to the opening in the adductor magnus it lies to the outer side, and close to the artery. It is joined by branches corresponding with those of the artery, as well as by the short saphenous vein (fig. 203).

The popliteal Nerves (fig. 202) are the two large trunks derived from the division of the great sciatic in the thigh; they are named internal and external from their relative position. In the popliteal space each furnishes cutaneous and articular offsets, but only the inner one supplies branches to muscles.

The internal politeal verve $\left({ }^{2}\right)$ is larger than the external, and occupies the middle of the ham : its connections are similar to those of the artery, that is to say, it is partly superficial and partly covered by the gastrocnemius. Like the vessel it extends through the back of the leg, and retains the name popliteal only to the lower border of the popliteus muscle. Its position to the vessels has been already noticed. 'The branches arising from it here are the following :-

a. Two small articular twigs (fig. $203,{ }^{9}$ ) are furnished to the knee joint with the vessels. One which accompanies the lower internal articular artery to the fore part of the articulation is the largest ; and another takes the same course as the azygos artery, and enters the back of the joint with it.

b. Muscular branches arise from the nerve between the heads of the gastrocnemius. One supplies both heads of the gastrocnemius and the plantaris. Another descends beneath the gastrocnemius, and enters the cutaneous surface of the soleus. And a third penetrates the popliteus at the under aspect, after turning round the lower border.

c. The external saphenous nerve (fig. $207,{ }^{5}$ ) (ram. communicans tibialis) is the largest branch, and is a cutaneous offset to the leg and foot. It lie's on the surface of the gastrocnemius, but beneath the fascia, as far as the middle of the leg, where it becomes cutaneous, and will be afterwards seen.

The external popliteal Nerve (fig. $202,{ }^{3}$ ) (peroneal) lies along the outer boundary of the ham as far as the knee joint; at that level it leaves the space and follows the edge of the biceps muscle for two inches, till it is below the head of the fibula. There it enters the fibres of the peroneus longus, and divides beneath that muscle into three-nusculocutaneous, anterior tibial, and recurrent articular. Its branches whilst in the popliteal space are cutaneous and articular.

a. The articular nerve, arising high in the space, runs with the upper external artery to the outer side of the knee, where it sends a twig along the lower articular artery: both enter the joint.

b. The peroneal communicating branch (fig. 207, ${ }^{4}$ ) (ram. communicans fibularis) joins the external saphenous branch of the internal popliteal about the middle of the leg. It soon becomes cutaneous, and offsets are given by it to the back of the leg.

$c$. One or two cutaneous nerves are furnished by the external popliteal to the integument on the outer side of the leg in the upper half.

The articular branch of the obturator nerve (fig. $203,{ }^{7}$ ) perforates the adductor magnus, and is conducted by the popliteal artery to the back of 
the knee joint. After supplying filaments to the vessels, the nerve enters the articulation through the posterior ligament.

The lymphatic glands of the popliteal space are situate around the large arterial trunk. Two or three are ranged on the sides; whilst one is superficial to, and another beneath the vessel : they are joined by the deep lymphatic vessels, and by the superficial set with the saphenous vein.

\section{TIIE BACK OF THE THIGH.}

Dissection (fig. 203). Now the popliteal space has been examined, the student may proceed with the dissection of the back of the thigh. The piece of skin between the buttock and the popliteal space should be divided, and reflected to the sides. In the fat on the sides of the limb fine offsets of the internal and external cutaneous nerves of the front of the thigh may be found; and along the middle line some filaments from the small sciatic nerve pierce the fascia.

Remove the deep fascia of the limb, taking care of the small sciatic nerve and its artery. Lastly, clean the hamstring muscles; trace out the perforating arteries to the front of the thigh, and clean the branches of the great sciatic nerve and profunda artery to the muscles.

Muscles. The muscles behind the fenur act mainly as flexors of the knee joint. They extend from the pelvis to the bones of the leg, and are named hamstrings from their cord-like appearance on the sides of the ham : they are three in number, viz., biceps, semitendinosus, and semimembranosus. The first of these lies on the outer, and the others on the inner side of the popliteal space.

The BICEPS (fig. 203, D) has two heads of origin, long and short, which are attached to the pelvis and the femur. The long head arises from an impression on the back of the ischial tuberosity, in common with the semitendinosus muscle. The short head is fixed to the femur below the gluteus maximus, viz., to all the linea aspera, to nearly the whole of the line leading inferiorly to the outer condyle, and the external intermuscular septum. The fibres end inferiorly in a tendon, which is inserted into two prominences on the head of the fibula by slips which embrace the external lateral ligament; and a slight piece is prolonged to the head of the tibia.

The muscle is superficial, except at the origin, where it is covered by the gluteus: it rests on the upper part of the semimembranosus, and on the great sciatic nerve and the adductor magnus muscle. On the inner side is the semitendinosus as far as the ham. Its tendon gives offsets to the deep fascia of the limb.

Action. It can bend the knee if the leg-bones are not fixed, and afterwards rotate out the tibia; and the long head, which passes upwards beyond the femur, will extend the bent hip joint when the knee is straight.

'The leg being supported on the ground, the long head will assist in balancing and ereeting the pelvis; and the short head will draw down the femur so as to bend the knee in stooping.

The semitendinosus (fig. $203,{ }^{\mathrm{E}}$ ) is a slender muscle and receives its name from appearance. It arises from the tuberosity of the hip bone with the long head of the biceps, and by fleshy fibres from the tendon of that muscle. Inferiorly it is inserted into the inner surface of the tibia, c'ose below the gracilis, and for a similar extent.

'Tinis muscle, like the biceps, is partly covered by the giuteus maximus. 
About its middle a tendinous intersection may be observed. It rests on the semimembranosus, and on the internal lateral ligament of the kneejoint. The outer border is in contact with the biceps as far as the popli-

Fig. 203.

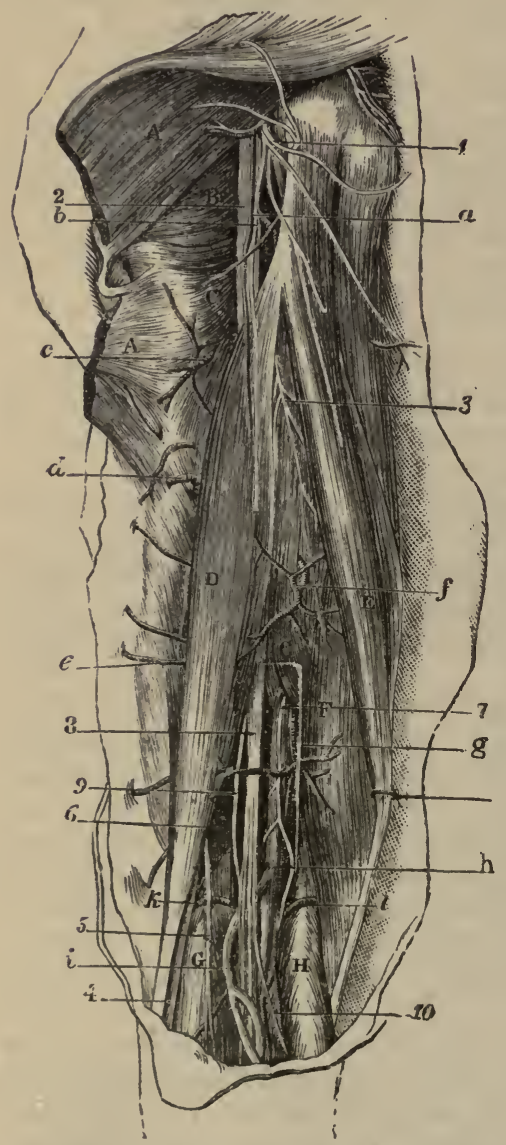

Muscles:

A. Gluteus maximus, cut below, and partly raised.

B. Quadratus femoris.

c. Adductor magnus.

D. Biceps.

E. Semitendinosus.

P. Semimembranosus.

G. Outer, and

H. Inner head of the gastrocnemius.

Arteries :

a. Small sciatic, cut.

b. Ending of internal circumflex to the hamstrings.

c. First, $d$, second, and $e$, third perforating of profunda.

f. Muscular branch of profunda.

g. Popliteal trunk.

$h$. Popliteal vein.

i. Short saphenous vein.

$k$. Upper external, and $l$, upper internal articular artery.

\section{Nerves :}

1. Small sciatic.

2. Large sciatic.

3. Branch to hamstrings from large sciatic.

4. External popliteal; and

5. Communicating peroneal.

7. Articular branch of obturator to knee.

8. Internal popliteal.

9. Articular branch to knee of the internal popliteal.

10. Short saphenous.

DIASECTION OP TAE BACK OP THE THIGH (II] ustrations of Dissections).

teal space. $\Lambda$ s the tendon turns forwards to its insertion, an expansion is continued from it to the faseia of the leg; and it is attached, with the gracilis, below the level of the tubercle of the tibia, the two being separated from the tendon of the sartorins by a bursa (p. 567).

Action. If the leg is movable the musele bends the knee; and continuing to contract, rotates towards the tibia. Supposing the knee-joint straight but the hip-joint bent, the femur can be depressed, and the hip extended by the semitendinosus and the other hamstrings.

Should the limbs be fixed on the ground, the muscle will assist in balancing the pelvis, or in erecting the trunk from a stooping posture.

The semmembraxosus muscle (fig. $203,{ }^{\text {F }}$ ) is tendinous at both ends, and its name is given from the membraniform appearance of the upper 
tendon. 'The muscle is attached above to the highest impression on the back of the tuber ischii, above and external to the semitendinosus and biceps; and it is inserted below into the hinder and inner part of the head of the tibia.

The musele is thick and fleshy inferiorly, where it bounds the popliteal space. On it lies the semitendinosus, which is lodged in a hollow in the upper tendon; and beneath it is the adductor magnus. Along the outerborder lie the great sciatic, and internal popliteal nerves. Between its tendon and the inner head of the gastrocnemius is a large bursa. The insertion of the muscle will be dissected with the knee-joint.

Action. This hamstring is united with the preceding in its action, for it bends the knee and rotates in the tibia; and with the knee straight it will limit flexion of the hip, or extend this joint after the femur has been carried forwards.

When the foot rests on the ground, the semimembranosus acts altogether on the pelvis.

The Great Sciatic Nerve (fig. $203,{ }^{2}$ ) lies on the adductor magnus muscle below the buttock, and divides it into the two popliteal nerves about the middle of the thigh, though its point of bifureation may be earried upwards as far as the pelvis. In this extent the nerve lies along the outer border of the semimembranosus, and is crossed by the long head of the biceps.

Branches. At the upper part of the thigh it supplies large branches to the flexor muscles, and a small one to the adduetor magnus.

Small sciatic nerve (fig. $203,{ }^{1}$ ). Between the gluteus maximus and the ham this small nerve is close beneath the fascia; but it becomes cutaneous below the knee, and accompanies the external saphenous vein for a short distance.

Small cutaneous filaments pierce the fascia of the thigh ; and the largest of these arises near the popliteal space.

Dissection. To see the posterior surface of the adductor magnus, and the branches of the perforating and muscular arteries, the hamstring muscles must be detached from the hip bone and thrown down; and the branches of arteries and nerves they receive are to be dissected out with care. All the parts are to be cleaned.

Adductor magnus muscle (fig. $203,^{\mathrm{c}}$ ). At its posterior aspect the large adductor is altogether fleshy, even at the opening for the femoral artery; and the fibres from the pubic arelı appear to form a part almost distinct from those connected with the tuberosity of the hip bone. In contact with this surface are the hamstring muscles and the great sciatic nerve.

Ending of the perforating arteries (fig. 203, $c, d, e$ ). These branches of the profunda appear through the adduetor magnus elose to the femur, and are directed out through the short head of the biceps and the outer intermuscular septum to the vasti muscles; but as the first branch is placed higher than the attachment of the biceps, it pierces the glutens maximus in its course. In the vasti they anastomose together, and with the descending branches of the external circumflex artery.

Muscular branches are furnished by the perforating arteries to the heads of the biceps; and a cutaneous offset is given by each to the teguments of the outer part of the thigh, along the line of the outer intermuscular septum. 
Muscular branches of the profunda (fig, 203, $f$ ) pierce the adductor magnus internal to the preceding, and at some distance from the femur. Three or four in number, the highest appears about five inches from the pelvis, and the rest in a line at intervals of about two inches from one another: they are distributed to the hamstring muscles, especially the semimembranosus, and communicate below with offsets of the popliteal trunk.

The HIP-JOINT (fig. 204). This articulation is a ball and socket joint, the head of the femur being received into the acetabulum or the cupshaped hollow of the innominate bone. Connecting the bones are the following ligaments:-one to deepen the receiving cavity, which is named cotyloid; another between the articular surfaces of the bones-the interarticular; and a capsule around all.

Dissection. The muscles are to be taken away from the back of the hip-joint, and the upper and lower attachments of the capsular ligament are to be especially cleared from areolar tissue.

Next, the front of the joint should be cleaned and examined in the

Fig. 204.

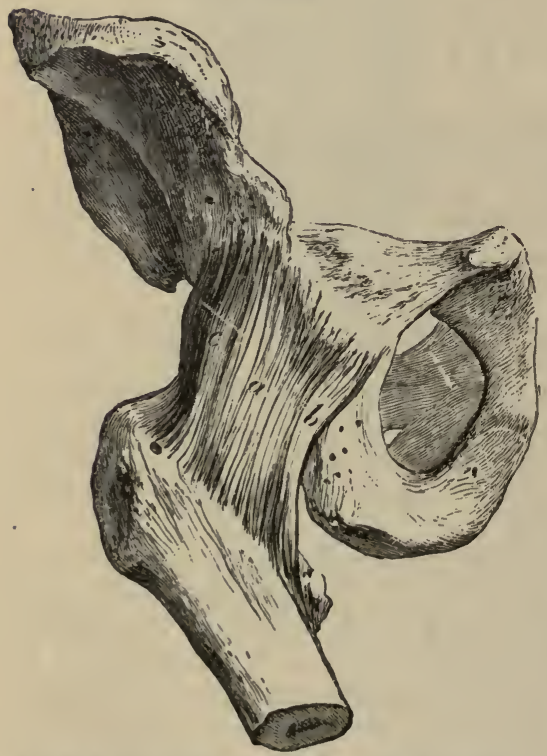

For bart of the CAPSULE OP THE IIIP-JoINT.

a. Illo-femoral ligament.

b. Pubio-femoral edge or band.

c. Ilio-trochauterlc band. same manner, with the body turned over for a short time, if this change in position does not interfere with the other dissections.

In the capsule itself the student has to define a wide thick part in front, and a transverse band near the neck of the femur behind.

The capsular ligament (fig. 204 ) is a thick fibrous case, which is strong enough to check the movements of the joint. Its upper margin is attached to the circumfererence of the acetabulum at a short distance from the edge, as well as to a transverse ligamentous band over the notch at the inner side of the eavity. Its lower margin is inserted in front into the anterior intertrochanteric line (fig. 204); behind, by a very thin piece, into the neck of the femur about a finger's breadth from the small trochanter and the posterior intertrochanteric line (fig. 205); and above, into the neck, near the great trochanter. The capsule differs much in strength, and in the arrangement of the fibres at the fore and hinder surfaces.

On the front it is strengthened by a wide layer of longitudinal fibres (fig. 204, $a, b, c)$. The central portion-the ilio-femoral ligament $(a)$, is fixed above by a narrow piece to the lower anterior iliac spinous process, and below where it widens, into the anterior intertrochanteric line. By its strength it can arrest extension of the joint; and the femur being 
fixed, it will prop the pelvis. The outer edge (e) (ilio-trochanteric band) extends from the hip bone opposite the outer head of the rectus, to the upper and fore part of the great trochanter and neck of the femur; its use is to elheck adduction of the femur. The inner edge $(b)$ (pubio-femoral band) is attached superiorly to the prominent pubic portion of the hip bone inside the acetabulum, and inferiorly to a roughened surface at the lower part of the neck of the femur on a level with, and in front of, the small trochanter : this band controls the abductory movement of the joint.

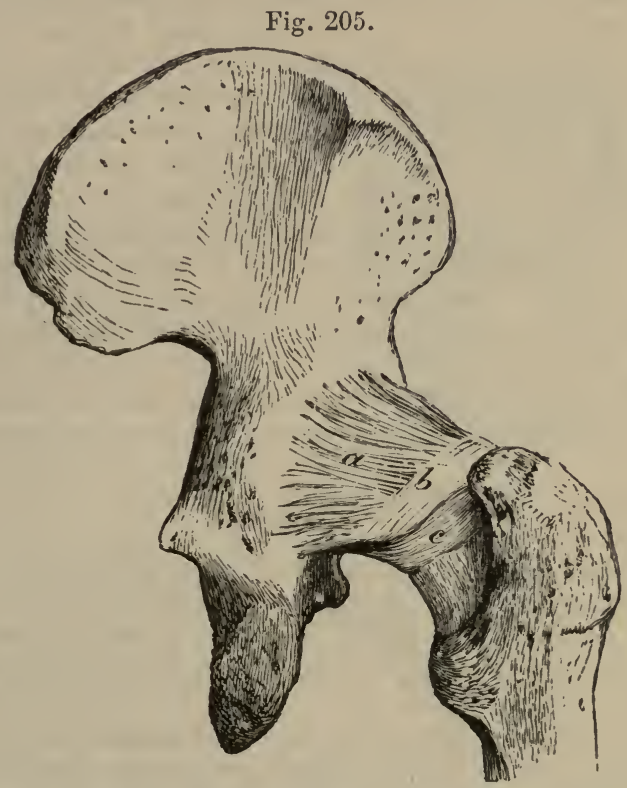

Hinder Part of the Hip-Joint Capgule.

a. Longitudinal fibres.

b. Transverse band. c. Thin piece attached to the neck of the femur about half way down.

At the back of the capsule close to the neck of the femur is a band of transverse fibres (fig. 205, $b$ ), about as wide as the little finger, which arches like a collar over the neck of the bone. By its lower edge it is united to the cervix femoris by a thin layer $(c)$ of fibrous tissue and synovial membrame; at the upper edge it is joined by the longitudinal capsular fibres (1 1 ). It gives insertion to the longitudinal fibres of the capsule, and prevents that restriction of the swinging movement which would result from their insertion into the hinder part of the neck.

Posteriorly the joint is covered by the external rotator muscles; and anteriorly by the psoas and iliacus, a bursa being between it and them. Above is the gluteus minimus, whose tendon is united with the upper and outer band of the capsule; and below is the obturator externus.

Dissection (fig. 206). The eapsular ligament is to be now divided over the prominence of the head of the femur, and this bone being disarticulated but not detached, the cotyloid and interarticular ligaments inside it will appear.

The interarticular or round ligament is attached to the aceiabulum by 
two pieces; and to bring these into view, the synovial membrane and areolar tissue must be removed. The transverse ligament over the notch is also to be defined.

The cotyloid ligament (fig. 191, g) is a narrow band of fibro-cartilage, which is fixed to the margin of the acetabulum, and is prolonged across the notch on the inner side, so as to form part of the transverse ligament. Its fibres are not continued around the acetabulum, but are fixed to the margin of the carity, and cross one another in the band. It is thickest at its attachment to the bone, and becomes gradually thinner towards the free margin, where it is applied to the head of the femur.

This ligament fills up the hollows in the rim of the acetabulum, and deepens the socket for the femur in the same manner as the glenoid ligament increases the surface for the reeption of the head of the humerus.

The transverse ligament (fig. $191, h$ ) is a firm but narrow band, which reaches across the upper part of the notch at the inner side of the acetabulum. It consists partly of deep special fibres $(h)$ which are attached to the margins of the notch; and partly of a superficial bundle from the cotyloid ligament $(g)$. Beneath it is an aperture by which vessels and nerves enter the acetabulum to supply the synovial membrane, and the fat in the bottom of that hollow.

The interarticular or round ligament (fig. 206, b) (ligam. teres) is a slight band about an inch long, connecting the femur with the innominate bone.

One extremity is roundish, and is inserted into the pit in the head of the femur.

The other is flattened, and divides into two parts opposite the transverse ligament. The anterior piece (c) (pubic) is attached with the transverse ligament to the pubic edge of the notch. The posterior part $(d)$ (ischial) is inserted behind the transverse ligament into the ischial border of the cotyloid notch.

Dissection. To see its condition in the different movements of the articulation, it should be examined in a joint in which the capsule is entire, and the bottom of the acetabulum has been eut out with a chisel inside the pelvis.

When the joint is in the extended state, the ligament is generally lax, the two end attachments being near each other; but if the femur is adducted, the ischial part of the ligament is rendered tight because the head of the femur rises.

In flexion of the joint the ligament is tighter than in extension, as the femoral insertion is removed firom the acetabular; and if, in the bent state, the femur be rotated out or adducted, the round ligament will be most stretched.

A synovial membrane lines the capsular ligament, and is continted along it to the acetabulum and the head of the femur. In the bottom of the cotyloid cavity it is reflected over the fat in that situation; and it surrounds the ligamentum teres.

Dissection. To see the surface of the acetabulum the lower limb is to be separated from the trunk by dividing the interarticular ligament, ard by cutting through any parts that connect it to the pelvis: at this stage the pelvic attachments of the round ligament can be better seen.

Surfaces of bone. 'The articular surfaces of the bones are not completely covered with cartilage. 
In the head of the femur is a pit into which the round ligament is inserted.

The acetabulum is coated with cartilage at its circumference, except opposite the notch, and touches the head of the femur by this part : the articular suriace is deep above, but gradually decreases towards the notch.

Within the cartilage and close to the notch, is a mass of fat (fig. 191, $f$ ) corering about one third of the area of the cotyloid cavity, which constitutes the gland of Havers : it communicates with the fat of the thigh beneath the transverse ligament.

Movement. In this ball and socket joint there are the same kinds of movement as in the shoulder, viz., flexion and extension, abduction and adduction, circumduction, and rotation.

Flexion and extension. In the swinging movement flexion is freer than extension, the thigh being capable of such elevation as to touch the belly.

During swinging the head of the femur revolves in the bottom of the acetabulum, rotating around a line corresponding with the axis of the head and neck; and the rapidity and extent of the move. ments do not endanger the security of the joint, the head of the bone not having any tendency to escape.

In flexion, the back of the capsule and the ilio-trochanteric band are put on the stretch; and in extension, the strong ilio and pubio-femoral bands are tightened.

Fig. 206.

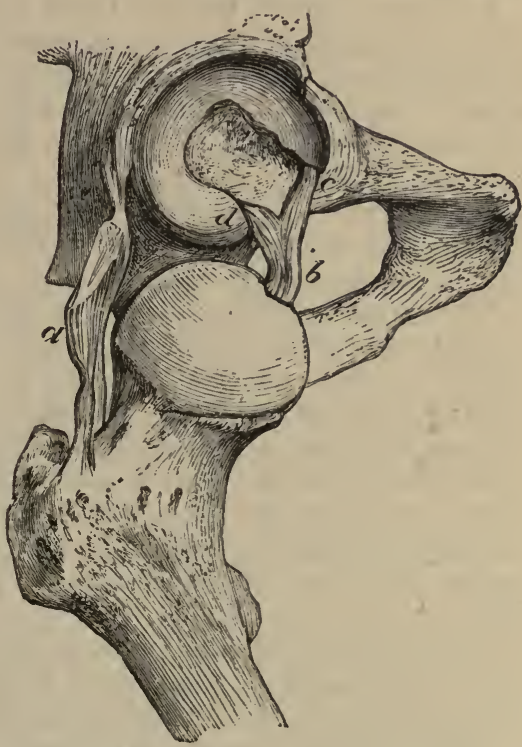

HIP JOINT OPENED, to show the interarticular or round ligament.

a. Part of the capsule.

b. Ligamentum teres: $c$, its pubic, and, $d$, its ischial attachment.

In abduction and adduction the femur is removed from, or brought towards the middle line of the body. Of the two, abduction is the most extensive, because the limb may soon meet its fellow when it is moved inwards, though, if it is carried in front of the other, adduction is considerable.

In both states the head moves in the opposite direction to the shaft. Thus, as the femur is abducted, the head descends, and the greater part of the articular surface projects below the acetabulum; and when the limb is raised to its utmost the great trochanter comes to rest on the margin of the acetabulum, so as to limit farther motion. As the limb descends and approaches the other, the head rises into the socket of the joint, and is securely lodged, finally, in the deepest part of the cavity.

In abduction, the inner band of the capsule is tightened over the projecting head of the femur, the upper part being relaxed. And in adduction, the outer band of the capsule is rendered tense enough to arrest the movement. 
Dislocation may take place in both these lateral movements, the edge of the cotyloid cavity serving as the fulcrum by which the femur can be lifted out of the hollow ; in the one case (adduction) the neck of the femur rests on the brim of the acetabulum, and in the other (abduction) the great trochanter is supported on the margin of the joint-socket. After a dislocation has been reduced, the state of adduetion, with the knees fastened together, is the securest position in which the limb can be placed, inasmuch as the head of the femur then occupies the deepest part of the acetabulum.

In circumduction, the four kinds of angular motion above noticed take place in succession, viz., flexion, abduction, extension, and adduction ; and the limb describes a cone, whose base is at its extremity, and apex at the union of the neck with the shaft of the fenur. This movement is less free than in the shoulder-joint, because of the greater bend between the neck and shaft of the femur.

There are two kinds of rotation, internal and external: in the former, the great toe is turned in; and in the latter, the more extensive of the two, it is moved outwards.

In rotation inwards, the head of the femur rolls backwards horizontally across the acetabulum, the great trochanter being put forwards; and the shaft of the bone revolves around a line inside it, which passes from the head to the inner condyle. During this movement the posterior half of the capsule is put on the streteh, and the anterior is relaxed.

In rotation out the head of the bone rolls forwards across the cotyloid cavity, and the great trochanter is brought backwards, whilst the shaft of the femur moves round the line on its inner side before noticed. The fore part of the capsule is now put on the stretch, and the hinder is rendered loose.

The movement of rotation is destroyed by fracture of the neck of the bone. Its degree is proportioned to the length of the neck, and is therefore greater in the femur than in the humerus.

Use of bend of femur. By means of the angle at the union of the neck with the shaft, the pelvis is more firmly propped than it would be if the neck was in a line with the rest of the femur. It permits also greater surface contact between the head of the femur and the hip bone, since the whole head can be lodged in the cotyloid eavity in progression; and gives greater security to the joint in flexion and extension, for if the neek and shaft of the bone were in a line, only half of the articular surface could enter the socket of the innominate bone in walking, and running. 'The important movement of rotation is also due to this angle; and greater space is obtained through it for the location of the ardductor muscles on the inner side of the femur.

Dissection. After the limb is removed, the attachments of all the museles in the thigh are to be examined more minutely beiore the dissection of the leg is undertaken. The muscles should not be removed from the femur, but about two inches of each should be left for after study. 


\section{SECTION IV.}

THE BACK OF THE LEG.

Directions. Before the dissection of the leg is begun, the student should make himself acquainted, as in the thigh, with the prominences of bone and muscle on the surface, and with the markings which lead to the position of the larger vessels.

Prominences of bone. The bones of the leg can be traced beneath the skin from the knee to the ankle-joint. On the inner side is the tibia, which is subcutaneous in all its extent, and is limited in front and behind by a sharp ridge: above, it presents in front a prominent tubercle into which the ligament of the patella is inserted; and below, it ends on the inner side of the ankle in the internal malleolar projection. On the outer side of the leg the lower half of the fibula may be felt with ease, but the upper half with more difficulty in consequence of the prominence of the muscles of the calf. The head of this bone may be recognized below the knee; and the lower end forms the eminence (malleolus) on the outer side of the ankle joint.

On the side of the ankle joint are the prominent malleoli; and when the joint is extended, the liead of the astragalus can be felt below the tibia.

Muscles and vessels of the leg. On the back of the leg is the swell of the calf: this is formed by the superficial muscles, and from it descends the firm band of the tendo Achillis, by which those muscles are connected with the heel. Between the tendon and the edge of the tibia, but nearest the former, is placed the superficial part of the posterior tibial artery. In front between the tibia and fibula are the flexor muscles of the foot and the extensors of the toes, amongst which the anterior tibial artery lies deeply ; the position of the vessel will be indicated by a line from the centre of the ankle-joint to the inuer side of the head of the fibula.

Prominences of the foot. At the inner border of the foot, about an inch in front of the internal malleolus, is the prominent scaphoid bone pointing out the spot at which un amputation (Chopart's) is practised; whilst one inch and a half farther forwards is a slight depression marking the articulation between the internal cuneiform and the metatarsal bone of the great toe. About the centre of the outer border of the foot is the eminence of the tursal end of the fifth metatarsal bone. A line over the dorsum of the foot, from the centre of the ankle joint to the interval between the inner two toes, will lie over the position of the main artery.

Position. For the dissection of the back of the leg, the limb is to be placed on its front, with the foot over the side of the dissecting-table; and the muscles of the calf are to be put on the stretch by fastening the foot.

Dissection. For the removal of the skin, one cut may be made along the middle of the leg to the sole of the foot, where a transverse incision is to be carried over the heel. The two resulting flaps of skin may be raised, the outer one as far as the fibula, and the other as far as the inner margin of the tibia.

In the fat the cutaneous nerves and vessels are to be followed. On the inner side, close to the tibia, is the internal saphenous vein with the nerve of the same name, together with twigs of the internal cutaneous near the 
knee. In the centre of the leg lies the external saphenous vein, with the small sciatic nerve as its companion above, and the external saphenous nerve below the middle of the leg. On the outer side, in the upper third, cutaneous offsets of the external popliteal nerve will be met with.

The superficial fascia, or the fatty layer of the back of the leg, is least thick over the tibia. Over the line of the superficial vessels it may be separated into two layers.

Superficial Veins. Two veins appear of the dissection of the back of the leg, which are named saphenous-inner and outer.

The internal saphenous vein (fig. 208, d) begins in an arch on the dorsum of the foot. Ascending along the leg in front of the inner ankle, and then behind the inner edge of the tibia, it reaches the thigh (p. 555). In the leg the vein is joined by superficial brauches, and by deep roots from the tibial veins.

The external saphenous vein (fig. 207,c) begins at the outer end of the arch on the dorsum of the foot, and appears below the outer ankle. The vein then courses along the back of the leg to the ham, where it ends in the popliteal vein. It receives large branches about the heel, and other's on the back of the leg, communicating with the internal saphenous. leg.

Cutaneous arteries accompany the superficial veins and nerves of the

Cutaneous Nerves (fig. 207). The nerves in the fat of the back of the leg are prolongations of branches already examined in part, viz. the internal and external saphenous, external popliteal, small sciatic, and internal cutaneous of the thigh.

The internal saphenous nerve (fig. $207,{ }^{7}$ ) accompanies the vein of the same name beyond the knee (p. 573), and terminates at the middle of the inner border of the foot. In the leg the nerve gives off lateral cutaneous offsets, and the outer of these turn over the tibia to the anterior aspect.

The external saphenous nerve $\left(207,{ }^{5}\right)$ is a branch of the internal pop-

- liteal. Perforating the deep fascia about the middle of the leg, it is continued with the external saphenous vein below the outer ankle, and is distributed to the outer side of the foot and little toe. As soon as the nerve enters the fat it is joined by the communicating branch of the external popliteal; and near the heel it gives large and long branches to the integunients.

Cutaneous nerves of the external popliteal. One branch of the external popliteal trunk, viz., communicating peroneal (fig. $207,{ }^{4}$ ), joins the external saphenous nerve about the middle of the leg; but not uncommonly this branch extends as a distinct nerve as far as the heel. One or two other small cutaneous offisets of the external popliteal terminate over the fore part and outer side of the leg in the upper half.

The small sciatic nerve (fig. $207,{ }^{6}$ ) perforates the fascia near the popliteal space, and reaches to about the middle of the leg with the external saphenous vein: it ramifies in the integuments, and joins the external saphenous nerve.

Offset of the internal cutaneous (fig. 207, ${ }^{8}$ ). The inner branch of the internal cutaneous of the thigh (p. 556) extends to the middle of the leg, and communicates with the internal saphenous nerve.

Dissection. The deep fascia will be seen by removing the fat. The superficial vessels and nerves may be either cut or turned aside.

The special or deep fascia on the posterior aspect of the leg covers the muscles, and sends a thick process between the deep and superficial layers. 
Fig. 207.

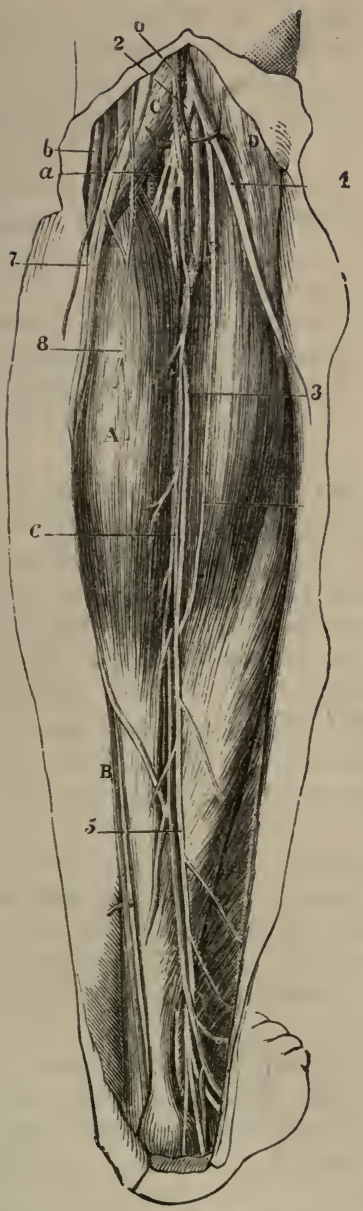

First ViBW OF THE BAC OF THE LEa (Illustrations of Dissections).

Muscles:

A. Gastrocnemius.

B. Soleus.

C. Semimembranosus.

D. Biceps.

Vessels :

$u$. Popliteal artery.

b. Inner saphenous vein.

c. External saphenous vein.

Nerves:

1. External, and 2, internal popliteal.

3. Short saphenous.

4. Communicating peroneal.

5. Common trunk of short saphenous.

6. Sinall sciatic.

7. Internal saphenous.

8. Internal cutaneous (inner) piece.
Fig. 208.

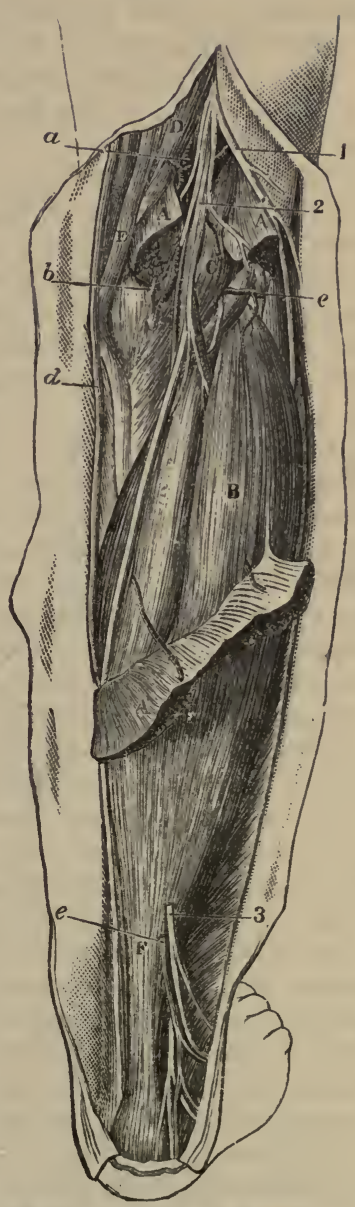

SeCOND TEW OF THE BACK OF THEL"G (Illustrations of Dissections).

Muscles:

A. Gastrocnemius, cut.

B. Soleus.

c. Plantaris.

D. Semimembranosu \&.

R. Semitendinosus.

F. Tendo Achillis.

Vessels:

a. Popliteal artery.

b. Inner lower articular.

c. External lower articular.

d. Internal saphenous vein.

e. External saphenous veín.

Nerves:

1. External popliteal.

2. Internal popliteal.

3. Short saphenous, cut.

4. Cutaneous plantar branch. 
Above, it is continuous with the investing membrane of the thigh, and receives offsets from the tendons about the knee; and below, it joins the two annular ligaments. Externally it is continued uninterruptedly from the one aspect of the limb to the other, but internally it is fixed to the edge of the tibia. Veins are transmitted through it from the deep to the superficial vessels.

Dissection. The fascia is to be divided along the centre of the leg as far as the heel, and is to be taken from the surface of the gastrocnemius muscle. By fixing with a stitch the inner cut head of the gastrocnemius, the fibres of the muscle will be more easily cleaned.

Superficial Layer. of Muscles. In the calf of the leg there are three muscles, gastrocnemius, soleus, and plantaris, which extend the ankle. The two first are large, giving rise to the prominence on the surface, and end below by a common tendon; but the last, inconsiderable in size, is chiefly tendinous.

The Gastrocsemus (fig. 207, ${ }^{\wedge}$ ), the most superficial muscle, is tendinous along the middle, and has above two distinct pieces or heads, which connect it with the condyles of the femur. The inner head of oriqin is attached by a large tendon to an impression at the posterior aspect of the inner condyle, behind the insertion of the adductor magnus, and by fleshy fibres to the line above the condyle. The outer head is fixed by tendon to a pit on the outer surface of the corresponding condyle, above the attachment of the popliteus muscle, and to the upper and back part of the same condyle. The fleshy fibres of the heads are united along the middle line by a narrow thin aponeurosis, and terminate inferiorly with the soleus in the common tendon of insertion.

One surface is covered by the fascia. The other is in contact with the soleus and plantaris, and with the popliteal vessels and the internal popliteal nerve. The heads, by which the muscle arises, assist to form the lateral boundaries of the popliteal space: and the fleshy inner head descends lower than the onter. In the outer head a piece of fibro-cartilage or a sesamoid bone may exist.

Action. When the foot is unsupported, the gastrocnemius extends the ankle; and when the toes rest on the ground, it raises the os calcis and the weight of the body, as in standing on the toes, and in progression.

Taking its fixed point at the os calcis, the muscle draws down the femur so as to bend the knee joint.

Dissection. To see the soleus, the gastrocnemius is to be reflected by cutting across the remaining heal, and the vessels and nerves it receives. After the muscle has been thrown down, the soleus and plantaris must be cleaned.

The soleus (fig. $208,{ }^{\mathrm{B}}$ ) is a large fat muscle, which is attaclied to both bones of the leg. It arises from the head, and the upper third or half of the posterior surface of the shatt of the fibula ; from the oblique line across the tibia, and from the posterior edge of this bone in the middle third; and between the bones from an aponeurotic arch over the large bloodvessels. Its fibres are directed downwards to the common tendon.

'The superficial part of the soleus is in contact with the orastrocnemius ; and the opposed surfaces of the two are aponeurotic. Beneath the soleus lie the bones of the leg, the deep layer of muscles, and the vessels and nerves. 
Tendo Achillis (fig. 208, ${ }^{\mathbf{F}}$ ). The common tendon of the gastrocnemius and soleus is one of the strongest in the body. About three inches wide above, it commences at the middle of the leg, though it receives fleshy fibres on the under surface nearly to the lower end : below it is narrowed, and is inserted into the lower half of the tuber calcis at the posterior aspect. A bursa intervenes between it and the upper part of the tuberosity. The tendon is close beneath the fascia; and lying along its outer side, but superficial to it, are the external saphenous vein and nerve.

Action. In its action on the foot the soleus, like the gastrocnemius, extends the ankle and points the toes when the foot is free to move, and raises the heel if the toes rest on the ground. By the sudden and powerful contraction of the fibres of both muscles, the common tendon is sometimes broken across.

If it acts from the os calcis, it will draw back the bones of the leg into a. vertical position over the foot, as the body is raised to the erect posture after stooping.

The PLANTARIS (fig. $208,{ }^{\mathrm{c}}$ ) is remarkable in having the longest tendon in the body, which takes the appearance of a ribbon when it is stretched laterally. About three-quarters of an inch wide, the muscle arises from the line above the outer condyle of the femur, and from the posterior ligament of the knee-joint; and the tendon is inserted into the os calcis with or by the side of the tendo Achillis, or into the fascia of the leg.

The belly of the muscle, about three inches in length, is concealed by the gastrocnemius, but the tendon appears on the inner side of the tendo Achillis about the middle of the leg. This little muscle crosses the popliteal vessels, and lies on the soleus.

Action. It assists the gastrocnemius to extend the ankle if the foot is not fixed; and to bend the knee-joint if the foot is immovable.

Dissection (fig. 209). The soleus is to be detached from the bones of the leg, and the muscles and nerves entering it are to be divided; but in raising it, the student should take care not to injure the thin deep fascia and the ressels and nerves beneath. The superficial muscles may be next removed by cutting through their tendons near the os calcis; and the bursa between the tendo Achillis and the os calcis should be opened.

The piece of fascia between the muscles of the superficial and deep layers is next to be cleaned; and the integuments between the inner ankle and the heel are to be taken away to lay bare the annular ligament, but a cutaneous nerve to the sole of the foot, which pierces the ligament, is to be preserved.

Deep part of the fascia. This intermuscular piece of the fascia of the leg is fixed to the tibia and fibula, and binds down the deep layer of flexor muscles. Beneath the soleus it is thin and indistinct; but lower in the limb it is much stronger, and is marked by some transverse fibres near the malleoli, which gives it the appearance and office of an annular ligament in that situation. Inferiorly it joins the internal annular ligament between the heel and the inner ankle.

Dissection. The deep layer of muscles (in part), the posterior tibial nerve, and the trunk and offsets of the posterior tibial vessels, will be laid bare by the removal of the fascia and the areolar tissue. A muscle between the bones (tibialis posticus) is partly concealed by an aponeurosis which gives origin to the two muscles (flexor communis and flexor pollicis) 
on the sides; and it will not fully appear until after the membrane covering it has been divided longitudinally, and reflected to the sides.

To prepare the peroneal artery, evert and partly divide the flexor pollicis in which it is contained; then follow branches of it to the fore part of the leg, the outer side of the foot, and to join the posterior tibial artery.

Deer Layer of Muscles (fig. 209). The deep muscles at the back of the leg are four in number, viz., popliteus, flexor longus pollicis, flexor longus digitorum, and tibialis posticus. The first of these is close to the knee-joint ; it crosses the bones, and is covered by a special aponeurosis The flexors lie on the bones, the one of the great toe resting on the fibula, and that of the other toes on the tibia. And the tibialis cover's the interosseous membrane. With the exception of the popliteus, all enter the sole of the foot, and have a fleshy part parallel to the bones of the leg, and a tendinous part beneath the tarsus.

The popliteus (fig. $209^{\wedge}$ ) arises by tendon, within the capsule of the knee-joint, from the fore part of an oblong depression on the outer surface of the external condyle of the femur; and external to the capsule of the joint, some fleshy fibres arise from the posterior ligament. The tendon gives rise to fibres, which are inserted into the tibia above the oblique line on the posterior surface.

The muscle rests on the tibia, and is covered by a fascia derived in great part from the tendon of the semimembranosus muscle: on it lie the popliteal ressels and nerve, and the castrocnemius and plantaris. Along the upper border are the lower articular vessels and nerve of the inner side of the knee; and the lower border corresponds with the attachment of the soleus on the tibia. The origin will be seen with the dissection of the ligaments of the knee-joint.

Action. The leg being free, the muscle bends the knee-joint, and then rotates inwards the tibia. The foot resting on the ground, it will support the knee. According to the view of Theile, it retracts the external semilurar fibro-cartilage.

The FLEXOR LONGuS POLLICIS (fig. $209,{ }^{F}$ ) arises below the soleus from the lower half or two thirds of the posterior surface of the fibula; from the intermuscular septum between it and the peronei muscles; and from the aponeurosis over the tibialis. Inferiorly the tendon of the muscle enters a groove in the astragalus, and crosses the sole of the foot to its insertion into the great toe.

In part the muscle is covered by the soleus; but in part it is superficial, and is in contact with the fascia. It lies on the fibula and lower end of the tibia, and eonceals the peroneal vessels. Along the inner side are the posterior tibial nerve and vessels : and contiguous to the outer margin, but separated by fascia, are the peronei museles.

Action. 'The foot being unsupported, the flexor bends the last phalanx of the great toe, and extends then the ankle.

The foot resting on the ground, the muscle raises the heel ; and it moves back the fibula as the body rises from stooping.

The FLEXOR LONGUS Digitorum (fig. 209, ${ }^{\mathrm{F}}$ ) (flexor perforans) arises from the posterior surface of the tibia, extending from the attachment of the soleus to about three inehes from the lower extremity; and from the aponeurosis covering the tibialis posticus. Its tendon enters a partition in the annular ligament, which is superficial to the sheath of the tibialis ; and it divides in the sole of the foot into tendons for the four outer toes.

'The muscle is narrow and pointed above, and is placed beneath the 
soleus; but in the lower half it is in eontact with the fascia, and the posterior tibial vessels and nerve lie on it. The deep surface rests on the tibia and the tibialis posticus.

Action. The muscle bends the farthest phalangeal joints of the four smaller toes, and then extends the ankle.

Fig. 209.

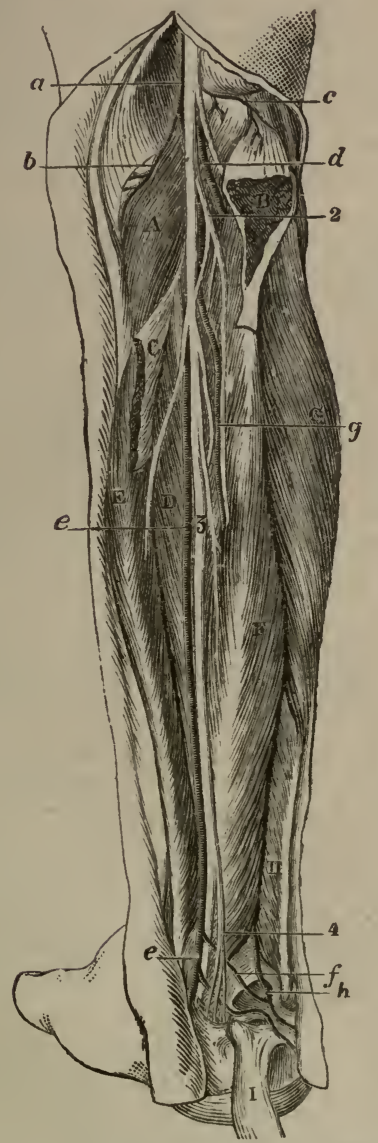

Muscles:

A. Popliteus.

B. Outer, and c, inner part of soleus, cht.

D. Tibialis posticus.

E. Flexor digitorum.

F. Flexor pollicis.

G. Peronens longus.

H. Peroneus brevis.

1. Tendo Achillis.

Arteries :

a. Popliteal.

$b$. Inferior internal, and $c$, inferior external articular.

d. Anterior tibial.

e. Posterior tibial, and $f$, its communicating branch to peroneal.

g. Peroneal artery.

$h$. Contiuuation of peroneal to outer side of the foot.

Nerves :

1. Internal popliteal.

2. Muscular branch of posteriur tibial.

3. Posterior tibial.

4. Cutuneous plantar.

DEBP Dissection OF THE BACK OF THE LFA (Illustrations of Dissections).

If the toes are in contact with the ground, the flexor helps to raise the heel in walking; and to move back the tibia in the act of rising from stooping.

The TIBIAlis posticus (fig. $209,{ }^{D}$ ) occupies the interval between the bones of the leg, but it crosses over the tibia inferiorly to reach the inner side of the foot. The muscle arises from an aponeurosis superficial to it, and from the interosseous membrane, except about one inch below : from an impression along the outer border of the tibia, which reaches from the head of the bone to rather beyond the attachment of the flexor longus digitorum; and from the adjacent inner surface of the shaft of the fibula 
as far down as the lowest fifth. In the lower part of the leg the muscle is directed beneath the flexor digitorum; and its tendon, entering the inner space in the annular ligament, reaches the inner side of the foot to be inserted into the scaphoid and other bones (p. 625).

The tibialis is concealed by the aponeurosis before mentioned, and is orerlapped by the neighboring muscles; but in the lower part of the leg it is placed between the tibia and the long flexor of the toes. On the muscle are the posterior tibial vessels and nerve. The upper part presents two pointed processes of attachment - that to the tibia being the highest-between which the anterior tibial vessels are directed forwards.

Action. Its action on the movable foot is to shorten the inner border by drawing down the scaphoid bone, and to extend the ankle joint. The toes resting on the ground, it will aid the muscles of the calf in raising the heel in the progression of the body.

In standing, the muscle can raise the inner border of the foot with the tibialis anticus, so as to throw the weight of the body on the outer edge.

As the body rises from stooping, the tibialis draws back the bones of the leg, with the soleus.

The aponeurosis covering the tibialis is attached laterally to the bones, but has a defined border inferiorly over the muscle. By one surface it gives origin to the flexors of the toes, and by the other to the tibialis.

The POSTERIor tibial artery (fig. 209,e) is one of the branches resulting from the bifurcation of the popliteal trunk. The vessel extends from the lower border of the popliteus muscle to the lower part of the internal annular ligament, where it ends in two plantar branches for the sole of the foot.

At its origin the artery lies midway between the tibia and fibula, but as it approaches the lower part of the leg it gradually inclines inwards; and at its termination it is placed below the tibia, internal to the centre of the hollow between the heel and the inner ankle.

As far as the middle (in length) of the leg the vessel is concealed by two muscles of the calf, viz., gastrocnemius and soleus; but below that spet, as it lies between the tendo Achillis and the inner edge of the tibia, it is covered only by the teguments and the deep fascia. At its termination it is placed beneath the anuular ligament. For its upper half the trunk lies over the tibialis posticus, but afterwards on the flexor digitorum. and on the lower end of the tibia and the ankle-joint. On the outer side is the flexor pollicis.

Between the heel and the ankle, the artery is placed between the tendons of the common flexor of the digits and special flexor of the great toc.

Venæ comites closely surround the vessel. The posterior tibial nerve is at first internal to the artery ; but at the distance of one inch and a half it crosses to the outer side, and retains that position throughout.

This artery supplies branchies to the muscles and the tibia, and a large peroneal trunk to the outer side of the leg.

a. Muscular branches enter the deep layer of muscles, and the soleus; and an offset from the branch to the soleus pierces the attachment of that muscle to the tibia, and ascends to the knee-joint.

b. A nutritious artery of the shaft of the tibia is uncertain in its place of origin ; penetrating the tibialis, it enters the canal on the posterior surface of the bone, and ramifies in the interior.

c. A communicating branch $(f)$ arises opposite the lower end of the 
tibia, and passes outwards beneath the flexor pollicis, to unite in an arch with a corresponding offset of the peroneal artery.

d. Articular branches arise from the artery opposite the ankle-joint, and enter the articulation.

e. Cutaneous offsets appear through the fascia in the lower half of the leg; the largest of these pierces the annular ligament, and accompanies the cutaneous plantar nerve to the sole of the foot.

Peculiarities. If the posterior tibial artery is smaller than usual, or absent, its deficiencies in the foot will be supplied by a large communicating braneh from the peroneal artery, which is directed inwards at the lower end of the tibia, and either joins the smali tibial vessel, or runs alone to the sole of the foot.

The PERONeal artery (fig. 209, $g$ ) is often as large as the posterior tibial, and arises from that vessel about one inch and a half from the beginning. It takes the fibula as its guide, and lying close to that bone in the fibres of the flexor pollicis, reaches the lower part of the interosseous membrane. At this spot it sends forwards a branch to the front of the leg (anterior peroneal); and it is directed onwards over the articulation between the tibia and fibula $(h)$ to the outer side of the heel, where it terminates in branches, which anastomose with offsets of the posterior tibial, and with the tarsal and external plantar arteries.

Two companion veins surround the artery; and the nerve to the flexor pollicis lies on it generally.

Branches. Besides the anterior peroneal, it furnishes muscular, nutritious, and communicating offsets.

a. Nuscular branches are distributed to the soleus, tibialis posticus, and flexor pollicis; and some turn round the fibula to the peronei muscles, lying in grooves in the bone.

$b$. The uutritious artery is smaller than that to the tibia, and is transmitted throngh the tibialis posticus to the aperture about the middle of the fibula.

$c$. The anterior peroneal branch passes forwards through an opening in the lower part of the interosseous membrane, and is continued to the dorsum and outer part of the foot ; on the fiont of the leg and foot it anastomoses with the external malleolar and tarsal branches of the anterior tibial artery.

d. A communicating offset near the ankle joins in an arch with a similar branch of the posterior tibial. Sometimes there is a second arch between the same vessels.

Peculiarities. The anterior branch of the peroneal may take the place of the anterior tibial artery on the dorsum of the foot.

A compensating prineiple may be observed amongst the arteries of the foot as in those of the hand, by which the deficiency in one is supplied by an enlarged offset of another.

The posterior tibial verss begin on the inner side of the foot by the union of the plantar: they ascend one on each side of the artery, and unite with the anterior tibial at the lower border of the popliteus to form the large popliteal vein. They receive the peroneal veins, and branches corresponding with the offsets of the artery; branches connect them with the saphenous veins.

The posterior tribial NerVe (fig. $209,{ }^{3}$ ) a continuation of the internal popliteal, reaches, like the artery, from the lower border of the popliteus muscles to the interval between the os calcis and the inner malleolus. 
Whilst beneath the anuular ligament, or somewhat higher than it, the nerve divides into the internal and external plantar branches of the foot.

Its connections with surrounding parts are the same as those of the artery ; but its position to the vessel changes, for it lies on the inner side above the origin of the peroneal offset, but thence to the termination, on the outer side. Its branches are muscular and cutaneous.

Muscular branches enter the deep flexors, and arise either separately along the trunk, or together from the upper part of the nerve. 'There is an offset for each of the muscles except the popliteus; but the branch for the tibialis is the largest, and that for the flexor pollicis lies on the peroneal artery.

A cutaneous nerve of the sole of the foot (fig. 209, 4) begins above the os calcis, and piercing the internal annular ligament as two or more pieces, ends in the integuments of the inner and under parts of the heel ; this nerve will be followed to its termination in the dissection of the foot (fig. 210).

The internal annular ligament stretches between the heel and the inner ankle, and serves to confine the tendons of the deep layer of muscles of the foot and toes. Attached by a pointed piece to the internal malleolus, the fibres diverge, and are inserted into the os calcis. One border (upper) is continuous with the fascia of the leg; and the opposite gives attachment to the abductor pollicis muscle of the foot.

Beneath it are sheaths for the tendons. The innermost incloses the tibialis posticus, lodged in a groove in the malleolus. Immediately behind this is another space for the flexor digitorum. And about threequarters of an inch nearer the os calcis is the interval in which the flexor pollicis lies, resting in a groove in the astragalus. Each sheath is lined by a synovial membrane.

Between the tendons of the two flexors of the digits are placed the tibial vessels and nerve.

\section{Sectron V.}

\section{SOLE OF THE FOOT.}

Position. The foot is to be placed over a block of moderate thickness with the sole towards the dissector; and the part is to be made tense by fixing the heel with hooks, and by separating, and by fastening apart the toes.

Dissection. The skin is to be raised as two flaps, inner and outer, by means of one incision along the centre of the sole from the heel to the front; and by another across the foot at the root of the toes. Afterwards the skin is to be removed from each toe, and the digital vesseis and nerves on the sides are to be dissected out at the same time.

In the fat near the heel the student shonld follow the cutaneous nerve of the sole (supra); and he may trace out, at a little distance from each border of the foot, some small branches of the plantar nerves and arteries.

The subcutaneous fat is very abun int. and forms a thick cushion over the parts that press most on the ground in standing, viz., over the os calcis, and the metatarso-phalangeal articulations. 
Dissection. The fat should be now removed, and the plantar fascia laid bare. Beginning the dissection near the heel, follow forwards the fascia towards the toes, to each of which a process is to be traced. In the intervals between those processes the digital nerves and arteries will be detected amongst much fatty and fibrous tissues; but the vessels and nerves to the inner side of the great toe and outer side of the little toe, pierce the fascia farther back than the rest.

The student is next to define a transverse fibrous band between the toes, over the digital vessels and nerves; and when this has been displayed, he may remove the superficial fascia from the toes to see the sheaths of the tendons.

Plantar fascia. The special fascia of the sole of the foot is of a pearly-white color and great strength, and sends septa between the muscles. Its thickness varies in different parts of the foot; and from this circumstance, and the existence of longitudinal depressions over the two chief intermuscular septa, the fascia is divided into a central and two lateral parts.

The central part, which is much the thickest, is pointed at its attachment to the os caleis, but widens and becomes thinner as it extends forwards. A slight depression, corresponding with an intermuscular septum, marks its limit on each side: and opposite the heads of the metatarsal bones it divides into five processes, which send fibres to the teguments near the web of the foot, and are continued onwards to the toes, one to each. Where the pieces separate firom each other, the digital vessels and nerves and the lumbricales muscles become superficial; and transverse fibres arch over them.

If one of the digital processes be divided longitudinally, and its parts reflected to the sides, it will be seen to join the sheath of the flexor tendons, and to be fixed laterally into the margins of the metatarsal bone, and into the transverse metatarsal ligament.

The lateral pieces of the fascia are thinner than the central one. On the inner margin of the foot the fascia has but little strength, and is continued to the dorsum; but on the outer side it is increased in thickness, and presents a strong band between the os calcis and the projection of the fifth metatarsal bone.

Dissection. To examine the septa, a longitudinal incision may be made along the middle of the foot through the central piece of the fascia, and a transverse one near the calcaneum. On detaching the fascia from the subjacent flexor brevis digitorum, by carrying the scalpel from before backwards, the septal processes will appear on the sides of that muscle.

The intermuscular septa pass down on the sides of the flexor brevis digitorum, and a piece of faseia reaches across the foot from the one septum to the other, beneath that flexor, so as to isolate it.

'The inner septum lies between the short flexor and the abductor pollicis; and the internal plantar nerve and vessels, and the tendon of the flexor pollicis longus, pass through it.

The outer partition between the short flexor and the abductor minimi digiti, is pierced by the digital nerve and vessels for the outer side of the little toe.

The superficial transverse ligament crosses the roots of the toes, and is contained in the skin forming the rudimentary web of the foot. It is attached at the ends to the sheath of the flexor tendons of the great and 
little toes, and is connected with the sheath of the others as it passes over. Beneath it the digital nerves and vessels issue.

The sheaths of the flexor tendons (fig. 211, ") are similar to those of the fingers, though not so distinct, and serve to confine the tendons against the grooved bones. The sheath is weak opposite the articulations between the phalanges, but is strong opposite the centre of both the metatarsal and the next phalanx. Each is lubricated by a synovial membrane, and contains the tendons of the long and short flexor muscles.

Dissection (fig. 210). . In the sole of the foot the muscles are numerous, and have been arranged in four layers. To prepare the first layer all the fascia must be taken away; but this dissection must be made with some care, lest the digital nerves and vessels, which become superficial to the central muscle towards the toes, should be injured.

The tendons of the short flexor musele are to be followed to the toes, and one or more of the sheaths in which they are contained should be opened.

First layer of Muscles. In this layer are three muscles, viz., the flexor brevis digitorum, the abductor pollicis, and abductor minimi digiti. The short flexor of the tues lies in the centre of the foot; and each of the others is in a line with the toe on which it acts.

The ABDUCTOR POLLICIS (fig. 210, ${ }^{\mathbf{A}}$ ), the most internal muscle of the superficial layer, takes origin from the inner part of the larger tubercle on the under surface of the os ealeis; from the plantar fascia; and from the lower border of the internal annular ligament, and the inner side of the foot as far as the scaphoid bone. In front the muscle ends in a tendon, which is joined by fibres of the short flexor, and is inserted into the inner side of the base of the metatarsal phalanx of the great toe.

The cutaneous surface of the muscle is in contact with the plantar fascia ; and the other touches the tendons of the tibial muscles, the plantar vessels and nerves, and the tendons of the long flexors of the toes with the accessorius muscle.

Action. This abductor acts chiefly as a flexor of the metatarso-phalangeal joint of the great toe, but it will abduct slightly that toe from the others.

The Flexor BREvis Digitorum (fig. $210,{ }^{\text {B }}$ ) (flexor perforatus) arises posteriorly by a pointed process from the inner part of the larger tubercle of the os calcis, and from the plantar fascia and the septa. About the centre of the foot the muscle ends in four small tendons, which are directed forwards over the tendons of the long flexor, and entering the sheaths of the four smaller toes, are inserted into the middle phalanges. In the sheath of the toe the tendon of this muscle lies at first (in this position of the foot), on the long flexor; opposite the centre of the metatarsal phalanx it is slit for the passage of the other, and is attached by two processes to the sides of the middle phalanx.

The short flexor of the toes is contained in a sheath of the plantar fascia, and occupies the middle of the foot. It conceals the tendon of the long flexor of the toes, the accessory muscle, and the external plantar vessels and nerve.

Action. It bends the nearest phalangeal joint of the four smaller toes, like the flexor sublimis in the upper limb, and approximates the toes at the same time.

The ABDECTor Minmi Digiti (fig. $210{ }^{\circ}$ ) has a wide origin behind from the outer tubercle of the os calcis, from the fore part of the inner 
tubercle, and from the plantar fascia and the external intermuscular septum. It ends anteriorly in a tendon which is inserted into the outer side of the base of the metatarsal phalanx of the little toe.

The muscle lies along the outer border of the foot, and conceals the flexor accessorius, and the tendon of the peroneus longus. On its inner side are the external plantar vessels and nerve. Sometimes a part of the muscle is fixed into the projection of the fifth metatarsal bone.

Action. Though it abducts the little toe from the others, as the name signifies, its chief use is to bend the metatarso-phalangeal joint.

Dissection. To bring into view the second layer or muscles and the plantar vessels and nerves, the muscles already examined must be reflected. Cut through the flexor brevis at the os calcis, and as it is raised, notice a branch of nerve and artery to it. Dividing the abductor minimi digiti near its origin, and turning it to the outer side of the foot, seek its nerve and ressel close to the calcaneum. The abductor pollicis can be drawn aside if it is necessary, but it may remain uncut till afterwards.

Next the internal plantar vessels and nerve are to be followed forwards to their termination, and backwards to their origin; and the external plantar ressels and nerve, the tendons of the long flexors of the toes, the accessory muscle, and the small lumbricales, should be freed from fat.

The plantar arteries (fig. 210) are the terminal branches of the posterior tibial trunk, and supply digital offsets to the toes. They are two in number, and are named external and internal from their relative position in the sole of the foot. Of the two the external is the larger, and forms the plantar arch of arteries.

The internal artery $(b)$ is inconsiderable in size, and accompanies the internal plantar nerve, under cover of the abductor pollicis, as far as the middle of the foot, where it ends in four superficial digital branches. lustrations of Dissections, p. 404.)

Brunches. The artery furnishes muscular branches, like the nerve, to the abductor pollicis and flexor digitorum perforatus, and to the flexor brevis pollicis and the two internal lumbricales. Its superficial digital branches accompany the digital nerves of the internal plantar, and are thus disposed of:-

The first is distributed to the inner side of the foot and great toe; the second lies over the first interosseous space; the third corresponds with the second space; and the fourth is placed over the third space. At the root of the toes the last three join the deeper digital arteries in those spaces.

The extemal artery $(a)$ takes an arched course in the foot, with the concavity of the arch turned inwards. The vessel is first directed outwards across the sole, and then obliquely inwards towards the root of the great toe, so that it crosses the foot twice. In the first half of its extent, viz., from the inner side of the calcaneum to the base of the metatarsal bone of the little toe, the artery is comparatively superficial; in the other half, between the little and the great toe, it lies deeply in the foot, and forms the plantar arch.

Only the first part of the artery is now laid bare; the remaining part, supplying the digital branches, will be noticed after the examination of the third layer of muscles (p. 623).

As far as the metatarsal bone of the little toe, the vessel is concealed by the abductor pollicis and the flexor brevis digitorum; but for a short distance near its termination it lies in the interval between the last muscle 
and the abductor minimi digiti. In this extent it is placed on the os calcis, and the flexor accessorius: and it is accompanied by vena comites, and the external plantar nerve.

Fig. 210.

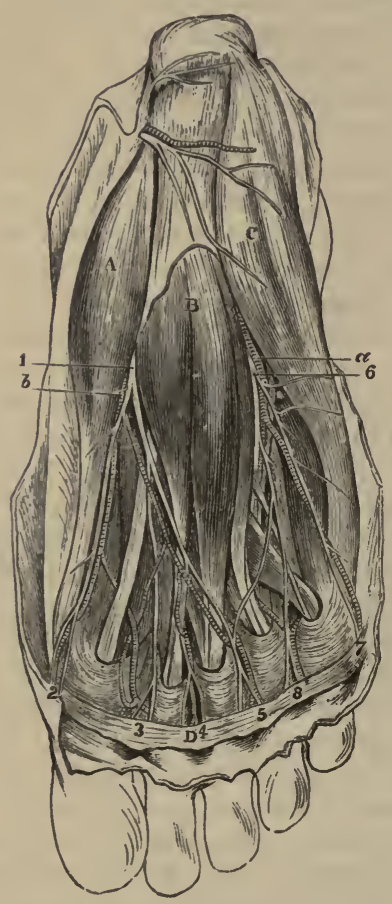

First View OF THE SOLE OF THE FoOT (Illustrations of Dissections).

\section{Muscles :}

A. Abductor pollicis.

B. Flexor brevis digitorum.

c. Abductor minimi digiti.

D. Ligament of the toes.

Arteries:

a. External plantar.

b. Internal plantar.

\section{Nerves:}

1. Internal plantar, with its four branches; $2,3,4$ and 5 , for three toes and a half.

6. External plantar nerve, with two digital branches; 7 and 8 , for one toe and a half.
Fig. 211.

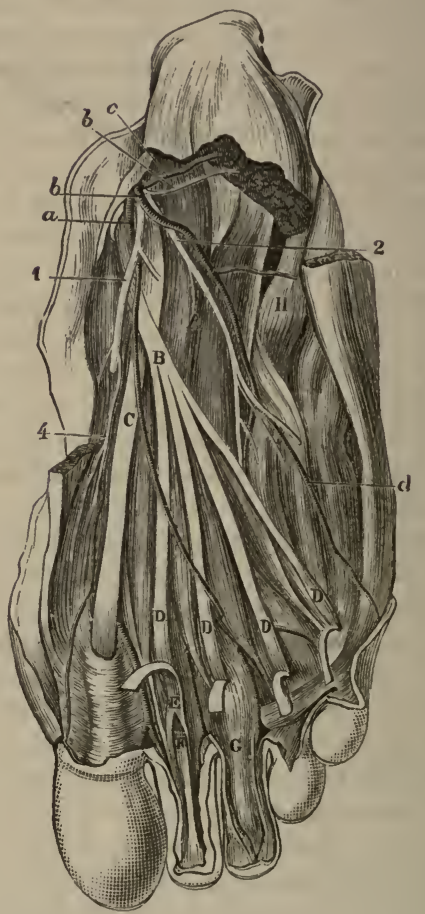

Second View of THE SOLE of the Foot (IIlustrations of Dissections). Muscles :

A. Musculus accessorius.

B. Tendon of flexor digitorum longus.

C. Tendon of flexor longus pollicis.

D. Marks the four lumbricales muscles, but the letters are put ou the tendons of the flexor digitorum perforans.

E. Tendon of flexor perforatus.

r. Tendon of flexor perforans.

a. Sheath of flexor tendons.

н. Tendon of peroneus longus.

Arteries :

a. Internal plantar.

b. External plantar.

c. Branch to abductor minimi digiti.

d. Branch to outer side of little toe.

\section{Nerves :}

1. Internal, and 2, external plantar.

4. Branch to flexor brevis pollicis.

It supplies offsets to the muscles between which it lies, and others to the outer side of the foot for anastomosis with the peroneal artery. 
The plaxtar Nerves (fig. 210) are derived from the bifurcation of the posterior tibial trunk behind the inner ankle. They are two in number, like the arteries, and have the same anatomy as those vessels, for each accompanies a plantar artery; but the larger nerve lies with the smaller bloodvessel.

The interial plantar nerve $\left(^{1}\right)$ courses between the short flexor of the toes and the abductor pollicis, and giving but few muscular offsets, divides into four digital branches $\left({ }^{2},{ }^{3},{ }^{4}, 5\right)$ for the supply of both sides of the inner three toes, and half the fourth; it resembles thus the median nerve of the hand in the distribution of its branches.

Muscular offsets are given by it to the short flexor of the toes (perforatus) and the abductor pollicis; and a few superficial twigs perforate the fasciat.

The four digital nerres have a numerical designation, and the first is nearest th: inner border of the foot. The branch $\left({ }^{2}\right)$ to the inner side of the great toe is undivided, but the others are bifureated at the cleft between the toes.

Muscular branches are furnished by these nerves before they reach the toes; thus, the first (most internal) supplies the flexor brevis pollicis; the second gives a branch to the inner lumbrical muscle, and the third, to the next lumbricalis.

Digital nerves on the toes. Each of the outer three nerves, being divided at the spot mentioned, supplies the contiguous sides of two toes, whilst the first belongs alone to the inner side of the great toe; all give offsets to the teguments, and the cutis beneath the nail, and articular filaments are distributed to the joints as in the fingers.

The external plantar nerve $\left({ }^{6}\right)$ is spent chiefly in the deep muscles of the sole of the foot, but it furnishes digital nerves to both sides of the little toe, and the outer side of the next. It corresponds in its distribution with the ulnar nerve in the hand.

It has the same course as the external plantar artery, and divides at the outer margin of the flexor brevis digitorum into a superficial and a deep portion :- the former gives origin to two digital nerves; but the latter accompanies the arch of the plantar artery into the foot, and will be dissected afterwards.

Whilst the external plantar nerve is concealed by the short flexor of the toes, it gives muscular branches to the abductor minimi digiti and the flexor accessorius.

The digital branches of the external plantar nerve $\left(7,{ }^{8}\right)$ are two. One $\left.{ }^{7}\right)$ is undivided; it is distributed to the outer side of the little toe, and gives offsets to the flexor brevis minimi digiti, and oftentimes to the interosseous muscles of the fourth space. The other $\left({ }^{8}\right)$ bifurcates at the eleft between the outer two toes, and supplies their collateral surfaces: this nerve communicates in the foot with the last digital branch of the internal plantar nerve.

On the sides of the toes the digital nerves have the same distribution as those from the other plantar trunk, and end like them in a tuft of fine branches at the extremity of the digit.

Dissection (fig. 211). To complete the preparation of the second layer of muscles, the origin of the abductor pollicis should be detached from the os calcis, and the muscle should be turned inwards. The internal plantar nerve and artery, and the superficial portion of the external plantar nerve, are to be cut across and thrown forwards; but the external plantar artery 
and the nerve with it are not to be injured. All the fat, and the loose tissue and fascia, are then to be taken away near the toes.

SECONd LAYER OF MUSCles (fig. 211). In this layer are the tendons of the two flexor muscles at the back of the leg, viz., flexor longus digitorum and flexor longus pollicis, which cross one another. Connected with the former, soon after it enters the foot, is an accessory muscle; and at its division into pieces four fleshy slips (lumbricales) are added to it.

The tendon of the FLexor Loxgus Digitorum (fig. 211 , $^{\mathrm{B}}$ ), whilst entering the foot beneath the annular ligament, lies on the internal lateral ligament of the ankle joint. In the foot it is directed obliquely towards the centre, where it is joined by the tendon of the flexor longus pollicis and the accessory muscle, and divides into tendons for the four outer toes.

Fach tendon enters the sheath of the toe with, and beneath a tendon from the flexor brevis, E. About the centre of the metatarsal phalanx the tendon of the long flexor, $\mathrm{F}$, is transmitted through the other, and passes onwards to be inserted into the base of the ungual phalanx. Uniting the flexor tendons with the two nearest phalanges of the toes are ligamentous bands (lig. brevia), one to each, as in the hand; and the one fixing the flexor perforans is anterior ( $p .277$ ).

Action. It flexes the last phalangeal joint, and combines with the short flexor in bending the metacarpo-phalangeal joint. If it acted by itself it would tend to bring the toes somewhat inwards, in consequence of its oblique position in the foot.

'The lumbricales (fig. 211, D) are four small muscles between the tendons of the flexor longus digitorum. Each arises from two tendons, with the exception of the most internal, and this is connected with the inner side of the tendon to the second toe. Each is inserted by a slip into the tibial side of the base of the metatarsal phalanx in the four outer toes, and sends an expansion to the aponeurotic covering on the dorsum of the phalanx. The muscles decrease in size from the inner to the outer side of the foot.

Action. These small muscles will assist the flexors in bending the metatarso-phalangeal joint of the four outer toes; and through their union with the long extensor tendon they will aid that muscle in straightening the two phlangeal joints.

The accessorius muscle (fig. $211^{\wedge}$ ) has two heads of origin :-One is mostly tendinous, and is attached to the under or the outer surface of the os calcis, and to the ligamentum longum plantæ; the other is large and fleshy, and springs from the inner or concave surface of the calcaneum. The fibres end in aponeurotic bands, which join the tendon of the flexor longus digitorum about the centre of the foot, and contribute slips to the pieces of that tendon going to the second, third, and fourth digits (Turner).

The muscle is bifurcated behind, and the heads of origin are separated by the long plantar ligament. On it lie the external plantar vessels and nerve; and the flexor brevis digitorum conceals it.

Action. By means of its offsets to the tendons of certain digits the muscle helps to bend those toes.

And from its position on the outer side and behind the long flexor to which it is united, it will oppose the in ward action of that muscle, and will assist the other flexors in bending the toes direetly batek.

The tendon of the FLExor Loxgus poldicis (fig. $211,^{\circ}$ ) is deeper in the sole of the foot than the flexor longus digitorum; and, directed to the root of the great toe, it enters the digital sheath, to be inserted into the base of the ungual phalanx. It is united to the long flexor tendon by a 
strong tendinous process which, joined by bands of the accessorius, is continued into the pieces of that tendon belonging to the second and third toes (Turner).

Between the calcaneum and the internal malleolus this tendon lies in a groove in the astragalus; and in the foot it occupies a hollow below the inner projection (sustentaculum tali) of the os calcis, being enveloped by a synovial membrane.

Action. For the action of this muscle on the great toe, see page 610 . Through the slip that it gives to the tendons of the flexor longus going to the second and third toes, it may bend those digits with the great toe.

Dissection (fig. 212). For the dissection of the third layer of muscles, the accessorius and the tendons of the long flexor are to be cut through near the calcaneum, and turned towards the toes. Whilst raising the tendons the external plantar nerve and artery are not to be interfered with; and two small nerves and vessels to the outer two lumbricales are to be looked for. Afterwards the areolar tissue is to be taken from the muscles now brought into view.

Third layer of Muscles (fig. 212). Only the short muscle of the great and little toes enter into this layer. On the metatarsal bone of the great toe the flexor brevis pollicis lies, and external to it is the adductor pollicis ; on the metatarsal bone of the little toe is placed the flexor brevis minimi digiti. Crossing the heads of the metatarsal bones is the transversalis pedis muscle.

The fleshy mass between the adductor pollicis and the short flexor of the little toe consists of the interossei muscles of the next layer.

The Flexor brevis pollicis muscle (fig. $212,{ }^{\Delta}$ ) is tendinous and pointed posteriorly, but bifurcated in front. It is attached behind to the inner part of the under surface of the cuboid bcne, and to a prolongation from the tendon of the tibialis posticus. Near the front of the metatarsal bone of the great toe it divides into two heads, which are inserted into the sides of the base of the metatarsal phalanx.

Resting on the muscle at one part, and in the interval between the heads at another, is the tendon of the flexor longus pollicis. The inner head joins the abductor, and the outer is united with the adductor pollicis. A sesamoid bone is developed in the tendon connected with each head.

Action. By its attachment to the first phalanx it flexes the metatarsophalangeal joint of the great toe.

The ADDECTOR pollicis (fig. $212,{ }^{\mathrm{B}}$ ), which is larger than the preceding muscle and external to it, arises from the sheath of the tendon of the peroneus longus, and from the bases of the second, third, and fourth metatarsal bones. Anteriorly the muscle is united with the outer head of the short flexor, and is inserted with it into the base of the metatarsal phalanx of the great toe.

To the inner side is the flexor brevis ; and beneath the outer the external plantar vessels and nerve are directed inwards.

Action. Its first action will be to adduct the great toe to the others, and it will help afterwards in bending the matatarso-phalangeal joint of the toe.

The TrANSversalis PEDIs (fig. $212,{ }^{D}$ ) is placed transversely over the heads of the metatarsal bones. Its origin is by fleshy bundles from the capsule of the metatarso-phalangeal articulations of the four outer toes (firequently not from the little toe), and from the fascia covering the interossei muscles. Its insertion into the great toe is united with that of the adductor pollicis. 
Fig. 212.

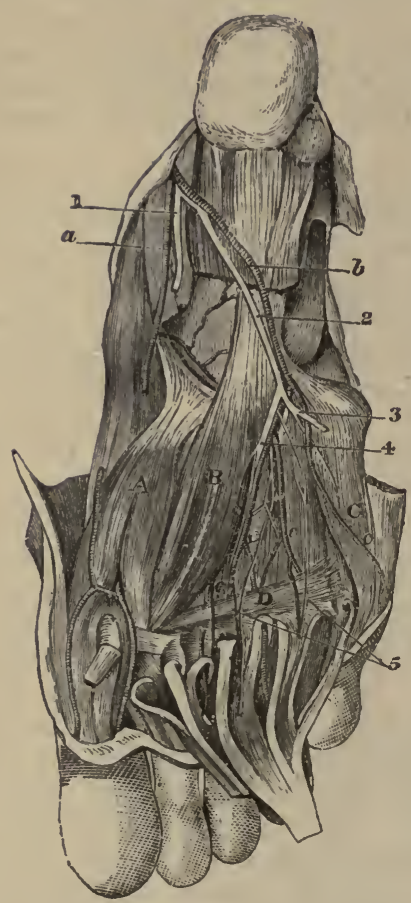

THIRD Vif.W OF THR SOLE OF THE Foot. (Illustrations of Dissections.)

Muscles :

A. Flexor brevis pollicis.

B. Adductor pullicis.

c. Flexor brevis minimi digit1.

D. Transversalis pedis.

Arteries :

a. Internal plantar, cnt.

๖. External plantar; and

c. Its four digital branclies.

Nerves :

1. Internal plantar.

2. External plantar.

3. Its superficial part, cut.

4. The deep part, with the plantar arch.

5. Two offsets to the outer two lumbricales inuscles.
Fig 213.

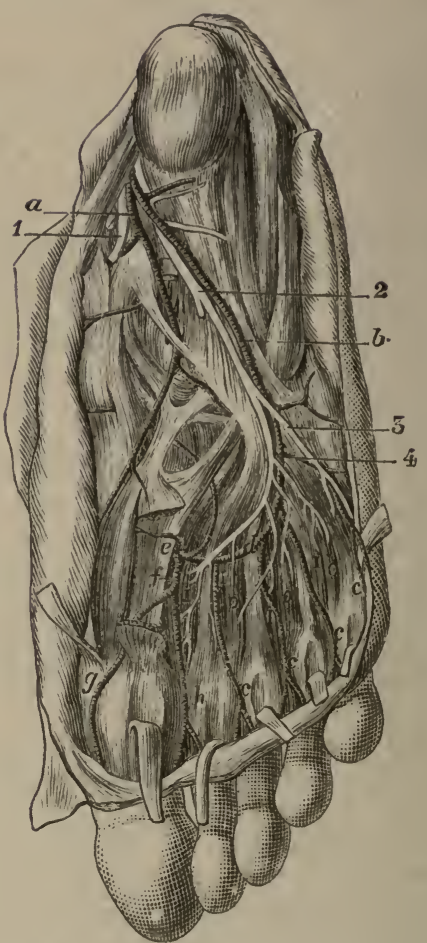

Fockth View of the Soter of the foot. (Illustrations of Dissections.)

\section{Muscles :}

o. Three plantar interossei.

I. Four dorsal interossel.

Arteries :

a. Internal plantar, cut.

b. External plantar.

c. Its four digitul branches.

d. Plantar arch.

$e$. Anterior tiblal entering the sole.

$f$. Arteria mugna pollicis.

s. Branch to inner side of great toe.

$h$. Brauch for the supply of great toe and the next.

Nerves:

1. Internal plantar, eut.

2. External pluntar.

3. Its superficial; und

4. Its deep part, both eut; the latter supplying offsets to the interossel inuscles.

The cutaneous surface is covered by the tendons, and the nerves of the toes; and the opposite surface is in contact with the interossei muscles and the digital vessels.

Action. It will adduct the great toe to the others, and then approximate the remaining toes. 
The Flexor brevis MiNimi Digiti (fig. $212,{ }^{\mathrm{c}}$ ) is a small narrow muscle on the metatarsal bone of the little toe, and resembles one of the interossei. Arising behind from the metatarsal bone and the sheath of the peroneus longus, it blends with the inferior ligament of the metatarsophalangeal articulation, and is inserted into the base of the metatarsal vhalanx of the toe; it is united also by fleshy fibres with the fore part of the metatarsal bone.

Action. Firstly it bends the metatarso-phalangeal joint, and nextly it draws down and adducts the fore part of the fifth metatarsal bone.

Dissection (fig. 2:3). In order that the deep ressels and nerves may be seen, the flexor brevis and adductor pollicis are to be cut through at the posterior part, and thrown towards the toes; but the nerves supplying them are to be preserved. Beneath the adductor lie the plantar arch, and the external plantar nerve, with their branches; and in the first interosseous space is the part of the dorsal artery of the foot that enters the sole. All these ressels and nerves with their branches require careful cleaning.

The muscles projecting between the metatarsal bones are the interossei; the fascia covering them should be removed.

The plaxtar arch (fig. $213, d$ ) is the part of the external plantar artery which reaches firom the base of the metatarsal bone of the little toe to the back of the first interosseous space: internally the arch is completed by a communicating branch from the dorsal artery of the foot $(e)(p .624)$. It is placed across the tarsal ends of the metatarsal bones, in contact with the interossei, but under the flexor tendons, and the adductor pollicis to which it gives many branches.

Vena comites lie on the sides of the artery, and the external plantar nerve accompanies it.

From the front or convexity of the arch the digital branches are supplied, and from the opposite side small nutritive branches arise.

Three small arteries, the posterior perforating, leave the under part: these pass to the dorsum of the foot through the three outer metatarsal spaces, and anastomose with the dorsal interosseous branches of the anterior tibial artery.

The digital branches $(c)$ are four in number, and supply both sides of the three outer toes, and half the next. One to the outer side of the little toe is single; but the others lie over the interossei in the outer three metatarsal spaces, but beneath the transversalis pedis (fig. 212), and bifurcate in front to supply the contiguous sides of two toes. They give fine offsets (fig. 212) to the interossei, to some lumbricales, and the transversalis pedis; and at the point of division they send small communicating branches-anterior perforating, to join the interosseous arteries on the dorsum of the foot.

The first digital runs on the outer side of the little toe, supplying the flexor brevis minimi digiti, and distributes small arteries to the teguments of the outer border of the foot.

The second belongs to the sides of the fifth and fourth toes, and furnishes a branch to the outer lumbrical muscle.

'The third is distributed to the contiguous sides of the fourth and third toes, and emits a branch to the third lumbricalis.

The fourth, or most internal, corresponds with the second interosseous space, and ends like the others on the third and second digits; it may assist in supplying the third lumbricalis. 
The last two digital are joined by superficial digitalis branches of the internal plantar at the root of the toes.

On the sides of the toes the disposition of the arteries is like that of the digital in the hand. They extend to the end, where they unite in an arch, and give off'sets to the sides and ball of the toe: and the artery on the second digit anastomoses with a branch from the anterior tibial artery. Near the front of both the metatarsal and the next phalanx, they form anastomotic loops beneath the flexor tendons, from which the phalangeal articulations are supplied.

The DORSAL ARTERY OF THE FOOT (fig. $213, e$ ) enters the sole at the posterior part of the first (inner) metatarsal space, and ends by inosculating with the plantar arch. By a large digital artery it furnishes branches to both sides of the great toe and half the next, in the same manner as the radial artery in the hand is distributed to one digit and a half.

The digital branch $(f)$ (art. magna pollicis) extends to the front of the first interosseous space, and divides into collateral branches $(h)$ for the contiguous sides of the great toe and the next; near the head of the metatarsal bone it sends inwards, beneath the flexor muscles, a digital branch $(g)$ for the inner side of the great toe.

These arteries have the same arrangement along the toes as the other digital branches; and that to the second digit anastomoses at the end of the toe with a branch of the plantar arch.

External. plantar Nerve (fig. $213,{ }^{2}$ ). The deep branch $\left({ }^{4}\right)$ of this nerve accompanies the arch of the artery, and ends internally in the adductor pollicis. It furnishes branches to all the interossei; to the transversalis pedis; and to the two external lumbrical muscles. The nerve corresponds with the deep portion of the ulnar nerve in the hand.

Dissection. It will be needful to remove the transversalis pedis muscle, to see a ligament across the heads of the metatarsal bones.

The transverse metatarsal ligament is a strong fibrous band, like that in the hand (p. 280), which connects together all the metatarsal bones at their anterior extremity. A thin fascia covering the interossei muscles is connected to its hinder edge. It is concealed by the transversalis pedis, and by the tendons, vessels, and nerves of the toes.

Dissection. 'To complete the dissection of the last layer of museles, the flexor brevis minimi digiti may be detached and thrown forwards. Dividing then the metatarsal ligament between the bones, the knife is to be carried directly backwards for a short distance in the centre of each interosseous space, except the first, in order that the two interossei muscles may be separated from each other. All the interossei are visible in the sole of the foot.

The fascia covering the muscles should be taken away if any remains, and the branches of the external plantar nerve to them should be dissected out.

Fovrti layer of muscles (fig. 213). In the fourth and last layer of the foot are contained the interossei, and the tendons of the tibialis posticus and peroneus longus.

The interossiri Muscles (fig. 213) are situate in the intervals between the metatarsal bones: they consist of two sets, plantar and dorsal, like the interossei in the hand. Seven in number, there are three plantar and four dorsal; and two are found in each space, except the innermost.

The plantar interossei, o, belong to three onter metatarsal bones (fig. 213), and are slender fleshy slips. They arise from the under and inner 
surfaces of those bones; and are inserted partly into the tibial side of the base of the metatarsal phalanx of the same toes, and partly by an expansion from each to the extensor tendons on the dorsum of the phalanx. These muscles are smaller than the dorsal, and are placed more in the sole of the foot.

The dorsal interossei, $\mathrm{I}$, one in each space, arise by two heads from the lateral surfaces of the bones between which they lie; and are inserted like the others into the side, and on the dorsum of the metatarsal phalanx of certain toes: Thus, the inner two muscles belong to the second toe, one to each side; the next appertains to the outer side of the third toe; and the remaining one to the outer side of the fourth toe.

The interossei are crossed by the external plantar artery and nerve, and their digital branches, and lie lieneath the transversalis pedis and the metatarsal ligament. The posterior perforating arteries pierce the hinder extremities of the dorsal set.

Action. Like the interossei of the hand (p. 281) they will contribute to the bending of the metatarso-phalangeal joints by the flexors, and will help the extensors to straighten the last two phalangeal joints.

They can act also as abductors and adductors of the toes. Thus the plantar set will bring the three outer towards the second toe; and the dorsal muscles will abduct from the middle line of the second toe-the two attached to that digit moving it to the right and left of the said line.

Dissection. Following the tendon of the tibialis posticus muscle from its position behind the inner malleolus to its insertion into the scaphoid bone, trace the numerous processes that it sends forwards and outwards. Open also the fibrous sheath of the tendon of the peroneus longus, which crosses from the outer to the inner side of the foot.

The tendon of the TIBIALIS posticus is continued forwards over the internal lateral ligament of the ankle joint, and over the astragalo scaphoid articulation to be inserted into the prominence of the scaphoid bone. From its insertion processes are continued to many of the other bones of the foot: One is directed backwards to the margin of the groove in the os calcis for the tendon of the flexor longus pollicis. Two offsets are directed forwards; one to the internal cuneiform bone; the other, much the largest, is attached to the middle and outer cuneiform, to the os cuboides, and to the bases of the second, third, and fourth metatarsal bones. In other words, pieces are fixed into all the tarsal bones except one (astragalus); and into all the metatarsal bones except two (first and fifth).

Where the tendon is placed beneath the articulation of the astragalus, it contains a sesamoid bone, or fibro-cartilage.

'The tendon of the PERoNeus LONGUS MUSCLE winds round the cuboid bone, and is continued inwards in the groove on the under surface, to be inserted into the internal cuneiform bone, and the base of the metatarsal bone of the great toe; and sometimes by a slip into the base of the second metatarsal bone.

In the sole of the foot (fig. 213), it is contained in a sheath which is crossed, towards the outer part, by the fibres of the long plantar ligament prolonged to the tarsal ends of the third and fourth metatarsal bones; but it is formed internally only by areolar tissue. A separate synovial membrane lubricates the sheatl.

Where the tendon turns round the cuboid bone it is thickened, and contains fibro-cartilage or a sesamoid bone. 


\section{Section VI. \\ THE FRONT OF THE LEG.}

Position. The limb is to be raised to a convenient height by blocks beneath the knee, and the foot is to be extended in order that the muscles on the front of the leg may be put on the stretch.

Dissection. 'To enable the dissector to raise the skin from the leg and foot, one incision should be made along the middle line from the knee to the toes, and this should be intersected by cross cuts at the ankle and the root of the toes.

After the flaps of skin are reflected, the cutaneous vessels and nerves are to be looked for. At the upper and inner part of the leg are some filaments from the great saphenous nerve; and at the outer side others, still smaller, from the external popliteal nerve. Perforating the fascia in the lower third, on the anterior aspeet, is the musculo-cutaneous nerve, whose branches should be pursued to the toes.

On the dorsum of the foot is a venous arch, which ends laterally in the saphenous veins. On the outer side is the external saphenous nerve; and about the middle of the instep the internal saphenous nerve ceases. In the interval between the great toe and the next is the cutaneous part of the anterior tibial nerve.

The digital nerves should be traced to the ends of the toes by removing the integuments: and after the several vessels and nerves are dissected, the fat is to be taken away, in order that the fascia may be seen.

The venous arch on the dorsum of the foot has its convexity turned forwards, and receives digital branches from the toes; at its concavity it is joined by small veins from the instep. Internally and externally it unites with the saphenous veins.

The internal saphenous vein begins at the inner side of the great toe, and in the areh. It ascends along the inner side of the foot, and in front of the inner ankle to the inside of the leg (p.606). Branches enter it from the inner side and sole of the foot.

The external saphenous vein begins on the outside of the little toe and foot, as well as in the venous areh; and it is continued below the outer ankle to the back of the leg (p. 606).

Cutaneous Nerves (fig. 214). The superficial nerves on the front of the leg and foot are derived mainly from branches of the popliteal trunks, viz., from the musculo-cutaneous and anterior tibial nerves of the external popliteal, and from the external saphenous nerve of the internal popliteal. Some inconsiderable offsets ramify on the sides of the leg from the internal saphenous and external popliteal.

The musculo-cutaneous nerve $\left({ }^{2}\right)$ ends on the dorsum of the foot and toes. Perforating the fascia in the lower third of the leg with a cutaneous artery, it divides into two prineipal branches (inner and outer), which give dorsal digital nerves to the sides of all the toes, except the outer part of the little toe and the contiguous sides of the great toe and the next. The branches may be traced in the integument as far as the end of the last phalanx.

The inner branch $\left({ }^{3}\right)$ communieates with the internal saphenous nerve, 
and supplies the inner side of the foot and great toe: it joins also the anterior tibial nerve.

The outer branch $\left({ }^{4}\right)$ divides into three nerves; these lie over the three outer interosseous spaces, and bifurcate at the web of the foot for the contignous sides of the four toes corresponding with those spaces; it joins the external saphenous nerve on the outer part of the foot.

The anterior tibial nerve (') becomes cutaneous in the first interosseous space, and is distributed to that space, and to the opposed sides of the great toe and the next. The musculo-cutaneous nerve joins it, and sometimes assists in supplying the same toes.

The external saphenous nerve (fig. $207,{ }^{5}$ ) comes from the back of the leg below the outer ankle, and is continued along the foot to the outside of the little toe; all the outer margin of the foot receives nerves from it, and the offsets towards the sole are larger than those to the dorsum. Occasionally it supplies both sides of the little toe and part of the next.

Internal saphenous nerve $\left({ }^{5}\right)$. A part of this nerve is continued along the vein of the same name to the middle of the instep, where it ceases mostly in the integuments, but some branches pass through the deep fascia to end in the tarsus.

The fascia of the front of the leg is thickest near the knee joint, where it gives origin to muscles. It is fixed laterally into the tibia and fibula. Intermuscular septa are prolonged from the deep surface; and one of these, which is attached to the fibula, separates the muscles on the front from those on the outer side of the leg. Superiorly the fascia is connected to the heads of the leg bones, but inferiorly it is continued to the dorsum of the foot.

Above and below the ankle joint it is strengthened by some transverse fibres, and gives origin to the two parts of the anterior annular ligament ;

Fig. 214.

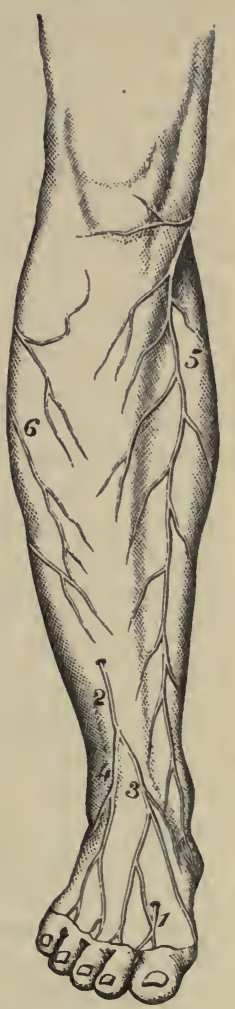

Cutankous Nerves of the FRONT OF THE LEG AND Foot.

1. Anterior tibial.

2. Musculo-cutaneous, with 3, its inner, and 4 , its outer piece; the usual distribution is not shown in the cut.

5. Internai saphenous.

6. Offsets of external popliteal. and below the end of the fibula it forms another band, the external annular ligament.

Dissection. The fascia is to be removed from the front of the leg and the dorsum of the foot, but the thickened band of the annular ligament above and below the end of the tibia is to be left. In separating the fascia from the subjacent muscles, let the edge of the scalpel be directed upwards.

In like manner the fascia may be taken from the peronei museles on the outside of the fibula, but without destroying the band (external annular ligament) below that bone.

On the dorsum of the foot the dorsal ressels with their nerve are to be displayed, and the tendons of the short and long extensors of the toes are 
to be traced to the ends of the digits. In the leg the anterior tibial nerve and vessels are to be followed from the dorsum into their intermuscular space, and are then to be cleaned as high as the knee.

The anterior annular ligament consists of two parts, upper and lower, which confine the muscles in their position : the former serving to bind the fleshy parts to the bones of the leg, and the latter to keep down the tendons on the dorsum of the foot :-

The upper part (fig. $215,{ }^{3}$ ), above the level of the ankle-joint, is attached laterally to the bones of the leg; it possesses one sheath with synovial membrane for the tibialis anticus.

The lower part is situate in front of the tarsal bones. It is inserted externally by a narrow piece into the upper surface of the os calcis, in front of the interosseous ligament; and internally, where it is thin and widened, into the plantar fascia and the inner malleous. In this piece of the ligament there are three sheaths : an inner one for the tibialis anticus ; an outer for the extensor longus digitorum and peroneus tertius; and an intermediate one for the extensor pollicis. Separate synovial membranes line the sheaths.

'The external annular ligament is placed below the fibula, and is attached on the one side to the outer malleolus, and on the other to the os calcis. Its lower edge is connected by fibrous tissue to the sheaths of the peronei muscles on the outer side of the os calcis. It contains the two lateral peronei muscles in one compartment; and this is lined by a synovial membrane, which sends two offsets below into the sheaths of the peronei muscles.

The MUSCles oN the FRONT OF THE LEG (fig. 215) are three in number. The large muscle next the tibia is the tibialis anticus; that next the fibula, the extensor longus digitorum; whilst a small muscle, apparently the lower part of the last, with a separate tendon to the fifth metatarsal bone, is the peroneus tertius. The muscle between the tibialis and extensor digitorum, in the lower half of the leg, is the extensor pollicis.

On the dorsum of the foot only one muscle appears, the extensor brevis digitorum.

The tibialis antices (fig. $215,{ }^{1}$ ) reaches the tarsus: it is thick and fleshy in the upper, but tendinous in the lower part of the leg. It arises from the outer tuberosity and the upper lalf or more of the tibia; from the contiguous part of the interosseous ligament; and from the fascia of the leg and the intermuscular septum between it and the next muscle. Its tendon begins below the middle of the leg, and passes through compartments in the pieces of the annular ligament, to be inserted into the internal cuneiform bone, and the metatarsal bone of the great toe.

The muscle is subaponeurotic. It lies at first outside the tibia, resting on the interosseous membrane, but it is then placed, successively, over the end of the tibia, the ankle-joint, and the inner tarsal bones. The outer border touches the extensor muscles of the toes, and conceals the anterior tibial vessels.

Action. Supposing the foot not fixed, the tibialis bends the ankle, moves the great toe towards the middle line of the body, and raises the inner border of the foot.

If the foot is fixed it can lift the inner border with the tibialis posticus, and support the foot on the outer edge.

If the tibia is slanting backwards, as when the advanced limb reaches the ground in walking, it can bring forwards and make steady that bone. 
The EXTENSOR PROPRIUS POLlicis (fig. 215) is deeply placed at its origin hetween the former muscle and the extensor longus digitorum, but its tendon becoms superfieial on the dorsum of the foot. The muscle arises from the middle three-fifths of the narrow anterior part on the inner surface of the fibula, and from the interosseous ligament for the same distance. At the ankle it ends in a tendon, which comes to the surface through a sheath in the lower piece of the annular ligament, and continues over the inner part of the tarsus to be inserted into the base of the last phalanx of the great toe.

The anterior tibial vessels lie on the inner side of the muscle as low as the sheath in the ligament, but afterwards on the outer side of its tendon, so that they are crossed by it beneath the ligament.

Action. It straightens the great toe by extending the phalangeal joints, and afterwards bends the ankle.

When the foot is fixed on the ground and the tibia slants backwards, the muscle can draw forwards that bone.

The extexsor longus digitorum (fig. $215,{ }^{2}$ ) is fleshy in the leg, and tendinous on the foot, like the tibial muscle. Its origin is from the head and three-fourths of the narrow part of the inner surface of the fibula; from the external tuberosity of the tibia, and about an inch (above) of the interosseous membrane; and from the fascia of the leg and the intermuseular septum on each side. The tendon enters its sheath in the annular ligament with the peroneus tertius, and divides into four pieces. Below the ligament these slips are continued to the four outer toes, and are inserted into the middle and ungual phalanges:-

On the metatarsal phalanx the tendons of the long and short extensor join with prolongations from the interossei and lumbricales to form an aponeurosis; but a tendon from the short extensor is not united to the expansion on the little toe. At the further end of this phalanx the aponeurosis is divided into three parts - a central and two lateral ; the central piece is inserted into the base of the middle phalanx, while the lateral unite at the front of the middle, and are fixed into the ungual phalanx.

In the leg the muscle is placed between the peronei on the one side, and the tibialis anticus and extensor proprius pollicis on the other. It lies on the fibula, the lower end of the tibia, and the ankle-joint. On the foot the tendons rest on the extensor brevis digitorum; and the vessels and nerve are internal to them.

Action. The muscle extends the joints of the four outer toes from root to tip, as in the fingers; and still acting, bends the ankle-joint.

If the tibia is inclined back, as when the foot reaches the ground in walking, it will be moved forwards by this and the other museles on the front of the leg.

The peroneus tertius is situate below the extensor longus digitorum, from which it is seldom separate. It arises from the lower fourth of the narrow part of the inner surface of the fibula, from the lower end of the interosseous ligament, and from the intermuseular septum between it and the peroneous brevis musele. And it is inserted into the tarsal end (upper surface) of the metatarsal bone of the little toe.

This muscle has the same conneetions in the leg as the lower part of the long extensor, and is contained in the same space in the annular ligament.

Action. The muscle assists the tibialis in bending the ankle, and in 
drawing forwards the fibula when the leg is advanced to make a step in walking.

The anterior tibjal artery (fig. $215,{ }^{4}$ ) extends from the bifureation of the popliteal trunk to the front of the ankle joint. At this spot it becomes the dorsal artery of the foot.

Fig. 215.

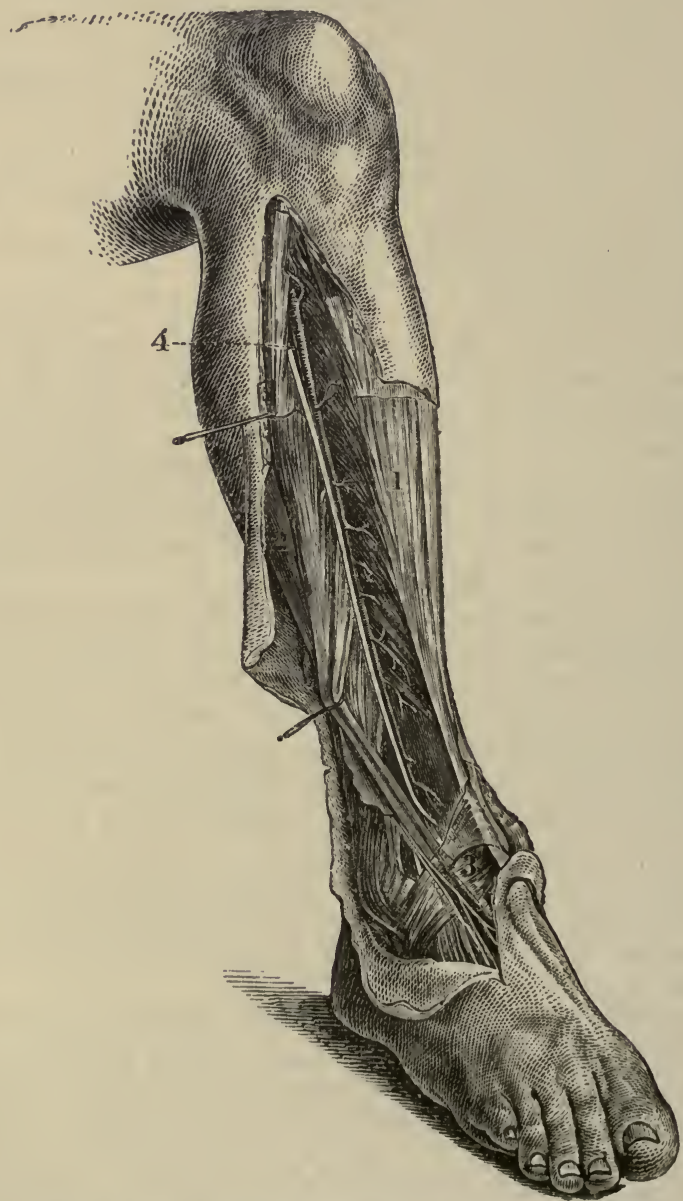

Anterior Tibial Vesszl and Múcles (Quain's Artęries).

1. Tibialus anticns muscle.

2. Extensor pollicis and extensor longus digitorum drawn aside.
3. Part of anterior annular ligament.

4. Anterior tibial artery : the nerve outside it is the anterior tibial.

The course of the artery is forwards through the aperture in the upper part of the interosseous membrane, along the front of that membrane, and over the tibia to the foot. A line from the inner side of the head of the fibula to the centre of the ankle will mark the position of the vessel.

For a short distance (about two inches) the artery lies between the 
tibialis anticus and the extensor longus digitorum; afterwards it is placed between the tibial muscle and the extensor proprius pollicis till near the lower end, where the last muscle becomes superficial, and erosses to the inner side. The vessel rests on the interosseous membrane in two-thirds of its extent, being overlapped by the fleshy bellies of the contiguous muscles, so that it is at some depth from the surface; but it is placed in front of the tibia and the ankle joint in the lower third, and is comparatively superficial between the tendons of the muscles.

Venæ conites entwine around the artery, covering it very closely with cross branches on the upper part. The anterior tibial nerve approaches the tibial vessels about the middle third of the leg, and continues with them, crossing once or twice : at the lower end of the artery the nerve lies on the outer side.

Branches. In the leg the anterior tibial artery furnishes mostly muscular offsets, but near the knee and ankle the following named branches take origin.

a. Cutaneous branches arise at intervals; and the largest accompanies the musculo-cutaneous nerve, and supplies the contiguous muscles.

b. A recurrent branch arises as soon as the trunk appears above the interosseous menbrane, and ascends in the tibialis anticus to the knee joint : on the joint it anastomoses with the other articular arteries.

c. Malleolar arteries (internal and external) spring near the ankle joint, and are distributed over the ends of the tibia and fibula. The internal is the least regular in size and origin; the external anastomoses with the anterior peroneal artery.

$d$. Some small articular branches are supplied from the lower end of the artery to the ankle joint.

The DORSAL ARTERY of the foot is the continuation of the anterior tibial, and extends from the front of the ankle joint to the posterior part of the first interosseous space: at this interval it passes downwards between the heads of the interosseous muscle, to end in the sole (p. 624).

The artery is supported by the inner row of the tarsus, viz., the astragalus, and the scaphoid and cuneiform bones; and it is covered by the integuments and the deep fascia, and by the inner piece of the extensor brevis musele. The tendon of the extensor pollicis lies on the inner side, and that of the extensor longus digitorum on the outer side, but neither is near the vessel.

The veins have the same position with respect to the artery as in the leg; and the nerve is external to it.

Peculiarities. On the dorsum of the foot the artery is often removed further outwards than the line from the centre of the ankle to the posterior part of the first interosseous space. Further, the place of the artery may be taken by a large anterior peroneal branch.

Branches. Offsets are given to the bones and ligaments of the foot: those from the outer side of the vessel are named tarsal and metatarsal from their distribution. A small interosseous branch is furnished to the first metatarsal space.

a. The tarsal branch arises opposite the scaphoid bone, and runs beneath the extensor brevis digitorum to the outer side of the foot, where it divides into twigs that inosculate with the metatarsal, plantar, and anterior peroneal arteries: it supplies offsets to the extensor muscle beneath which it lies. 
b. The metatarsal branch takes an arched course to the outer part of the foot, near the base of the metatarsal bones and beneath the extensor muscle, and anastomoses with the external plantar and tarsal arteries.

$c$. From the arch of the metatarsal branch three dorsal interosseous arteries are furnished to the three outer metatarsal spaces; and the external of these sends a branch to the outer side of the little toe. They supply the interossei muscles and divide at the cleft of the toes into two small dorsal digital branches.

At the fore part of the metatarsal space each interosseous branch joins a digital artery in the sole of the foot by means of the anterior perforating twig; and from the beginning of each a small branch, posterior perforating, descends to the plantar arch.

$d$. The first interosseous branch (art. dorsalis pollieis) arises from the trunk of the artery as this is about to leave the dorsum of the foot; it extends forwards in the space between the first two toes, and is distributed by dorsal digital pieces like the other dorsal interosseous offsets.

'The anterior tibial veins have the same extent and connections as the vessel they accompany. They take their usual position along the artery, one on each side, and form loops around it by cross branches; they end in the popliteal vein. 'The branches they receive correspond with those of the artery; and they communicate with the internal saphenous vein.

Dissection. To exannine the extensor brevis digitorum on the dorsum of the foot, cut through the tendons of the extensor longus and peroneus tertius below the annular ligament, and throw them towards the toes. The hinder attachment of the muscle to the os calcis is to be defined.

The EXTENSOR BREVIS DIGITORUM urises from the outer surface of the os calcis in front of the sheath for the peroneus brevis muscle, and from the lower band of the anterior annular ligament. At the back of the metatarsal bones the musele ends in four tendons, which spring from as many fleshy bellies, and are inserted into the four inner toes. The tendon of the great toe has a distinct attachment to the base of the metatarsal phalanx; but the rest are united to the outer side of the long extensor tendons, and assist to form the expansion on the metatarsal phalanx (p. 629).

The muscle lies on the tarsus, and is partly concealed by the tendons of the long extensor. Its inner belly crosses the dorsal artery of the foot.

Action. Assisting the long extensor, it straightens the four inner toes, separating slightly from each other.

Dissection. The branches of artery and nerve which are beneath the extensor brevis will be laid bare by dividing that muscle near its front, and turning it upwards.

By cutting through the lower band of the annular ligament over the tendon of the extensor pollicis, and throwing outwards the external half of it,-the different sheaths of the ligament, the attachment to the os calcis, and the origin of the extensor brevis digitorum from it may be observed.

The anterior tibial and musculo-cutaneous nerves are now to be followed upwards to their origin from the external popliteal : and a small branch to the knee-joint from the same source is to be traced through the tibialis anticus.

Nerves to the front of the leg. Between the fibula and the peroneus longus muscle the external popliteal nerve divides into recurrent articular, musculo-cutaneous, and anterior tibial branches. 
The recurrent articular branch is small, and takes the course of the artery of the same name through the tibialis anticus muscle to the knee joint.

The musculo-cutaneous nerve is continued between the extensor longus digitorum and the peronei muscles to the lower third of the leg, where it pierces the fascia, and is distributed to the dorsum of the foot and the toes (p. 626). Before the nerve becomes cutaneous it furnishes branches to the two larger peronei muscles.

The anterior tibial nerve (fig. 215) (interosseous) is directed beneath the extensor longus digitorum, and reaches the tibial artery about the middle third of the leg. From this spot it takes the course of the vessel along the foot to the first interosseous space (p. 627). In the leg it crosses the anterior tibial vessels once or more, but on the foot it is generally external to the dorsal artery.

Branches. In the leg the nerve supplies the anterior tibial muscle, the extensors of the toes, and the peroneus tertius. On the dorsum of the foot it furnishes a considerable branch to the short extensor; this is enlarged, and gives offsets to the articulations of the foot.

Muscles on the outer part of the leg (fig. 209). 'Two muscles occupy this situation, and are named peronei from their attachment to the fibula ; they are distinguished by the terms longus and brevis. Intermuscular processes of fascia, which are attached to the fibula, isolate these muscles from others.

The Peroneus Longus (fig. $209,{ }^{G}$ ), the more superficial of the two muscles, passes into the sole of the foot round the outer border. It arises from the head, and the anterior surface of the shaft of the fibula for twothirds of the length, gradually tapering downwards ; from the external border nearly to the malleolus; and from the fascia and the intermuscular septa. Inferiorly it ends in a tendon which is continued through the external annular ligament, with the peroneus brevis, lying in the groove at the back of the external malleolus; and it passes finally in a separate sheath, below the peroneus brevis along the side of the calcis and through the groove in the outer border of the cuboid bone, to the sole of the foot. Its position in the foot, and its insertion are described before (p. 625).

In the leg the muscle is immediately beneath the fascia, and lies on the peroneus brevis. Beneath the annular ligament it is placed over the middle piece of the external lateral ligament of the ankle with the peroneus brevis, and is surrounded by a single synovial membrane common to both. The extensor longus digitorum and the soleus are fixed to the fibula laterally with respect to it, one being on each side.

Action. With the foot free the muscle extends the ankle: then it can depress the inner, and raise the outer border of the foot in the movement of eversion.

When the foot rests on the ground it assists to lift the os calcis, and the weight of the body as in standing on the toes, or in walking. And in rising from a stooping posture it draws back the fibula.

The PERoneus Brevis (fig. $209,{ }^{\text {H}}$ ) reaches the outer side of the foot, and is smaller than the preceding muscle, and inferior in position to it. It arises from the anterior surface of the shaft of the fibula for about the lower two-thirds, extending upwards by a pointed piece internal to the other peroneus; and from the intermuscular septum in front. Its tendon passes with that of the peromeus longus through the external annular ligament, and is placed next the fibula as it turns below this bone. Escaped 
from the ligament, the tendon enters a distinet fibrous sheath, which conducts it along the tarsus to its insertion into the projection at the base of the metatarsal bone of the little toe.

In the leg the muscle reaches in front of the peroneus longus. On the outer side of the os calcis it is contained in a sheath above the tendon of the former muscle; and each sheath is lined by a prolongation from the common synovial membrane behind the outer ankle.

Action. If the foot is unsupported this peroneus extends the ankle, and moves the foot upwards and outwards in eversion.

Like the long muscle, it is able if the foot is supported to raise the heel, and to bring back the fibula as the body rises from stooping.

\section{Section VII.}

\section{LIGANENTS OF THE KNEE, ANKLE, AND FOOT.}

Directions. In examining the remaining articulations of the limb, the student may take first the knee-joint, unless this has become dry; in that case the ligaments of the leg, ankle-joint, and foot may be dissected whilst the knee is being moistened.

Dissection. For the preparation of the ligaments of each articulation, it is sufficient to detach the muscles and tendons from around it, and to remove the areolar tissue or fibrous structure which may obseure or conceal the ligamentous bands. In the knee a kind of aponeurotic capsule is to be defined on the front of the joint.

Some tendons, namely, those of the biceps, popliteus, adductor magnus, and semimembranosus, are to be followed to their insertion, and a part of each is to be left.

Articulations of the knee. The knee is the largest hinge joint in the body, and is formed by the contiguous ends of the tibia and femur, with the patella. The articular surfaces of the bones are covered with cartilage, and are maintained in apposition by strong and numerous ligaments.

The capsule (fig. 216) is an aponeurotic covering on the front of the joint, which closes the wide intervals between the anterior and the lateral ligaments; and is derived from the fascia lata united with fibrous offsets of the extensor and flexor museles. It covers the anterior and the external lateral ligament, being inserted below into the heads of the tibia and fibula; and it blends on the inner side with the internal lateral ligament. It is not closely applied to the synovial membrane, but it is united below with the interarticular fibro-eartilages.

Dissection. Four external ligaments, anterior and posterior, internal and external, are situate at opposite points of the articulation. The posterior and the internal lateral ligament will appear on the removal of the areolar tissue from their surfaces; but the anterior and the external lateral are covered by the aponeurosis on the fore part of the joint, and will not be laid bare till this has been cut through. If there is a second external lateral band present, it is not concealed by the aponeurosis.

The external lateral ligament (fig. $216,{ }^{2}$ ) is round and cord-like. It is attached to the outer condyle of the femur below the tendon of the gas- 
trocnemius, and descends vertically between two pieces of the tendon of the biceps to a depression on the upper and outer part of the head of the fibula. Beneath the ligament are the tendon of the popliteus, and the external lower articular vessels and nerve.

Fig. 216.

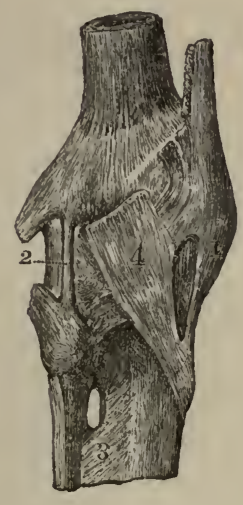

External Ligament of the KNeE-JOINT. (Bourgery).

1. Anterior ligament.

2. External lateral ligament.

3. Interosseous ligament.

4. Part of the capsule.
Fig. 217.

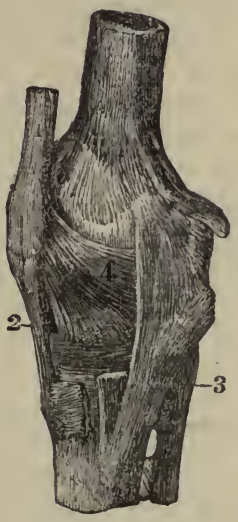

INTERNAL LIGAMENT OF THE KNEE-JOINT. (Bonrgery.)

1. Tendon of the extensur muscle ending below in the ligament of the patella, 2.

3. Internal lateral ligament.

4. Lateral part of the capsule.

A second fasciculus is sometimes present behind the other, but it is not attached to the femur: it is connected above with the gastrocnemius, and below with the posterior prominence of the head of the fibula.

The tendon of the biceps is inserted by two pieces into the points on the head of the fibula; and from the anterior of these there is a prolongation to the head of the tibia. The external lateral ligament passes between the pieces into which the tendon is split.

The tendon of the popliteus may be followed by dividing the posterior ligament. It arises from the fore part of the oblong depression on the outer surface of the external condyle of the femur. In its course to the outside of the joint, it crosses the external semilunar fibro-cartilage and the upper tibio-peroneal articulation. When the joint is bent, the tendon lies in the hollow on the condyle; but slips out of that groove when the limb is extended.

The tendon of the adductor magnus is inserted into a tubercle on the internal condyle of the femur, above the attachment of the internal lateral ligament.

The internal lateral ligament (fig. $217,{ }^{3}$ ) is attached to the condyle of the femur, where it blends with the capsule; but becoming thicker below, and separate from the rest of the capsule, it is fixed for about an inch into the inner surface of the tibia, below the level of the ligamentum patella.

The tendons of the sartorius, gracilis, and semitendinosus muscles lie over the ligament; and the tendon of the semimembranosus, and the internal lower articular vessels and nerve are beneath it. To the posterior edge some fibres of the tendon of the semimembranosus are added. 
The tendon of the semimembranosus muscle is inserted beneath the internal lateral ligament into an impression at the back of the inner tuberosity of the head of the tibia: between it and the bone is a synovial bursa. The tendon. sends some fibres to the internal lateral ligament, a prolongation to join the fascia on the popliteus muscle, and another to the posterior ligament of the knee joint.

The posterior ligament (liganent of Winslow), wide and membranous, covers the back of the joint between the two lateral, and is joined by fibres from the tendon of the semimembranosus, which are directed across the joint to the outer side. It is fixed below to the head of the tibia behind the articular surface, and above to the femur, but by strongest fibres opposite the intercondyloid notch. Numerous apertures exist in it for the passage of the vessels and nerves to the interior of the articulation ; and the tendon of the popliteus pierces it.

The anterior ligament (ligamentum patellæ) (fig. $217,{ }^{2}$ ), part of the tendon of insertion of the extensor muscle of the knee (p.571), is two inches long. Superiorly it is attached to the lower part of the patella, and to the depression on the inner surface of the apex ; and inferiorly it is inserted into the tubercle of the head of the tibia, and into an inch of the bone below it. An expansion of the triceps extensor covers it; and a bursa intervenes between it and the top of the tubercle of the tibia.

Dissection (fig. 218). To see the reflections of the synovial membrane raise the knee on blocks, and open the joint by an incision on each side,

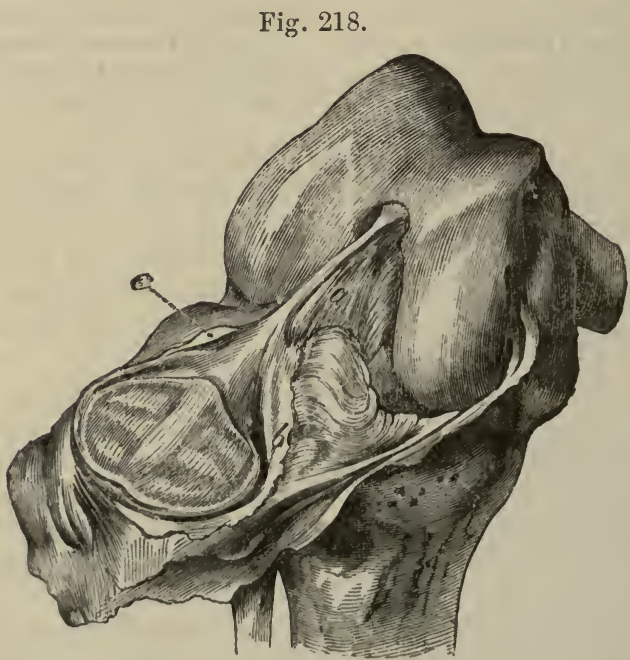

The Capsula of the Kanejoint cet across, and the Patella thrown down to show thb NAMED FOLDS OF THE SYNOVIAL SAC.

a. Mucous ligament.

b. Internal, and $c$, external alar ligament.

above the patella. When the anterior part of the capsule with the patella is thrown down, a fold (mucous ligament) will be seen extending from the intercondyloid fossa of the femur to a mass of fat below the patella. On each side of the knee-pan is a similar fold (alar ligament) over some fat.

The limb may be laid flat on the table, and some of the posterior liga- 
ment may be removed, to show the pouches of the synovial membrane which project behind over the condyles of the femur; but the limb is to be replaced in the former position before the parts are learnt.

The synovial membrane (fig. 218) lines the interior of the capsule, and is continued to the articular ends of the bones. It invests the interarticular cartilages after the manner of serous membranes, and sends a pouch between the tendon of the popliteus and the external fibro-cartilage and the head of the tibia; it is also reflected over the strong crucial ligaments at the back of the joint.

There are three named folds of the synovial membrane. One in the centre of the joint is the mucous ligament $(a)$, which contains small vessels and some fat, and extends from the interval between the condyles to the fat below the patella. Below and on each side of the patella is another fold-alar ligament $(b$ and $c$ ), which is continuous with the former below the patella, and is placed over a mass of fat : the inner $(b)$ is prolonged farther than the outer by a semilunar piece of the serous membrane.

At the back and front of the articulation pouches are prolonged beneath the tendons of muscles. Behind there are two, one on each side, between the condyle of the femur and the tendinous head of the gastrocnemius. On the front, the sac projects under the extensor muscle one inch above the artictilar surface; and if it communicates with the bursa in that situation, it will reach two inches above the joint surface of the femur. When the joint is bent there is a greater length of the serous sac above the patella.

Fut around the joint. 'Two large masses are placed above and below the patella, and some fat is located around the crucial ligaments.

The infra-patellar mass, the largest of all, fills the interval between the patella with its ligament and the head of the tibia, and gives origin to the ridges of the synovial membrane. From it a piece is continued around the patella: but it is larger at the inner margin of the bone, than on the outer, and overhangs the inner perpendicular facet. During extension of the joint the infra-patellar pad is applied to, and lubricates the articular surfaces of the femur.

The supra-patellar pad is interposed between the triceps extensor and the femur around the top of the synovial sac, and is greater on the outer than on the inner side.

Dissection (fig. 219). The ligamentous structures within the capsule will be brought into view, whilst the limb is still in the same position, by throwing down the patellat and its ligament, and clearing away the fat behind it. In this step the student must be careful of a small transverse band which connects anteriorly the interarticular cartilages.

The remains of the capsule and other ligaments, and the synovial membrane are next to be cleared away from the front and back of the crucial ligaments, and from the interarticular cartilages. Whilst cleaning the posterior crucial the limb is to be placed flat on the table with the patella down; and the student is to be careful of a band before it from the external fibro-cartilage, or of two bands, one before and the other behind it.

Ligaments within the capsule. The ligamentous structures within the capsule consist of the central crucial ligaments, and of two plates of fibrocartilage on the head of the tibia.

The crucial ligaments (fig. 219) are two strong fibrous cords between the ends of the tibia and femur, which maintain in contact the bones. They cross one another somewhat like the legs of the letter $\mathbf{X}$, and have 
received their name from that circumstance. One is much anterior to the other at the attachment to the tibia.

The anterior ligament $(f)$ is most oblique in its direction, and is smaller than the posterior. Inferiorly it is attached in front of the spine of the tibia, close to the inner articular surface, reaching back to the inner point of the spine: superiorly it is inserted by its posterior shorter fibres into the back of the outer condyle of the femur, and by the anterior or longer into the hinder part of the intercondyloid fossa.

The posterior ligament $(e)$ is almost vertical between the bones at the back of the joint. By the lower end it is fixed to the hindermost impression of the hollow behind the spine of the

Fig. 219.

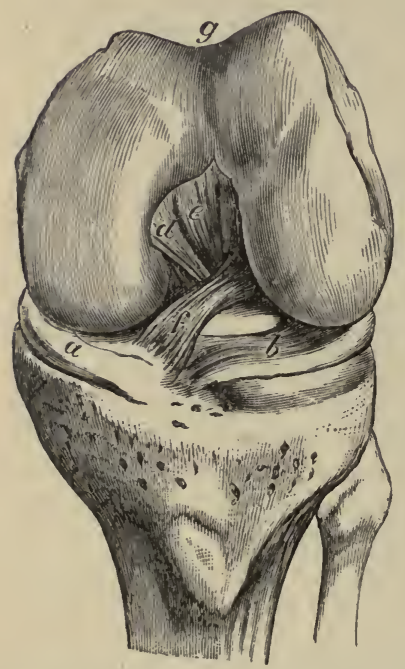

INTEKABTICULAR LIGAMENTS OF THE KNEB-JOINT.

a. Internal, and

b. External semilunar fibro-cartilage; the latter rather displaced by the bending of the joint.

e. Posterior crucial ligament, with $d$, the ascending ligamentous band of the external fibro-cartllage.

$f$. Anterior crucial ligament.

g. Patellar surface of the femur. tibia, near the margin of the bone; and above its posterior shorter fibres are inserted into the inner condyle along the side of the oblique curve, whilst the anterior and longer reach the fore part of the intercondyloid fossa.

The use of these ligaments in the movements of the joint, after the external ligaments have been cut through may now be studied.

As long as both ligaments are whole the bones cannot be separated from each other.

Rotation in of the tibia is stopped by the anterior crucial. Rotation out is not checked by either ligament ; for the bands uncross in the execution of the movement, and will permit the tibia to be put hind foremost.

Supposing the tibia to move, as in straightening the limb, the anterior. prevents that bone being carried too far forwards by the extensor tendon, or by force ; and the ligament is brought into action at the end of extension, because the tibia is being put in front of the femur. Its use is shown by cutting it across, and leaving the posterior entire, as then the tibial articulating surfaces can be placed in front of the femoral in the half bent state of the joint.

The posterior crucial arrests the too great movements backwards of the tibia by the flexors or by force; and it is stretched in extreme flexion, in which the tibia is being drawn back from the femur. This use will be exemplified by cutting across the posterior (in another joint or in another dissection) and leaving entire the anterior: when this has been done the articular surfaces of the tibia can be carried nearly altogether behind the condyles of the femur.

The interarticular or semilunar fibro-cartilages (fig. 220) cover partly on each side the articular surface of the tibia.

They are thick at the outer margin, where they are united by fibres to the capsule, and are thin at the inner edge; they are hollowed on the upper surface, so as to assist in giving depth to the fossæ for the reception 
of the condyles of the femur, but are flattened below. Inserted into the tibia at their extremities, they are coarsely fibrous at their attachment to the bone, like the crucial ligaments; and they become cartilaginous only where they lie between the articular surfaces. 'The synovial membrane is reflected over them.

The internal cartilage $(a)$ is ovoid in form, and is a segment of a larger circle than the external. In front it is attached by a pointed part to the anterior margin of the head of the tibia, in front of the anterior erucial ligament. At the back, where it is much wider, it is fixed to the inner lip of the hollow behind the spine of the tibia, between the attachment of the other cartilage and the posterior crucial ligament.

The external cartilage $(b)$ is nearly circular in form, and is connected to the bone within the points of attachment of its fellow. Its anterior part is fixed in front of the spine of the tibia, close to the outer articular surface, and opposite the anterior crucial ligament, which it touches; and its posterior extremity is inserted behind and between the two osseous points of the spine. This fibro-cartilage is less closely united to the capsule than the internal, for the fore part is in the centre of the joint, and the tendon of the popliteus muscle separates it behind from that membrane.

Fig. 220.

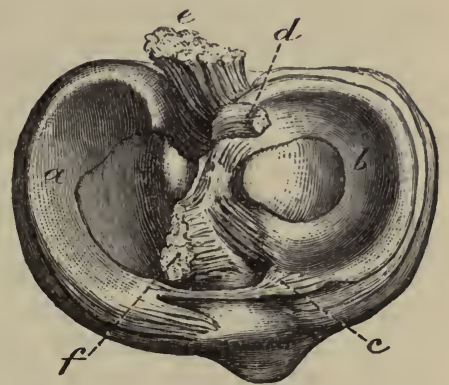

VIEW OF the Head of the TIBIa With THE Fibro.cARTILAGES ATtACHED: THE CRUCIAL LIGAMENTS HAVE BREN CUT THROUGH.

$a$. Inner, and $b$, outer semilunar fibro-cartilage.

$c$. Transverse, and $d$, ascending or posterior band (cut) of the external cartilage.

e. Posterior, and $f$, anterior crucial ligament.

The outer fibro-cartilage is provided with two accessory bands, one at the fore part, the other behind.

The anterior or transverse ligament $(c)$ is a narrow band of fibres between the semilunar cartilages at the front of the joint. Sometimes it is scarcely perceptible.

The posterior or ascending band $(d)$, thicker and stronger than the other, springs from the back of the outer fibro-cartilage, and is inserted into the femur as a single band (fig. $219, d$ ) in front of the posterior crucial, or as two bands-one being before, and the other behind that ligament.

Use. The fibro-cartilages deepen the sockets of the tibia for the reception of the condyles of the femur, and fill the interval between the articular surfaces of the bones at the circumference of the joint; they moderate the injurious effect of pressure of the one bone on the other; and cause the force of slıoks to be diminished in transmission.

In flexion and extension they move forwards and backwards with the tibia. During flexion they recede somewhat from the fore part of the joint, and surround the condyles of the femur; but in extension they are flattened out on the surface of the tibia. Of the two cartilages the external moves the most in consequence of its ends being less attached to the capsule. 
In rotation the fibro-cartilages follow the tibial movements, but the external is most displaced by the projecting outer condyle of the femur.

The accessory bands in front and behind serve to retain in place the least fixed external fibro-cartilage; thus the anterior ligament keeps for- wards the fore part of that cartilage in flexion, and the posterior secures the hack of the same from displacement in rotation.

Articular surfaces of the bones. The end of the femur is marked by a patellar and two tibial surfaces.

The patellar is placed in the middle line above the others; it is hollowed along the centre, with a slanting surface on each side, the outer being the larger of the two.

The surfaces for contact with the tibia, two in number, occupy the ends of the condyles, and are separated from the patellar impression by an oblique groove on each side. On the centre of each is a somewhat flattened part, which is in contact with the tibia in standing; and at the posterior third is a more convex portion, which touches the tibia in rotation.

The inner condyle of the femur is curved at its anterior third, the concavity being directed out : this has been named the "oblique curvature." Close to the curved part is a semilunar facet, which touches the perpendicular surface of the patella in extreme flexion.

On the head of the tibia are two slight articular hollows, the inner being the deeper and larger, which rise towards the middle of the bone, on the points of the tibial spine.

The joint-surface of the patella is marked by the following impressions. Close to the inner edge is a narrow perpendicular facet, and along the lower border is a similar transverse mark. Occupying the rest of the bone is a squarish surface, which is subdivided by a vertical and a transverse line into two pairs of marks-upper and lower. (Goodsir, Edinb. Med. Jour., 1855.)

Movements of the joint. The chief movements of the knee are two in number, bending and straightening, like the elbow ; but there is, in addition, rotation of the tibia when the joint is bent.

Flexion and extension. Each of these movements may be divided into stages for the purpose of particularizing changes in its direction.

In flexion the tibia with its fibro-cartilages moves backwards round the end of the femur; and its extent is limited by the extensor muscle, and by the meeting of the calf of the leg with the thigh.

For the anterior third of the movement the tibia is directed down and in along the oblique curve of the inner condyle, giving rise to rotation inwards of that bone; but for the posterior two-thirds, the tibia passes straight back over the condyles.

All the external ligaments are relaxed, except the anterior; and both crucials are put on the stretch towards the end of flexion.

In extension the tibia is carried forwards until it comes into a straight line with the femur, when the uniting ligaments prevent its farther progress.

In the hinder two-thirds of the movement the tibia has a straight course over the condyles of the femur; but in the anterior third the legbone is directed up and out over the oblique curve of the inner condyle, and is rotated out.

All the external ligaments except the anterior are tightened, and the crucial cords help to limit extreme extension. 
Rotation. A half bent state of the knee is necessary for this movement, for the purpose of relaxing the anterior crucial and the external ligaments; and the foot must be free. Then, the tibia with its fibro-cartilages rotates around a vertical axis, the great toe being turned in and out.

During rotation in the inner articular surface of the tibia touches the condyle of the femur and moves backwards; and the outer articular surface, separated by a slight interval from the thigh bone, passes forwards.

Both lateral ligaments are loose; but the anterior crucial is gradually tightened, and stops finally the motion.

In rotation out the opposite movement of the tibia takes place-the inner articular surface being directed forwards, and the outer backwards.

The internal lateral ligament controls the movement by its fibres being made tense. The crucials have not any influence on the motion (p. 637).

Movement of the patella. When the knee passes from flexion to extension the patella crosses it obliquely from the outer to the inner side, touching in succession different parts of the femoral articular surfaces.

In complete flexion the knee-pan lies on the outer side of the joint below the femur, where it is scarcely perceptible, and is fixed in its situation. It touches the semilunar surface on the inner condyle by its perpendicular facet, and the under part of the outer condyle by the upper and outer mark on its square surface.

When the joint is passing from flexion to extension, the upper pair of impressions on the square surface of the patella, and the lower pair rests successively on the pulley-surface of the femur.

In complete extension, the patella is situate at the upper and inner part of the knee-joint, where it is very prominent, with its apex and the ligament of the patella directed down and out to the tibia. For the most part the knee-pan articular surface is raised above the trochlea of the femur, which it touches only at the upper edge by its lower transverse facet.

Peroneo-tibial articulations. The tibia and fibula are united by ligamentous bands at the extremities, where they touch; and by an interosseous ligament between the shafts of the bones.

Dissection. The muscles are to be taken away from the front and back of the interosseous ligament; and the loose tissue is to be removed from a small band in front of, and behind the upper and lower ends of the tibia and fibula.

The UPPER ARTICULATION has very small movement, and the structures between the ends of the bones are two small bands, anterior and posterior.

The anterior ligament extends over the joint from the outer tuberosity of the tibia to the head of the fibula. 'The posterior ligament, thinner than the anterior, is attached to the bones behind the joint : it is covered by the tendon of the popliteus muscle and a prolongation of the synovial membrane of the knee-joint.

'The articular surfaces are eovered with eartilage: and a synovial membrane lining the articulation projects backwards so as to touch that of the knee-joint.

The lower articulation possesses an anterior and a posterior band, together with an inferior ligament between the ends of the bones.

The anterior ligament reaches obliquely from the lower end of the tibia to the fibula; and the posterior has attachments behind the articulation similar to those of the band in front.

The inferior ligament closes the space between the contiguous ends of 
the tibia and fibula, and consists of transverse yellowish fibres distinct from the posterior ligament. It is fixed on one side to the end of the fibula above the pit: and on the other it is inserted into the contiguous part of the tibia, and into the posterior edge of the articular surface so as to assist in deepening the hollow into which the astragalus is receiverl.

The interosseous ligament fills the interval between the bones of the leg, and serves as an aponeurotic partition between the muscles on the front and back of the leg. Its fibres are directed downwards for the most part from the tibia to the inner surface of the fibula : but some few cross in the opposite direction.

Internally it is fixed to the outer edge of the tibia : and externally, to the oblique line on the inner surface of the fibula along the upper threefourths, but to the posterior border along the lower fourth of that bone.

Both superiorly and inferiorly is an aperture which transmits vessels. The upper opening, about an inch in length, lies along the neck of the fibula, and gives passage to the anterior tibial vessels. The lower aperture is close to the fibula, about an inch above the lower end, and is only large enough for the small anterior peroneal vessels.

Some strong irregular bundles of fibres, the inferior interosseous ligament, extend between the bones below the aperture for the anterior peroneal artery. It may be seen after the examination of the ankle joint by sawing longitudinally the lower ends of the leg bones.

Movement. Very little movement is allowed in the tibio-peroneal articulations, as the chief use of the fibula is to give security to the ankle joint and attachment to muscles of the leg.

In the upper joint there is a slight gliding from before back. In the lower articulation the ligaments permit some yielding of the fibula to the pressure of the astragalus, as when the weight of the body is thrown on the inner side of the foot; but if the force is violent the lower fourth of

Fig. 221.

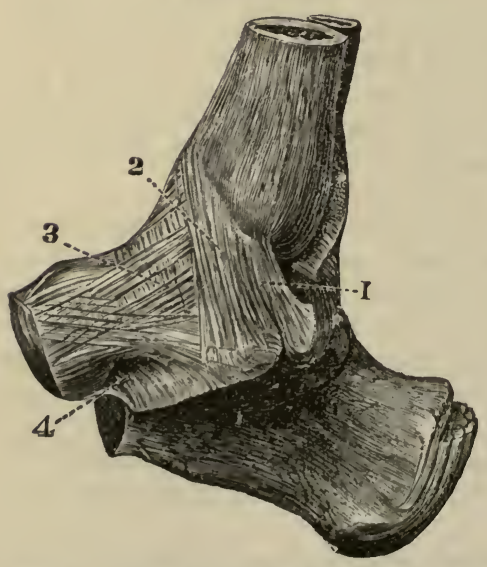

INTERNAL LATERAL LIGAMENT OF THB ANKLB (altered from Bourgery).

1. Posterior piece.

2. Middle piece.

3. Anterlor piece of the inner ligament.

4. Inferior calcaneo-scaphoid ligament. that bone will be fractured sooner than the ligaments.

Articulation of the ankle (fig. 221). Like the knee, the ankle is a ginglymoid or hinge joint. In this joint the upper surface of the astragalus is received into an arch formed by the lower ends of the tibia and fibula; and the four ligaments belonging to this kind of articulation connect together the bones.

Dissection. To make the dissection required for the ligaments of the ankle joint, the muscles, and the fibrous tissue and vessels must be removed from the front and back of the articulation.

For the purpose of defining the lateral ligaments, the limb must be placed first on one side and then on the other. 'The internal ligament is wide and strong, and lies beneath the tendon of the tibialis posticus. 
The external is divided into three separate pieces; and to find these the peronei muscles, and the remains of the annular ligament below the outer malleolus, should be taken away.

The anterior or tibio-tarsal ligament is a thin fibrous membrane, which is attached to the tibia close to the articular surface; and to the upper part of the astragalus near the articulation with the scaphoid bone. In the ligament are some rounded intervals and apertures for vessels. On the sides it joins the lateral ligaments.

The posterior ligament is thinner internally than externally; and it is inserted into the tibia and the astragalus, close to the articular surfaces of the bones. Towards the outer part it consists of transverse fibres, which are fixed into the hollow on the inner surface of the external malleolus.

The internal lateral or deltoid ligument (fig. 221) is attached by its upper or pointed part to the inner malleolus, and by its base to the astragalus, the os calcis, and the scaphoid bone, by fibres which radiate to their insertion in this manner:-The posterior $\left({ }^{1}\right)$ are directed to the hinder part of the inner surface of the astragalus; the middle $\left({ }^{2}\right)$ pass vertically to the sustentaculum tali of the os calcis; and the anterior $\left({ }^{3}\right)$, which are thin and oblique, join the inferior calcaneo-scaphoid ligament and the inner side of the scaphoid bone. The tendons of the tibialis posticus and flexor longus digitorum are in contact with this liganent.

The external lateral ligament (fig. 222) consists of three separate pieces, anterior, middle, and posterior, which are attached to the astragalus and the os calcis. The anterior piece $\left(^{1}\right)$ is a short flat band, which is directed from the fore part of the malleolus to the side of the astragalus in front of the lateral articular surface. The middle portion $\left(^{3}\right)$ is Hattened and descends from the tip of the malleolus to the outer surface of the os calcis, about the middle. The posterior $\left({ }^{2}\right)$ is the strongest, and is almost horizontal in direction; it is connected externally to the pit on the inner surface of the malleolus, and is inserted into the posterior part of the astragalus behind the upper articular surface, extending to the groove for the flexor pollicis tendon.

The posterior and middle fasciculi are placed beneath the peronei muscles. The middle part is but slightly in contact above with the synovial membrane of the ankle joint; and both it and the posterior piece touch the synovial membrane between the astragalus and the os calcis.

Dissection. Divirling the ligaments of the ankle joint, separate the astragalus from the bones of the leg, to see the osseous surfaces entering into the joint.

The synovial membrane of the joint lines the capsule, and is simple in its arrangement.

Articular surfaces. On the tibia there are two articular faces, one of which corresponds with the end of the shaft, and the other with the malleolus. On the fibula the surface of the malleolus which is turned to the astragalus is tipped with cartilage.

The astragalus has a central articular surface, wider before than behind and trochlear-shaped, which touches the end of the tibia : and on its siles are articular impressions for contact with the malleoli, but the outer one is the largest.

Movement. Only the movements of flexion and extension are permitted in the ankle: in the former state the toes are raised towards the fore part of the leg; and in the latter, they are pointed towards the ground.

In flexion the astragalus moves backwards so as to project behind; and 
all further motion is arrested by the meeting of the anterior edge of the tibia with that bone.

The posterior ligament is stretched over the projecting head of the astragalus, and the posterior and middle parts of the external lateral, and

Fig. 222.

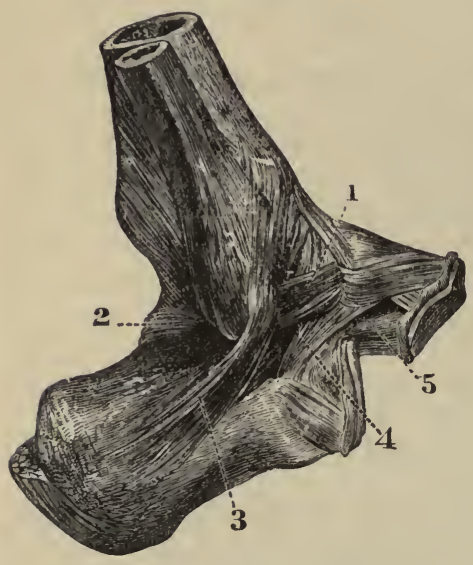

Externai Lateral Ligament of the ANKLE (altered from Bourgery).

1. Anterior part.

2. Posterior part.

3. Middle part of the outer ligament.

4. Interosseus of astragalus and os calcis.

5. External calcaneo-scaphoid ligament. the posterior piece of the internal lateral ligament, are made tense.

In extension the astragalus moves forwards over the end of the tibia, and projects anteriorly. A limit to the movement is imposed by the meeting behind of the astragalus with the tibia.

The lateral ligaments are partly made tight as in flexion, for instance the anterior piece of the external, and the fore and middle portions of the internal

When the joint is half extended so that the small hinder part of the astragalus is brought into the arch of the leg bones, a slight movement of the foot inwards and outwards may be obtained ; but if the foot is forcibly extended the portions of the lateral ligaments, attached to the astragalus, prevent this lateral movement by their tightness.

Dissection. The joints of the foot will be demonstrated by removing from both the dorsum and the sole all the soft parts which have been examined. Between the different tarsal bones bands of ligament extend, which will be defined by removing the areolar tissue from the intervals between them (fig. 223).

It will be more advantageous for the student to clean all the ligaments before he proceeds to learn any, than to prepare only the bands of one articulation at a time.

Articulation of tile astragalus and os calcis. These bones are kept together by two joints, and a strong interosseous ligament; and there are also thin bands at the outer side and behind.

The posterior ligament (fig. 223, a) consists of a few fibres between the bones, where they are grooved by the tendon of the flexor pollicis; and the external ligament $(b)$ is connected to the sides of the astragalus and os calcis, near the middle piece of the external lateral ligament of the ankle joint.

The interosseous ligament (fig. 223, $c$ ) consists of strong vertical and oblique fibres, which are attached above and below to the depressions on the contiguous surfaces of the two bones. This band extends across the bones, and its depth is greatest at the outer side.

In a subsequent stage of the dissection (p. 647) articular surfaces will be seen between the bones, viz., one behind the interosseous ligament, and one in front of it, with two synovial membranes.

Movement. Under the influence of the weight of the body, as in standing, the astragalus moves down and in (not straight forwards) with 
flattening of the arch of the foot, so that its head projects against the calcaneo-scaphoid ligament. In this state the interosseous ligament is put on the stretch.

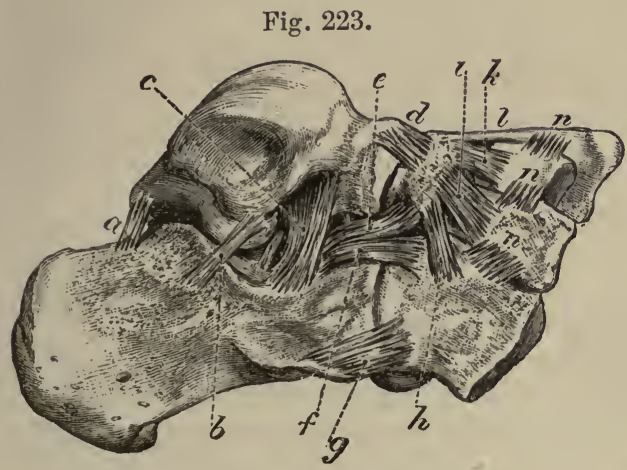

a. Posterior, $b$, external, and $c$, interosseous ligaments between astragalus and os calcis.

d. Astragalo-scaphoid.

e. External calcaneo-scaphoid.

$f$. Internal, and $g$, upper calcaneocuboid ligaments.

$h$. Dorsal scapho-cuboid band.

$i, k, l$. Dorsal external, middle, and internal scapho-cuneiform longitudinal bands.

$n$. Dorsal transverse bands between the cuneiform and cuboid bones.

VIEW OF THE DORSAL LIGAMENTS OF THE TARSER.

When the pressure of the leg is removed the astragalus is carried up and out by the tightened ligaments and muscles, and the arch of the foot is restored.

Astragalus witu the scaphoid bone. The head of the astragalus is received into the hollow of the scaphoid bone, and is united to it by a dorsal ligament; but the place of plantar and external lateral ligaments is supplied by strong bands between the os calcis and the scaphoid bone, which will be noticed below.

The dorsal astragalo-scaphoid ligament (fig. $223, d$ ) is attached to the astragalus close to the articulation, and to the dorsal surface of the scaphoid bone: its attachments will be better seen when it is cut through.

Dissertion. The external ligament of the articulation may be seen on the dorsum of the foot in the hollow between the os calcis and the scaphoid bone. Supposing the tendon of the tibialis posticus removed, the inferior ligament will be defined in the sole of the foot by cutting some fibro-cartilaginous substance from it.

The inferior ligament (fig. 225, c) (calcaneo-scaphoid) is attached behind to the fore part of the sustentaculum tali of the os calcis, and in front, to the hollow on the sustentaculum tali of the os calcis, and in front, to the hollow on the under surface of the scaphoid bone. In the upright posture of the body the tendon of the tibialis posticus is beneath it in the sole of the foot; and on it the head of the astragalus rests.

'The external calcaneo-scaphoid (fig. $223, e$ ) is placed outside the head of the astragalus, and serves as a lateral ligament to the astragalo-scaphoid articulation; it is about three-quarters of an inch deep. Behind, it is fixed to the upper part of the os calcis, between the articular surfaces for the cuboid bone and astragalus; and in front it is inserted into the outer side of the os scaphoides.

A synovial membrane serves for this articulation, and sends back a prolongation to the joint between the fore part of the os calcis and the astragalus.

Articular surfaces. The head of the astragalus has two articular faces ; a smaller, below, for the os calcis ; and a larger one, elongated transversely 
and larger externally than internally, for the scaphoid bone. The scaphoid bone is hollowed, and is widest externally.

Movement. The scaphoid moves down and in over the transversely elongated head of the astragalus, or up and out in the opposite direction.

As the bone is forced downwards, the upper and external ligaments of the joint are made tight; and when the seaphoid is moved in the opposite way the strong inferior ligament is put on the stretch.

Tine os calcis with the cuboid bone. The ligaments in this articulation are plantar and dorsal, the former being much the strongest ; and there is also an internal band.

The dorsal ligament (fig. 223,g) (superior calcaneo-cuboid) is a rather thin fasciculus of fibres, which is attached near to the contiguous end of the os calcis and the cuboid bone; it is sometimes divided into two parts, or it may be situate at the outer border of the foot.

At the inner side of the os cuboides is a stronger internal band (fig. 223, $f$ ) from the os ealcis, this is fixed behind to the upper part of the os ealcis external to the band to the seaphoid bone, and in front to the contiguous inner side of the os cuboides.

Fig. 224.

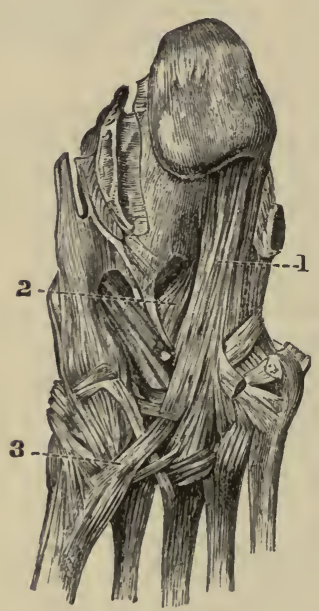

Plantar Ligaments of the Foot (Bourgery).

1. Long plantar ligament.

2. Deep portion of the inferior calcaneo-cu. boid ligament.

3. Tendon of the peroneus longus muscle.
Fig. 225 .

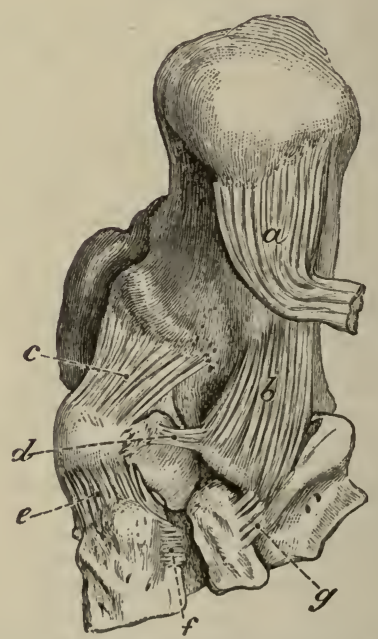

VIRW OF THE INFERIOR LIFAMENTS OF THE TARBAt Bones.

a. Long plantar, cut.

b. Short or deep inferior calcaneo-cuboid ligament.

c. Inferior calcaneo-scaphoid.

d. Plantar transverse scapho-cuboid ligament.

e. Dorsal inner scapho-cuneiform extending into the sole of the foot.

$f$. Plantar transverse ligament between the inner and middlecuneiform bones.

g. Plantar transverse band between the cuboid and outer cuneiform.

The inferior calcaneo-cuboid ligament in the sole of the foot (fig. 224) is much the strongest, and is divided into a superficial and a deep part :- 
The superficial portion, ligamentum longum plante $\left(^{1}\right)$ is attached to the under surface of the os calcis from near the posterior to the anterior tubercle : its fibres pass forwards to be connected with the ridge on the under surface of the cuboid bone; but the most internal are continued over the tendon of the peroneus longus muscle, assisting to form its sheath, and are inserted into the bases of the third and fourth metatarsal bones.

The deep piece of the ligament (fig. $225, b$ ), seen on division of the superficial, extends from the tubercle and the hollow on the fore part of the under surface of the os calcis, to the cuboid bone internal or posterior to the ridge.

A simple synovial membrane belongs to the articulation.

Articular surfaces. Both bones are flattened towards the outer part of the articulation; but at the inner side the os calcis is hollowed from above down, and the os cuboides is convex to fit into it.

Movement. In this joint the cuboid bone possesses two movements, viz., an oblique one, down and in, and up and out.

In the downward movement the internal lateral and the upper ligament are made tight : and in the upward, the calcaneo-cuboid ligaments of the sole are stretched.

Transverse tarsal articulation (fig. 223). The joints of the astragalus with the scaphoid, and os calcis with the cuboid bone, form a transverse articulation across the foot in which the movements of inversion and eversion take place.

In inversion the great toe is adducted; the inner border of the foot is shortened, and is raised from the ground so that the sole looks inwards.

The scaphoid bone passes down and in over the head of the astragalus, being approximated near to the inner malleolus; and the cuboid bone moves down and in on the os calcis. The cuneiform bones are raised at the same time and contribute to the movement (p. 648).

The ligaments connected with both joints on the dorsum of the foot are tightened.

In eversion the inner border of the foot descends and lengthens, the outer border is raised, and the great toe is abducted from the middle line of the body.

The same two tarsal bones are directed up and out, and the cuneiforms sink.

The ligaments in the sole of the foot of both joints now come into use to prevent over movement.

Dissection. Saw through the astragalus in front of the attachment of the interosseous ligament between it and the os ealcis; and remove the head of the bone for the purpose of observing the lower and outer calcaneoscaphoid ligaments.

Then the interosseous ligament uniting the astragalus and the os calcis is to be eut through, to demonstrate its attachments, the articular surfaces of the bones, and the synovial sacs (p. 644).

Articular surfaces of the two hinder tarsal bones. There are two articular surfaces, anterior and posterior, to both the astragalus and the os calcis. The hinder one of the os calcis is convex transversely and the anterior is concave; but sometimes the last is subdivided into two. The surface of the astragalus will have a form exactly the reverse of that of the os calcis, viz., the hinder one concave and the anterior convex; the anterior is seated on the head of the astragalus. 
Dissection. The calcaneo-cuboid joint may be opened to see the articular surfaces: and the student is to keep in mind that all the other articulations of the foot are to be opened for the like purpose, even should directions not be given.

Articulations of the scaphoid Bone. The scaphoid bone articulates in front with the three cuneiform bones, and laterally with the os cuboides.

In the articulation with the cuneiform bones (fig. 223) there are three longitudinal dorsal ligaments $(i, k, l)$, one to each bone; but the innermost is the strongest and widest, and extends round the inside of the articulation into the sole of the foot (fig. 225, e).

The place of plantar bands is supplied by processes of the tendon of the tibialis posticus.

A synovial membrane (common of the tarsus) lines the articulation, and sends forwards prolongation between the euneiform bones.

In the articulation with the os cuboides there is a dorsal oblique band of fibres (fig. $223, h$ ) between the contiguous surfaces of the bones; a plantar transverse band (fig. 225, d), which is concealed by the tendon of the tibialis posticus; and a strong interosseous ligament.

Where the bones touch, the surfaces are tipped with cartilage, and are furnished with a prolongation from the common synovial membrane of the tarsus.

Articulatoins of the cuneiform bones. These bones are united to one another by cross bands; and the external one articulates with the os cuboides after a similar manner.

The three cuneiform bones are connected together by short transverse dorsal bands (fig. $223, n$ ) between the upper surfaces. Similar plantar ligaments are wanting, except one between the two innermost (fig. $22.5, f$ ). There are also interosseous ligaments between the contiguous surfaces of the bones. Laterally there are articular surfaces between the bones, with offsets of the common synovial membrane.

Where the external cuneiform touches the cuboid bone the surfaces are covered with cartilage. A dorsal ligament (fig. $223, n$ ) passes transversely between the two; and a plantar ligament (fig. $225, g$ ) takes a similar direction. Between the bones there is also an interosseous ligament.

This joint is furnished either with a distinct synovial sac, or with a prolongation of the common tarsal synovial membrane.

The synovial membrane of the articulations of the cuneiform bones is common to many of the bones of the tarsus. Placed between the scaphoid and the three cuneiforms it sends one prolongation forwards between the inner and middle euneiform to the joints with the second and third metatarsal bones; another, outwards, to the articulation of the seaphoid with the cuboid bone; and sometimes a third to the joint between the external cuneiform and the os cuboides.

Articular surfaces. On the scaphoid are three articular faces, the inner being rounded, and the other two flattened. 'The three cuneiforms unite in a shallow elliptical hollow, which is most excavated internally.

Movement. The cuneiform bones glide up and out on the scaphoid in inversion of the foot, and down and in in eversion; and the inner one moves more than the others in consequence of the shape of the articular surfaces, and the attachment to it of the tibialis anticus.

When the bones pass down the dorsal ligaments are made tight: and 
as they rise the interosseous and transverse plantar bands will keep them united.

In standing and in progression these bones are separated somewhat from each other with diminution of the arch of the foot, and stretching of the transverse ligaments which connect them.

Articulation of the metatarsal bones. The bases of the four outer metatarsal bones are connected together by dorsal, plantar, and interosseous ligaments; and where their lateral parts touch, they are covered with cartilage, and have offsets of a synovial sac.

The dorsal ligaments (fig. 226) are small transverse bands from the base of one metatarsal bone to the next. The plantar ligaments (fig. 224) are similar to the dorsal. The interosseous ligaments are short, transverse fibres between the contiguous rough lateral surfaces: they may be afterwards seen by forcibly separating the bones.

Lateral union. The four outer bones touch one another laterally; the second metatarsal lies against the internal and external cuneiforms; and the fourth is in contact internally with the outer cuneiform. Those articulating surfaces are covered with cartilage; and are provided with synovial membrane, which is derived from the sacs serving for the articulation of the same four metatarsal with the tarsal bones.

The metatarsal bone of the great toe, like that of the thumb, is not united to the others at its base by any intervening bands.

The digital ends of the five metatarsal bones are united by the transverse metatarsul ligament; this has been described in page 624 .

Fig. 226.

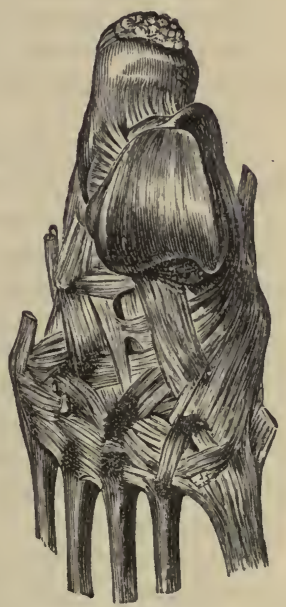

DORSAL LIgAMRNTS UNITING THB TARSUS TO THE Metatarsus, AND thB MeTATARSAL BONRS TO EACH OTHER BEHIND (Bourgery).

TARSAL With metatarsal bones. These articulations resemble the like parts in the hand, as there is a separate joint for the great toe, and a common one for the four outer metatarsals.

Articulation of the great toe. 'The articular ends of the bones are incased by a capsule, and are provided with an upper and a lower longitudinal band to give strength to the joint : the lower band is placed between prolongations firom the tendons of the tibialis anticus and peroneus longus.

A simple synovial membrane serves for the articulation.

The articular surfaces are oval from above down, curved inwards, and constricted in the middle; that of the great toe is excavated, and the other is convex.

Movement. There is an oblique movement of the metatarsal bone down and in and up and out, like that of the internal cuneiform with the seaphoid bone; and this will contribute some motion to inversion and eversion of the foot.

The joint possesses likewise slight abductory and adductory movement. Articulation of the four outer toes. 'The three outer 'tarsal bones of the last row correspond with four metatarsals ; - the middle cuneiform being opposite the second metatarsal bone, the external cuneiform touching that of the third toe, and the os cuboides carrying the two outer bones. 
The bones in contact are tipped with cartilage, and have longitudinal dorsal, plantar, and lateral ligaments, with some oblique in the sole.

The dorsal ligaments (fig. 226) are thin bands of fibres, which are more or less longitudinal as they extend from the tarsal to the metatarsal bones. Fach metatarsal bone receives one ligament, except that of the second toe, to which there are three; - the three bands to the second coming from all the cuneiform bones, one from each. The third bone obtains a ligament from the external euneiform; and the fourth and fifth have a fascieulus to each from the os cuboides.

Plantar ligaments (fig. 224). There is one longitudinal band from each of the two outer cuneiform to the corresponding metatarsal bone; but between the cuboid and its metatarsal bones there are only some scattered fibres.

The lateral ligaments are longitudinal; they lie deeply between the bones, and are connected with the second and third metatarsals : they will be better seen by cutting the transverse bands joining the bases of the bones. To the bone of the second toe there are two bands, one on each side :- the inner is strong and is attached to the internal euneiform; and the outer is fixed into the middle or the outer cuneiform bone. The metatarsal bone of the third toe is provided with one lateral slip on its outer side, which is inserted above into the external cuneiform bone.

Oblique plantar ligaments. A fasiculus of fibres extends across from the front of the internal cuneiform to the second and third metatarsals ; and from the external cuneiform there is another slip to the metatarsal bone of the little toe.

Line of the articulation. The line of the articulation between the tarsus and metatarsus is zigzag, in consequence of the unequal lengths of the cuneiform bones. To open the articulation, the knife should be carried obliquely forwards from the tuberosity of the fifth to the base of the second metatarsal bone; then about two lines farther back for the union of the second metatarsal with the middle cuneiform; and finally, half an inch in front of the last articulation, for the joint of the internal cuneiform with the first metatarsal bone.

Two synovial membranes are present in these tarso-metatarsal articulations.

There is one between the cuboid and the two outer metatarsals, which serves for the adjacent lateral articular surfaces of the bones; this is not always separate from the following.

The second is placed in the joint between the external and middle cuneiforms with their metatarsal bones (third and second), and is an offset of the common synovial membrane belonging to the articulation of the scaphoid with the cuneiform bones (p. 648): prolongations from it are furnished to the lateral articular surfaces of the second, third, and fourth (inner side) metatarsals.

Articular surfaces. The osseous surfaces are not flat; for the metatarsal bones are undulating, and the tarsal are uneven to fit into the others.

Movement. From the wedge-shaped form of the metatarsal bones a slight movement from above down is obtainable; and this is greatest in the little toe and the next.

In the little toe there is an abductory and adductory motion; and a small degree of the same exists in the fourth toe.

Dissection. All the superficial ligaments having been taken away, the interosseous ligaments of the tarsus and metatarsus may be seen by sepa- 
rating forcibly the cuneiform bones from one another and from the os cuboides; the latter bone from the os scaphoides: and the hases of the metatarsals from one another. The dissector will find that, in using force, the bones will sometimes tear sooner than the ligaments.

Metatarsus with phalanges. These are condyloid joints, in which the head of the metatarsal bone is received into the eavity of the phalanx.

Each articulation has two lateral and an inferior ligament, as in the hand; and the joint is further strengthened above by an expansion derived from the tendons of the extensors of the toes. A distinct synovial membrane exists in each joint.

In the articulation of the great toe there are two sesamoid bones, which are connected with the inferior and lateral ligaments.

All these structures are better seen in the hand, where they are more distinct; and their anatomy is more fully described with the dissection of that part. (See page 301.)

Surfaces of bone. The metatarsal bone has a rounded head, which is longest from above down, and reachest farthest on the plantar surface. On the end of the phalanx is a cup-shaped cavity.

Movement. In this condyloid joint as in the hand, there is angular motion in four different directions, with circumduction.

Flexion and extension. When the joint is bent the phalanx passes under the head of the metatarsal bone; and when it is extended the phalanx moves back beyond a straight line with the metatarsal bone.

A limit to flexion is set by the meeting of the bones, by the stretching of the fore part of the lateral ligaments, and by the extensor tendon; and to extension, by the tightness of the inferior, and the hinder part of each lateral ligament, and by the flexor tendons.

Lateral movement. 'The phalanx passes from side to side across the end of the metatarsal bone. Its motion is checked by the lateral ligament of the side from which it moved, and by the eontact with the other digits.

Circumduction, or the revolving of the phalanx over the rounded head of the metatarsal bone, is least impeded in the great toe joint; but these movements in the foot are not so free as in the hand.

Articulations of the phalanges. There are two phalangeal joints to each toe, except the first.

Ligaments similar to those in the metatarso-phalangeal joints, viz., two lateral and an inferior, are to be recognized in these articulations. The joint between the last two phalanges is least distinct; and oftentimes the small bones are immovably united by osseous substanee. These ligaments receive a more particular notice with the dissection of the hand (p. 302).

A simple synovial membrane exists in each phalangeal articulation.

Articular surfaces. In both phalangeal joints, the nearest phalanx presents a trochlear surface; and the distal one is marked by two lateral hollows or cups with a median ridge.

Movement. Only flexion and extension are permitted in the two phalangeal joints of the toes, as in the hand.

In flexion the farther phalanx glides under the nearer; and in extension the two are brought into a straight line.

The bending is checked by the lateral ligaments and the extensor tendon; and the straightening is limited by the inferior ligament and the flexor tendons. 


\section{TABLE OF THE ARTERIES OF THE LOWER LIMB.}

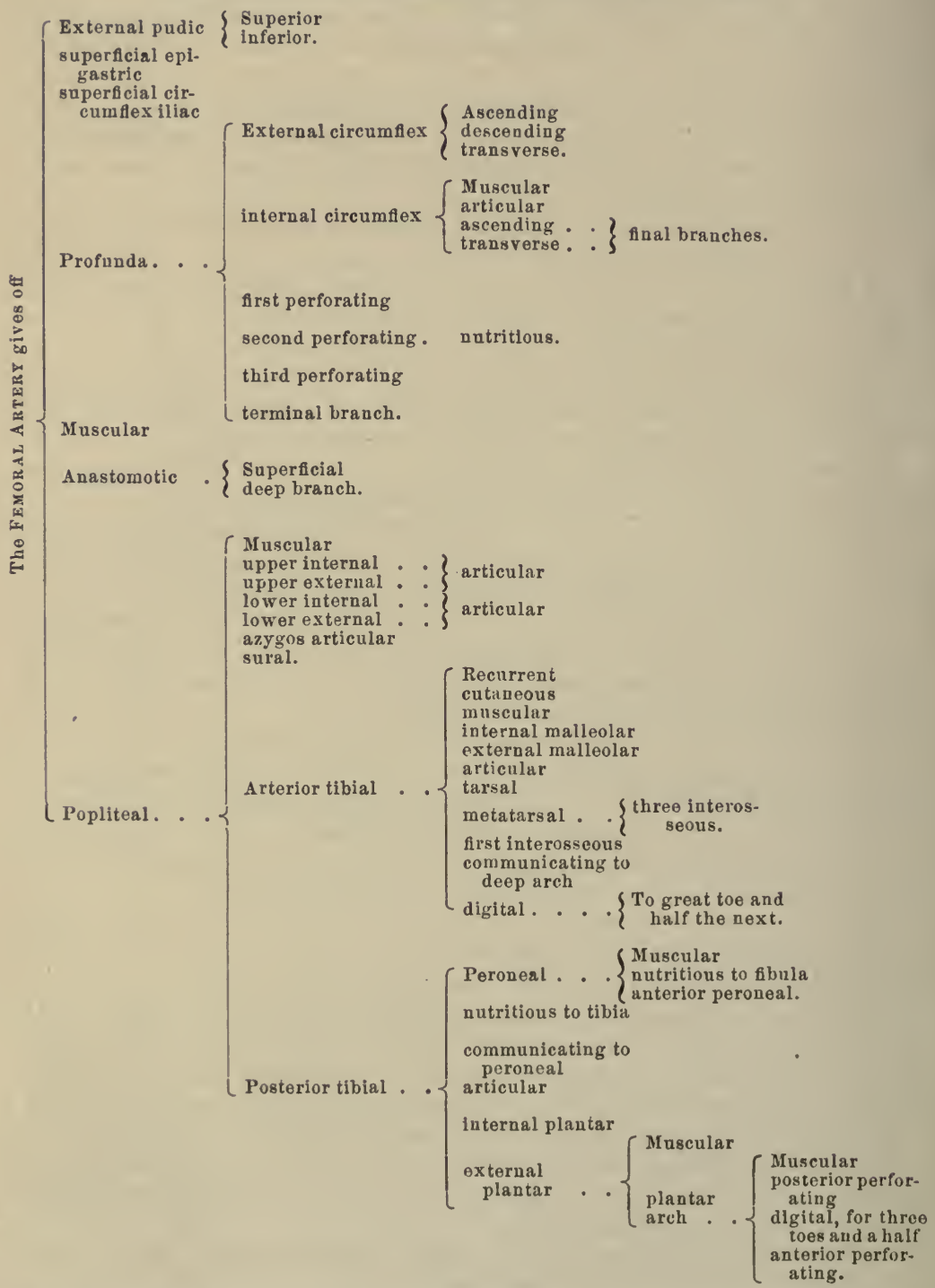

N. B. The branches of the Internal iliac artery which end in the limb, will be found in the table of the arteries of the abdomen. 
TABLE OF THE VEINS OF THE LOWER LIMB.

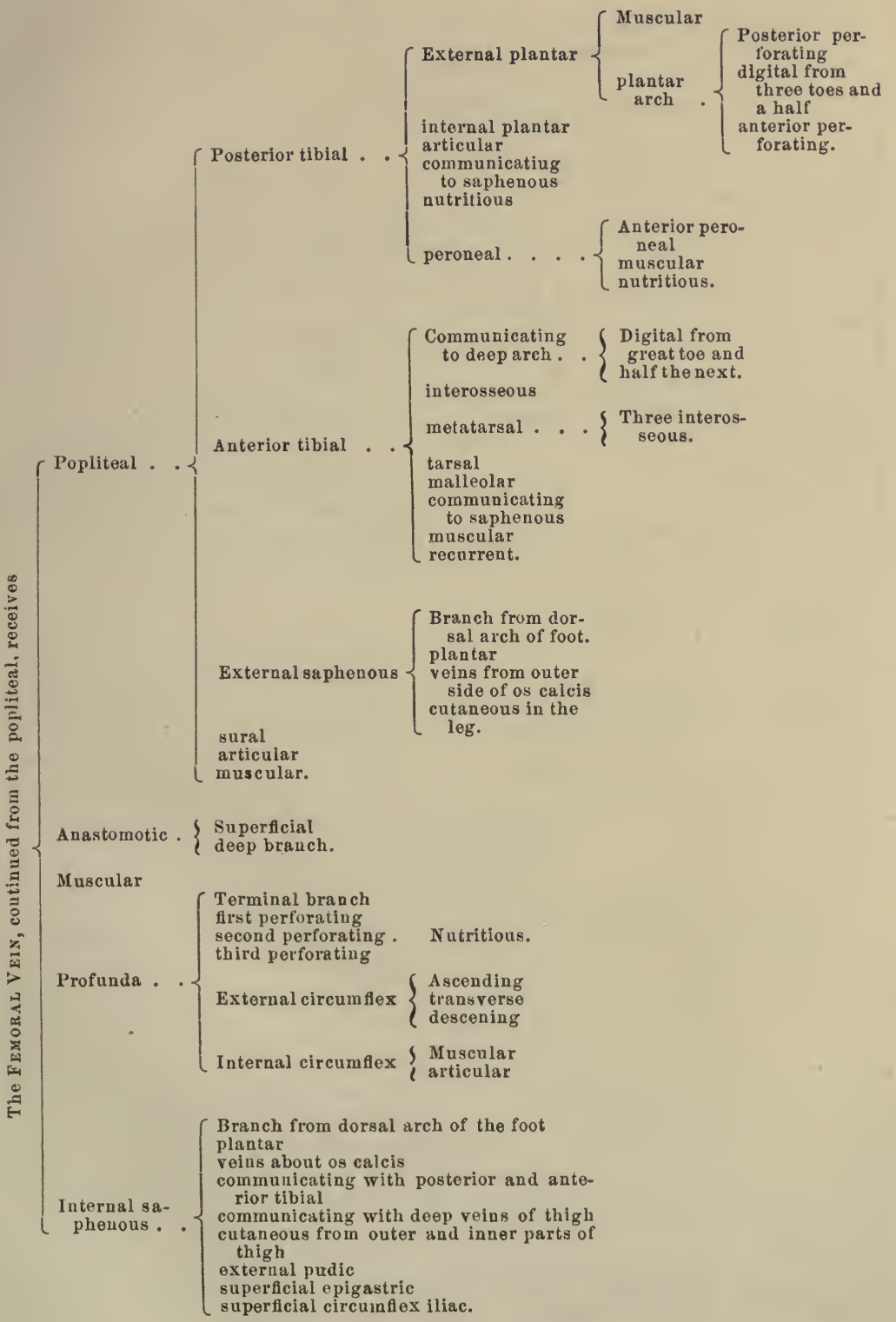




\section{TABLE OF THE NERVES OF THE LOWER LIMB.}

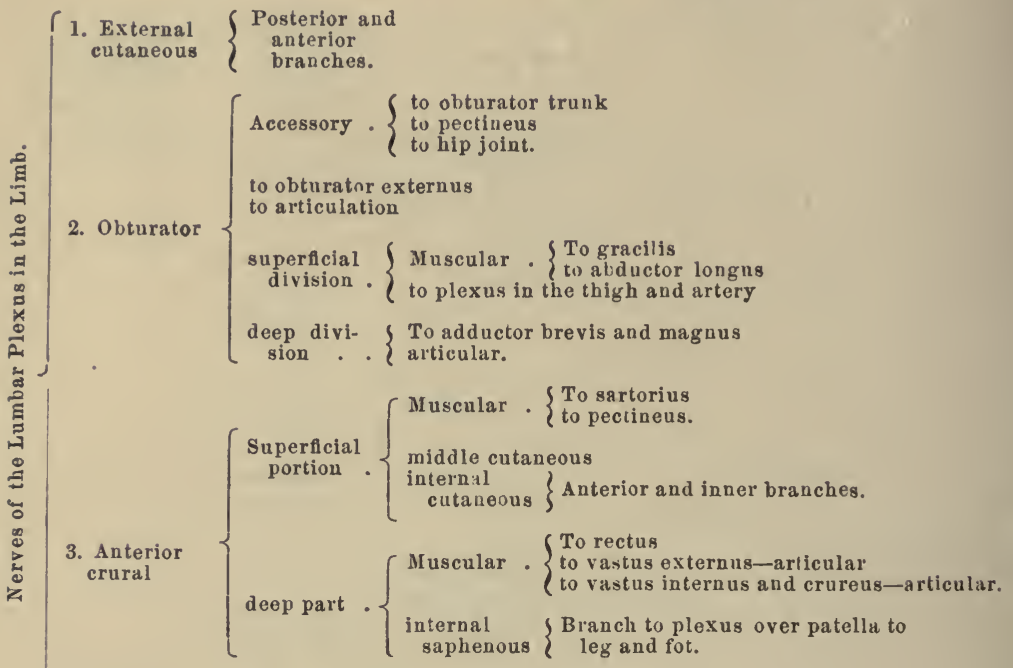

$\left.\begin{array}{c}\text { 4. Branch } \\ \text { of genito- }\end{array}\right\} \begin{gathered}\text { To integu- } \\ \text { ments. }\end{gathered}$

( crural.. $\}$ ments.

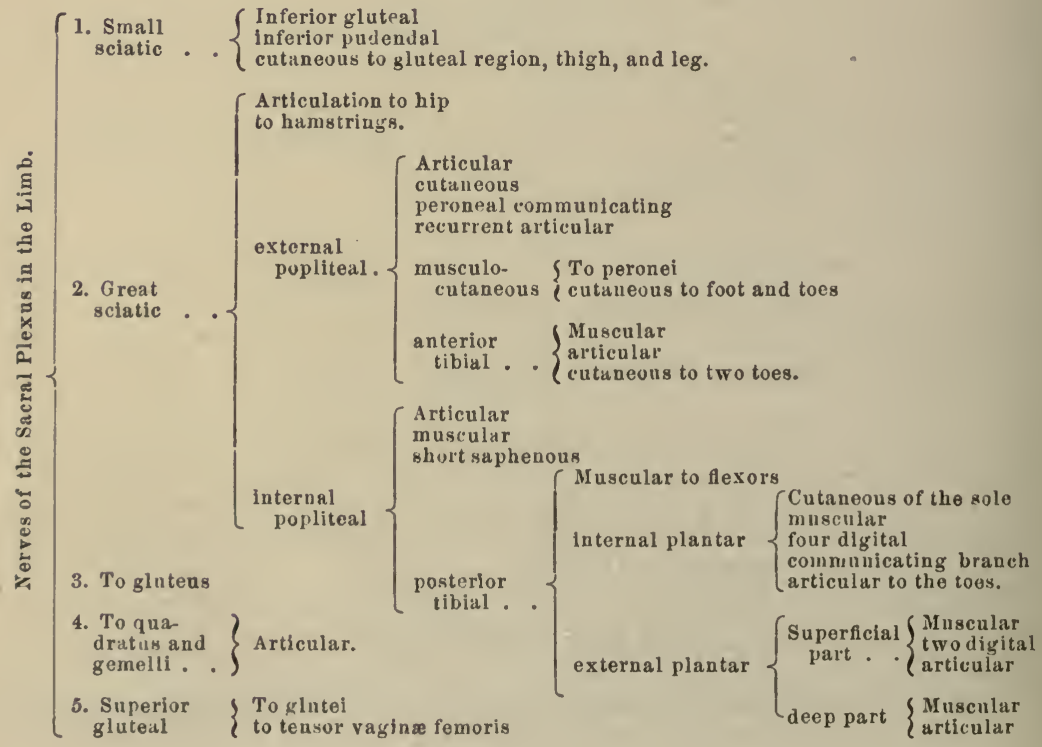




\section{CHAPTER X.}

\section{DISSECTION OF THE EYE.}

Tre eyeball is the organ of vision, and is lodged in the orbit. Supported in that hollow on a mass of fat, it is surrounded by muscles which impart movement to it; and a thin membrane (tunica vaginalis oculi) isolates the ball, so as to allow free movement.

Two lids protect the eye from external injury, and moderate the degree of light almitted into the interior; and the anterior or exposed surface is covered by a mucous membrane (conjunctiva).

Directions. In the absence of specimens of the human eye, the structure may be learnt on the eye of the ox or pig. Let the student procure half a dozen eyes of the ox for the purpose of dissection. One or two shallow basins will be needed; and some wax or tallow in the bottom of one, or in a deep plate, may be useful.

Dissection. 'l'o see the general form of the ball of the eye, and the outer surface of the external coat, the attachments of the different muscles are to be taken away; and the loose mucous membrane is to be removed from the front.

The ball of the eye (fig. 227) is roundish in form and consists of two parts, which differ in appearance, viz., an opaque posterior portion, forming five-sixths of the whole, and a smaller transparent piece (cornea) in front; these two parts are segments of different-sized spheres, the anterior belonging to the smaller sphere. To the back of the eye the optic nerve is attached, rather to the inner side of the axis of the ball; and around it nutritive vessels and the nerves enter.

The antero-posterior diameter of the ball amounts to nearly an inch ( ${ }^{9} 0$ ths), but the transverse measures an inch.

'The organ of vision is composed of' central transparent parts, with inclosing membranes or coats. The coats, posited one within another, are named sclerotic, choroid, and retina. The transparent media in the interior are likewise three, viz., the lens, the aqueous humor, and the vitreous body.

Dissection. To obtain a general idea of the structures to be dissected, the student may destroy one eyeball by cutting through it circularly: he will then be able to recognize the arrangement of the parts mentioned above, with their strength and appearance: and will be better prepared to follow the directions that are afterwards given.

Fibrous coat of the Eyeball. The outer casing of the eye consists of an opaque hinder part called sclerotic, and of an anterior transparent portion, the cornea.

The sCLERotic (cornea opaca) is the firm, whitish, and opaque portion of the external stratum of the eyeball, which supports the more delicate structures within.

Dissection. 'To examine the inner and outer surfaces of this layer it will be necessary to cut circularly with a scissors through the cornea close 
to the sclerotic, and to remove the cornea from the front of the eyeball ; on piercing the cornea the aqueous fluid escapes from the containing chamber. The outer structures may be then abstracted from the interior of the sclerotic covering, and may be set aside with the cornea for subsequent use.

Fig. 227.

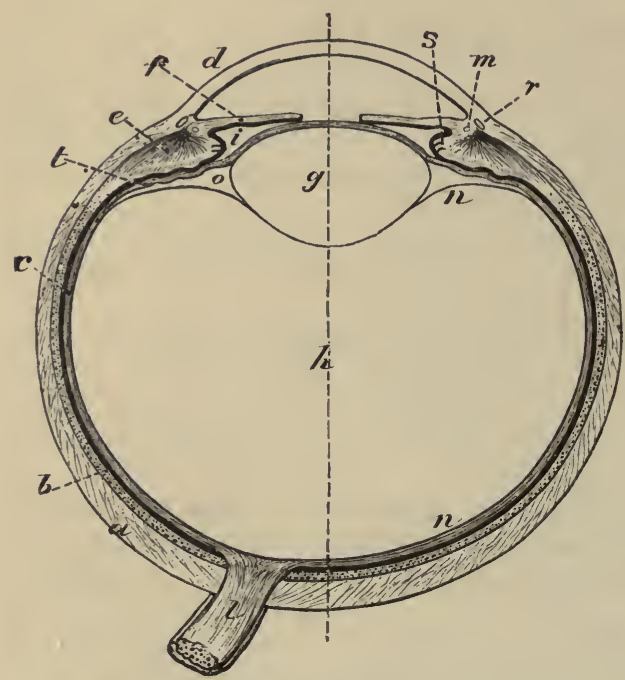

a. Outer or sclerotic coat, and $d$, the curnea.

b. Middle or choroidal coat.

$m$. Ciliary ligament.

s. Cillary process.

e. Ciliary muscle, and $f$, iris.

c. Inner $c$ at of retina, continuous with the optic nerve behind, with a dark layer outside it.

g. Lens.

$t$. Suspensory ligament of the lens.

$h$. Vitreous body.

$n$. Hyaloid membrane.

i. Posterior chamber.

o. Canal of Petit

$r$. Sinus circularis iridis.

l. Optic nerve.

The dotted line through the centre is the longitudinal axis of the ball.

Diagram of a Horizontal Section of the Eyeball.

The sclerotic tunic of the eye (fig. 227, a) is bell-shaped, and extends from the entrance of the optic nerve to the margin of the cornea, forming about five-sixths of the ball.

At its back, and a little to the inner side of the centre (one-tenth of an inch), the optic nerve $(l)$ is transmitted through an aperture in it: this opening decreases in size from without inwards, and is cribriform when the nerve is drawn out-the lattice-like condition being due to the bundles of fibrous tissue between the funiculi of the nerve. Small apertures for the passage of vessels and nerves are situate around the optic nerve; and there are others for vessels at the front and centre of the ball. Anteriorly the sclerotic is continuous with the transparent cornea.

On the outer surface this coat is smooth, except where the muscles are attached; on the inner aspect it is of a dark color with floceuli of fine areolar tissue (membrana fusca) uniting it to the next coat, and with the ends of ruptured vessels and nerves.

The sclerotic covering is thickest at the back of the eyeball, but it becomes thinner and whiter about a quarter of an inch from the cornea, where it is visible as the "white of the eye." Where it joins the cornea it becomes again somewhat thickened. In its substance near the union with the cornea is a small flattened venous space, the canal of Schlemm (sinus circularis iridis) (fig. $235,{ }^{r}$ ).

Structure. The sclerotic coat is formed of layers of white fibrous tissue, collected into bundles, with a fine network of yellow or elastic fibres. In 
it are scattered nucleated cells, fusiform in shape, or possessing rays, and some with pigment. Though interlaced with one another, the fibres have rather a longitudinal direction towards the back of the ball, and a transverse one at the outer surface near the cornea. Only a few vessels ramify in the membrane, and end in capillaries with large meshes. The presence of nerves in it is a subject for inquiry.

Cornea (fig. $227, d$ ). This firm transparent membrane (cornea pellucida) forms about one-sixth of the eyeball, and measures about half an inch transversely, but rather less from above down. Its shape is circular; though, when viewed in front, it appears largest in the transverse direction, in consequence of the opaque sclerotic structure encroaching further on it above and below than on the sides.

It is convex anteriorly, but concave posteriorly ; and it is $\frac{1}{20}$ th to $\frac{1}{30}$ th of an inch in thickness. Its anterior is of rather less extent than its posterior surface. At the circumference it is thinned, and is blended with the sclerotic coat by continuity of tissue. Supported by the aqueous humor, it deflects the light transmitted to the eye, and influences by its greater or smaller convexity degrees of sight at different distances. After death it becomes flaccid from the transudation of the aqueous humor; or, if the eye is immersed in water, it is rendered opaque by infiltration of the tissue by that fluid.

Structure (fig. 228). 'The cornea is laminar in texture. It is constructed of a special thick part called cornea proper : in front of this is the conjunctiva, and behind is the membrane of Demours.

The cornea proper, a (lamellated cornea), is made up of a series of superposed layers, about sixty in number, which join one another at numerous points, and cannot therefore be detached for any distanee. The laminæ are formed of fibres, continuous with those of the sclerotic, and are flattened into membranous layers, arranged one over another. 'This structure possesses great toughness; and its transparency is destroyed by disturbance of the position of the strata. The tissue when boiled gives chondrin.

Between the corneal layers are flattened irregular spaces, which join freely with one another; and these intervals are occupied by nucleated stellate cells, called corneal corpuscles.

In the healthy condition bloodvessels do not permeate it, but cease in capillary loops at the circuinference. Nerves ramify in it, after losing their opacity at the eircumference; they are said to form a subepithelial plexus on the anterior surface, from which varicose fibrils are prolonged amongst the pieces of the epithelium.

The membrane at the back of the cornea (fig. 228)-membrane of Demours - consists of a basement layer covered by epithelium.

Fig. 228
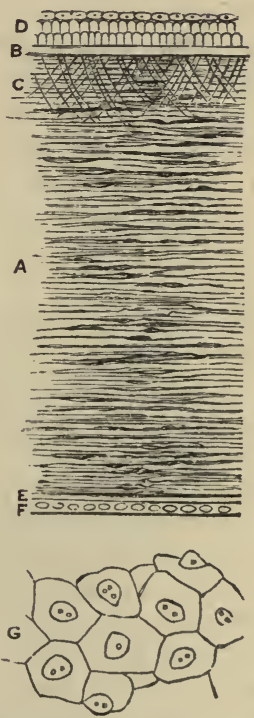

Vertical Section A of the Corsea.

b. Basement layer of cornea, with $d$, the conjunctival epithelium on it.

c. Oblique fibres from it to the layers of the cornea.

e. Basement lamina, with $f$, epithelium on it of the membrave of Demours.

G. Surface view of the epitheliurn of the membrane of $\mathrm{De}$ mours. 
The basement layer, $\mathbf{E}$ (posterior elastic lamina, Bowman), may be peeled off after a cut has been made across the cornea. It is dense; hard and elastic, measuring $\frac{1}{300}$ th $^{\text {th }}$ to $\frac{1}{200}$ th of an inch in thickness; it is very brittle, tearing readily when an attempt is made to separate it, and curls up when it is free, with the attached surface innermost. Though very elastic, the structure is destitute of fibres. It is always transparent, and remains so after boiling, after the action of acids, and even after maceration.

At the edge of the cornea this lamina breaks up into processes ("pillars of the iris") which blend with the outer margin of the iris, and with the selerotic and choroid coats.

A laminar epithelium, $\mathrm{G}$, like that on serous membranes, clothes its free surface (fig. 228).

The conjunctiva in front of the cornea (fig. 228) consists chiefly of epithelium cells, though there is a subjacent basement-like stratum.

The basement layer, B (ant. elastic lamina, Bowman), is transparent, and only from $\frac{1}{2000}$ th to $\frac{1}{1000}$ th of an inch thick. It seems to be a somewhat hardened piece of the cornea, without corneal spaces and corpuscles. From it fine threads are prolonged into the proper corneal layers, as in the woodcut.

The epithelium, $\mathbf{D}$, is formed of three or four layers of scales, the deeper being columnar, but the superficial laminar in form.

Vascular Coat of the Eyeball (fig. 231). The next covering is situate within the selerotic, and is formed chiefly of bloodressels and pigment cells : the muscles in the interior of the ball are connected with it.

It is constructed of three parts : a posterior (choroid) corresponding with the sclerotic ; an anterior (iris) opposite the cornea ; and an intermediate ring (ciliary muscle) on a level with the union of the sclerotic and cornea.

Dissection. Supposing the cornea of an eye cut through circularly, as before directed ( $p .656$ ), it will be necessary to take away the sclerotic to lay bare the choroid coat. With the point of the scalpel, or with a shut scissors, detach the fore part of the sclerotic from the front of the choroid by breaking through a soft whitish structure uniting them. Then, the eye being put into water, the outer coat is to be removed by cutting it away piece-meal with a scissors; in taking it off the slender vessels and nerves beneath are to be preserved. The white ring around the eye in front, which comes into view during the dissection, is the ciliary muscle.

For the purpose of obtaining an anterior view of the eiliary processes, which are connected with the anterior termination of the choroid coat, let the cornea be removed as before on another eyeball. Detach next the fore part of the sclerotic from the choroid ; and after two or three cuts have been made in it towarls the optic nerve, the resulting flaps may be pinned out, so as to support the eye in an upright position. On removing with care the iris, taking it away from the centre towards the circumference, the ciliary processes beneath will be displayed. A posterior view of the processes may be prepared on another ball by cutting through it circularly with a scissors, about one-third of an inch behind the cornea, so that the anterior can be removed from the posterior half; on taking away the portion of the vitreous mass adherent to the anterior part of the ball, and wiping off the pigment from the back of the iris, the small processes will be made manifest. By means of this last dissection the interior of the choroid coat may be seen. 
If a vertical section is made of another eyeball, it will show the ciliary processes in their natural position, and will demonstrate the relative situation of all the parts. This section, which is made with difficulty, should be attempted in water with a sharp large knife, and on a surface of wax or wood, after the cornea and selerotic have been cut with a scissors. When the eye has been divided, the halves should remain in water.

The CHOROID COAT (fig. $227, b$ ) is a thin membrane of a dark color, and extends from the optie nerve to the fore part of the eyeball. When viewed on the eye in which the ciliary muscle is entire, it appears to terminate there; but it may be seen in the other dissections to bend inwards behind that muscle, and to end in a series of projections (ciliary processes) behind the iris.

'This covering is rather thicker and stronger behind than in front. It is supported at the bottom of the eyeball by its close connection to the sclerotic coat, and in front by the ciliary muscle. Posteriorly it is pierced by a round aperture for the passage of the optic nerve; and anteriorly it is united with the iris.

The outer surface (fig. 231) is flocculent, and is covered by the remnants of a thin membranous layer (membrana fusca, supra-choroidea) between it and the sclerotic eoat: on it may be seen small veins arranged in arches, and the eiliary arteries and nerves. The inner surface is smooth, and touches the thin dark pigmentary layer of the retina (fig. 227).

Fig. 229.

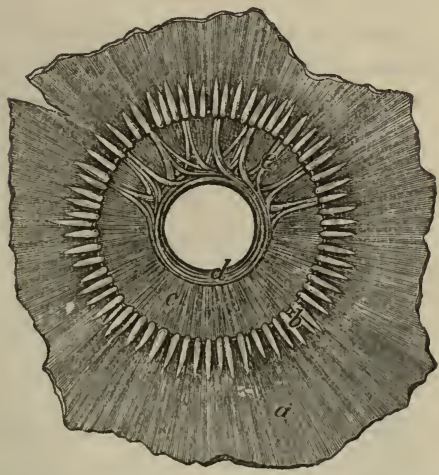

INNER VIKW OF THE FroNt OF THE CHOROHD

Coat with its Ciliary Processes, and the BACK OF THE JRIs.

a. Anterior piece of the choroid coat

b. Ciliary processes.

c. Iris.

d. Sphincter of the pupil.

$\epsilon$ Bundles of fibres of the dilator of the pupil.
Fig. 230.
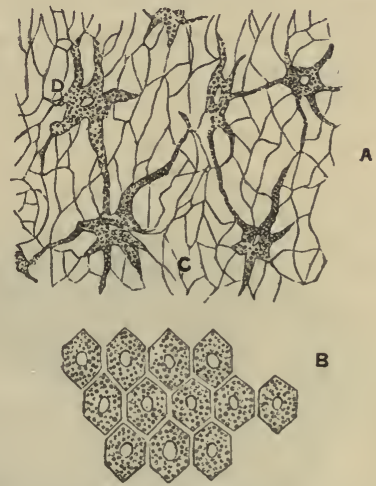

Pigment Celis of the Exeba 1.r. (Kölliker). A. Ramified pigment cells of the choroid coat. B. Front view of the bexagonal cells of the pigmentary membrane.

The ciliary processes (fig. $229, b$ ) are solid projections on the inner surface of the choroidal coat, and are arranged in a circle. About $85 \mathrm{in}$ number, they lie side by side, and consist of larger and smaller eminences; at their inner extremity they are united by transverse ridges.

About $\frac{1}{10}$ th of an inch in length, they increase in depth internally, and projecting around the lens, bound circumferentially by their free ends the 
space (fig. 227, $i$ ) (posterior chamber) behind the iris. In front they correspond with the back of the iris towards their inner end, but are separated from it by pigment; and behind they are closely connected with the membrane $(t)$ (suspensory ligament) on the front of the vitreous body, and fit into hollows between eminences on the anterior surface of that membrane.

Structure The choroid coat and its ciliary processes are formed principally of bloodvessels. Ramified pigment cells make up most of the rest of the coat.

The stroma or web of the choroid is formed by the outrunners of special pigment cells (fig. $230,{ }^{\mathrm{c}}$ ) which unite together, and construet, with fine areolar tissue, a fibrous network. Its meshes are finer towards the inner than the outer surface of the choroid. On the exterior and interior of the fibrous web the vessels ramify, with the following difference in their arrangement.

At the outer part (fig. 231) the larger branches of both arteries and veins are situate; and the veins $(h)$ form curves (vasa rorticosa) as they end in four or five chief efferent trunks. In the interspaces of the vessels are the ramified pigment cells (fig. $230,{ }^{\boldsymbol{A}}$ ), which contain a nucleus and molecular grains of dark-brown eoloring matter. At the inner part of the choroid the vessels form a network of eapillaries without pigment cells, and with meshes smaller than elsewhere, whose interstices are rather less towards the back than the front of the eyeball : this part of the choroidal coat is described sometimes as a separate layer (tunica Ruyschiana).

In the ciliary processes there is a similar texture of ramified bloodvessels, though with larger capillary meshes than in the choroid; and

Fig. 231.

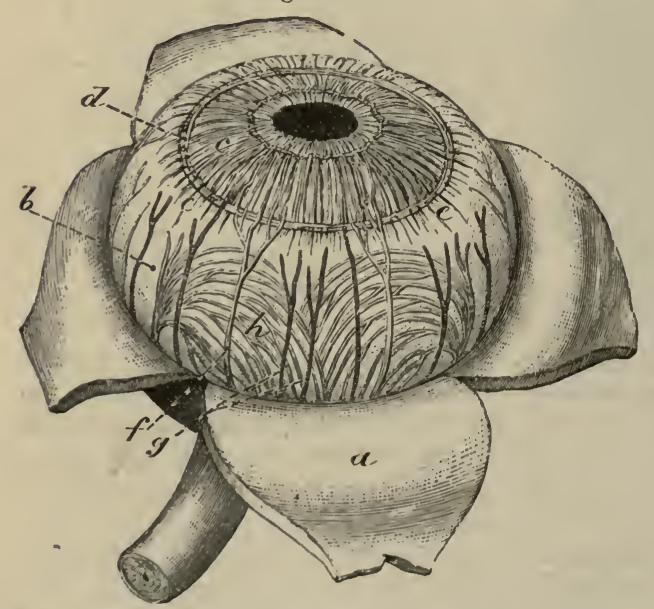

a. Sclerotic, cut, and reflected.

b. Chorold coat.

c. Iris.

d. Circular.

$e$. Rudiating fibres of ciliary muscle.

$f$. Ciliary nerves, and $g$, ciliary arteries between the two onter coats.

h. Veins of the choroid coat.

View of thB Front of the Choroidal CoAt and IRIS-external gURFACE (Zinn).

the intermixed pigment cells lose their coloring matter towards the free ends.

Ciliary muscle (fig. 231). In the eye from which the sclerotic coat has been removed, the white band of the ciliary muscle (e) (annulus 
albidus), may be seen in its natural position outside the front of the choroid coat.

It consists of unstriated fibres, and forms a grayish layer, about $\frac{1}{10}$ th of an inch wide, on the surface of the choroid coat (fig. 231,e): there are two sets of fibres in it, radiating and circular :-

The radiating or superficial, arise in front from the sclerotic coat (fig. $227, a$ ), and the fibres of the posterior elastic layer of the cornea ; its fibres are directed backwards, and are inserted into the choroid coat opposite to, and rather behind the ciliary processes. The nerves to the iris pierce it.

The circular fibres (eiliary ligament, fig. $231, d$ ) are internal to the radiating, and form a ring about $\frac{1}{40}$ th of inch wide around the edge of the iris : they are not attached to the selerotic or the cornea.

Use. The radiating fibres of the muscle draw forwards the fore part of the choroid coat, and the ciliary processes, and thereby relax the suspensory ligament of the lens. The circular fibres are said to compress the outer part of the lens.

The IRIs (fig. $231, c$ ) is a vascular and muscular structure, about half an inch in diameter, whose vessels are continuous with those of the choroidal coat. Its position and connections may be observed in the different dissections that have been prepared.

Placed within the ring of the ciliary muscle, it is suspended in front of the lens (fig. $227, f$ ), and is pierced by an aperture for the transmission of the light. It is cireular in form, is variously colored in different persons, and is immersed in the aqueous humor. By its circumference it is connected with the choroid coat and the posterior elastic layer of the cornea. The anterior surface is free in the aqueous humor, and is marked by lines converging towards the pupil. The posterior surface is covered with a thick layer of the pigment (fig. 227 ), to which the term uvea has been applied.

The aperture in it (fig. 231) is the pupil of the eye; this is slightly internal to the centre, and is nearly circular in form, but its size is constantly varying (from $\frac{1}{20}$ th to $\frac{1}{3}$ of an inch) by the contraction of the muscular fibres, according to the degree of light acting on the optic nerve.

Membrane of the pupil. In the foetus the aperture of the pupil is closed by a vascular transparent membrane, which is attached to the edge of the iris, and divides into two distinct chambers the space in which the iris is suspended. The ressels in it are continuous behind with those of the iris and the ease of the lens. About the eighth month the vessels become impervious, and at the time of birth only fiagments of the structure remain.

Structure (fig. 229). The stroma of the iris is composed of fibres of areolar tissue, arranged circularly at the eircumference, and as radiating bundles passing like rays towards the pupil. In it are involuntary muscular fibres, both circular and radiating, together with pigment cells. Vessels and nerves ramify through the tissue.

Muscular fibres. 'The sphincter of the pupil (fig. 229, $d$ ) is a narrow band about $\frac{1}{30}$ th of an inch wide, which is close to the pupil, on the posterior aspect of the iris. The dilator of the pupil $(e)$ is said to begin at the outer border of the iris, and to consist of bundles of fibres which join one another, and end internally in the sphineter.

Action. Enlargement of the pupil is effected by shortening of the radiating fibres; and diminution, by contraction of the eircular ring. The 
movements of the iris are involuntary and regulate the admission of light into the ball.

The pigment cells are spread out in the stroma, and are disposed also on both surfaces. In the stroma they are ramified and irregular, as in the choroid (fig. 230, $\mathbf{A}$ ), and may eontain yellow, brown, or very dark pigment. On the front they are ovalish or rounded, but still ramified; and behind, where there is a thick layer (uvea), the cells are round without outrunners, and are filled with granules. The color of the iris is dependent upon the tint, and the position of the pigment.

The arteries of the iris (fig. $232,{ }^{\mathrm{B}}$ ) have a looped arrangement: they are derived chiefly from the long and the anterior ciliary branches $(d)$,

Fig. 232.
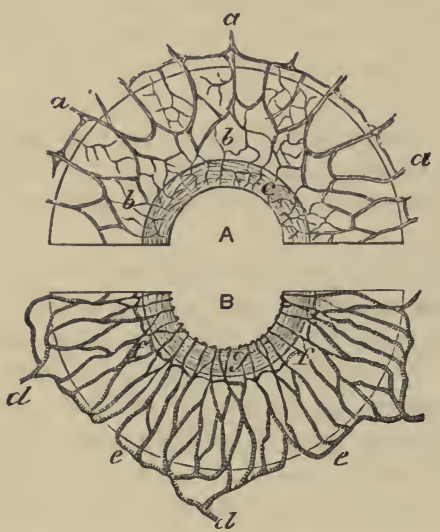

Distribetion of the NRRVFs and VesSELS OF THE IRIS.

A. Half of the iris representing the nerves.

$a$. Nerves entering the membrane, and uniting in a plexus, $b$, within it (Kölliker).

B. Half of the iris with a plan of the vessels.

d. Ciliary arteries.

e. Arch of vessels at the onter edge of the iris.

f. Inner circle of vessels in the iris.

g. Sphincter of the iris.

but some come from the vessels of the ciliary processes. On arriving at the eiliary muscle the long eiliary arteries form a circle $(e)$ around the margin of the iris; from this loop other anastomotic branches are direeted towards the pupil, near which they join in a second arterial circle $(f)$. From the last circle capillaries run to the pupil, and end in veins.

The veins resemble the arteries in their arrangement in the iris, and terminate in the veins of the choroidal coat.

The nerves of the iris (fig. 232, a) divide into branches, which communicate, and extend towards the pupil; they are withont dark outline, and their ending is not known.

Ciliary Vessels and Nerves (fig. 231). The ciliary arteries are off'sets of the ophthalmic (p. 56), and supply the choroid, the ciliary processes, and the iris. They are classed into posterior and anterior, and two of the first set are named long ciliary; but they will not be seen without a special injection of the vessels of the eye.

The posterior ciliary branches $(g)$ pierce the sclerotic coat around and close to the optic nerve, and running forwards on the choroid, enter its substance at different points.

Two of this set (long ciliary) are directed forwards, one on each side of the eyeball, and form a eirele around the iris in the ciliary muscle, as before explained. In the ball the outer one lies rather above, and the inner, rather below the mirldle.

The anterior ciliary arteries, five or six in number, are smaller than the posterior, and arise at the front of the orbit from muscular branches ( $p$. $56)$; they pierce the sclerotic coat about a line behind the cornea, supply the eiliary processes, and join the circle of the long ciliary vessels. In inflammation of the iris these vessels are enlarged, and offsets of them form a ring around the cornea. 
The posterior ciliary veins (fig. 231) are commonly four in number, and the branches entering these trunks form arches $(h)$ (vasa vorticosa) on the surface of the choroid coat: they perforate the sclerotic layer at separate points, midway between the cornea and the optic nerve, and end in the ophthalmic vein.

Anterior ciliary veins begin in a plexus within the ciliary muscle, and accompany the arteries through the sclerotic to end in the ophthalmic: they communicate with the venous space of the sinus circularis iridis (p. $656)$.

The ciliary nerves (fig. $231, f$ ) are derived from the lenticular ganglion, and the nasal nerve (p. 55). Entering the back of the eyeball with the arteries, they are continued with the vessels between the sclerotic and choroid, nearly as far as the ciliary muscle: at this spot the nerves send offsets to the cornea, and piercing the fibres of the ciliary muscle, enter the iris, but their manner of ending is unknown. Offsets from the nerves supply the ciliary muscle and the choroid.

Cimamer of the Aqueous Humor (fig. 227). The space between the cornea in front and the lens behind, in which the iris is suspended, contains a clear fluid named the aqueous humor. In the foetus before the seventh month this interval is separated into two by the iris and the pupillary membrane, but in the adult it is only partly divided, for the two communicate through the pupil. The boundaries of the two chambers may be seen in the eye on which a section has been made.

The anterior chamber is the larger part of the space; it is limited in front by the cornea, and behind by the iris.

The posterior chamber $(i)$ is a narrow interval behind the iris at the circumference, which is bounded in front by the iris; behind by the lens capsule, and by a piece of the membrane (suspensory ligament of the lens) on the front of the vitrenus humor: and at the circumference by the ciliary processes.

The aqueous humor is quite transparent, and consists nearly of pure water. A small quantity of chloride of sodium, with some extractive matter, is in solution in it.

The Retixa (fig. 227, c). This layer (tunica nervea) is in part continous with the optic nerve, and is the most delicate of all the coats of the eyeball. On it the image of objects is formed in the bottom of the eye.

Dissection. The retina can be satisfactorily examined only on an eye which is obtained within forty-eight hours after death. To bring it into view on the eyeball in which the choroid coat was dissected, the choroidal covering must be torn away earefully with two pair of forceps, whilst the eye is immersel in water or spirit.

The retina is the most internal of the three concentric strata in the globe of the eye, and is situate between the choroid coat and the transparent mass (vitreous) in the interior. It is moulded upon, and supported by the vitreous body; and its form is that of a segment of a sphere, with a large aperture in front. Beginning behind at the optic nerve (fig. 227, c) the thin layer extends forwards to the ciliary processes (their outer extremities), where it ends in a wavy border-the ora serrata.

Where the retina ceases in front, a thin gray layer (ciliary part of the retina) composed of elongated nucleated cells, which are not nerve elements, is continued on as far as the tips of the ciliary processes (fig. 235, j) on which it ends.

The retina is of a pinkish gray color, and is semitransparent when 
fresh, so that an image can be seen on it when the two external coats of the eye have been removed; but it soon loses this translucency, and is moreover rendered opaque by the action of water and other substances. Its thickness is greater at the posterior than the anterior part of the eyeball, being $\frac{1}{50}$ th of an inch in the former, and $\frac{1}{5} \frac{\text { th }}{0}$ in the latter situation.

On the outer surface of the dissected retina are some fine fragments of a structure (Jacob's membrane) to be noticed presently, which float in the

Fig. 233.

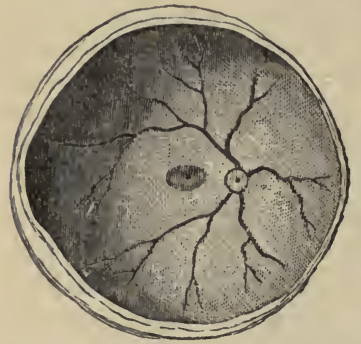

OBJECTS ON THE INNER SURFACE OF THE RETIXA. In the centre of the ball is the yellow limbus luteus, here represented by shading; and in its middle the dark spot. To the inner side is the nerve, with its accompanying artery. (Sømmerring.) fluid in which the preparation may be placed. The inner surface is smooth; it is covered with folds in a preparation of an eye cut in two, but these are accidental, in consequence of the membrane having lost its proper support. At the spot where the optic nerve expands (porus opticus) is the central artery of the retina (fig. 233).

In the anterior of the human eye, in the axis of the ball, is a slightly elliptical yellow part (fig. 233), $\frac{1}{12}$ th of an inch in diameter, which is named the yellow spot (limbus luteus of Sömmerring). Almost in the centre of that spot is a minute hollow, the fovea centralis, which appears black in consequenee of the thinness of the wall allowing the dark pigment outside to be seen.

Structure (fig. 234). In the retina are layers or strata with bloodvessels, viz., an inner $\left({ }^{3}\right)$, composed of nerve elements; an outer (1) (Jacob's membrane), formed of peculiar bodies; and an intermediate or granular layer $\left({ }^{2}\right)$; and outside all is a pigmentary stratum. Passing through the layers of the retina except Jacob's and the pigmentary, are minute threads-fibres of Müller.

The layer of nerve substance $\left({ }^{3}\right)$ is made up of nerve fibres, and of a molecular matrix containing different-sized nerve cells; these constituents have the following arrangement:-

The tubules of the optic nerve (h), having become solid in texture and gray in color from the absence of the white substance of Schwann, radiate in bundles from the end of the optic trunk, and communieate together to construct a thin web at the inner aspect of the nervous layer; this delicate network with lengthened meshes diminishes in strength as it is followed forwards.

Outside the nerve fibres is a stratum of molecular material $(f)$ with large pale pyriform and roundish nucleated nerve cells $(g)$ with offsets; this layer begins at the entrance of the optic nerve, and becomes thimner as it extends forwards. Around the optic nerve the cells are arranged in a layer one deep, but over the yellow spot they are about six or eight deep; near the ora serrata they are seattered in clusters. An offset from each cell is supposed to join internally the nerve fibres; and other offsets are prolonged externally into the molecular material $(f)$ and seem lost in it.

The outer or columnar stratum (') (Jacob's membrane) consists of two different elements_rods and cones, which are arranged with their ends inwards and outwards. 
The rods $(a)$ are elongated solid and grooved particles, which are pointed at the inner end, and are more numerous than the other elements; from their inner ends fine threads (rod-fibres) are prolonged through the outer set of granules $(c)$, and are there connected with elliptical transversely striated bodies. The cones $(b)$ are shaped like a flask with a long neck, and have the larger end turned inwards : they do not project so far out as the rods. When viewed on the outer surface, they form large isolated swellings $(m)$ amongst the ends of the rods, and at a deeper level. By their inner ends they are united with a pear-shaped cell (fig. 234) in the outer part of the granular layer, and are said to reach as far as the molecular material $(d)$ between the two sets of granules.

Fig. 234.

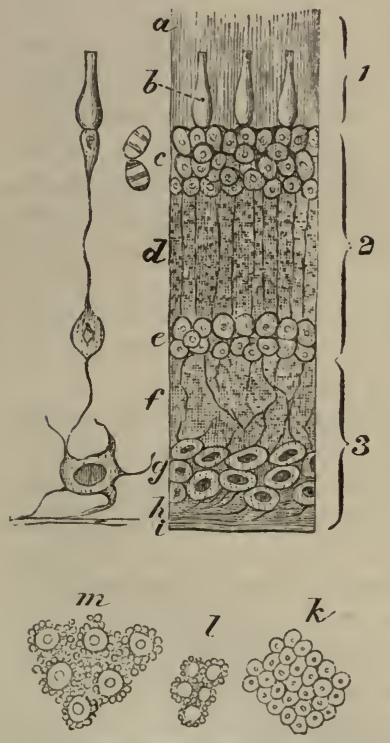

1. Columnar layer with rods $a$, and cones, $b$.

2. Granular layer with outer, $c$, and inner granules, $e$, and intermediate non-granular part, $d$.

3. Nervous layer with $g$, nerve cells, and $h$, nerve flbres: ontside the cells is a finely granular part, $f$, and inside the fibres a limiting layer, $i$, formed by fibres of Müller.

On the left of the vertical section is an ideal plan of the counection of the several parts. Thus the nerve cell, $g$, nnites interually with nerve fibres, and externally with the inner granular layer; and the inner granular layer is further joined by a thread (fibre of Mïller) to the outer granular layer.

$k$. Microscopic appearance of the outer surface of the retiua over the yellow spot, where there are only cones.

l. Appearance of the retina near the yellow spot-a single circle of rods surrounding each cone.

m. Appearance of the middle of the retina, a large number of the rods surrousding each cone. In all three figures the larger rings represent the cones, and the smaller ones the rods seen endwise.

Magnified Vertical, Section of the Retina (altered from Kölliker).

In the fresh state both are soft, clear, and homogeneous, with a glistening appearance, but these characters are soon destroyed by water and other fluids : their structure is very uncertain.

The granular layer $\left({ }^{2}\right)$ consists of innumerable rounded and ovalish bodies with nuclei, which are collected into two sets ( $c$ and $e$ ), with an intervening fine molecular material $(d)$.

In the inner set $(e)$ there are several kinds of cells, whose nature is unknown : two sets, which are oval and nucleated, are said to have this arrangement: One is connected with the fibres of Miuller; and the other has a fibre at each end, like a bipolar ganglion-cell, which is continued outwards and inwards into the molecular material $(d$ and $f$ ).

In the outer set $(c)$ two kinds of nucleated cells are to be recognized, which are connected with the fibres from the rods and cones in Jacob's layer. 'The cells of the rod-fibres, the most numerous, are elliptical, and marked by cross striæ (Henle) (fig. 234). The cells of the cone-fibres are 
pyriform in shape, with the base turned to the cone, but are free from cross stripes.

The intervening molecular portion $(d)$ has a striated appearance, from the passage of the fibres of Müller through it.

The fibres of Miiller (fig. 234) are extremely fine threads, which pierce the substance of the retina, supporting and miting the several layers, as with a framework of areolar tissue ; they reach from Jacob's layer to the inner surface. As they pass through the granular layer they are connected with very fine threads amongst both set of granules $(c$ and $e$ ) ; and in the inner set $(e)$ each possesses an oval nucleated cell. At the inner surface of the retina they are dilated and joined to each other, so as to construct a bounding stratum (i) (membrana limitans interna); and at the outer part they are united into another boundary line at the bases of the cones (membrana limitans exterua).

Structure of the yellow spot. The strata of the retina undergo modifications in the yellow spot, the chief of which are the following: externally, the rods of Jacob's layer $\left(^{1}\right)$ are absent, and the cones become less bulged and longer towards the centre. Internally, the nerve cells $(g)$ of the layer $\left(^{3}\right)$ are six to eight deep, but the nerve fibres $(h)$ gradually cease at the edge of the central fovea. 'The intermediate strata thin gradually towards the fovea centralis, where they are so blended as to render difficult their identification.

The yellow eolor is due to pigment diffused through the substance, except through Jacob's membrane and the outer set of cells $(c)$ of the granular layer. Water removes it easily.

Capillary vessels occupy the yellow spot, whilst the larger branches pass around on each side.

The pigmentary layer (fig. 227) covers the outer surface of the retina, and is eonnected thereto by fine processes. ${ }^{1}$ It consists of a single stratum of six-sided nucleated cells, with granular contents (fig. $230,{ }^{\mathrm{B}}$ ) which are applied to each other by the edges. Each possesses a nucleus towards the outer surface, and much pigment towards the inner. From the inner surface of the cells project very fine threads, which penetrate between the rods and cones of Jacob's layer. The pigmentary layer is constituted thus as far as the ora serrata of the retina; but in front of that line, viz., on the ciliary portion of the retina, the cells are rounded and full of pigment, and are continuous with the pigment on the ciliary processes and back of the iris.

Vessels of the retina. The central artery of the retina, accompanied by its vein, enters the eyeball through the optic nerve (p. 56). Inside the retina the vessel divides into four or five branches; these pierce the stratum of fibres (fig. $234, h$ ), and end in a network of capillaries amongst the ganglion cells $(g)$, and in the molecular material outside them $(f)$. In the yellow spot only capillaries exist.

In the foctus a branch of the artery passes through the centre of the vitreous mass to supply the lens capsule.

Vitreovs Bony. A transparent mass fills the greater part of the space within the coats of the eyeball (fig. $227, h$ ), which has been named vitreous body, from its resemblance to glass; it consists of a clear aqueous

1 In the dissection of the retina this pigmentary layer is removed with the choroid through the rupture of those prosesses; and it has therefore been commonly described as part of the choroid coat. 
fluid, contained in a translucent membrane, and has the consistency of jelly.

Dissection. The vitreous body may be seen on the eye on which the retina was dissected, by taking away the retina, the iris, and the ciliary muscle and processes.

To obtain a view of its anterior part, with the lens in situation, an eyeball should be fixed upright; the sclerotic and choroid coats are to be cut through about a quarter of an inch behind the cornea; and on removing carefully the cornea, ciliary muscle, and processes with the iris, the vitreous body will be apparent.

The vitreous body (fig. 227, $h$ ) is globular in form, and fills four-fifths of the ball, supporting the retina. In front it is slightly hollowed, and receives the lens with its capsule $(g)$, to which it is closely united. The fluid of the vitreous body has nearly the same composition as the aqueous humor with some salts and albumen. Enveloping the whole is a thin membrane named liyaloid.

The hyaloid membrane $(n)$ is a fine transparent covering of the vitreous body. It passes continuously over the surface; and in the foctus it is eonnected with fibres which penetrate the mass. On the inner aspect are a few delicate nuclei.

At the fore part it is joined by the membrane $(t)$ supporting the lens; and it ends by uniting with the back of the lens capsule. At the bottom of the ball the membrane is closely connected with the retina.

Structure of the vitreous mass. From the slow escape of the fluid after puncture of the hyaloid membrane, it has been supposed that there are membranous partitions, dividing the vitreous mass into compartments which communicate with each other. In the centre of the vitreous body is a fibrous canal, which reaches to the back of the lens, and transmits a branch from the central artery of the retina to the lens capsule in the foetus.

The vitreous mass and the hyaloid membrane are extra-vascular, and receive their nutritive material from the vessels of the ciliary processes and retina.

Suspensory ligament of the lens (Zonule of Zinn). This is a transparent membranous structure (fig. $23 \overline{5}, t$ ), placed around the lens eapsule,

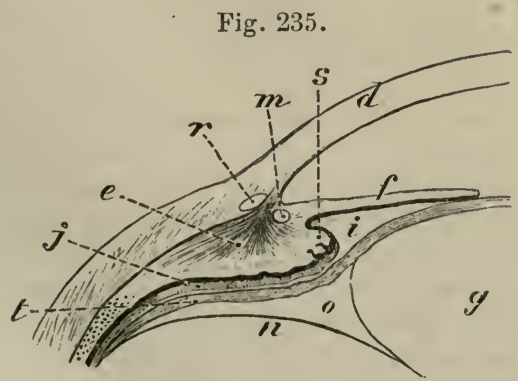

d. Cornea.

e. Ciliary muscle.

f. Iris.

g. Lens.

i. Posterior chamber.

j. Ciliary part of the retina.

$m$. Ciliary ligament.

$n$. Ilyaloid membrane.

o. Canal of Petit.

$r$. Sinus circularis iridis.

s. Ciliary process.

$t$. Suspeusory ligament of the lens.

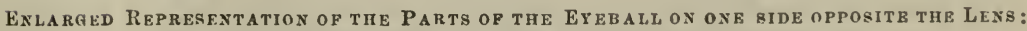
THE LETTERS REFER TO THE SAME PARTS AS IN THE WOODCUT, 227.

which joins externally the hyaloid membrane opposite the anterior termination (ora serrata) of the retina. After the ciliary processes of the choroid coat are detached from it, dark lines of pigment cover the surface; 
and when these are washed away plaits (ciliary processes) come into view, which are less prominent and longer than the processes of the choroid coat, and do not quite reach the lens capsule internally. The two sets of prominences are dovetailed together-the projections of one being received into hollows between the other. In this membrane are stiff longitudinal and elastic fibres. The tenseness or laxness is influenced by the state of the ciliary muscle; for during its contraction the membrane is rendered lax by the drawing forwards of the ciliary processes.

Canal of Petit. Around the margin of the lens capsule is a small canal (fig. 235, o) about one-tenth of an inch across, which is situate between the suspensory ligament and the front of the hyaloid membrane. When the canal has been opened, and filled with air by means of a blowpipe, it is saceulated at regular intervals, like the large intestine, by the inflation of the plaits of the anterior boundary. 'The margin of the capsule of the lens projects into the space.

Lexs AND ITS CAPSULE. The lens is situate behind the pupil of the eye (fig. $227, g$ ), and brings to a focus on the retina the light passing through that aperture.

The capsule is a firm and very elastic transparent case, which is permeable to fluid, and closely surrounds the lens: it is seated in a hollow on the front of the vitreous body. The anterior part projects towards the pupil; whilst the posterior is received on the vitreous mass, to which it is inseparably united. 'The circumference of the case corresponds with the canal of Petit $(o)$.

Its anterior surface (fig. 227) is free, and touches the iris $(f)$, but is separated from it by a slight space at the outer part (posterior cliamber, $i$ ); it gives attachment towards the circumference to the suspensory lig:tment $(t)$.

The fore part of the capsule is four or five times thicker than the posterior, as far outwards as the attachment of the suspensory ligament, and supports itself after the removal of the lens; it is firm and quite transparent, and remains clear for some time when immersed in spirit, acids, and boiling water. like the elastic layers of the cornea. The posterior part of the capsule is thin and membranous, and decreases in thickness towards the centre: it is joined by the hyaloid membrane $(n)$ of the vitreous body.

In the adult human eye the capsule of the lens is not provided with bloodvessels; but in the foetus a branch of the central artery of the retina supplies it (p. 666).

Dissection. The lens will be obtained by cutting across the thin membranous capsule in which it is inclosed.

The lens is a solid and transparent doubly convex body ; but the curves are unequal on the two surfaces (fig. 227, g), the posterior being greater than the anterior. Its margin is somewhat rounded; and the measurement from side to side is one-third of an inch, but from before back about onefourth of an inch. The density increases from the circumference to the centre; for whilst the superfieial part may be rubbed off with the finger, the deeper portion is firm, and is named the nucleus.

On each surface are three lines or stella diverging from the centre, and reaching towards the margin; they are the edges of planes or septa, and are so situate that those on one side are intermediate in position to those on the other. In the human eye they are not distinctly seen, because they bifurcate repeatedly as they extend outwards. 
Covering the anterior surface of the lens, and connecting it with the capsule, is a layer of very transparent nucleated polygonal cells, which can be recognized only in a fresh eye : these become elongated towards the circumference of the lens, and seem to pass into the superficial lens-fibres.

Structure. After the lens has been hardened by spirit or boiling, it may be demonstrated to consist of a series of layers (fig. 236) arranged one within another, like those in an onion. Under the microscope each layer may be seen to be constructed of minute parallel fibres. It consists mostly of albumen, and no bloodvessels are found in its texture.

The lamina of each surface have their apices in the centre, where the septa meet; they may be detached from one another at that spot, and may be turned outwards towards the equator of the lens.

The constituent fibres of the lamina are about $\frac{1}{5000}$ th of an inch in diameter, solid, and flattened at the margin of the lens; and the deeper

Fig. 236.

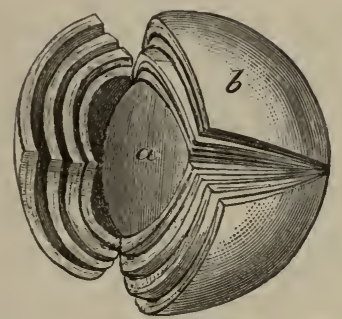

A RePresentation of THE LAMIN I IN A HARDENED LENS.

a. The nucleus.

b. Superficial laminæ.

fibres are narrowed and less distinct. In the superficial softer fibres are contained granular nuclei (fig. 237, a).

The edges are slightly waxy; and each fibre touches six others (fig. $237, b$ ), viz., two on each side, with one above, and another below : contiguous fibres are therefore dovetailed together, and this interlocking is best seen in the lens of the codfish.

The ends are soft and not well-defined, and are connected with the partitions on the opposite surfaces of the lens in this way:-those attached at the union of two septa on the one aspeet, are fixed to the extremity of a septum on the other aspect: and the fibres passing between two septa are nearer to the pole at one end, and farther from it at the other, while the middle ones are at the same distanee from the ends of the septa on both aspects.

Changes in the lens with age. 'The form of the lens is nearly spherical in the finetus; but its convexity decreases with age, particularly on the anterior aspect, until it becomes flattened in the adult.

In the foetus it is soft, is reddish in color, and is not quite transparent; in mature age it is firm and elear; and in old age it becomes flatter on both surfaces, denser, and of a yellowish color. 


\section{CHAPTER XI.}

\section{DISSECTION OF THE EAR.}

THE organ of hearing is made up of complex parts, which are lodged in, and attached to the surface of the temporal bone.

The fundamental structure, as in the eyeball, is an expansion of a special nerve over a membrane containing fluid. This delicate apparatus is inclosed in bone for its protection; and it is surrounded by certain accessory bodies which convey to it the vibrations produced by the sonorous undulations of the air.

The auditory apparatus may be arranged into the parts outside, and those within the substance of the temporal bone.

In the ExTERNaL seT which may be first examined, are included the pinna or auricle, and the auditory canal: the former has been noticed at p. 45, and the latter is described below.

The AUDITORY CANAL (fig. 238) (meatus auditorius externus) is the passage which leads from the pinna to the cavity (tympanum) in the temporal bone, and transmits inwards sonorous undulations of the air.

Dissection. To obtain a view of this canal, a recent temporal bone is to be taken, to which the cartilaginous pinna remains attached. After the removal of the soft parts, the squamous piece of the bone in front of the Glaserian fissure is to be sawn off; and the front of the meatus, except a ring internally which gives support to the thin membrana tympani, is to be cut away with a bone forceps.

The canal (fig. 238) is about one inch and a quarter in length, and is formed partly by bone, and partly by cartilage. It is directed forwards somewhat obliquely. In shape it is rather flattened from before backwards; and it is narrowest in the osseous part. The outer extremity is continuous with a hollow (concha) of the external ear, and the inner is closed by the membrana tympani.

The cartilaginous part $(u)$ is largest. It is about half an inch in length, and is formed chiefly by the pinna of the outer ear which is attached to the margin of the meatus; but at the upper and posterior aspect the cartilage is deficient, and the tube is closed by fibrous tissue. One or two fissures (fissures of Santorini) cross the piece of cartilage.

'The osseous part $(b)$ is about three-quarters of an inch long in the ardult, and is constricted sometimes about the middle. Its outer extremity is dilated, and the posterior part projects farther than the anterior; the greater portion of the margin is rough, and gives attachment to the cartilage of the pinna. The inner end is smaller, and is marked in the dry bone by a groove for the insertion of the membrane of the tympanum; it is so sloped that the anterior wall juts beyond the posterior by about two lines.

In the foetus the osseous part of the meatus is absent. After birth it grows out of the osseous ring (tympanic bone) which supports the membrana tympani.

Lining of the meatus. A prolongation of the integument lines the 
auditory passage, and is united more closely to the osseous than the cartilaginous portion; it is continued over the membrane of the tympanum in the form of a thin pellicle. At the entrance of the meatus are a tew hairs. In the subcutaneous tissue of the cartilaginous part of the meatus lie some ceruminous glands of a yellow-brown color, resembling in form and arrangement the sweat glands of the skin; these secrete the ear wax, and

Fig. 238.

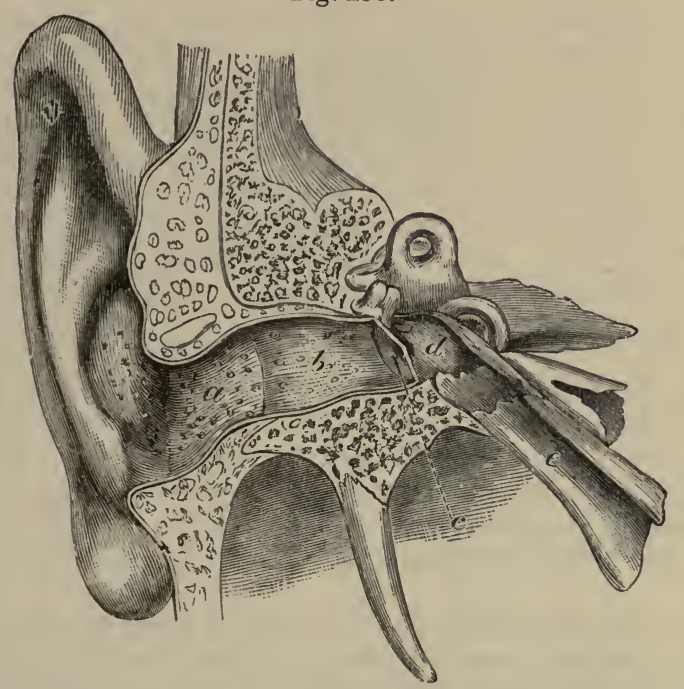

Vkrtical, Section of the Meatus A editorits and Tympandu (Scarpa).

$a$. Cartilaginous part of the meatus.

$d$. Cavity of the tympanum.

b. Osseous portion.

e. Eustachian tube.

c. Membrana tympani.

open on the surface by separate orifices: they are absent in the osseous part, and are most abundant in that portion of the tube which is formed by fibrous tissue.

Vessels and nerves. The meatus receives its arteries from the posterior auricular, the internal maxillary, and the temporal branch of the external carotid. Its nerves are derived from the auriculo-temporal branch of the fifth nerve, and enter the auditory passage between the bone and the cartilage (p. 96).

INNER PARTS OF THE EAR. The internal constituents of the organ of hearing are inclosed within the temporal bone, and consist of two large spaces-tympanum and labyrinth, with their accessory parts.

The trmpaxum, or drum of the ear (fig. $238, d$ ), is a hollow interposed between the meatus auditorius and the labyrinth. It communicates with the pharynx by a tube (e) (Eustachian), through which the air has access; and it is traversed by a chain of small bones, with which special muscles and ligaments are connected. Minute vessels and nerves are contained in the space.

Dissection. The tympanic cavity should be examined in both a dried and a recent bone.

On the dry temporal bone, after removing most of the squamous portion 
by means of a vertical cut of the saw through the root of the zygoma and the Glaserian fissure, the tympanum will be brought into view by cutting away with the bone forceps some of the upper surface of the petrous portion, and the anterior part of the meatus anditorius.

In the recent bone prepare the dissection as above, but without doing injury to the membrana tympani, the chorda tympani nerve, and the chain of bones with its muscles.

Form. The cavity of the tympanum has the form of a slice of a small cork, about a quarter of an inch thick, the outer and innel boundaries being flattened and the circumference circular. Its size is greater across the space, than from without inwards; in the former direction it measures about half an inch, but in the latter not more than a quarter of an inch.

The inner boundary (fig. 239) is of greater extent than the outer, and on it the following objects are to be noticed. Occupying nearly the whole surface is the large projection of the promontory $(a)$; this is pointed posteriorly, and is marked by two or three minute grooves $(d)$ which lorlge the nerves in the anastomosis of Jacobson. Above and below the narrowed part of the promontory is a large aperture; both lead into the labyrinth.

The upper opening $(b)$, semicircular in shape, with the convexity placed upwards, is named fenestra ovalis: into it the inner bone (stapes) of the osseons clain is fixed. The lower aperture (c)-fenestra rotunda, is rather triangular in shape, and leads into the cochlea ; it is situate within a hollow, which is somewhat semi-elliptical in form. In the recent state it is closed by a thin membrane, the secondary membrane of the tyinpanum.

The outer houndary of the cavity is formed by the membrana tympani (fig. 238, c), and the surrounding bone. Above and in front of the membrane, is the Glaserian or glenoid fissure, which is occupied in the fresh condition by the long process of one of the small bones (malleus); by a small muscle, the laxator tympani; and by tympanic vessels. Crossing the membrane towards the upper part is the chorda tympani nerve, which issues through the Glaserian fissure.

The circumference of the tympanum is circular, and is rough and uneven on the surfice here and there: round it the student may observe the following facts.

The roof is flattened, and consists of the thin osseous plate separating the tympanic cavity from the cranium. The floor is narrower than the roof, and is curved over the subjacent jugular fossa ; it has more or less of an areolar condition, and some small apertures communicate with the fossa beneath.

At the posterior part of the circumference, towards the roof, is one large with other small apertures, leading into the mastoid cells (fig. 239). Below those apertures, but near the inner wall and on a level with the narrowed part of the promontory, is the small conical hollow projection (e) of the pyramid; this is perforated by an aperture, and contains the stapedius muscle; a minute canal connects its cavity with the aqueduct of Fallopius: sometimes a small round spiculum of bone attaches the pyramid to the promontory. In a line with the pyramid, and arching upwards above the fenestra ovalis, is a bony ridge $\left(f^{*}\right)$ marking the situation of the aqueduct of 'Fallopius.

The front of the tympanic cavity corresponds with the carotid artery, only a thin piece of bone intervening. In it are the apertures of two 
canals which lie on the outer side of the passage for the vessel : the upper (h) contains the tensor tympani muscle; and the lower ( $i$ ) is the Eustachian tube. Between the two canals is a thin osseous lamina, which is hollowed above and dilated at the inner end, and is named processus cochleariformis.

Fig. 239.

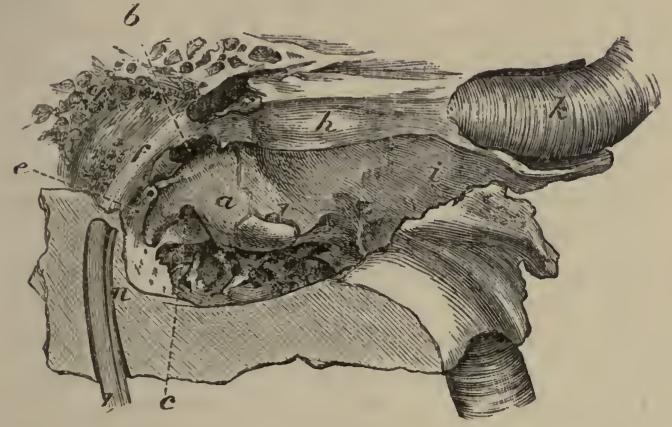
a. Promontory.
b. Fenestra ovalis.
c. Fenestra rotunda.
d. Nerve grooves on the promou- tory.
e. Pyramid.
$f$. Prominence of aqueduct of Fallopins.
g. Openings of mastoid cells.
$h$. Canal of tensor tympani.
i. Osseous part of Eustachian tube.
k. Internal carotid artery.
l. Facial nerve.

View of the InNer Wali of the TrMpaNum ENLARged (Bowman).

Some parts that have been referred to above, viz., the membrana tympani, the Eustachian tube, and the secondary tympanic membrane, require separate notice.

The membrana tympana (fig. $240, a$ ) is a thin translucent stratum between the meatus auditorius and the cavity of the tympanum. It measures one-third of an inch across, is oral in form, and is attached by its circumference to a groove at the inner end of the auditory passage; but in the foetus it is fitted into a separate osseous ring, the tympanic bone. The membrane is placed very obliquely so that it forms with the floor of the meatus an angle of 45 degrees, and the outer surface is directed downwards. Towards the auditory canal the surface is concave; but in the tympanum it is convex, and attached to its upper half is the handle of the malleus $(b)$-one of the ossicles.

Structure. The membrane is formed of three strata-external, internal, and middle. The outer one is continuous with the integuments of the meatus auditorius; and the inner is derived from the mucous membrane of the tympanum. The middle layer is formed of white and yellow fibrous tissues, and is fixed to the groove in the bone. From its centre, where it is connected with the handle of the malleus, fibres radiate towards the circumference; and near the margin, at the inner surface, lies a band of stronger circular fibres (fig. 240, $c$ ), which bridges across the notch at the upper part of the tympanic bone.

The Eustachian tube (fig. $238, e$ ) is the channel through which the tympanic cavity communicates with the fauces. It is about an inch and a half in length, and is directed downwards and inwards to the pharynx. Like the meatus auditorius, it is partly osseous and partly cartilaginous in texture.

The osseous part is rather more than half an inch in length, and is narrowed at the middle. Its course in the temporal bone is along the angle 
Fig. 240.

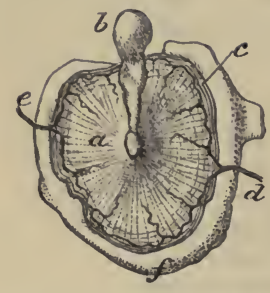

INAER VIEW OF THE MEMBRANA TYMPANI IN THE FotTs, WITH THE MALLKES ATTACHED.

a. Membrane or drum of the tympanum.

b. Malleus.

c. Band of circular fibres at the circumference.

$d$. Inferior, and $e$, superior tympanic artery.

$f$ Tympanic bone.

of union of the squamous and petrous portions, outside the passage for the carotid artery. Exterually it ends in a dilated and somewhat oval opening, with an irregular margin, which gives attachment to the cartilage.

The cartilaginous part of the tube is nearly an inch in length, and extends from the temporal bone to the interior of the pharynx (p. 126).

Through this tube the mucous membrane of the drum of the ear is continuous with that of the pharynx; and through it the mucus escapes.

The secondary membrane of the tympanum is placed within the fenestra rotunda, and is rather concave towards the tympanum, but convex towards the cochlear passage, which it closes.

It is formed of three strata, like the membrane on the opposite side of the cavity, viz., an external or mucous, derived from the lining of the tympanum; an internal or serous, continuous with that elothing the cochlea; and a central layer of fibrous tissue.

Ossicles of the Tympanum (fig. 242). 'Three in number, they are placed in a line across the tympanic cavity. The suter one is named malleus from its resemblance to a mallet; the next, incus, from its similitude to an anvil; and the last, stapes, from its likeness to a stirrup. For their examination the student should be provided with some separate ossicles.

The malleus (fig. 241) is the longest bone, and is twisted and bent. It is large at one end (a) (head) and small and pointed at the other $(c)$ (handle); and it has two precesses with a narrowed part or neck. The head or capitulum $(a)$ is free in the cavity, is oral in shape, and is smooth except at the back, where there is a depression for articulation with the next bone. The neck $(b)$ is the slightly twisted part between the head and the processes. The handle or manubrium $(c)$ decreases in size towards the tip, and is flattened from before backwards; but at the extremity it is compressed from within outwards : to its outer margin the special fibrous stratum of the membrana tympani is connected.

The processes of the bone are two in number, long and short. The short one (e) springs from the root of the handle on the outer side, and touches the membrane of the tympanum. The long process-processus gracilis $(d)$, is a flattened slender piece of bone, which projects from the neck of the malleus at the anterior aspect, and extends into the Glaserian fissure: in the adult this process is joined with the surrounding bone, and cannot be separated from it.

The incus is a flattened bone (fig. 241), and possesses a body and two processes. The body $(b)$ is hollowed at the upper and anterior part to articulate with the malleus. The two processes (short and long) extend from the side opposite to the articulation:-The shorter process $(c)$ is somewhat conical, and is received into the large aperture of the mastoid cells: the long process $(d)$ decreases towards the extremity, where it curves, and ends in a rounded point (e), the orbicular process.

The stapes (fig. 241) has a base or wider part, and a heal, with two sides or crura, like a stirlup. The base $(d)$ is formed by a thin osseous 
plate, which is convex at one margin and almost straight at the other, corresponding with the shape of the fenestra ovalis: the surface turned to the vestibule is convex, while the opposite is excavated. The head $(a)$ is marked by a superficial depression which receives the orbicular process of the incus; and below it is a constricted part (b), the neck of the bone. The crura $(c)$ extend from the neck to the base, and are grooved on the inner surface: the anterior crus is shorter and straighter than the other.

Fig. 241.
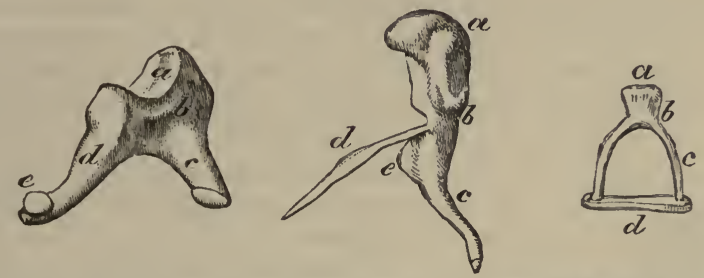

THE THRE OSgICLES OF THE TyMPANUM: THE CENTRAL BONB IS THE MALLEUS, THE LEFT-HAND BONE INCUS, AND THE RIGHT-HAND ONE STAPES.

Malleus : $a$. head; $b$, neck; $c$, long handle; $d$, long, and $e$, short process.

Incus : $a$, bead; $b$, body ; $c$, short process ; $d$, long process ; $e$, orbicular process.

Stapes: $a$, head ; $b$, neck ; $c$, crus ; $d$, base.

Position of the ossicles (fig. 242). The malleus $(d)$ is placed vertically in the tympanum, with the head upwards, and the joint surface turned backwards to articulate with the incus. Its handle is inserted externally into the membrana tympani between the mucous and fibrous layers; and its long process is directed forwards into the Glaserian fissure.

The incus $(e)$ is also upright, so that the long process is vertical, and the short one horizontal. Externally it is united with the malleus; the short process is received into the large aperture of the mastoid cells; and the long one descends, like the handle of the malleus, but rather posterior to it and nearer the inner wall of the cavity, to join inferiorly with the stapes.

The stirrup bone $(f)$ has a horizontal position, with the crura directed forwards and backwards : its base is fixed over the fenestra ovalis, and its head is united with the long process of the incus.

Ligaments of the ossicles. The small bones of the tympanic cavity are united into one chain by joints, and are further kept in position by ligaments fixing them to the surrounding wall.

Joints of the bones. Where the ossicles touch they are connected together by articulations corresponding with the joints of larger bones; for the osseous surfaces are covered with cartilage, are surrounded by a thin capsular ligament of fibrous tissue, and lubricated by a synovial sac. One articulation of the nature above described exists between the heads of the malleus and incus, and a second between the extremity of the long process of the incus and the head of the stapes.

Union of the bones to the wall. The bones are kept in place by the reflection of the mucous membrane over them, and by special ligaments. From the head of the malleus a short suspensory band of fibres is directed upwards to the roof of the tynpanum. Another ligamentous band (posterior) passes backwards from the incus, near the end of its short process, 
to the bone supporting it. And the base of the stapes is connected to the margin of the fenestra ovalis by fibres constituting an orbicular ligament.

In the recent bone the thin mucous membrane closes the interval between the crura of the stapes, and is attached to the groove on the inner aspect.

Muscles of the ossicles (fig. 242). Three muscles which possess transversely striated fibres are connected with the chain of bones; two of these are attached to the malleus. the other to the stapes.

The tensor tympani (fig. $242, h$ ) (internal muscle of the malleus) is the largest and must distinct of the muscles of the tympanum, and takes the shape of its containing tube, which must be laid open to see it completely. The muscle arises from the surface of its bony canal, also slightly in front from the cartilage of the Eustachian tube. Posteriorly it ends in a ten-

Fig. 242.

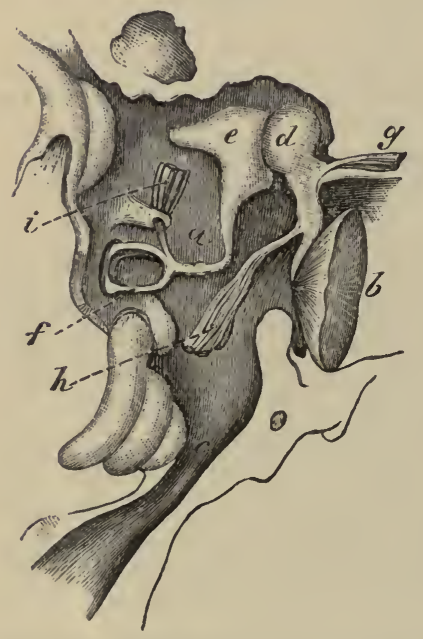

Plan of the Osgicies in Position in THE TYMPANCM WITH THEIR MUSCLES.

a. Cavity of the tympanum.

b. Membrana tympani.

c. Eustachian tube.

d. Malleus.

e. Incus.

f. Stapes.

g. Laxator tympani muscle.

h. Tensor tympani.

i. Stapedius. don which is reflected over the end of the cochleariform process, and is inserted into the inner border of the handle of the malleus, near its base.

Action. The muscle draws inwards the handle of the malleus towards the inner wall of the tympanic cavity, and tightens the membrane of the tympanum; and as the long process of the incus is moved inwards with the malleus the base of the stapes will be pressed into the fenestra ovalis.

Laxator tympani (fig. $242, g$ ) (external muscle of the malleus). It is connected externally with the spinous process of the sphenoid bone; and passing through the Glaserian fissure, it is attached to the neck of the malleus above the processus gracilis.

Action. The muscle draws inwards and forwards the upper part of the malleus, and tilts outwards the handle, so as to relax the membrana tympani; and on the cessation of the contraction of the tensor, it can move out the bony chain.

The stapedius (fig. $242, i$ ) is lodged in the canal holowed in the interior of the pyramid. Arising inside the tube, the muscle ends in a small tendon, which issues from the pyramid, and is inserted into the neck of the stapes at the posterior part.

Action. By directing the neck of the stapes backwards the muscle raises the fore part of the base out of the fenestra ovalis, diminishing the pressure on the fluid in the vestibule; and supposing it to contract simultaneously with the tensor, it would prevent the sudden jar of the stapes on that fluid.

Mucous membrane of the tympanum. The mucous lining of the tympanic cavity adheres closely to the wall; it is continuous with that of the 
pharyn $x$ through the Eustachian tube, and is prolonged into the mastoid cells through the apertures leading into them.

It assists to form part of the membrana tympani, and of the secondary membrane in the fenestra rotunda; it is reflected also over the chain of bones and the muscles, ligaments, and chorda tympani nerve. In the tympanum the membrane is thin, not very vascular, and secretes a watery fluid; but in the lower part of the Eustachian tube it is thick and more vascular, is provided with numerous glands, and its epithelium is laminated.

Its surface is covered with a columnar ciliated epithelium, but on the membrana tympani and the ossicula it is laminar and unciliated.

Bloodvessels. The arteries of the tympanum are furnished from the following branches of the external carotid, viz., internal maxillary, posterior auricular, ascending pharyngeal ; and some offsets come firom the internal carotid in the temporal bone. The veins join the middle meningeal and paryngeal branches.

The internal maxillary artery supplies the tympanic branch (fig. 240, d) (inferior, p. 93), which enters the cavity through the Glaserian fissure, and distributes a branch to the membrane of the tympanum.

The stylo-mastoid branch of the posterior auricular artery (p. 86), entering the lower end of the aqueduct of Fallopius, gives twigs to the back of the cavity, and the mastoid cells. One of this set, superior tympanic (fig. $240, e$ ), anastomoses with the tympanic branch of the internal maxillary artery, and forms a circle around the membrana tympani, from which offsets are directed inwards.

Other branches from the ascending pharyngeal, or from the inferior palatine artery, enter the fore part of the space by the Eustachian tube.

Nerves. The lining membrane of the tympanum is supplied from the plexus (tympanic) between Jacobson's and the sympathetic nerve: but the muscles derive their nerves from other sources. Crossing the cavity is the chorda tympani branch of the facial nerve.

Dissection (fig. 243). The preparation of the tympanic plexus will require a separate fresh temporal bone, which has been softened in diluted hydrochloric acid, and in which the nerves have been hardened afterwards in spirit.

The origin of Jacobson's nerve from the glosso-pharyngeal is first to be sought close to the skull; and the fine auricular branch of the pneumogastric may be looked for at the same time (p.113). Supposing the nerve to be found, the student should place the scalpel on the outer side of the Eustachian tube, and carry it backwards through the vaginal and styloid processes of the temporal bone, so as to take away the outer part of the tympanum, but without opening the lower end of the aqueduct of Fallopius.

After the tympanum has been laid open, Jacobson's nerve is to be followed in its canal; and the branches in the grooves on the surface of the promontory are to be pursued: one of these arching forwards joins in the carotid plexus; and two others directed upwards, unite with the large superficial petrosal, and the facial nerve.

The connections of the chorda tympani nerve can be seen on the preparation used for the muscles.

The tympanic branch of the glosso-pharyngeal nerve (fig. $243,{ }^{2}$ ), nerve of Jacobson (p. 112), enters a special aperture in the temporal bone, to 
reach the inner wall of the tympanum. In this eavity the nerve supplies filaments to the lining membrane and the fenestra rotunda ; and it terminates in the three undermentioned branches, which are contained in grooves on the promontory, and connect this nerve with others. ${ }^{1}$

Fig. 243.

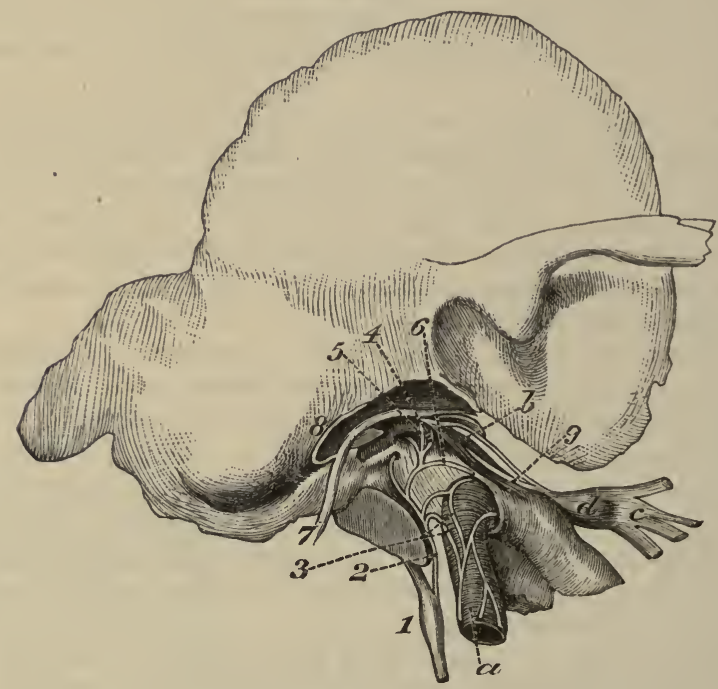

Jacobsox's Nerve in the TyMPANUM (Breschet).

a. Carotid artery.

b. Tensor tympani muscle.

c. Inferior maxillary trunk of the fifth nerve.

d. Otic ganglion.

\section{Nerves :}

1. Petrous ganglion of the glosso-pharyngeal.
2. Nerve of Jacobson.

3. Sympathetic on the carotid.

4. Small superficial petrosal nerve.

5. Offset to join large petrosal nerve.

6. Brauch to Eustachian tube.

7. Facial nerve; 8, chorda tympani branch.

9. Nerve of the otic ganglion to the tensor muscle.

Branches. One branch is arched forwards and downwards, and enters the carotid canal to communicate with the sympathetic $\left({ }^{3}\right)$ on the artery (p. 116).

A second $\left({ }^{5}\right)$ is directed upwards to join the large superficial petrosal nerve in the hiatus Fallopii.

And the third ( $\left.{ }^{4}\right)$ has the following course: It ascends in front of the fenestra ovalis, and near the gangliform enlargement on the facial nerve, to which it is connected by filaments (fig. 35). Beyond the union with the facial, the nerve is named small superficial petrosal, and is continued forwards through the substance of the temporal bone, to end in the otic ganglion (p. 144).

Nerves to muscles. The tensor tympani muscle is supplied by a branch from the otic ganglion (fig. $243,{ }^{9}$ ) : the stapedius receives an offset from the facial trunk ; and the laxator tympani from the chorda tympani nerve (?).

1 Instead of viewing these filaments as offsets of the nerve of Jacobson they may be supposed to come from the other nerves: according to this view the tympanic plexus would be derived from several sources. 
The chorda tympani (fig. $243,{ }^{8}$ ) is a branch of the facial nerve (p. 144). Entering the cavity behind, it crosses the membrana tympani, and issues from the space by an aperture in, or internal to the Glaserian fissure; it joins the gustatory nerve, and its farther course to the tongue is described at page 97.

The auricular branch of the vagus nerve, though not a nerve of the tympanum, is an offset to the outer ear, and may be now traced in the softened bone. Arising in the jugular fossa (p. 113), the nerve enters a special canal, and crosses through the substance of the temporal bone to the back of the ear.

The Labyrintir. The inner portion of the organ of hearing is so named from its complexness. It consists of three spaces surrounded by dense bone; and of sacs containing fluid for the expansion of the auditory nerve, which are contained within the former.

The ossEous LABYRINTn includes the vestibule, the semicircular canals, and the cochlea: these communicate externally with the tympanum, and internally, through the meatus auditorius internus, with the cranial cavity.

The Vestibure (fig. 244), or the central cavity of the osseous labyrinth, is placed behind the cochlea, but in front of the semicircular canals.

Fig. 244.

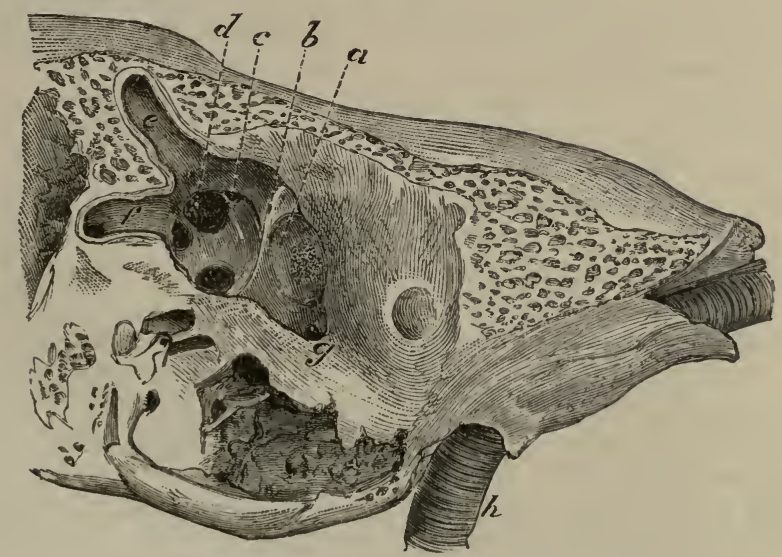

View of the Vestibele obtainen by ctetivg away the outer bodndarin a Fetus, enlarged thres times.

a. Fovea hemispherica.

b. Crista vestibuli.

c. Aperture of aqueduct of the vestibule.

d. Common opening of two semicircular canals. e. Upper vertical semicirular canal partly laid open.

$f$. Horizontal semicircular can 2 , partly opened.

g. Opening of the scala vestibuli.

Dissection. This space may be seen on the dry bone which has been used for the preparation of the tympanum. The bone is to be sawn through vertically close to the inner wall of the tympanum, so as to lay bare the fenestra ovalis leading into the vestibule. By enlarging the fenestra ovalis a very little in a direction upwards and forwards, the vestibular space will appear; and the end of the superior semicircular canal will be opened. 
Other views of the cavity may be obtained by sections of the temporal bone in different directions, according to the knowledge and skill of the dissector.

The vestibular space (tig. 244) is ovoidal in form, and the extremities are directed forwards and backwards. The larger end is turned back, and the under part or floor is more narrowed than the upper part or roof. It measures about $\frac{1}{5}$ th of an inch in length, but it is narrower from without inwards. The following objects are to be noted on the boundaries of the space.

In front, close to the outer wall, is a large aperture $(g)$ leading into the cochlea; and behind are five round openings of the three semicircular canals $(d, e, f)$.

The outer wall corresponds with the tympanum, and in it is the aperture of the fenestra ovalis. On the inner wall, nearer the front than the back of the cavity, is a vertical ridge or crista $(b)$. In front of the ridge is situate a circular depression, fovea hemispherica (a), which is pierced by minute apertures for nerves and vessels, and corresponds with the bottom of the meatus auditorius internus. Behind the crest of bone, near the common opening of two of the semicircular canals, is the small aperture of the aqueduct of the vestibule $(c)$, which ends on the posterior surface of the petrous portion of the temporal bone.

The roof is occupied by a slight transversely oval depression, forea semi-elliptica ; this is separated from the fovea hemispherica by a prolongation of the crista $(b)$ on the inner wall before mentioned.

The semicircular Canals (fig. 245) are three osseous tubes, which are situate behind the vestibule, and are named from their form.

Dissection. These small canals will be easily brought into view by the removal of the surrounding bone by means of a file or bone forceps. Two may be seen opening near the aperture made in the vestibule, and may be followed thence; but the third is altogether towards the posterior aspect of the petrous portion of the temporal bone.

The canals are unequal in length, and each forms more than half an ellipse. They communicate at each end with the vestibule, but the contiguous ends of two are blended together so as to give only five openings into that cavity. Each is marked by one dilated extremity which is called the ampulla. When a tube is cut across it is not cireular, but is compressed laterally, and measures about $\frac{1}{20}$ th of an inch, though in the ampulla the size is as large again.

From a difference in the direction of the tubes, they have been named superior vertical, posterior vertical, and horizontal.

The superior vertical canal $(a)$ crosses the upper border of the petrous part of the temporal bone, and forms a projection on the surface. Its outer end is marked by the ampulla, whilst the inner is joined with the following.

The posterior vertical tube $(b)$ is directed backwards from its junction with the preceding towards the posterior surface of the temporal bone; the upper end is united with the superior vertical canal, and the lower end is free and dilated.

The horizontal canal $(c)$ has separate apertures, and is the shortest of the three. Deeper in position than the superior vertical, it lies in the substance of the bone nearly on a level with the fenestra ovalis; its dilated end is at the outer side close above that aperture. 
Lining membrane of the osseous labyrinth. A thin fibrous periosteal membrane lines the vestibule and the semicircular canals, and is continuous with the fibrous process in the aqueduct of the vestibule. On the outer wall of the cavity it stretches over the fenestra ovalis; and in front it is prolonged into the cochlea through the aperture of the scala vestibuli (fig. $244, g)$.

Fig. 245 .

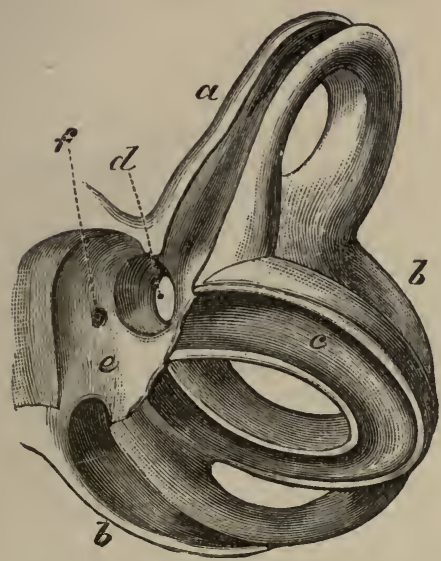

a. Upper vertical; $b$, posterior vertical; und $c_{\text {, }}$ horizontal canal.

d. Common opening of the two vertical canals.

e. Part of the vestibular cavity.

$f$. Opening of the aqueduct of the vestibule.

RePresentation of The SEMicircular CANALS ENLARged

(from a model in University College Museum).

The outer surface of the membrane is adherent to the bone; but the inner is covered by a single layer of flattened epithelium. It is supplied by the vestibular vessels; and it secretes a thin serous fluid, liquor Cotunnii, or perilymph, which surrounds the membranous labyrinth, and occupies for a short distance the aqueduct: this fluid also fills the scalæ of the cochlea.

Cochlea. This part of the osseous labyrinth has a position anterior to the vestibule, and has received its name from its resemblance to a spiral shell.

Dissection. To obtain a view of the cochlea it will be needful to cut or file away the bone between the promontory of the tympanum and the meatus auditorius internus, on the preparation before used for displaying the vestibule; or this section may be made on another temporal bone in which the semicircular canals are not laid bare. For the same dissection in the recent state, a softened bone should be used.

The cochlea (fig. 246) is conical in form, and is placed almost horizontally in front of the vestibular space. The base of this body is turned to the meatus auditorius internus, and is perforated by small apertures; whilst the apex is directed to the inner wall of the tympanum, opposite the canal for the tensor tympani muscle. Its length is about a quarter of an inch, and its width at the base is about the same. Resembling a spiral shell in construction, the cochlea consists of a tube wound round a central part or axis; but it differs from the shell in having its tube subdivided by a partition.

The axis or modiolus (fig. $246, a$ ) is the central stem which supports 
the windings of the spiral tube. Conical in shape, its size diminishes rapidly towards the last half turn of the tube, but it enlarges at the tip of the cochlea, forming a second small cone $(b)$, which is bent. 'The axis is perforated by canals as far as the contracted part in the last half turn, and the central one is larger than the others; these transmit vessels and nerves in the fresh state.

Fig. 246.

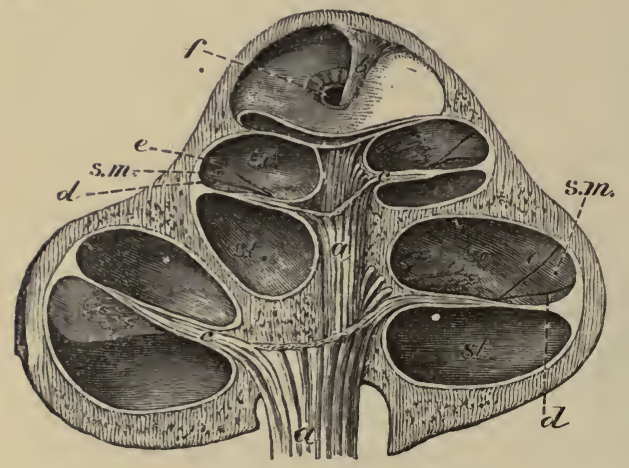

a. Axis with its canals.

b. Infundibulum or enlarged upper end of the axis.

c. Septum of the cochlea.

d. Membrane of Corti.

e. Membrane of Reissner.

$f$. Hiatus or helicotrema.

s.t. Scala tympani.

s. v. Scala vestibuli.

Section through the Cochlea (Breschet).

The spiral tube forms two turns and a half around the stem, and terminates above in a closed extremity named the cupola. When measured along the outer side, it is about one inch and a half long. Its diameter at the beginning is about one-tenth of an inch, but it diminishes gradually to half that size towards the opposite end.

Of the coils that the tube makes, the first is much the largest; this projects towards the tympanum, and gives rise to the eminence of the promontory on the inner wall of that cavity. The second turn is included within the first coil. The last half turn bends sharply round, and presents a free margin $(b)$ (the edge of the axis).

In the recent bone the tube is divided into two main passages (scalie) by the septum (fig. $246, c$ ). In the dry bone a remnant of this partition is secn in the form of a thin plate bone,-lamina spiralis, projecting from the axis; and on the outer wall is a slight groove, opposite the ridge of bone.

Septum of the spiral tube (fig. 247). The partition in the recent bone dividing the tube of the cochlea into two passages, consists of an osseous and a membranous portion :-

The osseous part $\left({ }^{3}\right)$, formed by the lamina spiralis, extends about half way across the tube. Inferiorly it begins in the vestibule, where it is wide, and is attached to the outer wall so as to shut out the fenestra rotunda from that cavity ; and diminishing in size ends above in a point,_the hamulus, opposite the last half turn of the cochlea. Between the hamulus and the axis a space, which is eonverted by the membranous part of the septum into a foramen (fig. 246, $f$ ) (hiatus, helicotrema), and allows the intercommunication of the two chief passages of the cochlear tube.

The lamina spiralis is formed by two plates of the bone, which inclose canals for vessels and nerves, and are separated farthest from each other at 
the axis. The surface of the lamina which is turned to the lower of the two cochlear passages (ST) is most pierced by apertures. The opposite surface is covered in the outer fifth of its extent by a border or limbus of fibrous structure $(a)$, which ends in wedge-shaped teeth near the margin of the bony plate (denticulate lamina, Bowman).

Fig. 247.

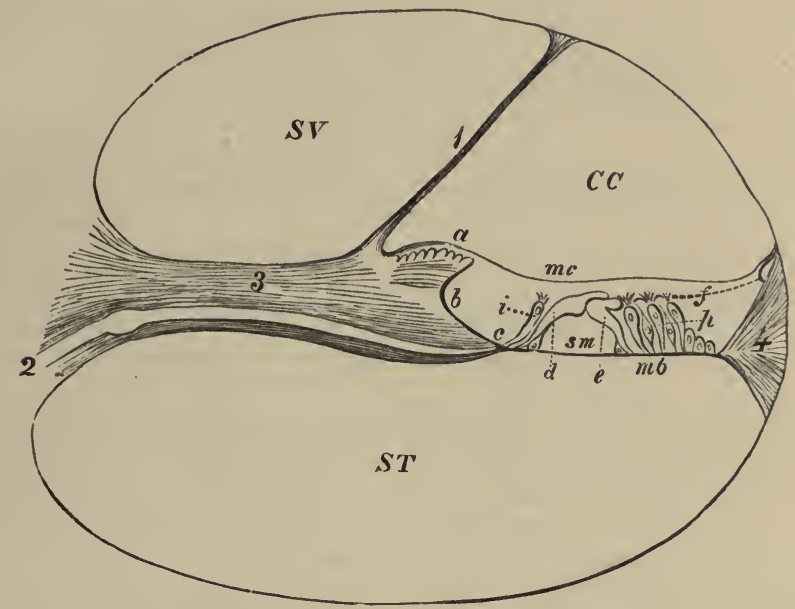

a Diagram of a Section of the Tcbe of the Cochlea Exlarged (modified from Henle).
SV. Scala vestibuli.
ST. Scala tympani.
CC. Canal of the cochlea.
1. Hembrane of Reissner.
2. Cochlear branch of the auditory neive.
3. Lamina spiralis ossea.
4. Ligamentum spirale.
a. Lamiua denticulata.
b. Sulcus spiralis.

c. Tympanic lip of the sulcus spiralis.

d. Inner rods of Corti.

e. Outer rods of Corti.

$f$. Lamina reticularis.

$i$. Inner hair cells.

$m b$. Membrana basilaris.

$m$ c. Membrane of Corti.

p. Outer hair cells.

$s m$. Central space between the rods.

Between the teeth and the underlying bone is a channel $(b)$, lined by flat epithelium, which is called sulcus spiralis : its edges are named vestibular $(a)$ and tympanic $(c)$.

The membranous part of the septum (membrana basilaris, fig. 247, $m b$ ) reaches from the lower edge $(c)$ of the laminx spiralis to the groove in the outer wall of the cochlear tube. Its width varies:-near the base of the cochlea it forms half of the partition across the tube, but at the apex, where the bony part is wanting, it constructs the septum altogether.

This membrane is firm and stiff, and possesses fine radiating lines which cause it to split in shreds. At its outer attachment there is a fibrous band $\left({ }^{4}\right)$ (ligamentum spirale, Kölliker) fixing it to the bone.

Scala of the cochlea (fig. 246). The tube of the cochlea is divided by the septum into two primary passages, of which one is the scala tympani (sT) and the other scala vestibuli (Sv); but the latter is rendered smaller by two canals being cut off from it by membranes.

These passages are placed one above another, the scala vestibuli ( $\mathrm{sr}$ ) being the nearest the apex of the cochlea. Above they communicate through the aperture named helico-trema $(f)$. Below they end differently, 
as the names express:-the scala vestibuli opens into the front of the vestibule (fig. $244, g$ ); but the scala tympani is shut out from the vestibular cavity by the lamina spiralis of the septum cochlex, and is closed below by the membrane of the fenestra rotunda, though in the dry bone it opens into the tympanum.

Each has certain peculiarities. The restibular scala extends into the apex of the cochlea; whilst the tympanic scala is largest near the base. Connected with the last is the small aqueduct of the cochlea, which begins close to a ridge near the lower end of the scala, and opens at the lower border of the petrous portion of the temporal bone.

The scalæ are clothed with a thin fibrous membrane, continuous with that in the restibule: in the scala tympani it helps to close the fenestra rotunda, forming the inner layer of the secondary membrane of the tympanum, and joins the fibrous process in the aqueduct of the cochlea. The fluid of the vestibular space fills both scala.

Sualler partitions of the cochlea (fig. 247). Two thin membranes are prolonged from the septum to the outer wall, across the vestibular scala. One is called membrane of Reissner, and the other membrane of Corti.

The membrane of Reissner $\left({ }^{1}\right)$, which is easily torn, is prolonged $\mathrm{ob}$ liquely from the upper surface of the septum cochlea, where the limbus ends, to the outer part of the tube of the cochlea, so as to cut off a small channel,-_the canal of the cochlea (Cc). It is formed by a very thin layer of fine connective tissue with blood capillaries, and is covered by the epithelial lining of the two spaces between which it intervenes.

The membrane of Corti ( $m c)$ (membrana tectoria) stretches horizontally across the tube of the cochlea near, and parallel to the membrana basilaris. Internally it is attached to the limbus of the septum cochlex, and externally to the wall of the cochlear tube. It is a thin elastic transversely-fibred layer, which is thicker internally than externally. Between it and the membrana basilaris $(m b)$ is a narrow channel for the reception of the organ of Corti.

Smaller passages of the cochlea (fig. 247). The spaces resulting from the subdivision of the scala vestibuli by the membranes above noticed are two in number, viz., canal of the cochlea, and space for the organ of Corti.

The canal or duct of the cochlea (CC) is the narrow interval towards the outer part of the cochlear tube, between the membrane of Reissner $\left({ }^{1}\right)$, and the membrane of Corti $(m c)$. It extends from apex to base of the cochlea like the larger scalæ; it is lined by epithelium, and contains a fluid (endolymph).

Above it reaches into the cupola and is closed. Below it is joined by a very small tube (fig. $248, c$ ) (canalis reuniens Hensen), with the saccule in the vestibule.

The space for the organ of Corti is a narrow interval corresponding in in depth with the sulcis spiralis, and is placed between the membrane of Corti $(m c)$ and the membrana basilaris $(m b)$. It contains the organ of Corti and is filled with fluid.

The organ of CoRTI (fig. 247) rests on the membrana basilaris $(\mathrm{mb})$, and occupies a narrow space between this and the membrane of Corti; it consists of rods and cells placed vertically on each side of a median space $(\mathrm{sm})$.

The rods are firm peculiar bodies, which are arranged in two rows ( $d$ 
and $e$ ) over an intervening triangular space; they slant towards each other above, and are separated below like the rafters in a roof. The inner row, the more numerous, are in contact with each other on the sides, and the outer set touch only by the upper end. Where they touch above they are flattened and directed out, the inner $(d)$ overlapping the outer $(e)$; and where' they rest on the membrana basilaris each is provided with a nucleuslike body.

The cells, somewhat like columnar epithelium (fig. 247), are nucleated, and are arranged vertically on the sides of the rods: the lower end is furnished with a process which directed to the membrana basilaria, and the other placed upwards, is provided with stiff filaments or hairs.

The inner set $(i)$ stand in a single line between the sulcus spiralis and the inner row of rods. The other set $(p)$ four deep, are flattened and riband-like, and bulged below, and are placed outside the external row of rods; the filaments at the upper end project through apertures in the layer $(f)$ above them.

Lamina reticularis $(f)$ (Kölliker). A very thin layer of flattened particles (phalanges) is continued over the outer half of the organ of Corti ; in it are apertures, through which pass the filaments at the end of the cells. And around the upper ends of the inner cells is a similar uniting material. It is supposed to fix and keep in place the cells.

An epithelial layer, consisting of cubical cells, covers the floor of the space outside and inside the organ of Corti.

The MEMBRANous LABYRINTH (fig. 248) is constituted of sacs, containing fluid, in which the auditory nerve is expanded. The sacs are two in number, viz., utricle and saccule, and have the general shape of the surroundiug bony parts; they are confined to the vestibule and the semicircular canals. Surrounding them is the perilymphic fluid.

Dissection. 'The delicate internal sacs of the ear, with their nerves, cannot be dissected except on a temporal bone which has been softened in acid, and afterwards put in spirit. The previous instructions for the dissection of the osseous labyrinth will guide the student to the situation of the membranous structures within it, but the surrounding softened material must be removed with great care.

A microscope will be needed for the complete examination of the sacs. For the display of the hloodvessels a minute injection will be required.

The utricle (fig. $248, d$ ), or the common sinus, is the larger of the two sacs, and is situate at the posterior and upper part of the vestibule, opposite the fovea semi-elliptica in the roof. It is transversely oval in form, and connected with it posteriorly are three looped tubes, which are prolonged into the semicircular canals.

These prolongations $(y)$ are smaller than the osseous tubes, being only one third of their diameter; and the interval between the bone and the membrane is filled by fluid-the perilymph. In form they resemble the bony cases, for they are marked at one end by a dilatation $(f)$ corresponding with the ampulla of the osseous tube; and further, two are blended at one end, like the canals they occupy : they communicate with the utricle by five openings, and are filled with the fluid of that sac. At each ampullary enlargement there is a transverse projection into the interior of the cavity, and at that spot a branch of the auditory nerve enters the wall.

The utricle and its offsets are filled with a clear fluid, like water, which 
is named endolymph; and in the wall of the sac is a small calcareous deposit (otolith, $e$ ) opposite the entrance of the nerve into it.

The saccule (fig. $248, a$ ) is a smaller and rounder cyst than the utricle, and is placed in front of it in the hollow of the fovea hemispherica. It is separate from the other; but it is continuous, below, by a short and small duct ( $c$, ductus reuniens) with the canal of the cochlea.

Like, the larger sac it has a translucent wall, in which is an otolith $(b)$ opposite the entrance of the nerve; it is also filled by endolymph, which is continued into the canal of the cochlea through the ductus reuniens.

Fig. 248.

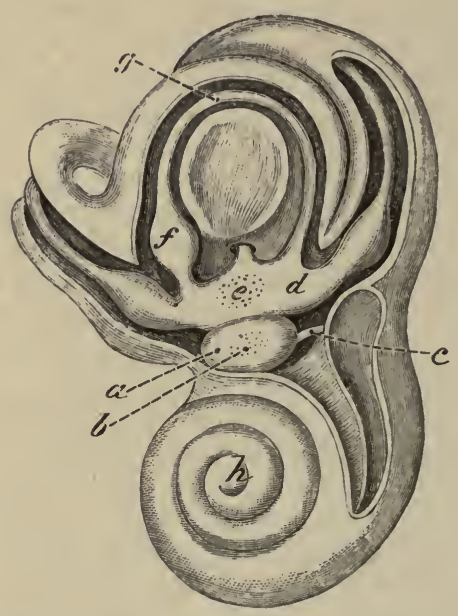

Petrous Bone partuy removed to show the Membranods labyrinth in place (Breschet).
a. Small sac.
b. Its otolith.
c. Ductus reuniens.
d. Large sac, or utricle.

e. Its otolith.

f. Ampullary enlargement on a semicircular tube.

g. Semicircular tube.

Structure of the sacs. The wall of the sacs of the membranous labyrinth is translucent and tirm; but it is more opaque where the vessels and nerves enter it. Three strata enter into its construction, together with bloodvessels and nerves.

The outer fibrous covering is loose and floceulent, is easily detached, and contains irregular pigment cells with ramifying bloodvessels. The middle stratum (tunica propria) is clear and tough, like the hyaloid membrane in the interior of the eyeball: its inner surface is irregular, owing to slight eminences; and in the ampulla of the semicircular tube this layer forms a transverse projection. The inner one is formed by a layer of flattened nucleated cells, which are continued over the eminences in the interior. In the region of the nerve, both in the ampulla of the semicircular canal and in the sacs, the epithelium is said to become columnar, and to have intermixed spindle-shaped nucleated cells (like the olfactorial), which possess hair-like processes at their free ends.

'The small calcareous masses, or the otoliths, consist of minute, elongated, six or eightit-sided particles of carbonate of lime, which are pointerl at the ends, and are situate in the inner part of the wall of the utricle and 
saccule. Within the enlargement of each semicircular tube there is also a calcareous material.

Bloodvessels. The membranes of the labyrinthic cavity receive their blood from an artery which enters the internal auditory meatus with the nerve; but some offsets from the posterior auricular supply their hinder part.

The internal auditory artery arises from the basilar trunk within the skull (p. 175), and enters the internal meatus with the auditory nerve. In the bottom of that hollow it divides into two branches-one for the vestibule, the other for the cochlea.

The branch of the vestibule, after piercing the wall of the cavity, subdivides into small offsets, which supply the lining membrane, and ramify over the sacs, and the tubes occupying the semicircular canals. The vessels end in a network of capillaries on the exterior of the special layer of the sacs, and this is most developed about the termination of the nerves.

The branch of the cochlea subdivides into twigs which enter the canals in the modiolus. Small offsets from these are directed outwards through canals in the lamina spiralis, and communicate together in loops near the margin of that osseous zone. From this anastomosis vessels are supplied to the basement layer and the membrane lining the scalæ; but the septum cochlex prevents the communication of the vessels of the two passages.

A longitudinal vessel is placed on that surface of the membranous part of the septum cochlex which is turned to the scala tympani, and is opposite the outer rods of Corti ; it communicates here and there with the arterial loops before mentioned.

The vein. One branch of vein is derived from the cochlea, and another from the membranous labyrinth: the two are united near the cochlea, and the trunk ends in the superior petrosal sinus in the base of the skull.

Nerve of the Labyrinth (fig. 249). Only one special nerve, auditory (p. 181), is distributed to the labyrinth. Entering the internal anditory meatus, the nerve divides into two branches, like the artery, viz., an anterior for the cochlea, and a posterior for the membranous labyrinth. In the trunk and in both branches nerve cells are contained: in the branch to the labyrinth they form a swelling (intumescentia ganylioformis of Scarpa).

The cochlear branch divides at the base of the modiolus into twigs, which enter it, and are directed outwards with the vessels into the canals in the lamina spiralis (fig. $247,{ }^{2}$ ). As they enter the lamina they form a plexus with ganglion cells, and are continued onwards near the scala tympani, being still plexiform, as far as the edge of the bone. At this spot they leave the bone, and are arranged in conical bundles, which pass through apertures at the junction of the bone with the lamina basilaris of the septum cochlex: consisting now of axis cylinders they are directed towards the cells of the organ of Corti, but their ending is unknown.

The vestibular branch ends in three nerves for the membranous labyrinth; these pierce the cribriform plate in the bottom of the meatus, and are thus distributed:-One appertains to the utricle, (c), and to the enlargements on the tubes contained in the superior vertical and horizontal semicircular cinals (fig. 249); a second ends in the saccule (a); and the third 
belongs to the ampullary swelling $(b)$ on the tube in the posterior vertical semicircular canal.

On the sacs the nerve divides, and its filaments separate, some fibrils passing through the otolith and others outside it, and pierce the middle layer of the wall. Now becoming axis cylinders they divide, and unite

Fig. 249.

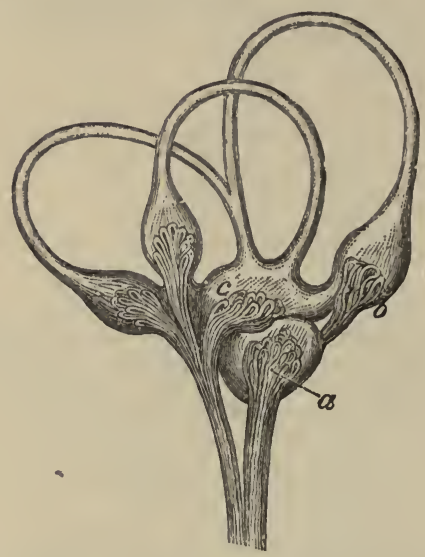

Distribution of Nerves to thi Mrmbrarous Labrinth (Breschet).

a. Nerve to the saccule.

b. Nerve entering the ampullary enlargement on a semicircular tube. c. Brancb of nerve entering the large sac or utricle.

in a plexus beneath the epithelium, and are thought to be united with the deep ends of the spindle-shaped cells with hair-like processes.

On the ampullary enlargement of the semicircular tubes the nerve enters the external side, where it forms a forked eminence (Steifensand), corresponding with the projection in the interior. The nerve divides at first into two chief pieces, which are directed to the sides; and it is doubtful whether filaments extend into the tube beyond those eminences. Finally it pierces the wall of the tube, and forming a sub-epithelial plexus of nerve-fibres, as in the utricle, is supposed to be connected with similar auditorial cells. 


\section{N D E X.}

The letter (o) refers to the origin, (c) to the course, and (d) to the distribution of a nerve or vessel which is described in different pages.

A BDOMEN, 430

A surface of, 404

Abdominal aorta, 444, 489

cavity, 430

hernia, 420

regions, 431

ring, external, 410 internal, 417,422

Abducens nerve, (o) 180, (d) 57, (c) 32 Abductor minimi digiti, 278 pedis, 616

pollicis manûs, 277 pedis, 616

Accessorius muscle, 620

ad sacro-lumbalem, 362

Accessory nerve of the obturator, (o) 495, (d) 577

pudic artery, 399, 516

spleens, 465

Acini of the liver, structure, 469

Acromial artery, inferior, 234

cutareous nerves, 242

thoracic artery, 233

Acromio-clavicular articulation, 246

Adductor brevis, 577

longus, 576

magnus, 579, 599

minimi digiti, 278

oculi, 57

pollicis manûs, 278

$$
\text { pedis, } 621
$$

Air-cells of the lung, 335

Alar ligaments of the knee, 637

Alveolar plexus, 142

Ampullæ, membranous, 685

of the semicircular canals, 680

Amygdaloid lobe of cerebellum, 213

Anastomotic artery of brachial, 254 femoral, 568

Anconeus muscle, 285

Angular artery, 40

vein, 40

Ankle-joint, 708

Anuular ligament of ankle, anterior, 628 external, 628 internal, 614

of wrist, anterior, 282 posterior, 282,289

protuberance, 188
Annulus of Vieussens, 316

Anterior elastic layer of cornea, 658 commissure, 207

medullary velum, 214

Anti-helix, 46

Anti-tragus, 46

muscle, 46

Anus, 386

Aorta, 320325

abdominal, 441,489

thoracic, 326,336

Aortic arch, 350

opening, 487

plexus, 443

sinus, 327

Aperture of the aorta, 321

of the cavæ, 316

of Eustachian tube, 127

for the femoral artery, 579

of the heart, 315,320

$\operatorname{larynx}, 128$

mouth, 128

nares, 126

œsophagus, 128

pulmonary artery, 318

veins, 319

thorax, 120

Aponeurosis, epicranial, 18

of external oblique, 409

over femoral artery, 568

of internal oblique, 412

lumbar, 357

palmar, 274

of the pharynx, 124

plantar, 615

of the soft palate, 129

temporal, 19

of the transversalis muscle, 414

vertebral, 360

Appendages of the eye, 43

Appendices epiploïcæ, 434

Appendix auriculæ, 315, 319

сæci, 463

vermiformis, 462

Aqueduct of the cochlea, $6 \$ 4$

of Sylvius, 206

of the vestibule, 680

Aqueous humor, 663

Arachnoid membrane of the brain, 172 
Arachnoid membraneof the cord, 375

Arbor vitæ cerebelli, 217

Arch of aorta, 326

crural or femoral, 559

of diaphragm, 486

palmar, deep, 280 superficial, 273

plantar, 623

of soft palate, 128

Arciform fibres, 186

Areola of the mamma, 226

Arm, dissection of, 248

veins of, 249

Arter. acromialis inferior, 234

anastomotica brachialis, 254 magna, 568

angularis faciei, 40

aorta abdominalis, 444,489

thoracica, 326, 336

articulares inferiores, 595

superiores, 595

articularis azygos, 595

articularis posterior, 21,86

auditoria, $] 76,687$

axillaris, 232

basilaris, 175

brachialis, 253

brachio-cephalica, 327

bronchiales, 336, 337

buccalis, 94

capsularis inferior, 489

media, 474, 489

superior, 491

carotis communis dextra, 81 sinistra, 120, 328

externa, 83

interna, 33, 106, 176

carpi ulnaris anterior, 269 posterior, 269

radialis anterior, 266 posterior, 289

centralis retinæ, 56, 666

cerebelli inferior, 175

inferior anterior, 175 superior, 175

cerebri anterior, 176

media, 176

posterior, 175

cervicalis ascendens, 78 profunda, 78,368

shoroidea cerebri, 175, 176

ciliares anteriores, 56, 662 posteriores, 56,662

circumflexa anterior, 235, 244 externa, 572

ilii interna, 420, 491, 590

superficialis, 407,554

interna, 578, 590

posterior, 235, 244

coccygea, 588

cochlearis, 687

cœliaca, 446

colica dextra, 441
Arter. colica-

media, 441

sinistra, 442

comes nervi ischiadici, 588

phrenici, 330

communicans cerebri anterior, 176 posterior, 176

palmaris, 274

plantaris, 623

coronaria dextra, 313

labii inferioris, 40 superioris, 40

sinistra, 314

ventriculi, 447

corporis bulbosi, 399,532

cavernosi, 399, 534

cremasterica, 418

cryco-thyroidea, 85

cystica, 448

deferentialis, 515

dentalis anterior, 105

inferior, 93, 97

superior, 94, 105

diaphragmatica, 490

digitales manûs, 274 pedis, 623

dorsales pollicis, 289

dorsalis carpi radialis, 289

carpi ulnaris, 269

indicis, 289

linguæ, 101

pedis, 624,631

penis, 399,408

pollicis, 289

scapulæ, 235, 248

epigastrica, 419, 491

superficialis, 407,554

ethmoidalis, anterior, 57, 142 posterior, 57, 142

facialis, 40,85

fernoralis, 564, 568

frontalis, 20,57

gastricæ, 447,455

gastro-epiploïca dextra, 447 sinistra, 447

glutea, 515,586

hemorrhoidalis inferior, 390 media, 515

superior, 443, 516, 533

helicinæ, 531

hepatica, 447,471

hyoidea lingualis, 101

thyroidex, 85

hypogastrica, 513

iliaca communis, 490

externa, 491

interna, 513

ileo-colica, 441

lumbalis, 513

incisoria, 90

infra-orbitalis, 94, 104

scapularis, 235,241

innominata, 327

inter(ostales anteriores, 239, 337 
Arter. intercostales-

rami anteriores, 337,419 posteriores, 368,379

intercostalis superior, 78,337 interossea, 269

anterior, 271

posterior, 288

interosseæ manûs, 280, 289 pedis, 632

intestinales, 440

intra-spinales, 385

ischiadica, 516, 588

labialis inferior, 40

lachrymalis, 45,56

laryngealis inferior, 158 superior, 85,158

lingualis, 85,101

lumbales, 498 rami anteriores, 498 posteriores, 368

magna pollicis manûs, 280 pedis, 624

malleolares, 631

mammaria, externa, 234 interna, 77, 239, 330, 419

masseterica, 94

maxillarus interna, $89,93,142$

mediana, 271

mediastinæ, 239, 337

miningea media, 29, 93 parva, 29,93

meningeæ anteriores, 29 posteriores, $30,80,174$

mesenterica inferior, 441 superior, 439

metacarpa radialis, 289 ulnaris, 269

matatarsea, 632

musculo-phrenica, 239, 490

mylo-hyoidea, 93

nasalis, 57, 142

lateralis, 40 septi, 40

nutritia femoris, 578

fibulæe, 613

humeri, 254

tibiæ, 612

obturatoria, 515,580

occipitalis, $21,86,368$

œsophageales, 337, 447

ophthalmica, 33,56

ovariana, 490,516

palatina inferior, 86 superior, 142

palmaris profunda, 274

palpebralis inferior, 45, 57 superior, 45,57

pancreaticæ, 447

pancreatico-doudenalis, 440, 448

perforantes femorales, 578, 599

mammariæ internæ, 239

manûs, 280

pedis, 623,632

pericardiacæ, 337
Arter.-

perinæi superficialis, 393

peronea, 613 anterior, 613

petrosa, 29

pharyngea ascendens, 109

phrenicæ inferiores, 490 superiores, 330,490

plantaris externa, 617, 623 interna, 617

poplitea, 594

princeps cervicalis, 360 pollicis, 280

profunda cervicis, 71,368

femoris, 565,578

inferior, 254

superior, 254, 258

pterygoideæ, 94

pterygo-palatina, 142

pudenda externa, 554,565

interna, 390, 398, 408, 516

pulmonalis, $318,325,335$

dextra, 325

sinistra, 325

pylorica inferior, 448 superior, 447

radialis, $265,280,289$ indicis, 280

ranina, 101

receptaculi, 33

recurrens interossea posterior, 288 radialis, 262

tibialis, 631

ulnaris anterior, 268 posterior, 269

renales, 476,489

sacro-media, 489,517

sacro-lateralis, 515

scapularis posterior, $78,248,359$

sciatica, 516, 588

sigmoidea, 442

spermatica, 484, 489

spheno-palatina. 142

spinales posteriores, 174,380

spinalis anterior, 174,379

splenica, 447,466

sterno-mastoidea, 85

stylo-mastoidea, 86

subclavia dextra, 75

sinistra, 118, 328

sublingualis, 101

submentalis, $86^{\circ}$

subscapularis, 235

superficial is cervicalis, 359

perinæi, 393

volæ, 266

supra-orbitalis, 21,56

scapularis, $78,247,359$

spinalis, 248

tarsea, 631

temporales profund, 94

temporalis, 86

anterior, 21

media, 87 
Arter. temporalisposterior, 21 superficialis, 20,86

thoracica acromialis, 234 alaris, 234 longa, 234 suprema, 234

thyroidea ima, 121 inferior, 78,121 superior, 85,121

tibialis antica, 630 postica, 612

tonsillaris, 86

transversalis colli, 78, 363 faciei, 41, 87 perinæi, 393 pontis, 175 pubis, 419

tympanica, 93 inferior, 677 superior, 677

ulnaris, 267

umbilicalis, 513

uterina, 516

vaginalis, 516

vertebralis, $77,165,174$

vesicalis inferior, 515,526 superior, 515

vestibuli, 687

vidiana, 142

Articular popliteal arteries, 595 nerves, 596

Articulation, acromio-clavicular, 246

astragalo-scaphoid, 645

astragalus to os calcis, 644

atlo-axoidean, 165,170

of bones of the tympanum, 675

calcaneo-cuboid, $646^{\circ}$ scaphoid, 645

of carpal bones, 298

carpo-metacarpal, 300

of cervical vertebræ, 166

chondro-costal, 346

sternal, 345

of coccygeal bones, 544

coraco-clavicular, 246

of costal cartilages, 346

costo-clavicular, 171

vertebral, 346

crico-arytænoid, 162 thyroid, 161

of cuneiform bones, 648

cuneiform to cuboid, 648 to scaphoid, 648

of dorsal vertebræ, 346

femoro-tibial or knee, 634

humero-cubital or elbow, 293

ilio-femoral or hip, 600

of lower jaw, 90

of lumbar vertebræ, 346

of the metacarpal bones, 299

metacarpo-phalangeal, 301

metatarsal, 649

metatarso-phalangeal, 651
Articulation-

occipito-atloidean, 168 axoidean, 168

os calcis to cuboid, 646 to scaphoid, 645

peroneo-tibial, 641

phalargeal of fingers, 302 of toes, 651

of pulic symphysis, 546

radio-carpal or wrist, 296 cubital inferior, 297 superior, 294

sacro-coceygeal, 544 iliac, 544 vertebral, 544

scaphoid to cuboid, 648 to cuneiform, 648

scapulo-humeral, 290 sterno-clavicular, 170 sternum, pieces of, 346 tarsal transverse, 647 tarso-metatarsal, 649 temporo-maxillary, 90 thyro-arytænoid, 162 of the thumb, 300 tibio-tarsal or ankle, 642 of vertebræ, 346

Arcus externus diaphragmatis, 487 internus diaphragmatis, 487

Arytæno-epiglottidean folds, 157

Arytænoid cartilages, 160

glands, 131

muscle, 153

Ascending cervical artery, 78 vein, 77 colon, 433

pharyngeal artery, 109 vein, 110

Attollens aurem, 18

Attrahens aurem, 18

Auditory artery, 175, 687

tube, external, 670

nerve (o) 191, (d) 687

nucleus, 188

Auricle of the ear, 45

Auricles of the heart, 313 left, 319 right, 315

structure of, 322

Anricular, 315, 319

A uricular artery, posterior, (d) 21, (o) 86 nerves, anterior, 23 great, 23,66 inferior, 96 posterior, 23, 48

vein, 21,86

nerve of vagus, (o) 113 , (d) 679

Auriculo-temporal nerve, 23, 96 ventricular aperture, left, 320 right, 318 rings, 321

Auricularis magnus nerve, 23, 66 A xilla, 228

dissection of, 228 
Axillary artery, 232

glands, 230

plexus, 235

vein, 235

Axis, coliac, of artery, 446, 485

of cochlea, 681

thyroid, of artery, 78

Azygos, artery, 595

veins, (o) 498, (d) 338,495

uvulæ muscle, 131

RACK, dissection of, 353

B Base of brain, 190

Base of the skull, arteries of, 29, 33 dissection of, 26

Basilar artery, 175 nerves of, 30,33

membrane, 683

sinus, 29

Basilic vein, 249

Biceps femoris musele, 597, 635 flexor cubiti, 252, 271, 291

Bicuspid teeth, 133

Bile ducts, 450, 457. 472 structure, 461,472

Biventral lobe, 213

Bladder, 512, 524 interior of, 525

connections of, 506,512

ligaments of, 503, 504

structure of, 524

Bones of the ear, 674

ligaments of, 675

muscles of, 676

Brachial aponeurosis, 251

artery, 253

plexus, 79, 235

veins, 255

Brachialis anticus, 256, 271

Brachio-cephalic artery, 327

vein, left, 329 right, 328

Brain, base of, 190 examination of interior, 199 membranes of, $24,26,172$ origin of nerves, 177

preservation of, 26

removal of, 25

vessels of, 173

Breast, 226

Broad uterine ligament, 510

Bronchial arteries, (d) 336, (o) 337 glands, 340

veins, 336,337

Bronchus, left, 333

right, 333

structure of, 333

Brunner's glands, 460

Buttock, dissection of, 581

muscles, 583

vessels and nerves, 586, 588

Buccal artery, 94

nerve, 95
Buccinator muscle, 39

Bulb of the urethra, 509

artery of, 399 , (d) 532

nerve of, 399

Bulbi vestibuli, 536

Bulbous part of the urethra, 509, 527

Bulbus olfactorius, 179

CACUM coli, 462

connections of, 433

structure of, 463

Calamus scriptorius, 217

Calices of the kidney, 477

Calciform papillæ, 148

Canal of cochlea, 684

of Petit, 668

of spinal cord, 382

of the tensor tympani, 673

Canine teeth, 133

Capsular arteries, inferior, 489 middle, 479,489

superior, 490

ligament of the hip, 600

knee, 634

shoulder, 290

thumb, 300

Capsule of crystalline lens, 668

of Glisson, 469

suprarenal, 478

Caput coli, 462

gallinaginis, 526

Cardiac nerve, inferior, (o) 117, (d) 332 middle, (o) 117 , (d) 332

of pnemmogastric, (o) 114, 331, (d) 332

superior, (o) 117, (d) 314,333

plexus, 314,332

veins, 314

Carnæ columnæ, 317, 320

Carotid artery, external, 83 internal, (d) 33,176 , (c) 106

left common, 120, 328

right common, 81

plexus, 33

Carpal artery, radial anterior, 266 posterior, 290

ulnar anterior, 268 posterior, 269

Carpo-metacarpal articulations, 300

Cartilage, arytænoid, 160

cricoid, 159

cuneiform, 160

of the ear, 46

thyroid, 159

triangular of the nose, 134

Cartilages of the eyelids, 44

of the nose, 42

of Santorini, 160

of trachea, 167

Caruncula lachirymalis, 45

Caruncula myrtiformes, 535

Cauda equina, 379

Cava, inferior, 317, 444, 492 
Cava-

superior, 316

Cavernous body, 508, 530

plexus, 33

$$
\text { artery of, (o) } 399 \text {, (d) } 530
$$

sinus, 28

structure, 530

Cavity of the omentum, 437

Central artery of the retina, (o) 56, (d) 666

ligament of cord, 376

lobe of brain, 194

pillar of cochlea, 681

point of the perineum, 394

Central ovale cerebri, 199

Cephalic vein, 250

Cerebellar arteries, inferior, 175 superior, 175

Cerebellum, form of, 211

lobes of, 213

structure of, 214

Cerebral artery, anterior, 176

middle, 176

posterior, 175

Cerebrum, division into lobes, 193

convolutions, 195

form of, 189

interior, 199

structure, 209

Cervical fascia, 63,68

ganglion, inferior, 117

middle, 117

superior, 116

glands, 81

nerves, anterior branches, 79, 165 posterior, 165

plexus of nerves, 80

deep branches, 80

superficial, $66,68,80$

Cervicalis ascendens artery, 78 muscle, 363

veins, 79

profunda artery, (o) 78 , (d) 368

veins, 77, 368

superficialis nerve, 66,68

Cervico-facial nerve, 50

Cervix uteri, 537

vesicæ, 506

Chamber of the eye, anterior, 663 posterior, 663

Check ligaments, 168

Cheeks, 132

Chiasma of the optic nerves, 179

Chondro-costal articulations, 346 glossus muscle, 149 sternal articulations, 345

Chorda tympani nerve, (o) 144 , (d) 97 , 102

Chordæ tendineæ, 318, 320 vocales, 156

Willisii, 32

Choroid artery of the brain, 175 coat of the eye, 658

plexuses of the brain, 205, 218
Choroid-

veins of the eye, 663

Cilia, 44 brain, $206^{\circ}$

Ciliary arteries, (o) 56, (d) 662

ligament, 661

muscle, 660

processes of the choroid, 659 of the suspensory ligament, 668

nerves of nasal, (o) 54 , (d) 663 of lenticular ganglion, 55, 663

Circle of Willis, 176

Circular sinus, 29 ,

Circumflex artery, anterior, 235, 244 external, 572

internal, 578, 590

posterior, 235,244

iliac artery, deep, 420, 491 superficial, 554, 565

nerve, (o) 236 , (d) 244

Clavicular cutaneous nerves, 66,80

Clitoris, 534

Coccygeal artery, 588

muscle, 501

nerve, 519

Cochlea, 681

canal of, 684

nerve of, 687

vessels of, 687

Coliac artery, 446 plexus, 451

Colic artery, left, 442

middle, 441

right, 441

Colon, 433, 461 course of, 433 structure of, 463

Columnar layer of retina, 664

Columnæ carneæ, 317,320

Columns of the cord, 382 vagina, 536

Comes nervi ischiatici artery, 588 phrenici artery, 330

Commissure, anterior, 207 of the cerebellum, 213,215 of the cord, 382

great, 200

of the optic nerves, 179

posterior, 216

soft, 206

Cornmissural fibres of the medulla, 186

Communicating artery of anterior cerebral, 176

of posterior cerebral, 176

in the palm, 273

peroneal nerve, 606

Complexus muscle, 364

Compressor of the bulb, 395

of the nose, 35

Coniarmm, 208

Concha, 45

Cones of the retina, 664

Congenital hernia, 424

Coni vasculosi, 482 
Conical papillæ, 146

Conjoined tendon, 414

Conjunctiva, 45

Conoid ligament, 246

Constrictor inferior, 124

of the fauces, 130

middle, 124

superior, 124

urethræ, 397, 403

Conus arteriosus, 317

Convolutions of the brain, 195, 198 of hemisphere, 195 of longitudinal fissure, 197

Coraco-brachialis muscle, 252 clavicular articulation, 246 humeral ligament, 291

Cordiform tendon, 480

Cords of the abdominal wall, 427

Cornea, 657 structure, 657

Cornicula laryngis, 160

Cornua of gray erescent, 382 of lateral ventricle, 200

Corona glandis, 509 radiata, 209

Coronary vessels of the heart, 313 of the lips, 40

artery of the stomach, 447

ligament of the liver, 438

plexus of the stomach, 451

plexuses of the heart, 314

sinus, 314, 329

vein of the stomach, 448

Corpora albicantia, 192

Arantii, 318, 321

cavernosa, 508, 530

geniculata, 207, 211

Malpighiana, 466, 474

mamillaria, 192

olivaria, 183, 185

pyramidalia anteriora, 183, 185 posteriora, 183, 184

quadrigemina, 208

Corpora restiformia, 183, 184

Corpus callosum, 192, 199

dentatum cerebelli, 215 medullæ, 188

finbriatum uteri, 541

geniculatum externum, 207 internum, 207

Highmori, 481

luteum, 540

olivare, 183, 184, 208

spongiosum urethræ, 509, 531

striatum, 203, 211

thyroideum, 120

Corpuscles of Malpighi, 466, 475

Corrugator cutis ani, 389

supercilli muscle, 37

Cortex of the tongue, 149

Cortical substance of the kidney, 474

Costo-clavicular ligament, 171

coracoid membrane, 232

transverse ligaments, 345
Cotunnius, nerve of, 140

Cotyloid ligament, 602

Covered band of Reil, 199

Cowper's glands, 398

Cranial aponeurosis, 18

nerves, 30, (o) 137

Cremaster muscle, 412

Cremasteric artery, 418, 420

fascia, 413

Cribriform fascia, 55.5

Crico-arytænoid articulation, 162

muscle, lateral, 153

posterior, 152

thyroid articulation, 161

membrane, 161

muscle, 152

Cricoid cartilage, 159

Crista vestibuli, 680

Crucial ligaments, 637

Crura cerebelli, 215

cerebri, 190, 211

of the diaphragm, 486

of the fornix, 203

Crural arch, 559

deep, 419,560

canal, 560

hernia, 429, 562

nerve, (o) 497, (d) 572

ring, 428, 561

sheath, 428, 560

Crypts of Lieberkühn, 459

Crystalline capsule, 668

lens, 668

structure, 669

Cuneiform eartilages, 160

Cupola cochleæ, 681

Curve of the urethra, 508

Cutaneous nerves of the abdomen, 406

of the arm, 249

of the back, 354

of the buttock, 581

of the face, 48

of the foot, back, 626 sole, 624

of the forearm, 261

of the hand, back, 261 palm, 272

of the head, 21

of the leg, back, 606 front, 626

of the neck, behind, 354 fore part, 68

of the perinzum, 391, 393

of the shoulder, 242

of the thigh, front, 556

of the thorax, 225

Cystic artery, 448

duct, 473

plexus of nerves, 452

DARTOID tissue, 408

Decussation of the oblong medulla, $186^{\circ}$ 
Deep cervical artery, (o) 78, (d) 368 crural arch, 449,560

transverse muscle of perinæum, 395 , 400

Deferential artery, 523

Deltoid ligament, 643 muscle, 242

Dens sapientiæ, 133

Dental artery, anterior, 105 inferior, 93, 97 superior, 94, 105

nerve, anterior, 105 inferior, 96 posterior, 104

Dentate, lamina, 204

ligament, 376

Denticulate lamina, 683

Depressor anguli oris, 39

epiglottidis, 154

labii inferioris, 38

alæ nasi, 36

Descendens noni nerve, 83, 115

Descending colon, 434

Diaphragm, 305, 484

arteries of, 490

plexus of, 451

Digastric muscle, 82 nerve, 48

Digital arteries of plantar, 624 of radial, 280 of tibial, anterior, 624 of ulnar, 274

nerves of median, 275 of plantar, 617

of radial, 262 of ulnar, 274

Dilator of the nose, 35 of the pupil, 661

Discus proligerus, 540

Dissection of the abdomen, 404 of the abdominal cavity, 430 of the anterior commissure, 207 of the arm, 225,248 of the axilla, 227

of the back, 253

of the base of the skull, 26

of the brain, membranes, and nerves, 172

of the buttock, 581

of the cardiac plexus, 331

of the carotid artery, internal, 106 of the carotid plexus, 33

of the cerebellum, 211

of the cerebrum, 189

of the corpus callosum, 192, 199

of the corpus striatum, 203, 211

of the crus cerebri, 191

of the external ear, 45,670

of the internal ear, 671

of the eighth nerve, 110

of the eye, 655

lids, 43

of the face, 34

of fascia lumborum, 357
Dissection-

of femoral hernia, 427, 559

of the fifth ventricle of the brain, 202

of the foot, back, 626 sole, 614

of the forearm, 260

back, 282

front, 260

of the fourth ventricle, 215

of the glosso-pharyngeal nerve, 110

of the hand, back, 261 palm, 272

of the head, external parts, 17 internal parts, 24

of the heart, 311

of the hypogastric plexus, 444

of the inferior maxillary nerve, 94

of inguinal hernia, 420

of the internal maxillary artery, 93

of Jacobson's nerve, 112, 677

of the labyrinth, 679

of the larynx, 151

cartilages, 158

muscles, 151

nerves, 157

of the lateral ventricles, 200

of the left side of the neck, 118

of the leg, back, 605 front, 626

of the ligaments of atlas and axis, 167

atlas and occiput, 168

axis and oeciput, 168

clavicle and scapula, 246

lip joint, 600

jaw, 91

lower limb, 634

pelvis, 543

ribs, 344

shoulder, 290

upper limb, 290

the vertebræ, 347

of the lower limb, 634

of Meckel's ganglion, 138

of the medulla oblongata, 182

of the neck, 61

anterior triangle, 69

left side, 118

posterior triangle, 63

of the ninth nerve, 115

of the nose, 133

of the ophthalmic of the fifth nerve,

31,51

of the orbit, 50

of the otic ganglion, 144

of the pelvis, female, 509 side view, 511

male, 499 side view, 500

of the perinæum, female, 401 inale, 386

of the pharynx, 122

of the pneumo-gastric nerve, 110 , 
Dissection-

of the pons, 188

of the popliteal space, 593

of the portio dura nerve, 47, 143

of the prevertebral muscles, 163

of the pterygoid region, 88

of the sacral plexus, 518

of the saphenous opening, 559

of the semilunar ganglia, 451

of the shoulder, 241

of the soft palate, 128

of the solar plexus, 451

of the spinal cord, 374 accessory nerve, 110

of the subclavian artery, 73

of the submaxillary region, 97

of the superior maxillary nerve, 103

of the testis, 479

of the thigh, back, 592, 597 front, 552

of the third ventricle, 206

of the thorax, 305

of the tongue, 146

of the triangular space of the thigh, 560

of the tympanurn, 671 vessels and nerves, 677

of the upper limb, 224

of the vena cava inferior, 444 , 492

of the vidian nerve, 141

Dorsal artery of the foot, 624,631 of the penis, 399 , (d) 408 of the tongue, 101 of the scapula, (o) 235 , (d), 248

nerves, anterior branches, 239,343 , 378

posterior branches, 367,378

cutaneous of the hand, 262

of the penis, (o) 399, 408

Ductus ad nasum, 61

arteriosus, 325

communis choledochus, 450,457 , 461

cysticus, 473

ejaculatorius, 523

hepaticus, 450,472

lymplaticus, 79,340

pancreaticus, 465

reuniens, $686^{\circ}$

Ductus Riviniani, 103

Stenonis, 41

thoracicus, 118, 339, 494

venosus, 469

Whartonii, 98, 103

Duodenum, connections, 445 peritoneum of, 438

Dura mater, 24, 26 of the cord, 374

nerves of, 30,377

vessels of, 29,377
TAR, external, 45, 670.

B internal, 671

Eighth nerve, 32, (o) 181

Ejaculator urinæ, 394

Elastic layers of cornea, 658

Elbow joint, 293

Eminentia collateralis, 202 teres, 217

Encephalon, 173

Endocardium, 324

Endolymph, 686

Ependyma ventriculorum, 200

Epididymis, 483

Epigastric artery, 419, 491 superficial, 407,565

region of the abdomen, 431 veins, 429

Epiglottidean glands, 157

Epiglottis, 160

Erector clitoridis, 408

penis, 394

spinæ, 362

Ethmoidal arteries, 56

Eustachian tube, cartilaginous part, 126

osseous part, 674

valve, 317

Extensor carpi radialis brevis, 284 longus, 284

carpi ulnaris, 285

digiti minimi, 285

digitorum brevis, 632

communis, 285

longus pedis, 629

indicis, 288

ossis metacarpi, 287

proprius pollicis, 629

primi internodii policis, 287

secundi internodii policis, 287

External cutaneous nerves of arm, 250, 262

of thigh, (o) 497 , (d) 556

saphenous nerve, $596,606,627$ vein, 606,626

Eye ball, 665

brows, 43

lashes, 43

lids, 43
muscles of, 44
nerves of, 45
structure, 43
vessels, 45

FACE, dissection of, 34

F Facial artery, (d) 40 , (o) 85 nerve, (d) 46, (c) 645, (o) 180

Falciform ligament of the liver, 439

border of saphenous opening, 559

Fallopian tube, 511, 540

Falx cerebelli, 27

cerebri, 25

Fascia, axillary, 225

brachial, 250 


\section{Fascia-}

cervical deep, 63,68

costo-coracoid, 232

cremasteric, 412

cribriform, 555

of the forearm, 262

iliac, 428, 494

intermuscular of the humerus, 258 of the thigh, 572

lata, 557, 593

of the leg, 606, 627

lumborum, 357

obturator, 500

palmar, 272

pelvic, 500

perinæal, deep, 396 superficial, 393

plantar, deep, 615

propria, 562

recto-vesical, 502

spermatic, 423

temporal, 19

transversalis, 417, 428

Fasciculus teres, 277

Femoral artery, 554, 568

hernia, 427,562

ligament, 559

vein, 565,568

Femoro-tibial articulation, 634

Fenestra ovalis, 672

rotunda, 672

Fibres of the cerebrum, 209

of the cerebellum, 215

of Müller, 665

Fibro-cartilage. See Interarticular.

of heart, 322

of tongue, 148

Fibrous coat of eye, 655

Fifth nerve, 31, 180

ventricle of brain, 202

Filiform papillæ, 146

Fillet of the corpus callosum, 200 of the olivary body, 186, 188, 208

Filum terminale, 372

Fimbrix of the Fallopian tube, 541

First nerve, (o) 134, (c) 30, (d) 137

Fissure, longitudinal, 193

parieto-occipital, 193, 198

of Rolando, 193

of Sylvius, 193

transverse of brain, 204

Fissures of Santorini, 47

of the cord, 381

of the cerebrum, 193

Flexor accessorius muscle, 620

brevis minimi digiti, 278

$$
\text { perlis, } 623
$$

carpi radialis, 264,282 ulnaris, 265

digitorum brevis pedis, 616 longus pedis, 610,620 profundus, 270,276 sublimis, 266, 276

pollicis longus, 270,277
Flexor pollicis longus-

brevis, 278 pedis, 621

Flocculus cerebelli, 214

Follicles, Meibomian, 44 ceruminous, 740 solitary, 459, 464

Foot, dorsum, 626 sole, 614

Foramen of Monro, 203 ovale, 317

for vena cava, 487 of Winslow, 437

Foramina Thebesii, 316

Forearm, dissection of, 260 cutaneous nerves, 261 veins, 262

Fornix, 202

Fossa, ischio-rectal, 387 navicular of the urethra, 528 of the pudendum, 534 ovalis, 316

Fossæ of abdominal wall, 427

Fourth nerve, (o) 180 , (c) 31 , (d) 51 ventricle, 217

Fovea centralis, 664 hemispherica, 680 semi-elliptica, 680

Foveæ of fourth ventricle, 217

Frænulum labii, 534

Frænum linguæ, $146^{\circ}$ præputii, 509

Frontal artery, 21, 57 nerve, 51

lobe, 193 vein, 21

Fungiform papille, 146

Funiculus gracilis, 184 lateralis, 183

CALACTOPHORUS ducts, 221

$\mathrm{U}$ Galen, veins of, 206

Gall bladder, 472 structure, 472

Ganglia, cervical, inferior, 117, 122 middle, 117,122 superior, 116,122

lumbar, 497

sacral, 519

semilunar, 451

of spinal nerves, 378

thoracic, 339

Ganglion of the vagus, 112

Gasserian, 31

impar, 519

jugular, 111

lenticular, 55

Meckel's, 139

ophthalmic, 55

otic, 144

petrosal, 111

spheno-palatine, 139 
Ganglion -

submaxillary, 101

thyroid, 117

Gastric arteries, 447 vein, 449

Gastro-colic omentum, 437

Gastro-epiploïc arteries, 447, 448 vein, 449

hepatic omentum, 437

Gastrocnemius muscle, 608

Gemellus inferior muscle, 589 superior muscle, 589

Geniculate bodies, 207, 211

Genio-hyo-glossus, 101, 149 hyoid muscle, 100

Genital organs, 507, 533

Genito-crural nerve, (o) 497, (d) 413, 556

Gimbernat's ligament, 411, 560

Gland, lachrymal, 51

parotid, 48

prostate, 507, 521

sublingnal, 103

submaxillary, 97

Glands, arytænoid, 131

axillary, 230

Bartholine's, 537

bronchial, 340

Brunner's, 460

eardiac, 339

ceruminous, 671

cervical, 66,67

concatenate, 67

Cowper's, 398, 508

Haver's, 603

inguinal, 407, 554

intercostal, 340

intestinal, 443

labial, 133

laryngeal, 157

lingual, 150

lumbar, 495

mammary, 226

mediastinal, 339

Meibomian, 44

mesenteric, 441

molar, 42

œsophageal, 339

of Pacchioni, 24

pelvis, 522

Peyer's, 459

popliteal, 597

solitary, 459,464

sternal, 339

tracheal, 339

Glans of the clitoris, 534

of the penis, 509

Glaserian-fissure, 672

Glenoid ligament, 209

Glisson's capsule, 469

Globus major epididymis, 483

minor epididymis, 483

Glomerulus, 475

Glosso-pharyngeal nerve, (o) 111, (d) 151,181
Glosso-pharyngeal-

Glottis, 155

nucleus, 188

Gluteal artery, (o) 515 , (d) 586

nerve, superior, 496,586

nerves, inferior, 588

Gluteus maximus muscle, 583 medius muscle, 585

minimus muscle, 586

Graafian vesicles, 540

Gracilis muscle, 574

Granular layer of retina, 666

Gray commissure of the cord, 382

crescent of the cord, 382

substance of the corpus striatum, 203

of the medulla oblongata, 186, 217

of the third ventricle, 206

tubercle of Rolando, 187

Great omentum, 437

Gustatory nerve, 97 , (d) 101,151

Gyrus fornicatus, 197

I ANORRHOIDAL artery, inferior, 390 middle, 515

superior, (o) 443, 516, (d) 533

nerve, inferior, 443

plexus, 520

veins, 517

Ham, 592

Hamulus laminæ spiralis, 682

Hand, dissection of, 272

Head, dissection of, 17

Heart, 312

constituents, 312

dissection of, 315

position, 312

structure of, 312

Helicine arteries, 531

Helicis major muscle, 46 minor muscle, 46

Helicotrema, 682

Helix, 46

Hepatic artery, 447, (d) 471

cells, 459

ducts, 450 , (o) 472

plexus, 451

veins, 471, 492

Hernia, crural or femoral, 429, 562 inguinal, external, 421 internal, 424

umbilical, 426

Hiatus cochleæ, 682

Hip joint, 600

Hippocampus major, 204 minor, 204

Hollow before elbow, 263

Humero-cubital articulation, 293

Hunter's canal, 568

Hyaloid membrane, 667

Hymen, 535

Hyo-glossus muscle, 99,148 
Hyo-glossal membrane, 148

Hyoid bone, 159

Hypochondriac region of abdomen, 431

Hypogastric artery, 513

plexus of nerves, 444

region of the abdomen, 431

Hypoglossal nerve, (o) 182, (c) 115, (d) nucleus, 187

JEO-C ÆCAL valve, 462

Ileo-colic artery, 441

valve, 462

Ileum intestine, connections of, 433 structure of, 456

Iliac artery, common, 490 exterual, 491 internal, 513

fascia, 495

region of the abdomen, 431

vein, common, 491

external, 491

internal, 513

Iliacus muscle, 493,580

Ilio-costalis, 362

femoral articulation, 600

hypogastric nerve, (o) 416, 492, 582

inguinal nerve, (o) 497 , (d) 416,556

lumbar artery, 514

Incisor branch of nerve, 96 teeth, 133

Incus, 674

Indicator muscle, 288

Infantile hernia, 424

Inferior cornu of the lateral ventricle, 200

maxillary nerve, (o) 32 , (d) 94

Infra-costal muscles, 342

orbital artery, 94,105

nerves, 49, 104

vein, 105

seapular artery, 235, 242

trochlear nerve, 54

Infra-spinatus inuscle, 244

Infundibulum of the brain, 192

Inguinal canal, 421

glands, 407, 554

hernia, external, 421

internal, 424

region of the abdomen, 431

Innominate artery, 327

veins, 328

Inter-articular cartilage of the jaw, 91 of the hip, 602

of the knee, 638

of the ribs, 344

of the scapula, 246

sacro-iliae, 544

sterno-elavicular, 170

of the symphysis pubis, 547

of the vertebræ, 347

of the wrist, 296

Interclavicular ligament, 170
Interoolumnar fascia, 410

fibres, 410

Intercostal arteries, anterior branches, 240, 337, 419

posterior branches, 368,384

artery, superior, (o) 78, (d) 338

muscle, external, 237, 342

internal, 238, 342 .

nerves, 237, 343, 416

cutaneous, anterior, 226

lateral, 226

veins, superior, 338

Intereosto-humeral nerve, (o) 226 , (d) 256,343

Intermediate tract, 383

Intermuscular septa of the arm, 258

of the foot, 615

of the thigh, 572

Internal cutaneous nerve of arm, 236, 250,256

of thigh, (o) 573 , (d) 556

saphenous vein, 557,626

nerve, $596,606,627$

Interosseous arteries of the foot, 631 of the hand, 280,259

artery, anterior, 244,271 posterior, 288

ligament of the arm, 295 of the leg, 642

muscles of the foot, 624 of the hand, 281

nerve, anterior, 271 posterior, 289

Interspinal muscles, 370

Intertransverse muscles, 165, 370

Intervertebral ganglia, 378 substance, 347

Intestinal arteries, 440 canal divisions, 433 structure, 456,463

Iutestine, large, 433, 461 small, 433, 456

Intra-spinal arteries, 384 veins, 385

Iris, 661 nerves of, 662

structure of, 661 vessels of, 662

Ischio-reetal fossa, 388

Island of Reil, 194

Isthmus faucium, 128 of the thyroid body, 120 of the uterus, 538

Iter a tertio ad quartum ventriculum, 206

ad infundibulum, 206

JACOB'S membrane, 664 structure, 664

Jacobson's nerve, (o) 112, (d) 678

Jejunum, connections of, 433 structure, 456

Joint, ankle, 642 
Joint-

elbow, 293

great toe, 649

hip, 600

knee, 634

lower jaw, 90

shoulder, 290

thumb, 300

wrist, 296

Jugular ganglion, 111

vein, anterior, 71

external, 42,62

internal, 82, 109

IIDNEY, 473

connections of, 435

structure, 475

vessels of, 476

Knee of the corpus callosum, 192 joint, 634

T ABIA pudendi externa, 534

L interna, 535

Labial glands, 133

artery, inferior, 40

nerve, 96

Labyrinth, 679

lining of, 681

membranous, 685

osseous, 679

Lachrymal artery, 45, 56

canals, 60

duct, 61

gland, 51

nerve, 52

point, 43,60

sac, 61

Lactiferous ducts, 227

Lacunæ of the urethra, 528

Lamina cinerea, 192

dentata, 204

reticularis, 685

spiralis cochleæ, 683

Laminæ of cerebellum, 213 of the lens, 669

Large intestine, connections, 433 structure and form of, 461

Laryngeal arteries, 158

nerve, external, 113

$$
\begin{aligned}
& \text { inferior, (o) } 114,331, \\
& 158 \\
& \text { superior, (o) } 113 \text {, (d) } 158
\end{aligned}
$$
pouch, 156

Larynx, 151

aperture of, 128, 155

cartilages of, 159

interior of, 154

ligaments, 160

muscles, 151

nerves, 158

ventricle, 156

vessels, 158
Lateral column of the medulla, 183, 185

of the cord, 382

Lateral sinus, 28

ventricles, 200

Latissimus dorsi, 236, 356

Laxator tympani, 676

Leg, dissection of the back, 605

$$
\text { front, } 626
$$

Lens of the eye, 668 structure of, 669

Lenticular ganglion, 55

Levator anguli oris, 38 scapulæ, 358

ani, 390,501

glandulæ thyroideæ, 120

labii superioris, 38 inferioris, 38 alæque nasi, 35

palati, 129

palpebræ superioris, 53

Levatores costarum, 373

Lieberkühn's crypts, 459

Ligament of the lung, 307

Ligaments of the bladder, 503, 504

of the larynx, 261

of the ovary, 512

of the pinna, 47

of the uterus, 510

Ligament, acromio-clavicular, 246

alar of the knee, 637

annular, anterior of the ankle, 628 external of the ankle, 628

internal of the ankle, 614

anterior of the wrist, 282

posterior of the wrist, 282,289

anterior, special, of ankle, 643 of elbow joint, 283 of knee joint, 636 of wrist joint, 296 of carpus, 298

astragalo-scaphoid, 645

alto-axoid, anterior, 167 posterior, 167

transverse, 169

calcaneo-astragaloid, 644 cuboid, 646

scaphoid, 645

capsular of the hip, 600

of the knee, 634

of the shoulder, 290

of the thumb, 300

carpal, dorsal, 297 palmar, 297

carpo-metacarpal, 300

clondrö-sternal, 345

common, anterior, of vertebræ, 346

common, posterior, 347

conoid, 246

coraco-acromial, 246

clavicular, 245

humeral, 291

costo-clavicular, 171

coracoid, 232 
Ligament-

costo-transverse, anterior, 345

middle, 345

posterior, 345

vertebral, 344

xiphoid, 346

cotyloid, 602

crico-thy roid, 161

crucial, 637

deltoid, 643

dorsal of the carpus, 298

of Gimberuat, 416, 560

glenoid, 290

ilio-femoral, 600

lumbar, 546

interarticular, of the clavicle, 171 of the hip, 602

of the jaw, 91

of the knee, 638

of the pubes, 546

of the ribs, 344

of the wrist, 296

interclavicular, 170

interosseous of astragalus and os calcis, 637

of carpus, 298

of cuneiform bones, 648

of metacarpal bones, 299

of metatarsal bones, 649

of radius and ulna, 294

of the scaphoid and cuboid, 648

of the tibia and fibula, 642

interosseous, inferior, of the tibia and fibula, 642

interspinal, 350

intertrausverse, 350

intervertebral, 347

lateral, external of the ankle, 643 interual, 643

external of the carpus, 298

internal, 298

external of the elbow, 293

internal, 293

phalangeal of the foot, 651 of the hand, 308

external of the jaw, 90

internal, 91

external of the knee, 634

internal, 634

external of the wrist, 296

internal, 296

long plantar, 647

metacarpal, dorsal, 299

palmar, 299

metatarsal, dorsal, 649

plantar, 649

mucous, 637

obturator, 546

occipito-atloid, anterior, 168 posterior, 168

occipito-axnid, 168

odontuid, 169

orbicular of the radius, 294

of the patella, $636^{\circ}$
Ligament-

palmar of carpus, 298

peroneo-tibial, 641

of Poupart, 411, 559

posterior of ankle, 643

of carpus, 298

of elbow, 293

of knee, 636

of scapula, 247

of wrist, 296

proper of the scapula, 246

pubic anterior, 546

superior, 547

round of the hip, 602

round of the radius and ulna, 295

sacro-coccygeal, anterior, 544 posterior, 544

sacro-iliac, anterior, 544 posterior, 544

sacro-sciatic, large, 545, 592 small, 545, 592

sacro-vertebral, 544

of the scapula, anterior, 247 posterior, 247

sterno-clavicular, 170

stylo-hyoid, 106

maxillary, 91

subpubic, 547

supraspinous, 350

suspensory of penis, 408

tarso-metatarsal, dorsal, 650 lateral, 650, plantar, 650

thyro-ary tænoid, 162

epiglottidean, 162 hyoid, 161

tibio-tarsal, 643

transverse of the atlas, 170

of the fingers, 273

transverse of the hip, 602

of the knee, 639

of metacarpus, 280

of metatarsus, 624

of the toes, 615

trapezoid, 246

triangular of the abdomen, 411

of the urethra, 396

of Winslow, or posterior, 636

Ligamentum arcuatum, 486

denticulatum, $376^{\circ}$

dûctus arteriosi, 325

latum pulmonis, 307

longum plantæ, 647

mucosum, 637

nuchæ, 356

patellæ, 636

spirale, 683

subflavum, 34

teres, 602

Limb, upper, 224

lower, 552

Limbus cochleæ, 683

luteus, 664

Limiting membrane of retina, 666 
Linea alba, 410

$$
\text { semilunaris, } 416
$$

Lineæ transversæ, 415

Lingual artery, 85, 101 glands, 150

nerve, $97,101,150$

vein, 101

Lingualis muscles, 149

Lips, 133

Liquor Cotunnii, 681

Lithotomy, parts cut, 399

Liver, 466

connections of, 435

ligaments, 438

structure, 469

vessels, 471

Lobes of the cerebellum, 213 of the cerebrum, 193

Lobules of the testis, 482 of the liver, 469

Lobulus auris, 46 caudatus, 467 quadratus, 467 Spigelii, 467

Locus niger, 191 perforatus anticus, 192 posticns, 191

Longissimus dorsi, 363

Longitudinal fibres of the brain, 210 fissure of the liver, 468

sinus, inferior, 27 superior, 25

Longus colli muscle, 163

Lumbar aponeurosis, 357 arteries, 498 anterior branches, 498 posterior branches, 368 ganglia, 497 glands, 495 nerves, anterior branches, 495 posterior branches, 368,384 plexus, 496 region of the abdomen, 431 veins, 492,498

Lumbo-sacral nerve, 495

Lumbricales of the foot, 620

Lungs, 333 of the hand, 277

connections, 308

physical characters, 334

structure, 334

vessels and nerves, 335

Lymphatic duct, 119,340 right, 79,340

Lymphatics of the arm, 250

of the axilla, 230

of the groin, 407,554

of the lungs, 336

of the mesentery, 441

of the neck, 67

of the pelvis, 520

of the popliteal space, 597

of the thorax, 339

Lyra, 203
IIALLEOLAR arteries, 637

II Malleus, 674

muscles of, 676

Malpighian corpuscles of spleen, 466 of kidney, 475

Mamillæ of the kidney, 474

Mamma, 226

structure of, 227

Mammary artery, internal, (o) 77, (c) $239,330,419$

gland, 226

Masseter muscle, 87

Masseteric artery, 94 nerve, 95

Mastoid cells, 672

Maxillary artery, internal, 93, 142

nerve, inferior, (o) 32 , (d) 94 superior, 32, 104

vein, internal, 94

Meatus auditorius externus, 670 nerves of, 671 vessels of, 671

urinarius, 527

Meatuses of the nose, 134

Meckel's ganglion, 139

Median basilic vein, 249

cephalic vein, 249

nerve, (o) 236 , (c) 255 , (d) 269,274

vein, 249,261

Mediastinal arteries, 239, 337

Mediastinum of thorax, 307

Mediastinum of testis, 481

Medulla oblongata, 18:2 gray matter of, 186,317

Medulla spinalis, 380

Medullary substance of the kidney, 474 velum, anterior, 215 posterior, 215

Meibomian follicles, 44

Membrana basilaris, 683 granulosa, 540

pigmenti, 666

pupillaris, 661

reticularis, 685

sacciformis, 297

tympani, 673

Membrane of Corti, 684

of Demours, 657

hyaloid, 667

Jacob's, 664

of the labyrinth, 681

of Reissner, 684

Membranes of the brain, 24, 172 of spinal cord, 374

Membranous labyrinth, 685 part of the cochlea, 683 of the urethra, 527

Meningeal artery, anterior, 29 middle, 29,93 posterior, 29, 86, 174 small, 29, 93

nerves, 30

Mesenteric artery, inferior, 441 superior, 439 
Mesenteric-

glands, 441

plexus inferior, 443 superior, 443

vein, inferior, 443 superior, 441

Mesentery, 438

Meso-cæcum, 438

colon left, 438

right, 438

transverse, 438

rectum, 438, 503

Metacarpal arteries, 269, 289

Metatarsal artery, 632

Mitral valve, 320

Modiolus of the cochlea, 682

Molar teeth, 133

$$
\text { glands, } 4 \cdot 2
$$

Mons Velleris, 534

Motor oculi nerve, (o) 180 , (c) 30 , (d) 54,57

Mouth, cavity of, 132

Mucous ligament, 637

Multifidus spinæ muscle, 370

Muscularis inucosæ, 457

Musculi papillares, 318,320

pectinati, 316,319

Musculo-phrenic artery, 239, 490

cutaneons nerve, (o) 633, (d) 626

of the arm, (o) 234, (c) 256 , (d) 259

spiral nerve, 236,259

Musc. abductor digiti minimi, 278

indicis, 280

pedis, 616

pollicis, 277

pedis, 616

accessorius pedis, 620

ad sacro-lumbalem, 362

adductor brevis, 577

digiti minimi, 279

longus, 576

magnus, 579, 599

policis manus, 278 perlis, 621

anconeus, 285

anti-tragicus, 46

arytænoideus, 153

attollens aurem, 18

attraheus aurem, 18

azygos uvulæ, 131

biceps femoris, 597,635

flexor, cubiti, 252, 271, 291

brachialis anticus, 256, 271

buccinator, 39

cervicalis ascendens, 363

chondro-glossus, 148

ciliaris, $66^{\circ} 0$

circumflexus palati, 129

coccygeus, 501

complexus, 364

compressor naris, 35

constrictor inferior, 124

isthmi faucium, 130
Musc. constrictor-

medius, 124

superior, 124

urethræ, 397, 403

coraco-brachialis, 252

corrugator cutis ani, 389

supercilii, 37

cremastericus, 412

crico-ary tænoideus lateralis, 153

posticus, 152

thyroideus, 152

deltoides, 242

depressor anguli oris, 39

epiglottidis, 154

labii inferioris, 38

alæ nasi, 36

diaphragma, 484, 343

digastricus, 82

dilatator naris, 35

pupillæ, 661

ejaculator urinæ, 394

erector clitoridis, 403

penis, 394

spinæ, 362

extensor carpi radialis brevior, 284 longior, 284

ulnaris, 285

digiti minimi, 285

digitorum brevis pedis, 632 communis, 285

longus pedis, 629

indicis, 288

ossis metacarpi pollicis, 287

pollicis proprius, 629

primi internodii pollicis, 287

secundi internodii pollicis, 287

flexor accessorius, 620

brevis digiti minimi, 278 pedis, 620

carpi radialis, 264, 282 ulnaris, 265

digitorum brevis pedis, 616

longus pedis, 610,619

protundus, 270,276

sublimis, 266,276

pollicis longus, 270, 277 pedis, 610,620

pollicis brevis, 278 pedis, 621

gastrocnemius, 608

gemellus inferior, 589

superior, 589

genio-hyo-glossus, 100, 149

hyoideus, 100

glosso-pharyngeus, 149

gluteus maximus, 583

medius, 585

minimus, 586

gracilis, 574

helicis major, 46

minor, 46

hyo-glossus, 99,182

iliacus, 493, 580

ilio-costalis, 362 
Musc.-

indicator, 288

infra-costalis, 342

infra-spinatus, 244

intercostales externi, 237, 342

interni, 238, 342

interossei manus dorsales, 281 palmares, 281

pedis dorsales, 625 plantares, 624

interspinales, 370

intertransversales, 165,370

kerato-cricoideus, 153

latissimus dorsi, 236, 356

laxator tympani, 676

levator anguli oris, 38 scapulæ, 358

ani, 390, 501

glandulæ thyroideæ, 120

labii superioris, 38 alæ nasi, 36 inferioris, 38

palati, 129

palpebræ, 53

uvulæ, 181

levatores costarum, 373

linguales, 149

longissimus dorsi, 363

longus colli, 163

Iumbricales manus, 277 pedis, 620

mallei externus, 676

internus, 676

massetericus, 87

multifidus spinæ, 371

mylo-hyoideus, 98

obliquus abdominis externus, 409 internus, 411

capitis inferior, 369 superior, 369

oculi inferior, 59 superior, 53

obturator externus, 580,591

internus, 543, 590

occipito-frontalis, 18

omo-hyoideus, 72,359

opponens digiti minimi, 279 pollicis, 278

orbicularis oris, 37

orbitalis, 60 palpebrarum, 36

palato-glossus, 130,148 pharyngeus, 130

palmaris brevis, 272 longus, 265

pectineus, 576

pectoralis major, 230, 231 minor, 231

peroneus brevis, 633 longus, 625,633 tertius, 629

plantaris, 609

platysma myoides, 62,67

popliteus, 610,635 pronator quadratus, 271

radii teres, 264

psoas magnus, 492,580

parvus, 493

pterygoideus externus, 89

internus, 90

pyramidalis abdominis, 416

nasi, 34

pyriformis, 542,587

quadratus femoris, 590

lumborum, 494

rectus abdominis, 415 capitis anticus major, 164 minor, 165

lateralis, 115

posticus major, 369 minor, 369

femoris, 570,587

oculi externus, 57

inferior, 57

internus, 57

superior, 53

retrahens aurem, 18

rhomboideus major, 358

minor, 358

risorius Santorini, 39

rotatores dorsi, 369

sacro-lumbalis, 362

salpingo-pharyngeus, 126

sartorius, 566

scalenus anticus, 75

medius, 75

posticus, 75

semi-spinalis colli, 370 dorsi, 370

semi-membranosus, 598, 636

semi-tendinosus, 597

serratus magnus, 236

posticus inferior, 360 superior, 360

soleus, 608

sphincter ani externus, 389 internus, 389

pupillæ, 661

vaginæ, 402

spinalis dorsi, 362

splenius capitis, 361 colli, 361

stapedius, 676

sterno-cleido-mastoideus, 71

hyoideus, 72

thyroideus, 73

stylo-glossus, 100, 148

hyoideus, 83

pharyngeus, 106

subanconeus, 259

subclavius, 232

subcrureus, 572

subscapularis, 241

supinator radii brevis, 288

longus, 284

supraspinatus, 247

temporalis, 20,88 
Musc. -

tensor palati, 129

tarsi, 59

tympani, 676

vaginæ femoris, 570

teres major, 245 minor, 245

thyro-ary tænoideus, 153 hyoideus, 73

tibialis anticus, 628 posticus, 612,625

trachelo-mastoideus, 363

tragicus, 46

transversalis abdominis, 413 colli, 363

transversus auris, 47

linguæ, 149

pedis, 621

perinæi, 395, 403 alter, 395

profundus, 398,403

trapezius, 354

triangularis sterni, 238

triceps extensor cruris, $\mathbf{5 7 0}$ cubiti, 258

vastus externus, 571 internus, 571

zygomaticus major, 39 minor, 39

Mylo-hyoid artery, 93

muscle, 98

nerve, 96

NARES, 42, 126

Nasal artery, internal, 57, 142

artery, lateral, 40

cartilages, 42

duct, 61

fossæ, 134

nerve, (d) 54 , (c) 141 , (o) 57

Naso-palatine nerve, 140

artery, 142

Neck, anterior triangle of, 69 posterior, 63

dissection of, 61

Nerve of Jacobson, 112, 678

Wrisberg, (o) 236, (d) 251, 256

Nerve to the inferior gemellus and quadratus, 589

latissinuus, 236

levator anguli scapulæ, 80

obturator internus, 518, 590

pectineus, 573

pterygoid, internal, 145

pyriformis, 518

rhomboid muscle, 80,359

scaleni, 80

serratus muscle, (o) 79 , (d) 236

subclavius, 80

superior gernellus, 589

tensor palati, 145

tympani, 145

vaginæ femoris, 574
Nerve-

teres major, 236

minor, 245

vastus externus, 573

internus, 573

Nervous tunic of eyeball, 663

Nerv. abducens, (o) 180 , (c) 32 , (d) 57

accessorius obturatorius, 497,577

spinalis, 114, 181, 356

acromiales, cutanei, 80, 242

articulares poplitei, 596

articularis poplitei obturatorius, 577,596

auditorius, 181, 680

auriculares anteriores, 23

auricularis magnus, 23,66

pneumogastricus, (o) 113, (d) 679

inferior, 96

posterior, 23,48

auriculo-temporalis, 23,90

buccales, 50,95

buccinatorius, 95

cardiacus inferior, (o) 117, 122, (d) 332

medius, (o) 117, 122, (d) 332

pneumogastrici, 114, 331, (d) 332

superior, (o) 11,122 , (d) 314 , 333

cervicales nervi facialis, 68

rami anteriores, 79,165

posteriores, 165,365

cervicalis superficialis, 66,68

cervico-facialis, 50

chorda tympani, 97, 144

ciliares ganglii ophthalmici, 55, 663 ciliaris nasalis, 54, (d) 663

circumflexus, 236, 244

claviculares cutanei, 66

coccygealis, 373,518

cochlearis, 687

communicans fibularis, 606

corporis bulbosi, 399

cruralis, (o) 497 , (d) 572

cutanei abdominis, anteriores, 407 laterales, 406

cutaneus externus brachialis, (o) 235 , (c) 256 , (d) 262

lumbalis, (o) 497, (d) $556^{\circ}$ musculo-spiralis, 250, 259, 262

internus brachialis, major, 236, 256,261

minor, 236, 251, 256

femoris, (d) 556 , (o) 573

musculo-spiralis, 250,259

medius femoris, (o) 573, (d) 556

musculo-cutaneus, 250

palmaris, 269,272

plantaris, 613

radialis, 262

dorsalis manûs, 269 
Nerv.-

dentales posteriores, 104

dentalis, anterior, 104 inferior, 96

descendens noni, 83, 115

diaphragmaticus, (o) 80 , (d) 330

digastricus 48

digitales mediani, 275

plantares, 619

radiales, 262

ulnares, 274

dorsales, rami anteriores, 239, 343, 378

posteriores, 367,378

dorsalis penis, 399,408

ulnaris, 262, 269

facialis, 47,143

frontalis, 52

genito-cruralis, 497,556

ramus fernoralis, 556 genitalis, 418

glosso-pharyngeus, 111, 151, 181

glutei inferiores, 588

gluteus superior, 496,586

gustatorius, 97, 101, 151

hæmorrhoidales superiores, 443

hæmorrhoidalis inferior, 390

hypoglossus, $83,102,115,151$

ilio-hypogastricus, $416,496,582$ inguinalis, $416,497,556$

incisorius, 96

infra-maxillares faciales, 50,68 orbitales nervi facialis, 49 orbitalis, 60, 104

trochlearis, 54

intercostales, 239,416

intercosto-cutanei anteriores, 226, 406

laterales, 206, 406

humeralis, $254,226,251$

interosseus anticus, 271 posticus, 289

labialis, 96

lachrymalis, 52

laryngeus externus, 114

inferior, (o) 331 , (d) 114,158 superior, (o) 113 , (d) 158

lumbales, rami anteriores, 495 posteriores, 369,384

Iumbo-sacralis, 496

malares nervi facialis, 48

massetericus, 95

maxillaris inferior, (o) 32 , (d) 94 superior, 31, 104

medianus, 236, 255, 269, 274

meningei, 30

molles, 116

motor oculi, (o) 180 , (c) 32 , (d) 54, 57

musculo-cutaneus brachii, 236,256 , 262

cruris, 633, (d) 626

musculo-spiralis, (o) 236, (d) 259

mylo-hyoideus, 96
Nerv.-

nasalis, (o) 51, (d) 54, 57, 141

naso-platinus, 141

obturatorius, (o) 497 , (d) 577 articularis, 577, 596

occipitalis, major, 366 minor, (d) 24 , (o) 66

œsophageales, 331

olfactorius, (c) 30, (d) 136, (o) 178

ophthalmicus, 32,51

opticus, 57, 137, 179, 207

orbitalis, (d) 60 , (o) 104

palatinus magnus, 140

medius, 141

minor, 141

palpebrales, 45, 104

pulmaris cutaneus, 272 ulnaris profundus, 280 superficialis, 274

patellaris, 557,573

perforans Casserii, (o) 236, (c) 256 , (d) 262

perinæales superficiales, 391, 394

peronealis, 596

petrosus superfieialis externus, 33

magnus, 33,141

parvus, 33,678

pharyngei, 112, 117

pharyngeus, 113

phrenicus, 80,330

plantaris externus, 619 profundus, 624

internus, 624

pneumo-gastricus, 112, 181, 330, 452

popliteus externus, 596 internus, 596

portio dura, $47,142,180$

mollis, (o) 181, (d) 687

pterygoidei, 95

pterygoideus internus, 95, 145

pudendus inferior, (o) 589 , (d) 394 , 407

internus, (o) 518, (d) 391, 399, 408

pulmonares anteriores, 331 posteriores, 331

radialis, 263,270

recurrens, 114, 331, (d) 158 articularis, 633

sacrales, rami anteriores, 518 posteriores, 372,581

saphenus externus, 606 , (d) 627 internus, (o) 573, (d) 557, 573, 627

sciaticus magnus, 519, 589. 599

parvus, $519,582,584,599$

spermatici, 443

spheno-palatini, 104

splanchnicus major, (o) 342,452

minor, (o) 342 , (d) 452

minimus, (o) 342 , (d) 452

splenici, 451

stylo-hyoideus, 48 
Nerv.-

suboccipitalis, ramus anterior, 115 posterior, 368

subscapulares, 236

superficialis cordis dexter, (o) 117 (d) 332

sinister, (o) 122 , (d) 314

supramaxillares nervi facialis, 48

orbitalis, 22,51

scapularis, $80,248,359$

trochlearis, 23, 52

sympatheticus abdominis, 443, 450, 497

cervicis, 115

pelvis, 519

thoracis, 331, 341

temporales nervi facialis, 48 profundi, 95 superficiales, (d) 23, (o) 48

temporo-facialis, 48

malaris, 60, 104

thoracici, anteriores, 236

laterales, 226

thoracicus posterior, 80, 236

tibialis anticus, 627, (d) 632

posticus, 613

trigeminus, 31, 180

trochlearis, $31,51,180$

tympanicus, (o) 112 , (d) 678

ulnaris, 255, 269, 274

uterini, 520

vaginales, 520

vestibularis, 687

vidianus, 141

Ninth nerve, (o) 182 , (c) 115 , (d) 83 , 102

Nipple of the breast, 226

Nodule, 212

Nose, cartilages, 42 cavity of, 133

meatuses of, 134

nerves and vessels, of, 139,141

Nuclei of medulla oblongata, 187

Nucleus caudatus, 203

lenticularis, 203

Nutritious artery of fibula, 613

of femur, 578

of humerus, 254

of tibia, 612

Nymphæ, 534

BLIQUUS abdominis externus, 409 internus, 411

capitis inferior muscle, 369 superior muscle, 369

oculi inferior, 59 superior, 53

Obturator artery, (o) 515 , (d) 580

fascia, 500

ligament, 546

membrane, 546

muscle, external, 58ก, 591

intermal, 543, 590
Obturator nerve, (o) 497, (d) 577

Occipital artery, (o) 86, (c) 368, (d) 21

lobe, 194

vein, 21,86

sinus, 27

Occipito-atloid articulation, 168 ligaments, 168

Occipito-axoid ligaments, 169

frontalis muscle, 18

Odontoid ligaments, 169

Esophagus, connections of, 121, 339 strueture, 131, 339

Esophageal arteries, 337, 447

nerves, 331

opening of diaphragm, 487

Olfactory bulb, 179

cells, 137

nerve, (o) 178 , (d) 138

region, 137

Olivary body, 183, 185

commissure, 186

fasciculus, $186,189,208$

Omentum, great, 437

small, 437

splenic, 437

Omo-hyoid muscle, 72

Ophthalmic artery, 33, 56

ganglion, 55

nerve, (o) 31 , (c) 32 , (d) 51

vein, 57

Opponens pollicis muscle, 278

Optic commissure, 179

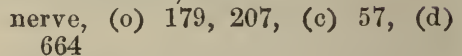

thalamus, 207, 211

tract, 179

Ora serrata, 663

Orbicular ligament of the radius, 294

Orbicularis oris, 37 palpebrarum, 36

Orbit, 50

muscles of, 53,57

nerves, 51

periosteum of, 50

vessels, 56

Orbital branch of nerve, (d) 60 , (o) 104

Organ of Corti, 684

of Giraldès, 483

Orifice of the urethra, 535

of the uterus, 535

of the vagina, 535

Os hyoides, 159

Ossicles of the tympanum, 674

Os tincæ, 537

Os uteri externum, 537

Otic ganglion, 144

Otoliths, $686^{\circ}$

Outlet of the pelvis, 386

Ovaries, 512, 539

appendage to, 540

arteries of, 490,516

Ovicapsule, 540 
Ovisacs, 540

Ovum, 539

PALATE (soft), 128

Palatine, arteries, superior, 143 artery, inferior, 86

nerve, external, 141

large, 140

small, 141

Palato-glossus, 130, 148

Palato-pharyngeus, 130

Palm of the hand, 272

cutaneous nerves of, 272

Palmar arch, deep, 280

superficial, 273

nerve of the ulnar, deep, 280

superficial, 274

cutaneous nerves, 272

fascia, 272

Palmaris brevis muscle, 272 longus muscle, 265

Palpebræ, 43

Palpebral arteries, 45, 57

ligament, 44

nerves, 45

veins, 45

Pancreas, 464 connections, 446 structure of, 464

Pancreatic arteries, 447 duct, 457,465 veins, 448

Pancreatico-duodenal arteries, 440, 448

Papilla lachrymalis, 45

Papillæ of the tongue, 146

Parietal lobe, 194

Parovarium, 540

Parotid gland, 41 arteries, 86

Patellar nerve, 573 plexus, 557

Pectineus muscle, 576

Pectoralis major muscle, 230, 231 minor muscle, 231

Peduncle of the cerebellnm, inferior, 215

middle, 215

superior, 215

of the cerebrum, 190, 211

of the pineal body, 208

Peduncular fibres, 207

Pelvis, female, dissection of, 509 male, 499 dissection of, 499

Pelvic cavity, 499 fascia, 500 plexus, 519

Penis, 508 integument of, 407

structure of, 529

vessels of, 532

Peptic glands, 455
Perforating arteries of the femoral, 578, 599

of internal mammary, 239

of the palm, 280

of the sole, 623,632

Perforans Casserii nerve, (o) 236, (c) 256, (d) 262

Pericardium, 310

vessels of, 311,337

Perilymph, 681

Perinæum, female, 400 male, 386

Perinæal artery, superficial, 393 fascia, deep, 396 superficial, 392 nerves, superficial, 391, 393, 399

Periosteum of the orbit, 50

Peritoneal prolongation on the cord, 417

Peritoneum, 435 of female pelvis, 510 of male pelvis, 504

Peroneal artery, 613 anterior, 613 nerve, 596

Peroneus brevis muscle, 633 longus muscle, 625,633

tertius muscle, 629

Peroneo-tibial articulations, 641

Pes hippocampi, 204

Petrosal ganglion, 111 sinus, inferior, 29 superior, 29

nerve, large, 33,141 small, 33, 678 external, 33

Peyer's glands, 459

Pharynx, 124

interior, 126

muscles of, 124

openings of, 126

Pharyngeal ascending artery, 109 nerve, 113

vein, 112

Pharyngeo-glossal muscle, 149

Phrenic artery, 330, 490 nerve, 80,330

Pia mater of the brain, 173 of the cord, 376

Pigmentary layer of retina, 666

Pigment cells of choroid, 660 iris, 662

Pillars of the abdominal ring, 410 of the fornix, 208

of the iris, 658

of the soft palate, 128

Pineal body, 208

Pinna, or auricle of the ear, 45

Pituitary body, 192

Plantar aponeurosis, 615 arch of the artery, 623 arteries, 617,623

ligament, long, 647

nerve, external, 619, 624 
Plantaris muscle, 609

Platysma myoides muscle, 62, 67

Pleura, 307

Plexus, aortic, 443

brachial, 79,235

cardiac, superficial, 314 deep, 332

carotid, 33

cavernous, 33

cervical, 80 posterior, 367

choroides cerebri, 205 cerebelli, 218

coliac, 451

coronary, anterior, 314 posterior, 314

coronary of the stomach, 451

diaphragmatic, 451

gulæ, 331

hepatic, 451

hæmorrhoidal, 520

hypogastric, 444

lumbar, 496

mesenteric, inferior, 443

mesenteric, superior, 443

œsophagean, 331

ovarian, 520

patellar, 557

pelvic, 519

pharyngeal, 113

prostatic, 520

pterygoid of veins, 94

pulmonary anterior, 331

renal, 451 posterior, 331 supra, 451

sacral, 518

solar, 451

spermatic of nerves, 443 of veins, 492

splenic, 451

tympanic, 678

uterine, 520

vaginal, $\mathbf{5 2 0}$

vesical, 520

vertebral, 117, 166

Plica semilunaris, 45

Pneumogastric nerve, (o) 181, (d) 112, $181,330,452$

Pons Tarini, 191

Varolii, 188 structure of, 184

Popliteal artery, 594

glands, 597

nerve, external, 596

internal, 596

space, 593

vein, 596

Popliteus muscle, 610, 635

Portal veins, 448

Portio dura, (c) 144, (d) 47, (o) 180 mollis, (o) 181, (d) 687

Porus opticus, 664

Posterior commissure, 207
Posterior-

elastic layer of cornea, 658

ligament of knee, 636

medullary vellum, 214

pyramid, 184, 186

triangle of the neck, 63

vesicular column, 383

Poupart's ligament, 411, 559

Pouch, laryngeal, 155 of the auricula, 316,319

Prepuce, 583

Princeps cervicalis artery, 368 pollicis artery, 280 of the foot, 624

Processus cochleariformis, 673 vermiformis, 212,216

Profunda artery, inferior, 254 of the neck, (o) 78 , (d) 368 of the thigh, 565,578 superior, (o) 254, (d) 258

Promontory, 672

Pronator quadratus muscle, 271 radii teres muscle, 264

Prostate gland, 571 connections, 507 structure, 521

Prostatic part of the urethra, 526 sinuses, 527

Psoas magnus muscle, 492, 580

Psoas parvus muscle, 493

Pterygoid arteries, 95 nerve, external, 95 internal, 95, 145 plexus of veins, 94

Pterygoideus externus muscle, 89 internus muscle, 90

Pterygo-maxillary ligament, 124 region, 87

palatine artery, 142

Pubic region of the abdomen, 431 symphisis, 546

Pudendal inferior nerve, (o) 589, (d) 394

Pudic arteries, extermal, 554, 565 artery, internal, (d) 390 , (c) 398 , 408 , (o) 516 nerve, internal, (o) 408, (d) 390 , 398

Pulmonary artery, (d) $318,325,335$ nerves, 331 veins, 319, 329, 336

Puncta lachrymalia, 43, 60

Pupil, muscles of, 661

Pylorus, 453

Pyloric arteries, 447

Pyramid, anterior, 183, 185 decussation of, 186

of the cerebellum, 213

of the thyroid body, 120

of the tympanum, 672

posterior, 184, 186

Pyramidal fibres of the medulla, 185 masses of kidney, 474

Pyramidalis abdominis muscle, 416 nasi muscle, 34 
Pyramids of Malpighi, 474

Pyriformis muscle, 542, 587

(UADRATUS femoris muscle, 590 lumborum muscle, 494

RADIAL artery, 265, (d) 280, 289

R nerve, 262, 270

veins, 266 cutaneous, 261

Radialis indicis artery, 272

Radio-carpal articulation, 296

Radio-ulnar articulations, 294, 297

Ranine artery, 101 vein, 101

Raphe of the corpus callosum, 200 of the medulla, 186 of the perinæum, 386

Receptaculum chyli, 495

Recto-vesical fascia, 5,02 pouch, 504

Rectus abdominis muscle, 415 capitis anticus major, 164 minor, 165

posticus major, 369 minor, 369

lateralis, 115

femoris, 570,587

Rectus oculi externus, 57

inferior, 57

internus, 57

superior, 53

Rectum, connections of, in the female, 510

connections of, in the male, 504 structure, 532

Recurrent interosseous artery, 288 radial, 266

tibial, 631

ulnar, anterior, 268 posterior, 268

Recurrent nerve of pneumogastric, (o) 114,331 , (d) 158 nerve of the tibial, 631

Renal artery, (d) 476, (o) 489 plexus, 451

vein, (o) 476 , (c) 492

Restiform body, 184, 186

Rete testis, 482

Retina, 663 structure, 664

Retrahens aurem, 18

Rhomboideus major muscle, 358 minor, 358

Rima of the glottis, 155

Ring, abdominal, external, 410 internal, 417,421

Risorius Santorini muscle, 39

Rods of retina, 665

Root of the lung, 309, 310

Roots of the nerves, 307,383

Rotatores dorsi, 371
Round ligament of the hip joint, 602 of the liver, 468

of the uterus, $418,512,539$

SACCULE of the ear, 686

Sacculus laryngis, 155

vestibuli, 685

Sacral artery, lateral, 515

middle, 489,517

ganglia, 519

nerves, anterior branches, 518 posterior branches, 372,581 , 584

plexus, 518

Sacro-coccygeal articulation, 544

iliac, 544

vertebral, 544

lumbalis muscle, 362

sciatic ligament, large, 545, 592 small, 545, 592

Salpingo-pharyngeus muscle, 126

Salvatella vein, 361

Saphenous vein, external, 606, 626 internal, 555, 573, 606, (o) 626 opening, 559 nerve, external, 606, (d) 627 internal, (o) 573 , (d) 557 , 573,627

Sartorius muscle, 566

Scala tympani, 684 vestibuli, 684

Scalenus anticus muscle, 75 medius, 75 posticus, 75

Scapular artery, posterior, 78, 248, 359 ligaments, 247 muscles, 240, 244

Scapulo-clavicular articulation, 246 humeral, 290

Scarpa's triangle, 563

Schneiderian membrane, 135

Sciatic artery, (o) 516, (d) 588

nerve, large, 519, 589, 599 small, 519, 582, 588, 599

Sclerotic coat of the eye, 655 structure, 656

Scrotum, 408

Second nerve, (o) 179, (c) 57, (d) 664

Secondary membrane of the tympanum, 672,673

Segments of the cord, 382

Semicircular canals, 680

Semilunar cartilages, 638 ganglia, 451

valves of aorta, 321 of pulmonary artery, 318

Semi-bulbs of vagina, 527

Semi-membranosus muscle, 598, $630^{\circ}$

Seminal duets, 523

Seminiferal tubes, 481

Semi-spinalis colli muscle, 370 dorsi muscle, 370

Semi-tendinosus muscle, 597 
Septum auricularum, 322

(ochleæ, 682

crurale, 428,561

intermuscular, of the arm, 258 of the thigh, 572

lucidum, 202

nasi, 134

pectiniforme, 530

scroti, 408

of the tongue, 147

ventriculorum, 324

Serratus magnus muscle, 236

posticus inferior, 360

superior, 360

Seventh nerve, (o) 180 , (c) 142 , (d) 47

Sheath of the fingers, 273

of the rectus, 415

of the toes, 616

Shoulder joint, 290

Sigmoid artery, 442

flexure of the colon, 434

valves, 318,321

Sinus, basilar, 29

of the bulb, 527

cavernous, 28

circular, of Ridley, 29

coronary, 314

lateral, 28

longitudinal, inferior, 27 superior, 25

occipital, 27

petrosal, inferior, 29

superior, 29

pocularis, 526

prostaticus, 527

straight of the skull, 27

torcular, 27

transverse, 29

of Valsalva, 321

Sixth nerve, (o) 180 , (c) 32 , (d) 57

Small intestine, 429,456

omentum, 438

Socia parotidis, 41

Soft commissure, 206

Soft palate, 128 museles of, 128

Solar plexus, 451

Sole of the foot, dissection of, 614

Soleus muscle, 608

Solitary glands, 459, 464

Spermatic artery, (o) 489, (d) 484 cord, 418

fascia, 423

plexus, 443

veins (o) 484, (c) 492

Spheno-palatine artery, 142 ganglion, 139 nerves, 104

Sphincter ani externus, 389 internus, 389

of the pupil, 661

vagin $x, 402$

vesicæ, 525

Spigelian lobe, 467
Spinal accessory nerve, (o) 181, (d) 114 nucleus, 187

arteries, 174, 379

cord, 380

membranes of, 374 structure, 382

nerves, 377

filaments of origin, 383 roots of, 377

veins, 385

Spinalis dorsi muscle, 362

Spiral tube of the cochlea, 682

Splanchnic nerve, large, 342, (d) 452 small, 342 , (d) 452 smallest, 342 , (d) 452

Spleen, 465

connections, 435

structure, 465

Splenic artery, 447,466

omentum, 447

plexus of nerves, 451

vein, 448

Splenius capitis muscle, 361 colli, 361

Spongy bones, 134 part of the urethra, 527

Stapedius muscle, 676

Stapes bone, 675

Stellate ligament, 344

Stenson's duct, 41

Sterno-clavicular articulation, 170

cleido-mastoid muscle, 71

hyoid muscle, 73

thyroid, 73

Stomach, form and divisions, 453

connections of, 431

structure of, 453

Straight sinus, 27

Striate body, 203, 211

Stylo-hyoid ligament, 106 muscle, 83 nerve, 48

glossus muscle, 99, 148

mastoid artery, 94

maxillary ligament, 91

pharyngeus muscle, 106

Subanconeus muscle, 259

Subarachnoid space, 376 of the cord, 376

Subclavian artery, left, 118 , (o) 328 right, 75 vein, 79

Subclavius muscle, 232

Subcrureus, 572

Subcutaneous malar nerve, 60, 104

Sublingual artery, 101 gland, 103

Submaxillary ganglion, 101 gland, 97 region, 97

Submental artery, 86

Suboccipital nerve, anterior branch, 115 posterior branch, 367

Subpeduncular lobe, 214 
Subperitoneal fat, 417,561

Subpubic ligament, 547

Subscapular artery, 235 nerves, 236

Subscapularis muscle, 241

Substantia gelatinosa, 383 perforata antica, 192

Sulci of brain, 193, 213

Sulcus, longitudinal, of the liver, 467 spiralis, 683

transverse, 467

Superficial fascia of the abdomen, 405 of the perinæum, 392 of the thigh, 553,554

Superficialis cervicalis artery, 359 volæ artery, 266

Supinator radii brevis, 288 longus, 284

Supra-orbital artery, 21, 56 nerve, 22,52

renal capsule, 478 plexus, 451

scapular artery, 78, 247, 359 nerve, 80, 248, 359

spinal artery, 248

spinatus muscle, 247

trochlear nerve, 23, 52

Suspensory ligament of the lens, 667 of the liver, 439 of the penis, 408

Sympathetic nerve in the abdomen, 443 , 452

in the head, 33

in the loins, 497

in the neck, 115

in the pelvis, 519

in the thorax, 341

Symphysis pubis, 446

Synovial gland of Havers, 603

TENIA hippocampi, 204 semicircularis, 204

Tarsal artery, 631 articulation, 647

cartilages, 44

Tarso-metatarsal articulations, 649

Taste buds, 147

Teeth, 132

Tegmentum, 191

Temporal aponeurosis, 19 artery, 86 deep, 94 middle, 87 superficial, 21

fascia, 19

muscle, 20,88

nerves, deep, 95 superficial, 23,48

vein, 21,88

Temporo-fascial nerve, 48 malar nerve, 60 maxillary articulation, 90 sphenoidal lobe, 194
Tendo Achillis, 609 palpebrarum, 40

Tendon of triceps extensor, 566

Tensor palati muscle, 129

tarsi, 59

tympani, 676

vaginæ femoris, 570

Tentorium cerebelli, 27

Teres major muscle, 245 minor, 245

Testes, 479

Thalamus opticus, 207, 211

Thebesian foramina, 316 valve, 317

Thigh, dissection of, back, 592, 597 front, 552

Third nerve, (o) 180 , (d) 54,57 , (c) 30 ventricle, 206

Thoracic duct, 118, 339, 494 ganglia, 339

Thoracic-acromial artery, 234 alar, 234

humeral, 234

long, 234

superior, 234

Thorax, boundaries of, 305 parietes of, 342, 237

Thymus body, 308

Thyro-arytænoid articulation, 162 ligaments, 156

arytænoideus muscle, 153

epiglottidean ligament, 162

hyoid membrane, 161 muscle, 73

Thyroid artery, inferior, 78, 121 superior, 85,121

axis of artery, 78

body, 120

cartilage, 159

plexus of veins, 121

vein, inferior, 78, 121

middle, 82 superior, 85

Tibial artery, anterior, 630 posterior, 612

nerve, anterior, 683, (d) 627 posterior, 613

veins, anterior, 632 posterior, 613

Tibialis anticus muscle, 628 posticus, 611,625

Tibio-tarsal articulation, 643

Tongue, 146

muscles of, 148

nerves of, 151

vessels of, 101

Tonsil, 131

Tonsilitic artery, 86

Torcular Herophili, 27

Trachea, connections of, 121, 333 structure of, $16^{\circ} 2$

Trachelo-mastoid muscle, 363

Tractus intermedio-lateralis, 383

Tragus, 46 


\section{Tragus-}

muscle, 46

Transverse colon, 434

fissure of the cerebrum, 204 of the liver, 468

ligament of the acetabulum, 602 of the atlas, 169 of the fingers, 273

of the knee, 639 of the metacarpus, 280 of the metatarsus, 624 of the toes, 615

perinæal artery, 393

sinus, 29

tarsal articulation, 647

Transversalis abdominis muscle, 413 cervicalis artery, (o) 78, (d) 359 colli muscle, 363

faciei artery, (o) 87 , (d) 41 fascia, 417,428

Transversus auriculæ muscle, 47 linguæ, 150

pedis, 621

perinæi, 395, 403 deep, 397, 403

Trapezius muscle, 354

Trapezoid ligament, 246

Triangle of the neck, anterior, 69 posterior, 69

Triangular cartilage of the nose, 134

fibro-cartilage of wrist, 297

ligament of groin, 411 of the urethra, 396

space of the thigh, 562

surface of the bladder, 525

'Triangularis sterni muscle, 238

Triceps extensor cruris, 570 cubiti, 258

Tricuspid valve, 318

Trigeminal nerve, (o) 180 , (c) 31

Trigonum vesicæ, 525

Trochlea, 53

Trochlear nerve, infra, 54 supra, 23,52

Tube of the cochlea, 681

Tuber cinereum, 206

Tubercle of Rolando, 187

'Tubules of the stomach, 454 of small intestine, 459 of large intestine, 463

Tubuli seminiferi, 481 uriniferi, 474

Tunica albuginea testis, 481

Ruyschiana, 660

vaginalis, 480 oculi, 655

vasculosa testis, 481

Turbinate bones, 134

'Twelfth intercostal nerve, 416, 498, 581

Tympanic artery, 93

'Tympanum, 674

arteries of, 674

lining membrane, 677

nerves of, 677
ULNAR artery, 267, 273

nerve, (o) 236, (c) 255 , (d) 269,274

veins, 268

cutaneous anterior, 261 posterior, 261

Umbilical hernia, 426

region of the abdomen, 431

vein, 469

Umbilicus, 404

Ureter, 477, 510, 526

Urethra, female, 541 comnections, 512

orifice of, 535

structure, 542

male, interior, 526 connections, 507, 526 structure, 527

Uterine arteries, $51 i, 539$

plexus of nerves, 520

veins and sinuses, 517

Uterus, 537

interior of, 538

ligaments of, 510

connections of, 511

structure of, 538

Utricle of the ear, 685

Uvea iridis, 661

Uvula cerebelli, 212

palati, 128

vesicæ, 574

VAGINA, connections, 512, 535

structure and form, 536

Vaginal arteries, 516, 537

plexus, 520

veins, 517

Vagus nerve, 112, 181, 330, 452

nucleus, 187

Vallecula, 212

Valve, Eustachian, 317

of cæcum, 462

mitral, 320

semilunar, 318, 321

of Thebesius, 317

tricuspid, 318

of Vieussens, 215

Valvulæ conniventes, 457

Vas deferens, 418, 483, 523

aberrans, 483

Vasa aberrantia, 250

brevia, 447

efferentia testis, 482

rete testis, 482

vorticosa, 660 .

Vascular coat of eye, 658

Vastus externus muscle, 571

intermus muscle, 571

Vein, alveolar, 142

angular, 21

ascending cervical, 78

lumbar, 498

pharyngeal, 110

auditory, 687 
Vein-

auricular, posterior, 21

axillary, 235

azygos, large, 338,495

small, 338, 494

superior, left, 338

basilic, 249

brachial, 255

brachio-cephalic, left, 329 right, 328

bronchial, left, 336,337 right, 336,337

cardiac, anterior, 314 great, 314 small, 314

cava, inferior, $329,444,492$ superior, 328

cephalic, 250

cerebellar, 177

cerebral, 177

choroid, 206

ciliary, anterior, 662 posterior, 663

circumflex iliac, 420, 491

coronary of the heart, 314 of the stomach, 448

of the corpus cavernosum, 531 striatum, 206

deep cervical, 77, 368

diaphragmatic, inferior, 492

dorsal, of the penis, 517

dorsi-spinal, 369

emissary, 21

emulgent, 476, 492

epigastric, deep, 420, 491 superficial, 407,554

facial, 40 , $\varepsilon 6$

femoral, 565,568

frontal, 21

of Galen, 208

gastro-epiploïc, left, 449

hemorrhoidal, 517, 533

hepatic, 471, 492

iliac, common, 491 external, 491 internal, 517

infraorbital, 40,105

innominate, 328

intercostal, 338 posterior branch, 368,385 superior, left, 338 right, 338

intraspinal, 385

interlobular, 471

intralobular, 471

jugular, anterior, 71 external, 42, 62

internal, left, 118 right, 82, 109

laryngeal, 158

lingual, 101

longitudinal, of the spine, anterior, 385

lumbar, 369, 498
Vein-

mammary, internal, 238, 329

median, of the arm, 249, 261

basilic, 249

cephalic, 249

maxillary, internal, 94, 329

anterior, internal, 40, 142

mesenteric, inferior, 443 superior, 441

occipital, 21, 80, 369

ophthalmic, 57

ovarian, 492

palpebral, inferior, 40

pancreatic, 448

perineal, superficial, 393

pharyngeal, 110

phrenic, inferior, 492

popliteal, 596

portal, 448, 471

posterior, spinal, plexus of, 385

profunda of the thigh, 579

pterygoid plexus, 94

pudic external, 555

internal, (o) 399, (c) 532

pulmonary, 319, 329

radial cutaneous, 261

ranine, 101

renal, 476,492

sacral, lateral, 517 middle, 517

saphenous, external, 606, (o) 626 internal, 555, (o) 626

spermatic, 484, 492

spinal, 380

splenic, 448

subclavian, 79

sublobular, 471

supra-orbital, 21 renal, 479,492

scapular, 78, 248, 359

temporal, 21,87

superficial, 21

thyroid, inferior, $78,121,329$

middle, 82

superior, 85

tibial anterior, 632 posterior, 613

transverse cervical, 78,359

ulnar, 267

cutaneous, anterior, 261 posterior, 261

umbilical, 469

uterine, 517

vaginal, 517

vertebral, 77, 166

vesical, 517

of the vertebræ, 385

Velum interpositum, 205

pendulum palati, 128

Vena cava, inferior, 444, 492 superior, 328

portæ, 449 , (d) 471

Venæ cavæ hepaticæ, 471

Venous arch of the foot, 626 
Venous arch-

of the hand, 261

Ventricles of the brain, 200

fifth, 202

fourth, 217

lateral, 200

third, 206

of the heart, 313

left, 320

right, 317

structure of, 321

of the larynx, 156

Vermiform appendix, 433,462 processes, 212, 216

Vertebral artery, (o) 77, (c) 165 , (d) 174 plexus, 117, 166 vein, $77,(\mathrm{o}) 166$

Veru montanum, 526

Vessels of the brain, 174 of the dura mater, 29,376

Vesica urinaria, 524

Vesical artery, inferior, 515 superior, 515

plexus of nerves, 520 veins, 517

Vesicula prostatica, 526

Vesicular column of cord, 383

Vesiculæ seminales, connections, 507 structure, 522
Vestibule of the ear, 679 artery of, 687 nerve of, 687 of the vulva, 535

Vestigial fold of pericardium, 311

Vidian artery, 142 nerve, 141

Villi, intestinal, 458

Vitreous body, 666 fluid, 667

Vocal cords, 156

Vulva, 533

W HARTON'S duct, 98, 103

White commissure of the cord, 382

Winslow's foramen, 437

Wrisberg's nerve, 236, 251, 256

Wrist-joint, 296

$Y^{\text {ELLOW spot of eyeball, } 664,666}$

TONULE of Zinn, 667

Zygomaticus major muscle, 39 minor muscle, 39 


\section{HENRY O. LEA'S}

(LATE LEA \& BLANCHARU'S)

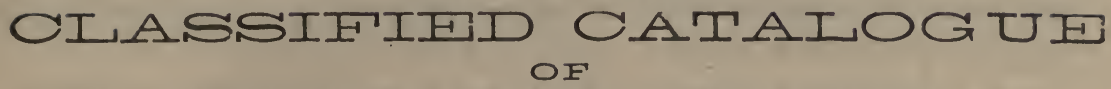

MEDICAL AND SURGICAL PUBLICATIONS.

In asking the attention of the profession to the works advertised in the following pages, the publisher would state that no pains are spared to secure a continuance of the confidence earned for the publications of the house by their careful selection and accuracy and finish of execution.

'The printed prices are those at which books can generally be supplied by booksellers throughout the United States, who can readily plocure for their customers any works not kept in stock. Where access to bookstores is not convenient, books will be sent by mail post-paid on receipt of the price, and as the limit of mailable weight has been removed, no difficulty will he experienced in obtaining through the post-office any work in this catalogue. No risks, however, are assumed either on the money or the books, and no publications but my own are supplied, so that gentlemen will in most cases find it more convenient to deal with the nearest bookseller.

An Itlustrated Catalogue, of 64 octavo pages, handsomely printed, will be forwarded by mail, post-paid, on receipt of ten cents.

HENRY C. LEA.

Nos. 706 and 708 SANSOM St., PhiladelPhia, July, 1879.

ADDITIONAL INDUCEMENT FOR SUBSCRIBERS TO

\section{THE AMERICAN JOURNAL OF THE MEDICAL SCIENCES.}

THREE MEDIOAL JOURNALS, containing over 2000 LARGE PAGES,

Free of Postage, for SIX DOLLARS Per Annum.

TERMS FOR 1879.

The American Journal, of the Mrdical, Sciences and Five Dollars per annum,

The Medical News and Library, both free of postage, $\}$ in ądvance.

\section{OR}

The American Journal of the Medical Scrences, published quar- ) Six Dollars, terly (1150 pages per annum), with

The Medical, News and Library, monthly ( $384 \mathrm{pp}$. perannum), and per annum, annum).

SEPARATE SURSCRTPTIONS TO

in advance.

The American Joursal of the Medical S'ciexces, when not paid for in advance. Five Dollars.

The Medical News and Library, free of postage, in advance, One Dollar.

'The Montuly Abstract of Medical Science, free of postage, in advauce, Two Dollars and a Hulf.

* * Advance paying subscribers can obtain at the close of the year cloth covers, gilt-lettered, for each volume of the Journal (two annually), and of the Abstract (one annually), free by mail, by remitting ten cents for each cover.

In commencing the second year of the second half century in the career of the "American Journal of the Ml kidcar. Sciences," the publisher has much pleasure in assuring its wide circle of readers, that at no former period has it had the prospect of a more extended sphere of usefulness. Sustained as it is by the profession of the whole United States, and with a circulation extending to every country in which the English language is read, the effurts of the editors will be directed, as heretofore, to render it in every way worthy of its reputation, and of the universal favor with which it is received. With its attendant periodicals, the "M EDICAI. News AND Library" and the "MoNTHLY A bSTRACT OF MEDical SCIEnCE," it combines the advantages of the elaborate preparation which can be given to a quarterly, and the prompt conveyance of intelligence by the monthly, while, the whole being under a single editorial supervision, the subscriber is secured against the duplication of matter inevitable under other circumstances. 'T'hese efforts the publisher seeks to second by offering these periodicals at a price unprecedentedly low - a price which places them within the reach of every practitioner, and gives the equivalent of three or four large octavo volumes for the comparatively trifling 
cost of Six Doluars per annum.

'The three periodicals thus offered are universally known for their high professional standing in their several spheres.

I.

\section{THE AMERICAN JOURNAL OF THE MEDICAL SCIENCES,} Edited by I. MINIS HAYS, M.D.,

is published Quarterly, on the first of January, April, July, and October. Fach number contains vearly three hundred large octavo pages, appropriately illustrated wherever necessary. It has now been issued regularly for over FIFTY years, during the whole of which time it has been under the control of the present senior editor. 'Throughout this long period, it has maintained its position in the highest rank of medical periodicals both at home and abroad, and has received the cordial support of the entire profession in this country. Among its Collaborators will be found a large number of the most distinguished numes of the profession in every section of the United States, rendering its original department a truly national exponent of American medicine.*

Following this is the "Review Departaent," containing extended and impartial reviews of important new works, together with numerous elaborate "ANALYTICAL AND Bibiographical Noticks" giving a complete survey of medical literature.

'I'his is followed by the "Quarteri,y Sumiary of Improvenents and Discoveries ix the Medicar. Scruxces," classified and arranged under different heads, presenting a very complete digest of medical progress abroad as well as at home.

'Thus, during the year 1878, the "Jou RNaL" furnished to its subscribers 77 Original (Jommunications, 133 Reviews and Bibliographical Notices, and 255 articles in the Quarterly Summaries, making a total of Four Hundred and Sixtry-Five articles illustrated with 48 maps and wood engravings, emanating from the best professional minds in America and Europe.

'That the efforts thus made to maintain the high reputation of the "Joursat" are successful, is shown by the position accorded to it in both America and Europe as a leading organ of medical progress:-

This is unirersally acknowledged as the leading $\mathrm{Onr}$ venerablè contemporary has our best wishes, American Journal, and has been cunducted by Dr. and we can onlyexpress the hope that it may conHays alone until i 869 , when his son was associated tinue its work with as much vigor and excellence fur with him. We quite agree with the critic, that this the next fifty years as it has exhibited in the past. juurnal is seeond to none in the lauguage, and cheer- -London Lancet, Nov. 2t, 1877.

fully accord to it the first place, for nowhere thall wo find more able and more impartial criticism, and nowhere such a repertory of able original articles. Indeed, now that the "Britibh and Foreign MedicoChirurgical Review" has terminated its career, the
Aneriean Journal stands without a rival.-London Med. Times and Gazette, Nov. 24, 1877.

The best medical jourual on the continent - Boston Med and Surg. Journal, April 17, 1879.

The Philadelphia Medical and Physical Journal issued its first number in 1S20, and, after a brilliant career, was succeeded in 1827 by the American Journal of the Medical Sciences, a periodical of world-wide reputation; the ablest and one of the oldest periodicals iu the world-a journal which has an unsullied record.-Gross's History of American Med. Literature, 1876 .

This is the medical journal of our country to which

It is universally acknowledged to be the leading the American physician abroad will point with the American medical jomrnal, and, iu our opinion, is greatest satisfaction, is reflecting the state of medical recond to none in the language.-Boston Med. and culture in his country. For a great many years it surg. Journal, Oct. 1 s77.

has been the medium throngh which our ablest writ-

The present number of the American Journal is a ers have made kuown their discoveries and observa. exceedingly good one, and gives every promise of tions - Address of L. P. Yandell, M.D., before Inter unaincaining the well-earned reputation of the review. national Med. Congress, Sept. 1876.

And that it was specifically included in the award of a medal of merit to the Publisher in the Vienna Exhibition in 1873.

'The subscription price of the "American Journal, of the Medical, Sciences" has never been raised during its long career. It is still Frve Doltans per annum; and when paid for in advance, the subscriber receives in addition the "MEDICAL NEWS AND Library," making in all about 1500 large octavo pages per annum, free of postage.

II.

\section{THE MEDICAL NLWS AND LIBRARY}

is a monthly periodical of 'Thirty-two large octavo pages, making 384 pages per annum. Its "Library Department" is devoted to publishing standard works on the various branches of medical science, paged separately, so that they can be detached for binding, when complete. In this manner subscribers have received, without expense, such works as "Watson's Practice," "Wrst on Chimdren," "Malgaigne's surgery," "Stokes on Fever," Gosshin's "Clinical Lectures on Surghry," and many other volumes of the highest reputation and usefulness. With July, 1878, was cummenced the publication of "Lectures on Diseases of the Nervous System," by J. M. Cinarcot, Professor in the Faculty of Medicine of Paris, translated from the French by George Signason, M.D., Lecturer on Biology, etc., Catholic Univ. of

* Communicalions are invited from gentlemen in all parts of the country. Elaborate articles inserted by the Editor are puid for by the P'ublisher. 
Ireland (see p. 17), which will be continued to completion during 1879. New subscribers, commencing with January, 1879, can prociure the previous portion by a remittance of 50 cents, if promptly made.

'The "NFws DFpartanent" of the "Mfidical News and Library" presents the current information of the month, with Clinical Lectures and Hospital Gleanings. A new and attractive feature of this will be found in an elaborate series of ORIGINAI. American Crinical Lectures, specially contributed to the News by gentlemen of the highest reputation in the profession throughout the United States. During 1878 there have appeared Lectures by

S. D. Gross, M.D., Prof. of Surgery, Jefferson Med. Coll., Philada.

'T'. Gailliard 'Thomas, M.I., Prof. Obstetrics, \&c., Coll. Phys. and Surg., N. Y.

William Pepper, M.D., Prof. Clin. Medicine, Univ. of Penna.

Lewis A. Sayre, M.I., Prof. Orthopædic Surg., Bellevue Hosp. Med. Coll., N. Y.

Roberts Bartholow, M.D., Prof. Theory and Practice of Med., Med. Coll. of Ohin.

'T'. G. Richardson, M.D., Prof. Genl. and Clin. Surg., Univ. of La., New Orleaus.

S. W. Gross, M.D., Surg. to Philada. Hospital.

F. Peyre Porcher, M.I., Prof. of Mat. Med. and Clin. Medicine, Med. Coll. of S. C.

William Gooderil, M.D., Prof. Clin. Gynecolngy, Univ. of Penna.

N. S. D Avis, M.D., Prof. Prin and Prac. of Med., Chicago Med. Coll.

W. H. Van Burnn, M.D., Prof. Surgery, Bellevue Hosp. Med. Coll., N. Y.

'To be followed by others of' similar value from

A ustin Flint, M.D., Prof. Prin. and Prac, of Med., Bellevue Hosp. Med. Coll., N.Y.

Fordych Barker. M.D., Prof. Clin. Midwifery; \&c., Bellevue Hosp. Med. Coll., N.Y.

L. A. Duhring, M.D., Clin. Prof. of Diseases of the Skin, Univ. of Penna.

'I'h hophilus Pa Rvin, M.D., Prof. Obstetrics, \&c., Coll. Phys. and Surg., Indianapolis.

J. P. White, M.D., Prof. of Obstetrics, \&c., Univ. of Buffilo.

John Asmurst, Jr., M.D., Prof. of Clin. Surg., Univ. of Penna.

D. Warren Bricket.l, M.D., Prof. Obstetrics, \&c., Charity Hosp. Med. Coll., N. O. J. Lewis Smith, M.D., Clin. Lec. on Dis. of Chil., Bellevue Hosp. Med. Coll., N. Y. William F. Norris, M.D., Clin. Prof. of Diseases of the Eye, Univ. of Penna.

P. S. Conner, M.D., Prof. of Anat. and Clin. Surgery, Med. Coll. of Ohio, Cin.

S. Weir Mitcheld, M.D., Phys. to the Infirmary for Nervous Diseases, Philada.

J. M. DaCosta, M.D., Prof. Prin. and Prac. of Med., Jeff. Med. Coll., Philada.

Thomas G. Morton, M.D., Surgeon to Penna. Hospitrl, Philada.

F. J. Bunstead, M.D., late Prof. of Venereal I)is., Coll. Phys. and Surg., N. Y.

J. H. Hutchisson, M.D., Physician to Penna. Hospital.

Christopher Johnson, M.D., Prof. of Surgery, Univ. of Md., Baltimore.

Wrltian 'I'nomson, M.D., Lecturer on Ophthalmology, Jeff. Med. Coll., Philada.

With contributors such as these, representing every portion of the United States, the publisher feels safe in promising to the subscriber a series of practical lectures unsurpassed in variety, interest, and value.

As stated above, the subscription price of the "Mrodcal News and Library" is ONe Dolia R per annum in advance; and it is furnished without charge to all advancepaying subscribers to the "American Journal of the Medical Scrences."

III.

\section{THE MONTHLY ABSTRACT OF MEDICAL SCIENCE}

is issued on the first of every month, each number containing forty-eight large octavo pages, thus furnishing in the course of the year about six hundred pages. "The aim of the "ABSTRACT" is to present-without duplicating the matter in the "Journal" and "News"-a careful condensation of all that is new and important in the medical journalism of the world, and all the prominent professional periodicals of both hemispheres are at the disposal of the Editors. 'T'o show the manner in which this plan has been carried out it is sufficient to state that during the year 1878 it contained-

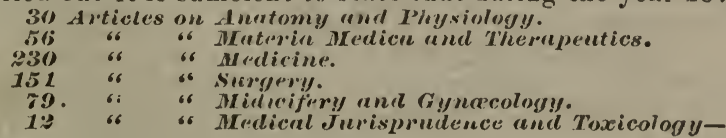

making in all 558 articles in a single year.

'The subscription to the "Nonthly Abstract," free of postage, is Two Dollaars AND A HALF a year, in advance.

As stated above, however, it will be supplied in conjunction with the "AMEricas Journal of the Medical Sciences" and the "Medical News and Library," making in all abont 'Twenty-one Hundred pages per anuum, the whole free of postage, for Six Dorisars a year, in advance.

In this effort to bring so large an amount of practical information within the reach of every member of the profession, the publisher confidently anticipates the friendly 
aid of all who are interested in the dissemination of sound medical literature. He trusts, especially, that the subscribers to the "Amrrican Medical Journal" will call the attention of their acquaiutances to the advantages thus oflered, and that he will be sustained in the endeavor to permanently establish medical periodical literature on a footing of cheapness never heretofore attempted.

\section{PREMIUM FOR OBTAINING NEW SUBSORIBERS TO THE "JOURNAL,"}

Any gentleman who will lemit the amount for two subscriptions for 1879 , one of which must be for a new subscriber, will receive as a PRFMIUN, free by mail, a copy of "Holofn's Landmarks, Medical axd Surgical" (for advertisement of which see p. 6), or of Fothergilla's "Antagonism of Medicines" (see p. 16), or of "Browne on the Use of the Ophthalmoscope" (Eee p. 2!!), or of "Fint's Essays on Conservatite Medicine" (see p. 15), or of "Sturges's Clinical M fidicine" (see p. 14), or of the new edition of "Swayne's Obstetric Aphorisus" (see p. 21), or of "T'anner's Cuinical Manual" (see p. 5), or of "Chambers's Restorativg Medicine" (see p. 18), or of "WESt on Nervous IISORDers of Childien" (see p. 20 ).

* * Gentlemen desiring to avail themselves of the advantages thus offered will do well to forward their subscriptions at an early day, in order to insure the receipt of complete sets for the year 1879 .

C. The safest mode of remittance is by bank check or postal money order, drawn to the order of the undersigned. Where these are not accessible, remittances for the "Journal" may be made at the risk of the publisher, by forwarding in REGIsTERED letters. Address,

Henty C. LFa, Nos. 706 and 708 Sansom St, Philadelphia, Pa.

DUNGLISON (ROBLEY), M.D.,

Late Professor of Institutes of Medicine in Jefferson Medical College, Philadelphia.

MediCAL LEXICON; A Dictionary of Medical Science: Cor. taining a concise explanation of the various Subjects and Terms of Anatomy, Physiolngy. Pathology, Hygiene, Therapeutics, Pharmacology, Pharmacy, Surgery, Obstetrics, Medical Jurisprudence, and Dentistry. Notices of Climate and of Mineral Waters; Formulø for Officinal, Empirical, and Dietetic Preparations; with the Accentuation and Etymology of the Terms, and the French and other Synonymes; so as to constitute a French as well as English Medical Lexicon. A New Edition. Thoroughly Revised, and very greatly Modified and Augmented. By Richard J. Iunglison, M.D. In one very large and hand. some royaloctavo volume of over 1100 pages. Cloth, $\$ 650$; leather, raised bands, $\$ 750$. (Just Issued.)

The object of the author from the outset has not been to make the work a merelexicon or dictionary of terms, but to afford, undereach, a condensed view of its various medical relations, and thus to render the work an epitome of the existing condition of medical science. Starting with this view, the immense demand which has existed for the work has enabled him, in repeated revisions, to augment its completeness and usefulness, until at length it has attained the position of a recognized and standard authority wherever the language is spoken.

Special pains have been taken in the preparation of the present edition to maintain this en. viable reputation. During the ten years which have elapsed since the last revision, the additions to the nomenslature of the medical sciences have been greater than perhaps in any similar period of the past, and up to the time of his death the authorlobored assiduously to incorporate every. thing requiring the attention of the student or practitioner. Since then, the editor has been equally industrious, so that the additions to the vocnbulary are more numerous than in any previous revision. Especial attention has been bestowed on the accentuation, which will be found marked on every word. The typ igraphical arrangement has been much improved, rendering reference much more easy, and ev $\rightarrow$ ry care has been taken with the mechanical execution. The work has been printed on new type, small butexceedingly clear, with an enlarged page, so that the additions have been incorporated with an increase of but little over a hundred pages, and the volume now contains the matter of at least four ordinary octavos.

A book well known to our readers. and of which | ssience so extensive, and with such collaterals as medi. every American ought to be proud. When the learned :ine, it is as much a neressity also to the practising author of the work passed away, probably all of us physician. To meet the wants of students and most feared lest the bonk should not maintain its place physitians, the dictionary must be condensed while in the advancing science whose terms it defines. For- somprehensive, and practical while perspicacious. It tunately, Dr. Richard J. Dunglisnn, having assisted lis sas because Dunglison's met these indications that it father in the revision of several editions of the work, became at once the dictionary of general use wherever and having been, therefore, trained in the methods and medicine was studied in the English lauguage. In no l mbued with the spirit of the bnok, has been able to former revision have the alterations and additions been edit it, not in the patchwork manner so dear to the lo great. Murethan six thousand new subjects snd ternıs heart of book editors, so repulsive to the taste of intel. have been added. The chief terms have been set in black ligent book readers, but to editit as a work of the kind letter, while the derivatives follow in small caps; an should be edited-to carry it on steadily, without jar arrangement which greatly facilitates reference. We or interruption, along the groeves of thoupht it has may cofely confirm the hope ventured by the editor travelled during its lifetime. To show the magnitude "that the work, which posesses for him a filial as well of the task which Dr. Dunglison has assumed and car. aq an indivilual interest, will be found worthy a con. ried throush, it is only necessary to state that more linuance of the position so long accorded to it as a than six thousand new guhjects have been adder in the 3tandard authoritv."-Cincinnati Clinic. Jan. $10,1874$. present edition.- Phila. Med. Times, Jan. 3, 1874 .

About the first book purchased by the medical stu. dent is the Yedical Dictionary. The lexicon explang. Ceferences_London Madical Garatte. tory of technical terms is simply a sine qua non. In a 
A CENTURY OF AMERICAN MEDICINE, 1776-1876. By Doctors E. H.

Clarke, H. J. Bigelow, S. D. Gross, T. G. Thounas, and J. S. Billings. In one very hand. some $12 \mathrm{mo}$. volume of about 350 pages : cloth, $\$ 225$. (Just Ready.)

This work appeared in the pages of the American Journal of the Medical Sciencesduring the year 1876. As a detailed account of the development of medical science in America, by gentlemen of the highest authority in their respective departments, the profession will no doubt welcome it in a form adapted for preservation and reference.

$H^{O B L Y N}(R I C H A R D \quad D),. M . D$.

A DICTIONARY OF THE TERMS USED IN MEDICINE AND THE COLLATERAL SCIENCES. Revised, with numerous additions, by JsAAC HAYs, M. D., Editor of the "American Journal of the Medical Sciences." In one large royal $12 \mathrm{mo}$. volume of over 500 double-columned pages; cloth, $\$ 150$; leather, $\$ 200$

It is the best book of definitions we have, and ought always to be npon the student's table. - Southern Med. and Surg. Journal.

ROD WELL (G.F.), F.R.A.S., \&.c.

A DICTIONARY OF SCIENCE: Comprising Astronomy, Chemistry, Dynamics, Electricity, Heat, Hydrodynamics, Hydrostatics, Light, Magnetism, Mechanics, Meteorology, Pneumatics, Sound, and statics. Preceded by an Essay on the History of the Physical Sciences. In one handsome octavo volume of 694 pages, and many illustrations: cloth, $\$ 5$.

NEILL (JOHN), M.D., and

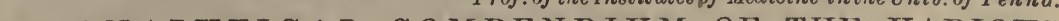

AN ANALYTICAL COMPENDIUM OF THE VARIOUS BRANCHES OF MEDICAL SCIENCE; for the Use and Examination of Students. A new edition, revised and improved. In one very large and handsomely printed royal $12 \mathrm{~m}($. volume, of about one thousand pages, with 374 wood-cuts, cloth, $\$ 4$; strongly bound in leather, with raised bands, $\$ 475$.

HARTSHORNE (HENRY), M.D.,

Professor of Hygiene in the University of Pennsylvania.

A CONSPECTUS OF THE MEDICAL SCIENCES; containing Handbooks on Anatomy, Physiology, Chemistry, Materia Medica, Practical Medicine, Surgery, and Obstetrics. Second Edition, thoroughly revised and improved. In one large royal $12 \mathrm{mo}$. volume of more than 1000 closely printerl pages, with 477 illustrations on wood. Cloth, $\$ 425$; le ather, $\$ 500$. (Lately Issued.)

We can say with the strictest truth that it is the dents, but to many others whomay desire torefresh best work of the kind with which w' artacquainted. their memories with the smallest possible expendi. It embodies in a cordensed form all recent coutribu- tare of time.-N. Y. Med. Journal, Sept. $187 t$.

tions to practical medicine, and is therefore useful Thestudent will find this the most convenient and to every busy practitioner throughout our country, useful book of the kind on which he can lay his besides being admirably adapted to the use of stu- band.-Pacific Med. and Surg. Journ., Aug. 1S74.

dents of medicine. The book is faithfully and ably executed.-Charleston Med. Journ., A pril, 1875.

The work is intended as an aid to the medical stulent, and as such appears to admirably fulfil its object by itsexcellent arraggement, the full compilation of facts, the perspicuity aud terseness of language, and the clear and instrnctive illustrations in sume parts of the work.-American Journ. of Phairmacy, Philadelphia, July, 187t.

The volume will be found useful, not only to sta.

This is the best book of its kind that we have ever examined. It is an honest, accurate, and concise compend of medical sciences, as fairly as possible representing their present condition. The changes and the additions have been so judicious and thorough as to render it, so far as it goes, entirely trustworthy. If students mast have a conspectus, they will be wise to procure that of Dr. Hartshorne.Detroit Rev. of Med and Pharm., Aug. 1874.

UDLOW (J.L.), M.D.

A MANUAL OF EXAMINATIONS upon Anatomy, Physiology, Surgery, Practice of Medicine, Obstetrics, Materia Medica, Chemistry, Pharwacy, and Therapeutics. To which is added a Medical Formulary. Third edition, thoroughly revised and greatly extended and enlarged. With 370 illustrations. In one handsome royal $12 \mathrm{mo}$. volume of 816 large pages, cloth, $\$ 325$; leather, $\$ 375$.

The arrangement of this volume in the form of question and answer renders it especially suit. able for the office examination of students, and for those preparing for graduation.

TANNER (THOMAS HA WKES), M.D., \&c.

A MANUAL OF CLINICAL MEDICINE AND PHYSICAL DIAG. NOSIS. Third American from the Second London Edition. Revised and Enlarged by Trlbury Fox, M. D., Physician to the Skin Department in University College Hospital, \&c. In one neat volume small $12 \mathrm{mo}$, of about 375 pages, cloth, $\$ 150$.

** On page 4, it will be seen that this work is offered as a premium for procuring new subseribers to the "Amemican Journal of the Medical Sciences." 
GRAY (HENRY),F.R.S.,

Lecturer on Anatomy at St. George's Hospital, London.

ANATOMY, DESCRIPTIVE AND SURGICAL. The Drawings by H. V. CARTER, M.D., and Dr. WestracotT. The Dissectionsjointly by the Autror and Dr. Carter. With an Introduction on General Anatomy and Developuent by $T$. HolmBs, M.A., Surgeon to St. George's Hospital. A new American, from the eighth enlargec and improved London edition. To which is added "LANDMarks, Medical and Surgical," by Luther Holden, F.R.C.S., author of "Human Osteology," "A Manual of Dissections," etc. In one magnificent imperial octavo volume of 983 pages, with 522 large and elaborate engravings on wood. Cloth, $\$ 6$; leather, raised bands, $\$ 7$. (Just Ready.)

The author has endeavored in this work to cover a more extendedrange of subjects than is cue. tomary in the ordinary text-books, by giving not only the details necessary for the student, but also the application of those details in the practice of medicine and sargery, thusrendering it both a guide for the learner, and an admirable work of reference for the active practitioner. The engravings form a special feature in the work, many of them being the size of nature, nearly all original, and having the names of the various parts printed on the body of the cut, in place of figures of reference, with descriptions at the foot. They thus form a complete and splendid series, which will greatly assist the studentin obtaining a clear idea of Anatomy, and will also serve to refresh the memory of those who may find in the exigencies of practice the necessity of recalling the details of the dissecting room; while combining, as it does, a complete Atlas of Anatomy, with a thorongh treatise on systematic, descriptive, and applied Anatomy, the work will be found of essentinl use to all physicians who receive students in their offices, relieving both preceptor and pupil of much labor in laying the groundwork of a thorough medical education.

Since the appearance of the last American Edition, the work has received three revisions at the hands of its accomplished editor, Mr. Holmes, who has sedulously introduced whatever has seemed requisite to maintain its reputation as a complete and authoritp tive standard text-book and work of reference. Still further to increase its usefulness, there $h_{2}$ s heen appended to it the recent work by the distinguished anatomist, Mr. Luther Holden- "Landmarks, Medical and Surgical" - which gives in a clear, condensed, and systematic way, all the information by which the praetitioner can determine from the external surface of the body the position of internal parts. Thus complete, the work, it is believed, will furnish all the assistance that can be rendered by typeand illustration in anatomical study. No pains have been spared in the typographical execution of the volume, which will be found in all respects superior to former issues. Notwithstanding the inerease of size, amounting to over 100 pages and 57 illustrations, it will be kept, as heretofore, at a price rendering it one of the cheapest works ever offered to the American profession.

The recent work of Mr. Holden, which was no- to consult his books on anatorny. The work is ticed by us on p. 53 of this volume, has been added simply indispensable, especially this present Ameris an appendix, so that, altogether, this is the most ican edition.-Va. Med. Monthly, Sept. 1s7s,

practical and complete a natomical treatice a vailable to A merican students and physicians. The former finds in it the necessary guide in making dissectíns: a very comprehensive chapter on mirute anatomy; and about all that can be taught bim on general and special anatomy; while the latter, in its treatment of each region from a snrgieal point of view, and in the valuable edition of Mr. Holden, will find all that will bo essential to him in his practice-New Remedies, Aug. 1878.

This work is as near perfection as one could possibly or reasonably expect any book intended as a text-book or a general reference book on anatomy to be. The American publisher deserves the thanks of the professiou for sppending the recent work of Mr. Holden, "Landmarks, Aredical and Surgical," which has already been commended as a separate book. The latter work-treating of topographical anatomy-lias become an essential to the library of every intelligent practitioner. We know of no book that can take its place, written as it is by a most distinguinhed anatomist. It would be simply a waste of words to say anything further in praise of Gray's Anatomy, the text-book in almost every medical college in this country, and the duily refer. ence book of every practitioner who has occasion

The addition of the receut work of Mr. Holden, as an appendix, renders this the most practical and complete treatise available to American students, who find in it a comprehensive chapter on minute anatomy, about all that can be taught on general and special anatomy, while its treatment of each region, from a surgical point of vie ${ }^{2}$, in the valuable section by Mr. Holden, is all that will be essential to them in practice.-Ohio Medical Recorder, Aug. $157 \mathrm{~S}$.

It is difficult to speak in moderate terms of this new edition of "Gray." It seems to be as nearly perfect as it is possible to make a book devoted to any branch of medical science. The labors of the eminent men who have successively revised the eight editions thrungh which it lias passed, would seem to leave nothlng for future editors to do. The addition of Holden's "Landmarks" will make it as indispensable to tl:e practitioner of medicine and surgery as it has been heretofore to the student. As regards completeness, ease of reference, utility, beanty, and cheapness, it has no rival.' No student should enter a medical school withont it; no physician can afford to bave it absent from his library. - St. Louis Clin. Record, Sept. $187 \mathrm{~S}$.

ALSO FOR SALE SEPARATE-

HOLDEN (LUTHER), F.R.C.S. Surgeon to St. Bartholomew's and the Foundling Hospitals.

LANDMARKS, MEDICAL AND SURGICAL. From the 2d London Ed. In one handsome volume, royal $12 \mathrm{mo}$, of 128 pages : cloth, 88 cents. (Now Ready.)

EATH (CHRISTOPHER), F.R.C.S. Teacher of Operative Surgery in University College, London.

PRACTICAL ANATOMY: A Manual of Dissections. From the Second revised and improved Londonedition. Edited, with additions, by W. W. KEEN, M. D., Lecturer on Pathological Anatomy in the Jefferson Medical College, Philadelphia. In one handsome royal $12 \mathrm{mo}$.volume of 578 pages, with 247 illustrations. Cloth, $\$ 350$; leather, $\$ 400$. 
A LLEN (HARRISON), M.D.

Professor of Physiology in the Univ. of $P a$.

A SYS'TEM OF HUMAN ANATOMY: INCLUDING ITS MEDICAL and Surgical Relations. For the Use of Practitioners and Students of Medicine. With an Introductory Chapter on $\mathrm{H}$ istology. By E. O. SחAKESPEARE, M D., Ophthalmologist to the Phila. Hosp. In one large and handsome quarto volume, with several hundred original illustrations on Jithographic plates, and numerous wood-cuts in the test. (Prepuring.)

In this elaborate work, which has been in active preparation for several years, the author has sought to give, not only the details of descriptive anatomy in a clear and condensed form, but also the practical applications of the science to medicine and surgery. The work thus has claims upon the attention of the general practitioner, as well as of the student, enabling him not only to refresh his recollections of the dissecting room, but also to recognize the significance of allvariations from normal conditions. The marked utility of the object thus sought by the author is self.evident, and his long experience and assiduous devotion to its thorough development are a sufficient guarantee of the manner in which his aims bave been carried out. No pains have been spared with the illustrations. Those of normal anatomy are from original dissectiuns, drawn on stone by Mr. Hermann Faber, with the name of every part clearly engraved upon the figure, after the manner of "Holden" and "Gray," and in every typographical detail it will bo the effort of the publisher to render the volume worthy of the very distinguished position which is anticipated for it.

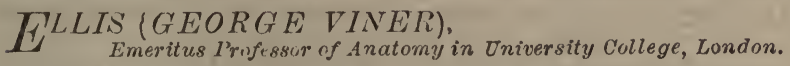

DEMONSTR A'TIONS OF ANATOMY; Being a Guide to the Knowledge of the Human Body by Dissection. By George Viner Ellis, Emeritus Professor of Anatomy in University College, London. From the Eighth and Revised London Edition. In one very handsome octavo volume of over 700 pages, with 256 illustrations. Cloth, $\$ 4.25$; leather, $\$ 5.25$. (Just Ready.)

This work has long been known in England as the leading authority on practical anatomy, and the favorite guide in the dissecting-room, as is attested by the numerous editions through which it has passed. In the last revision, which has just appeared in London, the accomplished author has sought to bring it on a level with the most recent advances of science by making the necessary changes in his account of the microscopic structure of the different organs, as dereloped by the latest researches in textural anatomy.

Ellib's Demonstrations is the favorite text-book its leadership over the English manuals upon disof the English student of anatomy. In passing secting.-Phila. Med. Times, May 24, 1879.

through eight editions it has been so revised and adapted to the needs of the student ihat it would seem that it had almost reached perfection in this special line. The descriptions are elear and the methods of pursuing anatomical investigations are given with such detail that the book is honestly entitled to its name.-St. Luuis Clinical Record, June, 1879.

The success of this old manual seems to be as well deserved in the present as in the past volnmes. The bouk seems destined to maintain yet for years

As a dissector, or a work to have in hand and studied while one is engaged in dissecting, we re gard it as the very best work extant, which is certainly saying a very great deal. As a text-book to be studied in the dissecting-room, it is superior to any of the works upon anatomy.-Cincinnuti Med. News, May $2 t, 1879$.

We most unreservedly recommend it to every practitioner of medicine who can possibly get it.Va. Mer. Monthly, June, 1879.

MILSON (ERASMUS), F.R.S.

A SYSTEM OF HUMAN ANATOMY, General and Special. Edited by W. H. Goвrecut, M.D, Professor of General and Surgical Anatomy in the Medical Col. lege of Ohio. Illustrated with three hundred and ninety-seven engravings on wood. In one large and handsome octavo volume, of over 600 large pages; cloth, $\$ 4$; leather, $\$ 5$.

SITH (HENR Y H.), M.D., and $H_{\text {Prof. of Surgery in the Univ. of Penna., \&e. }}^{O R E R}$ (WILLIAM E.), M.D.,
Late Prof. of Anatomy in the Univ. of Penna.

AN ANATOMICAL ATLAS, Illustrative of the Structure of the Human Body. In one volume, large imperial octavo, cloth, with about six hundred and fifty beautiful figures. $\$ 450$.

SCHAFER (EDWARD ALBERT), M.D., Assistant Profersor of Physiology in University College, London.

A COURSE OF PRACTICAL HISTOLOGY: Being an Introduction to the Use of the Microscope. In one handsome royal $12 \mathrm{mo}$. volume of 304 pages, with numerous illustrations: cloth, $\$ 200$. (Just Issued.)

HORNER'S SPECIAL, ANATOMY AND HISTOLOGY. Eighth edition, extensively revised and modified. In 2 vols. 8 vo., of over 1000 pages, with 320 wood-cuts: cloth, $\$ 600$

SHARPEY AND QUAIN'S HUMAN ANATOMY. Revised, by JosEPH LEIDY, M. D., Prof of Anat. in Uuiv. of Penn. In two octavo vols. of about 1300 pages, with 511 illustrations Cloth, $\$ 600$.
BELLAMY'S STUDENT'S GUIDE TO SURGICAL ANATOMI : A Text book for Stadents prepariog for their Pass Examination. With engravings on wood. In one handsome royal $12 \mathrm{mo}$. volume. Cloth, \$2 25.

CLELAND'S DIRECTORY FOR THE DISSECTION OF THE HUMAN BODY. In one sinall volume, ruyal $12 \mathrm{mo}$. of 182 pages: cloth, $* 125$. 
CARPENTER (WILLIAM B.), M.D., F.R.S., F.G.S., F.L.S., Registrar to University of London, etc.

PRINCIPLES OF HUMAN PHYSIOLOGY; Edited hy HENRY POWER, M.B. Lond., F.R.C.S., Examiner in Natural Sciences, University of Oxford. A new American from the Eighth Revised and Enlarged English Edition, with Notes and Additions, by Francis G. Sмгтв, M.D., Professor of the Institutes cf Medicine in the University of Pennsylvania, etc. In one very large and hand some octavo volume, of 1083 pages, with two plates and 373 engraving on wood; cloth, $\$ 550$; leather, $\$ 50$. (Just Issued.)

Thegreat work, the crowning labor of the distinguished author, and through which so many generations of students have acquired their knowledge of Physiology, has been almost meta. morphosed in the effort to ac'apt it thoroughly to the requirements of modern science. Since the appearance of the last American edition, it has had several revisions at the experienced band of Mr. Power, who has modified and enlarged it so as to introduce all that is important in the investigations and discoveries of England, France, and Germany, resulting in an enlargement of about one-fourth in the text. The series of illustrations has undergone a like revision, a large proportion of the former ones having been rejected, and the total number increased to nearly four hundred. The thorough revigion which the work has so recently receired in England, has rendered unnecessary any elaborate additions in this country, but the American Editor, Professor Smith, has introduced such matters as his long experience has shown him to be requisite for the student. Every care has been taken with the typographicalexecution, and the work is presented, with its thousand closely, but clearly printed pages, as emphatically the text-book for the student and practitioner of medicine-the one in which, as heretofore, especial care is directed to show the applications of physiology in the various practical branches of medical science. Notwithstanding its very great enlargement, the price has not been in. creased, rendering this one of the cheapest works now before the professiun.

We have been agreeably surprised to find the vol- subject, perfectly certain of the fulness of information ume so complete in regard to the structure and func- it will convey, and well satisfied of the accuracy with tions of the nervous system in all its relations, a which it will there be found stated.-London Med. lions of the throus system in all its relation, a subject that, in many respects, is one of the most diffiwhich to produce a full and satisfactory treatise of the class to which the one before us belongs. The additions by the American editor give to the work as it is a considerable value beyond that of the last English edition. In conclusion, we can give our cor dial recommendation to the work as it now appears. The editors have, with their additions to the only work on physiology in our language that, in the fnllest sen:- of the word, is the production of a philosopher as well as a physiologint, brought it up as fully as could be expected, if not desired, to the standard of our knowledge of its subject at the present day. It will deserved ly maintain the place it has always had in the favor of the medical profession.-Journ. of Nervous and Mental Disease, April, 1 S77.

"Good wine needs no bush" says the proverb, and an old and faithful servant like the "big" Carpenter, as carefully brought down as this edition has been by Ir. lienry Fower, needs little or no commendation by us. Such enormous advances have recently been inade in rur physiological knowledge, that what was perfectly new a year or two ago. looks now as if it had been a received and established fact for years. In this encyclopædic way it is unrivalled. Ilere, as it seems to us, is the great value of the book: one is are in sending a student to it for information on slmost any given

Times and Gazette, Feb. 17, 1877.

Thus fully are treated the structure and functions of all the important organs of the body, while there are chapters on sleep and somnamiulism; chapters on eth nology, a full section on generalion. and abundant references to the curiosilies of physiology, as the evolv tion of light, heat, electricity, ete. In short, this new edition of Carpenter is, as we have said at the start, a very encyclopedia of modern physiology.-The Clinic, Feb. 24, 1877.

The merits of "Carpenter's Phy siology" are so widely known and appreciated that we need only allude briefly to the fact that in the latest edition will be found a conprehensive embodiment of the results of recent physio logical investigation. Care has been taken to preserve the practical character of the original work. In fact the entire work has been brought up to date, and bear evidence of the amount of labor that has been bestowed upon it by its distinguished editor, Mr. Ilenry Power. The American editor bas msde the latest additions, in order fully to cover the time that has elapsed since the last English edition. $-N$. Y. Med. Journal, Jan. 1877.

A more thorough work on physiology could not be found. In this all the facts discovered by the late researches are noticed, and neither student nor practitioner should be without this exhaustive treatise on au important elementary branch of medicine.-Atlanta Med. and Surg. Journal, Dec. 1876.

\section{KIRKES (WILLIAM SENHOUSE), M.D.}

a MANUAL OF PHYSIOLOGY. Edited by W. Morrant Bakfr, M.D., F.R.C.S. A new American from the eighth and improved London edition. With about two hundred and fifty illustrations. In one large and handsome royal $12 \mathrm{mo}$. vol. ume. Cloth, \$3 25; leather, \$3 75. (Lately Issued.)

On the wbole, there is very little in the book whicheither thestudent or practitioner will not find of practlcal $v$ alue and consistent with our present knowledge of this rapidly changing science; and we hare no hesitation in exprersing our opinion that this eighth edition is one of the best handbooks on pliysiology which we havein our language. $-N . Y$. Med Record, April I5, 1873

In its enlarged form it is, in ouropinion, still the best book on phyiology, most useful to thestudent. -Phila. Med. Times, Ang. 30, 1873.
HAPTSHORNE'S HANDBOOK OF ANAT MMY AND PIIYSIOLUGY. Second edition, revised. In one roval 12 mo. vol., with 220 wood-cuts; cloth, \$1 $1: 5$.

LEHMAN'S MANUAL, OF CHEMICAL PHYSIOL. U iY. T'ramslated from the German, with Nutes and Additions, by J. Cheston Morkis M.D. Wlth lllustrations on wood. In one octavo volume of 336 pages. Cloth, \$2 25.

LEHMANN'S PHYSIOLOGICAL CBEMISTRY Com. plete in two large octavo volumes of 1200 pages, with 200 illustruti ins; cloth, $\$ 3$. 
DALTON (J. C.), M.D.,

Professor of Physiology in the Collegenf Physiclans and Surgeons, Now York, \&c.

A TREATISE ON HUMAN PHYSIOLOGY. Designed for the use of Studentsand Practitioners of Medicine. Sixth edition, thoroughly revised and enlarged, with three huldred und sixteen illustrations on wood. In one very beautiful octavo vol. ume, of over 800 pages. Cloth, $\$ 550$; leather., $\$ 650$. (Just Issued.)

During the past few years several new works on phy- This popular texl-book on physiology comes to us in siology, and new editions of old works, hare appeared, completing for the favor of the medical student, but none will rival this new edition of Dalton. As now enlarged, it will be found also to be, in ceneral, a satisfactory work of reference for the prastitioner.-Chicago Med. Journ, and Examiner, Jan. 1876.

Prof. Dalton has discussed conflieting theorles and conclusions regarding physiological questions with a fuirness, a fulness, and a conciseness which lend freshness and vigor to the entire book. But his discusgions have been so guarded by a refusal of admission to those speculative and theoretical explanations, which at best exist in the minds of observers themselves as only probabilities, that none of his readers need be led into grave errors while making them a study.-The Medical. Record, Feb. 19, 1876.

The revision of this great work has, brought it forward with the physiological advances of the day, and renders it, as it has ever been, the finest work for studenis extant.-NTashville Journ. of Med. and Surg., Jan. 1876.

For clearness and perspicuity, Dalton's Physiology commended itself to the student years ago, and was a pleasant relief from the verbose productions which it supplanted. Physiology bas, however, made many advances since then - and while the style has been preserved intact, the work in the present edition has been brought up fully abreast of the tines. Thetew chemical notation and nomenclature have also been introduced into the present edition. Notwithstanding the multiplieity of text-books on physiology, this will lose none of its old time popularity. The mechanical execution of the work is all that could be desired.-Peninsular Journal of Medicine, Dec. 1875. of new matter, chiefly in the departments of pathologieal chemistry and the nervous system, where the principal advances have been realized. With so tho. rough revisiou and additions, that keep the work well up to the times, its continued popularity may be confidently predicted, nntwithstanding the competition it may encounter. The publisher's work is admirably done.-St. Louis Med. and Surg.Journ., Dec. 1875.

We heartily welcomethis, the sixth edition of this admirable text book. than which there are none of equal brevity more valuable. It is cordially recommended by the Professor of Physiolory in the University of Louisi. ana, as by all competent teachers in the United States, and wherever the English language is read, this book has been apprecialed. The present edition, with its 316 admirably executed illustrations, has been carefully revised and very much enlarged, although its bulk does not seem perceptibly increased.-New Orleans Medical and Surgical Journal, March, 1876.

The present edition is very much superior to every other, not only in that it brings the subject up to the times, but that it. doas so more fully and satisfactorily than any previouseditinn. Takeit al togetherit remains in our humble opinion, the best text book on physiology in any land or lanuuage. - The Clinic. Nov. 6, 1875.

As a whole, we cordially recommend the work $\mathbf{a s}$ a text-book for the student. and as one of the best.The Joumal of Nervous and Mental Disease, Jan. 1876.

Still holds its position as a masterpiece of lucid writing, and is, we believe, on the whole, the luest book to place in the hands of the student.-London Students' Journal.

CLASSEN (ALEXANDER), Professor in the Royal. Polytechnic School, Aix la-Chapelle.

ELENENTARY QUAN'TITATIVE ANALYSIS. Translated with notes and additions by Edgar F. Smith, Ph.D., Assistant Prof. of Chemistry in the Towne Scientific School, Univ. of Penna. In one handsome royal $12 \mathrm{mo}$. volume, of 324 pages, with illustrations; cloth, $\$ 200$. (Just Ready.)

It is probably the best manual of an elementary / advancing to the analysis of minerals and such pronature extant, insomuch as its methods are the best. ducts as are met with in applied chemistry. It is It teaches by examples, commencing with single an indispensable book for students in chemistry.determinations, followed by separations, and theu Boston Journ. of Chemistry, Oct. 1878.

GALLOIVAY (ROBERT), F.C.S., Prof of Applied Chemistry in the Royal College of Sciencefor Ireland, etc.

A MANUAL OF QUAIITATIVE ANALYSIS. From the Fifth London Edition. In one neat royal 12 mo. volume, with illustrations; cloth, $\$ 275$. (Lately Issued.)

BOWMAN (JOHN E.), M.D.

INTRODUCTION TO PRACTICAL CHEMISTRY, INCLUDING ANALYSIS. Sixth American, from thesixth and revised London edition. With numerous illustrations. In one neat vol., royal $12 \mathrm{mo}$., cloth, $\$ 225$.

BY THE SAME AUTHOR.

PRACTICAL HANDBOOK OF MENICAL CHEMISTRY. New edition. In one neat volume, royal $12 \mathrm{mo}$. (Preparing.)

REMSEN (IRA), M.D., Ph.D.,

Professor of Chemistry in the Johns Hopkin \& University, Baltimore.

PRINCIPLES OF THEORETICAL CHEMISTRY, with sperial reference tn the Constitution of Chemical Compounds. In one handsome royal $12 \mathrm{mo}$. vol. of over 232 pages: cloth, $\$ 150$. (Just Issued.)

WOHLER AND FITTIG.

OUTLINES OF ORGANIC CHEMISTRY. Translated with Additions from the Eighth German Ed. By IRA RKasen, M.D., Ph.D., Prof. of Chem. and Physics in Williams College, Mass. In one volume, royal 12 mo.of 550 pp., cloth, $\$ 3$. 
FOTNES ( GEORGE), Ph.D.

A MANUAL OF ELEMENTARY CHEMISTRY; Theoretical and Practical. Revised and corrected by HENRY WATTs, B.A., F.R.S., author of "A Dictionary of Chemistry," etc. With a colored plate, and one hundred and serenty-seven illustrations. A new American, from the twelfth and enlarged London edition. Edited by ROBERT BRIDGEs, M.D. In one large royal $12 \mathrm{mo}$. volume, of over 1000 pages; cloth, \$2 75 ; leather, $\$ 325$. (Just Ready.)

Two careful revisions by Mr. Watts, since the appearance of the last American edition of "Fownes," have so enlarged the work that in England it has been divided into two volumes. In reprinting it, by the use of a suall and exceedingly clear type, cast for the purpuse, it has been found possible to comprise the whole, without omission, in one volume, not unlanily for study and reference. The enlargement of the work has induced the American Editor to confine his additions to the narrowest compass, and he has accordingly inserted only such discoveries as have been announced since the very recent appearance of the work in England, and has added the standards in popular use to the Decimal and Centigrade systerns employed in the original.

Among the additions to this edition will be found a very handsome colored plate, representing a number of spectra in the epectroscope. Every care has been taken in the typographical execution to render the volume worthy in every respect of its high reputation and extended use, and though it bas been enlarged by more than one hundred and fifty pages, its very moderate price will still maintain it as one of the cheapest volumes accessible to the chemical student.

This work, inorganic and organic, is complete in what formidable magnitude with its more than a one convenient volume. In its earliest editions it thousand pages, but with less thrn this no fair reprewas fully up to the latest advancements and theo- sentatiou of chemistry as it now is can be given. The ries of that time. In its present form, it presents, type is small but very clear, and the sectious are very in a remarkably convenient and satisfactory mau- lucidly arranged to facilitate study and reference.ner, the principles and leading facts of the chemistry Med. und Surg. Reperter, A ug 3, 1878.

of to-duy. Concerning the manner in which the various enbjects are treated, much deserves to be said, and mustly, too, in praise of the book. A review of such a work as Fownes's Chemistry within the limits of a book-notice for a medicul weekly is simply out of the question. - Cincinnati Lancet and Clinic, Dec. 14, 1878 .

When we state that, in our opinion, the present edition sustains in every respect the high reputation which its predecessors have acquired and eujoyed, we express therewith our full belief in its intrinsic ralue as a text-book and work of reference. $-A m$. Journ. of Pharm., Aug. IS7S.

The conscientious care which has been bestowed n pon it by the A merican and English editors renders it still, perhaps, the best book for the stndent and the practitioner who would keep alive the acquisitions of his student days. It has, indeed, reached a sone-

The work is too well known to American students to need any extended notice; suffice it to say that the revirion by the English editor has been faithfully done, and that Professor Bridges has added some fresh and raluable matter, especially in the inorganic chemistry. The book has always been a favorite in this country, and in its new shape bids fair to retain all its former prestige.-Boston Jour. of Chemistry, Ang. 1S78.

It will be entirely unnecessary for us to make any remarks relating to the general character of Fownes' Manual. For over twenty years it bas lield the foremost place as a text-book, and the elaborate and thorough revisions which have been made from time to timelea relittlechance for any wide awake rival to step before it.-Canadian Pharm. Jour., Ang. 1878.

As a manual of chemistry it is without a superior in the language.-Md. Med. Jour., Aug. 1s7S.

A TTFIELD (JOHN), Ph.D.,

Professor of Practical Chemistry to the Pharmaceutical Society of Great Britain, \&c.

CHEMISTRY, GENERAL, MEDICAL, AND PHARMACEUTICAL; including the Chemistry of the U.S. Pharmacopœia. A Manual of the General Principles of the Science, and their Application to Medicine and Pharmacy. Eighth edition revised by the author. In one handsome royal $12 \mathrm{mo}$. volume of 700 pages, with illustrations. Cloth, $\$ 250$; leather, $\$ 300$. (Just Ready.)

We have repeatedly expressed our favorable of chemistry in all the medical colleges in the opinion of this work, and on the appearance of a United States. The present eclition contains snch new edition of it, little remains for us to say ex- alterations and additiong ag aeemed necessary for cept that we expect this eighth edition to be as the demonstiation of the latest developments of indispenssble to us as the seventh and previons chemical principles, a ad the latesi applications of editions have been. While the general plan and chemistry to pharmacy. It is scurcely necessary arrangement have been adhered to, new matter for us to say that it exhibits chemistry in its prehas been added covering the observations made sent advanced state.-Cincinnati Medical Ntws, since the former edition The present differs from April, 1879.

the preceding one chlefly in these alterations and in about ten pages of useful tables added in the appendix -Am. Jour. of Pharmacy, Nay, $18^{-9}$.

A standard work like Attfleld's Chemistry need only be mentioned by its name, withont further comments. The present edilion contains such al. terations and additions as seemed necessary for the demonstration of the latest developments of chemical principles, and the latest applications of chemistry to pharmacy. The anthor has bestowed ardnons labor on the revision, and the extent of the information thas introduced may be estimated from the fact that the inder contains three bandred new references relating to additional material.-Druggists' Circular and Chemical Gazelte, May, 1879.

This very popular and meritorions work has now reached its elghth edition, which fact speaks in the highest terms in commendation of its excel lence. It has now become the principal text-book

The popnlarity which this work has enjoyed is owing to the original and clear disposition of the facts of the science, the accuracy of the details, and the omission of much which freights many treatises heavily without bringing corresponding instruction to the reader. Dr. Attfield writes for students, and primarily for medical students; he always has an eye to the pharmacopcia and its offcinal preparation's; and he is continually putting the matter in the text so that it responds to the questions with wich each section is provided. Thus the student learns easily, and can always refresh and test his knowled ge.-Mred and Surg. Reporter, A pri:19,'79.

We noticed only about two years and a half ago the publication of the preceding edition, and remarked upon the exceptionally valuable character of the work. The work now iacludes the whole of the chemistry of the pharnacopala of the United Strates, Great Britain, and India.-New Remedies, May, 1879 . 
FARQUHARSON (ROBERT), M.D.,

Lecturer on Materí Medica at St. Mary's Hospital Medical School.

A GUIDE TO THERAPEU'TICS AND MA'TERIA MEDICA. Second Amerisan edition, revised by the Author. Enlarged and adapted to the U. S. Pharmacopoia. By Fravk WoonbuRY, M.D. In one neat rojal $12 \mathrm{mo}$. volume of 498 pages: cloth, $\$ 2.25$. (Just Rendy.)

The appearance of a new edition of this conrenient aad handy book in less than two years may certrinly be takeu as an indication of its aseful ness. Its conrenient arrangement, and its terceness, and, at the sarne time, completeness of the information given, make it a handy book of reference.-Am. Journ. of Pharmacy, June, Is79.

The early appearance of a second elition of Dr. Farquharson's work bears sufficient testimony to the appreciation of it by $A$ merican readers. The plan is such as to bring the characier and action of drugs to the eye and mind with clearness The care with which both author and editor bave done their work is conspicnous on every page,-Med. and Surg. Reporter, May 31, 1579.

The second edition, enlarged and revised, is a happy medium between the first edition, which was rather too brief on some important matters, and the larga octavos of Wuod and Birtholow. It is bronght $a p$ to the most recent researches, one note referring to an article published in April of this year. The favorable reception accorded it, shown by this reissue in two years, was one well merited.-Louisville Med. New8, June 7, ]S79.

This work contains in moderate compass such well-digested facts concerning the physiological and therapenticat action of remedies as a re reason. ably established up to the present time. By a convenient arrangement the corresponding effects of each article in health and disease are presented in parallel cclumns, not only rendering reference easier, but also impressing the facts more strongly upon the mind of the reader. The book has been adapted to the wants of the American student, and copious notes have been introdnced, embodying the latest revision of the l'harmacopœia, togetber with the antidotes to the more promiuent poisons, and such of the newer remedial a gents as seemed neces. sary to the completeness of the work. Tables of weights and mearures, and a good alphabetical in dex, end the volume.-Druggists' Circular and Chemical Gazette, June, 1579.

It is a pleasure to think that the rapidity with which a second edition is demanded may be taken is an indication that the sense of apprectation of the value of reliable information regarding the ase of remedies i notentirely overwhelmed in the cultivation of pathological studies, characteristic of the present day. This work certainly merits the success it has so quickly achieved. -New Rernedies, July, '79.

BLOXAM (C.L.) Professor of Chemistryin King's College, London.

CHEMISTRY; INORGANIC AND ORGANIC. From the Second Lordon Edition. In one very handsome octavo volume, of 700 pages, with about 300 illus. trations. Cloth, $\$ 400$; leather, $\$ 500$. (Lately Issued.)

We have in this work a eompleteand most excellent text-book for the use of $6 \mathrm{chools}$, and can heartily recommend it as such.-Boston Med. and Surg. Journ., May 28, 1S7t.

The above is the title of a work which we can most conscientiously recommend to students of chemistry. It is as easy as a work on chemistry could be made, at thesametime that it presents a full account of that science as it now stands. We have spoken of the work as admirably adapted to the wants of students; it is quite as well suited to the requirements of practitioners who wish to review their chemistry, or have occasion to refresh their memories on any point relating to it. In a word, it is a book to be read by all who wish to know what is the chemistry of the present day.-American Prac titioner, Nov, 1873.

It would be difficult for a practical chemist and teacher to find any material fault with this most admirable treatise. The author has given us alinost a cy cloprdia within the limits of a convenient volume, and has done so without penning the useless paragraphs too commonly making up a great part of the bulk of many cumbrous works. The progressire scientist is not disappointed when he looks for the record of new and valuable processes ard discoveries, while the cantious conservative does not find its pages monopolized by uncertain theorles and speculations. A peculiar point of excellence is the crystallized form of expression in which great truths are expressed in very short paragraphs. One is surprised at the brief space allotted to an important topic, and yet, after reading it, he feels that little, if a $y$ more should have beeu said. Altogether, it is seldom you see a text-book so nearly faultless. - Cincinnati Lancet, Nov. 1873 .

CLOWES (FRANK), D.Sc., London.

Seninr Science-Master at the High School, Newcastle-under Lyme, etc.

AN ELEMENTARY TREATISE ON PRACTIC A L CHEMISTRY AND QUALITATIVE INORGANIC ANALYSIS. Specially adapted for Use in the Lahoratories of Schools and Colleges and by Beginners. From the Second and Revised Englisb Edition, with about fifty illustrations on wood. In one very handsome royal $12 \mathrm{mo}$. volume of 372 pages: cloth, $\$ 250$. (Now Ready.)

It is short, concise, and eminently practical. We are so simple, and yet concise, as to be interesting therefore heartily commendit to students, and expe- and intelligible. The work is nnincumbered with cially to those who are obliged to dispense with a theoretical deductions, dealing wholly with the master. Of course, a teacher is in every way desi- practical matter, which it is the alm of this comprerable, but a good degree of technical skill and prac- hensive text-book to impart. The accuracy of the tical knowledge can be attained with no other analytical methods are vouched fur from the fact instructor than the very valuable handbook now nuder consideration. - St. Louis Clin. Record, Oci. 1877 .

that they have all been worked through by the author and the menhers of his class, from the printed text. We can heartily recommend the work to the student of chemistry as being a reliable ard

The work is so written and arranged that it can be comprebended by the student without a tercher, aud the descriptions and directions for the various work comorehensive one.-Druggists' Advertiser, Oct. 15,1877 .

KNAPP'S TECHNOLOGY; or Chemistry Applied to the Arts, and to Manufactures. With American additions by Prof. WALTER R. JoInson. In two very handsome octavo volumes, with 500 wood engravings, cloth, $\$ 600$. 
PARRISH (EDWARD),

Late Professor of Materia Mredica in the Philadelphia College of Pharmacy.

A TREATISE ON PHARMACY. Designed as a Text-Book for the Student, and as a Guide for the Physician and Pharmaceutist. With many Formulæa anl Prescriptions. Fourth Edition, thoroughly revised, by Tromas S. Wiegand. In one hnndsome octavo volume of 977 pages, with 280 illustrations; cloth, $\$ 550$; leather, $\$ 650$. (Lately Issued.)

of Dr. Parrish's great work on pharmacy it only remuins to be said that the editor has accomplished his work so well as to maintain, in this fourth edl. tion, the high standard of excellence which it bad attained in prevlous editions, under the editorship of its accomplished author. This has not been accom. plished without much labor, and many additions and improvemeuts, iuvolting changes in the arrangement of the several parts of the work, aud the addition of much new inatter. With the modifications thas effected it constitutes, as now presented, a com. pendium of the science and art indispensable to the pharmacist, and of the utmost value to erery practitioner of medicine desirous of familiarizing himself with the pharmaceutical preparation of the articles which he prescribes forhis patients, - Chi. cago Med. Journ., July, 1874.

The work is eminently practical, and has the rare merit of being readable and interesting, while it preserves a strictly scientlfic character. The whole work reflects the greatest credit on author, editor. and nub. lisher. It will convey someidea of the liberality which has been bestowed upon its production when we mea. tion that therearenoless than 280 carefully executed illustrations. In conclusion, we heartily recommend

the work, not only to pharmacists, but also to the multitude of medical practitioners who are obliged to compound their ow medicines. It will ever hold an honored place on our own bookshelves. -Dublin Med. Press and Circular, Aug. 12, 1874.

We expressed our opinion of a former edition in terms of unqualified praise, and we are in no mood to detract from that opinion in reference to the pre. sent edition, the preparation of which has fallen into competent hands. It is a book with which no pharma. cist can dispense, and from which no physician can fail to derive much information of value to him in practice.-Pacific Med. and Surg. Journ., June,'74.

Perhaps one, if not the most important book apon pharmacy which has appeared in the English lan. guage has emanated from the transatlantic press. "Parrish's Pharmacy" is a well-known work on this side of the water, aud the fact shows ns that a really aseful work neverbecomes merely local in its fame. Thanks to the judicious editing of Mr. Wiegand, the posthumons edition of "Parrish" has been saved to the public with all the mature experience of $i t s a n$. thor, and perhaps none the worse for a dash of new blood.-Lond. Pharm. Journal, Oct. 17, 1874.

STILLÉ (ALFRED), M.D., Professor of Theory and Practice of Medicine in the Untversity of Penina.

THERAPEUTICS AND MA'TERIA MEDICA; a Systematic Treatise on the Action and Uses of Medicinal Agents, including their Description and History. Fourth edition, revised and enlarged. In two large and handsome 8vo. vois. of about 2000 pages. Cloth, $\$ 10$; leather, $\$ 12$. (Lately Issued.)

It is unuecessary to do mucli more than to an- of the present edition, a whole cyclopædia of theranounce the appearance of the fourth edition of this well known and excellent work.-Brit. and For. Med.-Chir. Review, Oct. 1875 .

For all whodesire a complete work on therapeutics and materia medica for reference, in cases involving medico-legal questions, as well as for information concerning remedial agents, Dr. Stillée's is "par excellence" the work. The work being out of print, by the exhanstion of former editions, the author has laid the profession under renewed obligations, by the careful revision, important additions, and timely re issuing a work not exactly supplemented by any other in the English language, if in any langnage. The mechanical execution handsomely sustains the well-known skill and good taste of the publisher. St. Louis Mert. and Surg. Journal, Dec. 1874.

From the publication of the first edition "Stille's Therapeutics" has been one of the classics; its absence from our libraries would create a vacuum which conld be filled by no other work in the language, and its presencesupplies, in the two volumes of the present edition, a whole cyclopædia of th
pentics.-Chicago Medical Journal, Feb. 1875.

The rapid exhanstion of three editions and the universal favor with which the work has been received by the medical profession, are sufflcient proof of its excellence as a repertory of practical and useful in. formation for the physician. The edition before us fally sustains this verdict, as the work has been carefully revised and in some portions rewritten, briug. ing it up to the present time by the admission of chloral and croton-chloral, nitrite of anyl, bichloride of methylene, methylic ether, lithium com. pounds, gelseminum, and other remedies. $-A m$. Journ. of Pharmacy, Feb. 1875.

We can hardly admit that it has a rival in the multitade of its citations and the fulness of its re. search into clinical histories, and we mnst assign it a place in the physician's library; not, indeed, as fully representing the present state of knowledge in pharmacodynamics, but as by farthe most complete treatise upon the clinical and practical side of the question.-Boston Med. and. Surg.Journal, Nov. 5 , 1874 .

GRIFFITH (ROBERT E.), M.D.

A UNIVERSAL FORMULARY, Containing the Methods of Preparing and Administering Officinal and other Medicines. The wholeadapted to Physiciar $\mathrm{s}$ and Pharmaceutists. Third edition, thoroughly revised, with numerous additions, by JoHN M. Maisch, Professor of Materia Medica in the Philadelphia College of Pharmacy. In one large and handsome octavovolume of about 800 pp., cl., $\$ 450$; leather, $\$ 550$. (Lately Issued.)

To the druggist a good formulary is simply indis-| A more complete formulary than it is in its pres. pensable, and perhaps no formulary has been more ent form the pharmacist or physician could bardly extensively used than the well-kuown work before desire. To the first some such work is indiepensa. n8. Many physlcians have to off ciate, also, as drug. ble, and it is hardly less essential to the practitioner gists. This 18 true especially of the country physi. who compounds lis own medicines. Nuch of what clan, and a work which shall teach him the means is contalned in the introduction ought to be com. by which to adninister or comhine his remedies in mitted to memory by every student of medicine. the most effcacious and plearant manuer, will al. As a help to physicians it will be found invalurble, ways hold its place upon his shelf. A formnlary of and doubtless will make its way into libraries not this kind is of benefit also to the city physicin in already supplied with a standard work of the kind. largest practice. - Cincinnati Clinic, Feb. 21, 1874. - The American Practitioner, Louisville, July, '74. 

and of Clinical Med. in Univ. of Pa.

THE NATIONAL DISPENSATORY : Containing the Natural History, Chemistry, Pharmacy, Aetions and Uses of Medicines, including those recognized in the Pharmacopoias of the United States and Great Brituin. In one very handsome octavo volume of 1628 pages, with over 200 illustrations. Extra cloth, $\$ 675$; leather, raised bands, $\$ 750$. (Now Ready.)

\section{EXTRACT FROM THE PREFACE.}

"In the rapid progress of modern research, few subjects have of late years received greater accessions of facts than the group of sciences connected with materia merlica and therapentics. The new resources thus placed at the command of the pharmaceutist and physician have seemed to the authors to justify an attempt to make, from the adranced stand-point of the present day, a concise but complete statement of all that is of practical importance to both professions-a digest in which that which is old and that which is new shall be so brought tngether as to give to the reader, within the most modernte practicable compass, all the details in pharmacology, pharuacy, and therapeutics, which he is likely to need in his daily avocations. In the almost infinite accumulation of material, this has required a careful and conscientious sifting to discard that which is obsolete, untrustworthy, or comparatively trivial, without impairing the practical completeness of the work. That they have wholly accomplished their object the authors do not venture to claim; but they can say that years of constant labor have been devoted to the task of producing a work to which the inquirer may refer with the certainty of finding everything which experience has stured up as worthy of confidence in the subjects embraced within its scope."

We intend to druw the attention of our brother pharmacists to this publication, which cannot fail ro exercise a widespread and $\mathrm{m} z \mathrm{r}$ ed infuence upon the discharge of the duties of their vocation. 'I be material embodied in the work is truly immense, as shown alone by the almost conntless number of subjects treated. We cungratulate the anthors apon their snccess in having brought to a close a work which must inevitably take its place as oue of the most Important con'ributions to uedical and pharin aceutical literature. $-A m$. Journ. of Pharm. мay, 1579 .

The aseociation of such distinguished authors as Professors Stillé und Maisch in the comiposition of a work of this character has excited the strongest interest and the bigliest expectations in the mind of every physiciau aud pbarmacist in the country. For once we can truly say that the promise of excellence ha, been $f u$ : flled to the letter, and the $\mathrm{N}$ tional Dispensatory has come almost perfect from the hands of its makers. The entire work is a most excellent one aud cannot fail to satisfy the purchaser. We can couscientious y recommend it to every student and practitioner of medicine and pharmacy.-St. Louis Clirical Record, A pr 1879.

This magnificent work has at last arrived, and we are at a loss for words to express our appreciation and to gise our readers an idea of it 'The subjest-matter is brought to date, showing that it has been the uncersing aim of the authors to kupply a much needed book, one that will contain all the important facts, and not dwell upon points that are of comparatively little interest to any but a specially interested student While this work, on account of its conciseness, is adapted to the pharmacal student, it is equally adapted to the medical student and practitiouer by its well arranged therapeutical index containing about 3750 reterences, while the materia medica index embraces about 10400 . The physician eees at a glance all medicines that are used for any certain class of disease.-Chicago Phar. macist and Chemist, A pril, 1879.

The present Dispensatory is arranged in alphabetical order from the commencement, the recent bdvances in chemistry are mentioned, and an effort made to include the late novelties in the review of the resources of the physician. This ls carried out with that sound conservalive judgment which characterizes all Prof. Stillé's work. T'he chemical and pharmaceutical seetions have, we may suppose, received the especial care of Prof. Maisch; and as he is fucile princeps in that branch, nothing can be said of them except in praise.-Med. and Surg. Reporter April 5, 1879.

It has been prepared by two gentlemen whose learning fully qualified them for the diftlcult task. and whuse eminence entitles them to be heard with the respect and attention dne to authority. The "raison d'être" of the book is modestly stated in

the preface, and now that it has been publisbed and opens to us its vast stores of information, we may add that it was almost a necessity; and this we eay withont meaning to impugn the great excellence of the works of similar chiriacter w hich have preceded it. All of the descriptions, whether medical, botanical, or pharmaceutical, are clear, in good Euglish, and unencumbered with obsolete and unintelligiblé terms. Those portions which have reference to therapentics form a convenient treatise on that subject, and are made the more valuable and available by a complete therapeutical index. The parely pharmacal part is as perfect as it is possible to make it, and less could not have been expected when we consider Prof. Maisch's great qualifications for work of that kind. $-N$. C Med. Journ., March, 1879.

The therapeutic part is as rich as would be expected of the author of the most compreheusive work on the subject in our language. The physiological effects of drugs receive due attention, and their influence over disease is stated succinctly. For the task of winnowing the immense accumulation of periodical literature, the experience and matured judgment of Prof. Stillé were eminen'ly fitted. No pharmacist or ductor will repent the purchase of a book which is at once a treasury of facts and the digest of a decision of a high court. - Louisville Med. Newos, March 29, 1879.

The pharmaceutical world has for a long time been ou the quivive, in expectation of the fortbcominf Dispensatory by Profs. Stillé aud Maisch, who have acquired euch a reputation in tbeir respective Departments that nothing but a satisfactory work cuuld be expected; this expectation bas been quite realized. We have examined the work with some care, and are very much pleased that we can pronounce it to be reliable, comprehensive, and inclnding the latest revegrches available to its authors. This is mure particularly true us regards the portion devoted to pharmacentical subjects. We are fully jnstiffed in statiog that it is, taken altogether, otie of the most important and creditable publications which have of late been issued by the Anerican press. It will be au Indispensable reference book both for the pharmacist and the physician. $-\lambda \in t$ Remedies, A pril, 1879.

A careful examination of the work calls forth $\mathrm{n} n$ qualified praise for its excellent arrangement, full yet concise information, its careful adherence to the best anthority on each particular topic, as well as the entire eliniuation of all unnecessary and obsolete data and particulars. The arrangement of all topics is purely alphabetical, and with surprising fidelity to the wants both of thephyslcian and phar. macentist. New remedies which have cune into recent use are here found noticed, with kuch fucts as have been colluted from careful investigation.Druggists' Circular and Chemical Gazette, Marcb, 1579 . 


CORNIL $(V),$.$\quad AND$
Prof. in the Faculty of Med, Paris. $\quad R_{\text {Prof. in the College of France. }}^{\text {ANVIER (L.), }}$

MANUAL OF PATHOLOGICAL HISTOLOGY. Translated. with Notes and Additions, by E. O. Smakespeane, M.D., Pathologist and Ophthalmic Surgenn to Philada. Hospital, Lecturer on Refraction and Operative Ophthalmic Surgery in Lniv. of Penna., and by IIExry C. Srmes. M D., Demonstrater of Fathological Ilistology in the Univ. of $\mathrm{Pa}$. In one very handsome octavo volume of about 600 pages, with o:er 300 illustrations. (S/hortly.)

So much has been done of late years in the elucidation of pathology by means of the microscope, and this subject now occupies so prominent a position as one of the most important branches of medical science, that the American profession cannot fail to welcome a translation of the present work, which, through its own merits and through the well-known reputation of its distinguished authors, is regarded in Europe as the standard text-book and work of reference in its department. Such investigations and discoveries as have been madesince its appearance will be introduced by the translator, and the work is confidently expected to assume in this country the same position which has been so universally accorded to it abroad.

FEN WICK (SAMUEL), M.D.,

Assistant Physician to the London Hospital.

THE S'TUDENT'S GUIDE 'TO MEDICAL DIAGNOSIS. From the Third Revised and Enlarged English Edition. With eighty-four illustrations on wood. In one very handsome volume, royal $12 \mathrm{mo}$, cloth, $\$ 225$. (Just Issued.)

of the miny guids-books on medical diagnosis, a re few books of this sizeon practical medicine that claimed to be written for the special instruction of contain so much and convey it sowell as che volume studeats, this is the best. The authoris evidently a before us. It is a book we can sincerely recommend well-read and accomplished physician,and he knows to the student fi)rdirectinstruction, and to the"prachow to teach practical medicine. The cbarm of sim. titioner as a ready a ud useful aid to his memors.plicity is not the least interesting feature in the man- Am. Journ. of Syphilography, Jan. 1874.

nerin which Dr. Fenwick conveyolnstruction. There

GREEN (T. HENRY), M.D., Lecturer on Pathology and Morbid Anatomy at Charing-Cross Hospital Medical School, ete.

PATHOLOGY AND MORBID ANATOMY. Third American, from the Fourth and Enlarged and Revised English Edition. In one very handsome octavo volume of 332 pages, with 132 illustrations; cloth, $\$ 225$. (Just Ready.)

This is unquestionably one of the best manuals on (ciently numerous, and usually well made. In the the subject of pathology and morbid anatomy that present edition, such new matier has been added as cun be placed in the student's hands, and we are was necessury to enubiree the later results ill pathoglad to see it kept up to the times by new editions. logical resenrch. No doubt it will continue to enjoy Ench edition is carefully revised by the author, with the favor it has received at the hands of the profesthe view of making it include the most recent ad. sion.-Ned and Surg. Reporter, Feb. 1, 1879.

vances in pathology, and of omitting whatever may have become obsolete.-N. Y. Med. Jour., Feb. 1879.

The treatise of Dr. Green is compact, clearly ex-

pressfd, up to the times, ind popular as a text-book,
buth in England and America. The cuts are suffi.

For practical, ordinary daily use, this is undoubtedly the best treatise that is offered to students of patholngy and inorbid auatomy.-C'incinnati Lancet and Clinic, Feb. 8, 1879 .

\section{DAVIS (NATHAN S.),}

Prof. of Principles and Prastice of Medicine, etc., in Chicago Mred. College.

CLINICAL LECTURES ON VARIOUS IMPORTAN'T DISEASES; being a collection of the Clinical Lectures delivered in the Medical Wards of Mercy Hospital, Chicago. Edited by Frank H. Davis, M.D. Second edition, enlarged. In one handsome royąl 12 mo. volume. Cloth, \$1 75. (Lately Issued.)

WHA'T 'TO OBSERVEATTHE BEDSIDE ATI AFTER Death iN Mspical Caseg. From the eecond London edition. I vol royal $12 \mathrm{mo}$., cloth. \$1 00 .

CHRISTISON'S DISPENSATORY. With copious additions, and 213 large wood engravings. By $\mathrm{R}$. KaLBSFikLD Grifrith, M.D. One vol. 8vo., pp. 1000 , cloth. $\$ 400$.

CARPENTER'S PRIZE ESSAY ON THE USE OF A LCOHOLIC LIQUORg in HBALTh AND DIsBAgE. NeW edition, with a Preface by D. F. ConDis, M.D., and explanations of scientiflc words. In oneneat $32 \mathrm{mo}$. volume, pp. 178 , cloth. 60 cents.

G LUGE'S ATLAS OP PATHOLOGICAL HISTOLOGY Translated, with Notes and Additions, by JosEPH LRIDY, M. D. In one volume, very large imperial quarto, with 320 copper-plate figures, plain and colored, cloth. $\$ 100$

LA ROCHE ON YELLOW FEVER, considered in its Historical, Pathological, Etiological, and Thera. peutical Relations. In two large and handsome octavo volumes of nearly $1500 \mathrm{pp}$, cloth. $\$ 700$.

HOLLAND'S MEDICAL NOTES AND REFLEC. TlONs. 1 vol. 8 vo., pp. 500, cloth. 350.
BARLOW'S MANUAL OF THE PRACTICE OF MEDICINE. With Additions by D. F. CONDIF, II D. 1 vol. 8 vo., pp. 600 , cloth. $\$ 250$.

TODD'SCLINICAL LECTORES OY CERTAIN ACUT $B$ Diseases. In one neat octaro volume, of $320 \mathrm{pp}$, cloth. \$2 50 .

STURGES'S INTRODUCTION TO THE STUDY OF CLINICAL MEDICINE. Being a Guide to the Investigation of Disease. In one handsome $12 \mathrm{mo}$. volume, cloth, \$1 2.5. (Lately Issued.)

STOKES' LECTURES ON FEVER. Edited by JонN Widila Moore, M. D., Assistant Physician to the Cork Street Fever Hospital. In one neat Sro. volume, cloth, $\$ 200$. (Just Issued.)

THE CYCLOPADDIA OF PRACTICAL MEDICINE : comprising Treatises on the Nature and Treatment of Disenspr, Materia Medica and Therapentics, Diseases of Women and Childreu, Medical Jurisprudence, etc. etc. By Dunalison, Forbes, Twerdis, and Coxolur. In fonr large super-royal octavo volumes, of 3254 double-columned pages, strongly and handsoinely bound in leather, \$15; cloth, \$11. 
GLINT (AUSTIN), M.D.,

Professor of the Principles and Practice of Medicine in Bellevue Med. College, N. Y.

A TREATISE ON THE PRINCIPLES AND PRACTICE OF MEDICINE; designed for the use of Students and Practitioners of Medicine. Fourth edition, revised and enlarged. In one large and closely printed octavo volume of ahout $1100 \mathrm{pp}$.; cloth, $\$ 600$; or strongly bound in leather, with raised bands, $\$ 700$. (I.ately Issued.)

By common consent of the English and American medical press, this work has been assigned to the highest position as a complete and compendious text-book on the most advanced condi. tion of medical science. At the very moderate price at which it is offered it will be found one of the cheapest volumes now before the profession.

This excollent treatise on medicine has acquired His own clinical stadies and the latest contribn. for itselfinthe United States a reputation similar to tions to medical literature both in this country and that enjoyed in England by the admirable lectures in Europe, have recelved careful attention, so that of Sir Thomas Watson. We have referred to many some portions have been entirely rewritten, and of the most importunt chapters, and find the revi. about seventypages of new matter bave been adsion spoken of in the preface is a gennine one, and ded.-Chicago M+d Jour., Jnne, 1873.

that theanthorhas very fairly brought up his matter to thelevel of the knowledge of the present day. The work has this greatrecommendation.that it is in one rolume, and therefore will not be so terrifying to the student as the bulky volumes which several of our English text-books of medicine have developed in to. -British and Foreign Med.-Chir. Rev., Jan. 187 .

It is of course unnecessary to introduce or enlogize this now standard treative. The present edition has been enlarged and revised to bring it up to the author's present level of experience and reading.

Has never been surpassed as a text-book for students and a book of ready reference for practition. ers. The furce of its logic, its simple and practical teachings, haveleft it without a rival in the fleld N. Y.-Med. Record, Sept. 15, 1874 .

It is given to very few men to tread in the steps of Austin Flint, whose single volume on medicine though bereand there defective, is a masterpiece or lucid condensation and of general grasp of an enor. mously widesubject.-Lond. Practitioner, Dec.'73.

\section{$B^{Y}$ THE SAME AUTHOR.}

CLINICAL MEDICINE; a Systematic Treatise on the Diagnosis and Treatment of Diseases. Designed for Students and Practitioners of Medicine. In one large and handsome octavo volume of about 900 pages. (In Press.)

It has been the object of the author in this volume to present the science and art of medicine in their most practical aspect, adapted to the necessities of the student and physician in the daily routine of duties at the bedside. By avoiding the discussion of questions relating to pathology and etiology, space is gained for the thorough consideration of diagnosis and treatment, embracing many points which escape attention in the ordinary text-books. In the arrangement of the work, diseases are classed according to the system of organs primarily affected; and affections closely related are grouped together so as to elucidate their differentiation, and the appropriate treatment is pointed out for each. The preparation of the work has occupied the author for several years, and is presented as embodying the results of prolonged observation and experience under opportunities more extensive than often full to the lot of the physician.

\section{$B$ Y THE SAME AUTHOR. \\ ESSAYS ON CONSERVATIVE MEDICINE AND KINDRED TOPICS. In one very handsome royal 12 mo. volume. Cloth, $\$ 138$. (Just Issued.)}

\section{$A_{\text {ARTSHORNE (HENR Y }), M . D .,}$ Professor of Hygiene in the University of Pennsylvania.}

ESSENTIALS OF THE PRINCIPLES AND PRACTICE OF MEDI. CINE. A handy-book forStudents and Practitioners. Fourth edition, revised and improved. With about one hundred illustrations. In one handsome royal $12 \mathrm{mo}$. volume, of about 550 pages, cloth, $\$ 263$; half bound, $\$ 288$. (Lately Issued.)

As a handbook, which clearly sets forth the Bssen- book, it cannot be inproved upon.-Chicago Med. TIALS of the PRIXCIPISE AND PRACTICF OP MEDICINE, Examiner, Nov. 15, 1874

we do not know of its equal. - Va. Med. Monthly. Without doubt the best book of the kind published

As a brief, condensed, but comprehensive hand- in the Englinh language. - St. Louis Med.and Surg. ,

- Journ., Nov. 1S7t.

W'ATSON (THOMAS), M.D., \&c.

LECTURES ON THE PRINCIPLES AND PRACTICE OF PHYSIC. Delivered at King's College, London. A new American, from the Fifth revised and enlarged English edition. Edited, with additions, and several hundred illustra. tions, by Henry Hartshorne, M.D., Professor of Hygiene in the University of Penn. sylvania. In two large and handsome 8vo.vols. Cloth, $\$ 900$; leather, $\$ 1100$. (Lately Publeshed.)

It is a subject for congratulation and for thank-, cate and important pathologicaland practical ques. ful lessthat Sir Thoms Watson, during a period of tions, the results of his clear insight and his calm cum parative leisure, after a long, laborions, and judgment are now recorded for the beneft of man. most honorableprofessional career, whlle retaining kind, in langnage which, for precision, vlgor, and full possession of his high mental faculties, should classical elegance, has rarely been equalled, and have employed the opportunity to submit his Lec- never surpassed The revision bas evidently been tures to a more thorough revisionthan was possible most carefully done, and the results appear in 8. . diring the earlier and busier period of his life. mostevery page.-Brit. Med. Journ., Oct. $14,1571$. Carefully passing in review some of the mostintri- 
RRISTOWE (JOHN SYER), M.D., F.R.C.P.,

\section{B Physician and Joint Lecturer on Medicine, St. Thomas's Hospital.}

A MANUAL ON THE PRACTICE OF MEDICINE. Edited, with Additions, by Janes H. II r chisson, M.D., Physician to the Penna. IIospital. In one hundsome octavo volume of over 1100 pages: cloth, $\$ 550$; leather, $\$ 650$. (Just Issued.)

This portly volume is a model of condensation. increased by the judicious notes of the Editor.In a siyle at once clear, interesting, and concise, Dr. Cincinnati Clinic, Jиn. 7, 1877.

Bristowe passes in review every conceivablesubject Auyone who wants a good, clear, condensed work connected with the practice of medicine. These upon Practice, quite ap with the most recent viewsin practitioners who purchase few books will find this pathology, will tiad this a most valuable work. The a most opportune publication, because to many top- additions made by Dr. Hntchinson are appropiate ics not usually embraced in a work on practice are and useful, andso well done that we wish there were adequately handled. The boek is a thoroughly gned more of thein.-Am. Practitioner, Feb. 1577.

une, and its nsefulness to American readers has been

WOODBURY (FRANK), M.D.,

Physician to the German Hospital, Philadelphia, late Chief Assist. to Med. Clinic, Jeff. College Hospital, etc.

A HANDBOOK OF THE PRINCIPLES AND PRACTICE OF Medicine; for the use of Students and Practitioners. Based upon IIusband's Handbook of Practice. In one neat volume, royal $12 \mathrm{mo}$. (In Press.)

HABERSHON (S. O.) M.D.

Senior Physician to and late Lecturer on the Principles and Practice of Merlicine at Guy's Hispital, etc.

ON THE DISEASES OF THE ABDOMEN, COMPRISING THOSE of the Stomach, and other parts of the Alimentary Canal, Esophngus, Cæcum, Intes. tines, and Peritoneum. Second American, from the third enlarged and rerised Eng. lish edition. With illustrations. In one handsome octavo volume of over 500 pages. Cloth, \$3 50. (Now Ready.)

This work has remained $\mathrm{s}$ ?me time out of print, owing to the careful and conscientious revision which it has enjoyed at the hands of the author, and which bas nearly doubled its size since the appearance of the first edition. Yet there is no work accessible to the profession to take its place, as a careful, practical guide on a class of diseases, which form so large and important a portion of the duties of the physician, and for which the author's position has given him almost unequalled opportunities for observation and experience.

We can do very little to add to the favorable reception which has already been given by the medicul press of the world to this well known treatise We commend to all practitioners a careful pernsal of Dr. Habershon's work. More especially, wedraw attenition to the number of intestinal diseases recorded in its pages, cases of extreme interest clinically und pathologically. This carefal record shows that the work is no compilation, but a careful exposition of the anthor's perzonal experience.-Canadian Med. and Surg. Journ., May, 1879.

As a work of reference, as well as daily study, no work yet emanating from the medical press is worthy of morecareful consideration by the general practitioner than the above. With the cureful revisinn given this edition, Dr. Habershon's work will sti l remain at the head of the list. and con tinue to be regarded as one of the best treatises on abdominal diseases extant-South. Practitioner. June, 1 S79.

There hare been many laborers in this department of special pathology, and among them no one has done better service than Dr. Habershon. The firkt editions were exhansted long since, and the author has revised the one now under cunsideration

with great care and thoroughness. The chapters on constipation and intestinal ubstruction sre of high value, and are worth many times the cost of the book, which, altogether, is a most excellent one.St. Louis Ulin. Record, June, 1879.

This valnable treatise on diseases of the stomach and uhdou en has been ont of print for several years, and is therefore not 80 well known to the profession as it desarves to be. It will be found a cyclopadia of information, systematically arranged, on all diseases of the alimeutary tract, from the mouth to the rectum A fair proportion of each chapter is devoted to symptoms, pathology, and therapeutics. The prekent edition is fuller i han former ones in many particulars, and has been thoroughly revised and amended by the author. Several new chupters have been added, bringing the work fully up to the times, and makingit a volume of interest to the practitioner in every field of medicine and kurgery. Perverted nutrition is in seme form associated with all diseases we have to combat, and we need all the light that can he ohtained on a subject so broad and general. Dr Habershon's work is one that every practiri ner should read and study for himself.-N. $Y$. Med. Juurn., A pril, 1 S79.

FOTHERGILL (J. MILNER), M.D. Fdin., M.R.C.P. Lond.,

Asst. Phys. to the West Lond Hosp.: Asst. Phys. to the City of Lond. Hosp., etc.

THE PRACTITIONER'S HANDBOOK OF TREA'TMENT; Or, the Principles of Therapeutics. In one very neat octavo volume of about 550 pages: cloth, $\$ 400$. (Now Ready.)

Our friends will find this a very readable book; and he knew how sugzestive and helpful it would be to that it sheds licht upon every theme it touches, causing him.-St. Louis Med. and Surg. Journ, April, 1877.

the practitioner to feel more certuin of his diagnosis in dificult cases. We confidently cominend the work to our realers as one worthy of careful perusal. It light the жиy over obscure and difficult passes in medical practice. The chapter on the circulation of the blood is the most exliaustive and instructive to be found. It is a book every practitioner needs, and would have, if

We heartily commend his book to the medical st udent as an honest and intelligent guide through the mazes of therapeutics, and assure the practitioner who has grown kray in the harness that he will derive pleasure and instruction from its perusal. Valuable sugrestions and material for thought abound throughout.- Boston Med. and Surg Journul, Mar. 8, 1877.

\section{$B^{Y}$ THE SAME AUTHOR.}

THE A NTAGONISM OF THER A PEUTIC $\Lambda$ GENTS, AND WHAT IT TEACIES. Being the Fothergillian Prize Essay for 1S78. In one neat volume, royal $12 \mathrm{mo}$. of 156 piges; cloth, \$1 00. (Just Ready.) 
REYNOLDS (J. RUSSELL), M.D.

Prnf. of the Principles and Practice of Medicine in Univ. College, London.

A SYSTEM OF MFidUine. With Notes and Additions by Hent Harts. Horne, M. D., late Professor of Hygiene in the University of Penna. In three large and hindsome octavo volumes, containing about 3000 closely printed double columned pages, with numerous illustrations. (In Press)

Reynolds's System of Medicine, recently completed, has acquired, since the first appearance of the first volume, the well-deserved reputation of being the work in which inodern British medicine is presented in its fullest and most practical form. This could scarce be otherwise in view of the fact that it is the result of the collaboration of the leading minds of the profession, each subject being treated by some gentleman who is regarded as its highest authority-as for instance, Diseases of the Bladder by Sir Henry Thompson, Malpositions of the Uterus by Graily Hewitt, Insanity by IIenry Maudsley, Consumption by J. IIughes Bennet, Dis. eases of the Spine by Charles Bland Radcliffe, Pericarditis by Francis Sibson, Alcoholism by Francis E. Asste, Renal Affections by William Roberts, Asthma by Hyde Salter, Cerebral Affections by 4 CaArlton Bastian, Gout and Rheumatism by Alfren Baring GarRon, Constitutional Syphilis by Jonathan Hutchinson, Diseases of the Stomach by Wilsox kox, Diseases of the Skin by Balmanno Squire, Affections of the Larynx by Moreli MaC. Fenzie, Diseases of the Rectum by Butzard Curling, Diabetes by Lauder Brunton, Intestinal Liseases by Jonn Syer Bristowe, Catalepsy and Sonnambulism by Thomas King ChaMBers, A poplexy by J. Hughlings Jackson, Angina Pectoris by Profeseor Gairdner, Emphysema of the Lungs by Sir WILLIAM JENNER, etc. etc. All the leading schools in Great Britain have contributed their best men in generous rivalry, to build up this monument of medical science. St. Bartholomew's, Guy's, St Thomns's, University College, St Mary's in London, while the Edinburgh, Glasgow, and Manchester schools are equally well represented, the Army Medical Sohool at Netley, the military and naval services, and the public health boards. That a work conceived in such a spirit, and carried out under such auspices should prove an indispensable treasury of facts and experience, suited to the daily wants of the practitioner, was inevitable, and the success which it has enjoyed in England, and the reputation which it has acquired on this side of the Atlantic, hare sealed it with the approbation of the two pre-eminently practical nations.

Its large size and high price having kept it beyond the reach of many practitioners in this country who desire to possess it, a demand has arisen for an edition at a price which shall render it accessible to all. To meet this demand the present edition has been undertaken. The five volumes and five thousard pages of the original will, by the use of a smaller type and double column., be compressed into three volumes of about three thousand pages, clearly and hand. somely printed, and offered at a price which will render it one of the cheapest works ever presented to the American profession.

But not only will the American edition be more convenient and lower priced than the English; it will also be better and nore complete. Some years having èlapsed since the appearance of a portion of the work, additions will be required to bring up the subjects to the existing condition of science. Some diseases, also, which are comparatively unimportant in England, require more elaborate trentment to adapt the articles devoted to them to the wants of the American physician; and there are points on which the received practice in this country differs from that adopted abroad. The supplying of these deficiencies has been undertaken by HeNrY HarTs HoRne, M.D., late Professor of Hygiene in the University of Pennsylvania, who will endeavor to render the work fully up to the day, and as useful to the Ameriean physician as it has proved to be to his English brethren. The number of illustrations will also be largely increased, and no effort will be spared to render the typographical execution unexceptionable in every respect. The preparation of the work is now proceeding as rapidly as is compatible with its careful execution, and its appearance may be expected at an early dıy.

\section{TOX (TILBURY), M.D., F.R.C.P., and T. C. FOX, B.A., M.R.C.S., Physician to the Department for Skin Diseases, University College Hospital.}

EPITOME OF SKIN DISEASES. WITH FORMULA. For STUDENTS AND Practitioners. Second édition, thoroughly revised and greatly enlarged. In one very handsome $12 \mathrm{mo}$. volume of 216 pages. Cloth, $\$ 138$. (Just Ready.)

The names of the authore are quite enfficient to exceeds in size, and surpusses in use, its predeces. commэnd this book, Dr. Tilbury Fox being well gor. The work is certainly a valuable addition to knowu as occupying a place in the front rank of the "handy volume" department of medical literadermatologists of the day.-Canarian. Jourual of ture.-The Med. Bulletin, May, Is7s

Med. Sci., May, 1:78.

For etudents a better book was never devised.Ti.e present edition of the Epltome considerably Cincinnati Lancet and Cinic, May, 1s79.

WILSON (ERASMUS), F.R.S.

THE STUDENT'S BOOK OF CUTANEOUS MEDICINE and DISRASES OF THE SKIN. In one very handsome royal $12 \mathrm{mo}$. volume. $\$ 350$.

HLLIER (THOMAS), M.D., Physician to the Skin Department of University College Hospital, ete.

HAND-BOOK OF SKIN DISEASES, for Students and Practitioners. Second Am. Ed. In one royal $12 \mathrm{mo}$. vol. of $358 \mathrm{pp}$. With Illustration, Cloth, \$2 25.

It is a cuncies, plain, practical treatise on the/dente ard practitioners. - Chicago Medical Exvar ous diseases of theskin; just euch a work, aminer, Mas 1865.

indeed. as wis much needed, both by medlcal stu- 
FINLA TSON (JAMES), M.I.,

Physician and Lecturer on Clinical Medi ine in the Glasgovo Western Infirmary, etc.

CLINICAL DIAGNOSIS; A Handbook for Stuclents and Practitioners of Medicine. In one bandsome $12 \mathrm{mo}$. volume, of $546 \mathrm{pages}$, with 85 illustrations. Cloth, \$2 63. (Just Ready.)

The concurrence of gentlemen specially fumiliar with the several subjects being requisite to the satisfactory development of a plan so extensive, Dr. Finlayson has securerl the co-operatinn of Prof. Gairiner, who has contributed the chapter on the Physiognomy of Disease ; Prof. Wm. Stephenson that on Disorders of the Female Organs; Dr. Alex. Robertson that on Insanity: Prof. Samson Gemmell those on the Sphygmograph and Physieal Diagnosis; and Dr. Joseph Coates those on the Fauces, Larynx, and Nares, and on the method of performing post-mortem examinations. Other chapters have enjnyed the advantage of revision by gentlemen specially versed in their several subjects; and the volume is presented as thoroughly on a level with the most advanced condition of knowledge in a department which has made such rapid strides of advancement within the last few years.

The book is an excellent one, clear, concise, conrenient, practical. It is replete with the very kuowledge the student needs when he quits the lecture room and the laboratory for the ward and sick-loom, aud does not lack in infurmation that will meet the wants of experienced and older men.-Phila. Med. Times, Jan. 4, 1879 .

The aim of the author is to teach a student and prictitioner how to exanine a case 80 as to u=e " a $l l$ his knowledye" in arriving at a diagnosis. All the varlons symptoms of the several systems are grouped together in such \& manner as $10 \mathrm{~m}$ ake their relations to a final dirgnosis clear and easy of apprehension. This work has been done by men of lirge experience and trained observation, who have beeu long recog nized as anthorities upon the suhj.cto which they treat. There is a profusion of illustrations to illustrute subjects under discussion. The application of electricitv, and iustruments of precision in diagnosis, is fully discussed. This book is all good. We com. mend it to all students and practitioners of medlcine as a work worthy of a place in their libraries. - Ohio Med. Recorder. Dec. 1878 .

\section{HAMLTON (ALLAN MCLANE), M.D., \\ Attending Physician at the Hospital for Epileptics and Paralytics, Blrckwell's Island, N: $Y$., and at the Out-Fatients' Department of the New York Hospital.}

NERYOUSDISEASES;THEIR DESCRIPTION AND TREATMENT.

In one handsome octavo volume of 512 pages, with 53 illus.; cloth, $\$ 350$. (Just Ready.)

This is unquertionably the best and most com plete text-book of nervois diseases thut hus yet appeared, and were interuational jealonsy in scientific affidirs at all possible, we night be excused $f+r$ ic feeling of chagrin that it should be of Americun parentage. This work, however, has been performed in New York, and has been so well performed that no ronm is left for anything but comtneudation. With great skill, Dr. Hamilton has presented 10 his readers a succinct and lucid survey of all that is known of the patliology of the nervous systern, viewed in the light of the most recent reserrehes. From the preliminary description of the methuds of examination and study, and of the instruments of precision employed in the investigation of nervous diseases, up till the final collection of formula, the hook is eminently practical. - Brain, London, Oct. 1 is.

The author tells us in his preface that it has been his object to produce a cuncise, praciical book, and we think he has been successful, considering the exteut of the sabject which he has nailertaken. In fact, it is mure extensive than the title pruperly or accurately indicates, embractag-besides what are usually regarded as nervous diseases-iuflammatory affections, both acute and chronic. liemorrliages and tumors of the cerebrum ind cerebellum, medulla oblongata, spinal cord and nerves, with thrumbosis aud embolism of the arteries, Ninuses, und veins. The reader may therefure expect Jufurmation, more or less full and satisfactory, on alinost every point
This is one of the really aseful books. It is attracive from priface to the final page, and onglit to he giveu a place on every otlice table, becanse it contains a coudensenform $x .11$ that is valuable in semelolugy nostics to be found in bulkier volumes, an unusually convenient for quick reference in any emergency that may coine upon the busy practitioner. C. Med, Juuv"n., Jan. 1579.

This is a most important work for stadents, and one that is destined to become rapidly popular. It composed of contributions from various eminent sources bearing npou this subject. The real secret of successtul pructice is the accurate diagnosis of This manual tenches the stadent to arrange his investigation in such system as to enable hin, with practice, to acquire this very desirable faculty. The division of the subject, as in this work, amons hest anthorilies living, is a good idea, and gives as in one compact form a series of monographs
written by masters.-Nasleville Journal of Med. and Surg., Jan. $18 \% 9$.

convected with the nervous system. We have no lesitation in sayiug that reliance uny be placed on Dr. Hamiltun's conscieutious performance of his sel tassinued task, on his roundness of judsinent, anc freedoin from empiricism.-Edinburgh Med. Journ. Oct. 1STS.

From a very careful examination of the whole work, we can juatly say that the ruthor has not only clearly and fully treated of diagnosia and treatment, but, ualikn most works of this class, it is very comprehensive in regard to etiology, and exposies the pathology of nervous disenses in the light of the very late-t experiments and discoveries. The drawing: are excellent and well selected. After this careful revision, we can heartily recoinmend this work to students and general practitioners in particular as boing a full expo-ition of aiseases of the nervons sy:tem, their pathology and treatment, to date. $-N$. $Y$. Med. Record, Aug. 3, 1sis.

As slated in the prefuce, the author's object has been to write a concise and practical book, for which there is certainly a place, and we think he has succeeled admilably in fulflling his object. The unual plan is adopted in the classification of the different diseaser, the book not being greatly unlike IIammond's in this respect, although it is very noticeable thronghout that the author's opin. Jons vary widely from those of Dr Hammond.- $\mathrm{Am}$. Supp. Osstet. Journ. Great Britain and Ireland, July, 18?S.

CYIARCOT (J. M.). Professor to the Fuculty of Med. Paris, Phys. to La Salpêtrière, etc.

LEC'UUES ON DISEASES OF'THE NERTOUS SYSTEM. TPanslated from the Second Elition hy George Stgerson, M.D., M.Ch., Lecturer on Biology, ete., Cath. Univ. of Ireland. With illustrations (Publishing in the Medical Neu's und Library, commencing with the July No. 1878 See page 2 ) 
BROWN (LENNOX), F.R.C.S. Ed.,

Senior Surgeon to the Central London Throat and Ear Hospilal, etc.,

'TIEE THROA'T' AND ITS DISEASES. With one hundred 'Typical Illustrations in colors, and fifty wood engravings, designed and executed by the author. In one very hanilsome imperial octavo volume of 351 pages; cloth, $\$ 500$. (Now Ready)

The author's rure artistic skill bas been utilized | are unusually accurate. In conclusion, we recom. in the production of one hundred beautiful illustri- mend this beautiful volnine as an acceptable additions in colors, the very best of the kind we bave tion to the library of those engaged in the treatment seen, a ud which have been distributed in teu plates. of diseases of the throat. $-N$. $\boldsymbol{Y}$. Med. Record, Nov. Fifty woud engravings, designed and executed by $9,18 i s$.

the aut:ior, appear in the body of the work-these

SEILER (CARL), M.D.,

Lecturer on Laryngoscopy at the Univ. of Penna, Chief of the Throat Dispensary at the Univ. Hospital, Phila., etc.

HANIBOOK OF DIAGNOSTS AND TREATMENT OF DISEASES OF THE THROAT AND NASAL CAVITIES. In one handsome royal 12mo. volume, of 156 pages, with 35 illustrations; cloth, \$1. (Just Ready.)

FLINT ( $A$ USTIN), M.D.

Prufessor of the Principles and Practice of Medicine in Bellevue Hospital Med. College, N. $Y$.

PHTIISIS: ITS MORBID ANATOMY, ETIOLOGY, SYMPTOMATIC EVENTS AND COMPLICATIONS, FATALITY AND PROGNOSIS, TREAT. MENT, AND PHYSICAL DIAGNOSIS; in a series of Clinical Studies. By Austin Flixt, M.D., Prof. of the Principles and Practice of Medicine in Bellevue Hospital Med. College, New York. In one handsome octavo volume: $\$ 350$. (Lately Issued.)

This book contains an analysis, in the author's lucid $\mid$ mend the book to the perusal of all interested in the style, of the notes which he has made in several hun- stady of this disease.-Boston Med. and Surg. Journal, dred cases in hospital and private practice. We com- Feb.10, 1876.

$B^{Y}$ THE SAME AUTHOR.

A MANUAL OF PERCUSSION AND AUSCULTATION; of the Plysical Diagnosis of Diseases of the Lungs and Heart, and of Thoracic Aneurism. In one handsoine royal $12 \mathrm{mo}$. volume: cloth, $\$ 175$. (Just Issued.)

\section{$B^{Y}$ THE SAME AUTHOR.}

A PRAC'TICAL TREATISE ON THE DIAGNOSIS, PATHOLOGY, AND TREATMENT OF DISEASES OF THE HEART. Second revised and enlarged edition. In one octavo volume of 550 pages, with a plate, cloth, $\$ 4$.

Dr. Flint chuse a difficult subject for his researches, and clearest practical treatise on those snbjects, a nd and has shown remurkable powers of observation should be in the hands of all practitioners and stu. and reflection, as well as greatindustry, in his treat. lents. It is a credit to American medical literature. inent of it. His book innst be considered the fullest -Amer. Journ. of the Med. Sciences, July, 1860.

$B^{Y}$ THE SAME AUTHOR.

A PRACTICAL TREATISE ON THE PHYSICAL EXPLORA. TION OF THE CHEST AND THE DIAGNOSIS OF DISEASES AFFECTING THE RESPIRATORY ORGANS. Second and revised edition. In one handsome octavo volume of 595 pages, cloth $\$ 450$.

WILLIAMS'S PULMONARY CONSUMPTION; its FULLER ON DISEASES OF THE LUNGS AND AIRNature, Varieties, and Treatment. With an Analysis of One Thousand Cases to exemplify its duration. In one neat octavo volume of about 350 pages; cloth, \$2 50.

SLADE ON DIPHTHERIA; its Nature and Treatment, with an account of the History of its Prevaleuce in various Conntries. Second and revised edition. In one neatroyal $12 \mathrm{mo}$. volume, cloth, \$1 25.

WALSHEON THE DISEASES OF THE HEART AND GREAT VESSELS. Third Ameriean Edition. In 1 vol. 8 vo., 420 pp., cloth, $\$ 300$.

I.ECTURES ON THE DISEASES OF THE STOMACH. With an Introduction on its A ratomy and Physio. logy. By WILLIAM BRINTON, M.D., F.R.S From the second and enlarged Londonedition. With illustrations on wood. In one havdsome octavo volume of about 300 pages: cloth, $\$ 325$.

LA ROCHE ON PNEUMONIA. 1 vol. 8vo., cloth, of 500 pages. Price, $\$ 300$.

LINCOLN'S ELECTRO-THERAPEUTICS; a Concise Manual of Medical Electricity. In one very neat. royal $12 \mathrm{mo}$. volume, clotb, with fllustrations, \$1 50.

CLINICAL OBSERVATIONS ON FUNCTIONAL NERVOUS DISORDERS. BY C. HANDFIELD JONE:, M.D., Physician to St. Mary's Hospital, \&c. Sec ond American Edition. In one had some octavo volume of 348 pages, cloth, $\$ 325$. PASSAGES. Their Pathology, Physical Diagnosis, Symptoms, and Treatment. From the second and revised English edition. In one handsome ocatro volume of about 500 pages: cloth, $\$ 350$.

CHAMBERS'S MANUAL OF DIET AND REGIMEN IN HEALTH AND SICKNESS. In one handsone octa vo volume. Cleth, $\$ 275$.

CHAMBERS'S RESTORATIVE NIEDICINE. An Harveian Aanual Oration. With Two Sequels. In one very handsome vol. small $12 \mathrm{mo}$, cloth, $\$ 100$.

PAVY'S 'TREATISE ON THE FUNCTION OF DIGESTION; its Disorders and their Treatment. From the second London odition. In one handsome volume, small octavo, clotl, $\$ 200$.

PAVY'S TREATISE ON FOOD AND DIETETICS. Physiologically and 'Therapentically Considered. In one handsome octavo volume of nearly 600 pages, cloth, $\$+75$.

S IITH ON CONSUMPTION ; ITS EARLYAND REII SDIABLESTAGES. 1 1 ol. 8vo,, pp. 254. \$2 2 K.

BASHAM ON RENAL DISEASES : a Clinical Guide to their Diagnosis and Treatment. With Illustrations Iu one $12 \mathrm{mo}$. vol. of $30 t$ pages, clnth, $\$ 200$. LECTURES ON THE STUDY OF FEVER. BY A. HuDsox, M.D., M.R.I.A., Physician to the Meath Hospital. In one vol. Svo., cloth, $\$ 250$.

A TREATISE ON FEVER. By ROBERT D. LYONF, K C C. In one octavo volumo of 362 pages, cloth, $\$ 225$. 
BUMSTEAD (FREEMAN J.), M.D., Professor of Venereal Diseases at the Col. of Phys. and Surg., New York, \&c.

THE PATHOI,OGY AND TREATMENT OF VENEREAL DIS. EASES. Including the results of recent investigation 8 upon the subject. Fourth edition, revised and enlarged. In one large and handsome octavo volume of over 700 pages. (Preparing.) CULLERIER (A.), and
Surgeon to the Hopital du Midi.

\section{BUMSTEAD (FREEMAN J.), Profeseor of Venereal Diseases in the Collegt of Physioiane and Surgeons. N. Y.}

AN ATLAS OF VENERFAL DISEASES. Translated and Edited by Framan J. Bumstrad. In cne large imperial 4 to, volume of 328 pages, double.columns. with 26 plates, containing about 150 figures, beautifully colored, many of them the size of life; strongly bound in eloth, $\$ 1700$; also, in five parte, stout wrappers, at $\$ 3$ per part.

Anticipating a very large sale for this work, it is offered at the very low price of THR a DoL. LARS a Part, thus placing it within the reach of all who are interested in this department of practice. Gentlemen desiring early impressions of the plates would do welf to order it without delay. A specimen of the plates and text sent free by mail, on receipt of 25 cents.

We wish for once that our province was not re- of illustrations of the venereal diseases. There is, strict d to methods of treatment, that we might say however, an additional interest and value pofsessf some:hlug of the exquisite colored plates in this by the volumebefore ur; for it is an American reprint volnme, -London Practitioner, May, 1869.

Other writers besideo M. Cullerier have given ns 8 good account of the diseases of which hetreats, ba no onê has furnished no with sach a completeseriel and translation of II Cullerier's work, with inc. dental remarks by one of the most eminent American sy philographers, Mr. Bumstead.-Brit and Fo: Medico-Chir. Review, July, 1869.

LEE (HENRY),

LEC'TURES ON SYPHILIS AND ON SOME FORMS OF LOCAL DISEASE AFFECTING PRINCIPALLY THE ORGANS OF GENERATION. In one handsome octavo volume: cloth; \$2 25. (Lately Published.)

HILL (BERKELEY), Surgeon to the Lock Hospital, London.

ON SYPHILIS AND LOCAL CONTAGIOUS DISORDERS. In one handsome octavo volume; cloth, $\$ 325$.

WEST (CHARLES), M.D., Physician to the Hospital for Sick Children, London, \&c.

LECTURES ON THE DISEASES OF INFANCY AND CHILD. HOOD. Fifth American from the six th revised and enlarged English edition. In one large and handsome octavo volume of 678 pages. Cloth, $\$ 450$; leather, $\$ 550$. (Lately Issued)

The continued demand for this work on both sides of the Atlantic, and its translation into German, French, Italian, Danish, Dutch, and Russian, show that it fills satisfactorily a want extensively felt by the profession. There is probably no man living who can speak with the authority derived from a more extended experience than Dr. West, nd his work now presents the results of rearly 2000 recorded cases, and 600 post-mortem examinations selected from among nearly 40,000 sases which have passed under his care. In the preparation of the present edition he has omitted much that appeared of minor importance, in order to find room for the introduction of additional matter, and the volume, while thoroughly revised, is therefore not increased materially in size.

Jf sll the English writers on the diseases of chil- | highestliving a uthorities in the difficult department dran, there is, no one so entirely satisfactory to us of medical science in which he is most widely as Dr. West. For years we have held his opinion known.- Boston Med. and Surg. Journal. as jadicial, and have regarded him as one of the

\section{B THE SAME AUTHOR. (Lately Issued.)}

ON SOME DISORDERS OF THE NERVOUS SYSTEM IN CHILDHOOD; being the Lumleian Lectures delivered at the Royal College of Physicians of London, in March, 1871. In one volume small $12 \mathrm{mo}$., cloth, $\$ 100$.

B THE SA YE AUTHOR.

LECTURES ON THE DISEASES OF WOMEN. Third American, from the Third London edition. In one neat octavo volume of about 550 pages, cloth, $\$ 375$; leather, \$4 75 .

CONDIE'S PRACTICAL TREATISE ON TIE DIS- SMITH'S PRACTICAL TREATISE ON TIIE WAST. EASES OF CHILDREN. Sixth edition, revised and augmented. In one large octavo volnme of nearly $8 r 0$ closely-printed pages, cloth, \$5 25; leather, 25 . ING DISEASES OF INFANCY AND CHILDHOOD. Second American, from the second reviked and enlarged English edition. In one haudsome octavo volame, cloth, $\$ 250$. 
SMITH (J.LEWIS), M.D.,

clinical Professor of Diseases of Children in the Bellevue Hospital Mred. Oollege, N $Y$.

A CONPLETE PRACTICAL TREATISE ON THE DISEASISS OF CHILDREN. Fourth Edition, revised and enlarged. In one handsome octavo volume of about 750 pages, with illustrations. Cloth, $\$ 450$; leather, $\$ 550$. (Now Ready.)

The very marked favor with which this work has been received wherever the English language is spoken, has stimulated the nuthor, in the preparation of the Fourth Edition, to spare no pains in the endeavor to render it worthy in every respect of a continuance of professional confidence. Many portions of the volume have been rewritten, and much new matter introduced, hut by an earnest effort at condensation, the size of the work has not been materially ncreased.

In the period which has elapsed since the third edition of the work, so exteusive have been the advauces that whole chapters required to be rewritten, and hardty a page conld pass without some materisi correction or addition. This labor has occupied the writer closely, and he has performed it conscientiously, so that the book may be considered a faithful portraiture of an exceptionally wide clinical experience in infantile diseases, currected by a careful study of the recent literature of the subject.Med. and Surg. Reporter, A pril 5, 1879:

It is scarcely necessary for us to say the work before us is a standard work upon diseases of children, and that no work has a higher atanding than it upon those affections. In consequence of its thorough revision, the work has been made of more value than ever, and may be regarded as fully abreast of the times. We cordially commend it to studants and physicians. There is no better work in the language on diseaves of children.-Cincinnati Med. Ntws, March, 1879.

Ihe anthor has evidently determined that it shall not lose ground in the esteem of the profession for want of the latest knowledge on that important department of medicine. He bas accordingly in. corporated in the present edition the useful and practical results of the latest study and experience, bith American and foreign, especially those bearing on therapeutics. Altogether the book has been greatly improved, while it has not been greatly iacreased in size. - New York Midical Journal, Jun $\theta, 1879$.

This excellent work is so well known that an ex ended notice at this time wunld be supertinoug. The author hrs tal en advantage of the demand for another new edition to revise in a most carelu manner the entirebook; and the numerons correetions and additious evince a determinution on bis part to keep fully ubreast with the rapid progress that is being made in the knowledge and treatment of children's diserses. By the adoption of a $8 \circ \mathrm{me}$. what closer ty pe, an increase in size of only thirty pages has been necestitated by the new snbject matter introduced.-Boston Med. and Surg. Jour., May $29,1879$.

Probably no other work ever published in this conntry upon a medical subject has reached such a heighth of popularity as has this well-known trea. tise. As a text and reference-book it is pre-eminently the anthority upon diseases of childrea. It stands deservedly higber in the estimation of the profession than any other work upon the same sub. ject.-Nashville Journ. of Med. and Surg., May, 1879 .

The author of this work has acquired an immense experience as pbysician to three of the large char. ities of New York in which childrea are treated. These asylums afford unsurpassed opportunities for observing the effcets of different plans of treatment, and the results as embudied in this volume may be accepted with faith, and should be in the possession. of all practitioners $n c w$, in view of the approaching season when the diseases of children al ways increase. -Nat. Med. Review, April, 1879.

SWA FNE (JOSEPH GRIFFITHS), M.D.,

Physician-Accoucheur to the British General Hospital, \&c.

OBSTETRIC APHORISMS FOR THE USE OF STUDENTS COMMENCING MIDWIFERY PRACTICE Second American, from the Fifth and Revised London Edition. with Additions by E. R. Hutchins, M.D. With Illustrations. In one neat $12 \mathrm{mo}$. volume. Cloth, $\$ 125$. (Lately Issued.)

** See p. 4 of this Catalogue for the terms on which this work is offered as a premium to subsoribers to the "American Journal of the Medical Sciences."

CHURCHILL ON THE PUERPERAL FEVER AND MEIGS ON THE NATURE, SIGNS, AND TREAT. OTHER DISEASES PECULIAR TO WOMEN. 1 Vol. 8vo., pp. 450, cloth. $\$ 250$.

DEWEES'S TREATISE ON THE DISEA SES OF FE. MALES. With illastrations. Eleventh Edition. with the Author's lastimprovementeand correct1ons. In one octavo volume of 536 pages, with plates, cloth. $\$ 300$.

MENT OF CHILDBED FEVER. 1 vol. 8vo., pp. 365 . cloth. $\$ 260$.

ASHWELL'S PRACTICAL TREATISE ON THE DIई.

EASES PECULIAR TO WOMEN. Third Americen, from the Third and revised London edition. I vol. 8 vo., pp. 528, cloth. $\$ 350$.

\section{$H^{O D G E}(H U G H L),. M . D$.}

Emeritus Professor "of Obstetrics, \&c., In the University of Pennsylvania.

ON DISEASES PECULIAR TO WOMEN; including Displacements of the Uterus. With original illustrations. Second edition, rerised and enlarged. In one beautifully printed octavo volume of 531 pages, cloth, $\$ 450$.

Professor Hodge's work is truly an original one / contribntion tothe study of women'sdiseases, 1tifrf from beginnling to end, consequently no one can pe. great value, and is abundantly able tostand on its

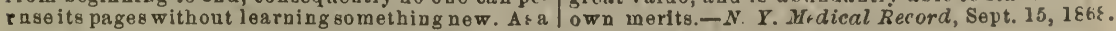

CHURCHILL (FLEETWOOD), M.D., M.R.I.A.

ON THE THEORY AND PRACTICE OF MIDWIFERY. A new A merican from the fourth revised and enlarged London edition. With notes and additiors by D. Francis Condiz, M.D., author of a "Practical Treatise on the Diseases of Chil. dren," \&c. With one hundred and ninety four illustrations. In onevery bandsome octaro volume of nearly 700 large pages. Cloth, $\$ 400$; leather, $\$ 500$.

MONTGOMERY'S EXPOSITION OF THE SIGNS RIGBY'S SYSTEM OF MIDWIFERY. With notes AND STMPTOMS OF PREGNANCT. With two and Additional illastrations. Second American exquisitecolored plates, and numeronswood cuts. Fition. One volume octavo, cloth, 422 pages, In 1 vol. 8 vo., of nearly $600 \mathrm{pp}$., clotb, $\$ 375$. 
ZHOMAS (T. GAILLARD), M.D.,

A PRACTICAL TREATISE ON THE DISEASES OF WOMEN. Fourth edition, enlarged and thoroughly revised. In one large and handsome octavo volume of 800 pages, with 191 illustrations. Cloth, $\$ 500$; leather, $\$ 600$. (Just Issued.)

The author has taken advantage of the opportunity afforded by the call for another edition of this work to render it worthy a continuance of the very remarkable favor with which it has been receired. Every portion has been subjected to a conscientious revision, and no labor has been spared to make it a complete treatise on the most advanced condition of its important subject.

$A$ work which has reached a fourth enlition. and isclassical withoutbeing pedantic, fullin the details that, too, in the short space of five yeurs, has achieved of anatomy and pathology, withont ponderons a reputation which places it almost beyond the reach translation of pages of Germanliterature, describes of criticism. and the favorableopinions which we have distinctly the details and difficulties of each opera. a ruady expressed of the former editions seem to re- tion, withont wearying and useless minntix, and is quire that we should do little more than announce in all respectsa work worthy of conflence, justifythis new issue. We cannot refrain from saying that, ing the high regard in which its distinguished auas a practical work, this is second to none in the Eng- thor is held by the profession. - Am. Supplement, lish, or, indeed, in any other language. The arrange- Obstet. Journ., Oct. 1874.

ment of the contents, the admirably clear manner in which the subject of the differential diagnosis of several of the diseases is handled, leave nothing to be desired by the practitioner who wants a thoroughly elinical work. nne to which he cun refer in difficult cases of doubtful diagnosis with the certainty of gaining light and instruction. Dr. Thomas is a man with a very clear head and decided views. and there seems to be nothing which he so much dislikes as bazy notions of diagnosis and blind routine and unreasonable therapentics. The student who will thoroughly study this b jok and test its principles by clinical observation, will certainly not be guilty of these faults.-London Lancet, Feb. 13, 1875 .

Reluctantly we are obliged to close this unsatis. factory notice of so excellent a work, and in conclusion would remark that, ns a teacher of gyamelogy, both didac!ic and clinical, Prof. Thomas has certainly taken the lead far ahead of his confrères, and as an a uthor he certainly has met with unnsual and merited success. -Am. Journ. of Obstetrics, Nov. 187 t.

This volume of Prof. Thomas in its revised form

Professor Thomasfairly took the Profession of the United States by storm when his book first made it 8 appearance early in 1S68. Its reception was simply enthusiastic, notwithstanding a few adverse criticisms from our transatlantic brethren, the first large edition was rapidly exhausted, and in six mouths a second one was issned, and in two years a third one was annonnced and published, and wo are now promised the fourth. The popularity of this work was not ephemeral, and its success was un precedented in the annals of American medical literature. Six year's is a long period in medical scentific research, but Thomas's work on "Diseases of Women" is still the leading native production of the United States. The order, the matter, the absence of theoretical disputativeness, the fairness of statement, and the elegance of diction, preserved throughout the entire ravge of the book, indicate that Professor Thomas did not overestimate his powers when he conceived the idea and executed the work of producing a new treatise upon diseases of women.-Prop. PALLEN, in Louis. nille Med. Journal, Sept. 1S74.

\section{BARNES (ROBERT), M.D., F.R.C.P.,}

\section{A CLINICAL EXPOSITION OF THE MEDICAL AND SURGI-} CAL DISEASES OF WOMEN. Second American, from the Second Enlarged and Revised English Edition. In one handsome netavo volume, of 784 pages, with 181 illustrations. Cloth, \$4 50; leather, \$50. (Just Ready.)

The call for a new edition of Dr. Barnes's work on the Diseases of Females has encouraged the authnr to make it even more worthy of the favor of the profession than before. By a rearrangement and eareful pruning space has been found for a new chapter on the Gynæcological Relations of the Bladder and Bowel Disorders, without increasing the size of the book, while many new illustrations have been introduced where experience has shown them to be needed. It is therefore hoped that the volume will be found to reflect thoroughly and accurately the present condition of gynacological science.

Dr Barnesstands at the head of his profession in the work is a valnable one, and shonld be largely the old conutry, and it requires bat scant serutiny consulted by the profession. Am. Supp. O'stetricril m.tster. It is plain, practical common sense; shows very deep research without being pedantic; is eminently calculated to inspire enthusiasm without in. culcating ra-hness; points out the dangers to be avolded as well as the success to be achieved in the various operationa connected with this branch of medicine; and will do much to smooth the rugged path of the young gynæculogist and relieve the perplexity of the man of rnature years. - Canadian Journ. of Med. Science, Nov. Ists.

Journ. Gt. Britain and Ireland, Ocl. 1578.

No other gynacological work holds a higher posi. tion, having become an authority. everywhere in diseases of women. The work has been bronght fully abreast of present knowledge. Every practitioner of mediclne should havo it upon the shelves of his library, and the student will find it a superior text-book.-Cincinnati Med. Neros, Oct. 1s7s.

This second revised edition, of course, deserves all the commendation given to its predecessor, with the additional one that it appears to include all or nearly

We pity the doctor who, having any considerable practice in dikeases of women, has no copy of "Barnes" for daily consultation aad instrnction. It is at once a book of great learning, research, and individual experience, and at the same time eminently practical. That it has been apprecisted by the profession, both in Great Britain and in this country, is show by the second edition following 60 soon upon the first. - Am. Practitioner, Nov. 1575 .

all the additions to our knowledge of its subject that have been made since the appearance of the first edition. The American references are, for au English work, especially full and appreciative, and we ean cordially recommend the volume to American readers.-Journ. of Nervous and Mental Disease, Oct. ] $87 \mathrm{~S}$.

This second edition of Dr. Barnes's great work comes to us containing many additions and ianprovements which bring it up to date in every feature.

Dr. Burnes's work is one of a practical character, largely fllustrated from cases in his own expertence, bui by 110 means confined to such, as will be learned from the fact that he quotes from no lers than 628 medical authors in numerous countries. Coming from such an author, it is not necessary to say that excellences of the work are too well known to require enumeration, and we hazard the prophecy that they will for many years maintain its high position as a standard text-book and guide book for students and practitioners. $-N$. C. Med. Journ., Oct. 1878. 
THUET (THOMAS ADDIS), M.D.

Surgeon to the Woman's Hospital, New York, etc.

THE PRINCIPLES AND PRAC'IICE OF G Y ECOLOGY, for the use of Students and Practitioners of Medicine. In one large and very handsome octavo volume of 850 pages, with 130 illustrations. Cloth, \$5; leather, \$6. (Just Ready.)

Dr Emmet is so widely known as among the most eminent of those who have made gynacology a peculiarly American science that the profession cannot fail to welcome a work in which he has condensed the results of his long and extensive experience. He has sought to consider the whole subject of the cliseases peculiar to females in a manner which will adapt the volume, not only to the wants of the student as a text-book, but to those of the practitioner as an aid in the emergencies of daily practice. A special feature of the work will be found in the numerous condensed tables, which convey at a glance, and within the narrowest compass, the conclusions to be drawn from the many thousand cases which have passed under the care of the author. With trifling exceptions, the illustrations are all original, and the rolume will be found in every point of typographical execution worthy of the distinguished position which is confidently anticipated for it.

It may be said that he bas had opportunities for observation and experience, for unfetterea and unrestrained experimentafion, and for testing the value of the original and dazzling operations first proposed and performed by his illastrious predecessors before referred to, and for devising $\mathrm{bew}$ operations and discovering pathological causes never before suspected or described, which no man in the profession has ever befure secured. We also think that the reader; of this work will agree with $u s$, after its careful perasal, that he has a rare capacity for discriminating analysis, and generally for philosophical deduction and the equally important quality of patient, honest, continued work. For the work as a whole, we have only praise. It deserves and will receive the careful study of all who desire to keep on a level with the progress of Gynæcology. It embodies a larger a monnt of carefully analyzed personal experience in a unique field for observation than any volume on Diseases of Women whicl has yet heen published. Its great merit cousists in this-coming as it does from a thoroughly honest, competent. and able specialist, who became a specialist only after an $\mathrm{x}$ xcellent training a ud experience as a general hospital phytician und surgeon. The book is not one to be histily glanced over, but will secu re the critical stujy of G yn cologists. Not only its style, which is individual and somewhat peculiar, but the new facts which it brings out, its original suggestions, its numerons and important statistical tables, ind, in someinstances, its unexpected deductions, will compel attention, and will form the basis for a great deal of Gyuxcological study and literature in the future. Ail who make themselves familiar with the contents of this volume, will feel assured that Dr. Emmet has well earned and well deserved the reputation which he has already won, as one of the great Gynæcolngists of the presant age.-The Am. Journ. of Obstetrics, April, 1579.

We have examined this book with something more than ordinary care, and now lay it asido captivated

by our impressions of it. From first to last, each page grows in interest, and one is struck with the practical tone of all that is said. It is indeed the gynæcological work for the practitioner. Its equal is not yet published, or at least we have not seen it. We cannot send this notice forward without relteratirg that, in our estimation, Emmet's Principles and Practice of Gynæcology is undoubtedly the best book for the student, as well as the general practitioner, which is at present published.-Va. LIed. Monthly, May, 1879 .

The advent of this important work has for some time been anxiously expected hy all who are interested in the subject of gynæcology, both here and abroad. The clinics held at the Woman's Hospital, and the minor writings referred to have acquired for Dr. Emmet a reputation for skill as an operator, and experience in the special branch to which he has exclusively coufined his attention, whlch is probably unrivalled by any one on this continent. The anticipations which have been awakened regarding the character of this extended treatise, are not likely to be disappointed, if one may judge from the very cursoly review we have made of its conteuts.-New Remedies, May, 1579

Few have had the rare opportunities of Dr. Em. met, and none have better improved that which was at their disposal. Sure are we that any practitioner of medicine, epecialist, or atherwisf, who will read carefully this volume, will find that be poscesses a clearer insight into a thousand problerns that have hitherto perplexed him. It is one of the best original works on the diseases of women published in this or any other land. We heartily commead it to the careful study of every medical man. -Detrrit Lancet, Iay, 1 s79.

We are satisfed that whoever reads the book carefully will agrea with os that it is the best work on gynæcology that has ever been written. This is high pralse, but we bare no hesitation in giving it. -St. Louis Cıin. Record, Muy, $1 \leqslant 79$.

CHADWICK (JAHES R.), A.M., M.D.

\section{A MANUAL OF THE DISEASES PECUIIAR TO WOMEN. In one} neat volume, royal $12 \mathrm{mo}$, with illustrations. (Preparing.)

America has contributed so largely to the advances which have made the treatment of Dis. eases of Women a distinctive department of medical science, that the student will naturally turn to American Books for the latest and most trustworthy instruction on the subject in its most modern aspect. Yet there has thus far been no attempt in this country to produce a handy manual, presenting in a condensed and convenient form the information requisite for the learner or for the general practitioner. This want it has been the effort of Dr. Chadwick to supply, and the special attention which he has devoted to the subject is a guarantee of the value of his labors. A distinguishing feature of the work will be a number of diagrammatic illustrations, facilitating greatly the oomprehension of the text.

WINCKEL $(F$.$) ,$

Professor and Director of the Gyncecologicul Clinic in the University of Rostock.

A COMPLE'TE TREATISE ON THE PATHOLOGY AND TREATMENT OF CHILDBED, for Students and Practitioners. Translated, with the consent of the author, from the Second German Edition. hy JAMES READ CHIDWICK, M.D. In one octaro volume. Cloth, $\$ 400$. (Lately Issued.) 
PLAYHAIR (W.S.), M.D., F.R.C.P.,

Professor of Obstetric Medicine in King's College, etc. etc.

A TRKATISE ON THE SCIENCE AND PRACTICE OF MIDWTFERY. Second Anerican, from the Second and Revised English Edition. Edited, with Additions, by Robert P. Harris, M.D. In one handsome octavo volume of 639 pages, with 182 illustrations. Cloth, \$4 00; Leather, \$5.00. (Just Ready)

In reprinting this work from the second London edition, the position which it has assumed in this country as an authoritative text-book seemed to eall for such additions as would render it more completely suited to the wints of the American stulent. A careful scrutiny on the part of the editor has shown that but little was required for this purpose; the work, though condensed, being very complete and accurate. With the exception of numerous short foot-notes, therefore, his additions have been confined to points in which the experience and practice of American obstetricians differ from those of England, and to one or two matters of recent interest. These are chiefly the Cxsarean Section; the varieties of forceps, nnd their use in the dorsal decubitus; dystocia from tetanoid uterine constriction; and the intra-venous injection of milk, as a substitute for the transfusion of blood.

The position which this work has so quickly taken in this country 48 an authoritative lext-book renders any extendel con-ideration of its rlan and scope nnnecessary. Its merits, which are many, havealready fonnd their way to the appreciation of students and practitioners alike in the leng!h and breadth of the land.-Am. Supp. Obstet. Journ. of Gt. Britain and Irtiand, Oct.1S7S.

This excellent text-book has been submitted to a thorough and careful revision, and will be found fully up to the limes in every department. The cotes by the American editor enhance the value of the work for the American student. Those ou the nse of forceps are particularly g sod, and constitute by themsel ves a valuable chapter. $-N . \boldsymbol{Y} . M \cdot d$. Journ., Nuv. 1sis.

The bast work on the subject ever published in the English lauguage It is written in a clear, pleasant style, withuut that verbosity which characterizes some modern and highly pretentions work8. The author is quite up with the times, both in practice and

theory. It is the best text-book we have for students, and sufficiently full of detail to supply all the wants of the practitioner. We would gladiy see it in the hauds of all who practise midwifery. - Canadian hauds of all who practise mid
Journ. of Med. Sci., Nov. $157 \mathrm{~s}$.

Probably this is the very best and most useful manual of midwifery now available to the profession. It is written in lucid, scholarly Englinh, which sorne of our cis-Atlantic writers would do well to imitate. There has been no attempt to swell the magnitude of the work by fine writing, or by lengthy discussions of obecure points of which uo trustworthy solution has yet been reached; on the contrary, the tendency is throughout obviously towards simplicity. Tho chapter upon the Nechanikm of Labor (which ong'st to be the crowning chnpter in a trea. tise ou obstetrics) is remarkably clear and good, and is divested of those features which in ilmust every other work we know lets only darkness instead of light iu upan the subject. - N. C. Med. Journ., Oct. $187 \mathrm{~s}$.

BARNES (FANCOURT), M.D.,

D Physician to the General Lying-in Hospital, London.

A MANUAL OF MIDWIFERY FOR MIDIVIVES. With numerous illustrations. In one neat royal $12 \mathrm{mo}$. volume. (In Press.)

TANNEK (THOMAS H.), M.D.

ON THE SIGNS AND DISEASES OF PREGNANCY. First American from the Second and Enlarged English Edition. With four colored plates and illustrations on wood. In one handsome octavo volume of about 500 pages, cloth, $\$ 425$.

\section{THE OBSTETRICAL JOURNAL." (Free of postagefor 1879.)}

THE OBSTETRICAL JOURNAL of Great Britain and Ireland; - Including Midwfery, and the Diseases or WoMen AND INFANTs. With an American Supplement, edited by J. V. INGHAM, M.D. A monthly of about 96 octavo pages, very handsomely printed. Subseription, Five Dollars per annum. Single Numbers, 50 cents each.

Commencing with April, 1873, the Obstetrical Journal consists of Original Papers by Brit. ish and Foreign Contributors; Transactions of the Obstetrical Societies in England and abroad. Reports of Hospital Practice; Reviews and Bibliographical Notices; Articles and Notes, Editorial, Historical, Forensic, and Miscellaneous; Selections from Journals; Correspondence, \&c Collecting together the vast amount of inaterial daily accumulating in this important and rapidly improving department of medical science, the value of the information which it presents to the subscriber may be estimated from the character of the gen. tlemen who have already promised their support, including such names as those of I)rs. AT. thill, Aveling, Robert Barnes, J. Henri Bennet, Natian Boznan, Thomas Ciambers, Fleetwood Ciurchill, Charles Clay, John Clay, Matthews Duncan, Arthur Farre, Robert Greenualgi, Graily Hewitt, Braxton Hicks, Alfred Meanows, W. Leishman, Alex. Simpson, Heywood Smith, Trler Smith, Edward J. Tilt, Lawson Tait, SPENCER WeLls, \&e. \&c. ; in short, the repregentative men of British Obstetrics and Gynæ. cology.

In order to render the OBSterrical Journal fully adequate to the wants of the American profession, each number contains a Supplement devoted to the advances marle in Obstetrics and Gyngeology on this side of the Atlantic. This portion of the Journal is under the editorial charge of Dr J. V. INGIAM, to whom editorial coinmunications, exchanges, books for retiew, \&c., may be addressed, to the eare of the publisher.

** Complete sets from the beginning can no longer be furnished, but subscriptions can comnence with January, 1879, or Vol. VII., No. 1, April, 1879. 
LEISHMAN (WILLIAM), M.D.,

Regius Professor of Midwifrry in the University of Glasgon, \&c.

A SYSTEM OF MIDWIFERY, INCLUDING THE DISEASES OF PREGNANCY AND THE PUERPERAL STATE. Third American edition, with additions by John S. PArRY, M.D., Obstetrician to the Philadelphia Hospital, \&c. In one large and very handsome octavo volume, with about two hundred illustrations. (Shortly.)

PARRY (JOHN S.), M.D.,

Obstetrician to the Philadelphia Hospital, Vice-Prest. nf the Obstet. S ciety of Philadelphin.

EXTRA-UTERINE PREGNANCY: ITS CLINICAL HISTORY, DIAGNOSIS, PROGNOSIS, AND TREATMENT. In one handsome octavo volume. Cloth, \$2 50. (Lately Issued.)

HODGE (HUGH L.), M.D.,

Emeritus Professor of Midwifery, \&c., in the University of Pennsylvania, \&c.

THE PRINCIPLES ANI PRACTICE OF OBSTETRICS. Illustrated with large lithographic plates containing one hundred and fifty-nine figures from original photographs, and with numerous wood-cuts. In one large and beautifully printed quarto volume of 550 double-columned pages, strongly bound in cloth, $\$ 14$.

The work of Dr. Hodge is something more than subject it is decidedly the best.-Edinb. Med. Jour., a simple presentation of his particular views in the Dec. 1864 .

de partment of Obstetrics; it is something more We have read Dr. Hodge's book with great than an rdinarytreatise on midwifery; it is, in fact, pleasure, and have much satisfaction in expressa cyclopædia of midwifery. He has aimed to $e m$. ing our commendation of it as a whole. It is cerbody in a ingle volume the whole science and art of tainly bighly instructive, and in the main, we be. Obstetrics. An elaborate text is combined with ac- lieve, correct. The great attention which the ancurate and varied pictorial illustrations, so that no thor has devoted to the mechanism of parturition, fact or principle is left nnstated or anexplained. taken along with the conclusions at which he has -Am. Med. Times, Sept. 3, 1864 . arrived, point, we think, conclusively to the fact

It is very large, profneely and elegantly illustrat - that, in Britain at least, the doctrines of Naegele ed, and is fitted to take its place near the works of have been too blindly received.--Glasgow Med. great obstetricians. Of the American works on the Journal, Oct. 1864.

*** Specimens of the plates and letter-press will be forwarded to any address, free by mail, on receipt of six cents in postage stamps.

$R$ AMSBOTHAM (FRANCIS H.), M.D.

THE PRINCIPLES AND PRACTICE OF OBSTETRIC MEDICINE AND SURGERY, in reference to the Process of Parturition. A new and enlarged edition, thoroughly revised by the author. With additions by W. V. KEATING, M. D., Professor of Obstetrics, \&c., in the Jefferson Medical College, Philadelphia. In one large and handsome imperial octavo volume of 650 pages, strongly bound in leather, with raised bands; with sixty-four beautiful plates, and numerous wood-cuts in the text, containing in all nearly 200 large and beautiful figures. $\$ 700$.

\section{STIMSON (LEWIS A.), A.M., M.D., Surgeon to the Presbyterian Hospital.}

A MANUAL OF OPERATIVESURGERY. In one very handsome royal 12 mo. volume of about 500 pages, with 332 illustrations; cloth, $\$ 250$. (Nov Ready)

The work before $\mathrm{us}$ is a well printed, profusely, performing them. The work is handsomely illus: illustrated manual of over four hundred and seventy trated, and the defcriptionsareclenrand well drawn. pages. The novice, by a perneal of the work, will It is a clever and nseful volume; every student gain a good idea of the general domain of oparative should possess one. The preparation of this work surgery, while the practical surgeon has presented does away with the necessity of pondering over to him within a very concise and intelligible form larger works on sargery for descriptions of operathe latest and most approved selections of operative tions, as it presents in a nnt-shell jnst what is wanted procedure. The precision and conciseness with which by the surgeon without an elaborate search to find the different operations are described enable the it.-Md. Med Journal, Ang. 1878.

anthor to compress an immense amount of practical The author's conciseness and the repleteness of informatiou in a very small compass. - N. Y. Melical the work with valuable illustrations entitle it to be Record, A $11 \mathrm{~g} .3,1578$.

This volume is devoted entirely to operative surgery, and is intended to familiarize the student with the details of operations and the different modes of

SKEX'S OPERATIVB SURGERT. In 1 Vol. 8vo cl., of 650 pages; with about 100 wood-cuts. $\$ 325$.

COOPRR'S LECTURES ON THE PRINCIPLES AND

Pa ACTICE OF SURGBY. In 1 vol. $8 v 0 . \mathrm{cl}^{\prime} \mathrm{h}, 750 \mathrm{p}$. $\$ 2$. GIBSON'SINBTITUTES AND PRACTICE OF SURG $B \mathbf{R}$. Eighth edit'n, improved and altered. With thirty-four plates. In two handsome octavo volnmes, abont 1000 p p.. leather, raised ba.bdf. \$6 50.

THE PRINCIPLES AND PRACTICE OF SURGERY. By Wil, la r PiRRik, F.R S. E., Profes r of Surgery in the University of Aberdeen. Edited by JонN classed with the text-books for students of operative surgery, and as one of reference to the prictitioner. - Cincinnati Lancet and Clinic, July 27, 1875.

Neilt, M. D., Professor of Surgery in the Penna. Medical College, Surg'n to the Pennsylvania Hos. pital, \&c. In one very handsome octavo vol. of 780 pages, with 316 illustrations, cloth, $\$ 375$.

MILLEI'S PRINCIPLES OF SURGERY. Fourtb A merican, from the Third Edinburgh Edition. In one large $8 v 0$. vol. of 700 pages, with 340 illustrations, cloth, $\$ 375$.

MILLER'S PRACTICE OF SURGERT. Fonrth American, from the last Edinburgh Fidion. Jievised by the A merican editor. In one large 8 vo. vol. of nearly 700 pages, with 364 illustration 8 : cloth, $\$ 375$ 
GROSS (SAMUEL D.), M.D., Professor of Surgery in the Jefferson Medical College of Philadelphia.

SYSTEM OF SURGERY: Pathological, Diagnostic, Therapentic, and Operative. Illustrated by upwards of Fourteen II undred Engravings. Fifth edition carefully revised, and improved. In two large and beautifully printed imperial octavo vol. umes of a bout $2300 \mathrm{pp}$., strongly bound in leather, with raised bands, 815. (Just Issued.)

The continned favor, shown by the exhaustion of successive large editions of this great work, proves that it has successfully supplied a want felt by American practitioners and students. In the present revision no pain have been spared by the author to bring it in every respect fully up tr the day. To effect this a large part of the work has been rewritten, and the whole en. arged by nearly one-fourth, notwithstanding which the price has been kept at its former very moderat rate. By the use of a close, though very legible type, an unusually large amount of matter is sondensed in its pages, the two volumes containing as much as four or five ordinary nctavo8. This, combined with the most careful mechanical execution, and its very durable bind ing cender, it one of the cheapest works accessible to the profession. Every subject properly belonging to the lomain of surgery is treated in detail, so that the student who possesses this work may e said to have in it a surgical library.

We have now brought ourtask to a conclusion, and e lition of Gros"s "Surgery," will confirm his title of have seldom read a work with the practical value of "Primus inter Pares." It is learned, scholar-like, mewhich we have been moreimpressed. Every clapter is thodical, precise, and exhaustive. We searcely think so concisely put together. that the busy practitioner, any living man could write socompleteand faultless a when in difficulty, can at once find the information he treatise, or comprehend more solid, instruetive matter requires. Il is work, on the contrary, is cosmopolitan, in the given number of pages. The labor must hare the surgery of the world being fully represented in it. been immense, and the work gives evidence of great The work, in fact, is so historically unprejudiced. and powers of mind. and the highest order of intellectual sn eminently practical, that it is almost a false compli- discipline and methodical disposition, aud arrangement ment to say that we believe it to be destined to occupy of acquired knowledre and personal experience.- $N$. $Y$. a foremost place as a work of reference, while a system of surzery like the present system of surgery is the practice of surgeons. The printing and binding of the work is unexceptionable; indeed. it contrasts. in the latter respect, remarkably with English medical and surgical cloth-bound publications, which are generally so wretehedly stitched as to require re-binding before they are any time in use.-Dub. Journ. of Med. Sci., March, 1874.

Dr.Gross's Surgery, a great work, has become still greater, both in size and merit, in its most recent form. I'he difference in actual number of pages is not more than 130 , but. the size of the page having been increased to what we believe is technically termed *ele. phant." there has been room for considerableadditions, which, torether with the alterations, are improveinents.Lond. Lancet, Nov. 16, 1872.

It combines, as perfectly as possible. the qualities of a text-book and work of reference. We think this last Med. Journ., Feb. 1873.

As a whole, we regard the work as the representative "System of Surgery" in the English language.-St. Louis Medical and Surg. Journ., Oct. 1872.

The two magnificent volumes before us afford a very complete view of the surgical knowledge of the day. Some years ago we had the pleasure of presenting the first edition of Gross's Surgery to the profession as a work of unrivalled excellence; and now we have the result of years of experience, labor, and study, all con. densed upon the great work before us. And to stullents or practitioners desirous of euriching theirlihrary with a treasure of reference, we can simply commend the nurchase of these two volumes of inmense research Cincinnati Lancet and O'sserver, Sept. 1872.

A completesystem of surgery-not a mere text-book of operations. but a scientifie aceount of surgical theory and practice in all its departments.-Brit. and For. Med Chir. Rev., Jan. 1873.

B T THE SAME AUTHOR.

A PRACTICAI, TREATISE ON THE DISEASES, INJURIES, and Malformations of the Urinary Bladder, the Prostate Gland, and the Urethra. Third Edition, thoroughly Revised and Condensed, by SAMUel W. Gross, M.D., Surgeon to the Philadelphia Hospital. In one handsome octavo volume of 574 pages, with 170 illus. trations: eloth, $\$ 450$. (Just Issued.)

For reference and general information, the physician lenses of the urinary organs.-Atlanta Med. Journ., Oct. orsurgeon can find nowork that meets their necessities 1876 .

more thoroughly than this, a reviked edition of an excellent treatise, and no medical library should be without it. Replete with havdsome illustrations and good ideas, it has the unusual advantage of being easily comprehended, by the reasonable and practical manuer in which the various subjects are syotematized and arranged We heartily recommend it to the professicn a a valuable addition to the important literature of dis-

It is with pleasure we now again take up this old work in a decidedly new dress. Indeed, it must be refarded as a new book in very many of its parts. The chapters on "Diseases of the Bladder," "Prostate Body," and "Lithotomy," are splendid specimens of descriptive writing; while the chapter on "Stricture" is one of the most concise and clear that we have erer read.-New York Med. Journ., Nov.1876.

\section{$B^{Y}$ THE SAME AUTHOR.}

A PRACTICAL TREATISE ON FOREIGN BODIES IN THE AIR-PASSAGES. In 1 vol. 8vo., with illustrations, pp. 468, cloth, $\$ 275$.

$D^{R}$ RUITT (ROBERT), M.R.C.S., \&c.

THE PRINCIPLES AND PRACTICE OF MODERN SURGERY. A new and revised American, from the eighth enlarged and improved London edition. Illus trated with four hundred and thirty-two wood engravings. In one very handsome octaro volume, of nearly 700 large and closely printed pages, cloth, $\$ 400$; leather, $\$ 500$.

All that the surgical student or practitionercould practice of surgery are treated, and so clearly and desire.-Dublin Quarterly Journal.

It is a most admirable book. We do not know when we have examined one with more pleasure. Bositon Med. and Surg. Journal.

In Mr. Druitt's book, though containingonly $80 \mathrm{me}$ seven hundred pages, both the principles and the perspicuonsly, as to elucidateeveryimportant topic. We nave examined thebook mostthoroughly, and can say that this success is well merited. His book moreover, possesses the inestimable advantages of having the subjects perfectly well arranged ard classified and of belng writen in a style at once clear and succlnct. - Am. Jourral of Med. Sciences. 
A SHHURST (JOHN, Jr. ), M.D.,

THE PRINCIPLES AND PRACTICE OF SURGERY. Second edition, enlarged and revised. In one very large and handsome octavo volume of over 1000 pages, with 542 illustrations. Cloth, \$6; leather, \$7. (Just Ready.)

Couscientiousness a ud thoronghness are two very Ashhurat's Surgery is too well known in this marked traits of character in the author of this country to require special commendation from hook. Ont of these truits largely has grown the This, jts second edition, enlurged and thoroughly success of his mental fruit in the past, and the pre- revised, brings it nearer onr idea of a model textsent offer scemsin nowise an exception to what has book than uny recently published treatise. Though gone before. The general arrangement of the vol. numerous additions have been made, the size of the ume is the sameas in the flrst edition, bat every part work is not materially increased. The main tronble bas been carefully revised, and much new matter of text-books of modern times is that they are too atded.-Phila. Med. Times, Feb. 1, 1 s79.

We have previously spoken of Dr. Ashburst's work in terms of praise. We wish to reiterate those terms here, and to add that no more satisfactory representation of modern snrgery lias yet fallen frow the press. In point of judicial fairuess, of power of condensation, of accuracy and conciseness of expression and thoroughly good Euglish, l'rof. Ashburst has no superior a inong the surgical writers in America.-Am. Practitioner, Jan. 1879.

The attempt to embrace iu a volnme of 1000 pages the whole field of surgery, general and special, would be a hopelens tank unless through the most tireless industry in collating and arranging, and the wisest judgment in condensing and excluding. These facilities have been abundantly employed by the author, and he has given us a most excellent treatise, brought up by the revisiou for the second edition to the latest drte. Of course this hook is not detigued for spocialists, but as a course of general surgical kuowledge and fur general practitioners, and is a text-book for students it is not surpassed by any that has yet appeared, whether of hume or foreign authorship. - N. Carolina Med. Journal, Jan. 1579 . cumbersome. The student needs a book which will furnish him the most information in the shortest time. In every respect this work of Ashburst is the model text-book - full, comprehensive and compact.-Nashville Jour of Med. and Surg., Jan.' 79.

The favorable reception of the first edition is a gnarantee of the popularity of this edition, which is fresh from the editor's hands with many enlsigements and improvements. The author of this work is deserredly popular as an editor and writer, and lis contributions to the literature of sargery bave gained for him wide reputation. The volume offered the profession will add new laurels to those already won by previous contributions. We can only add that the work is well arrang+d, flled with practical matter, and contains in brief and clear language all that is necessary to be learned by the studeat of surgery whilst in attendance upon lectures, or the general practitioner iu his daily routine practice.-Md. Med. Journal, Jan. 1579.

The fuct that this work has reached a second edition so very soon after the publication of the first one, speaks more lighly of its merits than anything we might say in the way of commendation. It seems to have immediately gained the faror of stu. deuts und physicians.-Cincin. Med. Neros, Jan. '79

BRYANT (THOMAS), F.R.C.S., Surgeon to Guy's Hospital.

THE PRACTICE OF SURGERY. Second American, fiom the Sec. ond and Revised English Edition. With Six Hundred and Seventy-two Engravings on Wood. In one large and very handsome imperial octavo volume of over 1000 large and closely printed pages. Cloth, \$6; leather, \$7. (Just Ready.)

This work has enjoyed the advantage of two thorough revisions at the hand of the author since the appearnnce of the first American edition, resulting in a very notable enlargement of size and improvement of matter. In England this has led to the division of the work into two rolumes, which are here comprised in one, the size being increased to a large imperial octavo, printed on a condensed but clear type. The series of illustrations has undergone a like revision, and will be found correspondingly improved.

The marked success of the work on both sides of the Atlantic shows that the author has succeeded in the effort to give to student and practitioner a sound and trustworthy guide in the practice of surgery; while the simultaneous appearance of the present edition in England and in this country affords to the American reader the benefit of the most recent advances made abroad in surgical science.

There are so many text-books of surgery, so many written by skilled and distinguished hands, that to ob. tain the honor of a third edition in England is no light praise. Mr. Bryant mexits this, by clearness of style, and good judgment in selecting the operations he re. commends, in his new editions he goes carefully over the old grounds, in light of later research. On these and many allied points, Mr. Bryant is a culm and unpartisan observer, and his book throughout has the great merit of muintuining the true scientific, judicial tone of mind. - Med. and Surg. Reporter, Mareh 22 , 1579.

The work before us is the American reprint of the last London edition, and has the advantage over the latter in being of more convenient size. and in being compressed into one volume. The author has rewrit. teu the greater part of the work, and has succeeded, in the amount of new matter added, in making it markedly distinctive from previous edi:ions. A few extra pages have been added, and also $u$ few new illustrations introduced. The publishers liave presented the work in a creditable style. As a concise and practicul manual of British surgery it is perhaps without an equal, rnd will doubtless ulways be a favorite text-book with the student und practitioner. $-N$. Y. Med. Record, March $2: 2$, isi 9 .

Another edition of this manual haring been callel for, the author has avriled himself of the oppurtunity to make no fow alterations in the substituce ar we 1 as in the arrangement of the work, and, will a view to its improvement, has reeast the materialo and re. vised the whole. We ourselves are of the opiniun that there is no better work on surgery extant Cin.cinnati Med. Nezos, Mareh, ls79

Bryant's Surgery has been favorably received from the first, and evideutly grows in the esteem of the profession with each succeeding edition. In glancing over the volume before us we find prouf in almox every chapter of the thorough revision which the work has undergone, many parts baving been cut out and replaced by matler entirely freoh. $-N$. $Y$. Med. . ourn., A pril, 1879.

Welcome as the new edition is, and as ruch as it is entitled to commendation, yet its appearance at this time is, in a certain sense, a matter of regret, as it will be in competition with another work, lately issued from the sume press. But, the difficalt task of forming a judgment as to the relative merits of Bryant, and Ashuurst we will not attempt, but predict that, considering the ligh excellence of buth, many others will likewise be rorced to hesitate long in making choice between them. - Cincinnati Lancet and Clinic, March 22, 1579. 


\section{ERICHSEN (JOHN E.),}

Professor of Surgery in Oniversity College, London, etc.

THE SCIENCE AND ART OF SURGERY ; being a Treatise on Surgical Injuries, Diseases, and Operations. Carefully revised by the antbor from the Seventh and enlarged English Edition. Illustrated by eight hundred and sixty two engravings on wood. Ir two large and beautiful octavo volumes of nearly 2000 pages: cloth, $\$ 850$; leather, $\$ 1050$ (Now Ready.)

In revising this standard work the a uthor has spared no pains to render it worthy of a continuance of the very marked favor which it has so long enjoyed, by bringing it thoroughly on a level with the advance in the science and art of surgery made since the appearance of the last edition. To accomplish this bas required the addition of about two hundred pages of text, while the illustrutions have undergone a marked improvement. A hundred and fifty additional wond-cuts have been inserted, while about fifty other new ones bave been substituted for figures which were not deemed sntisfactory. In its enlarged and inproved form it is therefore presented with the confident anticipation that it will maintain its position in the front runk of text-books for the student, and of works of reference for the practitioner, while its exceedingly moderate price places it within the reach of ull.

The seveuth edition is before the world as the last wurd ot aurgical ecieace. There may be mouographs which excel It "lpon certain points, but as a conspectus upun eurgical principles and practice it is narivalled. It will well reward practitiouers to read it, for it bas been a peculiar province of $\mathbf{M r}$. Erichsen to demonstrate the absolute interdependence of medical and surgical science We need sedrcely add, in conclusion, that wo heartily com. mend the work to students that they may be grounded in a sound faith, and to practitioners as an invaluable guide at the bedside.-Am Practitioner, A pril, 1878 .

It is ao ille compliment to say that this is the best edition Mr. Erichsen has ever produced of his wellknowu book. Besides iuheriting the virtues of i's predecessors, it possesses excelleuces quite its own. Having stated that Mr. Erichsen hs incorporated into this edition every recent improvement in the scieuce and art of surgery, it would be a supererogation to give a detailed criticism. In short, we unhesitatiugly arer that we know of no other single work where the student and practitioner can gain at oncesoclear an insight iuto the principles of surgery, and so complete a knowledge of the exigencies of surgical pructice.-Londun Lancet, Feb. It, $187 \mathrm{~s}$

For the past twenty years Erichsen's Surgery has maintained its place as the leadingtext-book, notonly in this country, but iu Great Britain. That it is able to hold its ground, is abundancly proven by the thoroughueos with which the present edition has begn revised, and by the large amount of valuable material that has been added. Asidefrom this, cne hundred und fifty new illnstrations have been inserted, including quite a number of microscopical appearances of puthil, gical processes. So marked is this change for the better, that the work almost appears as an entirely new oue.-Med. Record, Feb. 23, 1578.

Of the many treatises on Suryery which it has been our task to study, or our pleasure to read, there is none which in ull points has satisfied us so well as the classic treatise of Erichsen. His polished, clear style, his frecdom from prejudice and hobbies, his unsurpansed grasp of his subject, and vast clinical experience. qualify him admirably to write a model texi-book. When we wish, at the least cost of time, to learn the most of a topic in surgery, we turn, by preference, to his work. It is a pleasure, therefore, to see that the appreciation of it is general, and has led to the appenrance of anoiher edition.-Med. and Surg. Reporter, Feb. 2, 1978.

Notwithstanding the increase in size, we observe that much old matter has been omitted. The entire work has been thoroughly written $u p$, and not merely amended by a few extra chapters A great improvement has been made in the iliustrations. One hundred and fifty new ones huve been added, and many of the old ones have been redrawn. The author highly appreciates the favor wilh which his work has been received by A merican surgeons, and has endeavored to render bis latest edition more than ever worthy of their approval. That be has succeeded ad cnirably, must, we think, be the general opinion. We heartily recommend the book to both student and practitioner. $-\Lambda . Y$. Med. Journal, Feb. 1878 .

Erichsen has stood 80 prominently forward for years as a writer on surgery, that his reputation is world wide; and his name is as familiar to the medical student as to the accomplished and experienced surgeon. The work is not a reprint of furmer edi tions, but has in many places been entirely rewritteu. Recent improvenents in surgery have not escaped his notice, various new operations have been thoroughly analyzed, aud thelr merits thoroughly discussed. One huudred and fifty new wood-cuts add to the valne of this work. $-N$. U. Med. and Surg. Journal, Mareh, 1878.

\section{HOLMES (TIMOTHY), M.D.,}

SURGERY, ITS PRINCIPLES AND PRACTICE. In one handsome octavo volume of nearly 1000 pages, with 411 illustrations. Cloth, $\$ 6$; leather, $\$ 7$. (Just Issued.)

This is a work which bas been looked for on both its force and distinctness.-N. Y. Med. Record, April sicies of the Atluntic with much interest. Mr. Holmes $14,1876$.

is a surgeon of large and varied experience, and one of the best known, and perhaps the most brilliant writer upon surgical subjects in England. It is a book for students-and au admirable one-and for the busygeneral practitioner. It will give a student all the knowledge needed to pass a rigid examination. The book fuirl y justifies the high expectatlons that were formed of it. It s st yle is clear a ud forcible, even brilliant at times, and the conciseness needed to bring it withln its properlimits has notimpaired

It will be found a most excellent epitome of surgery by the general practitioner who has not the time to giveattention to more minute and extended works and to the medical student. In fact, we know of no one we can more cordially recommend. The anthor has sueceeded well in giving a plain and practical acconnt of each surgical injury and dis. ease, and of the treatment which is most com. monly advisable. It will no doubt become a popularwork in the profession, and especially as a textbook.-Cincinnati Med. New8, April, 1576.

ASHTON ON THE DISEASES, INJURIES, ANM MAI- I SARGENT ON BANDAGING AND OTHER OPERAFORMATIONS OF THE KECTUM AND ANUS: with remarks on Habitual Conetipation. Second A merican, from the fourth and enlarged London Editlon. With illustrations. In one 8vo, vol. of 287 pages, eloth, $\$ 25$. 
HAMILTON (FRANK H.), M.D.

Professor of Fractures and Dislocitions, in Bellevie Hosp. Med. College, New York.

A PRACTICAL TREATISE ON FRACTURES ANI) DISLOCATIONS. Fifth edition, revised and improved. In onelarge and handsome octavovolume of nearly 800 pages, with 344 illustrations. Cloth, $\$ 575$ : leather, $\$ 675$. (Lately Issued.)

This work is well known, abrnad as well as at home, as the highest authority on its important subject- $n$ authority recognized in the courts as well as in the schools and in practice-and again unifested, not only by the demand for a fifth edition, but by arrangements now in pro. gress for the speedy appearance of a translation in Germany. The repeated revisions which the a uthor has thus lad the opportunity of making have enabled him to give the most careful consid. eration to every portion of the volume, and he has sedulously endeavored in the present issue, to perfect the work by the aid of his own enlarged experience, and to incorporate in it whatever of value has been added in this department since the issue of the fourth edition. It will therefore be found considerably improved in matter, while the most careful attention has been paid to the typographical execution, and the volume is presented to the profession in the confident hope that it will more thun maintain its very distinguished reputation.

There is no better work on the subject in existence of its teachings, but also by reason of the medicn-legal than that of Dr. Hamilton. It should be in the posses- bearings of the cases of which it treats, and which have ion of every Am. Jnurn. of Obstetrics. Feb 1876.

The value of a work like this to the practical $\mu$ hysician and sur reon can hardly be over-estimuted. and the necessity of havine such a bonk reriged to the latest dates, unt merulynacenunt of the practicalinportance ton and others, is sufficiently obrious to every one. The present volume seems to amply fill all the requisites. We can safely recommend it as the best of its kind in the English language, and not excelled in any ntherJourn. of Nervous and Mental Disease, Jan.1876.

\section{$B^{R O W N E}(E D G A R A$.$) ,$}

Surgeon to the Liverponl Eye and Ear Infirmary, and th the Dispensary for Skin Disfass8.

HOW TO USE THE OPHTHALMOSCOPE. Being Elementary In. structions in 0phthalmoscopy, arranged for the Use of Students. With thirty-five illustra. tions. In one small volume royal $12 \mathrm{mo}$. of 120 pages : cloth, $\$ 1$. (Now Ready.)

This capital little work should be in the hands of could scarcely fail of understanding them. Equally ev ry medical student, and we had almostsuid every satisfactory are the directions for the use of tle in. general practitioner. Its explanation of the optical strument and the suggestions to aid in interpretiug principles on which the ophthalmoscope is founded, what is seen.-Detroit Med. Journ., Nov. 1877. is so clear and simple that the most stupid reader

CARTER (R. BRUDENELL), F.R.C.S., Ophthalmic Surgeon to St. George's Hospital, ,tc.

A PRACTICAL TREATISE ON DISEASES OF THE EYE. Elited, with test-types and Additions, by JoHN GREEN, M.D. (of St. Louis, Mo.). In one handsome octavo volume of about 500 pages, and 124 illustrations. Cloth, $\$ 375$. (Just Issued.)

It would be difficult for $\mathbf{M r}$. Carter to write an unin- manner, easy of comprehension, and hence the more structive book, and impossible for him to write au un- valuable. We would especially comnend, however, as interesting one. Even on subjects with which he is not worthy of high praise, the nanner iu which the therabound to be familiar, becan discourse with a rare degree peutics of disease of the eye is elaborated, for here the of clearness and effect. Our readers will therefore not author is particularly clear and practical, where other be surprised tc learn that a work by him on the Diseases writers are unfortunately too often deficient. The finul ot the Eve makes a very valuable addition to ophthal- chapter is devoted to a discussion of the useasnd selece. mic literature. . . . The book will remain one useful tion of spectacles, and is almirably compact, plain, und alike te the general and the special pructitioner.-Lon- useful, especially the paragraphs on the treatment of don Lancet, Oct. 30,1875 .

It is with great pleasure that we can endorse the work as a most valuable contribution to practical ophthalmology. Mr. Carter never deviates from the end he has in view, and presents the subject in a clear and concist presbyopia and myopia. In conclusion, our thanks are due the author for many useful hints in the great subject of ophthalmic surpery and therapeutics, a field where of late years we glean but a few grains of sound wheat from a mass of chaff - New York Medical kecord, Oct. 23,1875 .

WELLS ( J. SOELBERG), Professor of Ophthalmology in King's College Hospital, dc.

A TREATISE ON DISEASES OF THE EYE. Third American, from the Fourth and Revised London Edition, with additions; illustrated with numerous engravings on wood, and six colored plates. Together with selections from the Test-types of Jaeger and Snellen. In one large and very handsome octavo volume. (Preparing.)

LAUR FNCE (JOHN Z.), F.R.C.S.,

Editor of the Ophthalmic Review, \&c

A HANDY-BOOK OF OPHTHALMIC SURGERY, for the use of Practitioners. Second Edition, revised and enlarged. With numerous illustrations. In one very handsome octavo volume, cloth, \$2 75 .

LAWSON (GEORGE), F.R.C.S.Engl.,

Assistant Surgeon to the Rnyal London Ophthalmic Hospital, Moorfelds, \&c.

INJURIES OF THE EYE, ORBIT, AND EYELIDS : their Immediate and Remote Effects. With about one hundred illustrations. In ine very hand. some octavo volume, cloth, \$3 50 . 
URNETT (CHARLES H.), M.A, M.D., Aursl Surg to the Presb. Hosp., Surgeon-in-charge of the Infir for Dis. of the Ear, Phila.

THE EAR, ITS ANATOMY, PHYSIOLOGY, ANI DISEASE. A Practical Treatise for the Use of Medical Students and Practitioners. In one hand. sonie ootavo volume of 615 pages, with eighty-seven illustrutions : cloth, $\$ 450$; leather, $\$ 5$ 50. (Just Really.)

Recent progress in the inrestigation of the structures of the ear, and advances made in the modes of treating its diseases, would seem to render desirable a new work in which all the resources of the most advanced science should be placed at the disposal of the practitioner. This it has been the aim of Dr. Burnett to accomplish, and the advantages which he has enjoyed in the special study of the subject are a guarantee that the result of his labors till prove of service to the profession at large, as well as to the specialist in this denartment.

Foremost among the numerous recent contribu. ti, ns to aural literature will be ranked this work of Dr. Burnett. It is impossible to do justice to this volume of over 600 pages in a necessarily brief notice. It must suffice to add hat the boob is profasely and accurately illustrated, the references are conscientiously ucknowledged, while the result has been to produce a treatise which will henceforth rank with the clussic writing 8 of Wilde and Von Trólsch. - The Lond. Practitioner, Mнy, 1879

On account of the great advauces which have been made of late years in otology, and of the increased intfrest manifexted in it, the medical profession will welcome this new work, which preseuts clearly aud concisely its present aspect. whilst clearly indicating the direction in which further researches cau be must profitably carried on. Dr. Burn-tt from his own matured experience, and availing himself of the obserratious and discoveries of others, has prodaced a work, which as a text-book, stunds fucile princeps in cur langnage. We had marked several parsages as well worthy of quotation and the rttention of the general practitioner, but their number and the space at our commund torbid. Perhaps it is better, as the book ought to be in the hands of every

medical studeut, and its study will well repay the busy practitioner in the pleasule he will derire from the agreeable style in which many utherwise dry and mostly nnknown subjects are treated. To the specialist the work is of the highest value, and his seuse of gratitude to Dr. Burnect will, we hope, be proportionate to the amount of benefit he can obtuin from the careful study of the book, and a constaut reference to its trustworthy pages.-Edinbu gh Med. Jour., Ang. 1s7s.

The book is designed especially for the $u$ se of $t \mathrm{tu}$. dents and general practitioners, and places at their disposal much valnable material. Such a book ais the present one, we think, hrslong been needed, and we may congratulate the author un his success in fllling the gap. Both scudent and practitinner cau study the work with a great deal of benefit. It is profucely and berutifully illustrated. $-N$. Y. Hos. pital. Gazette, Oct. 15, 1877.

Dr. Burnett is to be commended for baving written the best book on the subject in the English language, and expecially for the care and attentiun he lins given to the xcientific side of the subject. -1 . 1 . Med. Journ., Dec. 1877.

\section{TAYLOR (ALFRED S.), M.D.,} Lecturer on Med. Jurisp. and Chemistry in Guy's Hospttal.

POISONS IN RELA'TION TO MEDICAL JURISPRUDENCE AND MEDICINE. Third Anerican, from the Third and Revised English Edition. In one large octaro volume of 850 pages; cloth, $\$ 550$; leather, $\$ 650$. (Just Issued.)

The present is based upon the two previous edi., being described which give rise to legal tuvestigations; "bntthe completerevisiun rendered necessmry tions. - Tne Clinic, Nuv. 6, 187.5.

by time has couverted it into on new work." This Dr. Taylor has bruught to bear on the compilatiou statement from the preface contains all that it is de- of this volume, stures of learning, experience, aud sired to kuow in reference to the new edition. The practical acqualitance with hissubject, probubly far workg of this author are already in the library of beyond what any other liviug rutliority ou toxiculevery phyeician who is liable to be called upon for ogy could have amassed or utilized. He bas fully medieu-legal testimony (and wh t inets not?), sothat sustained his reputation by the consummate skill all that is required to be know a ubut the present and legal acumen he has displayed in the arrange. book is that the athor bas kept it abreast with the ment of the subject-matter, and the renult is a work times. What makes it now, as always, especially ou Poisous which will beindispensable to every biavaluable to the practitioner is its couciseness and dentor practitioneriu law aud medicine.-The Dub. plactical character, only those puisonons substances lin Journ. if Med Sci., Uct. $157 j$.

\section{$B^{Y}$ TIIE SAME AUTHOR.}

\section{MEDICAL JURISPRUDENCE. Seventh American Edition. Edited} by Jonn J. Reese, M.D., Prcf. of .Med. Jurisp. in the Univ. of Penn. In one large octsvo volume of nearly 900 pages. Cloth, $\$ 510$; leather, $\$ 600$. (Lately Issued.)

To the members of the legal and medical profes. best ruthority on this specialty inourlanguage. Un sion, it is unaecersary to say anythiug commenda. this point, however, we will say that wecunsider $b_{1}$. tory of Trylur's Medical Jurisprudeuce. We might Taylor to be the safent uedico-legal authority tufol: us well undertske to speak of the nerit of Chitty's low, ingeneral, with which we are acquainted in any Pleadiligs. - Chieago Legal Neus, Oct. 16, 1s73. linguage.-Va Olin. Record, Nov. 1 s73.

It is beyond question the most attractive as well Thislastedition of the Manualisplobably the best as most reliable inanual of medical jurisprudence of ali, as it contain mure muterial aud is worked up published in the English language.-Am. Journal to the Iatest visws of the author asexpressed in the of Syphilngraphy, Oct. 1873.

last edition of the Principles. Dr. Retee, the editur

It is altogether superfluousfor us to offer anything of the slanual, has dune everything to make his in behalf of a work on medicaljurisprudence by a w wrk acceptable to his medicalcountrymen. $-N . Y$. author whoinalmost universally esteemed tobethe Med. Kecurd, Jan. 15, 1574.

\section{$B^{Y}$ THE SAME AUTHOR.}

\section{THE PRINCIPLES AND PRACTICE OF MEDICAL JURISPRU.} DENCE. Second Edition, Revised, with numerous Illustrations. In two large octavo volumes, cloth, $\$ 1000$; leather, $\$ 1200$

This great work is now recognized in England as the fullest and most authoritative treatise on every department of its important subject. In laying it, in its improred form, before the $A$ werican profession, the publisher trusts that it will assume the same position in this country. 
THOMPSON (SIR HENRY),

Surgeon and Professor of Clinical Surgery to University College Hospital.

LECTURES ON DISEASES OF THE URINARY ORGANS. With illustrations on wood. Second American from the Third English Edition. In one neat octavo volume. Cloth, $\$ 225$. (Just Lssued.)

$B^{Y}$ THE SAME AUTHOR.

UN THE PATHOLOGY AND TREATMENT OF STRIC'TURE OF

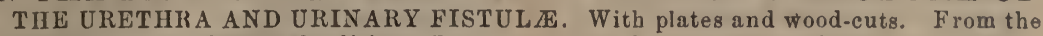
third and revised English edition. In one very handsome octavo volume, cloth, \$3 50. (Lately Published.)

ROBERTS (WILLIAM), M.D.,

12 Lecturer on Medicine in the Manchester School of Medicine, etc.

A PRACTICAL TREATISE ON URINARY AND RENAL DIS. EASES, including Urinary Deposits. Illustrated by numerous cases and engravings. Second American, from the Second Revised and Enlarged London Edition. In one large and handsome octavo volume of 616 pages, with a colored plate; cloth, $\$ 450$. (Lalely Published.)

TUKE (DANIEL HACK), M.D, roint author of "The Manual of Psychological Mledicine," \&c.

ILIUSTRATIONS OF THE INFLUENCE OF THE MIND UPON TIIE BODY IN HEALTH AND DISEASE. Designed to illustrate the Action of the Imagination. In one handsome octaro volume of 416 pages, cloth, $\$ 325$. (Lately Issued.)

BLANDFORD (G. FIELDING), M.D., F.R.C.P., Lecturer on Psychological Melicine at the Schonl of St. George's Hospital, \&c.

INSANITY AND ITS TREATMENT: Lectures on the Treatment, Medical and Legal, of Insane Patients. With a Summary of the Laws in force in the United States on the Confinement of the Insane. By IsAAC RAY, M. D. In one very handsome octavo volume of 471 pages; cloth, $\$ 325$.

It satisfies a wat which must have been sorely actually seen in practice and the appropriate treat felt by the busy general practitioners of this country. ment for them, we tind in Dr. Blandford'r work a It takes the form of a inual of clinicaldescription considerable adranceover previous writings on the of the various forms of insanity, with a description subject. His pictures of the varions forms of mental of the mode of examining persons snspected of in- disease are so clear and good that no reader can fail sanity. We call particularatention to this feature to be struck with their superiority to those given in of the book, as giving it a unique value to the gene- udinary manuals in the English language or (so $f 8 r$ ral practitioner. If we pass froin theoretical conside. as our own reading extends)inany otber. - London rations to descriptions of the varieties of insanity a. Practitioner, Feb. 1871.

LEA (HENRY C.).

SUPERSTITION AND FORCE: ESSAYS ON THE WAGER OF LAW. THE WAGER OF BATTLE, THE ORDEAL, AND TORTURE. Third Revised and Enlarged Edition. In one handsome royal $12 \mathrm{mo}$. volume of $552 \mathrm{pages}$. Cloth, $\$ 250$. (Just Reurly.)

The appearance of a new edition of Mr. Henry C. polemic. Though he obriously feels and thinks Lea's "superstition and Force" is a sign that our strongly, he succeeds in attaining impartiality. highest schular: hip is not without hunor in its ud- Whet er looked on as a picture or a mirror, y work tive coutry. Mr. Lea has met every fresh demand such as this has a lastiag value.-Lipyincott's for his work with a caleful revision of it, and the Magazine, Oct. 1s7s. present ecition is nut only fuller and, if possible, more accurate than either of the preceding. but, froin the thorough elaborition is more like a harmonions concert and less like a batch of studies.The Nation, Aug. 1, 1s7s.

Many will bo tempted to say that this, like the "Decliueand Fall,"isune of the uncriticizable books Its factsare in numerable, its deductions simple aud iuevitable, and its chevaux-de-frise of references bristling and dense enongh to make the keenest, stoutest, and best equipped assailant think twice before udvancing. Nor is there unything controversialin it to provuke assault. The author is no

Mr. Len's curious historical monographs, of which one (f the most important is here reproduced in an enlarged furm, have given him an unique positiun among English and Anerican Echolars. He is dis: tinguished for his recondite and afluent learning, his power of exhaustive historical analysis, the breadth and accuracy of his resedrches among the rarer sonrces of knowledge, the gravity and temperunce of his statements, combined with singular earnestness of conviction, and his warm attachneut to the cause of human freedoin and intellectual progress.-N. Y. Tribune, Aug. 9, 1s78.

$B^{Y \text { THE SAME AUTHOR. (Late y Published.) }}$

STUDIES IN CHURCIIISTORY-THE RISE OF THE TEM. PORAL POWER-BENEFIT OF CLERGY-EXCOMMUNICATION. In one large royal $12 \mathrm{mo}$. volume of $516 \mathrm{pp}$; ; oloth. $\$ 275$.

The story wa never told more calmly or with las a peculiarimportancefor the English 8 tudent, and gr vater learning or wiser thought. Wedunbt, indeed, is a chapter on Ancient Law likely to be regarded as it uny other study of this field can be cumpared with inal. We can hardly pass from our mention of such this for clearnes, uccucy, "Sacerdotal this for clearness, tccuracy, and power. - Chicago
Examiner, Dec. 1870 .

Mir. Lea's latest work, "Studiesin Church History," fally sustaius the prumise uf the first. It deal- with American honses is also the writer of some of its most three subjects- the Tein noral Power. Bejefit of origiual books. -London Athenaum, Jan. 7, 1871.

Clergy, and Excommunication, the record of which 


\section{N D EX TO CATALOGUE.}

American Jourual of the Medical Sciences A bstract, Montbly, of the Med. Sciencer. Allen's Antomy

Anatomical ptlus, by Sinith and Horner. Ashron on the hectum and Anue Attield's Chemistry

Ashwell on Diseases of Females Ashburst's surgery

Browae on () phithalmoscope Browue ou the Thruat

Burnett on the Ear.

Barnes on Direases of Wumen

Barnes' Midwifery

Bellamy's surgical A nutomy

Bryant s Practical Surgêry

Bloxanis Chemistry

blandford on Iusanity

Bdsham on Renal Diseases .

Brinton on the Siomach

Barlow's Pructice ol Medicine

Bowman's (John E.) Practical Chemistry.

Bowman's (John E.) Medical Chemistry.

Bristowe's Practice

birmstead on Venereal

B $11 \mathrm{mstead}$ and Cullerier's Atlas of Venereal Carpenter's Human Physiology

Cirpenter on the UBe and Abuse of Alcohol Cornil and Ranvier

Carter on the Eye.

Cleland's Diskector

Classen's Chemistry

Clowes' Chemistry

Century of Anerican Medicine

Chadwick on Diseases of Wumeu

Charcot on the Nervous System

Chrmbers on Diet and Regimen.

Chambers's Restorative Medicine

Christison and $\mathrm{Grifith}$ 's Uispensatory

Churchill's System of Midwifery

Churchill on ruerperal Fever

Condie on Diseases of Children.

Cooper's (B. B.) Lectares on Surgery

C.sllerier's Atlas of Venereal Dixeases

Cyclopadia of Practical Medicine

Dalton's Human Physiology

Davis's Clinical Lectures

Newees on Diseares of Females.

Druitt's ModernSurgery

Dunglison's Medical Dietionary

Ellis's Demonstratious in Aratomy

Erichsen's System of Snrgery

Eminet ou Diseases of Womeu

Farquharson's 'Therapeutics

Fe nwick's Diagnosis

Finlayson's Cliuicul Diagnosis

Flint on Respiratory Urgans

Flint on the Hesrt

Flint's Prilctice of Medicine.

Flint's Essays

Flint's Clinical Medicine

Flint on Phthisis

Flint on Percussion

Fothergill's Huadbook of Treatment

Fothergill's antagonism of Therapeutic Agents

F,wnes's Elementary Chemistry

Fox on Diserses of the Skin

Fuller on the Lungs. \&c.

Green's Pathology and Morbid Anatomy : Gibson's Surgery

Glage's Pathological Histology, by Leidy. Gray's Anatomy

Galloway's Analysis

Griffth's (R.E.) Univereal Formilary

Gross on Urinary Organs

Gross on Foreign Bedies in Air-Passages

Gross's Prlnciples and Practice of Surgery

Habershon on the Abdumen.

Hataliton on Dislocations and Fractures

Bartshorne's Essentials of Medicine

Hartshorne's Conspectno of the Medical Sciences

Hartahorne $\theta^{\circ} \Delta$ atomy and Physiology

Hamilton on Nervous Diseases

Herth's Practical Anatomy

Hublyn's Medical Dictionary
PACB

llodge on Women.

PAAR

3 tudge's Obsterric.

7 Iolland's Medical Notes and Reflectione. . 14

Holmes's surgery.

28 Holden's Landmarks

10 Iorner's Anatomy and Histology : $:$ :

3 Hadson on torer.

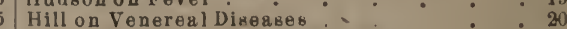

29 Hillier's Handbook of Skin Diseases : : 20

19 Jones (C. Handtield) on Nervous Disorders . 19

30 Kirkes' Physiulogy

22 Knapp's Chemical Technology .

24 Lea's Superstition and Force

7 Lea s Studies in Church History

27 Lee on Syphilis

11 Lincolu on Electro-Therapentics

31 Lisishman's Midwifery

19 La Rocheon Yellow Eever.

19 La Ruche on Pneumonia, \&c.

Lanrence and Moon's Ophthalmic Sargery

Lawson on the Eye

9 Lehmann's Physiological Chemistry, 2 vols. ., \&

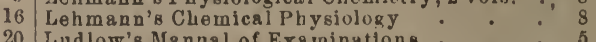

20 Ludlow's Manual of Exaininations : • • 5

20 Lyons on Fever . . . . . . . 19

8 Medical News and Library. . . . . 2

13 Meigs on Puerperal Fever : $\quad$ : $\quad$ : 22

14 Miller's Practice of Surgery . . . . 25

29 Miller's Principles of Surgery . . • , 25

7 Montgomery on Pregnancy : : . 21

9 Neill and Smith's Compendium of Med. Sclence 5

11 Obstetrical Journal • • • • . 24

5 Parry on Extra.Uterine Preguancy . . . 25

23 Pavjon Digestion . . . . . 19

18 Pavy on Food . . . . $\quad 19$

19 Parrish's Practical Pharmuey . . . . 12

19 Pirrie's System of Surgery - • • • • : 25

1t Playfair's Midwifery . . . . . 24

21 Quain and Sharpey's Anatony, by Leidy . . 7

21 Reynolds' Practice of I'edicine. . . I7

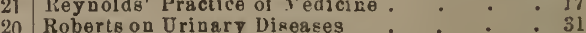

- 20 Robertson Urinary Diseases $\quad \cdot \quad \cdot \quad \cdot \quad \cdot 31$

19 Remsen's Principles of Chemistry

14 Rigby's Midwifery $\quad 0^{-} \cdot 21$

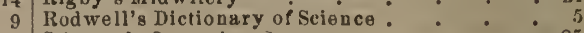

14 Stimson's Operative Sargery $\quad$ • . . 25

21 Swayne's Obstetric A phorisms : • • : 21

Seiler on the Throat

26 Seiler on the Throat $4:{ }_{4} \quad \cdot \quad \cdot \quad \cdot \quad 19$

Sharpey and Quain's Anatomy, by Leidy . . 7

2S Skey's Operative Surgery

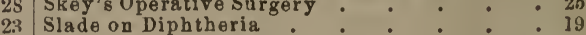

11 Schäfer's Histology $\quad: \quad \cdot \quad \cdot \quad \cdot \quad \cdot 7$

It Smith (J. L.) on Cliildren . • . . 21

1s Smith (H. H.) and Horner's Anatomical Atlas . 7

19 Smith (Edward) on Consumption . . . 19

19 Smith on Wasting Diseases in Children : . 20

15 Stille's 'Therapeutics . . . 19

15 Stillé \& Maisch's Dispensatory : $\quad \cdot \quad \cdot \quad \cdot 13$

15 Starges on Clinicál Medicine : • • : : 1

19 Stokes on Fever . . . . 14

19 Tanner's Manual of Clinical Medicine : :

16 Tanner on Pregnancy. : * : 24

16 Taylor's Medical Jurispradence $\bullet \quad: \quad * 30$

10 Taylor's Prínciples and Practice of Med Jurisp 30

17 Taylor on Poisons
19 Tuke on the Influence of the Mind $\quad \cdot \quad \cdot \quad \cdot 31$

14 Thomas on Diseases of Females. $::^{*}:{ }^{-} 22$

25 Thompson on Urinary Organg . . . . . 3]

- 14. Thompson on Stricture. $\quad \cdot \quad \cdot \quad \cdot \quad \cdot \quad \cdot \quad \cdot 31$

6 Todd on Acnte Diserses : $\quad$ : : : : 11

Woodbury's Practice . : . . . : 16

12 Walshe on the Heart :
26 Watson's Practice of Physic $\quad: \quad \cdot \quad \cdot \quad \cdot \quad \cdot 19$

26 Wells on the Eye. $\quad . \quad \div \quad: \quad \cdot 29$

26 West on Diseases of Females . • . . 20

$\begin{aligned} & 16 \\ & 29 \text { West on Diseases of Children }\end{aligned} \quad: \quad 20$

15 What to Observe in Medical Cases • : : It

Williams on Consumption..$\quad \cdot \quad 19$

Wilson' HamanAnatorny: : $: 7$

Wilson' Handbook of Cutaneous Medicine : 17

Wohler's Organic Chemlstry . - . :

Wlackel on Childbed . . : . : 23 




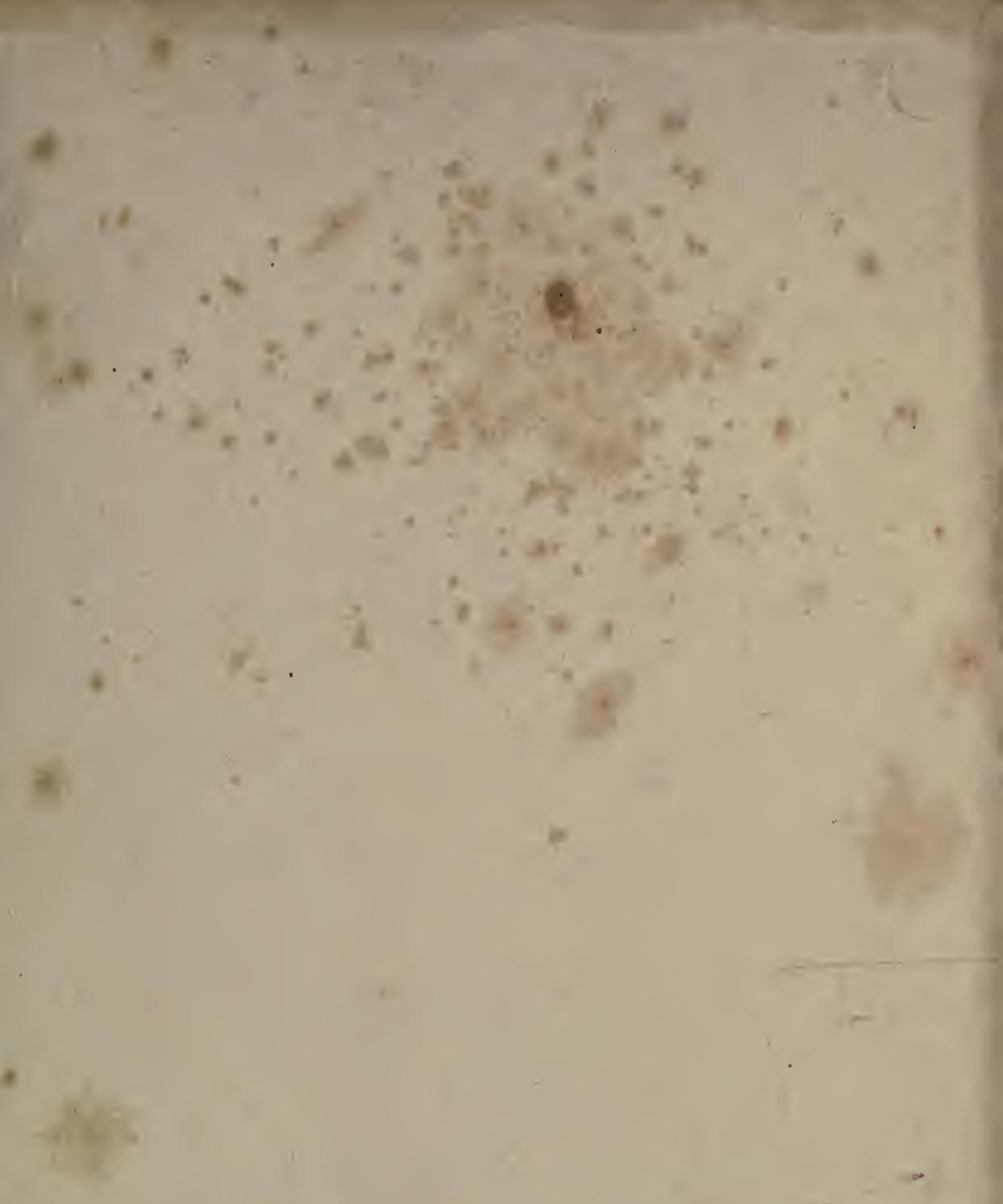



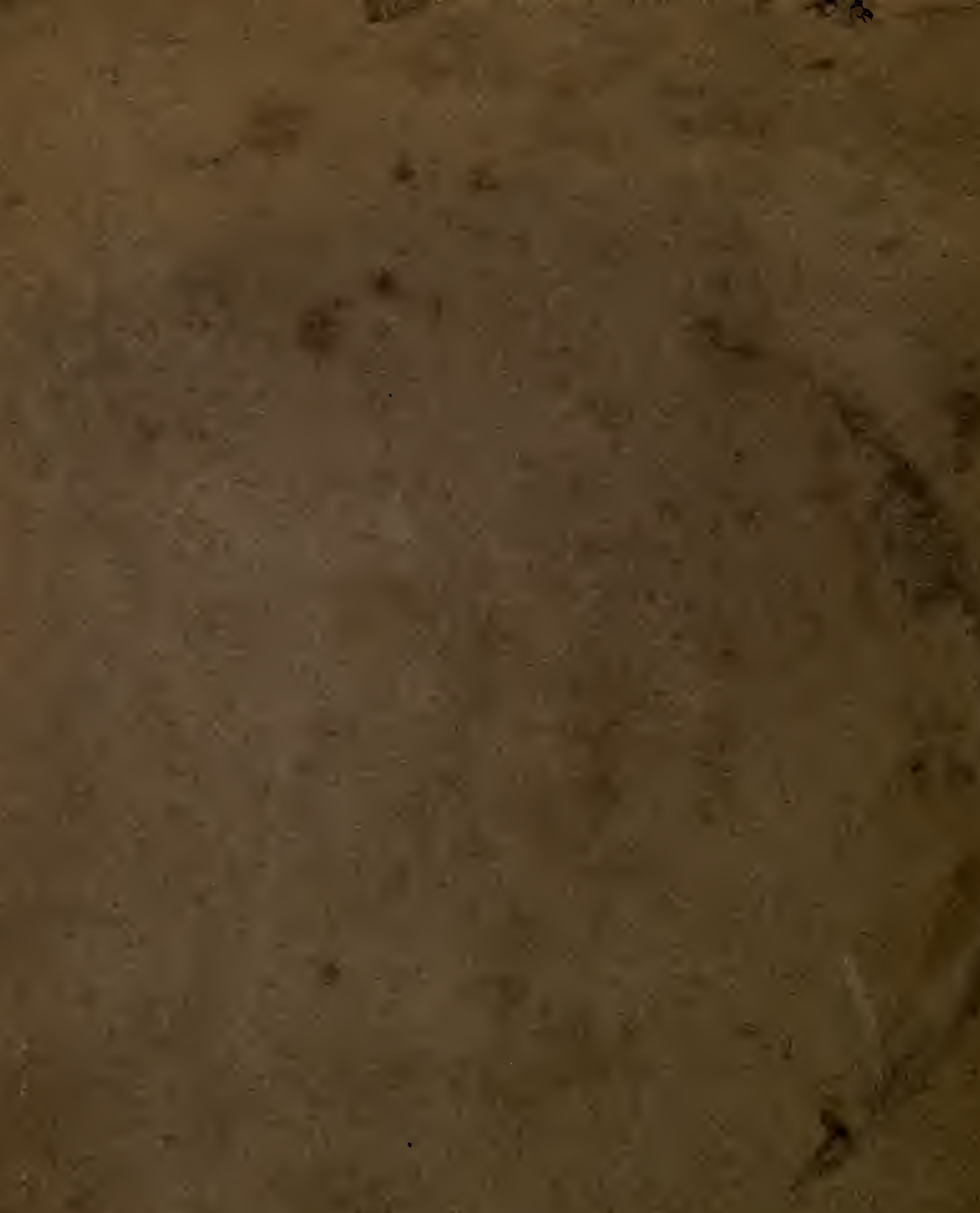